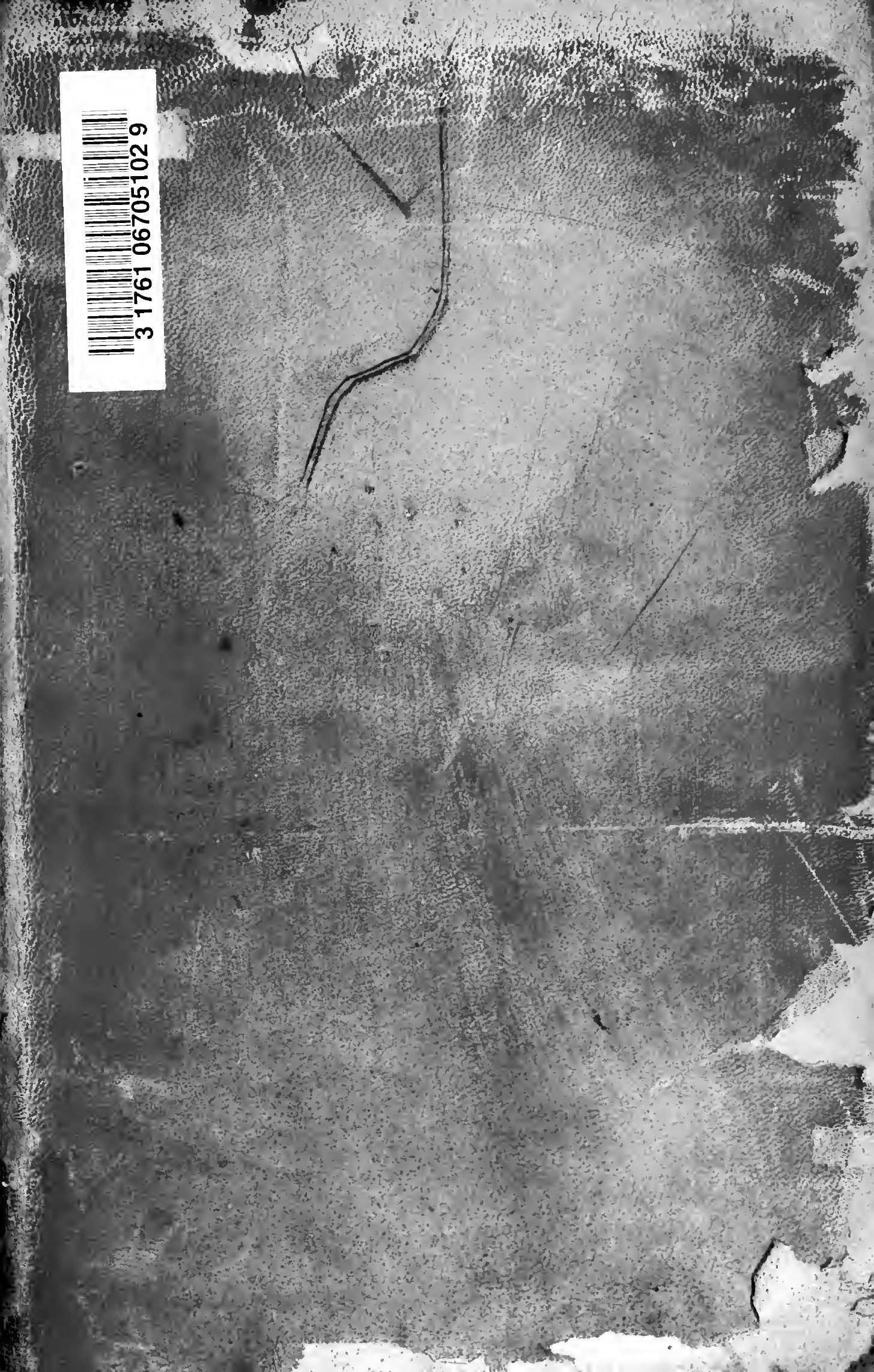




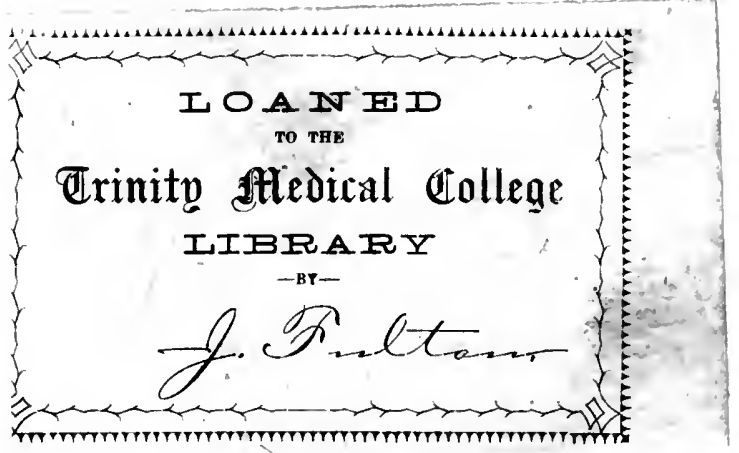


Digitized by the Internet Archive in 2007 with funding from Microsoft Corporation 




\section{A TREATISE}

ON THE

\section{PRINCIPLES AND PRACTICE \\ or}

\section{E D I C I N E;}

DESIGNED FOR TIIE USE OF

PRACTITIONERS AND STUDENTS OF MEDICINE.

BY

A USTIN FLINT, M.D.,

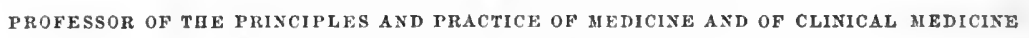
IN THE BELLEVUE HOSPITAL MEDICAL COLLEGE.

FOURTH EDITION, CAREFULLY REVISED.
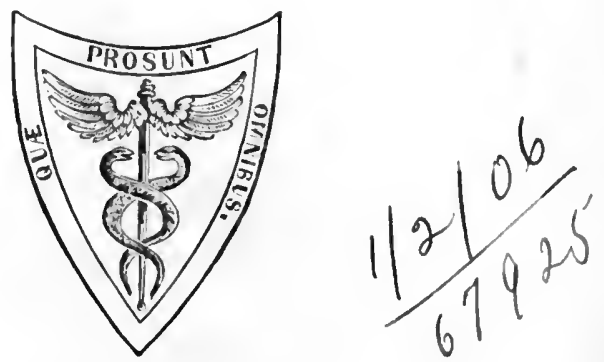

PHILA DELPH I A :

H $\mathrm{E} \mathrm{N} R \mathrm{Y}$ C. L E A. 
Entered according to the Act of Congress, in the year 1873, by H E N R Y C. L E A,

in the Office of the Librarian of Congress. All rights reserved. 


\section{PREFACE TO THE FOURTH EDITION.}

Since the publication of the Third Edition of this work, in October, 1868, the advances made in many departments of practical medicine have seemed to call for a thorough revision. In the performance of this duty the Author has sought to introduce the results of his continued clinical studies, together with the latest contributions to medical literature, both in Europe and this country, without changing the character of the volume as a condensed text-book and work of reference. This has required the rewriting of some portions, as well as numerous additions throughout, but more especially in the Section devoted to Diseases of the Nervous System, causing an increase in the text of about seventy. pages.

To accomplish this has required no little labor, and in return the Author hopes for the satisfaction derivable from a continuance of the favor with which the work has hitherto been received by the profession.

NEW YoRK, April, 1873. 


\section{PREFACE TO THE FIRST EDITION.}

THE object of this work is to present such a digest of the Principles and Practice of Medicine as will be serviceable alike to the pupil in the prosecution of his studies of disease, and to the physician engaged in the practical duties of his profession. The plan, at the outset, was to fulfil this object within the compass of one good-sized volume. In carrying out this plan, condensation has been necessary. As much conciseness as is consistent with clearness has been studied. Very little space will be found to be occupied with past opinions or doctrines which have become obsolete. Discussions relating to mooted pathological questions are rarely entered into. Illustrative cases have been introduced with reserve. As regards scope, the work embraces the subjects which generally enter into didactic teaching from the chair of the Principles and Practice of Medicine in the medical colleges of this country. Subjects belonging to other departments of instruction are, for the most part, omitted; hence, for information on matters relating to surgery, obstetrics, the diseases of women and children, cutaneous diseases, and the details of the Materia Medica, the reader is referred to other works. Finally, in writing the volume, the study has been to keep prominently in mind the practical applications of medical knowledge to diagnosis, prophylaxis, and therapeutical indications.

In submitting the work to the judgment of his fellow-teachers and practitioners, the author ventures to hope that it may be found to represent fairly the existing state of the science of Medicine with respect to the subjects of which it treats, and to reflect the views of those who exemplify, in their practice, the present stage of the progress of medical art. 


\section{O N T ENTS.}

\section{INTRODUCTORY CHA PTER.}

Scope of the term Medicine-Use of the term in contradistinction to Surgery and Obstetrics-Subdivisions of the different departments of medicine, or specialties - The general object of this work-Meaning of the phrase Principles and Practice of Medicine-Definition of Pathology-Division into General and Special Pathology-Nomenclature of Diseases-Subdivisions of General Pathology, viz., Morbid anatomy, and morbid changes of the fluids of the body; Etiology; Symptomatology; Diagnosis; Prognosis; Prophylaxis; and Therapeutics-Relations of these subdivisions to Special, as well as General, Pathology-Definition of Disease-Definition of Health-Relationship of Prthology to PhysiologyProgress of Pathological Knowledge . . . . . . . .

\section{P A R T I.}

\section{PRINCIPLES OF MEDICINE, OR GENERAL PATIIOLOGY.}

\section{H A P T E R I.}

\section{ANATOMICAL CHANGES IN THE SOLID PARTS OF THE BODY.}

Congenital Malformations-Three classes of Lesions, viz., Lesions of Qunntity, of Consistence, and of Composition-Hypertrophy and.Atrophy, Lesions of Quantity - Hypertrophy-True and False Hypertrophy-Atrophy-Atrophy with Increase and with Diminution of Volume-Lesions of Consistence-IndurationSoftening-Inflammatory and Non-Inflammatory Softening

\section{H A P T E R II.}

ANATOMICAL CHANGES IN THE SOLID PARTS OF THE BODY-(CONTINUED.)

Lesions of Composition-Exadations and Transudations-Fibrinous Exndation or Coagulable Lymph-Tubercle-Scrofula-Carcinoma or Cancer-Typhoid Deposit 


\section{H A P T E R I I.}

\section{ANATOMICAL CHANGES IN THE SOLID PARTS OF THE BODY-(CoNCLUdED.)}

Lesions of Composition, Continued-Transudation-Dropsies-Fluxes-Degenerations-Fatty Degeneration-Fatty Growth-Calcareous Degeneration-Lardaceous or Waxy Degeneration-New Formations or Morbid Growths-Pneumatoses -Animal and Vegetable Parasites

C H A P T E I V.

\section{MOBBID CONDITIONS OF THE BLOOD.}

General Remarks-Classification of the Constituents of the Blood-Morbid Conditions relating to the Organized or Corpuscular Elements-Plethora-AnæmiaApnca-Leucocythemia

\section{H A P T E R V.}

\section{MORBID CONDITIONS OF THE BLOOD-(ContinUEd.)}

Morbid Conditions relating to Fibrin, Hyperinosis, Hypinosis-Coagulated Fibrin within the cavities of the Heart-Coagulated Fibrin within the BloodvesselsEmbolism-Thrombosis-Morbid Conditions relating to Albumen-Dropsy as dependent on Diminution of Albumen-Morbid Conditions relating to Water in the blood-Hydræmia

\section{H A P T R VI.}

\section{MORBID CONDITIONS OF THE BLOOD-(CoNTINUED.)}

Morbid Conditions relating to Fat-Morbid Conditions relating to Sugar-Diabetes Mellitus-Morbid Accumulation of Excrementitious Principles in the BloodUræmia-Excess of Uric Acid in Gout, Uricæmia-Retention of Excrementitious Principles contained in the Vapor of Expiration-Retention of Excrementitious Principles contained in the Perspiration-Reabsorption of Bile, or CholæmiaCholesteræmia-Morbid Conditions relating to the Saline Constituents of the Blood

\section{H A P E R VII.}

\section{MORBID CONDITIONS OF THE BLOOD-(CONCLUDED.)}

Morbid Conditions due to the presence of morbid products, intrinsio and extrinsio -Pyæmia_Putrid infection of the blood, or Septicæmia-Matter of contagion -Virnses-Infectious Miasms-Venoms-Poisons, palpable, and impalpable or miasms-Zymosis-Considerations which lead to the inference of the prior existence of a constitutional or general morbid condition, or indeterminate bloodchanges, in various local affections 


\section{CH A P TER VIII}

\section{THE CAUSES OF DISEASE, OR ETIOLOGY.}

Etiology a branch of General and Special Pathology-Its Importance-Internal or Intrinsic and Extcrnal or Extrinsio Causes-Ordinary, and Special or Specifio Causes-Traumatic Causes-Spontaneous Diseases-Primnry and Secondary Causes-Complications and Intercurrent Diseases-Predisposing and Exciting Causes - Congenital and Inberited Predispositions to Disease-Co-operating Causes-Diathesis-Cacbexia or Dyscrasia-Endemic Diseases dependent on Emanations from the soil-Epidemio Diseases produced by Causes existing in the Atmosphere-The Causes of some Contagious Disenses exclusively derived from those affected with these Diseases, and other Diseases of this class produced, in some cases, by other Causes than Contagion or Infection-FomitesPortability of the special Causes of Diseases not Contagious nor InfectiousThe Causes of Contagious and Infectious Diseases probably Living Germs or Organisms

\section{H A P T E R IX.}

\section{SYMPTOMATOLOGY.}

Pathognomonic Symptoms-Diagnostic Symptoms-Subjective and Objective Symptoms-Signs-Clinical History of Diseases and the different Modes by which it is obtained-Stages of Disease-Precursory or Prodromic Events-SequelsDingnosis-Reasoning by way of Exclusion-Differential Diagnosis-PrognosisPrognostics . . . . . . . . . . • . .

\section{H A P T R X.}

\section{PROPHYLAXIS-GENERAL THERAPEUTICS.}

Experience the Basis of Thernpeutical Knowledge-Two Sources of Experience; Analysis of recorded Cases with reference to the Duration or Termination of Diseases and Observation of the Immediate effects of Therapentical MeasuresRational Inference as a means of arriving at Principles of Therapeutics-Importance of Knowing the Intrinsic Tendencies of Diseases toward Recovery or otberwise-Active Measures to be employed only when clearly indicated-The Expeotant Plan of Treatment-Conservative Medicine-Importance of Hygienic Measures of Treatment-Modes of Dying resolvable into Apncea and AstheniaClassification of Therapeutioal Measures, according to the Objects to be accomplished, into Prophylactic, Abortive, Curative, Palliative, Hygienic, and Sustaining Measures-Consideration of the Elementary Forms of Disease deferred . 


\section{PRACTICE OF MEDICINE, OR SPECIAL PATHOLOGF.}

\section{INTRODUCTION.}

Of the different aspects under which Individual Diseases are to be Considered, in treating of the Practice of Medicine, or Special Pathology-viz., Anatomical Characters, Clinical History, Pathological Character, Causation, Diagnosis, Prógnosis, Prevention, and Treatment-Sense of the Term Individual as applied to a Disease, and the several Grounds of Individuality-Varieties of a Disease-Definition of the Terms Acute, Subacute, and Chronic-The Symptoms or Events incidental to Disease sometimes, for convenience, considered as Individual Diseases -Self-limited Duration of Certain Diseases-Nosology-Nosological Arrangement adopted in this Work

\section{SECTION FIRST.}

\section{DISEASES AFFECTING THE RESPIRATORY SYSTEM.}

\section{CHA P T ER I.}

Division of the Diseases affecting the Respiratory System into Inflammatory and Non-inflammatory-Acute Pleuritis-Anatomical Characters-Clinical HistoryPathological Character-The Natural History of Inflammation, and our Knowledge of its essential nature-Causation of Pleuritis-Diagnosis-Prognosis

\section{CHAPTER II.}

Treatment of Acnte Pleuritis-Measures indicated in the First Stage-General Considerations relating to Bloodletting in Acute Inflammations $\rightarrow$ The use of Opium in Acute Pleuritis and other Acute Inflammations-Measures indicated in the Second and the Third Stage of Acute Pleuritis .

\section{H A P T E R III.}

\section{VARIETIES OF PLEURITIS.}

Chronic Plenritis-Anatomical Characters-Clinical History-Pathological Character-Causation-Prognosis-Treatment-Thoracentesis. Suppurative Pleuritis, or Empyæmia - Diagnosis-Prognosis-Treatment. Pleuritis with Pneumothorax - Anatomical Characters-Clinical History - Diagnosis - PrognosisTreatment. Pneumothorax. Circumscribed Pleuritis. Hydrothorax. • . 156 


\section{H A P E R I V.}

\section{PNEUMONITIS.}

Seat of the Inflammation. Varieties. Acute Lobular Pneumonitis-Anatomical Characters-Laws of the Disease-Clinical History-Pathological CharacterCausation-Diagnosis-Prognosis

\section{H A P T E R V.}

Treatment of Acute Lobular Pneumonitis. Pneumonitis in Young Children. Circumscribed Pneumonitis. Chronio Pneumonitis-Induration-Sclerosis and .Cirrhosis of the Lung. Pigment, or brown induration. Anthracosis. Siderosis. Lobular Paeumonitis-Atelectasis and Collapse of Pulmonary Lobules. Pleurodynia and Intercostal Neuralgia

\section{CHA P T R V I}

\section{BRONCHITIS.}

Acute Bronchitis affecting the Large Bronchial Tubes-Anatomical CharactersClinical History-Pathological Character-Causation-Diagnosis-PrognosisTreatment. Subacute Bronchitis. Acute Bronchitis in Young Children. Bronchitis affecting the small Bronchial Tubes. Epidemic Bronchitis. Bronchitis with Fibrinous Exudation. Circumscribed Brouchitis. Chronic Bronchitis .

\section{CH A P T R VII.}

Emphysema of the Lungs. Interlobular or Interstitial Emphysema. Subpleural Emphysema. Vesicular Emphysema or Dilatation of the Air-Cells-Anatomical Characters-Clinical History-Pathological Character-Causation-DiagnosisPrognosis-Treatment. Asthma-Clinical History-Pathological CharacterCausation-Diagnosis-Prognosis-Treatment. Pertussis, Whooping Cough .

\section{H A P E R VIII.}

Pulmonary Hemorrhage. Bronchorrhagia. Pneumorrhagia. Pulmonary Gangrene. Pulmonary Edema. Carcinoma within the Chest. Hydatids . . 246

\section{H A P T E R IX.}

Affections of the Larynx and Trachea. Points relating to the Anatomy and Physiology of the Larynx which are involved in the oonsideration of Diseases in this situation. Acnte Simple Laryngitis-Anatomical Characters-Clinical History-. Pathological Character-Causation-Diagnosis-Prognosis-Treatment. Subacute Laryngitis. Chronio Laryngitis. Laryngitis with Exudation of LymphClinical History-Patbological Character-Causation-Diagnosis-PrognosisTreatment. Edema of the Glottis. Spasm of the Glottis. Nerrous Aphonia. Morbid Growths 


\section{H A P T E R X. \\ PULMONARY TUBERCULOSIS.}

Definition and Scope of the Term Pulmonary Tuberculosis-Anatomical Characters

-Clinical History-Pathological Character-Causation . . . .

C H A P T E R XI.

PULMONARY TUBERCULOSIS-(CONCLUDED.)

Diagnosis-Prognosis-Treatment. Acute Pulmonary Tuberculosis

\section{SECTION SECOND.}

DISEASES AFFECTING THE CIRCULATORY SYSTEM.

\section{H A P T E R I.}

Infroductory Remarks. Pericarditis-Anatomical Characters-Clinical HistoryPathological Character-Causation-Diagnosis-Prognosis-Treatment. Chronic Pericarditis. Pneumo-Pericarditis . . . . . . . .

\section{H A P T E R II.}

Endocarditis-Anatomical Characters-Clinical History-Pathological CharacterCausation-Diagnosis-Prognosis-Treatment. Myocarditis . . .

\section{CH A P TER III. .}

VALVULAR LESIONS WITH ENLARGEMENT OF -THE HEART.

Anatomical Characters-Clinical History-Pathological Character-CausationDiagnosis-Prognosis-Treatment . . . . . . . . .

\section{CHA P T E I V.}

Enlargement of the Heart without Valvular Lesions. Hypertrophy. Dilatation. Atrophy of the Heart. Fatty Degeneration and Softening of the Heart. Rnpture of the Heart. Functional Disorder of the Heart. Enlargement of the Thyroid Body and Prominence of the Eyeballs associated with Functional Disorder of the Heart. Angina Pectoris. Thoracic Anenrism 


\section{SECTION THIRD. \\ DISEASES AFFECTING THE DIGESTIVE SYSTEM.}

\section{CH A P T E R I.}

Introductory Remarks. Sporadio Dysentery-Anatomical Characters-Clinical Ilistory-Pathological Character-Causation-Diagnosis-Prognosis-Treatment. Epidemic Dysentery. Chronio Dysentery. Inflammation of the Crecum. Perforation. Fecal Abscess. Painful Tumor near the Cæcum . . . . 366

\section{CHA P T E R II.}

Acute Enteritis-Anatomical Characters-Clinical History-Pathological Character-Causation-Diagnosis-Prognosis-Treatment. Subacute and Chronic Enteritis. Acute Gastritis-Anatomical Characters-Clinical History-Pathological Character-Causation-Diagnosis-Prognosis-Treatment. Subacute and Chronio Gastritis. Duodenitis. Pharyngitis. Parotiditis . . . .

\section{HA P TER III.}

\section{STRUCTURAL AFFECTIONS OF THE STOMACH.}

Induration. Softening. Gastrio Ulcer. Carcinoms of Stomach. Dilatation of

Stomach. Degeneration of the Gastrio Tubules

\section{CH A P TER IVे.}

Structural Affections of the Intestinal Canal involving Obstruction-InvaginationStrangulated Hernin within the Abdomen-Rotation or twisting of IntestineCompression and Stricture of Intestine. Obstruction from Impaction of Feces, Enterolithes, and Foreign Bodies. Functional Obstruction.

\section{CHA P T ER V.}

Functional Affections of the Stomach and Intestines-Dyspepsia-Polyphagia, Malacia or Pica, Polydipsia, Dipsomania. Gastrorrhagia . . . . . 431

\section{CHAPTER VI.}

FUNCTIONAL AFFECTIONS OF THE STOMACH AND INTESTINES-(CoNTINOED.)

Inanition. Gastrnlgin. Passage of Gall-Stones. Diarrbon. Enterorrhagia $\quad . \quad 450$ 


\section{CH A P T E R VII.}

FUNCTIONAL AFFECTIONS OF THE STOMACH AND INTESTINES-(CONTINUED.)

Constipation. Intestinal Colic. Enteralgia. Lead Colio . . . . . 469

\section{CHA P T E VIII.}

Sporadic Cholera-Clinical History-Pathological Character-Causation-Diagnoais-Prognosis-Treatment. Cholera Infantum. Epidemic Cholera-Anatomical Characters-Clinical History-Pathological Character-Causation-DiagnosisPrognosis-Prevention-Treatment

\section{H A P T E R IX.}

\section{INTESTINAL WORMS.}

Ascaris Lumbricoides. Ascaris or Oxyuris Vermicularis. Tricocephalus Dispar. Anchylostoma Duodenale. Ascaris Alata.'Tæniæ. Trichina Spiralis . . 612

\section{CHA P TER X.}

Acute General Peritonitis-Anatomical Characters-Clinical History-Pathological Character-Causation-Diagnosis-Prognosis-Treatment. Partial Peritonitis. Chronic Peritonitis. Hydro-Peritoneum . . . . . . . . 526

CHA P T R XI.

DISEASES AFFECTING THE SOLID VISCERA OF THE ABDOMEN.

Acute Diffuse- Hepatitis-Anatomical Characters-Clinical History-Pathological Character-Causation-Diagnosis-Prognosis-Treatment. Circumscribed Suppurative Hepatitis. Suppurative Portal Phlebitis. Cirrhosis of the Liver .

\section{H A P T R XII.}

\section{DISEASES AFFECTING THE SOLID VISCERA OF THE ABDOMEN-(CoNTINUED.)}

Fatty Liver. Waxy or Lardaceous Liver. Cancer of the Liver. Tuberculosis of the Liver. Acute. Atrophy of the Liver. Hydatid Tumors of the Liver. Pigmentary Deposit within the Liver. Hypertrophy of the Liver .

\section{CHA P TER XIII.}

\section{DISEASES AFFECTING THE SOLID VISCERA OF THE ABDOMEN-(CONCLUDED.)}

Diseases Affecting the Biliary Passages. Jaundice, or Icterns. Functional Affections of the Liver. Affections of the Spleen. Affections of the Pancreas . $\quad 579$ 


\section{SECTION FOURTH.}

\section{DISEASES AFFECTING THE NERVOUS SYSTEM.}

\section{H A P T E R I.}

Preliminary Remarks. Congestion of the Brain, Active and Passive. Cerebral Ansemia. A poplexy. Cerebral Hemorrhage and other Anatomical Conditions. Clinical History-Pathological Character-Causation-Diagnosis-PrognosisTreatment. Insolation, or Sunstroke. Congestion and Anæmia of the Spinal Cord. Hemorrhage within the Spinal Canal

\section{H A P T E R II.}

Pachymeningitis and Hematoma of the Dura Mater-Acute Cerebral MeningitisAnatomical Characters-Clinical History-Pathological Character-CausationDiagnosis-Prognosis-Treatment. Chronic Meningitis. Tuberculous Meningitis. Spinal Meningitis. Cerebro-Spinal Meningitis-Anatomical CharactersClinical History-Pathological Character-Causation-Diagnosis-PrognosisTreatment. Hydrocephalus. Hydrorachis . . . . . . .

\section{I A P T E R II.}

Cerebritis, and Abscess of the Brain. Structural Lesions within the Cranium. Induration or Sclerosis. Non-Inflammatory Softening of the Brain. Morbid Growths or Tumors within the Cranium. Psammona of the Brain. Hypertrophy and Atrophy of the Brain. Myelitis. Structural Lesions within the Spinal Canal

\section{CH A P T E R IV.}

Paralysis, General Remarks on. Paralysis of the Third Cranial Nerve. Paralysis of the Fourth Cranial Nerve. Paralysis of the Fifth Cranial Nerve. Paralysis of the Sixth Cranial Nerve. Paralysis of the Seventh Cranial Nerve. Paralysis of the Eighth Cranial Nerve. Paralysis of the Ninth Cranial Nerve. Local Paralysis of other than Cranial Nerves. Paralysis of the Cervical Sympathetic Nerve. Paralysis from Lead.

C H A P T E R V.

Hemiplegia. Paraplegia

\section{CH A P E R VI}

General Spinal Paralysis. Treatment of Paralysis. Glosso-labial Paralysis. General Cerebral Paralysis. Progressive Locomotor Ataxia. Prugressive Muscular Atrophy. Pseudo-hypertrophio, or myo-sclerotic paralysis. Scleroderma. Neuritis . 


\section{CH A P T E R VII.}

THE NEUROSES. NEURALGIA.

Trifacial Neuralgia. Cervico-Occipital Neuralgia. Cervico-Brachial Neuralgia.

Lumbo-Abdominal Neuralgia. Crural Neuralgia. Sciatio Neuralgia. Dermal-

gia. Myalgia. Cephalalgia. Vertigo . . • . . . . .

\section{H A P T E R VIII.}

Chorea. Anomalous Muscular Movements. Epilepsy

C H A P T E R IX.

Hysteria. Catalepsy. Eestasy. Somnambulism. Tetanus

C H A P T E R X.

Rabies. Delirium Tremens. Alcoholism. Nervous Asthenia. Hypochondriasis, Pathophobia

\section{SECTION FIFTH.}

\section{DISEASES AFFECTING THE GENITO-URINARY SYSTEM.}

\section{H A P T E R I.}

Suppurative Nephritis and Pyelitis-Anatomical Characters-Clinical HistorsPathological Character-Causation-Diagnosis-Prognosis-Treatment. Paroxysms of Pain caused by the Passage of Calculi along the Ureter, or Nephritio Colic, and Lithiasis or Gravel. Acute Desquamative Nephritis, Acute Albuminuria, Acute Bright's Disense, or Acute Inflammatory Dropsy-Anatomical Characters-Clinical History-Pathological Character-Causation-Diagnosis-Prognosis-Treatment

\section{CH A P T E I I.}

Degenerative Changes or Lesions of the Kidneys generally characterized by persistent Albuminuria, collectively termed Chronio Bright's Disease or Chronic Albuminuria-Anatomical Characters-Clinical History-Pathological Character-Causation-Diagnosis-Prognosis-Treatment

\section{CHA P TER III.}

Simple Renal Cysts. Hydatids in the Kidney. Renal Tuberculosis. Carcinoma of the Kidneys. Movable Kidney. Hæmaturia. Diabetes Mellitus-Anatomical Characters-Clinical History-Pathological Character-Causation-DiagnosisPrognosis-Treatment

\section{CHA PTER IV.}

Disease of the Supra-Renal Capsules_Addison's Disease. Involuntary Seminal Emissions, Spermatorrhœea. Inpotence 


\section{SECTION SIXTH. \\ FEVERS AND OTHER GENERAL DISEASES.}

\section{CHA P T E R I.}

Classification of Fevers. The General Pathology of Fever. Febricula. Typhoid Ferer-Anatomical Characters and Clinical History . . . . . . 861

\section{CH A P T E R II.}

Typhoid Fever Continued-Causation-Diagnosis-Prognosis. Typhns FeverAnatomical Characters-Clinical History-Causation-Diagnosis-Prognosis. Non-identity of Typhus and Typhoid Ferer . . . . . . .

\section{H A P T E R I I I}

Treatment of Typhus and Typhoid Fever. Relapsing Fever-Anatomical Characters-Clinical History-Causation-Diagnosis-Prognosis-Treatment. Erysipelatous Fever. Epidemic Fever characterized by mild Erythematic Pharyngitis

\section{CHA P T E R I V.}

Periodical Fevers. Intermittent Fever-Anatomical Characters-Clinical History -Causation-Diagnosis-Prognosis-Treatment. Pernicious Intermittent Ferer

\section{H A P T E R V}

Periodical Fevers, Conclnded. Simple Remittent and Typho-Malarial Fever-Anatomical Characters-Clinical History-Causation-Diagnosis-Prognosis-Treatment. Pernicious Remittent Fever. Yellow Fever-Anatomical CharactersClinical History-Causation-Diagnosis-Prognosis_Treatment . . . 933

\section{CHA P T E R V I.}

\section{ERUPTIVE FEVERS.}

Variola, or Smallpoz-Anatomical Characters-Clinical History-Causation-Diagnosis-Prognosis-Treatment. Varioloid, or Modified Smallpox. Vaccinia, or Cowpox. Varicella, or Chicken-pox.

CHA P TER VII.

\section{ERUPTIVE FEVERS-(CoNTINUED.)}

Scarlatina, or Scarlet Fever-Anatomical Characters-Clinical History-Causation -Diagnosis-Prognosis-Treatment 


\section{CH A P T ER VIII. ERUPTIVE FEVERS-(CoNCLCDED.)}

Rubeola, or Measles_Clinioal History-Causation-Diagnosis-Prognosis-Treatment. Roseola. Summary of the more important of the Differential Characters of Variola, Scarlatina, Rubeola, and Roseola. Dengue . . . . .

\section{H A P T E R IX.}

Diphtheria-Anatomical Characters-Clinical History-Pathological Character-

Causation-Diagnosis-Prognosis-Treatment . . . . . . . 1002

\section{CHA P T E R X.}

Acute Articular Rheumatism-Clinical History-Pathological Character-Causation-Diagnosis - Prognosis - Treatment. Subacute and Chronic Articular Rheumatism. Muscular Rheumatism . • • • . • . . 1015

\section{H A P E R XI.}

Gout-Anatomical Characters-Clinical History-Pathological Character-Causation-Diagnosis-Prognosis_Treatment. Rheumatic Gout . . . . 1034

\section{CHA P T E R XII.}

Scorbutus, or Scurvy-Anatomical Characters-Clinical History-Pathological Character-Causation-Diagnosis - Prognosis-Prevention - Treatment. Purpura Hemorrhagica. Hæmatophilia . . . . . . . . . 1050

INDEX 


\title{
PRINCIPLES AND PRACTICE
}

\author{
OF

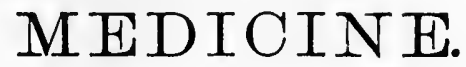

\section{N T ODUCTOR Y CHAPTER.}

\begin{abstract}
Scope of the term Medlcine-Use of the term in contradistinction to Surgery and ObstetricsSubdivislons of the different departments of medicine, or specialties-The general object of this work-Meaning of the phrase Principles and Practice of Medicine-Definition of Pathology-Division into General and Special Pathology-Nomenclature of Diseases-Subdivlsions of General Pathology, viz., Morbid anatomy, and inorbid changes of the fluids of the body ; Etiology ; Symptomatology ; Diagnosis; Prognosis ; Prophylaxis ; and TherapeuticsRelations of these subdivisions to Special, as well as General, Patlology-Definition of Dlsease-Defiultion of Health-Reiationship of Pathology to Physiology-Progress of Pathological Knowledge.
\end{abstract}

$\mathrm{M}$ EDICINE, in the largest sense of the term, comprehends everything pertaining to the knowledge and cure of disease. In a more restricted sense, the term is used in contradistinction to Surgery and Obstetrics. The latter are properly departments of medicine in the comprehensive sense of the term; and, although they may be cultivated separately, they cannot be disconnected from principles which are common to them and to medicine in its restrieted sense. The medical profession embraces all who devote themselves to the study and practice of medicine proper, surgery, and obstetrics, either separately or combined. The physician is a member of the profession who devotes his attention to the diseases which belong to the department of medicine proper, i.e., medicine in the restricted sense of the term. The physician may, or may not, undertake the duties which belong to surgery and obstetrics. In this country most physicians are, of necessity, to a greater or less extent, also surgeons and obstetricians, in other words, general practitioners. It is only in cities and large towns that practitioners can devote themselves exclusively, or chiefly, to surgery and obstetrics as separate departments of medicine. The distinction of physician, surgeon, and obstetrieian, in this country, is purely conventional. The only degree conferred by our universities and medical colleges is that of Doctor of Medicine, which authorizes the practice of either or all the departments, and the same is true of licenses to practise medicine.

The division of medicine into the three departments which have been named is natural, and has contributed to the knowledge acquired in each department. Subdivisions have also been found convenient and useful. The latter are commonly known as specialties, and they who devote themselves to particular subdivisions are called specialists. The more important of the subdivisions now recognized as specialties are affections of 
the eye and ear, diseases of the skin, syphilitic affections, diseases peculiar to females, orthopedic surgery, disorders of the mind and nervous system, and diseases of the chest. Specialties result from an increase of knowledge rendering it difficult or impossible for one mind to compass all that has been ascertained in either of the three departments of medicine. Doubtless the number of specialties will be greater as our knowledge continues to increase. The special cultivation of the several subdivisions of medicine leads to a further development of knowledge relating to each subdivision, and, hence, conduces to the progress of medicine. But, as the great principles of medicine are common to medicine proper, surgery, and obstetries, so with regard to the subdivisions, they cannot be completely isolated from the departments to which they respectively belong. A particular class of affections cannot be studied satisfactorily to the entire exclusion of others, and without reference to the general laws of disease. Directing the attention too exclusively to a specialty leads to the habit of attributing to it an undue relative prominence, and of regarding the affections belonging to it as of paramount importance when they may be secondary or merely incidental to others which, from being overlooked or not sufficiently appreciated, fail to receive appropriate treatment.

The object of this work is to present the outlines of Medicine proper, that is, of medicine in contradistinction to Surgery and Obstetrics. The Principles and Practice of Medicine is a title of this department considered as a province of medical teaching. This title is here adopted in preference to others, such as the Theory and Practice of Physic, General and Special Pathology, Pathology and Practical Medicine, or the Science and Practice of Medicine. The principles and practice of medicine comprehend everything pertaining directly to the knowledge and cure of those diseases which the physician is called upon to treat. The province of medical teaching thus designated properly enough embraces the prevention of disease, and it may include anything which concerns the conduct of the physician in the treatment of patients affected with disease.

The study of disease, as a province of scientific knowledge, is called Pathology. This province consists of two important divisions distinguished by the names General and Special Pathology. It is desirable to have a clear understanding of the terms which distinguish these two divisions of pathology. Diseases are presented in particular forms or species, constituting what are commonly known as individual diseases. The circumstances which give to the different diseases their individuality will be noticed hereafter. Now, the study of individual diseases constitutes special pathology. On the other hand, there are morbid conditions which are not peculiar to any individual disease, but are common to a greater or less number of diseases. The study of these conditions constitutes general pathology. Inflammation, for example, is a morbid condition which exists in a host of individual diseases. The study of inflammation, in so far as it is common' to different diseases, belongs to general pathology, while the study of the numerous individual inflammatory diseases belongs to special pathology. To take another illustration: a morbid condition which enters into a number of individual disenses is called fever. In this sense of the term fever, it belongs to general pathology ; but the study of the different forms of fever, or individual fevers, belongs to special pathology. The relation of general to special pathology is analogous to the relation of general to special anatomy, the former deseribing the several tissues which enter into the composition of the different organs of the body, and the latter describing the particular organs composed of the tissues. As the number of tissues is small in compari- 
son with the number of organs, so the morbid conditions belonging to general pathology are few as compared with the great number of diseases belonging to special pathology.

The province of medical teaching entitled the Principles and Practice of Medicine is made $u p$ of general and special pathology. 'The subjects which belong to the principles of medicine are derived from general pathology. The principles of medicine and general pathology are, in fact, synonymons terms, each term having the same scope of application. And, in like manner, the subjects which belong to the practice of medieine are derived from special pathology. These two terms relate to the same division of pathological knowledge, the former term being somewhat more comprehensive in its scope than the latter. The principles of medicine, thus, on the one hand, and the practice of medicine, on the other hand, constitute divisions which coincide with the two divisions of pathology distinguished as general and special. Moreover, these divisions are in accordance with the distinctions expressed by the terms Science and Art. "Science is knowledge reduced to principles; art is knowledge reduced to practice." The principles of medicine constitute medical science; the practice of medicine is the exereise of medical art. The object of this work being to present the outlines of both the principles and the practice of medicine, or of both general and special pathology, or, again, of medical science and art, it will be divided into two parts corresponding to these divisions. The outlines of the principles of medicine, or general pathology, will form the First Part of the work, and the Second Part will be devoted to the practice of medicine, or special pathology. In adopting this arrangement, however, I shall not be bound by it so closely as to treat of the topies belonging to general pathology exclusively in the first part of the work. It will be more convenient to defer the consideration of some of these topics, and to treat of them incidentally in connection with individual diseases. Moreover, I shall treat of general pathology in its relation to medicine, passing over, or noticing very briefly, those topies which are chiefly important in a surgical point of view. Although the general principles of pathology are common to both medicine and surgery, certain topies have relations especially to either the one or the other of these departments. The terms medical pathology and surgical pathology are used in conformity with this distinction.

The subjects of General Pathology, namely, the morbid conditions common to a greater or less number of individual diseases, are to be considered under various points of view; hence, this division of pathology admits of several subdivisions. One point of view relates to nomenclature, or the naming of diseases. The great desideratum in nomenclature, as applied to diseases, is, that the name of each disease shall express the morbid condition involved, and its situation. The names which were formerly applied to different forms of discase were frequently fanciful, and many of these are still in use, owing to the difficulty and inconvenience of displacing them after they have become established in medical literature; and in not a few instances it is by no means easy, with our existing knowlerlge of the essential character of morbid conditions, to substitute more appropriate names. Some approach, however, has been made toward a nomenclature which shall measurably secure the advantages derived from this source in other blanches of knowledge, more especially in chemistry. The existence of inflammation, which enters into so large a number of individual diseases, is expressed by the suffix

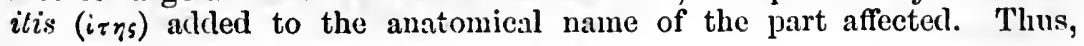


bronchitis, pneumonitis, pleuritis, peritonitis, etc. etc., are names denoting the inflammatory character of the diseases to which they refer, and the particular structure which is the seat of the inflammation. The suffix $\infty a(j \in \omega)$ denotes the existence of the morbid condition known as transudation, or flux, oceurring in a situation where the transuded liquid escapes upon a mucous surface. Examples are enterorrhœa, bronchorrhœa, gastrorrhœa, cystorrhœa, terms which have not, as yet, come sufficiently

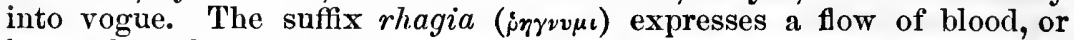
hemorrhage from a mucous surface. Examples are metrorrhagia, gastrorrhagia (hæmatemesis), enterorrhagia (melæna), bronchorrhagia (hæmoptysis), in like manner terms which have not displaced others in common

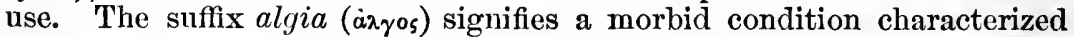
by pain without inflammation. Thus, neuralgia is a general term applied to this condition affecting any nerve or nerves; gastralgia, enteralgia, pleuralgia, etc., are terms severally expressing the neuralgic character

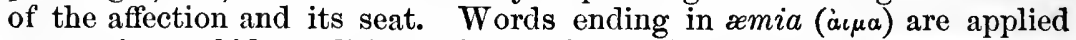
to certain morbid conditions of the blood. Examples are anæmia (impoverishment of the blood), uræmia (morbid accumulations of urea in the blood), septicæmia (putrid infection of the blood), and pyæmia (purulent infection of the blood). Words ending in uria (ovpov) are applied to certain morbid conditions of the urine. Examples are albuminuria,

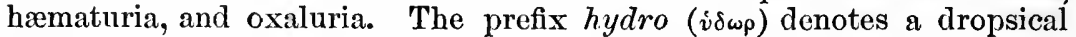
affection of the part named; as hydrothorax, hydrocephalus, hydro-peri-

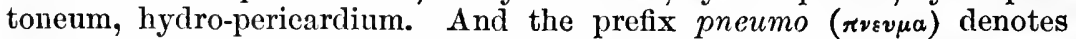
the presence of air in the part; as pneumothorax, pneumo-pericardium.

It is thus seen that, within late years, the effort to introdnce names expressive of the character and seat of morbid conditions has, in a measure, sueceeded. Further improvement in nomenclature will doubtless be made as our pathological knowledge increases.

An important subdivision of General Pathology relates to the appreciable morbid changes of the solids and fluids of the body. The study of all changes appreciable by the naked eye, or with the help of the microscope, constitutes a branch of pathology of great importance called morbid anatomy. Morbid anatomy is not confined to the study of the changes which occur in the tissues or solid parts; it embraces any changes which the eye can appreciate in the fluids of the body. The latter, not less than the former, are to be distinguished as anatomical, whereas those changes which are not visible either with the naked eye or by means of the microscope, but require for their detection processes of analysis or the employment of reagents, are usually considered as falling, not within the scope of morbid anatomy, but within the domain of animal chemistry. Prof. Charles Robin, however, considers the term anatomy to embrace investigation by means of analytical processes or reagents, as well as by means of dissection. Morbid changes not visible, or not yet ascertained with our present means of observation, are distinguished as functional, and are also said to be dynamic. Doubtless in all the socalled functional or dynamic deviations from health there are either molecular or cellular, that is, anatomical changes, at present inappreciable, or, at all events undiseovered, which may be hereafter ascertained by continued investigation with improved means of observation. Appreciable anatomical changes, which are either permanent or more or less persisting, are distinguished as lesions. The study of the minute anatomy of the tissues and fluids of the body with the microscope is called Histology, and the term Morbid or Pathological Histology is sometimes used to 
designate that part of morbid anatomy relating to those abnormal changes which are objects of microscopical research.

Anatomical changes, or lesions, belong to general pathology in so far as they are common to a greater or less number of individual diseases. Their consideration, as involved in individual diseases, enters into special pathology or the practice of medicine. Such is the extent, as well as importance, of morbid anatomy, that it constitutes a distinct branch of medical knowledge. Treatises are specially devoted to it, and these are to be studied, in conjunction with the examination of morbid specimens, in order to become fully and practically acquainted with the various changes in structure, form, size, etc., which are incident to disease. It is a fact, however, not to be lost sight of, that lesions do not constitute, but are the results of, disease. In other words, they are always due to underlying morbid actions or processes which may not be directly appreciable or well understood, but in which really consists the local affection. This fact, although obvious, is apt to be overlooked. Lesions are, of course, serious or otherwise, according to their character, their situation, and the amount of structural change involved. The plan of this work embraces, in Part First, the outlines of morbid anatomy in so far as it belongs to medical pathology, inclusive of known changes in the most important fluid of the body - the blood; and, in Part Second, a concise account of the lesions characterizing different individual diseases.

Another subdivision of General Pathology relates to the eansation of disease. The study of the causes of disease is called Etiology. As belonging to general pathology or the principles of medicine, this branch of medical knowledge will be considered in Part First of the work; and the causes involved in the production of each of the individual diseases will be embraced in the account of the latter in Part Second.

The great number and variety of phenomena or events to which disease gives rise constitute another subdivision of General Pathology. These phenomena or events are called symptoms, and their study constitutes a branch of medical knowledge called Symptomatology or Semeiology. Considerations relating to symptomatology, as belonging to general pathology, will claim attention in Part First; and the symptoms of individual diseases, respectively, forming, as they do, a highly important part of Special Pathology, or the practice of medicine, will be considered in Part Second.

Closely connected with symptomatology is another subdivision of General Pathology called Diagnosis. Diagnosis is the discrimination of diseases from each other. General considerations relating to this branch of medical knowledge will enter into Part First. In treating of individual diseases in Part Second, the means of discriminating them will be found to possess an importance second only to their treatment, and to be an essential prerequisite for the latter.

Another subdivision which will claim notice in both parts of this work is Prognosis, or the prediction of the termination of diseases.

The prevention of disease forms a branch of medical knowledge called Prophylaxis. This belongs alike to General and Special Pathology.

Lastly, the treatment of disease is called Therapeutics. General principles relating to the treatment of disease may be appropriately considered in connection with General Pathology. This portion of the subject is distinguished as General Therapeutics. I shall devote to it, together with prophylaxis, a chapter in Part First. It is hardly necessary to add that the treatment of individual diseases, distinguished as Special Therapeutics, is, in a practical view, the most important of the different aspects 
under which they are to be considered, being, in fact, the great end of both the principles and the practice of medicine.

It is thus seen that the foregoing subdivisions of General Pathology, which will be taken up successively in the first part of this work, represent also the different points of view under which individual diseases are to be considered in the second part of the work; that is, individual diseases, as well as the morbid conditions common to a greater or less number of diseases, are to be considered with reference to the morbid changes either of solids or fluids which they may respectively involve, together with their causes, their symptoms, their discrimination or diagnosis, their prognosis, their prevention, and their treatment. Morbid anatomy, etiology, symptomatology, diagnosis, prognosis, prophylaxis, considered as branches of medical knowledge, belong to General Pathology, and to these may be added general therapeutics; and, on the other hand, Special Pathology, or the practice of medicine, considers the truths contained in these subdivisions of general pathology in their application to individual diseases.

Pathology has been defined the study of disease; but disease has not yet been defined. The definition of disease is confessedly difficult. It is easier to define it by negation, to say what it is not, than to give a positive definition, that is, a definition based either on the nature or essence of the thing defined, or on its distinctive attributes. Disease is an absence or deficiency of health; but this is only to transfer the difficulty, for the question at once arises, how is health to be defined? And to define health is not less difficult than to define disease. If all the tissues and organs of the body have their normal integrity and properties, if the fluids of the body are in no respect abnormal, if all the functions of the organism are completely and harmoniously performed, health undoubtedly exists. But this perfection of health is purely ideal; it never actually exists. An examination of the bodies of the healthiest persons would probably reveal lesions of some kind; certain deviations from the normal composition of the different fluids are not inconsistent with the evidences of health in other respects; functions of different parts may be disordered to a certain extent without sufficient disturbance to constitute disease. Gradations of health are implied in the qualifications of this term in common use. If the term health expressed a well-defined state, it would be a pleonasm to add to the term, as is often done, the adjectives good, excellent, etc.; and, on the other hand, to speak of health as poor, bad, miserable, etc., would involve a solecism. In short, health and disease are so imperceptibly merged into each other, that the line of demarcation cannot be drawn with precision. And this is true of other departments of knowledge. It is not easy, for example, to settle upon the characters which mark the boundaries of the animal and the vegetable kingdom. But as there is rarely any practical embarrassment in distinguishing an animal from a vegetable, so with regard to health, if an important disease of any kind exist, the fact of its existence is in most cases sufficiently obvious. If, however, it be desirable to define disease otherwise than by saying that it is the absence or deficiency of health, the definition proposed by Chomel is, perhaps, as good as any other. According to this author, disease may be defined to be a notable disorder affecting more or less of the constituent parts of the living organism, as regards either their material constitution or the exercise of their functions.

1 "Un désordre notable survenu, soit dans la disposition matérielle des parties constituentes, du corps vivant, soit dans l'exercise des fonctions." For an enumeration of the varions definitions proposed by different writers. and some exeellent remarks on the subject, this author may be consulted: Elémens de Pathologie Générale, quatrième édition. 
By regarding disease as the absence or deficiency of health, we are led to the consideration of the relationship of Pathology to Physiology. Physiology studies the operations which go on in the healthy organism. The morbid conditions which are the subject of pathological study are these operations disordered or perverted. Pathology has been called morbid physiology. Both are, in fact, parts of one science, the science of life, or biology. Both are alike occupied with vital properties, actions, and processes, the difference being that physiology investigates them under the circumstances of health, and pathology under the circumstances of disease. The division is arbitrary, although sufficiently marked and appropriate.

Such being the relationship of pathology to physiology, it might be expected that the former would advance in proportion to the progress of the latter. This is measurably true. While our knowledge of pathological conditions does not consist of deductions from what is known of the operations within the organism in health, but is derived from the direct study of disease, every important physiological discovery sheds more or less light on the department of pathology. In striving to penetrate into the nature of morbid conditions, it is evident that the chief difficulty arises from the imperfection of our knowledge of the properties, actions, and processes of health. There will be frequent occasions, in the progress of this work, to remark that the pathologist may expect to be better able to explain the phenomena of disease, when the physiologist has succeeded in elucidating more fully the phenomena of health.

In proceeding now to present the outlines of medicine, the aim of the author will be to give a truthful representation of pathological knowledge as it exists at the present moment. The progress of pathological knowledge has wrought, within the few past years, much change in both the principles and practice of medicine. Concerning further progress and its effects, it would be in vain to speculate; but it is hardly to be expected that a faithful exposition of medicine as it exists at the present moment will serve as a lasting guide for the student and practitioner. And in the study of medicine, next in importance to an acquaintance with what is actually known, is a just appreciation of the limits of our present knowledge. The latter is often important as regards its bearing on the treatment of disease, and it conduces to a condition of mind most favorable for either contributing to, or keeping pace with, the continued progress of knowledge. 



\title{
P A R T I.
}

\section{PRINCIPLES OF MEDICINE, OR GENERAL PATHOLOGY.}

\author{
CH A P T E R I.
}

\section{ANATOMICAL CHANGES IN THE SOLID PARTS OF THE BODY.}

Congenital Malformations-Three classes of Lesions, viz., Lesions of Quantity, of Consistence,
and of Composition-Hypertrophy and Atrophy, Lesions of Quantity-Hypertrophy-True and
False Hypertrophy-Atrophy-Atrophy with Increase and with Diminution of Volume-Le-
sions of Consistence-Induration-Softening-Inflammatory and Non-inflanmatory Softening.

A MULTIPLICITY of anomalies, occurring during intra-uterine life, are embraced under the name congenital malformations. They consist of either the malposition, the absence, or the incompleteness of organs, deficiency or redundancy as regards bulk, deviations of form, supernumerary parts, want of the normal connection between parts, etc. They may be due to intrinsic defects in the ovum, to arrested or obstructed development from various extrinsic causes, and in some instances they arise from the diseases to which the fotus is liable. Passing by congenital malformations, this chapter will be devoted to certain definitions, distinctions, and general considerations relating to the changes in the solid parts, or lesions, which occur, as results of disease, after birth.

Anatomical changes in solid parts are recognized by means of morbid appearances, some of which are evident to the naked eye, and others require for their detection microscopical examination. The changes in color, form, etc., appreciable with the naked eye, constitute what are called the gross appearances of disease, and those discovered by the microscope are called the microscopical characters. Certain changes are determined by the touch; changes as regards softness or density, smoothness or roughness, alterations of form, etc. Increase and diminution in weight are also to be taken into account.

It is hardly necessary to add, but the fact is not always sufficiently considered, that the student must become familiar with the normal appearances (gross and microscopical) of the different structures and organs of the body before he is qualified to recognize and appreciate the alterations which proceed from disease.

A natomical changes, or lesions, may be arranged into three classes, as follows: 1st. Lesions of quantity; $2 \mathrm{~d}$. Lesions of consistence; and $3 \mathrm{~d}$. Lesions of composition. Lesions of quantity are of two kinds, viz. : first, a morbid increase, and, second, a morbid diminution of the substance or substances proper to the part affected. In the lesions which properly belong to this class the composition of the parts affected remains unchanged. The normal structure is unaffected ; the abnormal change is ptirely quantitative, not qualitative. A morbid increase of substance is called hyper- 


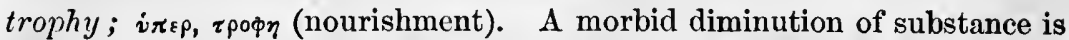
called atrophy, $\alpha, \tau$ гоф $\eta$. These two lesions of quantity claim separate consideration.

\section{Hypertrophy-Hyperplasia.}

The development of the different component parts of the body, the cessation of the growth of parts after an inherent principle of limitation, and the preservation of a definite size and form during the constant molecular changes incident to nutrition, are physiological mysteries which we cannot, with our present knowledge, undertake to explain. Hypertrophy involves an abnormal activity of nutrition, and an exaggeration of that principle or force which determines the normal size and form of the different parts of the body. The term hypertrophy is properly applied to enlargement from an increase of the normal materials of the parts affected, their structure remaining normal; in other words, the lesion consists in augmentation without any other change. Parts which become enlarged in consequence of a deposit of materials foreign to their normal composition, or from a disproportionate excess of certain of their normal constituents, or in which a structural change has taken place, are not, properly speaking, hypertrophied. For example, the liver augmented in size from fatty deposit, carcinoma, etc., is not an example of hypertrophy. The term hypertrophy has been applied to enlargement accompanied with change in composition or structure. True hypertrophy of a part proceeds from an excess of appropriation, by the part, of nutritive supplies, the process of nutrition being only increased, not perverted, whereas in false hypertrophy there is a morbid perversion of this process.

The enlargement of an organ, without the deposit of new material or structural change, does not always proceed from hyper-nutrition, and then it does not constitute hypertrophy. The hollow viscera-heart, stomach, bladder, etc.-may acquire more or less increase of volume from dilatation, the amount of substance being not greater, and perhaps less, than normal. Hence, it would not be a correct definition of hypertrophy to say that it consists in augmented volume without change in composition or structure,

True hypertrophy of a part generally proceeds from exaggerated and prolonged increase of the function of the part. Of the organs of the body, the heart is most frequently, perhaps, the seat of this lesion. And in the great majority of cases of hypertrophy of the heart, the enlargement is due to valvular lesions which, by interfering with the circulation of blood through this organ, induce a permanently increased power of its muscular action. The muscular walls, under these circumstances, increase in bulk by growth precisely as the voluntary muscles become enlarged when they are persistingly exercised. The heart may become lypertrophied to such an extent that it is five or six times heavier and larger than in health, yet there is a limit to its hypertrophic enlargement; that is, the increased growth does not go on indefinitely, but of necessity ceases after reaching a certain point of limitation, which point varies in different persons. Hypertrophy of the muscular tunic of the bladder in cases of long-continued urethral obstruction also illustrates the production of this lesion by prolonged increase of function.

It has been a much mooted question whether, in muscular hypertrophy, the increase of size is owing exclusively to enlargement of the primitive fibrillæe, or whether new fibrillæ are produced. Pathological authorities still differ in opinion on this point. It would seem to be probable, if not 
certain, that hypertrophy of the involuntary or unstriped muscular structure may involve the production of new fibres. Of this we have a physiological type in the development of the muscular walls of the uterus during gestation.

Hypertrophy of one of the kidneys sometimes ensues when the function of the other is either lost or greatly impaired. This is found to be the result of the removal of one of the kidneys in an inferior animal; undcr these circumstances the task of secreting the urine falls upon a single organ, its functional activity is doubled, and, as a result, the organ becomes enlarged. 'The enlargement in this case is abnormal, but, if it confer on the organ an increased capacity of secretion, it is a conservative lesion, that is, it conduces to the welfare of the body. This is certainly true of the examples of hypertrophy previously cited. Hypertrophie enlargement of the heart occurring in connection with valvular lesions is an important provision against evils which would otherwise ensue more quickly than they do. In this sense it is conservative. And this statement holds good with respect to hypertrophy of the muscular tunie of the bladder in cases of urethral obstruction. It is not an object of treatment in such instances to endeavor to remove or dimininish the hypertrophy so long as circumstances giving rise to it continue in operation.

The hyper-nutrition giving rise to hypertrophy does not always proceed from protracted, excessive functional exertion. In other than muscular and glandular structures, it may result from an undue determination of blood, from any cause, to the part affected. It may result from inflammation of a part. Hypertrophy of the fibrous and areolar tissues, perhaps, generally proceeds from inflammation. Hypertrophy of the adipose tissue, constituting corpulence, involves a general condition or diathesis, of which the undue growth of this tissue is a manifestation or local expression.

A distinction is made by Virchow between true or simple hypertrophy and enlargement due to multiplication of the anatomical elements of the affected parts. The latter kind of enlargement he distinguishes by the term hyperplasia. In hypertrophy, according to this distinction, there is simply swelling of structures from the addition of nutritive material. In hyperplasia new anatomical elements are generated, but without any other change than that relating to their number. 'Thus, a musele enlarged by the increase of the size of the primitive fibrillæ would be, in this sense of the term, hypertrophied; but the enlargement is hyperplastic if due to the production of new fibrillæ. This distinction is pathologically just.

\section{Atrophy.}

The term atrophy expresses a condition, as regards nutrition, the reverse of that expressed by the term hypertrophy. In hypertrophy the normal balance between the appropriation of nutritive material and destructive assimilation in the part or parts affected is disturbed by the former being in excess. In atrophy the destructive assimilation goes on, but the appropriation of fresh supplies is either suspended or diminished; hence, loss of the substance proper to the part ensues, and the struetures composing the affected parts undergo waste. Unless the space which was oecupied by the matter removed be filled with a morbid product of some kind, diminution of volume or bulk and of weight characterizes atrophy. Atrophy, however, may exist, not only without reduction, but with more or less increase, of size. For example, cirrhosis of the liver leads to atrophy of the proper tissue or parenchyma of this organ, and usually to diminu- 
tion of its volume; but in some cases, in conjunction with the atrophy due to the cirrhosis, there is a deposit of fat or some other material, the presence of which renders the organ as large as, or larger than, in health. Under these circumstances the organ is not less the seat of atrophy, although its size be not reduced, but even increased. Atrophy, thus, may exist with false hypertrophy. To cite another illustration, the heart is not unfrequently enlarged in consequence of the substitution of fat for the muscular substance contained within the myolemma, constituting what is called fatty degeneration. This substitution of fat involves atrophy of the muscular structure. Atrophy thus, it will be perceived, implies wasting of the tissue proper to the parts affected, either with or without a deposit of matter which alters the composition of the parts, and which, in proportion to its quantity, affects the volume.

Atrophy with diminution of volume is strikingly illustrated in that remarkable affection known as progressive muscular atrophy, in which different portions of the muscular system successively become greatly attenuated. It is illustrated in that variety of chronic disease of the kidneys in which these organs are greatly reduced in size. The liver much contracted from cirrhosis furnishes another striking illustration. Atrophy of membranous parts is illustrated by cases in which the valves of the heart become extremely thin and delicate, so that rupture is liable to occur. 'The walls of hollow viscera, the heart, stomach, etc., may be rendered abnormally thin by atrophy, either with or without increased volume by dilatation. Atrophy affecting the greater part of the body, and more especially the adipose tissue, constitutes the emaciation occurring in pulmonary tuberculosis and other chronic affections. This general atrophy is expressed by the terms phthisis, consumption, and marasmus. Progressive atrophy of most of the organs of the body, excepting the heart, is incident to old age, and is distinguished as senile atrophy.

Of the circumstances which give rise to atrophy, one is functional disuse of the part or parts affected. Atrophy induced in this way is seen in the wasted condition of muscles which have remained for a long time quiescent in cases of paralysis and chronic rheumatism. The other tissues, and even the bones, in these cases, become atrophied. Diminished supply of arterial blood is another causative circumstance. The ligature of an important distributing artery may produce wasting of parts which are thus deprived of a normal quantity of blood. Mechanical pressure is another circumstance. An aneurismal or other internal tumor which progressively increases in size occasions atrophy of the parts with which it is in contact. The atrophy in cirrhosis of the liver is probably caused by the pressure upon the lobules of this organ of a morbid product situated within the interlobular spaces. The general atrophy which occurs in pulmonary tuberculosis or other affections, and senile atrophy, are due to constitutional states unfavorable to nutrition.

As contrasted with hypertrophy, atrophic lesions are rarely, if ever, conservative; that is, they are not, as are hypertrophic lesions in most cases, conducive to the welfare of the economy in view of the circumstances under which they occur.

Morbid alterations as regards the consistence of parts constitute the second of the three classes of anatomical changes or lesions. These alterations are of two kinds, namely, increased consistence or induration, and diminished consistence or softening. Both induration and softening are frequently incidental to palpable changes in the composition of parts, and, under these circumstances, they are not properly included in the 
second class of lesions, but they belong to the third class. This second class embraces induration and softening, not occurring as results of morbid deposits of various kinds, degenerations of structure, acute intlammatory processes, etc., but constituting, in themselves, the primary, palpable lesions. As thus restricted, examples of each are not very numerous.

\section{Induration.}

A part is indurated when its density and firmness are abnormally increased. As just stated, this physical change is generally incidental to manifest changes of composition or structure involving usually the presence of morbid products in the parts affected. Thus, in cirrhosis of the liver, the morbid production of fibroid tissue in the interlobular spaces gives to this organ an abnormal density and firmness. The same is true of that variety of degeneration of the kidney characterized by contraction and hardness. In these and similar instances, induration is a result of other palpable changes. Induration as a sole or chief lesion occurs when certain parts are condensed by pressure, and when they are deprived, to a greater or less extent, of their normal quantity of liquid.

Induration due to condensation is exemplified, in a striking manner, by the carnified lung in cases of pleuritis with large effusion. 'The lung, in these cases, is compressed into a small, compact mass, bearing more resemblance to flesh than to the pulmonary organ in its natural condition, and hence it is said to be carnified. Yet the composition and structure are not materially altered; by means of insufflation after death, the condensed lung may be restored to its normal condition, and this also occurs during life when the liquid effusion is absorbed. A similar condensation of pulmonary lobules takes place, from collapse, in certain of the cases heretofore embraced under the name lobular pneumonia. Induration from deficiency of liquid is exemplified by the abnormal density and firmness of the brain and other organs after death from epidemic cholera, the loss of liquid in this disease being due to the intestinal transudation.

Induration of the substance of the brain has been observed in cases of acute and chronic lead poisoning; and the brain in these cases has been found to contain the sulphate of lead. The term sclerosis (sxinpos, hard) is used to denote induration of parts generally from hyperplasia of connective tissue, or a fibroid product.

\section{Softening.}

A part is softened when its density and firmness are abnormally diminished. The softening may be greater or less in degree. Portions of the brain or spinal cord are sometimes softened to liquefaction, but then, of course, there is total loss of structure, or disorganization. Softening, in most cases, is incidental to some manifest change of composition or structure, or it is an element of certain morbid processes, namely, inflammation and gangrene. An example of softening as incidental to an inflammatory deposit is afforded in pneumonitis. In the second stage of this disease the affected portion of lung, although solidified (hepatized), has less firmness than in health. It breaks down more readily under pressure with the finger than healthy lung, and hence, is said to be more frinble. The friability is still more marked in the stage of purulent infiltration, the softening then becoming so great that the structure gives way under moderate or slight pressure of the finger.

Softening of the brain or spinal cord is a result of inflammation extend- 
ing from the meninges, developed around a clot of blood or a tumor of some kind, or originating spontaneously within these nervous centres; but softening occurs here independently of inflammation, the cohesion of the nervous filaments and vesicles being diminished by defective nutrition, dependent on a deficient supply of blood or other causes. Non-inflammatory softening of the brain is frequently associated with degenerative changes of the coats of the cerebral arteries, in consequence of which the distribution of blood is impeded and nutrition impaired; and it occurs from arterial obstruction caused either by a mass of fibrin or a vegetation derived from the left side of the heart, eonstituting what is called an embolus or plug, or from coagulation of blood at the seat of the obstruction, when it is called a thrombus. Softening, as thus produced, as will be hereafter seen, is involved in certain cases of apoplexy and paralysis. If the softening be considerable, it involves a change in composition; there is either a decomposition analogous to gangrene, or the change consists in a granulo-fatty degeneration.

Softening is incidental to inflammation affecting any of the tissues. This result is especially marked in inflammation of the mucous membranes. These are sometimes completely disorganized and reduced to a pulp, so that portions are mechanically removed by the attrition of substances moving over their surfaces. This is seen in examinations after death in cases of dysentery. When the degree of softening of the mucous membranes is less, it is shown by the inability of tearing small portions away in strips with the forceps. Non-inflammatory softening of these membranes, elsewhere than in the stomach and small intestines, is certainly rare. And in the stomach, especially, the softening found after death, when inflammation has not existed, is, in most cases, probably, a post-mortem change due to the dissolving action of the gastric juice. Softening from the latter cause may exist in a great degree, affecting all the coats of the stomach, leading to perforation and softening of the adjacent viseera, in cases of death taking place suddenly while the process of digestion was going on. If the protection of the stomach against the action of the gastric juice, in life, depends, as considered by Bernard, on the presence of mucus, it is not improbable that softening from the same cause may sometimes take place after death, owing to deficiency of the secretion of mucus.

Softening occurs not only ir parts which are inflamed, but in proximate tissues not involved in the inflammation. Thus, in serous inflammations, the subserous areolar tissue loses its normal firmness and becomes brittle, so that the membrane is detached much more easily than in its healthy condition.

Softening of the muscular tissue is incidental to fatty degeneration, but it occurs, as the chief or sole palpable change in the heart, in certain cases of typhus and typhoid fever. Softening of the spleen, occurring in these fevers, is also an example of this change occurring without inflammation, or any well-defined change in composition or structure.

The softening which is non-inflammatory and independent of other palpable changes is, in general, referable to a defect in the nutrition of the affected parts. This defect may be dependent on eircumstances limited to the parts, as when cerebral softening arises from arterial obstruction; or it may proceed from a general condition, involving a deficiency of nutritive supplies in the blood, as when softening of the heart occurs in typhus or typhoid fever. If the softening consist only in diminished coherence of the component anatomical elements of the parts, without being sufficient to occasion disorganization, it is not irremediable no: 
necessarily even a grave lesion. Softening of the heart and spleen donbtless occurs in cases of fever which end in complete recovery ; and it is not improbable that in certain cases of softening of the brain, dependent on a temporary defect of arterial supply, as when it is occasioned by an embolus, the normal consistence may be restored after the re-establishment of the circulation.

To softening in a degree involving disorganization, resulting from the death and mortification of the part affected, Virchow applies the term necrobiosis.

\section{CH A PTER II.}

\section{ANATOMICAL CHANGES IN THE SOLID PARTS OF THE BODY- (CONTINUED.)}

Lesions of Composition-Exudations and Transudations-Fibrinous Exudation or Coagulable Lymph-Tubercle-Scrofula-Carcinoma or Cancer-Typhoid Deposit.

A LTERATIONS as regards the composition of parts constitute the A third class of anatomical changes. 'The lesions embraced in this class are more numerous and important than those included in the preceding classes. Of the changes belonging to this class, I shall notice those which are of importance in relation to medicine as distinguished from surgery, presenting, briefly, certain distinctions, etc., which should be understood by way of preparation for entering on the study of individual diseases. It does not fall within the scope of this work to enter into a full account of the various alterations which will be noticed. The student is expected, in addition to the outlines here presented, to pursue this part of the subject more fully by means of works which treat at length of morbid anatomy, including histology, and of general pathology.

It is appropriate to reckon among changes in composition, not only perversions of structure, but changes which consist in the addition of abnormal products, either solid or liquid, the tissues of the parts remaining unaffected. For example, the infiltration of liquid into the interstices of a part alters its composition, although there be no structural change in the part. To eite another example, in the hepatization which takes place in pneumonitis, there is a solid deposit within the air-cells, and the presence of this deposit alters, for the time being, the physical condition of the affected portion of lung. The composition of the lung is in fact changed. But the structure of the lung remains intact, and as soon as the morbid deposit clisappears, the normal condition of the lung is restored. In other examples, for instance, when the composition of the lungs is altered by the addition of the tuberculons products, the presence of these, and the changes which they undergo, lead to more or less injury or destruction of the pulmonary structure. In this instance, however, the morbid products were not, at the outset, substituted for, but added to, the anatomical constituents of the part, and the damage which is occasioned is a result more or less remote. On the other hand, there are changes in which morbid products supplant the substance proper to the parts affected, and thus involve perversions of structure. Here is an important 
distinction as regards changes in composition. Changes in composition may consist, then, 1 st, in the presence of morbid products not involving primarily, or of necessity, change of structure; or, $2 d$, they may consist in structural alterations. The latter are commonly called degenerations. Changes which do not affect the tissues entering the composition of parts, are not necessarily destructive; they may be temporary, and, after their disappearance, the parts may be in no wise damaged, as in the example of pneumonitis. But perversions of structure or degenerations involve, from the outset, disorganization of parts, and, in general, are permanent or incurable.

A distinction is to be made between morbid products which become solid or semi-solid, and those which remain in a liquid state. For the sake of distinction, the term exudations should be restricted to solid or semisolid products. On the other hand, the term transudations may be applied to constituents of the blood which escape through the coats of the vessels and preserve their liquid state. These terms will be used hereafter as now defined. An exudation is a solid or semi-solid morbid product derived from the blood; a transudation is a liquid effusion constituting, when retained in any part, a dropsy. 'The materials composing exudations, as well as transudations, of course, are derived from the blood, and are in a liquid form within the bloodvessels. In the exudative process there is, therefore, a derivation from the blood of certain of its constituents which assume a solid or semi-solid form after leaving the vessels. Now, regarding the act of exudation, there are certain pathological questions as yet unsettled. Does an exudation occur in consequence of a prior bloodchange, or does it depend on a process pertaining to the part in which the exudation takes place; or, again, are a blood-change and a local process conjointly involved? The doctrine which has been, and is still, held by many pathologists is, that exudations represent certain morbid conditions of the blood; and that they consist of blood-constituents which, after escaping through the coats of the vessels, undergo coagulation and subsequent changes. On the other hand, according to the so-called cellular pathology, now accepted by many, the act of exudation is exclusively a local process taking place through the agency of cells. Whatever pathological views may be entertained, the name exudation is appropriate enough as applied to a morbid product, solid or semi-solid, which is added to the tissues of a part, and which is distinct from either a morbid growth or the formation of new tissue. The term product will be frequently used as applicable alike to a morbid deposit and a morbid growth.

With these explanations; the consideration of the anatomical changes belonging to the third class will embrace exudations, transudations, or liquid effusions, lesions of structure or degenerations; and added to these are new formations or morbid growths, pneumatoses, or the abnormal presence of air or gas in parts, and parasitic productions, regetable and animal. The remainder of this chapter will be devoted to anatomical changes dependent on exudations.

\section{Fibrinous Exudation.}

An exudation which forms an important event in the natural history of certain inflammations is that distinguished as fibrinous. By the older writers it was called coagulable lymph, and it is still commonly known by

1 This distinction between an exudation and a transudation is not made by all writers. It was made especially by Lehmann. 
that name. Bennett calls it simple exudation. It is generally considered to be essentially the fibrin of the blood, or the blood-plasma, either simple or more or less modified, in a coagulated state; the liquor sanguinis, which contains fibrin in a liquid state, exudes, and the fibrin coagulating constitutes the deposit. It may also be distinguished as inflammatory exudation. It is perhaps correet to state that it is always a produet of inflammation; that is, it oceurs only as a result of an inflammatory process. It is the criterion by which the existence of inflammation in eertain structures is determined; its presence is proof of inflimmation in these struetures, and, if not found on examination after death, adequate evidence of inflammation having existed is wanting. It belongs especially to inflammation of the serous membranes, and will be found to constitute an important part of the history of pleuritis, periearditis, peritonitis, cte.

Examined shortly after it has exuded upon a serous surface, it presents the appearance of a semi-transparent, gclatinous semi-solid. Examined mieroscopieally, it is found to contain minute filaments, such as are found in the eoagulated fibrin of the blood, intermingled with corpuscles, to which various names have been applied, namely, plastic, pyoid, exudation-eorpuscles, etc. The latter are to be considered as belonging to a elass of corpuseles ealled leucocytes, embracing pus-globules, the white globules of the blood, mueous corpuscles, etc., all of which, aecording to mieroscopical observers, are essentially identical. At a later period the exudation beeomes more solid, frequently forming a thick, deuse layer, adherent mechanically to the serous surface, and having distinetly a fibrous arrangement. Detached and peeled off, it resembles a membrane, and is commonly ealled a false membrane. Not unfrequently the exudation forms a series of layers, which may be separated from each other like the coats of an onion. The coagulation of the fibrin contained in the exuded liquor sanguinis leaves a certain amount of serous liquid, which, in scrous inflammations, accumulates within the serous sac, so that, in examinations after death, more or less liquid is usually found together with the eoagulable lymph. Flakes of lymph, in greater or less abundanee, are contained in the liquid, and the latter is turbid, instead of being transparent as in purely dropsical effusions.

In the progress toward recovery, the liquid contained within the serous sac is absorbed. The serous surfaces then coming into contact may be agglutinated by means of the intermediate lymph. The latter is absorbed more slowly. A true membranous or organized strueture subsequently becomes developed, which is the medium of the permanent adhesions resulting from inflammation of serous struetures. An unfavorable progress is eharaeterized by the serum and lymph remaining unchanged for an indefinite period, or by the production in large quantity of the wellknown liquid called pus, which is eharaeterized by the presence of corpuscular bodies called pus-globules or lcucocytes.

Seleeting serous inflammations as typical, because they are distinguished by the constaney and abundance of inflammatory exudation, the brief deseription just given is an epitome of the natural history of this morbid product. But considerable variations are observed in different cases of serous inflammations. The relative proportions of lymph and serum vary widely. The quantity of lymph may be abundant or quite small, and the same is true of the liquid effusion. The constitution of the lymph varies. The formation of fibrille, or the fibrillation, may be greater or less, and the corpuseular constituents, or lencocytes, may be few or abundant. Paget reckons two varicties of lymph, one variety being characterized by the predominance of fibrillæ and the other by the 
abundance of corpuscles, with a small quantity of fibrilla. The former he ealls fibrinous, and the latter corpuscular, lymph. The fibrinous variety he regards as favorable for the production of organized tissue, and hence it is ealled plastic, while the corpuscular variety tends to the production of pus or suppuration.

The ordinary product of the inflammation of mucous membranes is mueus variously modified, and containing, in more or less abundance, the corpuseular bodies known as mucous globules, which are essentially identieal with pus-globules, or, in other words, belong in the category of leucocytes. Exceptionally, however, fibrinous exudation occurs on a mucous surface, and, disposed in the form of a false membrane, presents characters analogous to those which belong to the exudation in serous inflammations. 'This constitutes the characteristic feature of the inflammation of the larynx and trachea, sometimes extending to the bronchial tubes, in true croup, and of the inflammation of the fauces, air-passages, and in other situations, in the epidemic disease called diphtheria. Inflammation of a mueous surface or of the skin, when characterized by fibrinous exudation, is frequently distinguished as diphtheritic, after a term introduced by Bretonneau. Occasionally in bronchitis a false membrane is formed, and this variety of the disease is called, incorrectly, plastic bronehitis. Fibrinous exudation is not uncommon in cases of dysentery. The formation of new tissue does not take place in eonnection with a fibrinous exudation on mucous surfaces. The false membrane, in all cases, in the course of a few days becomes detached by a suppurative process beneath it, and is thrown off. This is the basis of a division, by Rokitansky, of inflammatory exudation into croupous and plastic. The term plastic, frequently applied to inflammatory exudation, denotes its capacity of developing within it organized struetures. This is also expressed by the term blastema, which was formerly applied to inflammatory exudation. The term cytoblastema, which has the same application, denotes the development of eell-formations within the exudation. 'These two terms (blastema and cytoblastema) have been used to denote an amorphous liquid in which anatomical elements, either cells or tissues, were supposed to be produced by auto-genesis or spontaneous generation. In pneumonitis an inflammatory exudation within the air-cells solidifies the lung, giving rise to the condition called hepatization. In this situation organic tissue is not formed, and the exudation is removed by absorption. A very small proportion, sometimes none whatever, is expectorated, and clinical observation shows that the large amount of exudation which takes place in this disease may be absorbed very rapidly, being all removed in the course of a few days. In other parenchymatous inflammations, fibrinous exudation sometimes takes place and is infiltrated among the component tissues. In the brain and spinal cord it is presented in the form of minute granules with which pns-globules or leucoeytes may be commingled. These products, in connection with softening, show the latter to be due to inflammation. In other parts, the presence of fibrinous exudation, which may persist without metamorphosis or change for an indefinite period, involves enlargement by false hypertrophy and induration; or it may sooner or later lead to suppuration; or, leading to the development of fibroid tissue, it may produce atrophy and disorganization, as is seen in eirrhosis of the liver and the hard contracted kidney.

It is thus seen that the fibrinous exudation in different situations, and in the same situation in different cases, presents variations as regards its constitution, the metamorphoses of which it is the seat, its behavior (to borrow a metaphorical term from the chemist), and its consequences. 
Our present knowledge does not enable us to say to what extent these diversities may be due to primary, essential differenees, that is, differences pertaining to the exudation itself, or how much is attributable to circumstances connected with the exuding process, or again, the amount of influence exerted by the surrounding parts upon the exudation after it has taken place. Much undoubtedly depends on the situation of the exudation; for example, it is never the seat of a newly-formed organized structure on a mucous surface. But it remains to account for the variations which are shown in different cases in the same situation, for instance on a serous surfice. Whether these variations depend on morbid conditions of the blood, or on local actions involved in inflammation, is at present an unsettled question.

A pathological question which must still be considered as sub judice relates to the development of organized tissue, and the production of corpuscular bodies in connection with fibrinous exudation; in other words, to this exudation regarded as a blastema and as a cytoblastema. The question is, whether the newly-developed tissues and pus-globules, which are undoubtedly produced, are products of a metamorphosis of the coagulated fibrin; in other words, is coagulable lymph converted into living structure, taking on organization, and is it transformed into pus? The view taken by Paget, and heretofore generally received by pathologists, is, that the coagulated fibrin or lymph is organizable, $i . e$., capable of being transformed into organized living structures; and also that it is formed into pus. The term plastic is used to distinguish the kind of lymph which is supposed to be especially prone to organization. On the other hand, Robin and others regard coagulable lymph in the light of an extraneous product, denying its capability of being transformed into either organized tissues or pus. According to the latter view, it either remains without essential change, or it is exfoliated, or it is absorbed, newlyformed tissue being produced by molecular combination during the act of exudation; consequently, alone it has no claim to be called a blastema, nor does it take part in the generation and multiplication of pus-globules or leucocytes." According to the cellular pathology of Virchow, morphological products, that is, cells or tissues, are never spontaneously generated; and hence, a blastema or a cytoblastema has no existence. The doctrine of Virchow is that all organized forms are produced by the proliferation or multiplieation of cells. This doctrine is formularized by the phrase omnis cellula e cellulâ. On the other hand, the doctrine advocated by Bennett, Robin, and otbers is, that cells and tissues may be produced by a formative force inherent in molecules and independent of cell-proliferation. A liquid in which such a force is exerted is a blastema or a cytoblastema. This is called by Bennett the molecular theory. This theory does not deny that morphological productions take place by the proliferation of cells, but, denying that tissues and cells are exclusively thus produced, it accounts for the development of both, to a greater or less extent, by the arrangement of molecules.

\section{Tubercle.}

The term tubercle, as generally used, embraces morbid products of great importance in view of the frequency of their occurrence and the extent to which they contribute to mortality. To the affections characterized by these products is applied the name tuberculosis; as, tuberculosis of the lungs, or pulmonary tuberculosis, tuberculosis of the liver, etc. 
They are also called tuberculous affections. The products occur in various situations, but especially in the pulmonary organs.

Two products especially have been embraced under the name tubercle, One of these is the product known as tuberculous, fibro-plastic, semitransparent, or miliary granulations, and the granulations of Bayle. This product may be conveniently distinguished as gray tubercle: It is presented in the form of small, spherical bodies of a grayish color, semitransparent, and of considerable resistance to pressure, the size being generally that of millet or hemp-seeds; but sometimes they are so minute as to be scarcely visible with the naked eye. They may be more or less abundant. Existing in the lungs in immense number, they characterize the affection called acute phthisis, life being destroyed by this affection in consequence of their accumulation sufficiently to compromise, to a fatal extent, the function of respiration. They occur elsewhere than in the pulmonary organs, being found in the liver, spleen, and other parts, as well as in the lungs. They give rise to the form of peritonitis called tuberculous, and to the so-called tuberculous meningitis of children. Examined microscopically, they are found to consist of grannles with minute free nuclei and cells, described by Robin under the name cytoblastions. According to Robin, cytoblastions are found in the lungs and in other parts in health; hence, he regards the gray tubercle as due to a hypergenesis of normal anatomical elements. Virchow considers this tuberculous product as a morbid growth or a neoplasm due to proliferation of the connective tissue cells. If the latter view be correct, the product is not an exudation in the sense in which this term has been defined.' By Bayle and others, gray tubercles were supposed to remain indefinitely without any notable change; but late microscopical researches seem to show that, after a time, they become opaque and soften, undergoing a granular and fatty metamorphosis. According to these researches, the primary seat of the gray tubercles in the lungs is without the aircells in the interstitial tissue around the small vessels and capillaries. They adhere so closely to the tissues that they are detached with considerable difficulty. Following Bayle, who first distinctly described these bodies under the name miliary granulations, some pathologists have denied their tuberculous character, regarding the product to be presently noticed as true tubercle; at the present time, however, distinguished German and French pathologists entertain an opinion directly the reverse of this, regarding the gray tubercle as the only truly tuberculous product.

As already stated, gray tubercles occur in various situations other than the lungs. Of the serous membranes, they occur in the pleura, peritoneum, arachnoid, pericardium, and the tunica vaginalis, enumerating these membranes according to the relative frequency of the occurrence in them. They are, however, not unfrequently found in several or all of these membranes, as well as in other parts, in the same case. They occur in the mucous membrane of the small and large intestine; in the trachea, larynx, and bronchial tubes, and, in some very rare instances, in the stomach and osophagus. They also occur in the lymphatic glands and vessels; in the liver, kidneys, and testicles, and in the bones. They have not been found in the muscular, cartilaginous, and tendinous structures, nor in the skin, nor in the mammary gland. Their occurrence in the salivary glands and pancreas is doubtful.

They are to be distinguished from cancerous granulations, coagulable lymph in a granular form, syphilitic or gummy tubercles, and the tuber- 
cles of glanders. For the differential characters involved in this discrimination, the reader is referred to works treating of morbid histology.

The other product embraced under the name tubercle, the product which by many has been heretofore considered as alone truly tuberculous, is commonly known as yellow tubercle. This is an exudation which, in the lungs, takes place within the air-cells or pulmonary alveoli. It is presented either in the form of small, isolated, round masses, constituting a variety of the so-called miliary tubercles; or it accumulates so as to form collections varying in size from a filbert to an orange, and these may be more or less numerous. The latter form is distinguished as infiltrated tubercle. In the state called crude tubercle, it is opaque; it has a yellowish color, and is of a cheesy consistence, being easily broken down with the fingers, i.e., friable. Examined microscopically, it is found to consist of anorphons granules, inclosing, in more or less abundance, corpuscular bodies which have been called tubereulous corpuscles. These were described by Lebert as specific morphological elements of tubercle. According to Virchow, they are the nuclei or fragments of epithelium which have undergone a granular and fatty metamorphosis. They are round, oval, or polyhedral, granular in appearance, devoid of nuclei or uncleoli, unaffected by water, rendered transparent by acetic acid, and extremely small, their average diameter being from $\frac{1}{20 \delta \sigma}$ to $\frac{1}{2 \sigma \delta}$ of an inch. According to Robin, they differ essentially from the cells, which he calls cytoblastions, found in gray tubercle. As regards chemical composition, this morbid growth consists of albuminous matter combined with fatty granules, crystals of cholesterin, and certain carthy salts, the latter being chiefly the phosphate and carbonate of lime. The relative proportion of animal matter and earthy salts varies in different specimens.

After a variable period, the masses or collections undergo softening, attributable partly to intrinsic changes, and, in part, to the permeation of fluid derived from the surrounding tissue; and they are at length converted into a creamy liquid, having a resemblance to pus. Microscopical examination now shows granular matter, the tuberculous corpuscles few in number, pus-globules or leucocytes, elastic fibres from the pulmonary structures, fatty granules, bronchial epithclium, and blood-crystals. Sooner or later these tuberculous abscesses, as they have been called, the vomicx of the older writers, open into the bronchial tubes. Exceptionally, they open into the pleural sac. These changes involve destruction of the pulmonary structure proportionate to the amount of the morbid product.

Both these morbid products are never the seat of newly formed tissue. They are not readily absorbed, but there are grounds for believing that the latter is sometimes completely removed by absorption. The animal portion may be absorbed, the mineral portion remaining, and in this way may be explained, at least in part, the formation of small calcareous masses, or calculi, not unfrequently found after death, and which are sometimes expectorated. When the process of absorption is going on, the tuberculous affection is said to be retrogressive, and the calculi formed in the lungs have been called obsolete tubercles.

'The relationship between the two products just described, has been heretofore much discussed, and at the present moment is under discussion. Laennec, rejecting the doctine of Bayle, that they are essentially different morbid products, considered each as composed of the same substance, the tuberculous matter, which is at first gray, and afterward becomes yellow tubercle. This view was generally adopted, until the microscope came to be employed in pathological researches. Among 
microscopical observers there has been, and still is, a difference of opinion respecting the identity of the two products, none doubting, however, the existence of a relationship between them. Without entering into historical details, I shall confine myself to a concise statement of certain recent views, which, although adopted by many pathologists, should be considered as, at present, sub judice. Following Virchow, the gray tubercle, meaning, by this term, the semi-transparent granulations of Bayle, is considered as the only true tuberculous product. Yellow tubercle is considered as a result of a degenerative change of different morbid products, inclusive of the gray tubercle; but, for the most part, it consists of the products of inflammation, which, in the lungs, are seated within the air-cells, these products having undergone what is called a cheesy metamorphosis. The so-called ycllow tubercle, as produced by the cheesy metamorphosis of inflammatory exudation, is not entitled to be called tuberculous, and the gray tubercle loses its tuberculous characteristics when it has undergone the granular and fatty degeneration. In some instances the so-called yellow tubercle is simply inspissated or concrete pus.

According to the views just stated, a true tuberculous affection of the lungs, or of any part, involves the production of the gray tubercles or granulations which have been described. Inflammation of the adjacent structures is generally, or frequently, associated; and, in the lungs, the collections of the so-called yellow tubercle, which soften, form abscesses, and result in cavities, denote parenchymatous inflammation, or, in other words, chronic pneumonia. In the majority of the cases of the affection known as chronic pulmonary tuberculosis, the clisease is, in fact, chronic pneumonia, associated with the production of the gray granulations. The association, however, of the pneumonia with the granulations is not invariable; the former takes place without the latter, and the latter is not always accompanied by the former. In general, the gravity of the affection is determined by the extent of the coexisting pneumonia ; but the granulations existing without pneumonia may give rise to grave symptoms, and produce death by accumulating in immense numbers.

Of the nature of the pathological connection existing between these two products, exactly opposite opinions are held by different pathologists at the present time. By some the gray tubercles are supposed to precede, and give rise to, the yellow product, that is, the pneumonia. Others consider the former as secondary to, and dependent upon, a granulo-fatty or cheesy metamorphosis of either yellow tubercle or analogous products in lymphatic glands within the chest and elsewhere. The production of the gray tubercles, according to the latter opinion, is due to the absorption of matter from degenerated inflammatory products. This doctrine receives some support from the facts recently ascertained with reference to the inoculability of tubercle, to which reference will presently be made. The former opinion, however, seems the most rational of the two. It is supported by the occurrence of gray tubercles, sometimes in great abundance in cases of acute tuberculosis, when no degenerated or cheesy masses are to be found either within the chest or elsewhere. Moreover, in cases of tuberculons meningitis and peritonitis, clinical observation appears to show that inflammation of the serous membrane follows, and is caused by, the production of gray tubercles. A third opinion is perhaps as rational as either of the foregoing opinions, namely, that the two products are dependent neither upon the other, but each upon a causative condition common to both. With our present knowledge, the wisest course is not to adopt unreservedly any of these opinions, but to consider the nature of the pathological connection between the two products as among the 
questions to be settled by future researches. It is to be added that some histologists still hold to the essential identity of the two products. It is conceded by those who deny their identity, that the gray tubercles, after they have undergone the granulo-fatty degeneration, cannot be distinguished, by their gross characters, from the so-called yellow tubereles, when these, from their size and form, are miliary; and, also, that the aggloneration and coalescence of gray tubercles may present appearances which render them not distinguishable from the so-called infiltrated tubercle.

The efforts to repudiate yellow tubercle as a tuberculous product, and to substitute the name pneumonia, have given rise to much confusion. 'I'o distinguish it from ordinary pneumonia, that is, lobar pneumonia, and also from chronic interstitial pneumonia, various terms are used, such as, chronic lobular pneumonia, chronic catarrhal pneumonia, cheesy pneumonia, and broncho-pneumonia. The propriety of these changes in nomenclature is doubtful, admitting the product to be, in fact, an inflammatory exudation. Admitting this, the behavior of the product differs widely from that of the exuclation in both lobar and interstitial pneumonit. The morbid growth in interstitial pneumonia remains without softening, and without leading to structural damage of the pulmontary substance, except from atrophy caused by the pressure of the adventitious tissue. In lobar pneumonia the exudation is absorbed completely, and often with rapidity, the structure of the lungs remaining intact, and the formation of abscesses being extremely rare. On the other hand, the exudation known as yellow infiltrated tubercle is rarely absorbed; and the clianges which it undergoes involve destruction, to a greater or less extent, of the parts in which it is seated. In view of these points of difference from other forms of pneumonia, and considering that, if they are not essentially identical, there is a close relationship of some kind existing between the gray and the yellow tubercle, if it be resolved to call the latter a product of pneumonia, it should be distinguished as a tuberculous pneumonia. It is to be arlded that by some, if not most, pathological histologists, the gray, as well as the yellow, tubercle, is regarded as an inflammatory product; and, according to this view, the former constitutes the anatomical characteristics of a variety of interstitial pneumonia.

Whatever views may be held respecting the relationship between the two products embraced under the name tubercle, and the pathological character of each, that is, of the nature of the process or processes involved in tuberculization, clinical facts which will be stated in treating of pulmonary tuberculosis, in the second part of this work, show that they are both to be "considered as the local effect or expression of a morbid constitutional state; in other words, they are the manifestations of a special diathetic condition. Whatever may be this antecedent constitutional state, clinical observation shows that, in many cases at least, it suffices for the occurrence of the tuberculous products, without requiring the co-operation of any local conditions which are discoverable; that is, the occurrence of the products is often the first appreciable local event. Moreover, since the tuberculous products in different situations do not essehtially differ, it follows that the local process, or the tuberculization, has more to lo with the diathetic condition than with the structure of the part in which it is seated. As already stated, the lungs are the seat of the tuberculous products much oftener than any other part; but not unfrequently they occur, simultaneously or successively, in various situations. The latter is observed in children oftener than in adults. Louis, by means of his statistical researches, ascertained this law, viz., whenever a tuberculous affection occurs in any part of the body, other than the lungs, after fifteen years of age, 
pulmonary tuberculosis coexists, and the latter is always the primary manifestation of the tuberculous cachexia. 'I'o this law, however, there are some exceptions. Other laws, together with certain questions recently raised relating to etiology and treatment, will be noticed when pulmonary tuberculosis is considered in the second part of this work.

Tubercle was formerly reckoned among morbid products distinguished as heterologous or heteromorphous, in contradistinction from those called homomorphous or homologous. The former names denote morbil products which are not identical with, nor analogous to, any of the normal constituents of the organism; the latter names were applied to morbid products which have normal prototypes, that is, they are identical with, or are analogous to, anatomical elements contained in the body in health. Laennec applied the term "accidental products" to those which were considered as heterologous or heteromorphous. But it appears to have been established by microscopical researches that there are no morbid products which are heterologous or heteromorphous in the sense of these names as just defined; in other words, all morbid products have their normal analogues in the anatomical history of the organism including therein embryonic life. In accordance with this doctrine, now accepted by the most distinguished pathologists, the different morbid products are accounted for by either an abnormal multiplication of normal anatomical elements of the body (hypergenesis, hyperplasia), or by abnormal modifications of their development and growth, or by an error of situation.

Finally, a discovery with respect to tubercle, of great interest and importance, in 1865, was communicated to the French Academy of Medicine, by M. Villemin. By a series of experiments M. Villemin demonstrated that tuberculous disease may be communicated from man to certain inferior animals by inoculation. The animals selected by him for these experiments were rabbits and guinea-pigs. Portions of each of the tuberculous products having been inserted beneath the skin, and the animals destroyed after a period varying from two to three months, in the lungs especially, but also in other parts, tubercles were found in more or less abundance. These experiments have been repeated with the same result by many other observers.

The inocilability of tubercle does not prove that the disease may be communicated by means of the breath or by emanations from the body; yet, the fact affords ground for the suspicion that tuberculous patients may sometimes communicate the disease to those with whom they are for a long time in close intercourse, as, for example, in the case of husband and wife. Villemin claims that his experiments showing inoculability prove the existence of a virus in the tuberculous products; and that tuberculosis expresses a specific disease in the same sense as, for examples, glanders and syphilis are specific communicable diseases. On the other hand, experiments made by different observers appear to show that inoculation with pus as well as other non-tuberculous morbid products, and with septic matter of various kinds, gives rise to the same result as the inoculation with tubercle. This militates against the doctrine of a tuberculous virus. The production of gray tubercles in the lungs and other organs by the introduction into the blood of tuberculous and other matter, gives, as already stated, some support to the opinion that the gray tubercles may be due to auto-infection, being caused by the absorption of matter from degenerated morbid products of different kinds seated in the lungs or in other parts of the body. 


\section{Scrofula.}

The term scrofula, as frequently used by medical practitioners and writers, has a comprehensive and an indefinite latitude of signification. In a restricted and definite sense it denotes an affection of the lymphatic glands, especially the superficial glands of the neck, occurring chiefly in children after the period of infancy, characterized by a morbid procluct which, if not truly tuberculous, bears a close resemblance to tubercle as regards its behavior and the morbid processes incident to it. The scrofulous product occasions more or less enlargement of the affected glands. It remains for an indefinite period apparently in a quiescent state, and it may be removed by absorption. If not absorbed, softening takes place, with but little evidence of inflammation; ulceration of the integument ensues, and the liquefied product is discharged. Finally, after a variable period, there is cicatrization resulting in an irregular, puckered, characteristic scar. The same series of events may occur in the bronchial glands, giving rise to what has been called bronchial phthisis. Clinical observation appears to show that they who suffer from a scrofulous affection of the glands of the neck in early life are not more liable than others to pulmonary tuberculosis in after-years.

According to the views of Virchow, Niemeyer, and others, the scrofulous enlargement of the lymphatic glands is due to a morbid increase of anatomical elements which these bodies contain in health, the process being a hyperplasia or morbid growth, to which inflammation is consecutive; and this product, like tubercle and other products, undergoes the cheesy metamorphosis. Cretefaction also may occur, as in the tuberculous product.

The absorption of the scrofulous product after it has undergone the cheesy metamorphosis, is by some supposed to be a cause of the development of tubercles in the lungs and other organs. The frequent existence of scrofula, however, without the occurrence of tuberculous disease in other situations, militates against, if it does not disprove, this supposition.

The existence of a constitutional state in children, called the scrofulons habit or diathesis, is generally recognized by practitioners and writers as manifested in affections occurring either with or without the affection of the glands, which has just been noticed. Enlargement of the head, tumefaction of the upper lip, or of the nose, swelling of the abdomen, and flaccidity of the muscles, are supposed to denote constitutional scrofula. Various affections are considered as local expressions of this diathesis, or, at least, as deriving from it important modifications. These affections are, certain of the cutaneous diseases, namely, eczema, impetigo, and lupus, especially when seated on the head or facc; chronic coryza, otorrhœe, conjunctivitis, and superficial ulceration of the cornea; chronic suppurative inflammation of the joints, formerly known as white swellings; caries and necrosis of bones ; bronchitis, and chronic inflammation of the intestinal mucous membrane. That these affections involve a morbid constitutional state, either congenital or acquired, is undoubtedly true ; but that they originate in a special cachexia is by no means certain. Even in children who have a scrofulous affection of the glands, the concurrence may be accidental. It is certain that the various affections just named are often observed in cases in which the glands are not affected; and, per contra, the glands are often the seat of scrofula without the coexistence of any of the other so-called scrofulous affections. 


\section{Carcinoma.}

In connection with the anatomical changes due to morbid products, may be noticed carcinoma or cancer. I shall pass by this product with a cursory notice. It is by no means unimportant. On the contrary, its importance and extent are such that it occupies a large space in pathology. and treatises are devoted specially to it. It will be found to give rise to a considerable number of individual diseases hereafter to be considered. But in a large proportion of cases the affection is seated either externally or in parts which are accessible, and the propriety of removal by the knife frequently becomes a question. Hence, it belongs to surgical rather than medical pathology, and is treated of at length in surgical works. For these reasons I shall devote to it but a few words. In most cases the carcinomatous product becomes the seat of more or less development into tumors which sometimes attain to a great size.

The terms carcinoma and cancer have been used heretofore with much looseness, having been applied to morbid growths differing from each other in essential points. In their present restricted scope of application, they embrace different forms or varieties which differ widely in appearances, but have in common certain characters which are distinctive. The general characteristics of the carcinomatous product, as regards its behavior during life, are as follows: It is a morbid development or growth which may go on indefinitely, giving rise to a tumor of greater or less size. It involves more or less destruction of the surrounding structures, partly by interfering with their nutrition, and in part by the inflammation which it excites. Sooner or later, if life be sufficiently prolonged, it leads to ulceration which becomes more or less extensive, eventuating in what is commonly called open cancer, and hemorrhage is apt to occur. The lymphatic glands in the neighborhood are secondarily affected in consequence, as gencrally supposed, of the transportation of morbid material to them by the lymphatics. Progressive destruction of parts and death therefrom (the product frequently occurring successively or simultaneously in different situations) is the rule to which there are few exceptions. Recovery is, however, possible, and it takes place by retrogressive processes which may involve either fatty or calcareous degeneration. As belonging to this general sketch, is to be added the liability to return, when situated in an accessible part and removed by the surgeon, either in the same part or in some new situation. In view of the salient points in its history just stated, the propriety of calling cancer a malignant disease is sufficiently apparent.

Anatomically considered, the different forms of cancer present notable points of difference. Three well-marked forms are generally recognized, viz., scirrhus or hard cancer, medullary or encephaloid, and colloid or alveolar, the two latter forms being distinguished from the first form as soft cancers. Each of these forms consists, in part, of a fibroid structure which is especially abundant in scirrhus, giving to this form its cartilagelike hardness. This structure is far less abundant in the two other forms. In medullary or encephaloid cancer the morbid growth bears a resemblance in its appearance to brain substance, as the names imply. In the colloid form, the filoroid structure is disposed in such a manner as to leave small spaces called areolæ or loculi, which are filled with a substance resembling glue. These forms are subdivided into numerous varieties which need not be noticed in this general account. The three forms, in addition to the fibroid tissue, or stroma, contain characteristic cell formations, or cancer cells. These are found on subjecting to microscopical examination scrapings from the cut surfaces of cancerous tumors, or the milky liquid 
known as the cancer juice, which exudes when these tumors are incised. The presence of this juice is a distinctive character of cancer. There has been much discussion of late years respecting the existence of cells specially distinctive of cancer, and their reliability as a diagnostic cricerion of cancerous growths. Without entering into the discussion, it may be stated that most microscopical observers, appear to believe in the existence of cells having characters distinctive of cancer, and that they are present in at least a large majority of specimens which are truly cancerous. The cells present a great variety of appearance, being round, caudated, fusiform, etc., and also varying largely in size. A cancer-cell is distinguished especially by the presence of a nucleus, or of nuclei, of large size, and by a large nucleolus. The nucleus, which varies in size, according to Bennett may occupy one-sixth to four-fifths of the volume of the cell. Frequently a cell contains more than a single nucleus, often two, and sometimes several nuclei; in the latter case it is sometimes called a mother cell. Acetic acid acts upon the cell-wall, rendering it transparent and sometimes dissolving it, while the nucleus is not affected. In some specimens the cells are few or wanting, but free nuclei exist in greater or less abundance. Whenever cells or free nuelei having the distinctive characters just stated are found, we are warranted in regarding them as evidence of the cancerous nature of the specimen, but it may be a question whether their absence constitutes negative proof sufficient to declare in all cases the non-existence of cancer. It is to be borne in mind that the minute quantity of matter which is placed under the field of the microscope may not contain cancer-cells in consequence of their being sparsely distributed throughout the morbid mass. They are-not to be pronounced absent until several portions of the mass have been examined. Cancerous formations have been considered as pre-eminently heterologous or heteromorphous productions. But if we accept the views of Robin, Virchow, and other pathologists, even cancer-cells do not exemplify a principle of the generation of new anatomical elements in disease. 'They regard these cells as resulting from a deformation of epithelium or other normal anatomical elements which, at the same time, are multiplied to a greater or less extent. Robin points out differences as regards the appearance of cancer-cells corresponding to differences of the structure in which the carcinomatous exuclation takes place.

Cancerons products are distinguished as primary and secondary. The first product which occurs is called primary, and products which subsequently take place are called secondary. It is generally supposed that secondary cancers are dependent on the primary, the latter furnishing germs which gain admission into the circulation, and, being deposited in other situations, become the point of departure for fresh manifestations of the disease. It. has been already stated that, not only are the structures in immediate proximity to the deposit involved and destroyed, but the affection extends to the neighboring lymphatic glands. It may, however, be doubted whether secondary cancers more or less remote from the site of the primary cancer proceed from the latter; in other words, it is by no means certain that the system becomes infected by the introduction into the circulation of matter derived from an existing cancerous affection. Various facts go to show that a primary cancer involves an antecedent or underlying unknown special pathological condition called a cachexia. A predisposition to this disease, or the cancerous diathesis, is undoubtedly in some cases inherent in the system from birtl, and inherited. Now, it is perhaps more probable that secondary cancers are dependent on the general or constitutional condition which led to the primary affection, than that 
they proeeed from an infection by the latter. This view is consistent with a fact which clinical experience appears to teach, viz., that secondary cancers are more apt to occur after the extirpation of a part primarily affeeted, than if surgical interference had not been resorted to. It is, however, proper to state that many, if not most, pathologists consider cancers as diffused by a cancerous infection derived from the part or parts primarily affected.

As regards the situation of cancers, there is a preference (so to speak) for eertain organs. Rokitansky gives the following list of parts which are liable to be the seat of this disease in the order of their enumeration. "First, the uterus, the female breast, the stomach, the large intestine, and especially the rectum; next comes the cancer of lymphatic glands, especially as retro-peritoneal cancer aceumulation in front of the vertebral column ; hepatic peritoneal cancer; bone-cancer; cancer of the skin, and of the lips; of the brain; of the globe of the eye; of the testis; of the ovary; of the kidneys; of the tongue; of the osophagus; of the salivary glands and the parotid."

In addition to the morbid products which have been noticed, namely, the fibrinous, the tuberculous, the scrofulous, and the carcinomatous, there are some which will be referred to in treating of individual diseases. One of these is the product which takes place in the. Peyerian and solitary glands of the small intestine, and in the corresponding mesenteric glands, constituting the anatomical characteristic of typhoid fever. Morbid deposits, solid, or semi-solid, which are either substituted for, or penetrate by infiltration, the normal substance of parts, are embraced under the name degenerations. Other anatomical changes are distinguished by morbid growths, or pathological new formations. Degenerations and new formations will be briefly considered in the next chapter, together with transudations, or dropsies, changes caused by the abnormal presence of air or gases, called pneumatoses, and, finally, those dependent on animal and vegetable parasites.

\section{H A P T E R III.}

\section{ANATOMICAL CHANGES IN THE SOLID PARTS OF THE BODY- (CONCLUDED.)}

Lesions of Composition continued-Transudation-Dropsies-Fluxes-Degenerations-Fatty Degeneration-Fatty Growth-Calcareous Degeneration-Lardaceous or Waxy Degeneration -New Formations or Morbid Growths-Pneumatoses-Animal and Vegetable Parasites.

\section{Transudations-Dropsies.}

A MORBID condition affeeting the composition of parts, consists in an abnormal accumulation of liquid, exterior to the vessels, derived from the blood. A liquid which escapes through the coats of vessels, which is devoid of congulated fibrin or lymph, but holding in solution more or less of the constituents of the serum of the blood, is distinguished as a transudation. Transudations preserve the liquid state, in this respect differing from exudations, the latter being solid or semi-solid. The act of transu- 
dation is physical, that is, it has no special relation to the vital endowments of the tissues, but only to their physical properties. 'The serous or watery portion of the blood percolates the walls of the vessels as any liquid confined in artificial tubes of leather or some porous material may transude. 'Transudations may not even involve the principle of exosmosis, which is operative to a consiclerable extent in living organisms. They take place as a lesult either of a morbid attenuation of the blood-serum, or of undue hydraulic pressure, or of both these physical causes combined; other circumstances, also, may co-operate by rendering the sides of the vessels more permeable. In a true transudation, however, there is no solution of continuity or rupture. Hemorrhage, therefore, is not a transudation unless it be admitted that the blood-corpuscles can pass through the pores of the vessels without the molecular coherence of the tissues being disturbed. Bloody serum, or serum colored with hæmatin, may transude, constituting what is called false hemorrhage.

Transudations may take place in different situations. Occurring in situations from which the liquids cannot escape, viz., within serous cavities and into the areolar tissue, they constitute dropsies. Occurring upon some internal surface whence the liquid is conveyed out of the body through an outlet, or, in other words, upon a mucous surface, they constitute fluxes. The latter do not now especially concern us, since they do not occasion lesions affecting the composition of parts. The transudations which constitute dropsies are alone to be considered in this connection.

The distinctive feature of a dropsy is, that the liquid which escapes through the conts of the vessels is not liquor sanguinis, but water colltaining more or less of the constituents of blood-serum. When liquor sanguinis or blood-plasma escapes, the results are coagulated fibrin or lymph, and certain metamorphoses or developments which were noticed in the preceding chapter. Blood-constituents which assume a solid or semi-solid form after leaving the vessels and become the seat of adventitious tissues, or of leucocytes, are exudations. On the other hand, liquid effusions devoid of coagulating material, undergoing no metamorphosis, not giving rise to new formations, in other words, in no sense blastemata or cytoblastemata, are transudations, and, if retained, such effusions constitute dropsies. It is proper to remark that this distinction is not always observed. Vogel, for example, applies the term "fibrinous dropsies" to accumulations of liquid which contain coagulated fibrin in greater or less abundance. According to the distinction just made, these are not correctly called dropsies; they are exudations, involving generally, if not always, inflammation. A true dropsy is not an effect of inflammation. Inflammation of serous membranes, it is true, frequently gives rise to more or less liquid effusion. But the effused liquid is turbid from the admixture of lymph, and contains more or less solid fibrin. In a dropsical accumulation, on the other hand, the liquid is generally clear, like the serum of the blood, and no solid fibrin or lymph is apparent. Again, while inflammatory effusion is due to a morbid condition (inflammation) of the serous membranc, in pure dropsy the morbid condition, on which the transudation depends, is situated elsewhere, the membrane itself being free from diseasc. Individual diseases excmplifying this contrast between inflammatory and dropsical effusions are pleuritis and hydrothorax, peritonitis and hydroperitoneum, true hydrocephalus and meningitis. The prefix hydro expresses the fact that the disease is dropsy.

In pure dropsy the effused liquid beirs a resemblance, more or less close, to the serum of the blood. But it differs in different cases, owing to variations as regards the ingredients held in solution. It has usually 
an alkaline reaction. It generally contains albumen in greater or less quantity. According to Lelimann, transudations into the pleural sac are richer in albumen than those into the peritoneal; there is still less albumen in dropsy of the arachnoid than in hydro-peritoneum, and effusions into the areolar tissue are the poorest in this constituent. When albumen is abundant, the liquid is viscid, and it may become so from the absorption of water. Dropsical effusions are sometimes red, from the presence of blood-pigment or hæmatin. Occasionally they are yellow or greenish, from the presence of bile-pigment. Fatty matter may be present in sufficient quantity to render the liquid opalescent or milky. Several of the saline ingredients of blood-serum are present, the chloride of sodium preponderating. Urea is occasionally found.

Dropsies receive different names according to their situation. Seated in the serous cavities, they are designated by prefixing hydro to the name of the membrane, as already stated. Dropsy of the areolar tissue of a part is called odema. This name is applied to effusions into the areolar tissue of internal organs as well as beneath the integument. An effusion into the pulmonary air-cells is called cedema of the lungs. When subcutaneous œdema is general, that is, extending over the body, it receives the name anasarca.

Dropsy is always dependent on some antecedent morbid condition. It is never a primary affection. It is, in fact, not a disease per se, but only a symptom of disease. It is produced by the affections which stand in a causative relation to it, in two ways: $1 \mathrm{st}$, by increased pressure from an accumulation of blood in the veins; and, $2 \mathrm{~d}$, by diminishing the density of the blood-serum. These two physical conditions comprise the mechanism of dropsical transudations. In all instances either there is an increase of hydraulic pressure in the veins from obstruction, or the blood is abnormally aqueons; and both these physical conditions may be combined. Dropsies seated in serous cavities may perhaps involve something more than simple transudation; that is, the effusion may not be purely a physical act. The small amount of liquid which serous cavities contain in health is not derived from the blood by transudation; for, inasmuch as the liquid differs in the different cavities, but is uniform in each cavity, there must be election and new combination of prineiples derived from the blood. Hence, the serous effusions in health are properly secretions ; and it is probable that, in certain eases of dropsy, in addition to mechanical causes, there is hyper-secretion. ${ }^{2}$

The terms general and local dropsy express an important distinction. In general dropsy, subcutaneous cedema is diffused to a greater or less extent; that is, anasarca exists, together with more or less effusion into certain of the serous cavities, the peritoneal and pleural especially. The œdema appears first either in the lower extremities, or on the face, or simultaneously in both these situations, and thence extends over the body. The anasarea may be accompanied with a small or a considerable accumulation in the serous cavities. Now, all cases of general dropsy involve either an obstruction seated at or near the central organ of the circulation, or a morbid condition of the blood, or both may be combined. General dropsy, due to obstruction, is usually a symptom of disease of the heart; occurring in this connection, it is distinguished as cardiac dropsy. The dropsy, under these circumstances, is generally associated with difficulty of breathing (dyspnoea) out of proportion to the amount of effusion into the pleural cavities ; also, with disturbed action of the heart, and more or less conges-

1 Vide Robin, Sur les Humeurs, Paris, 1867. 
tion of the surface, giving to the skin frequently a dusky hue. On the other hand, general dropsy due to a morbid condition of the blood, in the majority of cases, is connected with those affections of the kidneys embraced under the name Bright's disease. Occurring in this commection, it is called renal dropsy. Cases of this variety of dropsy are characterized by the presence of albumen in the urine (albuminuria), by pallor of the surface, and, as a rule, a less amount of dyspncea than in cardiac dropsy. Irrespective of disease of the kidneys, or albumimuria, the blood sometimes becomes attenuated so as to give rise to general dropsy. It is occasionally incidental to protracted intermittents. I have known anasarca to a great degree, with effusion into the cavities, to oceur in connection with anæmia due to lactation, without either renal or cardiac disease.

In local dropsy the effusion is limited chiefly to one serous carity, or, if in the areolar tissue, it is confined to one organ or to a part of the body. The most frequent of local dropsies is hydro-peritoneum, which is dependent on obstruction of the vena portre, generally as a result of hepatic disease, more especially the affection called cirrhosis. This, with other local dropsies, will be considered in comnection with individual diseases; oedema of internal parts, when restricted to any organ or to a limited space, is due to hyperæmia or congestion which may oceur under different circumstances. It may depend on venous obstruction, as when odema of the lungs is produced by cardiac lesions which involve mitral obstruction. It is incidental to inflammation. The swelling in the neighborlood of an inflamed spot on the surface of the body is due to serous infiltration. This occurrence is generally of little moment, but it becomes an event of very grave importance when œdema of the glottis is incidental to pharyngitis. Edema of the lungs is incidental to the congestion distinguished as hypostalic, due to gravitation of blood to the dependent parts from impairment of the forces carrying on the circulation; it occurs in the last stage of fevers or other diseases characterized by adynamia or great depression of the powers of life.

Transudations upon mucous surfaces, constituting fluxes, will be considered as entering into certain individual diseases, in the second part of this work.

\section{Degenerations-Fatty Degeneration.}

The lesions of composition which have been considered do not involve, primarily, alterations of structure, or textural changes. Certain of the products noticed under the head of Exudations, it is true, lead to the destruction of the tissues surrounding them. This occurs in the progress of cancer and tubercle, but the destructive effects of these products are due to pressure, to disrupture from their accumulation, and to the inflammation which they excite. In transudations the structures remain unaffected except that they may become attenuated from the distension occasioned by the liquid. But the lesions now to be noticed are characterized by the substitution of morbid deposits for the normal substance of the affected parts, or by changes in composition arising from morbid infiltrations in the cells or tissues. These are the distinctive features of the lesions of composition which are commonly known as degenerations. And of these lesions, one of frequent occurrence, is the substitution of fat for the normal substance of tissues, or falty degeneration. In the elucidation of this lesion and of other degenerative changes, the microscope, within late years, has rendered valuable service to pathology.

Different structures in various situations are found to present, in the 
place of the substance peculiar to their normal composition, fatty granules or oil-drops. Muscles are especially prone to this change. It is often observed in the muscular walls of the heart. Examined with the microscope, the characters of the muscular fibre are more or less impaired or lost, and those of fatty matter are present. In proportion as this change has taken place, the tissue becomes softened, rupture is liable to occur, and, instead of the healthy color, the affected parts present a yellowish or tawny appearance. Of course, also, in proportion as the muscular substance. is replaced by fat, the function proper to the structure is impaired; its power of contraction is diminished, and the affected organ is enfeebled.

An important clistinction is between fatty growth and fatty degeneration. In fatty growth the adipose tissue is increased. This may take place beneath the integument and in any part of the body containing more or less of this tissue. Increased generally throughout the body beyond a certain extent, the condition known as obesity or corpulency is produced. This may take place in a degree to constitute a disease. On the other liand, a decrease of the amount of adipose tissue which belongs to health occurs in emaciation produced by a deficiency of the alimentary supplies which contribute to fatty growth, and by various diseases. In obesity or corpulence there is, in fact, hypertrophy or abnormal growth of the adipose tissue, and in emaciation there is atrophy of this tissue.

Fatty growth may be limited to a particular organ. Thus, taking the heart for an illustration, this organ in health presents a certain amount of adipose tissue. Hypertrophy of this tissue, or, in other words, increase of the fat vesicles, may occur to such an extent that the whole surface of the organ is covered with a thick layer of fat; the fat resicles may also penetrate, in greater or less quantity, between the muscular fibres. Nevertheless, the muscular structure of the organ may remain unchanged. The heart is affected with obesity ; but this is not fatty degeneration. In fatty degeneration the fatty matter occupies the place of muscular substance; that is, fat is contained within the sarcolemma at the expense of the normal contents or the musculine.

Fatty degeneration of voluntary muscles occurs as a result of protracted disuse. In cases of complete paralysis, after the function of the muscles has been suspended for a long time, fatty granules are found to have taken the place of the muscular substance. The same is true of the nerves involved in paralyzed parts. These structures become, in fact, disorganized in so far as fatty degeneration has taken place, and, in proportion to the amount of this change, their ability to resume their functions is impaired. This fact is of great importance in its bearing on the treatment of paralysis. A notable interruption of the circulation of blood within a part occasions defect in nutrition, and, as a consequence of the latter, it may lead to decomposing changes which are embraced under the name fatty or granulo-fatty degeneration. An example of this is the softening of a portion of the brain-substance as a result of the occlusion of an arterial branch by a fibrinous plug brought from the heart, or by the coagulation of fibrin at the point of the obstruction. The same may occur in other situations.

Fatty degeneration of the coats of the arteries, especially of the minute cerebral arteries, not unfrequently occurs in aged persons and in middle life. This fact, ascertained within late years by the aid of the microscope, has explained the occurrence of apoplectic extravasation of blood in a large proportion of cases. The cohesion and resistance of the arterial coats being diminished by the displacement of their proper substance by fatty granules, they are liable to rupture; hence the occurrence of hemorrhage into the brain, giving rise to apoplexy and hemiplegia. 
Fatty matter is apt to be deposited, in abnormal quantity, within the secreting cells of important glands. Accumulating unduly within the hepatic cells, fatty liver is produced; and, in like mamner, fatty kidney, one of the forms of Bright's disease, is produced by the deposit of fat within the cells of the convoluted tubes. So, also, the epithelium lining the gastro-intestinal tubules and in other situations may be the seat of fatty degeneration.

Morbid exudations undergo changes whieh belong in this category. This is true of the exudation of fibrin, of the morbid products embraced under the name tuberele, and of the scrofulous product. As already stated, a mode in which it is possible for cancer to undergo retrogression and cure is by a fatty degeneration.

An interesting example of fatty degeneration is afforded by the cornea in some persons advancing toward old age. The outer margin of the cornea presents a pale, opaque ring, varying in width and extent, which is familiar to every observer as the arcus senilis. It has been ascertained that this is due to the presence of fat. Canton, of London, who was the first to attribute the arcus senilis to fatty degeneration, considered it as uniformly associated with a similar condition affecting internal parts, and more especially the heart. Hence, he regarded it as possessing much diagnostic value. Clinieal observation does not show it to be reliable evidence of the coexistence of fatty degeneration of the heart or other interual parts, nor, swhen associated with the latter, is it a eriterion of the amount of degeneration, as claimed by Canton; but taken in connection with other circumstances, it possesses a certain amount of diagnostic significance.

A variety of fatty degeneration, affecting especially the larger arteries and the valves of the heart, is called atheroma, a term signifying pap or pulp. This variety is highly important as leading to certain grave accidents and lesions pertaining to the parts affected. The affection consists, primarily, of a deposit beneath the lining membrane of the arteries, or the endocardium investing the valves of the heart, of a substance which presents a yellowish or whitish color, and is of a cheesy consistence. Examined microscopically, this substance is found to be composed of fatty granules, with crystals of cholesterin in abundance, and certain earthy ingredients. The accumulation of this deposit causes atrophy of the structure in contact with it, and may lead to softening and separation of the delicate membrane beneath which it is situated. In this way the parts are weakened, and hence occurs, in the arteries, especially the aorta and the large vessels connected with it, aneurismal dilatation. Situated in the valves of the heart, it may result in rupture, involving serious valvular lesions. It is apt to precede the deposit of calcareous matter in abundance, both in the arteries and within the heart, leading, in the latter, to what has been called ossification of the valves.

The importance of fatty degeneration, as regards its pathological effects, of course depends on the parts affected, together with and extent of the disorganization. In most situations the change of structure is irrecoverable.

\section{Calcareons Degeneration.}

A change in composition, due to an infiltration of earthy salts, in more or less abundance - the phosphate of lime and magnesia, and the carbonate of lime-constitutes what is called the calcareous degeneralion, calcification, or crelefaction. As just stated, this is apt to supervene upon the atheromatous deposit in the large arteries and within the heart, leading, 
in the latter situation, to various deformations of the valves. The term ossification is, in general, incorrectly applied to this change. Virchow prefers the name petrification. The calcareous deposit does not present the structure peculiar to bone, but consists of the earthy salts just named, without any structural arrangement. True bony structure, however, is sometimes produced in situations in which it does not exist normally. Thus produced it is called an osteophyte. The smaller arteries not unfrequently become calcified, especially in advanced life, giving rise, in some cases, to serious consequences. The gangrene of the feet and legs, which is peculiar to old persons, called gangræna senilis, is attributable, in certain cases at least, to obstruction of the circulation from calcarenus degeneration of the distributing arteries of the lower extremities. The arteries of the brain, becoming calcareous and consequently brittle, sometimes give way, and this is the source of the hemorrhage in certain cases of apoplexy and hemiplegia. The obstruction of the circulation due to calcification of the cerebral arteries, may be sufficient to give rise to non-inflammatory softening of the brain, by diminishing the amount of supplies requisite for healthy nutrition. By roughening the inner surfaces of the vessels it may occasion coagulation of the fibrin of the blood, or thrombosis.

Aside from the structures which have been mentioned, various others may be the seat of calcareous deposit. Not unfrequently the serous membranes present plates of bony-like substances, more or less numerous and large. I have met with a case of old pleuritis, in which the pleura had become hardened by calcareous deposit to such an extent that it was removed entire, together with several quarts of liquid which it contained, and, when open, and the liquid removed, it did not collapse, but retained the form of a solid box. Exudations and morbid growths may undergo the calcareous degeneration. This occurs in simple fibrinous exndation, in cancer and in tubercle. Ths pulmonary calculi, or obsolete tubercles, are masses of earthy salts which have taken the place of miliary tubercles.

\section{Lardaceous or Waxy Degeneration.}

Another change in composition has received several names, to wit, the lardaceous, waxy, albuminoid, and amyloid degeneration. 'The distinctive feature of this degenerative change consists in an infiltration by a morbid material, the nature of which is not yet satisfactorily ascertained. This material first appears in the walls of the small vessels, and thence pervades the adjacent structures. Affected parts are increased in volume and weight; there is also a notable increase of firmness or stiffness; the surfaces on section present a dull, translucent appearance, resembling fat bacon and wax, and hence, the significance of the terms lardaceous and waxy.

Wagner' found this lesion in 48 of 1200 autopsies. The organs which are most apt to be affected are the liver, the kidneys, the spleen, and the lymphatic glands; but it may be seated in any of the soft parts of the body. The names albuminoid and amyloid imply different views as to the nature of the infiltration. It is generally considered to be an albuminoid product, that is, resembling albumen. Virchow was led to suppose that it is a substance analogous to starch, or to cellulose, as expressed by the name amyloid. The evidence relied upon for the latter supposition consists in the change of color produced by iodine. If a little sulphuric acid be first cautiously added, the application of iodine is followed by

Archiv. der Heilkunde, 1861. 
either a blue or violet color. The iodine test is generally considered insufficient as evidence of the amyloid character of the substance, and very few at the present time accept the conclusion of Virchow. Iodine, without the previous application of sulphuric acid, however, is a test of the anatomical change under consideration: applied to healthy parts it gives a yellow tinge, whereas, a dark-brown color is produced if this change exist. With the present uncertainty respecting the nature of the material, the names lardaceous or waxy, which relate to the peculiar appearance of the affected parts, are to be preferred.

The lardaceous or waxy degeneration produces injury, in proportion to its amount, by deforming cells, encumbering tissues, and diminishing the calibre of the smaller arteries. The deposit does not undergo softening. Its presence does not appear to excite inflammation. It is doubtful if it be ever absorbed. The change is followed by serious consequences according to the parts affeeted, together with the degree and extent of the infiltration. It leads to evil results by obstructing the circulation in, and compromising the functions of, the parts affected. For example, in the kidneys, the elimination of urea is interfered with, and, if the infiltration be great, uramia may be the result. In like mamner, if the liver be affected, the functions of this organ are impaired in proportion to the amount of infiltration. Affecting thus an important organ, the lesion is of a grave character, more especially if it be permanent. According to Dr. Wilks, who analyzed nine cases oecurring under his own observation, the lardaceous or waxy infiltration implies a long standing and deep cachexia, which is seen in its most intense form after protracted caries and necrosis of bone, having its origin in tuberculosis or syphilis. In the great mass of cases it is associated with disease of bone, syphilis, or scrofula. Of sixtysix cases analyzed by Dickinson, fifty-one were associated with a profuse and long-continned drain of pus. It seems, in some cases, attributable to the malarial cachexia.

Interesting and important changes in the composition of parts are caused by the deposit within them of pigment derived from the blood. In general, the coloring matter in pigmentation, as it is called, is altered hematin from the blood-globules. An exception is the bile pigment; although this is hardly exceptional if it be true that the biliverdin is a product of the destruction of the blood-globules. Pigmentation will be referred to in connection with the morbid condition of the blood known as melanæmia, and, in the second part of this work, in connection with malarial fevers, Addison's disease, diseases of the lungs, and jaundice.

\section{New Formations or Morbid Growths.}

Changes in composition, of great practical importance, proceed. evidently, from new formations, neoplasms, or morbid growths. Some of the morbid prodncts already considered properly belong in this category, namely, gray tubercle and carcinoma; but, for the most part, the new formations or morbid growths now referred to are embraced under the head of tumors. They belong to surgical rather than medical pathology, inasmuch as they are often situated either externally or where they are accessible to manual examination, and may be removed with the knife or are treated with the appliances of surgery. This division of anatomical changes I shall therefore pass by, with scarcely more than a simple allu. sion to it, referring the reader to surgical treatises or to works devoted to morbid anatomy. Morbid growths have been heretofore considered by 
pathologists as consisting of analogous and non-analogous structures; the first being morbid structures, either identical with, or analogous to those found in the healthy body, that is, normal, and the second being structures essentially abnormal, in other words having no analogues among the healthy tissues of the body. As expressive of this distinction, the terms omologous or omorphous, and heterologous or heteromorphous, have been applied to morbid growths, as well as to morbid exudations. There is probably no just ground for this distinction. The so-called new formations are either normal structures abnormally situated, or the normal anatomical elements of a part developed and multiplied to such an extent as to constitute morbid growths or tumors. Virchow uses the term homologous or họmœoplastic, as applied to morbid growths, to denote those, the structure of which is identical with that of the parts in which they are developed; and he uses the term heterologous or heteroplastic to denote growths differing in structure from the parts in which they are seated. Examples of the latter are epithelial tumors in the walls of the heart, tumors of cerebral substance in the ovaries, cartilaginous growths in different situations, ete. The term Helerotopia is used to denote productions normal in character, but abnormal in situation. The division by Robin of the anatomical components of the body into fundamental and accessory elements renders intelligible the production of many morbid growths or tumors. The fundamental elements are those which are endowed with known functions, and which make up the greater portion of the body, viz., the muscular, nervous, osseous, glandular, etc., structures. 'The accessory elements are certain superadded constituents, the purposes of which are often not apparent. Among the accessory anatomical elements are various cellular or corpuscular bodies. Examples of the latter are the embryoplastic cells and nuclei of Robin, called also fibroplastic; cells found in the mucous tissue, the skin, serous membranes, and the lungs, called by Robin cyloblastions; myeolocytes, or cells found in the substance of the brain, the spinal eord, and in one of the beds of the retina; medulla cells, and myeoloplaxes, cells in the marrow of bones, etc. Now, these accessory elements are liable to be multiplied to a greater or less extent, and they are found to form the predominant and characteristic constituents of certain morbid growths or tumors. They do not grow, but they are multiplied; that is, the augmentation is of number, not of size. Hence, the proper designation of the process by which morbid growths and tumors are in this way produced is hypergenesis, or, after Virchow, hyperplasia. Thus, according to Robin, eytoblastions are found in abundance in certain mucous vegetations, in the gray semi-transparent granulations of the lungs, in the so-called tubereular or granular ineningitis, in the affection of the eyelids called chalazion, and in syphilitic tubercles; myeolocytes constitute the anatomical characteristic of certain affections of the retina, and of certain tumors of the brain, often called erroneously cancerous; fibroplastic cells give a distinctive feature to various tumors often confounded with cancer; myeoloplaxes are the point of departure for osteo-sarcoma, etc. According to Virchow, new formations or neoplasms are, in general, due to an abnormal proliferation of cells belonging to the connective tissue.

The different classes of tumors are designated by names expressive of their anatomical composition and disposition, to which is added the suffix

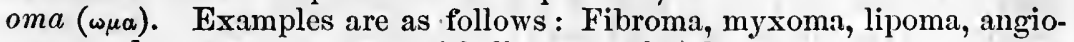
noma, adeñoma, cystoma, epithelioma, enchondroma, myoma, osteoma, carcinoma, neuroma, names denoting respectively fibrous, mucous, fatty, 
vascular, cystic, glandular, epithelial, cartilaginous, muscular, osseous, cancerous, and nervous tumors.

\section{Pneamatoses.}

In connection with morbid changes in the composition of parts, may be appropriately noticed those which depend on the presence of either air or gas in abnormal situations, and their abnormal amount in places in which they exist to a certain extent in health. Morbid conditions of this kind are collectively called pneumatoses. Air or gas diffused within the meshes of the areolar or connective tissue constitutes the morbid condition known as emphysema. This term is also applied to the over-accumulation of air in the air-resicles or cells in connection with their dilatation, eonstituting an affection called pulmonary or vesicular emphysema, which will be hereafter considered. Extravasation of air into the areol:ur or connective tissue which unites together the lobules of the lungs is distinguished as interlobular emphysema. The latter affection is due generally, if not always, to rupture of the air-tubes or cells. Subcutaneous emphysema extending more or less, and sometimes existing to such an extent as to increase enormously the volume of the body, is caused by rupture or a wound of the lungs or air passages, the movements of respiration forcing the air through the aperture, and diffusing it by means of the extensive relations of the areolar tissue. But gas may be generated within the areolar tissue of a part by putrefaction or other chemical changes. I have known emphysema of the leg to be produced in connection with suppuration and the formation of abscesses. This may occasionally occur in internal organs. In some eases of perforation of the intestine, the latter becoming adherent to the abdominal walls, diffuse cellulitis follows, and intestinal gas may be more or less diffused beneath the integument.

Air or gas may accumulate in serous cavities, either introduced from without in consequence of perforation, or generated within by chemical changes, or possibly by a proeess of secretion. Affections of serous membranes charaeterized by this feature are distinguished by the prefix pneumo; thus, pneumo-thorax, pneumo-perieardium, ete.

The different sections of the alimentary canal contain a variable quantity of gas in health. A morbid inerease gives rise to flatulency and tympanites. The former term is applied when the evidence of a morbid amount of gas is afforded by its movements (borborygmus) and its expulsion either by the mouth or anus; the latter, when it is retained suffieiently to cause distension with angmented resorance of the abdomen on percussion. Generally the gas is generated by chemical changes in the contents of the canal ; but it is diffieult to account for its production always in this way, and, hence, it has been inferred that gas may be secreted from a mucous surface. The latter supposition seems to be necessary in order to account for the large amount of gas suddenly developed in cases of hysteria and other nervous affections. Air may be swallowed in large quantity, as it is by horses affected with the habit called wind-sucking or crib-biting.

\section{Animal and Vegetable Parasites.}

Finally, in this division of anatomical changes may be included animal and vegetable parasites. IIere is a field in pathology which modern researches have shown to be extensive and highly interesting, but which must be passed by with a very eursory notice.

Microscopical examination of certain morbid products obtained from 
the skin and mucuos surfaces shows vegetable structures belonging to the series of cryptogamia, or flowerless plants propagated by spores, and to the class called Thallophytes, which includes lichens, alga, and fungi. Various species of algæ and fungi have been ascertained, some of which are characteristic, and thus constitute diagnostic criteria of certain forms of disease. I must content myself with a simple enumeration of the different species found first on the skin, and next on mucous surfaces. A species of fungus (trichophyton tonsurans) is found about the hair follicles in the affection of the scalp known as herpestonsurans. Another species (microsporon audouni) exists, in the same situation, in the affection of the scalp which has been called porrigo decalvans, or alopecia circumscripia. Another species (achorion Schœrleinii) belongs to the disease, affecting especially the scalp, but sometimes other parts of the skin, called favus, or the true tinea capitis. Another species (microsporon mentagrophytes) characterizes sycosis or mentagra, a disease affecting those portions of the face and neck which are covered with hair. Another species (microsporon furfur) exists in that variety of squamous disease, affecting different portions of the skin, but especially over the chest and abdomen, called pityriasis versicolor vel nigra. The presence of the cryptogamic plant appears to constitute an essential pathological element in these several contagious affections, and they are probably communicated by means of the germinal bodies called spores. The treatment of these affections involves measures to effect the destruction of the parasitic vegetable.

On mucous surfaces the following species have been ascertained: In aphthæ or thrush (muguet) and in diptheria, a fungus (oidium albicans) affecting the mouth, csophagus, intestines, and anus. The algous vegetable known as the yeast plant (crytococcus cerevisiæ) in the osophagus, stomach, and intestines. Another species of alga (merismopodia ventriculi), commonly know as the sarcina ventriculi, found in matters vomited, also in the fieces, and in gangrenous abscess of the lung. Another species (Leptothrix buccalis), found in coatings on the tongue and matter collecting between the teeth, a species of fungus found in the matter of expectoration in cases of tuberculosis described by Bennett.'

Animal parasites are divided into those inhabiting the exterior of the body, and those infesting internal parts. The former are called epizoa and the latter entozoa. Under the head of epizoa are included the insects which invade different portions of the surface and multiply in consequence of neglect and filth, without being associated with disease, viz., the flea, the head-louse, the body-louse, the crab-louse ; together with certain animals which penetrate the skin and give rise to more or less local inflammation, such as the West India chigoe. In addition to these, the cutaneous disease known as scabies, or vulgarly called itch, is characterized by the presence of a parasite called the acarus scabiei, or itch animalcule. It is by means of the transference of these minute insects, or of their ova, that this disease is communicated from one person to another. Another animalcular parasite called the acarus folliculorum inhabits the hair follicles and sebaceous glands, especially on the face, in healthy persons.

The human entoza are numerous, and much important information has been obtained, within late years, respecting their production and development. For this, the reader must consult more comprehensive pathological

1 The student desirons of studying the subject of vegetable parasites may consult Robin's treatise entitled IIistoire naturelle des végétaux parasites qui croissent sur l'homme et sur les animaux vivants. The nomenclature of Robin has been followed in the above enumeration. 
treatises, or works specially devoted to Helminthology. Suflice it to say that there are no longer grounds for maintaining the doctrine of equivocal generation, a doctrine which has heretofore been held by some. These parasites originate from either ova or animals which are introduced into the body from without, and which find in certain parts of the organism the conditions necessary for their development and growth. Certain of the entozoa are involved in individual diseases, and will be noticed hereafter in treating of the latter. In the following list of the more important of the entozoa of man, they are arranged in their proper zoölogical divisions. Helminthia hematoidea, thread or round worms: Filaria medinensis or Guinea-worm, found in the areolar tissue ; fllaria bronchialis, in the bronchial glands ; filaria oculi, in the crystallinc lens; Tricocephalus dispar, in the cæcum; spiroptera hominis, in the urinary bladder; strongylus gigas, in the kidneys; ascaris lumbricoides, in the small intestine; ascaris alata, in the small intestine; oxyuris vermicularis, in the rectum; anchylostoma duodenale, in the duodenum; trichina spiralis, in the muscles. Of this division, the worms which are chiefly important in a practical view are the trichina spiralis, ascaris lumbricoides, or lumbricoid, and the oxyuris vermicularis, or vulgarly pin-worm. The others are comparatively unimportant to the practitioner, either on account of their infrequency, or from their giving rise to little or no inconvenience. Trematodia, or worms with organs for suction: Distoma hepaticum, in the biliary ducts; distoma lanceolatum, or liver fluke, in the same situation; distoma oculi, in the crystalline lens; distoma hæmatobium, in the vena portæ. Cesroldea, or tapeworms: Botriocephalus latus, or tænia lata, in the small intestine; tænia solium, in the same situation; tænia nana, in the same situation; cysticercus cellulosus, in any of the organs or tissues of the body ; echinococcus hominis, in hydatid cysts found in the liver, ovaries, and other parts.

The trematoda or suction-worms are of rare occurrence in man, and comparatively unimportant. Those found in the bile-ducts are not uncommon in inferior animals. The intestinal cestoid or tapeworms are not very infrequent, and are of importance to the physician. They will be considered hereafter. The cysticercus and echinococcus are now shown to be essentially cestoid or tapeworms, which, from their situation within the solid structures of the body, fail to become developed; they are, in other words, immature tænia. The sacs or cysts, frequently of large size, which contain echinococci in greater or less number, are called hydatids or acephalocysts. The history of these, and of the cysticerci, although embracing points of much interest and practical importance, is not consistent with the scope of this work. 


\section{CH A P T E R I V. \\ MORBID CONDITIONS OF THE BLOOD.}

General Remarks-Classification of the Constituents of the Blood-Morbid Conditions relating to the Organized or Corpuscular Element-Plethora-Anæmia-Apnoa-Leucocythemia.

$\mathrm{H}$

A VING sketched in the preceding chapters the anatomical changes produced by disease in the solid parts of the body, morbid conditions of the fluids next claim attention. 'The fluids of the body, in health, consist of, first, the blood, and, second, various liquids separated or formed from the blood, constituting exhalations, secretions, and excretions. The latter class of fluids, viz., those derived from the blood, are liable to numerous morbid changes. These are often of intrinsic importance, and they form important events or symptoms in the history of different individual diseases. It will be sufficient to consider the morbid changes occurring in the exhaled, secreted, and excreted fluids, in conneetion with individual diseases in the second part of this work. But the morbid conditions of the blood are to be considered briefly by way of preparation for entering on the study of individual diseases.

As expressive of the importance of the blood, it is distinguished as the vital fluid. In literature and common parlance it represents life. "Life's blood" is a common expression. To have one's blood is to take life. The importance of the blood is shown by the fact that its presence in all the so-called vital organs is indispensable to the exercise of their functions. A striking and familiar illusiration of this fact is afforded by the temporary loss of the mental faculties and consciousness as a result of a momentary arrest of the supply of blood to the brain in syncope or fainting. The blood, in fact, may be said to be the grand condition of vitality. Its detention from a part occasions the molecular death of the part, $i . e$. , gangrene or sphacelus. Its abstraction, beyond a certain limit, from the body oceasions general or somatic death. The suspension of its distribution by an arrest of the heart's action for two or three minutes only is fatal. It forms a vital medium for all the organs essential to life, on which they are dependent, as the body or the blood itself is dependent on the surrounding atmosphere. The physiological relations of the blood to the solid parts being so intimate, it might reasonably be expected, $\grave{a}$ priori, that pathological changes in this fluid should give rise to corresponding morbid phenomena in the organs and tissues of the body. Observation shows this to be true. There are grounds for the belief that a large proportion of the morbid actions and changes which oceur in the solid parts are due to prior alterations in the blood. In many instances, as will be hereafter seen, the dependence of the former on the latter may be inferred or rendered probable, although not demonstrable with our existing knowledge. Supplies for the growth and repair of the whole body are contained in the blood. This fluid, therefore, represents in its constituents all the elements which enter into the composition of all the solid parts. It is taking but a step from the prosaic walks of scientific fact to the domain of fancy to say that the blood is the solid body in a liquid state. "The blood is the centre round which the general metamorphosis of animal 
matter revolves and in which it is perfected." It might be rationally predicted that morbid alterations in its composition and distribution lead to diseases seated in the solids, and this will be found to be the case. Another aspect foreshadowing the importance of the blood in its pathological relations, is its office as a reservoir for the accumulation of effete principles, the detritus of the tissues, which are to be climinated by excretion. Here is a source of disease, as will be presently seen. Again, the pliysiological activity, or mobility, of the blood is very great. In this respeet it is in striking contrast to the solid parts. It is the seat of unceasing changes, and yet, in health, maintains a uniform state as regards its organization and composition. New matter derived from the ingesta is daily added in considerable quantity, and a proportionate amount is derived from the decomposition of the tissues. I'ortions are appropriated by the clifferent structures. Other portions are secreted for various useful purposes in the economy. Other portions are thrown off or exereted. 'There is a constant interehange of gascous elements with the surrounding atmosphere by means of respiration and through the cutaneous surface. 'Thus it is the seat of constant and great changes, denoting wonderful aetivity, and yet its constitution remains the same. In this fact are admirably exemplified the precision and adaptation of the laws presiding over the safety and welfare of the organism. But this activity necessarily renders it more liable to morbid actions and conditions than the solid parts which in health are less active and more stable.

The blood is a complex fluid. It contains a large number of ingredients, preserving, however, certain fixed anatomical charneters. Anatomically considered, it consists of certain corpuseular bodies, viz., the red globules, the white globules or leucoeytes, and globulins which are suspended in a liquid called the liquor sanguinis, lympha sanguinis, blood-plisma, or inter-cellular fluid. These are resolvable by analysis into numerous elements, some of which are organic, $i . e .$, peculiar to organized bodies, and others inorganic or mineral. Examples of the organic elements are fibrin, albumen, hæmatin, etc. The inorganic elements embrace various saline ingredients, iron, water, and several gases. Further details belong to anatomy and physiology. It is necessary thus to glance at the composition of the blood in order to arrange its morbid conditions. 'The morbid conditions relate to the different corstituents of the blood, and, with a view to the consideration of pathological changes, these constituents may be distributed into three groups. 'The first group will embraee the corpuscular, distinguished also as the organized, constituents. The second group will consist of the organic elements. The third group will comprise the mineral substances. Morbid conditions affecting severally these three groups will be first considered in the foregoing order, and, afterward, morbid conditions due to the introduction into the blood of substanees which do not enter into its normal composition. Even with our present imperfect knowledge of the blood in health and disease, it is, in itself, a large field of study, which, considered as a distinct province of medieine, is called Hæmalology.

Of the corpuscular or organized constituents of the blood, the most abundant and important are the red globules. The known morbid ehanges affecting these relate, in the first place, to their number. They may be morbidly increased or diminished in number. An inerease of the number of red globules beyond the healthy limit constitutes the morbid condition called plelhora. A diminution below the limit of health constitutes the morbid condition called anæmia. 


\section{Plethora.}

The relative proportion of red globules to the other constituents of the blood may be increased by diminution of the latter. This obtains, in a marked degree, in epidemic cholera, owing to the draining away, through the intestinal canal, of the water of the blood, together with various elements held in solution by the transuded liquid. The density of the blood in this disease is notably increased; it becomes thick and heavy, and the circulation is mechanically impeded. Under these circumstanees, the red globules are relatively in abnormal excess, although they are actually less in number than in health. The term plethora is only applicable to an actual increase of the number of the red globules. This is now the significance of the term, without regard to the quantity of the mass of blood. An increase of the mass, causing over-repletion of the vessels, does not constitute plethora, although implied in the etymology of the term. This condition is called polyæmia. Its existence to an extent suflicient to constitute a morbid condition of importance is doubtful.

'The functions of the red globules in health are not fully understood. Their relative normal ratio to the other constituents of the blood differs considerably in different animals. They appear to sustain a certain relation to vigor, strength, and activity ; that is, they are abundant in races, breeds, and individuals, in proportion as the general attributes of the body just named are marked. Their importance is shown by the fact that animals bled nearly to death may be reanimated by injecting into the veins red corpuscles suspended in serum, and not by the introduction of the other constituents of the blood without the red corpuseles. From what is known of their physiological relations, it might be inferred that the effects of their morbid excess would be over-activity of the circulation and undue excitation of organs in proportion to their normal aetivity and the quantity of blood which they receive in health. The phenomena of plethora denote these effects. The power of the heart's action is inereased; the temperature of the body is raised; the brain is stimulated, giving rise to unusual mental energy and excitement. Sensibility and museular irritability are augmented. In comparing, however, different persons, it is not easy to draw the line of demareation between more or less intensity of the so-called sanguine temperament and plethora. A better idea of plethora, as a morbid condition, is formed by a comparison of the same person at different periods, and especially if the person have naturally a temperament not notably sanguine. He acquires more color in the prolabia and face; the mucous membranes are reddened; the pulse is full and strong; the heart's impulse is increased; the physical and mental powers are more active; the body is notably warm. Pain in the head is readily induced by stimulants or mental excitement, owing to the abnormal power of the circulation. This condition involves a liability to active cerebral congestion. It is supposed to constitute a predisposition to acute inflammations. It doubtless tends to render inflammations more intense, and to increase the symptomatic febrile movement. It may favor hemorrhages especially into the brain, by means of the increased force of the circulation. On the other hand, an abundance of red globules exempts from nervous disorders to which, as will presently be seen, a pancity of red globules predisposes.

The causes of plethora are, first, a constitutional tendency which may be congenital or inherited; second, over-feeding, with the use of generous wines and condiments; third, diminished expenditure of blood constituents in nutrition, incident to ease, idleness, and luxurious habits, the digestive and assimilative functions remaining active; and, fourth, the arrest of 
periodical or habitual hemorrhages, or some other drain to which the system had become accustomed. These several causes are frequently combined.

It is important for the physician to appreciate the condition of plethora, in order to avert, by appropriate management, the evils to which it tends; and, as an incidental element in different diseases, it is to be taken into account in considering the effects of therapeutical measures. It is relieved, for the time, most promptly and efficiently by bloodletting. An immediate effect of the abstraction of blood is a notable reduction in quantity of the red globules. Of course, the propriety of resorting to bloodletting will depend on the degree of plethora and the apparent inminency of evil results. Other means to diminish the excess of red globules are, a reduced diet as regards the quantity and quality of food, and exereise in order to increase the expenditure of blood-elements in repairing muscular waste and to render the amount of eliminated matter more abundant. Certain medieines appear to exert a direct effect upon the number of red globules. Mercury is such a remedy, as shown by the pallor which accompanies salivation. Mereurialization, however, is never indicated for the attainment merely of this object.

It is important not to confound plethora with other morbid conditions of the blood or circulation. Fulness of the vessels, due to some impediment to the circulation, has not unfrequently been considered as plethora. 'This may exist when the red globules are diminished, rather than increased. A pseudo-plethora, for example, is not uneommon in pregnaney, the red globules being diminished, and the proportionate quantity of water being notably increased, in this state. Bleeding was formerly employed with reference to this pseudo-plethoric condition, of course with an injurious effect on the constitution of the bloor. With pseudo-plethora, or fulness of the vessels, there is often evidence of deficient oxygenation of the bloorl, together with dulness and oppression, instead of heightened activity of the functions of the brain and other organs. True pletliora is to be determined by the symptomatic phenomena which have been mentioned, taken in connection with the evidence afforded by the pulse, and other symptoms, of an unobstructed, free circulation, with the activity of the digestive and assimilative functions, and the existence of one or more of the conditions under which this morbid.condition is known to be produced. A microscopical examination of the blood may suffice to determine the existence of plethora, if the observer be sufficiently practised to decide whether the red globules, in several successive speeimens, are in excess or not. It may be determined by a quantitative analysis, but the proeess is too tedious and delicate for ordinary clinical purposes.

As regards the essential pathological nature of plethora, all that can be said is, it consists in a hypergenesis of the most important of the organized or corpuscular constituents of the blood, the red globules. The pathologist might expect to explain this morbid condition more fully, if the physiologist were able to tell us where and by what process the red globules are normally produced.

\section{An mia.}

A morbid diminution of the red globules of the blood constitutes anæmia. The etymology denotes diminution of the mass of blood, but conventionally, the term is used to signify reduction of the quantity of red globules. Spanxmia is sometimes used in the same sense. In this definition of anæmia the sense of the term is restricted to a morbid condition of the 
blood. The term is also used to denote a deficiency of blood, and especially of arterial blood, in different organs. Thus, it is customary to say, anæmia of the brain, of the liver, ete. In this sense the term denotes a condition the opposite of hyperæmia or congestion. Ischæmia is a term used to denote a local anæmia. We are here to consider anæmia as a morbid condition of the blood.

The purest exemplification of anæmia is afforded by cases in which it has been produced by copious hemorrhages or repeated bloodlettings. It is not easy to effect, except for a transient period, a considerable reduction in the mass of blood. After a loss by hemorrhage or bloodletting, the quantity of liquid which has escaped is quickly replaced, but the red globules are not so speedily renewed, and, henee, the latter continue, for a greater or less period, to be deficient. 'This condition is one of the forms of so-called impoverished or poor blood. The degree of impoverishment varies. The proportion of red globules has been observed to fall below the normal range (120 to 130 in 1000 parts) to 70,60, and even 21 to 1000 parts.

Anæmia is of frequent occurrence. It is incident to a variety of diseases. It gives rise to a multiplieity of morbid phenomena. It is a condition highly important for the physician to appreciate and recognize. The knowledge of this condition, obtained within late years, constitutes one of the most striking of the characteristies of modern medicine, in view of its importance in medical practice. 'The condition occurs much more frequently than its opposite, viz., plethora.

In general terms, the pathological effects of anæmia are the reverse of those due to plethora. The power of the circulation is diminished, and there is a deficiency of the functional energy of different organs; the latter is more marked in different organs according to the quantity of blood which they receive in health. The phenomena denote these effects. The temperature is lessened. Anæmic patients have coolness of the surface, and especially cold extremities. They are not so able to resist eold as the plethoric: The action of the heart is feeble ; the pulse is small, weak, compressible. The action of the heart is easily disturbed, becoming rapid from slight causes, and frequently irregular. The mental energy is diminished; persons are not adequate to the intellectual efforts of which they are capable in health. The strength of will and determination of purpose are impaired. The vital functions are languidly performed. The muscular strength is diminished; fatigue follows moderate or slight exercise. The surfice is generally pallid from the deficiency of the hæmatin or coloring matter contained in the red globules. This pallor is apparent in the face, and especially the prolabia. The mucous membranes accessible to view have less redness than in health. The countenance may at once denote the existence of anæmia if the condition be marked. It is, however, important to state that in some persons who have an unusual vascularity of the face, anæmia may exist, as determined by physical signs and the pathological effects, not only without notable pallor, but even with a rosy complexion. In some cases pallor of the prolabia is noticeable in the morning, or when the patient is fatigued, disappearing under excitement. The urine frequently has a low specific gravity; owing to a deficiency of urea; and it is often notably limpid from a deficiency of pigment, a fact which may be cited as going to show that the coloring matter of the urine is derived from the red globules of the blood.

It induces a multiplicity of morbid phenomena, arising from disordered action of the nervous system. The relations of the blood to the functional activity of the nervous system are strikingly shown in the phenomena 
pertaining to the latter, which spring directly from morbid conditions of the former. And the special relations between'the red globules and the nervous system are shown by the phenomena ineident to anæmia. 'These phenomena are numerous and diversified. The more frequent and prominent are as follows: Mental depression, anxiety respecting health, hypochondriasis, irritable temper, want of buoyancy and energy, a feeling of lassitude, and a painful sense of inertia or indolence. There is ajt to be a feeling of incapacity for muscular exertion greater than the actual loss of muscular power. The physieal and mental powers are especially depressed during the process of digestion. Palpitations frequently oceur, so that organic disease of the heart may be suspected by those not conversant with physical means of diagnosis, and is greatly feared by the patient. Neuralgia in various situations is apt to occur, and in females hyperæesthesia of the abdominal walls, simulating peritonitis. 'The varied symptoms which have been heretofore described as belonging to spinal irritation, are likely to occur in connection with anamia. It sustains a causative relation to nearly all the functional affections of the nervous system embraced under the head of the neuroses. A large proportion of persons affected with any one or more of this class of maladies are anxmic; and, conversely, a large proportion of anæmic persons become affected with neurotic disorders. It is highly important that this pathological element be taken into account in the management of the neuroses. When it oceurs independently of the various affections with which it is connected incidentally, it is characterized especially by phenomena relating to the nervous system. These phenomena may be said to constitute the pathological expression of this morbid condition of the blood.

If it be asked, what is the explanation of the occurrence of these phenomena in consequence of a diminution of the red globules, the pathologist can only say that he may hope to answer the question when the physiologist is able to explain the normal relation between the presence of the red globules and the functions of the nervous system. Patlological facts show that an essential relation does exist between these two anatomical elements of the body; the nervous system depends on this blood-constituent for the manifestations of healthy life, and, hence, a deficiency occasions manifestations of disordered life, or morbid vital phenomena.

The causes of anæmia, when it exists independently of the various affeetions with which it is associated, are frequently obvious, but in some instances they are not assignable. It is a result of hemorrhages from wounds, flooding after labor and in cases of menorrhagia, or of injudicious bloodletting. It may proceed from deficient alimentation; the food being insufficient in quantity or not sufficiently rich in alimentary principles. It is caused by a loss of certain of those elements of the liquor sanguinis, or blood-plasma, which are necessary to the production of red globules. Thus, frequent causes are prolonged lactation, and a rapid succession of pregnancies. The obvious causes may be arranged into three elasses just stated, viz., First, eauses which involve an actual loss of red globules, as in hemorringes; second, causes involving a defective supply of materials for assimilation; and, third, causes which occasion expenditure of those constituents of the liquor sanguinis on which the production of red globules is dependent.

The causes are not always apparent. Anæmia is apt to oceur in females at or near the age of puberty, when there has been no loss of blood, no deficiency in alimentary supplies, and no unusual expenditure of bloodplasma. Under these circumstances, it is the prominent feature of the affection to which the name chlorosis was applied before the anxmic 
condition was fully understood. If this name be retained, it should be considered as denoting anæmia occurring under the circumstances just stated. It appears to be in some way connected with the evolution of the reproductive functions. In some cases it may be accounted for by the derangement of the assimilative functions at this period. In these cases the appetite is poor, the digestion disturbed, and there is apt to be a craving for indigestible, innutritive substances, such as chalk, slate, coal, etc. Addison has described cases of anæmia occurring without any obvious causation, accompanied by general debility which progressively increases, at length ending fatally without appreciable lesions of any of the vital organs. Cases of this kind are occasionally met with, especially in hospital practice. Addison distinguishes them as cases of "idiopathic fatal anæmia." In a certain proportion of these cases, the surface of the body, to a greater or less extent, assumes a dark discoloration or a bronzed appearance, and in several successive cases the suprarenal capsules were found to be more or less disorganized. Addison inferred from these facts a pathological connection between disease of the suprarenal capsules, and the bronzed hue of the skin. Clinical observation, however, shows that the two events are not uniformly associated.

In a large proportion of the eases in which anæmia exists, it is incidental to, or a pathological element of, some other affection. And, as thus associated, it may, or may not, claim the special attention of the practitioner. Of the great number of diseases with which it it conneeted, either constantly or frequently, the following list will embrace the more prominent :-

1. Tubereulosis.-Anæmia is generally early developed in tuberculous affections, and may preeede the deposit of tuberele.

2. Carcinoma.-The pale, waxy, or straw-colored complexion which characterizes some eases of careinomatous disease, denotes anæmia.

3. The affections embraced under the name Bright's disease.-A ssociated with odema of the face, the pallid complexion of anæmia becomes quite characteristic of these affections. The blood-changes which belong to these affections (to be hereafter considered) lead to diminution of the red globules.

4. A host of affections which involve expenditure of other constituents of the blood than the corpuscles, $i$. e., constituents of the liquor sanguinis. Such as chronic dysentery and diarrhœe, chronic pleurisy, purulent formations in any part of the body, leucorrhœa, etc.

5. Affections which involve loss of corpuseles, or hemorrhage, viz., menorrhagia, hemorrhoids, hæmatemesis, etc

6. Affections compromising the assimilative functions by occasioning indigestion, vomiting, loss of appetite, etc.

7. Certain affections of the liver, and especially cirrhosis. It has been supposed that the red globules are produced within the liver. If this be true, diseases of this viscus may lead to their diminution by interfering with their production. But in eirrhosis this effect is due, in a measure, to the obstruction to the introduction of fresh alimentary supplies brought by the portal vein.

8. The periodical fevers, if protracted. The special cause of these fevers may induce anæmia even where the fevers are not developed. Persons inhabiting regions caller malarious are apt to become anæmic, although they do not experience fever.

Certain mineral substances introduced into the system lessen the red globules in a notable degree. This is true of lead. Anæmia is a pretty constant element of saturine diseases; and it is observed in persons exposed to lead emanations before becoming affected with the characteristic 
diseases. The same is true of mercury. Mereurialization quickly reduces the quantity of red globules in a marked degree.

The diagnostic criteria of anæmia are: First, deficiency of the normal eoloration of the surface, due to the coloring principle which belongs to the hrematin in the red globules. The defective color, or pallor, is generally manifest on the ears, the face, and especially the prolabia; it is also apparent on the inner surface of the mouth and on the tongue. Second, weakness of the vital organs, denoted by symptoms which observation shows to be associated with pancity of the red globules. Third, more or less of the phenomena pertaining to the nervous system which have been mentioned. Fourth, the presence, frequently, of obvious adequate causes, such as loss of blood, lactation, frequent child-bearing; or, of affections, to the natural history of which anæmia belongs. Fifth, the microscopical appearance of the blood, the red globules appearing to be fewer than in bealth. Sixth, the evidence afforded by a quantitative analysis; but this is too difficult to be available for ordinary clinical purposes.

To the foregoing are to be added the following physieal signs. A bellows murmur at the base of the heart and in the larger arteries, carotid, the subclavian, etc. This murmur accompanies the first or sy'stolic sound of the heart, and is usually soft. To constitute evidence of anæmia, there must be wanting the signs of organic lesion of the heart and large vessels. In conjunction with this murmur, a continuous humming sound, sometimes musical, is heard when the stethoscope is applied over the neck, especially on the right side, due to the movement of the blood in the veins, ealler the venous hum, or, after the French, the bruit de diable. These murmurs are distinguished from those denoting lesions, as inorganic or auæmic murmurs. The arterial muruur is present in only a certain proportion of cases; but the venous hum may be found almost invariably, in cases of ansemia, if certain rules be olserved in looking for it. The rules referred to are the following: the patient should be sitting or standing with the head turned as far as possible to the left without turning the borly, and the stethoscope is to be applied posterior to the sterno-cleidomastoid muscle. Observing these rules, my experience has led me to consider the venous hum as a very reliable sign of anæmia; that is, it is almost invariably present if anemia exist, and it is generally winting if anzemia does not exist. Its disappearance, when cases of anæmia are under treatment, is evidence of the removal of the anæmic state, and in this way attention to this sign is of practical value.

With reference to therapeutics, anæmia constitutes a special indication for treatment when it occurs independently of other affections, or when it is associated, as a prior and causative morbid condition, with disorders of the nervous system. When it is an element of other affections, it contributes, in a greater or less degree, to their pathological effects, and claims a certain share of attention. When it exists alone, or with disorders to which it has given rise, it is generally a remediable condition; but it is otherwise when associated with such affections as tuberculosis, carcinoma, Bright's disease, etc. If not associated with affections of the kind just named, the nutrition of the body may not be impaired, and it is not incompatible with notable embonpoint. When it is the chief condition to be met therapentically, the first objects are to ascertain and remove, if practicable, the cause or causes on which it depends. The next object is to employ measures to restore the normal quantity of red globules. These measures consist of, first, a nutritious alimentation, into which meat shonld enter largely; second, the use of tonics and stimulants to render the digestive functions more active; third, iron as a special remedy-the effect 
of which is often remarkable; and, fourth, a regimen calculated to increase the energy of the assimilative functions, consisting of exercise in the open air, recreation, etc. These different measures are, of course, to be combined. Manganese appears to have an effect in cases of anæmia analogous to that of iron, and may be given, if chalybeate remedies are not well borne, or if they prove inetficient. $A$ s the anæmic condition may coexist with any disease; in other words, as persons affected by anæmia may contract a variety of diseases, its existence or otherwise is always to be considered, and its coincidence with different diseases may modify materially their treatment. Bloodletting, and other measures which tend to impoverish the blood, as a rule, are injudicious, whatever may be the disease, if it occur in an anæmic subject. And measures addressed to the anæmia may be called for in certain cases of clisease, when, occurring in a person not anæmic or plethoric, the same disease might claim measures of a quite different character.

When simple anæmia exists, and the cause producing it has ceased, as after a hemorrhage, the reproduction of red globules under efficient measures of treatment is rapid. Robin says, after bleeding an animal largely, the return of globules may be almost observed from hour to hour. Simon states that, in the case of a chlorotic girl, an analysis of the blood gave, of globulin, in a thousind parts, 30.860, and, of hæmatin, 1.431. In seven weeks, during which period she had taken two ounces of the tineture of iron and sixty-four grains of the metal, the proportion of globulin had increased to 90.810 , and of hæmatin to 4.598. "Before, she was pale and her lips colorless ; now, she presented really a blooming appearance." In two cases given by Andral and Gavarret, the red globules were increased, under the use of iron, in one case from 46 to 97 , in a thousand parts, in four weeks, and in the other case from 49 to 64 in three weeks. Pure auæmia, with our present knowledge of tonic and analeptic medieation, affords an excellent opportunity to display the resourees of medical art.

Of the manner in which the causes of anæmia, exclusive of hemorrhage, ocession diminution of the red globules, all that can be said, with our present knowledge, is, that they generally appear to act by impairing the liquor sanguinis. Further than this the pathologist cannot go until the physiologist explains how and where the red globules are formed from the elements of the blood-plasma in health.

Anæmia is of more frequent occurrence in the female than in the male. One reason for this is, the normal proportion of red globules is somewhat larger in the male. Another reason is to be found in the fact that, of the causes giving rise to this morbid condition, several are peculiar to females, viz., menorrhagia, leucorrhœa, lactation, and child-bearing.

\section{Changes in the Conformation and Composition of Red Globules.}

The foregoing morbid conditions relate to the quantity of the red globules. 'The question now arises, whether these bodies are not liable to changes as regards their conformation and their composition.

The red globules have a definite form and size, with a certain range of variation within the limits of health. They are circular and biconcave, with a regular contour. In size they vary from $\frac{1}{5 \sigma \delta \sigma}$ to $\frac{1}{30 \sigma \sigma}$ of an inch in diameter. Anatomists are not agreed whether they are cells or solid bodies. They are generally regarded as cells; but Robin and, also, other distinguished microscopists of the present time, regard them as solid or semi-solid bodies. When brought into contact with different 
substances out of the body, they are found to be readily altered in form, size, and, of necessity, in composition. They imbibe water by endosmosis, becoming swelled and globular. In some fluids they part with a portion of their water by exosmosis, becoming indented, losing their regular contour, and sometimes presenting a winkled appearance over their whole surface. The latter changes occur when the globules are contained in urine, and may be witnessed under the microscope by adding a few drops of perspiration. Again, the globules are composed of several constitnents, the more important being an albuminous substance externally, forming the cell-wall in the opinion of those who regard them as cells, and a substance, called hæmatin, in which resides the coloring matter, together with iron, fatty matter, etc. Now, the form, size, and composition of these organized bodies are doubtless adapted to certain physiological ends of more or less importance in the economy. And, since they are so easily altered out of the body, it may be inferred that changes in the liquid in which they are suspended (the liquor sanguinis) must, of necessity, give rise to various alterations of their size, form, and composition. This is a rational supposition, but, in the existing state of our knowledge, pathology is wanting in ascertained facts. 'l'heir respiratory function, that is, their capacity for absorbing oxygen, is destroyed by certain poisons, e.g. carbonic oxide, received with the inspired air, or added out of the body. The effects of various reagents out of the body have been studied; but the circumstances in the living body are so widely different, that we cannot infer the same effects to be produced when the same reagents are administered as remedies, or to lower animals for the sake of experiments. Indeed, experiments of injecting into the bloodvessels various substances have shown the results to be different from, and sometimes quite the reverse of, those produced by applying the same substinces out of the body. Future researches may lead to discoveries which will be found to have important pathological bearings; but, at present, no positive conclusions are warrantable.

A morbid condition of the red globules incident to inflammation may be here noticed. These bodies appear normally to exude a viscid or sticky substance in small quantity. This causes them to adhere in rolls or 'piles, presenting under the microscope an appearance compared to that of rows of coin. In the morbid state of the blood which belongs to inflammatory diseases, this exudation takes place in an abnormal quantity ; hence, there is a notable tendency to adhere in rows. A drop of blood under the microscope may thus furnish a diagnostic sign of inflammation.

\section{Apnœa.}

The blood contains in health certain gases, viz., oxygen, carbonic acid, and nitrogen. The red globules are agents which absorb and transport these gases. The oxygen of the blood exists exclusively in the globules, although the functional capacity of the latter to absorb this gas depends in a considerable degree upon the serum of the blood. Carbonic acid is held in solution by the blood-plasma as well as by the red globules, the capacity of the former to absorb this gas depending on the presence of the carbonate and phosphate of soda.' Oxygen and carbonic acid are received and expelled through the lungs, the interchange of gases with the atmosphere being necessary to the perfection of the blood, and constituting the function called HжMATosis. When these processes are interrupted,

1 Robin, Leçons sur les Humeurs. 
the normal condition of the blood, as respects the gases just named, is exchanged for a morbid condition. The blood does not undergo the conversion from venous into arterial; it presents, in the arteries, the dark color, and essentially the composition and properties of venous blocd. It is incapable of sustaining life. This morbid condition of the blood has been called asphyxia, a term signifying absence of pulse ; a more correct term, now more frequently used, is apnœa, signifying deficiency of breath. Any cause impeding respiration may occasion apnoea. It is the mode of dying in the fatal cases of a considerable number of diseases.

Deficiency of oxygen is the essential feature of apnoa, giving rise to phenomena which will be considered under the head of modes of dying. An excess of oxygen in the blood is probably never an element of disease. An excess of carbonic acid obtains whenever the respiratory function is compromised, and this is an element of apncea. It is difficult to say what pathological effects are due to an accumulation of carbonic acid, and what to deficiency of oxygen, but the latter undoubtedly has the larger amount of agency in giving rise to the phenomena of apnœea. Regarding apncea as consisting in a deficiency of oxygen in the red globules, the most complete type of this pathological condition is afforded in cases of death from the inhalation of the carbonic oxide, this gas, when absorbed by the globules, displacing completely the oxygen.

With our present knowledge of nitrogen in the blood, nothing is to be said of its pathological relations.

\section{Leucocythemia.}

Of the known morbid conditions affecting the organized or corpuscular constituents of the blood, those which relate to the red globules are the most frequent. 'There is but one morbid condition relating to the white or colorless globules as yet ascertained; this consists in their abnormal multiplication. The white globules are spherical bodies larger than the red globules, nearly double in size, and they are vastly less numerous. It is estimated that in health they are in the proportion of one to from 300 to 450 red globules. A point of importance, as regards its pathological bearing, is their resemblance to pus-globules. So closely do the two resemble each other that the most practised microscopical observers declare their inability to distinguish the one from the other. Robin and others regard them as essentially identical, and, as already stated, include both under the name leucocytes. According to the observations of Conheim, which seem to have been corroborated by those of other histologists, whenever suppuration takes place in any part, the pus consists, in part at least, of the white corpuscles of the blood, $\mathrm{or}$ leucocytes, which migrate through the walls of the vessels, without solution of continuity. Conheim and others affirm that this migration is demonstrable by direct microscopical observation. If this be established it is certainly a highly important discovery, and it settles, of course, the question concerning the identity of the white corpuscles of the blood with the pus-globules; the latter are, in fact, the white corpuscles situated without the vessels.

For our knowledge of the morbid condition consisting in an increase of the number of white globules, we are indebted to the microscope. The existence of such a condition was pointed out by two microscopical observers who were led to the discovery, independently of each other, in the same year (1845), viz., Bennett and Virchow. Virchow designated the condition Leukomia, a term signifying white blood. Bennett applied to it the name Leucocythemia, signifying white blood-cells. The latter is the 
better term, and is in common use. Both observers at first supposed the abnormal appearance of the blood to be due to the presence of pus.

A certain amount of increase in the number of white globules is not uncommon in pregnancy and in various affections. In order to constitute leucoeythemia, the increase should be considerable, amounting to a ratio of, at least, 1 to 20 of the red globules. Cases have been observed in which the ratio was much larger than this, amounting to an equal number of both, and the white even preponderating over the red globules. In all cases the red globules are diminished, and the relatively morbid disproportion is owing in part to this fact. In well-marked cases the gross appearance of the blood undergoes a notable change. It acquires a reddishgray or a chocolate color. This is particularly observable, after death, in the blood contained in the cerebral veins and sinuses.

This morbid eondition of the blood is associated very constantly with enlargement of the spleen, frequently with enlargement of the liver, and, in a certain proportion of cases, the lymphatic glands of the neek, axilla, groin, etc., become more or less enlarged. A distinction has been made by Virchow into splenic and lymphatic lencocythemia, the former being characterized by enlargement of the spleen, and the latter by enlargement of the lymphatic glands. Some cases, however, are characterized by enlargement of both the spleen and the lymphatic glands. In a majority of cases the spleen is alone affected. The lymphatic glands are rarely affected alone, that is, without splenic enlargement. It is stated that the lymphatic variety is also characterized by notable augmentation of the small white bodies.called globulins; these are also inereased in number, but in a less degree, in the splenic variety. Virchow states that in the splenic variety the white globules are voluminous and have nuclei; whereas, in the lymphatic variety the globules are small. A pathological connection of some sort undoubtedly exists between the enlargement of the parts named and the morbid condition of the blood. What the nature of this connection is, with our present knowledge, can only be conjectured.

Patients affected with leucocythemia are, as already stated, anæmic. They present the pallor of anemia, together with the phenomena symptomatic of that condition. Febrile movement characterizes certain cases, and in these eases the vital forces are rapidly impaired, and death takes place by exhanstion. Diarrhoea is apt to occur in this class of cases. Other cases are characterized by hemorrhages occurring from the nasal passages or in other situations ; and the loss of blood may be sufficient to prove the immediate cause of death. Dyspnoea, independently of any appreciable lesions of the chest, has been observed. Diminution of the vital forces, progressing more or less rapidly, belongs to the history of this condition, which ends fatally sooner or later. It is doubtful if recovery ever takes place in eases in which the condition is well marked. The average duration, before a fatal result, is estimated by Trosseau to be about thirteen or fourteen months.

This condition may be suspected when the appearance and other phenomena of anæmia are associated with considerable enlargement of the spleen or lymphatic glands. It is, however, to be borne in mind that simple anæmia may occur under these eircumstances withont leneocythemia. Enlargement of the spleen, as is well known, occurs not very unfrequently as a sequel of intermitting fever, and anæmia generally coexists ; but observation shows that leucocythemia is developed very rarely in these cases. The diagnostic criterion is the evidence of the augmented number of white globules afforded by microscopical examinations of the blood. Several specimens should be examined under the microscope; 
and it is to be recollected that, to constitute leucocythemia, the proportion of white to red globules should at least be as great as 1 to 20 . An increase, in the number of white globules more or less, but not sufficiently to constitute lencocythemia, is not uncommon. According to Virchow, the number is increased whenever the lymphatic glands are the seat of irritation. An increase, to cite examples, takes place in typhoid fever, in cases of cancer when the lymphatic glands become involved, and in cases of malignant erysipelas with enlargement of these glands.

After death, in cases of leucocythemia, the right cavities of the heart and the large vessels are sometimes found to contain yellow or yellowishgreen collections, soft and viscous, resembling very thick pus. These are formed chiefly by an aggregation into masses of white globules. Similar puriform collections are found in the veins in different situations, but especially in the veins of the cerebral meninges. If blood be drawn during life, the coherent white globules form soft masses, or a laycr, of a gray or reddish-gray color, attached to the fibrinons crust at the upper surface of the red clot; and if the fibrin be separated by beating the blood with twigs, the residue being left at rest for some time in a narrow vessel, the white globules collect into a milky or puriform layer at the upper part of the vessel, the red globules, from their specific gravity, falling to the bottom. of the vessel.

The question arises, whether there are sufficient grounds to consider this morbid condition as constituting a special disease, or whether it is to be regarded as merely incidental to certain affections. That it is a morbid eondition of grave import, is certain ; but how much importance belongs to it per se, is uncertain. With our present knowledge, we are not warranted in considering it as more than a pathological element of a cachexia, the essential nature of which remains to be determined. The pathologist may expect to be able to understand more fully the nature of this morbid condition when physiologists have established the source and the functions of the white globules of the blood. Robin remarks that leucocythemia is a return to the fotal state as regards the predominance of these bodies, they being much more abundant in embryonic life, and that this fact affiliates this morbid condition with certain morbid growths characterized by a hypergenesis of anatomical elements which are more abundant in the fœetus.

Since the occurrence of a morbid increase of the white corpuscles of the blood was ascertained by Bennett and Virchow, cases have been reported in which all the phenomena of leucocythemia were present excepting the increase of the white corpuscles. These cases have been called cases of pseudo-lencocythemia. They render it probable that the leucocythemic condition does not constitute the disease, but is merely an incidental feature. ${ }^{1}$

A third constituent of the blood, belonging to the class of corpuscular elements, is called, after Donné, globulins. These minute, spherical bodies, found also in chyle and in the liquid contained in the lymphatic vessels, are notably increased in certain cases of leucocy themia. This single fact is all that is at present known respecting them as entering into morbid conditions.

1 Vide article by Horatio C. Wood, Jr., in American Journal of Medical Sciences, No. for Oct. 1871. 


\section{CH A P TER V.}

\section{MORBID CONDITIONS OF THE BLOOD-(CONTINOED.)}

Morbid Conditions relating to Fibrin, Hyperinosis, Hyplnosis-Coagulated Fibriı within the cavities of the Heart-Coagulated Ftbrin within the Bloodvessels-Embolism-TlurombosisMorbid Conditions relating to Albumen-Dropsy as dependent on Diminution of AlbumenMorbid Conditions relating to Water in tlie blood-Hydræula.

$\mathrm{H}^{\prime \prime 2}$ A VING considered, in the preeeding eliapter, the morbid eonditions relating to the organized or corpuseular elements of the blood, it remains to eonsider morbid conditions relating to the other two divisions of blood-constituents, viz., the organic and mineral substances which enter into its eomposition. Direeting attention to the constituents distinguished as organie, the more important of these are fibrin and albumen. What are the known morbid eonditions relating to these constituents? Proceeding to answer this inquiry, the morbid eonditions relating to fibrin will first claim attention.

The fibrin is that portion of the liquor sanguinis, or blood-plasma, whieh solidifies when the blood is withdrawn from the vessels. In its normal state it is a liquid. It solidifies by an inherent proeess ealled coagulation. It forms the eoagulum or elot which is observed when a quantity of blood is drawn into a vessel and allowed to remain for a few moments. In the process of coagulation, the corpuseular elements become imprisoned, and are retained within the elot; henee the red color. Separated from the eorpuscles and other constituents, it is an elastie substance, more or less resisting to pressure, and, examined microseopically, presents an abundance of minute filaments irregularly distributed and interlaeed, forming a reticular arrangement: this is called fibrillation. With respect to its sourees and uses, physiologists are not agreed; some regarding it as transformed albumen, and constituting, par excellence, a nutritive element of the blood; while others suppose it to be effete matter whieh is to be excreted. The eongulable lymph exuded in certain inflammations is essentially fibrin, and this is probably the basis of all the exudations. With respeet to its capability of becoming organized, after it has exuded, pathologists differ, as has been already stated in treating of exudations. In view of the indeterminate state of our knowledge of fibrin, in its physiologieal relations, the amount of our knowledge of it in a pathological point of view must needs be limited. The only morbid conditions as yet well ascertained have reference to the quantity of fibrin. In eertain diseases the fibrin is increased, and in other diseases it is diminished in quantity.

\section{H yperinosis.}

An abnormal inerease of the fibrin of the blood eonstitutes a condition called after Franz Simon, hyperinosis. This condition characterizes aeute inflammations. The average proportion of desiccated fibrin in health is about two and a half in a thousand parts. In different cases of aeute inflammation, the increased proportion is found to vary from three to twelve parts in one thonsand. Cases of acute artieular rheumatisin present the 
largest increase of fibrin; pneumonitis ranks next as regards this feature, and capillary bronchitis next. The increase of fibrin is not found to bear any relation to the previous health and vigor of the patient; it is not less in feeble and sickly persons than in the strong and vigorous, when attacked with acute inflanmation. The increase takes place when inflammation is developed as an intercurrent affection in the course of diseases in which, if not complicated with inflammation, the fibrin is diminished, e.g., the continued fevers. The hyperinosis has not been ascertained to precede the development of inflammation. The latter is not the effect of the former. The converse is probably true; but, with our present knowledge, it is better to say that the augmentation of fibrin is a concomitant of inflammation, a pathological connection of some kind existing between them. We know too little of the physiological relations of fibrin to understand the nature of this pathological connection. According to Virchow, the hyperinosis in inflammation is due to the production of fibrin in the inflamed parts; and there is a notable difference as regards the amount of fibrin produced in different parts when they are inflamed. This difference Virchow states is in relation with the number of lymphatic vessels and glands in parts; hyperinosis is marked in proportion to the abundance of these in the parts inflamed. Hence, inflammation of the brain is not accompanied by hyperinosis; but the fibrin in the blood is notably increased in pulmonary inflammation, because the lungs abound in lymphatic vessels and glands. According to Virchow, the white globules of the blood are multiplied whenever the fibrin is morbidly increased.

The nature and extent of the pathological relations of hyperinosis not being settled, it is difficult to decide, upon rational grounds, how far this element of inflammation is a source of therapeutical indications. Bloodletting is found to increase the fibrin of the blood, and hence, is not an appropriate remedy for inflammations so far as this element is concerned. Animal diet, in health, more than vegetable, render's the fibrin abundant; hence, theoretically, farinaccous food is suited to the treatment of inflammation. But in starving animals the fibrin increases above the normal proportion; ergo, a great reduction of diet is not suited to the treatment of inflammation. The supposition that certain remedies diminish the fibrin of the blood appears to be based on their power of effecting the solution of fibrin out of the body. These effects are quite different. The fibrin in the blood is in a liquid state, and its diminution has nothing in common with its solution after coagulation has taken place. To preserve in the vascular system the liquidity of fibrin, in other words, to prevent coagulation within the heart or vessels, is an object in therapeutics, as will be presently seen.

\section{Hypinosis.}

An abnormal diminution of fibrin is called, after Simon, hypinosis. This is less frequent than an increase of fibrin, but it probably constitutes a graver morbid condition. It occurs frequently, not constantly, in the continued fevers, provided they are not complicated with acute inflammation of any part. As a rule, the diminution of fibrin is progressive as fevers advance in their career; and the amount of diminution is in proportion to the degree of exhaustion of the vital forces, or adynamia. In the frequent occurrence of hypinosis in these fevers, we have a ground of pathological distinction between them and inflammations.

The decrease of fibrin does not occur as frequently in the eruptive fevers. In variola, owing to the cutaneous inflammation, the quantity is generally 
above the normal average. In rubeola it is neither increased nor diminished. In scarlatina it is usually a little below the normal average. It is not diminished in the intermittent fevers.

It is difficult to say how far, if at all, the state of hypnosis constitutes an indication for treatment in the continued fevers. It is not improbable that the importance of animal food in these fevers may have relation to this state, and it has been conjectured that the mineral acids are useful in consequence of their tending to increase the quantity of fibrin in the blood.

\section{Qualitative Changes of Fibrin.}

The foregoing changes, relating to fibrin, are quantitative. Qualitative alterations are inferred, but they have not been satisfactorily ascertained. It has been supposed that certain morbid characters in exudations denote perversions of fibrin, but the latter have not been demonstrated. The tuberculous exudation, for example, has been considered as morbidly changed fibrin; this view, in the present state of our knowlerlge, is hypothetical. The existence of prior blood-changes of any kind, in this or any exudation, has not yet been proven. In short, we have no positive knowledge of morbid changes in the quality of the fibrin of the blood, nor is this knowledge easily obtained, since the characters of this constituent, as it cxists in the liquor sanguinis, are with difficulty studied. It is obtained, isolated from other constituents, only when coagulated, and is then in an abnormal state. It is not certain that variations observed in coagulated fibrin, either within or without the vessels, represent morbid changes which existed when it was liquid and in circulation.

\section{Thrombosis-Heart-Clot-Embolism.}

The recent study of the morbid conditions denoted by, and associated with, the names which form the heading of this section, has led to developments of much pathological interest and practical importance. The writings of some of the olcler medical authors, namely, Boerhaave, Vansweten, Bonet, and, in the early part of the present century, of Magendie, with several of his contemporaries in France, embrace striking observations and experiments relating to these conditions; but the developments during the past few years, which will be reckoned among the most prominent of the events distinguishing the progress of pathology in our day, are to be dated from the researches of Virchow, in 1847, and those of Kirkes, in 1852. The names thrombosis and embolism were introduced by Virchow. Thrombosis means the formation of a fibrinous clot in an artery or a vein; and the term may also be applied to the coagulation of fibrin within the cavities of the heart. Embolism is an obstruction of a vessel, or of vessels, by fibrin or some other substance which has been transported by the current of the blood from either the heart or some part of the vascular system more or less distant from the seat of the obstruction. A thrombus which obstructs an artery or a vein of sufficient size to be appreciable, is a stationary plug; an embolus obstructing such an artery or vein is a migratory plug. A thrombus becomes an embolus when the obstructing body ceases to be stationary, and is carried along by the blood-current until its course is arrested in a vessel not large enough to admit its passage further onward. It is plain that a migratory plug, or an embolus, can hardly be arrested in its course through systematic veins, since the course of the blood-current is from smaller to larger 
vessels. Embolism, therefore, aside from the heart, relates to the arteries with the single exception of the vena porta.

Thrombosis occurring in the cavities of the heart, or heart-clot, first claims notice. The vegetations, concretions, or excrescences which are so often observed after death on the valves, especially within the left cavities of the heart, consist, at least in part, of coagulated fibrin. These constitute a large majority of the arterial emboli, exclusive of those which obstruct the branches of the pulmonary artery. Aside from these examples of cardiac thrombosis, fibrinous masses or clots of considerable size are liable to form in the different cardiac cavities. They were formerly called polypi of the heart. There are two varieties of heart-clot which are easily distinguished from each other. In one variety the clot is essentially identical with that which is formed by the coagulation of blood, in mass, without the body, as after venesection. It is a dark, soft coagulum with, perhaps, a layer of fibrin, the latter being identical with the "buffy coat," as the fibrinous layer in the clot formed after venesection was formerly called. This kind of clot lies free in the cavities of the heart. That is, it is not attached to the endocardium, nor intertwined with the tendinous cords and papillary muscles. It may be formed either after death or during the last moments of life. Yet it is altogether probable that a heart-clot of this kind is sometimes formed when the patient is not in a moribund state, and that it may occasion sudden death. I have met with an example, in a case which will be referred to in connection with articular rheumatism, in which a clot of this kind occurred, and, forming an embolus, obstructed the pulmonary artery, causing sudden death. In general, however, heart-clots of this kind are formed either post-mortem or during the moribund state. Forming in the latter state, they doubtless, not unfrequently, prove the immediate cause of death.

The other variety is of grcater pathological and practical interest, because it is the kind which generally exists when death is attributable to heart-clot. To this variety more especially the term thrombosis is applicable. It is distinguished from the other variety by the fact that the clot consists of isolated fibrin, that is, of fibrin, for the most part, free from other blood constituents. The clot is white, dense, adherent to the endocardium, or intertwined with the chordæ tendinæ; and it may show grooves caused by the action upon it of the current of blood. Such a clot must have been formed ante-mortem; and its formation is not unfrequently a cause of death. In this variety of heart-clot, the coagulation does not, as in the first variety, take place at once, but the fibrin is isolated more or less slowly, being deposited in layers, or stratified somewhat as it is within an aneurismal sac. It is evident, however, that the action of the tendinous cords and papillary muscles may be instrumental in the formation of the clot or thrombosis, the fibrin being whipped out of the blood as in the process of obtaining this constituent from blood which has been drawn from the vessels.

The conditions involved in the formation of heart-clot are, first, overdistension of the cavities of the heart from an undue accumulation of blood; second, an excess of fibrin in the blood or hyperinosis. A third condition may perhaps be added, namely, an abnormal coagulability of the fibrin, a condition to which the name inopexia has lately been applied. The accumulation of blood occasions a certain amount of paralysis from distension; hence, the contents of the cavities are but partially expelled by the contraction of the muscular walls. There is more or less stagnation in the cavities. Under these circumstances the fibrin may coagulate, and it is isolated by the coagulation taking place slowly, and by the action 
of the cords and muscles, especially if the fibrinous constituent of the blood be in morbid excess. 'L'hese conditions are apt to exist in cases of pneumonitis, and especially when more than a single lobe is involved. 'I'he formation of heart-clot will be referred to in the second part of this ivork, as an accident which occasions death in a considerable proportion of the fatal cases of that disease. It is an accident liable to occur, also, in cases of the disease known as capillary bronchitis, in pyrmia, and, occasionally, in various aflections. In addition to the conditions which have been named, there may be others which at present are unknown. Heart-clot, or thrombosis, occurring in cases of pneumonitis and capillary bronchitis, are formed in the cavities of the right side of the heart, because, owing to the obstruction to the pulmonary circulation in these affections, the over-accumulation of blood and stagnation are in the right ventricle and auricle. When the heart-clot or thrombosis is in the cavities of the left side of the heart, there is generally weakness of the walls of the left ventricle and auricle, from either dilatation or fatty degeneration, the over-accumulation and stagnation being dependent on the loss of inuscular power incident to these lesions. Prolonged syncope may, perhaps, sometimes lead to a suddenly-formed, soft, dark coagulum, in any of the cavities of the heart. In this way, in 1849, the late Prof. Meigs accounted for sudden death shortly after labor attended with profuse flooding. The late Dr. Simpson, of Edinburgh, reported several similar cases. Other cases have been communicated by Dr. Barnes and Dr. Playfair, of London. ${ }^{1}$ Cases of sudden death in diphtheria may be thus explained. Of 580 cases of sudden death analyzed by Ogston, thrombosis of the heart and large vessels therewith connected was present in 63 (10.8 per cent.), and was the cause of death in fourteen. ${ }^{1}$

Passing now to thrombosis of arteries or veins, it involves coagulation of fibrin at the seat of the obstruction, and the latter may be either complete, or more or less incomplete. The conditions learling thereto are, first, retardation of the circulation; and, second, a lesion, of some kind, of the vessel at the seat of the thrombosis. The diminution of the calibre of a vessel from external pressure, and a partial or complete occlusion by an obstructing substance within the vessel, are causative circumstances; but the rationale of thrombosis, under these circumstances, is the local retardation of the circulation which they occasion. Thrombosis, arising chiefly from feebleness of the heart's action, very rarely, if ever, occurs in arteries; and retardation of the current from this cause is rarely sufficient, excepting in veins at a distance from the heart.

Thrombosis of the iliac or femoral vein constitutes the affection formerly known as phlegmasia alba dolens, occurring especially in puerperal eases; but, also, in some cases of phthisis, and occasionally irrespective of these pathological connections. 'The chief local effect is œdema, but it may contribute to erysipelas or erythema, hemorrhage, and even gangrene. In arterial trunks, thrombosis is generally a result of atheroma or a calcareous degeneration where the thrombus is formed; these lesions ronghening the inner surface, and also, perhaps, diminishing the calibre of the artery. The cerebral arteries are those most liable to thrombosis. In this situation it enters into the causation of hemiplegia and softening of the brain. Thrombosis is often an effect of embolism; that is, a thrombus is formed at the situation in which an artery is occluded by an embolus. Moreover, a thrombus having formed in a vein, the clot subsequently becomes extended in both directions, that is, toward the periphery of the venous circulation,

1 Vide art. by Dr. Lidell, in Am. Jour. of Mled. Sciences, Oct. 18r2. 
and, also, toward the heart. It is easy to see how the stasis in the former of these two directions leads to further coagulation. In like manner the retardation of the circulation in the latter of the two directions may be sufficient to occasion a prolongation of the thrombus. Thus, a clot in the crural vein on one side may lead to secondary coagulations extending to the vena cava of the same side; and these, progressing in a retrograde direction, may extend even to the iliac and the crural vein of the opposite side. These secondary coagulations, in a direction toward the heart, are important as sources of emboli.

The important facts pertaining to the subsequent history of thrombi are as follows: First, in the veins they may become dislodged in fragmentary portions, or in mass, and being transported toward the heart, become emboli, which are carried into the pulmonary circulation. Second, in either veins or arteries, they may remain indefinitely without essential change, being analogous to foreign bodies. Third, they may become organized, then, of course, remaining permanently. Fourth, they may undergo granulo-fatty degeneration, forming a puriform detritus which is carried into the circulation. This last named event is important as giving rise to capillary embolisms, infarctus, and events which belong to the history of pyæmia. Referring the reader, for histological details, to works which enter into the subject more fully than is consistent with the plan of this treatise, I pass to the consideration of embolism. It is to be added, that the local effects, immediate and remote, of arterial thrombosis, may be, in the main, the same as those of embolism, and it will sufflce to notice their effects in connection with the latter. The chief point of difference, as regards these effects, between arterial thrombosis and embolism is, that the latter occurs suddenly and the former more or less slowly.

Recalling the definition of embolism, namely, complete or partial vascular obstruction, the obstructing body or material having been transported from a situation within the circulatory system more or less distant, a point of difference, in the first place, relates to the size of the vessels obstructed. An embolus may obstruct an artery of large, considerable, or moderate size. On the other hand, granular matter, the detritus of thrombi, or other morbid products, may be brought to parts in sufficient quantity to accumulate and obstruct either arteries of small size or the arterioles. The latter, which is distinguished as capillary embolism, will be noticed separately after having considered embolism in arteries of either large, considerable, or moderate calibre. The emboli in these cases differ as regards their source, the direction which they take, their size, composition, and the morbid effects, immediate and remote, to which they give rise.

The larger proportion of the emboli now referred to are derived from the heart-cavities. 'They consist of vegetations, masses of fibrin, calcareous matter, or fragments of tissue which are detached and washed away by the current of blood. Generally these are from the left cavities of the heart, and the direction which they take is, of comrse, into the aorta and its branches. They are carried onward until they reach an arterial branch too small to permit their passage. Here they are arrested, often becoming fixed at a bifurcation, and they obstruct, partially or completely, the flow of blood beyond this point. The vessels into which emboli are especially apt to enter, are the following, enumerated according to their relative liability : The splenic, renal, iliac, carotid, infra-clavicular, mesenteric, and coeliac arteries. Of the carotid arteries, emboli go oftener into the left than the right, the route from the heart through the former being the most 
direct. The left iliac artery is more liable in consequence of the iliac vein crossing the artery on the right side.

Emboli of the same description, that is, obstructing arteries of large or moderate size, may also be derived from the right eavities of the heart. The deposit of fibrin in considerable or large masses, and the formation of coagula, that is, heart-clots, occur oftener in the right than in the left cavities. Fragments from the former and the latter in mass may become em. boli, which, of course, are carried into the pulmonary artery, obstrueting either this artery, its primary divisions, or the subdivisions of the latter. Reference has been made already to embolism of the pulmonary artery, by a recent clot formed in the right ventricle, the effect being sudden death. I have met with an example of complete obstruction of one of the primary divisions of this artery, by a calcareous embolus which was broken off from a similar mass in the right ventricle. ${ }^{1}$ Smaller emboli produce, according to their size, obstruction of branches of the pulmonary artery of greater or less calibre. They are more likely to go to the right than the left lung, because the right division of the pulmonary artery is the largest.

Venous thrombosis is another source of emboli, either of the pulmonary or of the systemic circulation. Thrombi in the puhmonary veins, transported first to the heart and thence into the arteries, have, of course, the same direction as those which are formed within the left carities of the heart ; they enter the aorta and become fixed at different points, namely, in the arteries of the spleen, kidneys, brain, etc. It is, however, rare for emboli sufficiently large to obstruct vessels of a considerable or moderate calibre, to originate in the pulmonary veins. On the other hand, it is not uncommon for thrombi formed in systemic veins to become detached, and, carried by the venous eurrent to the right side of the heart, to obstruct the pulmonary artery or its branches. Repeated instances have occurred in which the thrombus, in cases of plegmasia dolens, has furnished emboli of sufficient size to plug the pulmonary artery. The fibrin or clot formed in aneurisms is another source of emboli which take the direction of the systemic arteries. Masses consisting of the white or the red globules of the blood agglutinated together form a rare variety of emboli. Other rare varieties are portions of carcinomatous, cartilaginous, and other morbid growths, which, by ulceration into veins, find their way into the circtllatory system. Finally, foreign bodies, through wounds, may become emboli. In another work I have related a case in which a musket ball, inflicting a wound in the neck, passed into the jugular vein, and thence to the heart, becoming imbedcled beneath the muscular trabeculæ at the apex of the right ventricle, where it had doubtless remained without doing harm for many years.

The local morbid effects of embolism depend on the size, situation, and vascular connections of the obstrueted artery. An embolus obstructing completely the pulmonary artery destroys life in a few moments. One of the primary divisions of this artery, however, may be completely occluded, not only without a fatal result, but without grave symptoms. In the instance which came under my observation, there was nothing to excite suspicion of anght except the tuberculous discase, with which the patient died; and the embolus had evidently obstructed the artery for a considerable time. An obstruction of one of the carotid arteries does not occasion symptoms of any gravity; for the communication through the circle of Willis is so free between the arteries on both sides, that the obstruction is readily compensated for. An obstruction, however, of an artery beyond

1 Vide Treatise on Diseases of the Heart, by the author, 2d edition. 
the circle of Willis, owing to a deficiency of anastomosing branches, may give rise, as will be hereafter seen, to apoplexy and hemiplegia. Embolism of the femoral artery may be followed by gangrene of the limb. In this situation sudden obstruction is characterized by intense neuralgic pain. This was illustrated in the case of the late Valentine Mott. ${ }^{1}$ Local effects here and in other situations will enter into the consideration of particular diseases in the second part of this work. The immediate effects proceed from the sudden interference with the circulation within the area of the arterial branches beyond the point where the embolus is fixed. Circumscribed ischæmia or anæmia is the primary morbid effect; hence, temporary loss or impairment of functions in the part in which the circulation is suddenly interrupted. This is especially marked in cases of cerebral embolism, in which there is sudden loss of consciousness, accompanied with paralysis. The degree, or intensity of the immediate effects in the brain and elsewhere at the instant when embolism takes place, will depend on whether the obstruction be at once complete or incomplete. The embolus may either fill so completely the artery that the circulation is entirely arrested, or the obstruction may be but partial. If, however, the latter be the case, the obstruction usually becomes, in a short time, complete, in consequence of coagulation caused by the embolus. The immediate effects, under these circumstances, undergo an increase in degree or intensity, the latter being due to the thrombus which is added. Frequently, in examinations after death, emboli, proven to be so by a correspondence as regards their composition and characters with vegetations in the heart or thrombi situated in either the heart or vessels at a distance, are imbedded in a clot evidently formed at the seat of the obstruction. To distinguish, clinically, embolism which is at first partial and becomes complete, from an obstruction which is entirely due to thrombus, is not always practicable.

The loss or impairment of functions within the area in which the circulation is interrupted is sometimes only temporary. The circulation may be more or less speedily restored through anastomosing branches, and thereby the function is recovered. The restoration depends on the abundance of the anastomosing vessels. In general terms, the restoration of the circulation is more difficult, or less liable to take place, in proportion to the distance of the obstructed vessel from the heart, or, in other words, the small size of the artery in which the embolus is seated. The arteries, however, in different organs differ much as respects free communication with each other through anastomosing vessels. In the brain and other internal organs the ischæmia or local anæmia, which is the primary effect of embolism, is a matter of inference; but within the eye it is open to direct observation by means of the ophthalmoscope. In a patient who was seized suddenly with amaurosis of one eye, the late distinguished oculist, Von Græfe, observed, through the ophthalmoscope, a notable exsanguine condition of the retina. On this he based the conclusion that the central artery of the retina was the seat of an embolus. This truly brilliant diagnosis has been corroborated by instances in which, under similar circumstances, the fact of embolism of this artery has been determined after death. It appears, however, to have been ascertained that, at least in certain situations, the ischæmia or local anæmia caused by an embolus, is of short duration. The vessels beyond the seat of the obstruction are filled by collateral arterial branches, and perhaps by a reflux of blood from the veins; but, owing to the loss of the vis a tergo beyond the seat of the embolus, the blood stagnates in the vessels; there is a passive

I Vide New York Medical Record, 1865. 
congestion, which may cven occasion rupture and extravasation. This congestion, however, does not compensate for the arrest of the circulation, and, notwithstanding the hypernemia beyond the embolus, there is loss or impairment of function, and certain changes incident to defective nutrition are liable to occur. These changes belong more especially to capillary embolism, but they occur particularly in the brain, where an artery of moderate size, as the middle cerebral artery, is oceluded. They are due to either defective nutrition or necrobosis, that is, the loss of vitality within the space in which nutrition is arrested. Softening, sometimes to liquefaction, exemplifies these changes in the brain-substance.

An embolus sometimes excites inflammation of the surrounding tissues, which may lead to suppuration. On the other hand, it may undergo, like thrombi, degenerative changes, being reduced thereby to a detritus which, carried away by the current of blood, may become capillary emboli. In this way, sometimes, the channel of the artery is restored. In general the embolus remains permanently fixed without exciting local inflammation, and the obstruction of the vessel is permanent.

Passing now to capillary embolism, under this name more is comprehenderl than the name would imply. This variety of embolism is not limited to the capillary vessels, nor even to the arterioles, but it embraces embolic obstruction of the smaller arteries. The variety just considered, in which arteries of large or moderate size are obstructed, may be called, by way of distinction, arterial embolism. Capillary embolism, as thus defined, affords an explanation of certain morbid changes occurring in different organs, namely, hemorrhage, softening from granulo-fatty degeneration, abscess, gangrene; and it enters largely into events heretofore considered as characteristics of pyæmia.

The sources of capillary emboli are as follows: 1st. Thrombi in veins, small fragments detached from a venous clot, and the detritus which is left when clots are disintegrated, become capillary emboli. Venous Thrombi in the crural, iliac, and some other veins, have been already referred to; but venous thrombosis belongs to inflammatory affections in different organs, to local injuries, to surgical operations, and other eauses. Wherever coagulation in veins takes place, the clot, if it undergo either fragmentary division or disintegration, may lead to capillary embolism. Emboli, thus produced in the systemic veins, are carried by the bloodcurrent to the right side of the heart, and thence into the branches of the pulmonary artery. The morbid effects are manifested in the lungs; but it is conjectured that sometimes embolic matter may pass through the capillary vesscls of the lungs, and, gaining entrance into the systemic arterial system, be arrested in other organs ; for example, the kidneys in which the arterioles are of smaller size than in the pulmonary organs. 'The detritus from thrombi formed in the pulmonary veins will, of course, be carried to the left side of the heart, and thence into the arteries, through which it reaches, and is arrested in, different organs, the spleen, kidueys, etc. Thrombi, formed in the portal system of veins, may furnish fragments and detritus which are carried into the vena portre, and occasion embolism of the smaller vessels of the portal vein within the liver. $2 d$. Thrombi in the heart-cavities. Thrombi in the right side of the heart lead to capillary embolism within the lungs, and those in the left side lead to embolism of various other organs. 3d. Thrombi in arteries, and also emboli, becoming disintegrated, furnish small fragments and detritus, which give rise to capillary embolism. It is a reasonable conjecture that an arterial embolus of considerable size may be arrested at a bifurcation, 
and here be broken up into small fragments by the force of the blood-current, the fragments becoming capillary emboli. Finally, according to Weber, the free oil in the blood sometimes accumulates in the vessels of the lungs to such an extent as to occasion capillary embolism; and air in the blood, which destroys life by obstructing the circulation through the pulmonary capillaries, may also be considered as giving rise to embolism.

The morbid local effects of capillary embolism have been already stated, namely, hemorrhage, softening from degenerative changes, abscess, gangrene. It appears to have been determined by experiments in which small foreign substances were introduced into the circulation, and the abdominal organs exposed to view, that, very quickly after embolism of the smaller arteries is produced, hyperæmia or congestion occurs within circumscribed small pyramidal spaces corresponding to the distribution of the vessels. The pressure of the blood within the hyperæmic spots leads to rupture and extravasation. This is now known as hemorrhagic infarctus. By German and French writers, however, the term infarctus is used to embrace the other local changes following capillary embolism, that is, softening, suppuration, abscess, and gangrene. How are these changes to be explained? Impaired nutrition and loss of vitality (necrobiosis) account for the softening and gangrene. Blood extravasated becomes virtually a foreign substance, and its presence may excite suppurative inflammation. But, probably, in some, if not in most, cases, capillary emboli have a pathogenetic agency which is not merely mechanical, that is, their effects are not due to obstruction alone. It is a reasonable supposition that they may have septic or poisonous properties which will measurably explain the local morbid processes attributable to their presence. Different morbid products differ as regards the nature of these morbid properties; and particles of matter, in other words, capillary emboli derived from a part wherein a certain morbid process is going on, will be likely to excite a similar process in the places in which, having been transported thither by the current of blood, they are arrested.

The embolic changes embraced under the name infarctus, in the comprehensive sense of this term, are especially liable to occur in the spleen, kidneys, lungs, liver, and brain. In the spleen, the wedge-like portions so often met with, or "fibrinous blocks," which usually undergo degenerative changes and are at length absorbed, are the sequelæ of hyperæmic stasis and hemorrhagic extravasation. Suppuration and gangrene are here rare results. Essentially the same changes may take place in the kidneys, but small abscesses are less infrequent. They are situated in the cortical portion. In the lungs small extravasations of blood, circumscribed gangrene, lobular pneumonia, and little abscesses, the latter formerly called "metastatic," are local events attributable to pulmonary embolism. In the liver, hemorrhagic infarctus does not occur, but the effects of embolism are the well-known "multiple abscesses" in this viscus. In the brain the embolic changes are punctated or capillary hemorrhage, softening and liquefaction, the latter denoting complete disorganization or necrobiosis. These changes in the several organs just named will enter into the consideration of the local affections therein seated; and capillary embolism will also be referred to in treating of pyæmia.

I have endeavored in this brief section to present an outline of the developments, up to this time, in relation to thrombosis and embolism. At the date of the publication of the first edition of this work, in 1866, the importance of these topics was not fully appreciated by the majority of 
medical writers and practitioners. Since that date, facts previously observed have been corroborated, and important additions to our knowledge have resulted from experiments on animals and from clinical obscrvation. I coneluded this section in the third edition (1868) by saying that facts were constantly accumulating which tended to show the importance of these topics. At the present time (1872) this statement may be repeated. Much is to be expected from continued researches respecting thrombosis and embolism.

\section{Increase and Diminution of $A 1 b u m e n$.}

Having considered the pathological relations of fibrin, I pass to the other of the two most important organic constituents of the liquor sanguinis, viz., albumen. This exists in the serum of the blood after the separation of fibrin. It exists normally in a liquid state. It does not, like fibrin, coagulate spontaneously, but may be coagulated by heat or the addition of mineral acids, and is then presented as a white deposit. It is found also in ehyle, lymph, dropsieal effusions, and in various fluids of the body. It is much more abundant in the blood than fibrin, existing in the proportion of from 60 to 70 parts in 1000 . The serum of the bloor owes to it much of its density, which in health is represented by about 1030. Albumen is doubtless a very important constituent of the blood, but its precise physiological uses and relations are not satisfactorily ascertained. There is reason to believe that it furnishes the pabulum for the development and renewal of the organized or corpuscular constituents, and it undoubtedly constitutes the material for the nutrition of many of the tissues. It has been supposed that the fibrin is formed from the albumen. The two substances are nearly identical as regards their chemical composition.

Albumen is increased in certain diseases, viz., acute rheumatism, pneumonitis, pleuritis, etc.; but, with our present knowledge, it is impossible to say how much pathological importanee belongs to this fact. It does not appear to be uniformly inereased in inflammation, as fibrin is. It has not, therefore, the same significance as a criterion of inflammation. In short, there are no known special pathological relations of an excess of albumen; and there is no name to denote such a condition.

The pathological relations of an abnormal diminution of the quantity of albumen are more apparent. This constitutes an important condition incident to affections of the kidneys, characterized by the presence of albumen in the urine, or albuminuria. This blood-constituent eseaping constantly with the urine, the quantity in the blood is proportionately lessened; hence the density of the serum is lowered. Bright reported a case in which the specific gravity of the scrum was reduced, by a deficiency of albumen, as low as 1013 .

An important morbid effect frequently follows diminution of the albumen and, thereby, of the density of the blood. Aqueous or serous transudation is apt to occur in the interstices of the areolar tissue and into the serons cavities, giving rise to œdema and anasarca. It thus occasions general dropsy. This effect is doubtless in a great measure due to the diminished density of the blood serum; it becomes unduly aqueous, and filtrates more readily through the tissues. But the transudation is in a measure due to an obstruction to the free passage of the blood through the capillary vessels. Experiments have shown that the presence of fibrin and albumen in the blood facilitates its circulation in the capillary system; hence, when the blood is deprived of a considerable portion of 
its albumen, it accumulates in the capillary vessels, and the increased pressure favors transudation, or dropsy.

Diminution of albumen has been observed in various cachectic affections, in some cases of inflammation, in typhus, notably in scorbutus, in puerperal fever, dysentery, etc. This condition occurs whenever the body is insufficiently nourished, either from too little or poor food, or defective appropriation from any cause; also, whenever there is an abnormal expenditure of blood-constituents, as in chronic diarrhœa, copious purulent discharges, etc. The loss of albumen does not bear any constant relation to the number of red globules. It is neither increased nor diminished in chlorosis or anæmia. Nor does it appear to sustain any relation to fibrin. Bleeding, which increases fibrin, appears to lessen albumen, but not in a marked degree.

Of morbid alterations of the quality of albumen, we know nothing positive. That important qualitative changes do occur is highly probable. But, in the present state of knowledge, all is conjectural. When the physiologist develops further information respecting the uses of albumen in health, its metamorphoses, and its relations to other elements of the blood, and to the nutrition of the tissues, the pathologist may hope to understand better than now to what extent and in what modes it is involved in disease.

A substance analogous to albumen, distinguished by not coagulating under the influence of heat, and coagulating imperfectly with the addition of nitric acid, is called albuminose. It exists in the blood in the proportion of 4 to 6 parts in 1000 . It is found to disappear in the course of diseases in which albumen is diminished. It bas no known pathological relations.

\section{Increase and Diminution of Water.}

Water constitutes a large proportion of the mass of blood, viz., about 790 of 1000 parts. Obviously a certain proportion of water is essential. If abnormally deficient or superabundant, evils pertaining to the circulation and to changes in the blood itself are likely to follow. These morbid conditions of quantity are incidental to disease. It is doubtful if an excess of water ever occurs save as an incidental morbid condition. The quantity of blood, as already stated, is probably not subject to much fluctuation. It is, however, by no means probable, as stated by Lehmann and others, that the mass is never increased nor diminished. In phthisis and other wasting diseases it is undoubtedly diminished; and it is probable that the amount is sometimes increased. But, as a rule, its normal fluctuations are inconsiderable. If too much liquid be ingested and absorbed, the augmented presșure within the vessels causes exhalation from the pulmonary mucous membrane, the skin, and more especially into the uriniferous tubes of the kidneys, and the balance is speedily restored. On the other hand, if loss be sustained, the deficiency is speedily made good by the absorption of. water. In the latter mode the blood may become unduly watery, constituting hydræmia. If the globules be reduced in number, the space is filled with water. If albumen be drained away, the serum becomes watery. Hydræmia, therefore, is a substitution, of more or less of the other constituents of the blood by water, and not the blood normally constituted, plus a certain amount of water; it is thus incidental to other morbid conditions of the blood. It is doubtful if water ever remain in excess while the other constituents continue unaffected. Deficiency of water in the blood in a notable degree is incidental to epidemic 
cholera. The density of the blood in this disease is greatly inereased; it becomes thiek, and is with difficulty eirculated.

The morbid conditions and relations of several of the most important of the constituents of the blood have now been briefly eonsidered, to wit, the red and colorless globules, or the organized elements, the fibrin and albumen of the liquor sanguinis, earbonie acid gas and oxygen, and water. Numerous constituents yet remain to be noticed. Of these, some are organic, and others inorganic or mineral. Of the remaining organie elements, there are the fatty and extractive matters, sugar and certain principles embraced under the head of excrementitious substances. In the next chapter I shall notice these remaining constituents as arranged in the groups just named.

\section{CHA P T E R V I.}

\section{MORBID CONDITIONS OF THE BLOOD-(CONTINUED.)}

Morbid Conditions reiating to Fat-Morbjd Conditlons relating to Sugar-Diabetes MeliitusMorbid Accumulations of Excrementitious Principles in the Blood-Urxemia-Excess of Uric Acid in Gout, Uricania-Retention of Excrementitious Principles contajned in the Vapor of Expiration-Retention of Excrementitious Princlples contained in the Perspiratlon-Reab. sorption of Bile, or Cholæmia-Cholesteræenia-Morbid Conditions relating to the Saline Constituents of the Blood.

\section{Increase and Diminution of Fat.}

$\mathrm{F}$ AT enters into the composition of the corpuseular constituents and the liquor sanguinis, the average amount in the whole mass of blood being estimated to be a little over 2 in 1000 parts. 'The greater part is saponified, that is, in combination with alkalies. Of the fats which are non-saponifiable, with our existing knowledge, the most important is eholesterin. An excess of fat leads to the presence of free fat, in greater or less quantity, in the form of oil-globules. These have been repeatedly observed in diseases. In health the blood-serum is turbid or milky for several hours after a full meal. This appearance has been observed when venesection has been practised under these cireumstances. It is due to the products of lacteal absorption which are at this time poured into the blood. This is the explanation of some of the cases of white or milky blood which have been reported by medical practitioners.

The pathological relations of an excess of oil in the blood are very imperfectly known. Clinical observations have shown that it is more or less abundant in acute affections generally. Under these circumstances, it must be chiefly derived from the adipose tissue within the body; hence, the emaciation which accompanies acute affections. It accumulates in the blood of drunkards, leading to that amount of deposit in the adipose structure which constitutes obesity. Lehmann states that this result is not due directly to the introduction of alcohol, but that it occurs only when the liver becomes affected.

Nothing is to be said with respect to our positive knowledge of a mor- 
bid deficiency of oil in the blood. Yet, probably, such a morbid condition exists, and has more or less importance in its relation to diseases. This is certainly a fair inference from the benefit derived from cod-liver oil and other fatty substances in tubereulosis, scrofula, and other affections.

Of the non-saponifiable fats, cholesterin alone, with our present knowledge, has important pathological relations. Experiments and clinical observation have shown that this is an excrementitious substance, and a notice of its abnormal accumulation in the blood will therefore come under another division.

Therapeutically, it would seem to be an object to increase the amount of oil in the blood in certain diseases, especially in tuberculosis, and, on the other hand, to dimimish the amount in cases of obesity. The latter is also an object in cases of fatty degeneration of the heart and arteries, if it be true, as is highly probable, that this structural change is either dependent upon, or favored by, an excess of oil in the blood. The diminution of oil in the blood is effected by restricting the diet, in a great measure, to nitrogenized substances, avoiding butter, fat meats, sugar, and limiting the amount of farinaceous food. Obesity is not uncommon in middle age, developed to an extent to constitute a morbid condition; and it occurs sometimes in early life, evidently from a constitutional tendency, and may reach an excessive degree, as seen in persons exhibited as objects of curiosity on account of the enormous size and weight to which they attain. Whenever it exceeds the limits of health, it may appropriately claim treatment; and it may often be notably lessened by excluding from the diet fatty articles, and limiting the ingestion of substances readily converted into fat, viz., sugar and starch. ${ }^{1}$

To effect or promote an increase of oil in the blood, oleaginous remedies, and a diet the opposite of that just indicated, are to be employed.

As regards the substances embraced under the head of extractive matters, a few words will suffice. This division includes a variety of undetermined constituents of the blood - undetermined with respect to their nature, source, metamorphoses, relation to different organs, and their uses. Of course they have not, in the present state of our knowledge, any well determined pathological relations. It is not improbalule that they play an important part in morbid processes, but nothing has as yet been ascertained.

\section{Abnormal Accumulation of Sugar-Glycohmmia.}

Sugar is an organic substance which exists in the blood in health. It is a constituent of the blood in certain portions only of the vascular system, viz., within the portal vein, but more abundantly within the hepatic vein, the vena cava ascendens, the right cavities of the heart, and the pulmonary artery. It is sometimes, but not uniformly, found in the blood generally; and, excepting in the parts just named, when found, the quantity is very small, save as a morbid condition. With respect to the existence and production of sugar within the organism, the discovery by Bernard is of great interest and importance. This distinguished phys-

1 Mr. Banting, an English non-medical writer, published, some years since, a "Letter on Corpulence," which attracted considerable popular attention. By a system of dietetic management, he reduced his weight within a year $46 \mathrm{lbs}$. I could cite a case in which, several years ago, I obtained a success not less remarkable. The management of obesity has undoubtedly received too little attention, but it is to be borne in mind that a system of diet suited to diminish an undue accumulation of fat may be, not only inappropriate, but hurtful to persons who do not suffer from corpulence. 
iologist has shown that its production is one of the functions of the liver. A certain quantity is received from the sugar and starch contained in the ingesta. But the hepatic vein contains much more than the portal; and it is contained in the former when the ingesta are devoid of both saccharine and amylaceous substances, and when no sugar is contained in the portal blood. It is destroyed during the passage of the blood through the lungs. How is it destroyed? 'This is a physiological question, but it has important pathological bearings. The explanation which seems most probable is, that it is converted into lactic acid, the latter combining with soda, lime, and potash, forming lactates which are again converted into carbonates, and then again decomposed by the pneumic acill. ${ }^{1}$

It should be stated that Pavy, MeConnell, and others deny that a normal function of the liver is the production of sugar. They affirm that during life the liver does not contain sugar, but produces an amyloid substance (hepatic dextrine or starch) which is converted into glucose by the contact of warm saliva, pancreatic juice, or diastase. Pavy claims that the conversion of this substance into sugar is always pathological. It may be added that, according to Robin, the glycogenic function of the liver is performed by the hepatic lobules, the bile being secreted by the membrane lining the hepatic ducts.

In health, sugar is not eliminated from the body, but, as a symptom of disease, it may exist in great abundance in the urine, in other secretions, and in exhalations; and under these circumstances, the blood everywhere throughout the body contains it. This constitutes the morbid condition commonly known as diabetes mellitus.

In cases of diabetes, sugar exists in the urine, in other secretions, and in transuded liquids, in consequeuce of its existence in the whole mass of blood. Diabetes is not a disease of the kidneys, as it was formerly regarded ; these organs merely excrete sugar contained in the blood brought to them by the renal arteries. The sugar in the renal blood increases the functional activity of the kidneys, acting like a diuretic, and, hence, the quantity of urine is greatly increased. $\Lambda \mathrm{n}$ increased quantity of urine containing sugar in abundance thus becomes the diagnostic criterion of diabetes. The abnormal presence of sugar in the blood is expressed by the term glucohæmia or glycohæmia.

Why is it that the blood in the general circulation becomes saccharine in this disease? Either the liver produces too much sugar, or the processes by which it should be made to disappear from the blood in the lungs are imperfect. Our present knowledge does not perhaps cnable us to decide between these two explanations, and both may concur in some cases ; the first, however, is the more rational, if it be true, as estimated by Bernard, that in some cases of diabetes a much larger amount of sugar is excreted by the kidneys than is formed by the liver in health.

Moreover, certain facts render it not improbable that a prior morbid condition of the nervous system may be involved. Bernard ascertained that, by irritating the medulla oblongata at the point of connection of the pneumogastric nerve, sugar appears in the urine. A transient attack of diabetes may in this way be produced at will in an inferior animal. Hence, it may be conjectured that diabetes, occurring spontaneously, is an affection of the nervous centre, an abnormal influence being transmitted to the liver through the pneumogastric nerve.

Were the seat and nature of diabetes established, our knowledge might, perhaps, lead to rational indications for treatment. $A s$ it is, the thera-

1 Robin and Verdeil, Chimie Anatomique. 
peutics of the disease is quite unsettled. It is a very grave disease, persisting in the great majority of cases, and proving fatal sooner or later. A temporary and partial relief is, in general, only attainable; and this is effected by different means, so that the success of a particular mode of treatment does not reflect much light on the pathology of the disease. Inasmuch as a considerable portion of the sugar is derived from the ingesta, one point in the management is to cut off or diminish the supply of sugar in the food. In this way, the quantity of urine, and the amount of sugar which it contains are generally lessened. But this measure of treatment is directed, not against the primary or essential morbid condition, but only against an effect or symptom; for the presence of the sugar in the blood is dependent on some prior morbid condition. The saccharins blood is the ultimate, appreciable, morbid condition, in the existing state of our knowledge, but this is evidently a result of some deeper change which has not yet been ascertained. Alkaline remedies, continued, with occasional intermissions, for a long time, as recommended by Trousseau, I have known to prove remarkably uscful, but in some cases they are attended with no benefit. This treatment is based on the hypothesis that the sugar received into and formed within the system is not destroyed owing to the want of alkalies in the blood. Rennet has been proposed with a view to promote lactic fermentation, and its usefulness is said to be shown by the results of clinical experience. Remedies addressed to the nervous system are found to diminish the quantity of sugar excreted. Opium is generally beneficial. Strychnia is sometimes notably so.

The treatment of this disease will be more fully considered in the second part of this work. These different measures are here mentioned in illustration of the fact that clinical experience appears to show the utility of methods of treatment based on different views of the pathology and seat of the disease.

Sugar may appear transiently in the urine, in small quantity, in various diseases, and, under these circumstances, has no more pathologieal importance than the occasional appearance of a trace of albumen. The statement made a few years ago by Reynoso that the urine is habitually saccharine in affections which compromise the respiratory function, has not been conflrmed by the observations of others. A trace of sugar may be found in the urine of healthy persons after certain kinds of food, after the inhalation of chloroform, and when certain remedies have been given, especially the ethers. It is also found sometimes in considerable abundance for a short period when the system is disturbed by strong mental emotions.

\section{- Accumulation of Excrementitious Principles in the Blood-Uræmia.}

Certain morbid conditions of the blood consist in an undue accumulation of excrementitious substances. And, of these, first in importance are the organic constituents of the urine, viz., urea and uric acid. These, as well as all excretory products, are pre-formed in the blood, not produced within the glands, as was formerly supposed.' They exist, therefore, in the blood in health, and it is their accumulation in abnormal quantity which consti-

1 It has been claimed that experiments made by Oppler, Schottin, Perls, and Zalesky, go to show urea and uric acid to be produced within the kidneys; and that the morbid phenomena attributed to uræmia depend on the accumulation in the blood and tissues of creatine, creatinine, and other primary products of tissue metamorphosis. Vide Roberts's Treatise on Renal and Urinary Diseases. 
tutes the morbid eondition. The exerementitious substances just named are distinguished as nitrogenized; and consist of effete matter derived from the nitrogenized tissues of the body. 'Their existence in the blood in health has been fully demonstrated ; and their undue accumulation in certain affections of the kidneys has been proved by direct observation. Deficient exeretion of urea occurs in acute inflammation of the kidneys or nepluritis, in the affection commonly known as acute albuminuria, and as a result of the lesions embraced under the name chronic Bright's disease. Under these circumstances the urea accumulates in the blood, and gives rise to the condition called uræmia. There is no evidence that this condition is ever due to an excessive production of urea of the blood. In all cases it is a consequence of the excretory function of the kidneys being either arrested or inadequate to a proper elimination of this excrementitious substance.

Uræunia is a form of blood-poisoning, or toxæmia, entering largely into the causation of morbid phenomena, and consequently of great importance in its practical relations. Our present knowlelge of it has been recently acquired, and although by no means so complete as could be desired, constitutes one of the most prominent of the characteristics of medicine at the present time, as compared with the past. 'The effects of this morbid condition are manifested especially in phenomena pertaining to the nervous system, coma and convulsions of an epileptiform character constituting the graver of these. These phenomena are apt to precede a fatal termination. Coma and convulsions occurring either with, or subsequent to, scarlatina with renal complication, in nephritis, in Bright's disease, and puerperal eclampsia, are due to uræmic poisoning. The urea accumulating beyond certain limits acts upon the nervous system in a manner analogous to certain poisons introduced from withont the body-for example, strychnia. Its mode of action is not explicable with our present knowledge. The question has arisen whether it acts directly as urea, that is, without having undergone in the blood any change. According to Frerichs, it is converted in the blood into earbonate of ammonia, a substance with which it is chemically nearly identical, and it becomes poisonous only after this conversion has taken place. Frerichs claims to have established the correctness of his opinion by experiments on inferior animals and clinical observation. The admirable experiments of Hammond, however, disprove this hypothesis. ${ }^{1}$

Clinical observation shows that various inflammations are apt to be developed as results of uræmia, more especially serous inflammations, viz., pleuritis, pericarditis, and peritonitis. It is supposed to stand sometimes in a causative relation to pneumonitis. A maurosis is an occasional result. It is one of the pathological conditions under which neuralgia occurs. Other results denote efforts for the vicarious elimination of urea. Persistent vomiting and purging belong to the clinical history of urremia ; and these symptomatic events are due to efforts of elimination of the urea through the gastro-intestinal mucons membrane. The experiments of removing the kidneys of inferior animals, by Prevost and Dumas, Bernard and Barreswill, Hammond and others, show that, under these circumstances, urea accumulates in the blood; after a time vomiting and purging oecur, and either urea or the carbonate of ammonia is found in the contents of the alimentary canal; finally, this vicarious elimination being insufficient to prevent an accumnlation from reaching the point necessary for the production of poisonous effects, convulsions and coma ensue, and death 
speedily follows. . The same consequences take place when the excretion of urea is prevented by certain diseases of the kidneys.

in order to ascertain the existence of uræmia, an analysis of the blood may be made; but this mode is hardly available for ordinary clinical purposes. The existence of uræmia may be inferred whenever, in connection with its characteristic pathological effects, the secretion of urine is suppressed, or the quantity greatly diminished. Urea, however, may be wanting in the urine, although the quantity of the latter be not much, if at all, diminished. 'The urine is of a low specific gravity when the urea is deficient, and, as the deficiency of urea is generally associated with albuminuria, the presence of albumen in the urine, with diminished density, renders it probable that uræmia exists. For positive proof, the urine may be analyzed with reference to the quantity of urea which it contains. If not adequately excreted by the kidneys, urea must accumulate in the blood, and if not eliminated vicariously through some other channel, the pathological effects of uræmia must sooner or later ensue.

The therapeutical indications pertaining to uræmia are, first, to endeavor to promote the excretion of urea by the appropriate emunctories, viz., the kidneys, and, second, to favor its vicarious elimination. The first indication relates to the use of diuretic remedies, which, unhappily, are often inoperative when the kidneys are much diseased. The second indication calls for hydragogue cathartics and sudorific measures, urea being eliminated through the intestinal canal and the skin. These measures are to be relied upon whenever the prompt and efficient elimination of urea is indicated. The spontaneous efforts of elimination through the stomach and bowels are not to be too much interfered with. There are no known means of neutralizing the urea in the blood, or protecting the system against its poisonous effects.

\section{Uricæmia.}

Uric acid, the other important organic constituent of the urine, exists in a small quantity in the blood in health, as shown by the researches of Garrod. Garrod has also shown that it exists in an abnormal quantity in gout. He regards this morbid condition as sustaining a special causative relation to the phenomena of gout. ${ }^{1}$ Uric acid enters into the composition of the deposit around and within the joints, in the tubuli of the kidneys, and sometimes in other situations, which is characteristic of this disease. Moreover, Garrod's researches show that the excretion of uric acid in the urine is often notably diminished during a paroxysm of gout, and that this constituent of the urine is habitually lessened in the chronic form of the disease. The kidneys in persons affected with gout seem to lose, to a greater or less extent, the faculty of excreting uric acid, the faculty of excreting urea remaining intact. It is an interesting fact that in acute rheumatism the uric acid in the blood is not in excess, and the quantity in the urine is apt to be increased. This fact, according to the author just named, goes to establish the non-identity of gout and rheumatism, although as regards their local manifestations, these two diseases bear to each other a close analogy.

Assuming that the pathology of gout involves an excess of uric acid in the blood, the question arises whether this excess be due to an accumulation from insufficient excretion, or whether the uric acid may not be produced

1 The Nature and Treatment of Gout and Rheumatic Gout, by Alfred Baring Garrod, F.R.S., etc. London, 1859. 
in too large quantity. Garrod holds the opinion that both explanations are applicable. He thinks, also, that a reduction of the alkaline condition of the blood favors the deposition of the urates, which experiments show to be precipitated out of the body in a solution to which a weak acid is added, whereas they are held in solution so long as the fluid remains alkaline. 'This view of the pathology of gout is rendered highly probable by' the facts which Garrod's researches appear to establish. It remains to determine whether an excess of uric acid in the blood be not a morbid condition existing in other affections as well as in gout. Garrod has found it to exist in cases of lead-poisoning Continued research may show that this is not the only exception to its being a condition peeuliar to gout, and it may be found to stand in a causative relation to other phenomena than those which characterize this disease. An excess of uric acid (in the form of urates) in the blood, constituting a conclition differing from uremia, it is desirable to distinguish it by a name, and if I might venture to coin a term, I would propose for this purpose, uricæmia.

The therapeutical indications in this condition are to introduce alkalies into the blood, in order to hold the urates in solution, and to promote elimination through the kidneys by diuretic remedies. Clinical experience appears to sustain the correctness of these indications. Dr. Buckler, of Baltimore, has suggested, as a remedy for gout, on chemical grounds, the phosphate of ammonia, the ammonia being supposed to combine with the uric acid, and the phosphorus with the soda, forming two soluble compounds which are readily eliminated by the kidneys. Various clinical observers have borne testimony to the utility of this remedy.

\section{Accumalation of the Excrementitious Principles of Ex-} pired Air, Perspiration, and Bile-Cholmmia-Cholester $\boldsymbol{m}$ ia.

In addition to the kidneys, the lungs, the skin, the liver, and, perhaps, the mucous membrane of the intestinal canal, take part in the elimination from the body of excrementitious principles; and an arrest or impairment of the excretory functions of these organs may lead to an accumulation of noxious matters in the blood. It remains to notice briefly the supposed morbid conditions thus produced.

The most important purpose of respiration, so far as excertion is concerned, is the elimination of carbonic acid. The undue retention of this gas has already been noticed as an element of apnœa. The vapor of the expired breath, called the pulmonary transpiration, contains other exerementitious principles. It is well known that various medicinal substances and certain constituents of articles of food escape through this channel; and the retention of the indeterminate substances contained in the extractive matters of the expired moisture may give rise to noxious effects, either directly or by inducing catalytic changes in the blood. 'But we have no positive knowledge with regard to blood-poisoning from this source.

The chief purposes of the perspiration appear to be the elimination of water and the regulation of temperature. The proportion of solid matter in the perspired fluid is small, chloride of sodium being the largest ingredient. The excrementitious principles which, if retained, may give rise to morbid conditions of the blood, must be contained in the extractive matter of the sweat. The odor frequently accompanying emanations from the skin shows that something is eliminated, which, we may imagine, would be noxious if retained. It is generally thought that important excrementitious principles are eliminated by the skin; but this is a con- 
jecture rather than a conclusion based on actual knowledge. Observation appears to show that disease not unfrequently originates from suppression of the cutaneous transpiration. It is a popular notion that many diseases are thus produced. The importance of this as a source of disease is doubtless vastly overrated; yet the general opinion is not devoid of foundation. The occurrence of disease, however, in consequence of the action of cold on the surface, is not to be explained exclusively on the hypothesis of the retention of excrementitious principles. When the functions of the skin are arrested by cold, there are two other modes in which we may suppose disease to be produced. One of these relates to the circulation. The blood, being driven from the surface, must accumulate in the internal organs, giving rise to congestion. The other mode relates to the increased supplementary activity of certain organs, to compensate for the deficient elimination from the skin. The kidneys and lungs antagonize the skin in this regard, and if the kidneys fail, the labor of performing the eliminative function of the surface falls upon the lungs; hence, disease of the latter-an excessive physiological activity of an organ being liable to end in a pathological condition. Perhaps these two modes of operation will account for the production of disease in consequence of suppressed cutaneous transpiration, in the majority of the cases in which disease is thus produced.

With our present knowledge respecting bile, it is to be considered as embracing both excrementitious and recrementitious principles. The biliverdell, or coloring matter, and the salts peculiar to the bile, viz., the glyco-cholate and tauro-cholate of soda, do not exist preformed in the blood, but they are produced within the liver. On the other hand, cholesterin, which enters into the composition of bile, does not exist in the blood. This constituent, there is reason to believe, is excrementitious. Late experimental researches appear to have established that it is produced in the destructive assimilation of nervous tissue. It is contained more abundantly in the venous blood coming from the brain than in the arterial blood going to the brain. It is found in larger quantity in the portal than in the hepatic vein; and this fact, taken in connection with the fact of its presence in the bile, goes to show that one of the functions of the liver is the elimination of this substance. It appears in the feces in the form of another variety of non-saponifiable fat, called serolin, which it has recently been proposed to call stercorin.. Other substances, viz., leucine and tyrocine, have been found in bile; and it is probable that these are excrementitious, but their physiological and pathological relations are not yet satisfactorily ascertained.

The presence of bile in the blood constitutes the morbid condition called cholæmia. It will be considered, hereafter, as an individual disease, under the name icterus or jaundice; but the condition is incidental to varions affections. Cholæmia is manifested by the yellow or greenish coloration of the conjunctiva and cutaneous surface. As in the case of sugar in the general circulation, the bile is eliminated, to a greater or less extent, by the kidneys, and it is therefore found in the urine in abundance. In the great majority of cases, the condition depends on obstruction of the passage of the bile from the liver to the duodenum. It rarely accompanies certain structural affections of the liver, such as cirrhosis, fatty deposit, carcinoma,

'Vide article entitled "Experimental Researches into a new Excretory Function of the Liver, consisting in the Removal of Cholesterin from the blond, and its Discharge from the Body in the form of Stercorin," by Austin Flint, Jr., M.D., etc., American Journal of the Medical Sciences, October 1862. 
tuberculosis, ctc. These facts go to show that it occurs from the re-absorption of bile after the seeretion has taken place, and not from defective secretion. Defective secretion, however, either with or without obstruction and re-absorption, doubtless occurs, and, under these circumstances, the excrementitious constituents of the bile, which are performed in the blood, e.g., cholesterin, will accumulate if not vicariously eliminated. 'The non-secretion of bile has been called acholia. Occurring either in cases of icterus or independently of the phenomena of the latter, it may give rise to a condition of the blood more immediately serious than the condition caused by the re-absorption of bile, or cholæmia.

Cholremia does not, in general, oceasion serious effects. Patients are not always greatly incommoded by this condition. The re-absorbed bile appears to exert a narcotic inflience, more or less marked, on the nervous system, producing dulness of the mental faculties, a disposition to sleep, slowness of the pulse, and torpor of the functions gencrally. If, however, the condition be prolonged, hemorrhages are apt to occur, and the kidneys may become affected so as to lead to uræmia.

All clinical observers have been led to observe exceptional cases of choliemia, in which the bile appears to exert an intensely noxious influence on the nervous system, indueing coma and death. Excluding cases in which the supervention of uræmia may account for these results, they may be attributed to the retention and aecumulation in the blood of the excrementitious principles of the bile, and perhaps especially cholesterin. There are grounds for believing that the aceumulation of cholesterin in the blood gives rise to a special form of blood-poisoning, to which the name of cholesteræmia has been applied. The grave consequences of aeholia may be explained in this way. It is to be borne in mind that acholia is not necessarily associated with eholæmia ; in other words, there may be non-secretion of bile without, as well as with, obstruction and re-absorption, the latter only giving rise to the phenomena of jaundice. It is not improbable that a deficient elimination of cholesterin may occasion more or less of those indefinite symptoms which are commonly embraced under the name biliousness, and whieh are relieved by remedies supposed to act upon the secretory function of the liver. ${ }^{2}$

It has been supposed that one of the functions of the intestinal canal is elimination of excrementitious principles from the blood. The fluid formed by the glands of Liebcrkuhn, in the small and large intestine, may be in part excretory. This, however, is at present only a conjeeture. There are no known facts which show that the retention of principles contained in the intestinal juice constitutes a morbid condition of the blood.

\section{Saline Constituents of Blood.}

Morbid conditions pertaining to the saline constituents of the blood will claim very brief notice, because, although it is highly probable that they are the seat of important changes, we have very little positive knowl-

' M. le Dr. Picot, Professor of Histology, in Tours, has reported a fatal case of malignant or grave icterus, the history charncterized by notable torpor of the mental faculties, with mild delirium, and, at length, complete coma. the post-mortem examinations having been made with special reference to the existence of cholesteræuia. A cliemical examination of the blood and the brain, in this crse, showed a large accumulation of cholesterin. Dr. Picot concludes that this case goes to confirm the existence and the importance of clolesteræmia as a morlid condition of the hlood. Vide. Journal de l'Anatomie et de la Physiologie; publié par M. Charles Robin, No. 3, Mai et Juin, 1872. Paris. 
edge respecting them. Enumerated in the order of their relative quantity, the more important of the salts in the blood are, the chloride of sodium, carbonate of soda, chloride of potassium, phosphate of soda, and sulphate of potassa. 'The average amount of the salts, collectively, in healthy blood, is estimated to be from 7 to 8 in 1000 parts. Our knowledge of the physiological changes and uses of these constituents is far from complete, but they are manifestly important as supplying matter required for the several tissues and for various secreted fluids. Moreover, they are concerned in the preservation of the red globules, in the solution of albumen, in maintaining the alkalinity of the blood, in the regulation of the passage of liquids through the coats of the vessels in the processes of endosmosis and exosmosis, etc.

The alkaline salts (carbonate of soda and potassa) have been found to undergo considerable fluctuations in disease. They are much diminished in inflammations, and increased in the eruptive and continued fevers. The supposed utility of mineral acids in the essential fevers is consistent with a supcr-alkalinity of the blood. As already stated, a deficiency of the alkaline salts is supposed by Garrod to be concerned in the deposition of uric acid in gout. In articular rheumatism the employment of alkaline remedies, as advocated by Fuller and others, is based on the theory of neutralizing an acid, supposed to be the lactic, in the blood. The pathology of diabetes has been thought to involve a deficiency of alkalies in the blood, and hence the value of alkaline remedies in certain cases.

The researches of Garrod appear to show that a deficiency of potassa constitutes an important element in scorbutus; and, according to this pathological view, the efficacy of lemon-juice and the various anti-scorbutic vegetables is due, not to the presence of an acid, but to the salts of potassa which they contain. This view is snstained by the usefulness of the salts of potassa employed as remedies in this disease.

Finally, the phenomena pertaining to the blood and circulation in that remarkable disease, epidemic cholera, are measurably due to the loss of saline constituents contained in the serous transudation into the alimentary canal.

\section{CHAPTER VII.}

\section{MORBID CONDITIONS OF THE BLOOD-(CONCLUDED.)}

Morbid conditions due to the presence of morbid products, intrinsic and extrinsic-PyæmiaPutrid infection of the blood, or Septicamia-Matter of contagion - Viruses - Infectious Miasms-Venoms-Poisons, palpable, and impalpable or miasms-Zymosis-Considerations which lead to the inference of the prior existence of a constitutional or general morbia con. dition, or indeterninate biood-changes, in various local affections.

THE morbid conditions of the blood which have been thus far considered relate to its normal constituents. The presence in the blood of morbid products gives rise to conditions which are now to be noticed. The morbid products which may be contained in the blood are of two kinds, viz., lst, those formed within the body of the person affected, and, $2 d$, those derived from the body of another person. 


\section{Pyæmia-Septic mia.}

A brief enunciation of views secmingly most consistent with our present knowledge of the morbid conditions to which the above terms relate, is all that the seope of this work will allow. More than this is hardly required in a treatise on the principles of medicine, inasmuch as these morbid conditions enter so largely into surgical pathology.

'The name pyæmia signifies pus in the blood. Purulent infection of the blood is a synonymous phrase. When the name was introduced by Piorry the absorption of pus was supposed to take place without disintegration of its corpuscular constituents. And it was supposed that after gaining entrance within the bloodvessels the pus was deposited in different situations, and that in this way were produced the purulent collections in the lungs and elsewhere known as "metastatic abscesses." After the advent of microscopy into pathological researches, it appeared to be clear that the size of the pus-corpuscles must prevent their passage from without into the blood otherwise than through openings into the vessels resulting from wounds or ulceration, and, also, that, owing to their size, they could not pass through the coats of vessels and be deposited in different organs. Moreover, it became apparent that pus-corpuseles and the white corpuseles of the blood are identical, and the name leucocytes was applied to both. Until within a late period, suppurative phlcbitis was supposed to occur not unfrequently, and, pus being produced in consequence within the veins, its presence in the blood was often thus accounted for. This view, held by many when the first edition of this work was published (1866), seems now to have been in a great measure abandoned by most pathologists, the researches of Virchow and others appearing to prove that inflammation of the lining membrane of the veins is rare. It seems, also, to be proven that coagulation of blood in veins is not, as was formerly held, an effect of phlebitis, but, when the latter is associated it is secondary to the coagulation, and that the lining membrane of the veins is not the one most likely to become inflamed. Again, the facts which have been ascertained respecting thrombosis and embolism (vide page 71 , et seq.), have secmed to account, in a great measure, for the production of "metastatic abseesses," these constituting a characteristic feature of pyæmia. These results of pathological studies have, of late, unsettled the views concerning pyæmia which were held when the name was introduced. In so far as concerns pus-corpuscles being present in the blood, it is denied that this constitutes the essential morbid condition in cases of so-called pyæmia. Admitting pus-corpuscles and the white corpuseles of the blood to be identical, their accumulation in excessive quantity is the morbil condition already noticed under the name lencoeythemia, and in this affection the symptomatic phenomena attributed to purulent infection of the blood are not present.

On the other hand, as supporting the doctrine of pyæmia, microscopical observations still more recent than those just referred to, seem to have ascertained that the purulent collections, that is, abscesses, in different situations consist, at least in part, of the white corpuscles of the blood, or lencocytes, which, under conditions of disease, do pass through the coats of the vessels without any solution of continuity, and migrate into the surrounding tissues. It is claimed that passage from within outward, and vice versâ, has been demonstrated by direct observation. Admitting this, the absorption of pus, together with its disposition to form "metastatic abscesses," should no longer be considered a physical impossibility. That pus sometimes enters into the circulation through openings into 
vessels by orifices resulting from wounds and ulceration is certain; and clinical observation shows that the symptomatic phenomena of pyæmia are most likely to be developed when the entrance of pus in this way is especially apt to occur, namely, in certain diseases of the bones. Again, although pus-corpuscles are identical, as far as microscopical characters are concerned, with the white corpuseles of the blood, it is easy to understand that the former may have acquired toxical properties not belonging to the latter, properties not appreciable by the mieroscope, nor by chemical examination. In these toxic properties may consist their pathogenetic power. Moreover, it is evidently incorrect to consider the corpuscular component as alone constituting pus. The liquor-puris is an essential constituent of a purulent liquid, as much as the liquor-sanguinis is of blood. Now, it is not unlikely that the toxic properties of pus belong especially to the liquor-puris. Finally, by the injection of pus into the blood, in inferior animals, the phenomena of pyæmia, during life, and the anatomical characters after death, may be produced. These facts and considerations go far to sustain the doctrine that in cases of so-called pyamia there is, in fact, a purulent infection of the blood.

Regarded from a clinical point of view, pyæmia denotes an affection undoubtedly involving a special morbid condition of the blood, and presenting certain distinetive features relating to its causation, its symptoms, its course, and the appearances after death. In general, the causation is distinetly traumatic. In most cases it follows an accidental wound or a surgical operation, and more especially wounds or operations involving a bone of considerable or large size, for example, the femur. It may follow inflammations which are not of traumatic origin. It is one of the affections which are embraced under the name puerperal fever, that is, it may arise from uterine morbid conditions shortly after parturition. Occasionally it occurs when there is no apparent causation. It is then distinguished as idiopathic or spontaneous pyæmia. In typical cases the local causation being obvious, it is developed consecutively to suppuration; henee, the pyæmic phenomena are not manifested until after several days from the date of a wound or surgical operation, which, meanwhile, has become notably tender and painful, with more or less diffusion of the inflammation.

The clinical history is characterized by repeatedly recurring chills, more or less pronounced, occurring irregularly, and followed by profuse sweating. Fever is persistent, varying, as slown by the thermometer, in different cases, the greatest amount of heat being directly after the chill, the mereury rising, at this time, from $104^{\circ}$ to $108^{\circ}$. The skin becomes sallow, and not unfrequently distinctly jaundiced. The intelligence is but little or not at all disturbed. There may be pain in the chest, with cough and frequent respiration; and physical exploration may show pleuritis with effusion, or bronchial râles with broncho-vesicular respiration at different points on either side of the chest, or on both sides. Pain may. be felt in the knee and other joints, and effusion within the synovial membrane may be discovered. At length persistent collections, not preecded by the usual local symptoms of phlegmonons inflammation, "cold abscesses," as they are sometimes called, may be found in different situations, sometimes in one or more of the limbs, and sometimes on the trunk. The formation of purulent matter in these situations is sometimes extremely large. Suppurative inflammation of the eye, leading to sloughing and the evacuation of the humors, is an occasional event. This has been observed chiefly in cases of pyremia following parturition. The affection in most cases runs a rapid course, the duration being from eight to ten 
days. With less intensity the disease may continue from two to four weeks, and occasionally it becomes chronic, lasting for several months. In the latter cases the duration depends on the secondary abscesses, which, by their continuance, become properly sequels of the pyramia. In the vast majority of cases the affection ends fatally. Death seems, in some cases, to be attributable to asthenia incident to the morbid condition of the blood, sometimes to heart-clot, and sometimes to the secondary affections of the viscera. In the cases which recover, the local effects consist chiefly of subcutaneons purulent collections and arthritic suppuration. The foregoing enumeration of the leading symptomatic events embraces points by means of which, generally, the discrimination of pyæmia can be made without difficulty.

The most characteristic lesions found after death are circumscribed inflammations and multiple abscesses in different internal organs, but more especially in the lungs. In these organs they generally vary in size from that of a filbert to that of a pea. 'They vary in number in different cases. They are situated especially in the lower portion generally of both lungs, but they are sometimes limited to one lung. The appearances differ in different cases, and the differences are sometimes exemplified in the same case. In the portions affectel, there may be intense congestion or an extravasation of blood. The appearances may be those of pneumonia in the second stage; collections of purulent matter may be found, as expressed by the term "multiple abscesses," and the tissues are sometimes in the state of gangrene or sloughing. Patches of eongestion, inflammatory exudation and suppuration, varying in size from a hen's egg to a filbert, and more or less numerous, are disseminated throughout the liver in certain cases. This organ sometimes contains an enormous amount of purulent matter. The kidneys, in some cases, contain small abscesses, situated in the cortical portion. In the spleen, either extravasations of blood or the so-called fibrinous blocks are often found. Numerous extravasations of blood may be found on the surface of the heart. Ulcerations of the crecum and colon are sometimes observed. The anatomical characters of peritonitis, pleuritis; and pericarditis are not unfrequently present, the inflammation, however, of these membranes being probably dependent on a prior affection of the solid viscera which they invest, viz., the lungs, heart, liver, spleen, and kidneys. Circumscribed softening of the brain is sometimes observed. Fibrinous clots of ante-mortem formation are often found in the right cavities of the heart.

The lesions just enumerated are, in great measure, those already noticed as incident to embolism (vide page 71, el seq.). Now, does this fact prove that pyæmia is neither more nor less than embolism? A negative answer to this question is based on the following considerations: Cases of embolism by no means always present the clinical history of pyæmia. The socalled infarctions occur in different viscera without the constitutional symptoms which are considered as denoting a purulent infection of the blood. In cases of pyæmia, the constitutional symptoms-pulse, temper:1ture, etc.-are out of proportion to the local effects, that is, the multiple abscesses, etc., and life may be destroyed apparently as a direct consequence of the blood-poisoning irrespective of these local effects, although, doubtless, the latter react upon the system and give rise to more or less increase of constitutional disturbance. In some well-marked cases of pyremia these local effects are wanting. Certain of the local secondary affections are not explained by the theory of embolism; for example, the inflammation within the joints and the extensive subcutaneons abscesses. Lesions which, it is admitted, involve embolism, seem often to involve something more than 
the effects of simple obstruction of the vessels. This statement applies to active inflammation and sloughing in the lungs and elsewhere. It appears to be a fair conclusion from these considerations that, although embolism forms an important element in many, if not most, cases of pyæmia, the morbid condition of the blood denoted by the latter term embraces something more than the presence of either plugs or disintegrated thrombi, which produce their local morbid effects by a mechanical obstruction of arteries. Pure embolism is not properly a toxæmia, whereas the phenomena of pyæmia point to an extremely grave toxæmic condition.

The evidence that in pyæmia there is a purulent infection of the blocd is derived from the following facts: The affection occurs connectively to suppurative inflammation. It occurs when, from the situation of the suppuration, as in osteo-myelitis, it is apparent that pus is liable to enter the circulation. The phenomena may be produced by injections of pus into the veins in inferior animals. The experiments of Savory, it may be here stated, show that even the local effects of embolism in the lungs, that is, multiple abscesses and gangrene, may be produced by the injection of pus serum, filtered so as to exclude all solid particles which might act as capillary emboli. ${ }^{1}$

Embolism, occurring in pyæmia, is doubtless dependent on thrombosis of veins, either within, or in the neighborhood of, the parts affected with suppurative inflammation. Phlebitis, whether it be a cause or an effect, of thrombosis, doubtless occurs in a certain proportion of cases. The emboli, under these circumstances, may become the carriers of a special toxic principle. In addition, the pus in mass, or the liquor puris, may gain entrance into the blood, either through open orifices into veins or by absorption. In what consists the special morbific agency of pus in cases of pyrmia, whether this agency be due to chemical changes or to the presence of animal or vegetable organisms, are points of inquiry which further researches are to elucidate.

The question as to the contagiousness of pyæmia is, of course, one of not only pathological interest, but practical importance. With reference to this question, the view which seems most rational is, that emanations from suppurating parts contain a materies morbi which, by coming into contact with existing wounds or external inflammations, and exciting therein a morbid action, leads to pyæmia. According to this view, pyæmia is not communicated, like smallpox, typhus, etc., by an infectious miasm entering into the system through the lungs or the alimentary canal; but it is, nevertheless, communicated by means of a virulent morbid product acting primarily on a part already suppurative. This morbid product diffused in the atmosphere, attached to the walls of hospital wards, to bedding, the clothing of medical attendants, etc., occasions the production of the disease in those suffering from wounds, or after surgical operations. Hence, the importance of ventilation, the efficient use of disinfectants, and the isolation of cases treated in hospitals.

Septicæmia.-This term denotes the presence in the blood of septic matter, that is, a product of the putrefaction of animal substance. The term is synonymous with "putrid infection of the blood." Putrescent matter is supposed to enter the blood either through the lymphatics or the veins, in certain cases, after wounds and surgical operations. Typical examples are afforded by these cases, and, moreover, they form the great

1 Vide articles by William S. Savory in St. Bartholomew's Hospital Reports, vol. 1,1865 : also vol. 3,1867 . 
majority of the cases of septicamia. Hence, this morbid condition belongs rather to surgical than to medical pathology. 'The absorption of putrid matter from within the uterus is supposed to take place sometimes after parturition, especially if portions of the placenta be retained. A certain proportion of the cases of so-called puerperal fever, thus, are cases of septicrmia. This morbid condition of the blood nay also be produced by the absorption of putrid matter from situations in which spontaneous, that is, non-traumatic, sloughing, or gangrene occurs.

Clinically, the symptoms which are recognized as characterizing septicæmia, present certain features, by means of which it may be differentiated from pyæmia. With or without an initial chill, fever occurs, and the thermometer indicates speedily a considerable rise of temperature. There is no recurrence of chill, if it have once occurred, but perspirations early in the disease are not infrequent. Diarrhœea is generally present, and it is often profuse. Delirium often occurs, which may. be manifested by incoherence and muttering, or sometimes by mania. Aside from delirium, somnolency and indifference are mental symptoms commonly present. Generally before death the extremities become cold, and the thermometer shows a decline of temperature which sometimes falls below the minimum of health. It is supposed that septic poisoning, in a mild or moderate degree, not infrequently occurs and ends in recovery; but in the cases in which the symptoms denote a grave affection, the proportion ending fatally is very large. The duration in fatal cases is short, in general only a few days, but it is sometimes prolonged to several weeks.

As regards the appearances after death, the infarctions attributable generally to embolism, and the multiple abscesses so often found after death from pyæmia, are wanting. Evidence of pleuritis and pericarditis is sometimes found. 'The spleen is enlarged and softened. Suppuration beneath the skin and gangrene are, in some cases, secondary affections. The intestinal mucous membrane is often swollen and softened.

The evidence of the existence of a putrid infection of the blood is derived from, 1st, the occurrence of a group of symptoms, forming a welldefined and recognizable acute general disease which is developed in connection with putrefactive decomposition incident to wounds, surgical operations, parturition, and spontaneous gangrene; and, $2 \mathrm{~d}$, the similarity of symptoms and appearances after death, when putrid liquids are injected into the veins of inferior animals.

If it be true, as is now held by many, that putrefaction always involves the agency of minute organisms, then, of course, it follows that the causation of septicæmia involves the presence of these organisms. This view of the causation will account for the efficacy of disinfecting agencies and topical applications which are destructive to infusorial life; and this view enforces the importance of preventing the access of putrescent germs contained in the atmosphere to parts which are the seat of tranmatic or idiopathic inflammation, and, if this cannot be done, of employing carbolic acid or other means of effecting their destruction. It is affirmed by L. Coze and V. Feltz, that the organisms known as bacteria are found in the blood in abundance in cases of septicamia, and that herein consists an essential pathological distinction between this affection and pyæmia, these organisms not being found in the latter affection.'

Although there are sufficient grounds for considering pyæmia and

1 Recherches cliniques et experimentales sur les maladies infectieuses etudiées spécialment au point de vue de l'état du sang et de la presence des ferments. Paris, 1872. 
septicæmia as distinct toxhæmic conditions, yet clinical observation shows that the phenomena of both may be combined. After wounds, surgical operations, and parturition, as well as in connection with sloughing or grangrene when not produced by traumatic causes, in some cases, both putrid and purulent infection of the blood ensue, the symptoms and the morbid appcarances belonging to exch being associated.

\section{Melanæmia.}

Microscopical observers within late years have described a morbid condition of the blood consisting of the presence, in more or less abundance, of pigmentary granules and dark-colored cells. The granules are black or brown particles, rounded or angular in form, existing in the blood isolated and collected in masses. The cells resemble the white globules of the blood in form and size, their dark color being due to the pigmentary granules which they contain. Robin considers them to be the white globules, or leucocytes, containing dark-colored granules. To this morbid condition the name melanæmia has been applied.

Melanæmia is observed both after death and in blood drawn during life, in cases of malarious disease. Certain organs of the body, in examinations after death, in cases of periodical or malarial fever, present notable discoloration from an accumulation in the capillary vessels of pigmentary granules and the dark cells. The liver and the spleen are the organs in which this discoloration is especially observed, but it may extend to the brain, spinal cord, kidneys, lungs, and other parts.

The pathological effects of the melanæmic condition cannot be considered as established. Frerichs supposes that the accumulation of the granules and cells in different organs may occasion obstruction to the circulation sufficient to give rise to congestion and stasis, and to interfere materially with the functions of the organs. affected. The accumulation of pigment in the liver, he thinks, may explain, in some cases, the occurrence of intestinal hemorrhage, diarrhœa, and dropsical effusion into the peritoneal cavity. Cerebral phenomena-stupor, delirium, convulsions, or paralysis - may possibly be due to the accumulation of pigment in the brain ; and the accumulation in the kidneys may cause albuminuria and general dropsy. The production of anæmia is attributed by Frerich to the accumulation of pigment in the spleen.

The source of the pigment is supposed to be the hematin or coloring matter of the blood; hence, melanæmia involves destruction and consequent paucity of the red globules, or anæmia. Virchow supposes that the pigmentary granules and the cells are derived from the spleen, and, hence, melanæmia is a result of disease of that organ. The melanæmic condition will be referred to in connection with the morbid appearances found after death from malarial fever, in the second part of this work.

\section{Viruses-Miasms-Venoms-Pois ons.}

Of the morbid conditions of the blood induced by morbid products originating within the bodies of the persons affected, that is, intrinsic products, there are doubtless many in addition to those which have been noticed. We do not possess demonstrative nor even inferential knowledge of the different kinds of these morbid products existing in the blood, the sources whence they are derived, the manner of production, etc. We only know, by means of clinical observation, some of the circumstances under which they originate, and the phenomena to which they give rise. They 
belong to what may be called the indeterminate morbid conditiors of the blood. Deferring, for the present, the consideration of these, the morbid conditions of the blood indueed by morbid products derived from other bodies, and those induced by extrinsic matters other than morbid products, claim attention. Morbific substances, of various kinds, developed exterior to the body, i. e., extrinsic, may gain access to the blood, and give rise to disease, either directly, by their immediate action on one or more parts to which they are transported in the blood, or indirectly, by occasioning morbid changes in the blood itself.

In this category are embraced morbid products by means of which certain diseases are commumicaterl from those affected with them to others in health. 'These diseases are called either contagious or infectious. The morbid product which is the efficient agent in the communication of a contagious or an infectious clisease is called a virus. $A$ virus is a morbific principle, produced in the body of a patient having a particular disease, which is capable of producing the same disease when it enters the blood of another person. As thus defined, a virus is sometimes contained in matter which is appreciable, and this matter may be either liquid or solid. Examples are the fluid contents of a variolous vesicle, and the crust or seab which is formed by desiccation-both contain a morbific principle which is capable of producing smallpox. In this disease there are emanations from the boc:y, not apparent to the senses, which contain, also, the virus, inasmuch as, getting into the blood, probably through the inspired atmosphere, they are capable of communicating the discuse. The insensible emanations from the body belong among the morbific matters distinguished as miasms. A miasm may be defined to be morbific matter not recognizable by the senses. This term, however, is not limited to emanations from the body; all inappreciable forms of morbifie matter may be called miasms. Of course, then, there are miasms which do not contain a virus, since a virus is always a product of disease. A familiar example of a miasin of this kind is the malaria which causes periodical fever, or marsh miasm, as it has been called.

In this connection the terms contagion and infection may be defined. These terms have been, and still are, used without much precision as regards the relative signification of each. They are often used as synonyms. The definitions of different writers do not agree. It is not easy to settle upon definitions which are in all respects satisfactory. The term contagion, in its etymology, implies communication of a disease by contact. If, however, the sense of the term be extended by considering that the contact may be immediate or mediate, it may embrace all modes of communicability, including emanations, the medium of contact, in the latter, being the atmosphere. If it be desirable to give a precise definition to each term, conlagion may be restricted to the communication of a discase by means of a virus contained in a palpable morbid product, viz., serum, lymph, pus, or solid matter. On the other hand, infection may be used to denote communication by means of an inappreciable emanation or miasm. With this use of the terms, some diseases are propagated by contagion alone, for example, syphilis; some by both contagion and infection, for example, smallpox; and others by infection alone, for example, pertussis, or hooping-cough. It is, however, customary and convenient to treat of the diseases which are diffused exclusively by infection-scarlatina, typhus-fever, etc.-as contagious diseases. Moreover, the term infection is often applied to miasms which do not contain a virus.

Fach contagious or infections disease has its own peculiar and specific morbid product. The virus of smallpox, the miasın of scarlatina, for ex- 
amples, produce respectively these two diseases, and no other form of disease. And in this fact we have a grand criterion of the special character of a disease, and of determining the identity or non-identity of diseases which may have more or less phenomena in common. Typhus and typhoid fever, for instance, have been considered by some identical, and by others non-identical. Now, when it is conclusively shown that the infectious miasm of typhus gives rise always to this form of disease, and never to typhoid fever, and, conversely, that the infectious miasm of typhoid fever gives rise to this disease, and never to typhus, we have the best of evidence that they are not one disease, but two distinct diseases.

Of the essential nature of the different morbific principles which constitute viruses, that is, in what consists their faculty of producing each its own disease, and of reproducing the same virus, we have not, as yet, positive knowledge. The hypothesis which has of late years been generally entertained_-an old doctrine revived by Liebig-is, that a virus, received into the blood, acts as a ferment, exciting therein processes called catalytic. The grounds for this hypothesis are the inappreciable amount of material substance which suffices to communicate a disease, and the occurrence of days or weeks between its introduction into the blood and the manifestation of any morbid phenomena. Diseases supposed to be thus produced have been distinguished as zymotic, a term denoting a ferment, which was introduced and made the basis of a nosological arrangement of diseases by Farr. All contagious or infectious diseases were considered as zymotic, and also all diseases which prevail as either epidemics or endemics. For information respecting the processes embraced under the vague term catalysis - a term which has served chiefly to mask our ignorance of the nature of the processes which it embraces-the reader is referred to other works. Suffice it to say, with regard to fermentations, it seems to be well established that they are not mere chemical reactions excited by the presence of what is known as the ferment; but they are effects of the functional activity of living organisms, either animal or vegetable. At the present moment it is a question whether the doctrine of zymosis be not, in a great measure, resolvable into what is now known as the germ thcory of disease. According to this theory, in certain diseases, morbid fermentative or catalytic processes take place without any reproduction of a materies morbi, that is, a virus capable of dereloping the disease in others. These processes may be compared to the action of diastase, which, placed in a solution of starch, changes the starch into glucose; but the resulting compound has no power to effect the same change in another solution of starch. The so-called zymotic diseases to which this analogy is applicable, not giving rise to a contagious or infectious virus, are not communicable. On the other hand, certain diseases involve in their causation and pathology fermentations in which the ferment, that is, the virus, is reproduced. A comparison applicable to these discases is the alcololic fermentation, the power of inducing the same process being transmitted to the yeast through successive generations. The diseases characterized by fermentations of the latter kind are contagious or infectious. Further notice of this theory will be made at the end of the next chapter in connection with the causation of diseases.

An extrinsic morbid product, giving rise to a morbid condition of the blood, may come from an inferior animal. Hydrophobia is thus derived from the dog or cat, and glanders from the horse. Disease in man may, also, be produced by the introduction into the blood of a healthy product of secretion derived from an inferior animal. Such a product is not a virus, but a venom. Venomous animals produce, physiologically, a pro- 
duct which, received into the system of man, or another animal, gives rise to disease. Thus, certain serpents and insects have speeial glands furnishing secretions which cause disease and even death when incroduced in sufficient quantity into the human body. 'These secretions, normal as regards the animals which furnish them, are venomous.

Various morbific substanees which are neither viruses nor venoms are embraced under the name poisons. They are derived from the mineral and the vegetable kingdom. Their morbid effects are, in general, proportionate to the quantity of poison received into the system. Here is a striking point of difference as contrasted with viruses. The morbid effects of the latter bear little or no proportion to the amount received. The effeets of some poisons are cumulative; that is, they are manifested suddenly, as it were, by a kind of explosion, when the quantity has increased to a certain amount. Lead, for example, may be taken into the systern for a long period without any manifestation of morbid effects, but at length, when the aceumulation has reached a certain point, its poisonous results are suddenly declared.

Extriusic poisons are palpable and impalpable. Palpable poisons admit of examination, and their source and nature are understoor, but not always the mode of their operation. Impalpable poisons belong among the miasms. The physical and chemical characters of these have not been aseertained, and their sourees are not always known.

The palpable poisons are numerous. Their study constitutes an important branch of scientific inquiry, called Toxicology. Eximples of the palpable poisons are, arsenic, lead, mercury, prussic acid, etc. etc. It is now gencrally admitted that they act by getting into the blood, and not by means of sympathetic influences propagated from the part with which they first come into contaet, as was formerly supposed. They offer marked differences as regards the gravity of their effects. Some are quickly fatal in a small quantity, as prussic acid, strychnia, aconite, etc. Others act more slowly and with less virulence, as mercury, lead, etc.

The effects of extrinsic poisons are manifested in different parts of the body. Certain poisons exert effects on particular parts. Thus, alcohol and opium exert their effects on the brain, arsenic and oxalie acid on the heart, strychnia, woorara, and conia on the spinal cord, mercury on the mouth, lead on the muscles, etc. Many, however, act simultaneously on a greater or less number of parts. As regards their effects on the blood, some are merely mixed, or in solution, and act directly on parts to whieh they are carried in the circulation. Oxalic acid, arsenic, mercury, lead, prussic acid, alcohol, ete., have been discovered in the blood. In other instances chemical combinations take place in the blood. A striking illustration of this is afforded by one of the experiments of Bernard. Emmlsin and amygdalin are not poisonous separately, but they combine and form prussic acid. Injected separately into the veins in different animals, they do no harm. If, however, they are suceessively injected into the reins of the same animal, they combine in the blood to form prussic acirl, and the animal dies as if struck by lightning. On the other hand, the blood prevents certain combinations which take place readily out of the body. An experiment of Bemard illustrates this fact. Cyanuret of potassium and lactate of iron in combination form Prussian blue. Injected successively in the veins, they do not combine in the blood, but the combination takes place and Prussian blue is formed after both have entered the urine, or within the intestinal canal.

Examples of impalpable poisons are the miasms giving rise to the periodical fevers, epidemic cholera, cholera infantum, epidemic dysentery, yel- 
low fever, etc. All the extrinsic causes of disease which are special in their character, and not of the nature of virus or venom, belong anong the miasms. By the term special is meant causes, each of which produces exclusively a particular disease. Now, the existence of a special cause, as just defined, may be inferred from the special character of a disease. Whenever, therefore, the special character of a disease is established, we may attribute it to a toxical origin. All endemic and epidemic diseases have a special character; in other words, the events which make up their clinical history are so definite and are regulated by such fixed laws, that they undoubtedly proceed from special causes, which it is certain are produced, not within, but without, the body. These special causes are not of the nature of virus or venom; they are impalpable, or miasms. But their nature and source are very imperfectly known, nor does our present knowledge enable us to understand the morbid conditions of the blood which they occasion. Of course, these morbid conditions and the special causes differ in different epidemic or endemic diseases. The condition and the cause in yellow fever, for example, cannot be the same as in epidemic cholera, in view of the differences as regards the phenomena and laws between these two diseases. But there is this striking point of contrast between the operations of poisonous miasms and the virus of contagion and infection: Poisonous niasms do not lead to a reproduction, by the processes of disease, of the poison, and, hence, the diseases to which they give rise are not communicable.

\section{Indeterminate Blood-Changes.}

The morbid conditions of the blood, so far as they are at present known to the pathologist, liave now been passed in review. It is evident that our existing knowledge is very incomplete. Were all the morbid conditions of the blood fully understood, there is reason to believe that we should have a thread guiding us far through the labyrinth of pathology. This knowledge would probably do much toward elucidating the greater part of the morbid conditions of the solid structures. The most rational view of the source of disorders referable to the eirculation, to the various secretions and excretions, nutrition, together with morbid states of the muscular and nervous systems, is, that they proceed often, if not generally, from blood-changes. The blood offers at the present moment a most interesting and promising field for the scientific inquirer. It is here that important discoveries are to be made which will shed light on the nature and source of diseases now imperfectly understood. In view of the physiological relations of the blood, and what has been already ascertained respecting its pathological relations, it is safe to prophesy that future revelations in pathology are mainly to come from analytical, microscopical, and experimental researches in this direction.

The remark has been already made that the existence of numerous morbid conditions of the blood may be logically inferred, although, with our present knowledge, they cannot be demonstrated. In other words, there are many indeterminate blood-changes involved in different diseases. What are the consiclerations which warrant this conclusion? I shall devote the remainder of this chapter to the answer of this question.

It is a reasonable supposition that all the diseases distinguished as general, or constitutional, or of uncertain seat, involve blood-changes. In fevers, generally, if not always, these changes are occasioned by extrinsic morbific matter, either a virus, a miasm, or a poison. But many other diseases evidently involve a general or constitutional pathological condition, 
called a cachexia or dyscrasia. Now, it is more rational to refer this condition to the blood than to any other portion of the body. Exclusive of the blood, there is only one anatomical system sufliciently extensive in its relations to warrant a suspicion of its being the seat of the essential pathological condition in general or constitutional affections; this is the nervous system. But the nervous system, as a generator of force, depends on the blood. Without blood it develops nothing, and its functions are soon lost. Morbid conditions of the blood voluntarily produced affect powerfully the nervous system. For examples, alcohol injected into the veins produces the phenomena of inebriation, chloroform or ether extinguishes sensation, strychnia excites the motor fibres of the spinal cori, woorara paralyzes them, etc. 'These substances exert their effects either by being transported in the blood and acting upon the organs affected, or by means of modifications which they produce in the blood. Clinical observation, moreover, shows that a large proportion of nervons affections are due to prior blood-changes. As between the blood and nervous system, therefore, the essential pathological conditions which constitute tle cachexia or dyscrasia must relate primarily to the former.

Assuming the correctness of the statement just made, it is enough, with regard to any local affection, to show that it is dependent on a general or constitutional morbid condition, in other words, that it involves a cachexia or dyserasia, to render it probable that it proceeds from a blood-change of some kind. It only remains then to inquire what eircumstances authorize the inference that a local affection depends on a general or constitutional condition. 'The'following are grounds for such an inference:-

1. A local affection not due to the action of an appreciable cause acting on the part affected, is said to be spontancous. Of course, it is not so called with strict propriety. Every affection must have an adequate determining cause. 'The distinction implied by the term is, the cause is internal and inappreciable, and the term is used in contradistinction from causes called traumatic. Now, the internal determining causes involved in the production of the so-called spontaneous diseases, it is not probable, are exclusively local; they are not generated within the parts affected, but, originating within the system somewhere, they are brought to bear upon the organs or structures in some particular situation. For exanple, a person, exposed to no obvious cause of disease, is attacked with pleuritis ; whence comes the internal cause determining this disease? It is reasonable to conclude that the disease is a local expression of some prior more general morbid condition. Clinical researches have shown this to be true in certain cases in which pleuritis, occurring in the course of Bright's disease, is fairly attributable to the accumulation of urea in the blood. 'The so-called spontaneity of any disease is presumptive evidence of its being a result of the localization of a morbid condition seated in a movable element of the body, that is, the blood. Going no further than the simple fact of a local affection cleveloped without any appreciable local cause, the existence of a blood-change is a logical inference from this fact. That most local affections distinguished as spontaneous do proeeed from some general or constitutional morbid condition, is rendered probable, in addition to the consideration just presented, by others which are to follow.

2. Local affections characterized by morbid deposits are, from this fact, rationally attributable to a morbid condition of the blood. 'The production, for example, of purulent matter in great abundance, having the property of virus in smallpox, or the morbid product within the Peyerian, solitary, and mesenteric glands in typhoid fever, denotes a blood-change proper to each of these diseases. In like manner, all the cutaneous erup- 
tions involving morbid products, especially when not due to obvious local causes, in other words, when spontaneous, imply blood-ehanges. The old medical philosophers, guided by common sense, before the discovery of the circulation, attributed most diseases to the presence of certain peccant humors, which were to be concocted and expelled before recovery could take place. They regarded the local and general phenomena of diseases as proceeding from the efforts of nature at coction and elimination. Morbid proeesses, therefore, were, in a great measure, conservative. These notions gave way under the influence of an exelusive solidism, developed and fostered by the study of morbid anatomy which is occupied, not with morbid aetions, but their results ; disease itself was overlooked, the attention being engrossed with its effeets. But in the ancient common-sense notions lies the germ of true pathology, as modern researehes are constantly exemplifying more and more. With our present knowledge, the old humoral doctrine is, in the main, applicable to the diseases which have been numed ; and its application may be extended to other diseases characterized by morbid deposits, such as gout, tuberculosis, carcinoma, and perhaps even to certain inflammations attended by the exudation of lymph.

3. Disorders of the different secretions and excretions denote bloodchanges. This is true more especially of excretions, inasmuch as excreted matters exist formed in the blood, and are simply eliminated by the excretory organs.

4. The fact of local affections occurring simultaneously or in quick succession in different situations, is evidence of a general or eonstitutional morbid condition. Examples are, multiple abscesses in so-called pyæmia, tubercle deposited in various organs, caneer, ete. Shifting of the loeation of the manifestations of disease, as in acute articular rheumatism, is ananother point of evidence.

5. The occurrence of a local affection on the two lateral sides of the body, and a correspondence of the affection of the two sides as regards the character of the affection, its situation, extent, etc., constitute a very strong point in evidence of a constitutional morbid condition, involving a blood-ehange. A striking law of symmetry characterizes certain diseases. They may be distinguished as symmetrical diseases. Examples are various cutaneous diseases, articular rheumatism, Bright's disease of the kidneys, pulmonary tuberculosis, ete. We cannot well conceive that the internal determining conditions which give rise to the local manifestations in these discases are seated elsewhere than in the blood. We may assume it to be a rational conclusion that the essential pathology of all symmetrieal diseases pertains to the blood; and the fact that the law of symmetry is exemplified in any disease, suffices to render it probable that it is a blood-disease.

6. The modus operandi, so far as known, of remedies found to be useful in the treatment of a great number of local affections, shows their source to be in the blood. The remedies referred to are those which act by being absorbed into and modifying the blood. These remedies have been significantly termed alteratives. Their immediate effects in the blood are unknown; we can only say that they alter in some way and affect sensibly the condition of the blood. Examples are mercury and iodine.

The foregoing are some of the considerations which establish, by logical inference, the general or constitutional origin of local affections, the nature of the general or constitutional morbid condition being unknown, but consisting, as is rationally probable, in indeterminate pathological changes in the blood. Other considerations which might be adduced are omitted, the aim bcing, not to enter into a discussion of this topic, but only to furnish suggestions for the inquiries and reflections of the reader. 
The general doctrine that the primary morbid conditions in most diseases are seated in the fluids, distinguishes what has been ealled the humoral pathology, in contradistinction from solidism, the doctrine expressed by the latter term being that diseases originate in the solid parts or tissues. The view which has just been presented respecting the great extent to whioh blood-changes are involved in disease, does not require the belief" that these changes are necessarily humoral in their origin. There is ground for the belief that many, if not most, of the morbid changes of the blood are secondary to, and dependent upon, local morbid processes in solid parts. When the physiological changes in the blood are considered-several pounds of fresh material being daily added and an equal amount parted with in the processes of exhalation, secretion, exeretion, and nutrition-it is difficult to conceive how definite morbid conditions here can be self-persisting, that is, continue from the operation of laws inherent in, and limited to the blood. Moreover, it is to be considered that the blood in health is not self-existing and self-renewing, but that it is dependent upon the action of solid organs. The indeterminate bloorlchanges which constitute the essential pathological conditions in the different cachexi:e or dyscrasix, may not originate in the blood, but may owe their origin to the continued introduction into the blood of noxious matter derived from local sources, or to effects of some kind prodnced by certain organs.' In the existing state of our knowledge, the doctrine that diseases are primarily humoral, is no more to be inculcated than the doctrine of exclusive solidism; but, appreciating the limits of our knowledge, the mind should be prepared to accept the development or establishment of truth without being biased by an attachment to either doctrine.

\section{H A P T E R V I I.}

\section{THE CAUSES OF DISEASE, OR ETIOLOGY.}

Etiology a braneil of General and Special Pathology-Its importanee-Internai or Intrinsie and External or Extrinsie Causes-Ordindry, and Special or Speeilic Causes-Traumatic CausesSpontaneous Diseases-Prinary and Secondary Causes-Complications and Intercurrent Diseases-Predisposing and Exeiting Causes-Congenital and Inherited l'redispositions to Disease-Co-operating Causes-Diathesis-Caehexia or Dyscrasia-Endemie Diseases dependent on Emanations from the Soil-Epidemic Diseases produced by Causes existing in the At mosphere-The Causes of some Contagious Diseases exelusively derived from those affeeted with these Dise:ises, and other Diseases of this class produced, in some cases, by other Causes than Contagion or Infeetion-Fonites-Portability of the speeial Causes and 1)iseases not Contagious nor Infeetious.-The Causes of Contagious and Infeetious Diseases probabiy Living Germs or Organisıns.

$\mathrm{E}$ TIOLOGY is that branch of medicine which treats of the causes of disease. As one of the divisions of the Principles of Medicine, or General Pathology, it treats of causes considered with referenee, not to individual affections, but to disease in general or in different groups of diseases. The consideration of the causes of individual diseases forms

1 Vide Virchow's Cellular Pathology, Lecture VI. 
an important part of special pathology, or the practice of medicine. Etiology, therefore, enters into the range of topies belonging to the first and second parts of the work. Following the general plan pursued in the foregoing chapters, I shall limit myself here to eertain distinctions and definitions whieh are to be understood as preliminary to entering on the study of individual diseases.

Knowledge of the causes of disease is highly important, in a practical view, as the basis of prophylaxis, or the prevention of disease. It is obvious that in proportion as we are able to trace diseases to their sources, we may expect to extinguish causes or obviate their morbifie influence. Knowledge of causes also is important as entering into the management of diseases; for it not unfrequently happens that causes continue to be operative after disease has been produced, and their removal is, of course, a prime object in order to effeet a cure. It will be apparent, when we come to inquire into the causes of individual diseases, that the amount of our present knowledge in this direetion is extremely limited. It is true that modern researches have developed mueh information, and not a little progress has been made in the prevention of diseases in eonsequence of increased knowledge of etiology, but there yet remains a wide field for further developments.

In treating of morbid conditions of the blood, reference has been made to certain causes of disease originating within the bodies of persons affected, and to other causes which are received from without. Here is one ground of distinction. Causes are intrinsic or internal, and extrinsic or external. Examples of external or extrinsic causes are the different kinds of miasm, infectious or poisonous, the various species of virus, venous, palpable poisons, together with the numerous ageneies of disease by means of wounds or injuries. On the other hand, examples of internal and intrinsic eauses are, an accumulation of urea in the blood, an excess of uric acid in gout, a morbific principle in rheumatism supposed to be lactie acid, etc.

Among the internal causes belong those which originate in the mind; and these play an important part in the production of morbid phenomena. Over-exertion of the faculties of the mind, mental application unduly prolonged, and intense or long-continued emotional excitement, lead to disorders of the nervous system, together with more or less impairment of the vital forces. Insanity and minor forms of mental derangement may not unfrequently be traced to these causes. The bodily functions are weakened, partly from direct influences pertaining to the nervous system; and, in part, because the operation of these causes involves neglect of hygienic conditions of health which relate to diet, muscular exercise, recreation, etc. Long-continued mental depression, whether produced by the causes just named, or other causes, often stands in a causative relation to disorders of digestion and anæmia. An absorbing sentiment or passion may induce disorders by leading to neglect of the means of preserving health. Finally, a concentration of the attention on the bodily functions, over-anxiety concerning health, and a craving for the interest or sympathy which illness is expected to awaken in others, are important elements entering into many cases of disease, espeeially among females. The knowledge and tact of the physician are often called into requisition in estimating the agency of these various causes referable to the mind.

Causes may be distinguished as ordinary, and special or specific. Orainary causes are those to which all persons are more or less exposed, and which may give rise to two or more forms of disease. Atmospherical vieissitudes are ordinary causes. They are generally supposed to be involved in the causation of disease very frequently, and to give rise to a 
great variety of diseases. Special or specific eauses are extraorlinary in their occurrence, that is, persons are exposed to them only at certain times or in certain situations, and each cause gives rise uniformly to one particular form of disease. 'The poisonous mi:sm which produces periodical fever, the infectious miasm which causes typhus, the virus which gives rise to smallpox, etc., are examples of special or specific causes. The miasm which produces periodical fever is not capable of producing typhus, the infection producing typhus will never give rise to periodical fever, and the same is true of all other ctuses belonging in this category. The existence of a special or specitic cause may be inferred from the specific character of a disease, although the nature and source of the causative agent are not understood. It is certain, for instance, that diseases possessing phenomena and laws so distinetive and uniform as those which belong to the clinical history of epidemic cholera, typhoid ferer, yellow fever, etc., must each have its own special or specific cause, although the causes of these diseases have thus far eluded investigation.

Certain causes are distinguished as traumatic; and this name is applied also to distinguish the diseases produced by this class of causes. 'Traumatic causes are those which give rise to disease by acting in an appreciable manner on the part affected. 'Iraumatic diseases are those thus produced. Anything which occasions an injury or wound of a part, and consequently disease of that part, is a traumatic cause. The name signifies a wound. A bronchitis caused by the inhalation of an irrititing vapor is produced traumatically. A calculus in the pelvis of the kiclney which gives rise to pyelitis, and a stone in the bladder which oceasions cystitis, are traumatic causes. The causes thus distinguished, therefore, may be either internal or external, although, in the great majority of cases, they belong to the latter class.

Diseases not traumatic, and which do not proceed from any appreciable causative agency, are said to be spontaneous. 'This term, as thus applied, is to be taken in a conventional, not a literal sense. It is obviously ineorrect to say of any disease that it originated spontaneously. Every disease must have its adequate determining canse. But many diseases are developed without our being able, in the existing state of knowledge, to refer them to their causes; and the term spontaneous, applied to those discases, means simply that they proceed from causes which our present knowledge does not enable us to appreciate. Pleuritis, pericarditis, and other affections developed frequently in the course of 13right's disease, were formerly considered as spontaneous; but now that their development is attributable to the action of urea accumulating in the blood, the term has ceased to be applicable to them. The number of so-called spontaneous diseases will diminish in proportion as our knowledge of etiology advances.

Causes are distinguished as primary and secondary. A secondary cause is an effect of disease. Urea, for example, aceumulating in the blood, gives rise to various local affections-pleuritis, pericarditis, etc. But the accumulation of urea is an effect of some disease of the kidneys, which interrupts the eliminative function of these organs. Urea in excess in the blood, is thus a secondary cause of disease. A primary cause, on the other hand, does not proceed from a prior disease. All external traumatic causes, for instance, are primary. The same distinction is made among diseases; that is, some are primary, and others secondary.' Primary diseases proceed from primary causes, and those called secondary

1 Protopathic and deuteropathic. 
are due to the effects of pre-existing diseases; in other words, to secondary causes. A large proportion of the diseases, the existence of which is first ascertained, are secondary; they involve in their causation antecedent affections. Not unfrequently a number of diseases are thus linked together in a consecutive series. In the examination of patients, therefore, it is not sufticient to have discovered a disease ; but, having discovered one disease, it remains to be ascertained whether one or more additional diseases do not exist, sustaining to the disease first discovered a relation either of cause or effect. Clinical researches have developed important facts with respect to the causative relations existing between different diseases, and these facts are of great utility in medical practice.

Secondary and subordinate affections are distinguished as complications. A complicating disease is one which occurs in the course of another disease with which it is connected by some pathological relation. Pnenmonitis, for example, developed in the course of typhoid or typhus fever, is a complication of the latter. It is consecutive to the fever, and to a greater or less extent dependent thereon for its production. Pneumonitis gęnerally has a complication, viz., circumscribed pleurisy. A disease is said to be intercurrent when it occurs during the progress of another disease, without necessarily having any dependence on the latter. Lobar pneumonitis, for example, occurring in a patient affected with pulmonary tuberculosis, is an intercurrent affection.

As just stated, certain causes of disease are themselves effects of disease; so that certain diseases may be said to stand in a causative relation to other diseases. On the other hand, some diseases afford protection against others. As an illustration, emplysema of the lungs and pulmonary tuberculosis may be cited. The latter disease occurs in patients affected with the former so rarely as to show that it exerts a protective influence. Another illustration is afforded by disease of the heart and tuberculosis of the lings. A tuberculous deposit occurs so rarely, when cardiac disease occasions much disturbance of the circulation, that the latter must be considered as affording more or less exemption from the former.

Another division is into predisposing and exciting causes. Predisposing causes induce a liability or tendency to certain forms of disease. Exciting causes, acting on persons already predisposed to certain disenses, determine their occurrence. Predisposing causes may be alone sufficient to give rise to disease, or they may only suffice to place the system in a condition favorable for the occurrence of clisease ; in the latter case, the disease occurs when exciting are superadded to the predisposing causes. To cite an instance: A person under the influence of predisposing causes is in a condition favorable for the occurrence of acute rheumatism ; in this condition he is exposed to atmospherical vicissitudes, and the latter determine an attack of this disease. The exciting cause, viz., the atmospherical vicissitudes, would not, alone, have been adequate to give rise to an attack of rheumatism; and the former, viz., the predisposing causes, alone, might have been inadequate; but, conjointly, they occasion this disease. Fxciting causes act the part of the match when everything is ready for an explosion. It is thus seen that predisposing causes determine the nature of the disease, and exciting causes the date of its occurrence.

A predisposition to some form of disease may be inherent in the constitution, or congenital. Some persons have inborn tendencies to certain diseases. This fact is strikingly illustrated by the occurrence of phthisis successively in different members of the same family-a large number of brothers and sisters being carried off by this diseuse. 'This congenital 
predisposition may remain completely latent until the period of life in which the disease is most apt to be developed; and we sometimes see a whole family of children, one after the other, fall victims to this clisease, when they severally reach a certain age. A predisposition to certain forms of disease may not only be congenital, but inherited; a constitution involving a tendeney to disease is transmitted from parent to offspring. Among the diseases perpetuated in this way are phthisis, gout, cancer, asthma.

The predisposition to a disease, whether congenital or acquired, may be strong or feeble. Various circumstances may act as co-operating causes; that is, acting in furtherance of the constitutional tencleney. Thus, in a person predisposed to phthisis, the development of this disease may be greatly promoted by unfavorable hygienic circumstances, such as sedentary habits, deficient ventilation, and inadequate alimentation. These co-operating causes, taken in conjunction with an existing predisposition, may serve to develop the disease, when, without their aid, the predisposition might not have been sufhicient. There is reason to believe that persons with a feeble predisposition to this disease often escape, if they be exempt from the operation of co-operating causes. The latter, moreover, are to a greater or less extent controllable, while the predisposition, especially if it be congenital, is entirely beyond control. Diseases thus are preventable, notwithstanding a predisposition to them, in so far as they depend on the union of co-operating causes. Herein lies a truth of great practical importance.

Diseases which originate from special causes often appear to require for their production co-operating causes. Facts seem to show, for examples, that yellow fever, periodical fevers, and epidemic cholera, which undoubtedly involve the agency of special causes, would in many cases not have occurred, had not other than the special causes contributed to their occurrence. By eradicating, as far as possible, all unfitrorable hygienic influences, the special causes of these diseases may be rendered, to a great extent, or perhaps completely, inoperative. The speeial causes of disease and the co-operating causes are, to quote the comparison of the late Dr. Barton, like the two blades of a pair of scissors-conjoined they may be effective, when disunited they effect nothing. The special causes of these diseases we may not be able to remove; but co-operating causes are, to a great extent, within our control; and, by removing the latter, the diseases are rendered preventable.

A constitutional predisposition to a particular form of disease constltutes what is called a diathesis. A diathesis, therefore, may be either congenital or acquired. And the diseases which are considered as generally, if not always, involving a constitutional predisposition, or diathesis, are sometimes distinguished as diathetic diseases. A cachexia or dyscrasia involves a diathesis, and something more. These terms denote, not merely a constitutional predisposition to disease, but that conclition of the system which exists when the disease is actually developed. A person born with a tendency to phthisis, for example, has the tuberculous diathesis; and this diathesis eventuates in the tuberculous cachexia when the person becomes affected with phthisis.

Our present knowledge, for the most part, of the special or specific causes of disease, is based on inference rather than on demonstration. But certain conclusions respecting their origin and diffusion may be logically determined. One of these conclusions is, that some poisonous miasms emanate from the soil. This may be inferred with respect to the special causes giving rise to diseases called endemic. Endemic diseases are those 
which prevail within circumscribed territorial limits ; that is, their prevar lence does not extend beyond sectional boundaries. Now, this fact is sufficient for the inference that the source of the causative miasms is in the soil ; because, of the elements which enter into climatic influences, those peculiar to any particular district are terrestrial. Again, in the diseases called epidemic, viz., those which prevail successively or simultaneously in different and often wide-spread territorial distriets, the poisonous miasms, whatever may be their source, must be diffused through the atmosphere. 'The causes of epidemic disease are migratory. In some instances they traverse successively almost every portion of the habitable globe. This is true of epidemic bronchitis or influenza, and of epidemic cholera. It is altogether improbable that the special causes in these and other epidemics are developed in the different sections of country over which their prevalence extends. They are produced in the situation whence they take their point of departure, and travel, in some way, through the atmosphere. The special causes which give rise to contagious and infectious diseases are derived from the bodies of those affected with these diseases; and, with respect to certain of these diseases, it is probable that the special causes are exclusively thus derived. It may fairly be doubted whether smallpox ever originates from any other souree, notwithstanding the occurrence of cases in which it cannot be traced to contagion or infection. Yet, the first case of smallpox which ever existed must, of course, have been an exception to this law, and it is possible that there are still occasional exceptions. With respect to certain of the diseases which are communicable, it is probable that they not unfrequently originate otherwise than by contagion or infection. 'This is true of typhoid, and perhaps also of typhus fever.

Diseases are communicated, not only by viruses received by contact or diffused in the atmosphere, but by means of what are called fomites. This term is applied to any inanimate substances, such as clothing or articles of merchandise, to which contagious or infectious matter has adhered. In this way certain diseases, such as smallpox and scarlatina, may be disseminated at points far distant from the source of their special causes. The term fomites is restricted to the transmission of the matter of contagion or infection. But it is probable that other special causes of clisease may be transmitted in the same way. Facts, for example, appear to show that the special cause of yellow fever is capable of transportation. And if this be true, it is correct to say that a disease may be portable, although not contagious nor infectious.

Special causes of disease, embraced under the names contagion and infeetion, have been noticed in giving some account of viruses (vide p. 97). As stated in that connection, the theory which seems most consistent with our present knowledge is, that the essential morbific principles (the contagium) in these causes, are living germs or organisms, either vegetable or animal. This is, by no means, a new theory. The doctrine that the source of epidemic diseases is in animalcule life was advocated many years ago (1839) by Sir Henry Holland, and in a volume by the late Professor J. K. Mitchell, of Philadelphia, published in 1847, were presented facts and cogent arguments in behalf of the cryptogomic origin of malarious and epidemic fevers. Indeed, the theory was advanced very early in the history of medicine, and in modern times was elaborately advocated by the great botanist, Linnæus. Very lately, however, the microscopical study of the lowest appreciable forms of life, and discussions relating to the long mooted question as to spontaneous or equivocal generation, have 
led to the accumulation of weighty evidence in its support; so that the probalility of its truth seems vastly greater now than hitherto.

Assuming the causes of contagious and infectious diseases to be living germs or organisms, a certain period is required for their derelopment and multiplication within the boly sufliciently to give rise to these diseases. In this way, better, perhaps, than in any other, may be explained the fact, that in contagious and infectious diseases, as well as in some which are not communicable, for a period which is sometimes extended to weeks, months, and even years, the special cause remains in the body completely latent as regards any morbid manifestations. The reproduction of germs and organisms within the body affords an intelligible explanation of the formation of viruses, or the morbific principles, by means of which, diseases are communicated. The self-limited duration of these diseases is explained by supposing that, after successive generations, the organisms die out, or they are in some way either destroyed or eliminated from the body. 'The germ theory affords a satisfactory explanation of another fact, namely, that an inappreciable quantity of virus suflices to give rise to the disease of which it is the special cause; and, moreover, that the intensity of the disease bears no proportion to the quantity of virus received. For example, the severest form of small pox may follow the reception of an almost infinitessimal quantity of virus in infection, while, on the other hand, the disease may be mild after the utmost amomnt of exposure. In this point of view, these causes are in striking contrast with poisons, the effects of which are in proportion to the quantity received into the system.

A remarkable fact relating to the causes of contagious and infections diseases is, that, as a rule, they exert their morbid effects but once in the same person. 'These diseases are rarely experienced a second time. Evidently this is because the susceptibility to the causes is destroyed, since it is not to be supposed that the latter lose their efficieney, and they undoubtedly are received into the system as readily after, as before, the disease had occurred. With our present knowledge, the mode in which the susceptibility to these causes is destroyed, cannot be explained. Assuming the causes to be germs, however, it is well known that the evolution and development of organisms therefrom require certain conditions. The seod must have its appropriate soil, and various circumstances may, on the one hand, promote, and, on the other hand, arrest its germination. In some inconceivable mode, contagious and infectious diseases effect a permanent destruction of the unknown conditions within the body, on which depend the development and multiplication of germs which, it is assumed, constitute the causes of these diseases. It should be added, that this is not exclusively an effect of diseases which are communicable. Yellow fever, for example, is rarely experienced a second time. $\Lambda$ deficient susceptibility to the causes of contagions and infections diseases is sometimes a natural defect. 'There are persons who, from birth, and during life, are in no degree affected by the virus of smallpox, and the same is true with respect to the causes of other diseases.

The demonstration of the germ theory requires, 1st, the proof afforded by direct observation of the existence of germs or organisms in the blood; $2 d$, that certain living forms are always present in connection with certain diseases; and 3d, that these forms are not to be found in the blood when the diseases do not exist. It is claimed by microscopical observers that these requirements have been fulfilled. The micrococci of Hallier, the microzymes of Béchamp, the bioplasts of Beale, and the several fornis described by Salisbury are considered, by these observers, as characterizing 
particular diseases. The studies of Davaine, Coze, and Feltz, and others appear to establish conclusively the fact that bacteria in certain diseases are found in the blood. As tending to prove that organisms may gain entrance into the blood from the alimentary canal, the experiments of Dr. Joseph G. Richardson, of Philadelphia, are especially interesting. They consisted in drinking putrescent liquids abounding in bacteria and vibriones, and afterward examining the blood at stated intervals, taking pains to prevent the introduction of infusoria into the specimens of blood after it had been drawn. These experiments showed the presence in the blood, within a short period after the ingestion of the liquid, of the same organisms contained in the latter; they showed that the number of bacteria and vibrios in the blood corresponded to the quantity of the liquid drank; that they disappeared from the blood after a certain number of hours, and that, introduced into blood after it had been drawn, they distinctly increased in size during a period of twenty-three hours.' $\mathrm{T}$ 'o undertake to give even a condensed account of the varied experiments and observations relating to the germ theory, which are to be found in recent medical literature, would not only call for too much space, but it would be here out of place. Moreover, to judge of the accuracy of the microscopical observations, to endeavor to interpret facts, and to discuss the various questions connected with the theory-questions concerning the source of morbific germs; whether they are of animal or vegetable beings; the extent to which the organisms produced are dependent on the laws of development inherent in the germs, or on extrinsic circumstances; and, lastly, the long-mooted question as to spontaneous generation-to do all this, were there no difficulty on the score of space or propriety, qualifications are required to which I cannot lay claim.

In conclusion, the observations which have shown the great abundance and the wide diffusion in the atmosphere of organic entities invisible to the naked eye; the researches which seem to show that putrefactive and fermentative processes are effected by the agency of living organisms; the analogy, long recognized, between the phenomena of fermentation and those arising from certain of the unknown special causes of disease; the differences between the action of these unknown causes and of the known vegetable and mineral poisons; the discovery, by means of the microscope, within late years, of cryptogenic formations in affections of the skin and mucous membrane, and, also, of parasitic animals; the detection of living organisms in the blood and other liquids of the body; the successful employment - with a view to the prevention of diseases - of carbolic acid and other articles which are especially destructive to the lower forms of animal and vegetable life, and the apparent remedial power of some of these articles in certain of the so-called zymotic diseases-these are grounds for the conjecture, if not expectation, that continued investigations may lead to results which will greatly enlarge the boundaries of our present knowledge, not only in etiology, but. in pathology and therapeutics.

1 Vide Am. Journ. of Med. Sciences, No. for July, 1868, p. 291. 


\title{
CH A PTER IX.
}

\author{
SYMPTOMATOLOGY.
}

Pathognomonic Symptoms - Diagnostic Symptoms - Subjective and Objective SymptomsSigns-Clinical History of Discases and the ditferent Modes by which it is obtained-stinges of Disease-Precursory or Plodromic Events-Sequels-Diagnosis-Reasoning by way of exelnsion-Differential Diagnosis-Prognosis-Prognosties.

THE study of the symptoms of disease forms a division of the principles of medicine, or general pathology, called Symplomatology or Semeivlogy. The consideration of symptoms will oceupy a large proportion of the space to be devoted to each individual disease, in treating of the practice of medieine, or special pathology, in the second part of this work. Continuing to pursue the plan adopted in the first part of this work, it will suffice to present in this chapter ecrtain distiuctions and definitions which should be understood before entering on the study of indiviclual diseases.

The term symptoms embraces all the appreeialle morbid phenomena occurring in conneetion with disease. 'The name signifies to fall together, and denotes concurrence of events. To reiterate the often quoted coinparison by Galen, symptoins accompany discases as the shadow follows the substance. By means of symptoms the existence of clisease is made apparent, and the character and seat of different affections are ascertained.

Certain symptoms are distinguished as pathognomonic. $\Lambda$ pathognomonic symptom is one which denotes invariably the existence of a particular disease. It derives this signifieance from the fact that it oecurs only in connection with one disease. Whenever such a symptom, therefore, is present, it represents the disease, and, in itself, suflices for the reeognition of the disease. The number of pathognomonie symptoms is quite small. As an illustration of the symptoms of this class, a semi-transparent, viscid, rusty-colored matter of expectoration belongs exclusively to pneumonitis. It is, therefore, a pathognomonic symptom, always denoting the existenee of that disease. In like manner the crepitant râle, obtained by auscultation, is so distinctive of the same disease as to be nearly pathognomonie. It would be diflicult to cite many examples in addition to the two just named.

A symptom is said to be diagnostic when it occurs more frequently in connection with a partieular disease than with other diseases. Just in proportion as it is restricted to a few diseases, is its diagnostic character and importanee marked; that is, it is of value as denoting the presenee of some one of these diseases, and, therefore, of value in diagnosis. Different symptoms differ as regards their respective diagnostie value. Some are highly diagnostic ; in other words, they point strongly to the existence of particular diseases. Others are moderately, and, again, others slightly diagnostic. The measure of diagnostic significance depends on the frequency of concurrence with a particular disease and the infrequency of concurrence with other diseases.

Symptoms are either suljective or objective. Subjective symptoms are those which relate to the sensations or feelings of the patient, and which, 
consequently, the physician can appreeiate only through the patient's description of them. Objective symptoms, on the other hand, are those which the physician observes for himself, and for a correct appreciation of which he is in no wise dependent on the patient. As regards subjective symptoms, the physician is exposed to error in consequence of inability on the part of the patient to describe them clearly. In young children this class of symptoms is not available. 'This is true, also, of idiots, the insane, and in the delirium incident to different diseases. As regards these symptoms, too, the physician is liable to be deceived by intentional misrepresentations on the part of patients; and he is liable to be deceived, unintentionally, by a tendency, on the one hand, to exaggeration, and, on the other hand, to depreciation of morbid sensations or feelings. Owing to these sources of error, much tact is often requisite in placing a proper estimate on subjective symptoms. Objective symptoms are not open to the same liabilities to error. The physician judges of these by the exercise of his own senses, and, if he fail to discover or place a proper estimate upon them, the fault consists in his own want of attention, judgment, or skill. Objective symptoms, thus, are much more reliable than subjective, in the investigation of cases of disease.

The exaggeration of pain or suffering of any kind, and of morbid sensations, is common, sometimes because patients are led to form an exaggerated idea of their subjective symptoms; sometimes with a view to awaken interest or sympathy; sometimes for malingering purposes, and sometimes to secure greater attention on the part of the physician with more efficient treatment. Factitious and exaggerated ailments enter pretty largely into the cases which the practitioner is called upon to treat, especially among females.

A symptom, as already defined, is any manifest morbid phenomenon or event. Occurring concurrently with a disease, it may, or may not, have a pathological connection with that disease. Its pathological connection with the disease may be such as to render it a pathognomonie symptom, ol more or less diagnostic; and, on the other hand, it may be present as a mere coincidence, or accidentally. In the latter case, it has no pathological connection with the disease, and is devoid of diagnostic signifeance. This difference among symptoms has been expressed by calling them signs when they are either pathognomonic or more or less diagnostic. According to this distinction, a symptom becomes a sign whenever it denotes, in a greater or less degree, the existence of a particular disease. The term signs, however, has come conventionally to be used in a different sense. It is usual to apply this term to the phenomena obtained by certain methods of investigation distinguished as physical, viz., percussion, auscultation, etc. These phenomena are called physical signs, but for convenience, the word physical is often omitted, and is considered as understood when the term sign is alone used. In this sense of the term signs, or, as restricted to the phenomena obtained by the physical methods just named, it is used in contradistinction to symptoms, the latter embracing all the other phenomena which are manifested in connection with disease. The phenomena thus included under the name signs, are, of course, objective, and will be found to be the most reliable of those by means of which the existence, seat, and character of certain diseases are ascertained.

Late researches have shown that by means of the thermometer symptomatic phenomena of much value may be ascertained. The variations in animal temperature in disease, as determined by the thermometer, have been studied, within the past few years, by Wunderlich in Germany, 
Ringer in Great Britain, and many others. For determining conveniently the heat of the body, a thermometer specially adapted to this purpose is requisite. A self-registering thermometer is to be preferrerl. The bulb of the instrument is to be placed deep within the axilla, and the arm of the patient folded across the ehest, the perspiration having been first wiped away. The instrument should remain in this situation for from five to ten minutes. It is a good practical rule to allow the thermometer to remain until a minute passes withont any further rise of the mercury, the physician pressing the shoulder of the patient in order to secure close contact of the integument on all sides of the instrument. 'The normal temperature is from $98^{\circ}$ to $99^{\circ} \mathrm{Fahr}$. in the great majority of persons; exceptionally it may be half a degree, or even a little more, either above or below this range. The normal fluctuations in different climates or seasons, and under different circumstances as regards exercise, diet, etc., are inconsiderable in comparison with the variations incident to certain diseases.

From observations on healthy persons, Dr. Wm. Ogle deduced the following conclusions respecting diurnal fluctuations of temperature in health: The temperature is at its minimum at about 6 o'clock A.M.; the maximum is reached late in the afternoon, and then there is a fall, which lasts until $6 \mathrm{~A} . \mathrm{M}$. The range of the ordinary diurnal fluctuations is not greater thin about one and a half degrees, Fahrenheit. Exercise canses a considerable rise. Food causes a rise, which is most marked after breakfast, less so after lunch; and after clinner there is a retarlation of the fall which would otherwise occur. Alcohol diminishes the temperature. 'I'ea causes an elevation.'

The introduction of the thermometer into clinical investigations is of recent date. That by this means highly important symptomatic phenomena are obtained, facts have sufficiently established. As a means of determining with accuracy the animal temperature, the thermometer is indispensable. The sensation eommunicated to the hand placed on the skin, on which physicians have heretofore relied for ascertaining the heat of the body, is not to be depended upon; the thermometer in the axilla may indicate an increase of heat, when, to the touch, the surface of the body does not seem to be warmer than in health. Still less reliable are the sensations of the patient; he may complain of coldness when the thermometer indicates increase of heat, and vice versâ. By the use of the thermometer, symptoms pertaining to animal hent are rendered objective, and determined with precision. Clinical observation has shown that the axilla is a much better situation in which to place the bulb of the instrument than under the tongue, and a properly eonstructed instrument may be very conveniently applied in that situation.

The study of the thermometric phenomena in different diseases has developed certain laws, the more important of which may be here stated.

In fevers and other acute diseases, the temperature of the body, as indicated by the thermometer, is always more or less raised, thus substantiating the propriety of the term fever, which signifies increase of heat. If the thermometer in the axilla do not indicate preternatural heat, it is certain that a patient is not affected with a febrile disease. Hence the physician, by means of the thermometer, may be enabled to avoid an error into which he might be led by other symptoms pointing to the existence of a fever or some acute disease. On the other hand, increased heat somc-

1 St. George's Hospital Reports, Vol. I. 1866. Also, Amer. Journ. of Med. Science, July, 1867. 
times precedes the development of a febrile disease, which may thus be predicted by means of the thermometer.

The range of the increase of heat in different febrile diseases extends to $110^{\circ} \mathrm{Fahr}$., and, as a rule, the amount of increase is a criterion of the intensity of the disease. An increase to $100^{\circ}$ or $101^{\circ}$, is evidence of mildness of the disease. If the thermometer indicate steadily $105^{\circ} \mathrm{Fahr}$, it is certain that the disease is severe. A persisting temperature above $105^{\circ}$ denotes great danger, and if the thermometer indicate an increase to $108^{\circ}$, $109^{\circ}$, or $110^{\circ} \mathrm{Fahr}$., death is almost inevitable. The greatest increase of heat has been observed in fatal cases of scarlet fever and tetanus.

The persistence of preternatural beat in any disease denotes its continuance. Whatever may be the other symptoms, the career of the disease is not ended as long as the thermometer indicates increase of temperature. A progressive increase of heat from day to day denotes a corresponding increase of the severity of the disease, and the reduction of heat to the normal standard, as a rule, is evidence of convalescence. The temperature, in some diseases, falls rapidly to the normal standard, and in other diseases the decline is gradual. 'The term defervescence was introduced by Wunderlich to express the decline of temperature toward the standard of health, meaning, in other words, the approach of convalescence. Some diseases are characterized during the period of defervescence by considerable oscillations of temperature between night and morning, the maximum being at night and the minimum at morning. Typhoid fever is especially characterized by these oscillations toward the end of the febrile career. Hence it is important to employ the thermometer, not only daily, but at least twice a day. The decrease of heat in the morning is favorable, whereas, an increase from night to morning is the reverse. The temperature in some cases of disease remains above the normal standard after the general symptoms denote convalescence; under these circumstances, the patient is in danger of a relapse or the supervention of some other affection. A relapse or the supervention of some other affection is denoted by a rise of temperature after either apparent or real convalescence. During the progress of a fever or any acute disease, a sudden, notable increase of temperature, not sufficient to denote impending death, is evidence of the development of some serious complication or intercurrent affection.

The abnormal variations of temperature which are most apt to occur consist of more or less increase. Diminution below the normal standard is comparatively rare, and has less significance; yet the latter occurs occasionally, and is not without importance. In the course of typhoid fever a sudden and notable decrease of temperature has been observed to precede the occurrence of intestinal hemorrhage. In some cases of disease the temperature is observed to fall notably without improvement in other symptoms, constituting, under these circumstances, an unfavorable symptom. In the collapsed stage of epidemic cholera the heat falls three or four degrees below the normal standard. In conclusion, while the introduction of the thermometer has enabled the practitioner to obtain highly important information concerning diagnosis and prognosis, this information is always to be considered in connection with other facts belonging to the clinical history, and the significance or value of the thermometric phenomena depend, in no small measure, upon the symptoms with which they are associated.

From time immemorial morbid characters of the arterial pulse have been ranked among the most important of objective symptoms. An instrument called the sphygmograph, was devised by a French physician, 
Marey, with the design to obtain information more extended and accurate than that afforded by the usual digital examination. The sphygmograph is an instrument ingeniously contrived to represent to the eye the movements of the pulse. Applied over the radial artery, a line traced in an upward direction represents the influx of blood from the systole of the left ventricle; from the summit of this line, a line downward and more or less oblique, represents the efflux of blood from the arterial system into the capillaries. The two lines connected at the summit make a diagram which Marey terms the form of the pulse. The upward line traced during the ventricular systole is called the line of ascent; the oblique line traced from above downward during the ventricular diastole is ealled the line of descent, and the junction of the two lines at the top is called the summit. 'The number of these diagrams within a given space, of course, shows the frequency of the pulse. Pulse-writing thus consists of a series of diagrams, each representing an arterial pulsation, that is, the movements occurring from one beat of the heart to another.

Comparative observations, by Marey and others, liave shown that the form of the radial pulse has certain traits as regards the line of ascent, the line of descent, and the summit, which are physiological, that is, they belong to health, and that certain variations in the form of the pulse are pathological, denoting morbid conditions relating to the circulation. These pathological variations constitute eharacters symptomatic of disease. Referring the reader to the work by Marey, and to other publications, for an account of the mechanism of the sphygmograph, the rules for its employment, and illustrations of the varied forms of the pulse in health and disease, the more important of the characters which have been ascertained will be here concisely introducel..' The characters relate respectively to the line of ascent, the line of descent, and the summit.

The line of ascent represents, measurably, the force of the systole of the left ventricle. Other things being equal, the length of this line is a criterion of the force with which the blood is propelled into the aorta. The length of this line is, however, much affected by the resistance, caused by the tension of the arteries, or, in other words, the amount of blood which the arteries contain at the time of the ventricular systole. In proportion as the tension is great, that is, the arteries filled, the line will be short, although the ventrieular systole be strong; and, on the other hand, the line is long in proportion as the tension is small, that is, the arteries unfilled, provided the heart's action be not weak. Bloodletting increases the length of this line, not by increasing the power of the heart's action, but by diminishing the arterial tension. Notable shortness of this line is observed if the heart's action be much enfeebled, as in an advanced stage of typhoid fever, or in eases of local inflammation in connection with a typhoid condition. The line is notably short, and varied with successive pulsations, in cases of mitral cardiac lesions with much insufficiency. A notable disparity in this regard between the two radial pulses exists in certain cases of aneurism affecting the aorta, the innominata, or the subclavian artery.

The line of ascent is either vertical or more or less oblique. If vertical, it denotes quickness of the contraction of the left ventricle; and in proportion to the obliquity the ventricular contraction is slow. $\Lambda$ vertical line of ascent, representing rapidity of the arterial movement, denotes a character of the pulse which, to the touch, may give a fallacious sensa-

' Physiologie Medicale de la Circulation du Sang, etc., par le Dr. E. J. Marey, Paris, 1863. 
tion of force. The shortness of the line may show want of force when, owing to the quickness of the movement, the pulse appears to be strong.

The line of ascent is not always a straight line, but being for a certain distance either approximatively or completely vertical, it then becomes more or less oblique, or the obliquity is increased. This denotes a difference between the first and latter part of the ventricular systole, as regards quickness-at the beginuing it is more or less quick, and towards the end, owing to the resistance of the blood in the arteries, it becomes more or less slow. The change is sometimes sudden, and causes a jog or notch toward the upper extremity of the line; this is observed in some cases of aortic cardiac lesions involving obstruction and regurgitation.

The line of descent represents the arterial movements from the cessation of one ventricular systole to the time when the next systole occurs. In general, no movement of the artery is appreciable by the touch during this period. The sigmoid valves of the aorta being closed, the movements of the artery correspond to the efflux of blood from the arteries into the capillaries. The line of descent connects the two succeeding lines of ascent extending from the upper end of one to the lower end of the other. The length of this line will thus depend on the interval of time between the ventricular contractions, that is, on the frequency or infrequency of the pulse. The line is more or less oblique according to the rapidity or slowness with which the efflux of blood from the arteries into the capillaries takes place. The line is sometimes straight and sometimes curved, the convexity looking upward. Morbid characters relating to obliquity and curvature are produced by an abnormal obstruction in the capillary system, and by an abnormal rigidity of the coats of the large arteries.

The line of descent in health has in its course an oscillation more or less marked corresponding to the dicrotic or rebounding pulsation which is sometimes felt by the touch in cases of disease. The sphygmograph shows dicrotism to be a normal character of the pulse, but the rebounding or oscillation, in health, is not sufficient to be felt by the finger. When appreciable by the touch, it is abnormally increased. Marey's observations show the dicrotic character of the line of descent to be marked in proportion as the ventricular systole is rapid, as denoted by a vertical line of ascent; and, on the other hand, the dicrotic character is slight if the contraction of the ventricle be slow, as denoted by obliquity of the line of ascent. Elasticity of the arterial coats is necessary to the presence of dicrotism in a marked degree; in cases of senile rigidity of the arteries it is either lost or but slightly apparent. Slowness of the effux of blood from the arteries into the capillaries, inducing slowness of the ventricular contraction, diminishes, whereas relaxation of the capillaries, increasing the freedom of this efflux, increases dicrotism. The dicrotism is more marked when the quantity of blood sent out with the ventricular systole is small, than when the quantity is large. Dicrotism is especially marked in continued fever, a fact which Marey attributes to the relaxation of the capillaries. In some cases of disease two or more oscillations may appear in the line of descent, showing a corresponding number of oscillations in the artery during the ventricular diastole. This occurs under circumstances favoring dicrotism, when the frequency of the pulse is notably diminished.

Certain characters relate to the summit, that is, to the connection between the upper end of the line of ascent and of the line of descent. The line of ascent ends when the ventricular systole is balanced by the resistance caused by the blood in the arteries and the arterial coats. Now, if 
the systole end directly this equilibrinm takes place, and the efflux of blood into the capillaries at once begin, the line of deseent forms with the line of ascent an angle more or less acnte; in other words, there is no intermediate space at the suminit. If, however, the ventrieular systole continue after the equilibrium is established, the summit is a plane of variable length. If the equilibrium be exact, the summit is represented by a line perfectly horizontal, its length representing the time between the cessation of the influx of blood into the arteries and the commencement of the efllux into the capillaries. If the equilibrium be not exact, the line forming the summit will not be perfeetly horizontal; it will have an inclination upward if the influx slightly exced the eflux, and, if vice vers $\hat{a}$, the line will incline downward. The extension of the ventricular systole beyond the ending of the line of aseent, as denoted by a line nearly or quite horizontal at the summit, occurs especially in connection with senile induration or ealeification of the arteries.

The study of the graphie characters of the pulse has already afforded important information concerning the circulation both in health and disease. The further prosecution of this study is desirable. 'The splyygmograph has undoubtedly enlarged our resources for obtaining data for diagnosis and prognosis. The delicacy of the instrument, however, the nieety required for its employment, and the numerous incidental circumstanees which are liable to affect its operation, must restrict very much its availability in the hands of the general practitioner.

By means of another instrument for clinical observation, namely, the ophthalmoseope, invented and describer by IIelmholz, in 1851, a new and important field has been added to the domain of symptomatology. 'The ophthalmoseope is an optical instrument which enables the physician to observe the interior of the eye. By means of a simple mirror, with a central perforation, a strong light is directed into the eye of the patient, and the observer, through the perforation, obtains a view of the interior of the organ. 'This is distinguished as the direct method of examination. The objects within the eye are seen in their natural position by this method. A nother method consists in interposing between the eye to be examined and the mirror, a bi-eonvex lens. By this method an aerial image of the parts within the eye is perceived, the image being inverted. The latter is called the indirect method of observation. For information respecting different instruments, their relative merits, the principles of opties involved in their construction and use, together with all details relating to practical ophthalmoseopy, the reader is referred to either special treatises, or to works on ophthalmology.

Aside from the study of the diseases of the eye, the opthalmoscope brings under observation morbid appearances which are importint as symptoms of cerebro-spinal morbid conditions. Certain of these morbid appear:tnces relate to the ressels of the retina and the optic disk. Congestion, or hyperremia, of these vessels, on the one hand, and, on the other hand, a defieieney of blood, or anæmia, to a certain extent represent corresponding morbid conditions of the nervous centres. An extravasation of blood into the retina may denote, under certain circumstances, changes of the intra-eranial vessels, involving liability to cerebral hemorrhage. Characteristic intra-ocular appearances have repeatedly led to the discovery of renal disease which had previously been unsuspected. Appearances denoting inflammation of the optic nerve and the retina, sclerosis and atrophy, are significant, in certain cases, of intra-cranial or intra-spinal diseases. On the nther hand, the ahsence of morbid appearances within the eye is so:nevimes of importanee as contributing to exclude 
lesions or inflammatory affections of the nervous centres. It is a noteworthy fact that observation by means of the ophthalmoscope may show significant intra-ocular morbid appearances when there is neither defect of vision nor any subjective symptoms referable to the eye. Undoubtedly, at the present time, the importance of oplithalmoscopic phenomena is exaggerated by the enthusiasm of some, as it is underrated by others. The careful study of the morbid appearances which are observed in different diseases, in connection with other symptomatic phenomena and post-mortem examinations, for a longer period, is necessary to determine the full amount of reliable information to be obtained from this source. It is certain, however, that the ophthalmoscope is a highly important acquisition of modern clinical medicine as a means of investigating, especially, diseases of the nervous system with reference to diagnosis and prognosis, sustaining, in relation to these diseases, in some measure, at least, the same relation as the stethoscope to the affections within the chest. In treating of the diseases of the nervous system, in the second part of this work, the symptoms furnished by the ophthalmoscope, according to the observations of those who have devoted attention to this field of clinical study, will be noticed. To distinguish with accuracy the varied morbid appearances which are to be observed by means of this instrument, requires an amount of skill which can only be obtained by giving to its use special attention, and by considerable experience. The student or practitioner who desires to become a skilful ophthalmoscopist should, if practicable, obtain practical instruction from an expert, and he will, of course, consult works which treat in extenso of ophthalmoscopy. ${ }^{1}$

The symptoms and signs which occur during the course of a disease, are the cvents which make up its Clinical history. This phrase is applied to a description of the manifest phenomena taking place from the beginning to the end of a disease, together with the order of their succession and the laws which govern them. Or, instead of clinical history, it is proper to say the natural history of a disease, when the disease is allowed to pursue its course unaffected by disturbing influences of any kind, and when no attempt is made to arrest, abridge, or modify its course by therapeutical measures.

How is the clinical or natural history of a disease to be obtained? There are three methods which may be employed. One method consists in selecting, as types of the disease, a few well-marked cases, and basing the description of the phenomena belonging to the disease on the events observed in these cases. This method is defective, because some symptoms belonging to the disease might be wanting, and some symptoms not belonging to the disease might be present in these few rcpresentative or type cases. Moreover, this method is inadequate to show the relative importance of particular symptoms. Another method is to make out a history from recollection, after having observed a greater or less number of cases. A history thus obtained must be imperfect from the obvious inability to retain in the memory :ll the events which occurred while the cases were under observation, together with the order and relative frequency of their occurrence. The third method is the only one by means of which an accurate clinical history of a disease is to be obtained. It consists, first, in the accumulation of a certain number of cases fully and carefully recorded; and, second, in an analysis of the recorded cases with

I The work by Dr. Allbut "On the Use of the Ophthalmoscope," etc., is the fullest on this subject in the English language. Dr. A. treats of the subject with commendable caution and candor. 
reference to the symptomatic phenomena which oceurred, the relative frequency of their occurrence, the order of their succession, the number of deaths and recoveries, the duration of the disease, etc. By this method are ascertained events which are constantly present, and, therefore, having an essential relation to the disease; events which are ineidental to the disease, being present more or less frequently, and events the presence of which is purely accidental. There are many diseases which have not, as yet, been sufticiently studied after this method, and here, therefore, is ample scope for further clinical labor. Among the diseases which have been studied after this method may be mentioned typhoid and typhus fever and pneumonitis. The knowledge of the clinical history of these and some other diseases obtained by means of this method within late years exemplifies its advantages.

The clinical history of a disease is divided into sereral distinct periods or stadia, which are commonly called stages. 'The first stage may be distinguished as the invasion or access of the disease. This period dates from the first morbid manifestations, and extends to the time when the disease may be considered as fully developed or established. The duration of this stage varies in different diseases and in different cases of the same disease. Generally the duration is governed by certain laws proper to different diseases. Some diseases are characterized by the abruptness of their development, the stage of invasion being very short or altogether wanting. The career or course of a disease extends from the date of its development, that is, from the end of the stage of invasion, to the time when the disease may be considered as ended. This period is frequently subdivided into two or more stages. The stage of convalescence embraces the period between the end of the disease and the complete restoration of health.

The clinical history of a disease properly embraces phenomena which may precede the stage of invasion or access. They are distinguished as precursory or prodromic events; and in some diseases is to be included a period which is to be called the period of incubation. The latter term, signifying hatching, relates to the time which elapses after the reception of certain special causes of disease, before any morbid manifestations take place. For example, after inoculation with the virus of smallpox, a certain number of days elapses before any morbid phenomena make their appearance. But, in the mean time, occult operations are doubtless going on in the system, and, when these operations are sufficiently advanced, the period of invasion or access occurs. The significance of the lerm incubation is thus apparent. Again, the clinical history of a disease should take in phenomena which may occur after recovery from a disease, provided they are dependent upon it. Certain diseases are liable to be followed by certain consequences, which are called sequelæ or sequels. Thus, after recovery from scarlatina, albuminuria and general dropsy oceur in a certain number of eases. These are results of this disease, and henee belong to its history as sequels. In like manner tuberculosis is apt to be a sequel of measles, motor paralysis follows diphtheria, etc.

As a general statement, diseases, in respect of the phenomena and laws belonging to the natural history of them, present in different times and places great uniformity; they are essentially the same in different parts of the world, and they undergo little or no material changes from age to age. This statement is believed to be correct, althongh it is in opposition to views of late somewhat prevalent. The different phases and modifications which the same diseases may present, not only at different times and places, but in different cases at the same time and place, are, for the most 
part, to be explained by the co-existence of affections which may have been antecedent or which are developed as complications.

\section{Diagnosis.}

One of the most important of the aspects under which individual diseases are to be considered, is their diagnosis. The term diagnosis means the discrimination of diseases, that is determining respectively their charaeter and seat. The practical importance of a correct discrimination of diseases, with reference to their management, is sufficiently obvious. How is it possible to bring to bear upon a case of disease established principles of treatment until the character and seat of the disease are ascertained? I) iagnosis and treatment are, in fact, the two practical ends of the study of medicine. Diagnosis is also important with reference to the acquisition of knowledge of the clinical history of diseases. In collecting cases for analysis, if different diseases be confounded, the results of the analysis will be vitiated. In like manner, correctness of diagnosis is the basis of therapeutical experience. Conclusions drawn from the effects of measures of treatment in a series of cases will, of course, prove fallacions if the series include cases of different diseases which have been confounded. Here is a source of not a little of what has been called false experience in practical medicine. Diseases which were formerly confounded are now known to be distinct and readily discriminated from each other. For example, the eruptive fevers, measles, scarlet fever, and smallpox were once considered as different varieties of one disease. Physicians did not undertake, until within a few years, to discriminate between pleuritis, pneumonitis, and bronchitis; now it is not only known that these diseases occur separately, but the diagnostic characters of each are well ascertained, and are sufficient for its recognition. Still more recently, typhus and typhoid fever have been confounded, and, indeed, are still by some considered as one disease.

Differences in skill and tact, among practitioners of medicine, relate especially to diagnosis. In medical consultations it is here chiefly that points for discussion and disagreement are apt to arise. It is here that the want of aid and counsel is oftenest felt by the physician. The diagnosis involves more embarrassment than the management of diseases. This fact is not appreciated by the people at large, many of whom, without any medical education, undertake to decide respecting the nature and seat of the disease whenever their friends are ill.

The diagnosis of a disease may be based on the presence of pathognomonic events. Thus, the characteristic expectoration, or the crepitant rale, denotes the existence of pneumonitis. But, as already stated, there are but few symptomatic phenomena which are truly pathognomonic. Moreover, the few pathognomonic events are not present in all cases of the diseases which they denote if they be present; hence their absence is by no means proof against the existence of the diseases. The characteristic expectoration and the crepitant rale, for example, are wanting in not a very small proportion of cases of pneumonitis. It is evident, therefore, that the diagnosis of a disease cannot often be based on pathognomonic events. And, next in importance, as regards the basis of diagnosis, is the presence of diagnostic symptoms. As a rule, in proportion to the degree of diagnostic' significance belonging to the symptoms present and their number, is the diagnosis of a disease easy and positive. In these respects different diseases and different cases of the same disease differ widely ; in some instances the diagnostic symptoms present are few in number and 
weak, while in other instances they are numerous and strong. It is with special reference to the relative diagnostic value of the different events which make up the clinical history of a disease, that this history is important to the physician if it have been obtained hy the true method of study, viz., the analysis of recorded cases.

'The laws of disease are often of importance in relation to diagnosis. For example, typhoid fever very rarely affects persons over fifty years of age. When, therefore, persons beyond this period of life are said to have typhoid fever, the chanees are that an error has been made in diagnosis. Certain diseases, as a rule, occur but once in the same person. The probabilities, therefore, in a case of doubtful diagnosis, are against the existence of a disease which might otherwise be suspected to exist, if this disease be one of this class, and it be ascertained that the patient has already had the disease. Certain physical signs situated within a circumseribed space at the summit of the chest, denote tuberculous disease, while the same signs, situated elsewhere, might point to some other affection, owing to the law that the tuberculous product, in the vast majority of eases, first occurs at or near the apex of the lung. Similar illustrations might be multiplied indefinitely ; the laws regulating the development of a rlisease, the succession of events, its duration, its sequels, cte, often furnish valuable aid in its diagnosis. Here, too, the practical importance of knowledge of the clinical history of diseases is apparent.

A highly effective method of reaching the diagnosis of a disease is called "reasoning by way of exclusion." In a case of doubt as regards diagnosis, the problem is generally to decide between a certain number of diseases. The existing disease is one of two, three, or more diseases which may be suspected to exist. Now, if it be difficult to decide which one of these is the existing disease, from the positive proof relating to diagnostic events and laws, it may be practicable to decide that there is insuflicient evidence of the existence of one or more, and therefore the latter are excluded. By this process of elimination, the number of diseases is diminished, and may be reluced even to one disease; the diagnosis then being made on the principle of exclusion. To illustrate the applieation of this method, the presence of lancinating pains in one side of the chest, as a prominent symptom of disease, is consistent with the existence of either pleuritis, pneumonitis, or pleurodynia. 'The question is, which one of these three diseases is the existing disease. In seeking to decide this question, we look for positive evidence of the existence of either one, that is, for pathognomonic or diagnostic symptoms. 'The characteristic expectoration of pneumonitis would be decisive, if present, but its absence is not proof that this disease does not exist. We eannot exclude this disease by the absence of that symptom. But pneumonitis is frequently accompanied by a crepitant rale, and always leads to solidification of ling, which is revealed by certain auscultatory signs. Suppose these signs to be wanting, we may exclude pneumonitis. The question now is between pleuritis and pleurodynia. Pleuritis is sometimes accompanied by a friction murmur on auscultation, and always, if primary, leads to more or less liquid eflusion, the presence of which is demonstratively determined by physical signs. Suppose these signs to be wanting, we may exclude pleuritis. We thus arrive, by way of exclusion, at the diagnosis of pleurodynia, assuning the problem to comprise only these three diseases.

In discriminating a disease from one of sereral diseases with which it has more or less symptomatic phenomena in common, the amount of positive evidence in behalf of its existence is to be considered, and, also, the evidence against the existence of the diseases from which it is to be dis- 
criminated. This comparison of one disease with other diseases, with reference to the positive and negative evidence of its existence, constitutes what is called differential diagnosis; and to discriminate a disease in the manner just described is to differentiate it. Thus, typhus and typhoid fever have many symptoms in common, but differ from each other in essential points. The differential diagnosis of these diseases has reference to the circumstances which denote the presence of one, and the absence of the other.

Diagnostic skill is required, not alone in differentiating diseases, but in the discrimination of cases in which diseases are either simulated or their existence fancied. The knowledge and tact of the physician are here not unfrequently called into requisition. The judgment must rest mainly on deviations from the established laws of different diseases as regards symptomatic phenomena, etc., and the absence of objective phenomena which in certain diseases are invariably present. It follows, that the practitioner, other things being equal, will be successful as a diagnostician in such cases in proportion to the accuracy and extent of his knowledge of the phenomena and laws of different diseases. Diseases are simulated for the attainment of certain ends. Thus, soldiers frequently feign diseases with a view to escape active duty, or to obtain a discharge from the service; prisoners feign diseases to secure pardon, or the comforts of a hospital; and cases are not rare of persons simulating various affections to excite sympathy and become objects of interest. Feigning diseases for these or other ends is called malingering, and persons attempting the deception are malingerers. One mode of malingering is to pretend that diseases which actually exist are more severe than they really are. Among the diseases which malingerers are apt to feign are, epilepsy, paralysis, phthisis, aphonia, amaurosis, and insanity. ${ }^{2}$

Practical skill in diagnosis requires mental qualifications which all do not possess in equal measure. Aside from these, the amount of skill which may be acquired will be proportionate to the knowledge of, and familiar acquaintance with, different diseases as regards their clinical histories. It would be absurd to suppose that diseases are to be recognized by an intuitive preception, and that some persons have a peculiar innate gift in this direction. A diagnosis not based on adequate pathological information is always open to more or less of the chances of error which pertain to guessing. The mental qualifications of the skilful diagnostician do not differ essentially from those required for other than medical. investigations, namely, accuracy of observation, the ability to reason correctly sound judgment and good sense.

\section{Prognosis.}

The division of medicine called prognosis treats of the means by which the course and termination of diseases may be foreseen. It is often important to form a correct judgment concerning the way in which diseases are to end, with reference to communications with patients and their friends. They may desire, and, if so, it is proper they should receive, information on this point. Nor should the physician be unmindful of the fact that people in general are apt to estimate his knowledge and ability by the correctness of his judgment in this regard. Recollecting this fact,

1 For an excellent account of malingering, as regards its various forms, the causes, etc. vide article by Dr. Roberts Bartholow in the medical volume of the Reports of the United States Sanitary Commission, 1868, edited by Austin Flint, M.D. 
the protection of his own interests, in addition to other considerations, will dictute a certain amount of reserve in his predictions. But a eorrect judgment as regards prognosis may be important with reference to treatment. Therapeutical measures are sometimes to be withheld in view of the complete hopelessness of the condition of the patient; and, under these eircumstanees, persistence in the use of remedies is not only superfluous, but likely to do harm. On the other hand, therapentical measures may be employed with reference to the danger of death, without regard to the nature or seat of the disease. A disease equal in all respects in different cases, may destroy the lives of some, while others recover, owing to the diversity which exists in different persons as regards the ability to sustain and overcome disease; and in the management of diseases, it is often the chief aim of the physician, in the language of Cullen, to obviate the tendency to death.

Prognostics are those eircumstances on which a prognosis is based. They relate, of course, mainly to the symptoms of disease. Formerly, before the nature, seat, anatomical changes, etc., of different forms of discase were as well understood as now, all the cireumstances connected with diseases were considered with direct and special referenee to their significance as prognostics. The consideration of these is appropriate in treating of the principles of medicine, or general pathology; but it will suflice to consider them incidentally in connection with individual diseases. A few points which relate to the physiognomy or obvious appearances, and which render the prognosis unfavorable, may be here enumerated, by way of illustration.

Notable emaciation and pallor, existing in conneetion with any chronic affection, are unfavorable prognostics. Lividity of the prolabia and face, if persisting, generally denotes great danger. Great frequency and feebleness of the pulse, exclusive of diseases of the heart, and especially in acute diseases, point to a fatal termination. Great prostration is usually an alarming symptom. Spasmodic inspiration, if not dependent on pulmonary disease, precedes the development of coma, exeept it be referable to hysteria. Coma, if it last more than one or two days, will be likely to end in death. Paralysis of the sphincters, leading to involuntary evacuations, and loss of the muscular power of deglutition, are apt to denote impending dissolution. Impaired capability of feeling the presence of liquid in the air-passages, and inability to make the efforts requisite for its removal by expectoration, are forerunners of the moribund state. Late researches have shown that notable increase of the animal heat, as determined by the thermometer placed within the axilla, if persisting, is to be reckoned among the prognostics denoting imminent danger. Finally, the characters pertaining to the physiognomy which constitute the Hypocratic countenance, so called because they were deseribed by the ancient father of medicine, denote the moribund state. These characters are, marked pallor with more or less lividity, pinching of the nostrils, sinking of the eyes, hollowness of the temples, coldness and transparency of the ears, dropping of the lower jaw, etc. The change in physiognomy in this state is so great, that persons are sometimes hardly recognizable. The features, to use a French expression, are decomposed, and a glance at the face often suffices to show that death is nigh at hand. 


\section{II A P T E R $X$ \\ PROPHYLAXIS-GENERAL THERAPEUTICS.}

Experience the Basis of Therapeutical Knowledge-Two Sources of Experience; Analysis of recorded Cases with reference to the Duration or Termination of Diseases, and Observation of the Immediate Effects of Therapeutical Measures-Rational Inference as a Means of ar. riving at Principles of Therapeutics-Importance of Knowilg the Intrinsic Tendencies of Diseases toward Recovery, or otherwise-Active Measures to be employed only when clearly indicated-The Expectant Plan of Treatment-Conservative Medicine-Importance of $\mathrm{Hy}$ gienic Measures of Treatment-Modes of Dying resolvable into Apnoea and Asthenia-Classification of Therapentical MIeasures, according to the Objects to be accomplisled, into Prophylactic, Abortive, Curative, Palliative, Hygienic and Sustaining Measures-Consideration of the Elementary Forms of Dlsease deferred.

THE prevention of disease is an object connected with pathological studies. It constitutes a division of medicine called prophylaxis. It is one of the several aspects under which individual diseases are to be considered. As regards the beneficent fruits of knowledge, it holds a place scarcely inferior to that of therapeutics. Many diseases are preventable by removing or obviating their causes; and it is with reference especially to this application, that the study of causes, or etiology, is of great practical importance. The knowledge already acquired of the causation of certain diseases, has proved of much value. A striking illustration of this is afforded by our present acquaintance with the poisonous effects of lead. It is only within late years that certain diseases have been traced to the action of this poison. The diseases thus produced were doubtless of frequent occurrence before their causation was understood. The physician is now able sometimes to determine that diseases are thus produced, although he may not be able to ascertain in what manner lead has been introduced into the system; characters pertaining to the diseases show that they have originated from this source. This statement is true of that form of paralysis ealled wrist-drop, and of the form of neuralgia known as saturnine colic.

The recognition of the "germ-theory" as affording the most rational explanation of certain of the special or specific causes of disease, has led to the employment, with great effect, for the prevention of epidemic and infectious diseases, of measures, the efficacy of which apparently depends on their agency in destroying the low forms of animal or vegetable life. In this agency consists the efficiency of certain of the so-called disinfectants. But without positive knowledge of the nature and origin of the causes of disease, we can, to a certain extent, secure exemption from their morbific effects by the knowledge which we possess of certain of the laws regulating their operation. Thus, the special cause of periodical fever, commonly known as malaria, may often be avoided by taking care to avoid exposure to the night-air and by sleeping in an upper story. These causes may be indirectly controlled, to a certain extent, by removing accessory or co-operating causes. It is in this way, probably, that sanitary measures are efficient in protecting against epidemic and endemic diseases. Much has been accomplished in behalf of prophylaxis by these measures, but much more remains to be accomplished. Sanitary reforms 
relating to ventilation, sewerage, cleanliness, etc., there is reason to believe, do very much toward the prevention of disease, and, hence, these are among the most important of the unodes by which philanthropic efforts may be rendered useful to mankind.

'The prospect of diminishing the mortality from certain diseases relates more to prevention than cure. This is true of that disease which contributes so largely to the death-rate in all countries, viz., plithisis. It is unquestionably true that phthisis is much more successfully managed now than heretofore, but it is probable that, under any system of treatment, the disease will prove fatal in a large proportion of cases, sooner or later. A great deal, however, is to be hoped for from the knowledge and avoidance of accessory causes which lead to the development of the disease by acting in conjunction with a congenital predisposition.

\section{Therapeutics.}

The division of medicine, devoted to the treatment of disease, is called therapeutics. The consideration of therapeutical measures, as regarcls the evidence of their usefulness, the rationale of their operation, the indications for their employment, etc., constitutes general therapeutics. 'The general principles of therapeutics are usually considered in connection with the principles of medicine, or general pathology. The consideration of the treatment of individual diseases enters into special pathology, or the practice of medicine, and constitutes special therapeutics. It will suffice here to offer a few remarks relating to general therapeutics, reserving the different therapeutical measures for consideration, in connection with the treatment of individual diseases, in the second part of this work.

It is needless to offer any remarks on the importance of therapeuties. The treatment of disease is the great end of all our studies relating to pathology, general and special. It is, however, the most difficult of all the branches of medicine for the teacher. It is so, not alone from uncertainty as regards the correctness of therapeutical principles, but because principles, undoubtedly correct in their general application, are to be greatly modified in adapting them to the varied circumstances pertaining to individual cases of disease. The same disease, under different circmmstances, may claim, not only widely different modifications of treatment, but therapeutieal measures directly opposite in character. There will be abundant occasions for citing illustrations of this fact in treating of individual diseases. It is impossible to formularize rules for the application of therapentical measures. If this were possible, the practice of medicine would be a mechanical, not a rational, art. All that the teacher and author can hope to do is to present general principles, together with the more important considerations involved in their application. And their successful operation requires, not only knowledge, but reasoning powers, judgment, good sense, and practical tact.

How are the facts and principles of therapeutics obtained? Mainly by experience. The question then arises, how are the results of experience, as regards the utility of therapeutical measures, obtained? There are two sources of experimental evidence in behalf of the utility of a particular method of treatment in any disease. One source of evidence relates to the termination and duration of the disease in a series of cases in which the method of treatment has been employed. The superiority of a method is shown by a larger number of recoveries and an average duration shorter in such a series than in other series of cases treated otherwise. This statistical investigation is open to certain fallacies. No two series of 
cases of a disease are in all particulars exactly alike. Cases differ in the degree of severity and extent of disease, in the constitutional condition of patients, in the existence, or otherwise, of complications, and in a great variety of circumstances pertaining to season, climate, age, habits, etc. The results of a comparison of different series of cases, in respect of termination and duration, are to be accepted as the basis of experience only on the ground that the differenees in the different series mutually compensate for each other. Hence, the number of cases embrneed in a series should be large, and the results of the comparison should be confirmed or corrected by being repeated with successive series of cases collected at different times and places and by different observers. Moreover, pains should be taken to group together cases resembling each other as closely as possible. Observing carefully all proper precautions, the results obtained by this kind of investigation are of great value, if deduced from data faithfully recorded by honest observers and competent diagnosticians. The memory is wholly inadequate for such an investigation. Moreover, recorded facts afford the only security against prejudice and bias. Unrecorded experience is by no means valueless, but it is of little relative value as compared with the results of the analysis of recorded data.

A nother source of experimental evidence relates to the immediate effects of therapentical measures irrespective of the termination or duration of diseases. The usefulness of remedial measures may be apparent from their immediate effects, although there be no proof that the disease has been abridged, and in cases which end fatally. A method of treatment may be useful in a certain number of the cases in a series, although in the majority of cases it may be injurious. For example, let it be assumed that in the larger number of series of cases of pneumonitis bloodletting does harm, it may nevertheless do good in a small number of cases; and, conversely, if it be assumed that this remedy is useful in the majority of cases, it may be pernicious in some cases. So with regard to other measures in other diseases.

It is thus evident that after the utility of particular therapeutical measures in certain diseases, as the rule, has been ascertained by means of a suffieient number of facts derived from the first source of experimental evidenee, it is vastly important for the physician to exereise discrimination in the application of these measures to individual cases. And it is to be borne in mind, that, in the practice of medicine, the physician deals with cases of disease separately, not in aggregates. The general principles of therapentics háve a relation to individual eases of disease, not unlike that of the rates of life insuranee to the liability to death of partieular persons. It is easy to calculate the probabilities of the duration of life in a large number of persons of a given age, and to determine exactly what it is worth to insure the lives of a large number, but no one thinks of applying to an insurance office to know how long he is to live!

Rational inference is a means of arriving at therapeutical principles. If, for example, it be known that a person has swallowed a corrosive poison, reason and common sense at once dictate the propriety of endeavoring either to expel it, or to neutralize it by an appropriate antidote. But, in such a case, experience must be resorted to to know whether either of these objects be practicable, which of the two is to be preferred, and the best means of effecting them. Analogical and $\grave{a}$ prior $i$ reasoning may lead to the employment of measures in the treatment of diseases, but the results of experience are necessary to establish their therapeutical value. Talent and genius may be shown in originating methods of cure, but the practical test of their efficacy is afforded by experience. It is true 
that false experience has abounded in medicine, but, if experience be open to fallacies, this is true to a far greater extent of theoretical or speculative reasoning. It is worthy of note that our knowledge' of the most important remedies is based wholly on experience, without our being able to offer any explanation of their modus operandi. Examples are, the salts of quinia as a remedy for periodical fever, mercury and iodine in certain forms of syphilis, etc.

The intrinsic tendency of a disease to recovery, or otherwise, is to be considered with reference to the value of therapeutical measures. Knowledge of the natural history of diseases is important as the true point of departure for therapeutics. Much has been acquired, within late years, in this branch of knowlerge, but much yet remains to be acquired. Diseases which were formerly supposed to tend to a fatal result, if not treated by means of active remedies, are now known to end generally in recovery, if let alone. Examples are, acute pleuritis and simple pneumonitis. The importance of increasing our knowledge of the natural history of diseases, by collecting cases which have pursued their course without active treatment, is to be kept in mind by those desirous of contributing to the advancement of therapeutics, and opportunities of accumulating such cases should not be neglected, always provided that the welfare of the patient be not compromised by withholding measures which we have good grounds for believing will prove useful.

It may be laid down as a golden rule in therapeutics that active measures of treatment are only to be employed in cases in which they seem to the physician to be clearly indicated. The severity of disease, and the danger of the patient, be they never so great, do not alone constitute grounds for the employment of active measures. If they be not useful, they will be likely to do harm. Therapentical measures, in proportion to their power, are powerful for either good or harm, and must, therefore, be either indicated or contra-indicated. In the language of another, the physician "should be content with doing nothing when ignorant how to do good."

The term expectant, as applied to the treatment of disease, is often used, and its true sense is not always appreciated. The treatment of a disease by expectation consists in watching carefully its progress, and meeting with appropriate measures unfavorable events as they arise. This plan is not inconsistent with the employment of active measures of treatment; but these are resorted to, not simply because the disease exists, but with reference to events connected with the disease. In this sense of the term, the treatment of a disease is expectant whenever the physician does not attempt to abridge or arrest it, but strives to aid in conducting it to a favorable termination.

The progress of medicine within late years, as regards therapeutics, is characterized by the development of a principle which may be distinguished as conservatism. This term may be applied to medical as to surgical practice, and denotes an object which has not heretofore been sufficiently considered, viz., the avoidance of useless and injurious therapeutical measures. Conservative medicine does not interdict the use of the most potential of remedial agencies; but it enforces discrimination and circumspection in their use, recognizing fully their potency for evil as well as for good. The conservative physician is by no means a mere spectator of the progress of disease, but, in wielding the resourees of therapeutics, he is careful to preserve the powers of life, following the injunction of Chomel not so much to treat disease, as patients affected with disease.'

' Vide "Conservative Medicine as anplied to Therapeutics," American Journal of the Medical Sciences, January, 1863. 
The management of cases of disease involves not only the exercise of judgment in the employment, or otherwise, of therapentic measures, but attention to hygienic regulations. And in a better appreciation of these, the practice of medicine at the present time offers a striking contrast with the past. It may perhaps safely be said that the greater success attending the management of diseases now, than heretofore, is due as much to improvements as regards diet, ventilation, etc., as to the more judicious use of remedial agencies.

In the treatment of diseases involving danger to life, the mode of dying is to be consiclered. It is often a useful inquiry at the bed-side, by what mode of dying will the case under treatment end fatally if the patient do not recover? The answer to this question will often furnish the indications for treatment, by means of which the danger may be forestalled; and here, it is plain, is the source of indications under circumstances in which it is the aim of the physician to "obviate the tendency to death." It suffices for all practical purposes to reduce the different modes of dying to two, viz., by apnoea and by asthenia.

Death by apnœa is produced by interruption of the function of respiration. The type of this mode of dying is furnished in cases of strangulation. Death by this mode occurs when produced by affections involving obstruction to the passage of air to the air-cells of the lungs, such as acute laryngitis, croup, œedema of the glottis, foreign bodies in the airpassage, and capillary bronchitis. It oceurs sometimes when a considerable portion of the pulmonary organs is rendered incompetent to receive air, as in pleuritis with effusion, pneumonitis affecting both lungs, and acute plithisis. It also occurs when the respiratory movements are arrested by an affection of the medulla oblongata, involving the suspension of the reflux influence by which these movements are sustained. When death occurs from coma, the mode of dying is by apnœa. In this mode of dying, the embarrassment of respiration is out of proportion to the disturbance of the circulation, and, when death takes place purely by this mode, the action of the heart continues for some time after the cessation of respiration.

Death by asthenia, on the other hand, is dne to the failure of the circulation. .The action of the heart may be suddenly arrested by paralysis from over-distension of the ventricles, by the pressure of blood from an aneurism opening into the pericardium, by pericardial effusion, and by the coagulation of blood within the cavities. Jut in all diseases which prove fatal by adynamia or exhaustion, the mode of dying is by asthenia, and, when deatli takes place purely by this mode, respiration continnes for a greater or less period after the pulse has ceased to be appreciable at the wrist. Apnœa, however, is frequently combined with asthenia in producing death; the diminished muscular power renders the patient unable to prevent the accumulation of liquid in the air-passages, and the respiratory function also may be interfered with by congestion and oedema of the lungs due to exhaustion. So, also, asthenia is frequently combined with apncea in fatal affections of the organs of respiration. In fact, in a large proportion of cases, the two modes of dying are combined, either the one or the other mode predominating.

With a view to reference in the second part of this work, a classification of measures of treatment, based on the general objects which the physician aims to accomplish in the management of individual diseases, will be convenient. These objects are embraced in the following classes:-

1. Prophylactic or preventive measures.-Vaccination is a preventive measure with respect to smallpox. Quinia, given to protect against inter- 
mittent fever, is another measure belonging to this class. Measures to eliminate urea from the blood when the deficiency of this excrementitious principle in the urine is ascertained, before the manifestations of uramia take place, is another example. 'The ejection of poisons from the stomach, and the employment of antidotal remedies, belong in the same category.

2. Abortive measures.-'The measures in this class are those which are employed with a view to arrest the progress of a disease at the outset; to cut it short, or strangle it. Quinia given in large doses in intermittent fever is an abortive remedy. It is not easy to cite many examples of this class. The range in which abortive measures are effective, with our present knowledge, is small. Here is a wide field for further developments in therapeuties.

3. Curative remedial measures.-Those measures which abridge the duration of a disease, diminish its severity or danger, and, in general terms, modify or influence it in a favorable manner, may be distinguished as curative. 'This class is limited to remedial measures, that is, it does not embrace hygienic measures. Quinia is a curative remedy in the cases of periodical fever in which it is not abortive, and, also, in certain cases of neuralgia. Mercury and iodine are eurative in eases of syphilis. Opiun is a curative remedy in peritonitis, in spasmodic affections like eolic, and in sporadic cholera. Numerous other examples of this elass might be cited.

4. Palliative measures.-The measures belonging to this elass are those which are employed with the object of relieving pain or suffering. These measures are directed, not to a disease per se, but to the symptoms or effects of a disease. But they may, to a greater or less extent, exert a curative influence. It is conceivable that relief of pain or suffering may conduce to the farorable termination of a disease in some cases.

5. Sanitary or hygienic measures.-The measures of this elass relate to pure air, temperature, diet, eleanliness, moral influenees, ete. Medieine has certainly derived as much advantage from this class, of late years, as from eurative remedial measures. The improvements which have taken plaee in the management of diseases, as regards air, water, food, exercise, etc., are very great, but there is room for still further improvement.

6. Sustaining or supporting measures.-The measures of this class consist of tonie remedies, stimulants, especially alcololic, and nutritious alimentation. The objects are to obviate a tenclency to death by asthenia, and to forestall a degree of prostration dangerous to life. Sustaining measures are applicable to the treatment of all diseases which, if they prove fatal, will destroy life by asthenia, and they are indicated with an urgency corresponding to the danger of death by this mode. These, together with palliative and hygienie measures, constitute the treatment of all diseases which cannot be arrested, and which are not amenable to curative measures. Not a few diseases will be found in this category.

The terms analeptic and restorative are applied to measures relating to diet, regimen, and remedies, when the object is, in general terms, to bring the system back to its normal condition. Supporting measures have reference more especially to acute diseases which threaten life. Analeptic measures are those called for when the general health is deteriorated, although there may be no disease which involves danger to life; when in other words, the system is below par. They are aften called building up measures, and are indicated in a large majority of disorclers and chronic ailments.

A very good division of therapentical indieations is adopted by Niemeyer, namely, 1st, the indications which relate to the removal or abate- 
ment of causes-these may be distinguished as Causal indications; $2 d$, Symptomatic indications, or those having reference to the symptoms, irrespective of the nature and seat of the disease; and 3d, Pathological indications, that is, the indications derived from a knowledge of existing diseases considered with reference to their character, situation, and intrinsic tendeneies.

Measures of treatment are sometimes classified and named from their aetion being in opposition to a particular morbid condition; thus, formerly, the term antiphlogistics was much in vogue, this name being applied to measures which were considered as opposed to inflammation. Of late, certain measures, the immediate effeet of which is the diminution of the temperature of the body, are often distinguished by the name antipyretic. Quinia and some other remedies are called antiperiodic, from their efficacy in arresting affections which are characterized by intermittency; remedies which are antagonistic to the effects of syphilis are called antisyphilitic, etc. It is, however, an error to suppose that it is a governing principle in therapeutics to employ remedies or measures, the action of which upon the body in health is the opposite of the effects or manifestations of disease. This prineiple is expressed by the term allopathy, a term applied by Hahnemann to denote a therapentical doetrine the reverse of homceopathy. Legitimate medicine recognizes no such restricted and exclusive dogma as these two terms alike denote; and, hence, the name allopathy is to be by no means accepted, but considered as a term of ummerited reproach.

An important precept in therapeutics is the observance of simplicity in prescribing remedies. As a rule, curative remedies differing in their effects, should not be given in combination or at the same time. Different remedies should be conjoined only when they co-operate for a common object, stich, for example, as a cathartic or a diuretic operation. This precept is important, not only because different remedies are likely to conflict with each other, but because it is difficult to judge of their efficacy severally. To observe the separate effects of particular remedies in individual cases is essential in order to obtain that experience which will be useful to the same patient at other times, and in other cases of the same disease. . The experience of the physician accustomed to prescribe together multifarious remedies, must, of necessity, be confused and uncertain. Moreover, complicated prescriptions are oljectionable on the score of their tending to invest the practice of medicine with an unworthy mystery. These remarks, of course, do not apply to uniting with a curative remedy medicines designed to correct incidental effects which it is desirable to avoid, and to diminish the repulsiveness of a remedy, or to secure for it a better tolerance. These objects, in the combination of medicines, are important. Not the least conspicuous of the modern improvements in medical practice relates to the use of alkaloids and concentrated remedies, together with other contrivances to divest remedies of a nauseonsness which was formerly, as a matter of course, associated with the idea of a medicine.

A considerable share of medical practice consists in the treatment, not of the individual diseases which are to be taken up in the second part of this work, but of disturbances of health which have no well-defined places in medical nosology. These disturbances often proceed from the operation of morbid causes, intrinsic or extrinsic, which it shonld be the aim of the physician to ascertain, and the removal of which, if possible, is to be effected. The development of serious diseases may, doubtless, in this way frequently be prevented. In many cases, the knowledge and skill of 
the physician are called into requisition to determine that no serious or well-defined disease exists. It is by no means the sole oflice of the physician to treat diseases. 'To indicate the causes of disease, more especially the voluntary violations of the laws of health ; to enjoin such a course of life as shall seem most likely to afford security against disease, and promote physical and mental vigor; to decide in cases of clisease against the employment of active therapeutical measures,-these are duties not less important than the employment of potent remedies whenever the latter are required.

In the management of cases of disease, mental influences are often of not a little importance. 'The physician may in many cases effect much by judicious encourngement, and by stimulating the voluntary efforts of the patient. Hopefulness and a strong will are curative agencies which are frequently powerful auxiliaries to medicinal remedies, and they are sometimes more potent than drugs; on the other hand, despondency and a lack of resolution have a depressing effect which, in some cases, tells greatly against recovery. Certain disorders may be produeed and prolonged indefinitely by mental eauses, the removal of which is essential to a cure. Too much concentration of the attention on the funetions of the bolly is a common souree of disorder among persons who, from want of occupation, unfortunately become unduly engrossed with their plyssical condition; and, in such cases, to divert the mind from the body is an olject not less important and difficult than, in other cases, to secure a proper degree of care for exising ailments. The ability to exert those mental influences which are suited to different cases, conduces, in no small measure, to success in practice; and this ability requires, in addition to natural powers, knowledge of human eharaeter, and a taet which is acquired by experience. A cheerful mien, and manners calculated to inspire conficlence, are by no means beneath the attention of the physician, but, on the contrary, they are to be cultivated as highly useful professional attainments. Medicine, in its practical aspect, involves knowledge, judgment, and skill, as regards, not only the employment of the several classes of the therapeutical measures which have been enumerated, but the mental influences suited to the circumstances peculiar to individual eases of disease.

The history of medicine shows many and great mutations in the prevailing doctrines of therapentics at different periods. T'he medical practice of the present day in many respects is in striking contrast to that current a half century ago; and it is by no means improballe that a comparison with the present day at the end of the next half century, will reveal points of contrast not less striking. These mutations, so far from being a reproach, illustrate the progressive character of medicine. Therapeutical prineiples can never become unchangeably fixed until the utmost limits of attainable pathological knowledge are reached, and nothing further remains to be ascertained by experienee. It would be absurd to clain for medicine this perfectibility ; but continued improvement in the management of disease may be reasonably expected. Future improvement will doubtless embrace the addition of new remedies and measures of treatment, more extended and precise knowledge of those already in use, and the correction of errors which probably now, as heretofore, render sometimes the efforts of the physician not only inadequate but prejudicial.

The uniformity of the laws of disease at different periods and places has been already referred to. The bearing of this on the stability of therapeutics is obvions. So, also, with reference to the same application, it is important to assume that the system of man is not liable to alterations 
which render the operation of therapeutical agencies widely different at periods not far removed from each other. 'The opinion held by some that such alterations have occurred within the memory of those now living, does not rest on any solid basis. Admitting to the fullest extent diversities attributable to race, climate, occupations, hereditary influences, etc., these do not invalidate the statement that the human constitution remains essentially unchanged, as regards the application of established therapentical principles. Were the truth otherwise, it would strike at the root of medical experience. If changes requiring a revolution in therapeutics were liable to occur with each successive generation, it is evident there could be no permanent principles of medical practice; the fruits of experience in our day, which so many are striving to develop, would be not only useless, but an injury to those who are to come after us.

This chapter concludes the first part of this work, the part devoted to the Principles of Medicine, or General Pathology. A succinct account of the changes belonging to morbid anatomy, and of the known morbid conditions of the blood, has been presented, together with certain distinctions, definitions, and general considerations relating to etiology, symptomatology, diagnosis, prognosis, prophylaxis, and therapeutics. These subdivisions of the principles of medicine, or general pathology, have been considered as fully as is deemed important by way of preparation for entering on the study of individual diseases. 'The latter will be considered in the remainder of the work, which is to be devoted to the Practice of Medicine, or Special Pathology.

Certain topics which might properly be included among those considered in the first part of this work, are reserved for consideration exclusively in connection with individual diseases. The morbid conditions constituting what may be called the elementary forms of disease, are thus reserved. Under the head of elementary forms of disease are embraced those morbid conditions, entering into a greater or less number of individual diseases, which, with our present knowledge, constitute the primary deviations from healthy conditions. They are, in other words, the simplest forms into which morbid conditions are resolvable. These elementary forms enter into different individual diseases, as the elementary tissues either constitute or combine to form the different organs of the body. Inflammation is an example of an elementary form of disease giving rise to a host of individual diseases. Fever, considered as an essential form of disease, is another example. The consideration of these and other elementary forms belongs appropriately to the principles of medicine, or general pathology, but it will suffice to consider them in treating of the individual diseases to which they give rise, or into which they enter. Our present knowledge of the elementary forms of disease is extremely limited. This will be apparent, in treating of individual diseases, when the inquiry arises respecting what is known of the primary, essential morbid conditions which they involve.

The consideration of certain measures and plans of treatment belong to general therapeutics. Thus, in the present chapter, bloodletting, the use of mercury, etc., as applied, not to any particular disease, but to groups of diseases, might with propriety have been considered. It will be more convenient, however, to offer such general considerations relating to these topics as may be deemed important, in treating of the application of therapeutical measures to the management of individual diseases. 


\section{P A R T I I.}

\section{PRACTICE OF MEDICINE, OR SPECIAL PATIIOLOGY.}

\section{INTRODUCTION.}

of the different aspects under which Individuai Diseases are to be considered, in treating of the Practice of Medicine, or Special Pathology-viz., Anatomieal Characters, Ciinical History. Pathological Character, Causation, Diagnosis, Prognosis, Prevention, and Treatment-Sense of the Term Individual as applied to a Disease, and the several Grouncis of IntlividualityVarieties of a Disease-Definition of the Terms Acute, Subacute, and Chronic-The Symptoms or Events incidental to Disease sometimes, for convenience, considered as Indlvidual Diseases-Self-limited Duration of Certain Diseases-Nosology-Nosological Arrangement adopted in this Work.

THE Practice of Medicine, or Special Pathology, treats of individual diseases. In this department of medical study, individual diseases are considered under several different aspects. One of these aspects relates to the appreciable changes found after death. These changes belong to morbid anatomy. Some diseases are characterized by morbid appearances in the parts affected, and other diseases are not accompanied by any lesions which are appreciable. The latter are distinguished as functional affections. Of the morbid appearances occurring in connection with different diseases, some are peculiar to one disease, and others are common to a greater or less number of diseases. The term anatomical characters of a disense expresses the changes, belonging to morbid anatomy, which occur in connection with that disease. This term will be adopted as the title of the first aspect under which will be considered generally, in the following pages, those diseases which are not functional.

Another aspect relates to the phenomena or events which compose the clinical history of a disease. Under this head will be embraced precursory or prodromic events, the symptoms which accompany the aecess, and those which occur during the career of the disease under consideration, the order of their suecession, the laws regulating the development and progress of the disease, etc. This will constitute the second of the different aspects under which individual diseases, severally, are to be considered.

A third aspect relates to the patholcgical character of a disease. Under this head will be presented what is known of the nature and sent of the morbid conditions which constitute, or enter into, the disease under consideration. In treating of each individual disease, the inquiry will arise under this head, what are the primary, essential deviations from the conditions of health; in other words, what morbid actions or processes constitute the point of departure for the manifestations of the disease? 'This inquiry will often lead to a confession of the imperfection of existing knowledge.

A fourth aspect relates to efiology, or the causation of a disease. Under this head the cause or eauses of each individual disease will be considered. 
Here, too, the imperfections of existing knowledge will be frequently apparent.

A fifth aspect relates to diagnosis, or the discrimination of a disease. The application of the methods of investigation which, by means of symptoms, laws, etc., enables the physician to recognize the disease under consideration, and to distinguish it from other diseases, will be considered under this head. Physieal signs, whenever these are available in the diagnosis of a disease, will be embraced under this head.

A sixth aspect relates to prognosis. Under this head will be considered the intrinsic tendency of a disease as regards its termination in death or reeovery. The circumstances which denote, on the one hand, a favorable, and, on the other hand, an unfavorable progress of the disease under consideration, will come under the head of the prognosis. And the consequences more or less remote, or sequels, may sometimes be conveniently embraced under this head.

A serenth aspect relates to the treatment and prevention of a disease. The consideration of the treatment of each disease will embrace the indications for therapentic measures, and the remedies which experience has shown to be useful in that disease. Hygienic measures also will enter into the consideration of treatment. Prophylactic measures of treatment will elaim consideration in treating of some diseases, and the measures whieh remove or obviate the eauses of disease, will often be found to be not less important than the judicions application of principles of therapentics.

Before entering upon the eonsideration of individual diseases, it may be well to inquire what is meant by this use of the term individual, and whence does a disease derive its individnality. An individual disease differs from other diseases sufficiently for it to be regarded as separate and distinct. The sense of the term individual, in this application, is analogons to that of species, in natural history. The individuality of a disease is established by distinctive features pertaining to its clinical history. These distinetive features may be derived exclusively from the seat of the disease. Thus, pleuritis and pericarditis are inflammatory affections of a serous membrane, and have essentially the same pathological charaeter; but the different anatomical relations of the pleura and pericardium furnish sufficient points of distinction to constitute each of these affections an individual disease. In other instances, distinctive features are derived from the actions or proeesses belonging to disease. Inflammatory affections, for example, differ essentially from those which are not inflammatory. Again, certain diseases derive each an essential dictinction from its dependence exelusively on a special cause. It is sufficient to show that a disease is produced by a special cause to establish its elaim to be regarded as an individual disease.

An individual disease is to be distinguished from individual cases of disease. An individual case of disease has a relation to an individnal disease, like that of a particular man to the species homo. 'The study of individual eases of disease belongs to clinieal medicine. Some individual diseases embrace several varieties. Empyema and pneumo-hydrothorax may be considered as varieties of pleuritis, both consisting in inflammation of the pleura, the former differing from ordinary pleuritis in the fact that the liquid eontained in the cavity of the pleura is purnlent, and the latter differing in the fact that air and liquid are contained in that eavity. The several types of intermittent fever, distinguished as tertian, quotidian, etc., are different varieties of one disease. In the progress of medieine, certain diseases have come to be considered as essentially distinct, which were formerly regarded as different varieties of one disease. This is true 
of all the cruptive fevers. It is probable that certain diseases now considered as individual will be found to be composed of two or more. Bright's clisease of the kidneys may be eited as an example. It is still a mooted question whether certain diseases are essentially distinct; for example, typhus and typhoid fever are by some considered as identical, differing suflleiently to be regarded as different varieties of one disease, but not enough to establish the individuality of each.

Differences as regards severity and duration constitute a basis of the division of a disease into varieties. The same disease may be either acule, subacute, or.chronic. A disease is aeute when it has a certain degree of intensity and runs a rapid eareer. In general, an acute disease is of sufficient gravity to confine the patient to the bed. 'The subacute variety of a disease has less intensity, frequently not compelling the patient to keep the bed, and perhaps not preventing him from being about, or even pursuing his usual occupation. The chronie variety exists when a disease is subacute and has continued for a considerable period. Acute, subaeute, and ehronic rheumatism may be cited as illustrating these three varieties of one disease. Some diseases are never chronic. 'This term, for instance, is not applicable to typhus or typhoid fever. 'The division into varieties, based upon the differences just named, is especially applicable to inflammatory affections.

The list of individual diseases embraces some, recognized as sueh for the sake of convenienee, which, in reality, are only effects or symptoms of diseases. Jaundice, for example, is an effeet or symptom oecurring in connection with different affections. Dropsy is in the same category. It is, however, convenient to consider jaundice and the different forms of dropsy as if they were individual diseases. In fact, if we were better acquainted with the primary or elementary forms of disease, it would probably appear that not a few of the affeetions now considered as individual, are only manifestations of morbid conditions at present oceultthe latter constituting the true diseases.

A point of distinction among diseases, interesting and of much practical importance, relates to duration. Some diseases continue for a rlefinite period, never exeeeding certain limits in this regard. The duration of certain diseases is regulated by fixed laws; that is, they never end until after the lapse of a certain number of days, and never exeed a certain number of days in their continuanee. The eontinued and eruptive fevers are examples of diseases, the duration of which has definite limits. Intermittent fever, on the other hand, has no fixed or definite duration, if its course be not interfered with. Diseases which tend intrinsieally to end after a certain time, are distinguished as self-limited. The laws of different diseases with respect to self-limitation are often to be taken into account in the employment of therapeutical measures, and it is obvions that these laws are to be considered in estimating the anount of eurative influence exerted by methods of treatment.

In treating of individual diseases, some elassification of them is essential. The classification of diseases constitutes a division of medicine called nosology. The history of medicine furnishes numerous nosological systems, the relative merits of which it would be quite useless to discuss. Undoubtedly, the most scientifie mode of classifying diseases would be to arrange them after essential points of distinction, relating to their pathological character. But, with our present knowledge of the pathological character of different diseases, to do this is impracticable. A nosologieal arrangement on this basis must be deferred for a future period, when pathology has advanced far beyond its condition at this time. 'l'he same 
remark is applicable to any arrangement based on differences as regards the causes of diseases. Our present knowledge of etiology is too limited to serve as the basis of a permanent nosology. Inasmuch as the chief object of a classification is convenience, the proper course to be pursued, evidently, is to adopt a provisional plan which will best subserve this objeet. And the most convenient classification, at the present time, is that which has of late years been adopted by the majority of writers and teachers. This consists in dividing diseases into, first, general, and, second, local. General diseases are those which involve more or less disturbance of the whole system, this disturbance not having been preceded by any known local affection, local affections, if they occur, being developed secondarily. To this class belong the essential fevers. Under the head of constitutional diseases, it is convenient to include some in which the local manifestations have no fixed seat, and sometimes differ in charucter. Rheumatism and gout are constitutional diseases, the chief manifestations consisting of local affeetions differently situated in different cases and at different periods in the same case. The local affection, however, is, in each, essentially the same wherever seated. Syphilis is another constitutional disease manifesting itself in local affections which differ, not only in situation, but in character. The want of fixedness or uniformity in the seat of local affections proceeding from constitutional disease, and their diversity in character in some instances, are the grounds for placing certain diseases in this class. A large proportion of the affections, nosologically considered as local, probably proceed from constitutional disease; but as the local affections are fixed and uniform, and our knowledge of the constitutional disease is, for the most part, inferential, it is more convenient to arrange them in the second division.

The second division, or local diseases, will embrace all which are not reckoned as general, or constitutional. The local diseases are far more n.umerous than those in the first division. It is necessary to arrange them into subdivisions. And the most convenient plan is to distribute them into groups, corresponding to the arrangement of the different organs of the body into physiological systems. According to this plan the different local diseases are subdivided as follows :-

1. Diseases affecting the respiratory system.

2. Diseases affecting the circulatory system.

3. Diseases affecting the digestive system.

4. Diseases affecting the nervous system.

5. Diseases affecting the genito-urinary system.

The foregoing arrangement of local diseases will be adopted, and they will be considered in sections corresponding to the groups just enumerated; afterward a section will be devoted to general diseases.

of the diseases belonging to these several groups, those only will be considered which are medical, as distinguished from surgical. T'he latter will include affections which are either external or in accessible situations. Thus, affections of the mouth, throat, and nasal passages, of the anus and rectum, of the urethra and bladder, of the eye and ear, and of the vagina and uterus, fall within the province of surgery. Diseases of the skin, being external, belong to surgery, notwithstanding they receive special attention from the physician perhaps oftener than from the surgeon. As an additional reason for not including them among the diseases to be considered, there are numerous treatises devoted specially to their consideration. 


\section{SECTION FIRST.}

DISEASES AFFECTING THE RESPIRATORY SYSTEM.

\section{H A P T E R I.}

Division of the Diseases affecting the Respiratory System into Inflammatory and Non-inflammatory-Acute Pleuritis-Anatomical Characters-Clinical History-Pathological Character - The Natural History of Inflammation, and our Knowledge of its Essential Nature-Causation of Pleuritis-Diagnosis - l'rognosis.

$\mathrm{F}$ the diseases affecting the respiratory system, an obvious division is based on a difference in pathological character, as regards the existence, or otherwise, of inflammation. 'The inflammatory affections in this group of diseases are seated respectively in the three most important of the struetures whieh are comprised in the organs of the respiratory apparatus, viz., the mueous membrane lining the air-passages, the serous membranes which invest the lungs, and the pulmonary parcuehyma. Exclusive of affections seated in the larynx and trachea, inflammation affecting the structures just named gives rise to three important diseases, viz., pleuritis $^{1}$ or pleurisy, pneumonitis or pneumonia, and bronchitis. Beginning with these inflammatory affections, they will be treated of in the order in which they have just been enumerated. The first disease to be considered, viz., pleuritis, may exist as an acute and as a subacute or chronic affection. These two varicties of the disease will claim separate consideration. Other varieties, to be considered separately, are empyema and puenmo-hydrothorax. It will be convenient to notice, in connection with these varieties of pleuritis, the dropsical affection known as hydrothorax.

\section{Acute Pleuritis.}

The pleura, anatomically, is essentially similar to other serous membranes. It is composed of white non-elastic and yellow elastic fibres. It is divided into two layers, viz., the visceral and parietal. The free surfaces of these two layers are smooth, polished, and moistened with halitus. It is transparent, receiving a certain number of blood-corpuscles, but not sufficiently aggregated to be visible. The nutrient vessels ramify in the sub-serous areolar tissue. It forms in each side a shut sac. Its oflices are to afford support and allow movement without injury from friction.

Anatomicat Characters of Acute Pleuritis.-The first noticeable appearance, could the inflamed part be seen during life, would doubtless be redness from an increased quantity of blood, chiefly contained in the vessels beneath the membrane. Redness here, as in other situations, may

1 With a view to uniformity as regards nomenclature, the terms pleuritis and pneumonitis are used instead of pleurisy and pneumonia. 
diminish and disappear after death. It is supposed that, for a brief period after the commencement of inflammation, the membrane is abnormally dry, from suppression of the halitus which moistens it in health. Liquor sanguinis, or blood plasma, begins soon to exude, the fibrin which it contains coagulates, and characters suflieient to show the existence of inflammation are now present.

If the disease prove fatal within two or three days, the pleural cavity contains a greater or less quantity of liquid, the amount varying considerably in different eases. The liquid is not everywhere clear, like the serum of the blood, or a purely dropsical effusion. 'The upper portion is usually clear, if the body have not been much disturbed in opening the chest, but near the bottom it is turbid from the admixture of coagulated fibrin or lymph. The pleural surfaces are more or less coated with lymph which, at this period of the disease, is soft and easily removed. The amount of lymph varies considerably in different eases. Its presence is essential as a criterion of inflammation. The membrane loses a portion of its transparency from serous infiltration beneath it; it is somewhat swollen, and its epithelium is exfoliated. Redness, from sub-serous congestion, may or may not be apparent. Small extravasations of blood or ecchymoses are not infrequently observed.

On examination after death occurring at a later period, the pleural sac is found to contain a certain quantity of liquid, varying from a few ounees to several pints, with floeculi of lymph at the bottom of the liquid. Lymph may be found to coat the greater part of, or even entirely, the pleural surfaces, or it may be limited to patches. It is usually more abundant on the parietal than on the visceral surface. It is more dense than at an earlier period, and is disposed in the form of a layer or a series of layers. The layers of lymph present a membraniform appearance without organization. A layer of lymph sufficiently dense to be stripped off is properly called a false membrane. The name false membrane, which is often applied to the adventitious tissue subsequently produced, is more correctly limited to an unorganized layer of lymph or fibrin. It may adlere with considerable tenacity to the pleural surface, and if the opposing surfaces be not separated by liquid, they may be pretty firmly agglutinated by means of the intervening lymph. The density of the lymph and the firmness of its adherence are evidenees that the exudation is not of recent oeeurrence.

The presenee of serum and lymph, in varying relative proportions, in other words, a sero-fibrinous liquid, thus, is characteristic of simple acnte pleuritis. The liquid in the pleural cavity occupies space at the expense of the volnme of the lung. As it accumulates, it eompresses the lung, the latter occupying a space reduced in proportion to the amount of liquid. If the pleural surfaces be free from old adhesions, and not agglutinated by lymph, the relative situations of the liquid and lung will vary according to the position of the body. If the patient be sitting or standing, the liquid gravitates to the bottom of the sac, and rises to a height proportionate to the quantity. If the patient be recumbent on the back, the liquor gravitates to the posterior part of the chest, and the lung will descend in front, more or less, in a direction toward the diaphragm. In like manner, if the patient lie on the face, the liquid occupies the now depending anterior part of the chest, and the lung will descend behind. These changes in relative situation are frequently available in determining, by physical signs, the existence of liquid in the pleural cavity.

If the disease progress favorably, the liquid diminishes progressively, with variable rapidity in different cases, by endosmosis or absorption. It diminishes often with considerable rapidity until it is reduced to a small 
or moderate quantity, and then the absorption takes place slowly-a clinieal fict probably due to the inereasing density of the liquid from the larger relative proportion of 'lymph as the serosity lessens, and to the increased density of the layers of lymph with which the pleurnl surfices are coated. The absorption of the lymph occupies a considerable period after all the liquid has been absorbed. In the mean time, the pleural surfaces, coming into contact over their entire area, are agglutinated by means of the intervening lymph, and this exudation is replaced by a newly-developed adventitious tissue which unites by permanent adhesions the surfaces in apposition. It is to be noted that the union by means of the adventitious tissue is not mechanical, as when the surfaces are agglutinated by lymph, but vital, that is, by an organized membrane. The adhesions may extend over the whole of the pleural surfaces, and then the serous cavity is obliterated, or they may be limited to certain portions, in the latter ease constituting ligamentous bands, which, being observed by some of the older anatomists, were supposed to have been produced by inordinate langhter! This true membranous tissue has been supposed to be organized fibrin or lymph. Many, if not most, pathologists at the present time have abundoned this view of its production. It is, however, a question eoncerning which pathologists are not agreed, whether the new tissue is generated spont:meously, within the exudation, the latter being then a blastema; or whether its production be due to proliferation, in other words, a vegetation or hyperplasiat of the areolar tissue of the pleura. This pathological question has been referred to in the first part of this work. ${ }^{1}$

The adhesions by means of organized tissue take place during convalescence. At first, they are weak and liable to be broken by active exercise, or in aets of coughing. Hemorrhage may in this way occur. They become stronger with time, so that when death oceurs after the lapse of severil weeks or morths, the lung is detached and removed from the chest with considerable difficulty. After a time, all the lymph disappears, and nothing remains but the newly-produced tissue. It may be remarked, in passing, that after the serous cavity is thus abolished, there is no space for the effusion of liquid to constitute hydrothorax, and cedema of the lung may take its place under circumstance which give rise to dropsical effusion in the opposite side. A case has come under my observation exemplifying this fact. It is also to be remarked that these adhesions, however extensive, occasion no appreciable disturbance of respiration. 'They are, in fact, innocuous, and perhaps confer exemption from recurrence of pleuritis on the same side. Nor do they give rise to any distinct physieal signs.

The aceumulation of liquid in acute pleuritis may take place in such quantity as to fill the cavity of the pleura, compressing the lung into a small solid mass. The compressed lung loses all the physical eharacters dependent on the presence of air in the pulmonary vesieles. It resembles a mass of flesh, and is therefore said to be carnified. 'The pulmonary' structure, however, remains intact, and it is capable of being inflated, after death, nearly or quite to the same extent as if it had not undergone this compression. A similar expansion may take place during life, after the absorption of the liquid. So large an accumulation of liquid, lowever, oceurs much oftener in chronic than in acute pleuritis.

In cases of acute pleuritis which pursue a favorable course, absorption of the liquid commences a few days after the accumulation has reached the maximum. Should the quantity remain stationary or the diminution take place very slowly, after the lapse of two or three weeks the disease has 
become chronic. The greater part of the liquid is sometimes absorbed, and vital adhesions take place between the larger portion of the plenritic surfaces, leaving a circumscribed space in which a certain quantity of liquid may remain for an indefinite period. The disease then eventuates in a circumscribed chronic pleuritis.

The foregoing description of the anatomical characters of acute pleuritis relates to its occurrence as a primary disease so far as any other pulmonary affection is concerned. Occurring independently of any prior disease of the lung, the inflammation is general, that is, it extends over the greater part or the whole of the serous membrane. It is otherwise when pleuritis occurs consecutively to another pulmonary disease. Occurring, as it does quite constantly, as a complication of pneumonitis and pulmonary tuberculosis, the inflammation is limited to a portion of the membrane; the pleuritis is circumscribed. And circumscribed pleuritis, developed thus as a secondary affection, is rarely accompanied by liquid effusion. It is distinguished from general pleuritis by this fact; the exndation of fibrin only occurs, and the pleuritis is said to be dry. Primary or idiopathic pleuritis is seated in the left oftener than in the right side.

Cuinical History.- - The most convenient division of acute pleuritis into stages, is to consider as the first stage the period from the attack to the time when an appreciable quantity of liquid effusion has taken place; the second stage will extend to the time when the liquid begins sensibly to diminish, and the third stage will comprise the period occupied in the removal of the liquid. The first stage, or the period anterior to an appreciable amount of liquid effusion, is usually of short duration. The physical signs may show the presence of liquid within a few hours from the date of the attack. The stage rarely extends beyond twenty-four hours. The second stage is of variable duration. The accumulation of liquid may go on with more or less rapidity. When the maximum amount is reached, the quantity may remain stationary for some time, or absorption may speedily begin. The liquid may diminish under the effect of treatment, and renewed effusion take place. The disease does not, of course, advance beyond the second stage in the cases in which it eventuates in chronic pleuritis. If a considerable quantity of liquid remain at the end of from two to three weeks, the disease is to be considered as having become chronic. In cases which pursue a favorable course, considerable progress should be made in the removal of the liquid after this lapse of time. The duration of the third stage, or the stage of absorption, is quite variable. Generally some liquid remains when the general symptoms denote convalescence, and even after the patient has apparently recovered. The physical signs may show the presence of lymph for some time after the date of convalescence. From what has been stated, it follows that the duration of the career of the clisease is from two to three weeks.

The invasion is sudden, and often without premonition. In a certain proportion of cases, however, some degree of pain or soreness is felt for one, two, or three days before the development of acute inflammation. This fact, which other observers have noted, has repeatedly occurred among the cases that have fallen under my observation. The development of acnte inflammation is characterized by symptoms which sufficiently mark the date of its occurrence. It is sometimes ushered in by a well pronounced chill, accompanied perhaps with rigors. Chill and rigors; however, are by no means so frequent, nor, as a rule, so marked as in cases of pneumonitis. Not unfrequently chilly sensations and slight shivering only are experienced. 
Pain in the affected side attends the onset of acute inflammation in a large majority of cases. The pain is usually intense. It is sharp, cutting, or lancinating in charaeter. It is felt especially in the aet of inspiration; it increases during inspiration, often becoming so severe that the act is instinctively arrested before being completed. Coughing and sneezing occasion severe pain. The movements of the body are painful, but in a far less degree than the respiratory movements. The pain is referred to the middle and lower third of the affeeted side of the chest, and is usually diffused over the anterior, the lateral, and sometimes the posterior portion. It is not, as a rule, circumseribed or limited to a small space. The morbid sensitiveness of the inflamed pleura seems to me adequate to account for the production of acute pain, as a result of the movements of the pleural surfaces upon each other, and the stretehing of the membrane, without requiring the hypothesis that either intercostal neuralgia, or an affection of the costal muscles, is superadded to the inflammation. Pain, with the characters just described, is almost uniformly present in acute pleuritis, but cases occur in which it is not marked, and even wanting, other symptoms showing the inflammation to be acute.

Febrile movement is coincident with the development of the inflammation. The pulse is increased in frequency and in strength. The temperature of the surface is raised. The thermometer in the axilla shows more or less intensity of fever, but the rise rarely exceeds $102^{\circ}$. As a rule, this criterion of fever denotes less than in cases of pneumonitis. The usual concomitants of symptomatic fever, viz., thirst, anorexia, pain in the head and loins, restlessness, muscular weakness, etc., are more or less marked. The intensity of the febrile movement, and of the general or constitutional disturbance, varies much in different cases of this, as of other inflammations, and is not to be taken as evidence of a corresponding grade of acuteness of the disease. It may be laid down as a fact applicable to inflammatory diseases in general, that the symptoms distinguished as constitutional have no fixed relation to the extent or intensity of the local affection; in other words, the same amount of acute pleuritis will give rise in one person to more, and in another person to less, than the average amount of constitutional disturbance.

Cough is usually present, but sometimes wanting. The pain in coughing leads instinctively to efforts to repress it, and this gives to the mode of coughing a character denoted by the term suppressed. The patient succeds, to a certain extent, in these efforts. The expectoration is slight or wanting, and, when present, it consists of simple mucus. 'The exceptions to this rule are the cases in which the development of pleuritis is preceded by bronchitis. When these affections are associated, it is merely a coincidence; there is no tendeney in either to give rise to the other.

The respirations are increased in frequency, but not greatly. Their increased frequency at this period is due to the arrest of the inspiration, before it is completed, in consequence of pain. The patient instinctively multiplies the respiratory acts to compensate for the want of a full inspiration. As a rule, the patient in lying chooses a position on the healthy side, the weight of the body upon the affected side inereasing the pain and soreness. This rule is by no means without exceptions. Not unfrequently the decubitus is either dorsal or diagonal.

These symptoms constitute the clinical history of this disease during the first stage, or prior to liquid effusion. They continue into the second stage, but, after a certain amount of effusion has taken place, they are materially modified. The pain is notably lessened, and may disappear, except on forced breathing. The acts of coughing are less painful, and 
the efforts at suppression are less apparent. The febrile movement diminishes, and may even cease during this stage. Other evidences of constitutional disturbance are less marked. 'The patient reports better, and may be able to sit up and perhaps walk about the room. The respirations are more or less frequent; their frequency now depends on the compression of the lung by liquid. Their frequency will be great, other things being equal, in proportion to the quantity of liquid and the rapidity of the effusion. If a considerable amount of the liquid be rapidly effinsed, the respirations are rapid, the patient suffers from a painful sense of the want of breath, or dyspnoa, and may be obliged to maintain the sitting posture. If the quantity be not large, and the effusion have not taken place rapidly, the patient will not be likely to suffer from dyspnœea while remaining quiet; but exertion will cause panting and a sense of the want of breath. Even with the pleural sac distended with liquid, the only effect on the breathing may be an increase of the frequency of the acts to twenty-five or thirty per minute, without suffering so long as the patient remains quiet. The diminution or cessation of pain is due to the restrained movements of the pleural surfaces upon each other, in consequence of the presence of the liquid and lymph, the latter perhaps agglutinating the portions of these surfaces which are in contact. The change as regards febrile movement and other constitutional symptoms, proceeds, in part, from the removal of the conditions eausing pain, and, partly, beeause, after exudation has taken place, the disease is mitigated from an intrinsic tendency to decrease. The patient now, as a rule, prefers to lie upon the affected rather than the healthy side, as in this position he is able to expand more freely the opposite lung. To this rule, however, there are exceptions; and the decubitus in this stage, as in the first stage, is frequently either dorsal or diagonal.

During the stage of absorption, pain is either wanting or produced only by muscular efforts; febrile movement has disappeared; cough diminishes or ceases; the respirations become less and less frequent, and are less hurried by exereise; the appetite and digestion return, and the normal condition of all the functions is gradually restored. During this stage the patient is convalescent ; he is able to be out of doors, and, perhaps, to resume in part his usual avocation and habits. Convalescence is declared, and the symptoms, both general and local, may appear to denote that recovery has taken place, while some liquid effusion still remains; the lymph is not yet fully absorbed, and the formation of new tissue leacling to permanent adhesions is going on. A sense of weakness in the affected side, together with some soreness or pain on unusual exertions, frequently continues for some time after the termination of the career of the disease.

Primary or idiopathic pleuritis, as already stated, affects, more or less, an entire pleural membrane; it is general, not partial nor eircumscribed. If it be partial or circumscribed, it is developed as a complication of some other pulmonary affection, as a rule. It will be seen hereafter that it occurs as a complication of pneumonitis and phthisis, but it is generally, under these circumstances, subacute. Primary pleuritis does not lead to the development of pneumonitis, except that the air-cells in immediate proximity to the pleura are sometimes involved; nor does it involve any tendency to bronchitis. It is a unilateral disease, that is, it affects the pleura of one side only. The exceptions to this rule are so unfrequent, that the disease, when bilateral, or double, may be presumed to be seconrlary to another pulmonary affection, generally tuberculosis. I have met with a case of double pleuritis, accompanied with a large effusion into both 
pleural cavities. Death took place by apnoa, in this case, and pleuritis was found to be consecutive to a small tuberculous deposit in both lungs. Such cases are extremely rare.

Pathological Character. - The disease under consideration is an example of acute inflammation affecting a serous membrane. Inflamm:tion is an elementary form of disease. Its phenomena and results are the same essentially whenever it affects a serous membrane. In different situatious it has points of difference, relating to the structure affeetel, ancl it presents diverse modifications dependent on other circunstances than its seat. Nevertheless, there are 'features common to acute inflummation wherever seated, and under all circumstances, suflicient to show the form of disease to be essentially the same. The phenomena of inflammation are best studied when it is situated externally, or in parts accessible to observation during life. It is therefore considered at length in treatises on surgical pathology and surgical diseases, to which the reader is referred for a full account of events which will be here very briefly noticed.

The sensible changes which first occur in an inflamed part are ineident to hyperæmia, or a morbid accumulation of blood in the part. Hence arise redness, heat, pain, and swelling, the four characters which have heretofore entered into the definition of inflammation. The accumulation of blood is a result of an undue determination of blood to the part; in other words, the part receives a larger amount of arterial blood than in health. There is also a retardation of the circulation through the inflamed part. So far, the condition is expressed by the term active congestion, called by Virchow fluxion. If the affection should go no further, there would hardly be sufficient evidence that inflammation has become developed, or it might be considered as incipient inflammation disappearing by delitescence. But inflammation rarely ceases at this incipient period. A variety of local events may follow, some of which always occur, different cases presenting very wide differences arising from the presence of some of the events and the absence of others. Of the local events succeeding to active congestion, one is death of the part. Complete stasis of the circulation may occur, and the affected part loses its vitality; it becomes gangrenous or sphacelated, and will slough away, if the life of the patient be sufficiently prolonged. This is a rare occurrence in plenritis and other serous inflammations. The escape of liquor sanguinis or blood-plasma is another event occurring constantly in serous inflammations, also in certain parenchymatous inflammations, and, exceptionally, in inflammation of mucous membranes. Coagulated fibrin, or lymph, is a characteristic product of inflammation. Another product, in like manner characteristic, is pus. The production of adventitious tissue is another event, the occurrence of which is illustrated by the morbid anatomy of pleuritis. This event occurs, as a rule, in serous inflammations. Extravasation of blood is an occasional event incidental to inflammation in various situations. Other events are, softening of the struetures affected, induration from the presence of lymph, ulceration, thickening, etc. These different events, belonging to the natural history of inflammation, will be referred to frequently in connection with the anatomical characters of different individual inflammatory diseases.

Waiving consideration of the historical events just enumcrated, the inquiry arises, in what consists the local devi:ttions from health which constitute inflammation? in other words, what is knowu of its essential nature? Reverting to the fact already stated, that the first appreciable change is an abnormal determination of blood, the answer to the foreguing 
inquiry involves an explanation of this fact. Why is more blood determined to an inflamed part than in health? We might answer this question satisfactorily, if the causes determining the quantity of blood which each organ and structure contains in health were known. The amount of blood which a part receives in health is by no means exclusively regulated by the forces which carry on the general circulation, and which chiefly belong to the heart. Parts receive more or less blood according to their state as regards functional activity. The stomach, for example, receives a much larger quantity of blood during the act of digestion than at other times. A very striking contrast, in this respect, is offered by the uterus before and after impregnation. The explanation of the cause or causes determining thus, in health, within certain limits, the amount of blood which different parts receive, devolves on the physiologist; and when this is understood, the pathologist may be able to explain why there is hyperæmia in an inflamed part. Witl our present knowledge, two explanations are offered. One of these attributes to each part an inherent attractive force between the blood and the tissues. The physiological operation of this force regulates the quantity of blood in different parts in health, and a morbid exaggeration of the force accounts for an overplus of blood in the congestive stage of inflammation. The other explanation relates to the vessels and the vaso-motor nerves. The regulation, in health, of the quantity of blood in parts being considered as due, measurably, to differences with respect to the contraction and relaxation of the vessels, lyyperæmia is attributed to relaxation from paralysis, and, on the other hand, a morbid deficiency of blood, or local anæmia, to contraction from spasm. Whichever of these two explanations be accepted, or, assuming each to be more or less involved, it is evident that to account for an increased quantity of blood in an inflamed part, is only to take one step, however important it may be, in the pathology of inflammation. Most of the events following the accumulation of blood in inflamed parts denote aberration of the molecular changes involved in nutrition. Inflammation, therefore, consists, in great part, of perversions of nutrition ; and when the physiologist is able to explain the local changes belonging to healthy nutrition, the pathologist may be able to explain the perversions which belong to inflammation. In conclusion, then, we may say that inflammation is a morbid deviation from those conditions of health which relate to the circulation and nutrition of the part affected.

After the foregoing brief account of inflammation, it will suffice, in treating of other inflammatory affections, to indicate, under the head of pathological character, the points which distinguish this elementary form of disease in the particular affection under consideration.

CaUsation.-Acute pleuritis may be produced traumatically by contusions, especially if accompanied with fracture of the ribs, and by penetrating wounds. It is remarkable, however, that severe injuries of the chest often occur without giving rise to general pleuritis. Persons receiving gunshot wounds frequently escape if the ball or other foreign material be not lodged within the pleural sac. The disease is attributed, in certain cases, to the action of cold. This has so long been considered as a frequent source of a great number of diseases, that patients at once are led to refer an attack to some exposure, often, doubtless, when it had no causative agency, or, at most, acted only as an exciting cause. Still it is probable that the disease sometimes originates in this way. In the larger proportion of the cases in which it is not traumatic, it is spontaneous, that is, it proceeds from an unknown internal cause. It occurs sufficiently often 
in persons affected with the diseases of the kidneys embraced under the name Bright's disease, to show a pathological connection; and in these cases it is referable to the accumulation of urea in the blood. It is developed occasionally in connection with acute articular rheumatism. It is an occasional sequel or concomitant of scarlatina, measles, and continued fever. It enters into the history of pyæmia and of septicæmia.

The liability to the disease is not equal at all periods of life. The disease is extremely rare in infancy. It is not uncommon in childhood. It occurs in youth and middle life much more frequently than in old age. It is as rare in old age as in infancy. 'The proportion of males over females, among cases of this disease, vastly preponderates. There do not appear to be any causative influences pertaining to constitution, season, climate, or locality. 'The aeute variety of pleuritis is not of very frequent oceurrence; the subacute or chronic variety, in my experience, is much more frequently met with. It is less frequent than pneumonitis, and, in relative frequency, bears no comparison with acute bronchitis.

Dragnosis.-Although the symptoms which enter into the clinical history of acute pleuritis are sufficiently characteristic to point to the existence of the disease, they are not, singly or collectively, sufficient for the diagnosis in all cases. The characteristic pain is not peculiar to the disease. This occurs in some cases of intercostal neuralgia and pleurodynia as strongly marked as in acute pleuritis; and these three affections have heretofore been often confounded in practice. Moreover, the pleuritic pain in some cases of pneumonitis is as intense as when the pleuritis is a primary disease, and the former disease has not infrequently been mistaken for the latter. On the other hand, acute pleuritis is liable to be overlooked in the cases in which pain is slight or wanting. More or less febrile movement is present in acute pleuritis, but this symptom may aceidentally be associated with intereostal neuralgia; it will be likely to be present in pleurodynia, and it exists, of course, in pneumonitis. The diagnosis is to be based on the physical signs, taken in connection with the symptoms. The latter are of importance, and, indeed, essential in determining whether the affection be acute or not. The differential diagnosis eonsists in discriminating between the disease under consideration and intercostal neuralgia, pleurodynia, and pneumonitis.

The diagnosis in the first stage, with the aid of physical signs, cannot always be made with positiveness. Examination of the chest by inspection will show restrained movements on the side to which the pain is referred; on percussion, there will be found to be no distinct disparity between the two sides, and on auscultation, the murmur of respiration will be more or less weakened on the affected side. But these signs are due to the fact that the pain leads instinctively to a diminished use of the lung on the side affected, while the use of the other lung is increased. These signs are equally present when the pain is incident to the affections from which this disease is to be discriminated. A slight friction murmur may be discovered in this stage ; if distinctly present, its diagnostic significance is important; it shows that pleuritis exists, and the discrimination then lies between pleuritis as a primary disease or as a complication of pneumonitis. To decide this point at once is not always easy. The diagnostic sign of pneumonitis, viz., the crepitant rale, if present, settles the question ; but as this sign is by no means present uniformly in pneumonitis, its absence does not authorize the exclusion of that disease. The same remark is applicable to the characteristic expectoration of pneumonitis. It must be confessed that, in a certain number of cases, this differential diagnosis 
requires a little delay. But a friction murmur is heard in only a very small proportion of cases during the first stage, and, in the absence of this sign, the signs belonging to the second stage are essential to render the diagnosis positive.

The signs belonging to the second stage are usually present without much delay. If the disease be idiopathic pleuritis, after the lapse of twelve or twenty-four hours, liquid effision will have taken place in sufficient quantity to be apparent. The signs now denoting the existence of the disease are those due to liquid effusion. The diagnosis is based upon these signs, in conjunction with the symptoms and history, and on the absence of the signs of pneumonitis.

The signs of liquid effusion are obtained by percussion, auscultation, palpation, and inspection. On percussion, dulness or flatness is found at the base of the chest, extending upward in proportion to the quantity of liquid, the patient being in a sitting posture. By the finger which serves as a pleximeter, also, is perceived an increased sense of resistance on percussion. The upper limit of the dulness or flatness corresponds to a horizontal line drawn across the anterior and lateral surface of the chest, this line denoting the level of the liquid. Changing the position of the patient to recumbency on the back, frequently induces a marked change in the relation of dulness or flatness and the resonance, as previously determined on the anterior surface of the chest, in the sitting posture; the liquid gravitating to the posterior part of the chest, the lung descends in front, and furnishes resonance for a greater or less distance below the line which was coincident with the level of the liquid while the patient was sitting. This latter test of the presence of liquid is not available in all cases. If the pleural surfaces above the level of the liquid be united by old adhesions, or aglutinsted by lymph, the liquid and lung will maintain the same relation in different positions of the body. The failure of this test, therefore, is not proof against the presence of liquid. The test, however, is available, according to my experienee, in a large majority of cases. The resonance over the lung above the level of the liquid, if the latter rise to a third, a half, or, perhaps, over two-thirds the height of the chest, is exaggerated, and acquires, in part, a tympanitic quality, constituting vesicnlo-tympanitic resonance.

On auscultation, the respiratory murmur is either suppressed or feebly appreciable below the level of the liquid. Above the liquid, it is usually weak, as compared with the murmur on the opposite side. If the lung be considerably reduced in volume by the amount of liquid, the respiratory sound will become broncho-vesicular, or rude, and the vocal resonance will be increased. If the liquid be sufficient to compress the lung into a solid mass, the latter will furnish the bronchial respiration. The bronchial respiration will be heard over the site of the compressed lung. It is usually limited to that site, but, in a certain proportion of cases, it is diffused over the greater part or the whole of the affected side. The normal vocal resonance is either suppressed or diminished over that portion of the affected side which corresponds to the space occupied by the liquid. But if the accumulation of liquid be large, bronchophony may be produced over the whole of the compressed lung, or at the portion in contact with the liquid. The bronchophony has sometimes a tremulous or bleating character, and is then æegophony. If the chest be filled with liquid, bronchophony is sometimes diffused over the whole of the affected side.

Vocal fremitus is either arrested or diminished by liquid effusion. From this fact is derived valuable evidence of the presence of liquid when the effusion is in the right pleural cavity, in consequence of the greater amount 
of fremitus over the right side in health. If the question be as to the presence of liquid in the right pleural cavity, and the fremitus be found to be greater on the left than on the right side, the evidence of liquid is strong.

If the quantity of liquid be large enough to distend the pleural sae, the intercostal depressions may be diminished or destroyed at the inferior, anterior, and lateral portions of the chest. This constitutes valuable evidence of the presence of liquid.

Other and marked signs of effusion are present if the quantity of liquid be sufficient to dilate the chest. This occurs exceptionally in acute plenritis. It occurs much oftener in the chronic variety of the disease, and the signs referred to will be noticed in connection with the diagnosis of the latter.

The physical signs not only lead to a positive diagnosis after the first stage, but they show the amount of liquid in the pleural sae, a point of importance with reference to treatment. By means of daily explorations, the physician may ascertain whether the effusion be increasing or diminishing, and the rate of either the increase or diminution. 'The information thus obtained is highly important as a guide to the employment of therapeutical measures. This information cannot be obtained from the symptoms. 'The signs, thercfore, are essential, not only to the diagnosis, but to a proper knowledge of the progress of the disease.

After absorption, to a greater or less extent, of the liquid, the pleural surfaces coming into contact over a larger area, and roughened by lymph which has now become dense and closely adherent, a friction murmur is frequently apparent. This is sometimes so loud as to be heard by the patient. It may continue into. convalescence, ceasing when the pleural surfaces have become united by means of newly-formed tissue. $A$ friction murmur, however, by no means occurs uniformly even at this stage of the disease.

Prognosis.-The prognosis in cases of aeute, idiopathic, simple pleuritis is always favorable, provided the disease be uncomplicated, and the constitution of the patient be not enfeebled. There is reason to believe that, without therapeutical interference, it would very rarely prove fatal. I have collected several cases in which the disease passed through its course favorably without any treatment. It may possibly prove fatal in consequence of a large amount of liquid effusion occurring very rapidly, death taking place by apnca. I have known one instance in which the loss of a hospital patient, suddenly and unexpectedly, seemed fairly attributable to the fact that the pleural sac was greatly distended with liquid. Developed as a sequel to other diseases, or in the course of a grave affection, such as Bright's disease of the kidneys, or in persons with broken constitutions, it may destroy life by asthenia. Acute pericarditis is sometimes developed simultaneously with, or during the progress of, pleuritis, and these associated diseases are apt to prove fatal. Their occasional coincidence is to be borne in mind, or the pericarditis, in this connection, if not carefully sought for, will be likely to be overlooked. As already stated, the development of pneumonitis, consecutively to pleuritis, is not to be expected, nor is there much, if any, danger that an acute pleuritis, after its development, will eventuate in suppuration, constituting the variety of the disease called empyema. In short, the intrinsic tendency of the disease is to recovery, a fatal termination being due very rarely to the disease per se, but to extrinsic circumstances. 


\section{CH A P T E R II.}

Treatment of Acute Pleuritis-Measures indicated ln the First Stage-General Considerations relating to Bloodletting in Acute Inflammations-The use of Opium in $\Lambda$ cute Pleuritis and other Acute Inflammations-Measures indicated in the Second and the Third Stage of Acute Pleuritis.

THE objects of treatment in acute pleuritis differ in its different stages, and the latter, therefore, claim, severally, distinct consideration with reference to therapeutic indications. The objects in the first stage are to arrest, if possible, the progress of the disease, to diminish its intensity, if it be not arrested, to limit the amount of morbid products, and to relieve suffering; in other words, the treatment relates to the employment of abortive, curative, and palliative measures. The indications in the first stage of this clisease are essentially the same as in the early period of most acute inflammations. The general principles which should govern the treatment in this stage, therefore, will be here considered, and simply referred to hereafter in connection with other diseases to which they are measurably applied.

Owing to its brief duration, patients are frequently not seen until this stage has passed. Can the disease be arrested at this stage if the opportunity offer and a positive diagnosis be made? Admitting that, formerly, physicians did sometimes succeed, as they believed, in cutting short the disease, it must be said, in answer to this question, that there are no known means which can be relied upon for this end. Bloodletting, and other socalled antiphlogistic measures, which were formerly employed for this object, have certainly not been successful in a sufficient number of cases to warrant their employment to an extent which will render them likely to do harm if they are not successful. Assuming that the disease goes on to the second stage, the question then is, what curative and palliative measures are to be employed? This question leads at once to the consideration of bloodletting.

A great change has taken place, within the last few years, with respect to bloodletting in the treatment of acute inflammations. This measure was formerly thought to be highly important, and was rarely omitted. It is now considered by many as seldom, if ever, called for. The infrequent use of the lancet now, contrasted with its frequent use forty years ago, constitutes one of the most striking of the changes in the practice of medicine which have occurred during this period. It can hardly be doubted that this measure was formerly adopted too indiscriminately, and often employed too largely ; but, the natural tendency being to pass from one extreme to another, the utility of bloodletting in certain cases, at the present time, is not sufficiently appreciated.

Experience and pathological reasoning combine to show that bloodletting does not exert a direct controlling effect upon an inflammatory disease. It may exert a powerful immediate effect as a palliative measure, but whatever curative power it may possess is exerted indirectly. Its therapeutic action, in general, consists in lessening the frequency and force of the heart's action; in other words, in diminishing the intensity of symptomatic fever. In the early period of an acute inflammation accom- 
panied by high febrile movement, as indicated by a pulse accelerated and of abnormal strength, the abstraction of blood affords relief, and may contribute to a favorable progress of the disease. It should enter into the treatment of a certain proportion of cases, provided other and more congervative means for the same ends are not available.

The evils of bloodletting arise from its spoliative effect upon the blood. It diminishes the red globules, and these, during the progress of an acute disease, are not readily reproduced. It induces, thus, the aniemic condition, and in this way impairs the vital powers. It will be likely to do harm, therefore, whenever it is important to economize the power's of life, and it may contribute to a fatal result in disenses, or cases of disease, which involve danger of death by asthenia.

The useful effects of bloodletting may frequently, if not generally, be obtained by other means which require less circumspection in their employment, because, if injudiciously resorted to, they are in a less degree hurtful. The mass of blood may be temporarily lessened by saline purgatives and diaphoretic remedies, conjoined with a restricted ingestion of food and liquids. Depletion is obtained in this way without spoliation or impoverishment of the blood. The frequency of the heart's action may be affected by nauseant sedatives, such as tartar-emetic, ipecacuanha, etc., and by direct sedatives, viz., digitalis, aconite, and the veratrum viride. By saline depletories and sedatives, the symptomatic fever may be modified without the expenditure of blood, and thus the evils of bloodletting avoided. The advantage of bloodletting consists mainly in the promptness of its operation. Several bours are required to secure results from the means employed in lieu of bloodletting, whereas, the effects of the latter are produced in a few moments.

In accordance with these views, bloodletting is never indicated by the fact simply that acute inflammation exists; it is a measure directed, not to the disease per se, but to circumstances associated with the disease. The state of the circulation, and other circumstances, furnish the inclications for the employment of this measure. It is admissible, if, with the development of inflammation, there exist high symptomatic fever, the patient being robust and in good health when attacked, and the disease not involving danger of death by asthenia. The measure is admissible, under the conditions just stated, whenever the promptness with which its effects are obtained renders it desirable to adopt it in preference to other measures producing the same effects with more or less delay. Per contra, bloodletting is not admissible when the development of inflammation is not accompanied by high symptomatic fever, when the patient was not in good health when attacked, nor when the constitution is feeble, nor when the disease involves danger of death by asthenia. These rules of practice, while they accord to bloodletting therapeutic value, undoubtedly restrict its use within narrow limits.

Applying these rules to the disease under consideration, a patient in the first stage of acute pleuritis, robust, perhaps plethoric, suffering from severe pain and a sense of oppression, with a strong, non-compressible pulse, will derive immediate relief from the abstraction of from ten to sixteen ounces of blood. The loss of this quantity of blood, under such circumstances, in a disease like this which does not tend to destroy life by asthenia, will give rise to no evil results, but will be likely to affect favorably the progress of the disease. On the other hand, a patient feeble, or anæmic, with a pulse more or less frequent, but denoting diminished power of the heart's action, should not be bled, notwithstanding the local symptoms would undoubtedly be thereby relieved. By impairing the 
vital powers, the loss of blood will do harm, and is not admissible under these circumstances merely as a palliative remedy. And, in the first case, if the local symptoms do not urgently call for immediate relief, other measures may be substituted for the bloodletting.

Before leaving the consideration of bloodletting, several incidental points may be briefly noticed.

This measure is perhaps more applicable to the treatment of inflammation affecting the pulmonary organs than to the treatment of other inflammatory affections, in consequence of the relations of the former to the circulation. The free passage of the blood through the pulmonary circuit seems to be promoted, and the functional labor which the lungs have to perform is diminished by the abstraction of blood. At all events, relief of the sense of oppression and dyspnœa attendant on the early stage of acute inflammation of any of the pulmonary structures is more quickly and effectually proeured by bloodletting than by other measures. Were it not for its ulterior effects, it would be invaluable as a palliative measure in pleuritis and other inflammatory affections within the chest.

In another mode the abstraction of blood may sometimes be peculiarly useful in affections of the lungs involving considerable or great obstruction to the passage of blood through the pulmonary circuit. This obstruction oecasions an accumulation of blood within the cavities of the right side of the heart; hence, congestion of the systemic veins, giving rise, if sufficient in degree, to lividity or cyanosis. The accumulation of blood in the cavities of the right side of the heart, under these circumstances, weakens the force with which the ventricle and auricle contract, and paralysis of this portion of the heart from over-distension may follow. The formation of a heart-clot may be a consequence of this accumulation. In certain cases, bloodletting may be advisable with a view to diminish the accumulation of blood in the cavities of the right side of the heart; but it is to be employed for that end only when the contraindications which have been mentioned are not present.

After bloodletting, the pulse sometimes appears to indicate increased power of the heart's action. The artery seems to strike against the finger with more force than before the abstraction of blood. Formerly practitioners were misled by this effect upon the pulse, and bloodletting was employed as a means of increasing the power of the heart's action. The sensation which.the finger receives, in these cases, is delusive, and is caused by the quickness of the movements of the artery. This has been shown by the sphygmograph to depend on the diminished tension of the arteries following the abstraction of blood. It is to be borne.in mind, in estimating the power of the heart's action by the sensible characters of the pulse, that the sense of resistance which is felt and the amount of pressure required to compress the artery are the evidences of strength.

To a certain extent the advantages of bloodletting, inclusive of the promptness of its operation, may be obtained by arresting temporarily or retarding the circulation in a part more or less remote from the seat of the inflammation. A ligature applied to one or more of the extremities will effect this object; so, also, will the removal of atmospheric pressure over a considerable space. There is reason to think that these measures as substitutes for bloodletting have not been sufficiently considered. It is probably owing to their effect in withdrawing temporarily a certain quantity of blood from the circulation, that dry cups and various so-called revulsive applications to the surface of the body, are of utility in the first stage of acute inflammation.

The evils of indiscriminate and excessive bloodletting are manifested 
by a larger rate of mortality in those diseases which tend to destroy life by asthenia, and it can hardly be doubted that the death-rate has been diminished by a much more sparing use of the lancet within late years. But the results of injudicious bloodletting are manifested in cases which end in recovery, as well as in those which end fatally. These results consist in protracted convalescence and subsequent feebleness. 'The cases of different inflammations treated formerly by bloodletting with other measures entering into the so-called antiphlogistic method, and the cases now treated otherwise, present a striking contrast as regards the conclition of patients during convalescence and after recovery.

The opinion held by some that diseases have undergone a notable change within the memory of those now living has been already referred to. ${ }^{1}$ They who hold this opinion suppose that bloodletting and other antiphlogistic measures are less appropriate now than formerly in consequence of such a change. With a professional experience of nearly forty years, I do not hesitate to express a conviction that acute inflammations at the present day are essentially the same as they were as far back as the time just named, and that antiphlogistic measures were neither more nor less appropriate then than now.

In addition to general bloodletting, or the employment of venesection, much importance was formerly attached to the abstraction of blood by cups or leeches applied in the neighborhood of the inflamed part. Local bloodletting, in some cases, is more convenient than general; but, so far as the abstraction of blood is concerned, it is diflicult to conceive that it is a matter of much importance from what part of the body or vaseular system it is taken. Whether it be abstracted by means of eups, leeches, or the lancet, the benefit or injury will depend on the quantity withdrawn in a given period. Whatever advantage may accrue from the removal of a certain amount of blood by cups or leeches, over the abstraction of the same amount by venesection, must be derived from the operation of the former as revulsive measures.

W'ith these remarks, leaving the consideration of bloodletting, and reverting to the question concerning the curative and palliative measures to be employed in the first stage of acute pleuritis, we are led to the consideration of opium. And, with respect to this remedy, certain considerations may here be presented, which will apply to the treatment of other inflammations.

A great change has taken place, within the last few years, with respect to the use of opium in acute inflammations. It was formerly used with much reserve, under the apprehension that, acting as a stimulant, its influence upon the loeal disease must be unfavorable. It was regarded as antagonistical to the antiphlogistic plan of treatment. Clinical experience and sounder pathological views, however, have led to the knowlerge of its great value in the treatment of inflammatory affeetions wherever situated. It is valuable, not alone as a palliative, but as a curative remedy. Its palliative efficacy is, of course, intelligible; and we can also, to some extent, understand its curative influence. By relieving pain, it diminishes the determination of blood to the inflamed part, for pain is alone sufficient to occasion an increased aflux of blood to a part. 'This fact is illustrated by cases of neuralgia affecting a superfieial nerve. Neuralgia of the supra-orbital nerve, for example, occasions a determination of blood to the conjunctiva, so that this membrane appears to be inflamed, and the congestion disappears directly the pain is relieved by a full opiate. Opium 
exerts also a curative influence by preventing or diminishing the constitutional disturbance which inflammations are apt to occision; it places the system in a condition to tolerate better the local affection. The severity of an inflammatory disease depends much on the amount of constitutional disturbance which it oceasions, and, with respect to the latter, different cases differ widely, although in each the degree and extent of the local affection be the same. Life is often destroyed, not in consequence of the injury done to the inflamed part, but because the vital powers are inadequate to bear the continuance of the disease. 'These considerations afford some insight into the utility of opium, especially when patients are of the class who are liable to take this remedy without inconvenience, there being, as is well known, a great difference among difterent persons in this regard. The evidence of the curative value of opium, of course, is derived from experience, and there is ample ground for the belief that, by its judicious use in acute inflammations, not only is their course divested of much of the suffering which would otherwise be experienced, but their intensity is lessened, and the danger of death diminished in those inflammations which tend to destroy life by asthenia. 'The immediate effect of this remedy is often very strikingly manifest in improvement as regards the local and general symptoms.

Considering now the use of opium in the first stage of acute pleuritis, after the abstraction of blood, if this be deemed advisable, or, after a saline purgative, if this be employed in lieu of bloodletting, it will generally be judicious to prescribe opium,' in some form, in doses sufficient to relieve pain, and place the system fairly under an anodyne influence. If the pain be severe, and the constitutional disturbance considerable, the use of opium need not be delayed for the operation of a purgative, but may be at once entered upon. 'This is, perhaps, the most judicious course in the majority of cases, a purgative being deferred until a distinct anodyne inpression has been produced and maintained for some hours by opium. Conjoined with the use of opium, sedative remedies, addressed to the circulation, may be employed. These should not be pushed to the extent of produeing disorder. If, for example, tartar-emetic be given, it should not be carried beyond the point of slight nausea; and the veratrum viride, if given, should occasion no appreciable effects beyond a reduction of the frequency of the heart's action. These remedies, it is to be borne in mind, are addressed, not to the disease per se, but to the symptomatic phenomena of the disease, viz., those belonging to the febrile morement. As a rule, digitalis and aconite are to be preferred to the sedatives just named, because their sedative effect upon the circulation is produced without depression.

Aconite is a most valuable sedative remedy in the early stage of this, as of other acute inflammations. The most effective mode of administration in order to secure promptly and fully its remedial effect, is that recommended by Ringer, namely, half a drop, or a drop, of the tincture to be given every ten minutes, or quarter of an hour, for two hours, and afterwards the dose repeated hourly. Smaller doses are to be given if the pulse become feeble, or there be much prostration.

A summary of the treatment of the first stage of acnte pleuritis, then, will embrace, bloodletting in some cases, the use of opium given sufficiently to relieve pain and tranquillize the system, a saline purgative, and remedies designed to act as sedatives upon the circulation. Blisters should

I The word opium is here used as a general term, including the alkaloids, morphia, and codeia. 
not be applied in this stage. They occasion general disturbance more than enough to counterbalince any effect by way of revulsion or substitution. They add an inflammation of the skin to the existing inflammation of the pleura. A sinapism, turpentine stupes, or fomentations to the chest, will secure all the benefit of vesication without the annoyance and other evils of the latter. Moreover, a blister is an obstacle in the way of those examinations of the chest which are of importance as affording the only reliable information respecting the progress of the disease. Niemeyer advocates, as a palliative measure safe and eflicient, the application of cold water over the affected side of the chest, the application being made by means of napkins dipped in cold water, which are to be renewed every five minutes. He states that the pain is promptly relieved by this measure. The application of cold water over the whole surface of the borly, either by means of the bath, or by wrapping the body in a wet sheet, employing, in short, cold water with a view to climinish the heat of the body, has been recommended by other elinical observers as perfectly safe, and, under judicious management, useful in this and other acute inllammatory aflections. For a fuller account of this measure, the reader is referred to the treatment of typhoid fever. Employed as an antipyretic measure, it is indicated only by a considerable elevation of temperature, as shown by the thermometer in the axilla. The immediate favorable effect is shown by decline of temperature, diminished frequeney of the pulse, and improvement in other respects. If the immediate effect be thus favorable, the measure may be repeated, at intervals, whenever the fever again becomes high, as shown by the axillary temperature and the pulse. Dry cups applied to the chest often afford prompt and marked relief of the pain and dyspnoea in the first stage of acute pleuritis, as well as in other pulmonary affections accompanied by these symptoms singly or combined. Preventing the respiratory movements of the chest on the affected side by meins of long strips of adhesive plaster encircling this side and extencling partially over the side not affected, affords notable relief of the lancinating pain felt in the acts of inspiration.

In the second stage, so long as the quantity of liquid effusion is small and a further aceumulation may be expected to take place, the indieations belonging to the first stage may continue. If the pain be still acute and the febrile movement have not abated, measures to relieve the former and lessen the latter are inclicated. Bloodletting is rarely called for, but depletion by purgatives may be useful, not only by lessening the felirile movement, but by restraining the amount of effusion. Diaphoretic remedies are useful by contributing to the two ends just stated. Opium is indicated in proportion to the pain and constitutional disturbance, and sedative remedies addressed to the circulation may be employed.

These measures cease to be appropriate when considerable effusion of liquid has taken place, when pain is no longer a prominent symptom, and the febrile movement has either ceased or beeome much diminisherl. The object of treatment now is to promote absorption of the effused liquid. The means which may be employed for this object are, hydragogue purgatives, diuretics, blisters, and the use of certain remedies which have been supposed to act directly as sorbefacients.

Hydragogue purgatives act most promptly and efficiently. The more active hydragogues, which are drastic in their operation, should be given circumspectly, and generally need not be employed. Reference is had to such remedies as elaterium and gamboge. The saline purgatives, such as the bitartrate of potassa, the citrate or sulphate of magnesia, are to be 
preferred as sufficiently effective and less severe. These will sometimes effect a rapid decrease of the liquid.

Diuretics are sometimes equally but less rapidly effective. They effect the object with less disturbance of the system and are less debilitating. The union of several diuretic remedies is more likely to prove effective than a single remedy of this class. The squill and digitalis may be combined, and, in addition, the diuretic salts of potassa, or the iodide of potassium, may be given dissolved in an infusion of some one of the various vegetable diuretics. The mustard whey is a favorite remedy of my colleague, Prof. McCready, and I have found it to act upon the kidneys sufficiently.

The regulation of the amount of drink ingested is an important point with reference to the promotion of absorption. The elimination of water by the bowels or kidneys is of little avail if the patient be allowed to take fluids into the system abundantly. The quantity of liquid ingested should be as small as is compatible with comfort. The treatment is often rendered inefficient by inattention to this point.

Blisters, in this stage of the disease, doubtless contribute to the object under consideration. If the other means are effective, however, they may be dispensed with. 'Their interference with examinations of the chest is a matter of considerable consequence, and the annoyance which they frequently oceasion is not an inconsiderable objection. If employed, small blisters successively applied in different situations, removed as soon as vesication commences, the vesicated surfaces being allowed to dry up rapidly, accomplish all the good to be effected in this way, with the smallest amount of inconvenience both to the physician and patient.

The remedies supposed to act as sorbefacients are mereury and iodine. Mercury I have long ceased to employ for this end. Of doubtful efficacy, the annoyance and other evils incident to mercurialization render it objectionable. The efficacy of iodine is, perhaps, equally doubtful, but it is not open to similar objections. It may be given internally, or applied externally in the form of either the ointment or tincture.

It is an important injunction not to continue perturbatory measures with a view to promote absorption too long, nor to push their use too far. The decrease of liquid effusion, after it has reached a certain point, is apt to go on slowly, for reasons already stated. Whenever this point is reached, cathartics and diuretics should be discontinued, or employed with great moderation, else they will be likely to do harm, rather than good, by weakening the patient. And if, at any time, these measures appear to impair the general conclition, the propriety of continuing them is doubtful. A leading object in the second stage of the disease is to maintain the constitutional strength. For this object the diet should be nutritious; tonic remedies are useful, and a little wine or spirit may be allowed. These measures should constitute the treatment, after those designed to promote absorption have been sufficiently carried out, and should take the place of the latter whenever there is evidence of the strength giving way.

It is very rarely the case that this disease tends to a fatal ending by asthenia. Should such a tendency be manifested by the symptoms, viz., frequency and feebleness of the pulse, muscular prostration, etc., the measures which constitute the supporting treatment are of course indicated. These measures, which form the most important part of the treatment of the diseases involving danger by asthenia, consist of alcoholic stimulants, nutritious alimentation, and tonic remedies. They will be noticed more fully in connection with diseases in the treatment of which they are oftener called for. 
A fatal result in acute pleuritis may be due to rapid and excessive effusion of liquid, as already stated. If the liquid accumulate sufficiently to endanger life, it should be at once withdrawn from the chest by the operation of thoracentesis. This operation is much more frequently indicated in chronic pleuritis, and will be considered in connection with the latter variety of the disease.

The measures which have reference to the promotion of absorption may be indicated in the third stage; that is, for a certain period during the progress of absorption. When these measures have been carried to a sufficient extent, and the amount of liquid is considerably reduced, the patient is to be considered as convalescent. During convalescence the object is to re-establish the normal health of the patient. This is to be done by tonic remedies, a nutritious diet, and other hygienic means. Solid food, embracing a fair proportion of meat, may be allowed whenever taken with relish and digested. Alimentation is important; and if the appetite be small and the digestion weak, remedies to improve both are indicated. No apprehension need be felt that a diet as nutritious as can be taken without inconvenience will do any harm; on the contrary, the more nutritious the food which can be taken and digested, the more rapid the convalescence. Wine or spirit, or beer, in small quantities, taken with meals, will expedite recovery. Going out of doors and gentle excrcise are to be encouraged. The patient will gain more rapidly as regards appetite, digestion, and strength, if he do not confine himself within doors ; and with proper precautions, no risk of harm is incurred. The removal of the liquid which remains, the absorption of lymph, and the development of new tissue leating to adhesions, will go on rapidly in proportion to the rapidity with which improvement in the general condition takes place.

In the foregoing account of the treatment of simple acute pleuritis, it is assumed that, exclusive of a small number of cases in which this disease destroys life either by asthenia or apnœa, its course is toward convalescence, without becoming chronic. 'This is the rule, to which there are exceptions. If the disease become chronic, the measures of treatment indicated are those which will be considered under the head of chronic pleuritis.

The prevention of acute pleuritis hardly claims consideration. The abrupt and rapid development of the disease precludes the employment of prophylactic measures; there are no premonitions which warrant our anticipation of its occurrence.

Marked dulness on percussion over the affected side, especially at the inferior portion, may continue for some time after the liquid effusion has disappeared, the symptoms, general and local, denoting recovery, and the vesicular murmur of respiration being heard over the whole of the side. This shows that lymph remains to be absorbed, and it need occasion no apprehensions. In a patient discharged from hospital on the day these remarks are penned, the liquid effusion has nearly or quite disappeared, as shown by vesicular breathing and vocal resonance extending to the base of the chest; yet considerable dulness remains, and will probably continue for several weeks. 


\section{CH A P T E R III.}

\section{VARIETIES OF PLEURITIS.}

Chronic Pleuritis-Anatomical Characters-Clinical History-Pathological Character-Causation-Prognosis-Treatment. Thoracentesis. Suppurative Pleuritis, or Empyema-Diagnosis -Prognosis-Treatment. Pleuritis with Pncumothorax-Anatomical Cliaracters-Clinleal History-Diagnosis-Prognosis-Treatment. Pueumothorax. Circumscribed Pleuritis. Hydrothorax.

$\mathrm{F}^{\mathrm{F}}$ the varieties of pleuritis, the first to be considered is the simple or ordinary ehronie form of the disease. Other varieties are suppurative pleuritis, or empyema, and pleuritis with pneumothorax. Cireumscribed pleuritis will elaim some consideration. This ehapter will be devoted to these affections, including a brief aceount of pneumothorax without pieuritis, and of hydrothorax.

\section{Chronic Plearitis.}

Anatomical Characters. - The anatomieal characters in simple chronic pleuritis are essentially the same as in the acute form. The pleural cavity contains serum and lymph in variable relative proportions, but, as a rule, the quantity of lymph is less, and the accumulation of liquid greater, than in aente pleuritis. Liquid effusion frequently takes place to such an extent that the affected side is more or less dilated. The lung is compressed into a small solid mass usnally situated at the upper and posterior part; the thoracic walls are expanded in every direction; the intereostal spaces are pushed out to a level with the ribs or even beyond this level, and show no depression with the act of inspiration; the diaphragm may be depressed, pushing downward the abdominal organs situated in proximity to it, viz., the stomach, spleen, and liver; the lateral pressure on the mediastinum may be sufficient to move the heart from its normal situation, and, if the effusion be in the left side, the heart may be carried quite into the space belonging to the right side of the chest.

The dilatation decreases as absorption of the liquid takes place, and contraction of the affeeted side succeeds. This arises from the fact that the compressed lung does not readily resume its former volume when the pressure of the liquid is removed, in consequence of the layers of lymph with which it is more or less invested, and, sometimes, beeause pleuritic adhesions have already taken place. Other things being equal, the extent of contraction is in proportion to the amount of the previous dilatation and its duration. Usually the dimensions of the affeeted side in every direction are lessened, the shoulder is lowered, and lateral spinal curvature is apt to ensue. In some cases in which the lung expands to a certain point and becomes fixed by permanent adhesions at that point, the removal of the liquid below leads to a deep depression; the chest presents an appearance as if the lower ribs had been crushed inward. The contraction succeeding a large effusion is generally permanent. It becomes less marked after several months or years, in some cases, and, if the patient be quite young, it may, in the progress of time, nearly disappear. In a 
patient who had chronic pleuritis with very large effusion, at the age of 17 years, followed by great contraction, I found, ten years afterwards, the two sides presenting only a slight disparity.

Chinione History.-Chronic pleuritis, in the great majority of cases, is a subacute affection from the first. Occasionally it follows the acute form of the disease. The chronic is a more frequent affection than the acute, a fact which shows that the latter does not usually precede the former, as has been stated by some authors. Of thirty-five recorded cases which I analyzed with reference to this point, in 1852, the inflammation was subacute from the first in twenty-nine. The left side is affected oftener than the right, as in acute pleuritis. Of forty-two cases, 1 the left side was affected in twenty-three. Of seventy-eight cases analyzed by Dr. Blakison, the left side was affected in fifty-eight.

This variety of pleuritis is often developed imperceptibly, and, so far as the symptoms are coneerned, is one of the most insidious and latent of diseases. It is habitually overlooked by those who do not employ the physical methods of examination. Its development is not, as in the vast majority of cases of acute pleuritis, characterized by pain. Pain is often either wanting or so slight as scarcely to attract attention. In some cases, however, the pain is moderate or considerable, but falling much short of that intensity which belongs to the acute affection. Tenderness over the chest is slight or moderate, and may be wanting.

Cough and expectoration are frequently wanting, and rarely prominent as symptoms. 'The cough, if present, is dry, short, hacking, and the matter of expectoration consists of mucus, transparent or slightly opaque. If there be considerable expectoration, and the matter expectorated be muco-purulent, either bronehitis coexists, or the pleuritis is associated with tuberculous disease.

The frequency of the respirations is increased, assuming the pleuritis to be accompanied by a large effusion of liquid. The increase, however, nay be but moderate while the patient is at rest, but this symptom becomes marked on excreise. Deficiency of breath is also shown by inability to speak with a sustained expiration, the speech being interrupted at short intervals for a fresh inspiration. Dyspnœa is rarely present except on exercise. Here, as in acute pleuritis, the embarrassment of breathing will depend much on the rapidity with which the effusion takes place. The suppression of the respiratory function of an entire lung, in this affection, does not usually occasion lividity of the face or prolabia.

The pulse is generally more or less accelerated, ranging from 80 to 120 per minute, and it is small and compressible. There is, however, considerable variation in different cases as regards these characters of the pulse. The surface of the body frequently presents more or less capillary congestion. Sweating is apt to occur, especially during the night, and may be profuse without having been preceded by a chill or febrile movement. Irregular, slight chills, however, are not unusual, leadling sometimes to the error of supposing the disease to be malarious.

The appetite is sometimes notably impaired, but in other eases it is preserved, and the digestion continues good. Diarrhœa occurs in some cases. Generally, the countenance is pallid, denoting anæmia, but this is not true of all cases; a healthy aspect is sometimes maintained. Emaciation is not usually marked, except when the disease is associated with tuberculosis. The strength is often retained in a remarkable degree. Patients are rarely obliged to take to the bed, and usually are able to be up and out of doors, even with one side of the chest filled with liquid. I 
have known repeatedly persons in this condition to continue pursuits which required active muscular exertion.

This sketch of the symptomatology applies, of course, to cases of chronic pleuritis not associated with other affections furnishing symptoms which are combined with those belonging to the disease under consideration. It may be associated with pulmonary tuberculosis, but not so frequently as is stated by some writers. Indeed, in my experience, tuberculous disease has existed in a small proportion of cases only. The coexistence of this disease will be likely to render cough more prominent as a symptom, and to give rise to a more or less abundant expectoration. Emaciation and muscular feebleness will also be more marked. Pericarditis exists in a small proportion of cases. With this complication, the circulation is more disturbed, there is more suffering from dyspnœea, and the symptoms in general denote greater gravity of disease. 'The affection may be developed in the course of either acute albuminuria or chronic degenerative disease of the kidneys; and, under these circumstances, general dropsy may be present, emaciation and feebleness are greater, and the tendency to asthenia is more apparent. In like manner, other affections which may be associated modify and add to the symptoms pertaining to the pleuritic inflammation.

The duration is variable, but the career rarely ends under several weeks, and generally it extends over several months. As regards stages, when acute pleuritis has not preceded, cases do not come under observation until more or less liquid effusion has taken place, and, it may be, not until the pleural sac is filled with liquid. It suffices to consider the career of the disease as consisting of two stages, one embracing the period during which the liquid either is accumulating or remains stationary, and the other embracing the period during which absorption of the liquid is going on. After absorption has made a certain amount of progress, the patient may be regarded as convalescent.

Pathological Character.-Ordinary or simple chronic pleuritis is a subacute inflammation, differing from the acute form of the disease only in the degree of inflammatory action. The inflammation has no special character, but is of a low grade of intensity, with a tendency to continuance for an indefinite period.

Causation.- The same causes are involved in the causation of chronic as of acute pleuritis. It may be produced traumatically. It may sometimes be due to cold. It occurs occasionally during the course of affections of the kidneys. But, in a large majority of cases, it proceeds from internal causes which are not understood, and it is then said to be spontaneous.

An important point of inquiry relates to its pathological relations to pulmonary tuberculosis. According to some authors, it is generally consecutive to, and dependent upon, a tuberculous deposit. There has been no evidence of this in a large majority of the cases which have come under my observation. Of forty-two cases analyzed several years ago, the evidence of coexisting tuberculosis was present in only three; and of a large number of cases which I have since observed, I am sure that the two affections have been assosiated in but a small proportion. As, however, there are grounds for believing that the occurrence of pleuritic effusion tends to retard, and contributes to the arrest of, the progress of tuberculosis, the association of the two affections may sometimes exist without being apparent. 
Chronic, as well as acute, plenritis occurs very rarely in infancy. It is not of infrequent occurrenee in childhood and early life, but it is most frequent between puberty and middle life. It is of rare occurrence in the aged.

Prognosis.-Simple chronic pleuritis, not associated with tuberculosis, nor complicated with any serious affection, and occurring in a healthy subject, tends to recovery. A fatal result may take place in consequence of a rapid and large accumulation of liquid, but instances of this are exceedingly rare. If death occur, it is generally from associated affections or complications.

Recovery takes place, leaving the affected side more or less contracterl; and sometimes the contraction is so marked as, in connection with the lateral curvature of the spine which it occasions, to produce an obvious and permanent deformity. In general, however, the contraction which follows is attended with little or no inconvenience, and is not observed unless the chest be denuled, and the two sides compared.

A permanent dislocation of the heart to the right side of the sternum is sometimes an effect of the removal of a large collection of liquid from the right pleural sac; and, in some cases of pleuritis, with large effusion in the left side, the heart, which was dislocated by the lateral pressure of the liquid, is found on the right side of the sternum after recovery from the pleuritis, adhesions having taken place which prevent it from returning to the left side after the liquid is removed.

Pulmonary tuberculosis becomes developed, sooner or later after recovery, in a certain proportion of cases. In some of the cases in which tuberculous disease appears to be a sequel, it may have existed prior to the pleuritis, and have undergone arrest. The proportion of cases in which tuberculous disease follows is not sufficiently large to show a predisposition to this disease derived from the pleuritis. The opinion, frequently and perhaps commonly entertained, that chronic pleuritis, when it does not supervene on a tuberculous deposit, predisposes strongly to the occurrence of the latter, I believe to be incorrect. This belief is based on my own experience. It is sustained by the statistics communicated by Dr. Blakiston. ${ }^{1}$ of fifty-three cases observed by this author, the patients remaining under observation for several years after recovery, not one became affected with pulmonary tuberculosis. This result is striking, for it might be expected that, out of so large a number of cases of any disease occurring, as does pleuritis, for the most part at an early age, a certain proportion would be likely, in the course of several years, to become tuberculous. In estimating the influence of this or any disease in determining the subsequent development of tuberculosis, the liability to the latter, irrespective of antecedent affections, is of course to be taken into account.

The idea has been suggested that, inasmuch as chronic pleuritis occurs oftener on the left than on the right side, its occurrence on the right sicle is evidence of its being connected with tubercle. This, however, appears to be disproved by the statistics reported by Dr. Bowditch. ${ }^{2}$ of fourteen patients affected with pleuritis seated in the right side, paracentesis being employed in all, 28.57 per cent. died, 64.28 per cent. were cured, and 7.14 per cent. remained doubtful. On the other hand, of eleven cases

1 Practical Observations on Certain Diseases of the Chest, etc. American edition. Blanchard \& Lea, 1848.

2 Fide American Journal of the Medical Sclences. Number for January, 1863. 
in which the left side was affected, paracentesis being employed in all, 45.45 per cent. died, 33.36 got well, and 18.18 were donbtful. These results disprove the idea just stated, because it is fair to conclude that the proportion of deaths would be greater, and of recoveries less, of those in whom the right side was affected, if a large number of this class of cases were tuberculous; whereas, these statistics show precisely the reverse.

The mortality from chronic pleuritis, even without regard to associated affections and complications, is not great. The rate of mortality in $\mathrm{my}$ own experience up to 1856 , was 17 per cent.

Recovery is understood to imply the removal, by absorption, of the liquid effusion, and adhesion of the plenral surfaces. The affection, however, may terminate in another way, leaving no local symptoms, and the patient regaining good health. This consists in the affected side of the chest remaining permanently filled with liquid. The following case, illustrative of this termination, came under my observation in the Charity Hospital of New Orleans in 1861 :-

A man, aged about 45, was admitted into a ward under the charge of my colleague, Dr. Fenner, stating that he had been ill and confined to the bed for four days, having previously been in good health. In answer to inquiries suggested by the marks of cupping on the left side, he stated that he had had pleurisy several years before. I saw the patient casually, and found the left side dilated, everywhere flat on percussion, and the respiratory murmur suppressed. Death occurred on the third day after his admission, and my notes do not contain data showing the probable cause of death. The man presented a robust appearance, and declared that he was quite well when seized with his present illness four days before his admission. His habits were intemperate.

On examination of the chest after death, the left pleural sac was found to be so resisting that it was removed entire, and brought to the college. The sac was then opened with some difficulty, and a large amount of turbid liquid, estimated to be two gallons, removed. After the liquid had escaped, the pleural sac was sufficiently rigid to retain the shape which it had before being opened. A chondroid formation, from one-eighth to one-quarter of an inch in thickness, lined the entire pleura. The parietal portion of the membrane could in some places be stripped from this chondroid formation. Calcareous matter was abundantly deposited in the latter. The lung was compressed into a solid, small mass, which resisted efforts at insufflation.

Another case, illustrative of a similar termination, so far as regards the permanency of liquid effusion, came under my observation in private practice. A lawyer, aged about 30 , had an attack of pleuritis, which confined him for some time to the bed, nine years before my first examination in 1861. Five years afterward he was in excellent health, being entirely free from any pulmonary symptoms except deficiency of breath on exercise. Flatness on percussion existed over the whole of the left side, with suppression of the respiratory murmur and vocal resonance, except at the summit where bronchial respiration and bronchophony existed. These signs were considered as denoting liquid in sufficient quantity to fill the pleural sac and compress the lung into a solid mass. The patient stated that he had been repeatedly examined since the attack of pleuritis nine years before, and there had been no change in the condition of the chest since his apparent recovery from that disease.

Diagnosis.-Owing to the latency of chronic pleuritis, as regards the subjective symptoms, in many cases, the existence of intra-thoracic disease is often overlooked by those who do not employ physical exploration. Of 
the cases which have come under my observation, in not a few the patients have been supposed to have dyspepsia, irregular intermittent fever, hepatic trouble, or some ailment not connected with the pulmonary organ. If the symptoms point to the existence of some affection within the chest, patients are often supposed to be tuberculous, and the congh, deficiency of breath, night perspirations, etc., rationally favor such a conclusion. A positive diagnosis can hardly be made without the aid of physical signs, but, with their aid, the existence of pleuritis is readily determined.

'The signs on which, taken in connection with the history and symptoms, the diagnosis is to be based, are those denoting pleuritic effusion. 'The signs of liquid effusion are the same in chronic as in acute pleuritis, and need not be again stated; but in chronic, more frequently than in acute, pleuritis, the accumulation of liquid is so large as to occasion more or less dilatation of the affected side, and then are superadded signs obtained by inspection and palpation, notice of which was deferred in treating of the acute affection.

Dilatation of the affected side by liquid is apparent to the eye, and may be ascertained by mensuration, either with graduated tape or eallipers. The intercostal spaces are pushed out to a level with the ribs, and nay be bulging. The side is motionless, or nearly so, in the acts of respiration, the morements of the opposite side being exaggerated. By applying the fingers within the intercostal spaces and employing peripheral and dianetrical percussion, a sense of fluctuation is sometimes obtained. The heart is moved from its normal situation, as ascertained either by palpation or auscultation. If the effusion be in the left side, the apex-bent or the maximum of the heart-sounds is on the right side of the sternum; and they are carried toward the left lateral portion of the chest if the effusion be in the right side. 'The diaphragm is depressed, pushing downward the abdominal organs in contact with it. The addition of these signs renders the diagnosis of pleuritic effusion still more complete and positive than the signs which denote the presence of liquid without dilatation.

During the stage of absorption, the signs show the progressive diminution of liquid until the quantity is no longer sufficient to occasion clilatation, and, from this point, the continued decrease in quantity is ascertained as in acute pleuritis. The contraction of the affected side which follows chronic pleuritis is more marked than that succeeding the acute affection.

Treatuent.-The main objects of treatment in simple chronic pleuritis are twofold, viz : First, to aid in the removal of the effused liquid ; and, second, to develop and sustain the powers of the system. For the first object, the measures which may be employed are the same as in the second and the third stage of acute pleuritis, consisting of the milder hydragogues, diuretics, small blisters applied in succession over different portions of the affected side, and the use of iodine intermally and externally. In view of the anæmic state which generally exists, and the effect of mereurialization on the blood, this measure is contra-indicated. Bloodletting, general or local, is never indicated in chronic pleuritis.

In pursuing measures with reference to the first object, the second is not to be overlooked nor neglected. Cases are often injudiciously treated in consequence of the attention being too much engrossed with the measures to promote absorption; these measures will do harm if continued too long or pushed too far. After a ecrtain amount of effect has been produced, if they cease to be further effective, they should be discontinued, at least for a time; nor should they be persisted in if they fail to have any effect. 
Harm is produced by their injudicious employment, in proportion as they debilitate.

The second object calls for tonic remedies, nutritious alimentation, the moderate use of alcoholic stimulants, and gentle exercise out of doors. These measures are always important, and they are pre-eminently so if, from associated affections, complications, or the general condition of the patient, the powers of the system are notably lowered. I have repeatedly seen a marked change for the better occur at once, followed by progressive improvement in all respects, on the discontinuance of measures having reference to the absorption of liquid, and the substitution of measures addressed to the system. Hygienic measures, viz., nutritious diet and exercise in the open air, under proper restrietions, are especially important.

A mode of effecting the first object, viz., the removing of the liquid, requires distinct consideration. Reference is now had to the operation of paracentesis or thoracentesis. The propriety of puncturing the chest when the accumulation of liquid is sufficient to place the patient in imminent danger, is unquestionable. But it is a question which of late has been much discussed whether it be not advisable to puncture in cases in which the chest is dilated by liquid, although the patient be not in great distress or danger. It is clearly an object to get rid of the liquid, and the point to be decided is whether it be better to resort to the direct mode, that is, puncture and withdraw it, or to effect the object indirectly by purgatives, diuretics, etc. This point is to be decided, of course, after a consideration of the advantages and disadvantages of the operation of thoracentesis.

Heretofore, this operation was performed only as a dernier resort, under circumstances when little was to be expected from any measure. It was deferred as long as possible, sometimes on account of doubt as to the diagnosis, and because the perforation and introduction of air were supposed to involve danger of an increase of the inflammation. A considerable opening was necessary in order to give free exit to the liquid, and it was not easy to prevent the air from entering the pleural cavity. Objection to the operation on the score of diagnosis is now removed by our present knowledge of physical signs. Moreover, the operation has been divested of all severity, and the liability to the introduction of air has been provided against, by the application of the suction-pump, first suggested by Dr. Morill $W y m a n$, in 1850, and since employed in a large number of cases by Dr. Bowditch. The introduction of air is not attended by the injurious effects formerly apprehended, but it is objectionable, because the presence of air is an obstacle to the full expansion of the lung after the liquid is removed. Its introduction is prevented by the use of the pump in withdrawing the liquid. The operation is rendered trivial because, with the suction force of the pump, a small exploring trocar suffices to make the puncture, which causes very little pain, and closes directly the canula is removed.

Dr. Bowditch operated in this way 250 times on 154 persons in the course of twenty years. In no instance was any permanent injury referable to the operation. It was in some cases repeated several times during the progress of the disease. In a considerable proportion of cases recovery appeared to be attributable to the operation. Immediate and great relief was obtained in cases which ultimately proved fatal. Dr. B. has been led by his experience to operate in all cases'in which the quantity of liquid induces either permanent or occasional dyspnœa of a severe character, and in all cases in which the pleural cavity is filled, if, after a reasonable amount of treatment otherwise, the liquid does not diminish.

My own experience in the practice of the operation has been conside- 
rable, and I can testify in behalf of its innocuousness, the relief which it affords, and its value as a means of rescuing patients from a condition of imminent danger tolife. And the conclusions of Bowditch, as just stated, are not only sustained by his large experience, but are consonant with common sense. If by an operation trivial with respect to pain or any evil effects, and easily performed, the liquid effusion may be withdrawn at pleasure, this mode is certainly to be preferred to mensures which are indirect, not very reliable, requiring considerable time, and producing more or less disturbance of the system and debility. An important advantage of the operation is, the liquid being removed before the lung has been subjected to long pressure, and has become adherent by dense layers of lymph, or, perhaps, organized tissuc, the expansion takes place more fully, and is followed by less deformity of the chest from contraction of the affected sile. It is a noteworthy fact that, direetly after the operation, the temperature deelines, and may fall to the normal standard.

Thoracentesis by means of a small trocar attached to the suction-pump, the puncture made at a convenient point either behind, laterally, or in front, is admissible whenever the pleural eavity remains filled with liquid after a brief trial of the measures designed to promote absorption; and the operation should not be delayed whenever the aceumulation of liquid is sufficient to involve danger or distressing dyspnoa.

For several years past, in place of the suetion-pump employed by $\mathrm{Wy}$ man and Bowditch, I have used a simpler apparatus, namely, a small trocar and canula fitted to screw upon the flexible suction-tube of Davidson's syringe. The eanula should be provicled with a stopcock. The trocar and canula being introduced into the chest, the troear is withdrawn and the canula attached to the syringe; the liquid is then removed by means of the expansion of the India-rubber suction-bag after its compression with the hand. I have used this apparatus in many cases with satisfactory results. It has also been used with suceess by many others. In general, after a certain quantity of liquid has been withdrawn, the patient is seized with cough, and experiences a distressing sense of constriction in the chest. These symptoms probably arise from the atmospheric pressure upon the thoracic wall and from the disruption of the pleural surfaces, united by lymph, by the expansion of the compressed lung. They may also be, in part, due to the suction force exerted laterally upon the lung of the healthy side. They may be delayed, and sometimes avoided, by withdrawing the liquid very slowly, thus allowing time for the expansion of the compressed lung, and the gradual accommodation of the parts to fill the vacuum occasioned by the removal of the liquid. When these symptoms oceur, the further removal of liquid had better be postponed for another operation. As regards the quantity removed at one operation, much will be gained by following the injunetion to withdraw the liquid slowly, especially when the patient begins to cough and complain of constriction. The advantage of this apparatus over the suction-pump, is its greater simplicity, the ease with which it is managed, and a less liability to get out of order. A French instrument called the aspirator has these modifications: The cylinder is fixed upon a stand in an upright position, and is of glass; at the lower part there are two tubes, provided with stopcocks, one connected with a flexible tube attached to the canula, and the other connected with a tube through which the contents of the eylinder are discharged. By means of a piston, with a rack and pinion movement, the air is withdrawn from the cylinder, after which the stopeock attached to the tube connected with the canula inserted into the chest is turned, and the liquid flows from the pleural cavity into the cylinder. After the 
cylinder is filled, its contents are expelled through the other tube, by turning the stopcock and allowing the piston to fall. 'This apparatus provides effectually against the introduction of air into the chest; but this is sufticiently provided for in the other instruments which have been described. The suction force operates steadily, and a very small trocar for penetrating the chest suffices. The cylinder being of glass, its contents are at once seen. The objections to the instrument are, first, that, being somewhat complicated, it is apt to get out of order ; and, second, it is expensive as compared with Wyman's instrument, and especially with that devised by me. A little appendage has lately been added to the trocar to be attached to Davidson's syringe, so that it may be in an instant connected with the latter. It is easy to secure more expansive force than is obtained by the ordinary syringe used for cnemata, by having an instrument made especially for thoracentesis, with a larger and thicker Indiarubber suction-bag.

The conclusions drawn by Bowditch from the data afforded by his large experience, with respect to the character of the fluid withdrawn, and its influence on the prognosis, are of practical importance. A sanguinolent fluid, at the first puncture, denotes the existence of malignant disease of the lungs or pleura, and, consequently, the case will end fatally. A case presenting a mixture of bloody, purulent fluid at the first operation is usually fatal. A fetid, gangrenous fluid is very rare; it denotes gangrene, and the case will end fatally. In all such cases the operation may afford great relief and prolong life.

\section{Empyema, Pyothorax, or Suppurative Pleuritis.}

These names denote a variety of pleuritis characterized by the accumulation, in the pleural cavity, of a purulent liquid. The accumulation of pus is a feature sufficiently distinctive and important to serve as a ground for constituting this a variety of pleuritis. The term empyema only expresses the existence of pus, without indicating its situation. Pyothorax is a better name, but this does not express the existence of inflammation. Suppurative pleuritis is a simple title suggested as more fully expressive of the affection.

Suppurative pleuritis may be either acute or subacute at the beginning, but it becomes chronic. It is therefore a variety of chronic pleuritis. The symptoms attending its development are the same as in simple or ordinary pleuritis, either acnte or chronic. There are no distinctive points pertaining to the symptomatology, and it is not necessary, therefore, to consider under a distinct head its clinical history. The anatomical characters, too, are essentially the same as in simple or ordinary pleuritis, excepting that, in the latter, the effused liquid is a mixture of serum and lymph, whereas, in the variety under consideration, the liquid contained in the cavity of the pleura is pus.

The pathological character of suppurative pleuritis is inflammation, acute or subacute, resulting in the accumulation of pus, or suppuration. This result of the inflammation is dependent on an intrinsic tendency existing at the outset. In other words, the accumulation of pus is not an accidental effect, nor does it depend on the duration of the inflammation. The inflammation in cases of suppurative pleuritis tends at once or speedily to suppuration. On the other hand, in cases of simple or ordinary pleuritis, the disease may continue for months and even years withont eventuating in the accumulation of pus. The affection is not at first simple 
and subsequently suppurative pleuritis, but it is a distinct variety of pleuritis from the begimning.

As regards its causation, it may be produced traumatically; and it occurs spontaneously, the internal causative agencies in the latter case being, with our existing knowledge, neither more nor less intelligible than when the inflammation is of the simple or ordinary kind. Why pleuritic inflammation in one case should lead to a collection of quarts or even gallons of pus, and, in another case, continue indefinitely without suppuration, we are unable to explain.

Diagnosis.-The signs, as well as symptoms, in suppurative pleuritis are not distinctive of this variety. They represent the presence of more or less liquicl in the pleural cavity, and they are essentially the same when the liquid is purulent as when it consists of serum and lymph. The point of inquiry, in regard of diagnosis, is, whether there be any means of determining that the liquid is purulent. This is an important point with reference to prognosis and treatment. The occurrence of chills, greater febrile movement, hectic paroxysms, etc., are not reliable in this differential diagnosis. As a rule, however, the axillary temperature is higher than in simple pleuritis. The symptoms and signs, in fact, exclusive of spontaneous perforation of the thoracic walls, will hardly warrant a positive diagnosis. But the purulent character of the liquid may be inferred with considerable confidence, if the quantity progressively continue to increase, notwithstanding the employment of measures to promote absorption. A serous effusion is generally either diminished or retarded by the use of hydragogues, diureties, etc.; but these measures are not likely to exert any influence upon a purulent liquid.

Spontaneous perforation of the walls of the chest occurs, after a time, in cases of suppurative pleuritis. When this occurs, before nleeration of the integument ensues, a soft fluctuating tumor makes its appearance over or near the point of perforation. The appearance of such a tumor, developed rapidly without having been preceded by inflammation exterior to the thoracic walls, is at once significant of this variety of pleuritis. The size of the tumor may be observed to vary with forcible acts of inspiration and expiration. The action of the heart upon the liquid within the chest may communicate a pulsation to the tumor, and this may at first lead to a suspicion of ancurism; but an examination of the chest, by furnishing the evidence of the pleural sac being filled with liquil, will show the character of the affection. If the tumor be not opened, inflammation of the integument, from distension, and ulceration superrene, and the pus makes its escape from the tumor and the pleural cavity. "Paracentesis from necessity," as it is called, has then taken place. "This result is almost exclusively due to suppurative pleuritis, but I have known an instance in which spontaneous perforation took place when the liquid was not purulent.

A positive diagnosis, however, may be made in cases in which spontaneous perforation of the chest does not occur, or without waiting for the occurrence of this event. A small exploring trocar may be introduced into the chest, and a few drops of the liquid withdrawn; the gross and microscopical characters of the specimen thus obtained will at once settle the question. This procedure is warrantable as a means of diagnosis in view of the bearing on the prognosis and treatment. Indecd, the operation is so slight as to be in no wise objectionable.

In a much smaller proportion of cases a spontaneous evacuation occurs in another direction. Perforation of the lung takes place, and the pus 
finds its way into the air-passages. The occurrence of this event is marked by a sudden and copious purulent expectoration ; this expectoration continues for an indefinite period. Perforation of both the walls of the chest and of the lung sometimes occurs.

Prognosis.-Duppurative inflammation of the pleura is a more serious affection that simple pleuritis, whether acute or chronic. Happily it is an unfrequent varicty of pleuritis. Inasmuch as the pus cannot be removed by absorption, but must be evacuated either spontaneously or by surgical interference, the condition is analogous to that of a large abscess which, after opening, continues to discharge through a fistulous orifice. After the purulent contents of the pleural cavity are evacuated, the membrane continues to furnish pus, and thus the discharge continues indefinitely. Emaciation, debility, hectic paroxysms, etc., result from long-continued suppuration, and death takes place by slow asthenia. An unfavorable termination may be due, as in eertain cases of simple pleuritis, to the coexistence of tuberculosis, pericarditis, or some other serious affection.

In the case of a patient ten years of age, which I saw with Dr. Dudley, of Brooklyn, sudden death followed perforation of the lung, the discharge of pus taking place rapidly, and causing suffocation by filling the bronchial tubes.

Recovery from suppurative pleuritis, however, is by no means unfrequent, and may reasonably be hoped for if the patient have a good constitution, and the affection be uncomplicated. The purulent discharge continues for weeks, months, and perhaps years, but progressively diminishes; pleuritic adhesions take place, the affection becoming more and more circumscribed, until, at length, they become universal, and the recovery is completed, leaving the affected side considerably or greatly contracted.

Treatment.-In the treatment of suppurative pleuritis, little or no reliance is to be placed on the employment of measures to promote absorption of the purulent liquid. If the pleural cavity be filled with pus, it will not be absorbed; but, if life be sufficiently prolonged, and thoracentesis be not resorted to, it will, sooner or later, make its way either into the air-passages or through the thoracic walls. Hydragogues, diuretics, blisters, etc., will not only prove unavailing, but they do harm, if persistingly employed, by impairing the powers of the system. Thoracentesis should be performed as soon as the purulent character of the liquid is ascertained, provided "paracentesis by necessity" have not already taken place. 'The suction-pump may be at first used, a larger trocar being advisable than when the liquid is serons, in consequence of the greater consistency of pus. The operation may be repeated as often as required, but, in the majority of cases, owing to the reproduction of pus, this mode of performing thoracentesis will prove unsatisfactory. An opening should then be made at the bottom of the pleural sac, allowing the pus to escape freely. Air will gain admission into the chest, and, since this is unavoidable, its ingress and egress should be unobstructed. A small orifice, not sufficient for the free escape of the pus, and allowing air to be pent up within the pleural cavity, is injudicious. The pus, becoming decomposed and fetid, acts as an irritant on the inflamed surfaces, whereas if it drain away as fast as it is formed, and the cavity of the pleura be well ventilated, this result does not follow.

If spontaneous perforation take place, as denoted by the development of a fluctuating tumor, the latter should be at once opened. If the per- 
foration be at a point so far above the bottom of the sac that a portion only of the pus is evacuated, more or less remaining constantly below the point of perforation, a counter-opening slould be made. It is desirable that the drainage should be so effectual that there will be little or no fetor connected with the discharge. If there be fetor, injections of tepicl water are useful. I have known these to be extremely useful in effecting the removal of fetid pus, when the perforation was not at a favorable point for the free discharge of the purulent contents of the pleural sac.

'To support the powers of the system is an object of still greater importance in the treatment of suppurative, than of simple, pleuritis. The importance is greater because therc is more danger of death by asthenia, and, in favorable cases, the duration of the affection is longer. The measures to be employed for this object are the same in both forms of the disease.

\section{Pleuritis with Pneumothorax, or Pneumo-hydrothorax.}

The term pneumo-hydrothorax denotes the presence of air or gas and liquid in the pleural cavity. As the liquid, however, is either sero-fibrinous or purulent, hydrothorax, which signifies a purely serous liquid, is not strictly appropriate ; the liquid is due to inflammation, and the affection is a variety of pleuritis. It would be more correct to say, pleuritis with pneumothorax. Pneumothorax denotes the presence of air without liquid; the latter affection is rare, but it occurs, and claims separate notice.

Pnenmothorax coexists with suppurative pleuritis, after a spontaneous perforation of the thoracic walls, or when a fistulous opening is made; and also in the rare cases in which the lung is perforated from the pleural surface, and a communication thus opened between the air-passages and the pleural cavity. But the term pneumothorax, with or without plenritis, is considered as denoting the presence of air in the cavity of the pleura, the air generally gaining admission from the air-passages through a perforation which, in most cases, takes place from within the lung. 'This perforation, in the vast majority of eases, is an accident occurring in the progress of pulmonary tuberculosis. It is possible that gas may be generated by the decomposition of pus in some cases of suppurative pleuritis, and that the affection under consideration may be thus produeed without perforation either of the lung or the parietes. Instances, however, of this kind, if they ever occur, must be extremely rare.

It is probable that perforation of the lung would oecur much oftener than it does, were it not for the constancy with which cireumscribed, dry pleuritis, leading to pleuritic adhesions, supervenes upon tubercle. Over the portions of lung in which the tuberculous affection is seated, the pleural surfaces usually become firmly united, requiring often considerable force for their separation on post-mortem examinations. These adhesions offer an obstacle to the evacuation of collections of liquefied tubercle into the pleural cavity, and the evacuation is usually into the bronchial tubes. But, occasionally, a collection of liquefied tuberele being situated near the superficies of the lung, at a point not protected by adhesions, the membrane gives way and the evacuation is into the pleural cavity. Pleuritis, under these circumstances, is at once excited, air finds its way through the perforation, and in this way the pneumothorax becomes developed. . The perforation is rarely at the summit, but is situated at the middle or inferior portion of the lung.

In like manner pleuritis with pneumothorax is produced in certain cases of circumscribed, pulmonary gangrene. The decomposed pulmonary tissues are usually evacuated into the bronchial tubes and expecto- 
rated ; but sometimes, being situated near the surface, the pleura at that point becomes involved in the gangrene, and gives way, leaving a perforation through which air enters the pleural eavity, and pleuritis is caused by the presence of the spliacelated matter. A few instances of this kind have come under my observation.

Anatomical Characters.-Gas escapes with more or less force after the knife penetrates the chest, in making the post-mortem examination. The gas consists of carbonic acid and nitrogen. The pleural sac contains liquid in variable quantity in different cases. The liquid is turbid from admixture with lymph. The pleural surfaces are more or less covered with lymph, which is soft or dense, according to the duration of the affection. The air and liquid are sometimes extremely fetid, and in other cases devoid of fetor. 'The fetor is excessive if the affection be associated with pulmonary gangrene. After the sternum is removed, if the nozzle of a pair of bellows be inserted into the trachen, and air forced into the lung of the affected side, bubbles rising in the liquid show the fact of perforation and its situation. The size of the perforation varies. It is usually small when the affection supervenes on tuberculosis; but it may be quite large in cases of gangrene. The lung on the affected side, if not prevented by old adhesions, is compressed into a solid mass, as in cases of simple pleuritis with large effusion.

Cinnicar History.-The occurrence of perforation is usually marked by acute pain denoting the devclopment of pleuritis. The introduction of air, and the speedy effusion of liquid, give rise generally to notable dyspnœa, with accelerated breathing, accompanied frequently with lividity. The pulse is in most cases rapid and feeble. These symptoms show the supervention of a serious affection of some kind, and point to the chest as its seat.

In some cases the accelerated breathing and dyspnœa continue to be prominent symptoms, the patient suffering greatly, and perhaps being unable to lie down (orthopnoa), until the affection ends fatally, which, under these circumstances, is usually after the lapse of a few days, and it may end fatally in the course of a few hours. But, in other cases, the violence of the symptoms subsides after a short time, and the dyspnoa may be moderate, or wanting, save on exercise. In some instances, even considerable exercise is practicable. As regards strength and the gencral condition, there is much variation in different cases. In the majority of cases, patients are confined to the room and bed, but sometimes they are able to be out of doors, preserving appetite, and not presenting a notably morbid aspeet. Much will; of course, depend on the condition as regards the affections of which this is a complication.

As an exception to the rule, pleuritis, with pneumothorax from perforation, is developed gradually and imperceptibly. It may remain for a greater or less period quite latent. In some instances the accumulation of liquid becomes large, the air disappears, and the affection is apparently converted into simple pleuritis with effusion. In several instances of this kind whieh have fallen under my observation, thoracentesis was resorted to, and the communication between the air-passages and pleural cavity, through the perforation, was resumed after the liquid was withdrawn.

Dragnosis. - When this affection is developed with marked symptoms in the course of pulmonary tuberculosis, its existence should certainly be at once suspected; but the symptoms are not sufficiently distinctive of 
this affection, as contrasted with simple pleuritis and effusion, to warrant a positive differential diagnosis. Moreover, as just stated, the affection is sometimes latent, the symptoms not warranting even the diagnosis of pleuritis. A positive diagnosis, in short, must be based on physical signs, and these are usually easily obtained and very explicit.

The presence of air in the pleural cavity renders the percussion-sound purely tympanitic. If the quantity of liquid be small or moderate, tympanitic resonance may extend over the whole of the affected side, being propagated from the level to the bottom of the liquid. If the liquid be large or considerable in quantity, clulness or flatness will be found at the base and extending upward (the patient sitting) to a certain height, tympanitic resonance existing above. Under these circumstances, the relation of resonance and dulness or flatness will be found to vary with the changed position of the body of the patient. The tympanitic resonance sometimes has an amphoric character.

Respiratory sound is sometimes wanting over the whole of the affected side. Over the compressed lung bronchial respiration may be found. The ordinary cavernous respiration is sometimes heard, but, in most cases, anphoric respiration and amphoric voice or echo are present, either limited or diffused over the whole of the affected side, and metallic tinkling is generally conjoined. The presence of these striking and distinctive signs renders the diagnosis easy and positive, taken in connection with other signs.

Succussion in most cases develops a splashing sound, frequently having the same kind of musical intonation as the respiration, voice and tinkling sounds. This proof of the presence of air and liquid is positive and rarely wanting.

The affected side is frequently dilated, and presents the same characters as in cases of dilatation from liquid in simple and suppurative pleuritis.

Proanosis.-The prognosis, in eases of pleuritis with pneumothorax, is always unfavorable. In this statement traumatic cases are exclucled; the prognosis in these cases is generally favorable. If the affection be connected with pulmonary gangrene, death takes place invariably and within a short period. Other things being equal, when it occurs in the course of pulmonary tuberculosis, a fatal termination is speedy in proportion to the progress which the latter affection has made. 'There is a wide variation in different cases as regards its duration before a fatal ending; in most cases it ends fatally within a few days or weeks, but sometimes life is prolonged for months or even years. It may continue for an indefinite period, the patient experiencing so little inconvenience as to consider himself in good health. The following remarkable case will serve to verify this statement, and also illustrate the latency of the affection occurring in connection with tuberculosis:-

I received from Dr. J. H. H. Burge, of Brooklyn, N. Y., the left lung which he had removed at a post-mortem examination made two or three days before. The patient, aged eighteen, an accountant, was seizel, four days before his death, with an acute affection which proved to be pneumonitis atfecting the lower lobe of the right lung. At the time of the attack he considered himself in good health. He was attended by Dr. Burge, who found the physical signs denoting pneumonitis, and the affeeted lobe was found after death in the second stage of this disease. On an examination of the chest, in addition to the pneumonitis, Dr. Burge discovered the signs of pneumo-hydrothorax affecting the left side. Metallic tinkling, and amphoric respiration and voice, were finely marked. The upper por- 
tion of the ehest on this side yielded a tympanitic, and the lower portion a dull resonance on percussion.

Oil inquiry as to the previous health, the patient stated that he was quite well when attacked with the acute disease; that is, four days before his death. On close questioning, however, it was ascertained that he had had a slight eough for a year, but so slight that he thought nothing of it. He was not conscious of any deficiency of breath, was not subject to pain in the side, and, in short, there were no symptoms pointing to so grave an affection as pleuritis with pneumothorax.

The left lung was completely condensed from compression. The lower lobe was readily inflated; the upper lobe but slightly, owing to the free escape of air through a perforation as large as a crow's quill, situated on the anterior aspect of this lobe, about midway from the upper to the lower extremity. On closing the aperture, this lobe was readily inflated. The perforation was gaping, the orifice being surrounded by a cartilaginous rim. On passing a probe into the aperture, it entered a small cavity of about the side of an American walnut. The cavity was smooth and lined by a membrane. This was the only cavity in the left lung, and there were no tubereulous masses. The whole surface of the lung was covered with lymph dense and elosely adherent. The pleural cavity contained over a quart of turbid liquid, which was not fetid. When this side of the chest was opened, air eseaped with foree, emitting no fetor. The upper portion of the right lung contained several tubereulous eavities, the largest of the size of an English walnut; also, small masses of crude tubercle.

I exhibited the perforated lung at a meeting of the New York Pathological Society, March 27, 1862.

Treatuent.-The treatment of pleuritis with pneumothorax, in most cases, embraces only palliative and sustaining measures. The severity of the pain and the distressing dyspnœa frequently call urgently for measures to procure relief. Opium, soothing applications to the chest, and the ethereal preparations are indieated for this object. The powers of the system are to be sustained by tonic remedies, alcoliolic stimulants, and a nutritions diet. These measures will generally only serve to mitigate the sufferings of the patient and prolong life; and in the rare eases in which the affection continues for months, or years, or indefinitely, and is not incompatible with comfortable health, there is no special treatment to be pursued, the main object being to invigorate and support the powers of the system.

If the patient suffer greatly from clyspnœa arising from dilatation of the affected side, the chest may be punctured as a palliative measure. I have resorted to this operation to the great relief of the patient. The air will be likely to reaccumulate, and the side become again dilated as before; but I have known this not to occur for some weeks, and the operation may be repeated as often as called for by the dyspnoa arising from the dilatation.

\section{Pneumothorax.}

The term pneumothorax denotes the presence of air, without liquid, in the pleural cavity. Under these eireumstances, pleuritis either does not exist, or it is circumscribed and not accompanied by liquid effusion. This affection is certainly extremely rare; its occurrence has been doubted, but its existence is sufficiently established. In cases of pneumothorax, without pleuritis, the perforation of lung is not incident to gangrene or tuber- 
culosis, but is probably connected generally, if not invariably, with emphysema, which may not be general, but circumscribed. Einphysematous dilatation of the air-cells, perhaps confined to a few lobules at the margin of a lobe, or an emphysematous bleb, may lead to a minute rupture through which air is forced, by the acts of breathing, into the cavity of the pleura. The perforation, as remarked by Dr. Gairdner, may be no larger than a pin-hole. 'The presence of the air does not excite pleuritis, but the accumulation may be sufficient to compress the lung into a solid mass, as in pleuritis with large effusion, dilating the affected side, and removing the heart from its normal situation.

Pneumothorax, without effusion from pleuritis, is characterized by the absence of the symptoms and signs denoting inflammation and the presence of liquid. The patient suffers chiefly from the mechanical effects of the presence of air. These effects relate exclusively to the respiration and circulation. There is little or no febrile movement; the appetite, digestion, and nutrition are not affected, and the vital powers are maintained. If the affection were to end fatally, it would be by apnoea, assuming that pleuritis is not developed sooner or later. It is unquestionable that this affection may end in recovery. Under the influence of compression, the perforation inay become sealed up by a circumscribed pleuritis with the exudation of lymph, or subsequently by the adhesion of the pleural surfaces at the point of perforation, and the lung again become expanded and resume its function. Dr. Gairdner has quoted the report of a remarkable case illustrative of the occurrence of pneumothorax without plemritis, and of its favorable course and termination. ${ }^{1}$ Dr. Gairdner regards this case as unique in respect of the distinctness of the clinical facts on which the diagnosis was based. A case equally striking, and not less distinct as regards the diagnostic phenomena, has fallen under my observation. The important points taken from the history of this case, which was recorded very fully, are as follows :-

$\Lambda$ Polish peddler, aged 29, was admitted into hospital, January, 29, 1856. He stated that twelve days before his admission, being apparently in perfect health, he was attacked suddenly with a sharp pain in the region of the left nipple, accompanied with shortness of breath. This occurred while he was carrying his pack, which weighed about 70 pounds. He had no cough nor expectoration. The pain soon disappeared, but the want of breath obliged him to give up his occupation and led him to the hospital. He was a vigorous man, weighing about 175 pounds. He presented a healthy aspect, complaining only of deficiency of breath on exercise. This difliculty did not prevent him from being up and walking freely about the ward.

The left side was dilated, and the intercostal depressions obliterated; the resonance over this side was purely tympanitic, and anseultation furnished a well-marked amphoric echo withont any respiratory sound. Subsequently amphoric respiration became extremely well marked, together with metallic tinkling. The heart-sounds showed the heart to be to the right of the sternum.

After a month there was considerable improvement as regards the deficiency of breath on exercise. The dilatation of the left side was no longer apparent, and the intercostal depressions became visible; vesieular respiration extended from the summit to the nipple, and the amphoric sounds, with the metallic tinkling, had disappeared.

Three weeks afterward, the patient felt sufficiently improved to leave

' Clinical Medicine. Edinburgh, 1862, page 381. 
the hospital and return to his occupation. The respiratory murmur on the left side was feeble, but every where appreciable, with no abnormal modification, save in intensity, and no amphoric sounds. 'The impulse of the heart was felt on the left side of the sternum.

The patient consulted me a month after his clischarge from the hospital. He had returned to his occupation of peddling, carrying his pack on his back as before. He had experienced no difficulty from want of breath until three days before his visit to me; but for three days the deficiency of breath on exercise had been considerable. On examination of the chest, the left side was everywhere tympanitic ; there was no respiratory murmur on that side, and the heart was on the right side of the sternum. No amphoric sounds. He complained of nothing but the want of breath; excepting this, he was well.

I did not see the patient afterward for eleven months. At the end of that time he called to see me with another patient. 'After his former call upon me, he gave up peddling and became a farm-laborer. - He now appeared and reported himself to be perfectly well. The respiratory murmur was everywhere heard over the left side, but was slightly weaker than on the right side. The apex-beat of the heart was in its normal situation. The left side latterly and posteriorly presented to the eye slight contraction.

This was eviclently a case of simple pneumothorax, without pleuritis. A pin-hole perforation at a weak point, or the rupture of an emphysematous bleb, probably took place under the muscular exertion of carrying his pack. He had nearly recovered when, on resuming his occupation, the perforation was reopened, and the pnenmothorax reproduced. Recovery again took place, and after tho lapse of sereral months, appeared to be permanent.

A sufficient number of cases of pneumothorax, without pleuritis, to serve as a basis for the clinical history and prognosis, are not yet available. The infrequency of the affection is such, that the collection of a considerable number of cases for analysis cannot soon be expected. The case just given, and the case recently reported by Gairdner, go to show that a favorable prognosis may be entertained.

As regards treatment, it is obvious that no special course of medication is called for. The compression of lung probably favors the healing up of the perforation, so that it would not be adrisable to puncture the ehest in order to allow the air to escape. Active exercise or muscular efforts, as in straining, are to be avoided, not only during the continuance of the affection, but for some time after recovery, lest the orifice may be reopened, as in the case just detailed. Soothing embrocations to the chest will be useful as palliatives; but nothing can be gained by active counter-irritation. A nutritious diet may be allowed. Cathartics, diuretics, and rubefacients are not indicated.

\section{Circumscribed Pleuritis.}

The frequency with which pleuritic adhesions, over a limited area, are found after death, is proof that circumscribed pleuritis often occurs. But the inflammation which results in these adhesions is often subacute, and generally occurs without giving rise to much pain or other disturbance. $A$ s already stated, circumscribed pleuritis is secondary to some pulmonary affection, especially tuberculous disease and pneumonitis, and it differs from a primary general pleuritis in not giving rise to pleuritic effusion. Fibrinous cxudation is sometimes small, and, perhaps, may be altogether 
absent, the adhesions taking place purely by a hyperplasia or proliferation of the connective tissue. Limited to the pleura investing the diaph:agm (diaphragmatic or phrenic pleuritis), inflammation is supposel to give rise to symptoms which are distinetive, viz., hiccough, attended with pain, and pain referable to the diaphragm in the acts of coughing. It may be doubted, however, if these symptoms are sufficient to mark the situation of inflammation, or to distinguish inflammation from pleurodyuia. If, from the symptoms, circumscribed pleuritis be suspected, the treatment indicated embraces moderate counter-irritation, soothing applications to the chest, and anodyne remedies. It may fairly be doubted whether acute, primary pleuritis be ever circumscribed. The suecessive attacks of circumscribed pleuritis which oceur during the progress of pulmonary tuberculosis, and which lead to firm adhesions, are seldom accompanied by much pain, and only call for palliative measures.

Cireunscribed collections of sero-fibrinous or purulent liquid are usually, if not alway's, preceded by general pleuritis which has led to the adhesions surromuding the space in which the liquid is contained. These collections may be situated at the lower part of the chest, between the lung and diaphragm, or between the lobes. The previous history and the appearances of the chest will generally show the prior existence of general pleuritis. The diagnosis of circumseribed collections of liquid is not always easy. For the physical signs, the reader is referred to works which treat more fully of thoracic diseases. The treatment embraces the measures for promoting absorption ; and, if there be grounds to suppose that the liquid is purulent, after this fact is demonstrated by means of the exploring trocar, it may be advisable to effect the removal of the liquid by paracentesis.

\section{Hydrothorax.}

Hydrothorax, using the term correctly, is not a variety of pleuritis, but it is noticed in this connection because, so far as certain symptoms and physical signs are concerned, it has points in common with pleuritis accompanied by liquid effusion. In the proper sense of the term, it denotes at dropsical affection. The effused liquid is serum devoid of lymph. The effusion is not a result of inflammation or any other affection of the pleural membrane, but proceeds from the conditions, relating to the blood and circulation, on which dropsical effusion in other situations depends. It very rarely occurs alone, but is generally an element of general dropsy; that is, dropsical effusion exists at the same time in the peritoneal cavity and in the subcutaneous areolar tissue, the latter constituting either œdema or anasarca. As a rule, in cases of general dropsy, the hydrothorax is subsequent to the anasarea and to hydroperitoneum.

The signs which denote the presence of liquid in the pleural cavity are alike applicable to inflammatory and dropsical effusion. The proof afforded by the change of level of the liquid, corresponding with changes of the position of the body, is more constantly available in hydrothorax, because lymph is not present to agglutinate the pleural surfaces and lead to permanent adhesions. But the distinctive feature of this affection is the existence of effusion in both pleural cavities. Hydrothorax is bilateral, provided the pleural cavity on one side be not abolished by universal adhesions due to a previous attack of pleuritis, whereas, pleuritis, when it is primary, is unilateral. The amount of dropsical effusion, however, in both sides, is generally not uniform. It is not uncommon to find in the pleural eavity on one side a quantity of liquid considerably larger than is contained in the cavity on the other side. It may then be laid down as a 
rule, that, if the physical signs show the presence of liquid in both sides, and general dropsy coexist, the affection is hydrothorax. A friction murmur is, of course, not developed in this affection.

Symptoms denoting inflammation, viz., pain, cough, febrile movement, do not belong to the elinical history of hydrothorax. The respirations are increased in frequency, and the patient suffers from deficiency of breath, or dyspnœa, in proportion to the amount of dropsical effusion. Existing in both sides, it is obvious that the embarrassment of respiration will be the same as if all the liquid were in one pleural cavity, and twice as much as if the effusion in one side were unaccompanied by an equal effusion in the other side. If there be considerable effusion, dyspnœa will be marked, amounting perhaps to orthopnœa, and death by apnœa may be due to the amount of effusion. Cardiac lesions, producing obstruction of the mitral orifice, especially favor the occurrence of hydrothorax, after these lesions have led to dilatation of the right eavities of the heart.

The measures of treatment in hydrothorax are those indicated in general dropsy, and these need not be considered in this connection. It is rarely the case that the affection oceurs under circumstances calling for paracentesis. This operation, however, is admissible, as a palliative measure, if the amount of effinsion be sufficiently large to occasion great suffering or endanger life. I have resorted to it in a case in which the danger of death by apnœa was imminent, great temporary relief being obtained by it, and the life of the patient being apparently prolonged. As there is no obstacle to the expansion of the compressed lung in cases of hydrothorax, all the effused liquid may be at once removed without the distress which is apt to accompany the operation of thoracentesis in cases of pleurisy.

It should be added that in some cases in which it is dependent on mitral obstruction, hydrothorax is greatly out of proportion to the amount of dropsical effusion in other situations; and I have repeatedly observed prompt, complete, and permanent relief procured by the hydragogue operation of elaterium in these cases.

\section{CH A PTER I V.}

\section{PNEUMONITIS.}

Seat of the Infiammation. Varieties. Acute Lobular Pneumonitis-Anatomical CharactersLaws of the Disease - Clinical History - Pathological Character-Causation -Diagnosis Prognosis.

$\mathrm{H}$

AVING considered in the preceding chapters inflammation affecting the serous membrane investing the lungs, inflammation seated in the pulmonary substance or parenchyma is next to be considered. The latter is called pneumonitis, or pneumonia. Pneumonia is the name commonly used; pneumonitis, however, is the name used in this work in comformity with the plan of distinguishing inflammatory affections by the suffix itis. The question at once arises, what constitutes the parenchyma, or substance of the Iungs. These terms relate to the air-cells, vesicles, or alveoli, 
together with the bronchioles or terminal bronchial branches. They are lined by a membrane differing materially from the mucous membrane lining the successive divisions of the bronchial tubes. The membrane which lines the air-cells and bronchioles is distinguished by its tenuity, by the absence of mucous follicles, and by a change of epithelium from the cylindrical and ciliated to the squamous, pavement, or tessellated variety. 'The air-cells and bronchioles make up the lobules, and these, united by means of areolar tissue, constitute the lobes into which each of the two lungs is divided. In acute pneumonitis, the membrane lining the air-cells and bronchioles is inflamed. 'The differences in the structure of this membrane, as compared with the bronchial mucous membrane, together with a difference of function, will serve to account for the fact that the inflammation in pneumonitis may be limited to the pulmonary substance, and, also, the fact that in bronchitis the inflammation rarely extends to the pulmonary parenchyma. This is in accordance with a pathological law, viz., that inflammation of a structure does not, as a rule, extend to another structure, however proximate, which has important anatomical differences, or which differs as regards its functions. Some striking examples of conservatism in disease will be found to hinge upon this pathological law. The fact, also, that the lining membrane of the air-cells is not, strietly speaking, a mueous membrane (since it is devoid of mucous follicles) will account for important differences pertaining to the inflammatory products of pneumonitis when compared with bronchitis and other mucous inflammations. The relation of the air-cells to the terminal branches of the pulmonary artery is also involved in the pathological phenomena of inflammation of the parenchyma of the lungs.

Acute pneumonitis, when it is not a complication of an existing pulmonary disease, usually affects, at least, an entire lobe; and this extension of inflammation is expressed by the term lobar pneumonitis. Developed in the course of another pulmonary disease, it may be more or less limited. When limited to a portion of a lobe, it maly be distinguished as circumscribed pneumonilis.

A distinct section will be devoted to chronic pneumonitis; and under this head will be noticed induration, sclerosis of the lung, together with anthracosis, siderosis, and pigment or brown induration. The terms lobular and disseminated pneumonitis have been used to denote an affection of isolated lobules, more or less in number, in both lungs. This will form the heading of a separate section, which will embrace some account of atelectasis and collapse of pulmonary lobules. Aćute lobar jneumonitis will be first considered. By German writers, this affection is called croupous pneumonitis; and, by some French writers, fibrinous pneumonitis.

\section{Acute Lobar Pneumonitis.}

Anatomical Cinaracters. - The first appreciable change resulting from acute inflammation is the same here as in other situations, viz., an abnormal accumulation of blood, or hyperæmia, due to active congestion or engorgement. The inflamed portion of the lung is heavier than in its healthy state; on section, the cut surfaces present a dark appearance, and blood flows in abundance, together with serous liquid more or less frothy. It would not be easy to discriminate between hyperæmia arising from inflammation and that due to hypostatie congestion, by the appearances. But the latter affects the portions which are dependent and is not limited to one lung, whereas the former is confined to one side and not always 
limited to the position into which the blood would accumulate by gravitation. The hyperæmia does not prevent air from entering the air-cells. Into the latter, however, soon, or from the first, is effused a little albuminous liquid. This condition lasts but for a short period.

Exudation speedily follows. A coagulating material escapes from the blood, and coagulates within the air-cells. The cells are filled and distended with the exuded matter. They cease to contain air. The lung is solidified, presenting an appearance not unlike that of the liver, and hence this condition has been called hepatization. In this condition usually the lung contains but little blood, and presents a pale, anæmic aspect; hence the term red hepatization is inaccurate. The red coloration, when present, denotes an umusual proportion of the red globules in the exudation within the cells. Closely examined, the cut surfaces have a granular appearance. When the hepatized part is divided, more or less liquid escapes and contains no air bubbles. The substance of the lung is softened, breaking down under the pressure of the finger more readily than in its healthy state; that is, it is more friable. Its weight is much increased; a single lobe solidified by inflammatory exudation increases in weight from one to two pounds, and an entire lung solidified may acquire an additional weight of four pounds. This increase in weight is due, not to an accumulation of blood, which is less than in health, but to the amount of solid matter withdrawn from the blood. Owing to the absence of air, portions of the solidified lung sink when thrown into water. 'The volume of the affected lobe or lobes is large in proportion to the amount of exudation, and no collapse occurs when the chest is opened. Examined with the microscope, the substance blocking up the air-cells is found to be composed of molecular matter which is probably amorphous fibrin or lymph in a granular form, epithelium, fatty granules, blood disks, and leucocytes.

If the progress of the disease be favorable, the exudation is remored mainly or exclusively by absorption. It may be absorbed with great rapidity. 'The statement by Rindfleisch, and others, that the greater part of the exuclation is expectorated, is certainly incorrect. The matter expectorated, in general, bears no proportion to the quantity of exudation; and cases are not rare in which the exudation disappears without any expectoration. After its removal, the air-cells are found to have sustained no damage. The pulmonary structure remains intact while the exudation remains, and its functional capacity is fully restored after the exudation disappears. The circulation, too, is again restored as in health, and the recovery of the affected part is complete.

If the progress of the disease be unfavorable, absorption of the morbid products within the air-cells does not take place, and the affected lobe or lobes are infiltrated with liquefied fibrin and pus. This condition is called purulent infiltration. The lung presents a grayish appearance. When divided, a purulent liquid flows freely from the cut surfaces. The substance is much softened, breaking down on slight pressure. Occasionally, collections of pus take place forming pulmonary abscesses. Gangrene of the affected portion of lungs sometimes occurs, but this, as well as the occurrence of abscess, is extremely rare.

Pleuritis, limited to the affected lobe or lobes, usually occurs and is developed coincidently with the pneumonitis. In a small proportion of cases the concurrent pleuritis is wanting. It varies much in degree in different cases, being sometimes slight and sometimes severe. The exudation of lymph on the pleuritic surface over the affected lobe or lobes, varies in amount. Much liquid effusion into the pleural cavity occurs only as an exception to the rule; the pleuritis, in most cases, is circum- 
scribed and dry. The terms peripneumonia and pleuropneumonia denote the coexistence of pleuritis and pneumonitis. Inasmueh as pleuritis is very rarely wanting, these terms are applicable to nearly all cases of pneumonitis. They may, however, be applied to distinguish eases in which the pleuritis is unusually prominent and attended with more or less liquid effusion. It is quite unnecessary to consider these terms as denoting a distinet variety of pneunonitis.

More or less bronchitis, affecting the bronchial tubes within the affected lobe or lobes, usually exists with pneumonitis. In some cases, however, the pneumonitis passes through its whole course without affording any evidence of this limited bronchitis. Bronchitis affecting the bronchial tubes of both lungs is sometimes, but rarely, present with pneumonitis. When these two affections are combined, it is accidental; bronchitis, as the primary affection, does not tend to the development of pneumonitis, and the latter has no tendency to give rise to bronchitis save within the affected lobe or lobes.

To the foregoing sketch of the anatomical characters may be added certain laws of the disease, which are best presented in this connection. One of these relates to the situation of the disease. Pneumonitis attacks, in the great majority of eases, the lower lobe, and the lower lobe of the right, oftener than of the left, lung. Exceptionally, it sometimes attacks primarily an upper lobe, and in these cases, as a rule, the disease is more severe; but to this rule there are exceptions. The disease very rarely, if ever, attacks two lobes simultaneously, but it invades, not infrequently, in succession a second and even a third lobe. The inflammation does not extend from one lobe to another, but, whenever a new lobe is affected, it is the seat of a new invasion. 'The lobes of one lung may be successively' invaded, or a single lobe on both sides; or, an entire lung being first affected, a lobe of the other lung may be attacked. In the two latter cases the pneumonitis is said to be double.

The inflammation extends at least over an entire lobe, as the name lobar pneumonitis implies, in the great majority of cases. There are exceptions to this rule. I have known some instances in which the physical signs clearly showed the occurrence of inflammation extending over a limited area, withont any evidence of the eireumscribed pneumonitis being a complication of any other pulmonary disease. In a lobe invaded secondarily, that is, one lobe being already affected, the inflammation is sometimes found after death to have extended over a portion only of the lobe. This fact I have repeatedly noted, but, perhaps, had the life of the patients been prolonged, the whole of the lobe would have been affected.

The whole of the lobe first invaded is not at once affected. The inflammation begins at a certain point, or at several distinet points, and extends from lobules to lobules until the entire lobe is involved. The point or points of departure may be at either the upper or lower extremity of the lobe, and at either the superficies or centre of the lobe. The diffision of the inflammation over the lobe takes place with more or less rapidity ; sometimes a few hours suffice, but in some cases it occupies several days. The progress of the solidification from the exudation may be determined very accurately from day to day, or from hour to hour, by means of physical signs.

The inflammation very rarely ends with the occurrence of engorgement only, without exudation. Solidification almost always occurs, and, when the inflammation is not secondary to another pulmonary disease, usually extends over to the lobe or lobes invaded.

With respect to the situation and extent of the affection, the following 
are the results of an analysis of 121 cases : 1 In 29 cases it was limited to the lower lobe of the right, and in 25 cases to the lower lobe of the left lung. It extended over the whole of the right lung in 27 , and over the whole of the left lung in 9 cases. It was limited to the upper lobe of the right lung in 8 , and to the upper lobe of the left lung in 3 cases. It was seated in the lower lobe of both lungs in 8 cases.

Clinical History.-The division of the career of acute pneumonitis into stages is based upon differences as regards the anatomical characters at different periods of the disease. The first stage embraces the period during which the affected lobe is in the state of active congestion or engorgement. This stage is called the stage of engorgement. The disease is considered as passing into the second stage when the affected lobe, or a greater part of it, has become solidified by the inflammatory exudation. This stage is called the stage of solidification or hepatization. In the third stage the affected lobe is in one of two conditions. If the disease pursue a favorable comrse, the third stage begins when it is evident that absorption of the exuded matter is going on, and convalescence takes place during this period. This may be called the stage of resolution. If the disease pursue an unfavorable course, the third stage is one of suppuration or purulent infiltration, and this stage may be called the purulent or suppurative stage. If this stage occur, the disease generally ends fatally.

The duration of each of these stages varies much in different cases. The stage of engorgement may last but for a few hours. I have known an entire lobe to be solidified by two pounds of exudation matter, as determined after death, in less than twelve hours. Not infrequently this stage does not extend beyond twenty-four hours. But sometimes the solidification occupies two, three, or four days, and in some cases it occupies even a longer period. In the majority of cases this stage is from twenty-four to forty-eight hours in duration. The stage of solidification may also be of short duration. I have known resolution to commence and make considerable progress in twenty hours. But its commencement may not be evident for two, three, or four days, or even for a considerably longer period. In the majority of cases the duration of this stage is from two to four days. The stage of resolution is still more variable. There is a notable difference in different cases as regards the rapidity or slowness with which the solidifying deposit is removed. It is very rarely the case that the resolution is completed in less than three or four days, and generally eight or ten days are required. In some cases two or three weeks elapse before the air-cells are restored to their normal condition, and oceasionally the resolving process is even more protracted. If the disease pass into the purulent stage, death usually takes place within a few days; but if the disease ends in recovery, many days and perhaps weeks elapse before the normal condition is restored.

In the great majority of cases, acute pneumonitis commences with a well-pronounced chill, frequently accompanied by rigors. 'The thermometer in the axilla at this period of the disease shows an increase of the temperature of the body, although coldness exists as a subjective symptom. The invasion is usually abrupt, with few or no premonitions. The attack is apt to occur during the night. Coincident with, or speedily following the chill, is the occurrence of pain. The pain is frequently severe. It is acute, lancinating, and in all respects identical with the pain

1 Clinical Report on Pneumonia, based on an Analysis of 133 Cases. By the Author. Vide American Journal of the Medical Sciences, January, 1861. 
in acute pleuritis. It proceeds, certainly in most cases, from the pleuritis developed in conjunction with the pneumonitis. As a rule, the intensity of the pain is in proportion to the amount of coexisting pleuritis; but it is true alike of pleuritis thus developed as a complication and when it occurs primarily that it is sometimes attended with little or no pain. Cases of pneumonitis differ considerably as regards this symptom; it may be quite prominent, or present in a moderate degree, or altogether wanting. The pain is usually referred to a circumseribed space at or near the nipple of the affected side. 'This limitation of the pain is a point distinetive of pneumonitis as compared with pleuritis.

Cough is usually present at, or soon after, the invasion. It is more or less prominent. It is sometimes wanting. It is painful in proportion as pain is a prominent symptom irrespective of the congh. 'The cough is frequently accompanied by expectoration. The matter at first expectorated is scanty, transparent, and viscid; and, in a certain proportion of cases, it soon assumes characters which are highly distinctive of the disease. It becomes semi-transparent, adhesive, and has a reddish tint like that of iron rust; hence it is commonly known as the rusty expectoration. This appearance is clue to a small quantity of blood which has become intimately mixed with the matter in its passage from the smaller to the larger bronchial tubes. The adhesiveness is such that when a considerable qnantity has accumulated, it adheres so closely to the bottom of the vessel as to remain when the vessel is inverted. The rusty expectoration is by no means uniformly present. Sometimes it is semi-transparent and adhesive without the reddish tint. It has sometimes a yellowish tint as if mixed with a little bile, which, however, is not the fact. It sometimes contains blood in abundance; and when the blood is of a dark color it gives rise to what is called the prune-juice expectoration. It is to be borne in mind that expectoration, in some cases of pneumonitis, is entirely wanting.

Febrile movement occurs with the invasion, together with pain in the head, loss of appetite, thirst, prostration, heat of skin, ete. 'The pulse varies much in frequency in different cases, ranging from 80 to 120 , and is more or less full and hard. 'The thermometer in the axilla indicates more or less increase of heat, the increase, in mild cases, not exceeding $104^{\circ} \mathrm{F}$; an increase above this amount always denotes great severity of the disease. A sudden increase of temperature denotes the invasion of a new lobe or some intercurrent affection. Cases differ greatly as regards the symptoms of general or constitutional disturbance, the system being much more tolerant of the disease in some persons than in others.

The respirations are increased in frequeney. This may depend on the pleuritic pain if it be marked, and also on the interruption of the function of hæmatosis in the portion of lung affected.

These are the important symptoms belonging to the clinical history at the commencement and during the first stage of the disease. During the stage of solidification, the symptoms undergo some changes. Pain, if it have existed, diminishes or ceases. Cough and expectoration continue, but the congh is less hard and painful, and the expectoration is effected with more facility. The matter expectorated loses its rusty appearance, becomes opaque, less adhesive, and more abundant. It is now the expectoration of resolving bronchitis. It is furnished chiefly by the bronchial tubes within the affected portion of lung, and is abundant in proportion as the bronchial mucous membrane is involved in the inflammation. On microscopical examination of the sputa, fibrinous casts of the minute bronchial tubes may be found. The febrile movement continues, but with 
diminished intensity. The pulse in this stage may be small and deficient in strength, especially if more than one lobe be involved, owing to the obstruction to the pulmonary circulation, which diminishes the quantity of blood received from the lungs by the left auricle; and owing also to the amount of material withdrawn from the blood and deposited in the aircells. The respirations are still increased in frequency in consequence of the solidified portion of lung taking no part in the respiratory function.

These are the modifications of the symptoms, assuming a single lobe only to be affected and that the course of the disease is favorable. The invasion of another lobe is rarely accompanied by a chill. The febrile movement persists or is increased, and the respirations become more frequent, accompanied perhaps by labor of breathing, marked dilatation of the alæ nasi, and dyspnoa. The affection, however, of an entire lung does not always give rise to symptoms denoting great gravity of disease. The febrile movement is not always intense, and may be slight; the respirations may be but little increased in frequency; cough and expectoration may be not only not prominent but even wanting, and the system may be but little disturbed. These remarks will also apply to double pneumonitis.

The symptoms during the stage of resolution denote progressive improvement. The febrile movement diminishes and ceases. The cough and expectoration become less and less, the respirations resume their normal frequency, the appetite returns, the strength is increased, and, in short, convalescence is declared. If, however, the disease pass into the stage of suppuration, the symptoms denote an unfarorable course. The pulse becomes more and more frequent and feeble. The expectoration is abundant and purulent. The respirations are frequent. The strength of the patient fails, and death takes place by asthenia, the accumulation of morbid products in the air-tubes frequently contributing to the fatal termination.

There are certain symptoms belonging to the clinical history of pneumonitis, which are not included in the foregoing sketch of its symptomatology. One of these relates to the urine. While the process of exudation is going on, and after solidification has taken place, until resolution begins, the chlorides frequently are greatly diminished, or disappear from the urine. This is ascertained by adding a solution of the nitrate of silver, having previously added a few drops of pure nitric acid. This delicate test shows the presence of a small quantity of the chlorides in the form of a cloudy precipitatc. During the period just stated, if the chlorides be deficient or absent, the addition of the nitrate of silver produces little or no effect upon the urine. The precipitate is manifest as soon as resolution commences, and increases until the condition of health in this regard is gained. The disappearance of the chlorides from the urine is not peculiar to pneumonitis. It is observed in other diseases. It is not, therefore, a diagnostic criterion of the disease, but it constitutes evidence either that the exudation is going on, or that resolution has not begun. It is stated that the chlorides are found in abundance in the matter expectorated during the time of their disappearance from the urine. The quantity of urine is in general more or less diminished. The urea eliminated exceeds the amount in health, and, owing to the diminished quantity of water, the urates are often deposited in abundance when the urine becomes cold. The increase of urea denotes an abnormal secondary or destructive assimilation. It is not uncommon for the urine to contain a trace or a moderate quantity of albumen. The presence of albumen is probably due to the systemic venous congestion dependent on the over-accumulation of blood 
in the right cavities of the heart. Albumen in abundance in the urine denotes coexisting renal disease.

Delirium not infrequently occurs in acute pneumonitis. It may be mild, and merely incidental to high febrile movement. But it is sometimes a prominent symptom. It may be active, the patient attempting to get out of the bed, resisting efforts of restraint, and perhaps using violence; or it may be of the low, incoherent, muttering kind, snch as occurs in typhoid fever. Delirium is more apt to occur if the upper lobe be affected, than if the affection be limited to the lower lobe. It is evidence of gravity of clisease in proportion as it is prominent, active, and persisting.

A circumscribed redness of one or both cheeks, the margins of the redness being abruptly defined, is frequently observed, and if the hectic flush of pulmonary tuberculosis be excluded, is quite distinctive of acute pneumonitis. When confined to one cheek, it does not follow, as was formerly supposed, that the pulmonary inflammation is seated on the same side. It is not uncommon for an herpetic eruption to occur upon the face; more especially around the mouth.

Slight jaundice is an occasional concomitant. Its occurrence is probably accidental. At all events, it is not associated often enough to show any pathological connection between pneumonitis and hepatic disease. 0 wing to the accumulation of blood in the right cavities of the heart, and the consequent congestion of the systematic veins, the liver sometimes becomes more or less enlarged.

The occurrence of abscess and gangrene is accompanied by symptomatic phenomena denoting these events. The collection of pus in an abscess leads to a sudden and copious purulent expectoration, if the life of the patient be sufficiently prolonged; and the existence of a cavity may be determinable by physical signs. This is a grave event, but it by no means necessarily renders the clisease fatal. It occurred in 4 out of 133 cases, which I recorded and analyzed, and two of these four cases ended in recovery. I have notes of a case, in addition to the four eases just referred to, in which a series of purulent collections werc expectorated, and the recovery was complete. The infrequency of gangrene is shown by its having occurred in but one of 133 cases. The disease proved fatal in that case. Since the analysis of these cases, I have known gangrene to occur, to a limited extent, in a case which ended in recovery. Its occurrenee is shown by the characteristic fetor attending expectoration, together with the appearance of decomposed pulmonary tissue in the matter expectorated.

The combination of other affections with acute pneumonitis will, of course, involve the addition of symptoms derived from the former. In malarious districts it may be conjoined with periodical fever. 'This is a most important complication as regards danger and therapentieal indications. The existence of the pneumonitis may serve to interrupt, to a greater or less extent, the regular succession of periodical paroxysms, and the latter may tend to obscure the phenomena of the pulmonary aflection. Pneumonitis occurring in the intemperate is liable to lead to the development of delirium tremens. The complication is always serious, and is important with reference to treatment. It occurred in 8 of 133 cases. Pericarditis is another grave complication. This occurred in 8 of 133 cases.

The supervention of certain symptoms is expressed by the term typhoid pneumonitis. This term denotes the existence of general symptoms analogous to those which belong to typhoid fever, of which the most prominent is low, muttering delirium. $A$ distinction is here to be made 
between typhoid pneumonitis and typhoid fever with pneumonitis as a complication. Pneumonitis is liable to be developed in the course of typhoid fever, the latter being the primary affection; but in the so-called typhoid pneumonitis, the latter is the disease, certain of the typhoid phenomena being superadded. In other words, the typhoid state may be developed in connection with pneumonitis, as with other local affections, but this typhoid state does not involve the essential fever designated as typhoid.

The duration of acute pneumonitis, when uncomplicated, varies within pretty wide limits. Dating from the attack to the time when the patient could be pronounced convalescent, in 30 cases, the shortest was 5 , and the longest 23 days. 'The mean duration was a fraction over 12 days. In 14 fatal eases, the shortest duration from the attack to the time of death, was 3 , and the longest 20 days, the mean duration being a fraction over 10 days.

A rotable decrease of temperature sometimes precedes the fall of the pulse and improvement in other symptoms; and the defervescence in this disease, if it be uncomplicated and pursue a favorable course, is usually rapid. The pulse during convalescence is not unfrequently below the normal frequency, falling in some cases to forty per minute.

Pathological Character.-Acute pneumonitis is an inflammation affecting a structure reckoned as a mucous tissue, but differing materially from the membrane lining the bronchial tubes, in respect of both anatomical composition and function. Inflammation in this situation is characterized by an exudation which does not lead to the production of new tissue and adhesions, as in pleuritis; which is not exfoliated and expectorated, in this respect differing from the exudation of lymph on other mucous surfaces, but which is removed by absorption, and sometimes absorbed with great rapiclity. The exudation in pneumonitis, doubtless, comes from the blood circulating in the branches of the pulmonary artery.

Causation.-Statistics show that no period of life involves either exemption from, or a notable proclivity to, acute lobar pneumonitis. It oceurs under 5 years of age, but not frequently. Cases are less infrequent between the ages of 5 and 10 . Of 118 cases, the patients all were over ten years of age, 13 were under $20 ; 44$ between 20 and 30 years ; 37 were between 30 and 40 years; 17 were between 40 and 50 years; 7 were between 50 and 60 years, and in no case was the age over 60. Cases, however, occur after the age last named. Cases occur much oftener anong males than females. The analysis of my cases with reference to occupation, does not show that any particular calling preclisposes to this disease, but the proportion of laborers engaged in out-door work over those employed within doors, shows that they are most liable to the disease who are exposed to the vicissitudes of the weather. Not infrequently the attack is fairly attributable to some unusual exposure, such as working in the cold and wet, or sleeping out of doors at night. The large proportion of hospital cases in which patients are addicted to excessive drinking shows that causative influences proceed from intemperance. In 10 of 37 cases in which the habits were noted, the attack followed a debauch. It is questionable whether the abuse of alcohol acts directly as a cause, or whether the disease may not be dne, in a great measure, to circumstances incidental to intemperance, such as exposure to cold, etc.

The association of periodical fever and pneumonitis has already been adverted to. My observations do not show the existence of any patho- 
logical connection between these affections. There does not seem to be ground for the conclusion that persons subject to intermittent fever are thereby more prone to pneumonitis, nor that the latter affection tends to reproduce an attack of the former. 'Their coexistence would appear to be due to the coincidence of the causes proper to each.

With respect to catusative influences pertaining to other pulmonary affections, facts show that bronchitis does not tend to eventuate in pneumonitis. The same is true of pleuritis. 'Tuberculous patients are not particularly prone to the disease, and when it does supervene, it may, or may not, affect the upper lobe, in which the deposit of tuberele is most abundant. Certain pulmonary affections, viz., emphysema, asthma, and ehronic pleuritis, are so infrequently associated with pneumonitis as to show that, instead of involving a predisposition to this disease, they afford more or less protection against it. I'neumonitis is not often developed in persons affected with organic dise:se of the heart.

Pneumonitis occurs as a complieation of other than pulmonary diseases. It is not infrequently developed in the course of typhus and typhoid fever, and in rubeoli. Degenerative affeetions of the kidneys have been supposed to stand in a causative relation to it. My experience does not furnish evidence of such a relation. In not one of the 133 eases analyzed was the disease preceled by albuminuria. I must, therefore, consider it doubtful whether pneumonitis is to be reckoned among the consecutive affections dependent on disease of the kidneys.

It occurs in certain seasons of the year in preference to other seasons. It occurs every where much oftener during the winter than during the summer months, and in our Northern States cases are more numerous in the spring months than at any other season. Forty-nine eases recorded at Buffalo were distributed among the different months as follows: January, 7 ; February, 2; March, 13 ; April, 9; May, 3; July, 3; A ugust, none ; September, 2; October, 1; November, 1 ; December, 5 . At New Orleans, cases are most numerous during the winter months. The analy'sis of 296 cases, by Grisolle, shows the largest number of cases in February, March, A pril, and May, in Paris, the relative proportion of cases in June, August, September, and October being small.

In this country the disease occurs in the Middle and Southern, much oftener than in the Northern States. It prevails much more in some year's than in others. In sections of the Southern States it prevails at times suffciently to be considered as endemic. It is emphatically the prevalent disease during the winter months in these States, and, effeeting especially the negro population, often proves the scourge of the sugar and cotton plantations of the South. It is a severer disease in the southern sections of the country than at the North, being more lialle to extend beyond a single lobe, and proving fatal in a larger proportion of cases. 'This liability is greater, in the same district, in some years than in others. In some years, also, there is an unusual tendency in the disease to attack primarily an upper lobe. Such a tendency is manifest at the time of writing these remarks. Within the last two months (October and November) in hospital practice, I have met with five cases in which the disease has been limited to an upper lobe, being about one-half the cases which have occurred under my observation during this period.

In a large proportion of the cases of acute pneumonitis, the disease is developed spontaneously ; that is, it is not referable to any obvious causative agency. An adequate, internal cause, of course, always exists, but its nature and source are not understood. Nor, indeed, are we able, with our present knowledge, to explain the development of the disease when it 
appears to follow an obvious cause, viz., exposure to cold. It is probable that the latter acts only as an exciting cause, co-operating with a cliathetic condition, or predisposition. Finally, the disease may be produced traumatically by injuries inflicted on the chest, and, thus produced, the inflammation rarely extends beyond a lobe, and may extend only over a portion of a lobe.

Dragnosis.-Acute pneumonitis, in some cases, is accompanied by symptoms which are highly disținctive. If a patient be seized with a chill, followed by febrile movement, together with a pleuritic stitch referred to a circumscribed space near the nipple, and the characteristic rusty expectoration occur, the diagnosis is readily made without the aid of physical signs. But these symptoms are by no means constantly present. 'The disease is not infrequently entirely latent as regards diagnostic symptoms, and it is often overlooked by those who do not employ physical exploration. Even if its existence be revealed by symptoms, without signs, the situation and extent of the affection can only be ascertained by means of the latter. With the aid of signs, the diagnosis, in the great majority of cases, is made without difficulty.

In the first stage, the signs on which the diagnosis is to be based are, slight or moderate dulness on percussion, and the crepitant rale. The latter, if persistent and well marked, is almost pathognomonic. Care must be taken, however, not to confound this rale with the subcrepitant, its distinctive characters being its fineness, dryness, and limitation to inspiration. With a distinct appreciation of these characters, it should never be confounded with the subcrepitant rale, which, however fine, is a moist, bubbling sound, and liable to be heard in expiration as well as in inspiration. These two rales sometimes exist in combination.

But the erepitant rale is not uniformly present. In its absence, a positive diagnosis may require some delay, until a sufficient number of lobules are solidified to give rise to an appreciable modification of the respiratory murmur. The modification is that which I have described under the name broncho-resicular respiration $;^{1}$ a modification approximating, more or less, toward the bronchial or tubular respiration. With the occurrence of this modification, the dulness on percussion becomes more marked. In most cases, if the disease be observed from the begimning, the signs of the second stage are soon declared. In hospital practice, and in many cases in private practice, the disease has already advanced to this stage when the patient is first examined.

When the solidification has become sufficient in degree, and has extended sufficiently over the affected lobe to furnish the signs of that condition, the diagnosis, if it have not been already fully made, is rendered clear by the presence of these signs. Bronchial respiration, bronchophony, and whispering bronchophony are the signs denoting the condition of solidification. They are first manifested over a limited portion of the affected lobe, and are thence diffused over its whole extent, either quickly or gradually, according to the rapidity or slowness with which the whole lobe becomes solidified. The progress of the second stage may be determined by the diffusion of these signs. They are generally all present, but if one be wanting, the others will be likely to be present; it is exceedingly rare for all to be absent. Dulness on percussion is now marked, amounting, perhaps, nearly or quite to flatness. This dulness or flatness extends over a space

1 Vide Physical Exploration of the Cliest and the Diagnosis of Diseases affecting the Respiratory Organs. Second Edition. Henry C. Lea, 1866. 
corresponding to that occupied by the solidified lobe; or, percussing anteriorly, the boundary line separating the dulness or flatness from the pulmonary resonance, is found to pursue a course coincident with the situation of the interlobular fissure, viz., obliquely upward and outward from the fourth or fifth costal cartilage toward the axilla. And this boundary line is the same whether the patient be sitting or lying upon the back. This is assuming that the pleural cavity does not contain liquid effusion, which, if it exist, is detcrmined by the signs indicated in connection with the diagnosis of simple pleuritis. The crepitant rale, if it have existed in the first stage, may continue, more or less diminished, during the second stage, or it may disappear after the affected side has become solidified. The moist and dry bronchial rales are liable to be hearl in this stage.

The invasion of a second or third lobe is denoted by dulness on pereussion and the auscultatory signs of solidification, the crepitant rale rarely occurring in the lobes which become sccondarily attacked. By means of the signs of solidification, viz., broncho-vesicular and bronehial respiration, and bronchophony with the loud and whispered roice, the extension of the affection to other lobes, in addition to the one first invaded, is speerlily ascertained. If the upper lobe become solidified, it is sometimes flat on percussion, and sometimes it yields a tympanitic resonance which occasionally has an amphoric intonation.

For an accomnt of the distinctive cliaracters of the signs which have becn named, and a fuller exposition of percussion and auscultation, together with the other methods of exploration in their application to the diagnosis of this as well as other pulmonary affections, the reader is referred to works which treat professedly of the subject. With a proper practical knowlerlge of the signs, the existence of the disease, the amount of lung involved, and the anatomical condition of the affected portions, are readily and accurately determined.

The commencement of resolution and its progress from day to day are shown by modifications of the signs denoting solidification. 'The bronchial respiration gives place to the broncho-vesicular, and the latter progressivcly approaches more and more to the normal vesicular murmur, into which it becomes finally merged when the resolution is completed. The characters of bronchophony are gradually lost. Dulness on pereussion becomes less and less marked, but some legree of dulness over the affected lobe or lobes continues for some time after the auscultatory signs show the exudation to have been removed. During the progress of resolution, the subcrepitant rale is frequently heard, and sometimes the crepitant rale reappears, constituting the crepitant rale redux.

If the disease pass into the stage of suppuration, the dulness or flatness on percussion continues, and the moist bronchial rales, due to pus in the air-tubes, are prominent. 'The auscultatory signs of solidification continue, but are less marked. If abseess of the limg take plice, and the patient's life be prolonged until a discharge of the pus into the bronchial tubes occur, cavernons respiration may become well marked.

A coexisting liquid effusion, occurring exceptionally, is shown by sigus already noticed. Under these circumstances, the affected side inay be dilated, with obliteration of the intercostal depressions, and obvious contraction of the chest may follow recovery.

Prognosis.-The prognosis, in cases of acute pneumonitis, will depend on the extent of lung involved, the diseases with which it may be connected as an intercurrent affection, its complications, the previous constitution of the patient, etc. These circumstances will affect the gravity 
and danger to such a degree that, in respect of the probable termination, different cases differ as much as if they were cases of clifferent forms of disease. Occurring as a primary disease, limited to a lower lobe, remaining uncomplicated, and the person affected having a fair constitution, the intrinsic tendency is to recovery ; indeed, recovery is not only the rule, but the exceptions are exceedingly unfrequent. Of sixty-nine deaths out of 750 cases reported by Dr. Dietl, of Vienna, in not one was the disease devoid of complications. Of the 133 cases which $I$ have analyzed, in only two of the fatal cases was the disease limited to one lobe, and not complicated or associated with other important affections. In one of these two cases the inflammation was seated in an upper lobe, and eventuated in abscess. In the other case the absence of complications was predicated on the examinations during life, a post-mortem examination not having been made.

Even if more than one lobe be involved, provided the disease be primary and uncomplicated, a favorable termination may reasonably be expected in a subject not enfeebled by age or other causes. I have known recovery to take place in a case in which an entire lung was involved, and the patient situated under as unfavorable hygienic circumstances as could well be imagined. In the case referred to the patient was attacked when working alone in a shanty, there being, at the time, two inches of water on the ground. The case occurred, during the winter season, in the swamp near New Orleans. After the attack he was unable to leave the bed for any purpose for a week, and during this time he was entirely alone. He had a quart of brandy, which he drank during the week. His habits, as he stated, were temperate. At the end of a week he was visited by some one (not a physician) who gave him thirty grains of calomel. After this he remained alone for ten days. A friend at length eame to him, gave him some doses of quinia, and removed him first to his own house, and afterward to the Charity Hospital of New Orleans. The physical signs, on his admission into the hospital, showed pneumonitis affecting the whole of the riglit lung, and resolution progressing. He remained in hospital six days, convalescence going on rapidly, and at the end of that time he was well enough to be discharged.

The 'gravity and danger, then, in cases of this disease, proceed, not so much from the disease per se, as from coexisting affections and other incidental circumstances. Developed in the course of continued fever, measles, or other diseases, it may lead to a fatal termination. In aged and feeble persons it may end fatally without any coexisting disease, especially if more than a single lobe be involved. Occurring in persons affected with organic disease of the heart, it is likely to prove a serions affection. I have known it to destroy life in the first stage when developed in this connection. The pulmonary symptoms are rendered more severe and the danger increased by antecedent emphysema.

The complications which are apt to render it fatal are pericarditis, intermittent fever, and delirium tremens. These complications in rest cases with much gravity and danger, but recovery takes place, in a certain proportion of cases, notwithstanding their existence. The gravity and danger from these complications are of course greater if the disease invade more than a single lobe.

There is a liability in the course of this disease to a fatal accident which claims especial notice, and which has not been sufficiently considered. Reference is had to the coagulation of fibrin in the right cavities of the heart. I have been led to regard this as an event of not unfrequent occurrence in fatal cases of pneumonitis. It is apt to occur in cases in which 
an entire lung becomes involved, or in cases of double pneumonitis. In such cases, the obstruction to the passage of blood through the lungs, caused by the presence of the exudation, involves an over-accumulation of blood within the right cavities of the heart. 'Ihe right ventricle and auricle are enfeebled by distension, and this condition, in conjunction with the increase of fibrin in the blood (hyperinosis), leads to coagulation. On examination after death in cases in which this accident has taken place, the right ventricle is found to contain a dense white clot closely intertwined with the tendinous cords, and perhaps adherent to the ventricular walls, sending prolongations into the pulmonary artery and through the tricuspid orifice into the auricle. A clot may also be formed in the auricular portion of the latter cavity. These clots are formed during life, and prove the immediate cause of death. Their formation may sometimes be determined with much confidence by the symptoms during life. In a case presenting no symptoms which denote imminent danger, a sudden change takes place for the worse ; the circulation is notably disturbed, as shown by frequency, feebleness, and irregularity of the pulse; the respiration becomes notably embarrassed, the expression haggard and anxious - the patient falls speedily into a moribund state, and this unexpected change is not connected with an extension of the disease to a new lobe or any newly-developed inflammatory complication. Under these circumstances the formation of a heart-clot is highly probable, and the probability of this accident is rendered still stronger if a newly-developed cardiac murmur be discoverable.

The following synopsis of a case which I observed in March, 1865, illustrates the sudden development of symptoms of great gravity, on a secondary invasion of a lobe of the opposite lung, or the occurrence of double pneumonitis :-

The patient, a banker, about sixty years of age, was a man of delicate constitution, of temperate, regular habits, and extremely eareful in everything relating to his health. He was attacked with pueumonitis March 28 , the lower lobs of the right lung being the seat of the disease. He appeared to be doing well, the affeeted lobe, however, presenting, wellmarked, the signs of solidification, when, on the evening of $A$ pril 1st, the fifth day of his illness, there was a sudden and notable change as regards the general symptoms. I saw him soon after this change occurred, and found the pulse very frequent and feeble, the skin cold and covered with a clammy sweat, the respirations spasmodic with tracheal rales. There was profound coma, the pupils scarcely responded to light, and liquid poured into the mouth was not swallowed. The patient was seen by Dr. Charles F. Heywood; we considered him as moribund, and there seemed to be scarcely an encouragement in the symptoms of the case to undertake to avert impending death. A fter persevering efforts'we succeeded in making him swallow a tablespoonful of brandy. In half an hour another tablespoonful was given with less diffieulty. After this he swallowed readily, and brandy was given at short intervals. After the lapse of several hours consciousness gradually returned, and all the symptoms denoted improvement. His prostration, however, was such that no examination of the chest was made until $A$ pril $3 d$, when were found the signs denoting invasion of the lower lobe of the left lung. Under supporting measures of treatment he passed safely through the double pneumonitis, and he has better health since his recovery than for a long time prior to his illness. Since this case was observed, I have met with cases in which a primary invasion, occurring in aged persons, produced such a degree of prostration and disturbance of the heart's action, that death by rapid asthenia. 
seemed imminent, life being apparently saved by free alcoholic stimulation and digitalis.

As regards anatomical changes pertaining to the lungs, gangrene, abscess, and the suppurative stage of the disease render the prognosis extremely unfavorable. Yet I have known recovery to take place in cases in which the symptoms denoted the occurrence of these events.

Symptoms which are unfavorable as prognostics are the following: Frequency and feebleness of the pulse, irrespective of the formation of a heart-clot. Great frequeney and labor of respiration. Lividity of the prolabia and face. An abundant purulent or mueo-purulent expectoration. Bloody, dark-colored sputa, commonly known as the prune-juice expectoration. Active, violent delirium. Low, muttering delirium, with prostration and subsultus tendinum, constituting the typhoid state. Cases presenting the symptoms last stated are often distinguished as cases of typhoid pneumonitis.

In the majority of fatal cases of acute pneumonitis, death takes place by asthenia in combination with apnoa, the former predominating. Death purely by apnœa may occur if two or three lobes become rapidly involved, but its occurrence is rare. The occurrence in some cases of an abundant pleuritic effusion, increases the danger from apnœa. So, also, its occurrence in persons affected with emphysema, and in cases of pertussis. In general, life is not lost in consequence of the extent of interference with respiration, but, owing to concomitant affections or other cireumstances, the vital powers give way, and the patient dies from exhaustion. This is an important fact in its bearing on therapentieal indications.

When convalescence takes place in this disease, it generally progresses until the recovery is complete. The tendeney to pass into a chronic form is exceedingly slight. Nor is there a tendency to relapse. It is eertainly rare for tuberculous clisease to become developed as a sequel of pneumonitis. When phthisis follows, the deposit of tubercle probably existed prior to the pneumonitis. When the upper lobe is primarily attacked, it has been supposed that the prior existenee of tuberculosis may be inferred. There may be some ground for this inference, but I have repeatedly known the inflammation to be limited to the upper lobe in cases in which the anterior and subsequent history furnished no evidence of phthisis.

\section{CHA P TER V.}

Treatment of Acute Lobular Pneumonitis. Pneumonitis in Young Children. Circumscribed Pucumonitis. Clronic Pncumonitis-Induration-Sclerosis and Cirrhosis of the Lung. Pigment, or brown Induration. Antluracosis. Siderosis. Lobnlar Pnenmonitis-Atelectasis and Collapse of Pulmonary Lobules. Pleurodynia and Intercostal Neuralgia.

THE different stages of acute pneumonitis furnishing different therapeuItical indications, the treatment of each stage is to be considered separately.

The question whether the disease may be arrested, relates to the first stage. Measures which have heretofore been considered as abortive, and which are still, perhaps, so considered by some, are bloodletting, eathartics, 
and other remedies entering into the so-called antiphlogistic metnod of treatment. Experience has abundantly shown that these measures are not to be relied upon for the arrest of this, more than other iuflammations. Admitting that they sometimes sueceed, the probability of sueeess is so small as not to warrant their employment under circumstanees which will be likely to render their operation hurtful should they not prove suecessful. The objects of treatment, then, in the first stage, are to diminish the intensity of the inflammation, to relieve symptoms, and place the system in a condition to tolerate the disease.

As regards bloodletting, its employment is to be regulated by the general considerations presented in Chapter II., Part II., to which the reader is referred. It is admissible in certain cases as a palliative, ancl, perhaps, to some extent, a curative measure, in view of the promptness of its operation. 'The circumstances whieh warrant its employment are: high febrile movement, the pulse more or less resisting compression, and the condition of plethora, or at least a robust constitution. It is contra-indicated whenever the febrile movement is not marked, when the pulse is frequent and weak, and if the patient be anæmic or have a feeble constitution. The relief of pain and embarrassment of breathing by bloolletting is often immediate and marked. The efficiency of this measure as a palliative is due in part to its effect on the general circulation, and to the diminution of the functional labor of the lungs; but the relief may be in part explained by the effect in diminishing congestion of the portions of the pulmonary organs not inflamed (the collateral fluxion of Virchow), and also by the effect in lessening the accumulation of blood in the cavities of the right side of the heart. In most of the cases, however, in which bloodletting would be admissible, provided the same ends could not be secured by other means, the latter are to be preferred. These consist of depletion by saline purgatives, and sedative remedies. After the operation of a saline purgative, if the skin be hot and the pulse frequent, tartar-emetic or some antimonial preparation may be given as a nauseant sedative; but the doses should not be carried to the extent of producing marked or distressing nausea. . The veratrum viride may be given under the same restriction. These remedies are not called for if the symptoms be not urgent, and they are contra-indicated by feebleness or a tendency to depression. A conite is an effective sedative remedy, and is to be preferred to the sedatives just named whenever it be desirable to avoid the depressing effects of the latter. Opium may be given with propriety and adrantage in the first stage, in doses sufficient to relieve the pain and tranquillize the system. IBlisters are injulicious, but dry cups, sinapisms, or stimulating liniments may be employed. Stupes, or warm fomentations, applied to the chest, are useful. In many cases, a saline purgative followed by some form of opium, the latter continued at intervals, together with soothing applications to the ehest, will meet the indications pertaining to the first stage. Niemeyer extols cold applications over the part of the chest corresponding to the affected portion of the lung, by means of napkins which have been dipped in cold water, and which are to be renewed every five minutes. He states that his advocacy of this measure is based upon the farorable effect in a large number of cases. Generally, after a few hours, patients are sensible of relief; and often both the respiration and the pulse become less frequent. Not unfrequently, the temperature of the body is notably reduced. In some cases, however, patients complain of the application, and, if so, it should not be continued. He does not claim for this measure that it arrests the disease, but that the duration of the disease may be abridged 
by it, and that it is valuable as a palliative measure. It by no means follows that because warm applications are useful, the application of cold is not also useful, or vice versa; each may have a favorable effect, the modus operandi of each differing from that of the other. Warm applications soothe and act as revulsives; the application of cold diminishes the determination of blood to the part, and relieves pain by obtunding the sensibility. The application of cold water to the entire surface of the body, by means of the eold bath or by wrapping the body in a wet sheet, has been found safe and useful in acute pneumonia as in other acute inflammations. If resorted to, its employment is to be regulated by the circumstances stated in connection with the treatment of acute pleuritis. Vide page 153.

The treatment in the second stage has reference to the promotion of resolution, palliation of symptoms, and supporting the powers of the system. Bloodletting in this stage is not admissible, and depletion by means of salines is not called for. It is not an object to divert the blood from the solidified lnng, for the exudation has already cleprived it of blood; and the amount of exudation involves the withdrawal from the blood of a pound or so of solid matter, even when the inflammation is limited to a single lobe. Can we give remedies to excite absorption, and thus shorten the duration of the second stage? Laennec extolled the use of tartaremetic in large doses for this object, after the plan of Rasori. But Laennec was not aware how speedily absorption sometimes begins after exudation has reached its maximum, and how rapidly the resolving process goes on in some cases, irrespective of any treatment; for, in his day, the natural history of this disease had not been studied. It is reasonable, therefore, to conclude that he mistook for the effect of this remedy the occurrence and progress of resolution due to the intrinsic tendency of the disease. With our present knowledge of the course of the discase in cases in which no active measures of treatment are employed, and after a large experience of the value of tartar-emetic as a sorbefacient, its utility in this way is, to say the least, doubtful. The same remarks are applicable to mercury given with a view to affect the system. I have now for many years ceased to employ these remedies for the purpose under consideration, and have seen no reason to be dissatisfied with discontinuing their use. The use of iodine externally or internally is not open to the objections of tartaremetic and mercury, on the.score of doing harm if it be not useful. A convenient mode of employing iodine is to apply the tincture to the affected side.

Repeated applications of the tincture of iodine will sccure a sufficient amount of counter-irritation, if any be desirable. Blisters are not advisable on account of the general disturbance which they are apt to occasion, and their interference with physical examinations of the chest. It is the custom in Bellevue Hospital to cover the chest with an oiled muslin jacket. This contributes to comfort by keeping the surface moistened with perspiration; and if a flannel covering be added, all the advantages of a poultice or water-dressing are thereby secured. If pain and soreness continue in this stage, stupes with warm water only, or with some stimulating liquid, as the spirits of turpentine, will afford relief. The latter will not be required if the tincture of iodine have been applied.

Opium is a most valuahle remedy in the second as in the first stage. It is indicated, not only by the continuance of pain, but by vigilance, restlessness, and symptoms denoting constitutional disturbance. I have repeatedly observed a rapid and notable diminution of the frequency of the pulse and of the respirations, with refreshing sleep and a conclition of comfort, to follow full doses of opium. This remedy has the advantage 
of not doing harm if it fail to do the goód which we expect from it. The free use of opium does not delay the commencement nor retarl the progress of resolution. An accumulation of mucous secretion in the bronchial tubes contra-indicates the use of opium in full doses.

Remedies to promote expectoration, as a rule, are not indicated. The use of remedies of this class is based on the erroneous idea that the matter of exudation is expectorated. Clinical observation shows that the removal of this matter may go on with great rapidity without any expectoration. The expectoration in the second stage of the disease is due to bronchitis usually limited to the affected lobe or lobes. The mucous products rarely accumulate in the bronchial tubes to an extent to occasion ineonvenience, except as a consequenee of a degrec of exhaustion suflicient to render the muscular power inadequate to eflicient efforts of expectoration; and, under these circumstances, expectorant remedies will not afford relief.

Sedative remedies, such as the tartar-emetic, in small doses, aconite, and the veratrum viride, are admissible in this stage, if there be eonsiderable or high febrile movement, without a tendency to asthenia. They should be cautiously given, so as not to incur risk of constitutional disturbance or depression.

To support the powers of life is the leading general indication in the second stage. Resolution will be sure to begin and continue if the life of the patient be suffieiently prolongerl. The danger is generally, not from the amount or persistence of the solidification of lung, but from failure of the vital powers before the resolution takes place. 'This disease belongs among those distinguished as self-limited; if uncomplicated and not attended by accidents, it runs a definite career ending in restoration, provided the powers of life hold out. These considerations, together with the results of clinical experience, enforec the importance of the supporting treatment.

The indication for supporting measures, as regards urgency, varies much in different eases. In general terms, it is urgent in proportion to the danger from asthenia. It should govern the treatment in eases sometimes characterized as asthenic, and whenever there are grounds for clistrust of the adequateness of the vital powers to earry the patient safely through the disease. It is a serious mistake to defer supporting measures until the symptoms denote imminent danger from failure of the powers of life. If deferred so long, they will probably be too late. The observing and skilful practitioner will foresee and endeavor to forestall a degree of prostration attended with imminent danger. The constitution of the patient, his previous health, and his habits, are to be taken into account in judging early of the ability. to sustain the disease. Other things being equal, in a warm climate patients are less able to sustain the disease than in cold or temperate climates; supporting treatment, therefore, is oftener and earlier ealled for in the former than in the latter. The plantation negro at the South is less able to sustain it than the white man, and, consequently, is more likely to need support. In eases which are distinguished as asthenic and typhoid, the reliance for successful management must be on supporting measures. These views are the more to be impressed because it is undoubtedly true that, until lately, the minds of medical men have been so much oceupied with the means of subduing inflammation, as to overlook the fact that means employed for this end, not only often confliet with those which are more important for recovery, but may be positively injurious, if not, indeed, destructive to life. The attention has been directed too much to the disease and too little to the patient.

The supporting treatment embraces tonic remedies, alcoholic stimulants, 
and nutritious diet. Of tonic remedies, quinia is to be preferred. It is not indicated in mild cases, but whenever there are grounds for anticipating undue depression of the powers of life, it may be given and continued during the progress of disease. Alcoholic stimulants form a very important part of the supporting treatment in this disease, as in all other diseases, whenever the great object is to keep the patient alive until the disease lias reached the end of its career and adranced into the stage of resolution. 'The principle is the same as in the essential fevers; and here, as in the management of the essential fevers, alcoholic stimulants are indicated to an extent commensurate with the danger from failure of the vital powers. In eertain cases of pneumonitis, as in typhus or typhoid ferer, and other affections, there is often a remarkable tolerance of alcohol, and the only guide, as regards quantity, is the effect as manifested by the symptoms. No abstract rules can be laid down, applicable to all cases, but careful observation must furnish the rule proper to each individual case. Here, too, as in the continued fevers, because alcoholic stimulants are vastly important in some eases, it is not be inferred that they are invariably indicated, nor that they can never do harm; on the contrary, if pushed to an injudicious extreme, they are as potent for evil as they are potent for good when judiciously used.

$\Lambda$ s regards the circumstances under which the use of alcoholic stimulants is to be commenced, they are always indicated as soon. as evidence appears of any tendency to failure of the powers of life. And of this, the action of the heart, as represented by the pulse, is the best critcrion. Feebleness, great frequency, and a pulse vibratory or thrilling but compresșible, denoting increased activity but diminished power of the ventricular contractions-these are the characters which inclicate supporting measures, of which alcoholic stimulants are an essential part. Given at first in small or moderate doses, the effect is to be watched, and the quantity increased in proportion to the urgency of the indication. The habits of the patient, as regards the habitual use of alcoholic drinks, are of course to be taken into account. Whenever the question arises, in the management of a case, whether alcoholic stimulants be advisable or not, it should be borne in mind that to begin earlier than they are required is far preferable to subsequent delay; for with proper care they can be suspended without any injury having been done, whereas the time lost by beginning too late cannot be regained.

Alimentation is an essential part of the supporting treatment. It is as important to nourish patients affected with pneumonitis as those affected with fever or any discase, whenever there is danger from failure of the vital powers. The statement that patients with pneumonitis should be encouraged to take nutritious food during the whole course of the disease is based on considerable experience, and alimentation should enter into the treatment in proportion as the symptoms denote a tendency to asthenia. Milk with farinaceous substances, with animal broths, should form the diet, thus combining a proper variety of alimentary principles. The desires and taste of the patient may generally be trusted.

These remarks on the supporting treatment are not specially applicable to pneumonitis, but apply alike to cases of any disease, the gravity or danger of which is manifested by symptoms denoting failure of the vital powers, and they will be referred to in connection with the treatment of other affections. As applied to pneumonitis, they relate chiefly to cases in which this disease is rendered grave or dangerous either by the extent of lung involved, or by coexisting affections. It will be borne in mind that in a large proportion of the cases in which the disease is limited to a 
single lobe, and disconnected from other affections, there is little or no intrinsic tendency to a fatal termination. In such cases no active treatment is required, either with a view to lessen the intensity of inflammation or to support the powers of the system. Patients pass through the disease satisfactorily under simple palliative measures.

In cases of pneumonitis associated with intermittent fever, quinia should be given promptly and in efticient doses. The paroxysms should be arrested as speedily as possible; the patient may be placed in great danger by their repetition. 'The existence of pneumonitis and the degree of symptomatic febrile movement in no wise conflict with this important indication; indeed, so far from conflicting with it, the sedative effect of full doses of quinia may be useful, aside from the arrest of the paroxysms. In a malarious region, or if the patient have been subject to intermittent fever, it is judicious to forestall the possible development of the latter affection by moderate doses of quinia. The malarious cachexia impairs the power of resisting the disease ; hence, it is more likely to prove fatal in malarious regions, even when uncomplicated with internittent fever.

Cases of pneumonitis complicated with delirium tremens call for the pretty free use of opium, together with alcoholic stimulants and a nutritious diet. Delirium and vigilance, if protracted, are likely to lead to a fatal termination. Pericarditis, as a complication, adds greatly to the gravity and the danger; but, by perseverance in the judicions employment of supporting measures, the patient may be carried safely through this combination of diseases. The occurrence of gangrene furnishes an additional indication for support. Pneumonitis oceurring in the course of the continued or eruptive fevers is to be treated by soothing and supporting measures. Depressing treatment by bloodletting, antimonial preparations, and purgatives, are very rarely, if ever, admissible under these circumstances.

During the progress of resolution, the principles of treatment which have just been presented are applicable until the improvement in the local and general symptoms, in connection with the physical signs, denote convalescence. When convalescence is established, there is little or no danger of a renewed attack. There is, therefore, no need of extreme precautions in order to prevent a liability to relapse. Experienec shows that a solid, substantial diet may be entered upon as soon as the patient is fairly on the road to recovery, and that the recovery is more rapid than if the appetite be too much restrained. As a rule, ordinary wholesome, digestible articles of food may be allowed when they are desired by the patient. Permitting or encouraging the patient to sit up will be found not to retard recovery, but, on the other hand, apparently to hasten the progress of resolution. Early going out of doors is not objectionable.

If the disease proceed to the suppurative stage, purnlent matter being either infiltrated or forming abscesses, the prognosis is extremely unfarorable, but perseverance in the employment of supporting measures is sometimes suecessful. It may be added, that timely and efficient support probably affords the best security against suppuration, which happily is extremely rare.

The liability to the formation of heart-clot in cases of pneumonitis suggests the important inquiry whether measures may not be employed to prevent this accident. Acting upon the conclusion, deduced by $\mathrm{Mr}$. B. W. Richardson from a large number of varied experiments, that the liquid form of the fibrin in the blood is due to the presence of ammonia, I have for several years advised the carbonate or muriate of ammonia during the progress of the disease with a view to prevent coagulation. Of course, it 
is difficult to obtain clinical proof of the protective efficacy of this or any other remedy. There can be no objection to the use of this remedy on therapentical grounds, since it does not in any manner affect unfavorably the progress of the disease. It may be added that the conclusion just stated has been abandoned by Dr. Richardson. Dr. A. Patton, of Indiana, in a paper advocating the free use of this remedy throughout the disease, that is, from 5 to 10 grains every three hours, states that, of 96 cases in which this constituted all the medicinal treatment, in only two cases was the disease fatal. ${ }^{1}$ It is probable that digitalis, given with a view to increase the power of the heart's action, is useful in preventing heart-clots. This remedy has seemed to me often signally useful when the action of the heart is feeble or irregular.

Acute pneumonitis, diffused over an entire lobe, sometimes involving a second lobe, and presenting after death the anatomical characters which have been described as belonging to this disease in the adult, occurs in young elildren, although infrequently as compared with its occurrence after this period of life. It is a much graver disease in young children than in adults, proving fatal in a large proportion of cases. From the absence of the subjective symptoms, and the difficulty of obtaining physical signs, the diagnosis is less easy, and the disease is not unfrequently over: looked.

The attack is sometimes accompanied by a convulsion. This may mislead by directing attention to the head. Dulness, drowsiness, or stupor sometimes accompanies the progress of the disease, and these symptoms may mislead in the same way, if the symptoms referable to the chest be not marked. The expectoration, being swallowed, cannot be observed in young ehildren. As regards marked local symptoms, the disease may be latent in the child, as it frequently is in the adult. But, in a certain proportion of eases, the existence of sharp pleuritic pain is manifested by the expression, and, also, by the cry in acts of coughing, or whenever a deep inspiration is taken. A diagnostic symptom, of frequent occurrence, is a moaning or grunting sound with the expiratory act. Attention to this symptom is especially important, as it points very strongly to the existence of pneumonitis. Increased frequency of the respirations and dilatation of the nostrils show the existence of some pulmonary affection compromising the respiratory function. The respirations, in some cases, are very frequert, numbering 40,50,60, and sometimes even many more per minute. One or both cheeks may present a circumscribed flush. If the progress of the disease be unfavorable, lividity of the prolabia and face becomes marked; more or less acceleration of the pulse occurs, and the frequency, in some cases, is very great, amounting to from 150 to 200 per minute.

When the existence of some acute affection of the chest is declared by the symptoms, the differential diagnosis lies between primary pleuritis, capillary bronchitis, and pneumonitis. The first of these affections being extremely rare under five years of age, the problem is usually to decide between the last two. In making this decision, the physical signs are to be relied upon, and the reliance is also upon these for the diagnosis in cases in which the symptoms denoting an acute thoracic affection are not marked. The physical signs are generally available, with care and patience, nowithstanding the difficulty of exploring the chest in the young child.

The crepitant rale is oftener wanting in pneumonitis affecting the child than the adult, but it is present in a certain proportion of cases, and is, of

I Am. Journ. of Med. Sciences, Oct. 1870. 
course, to be sought after. This sign belongs exclusively to pneumonitis, whereas the suberepitant rale belongs to capillary bronchitis. If there be cloubt as regards the discrimination between the erepitant and subcrepitant rale, it is to be borne in mind that capillary bronchitis is a bilateral disease, and the rale will be present in both sides; whereas, in the great majority of cases, pucmonitis in the child or in the adult is milateral, and the rale will be limited accordingly to one side. Dulness on pereussion is readily determinable in the child, and this is an important point in the differential diagnosis. Bronchial respiration and bronchophony may generally be obtained in the child by perseverance in auscultation, the cry answering for the voice. These signs do not belong to capillary bronchitis. In short, the diagnosis is to be based on the sime signs as in the adult, but patient efforts may be requisite to obtain them. As regards primary pleuritis, the sigus denoting effusion are avalable in the child as well as in the adult, and their absence warrants the exclusion of that affection.

The treatment of pneumonitis in children involves the same principles as the treatment of the disease in adults, with those modifications which therapentical measures require in their application to infantile life. It is questionable whether bloodletting be ever advisable in young children, even when the diagnosis is elear in the first stage of the disease. As a rule, this measure is not to be employed, and the exceptions to the rule, if there be any, are few; depletion by salines should take its place. Antimonial preparations, if given at all, are to be prescribed with great caution, and, in general, other and less depressing nauseant sedatives are to be preferred. 'The veratrum viride, if given, should be prescribed in small doses, and its effects very carefully watehed. Aconite, in children as well as adults, is a useful sedative in this affection. Blisters should not be employed. A sinapism or turpentine stupes may be applied to the chest, followed by a poultice or the water-dressing. I'articular attention should be given to the latter, in order to see that either the entire chest or the whole of the affected side is covered with several thicknesses of flamnel and a layer of oiled muslin. The spongio-piline is a good substitute for the poultice or water-dressing. Opium need not be withheld, but must be given with circumspection. Finally, as much depends, in severe cases, in the child as in the adult, upon the early, judicious, and persistent employment of supporting measures. In clildren, as in adults, under certain circumstances, there is a remarkable tolerance of alcoholic stimulants. I have notes of the case of a child of a medical friend (age fourteen montlis), presenting the utmost gravity of symptoms, the pulse 200 and the respiration 120 per minute, in. which brandy was increased to at least an ounce hourly, and, under this amount of stimulus, the pulse fell rapidly to 124 , and the respiration to 50 per minute, the carbonate of ammonia and a little morphia constituting the additional treatment. Recorery took place. This case is cited in illustration of the extent to which, in some cases, the employment of alcoholies may be carried. It does not follow that they are to be generally employed excessively or largely. The same rules are to be observed in treating cases of this disease in children as in adults, with a view, on the one hand, to secure the benefits of stimulants, and, on the other hand, to aroid the evils of their over-use.

\section{Circumscribed Acute Pneumonitis.}

Acute pneumonitis, when not incidental to an existing pulmonary affection, as a rule, extends over an entire lobe at least. To this rule, as already stated, there are exceptions. The exceptions are infrequent, yet perhaps 
they are not so rare as is supposed, for, if much circumscribed, the affection is overlooked without a careful physical exploration, and even the sigus may escape detection. One of my colleagues experienced a slight pain in the chest, and expectorated two or three rusty sputa. On careful examination by auscultation, the crepitant rale was discovered at the base of the chest, limited to a space not larger than half a dollar. This circumscribed pneumonitis, preceded by perfect health, did not prevent him from continuing his professional labors as usual. Such cases might easily be overlooked. But, in general, when pneumonitis is circumscribed, it is incidental to some previously existing pulmonary affection.

It is a not infrequent concomitant of tubereulosis. More or less of the lobules in proximity to the tuberculous product become inflamed. An examination of the chest, at this time, may disclose a erepitant rale within a limited area, and this rale, under these circumstances, is one of the accessory signs of phthisis. The exudation proceeding from the circumscribed pneumonitis renders the physical signs of solidification more marked and cxtensive than they had been found to be on previous examinations, and, after a time, when resolution has taken place, these signs diminish so as to represent simply the tuberculous affection. Cases exemplifying this statement have repeatedly come under my observation. The occurrence of such attacks of circumscribed pneumonitis will serve to account for changes, as regards the physical signs, which, if not understood, would seem to be inconsistent with the natural history of tubereulous disease. Circumscribed pneumonitis occurs in certain of the cases of so-called pyremia, and is manifested, if death take place early, by nodules, more or less numerous, varying in size from that of a pin's head to a walnut, of a dark color, the appearances being due, not to extravasation of blood, but to deep congestion. These nodules, when situated near the surface, are conoidal in form, the pleural portion being the larger. They represent the first stage of inflammation; and, if life continue, they become the sites of the purulent collections which were formerly called, incorrectly, metastatic.

Inflammation excited by a wound of the lung, as when a musket or pistol ball passes through the chest, may be more or less circumscribed.

Whether primary or otherwise, pneumonitis limited to a circumscribed space claims only simple palliative measures and hygienic precautions. The treatment in cases of pyæmia is to be directed to the general disease.

\section{Chronic Pnenmonitis-Indnration-Sclerosis and Cir- rhosis of the Lung-Pigment, or Brown Induration- Anthracosis-Siderosis.}

The term chronic pneumonitis is not here considered as embracing what has been commonly known as tuberculous infiltration of the lung. This statement is required, inasmuch as the latter is by some writers reckoned a variety of pneumonitis, and so named. This affection is considered in the first part of this work (see page 35), and in Part Second, Chapter X., under the name pulmonary tuberculosis. If it be designated pneumonitis, the adjective tuberculous should be prefixed, in order to distinguish it from other forms of inflammation of the lung, and, at the same time, to indicate a pathological connection, to say the least, with tubercle.

It has been seen that acute pneumonitis is characterized by the presence of inflammatory products within the pulmonary vesicles, cells, or alveoli. This characteristic, also, belongs to tuberculous infiltration. But, climinating the latter from present consideration, cases occur in which, with 
essentially the same kind of product within the air-cells, as in cases of acute pneumonitis, the symptoms and progress denote either a subacute or a chronic affection. Occurring as a complication of other diseases, pneumonitis is not infrequently a subacute affection. So, also, when primary, it is sometimes devoid of symptoms which denote acuteness; and yet the local morbid conditions, as determined by physical signs, extend over an entire lobe, and are as marked in degree as in acute cases. It is not very rare for patients to come to dispensaries for treatment in the second stage of the disease; and the disease may pass through its entire course without obliging the patient to keep the bed even for a day. On the other hand, in some exceptional cases, the acute symptoms disappear, but the solidification remains for many weeks, resolution at length taking place completely. The name chronic pneumonitis might, with propriety, be applied to the disease in such cases. A patient is admitted into hospital, having been confined to the bed for some time before almission. There are found the signs of solidification extending over the greater part or the whole of either an upper or a lower lobe. If the upper lobe be the seat of the affection, the disease is apt to be considered at first as tuberculous. But, atter a time, the solidification is found to diminish, and, finally, at the end of one or two months, it has disappeared, the normal signs return, and the patient is discharged well. Such a listory is of rare occurrence, but examples have fallen under my observation.

The name chronic pneumonitis, however, is applied to an affection quite different from that which exists in the eases just referred to. Exclusive of infiltrated tubercle, or tuberculous pneumonitis, the name is used to denote a morbid condition characterized by hyperthesia, or a morbid growth of the connective tissue which holds together the cells, vessels, and lobules of the lungs. It differs from the pneumonitis already considered in the absence of morbid products within the air-cells or alveoli. It is not an inflammation of the latter together with the bronchioles, but the morbid process is seated in the interstices of these and of the pulmonary lobules; hence the pertinency of the name given to the affection by Rokitansky "interstitial pneumonitis." In its seat and character, it is affiliated with a morbid growth of th 3 neuroglia constituting sclerosis of the brain and spinal cord, with cirrhosis of the liver, and with the fibroid or cirrhotic kidney. Corrigan described the affection, under the name "cirrhosis of the lung," in 1838. It has been called fibroid degeneration and fibrous induration of the lung; the latter term is still not infrequently applied to it.

$\Lambda$ s regards its pathological character, Bastian and others deny that it is inflammatory ; and, with this view of its pathology, the name pneumonitis is, of course, inappropriate. Whether it is to be considered an inflammation or not, will depend on the definition and scope given to this term. In a practical point of view, the question is not one of great importance. Suffice it to say, as has been already said, that the chief anatomical characteristic is an abnormal development of the pulmonary connective tissue.

As thus characterized, chronic pneumonitis may be secondary to other pulmonary affections, more especially tuberculous disease, and it oceurs as the predominant, if not a primary affection; in either case the elaracters which distinguish the lesion are the same. The pulmonary strueture is dense, resisting, and tough. The air is expelled from the vesicles by the pressure of the extra-vesicular morbid growth; and hence is produced more or less contraction of the lung. When incised, it creaks under the knife, and the cut surfaces, instead of presenting a granular appearance, like that in the second stage of acute pneumonitis, are smooth and shining. Little or no liquid escapes after an incision. The color is more or less 
dark or gray from the presence of pig'ment. These changes are doubtless permanent.

Circumscribed chronic pneumonitis, with the foregoing characters, is often found after death in cases of pulmonary tuberculosis. The portions of lung affected are in immediate proximity to tuberculous prociucts or cavities. The pneumonitis leads to dilatations of bronchial tubes, which are liable to be confounded with tuberculous cavities. It occasions, at the summit of the chest, the depression which is so common in tubcrculous disease. It renders the walls of cavities rigid, and is thus concerned in the causation of amphoric resonance on percussion, and amphoric respiration. It is probably, in one point of view, conservative; it forms a barrier preventing the enlargement of cavities by progressive destruction of the substance of the lung. It ocecurs, not only as secondary to tuberculous disease, but around cancerous nodules, extravasations of blood, and abscesses of the lungs. When secondary to these affections, it isolates and forms a barrier between the affected portions and healthy lung, preventing, perhaps, an extension of disease to the latter.

Chronic pneumonitis, as either a primary affection, or as constituting a particular disease, claims somewhat fuller consideration. It is almost always unilateral, and the whole, or the greatcr part, of one lung is apt to be affected. After a time, it leads to considerable, and sometimes to great contraction of the affected side, the external appearances being similar to those which often follow recovery from chronic pleuritis. The heart is dislocated by suction, as it is in certain cases of pleuritis after the absorption of the effused liquid. Plenritic adhesions are generally found after death-another point of similarity to chronic pleuritis.

Bronchicetasis, that is, dilatation of the bronchial tubes, is frequently, but not constantly, associated. Saccular dilatations from the size of a pea to a hen's egg and more or less numerous, are found usually in connection with cylindrical dilatations. The cavities which are found, however, are not always due to bronchicctasis; they are sometimes an effect of circumscribed gangrene, and of molecular disintegration clue to inflammation developer secondarily to the interstitial growth. The bronchiectasis has been attributed to the traction upon the bronchial tubes produced by the morbid growth, to the accumulation of mucus within the tubes, and to an expansion, compensative of the shrinking of the lung, caused by the dilatation of the chest in the inspiratory acts. The latter enters into the rationale, probably, more largely than the two former explanations.

The affection is of very rare occurrence. Its causation is obscure. In an exceedingly small proportion of cases, it has been preceded by acute pneumonitis; but in these cases it is perhaps most rational to regard the succession of the two affections as merely a coincidence. 'This may also be said of the cases in which it has been observed to follow bronchitis, in view of the fact that it is an unilateral disease, whereas bronchitis, when primary, is always bilateral ; it is hardly probable that such a sequel of the latter would be limited to one lung. In short, we have no positive knowledge of its etiology. In the majority of cases, the age of patients is under forty years.

The local symptoms of this disease are cough, with more or less expectoration, and want of breath on exercise. The matter expectorated is often notably fetid, and sometimes suggestive of gangrene. Circumscribed gangrene, as already stated, occasionally occurs; but the fetor generally is due to the decomposition of mnco-pus from its detention in the cavities incident to bronchiectasis. Hremoptysis, sometimes slight, but in some 
cases profuse, occurred in more than one-half of the 30 cases analyzed by Bastian.

The disease may exist for an indefinite period without notable emaciation or debility; but, sooner or later, even if there be no complication or intercurrent affection, hectic paroxysms occur, diarrhoa is not uncommon; the appetite fails, with of course a corresponding defect of nutrition. 'The fingers may become clubbed, as in some cases of phthisis and clisease of the heart. General dropsy occurs in a considerable proportion of cases. This is due to dilatation of the right sicle of the heart, an effect of the obstruction of the pulmonary circulation, that is, assuming renal cisease not to coexist.

The physical signs, aside from the contraction of the chest, to which reference has been already made, are dulness on percussion, rarcly flatness; and the resonance which is elicited is frequently purely tympanitic. The latter is marked in proportion as bronchiectasis is a concomitant lesion; and, of course, it is most marked when the dilated bronchial tubes are free from morbid products. Saccular dilatations may occasion crackedmetal and amphoric resonance. Either ordinary cavemous or amphorie respiration may be heard on auscultation in certain situations. In other situations the respiration may be bronchial or tubular. In some situations the characters of the cavernous and bronchial respiration may be combined. On the other hand, there may be absence of respiratory sound over more or less of the affected side. The vocal signs are those denoting soliclification of lung and cavities; namely, bronchophony, increased vocal resonance, and sometimes pectoriloquy. Bubbling and gurgling rales are often present. The dilatation of the right side of the heart may give rise to a tricuspid regurgitant murmur and to a jugular pulse.

As regards diagnosis, phthisis is to be excluded by the extent of the damage, taken in connection with the fact that the affection is unilateral, and with the absence of the emaciation which generally characterizes tuberculous disease. The affections with which it is most likely to be confounded, by those who take into account physical signs, are chronic pleurisy and carcinoma. Chronic pleurisy, however, after the removal of the effused liquid, does not furnish the signs of solidification, and perhaps cavities, which belong to the clinical history of this disease. Carcinoma of the lung is generally secondaly to carcinomatous disease in some other sitnation; the cancerous cachexia is often marked; there is progressive and perhaps rapid wasting of the body, and the progress of the disease is more rapid.

Chronic pneumonitis, or cirrhosis of the ling, is irremediable, and, sooner or later, it ends fatally. But, in most cases, life is destroyed by some complication or intercurrent affection. An acute pnenmonitis affecting a lobe of the sound lung, or an attack of acute bronchitis, may prove fatal. Tuberculous disease may become developed secondarily. The ability to resist any severe disease is of course impaired. Alone, the disease ends fatally by the slowly progressive exhaustion which it incluces; but, existing alone, it may be borne for a great number of years.

The treatment embraces, as the most important object, maintenance of nutrition and strength. The measures for this object are remedies to improve appetite and digestion, a nutritious and abundant alimentation, climatic and other hygienic influences, which tend to invigoration. All that the physician can aim to do is to aid in rendering the system tolerant of the disease as fully and as long as possible. Palliative measures, having reference especially to cough and expectoration, are indicated, with a view to a remedial influence on the bronchial mucous membrane when the expectoration is abundant. The inhalation of the vapor of turpentine is 
useful. Carbolic acid vapor or spray may be inhaled if the matter of expectoration be fetid.

\section{Brown or Pigment Induration of the Iungs.}

The lesion thus entitled and described by Virchow in 1847 , is referable to the long-continued passive congestion incident to obstruction at the mitral orifice of the heart. The appearances, as described by Virchow, are as follows: The lungs do not collapse on opening the chest as when they are healthy. They are compact, heavy, inelastic, crepitating on pressure but little; and they frequently have a peculiar yellow, brown, or reddishbrown color. On section, numerous red points, dark colored at the centre and lighter at the edges, are seen, and, also, spots which are brown, rusty, or black. In the interspaces the tissue is yellow, orange, or rust-colored. The microscope shows the parenchyma to be normal, with extravasated blood in the different stages of transformation into pigment.

This lesion has been studied by Dr. Francis Delafield, one of the curators of Bellevue Hospital. ${ }^{1}$ He found it more or less marked in 20 out of 60 cases in which there were cardiac lesions involving obstruction of the pulmonary circulation. He describes the pulmonary structure as dense and resisting pressure likc compressed lung, containing but little air, and very little blood or serum escaping on section. Under the microscope four pathological conditions were observed, namely, new pigment, hypertrophy of the walls of the air-cells, dilatation of the capillaries, and a morbid inerease of cellular elements within the alveoli. No extravasations of blood were observed. Dr. Delafield considers the filling of the alveoli with new cells as an important element in the lesion, although it is the one which has attracted the least attention.

'The symptoms denoting the lesion are not definite, but it must increase the dyspnœa which is connected more directly with the cardiac disease.

\section{Anthracosis, Siderosis.}

The pigment in the lesion just noticed is derived from the blood. It is, therefore, of autochthonous origin. Black pigment, however, may consist of particles. of coal derived from the atmosphere. The pigmentation which is more or less marked in the lungs of aged persons is measurably, thus, of external origin. The lesion caused by the inhalation of coal dust is called anthracosis. It is especially marked in the lungs of coal mincrs. The particles of coal, carried by the current of inspired air into the air vesicles, or alveoli, penetrate the tissues; and the microscope shows their presence in the areolar structure and in the bronchial glands. By their microscopical characters the carbon-pigment is readily distinguished from that derived from the blood; and sometimes the particles of coal are sufficiently large to be recognized by the naked eye.

Anthracosis, within certain limits, is hardly to be considered as a disease. The particles of coal may be innocuous, and, if the quantity be not large, there is no appreciable diminution of the respiratory function. On the other hand, by mechanical irritation, they may occasion interstitial pneumonitis, and, perhaps, sometimes, in circumscribed situations, suppurative inflammation of the pulmonary parenchyma. Suppuration of bronchial glands is an occasional effect, and, oftener, their destruction by atrophy. 
The term Siderosis denotes the accumulation in the pulmonary structures of the oxide of iron. This forms a very fine, brownish-red dust, and it is inhaled by workmen employed in dyeing, polishing mirrors, and other mechanieal arts in which it is used. Accumulating in the lungs, it causes a reddish pigmentation. $\Lambda$ German observer, Zenker, has studied its morbid effects, which are essentially the same as in anthracosis. ${ }^{1}$

\section{Lobular Pneumonitis-Atelectasis-Collapse of Pulmo- nary Lobules.}

The phrase lobular pneumonitis might, with propriety, be applied to the pulmonary affection which occurs in cases of pyæmia, and which, from embolism being involved in its causation, is also called "embolie pneumonia." This has been noticed under the heading "circumseribed pneumonitis" (page 195). 'The phrase, moreorer, is among the several names which have, of late, been applied to tuberculons infiltration, or tuberculous pneumonitis. Aside from these applications, it has been used to distinguish an affection oceurring especially in children under six years of age, but not exclusively confined to this period of life. It was deseribed by Gerhard and Rufz, Barthez and Rilliet, and by Valleix as a form of pneumonitis peeuliar to children, although, as regards the morbid appearances, presenting striking points of distinetion from lobar pneumonitis. Subsequent researches by Jörg, Legendre and Bailly, and by Fuchs, tended to show that the affection is not a form of inflammation of the pulmonary parenchyma; but that the appearances snpposed to denote pneumonitis are due to collapse of more or less of the lobules of the lungs. This is the pathological view most consistent with our present knowledge. The collapse of lobules is incident to bronchitis, and, hence, this so-called lobular pneumonitis is hardly entitled to be recognized as a distiuct affection. Other names are broncho-pneumonia, catarrhal pneumonia, atelectasis, and apneumatosis. The two first of these are objeetionable because the primary and essential condition is not an inflammation of the substance of the lungs. The significance of the two latter names will be apparent in giving some aceount of the anatomical characters.

The morbid appearances found after death are as follows: Solidified portions of lung, or nodules, are found, sometimes confined to one side, but almost always disseminated throughout both lungs. In general, these nodules vary in size from that of a pea to that of a filbert; but, exceptionally, a considerable portion of a lobe may be solidified. The number of nodules raries much in different cases. The situations in which they are most frequently found are the lower margins of the lower lobes, and the posterior surfaces of both the upper and lower lobes. They are generally of a dark red color, and they are abruptly defined by the interlobular boundaries. Usually the spaces on the superficies, corresponding to the nodules, are depressed; but sometimes this is not the case, and they may even projeet above the level of the adjacent lobules. This variation is due to a difference as regards congestion of the portions solidified. The solidified lobules are dense, resisting pressure, and sinking in water. The cut surfaces are smooth, not granular, and resemble musele. The pleura over the soliclified lobules, when these are near the superfieies, retains its normal appearance; that is, there is no evidence of pleuritis. The several points of contrast will be apparent on comparing these characters with those of lobar pneumonitis (page 175). The anatomical condition is essen-

1 Text-book of Pathological Histology, by Rindfleisch, 1872. 
tially the same as that of lung condensed by pressure. From the resemblance to muscle, the term carnification is used to express this condition.

'The solidified lobules in the so-called lobular pneumonitis correspond to those found in the bodies of children, dying shortly after birth, in whom complete expansion of the lungs had not taken place. In the latter cases the hard nodules are lobules which have never been inflated. They retain the fotal state. Jörg, who described this condition in 1832, applied to it the name atelectasis, a term signifying absence of inflation. He found that by insufllation after death the soliditied lobules disappeared, the air-cells being filled with air. Afterward Legendre and Bailly demonstrated that, in like manner, the solidified lobules in lobular pneumonitis could be made to disappear by insufflation; and, thus, the identity of the anatomical condition with atelectasis was established. Hence, the term atelectasis has been used to denote both congenitally solidified lobules and a return, after birth, of more or less of the lobules to a foetal state by collapse. The term "acquired atelectasis" is sometimes used to distinguish the latter. The term apneumatosis, signifying absence of air, properly embraces solidification, both from collapse and from the persistence of the fotal state, in other words, congenital and aequired atelectasis. This term was first used by Fuchs, in 1849 , and is adopted by Graily Ilewitt.'

Evidently the name lobular pneumonitis is incorrectly applied, if the collapse of pulmonary lobules accounts for all the morbid appearances. It appears, however, that the solidified lobules, often, if not generally, afford evidence of inflammation. This evidence consists in the presence of desquamated epithelium and leneocytes within the collapsed air-cells, without the presence of filmious exudation. Hence, the name catarrhal pneumonitis, which is used by some writers to distinguish a pneumonic inflammation without exudation, the name croupous pneumonia being used to denote inflammation with exudation. The pneumonitis, however, there is reason to believe, is secondary to the collapse. The collapsed lobules become eongested, and inflammation follows. The collapse, therefore, not only occasions the gross appearances, but it gives rise to the pneumonitis when this occurs. Under these circumstances, the names collapse, acquired atelectasis and apneumatosis are more appropriate than either lobular or catarrhal pneumonitis.

For a fuller account of atelectasis and collapse of pulmonary lobules, the reader is referred to works treating of the diseases of children. The etiological relation of the latter to bronchitis will be briefly considered in the next chapter, under the heading "acute bronchitis in young children."

Collapse of pulmonary lobules will enter into the etiology of pulmonary emphysema. (Vide Chap. VII.)

\section{Pleurodynia and Dorso-Intercostal Neuralgia.}

A brief consideration of these affections is included in this chapter, because, from similarity as regards pain, they are liable to be confounded with either acute pnemmonitis or pleuritis. Prior to the employment of physical exploration, this mistake was by no means uncommon, and it is still made by those who depend on symptoms alone. The term pleurodynia is applied to a painful affection of the muscles entering into the composition of the thoracic walls. Intercostal or dorso-intercostal neuralgia denotes a neuralgic affection of the intercostal nerves.

Both affections may be characterized by pain resembling that of either

1 Reynolds's System of Medicine. 
acute pneumonitis or pleuritis; that is, a lancinating pain felt especially in the act of inspiration. They may be accompanied by a dry cough, which is acutely painful. In cases of pleurodynia there may be febrile movement, and this may accirlentally exist in cases of intercostal neuralgia. How are these affections to be discriminated from inflammation of the pleura, either primary, or occurring as a complication of intlammation of the pulmonary parenchyma?

Intercostal neuralgia, as a rule, is not accompanied by febrile movement. But this affection has a diagnostic criterion which is readily available. It consists in the existence of tenderness usually in three isolated points, viz., behind near the dorsal vertebræ; laterally in one, two, or three intereostal spaces, and anteriorly in one or more intercostal spaces near the sternum or over the epigastrium. Sometimes tendemess exists in only two of these situations. The tenderness is frequently confined to a very limited space in each situation, a space perhaps small enough to be covered by the finger. On the opposite side of the vertebra, and between the tender points in the three situations, pressure or percussion is well borne. In addition to this diagnostic test, physical exploration fails to discover the evidence of either pleuritic or parenchymatous inflammation, viz., in primary pleuritis a friction murmur in some eases, and the signs denoting pleuritic effusion, and, in pneumonitis, the crepitant rale and the signs of solidification. The affection occurs much oftener on the left than on the right side. Both sides may be affected, but this is extremely rare.

Pleurodynia lacks a similar positive test, that is, the tenderness is not limited to isolated points, but is more or less diffused. The diagnosis, therefore, must rest on the exclusion of intercostal neuralgia by the absence of the evidence just stated, together with the absence of the signs of both pleuritis and pneumonitis. Pain in pleurodynia and intercostal neuralgia is more marked, as a rule, in movements of the body than in the respiratory movements, the reverse being true of the pain in pleuritis and pneumonitis. This remark applies more especially to pleurodynia. The tenderness in both affections varies, being sometimes slight and sometimes exquisitely acute.

Of the two affections, pleurodynia is much the less frequent. Before the diagnostic criterion of intercostal neuralgia hid heen pointed out by Bassereau and Valleix, cases of this affection were doubtless often considered as rheumatic, and hence, cases of the neuralgic affection have apparently increased of late at the expense of pleurodynia. Intercostal neuralgia is of frequent occurrence. Cases are numerous both in private practice and among patients treated at hospitals and dispensaries, but more especially the latter. The poorer classes are more liable to it than those in comfortable circumstances. It occurs oftener among females than males. J'ersons affected are frequently anamic. It is apt to be developed during the progress of pulmonary tuberculosis. It occurs especially as a sequel of intermittent ferer.

The degree of suffering from these affections varies much in different cases. They are sometimes so slight as merely to occasion annoyance, but in some cases the pain is sufficiently severe to shorten the inspirations, thus rendering the respirations frequent, as in acute pleuritis, and oecasioning great distress. In the neuralgic affection, exacerbations of pain are frequiently experienced. They vary greatly in different eases as regards duration, occurring sometimes as transient affections, but in some cases persisting for a long period. Pain and soreness in the chest are sometimes assumed by malingerers, but a ready proof of the reality of the affection is the diagnostic test, viz., the limitation of the tenderness to the three 
points on one side of the chest. This proof is reliable, unless the malingerer have had shrewdness enough to discover that this test is relied upon for the diagnosis.

'The treatment of both affections embraces measures to relieve pain if it be severe or considerable. Opium may be required internally for this object. Local applications may suffice if the pain be slight or moderate, for example, liniments containing chloroform or aconite, or the belladonna plaster. In cases of intercostal neuralgia, I have found quinia an effective remedy, whether the affection be a sequel of intermittent fever or not. The quinia should be given in doses sufficient to occasion slight cinchonism, and continued in such doses for one or two weeks. If anæmia exist, this condition claims appropriate treatment. The citrate of iron and quinia or the tincture of the chloride of iron may be prescribed with reference to this condition. In obstinate cases flying blisters are useful. Valleix considers these as most likely to prove effective. The application of dry cups will sometimes afford relief. Hypodermic injections of a solution of morphia, or of aconite, may be resorted to if the pain be severe, or morphia may be sprinkled upon a blistered surface.

\section{H y peræmia of Lungs, or Pulmonary Congestion.}

The terms hyperæmia and congestion denote an abnormal accumulation of blood in a part, their signification being the reverse of that of anæmia as applied to any organ or structure. The congestion is of two kinds. In one, the over-accumulation is due to an abnormal determination of blood to the part affected; more blood is either sent or attracted to the part than can be readily returned by the veins from the part. This kind of congestion is distinguished as active. Virchow calls it fluxion. In this kind, arterial blood predominates in the congested part. Active hyperæmia is the first appreciable morbid condition in all acute inflanmations. It constitutes a morbid condition, in certain cases, without eventuating in inflammation. In the other kind of congestion, the abnormal accumulation of blood arises from an obstruction to the return of blood from the part affected, the quantity of blood determined to the part not being increased. This is distinguished as passive congestion or stasis. Venous blood predominates in this kind of congestion.

Active congestion or hyperæmia of the lungs, irrespective of inflammation, is rarely, if ever, a primary morbid condition, and is hardly to be considered as an individual affection. It may be produced by increased power of the heart's action, arising either from transient causes, such as the ingestion of alcoholic stimulants and mental emotions, or from simple hypertrophy affecting the right ventricle, the latter being extremely rare. It may be caused by the inhalation of stimulating or irritating vapors. Whenever the circulation in a considerable portion of the lungs is interrupted, more or less abnormal determination of blood must take place in the portions to which the flow of blood is unobstructed, as in cases of emphysema, pleuritis, etc. Virchow calls this collateral fluxion.

Passive hyperæmia or congestion is of more frequent occurrence, but is never primary or idiopathic. It is an effect of mitral cardiac lesions causing obstruction or regurgitation, or both combined. It is also consequent on feebleness of the action of the heart, especially of the right ventricle, as in cases of dilatation or fatty degeneration. It is an important morbid condition as standing in a causative relation to pneumorrhagia, or pulmonary apoplexy, and to cedema of the lungs, affections to be presently considered. (Vide Chapter VIII.) In cases of general dropsy it occasions a dispro- 
portionate and sometimes an alarming amount of hydrothorax. It gives rise to brown or pigment induration of the lungs (sec p. 200).

Passive hyperamia is liable to occur in the dependent portions of the lungs in the course of chronic diseases which occasion general debility or asthenia. 'The accumulation of blood, under these circumstances, is the result of gravitation, as 'when it takes place in the dependent portions of the lungs and of other parts of the body after death; and it may be distinguished by the name applied to the latter, to wit, hypostatic. Hypostatic congestion of the posterior portions of the lungs, in feeble patients confined for a long period to the recumbent posture, frequently gives rise to œdema.

Congestion of the lungs, whether active or passive, compromises the respiratory function, first, by diminishing the capacity of the air-cells, and, second, by lessening the quantity of decarbonized and oxygenized blood returned from the lungs to the systemic circulation. The respirations are increased in frequency, and a sense of the want of air, or dyspnna, is felt, caleris paribus, in proportion to the degree and extent of the congestion. As regards physical signs, congestion, if marked or considerable, undoubtedly causes a certain amount of dulness or percussion; but this is not determinable if both lungs be alike congested, or in cases in which congestion of onc lung follows certain affections in the other side of the chest, as in pleurisy with effusion, pneumo-hydrothorax, etc. If the dulness on percussion be great, either odema or extravasation of blood is superadded. Congestion also undoubtedly diminishes the intensity of the respiratory murmur; but if the murmur be changed in character, that is, if it be bronchial or broncho-vesicular, there is solidification from some cause. It is not probable that simple congestion gives rise to adventitious sounds or rales; nor does it occasion any vocal signs. Dulness on percussion on both sides, and diminished intensity of the respiratory mumur, are effects of the brown or pigment induration; but these effects may not be determinable owing to the fact that both lungs are equally affected.

The treatment must of course, have reference to the causative conditions and the circumstances under which the congestion occurs. Active congestion calls for cardiac sedatives and revulsive measures. Passive congestion is generally cither hypostatic or an effect of disease of the heart. In cases of the latter, the measures are indicated by the cardiac lesions. Hypostatic congestion may be prevented or relieved by changing at intervals the position of the body, so that the same portions shall not be constantly dependent. 


\section{CHA PTER VI.}

\section{BRONCHITIS.}

Acute Bronclitis affecting the Large Bronchial Tubes-Anatomleal Characters-Clinical History -Pathological Character - Causation - Diagnosis - Proguosis-Treatment. Subacute Bronchitis. Acute Bronchitis in Young Children. Bronchitis affecting the small Bronchlal Tubes. Epidemic Bromchitis. Bronchitis with Fibrinous Exudation. Circumseribed Bronchitis. Chronic Bronchitis.

THE preceding chapters have been devoted mainly to the consideration of inflammation affecting the serous covering of the lungs and the lining membrane of the pulmonary cells. It remains to consider inflammation seated in the lining membrane of the bronchial tubes. Inflammation in this situation constitutes the disease called bronchitis. This, in its ordinary form, is the most frequent of the pulmonary inflammations. Ordinary bronchitis is a very common affection in all parts of the globe. A highly important variety of the disease is based upon the section of the bronchial tree affected. In ordinary bronchitis, the inflammation is limited to the large bronchial tubes. An infrequent and a much graver form of bronchitis is developed when the inflammation affects the small tubes. The latter form is generally, but, as will be seen, incorrectly called capillary bronchitis. Inflammation of the bronchial mucous membrane may be acute, subacute, or chronic, and varieties of the clisease are based on these differences as regards the degree and duration of the inflammation. Bronchitis may be primary or secondary, as regards other pulmonary affections; that is, it may be developed as a complication of certain diseases, such as "pneumonitis or tuberculosis, or it may not be preceded by any disease of the lungs. As a complication of other pulmonary affections, it is more limited than when it is the primary affection, and may be distinguished as circumscribed bronchitis. A form of the disease is characterized by the exudation of lymph, and may be denominated diphtheritic bronchitis. Finally, bronchitis occurs as an epidemic, and is then commonly known as influenza. These several varieties of the disease will claim separate consideration, after having considered the ordinary form, viz., bronchitis affecting the large bronchial tubes. The term catarrh has been used to denote inflammation of a mucous structure, giving rise only to a mucous, or a mucopurulent, product. As the term bronchial cartarrh expresses neither more nor less than bronchitis, it is superfluous, and may be dispensed with. Proceeding to treat first of ordinary acute bronchitis, it will be considered as occurring after infantile life, and afterward, certain points relating to the disease as it occurs in young children will be briefly noticed.

\section{Acute Bronchitis affecting the Large Bronchial Tubes.}

Anatomical Characters.-The opportunity of observing the appearances after death is not often offered, as this form of bronchitis does not in itself prove fatal. The mucous membrane differs from serous membranes and the lining membrane of the air-cells in being thicker, softer, much more vascular, and containing follicles which secrete mucus. Owing to 
these points of difference, the anatomical characters of inflammation differ from those which belong to the inflammatory affections already considered. The accumulation of blood takes place in the membrane itself, whereby it is reddened and swelled. The redness and swelling after deatl may be found to be uniform in the affected tubes, or more marked in patches or zones. The appearance with respect to redness after death here, as in other situations, is not to be consiclered as evidence of the condition as regards the amount of accumulation of blood during life, for, ill parts which are open to observation, for example, the conjunctiva, mouth, and throat, redness marked cluring. life is found to diminish or even to disappear after death. The inflamed membrane is more or less softened by inflammation, and the affected tubes are likely to contain more or less of the inflammatory products which were expectorated during life, viz., mucopurulent matter, with predominance of the characters of either mucus or pus. Inflammation below the trachea very rarely leads to ulceration.

In ordinary bronchitis, the inflammation, as already stated, is limited to the large bronchial tubes. Probably in most cases it does not extend beyond the bronchi exterior to the pulmonary organs; but, in cases unusually severe, the larger divisions within the lobes may be involved. The tubes on both sides are equally affected, provided the affection be not incident to an antecedent pulmonary disease. With this exception, bronchitis exemplifies the law of parallelism; it is a bilateral or symmetrical disease. In this respect it differs from pleuritis and pneumonitis.

Clinical History.-Acute bronchitis is generally preceded by inflammation of the mucous membrane of the nasal passages, or coryza. The inflammation commences in the nostrils and travels downward, either affecting, or passing by, the pharynx and larynx in its passage to the bronchial tubes. The period occupied in the passage varies from a few hours to one, two, or three days. In a certain proportion of cases, the bronchial tubes are attacked at once without any affection of the air-passages above.

The symptoms offer marked points of contrast with acute pleuritis and pneumonitis. Pain is not a prominent symptom, but the patient experiences a sense of constriction or tightness, with a feeling of soreness or rawness. These painful sensations especially accompany acts of cougling. The pain is of an obtuse or contusive character, and is situated beneath the sternum.

The attack is rarely accompanied by a distinct chill, but chilly sensations, followed by flaslies of heat, are frequently repeaterl during the carcer of the disease. The appetite may be more or less impaired, bit is not usually lost. Lassitude is complained of, with a general feeling of malaise.

The febrile movement is generally moderate. The pulse has not the frequency and strength which it has in pleuritis and pneumonitis. The heat of the surface is not notably raised.

The cough is at first painful, but not sufficiently so to be suppresser. It is at first dry, the secretion of mucus being for a time scanty. Deep) inspirations, breathing cold air, and the exercise of the voice in singing or speaking excite acts of coughing, which occur in paroxysms, and consist of a deep inspiration followed by a series of expiratory eflorts. If the paroxysms of coughing be frequent and severe, the traction of the diaphragm occasions pain and soreness referred to the false ribs and the ensiform cartilage. The patient feels as if relief would be afforded by a more abundant expectoration, and desires to have the cough loosenerl. The expectoration is at first small, glairy, frothy, and viscid, and occa- 
sionally streaked with blood. In the progress of the affection, after two, three, or four days, the expectoration becomes more abundant, and consists of thick, yellowish, or greenish sputa. The cough is then said to be loose, the acts of expectoration being easier, unattended by pain or soreness, and followed by a sense of comfort. The mucous secretion is rarely sufficient to accumulate in the tubes and occasion embarrassment of respiration. This may happen in young children, in the aged, and in feeble persons. The presence of a collection of mueus in the large bronchi or trachea is felt, and the patient is led to make voluntary efforts of coughing for its expulsion. The inereased amount of mucous secretion and the change in character, denote diminution or resolution of the inflammation.

Respiration is not sensibly affected. No portion of the lung is withdrawn from the exercise of its function as in pleuritis and pneumonitis. Dyspnœa is not produced save in the exceptional cases in which, from feebleness or the want of voluntary efforts of coughing, the mucous secretions accumulate sufficiently to obstruct the bronehial tubes.

The affection is not accompanied by much debility. Patients are generally not confined to the bed, and they may not confine themselves to the house.

The average duration of acute bronchitis is ten or twelve days. In severe cases, after five or six days patients are usually able to be out of doors.

The disease may be divided into two stages. The first stage embraces the period during which the expectoration is scanty, transparent, and viscid. The second stage extends from the time when the expectoration becomes abundant, opaque, and thick, to convalescence.

Pathological Cimaracter.-Acute, ordinary bronchitis is an inflammation affecting a mucous structure, leading to a secretion of mueus and the production of muco-pus in greater or less abundance. Resolution takes place in this situation without the occurrence of ulcerations. It belongs among the symmetrical diseases.

Causation.-Primary or idiopathic bronchitis is supposed to be frequently, if not generally, produced by the action of cold. This is implied in the name by which the affection is popularly known, viz., " a cold." Exposure to cold is supposed to produce this disease by interrupting the eliminative functions of the skin, whereby an increased duty is thrown upon the pulmonary mucous membrane, and by inducing internal congestion. This view of the cansation is inconsistent with the fact that great exposure, in a large proportion of cases, is not followed by bronchitis, and also with the fact that in a large proportion of the cases of bronchitis it is not traceable to any unusual exposure. There is abundant evidence, however, to show that exposure of a portion of the body to a current of air is liable to excite an attack of this disease. In general it is reasonable to conclude that the disease is due to a morbific agent in the atmosphere or some special atmospheric change. This is, of course, to be inferred when the disease prevails as an epidemic; but, when cases are not numerous enough to constitute an epidemic, it is a matter of common observation that a greater or less number of persons are apt to be simultaneously affected. Sometimes the occurrence of cases is connected with an obvious change in weather, but it would be useless to offer any speenlations as to the particular atmospherical condition which stands in a special causative relation to the disease.

Bronchitis occurs secondarily to, and forms an element of, other diseases. It belongs to the clinical history of rubeola or measles. In this 
connection it is frequently acute. It belongs to typhoid fever, but is rarely acute in connection with that disease. Developed in connection with diseases situated elsewhere than in the pulmonary organs, it is symmetrical, as when it occurs primarily ; but, developed as a complication of pneumonitis and tuberculosis, it may be limited to one side, that is, unilateral, and is confined within a circumscribed space.

It may be produced traumatically by the local action of irritating gases received with the inspired breath. Inhalation of chlorine gas will produce it. I have known a severe attack produced by inhaling the fumes of sulphuric acid. In some persons, owing to an idiosyncrasy, the bronchial membrane, becomes inflamed by constituents of inspired air which are innocuous when inhaled by most persons. The powder of ipecacuanha, emanations from newly-mown hay, and from feathers, certain morbific matters in the atmosphere during the summer and a portion of the autumnal season, the nature and source of which are not, as yet, ascertained, and even fragrant odors, will produce bronchitis in those constituted with a peculiar susceptibility of this membrane to be affected by these causes. Frequently, in these eases, to the bronchitis is added asthma, and this idiosynerasy will be noticed in connection with the latter affection. (Vide Chap. VII.)

As a rule, the liability to bronchitis is less in proportion as persons are habituated to free exposure in the open air. It is much more apt to attack persons who spend most of their time in warm apartments than those whose occupations keep them out of doors. Soldiers, surveyors, explorers, and hunters, who are accustomed to sleep at night in tents or in the open air, rarely "take cold," but it is a matter of frequent observation that, after having been for some time accustomed to camping out of doors, bronchitis occurs on resuming comfortable in-door quarters.

Draanosis.-Acute bronchitis is to be discriminated from pleuritis and pneumonitis, more especially the latter. The points, relating to symptoms, which are involved in this differential diagnosis are as follows: The absence of lancinating pain, and the pain which is felt being sub-sternal; the presence of more or less expectoration which is not rusty, but either devoid of blood or containing it in the form of streaks ; absence of accelerated breathing and dyspnoa; absenee of the circumscribed flush of cheeks, the pulmonary symptoms preeeded by coryza, with perhaps soreness of throat and subacute laryngitis. These points are chiefly negative, and they are not sufficient for a positive diagnosis in all cases, because the symptoms belonging to pleuritis or pneumonitis, which are wanting in bronchitis, are by no means uniformly present in cases of the two former diseases. The clinical history, however, in conjunction with the results of physical exploration, generally render the diagnosis sufficiently easy and positive.

The physical diagnosis is based chiefly on negative points. Percussion elicits the resonance of health. The vocal resonance is unaffected. The respiratory murmur may be weakened, but is not otherwise altered. Suppression of the murmur over a portion of the chest may occur temporarily from obstruction of a bronchial tube by an accumulation of mucus. These points warrant the exclusion of pleuritis and pneumonitis. The dry and moist bronchial rales may, or may not, be present at the time when the explorations are made. Their presence and diffusion, more or less, over both sides, are indicative of the existence of the disease, but the absence of the disease is by no means to be inferred because these signs happen to be wanting when the chest is examined. 
Prognosis.-Acute ordinary bronchitis, not associated with other affections, is a dangerous disease only when it occurs in the young, aged, and feeble. The danger, in these classes of patients, arises from the accumulation of the products of inflammation within the bronchial tubes, and from an occasional accident which will be noticed under the head of bronchitis in young children, viz., collapse of pulmonary lobules. The danger then relates to apneea. The disease does not lead to great prostration and danger from asthenia. Exclusive of the classes of patients just named, it is generally a disease of little or no gravity. In the great majority of cases it passes through its course and the recovery is complete, but it may eventuate in chronic bronchitis. Associated with certain other affections, it may give rise to much suffering and danger. Occurring, for example, in patients affected with pulmonary emphysema, it occasions more or less dyspnoa, and it may prove fatal if the amount of emphysema be great. So in cases of spinal paralysis affecting the costal respiratory movements, the accumulation of the bronchial secretions, from inability to expel them by the action of the diaphragm alone, may occasion death by slow apnœa. Prof Alfred Stillé has cited a case of this kind.'

$A \mathrm{~s}$ a rule, the disease has no tendency to induce either pleuritis or pneumonitis, and, contrary to a very general impression, it rarely precedes the development of pulmonary tuberculosis.

Treatment.-There is reason to think that acute bronchitis may be prevented, in some instances, on the appearance of coryza, by a full opiate and diaphoretic. A quarter of a grain of the sulphate of morphia, half a grain of codeia, a proportionate dose of any of the preparations of opium, or ten grains of the powder of opium and ipecacuanha, nay be given for this object at bedtime, accompanied by a hot pediluvium and some warm stimulating drink, such as weak punch or toddy, or an infusion of some of the aromatic herbs, and followed in the morning by a saline purgative. If this plan of treatment do not succeed, it may mitigate the severity of the disease.

If the affection become established, it does not claim very active measures of treatment, in view of the almost uniform tendency to recovery with mere attention to hygiene. Bloodletting is not called for except in some rare'cases in which the patient is plethoric, and the symptomatic febrile movement is umusually intense. Depletion by means of saline laxatives and reduced diet will generally suffice. If the attack be severe, confinement to the bed for a few days may be advisable in order to secure uniform warmth and moisture of the surface. Diaphoresis relieves the inflamed membrane, and, for this purpose, small doses of antimony or ipecacuanha may be prescribed. Patients with this disease are more comfortable when moderately perspiring. These remedies should be continued only during the first stage, that is, prior to the free secretion of mucus. In severe cases, an abundance of moisture in the apartment soothes the inflamed membrane, and renders the patient more comfortable. The Russian bath is palliative, and appears to abridge the duration of the disease.

Opium is thought by many to be contra-indicated in the first stage. It is supposed to interfere with the free secretion of mucus and render expectoration difficult. This is an inference from the effect of opium on the secretions in health; but, so far from these results being produced, opium appears to hasten the second stage. The free secretion of mucus is not

1 Vide American Journal of the Medical Sciences, July, 1866, p. 146. 
the cause, but the consequence, of an abatement of the inflammation; and by contributing to the latter, opium virtually acts as an expectorant. Opium, therefore, is indicated in the first stage of bronchitis, as it is in most acute inflammations. In the second stage, it is only indicated when the cough is out of proportion to the expectoration; that is, when the amount of congh existing is not needed to effect the removal of morbid products in the bronchial tubes. Opium is contra-indicated if, owing to the fecbleness of the patient, the efforts of expectoration are inarlequate to prevent accumulation in the bronchial tubes. 'T'he inhalation of pulverized warm water, or spray, affords relief and promotes the secretion of mucus.

During the acute stage, if pain and soreness of the chest be prominent symptoms, sinapisms or stimulating liniments are useful. These symptoms hardly claim blisters or other active measures of counter-irritation.

The stimulating so-called expectorants are not indicated in the first stage. In so far as they have the faculty of exciting the bronchial mucous membrane, they tend to increase the inflammation. They are rarely indicated in the second stage. If the affection linger and threaten to become chronic, the sulphate of quinia and other tonic remedies, together with a nutritious diet and an invigorating regimen, are the most efficient measures for recovery. I have been led by experience to regard the chlorate of potassa as a useful remedy in both the first and second stage of acute, as well as of chronic, bronchitis.

\section{Subacute Bronchitis.}

The difference, as regards symptoms and therapeutic indications, between the acute and subacute forms of bronchitis, is snch that a passing notice of the latter, under a distinct head, is called for.

Subacute bronchitis is, in common language, a slight cold, beginning usually with coryza, and running the same course as acute bronchitis, but with less severity, and perhaps not involving the same extent of the bronchial mucous surface. The affection is not of sufficient gravity to keep patients within doors, and, in the majority of cases, they do not call upon the physician, but either allow it to run its course, or resort to some domestic remedies.

This form of bronchitis may frequently be rendered abortive by a full opiate at night, given in conjunction with a diaphoretic stimulant. But, if not prevented, it claims only a saline laxative, followed by a little anodyne, such as one-eighth of a grain of the sulphate of morphia every four or six hours in syrup. It is not necessary to enjoin confinement within doors. As it is desirable not to exaggerate, as well as not to depreciate, the importance of diseases, it should be understood, by physicians and patients, that common colds do not tend to eventuate in inflammation of the lungs, or in phthisis. A certain amount of care is advisable, and mild remedies are useful, but it would be injudicious to subject patients to active measures of treatment.

\section{Acute Bronchitis in Young Children.}

Acute bronchitis in young children offers an important peculiarity in the greater liability to an accident to which reference has been already made, viz., collapse of a greater or less number of pulmonary lobules, called, also, atelectasis and apneumatosis (see page 195.)

Collapse of pulmonary lobules is incidental to bronchitis in young children, in consequence of the accumulation of mucus in the bronchial tubes. 
The tubes consisting of branching cylinders diminishing in calibre, it is easy to understand that plugs of viscid mucus, in certain situations, may obstruct the ingress of the inspired air into the air-cells, without obstructing the egress of air from the cells in expiration; hence, collapse occurs of the lobules to which the obstructed tubes are distributed. In expiration the plugs are moved by the current of air from the smaller into the larger tubes, but in inspiration the current moves them into a position in which the progress of air is interrupted. It is supposed that collapse may occur when the current of air in expiration as well as in the act of inspiration is obstructed, the air which is thus pent up in the lobules being absorbed. In proportion to the number of collapsed lobules, the respiratory function is compromised, and danger from apnoea may attend this accident. It is more liable to occur in young children than in adults, because children do not promote expectoration by voluntary efforts, and also because the respiratory acts are less vigorous. It occurs especially in feeble children. It does not, however, belong exclusively to acute bronchitis in young children; it occurs in aged persons, and may take place in those of any age who are constitutionally weak. It is also incidental to chronic bronchitis in children and adults, giving rise, as will be seen hereafter, to emphysema of the lungs.

The occurrence of collapse of pulmonary lobules may be strongly suspected in the course of acute bronchitis, if the respirations become frequent, with dilatation of the alæ nasi, accompanied perhaps with lividity, and if the symptoms and signs of pneumonitis be wanting. Physical exploration does not furnish definite signs of this accident. Relative dulness on percussion may be found on one side, if there be a marked disparity between the two lungs as regards the number of lobules which are collapsed. The respiratory murmur will be likely to be more feeble over the posterior than over the anterior portion of the chest, and, if relative dulness on percussion exist on one sicle, the murmur of respiration will be more feeble on that side. Mucous rales are more or less abundant.

In the treatment of bronchitis in young children, the liability to this accident is to be considered. Mild emetics are indicated with a view to promote the expulsion of mucus from the bronchial tubes. They are admissible because they occasion less disturbance than in adults. They are indicated because in young children the acts of coughing are for the most part purely reflex; that is, voluntary efforts to expectorate are not made. They should not, however, be repeated too frequently, and antimonial emetics should never be employed. Remedies to maintain nausea are contra-indicated on account of their depressing effect; an emetic should be given to produce prompt vomiting, and afterwards nauseant remedies withlield, until a repetition of the emetic be deemed advisable. And, in the mean time, tonic remedies, a nutritious diet, and perhaps alcoholic stimulants, are called for to support the general strength. Opium is to be given with great circumspection, since it may favor the accumulation of mucus in the bronchial tubes. The chief objects of treatment, in short, are twofold: first, to supply the want of voluntary efforts of expectoration by the judicious employment of emeties; and, second, by sustaining measures to maintain the force of the respiratory acts. The iodide of potassium, given pretty freely, has seemed to me to have a curative effect.

\section{Acute Bronchitis Affecting the Small Bronchial Tubes.}

An extension of inflammation from the larger and medium-sized tubes to those of small size, gives rise to the variety of bronchitis commonly 
called capillary bronchitis. This application of the term capillary, however, is not strictly correct. 'The truly capillary tubes, that is, the ultimate subdivisions of the bronchial tree, or the bronchioles, are not the seat of inflammation in this affection. 'They are involved in pneumonitis. The bronchial branches of small size, but not the sinallest, are affecter in so-called capillary bronehitis. The larger tubes are generally first affected, and the small tubes become involved by a more or less rapid extension of the inflammation. The affeetion has but recently come to be understood, and has beretofore been known by various titles, such as catarrhus senilis, peripneumonia notha, bastard pleurisy, suffocative catarrh, etc.

This affection differs widely from ordinary acute bronchitis, as regards its clinical history and danger. It is an exceedingly grave affection, proving fatal, especially when it attacks young children, in a large majority of cases. The danger proceeds from obstruction to the current of air to and from the air-vesicles. The obstruction is incidental to the small size of the affected tubes; the swelling of the mucous membrane, and the presenee of muco-purulent liquid, which, in the larger tubes, do not interrupt the free passage of air during the respiratory acts, here oceasion serious interference, and, as the tubes in both lungs are affected, fatal apnœea is liable to be produced.

The anatomical characters, as regard the mucons membrane, are essentially the same as in ordinary bronehitis. The membrane is more or less reddened, swollen, and softened, and the tubes are more or less filled with muco-pus which escapes in small drops when a section of the lungs is made. The obstruction due to the contents of the tubes may lead to collapse of a greater or less number of pulmonary lobules. Portions of the lungs are apt to become œdematous. A bnormal dilatation of the air-cells of the superior and anterior portions is another effect, and, in some cases, this condition, known as emphysema, becomes developed in a great degree. Niemeyer accounts for this as follows: The forcible exertion of the respiratory muscles is brought to bear chiefly upon the lower part of the chest; the effect upon the upper portion being mueh less. Hence, while the air is expelled from the lower portions of the lung, it accumulates in the upper portions and thus occasions dilatation of the cells. This dilatation may be temporary, disappearing after recovery from the bronchitis. Although the inflammation reaches so near the bronchioles and air-cells, it does not tend to extend into the latter; in other words, the so-called capillary bronchitis does not eventuate in pneumonitis. Cases in which these two affections become combined, if they oecur, are rare exceptions to the rule.

The mechanical obstruction, and the incidental circumstances just stated, afford an explanation of the symptoms which make up the clinical history of this variety of bronchitis; and, as regards its clinical history, it presents a striking contrast to acute bronchitis limited to the large tubes. The respirations are frequent in proportion to the severity of the case. In young children they may be increased to sixty or seventy per minnte. Ityspncea exists in a degree corresponding to the acceleration of breathing. In severe cases it amounts to orthopnœa, the patient keeping the sitting posture, and suffering extremely from the want of breath. The speech is short and jerking; the alæ nasi dilate; the face is congested and swollen, and the countenance expresses anxiety and distress. Lividity is marked in proportion to the extent to which the respiratory function is eompromised. There is great restlessness. The pulse is notably frequent, but not accelerated in proportion to the respirations. Cough and expectoration are more or less prominent; the former being difficult and ineffectual from the want of breath, the latter consisting of muco-purulent matter. Nie- 
neyer describes an expectoration as characteristic of this variety of bronchitis as follows: 'The secretion from the small tubes does not contain bubbles of air ; its specific gravity is greater than that of water, and it has sufticient consistence to preserve the form of the tubes. In its passage along the large tubes it becomes mixed with mucus from the latter which contains air and consequently floats on water. The characteristic expectoration consists of mucus floating on water, with which are connected filaments sinking below the surface.

The symptoms distinguish sufficiently capillary from ordinary bronchitis, but they do not always suffice for the discrimination of this from other affections. It should never be confounded with laryngeal affections occasioning obstruction (simple or diphtheritic laryngitis), for in these affections the voice is affected, the cough is characteristic, and the respirations are labored, but not notably accelerated. Attention to the points just named will show the affection to be seated within the chest. The diseases with which it is most liable to be confounded are pneumonitis, asthma, and ordinary acute bronchitis with collapsed lobules. It may generally be discriminated from these affections by means of physical signs, taken in connection with the symptoms. The resonance on percussion is not diminished in capillary bronchitis, and it may be greater than in health, owing to an emphysematous condition of the air-vesicles. The presence of mucopurulent liquid in the small tubes gives rise to the subcrepitant rale, commingled with coarse and fine mucous or bubbling sounds. As the affection is bilateral, these moist bronchial rales, produced in the small tubes and those of larger size, are heard on both sides, and especially on the posterior aspect of the chest. The respiratory murmur is weakened. I have known the anterior, superior, and middle portions of the chest, in a young child, to become largely dilated, presenting the characteristic deformity of longcontinued and great emphysema, and this appearance to disappear after recovery.

Pneumonitis is to be excluded by the absence of the symptoms and signs proper to that disease. Dulness on percussion, extending over one or more lobes, is wanting. Pneumonitis is generally abruptly developed, and is not, as a rule, preceded and accompanied by bilateral bronchitis. It is rare for pneumonitis affecting but one lobe to occasion so much disturbance of the respiration as takes place in severe cases of capillary bronchitis. The crepitant rale, if it be present, denotes pneumonitis, and, if this affection exist and have advanced far enough for the subcrepitant rale to become developed, the latter is confined to one side only, except in the rare instances in which the pneumonitis is double.

Asthma generally is accompanied with little or no febrile movement. The respirations are not rapid but labored, the labor being especially marked in expiration. They are accompanied by loud sibilant and sohorous rales. It is a paroxysmal affection, the dyspnoea not persisting as in capillary bronchitis ; and, unless the case happens to be one in which asthma is experienced for the first time, the liability of the patient to this affection is known.

The discrimination fiom ordinary acute bronchitis with collapsed lobules is. more difficult. Collapse of pulmonary lobules is incidental to capillary as well as ordinary bronchitis in young children but the extension of inflanmation to the smaller tubes involves, with this contingency, a far greater amount of danger than belongs to cases in which the inflammation is limited to the larger tubes. The differential diagnosis must be based on the gravity of the symptoms, the clearness of the resonance on percussion, and the diffusion, over both sides, of the subcrepitant rale. 
Pleuritis with large effusion may give rise to accelerated breathing, lividity, etc., but this attection is readily cxcluded by the absence of the signs denoting the accumulation of liquid in the pleural cavity.

Capillary bronchitis occurs especially in young children, and, next in frequency, in the aged, but it is occasionally met with in adults. It is always a disease of gravity, but more particularly so at the two extremes of life. In young children and the aged, it proves fatal in a very large proportion of cases. It destroys life, in some cases, very rapidly. I lave known it to prove fatal in less than twelve hours after the symptoms denoted gravity of disease. The average duration in fatal cases in children is from three to five days. The duration is longer in adults, and the disease is much less grave after childhood and cluring middle age. In cases ending in recovery, convalescence becomes established after a duration varying from one to two weeks. Happily, it is by no means a clisease of frequent occurrence even in young children. In the mode of dying, apnnea predominates; and the progress toward a fatal termination is denoted by increasing lividity, the pulse becoming more and more frequent and feeble, the skin covered with clammy perspiration, the labor and frequency of the respirations, together with cough and expectoration, diminishing toward the close of life, from muscular prostration. This variety of bronchitis sometimes, although very rarely, prevails sufficiently to be considered as an epidemic. Thrombosis, or the formation of a clot in the right side of the heart, is an occasional accident.

Treatment.-Bloodletting may be employed at the onset, under those conditions by which the employment of this remedy in other inflammatory affections is to be regulated. It is not admissible in feeble subjects, and in employing this or any other debilitating measure, the physician should not lose sight of the fact that it is important to economize the strength in order that the laborious efforts of breathing may be carried on for a length of time sufficient for the resolution of the clisease. The danger and distress incident to the disease arise chiefly from the deficiency of fresh supplies of atmospheric air in the air-cells; and if bloodletting be injudiciously practised, the patient suffers from the want of both blood and air. The importance of economizing strength should also enforce circumspection in the use of saline depletants and sedative remedies. These should not be pushed to the extent of producing prostration. The difficulty consists in mechanical obstruction, which neither these nor any therapeutical measures will immediately remove. The safety of the patient depends on the maintenance of life until the disease passes through its career. The inhalation of oxygen affords marked relief of the dyspnœa. The iodide of potassium should be given freely.

Emetics in young children are advisable for a reason already stated, viz., to supply the want of voluntary efforts of expectoration. Care, however, is to be taken not to repeat them so often as to occasion exhaustion. Laxatives are useful by diminishing the contents of the intestines, and thereby giving space for the more effective action of the diaphragm in respiration. Revulsive applications to the chest are useful, but blisters are of doubtful utility.

The great object being to carry the patient safely through the disease, supporting measures are early indicated, and they are important in proportion as the symptoms denote failure of the vital powers. 


\section{Epidemic Bronchitis.}

For the last four or five centuries, medical observers have noted the occurrence, from time to time, of an epidemic affection characterized by bronchitis. In most countries it is commonly known by the name infuenza, after a term introduced by the Italian writers in the seventeenth century. In France it is called la grippe. As an epidemic, it is remarkable for its extensive and rapid diffusion, sometimes extending, within a brief period, over many different and widely separated countries. During its prevalence a vast number of persons of all ages are simultaneously affected. In some epidemics the fatality has been large, but, in most fatal cases, other affections are dereloped, more especially pneumonitis. The fatality is limited chiefly to the aged and to persons with fecble constitutions.

Influenza differs materially from ordinary acute bronchitis. The bronchial affection is not more intense, but, commencing usually with coryza, the inflammation is apt to extend to the frontal and maxillary sinuses, to the lachrymal ducts and conjunctiva, and into the Eustachian tube. Frontal headache is a prominent symptom. It is accompanied by more marked general symptoms than ordinary bronchitis, viz., chills, febrile movement, lassitude, debility, anorexia, etc. It is apt to end in free perspiration or with diarrhœa. The disturbance of the system is out of proportion to the pulmonary symptoms. It is a general rather than a local disease. The bronchitis is not idiopathic or primary, but the local expression of a constitutional affection. It is an essential ferer, not merely a local inflammation with symptomatic febrile movement. It is a peculiar species of fever, running a brief career, with bronchial inflammation as its anatomical characteristic.

A special cause is involved in the production of this as of every other epidemic disease. The nature, source, and mode of action of the special cause of this, as of other epidemics, are unknown. It has been attributed to ozone in the atmosphere by Schœnbein and others, to animalculæe by Holland, and to cryptogamous productions by the late Prof. Mitchell, of Philadelphia. These different views respecting its causation rest solely on speculation. The special cause doubtless exists in the atmospherc, but it is independent of appreciable atmospheric changes. This fact was exemplified in the Massachusetts General Hospital during the extensive prevalence of this epidemic in 1832. Nearly all the patients in the hospital were affected, although the wards were kept day. and night at a uniform temperature.

The disease is generally mild, but, as already stated, it is liable to lesd to the development of local affections, other than bronchitis, and may in this way prove fatal. Dr. Gardiner's observations go to show that, during the prevalence of influenza, other diseases are usually severe, and the rate of mortality from all diseases is increased. The duration of an attack of influenza is from threc to six days.

In the treatment of epidemic bronchitis the physician must not regard the febrile movement as symptomatic fever. This error might lead to needless depletory measures. Bloodletting is rarely, if ever, required. In healthful, vigorous subjects, brisk purging has seemed to me uscful. This should not be resorted to if the patient be feeble or advanced in years. A side from purgation, diaphoretic remedies and opium will meet the theripeutic indications. Pneumonitis, or other associated affections, will claim appropriate treatment, but debilitating or depressing measures are to be employed with greate: circumspection than if the affections were primary. 
In aged and fecble persons, tonic and supporting measures may be highly important.

\section{Bronchitis with Fibrinous Exadation.}

A variety of bronchitis is characterized by the exudation of coagulable lymph on the inflamed mucous surface. 'Ihis characteristic feature is expressed by the names diphtheritic, pseudo-membranous, plastic, croupous or croupal, which have been applied to it. This variety of bronchitis is frequently associated with inflammation of the larynx and trachea, attended with a similar exudation, constituting the affection known commonly as true croup, and occurring also in certain cases of diphtheria. But reference is here made to bronchitis with this peculiarity, occurring independently of a similar affection of the larynx and trachea. Exclusive of the cases in which it accompanies croup and diphtheria, it is an extremely rare variety of bronchitis. The exudation is the same in character as that which takes place in the affections. just named. It forms a dense, membraniform layer, which at first is closely agglutinated to the mucous surface, but sooner or later, loosened and detached by a suppurative process, is at length expectorated, if the life of the patient be sufficiently prolonged. When expectorated, the false membrane, as it is called, either has the form of patches or strips, or it is thrown off entire from portions of the bronchial tubes, and, when carefully displayed, may be found to consist of casts representing more or less of the bronchial subdivisions, presenting an appearance like boiled maccaroni. A side from the form of cases of the tubes, the fibrinous character of the expectorated matter may be ascertained by microscopical examination and by chemical reagents. It presents the fibrillæ which characterize fibrin or lymph in other situations. Placed in a solution of acetic acid, it becomes swollen, whereas mucus contracts and is rendered more dense.

In a case reported by Dr. Stephen Rogers, of New York, casts of the bronchial tubes were repeatedly expectorated, and in a specimen presented by Dr. Rogers for the museum of the Bellevue Hospital Medical College, the size, length, and number of branches showed that they were formed in the bronchial tubes of an entire lobe, extending from the largest tubes to those of minute size. In this specimen, and in others from the same case, the casts were solid, being composed of concentric layers of fibrin. The patient finally died with symptoms and signs denoting malignant disease within the chest. ${ }^{2}$

The inflammation in cases of bronchitis with fibrinous exudation, is either acute, subacute, or chronic. Occurring in young children, it may occasion sufficient obstruction to compromise seriously respiration, and frequently proves fatal. Affecting adults, however, if not complicated with other grave affections, such as phthisis or pneumonitis, it rarely lcstroys life; and in the majority of cases the recovery is complete. I have met with a case in which it was associated with asthma and emphysema.

There are no symptoms or signs by means of which the diagnosis of this variety of bronchitis can be made out prior to the expectoration of false membrane. When this occurs, the character of the affection is, of course. manifest. This expectoration is preceded and accompanied by violent paroxysms of coughing. It is followed by a muco-purulent expectoration which may be bloody. The symptoms are relieved by the removal of the fibrinous exudations or casts. The expectoration of the latter may be re-

' Vide Trans. Med. Society of the State of New York, vol. for 1866, page 41. 
peated after intervals of days, weeks, months, or even years. The character of the disease, if it prove fatal before the expectoration of false membrane takes place, may be suspected, but the symptoms and signs only show the existence of bronchitis, with obstruction of bronchial tubes, and, perhaps, collapse of pulmonary lobules.

The principles of treatment are essentially the same as in cases of acute or chronic bronchitis without this peculiar feature, and need not be separately considered. Pulverized warm water, or spray, inhaled, will aid in the scparation of the false membrane.

\section{Circumscribed Bronchitis.}

As already stated, bronchitis, when circumscribed, is incidental to some other pulmonary disease. It is developed in pneumonitis, being limited to the lobe or lobes affected, and is the chief source of the expectoration in this disease if it do not go on to the suppurative stage. It occurs in pulmonary tuberculosis, and is limited to the tubes in proximity to the seat of the deposit of tubercle. The bronchitis, in this disease, furnishes the expectoration prior to the softening and discharge of the tubereulous product. The circumscribed bronchitis, in cases of tuberculosis, gives rise to physical signs of importance in diagnosis. As thus developed, bronchitis claims no special attention in a therapeutical point of view.

\section{Chronic Bronchitis.}

Subacute, persisting inflammation of the bronchial mucous membrane, constitutes chronic bronchitis. It is generally a sequel of the acute variety of the disease, but the inflammation may be subacute from the first. It is far less frequent than acute and subacute bronchitis. It occurs much oftener in aged persons than during the early and the middle period of life. In fact, exclusive of the cases in which it is associated with asthma and pulmonary emphysema, it is a rare affection prior to old age.

The anatomical characters are, redness, swelling, and softening of the membrane, these changes being either uniform within the affected tubes, or limited to irregular patches. The affected tubes are found after death to contain more or less muco-purulent matter like that expectorated during life. Contingent morbid changes are, solidified nodules caused by collapse of pulmonary lobules, dilatation of bronchial tubes and frequently dilatation of the air-cells, or emphysema, affecting especially the anterior superior portions of the lungs. The inflammation may be limited to the large, or it may extend more or less toward the small, tubes. The bronchitis associated with emphysema and asthma affects the smaller tubes, not, however, including those affected in the so-called capillary bronchitis.

The symptoms relating directly to the bronchial inflammation are essentially the same as in acute bronchitis, differing only in intensity, but connected with these symptoms, frequently, are others proceeding from associated affections.

Pain is generally wanting, or the patient complains only of a sense of uneasiness or constriction beneath the sternum. If cough be frequent and violent, soreness may be felt at the base of the chest on both sicles, or at the epigastrium, due to the traction of the diaphragm on the ribs. Febrile movement is slight or wanting. A slight exacerbation of fever at night is observed in some cases. The appetite may continue good, or it may be more or less impaired. The nutrition is frequently not much, if at all, affected. The loss of weight, with the continuance of the disease, 
is not marked as it usually is in cases of pulmonary tuberculosis. The patient usually does not present pallor or a notably morbid aspect.

More or less cough always exists. In frequeney and severity this symptom varies much in different cases. It is paroxysmal, violent, and difficult in proportion to the small quantity and tenacity of liquid prodncts within the affected tubes, and in proportion as the smaller tubes are involved. The expectoration also varies much in different cases. It is sometimes abundant, consisting of mucus with serous transudation. When the latter is copious, the affection has been called bronchorrhcea. It may consist of large, solid, greenish or ash-colored sputa, and in some cases small pearl-like masses are raised after much coughing; in cases characterized by the latter, the disease has been called, after Laennec, dry catarrh. The sputa may at times be streaked witl blood. In general, the expectoration is muco-purulent, the purulent characters not infrequently predominating, and sometimes it appears to consist of pure pus. Formerly it was thought to be highly important to determine whether the expectoration was purulent or not, the presence of pus being supposed to denote something more than bronchitis. This is now deemed a point of comparatively little importance, since it is ascertained that pus is formed on a mucous surface. A very copions expectoration of pure pus, however, does point to some other source than bronehitis, such as the discharge of a tuberculous abscess, the third stage of pneumonitis, abscess of liver evacuating through the lung, empyema with perforation, etc. The diversified characters which belong to the expectoration in different forms of the disease are of less value, in a diagnostic view, than was formerly supposed, since the knowledge which has been acquired of physical signs furnishes a far more reliable source of information respecting intra-thoracic conditions. The obvious differential characters by which mucus and pus may be distinguished from each other are as follows: Mucus is transparent or semi-transparent; it is more or less viscid; it is stringy and not miscible with water; it retains bubbles of air and consequently floats on water. Pus is opaque, less viseid than mucus, miscible in water, and in water sinks to the bottom. These differential characters are combined in varying proportions when the matter of expectoration is muco-purulent.

Chronic bronchitis, of course, involves the same canses as the acute in the cases in which it is preceded by the latter. It may be produced and maintained by the inhalation of irritating particles of stone or metal in the exercisc of certain oceupations. It is supposed to be one of the varied local affections attributable to the syphilitic poison. Its continuance, in some cases, is referable to organic affections of the heart, consisting of obstructive or regurgitant mitral lesions. These act by inducing pulmonary congestion. It is an affection incidental to old age, occurring without any obvious causative agencies. It occurs in youth and middle age, irrespective of apparent causes, and is often associated with asthma and emphysema. To these affections it stands in the relation of a cause rather than an effect. In cold latitudes it is sometimes manifestly dependent on climatic eauses, recurring with each successive winter, and disappearing during the summer season, or on removal to a warm climate.

It is not an affection which tends directly to destroy life. In the aged it may sometimes dispose to the development of capillary bronchitis, and thus, indirectly, prove serious. In connection with the feebleness of advanced years, it may lead to collapse of pulmonary lobules, and, in this way, shorten life. Its existence in a patient prostrated with any other disease involves danger from the accumulation of morbid products in the 
bronchial tubes, which may be the immediate cause of death by apnca. It may lead to the development of asthma and pulmonary emphysema, affections which, although not immediately dangerous, diminish the duration of life. A side from these contingencies, the evil to be apprehended relates to the permanency of the affection. It is liable to become established, persisting for years, and, in aged persons for the remainder of life. The existence of chronic bronchitis does not involve an increased liability to the clevelopment of pulmonary tubereulosis, but, perhaps, the reverse.

The diagnosis of chronic bronchitis involves discrimination from pulmonary tuberculosis. This would be difficult, and, indeed, sometimes impossible, were the investigation to be limited to the symptoms; hence, before physical signs were studied, these two affections were of necessity confounded. The importance of making the discrimination is obvious in view of the great difference between the affections as regards prognosis. The differential diagnosis cannot be fully considered without anticipating the consideration of pulmonary tuberculosis. It will suffice to state that it is to be based mainly on the absence of the symptoms and signs which are diagnostic of the latter disease. In other words, it is concluded that pulmonary tuberculosis does not exist, from negative evidence, or reasoning by way of exclusion. The results of physical exploration, as just stated, are mainly to be relied upon, but the absence of symptoms which belong to the clinical history of tuberculosis is also to be considered, such as progressive, marked emaciation, hæmoptysis, and accelerated breathing. These symptoms are wanting in chronic bronchitis. The physical signs in this variety of bronchitis are the same as in the acute form, viz., the dry and moist bronchial rales, the resonance on percussion and the vocal resonance unaffected, the respiratory murmur weakened and sometimes suppressed over a portion of the chest from temporary obstruction of bronchial tubes.

The coexistence of pulmonary emphysema is to be determined by symptoms and signs diagnostic of this affection, which will be considered hereafter.

In the treatment of chronic bronchitis, bloodletting or other depletory measures are very rarely, if ever, indicated. The affection seldom exists under circumstances which render it desirable to lower the powers of life. Counter-irritation, by means of croton oil or stimuiating liniments, is sometimes useful. The diet should be nutritious but not stimulating, consisting of milk, eggs, fowl, fish, and farinaceous articles. Meat, however, is not to be interdicted if the system be enfeebled. Wine and alcoholic stimulants are to be avoided, excepting when measures to support or strengthen the system are indicated. Attention to the surface of the body is important. In a cold or variable climate, woollen or silk garments should be worn next the skin. An undergarment of buckskin or chamois leather, worn over light woollen or silk, affords, during cold weather, great protection, and saves the inconvenience of an undue quantity of clothing. The object is to secure unformity of the temperature of the surface, and to maintain the functions of the skin.

Medicines may be employed with a view to palliation and cure. If cough be troublesome, exceeding the amount requisite for expectoration, soothing remedies are called for. Opium, however, is to be prescribed with circumspection, in the first place, lest the habit of using it be formed: this is a consideration to be taken into account in all chronic affections; and, in the second place, in feeble subjects, and under circumstances in which there may be danger from an accumulation in the bronchial tubes, serious consequences may sometimes follow the blunting of that sense of 
the presence of morbid products which leads to their removal by efforts of expectoration. Moreover, the use of opium tends to impair the digestive powers. For these reasons, other anodynes, such as hyoscyamus, conium, belladonna, and hydrocyanic acid, are generally to be preferred.

As a rule, the remedies which are given as expectorants are not indicated. The nauseant expectorants do harm by their depressing effect, and by disturbing the appetite and digestion. The stimulant expectorants, such as squill, senega, etc., are of doubtful efficacy, and, if not useful, are more or less hurtful.

Certain remedies, however, sometimes exert a curative effect. This is true of the balsam of copaiba. I have known this remedy to act almost as a specific; yet, in many cases, it has little or no effect. The iodide of potassium, also, in some cases is remarkably efficacious; but in other cases it is useless. The chlorate of potassa is sometimes a useful remedy. The muriate of ammonia is in some cases a curative remedy. I have notes of a case in which a cure was promptly effected by this remedy, the affection having existed for ten years. The bromide of potassium, or of ammonium, I have prescribed in some cases with marked benefit. Other remedies which have been found serriceable are the gum-ammoniac emulsion, arsenic in small doses not increased, naphtha, sulphur, and cubebs.

Frequently, marked benefit, as regards the bronchitis, is derived from tonic remedies in conjunction with measures to invigorate the system. The salts of quinia and the preparations of iron are often highly useful. These and other tonic remedies are indicated if the powers of the system be reduced, or the patient be anamic. A generous diet is to be conjoined, and wine or alcoholic stimulants may be taken moderately with advantage. Out-of-door life, in such cases, should be added. This plan of treatment is applicable to a pretty large proportion of the cases of chronic bronchitis ; to improve the general health, and restore vigor, are important objects in the treatment of this, as of any chronic local affection.

It is an object of treatment, in some cases, to diminish the amount of expectoration when this is excessive. For this end, astringent remedies may be prescribed, such as tannic acid, the persulphate or pernitrate of iron, and the preparations of zinc. After a bronchitis, however, has become habitual, especially in the aged, it sometimes appears to be, as it were, an element of health. At all events, patients complain of the discomfort arising from the suspension or diminution of the expectoration, and, for this reason, opiates do not procure relief. Under these circumstances, expectorants are useful as palliatives.

A late improvement in inhalations consists in the pulverization or atomization of liquids; that is, the production of spray, instead of vapor. By means of apparatus adapted to the purpose, not only is water in the form of spray brought more abundantly to the affected membrane, but medicated liquids may be employed more efficiently than when simply vaporized. The inhalation of spray medicated with various remedies has been found useful in cases of chronic bronchitis. The utility of the spray of warm water in acute bronchitis has been already referred to. In chronic inflammation the objects to be effected by the topical application of spray formed from medicated liquids are as follows:-

1. To check profuse expectoration by an astringent effect, and to modify favorably the inflamed condition of the membrane. For these objects, solutions of alum, tannin, sulphate of zinc, perchloride of iron and iodine have been found useful.

2. To promote mucous secretion and expectoration. Solutions of the 
hydrochlorate of ammonia and of the chloride of sodium may be used for these objects.

3. To produce a sedative effect on the inflamed membrane, solutions of opium, conium, hyoscyamus, and cannabis indica may sometimes be employed with advantage.

4. As antiseptic as well as modifying or alterative remedies, tar and turpentine may be employed. ${ }^{1}$

Change of climate is sometimes advisable. A removal from a cold and variable climate to a situation in which the temperature is mild and equable may be attended with marked relief, and perhaps lead to recovery. Benefit may be looked for especially in the cases in which either immunity from the disease or marked amelioration is experienced during the summer season. The trial of change of climate is particularly advisable in the cases in which emphysema is becoming developed in connection with the bronchitis.

\section{CHAPTER VII.}

Emplysema of the Lungs. Interlobular or Interstltial Emphysema. Subpleural Emphysema. Vesicular Emplyysema or Dilatation of the Air.Cells-Anatomical Characters-Clinical History - Patholocrical Character - Causation - Diagnosis - Prognosis - Treatment. AsthmaClinical History - Pathological Character - Causation - Diagnosis - Proguosis - Treatment. Pertussis, Whooping Cough.

T $\mathrm{Y}$ the order of pulmonary diseases, emphysema and asthma naturally follow bronchitis, from the fact that they involve the latter disease in the great majority of cases. Emphysema, elsewhere than within the chest, denotes the presence of air in the areolar or connective tissue. It may denote the same condition as applied to the lungs. A form of emphysema of the lungs consists of the extravasation of air into the areolar tissue which unites the pulmonary lobules. This is distinguished by the namc interlobular or interstitial emphysema. It arises from rupture of the air-cells, which causes a communication between the latter and the interlobular areolar tissue. The accumulation of air in this situation widens the interlobular spaces at the expense of the lobules, and thus diminishes the functional capacity of the latter. The air traversing the areolar tissue at the root of the lung may find its way into the subcutaneous areolar tissue of the neck, and become diffused over more or less of the entire body. This sometimes follows perforating wounds of the chest or injury of a lung by the extremity of a fractured rib. In cases of interlobular eniphysema the air extravasated is speedily absorbed, and recovery soon takes place. There are no indications for treatment except to enjoin avoidance of muscular exertions. Another situation of the same form of emphysema is the subpleural areolar tissue. In this situation the air sometimes detaches the pleura so as to form air-blebs or tumors of greater or less size. These are not infrequently found after death. They are occasionally quite large. I have seen a pleural air-tumor as large as an English walnut. Bonillaud bas reported a case in which a tumor existed

1 Vide On Inhalations in the Treatment of Diseases of the Respiratory Passages, by M. Da Costa, M.D. New York Med. Journal, Sept. and Oct. 1866 . 
as large as the stomach. Of course, in proportion to their size, they interfere with the full expansion of the lungs, and in this way interfere with the function of respiration. They are liable to rupture during life, and pneumothorax, with or without pleuritis, may thus be produced. Cases of pneumothorax, in which recovery takes place, probably exemplify this accident, and an instance, supposed to be of this kind, bas been given in a preceding chapter.

By the term emphysema, however, as applied to the pulmonary organs, is generally meant an abnormal accumulation of air within the air-vesicles. To distinguish this from the other forms just noticed, it is ealled pulmonary or vesicular emphysema. It has also been called rarefaction of the lungs. As the accumulation of air is an effect of a morbid increase of the size of the cells, the simple term dilatation of the air-cells expresses correctly the pathological condition. 'This condition is to be understood as constituting the affection under consicleration.

An emphysematous condition, limited to a few lobules, is frequently incidental to the deposit of tuberele, and occurs in various pathological conncetions. Under such cireumstances, it is not entitled to be regarded as constituting an individual disease. In the latter sense, the term emphysema denotes a greater diffusion of this condition, and, as a rule, the affection is bilateral or symmetrical. Emphysema more or less diffused is sometimes called lobar, and it is called lobular when limited to comparatively a few lobules.

A natomical Characrers.-As a rule, the lungs affected with lobar emphysema are increased in volume. They remain expanded when the chest is opened, not collapsing under atmospheric pressurc, as they do in a healthy condition. Their contractility is thus impaired. They crepitate on pressure less than healthy lungs. The cells are enlarged so as to be plainly visible with the naked eye through the serous covering in the superficial lobules. They may be dried without collapsing, and, on section, fiequently present spaces varying in size from a pin's head to a bean, and sometimes much larger. These are produced by rupture of the intercellular septa and coaleseence of more or less of the eells. In some specimens these cavities are so numerous and large that the affected organs resemble the lungs of the batrachian reptiles. According to Dr. Gairdner, traces of collapsed lobules may generally be found on careful examination. The enlargement of volume may be sufficient to cover the heart and depress it downward and forward toward the epigastrium. The diaphragm may be flattened and depressed, pushing downward the organs situated beneath it. These changes denote emphysema existing in a great degree and extensively diffused. Existing in a less degree and not embracing all the lobules of the affected lobes, the emphysematous portions are distinguished by their prominence, dryness, and exsanguine appearance. The brouchial tubes are sometimes dilated. Evidence of coexisting bronchitis is generally present. The right side of the heart is usually dilated if the emphysema be extensive. The emphysema is rarely equal in the two lungs, and the predominance is usually on the left side. The upper lobes are much oftener affected or affected to a much greater extent than the lower lobes, and the affection is more marked in the anterior than the posterior portion of these lobes.

In some cases the emphysematous condition appears to be due chiefly to destruction of the intercellular septa from atrophy, and the volume of the lungs instead of being increased may be notably diminished. Jenner calls this "small-lunged emphysema," and the ordinary form "large-lunged 
emphysema." The emphysema in these cases is peculiar to aged persons, and has been distinguished as senile emplysema. Other names are atrophous emphysema, and atrophy of lung. "Dilatation of the air-cells" is not an appropriate term as applied to this form of emphysema. The aircells are not dilated, but the enlarged spaces arc caused wholly by the coalescence of cells.

Emphysemi, developed in a person free from tubercle, undoubtedly diminishes the liability to tuberculous disease. The two affections are rarely associated, exclusive of the cases in which pulmonary lobules in the vicinity of tuberculous deposits become secondarily emphysematous. In these cases the emphysema is lobular. A reason for the protection against tubercle afforded by emphysema, may perhaps be found in the diminished quantity of blood within the emphysematous portions of lung, and in the fact that the portions affected with emphysema, namely, the upper lobes, are the primary seat of tubercle in the immense majority of cases. 'The condition of emphysema is, also, to some extent, incompatible with hæmoptysis. Pneumonitis, as a rule, is not developed in an emphysematous patient. I have met, however, with several exceptions to this rule.

Clinical History.-Emphysema, existing to an extent to compromise considerably the respiratory function, is characterized by habitually labored breathing, and inability to take active exercise without suffering from want of breath. The laborious breathing is manifested in both respiratory acts, but especially in expiration. The rhythm of the respiratory acts is altered; the inspiration is shortencd, and the expiration prolonged. As chronic bronchitis generally coexist, cough and expectoration are habitually more or less prominent as symptoms. The cough is usually violent, paroxysmal, and is characterized by a series of prolonged, spasmodic expiratory efforts, as in whooping-cough. The expectoration varies mueh in different cases as regards quantity and character. The acts of expectoration are difficult, and the sputa are frequently accompanied by an abundant, frothy, serous liquid, resembling soapsuds. Not infrequently sputa streaked with blood are cxpectorated.

The affection is chronic, and unaccompanied by febrile movement. The pulse is feeble and the body cool. Owing to an accumulation of blood within the right cavities of the heart, the surface of the body presents venous congestion, and this, together with deficient oxygenation of the blood, may give rise to a cyanotic hue. In extreme cases, lividity is marked. The paroxysms of coughing are accompanied with great congestion of the face and turgescence of the cervical veins. Symptomatic phenomena, aside from those referable to respiration and circulation, are accidental. The appetite and digestion may not be notably impaired; the body, for a considerable period, may be well nourished, but, after a time, slow, progressive emaciation takes place. The countenance has an expression of distress, which, conjoined with tumidity, and a dingy or livid hue, renders the physiognomy somewhat characteristic. At an advanced period anasarca may occur as a consequence of dilatation of the right side of the heart. Albumen in small quantity may be found in the urine, this being an effect of congestion of the kidney ; and congestion of the liver and spleen may render these organs appreciably larger than in health.

These symptoms accompany the affection when it exists to an extent to compromise considerably respiration. Existing in a moderate or slight degree, there is no habitual want of breath nor labor of breathing. But the respiration is disturbed by active exercise; the patient finds himself short-winded if he attempt to run or walk fast, and this difficulty is appa- 
rent, also, in speaking, singing, and especially in paroxysms of laughter. Cough and expectoration are usually present, having the same characters as when the affection exists to a greater extent, but less marked. Congestion, tumidity, and lividity are present, not habitually, but only during paroxysms of coughing or any violent physical exertion. The functions of the body generally may present little or no disturbance. Different cases exemplify all gradations between the two extremes, as regards the symptoms belonging to the affection.

Most persons affected with emphysema are subject to paroxysms of labored respiration and dyspnoea, which are due to another affection, viz., asthma. The two affections-emphysema and asthma-although so often associated, are, pathologically, distinct, and the latter will be considered under a distinct head. Acute ordinary bronchitis, occurring in a person affected with emphysema, occasions symptoms of embarrassed respiration, which do not belong to the clinieal history of bronchitis occurring in a healthy person. The reason of this is to be found in the pathological character of emphysema.

The development of emphysema is generally slow. In the majority of cases, the previous history shows the commencement of the affection, as indieated by defieiency of breath on exercise, to be dated back many years, frequently extending to childhood, and not infrequently beyond the recollection of the patient. Exceptionally, it may be developed with rapidity. I have known it to occur, in a marked clegree, during the progress of capillary bronchitis. The affection has no fixed duration, usually continuing, and progressively increasing, during the patient's lifetime.

Patrologrcal Character.-Exclusive of the senile or atrophous variety, emphysema is a lesion which consists in permanent dilatation of the air-cells, with, frequently, more or less destruction of the cell-walls and coalescence of the cells. In so far as destruction of cells takes place, it is obvious that the area of the respiratory surface is diminished, and the function of respiration in this way compromised. The dilatation of the cells involves a loss of the elasticity of the lungs, so that the accumulation of air within the cells offers an obstacle to the contraction of the chest in the act of expiration. Consequently, the air in the cells is not sufficiently renewed for the purpose of respiration; the air stagnates in the cells. In this way the affection compromises the respiratory function. The distension of the cells involves pressure upon the terminal branch of the pulmonary artery, and in this way the pulmonary circulation is obstructed. This obstruction is further increased by the diminished activity of the respiratory function. The obstruetion to the pulmonary circulation occasions an overaccumulation of blood in the right cavities of the heart; hence the venous congestion of the face and neck, and, to a greater or less extent, of the surface generally, with, in some cases, the phenomena of cyanosis. Hence, too, sooner or later, supervenes dilatation of the right side of the heart.

The pathological character of emphysema furnishes an explanation of the symptoms which make up the clinical history of the affection. When the respiratory function is so far compromised that the functional capacity of the lungs is insufficient while the respiratory acts are performed without effort, the want of breath is felt and the respiration becomes labored. But if the emphysematous condition be slight or moderate, no difficulty is experienced habitually, because there is still functional capacity enough for ordinary respiration. The quantity of lung in health exceeds the amount required; there is a reserve provided for any unusual demand on the respiratory function, as in muscular exercise. If, therefore, the 
extent to which the respiratory function be compromised do not exceed the amount of lung held in reserve, no difficulty is experienced, save when an extra demand is made on the function. Under these circumstances, exercise, which involves an extra demand, cannot be taken without the want of breath being felt. The patient, who is habitually using the lungs to their fullest functional capacity, is disturbed by any interference with respiration. Ordinary bronchitis, which in a healthy person only requires a little of the reserved lung to be brought into play (a fact of which the patient is not conscious), occasions, if there be no lung in reserve, difficulty arising from the slight diminution of the calibre of the larger bronchial tubes which is incident to this disease; hence, patients with emphysema existing to much extent suffer from dyspnœa and labored breathing in ordinary bronchitis. The cough in connection with emphysema arises from the coexisting bronchitis; and the difficulty of expectoration is in consequence of the impaired elasticity of the lungs, the expiratory efforts in coughing not being brought to bear readily on the contents of the bronchial tubes.

The venous congestion of the face and surface generally is due to obstruction caused by over-accumulation of the right cavities of the heart. The lividity is, in a great measure, due to the same condition, but in part, perhaps, to deficient oxygenation of the blood. The smallness and weakness of the pulse also proceed from an obstruction to the free passage of the blood through the pulmonary circuit.

Causation.-The mode in which dilatation of the air-cells is produced has given rise to much discussion. Laennec, who was the first to describe distinctly this lesion, attributed it to obstruction of the bronchial tubes from the products of bronchitis. Regarding the inspiratory act as more powerful than the expiratory, he supposed air to be forced through imperfectly obstructed tubes in inspiration, and to remain imprisoned in the cells in consequence of the want of sufficient expulsive force in expiration. The dilatation, according to this theory, is produced by the rarefaction of the imprisoned air by heat, and by the reaction of the force of expiration in conghing, etc., upon the cells already filled with air. This theory is certainly not tenable in view of the fact that, of the two acts of respiration, the expiratory is a third more forcible; and still more, when voluntary and spasmodic efforts are brought to bear upon it. The more powerful the efforts of expiration, in coughing, etc., the less ought to be the liability of accumulation of air in the cells communicating with obstructed tubes. An explanation of the mode of production has been advocated by Dr. Gairdner, of Glascow, which differs essentially from the theory of Laennec. Gairdner attributes the affection to obstruction of bronchial tubes incident to bronchitis. But he supposes that the obstruction from collections or plugs of mucus leads to collapse, instead of dilatation, of the cells which communicate with the obstructed tubes, in the manner already adverted to in the chapter on bronchitis. The current of air in the act of expiration may remove the mucus from its situation, in tubes of small size, into larger tubes, where the obstruction is less; but the effect of the inspired current will be to carry it back from the larger to the smaller tubes, the mucous plugs thus acting like a ball-valve upon the orifice of a syringe, obstructing the entrance of air to the cells, but permitting the escape of air from the cells. Moreover, owing to the greater force of the expiration, the expired air may be driven past obstructions which arrest the current of air in inspiration. Collapse of more or less of the pulmonary lobules being thus produced, a larger amount of air enters the cells 
communicating with unobstructed tubes, and these cells become consequently dilated. The clilatation of cells, in other words, is supplementary to the diminished volume of the lungs due to collapsed lobules, and is produced, under these circumstances, in the act of inspiration. In support of this explanation, Gairdner states that observation shows the tubes connected with emphysematous lobules to be unobstructed, and that close examination of lungs affected with emphysema will show the traces of collapsed lobules. Moreover, experiments of introducing into the airtubes, in inferior animals, forcign bodies, such as shot and wads of paper, have been found to lead to collapse of lobules connected with the tubes in which these bodics become lodged, and dilatation of lobules connected with other tubes. This explanation will account for the production of the emphysematous lobules so frequently found in proximity to tuberculous deposits, the latter inducing, in the first place, by collapse, diminished volume of the portion of the lungs in which they are situated.

There is reason to belicve that by prolonged, forcible efforts of expiration, as in long-continued exertions at lifting or pulling, or in voluntarily blowing throngh a partially obstructed tube, in the manner which has been to some extent practised as a means of preventing and curing consumption, the elasticity of the lungs may be diminished and permanent dilatation produced. It is well known that the chest may be in this way considerably enlarged, and deficiency of breath on exercise is sometimes a result. Expiratory efforts thus may sometimes co-operate with collapse of pulmonary lobules in the causation of emplyysema. The paroxysms of whooping-cough and the labored efforts of breathing in attacks of asthma may be more or less involved in the causation.

The mechanism, as explainerl by Mentlelssohn, and adopted by Niemeyer and others, to which reference has been already made, ${ }^{\prime}$ is as follows: During violent acts of coughing the glottis is contracted and offers an obstacle to the free escape of air; and the muscles involved in the acts of coughing exert their effect especially upon the inferior portion of the chest. Under these circumstances, the air not escaping through the glottis distends the upper lobes of the lungs, and this distension, often repeated, leads to dilatation of the air-cells in these lobes. This explanation accounts for the fact of the upper lobes being especially the seat of emphysema, and that the anterior portions of the chest, in some cases, project beyond the limits of a forced inspiration in health. It is obvious that this explanation applies to the prorluction of emphysema by forcible acts of expiration produced otherwise than in coughing. Accepting this explanation, the fict that emphysema, as a rule, is more marked in the left than in the right upper lobc, may be thus accounted for: In coughing and all forcible expiratory efforts the lower portion of the chest is more contracted on the left than on the right side, owing to the presence of the liver on the latter side.

The diminution of the elasticity of the ribs and costal cartilages, incident to advancing age, favors the production of emphysema. This change involves diminished extent and force of expiration, with undiminished, if not increased, power of inspiration. The result is, when the chest is expanded in inspiration, the lungs already contain a surplus of air. The lessened clasticity of the lungs in old persons has the same effect, keeping the organs permanently expanded.

In the production of senile, or atrophous emphysema, the point of departure appears to be atrophy and consequent rupture of the cell-walls,

- Vide chap. on Capillary Bronchitis. 
leading to coalescence of more or less of the cells. The atrophous condition of the cell-walls was attributed by Rainey to fatty degeneration. Other observers, however, have failed to find sufficient evidence of this degenerative change.

Clinical observation shows that, in the great majority of cases, emphysema is not only accompanied, but preceded, by bronchitis. It can hardly, therefore, be donbted that, whatever may be the modus operandi, bronchitis stands, in some way or other, in a causative relation to the affection.

Emphysema is to be reckoned among the hereditary affections. This fact was first established by the late Dr. James Jackson, Jr., of Boston, who found that in 18 ont of 28 cases, one or both parents had been similarly affected. Dr. Fuller found this to be true of 26 out of 43 cases. ${ }^{1}$ An analysis of a larger number of cases with reference to this point is desirable.

The mection may be developed at any period of life. In a large proportion of cases its commencement is in infancy or childhood. Its slowly progressive increase explains the fact that patients frequently attain to middle life before the affection has advanced sufficiently to occasion great inconvenience. In a certain proportion of cases it is developed in old age, in these cases the anatomical characters consisting chiefly in destruction of the cell-walls from atrophy.

Diagnosis.-The symptoms described under the head of the clinical history are of a character to render the existence of the affection probable in the cases in which it exists sufficiently to compromise considerably the respiratory function. The probability of the existence of the affection is increased by the coexistence of asthma in a large proportion of cases. The physical signs, however, render the diagnosis positive in these cases; and in the cases in which the affection exists in a moderate or slight degree, the signs are indispensable to the diagnosis. In the latter class of eases, if asthma have not become developed, patients are often supposed to have phthisis by those who rely exclusively or mainly on the symptoms in making the diagnosis. I shall content myself here, as in treating of the diagnosis of other pulmonary affections, with giving a synopsis of the signs, referring the reader for a fuller exposition to works which treat at length of physical diagnosis.

The enlarged volume of the upper lobes of the lungs, in certain cases of emphysema, gives rise to distinctive signs obtained by inspection. In the cases in which the characters of the "emphysematous chest" are strongly marked, the diagnosis may be at once made with the eye. The upper and middle thirds of the chest, on its anterior aspect, are expanded, and present a globular or barrel-shaped form. This projection extends from the summit to the sixth rib. The obliquity of the ribs is diminished, and the angle formed by the junction of the ribs with the costal cartilages is more obtuse than in health. The inferior portion of the chest is relatively and actually contracted. The dorsal spine becomes curved anteriorly. In extreme cases, these changes amount to a deformity which is highly characteristic. The superior costal respiratory movements are lessened, and in laborious breathing the ribs and sternum are raised together, as if they formed a solid bony case. The lower anterior portion of the chest is contracted, and the soft parts above the clavicles and sternal notch are depressed in inspiration. The chest is drawn perma-

1 Diseases of the Chest. London, 1862. 
nently upward by the sterno-cleido-mastoid muscles and the scaleni, so that the neck appears to be shortened. 'The upper part of the chest, during the act of expiration, is in the same eondition as at the end of a forced inspiration. The intercostal depressions are usually strongly marked. The heart's impulse is frequently seen and felt in the epigastrium, and is not appreciable in its normal situation.

These visible changes are present in cases in which the emphysema exists to an extent to compromise considerably the function of respiration, and in which the volume of the lungs is notably inereased. 'The signs are more marked on the side in which the emphysema is greatest; this side being, in $\mathrm{my}$ experienee, much oftener the left than the right. If the emphysema be comparatively slight or moderate, the size, form, and movements of the chest will not be sensibly altered. An abnormal relative fulness, however, may be observed on one side below, and perhaps above, the clavicle, with deficient superior costal movement. 'This disparity of the summit between the two sides, as regards size and mobility, is to be distinguished from a similar disparity due to contraction. of one side from tuberculous deposit.

Pereussion elicits an abnormally intense resonance, the character of the resonance being altered. The vesicular and the tympanitic quality are combined, that is, the resonance is vesieulo-tympanitic. This vesiculotympanitic resonance is more marked on the side corresponding to the lung which is most emphysematous. The exaggerated resonanee being usually greater on one side, care must be taken not to consider the resonance diminished on the side in which it is least exaggerated. The pitch of the resonance is always higher on the side which yields the greater resonanee, whereas, if the disparity be due to diminished resonanee or dulness on one side, the pitch is higher on that side.

The murmur of respiration, as a rule, is weakened in proportion to the degree of the emphysema. If the atfection exist to such an extent that the respiration is habitually labored, the inspiratory sound may be shortened (deferred) and the expiratory sound prolonged. 'The expiratory sound, however prolonged, is lower in pitch than the inspiratory, as in health. The ehanges in intensity and rhythm are the only modifications proper to emphysema. Not infrequently, over portions of the ehest corresponding to parts of the lungs which are not highly emphysematous, the labored efforts of breathing evolve an exaggerated vesicular murmur. 'The weakness and alteration of rhythm are likely to be more marked on the side corresponding to the greater degree of the emphysema. Sibilant and sonorous rales, due to coexisting bronchitis or asthma, are often present in cases of emphysema.

'The normal relation between the two sides, as regards vocal resonance and fremitus, continues. In some cases, owing to the displacement of the heart, the resonance on percussion within the præcordial region is abnormally clear.

In arriving at the diagnosis by means of the foregoing signs, it is to be considered that other affections, such as tubereulosis, pleuritis, pneumohydrothorax, are excluded by the absence of signs which should be found if these affections existed. The signs are also to be taken in connection with diagnostic points embraced in the clinical history, viz., the character of the congh and expectoration, the absence of marked emaciation, notwithstanding the duration of the pulmonary symptoms, and, in many cases, the coexistence of asthma.

In the foregoing account, reference las been had to the ordinary form of emphysema, namely, the form characterized by dilatation of the air-cells. 
The atrophous form, or senile emphysema, offers certain marked points of difference, especially as regards the physical signs on inspection. The size of the chest, instead of being inereased, may be notably diminished. The obliquity of the lower ribs may be much greater than in health, leaving sometimes but little space between the base of the chest and the pelvis. 'The resonance on percussion may be diminished.

Prognosis.-Emphysema, even in the cases in which it is most marked, rarely, if ever, iuvolves, in itself, immediate danger. It is not a fatal affection per se, but it diminishes the power in the system of resisting intercurrent or superadded diseases. It leads to enlargement of the heart, which further impairs the ability to overcome other diseases. But aside from danger to life, it is an affection which, if it exist to an extent to compromise respiration, incapacitates for physical exertion, and renders life uncomfortable. Existing in a lesser degree, it is a source of much inconvenience and annoyance from the deficiency of breath on exereise.

In so far as it involves destruction of cell-walls, and the coalescence of cells, it is an irremediable lesion. And, in the majority of cases, the dilatation of the cells which takes place is permanent; the tendency, in fact, is oftener to increase rather than to decrease. I have known, however, marked improvement to take place, as shown by the physical signs. In a case in which the enlarged volume of the upper lobes was sufficient to give rise to the characteristic deformity in a marked degree, the improvement was so great that the chest nearly resumed its normal form. In so far, therefore, as the lesion consists in dilatation of the cells, it is not necessarily irremediable.

Treatment.-The treatment of emphysema has reference, first and chiefly, to the bronchitis with which it is generally associated. The object is to relieve, and, if possible, remove the bronchial inflammation which stands in a causative relation to the affection, and contributes to its increase. In proportion as the bronchitis is relieved, the condition of the patient is rendered more comfortable, notwithstanding the emphysema continues undiminished; the emphysema is less likely to increase, and it may undergo diminution.

With reference to this object, the measures to be pursued are those indicated in chronic bronchitis not connected with emphysema. The treatment, in fact, so far as this object is concerned, resolves itself into the treatment of chronic bronchitis, which has been considered in the preceding chapter. Trial should always be made of the iodide of potassium. The effect of this remedy is in some cases truly marvellous, but in other cases it produces little or no effect. It may not be amiss to remind the inexperienced practitioner that some persons cannot continue the use of this remedy, in consequence of great irritation of the fauces; and $I$ have met with an instance in which it always produced violent vomiting and general disturbance. The eruptions on the face, to which it frequently gives rise, are an annoyance which may be submitted to, if it exert a curative effect as regards the bronchitis. When the remedy is well borne, it may be continued, in moderate doses, for a long period without any unpleasant consequences. I have known it to be continued in five grain doses, three times daily, for a year, the patient finding that, under its constant use, he was nearly free from all pulmonary symptoms. In this case the emphysema was notably lessened. The chlorate of potassa, the bromide of potassium, and the muriate of ammonia should also be tried if the iodide of potassium prove ineffectual. 
Other measures, already referred to in connection with chronic bronchitis, are to be resorted to, if the remedies just named fail of suceess. Tonic remedies, and the regulation of diet and regimen, with a view to invigorate the system, form an important part of the treatment. If these measures fail, change of climate, if practicable, should be advised.

As there is reason to believe that violent paroxysms of cough contribute to perpetuate and increase the dilatation of the cells, palliative remedies, for this symptom, are called for, provided the bronchitis, on which it depends, cannot be removed. 'The labored effects of breathing in paroxysms of asth:na probably have the same effect; and if this affiection coexist, it is desirable, with reference to the emphysema, to relieve the paroxysms as speedily as practicable. It is important for the patient to avoid, as far as possible, attacks of acute bronchitis, and when they occur they should be cured as quickly as possible. Flatulency of the stomach or bowels and constipation contribute to dyspnoea by mechanically interfering with the play of the diaphragm. They are to be avoided by a regulated diet and appropriate remedies.

Exercise, in so far as it can be taken without inconvenicnce, is not to be interdicted; but exertions which induce labored breathing are to be abstained from. Prolonged efforts of expiration, as in public speaking, singing, and straining at stool, are to be avoided.

'The form of emphysena distinguished as atrophous, or senile, admits of no permanent relief. Ethereal preparations and remedies to palliate dyspnœa, together with measures to support the system, comprise the treatment in these cases.

\section{Asthma.}

The term asthma has been applied,in a loose way, to dyspucea dependent on different pathological conditions. As the name of an individual disease, it should be limited to one pathologieal coudition, viz., obstruction of the smaller bronchial tubes from tonic spasm of the museular fibres forming a part of the anatomical constitution of the tubes. 'This will exclude dyspnœa due to disease of heart, ealled cardiac asthma, or to any affection which does not involve spasm of the bronehial muscular fibres. Asthma and emphysema have been confounded, and, indeed, eonsidered by some writers as one disease ; but, although very often associated, they are distinct affections, and each may exist withont the other. Vesicular emphysema denotes a particular pulmonary lesion, viz., either dilatation of the air-cells, or enlargement by coalescence resulting from destruction of the vesicular septa; asthma is a neuropathic affection, and does not involve necessarily any appreciable lesion. Asthma has no anatomical characters. It is true that, in the great majority of cases, pulmonary lesions are found after cleath, viz., those which belong to chronic bronehitis and emphysema; but this fact only shows the frequent coexistence of these affections with asthma.

Chinical History.-Asthma is essentially a paroxysmal affection. It is characterized by the occurrence of paroxysms, which recur more or less frequently, and hence it belongs in the category of diseases which exemplify the principle of periodicity. The clinical history will therefore consist of an account of the characters which distinguish asthmatic paroxysms, together with the laws of their recurrence, and the condition of health in the intervals.

A paroxysm of asthma may commence without warning, or it may be 
preceded by certain premonitions. The latter are frequently experienced, provided the paroxysm be not produced by some obvions external cause. The premonitions vary in different cases. Patients who are subject to the affection are able often to predict an attack for some hours before its occurrence, sometimes by a sense of drowsiness or heaviness ; sometimes, on the other hand, by an unusual excitation of the mind, and again, by sensations, the signification of which the patient has learned by experience, but which are not easily described. The paroxysm may be slowly developed, that is, an hour or two, and sometimes a longer period, may be required for its full development; or the attick may be sudden, and the intensity quickly reached. It takes place, in the great majority of cases, during the sleeping hours, and generally in the latter part of the night, or very early in the morning.

The paroxysm is characterized by laborious efforts of breathing, prompted by a painful sense of the want of air, or dyspncea. The patient is mable to lie down, but sits with the elbows on the knees, or resting on some solid support, the head thrown backward, the mouth open and gasping with each inspiration. The respirations are not increased, but may be less in number than in health. The inspiratory act is performed with a spamodic effort, and the expiratory act is prolonged and accompanied with a wheezing sound. Speech is difficult from the want of breath, and words are uttered with interrupted efforts. Movements of the body are dreaded, and changes of position are made slowly. Cold air, from its density, affords some relief, and the patient desires the windows to be opened, without regard to chilliness of the body. The face is pallid, and sometimes presents with the palor a cyanotic hue, giving to the face a death-like appearance. Frequently, perspiration is profuse. Limpid urine, in great abundance, is passed frequently, especially at the commencement of the paroxysm. The pulse is small and feeble, due, doubtless, to an aceumulation of blood in the right cavities of the heart, in consequence of obstruction to the pulmonary circulation. The surface of the body is cool or cold. The nostrils are widely dilated. The countenance denotes extreme anxiety and distress. Salter mentions a symptom which he has observed almost constantly, viz., an itching sensation under the chin, which the patient endeavors ineffectually to relieve by rubbing; and the same sensation is frequently felt on the sternum, and between the shoulders.

Paroxysms differ in severity. A patient suffering from a severe paroxysm presents a spectacle more distressing than can be well imagined; to one not familiar with the disease, he appears to be on the point of death. The suffering is considerable even when the paroxysms are so mild as not to present to the spectator a picture of great distress. Their duration differs. They may continue for a few minutes only, or for several days. Usually, after continuing for a few hours, either partial or complete relief occurs. If the relief be complete, or nearly so, tranquil sleep follows, which is peculiarly sweet and refreshing. With the diminution of suffering there is usually cough, and more or less expectoration. The expectoration is frequently small, consisting, in some cases, of a few mucous pellets, semi-transparent, and of a jelly-like consistence. Cough and expectoration, if they have not existed prior to the attack, generally continue for several days afterward, unless the paroxysm has been quite mild and brief. The expectoration is sometimes streaked with blood, and occasionally a true hemorrhage or hæmoptysis takes place.

The paroxysms recur after intervals varying greatly in duration in different cases. They recur in some cases with great regularity after a stated period, and in other cases the recurrenees are extremely irregular. Some 
patients suffer from a diurnal recurrence, and it is remarked by Salter that in these cases the affection is almost always associated with bronchitis or disease of the heart. Recurrence regularly after the interval of a week or a fortnight is not uncommon. Females sometimes have attacks only at the menstrual periods. Some persons experience an attack annually, and it may oceur either in the winter or summer season. Examples of the latter are cases of so-called hay asthma, which will be noticed presently under another head. When the paroxysms recur irregularly, they are sometimes attributable to a particular exciting cause, but in other cases the reason of their recurrence is not apparent.

In the intervals the condition varies, the variations depending on the existence, or otherwise, of associated affections. Asthma is not infrequently associated with persisting chronic bronchitis, and, under these circumstances, more or less cough and expectoration are habitual. Emphysema is also a frequent concomitant, and deficiency of breath on exercise, or constant dyspnœa in proportion to the amount of emphysema, will characterize these cases. The habitual dyspnœa in these cases is due to the empliysema, and is not properly asthmatic. Enlargement of the heart is another affection, not infrequently coexisting, which may give rise to more or less dyspnœa.

Patnological Character.-The pathological character of asthma has been already stated in defining the term as applied to an inclividual disease. It is a neuropathic affection, tonic spasm of the bronchial muscular fibres being induced by a morbid excitation through the nervous system. The exciting causes of the paroxysms doubtless exert their effect through the excito-motory or reflex function of the nervous system. It is true that bronchitis generally exists at the time of the occurrence of the paroxysms, but bronchitis exists often enough without astlıma; and cases occur in which the spasm takes place independently of bronchitis. In the latter cases, the affection has been distinguished as nervous asthma; but, with the pathological view just presented, asthma is always nervous, and these cases differ from the majority only in the absence of eoexisting bronchial inflammation. It is probable that inflammation renders the museular fibres more prone to spasmodic action, and, in this way, is involved in the production of the affection, but not constituting an essential part of it. The distinction between asthma and emphysema has been already sufliciently stated.

Causation.-The occurrence of asthma involves a peculiar susceptibility in the bronchial muscular fibres to take on spasmodic action. This susceptibility does not exist in all persons. It constitutes a predisposition to the affection which characterizes certain constitutions. 'The majority of mankind, no matter to what influences they may be exposed, never experience this affection. The predisposition may be transmitted by inheritance. Of those who become asthmatics, so large a proportion have had parents or progenitors endowed with the same peculiarity of constitution that there must be admitted to be, in some cases, an inborn and inherited predisposition. 'The fact of its being congenital is also shown by the occurrence of the affection not infrequently in early childhood and in ianey, independently of any appreciable external causes. Whether the peculiar susceptibility be always congenital, or whether it be acquired in a certain proportion of cases, it is impossible to say. That the affeetion frequently does not occur until after youth, and is sometimes deferred until old age, and that it follows some other pulmonary affection, such as whooping-cough, 
bronchitis, or measles (which is frequently observed), is not proof that it does not involve an innate peculiarity of constitution.

For the development of the affection, certainly in most cases, in addition to the predisposition, exciting causes are requisite. The exciting causes are varions, and the facts with regard to these show, in some persons, a remarkable susceptibility to particular agencies which upon most persons produce no morbid effect. A striking illustration of an idiosynerasy, in this regard, is the variety of the affection commonly called hay asthma. 'The emanations from newly-mown hay produce, in some persons, coryza, bronchitis, and asthma, and the latter affection may never occur save when produced by this partieular cause. The term hay asthma, however, is applied to cases occurring exclusively during the summer season, although the exciting cause may not proceed from hay. We meet occasionally with persons who suffer, for a certain period during the summer months, with asthma, associated with coryza and bronchitis, and during the remainder of the year they are entirely free from the affection. Sometimes the annual visitations in successive years occur precisely on the same date, and the duration is always about the same. For example, a medical friend of the author is attacked early in September of each year with coryza and bronchitis, and subsequently paroxysms of asthma occur during the night. He continues to suffer from the former affections and the nightly recurrence of the asthma for six weeks. The affections then disappear, and, excepting for the period just stated, his health is excellent. 'The exciting cause is doubtless in the atmosphere, and probably proceeds from something emanating from the vegetable kingdom, but the particular agent remains to be ascertained. Perhaps, in different cases, emanations from different vegetable products are involved. In the case just referred to, relief and exemption are obtained by removing, at the period when the affections occur, to a situation devoid of, and distant from, vegetation. On going to sea, the affections are sure to disappear after sailing a certain distance from land.

The affection known as "Hay Asthma," or "Hay Fever," first described by Dr. John Bostock, in 1819, under the name Catarrhus Astivus, has been studied, as it oceurs in the northern part of this country, by Dr. Morrill Wyman. ${ }^{1}$ There are two forms of annually recurring bronchial inflammation (catarrh) in persons with a peculiar idiosyncrasy. The first is often called the " rose cold," or "June cold," commencing in the latter part of May or early in June, and continuing until early in July. This corresponds with the affection known in England as "Hay Asthma" or "Hay Fever." The other form is called by Dr. Wyman Catarrhus Autumnalis, or Autumnal Catarrh. In this form the affection begins generally in the third or fourth week of Angust, and ends in the latter part of September or in October. It rarely, if ever, continues after the occurrence of black frost. Asthma occurs in a certain proportion of cases only. The local symptoms denote irritation and subacute inflammation of the nostrils and bronchial mucous membrane, together with some febrile movement, and more or less constitutional disturbance. The affection appears not to exist in the Southern States, nor in the colder regions of Canada. Dr. Wyman has collected a considerable number of cases showing that relief may be obtained by going to certain portions of the White Mountain region; also Mount Mansfield, in Vermont, one of the Green Mountains, and the Adirondac Mountains, in the State of New York. ${ }^{2}$

1 Autumnal Catarrh (Hay Fever). New York, Hurd \& Houghton, 1872.

- For further details respecting the parts of the country in which the affection occurs, and those which are exempt from it, together with its natural history, vide Dr. Wyman's work. 
A remarkable idiosyncrasy is exemplified in the author's personal experience. This consists in the produetion of eoryza, bronclitis, and asthma by an emanation from feather beds or pillows. I had suffered repeatedly from attacks of these affections on sleeping away from lome, especially in inns and steamboats, before the source was discorered. All feather pillows and even feather beds do not furnish the special emanation, and the eircumstances on which the latter depends I have not ascertained. The poisonous principle is not comnected with the odor which is sometimes perceived. I eannot determine beforehand whether a strange bed will excite an attack or not. If the feathers are of the (to me) poisonous kind, shortly after retiring, labored respiration, eough, and wheezing conlmence, and progressively increase until I am obliged to get up, when the difficulty passes off in a few hours, leaving a little bronchitis, which continues for a day or two. I have never experienced the least degree of asthma excepting as a result of an emanation from feathers.

Dr. Richard M. Crane, of Hagerstown, Mr., has communicated to me a case under his observation in which attacks of asthma were distinctly traced to sleeping beneath a feather bed made of duck feathers. The eause was not ascertained until after four years of suffering from the affection which had not existed previously, the patient being fifty years of age; and there was no return of the affection after an ordinary feather bed, made of geese-feathers, was substituted for the bed which furnished the causative emanation.

Inhalation of the powder of ipecacuanha in some persons produces similar effects. Several examples of this idiosyncrasy have been reported. On relating my own experience at a meeting of a medical society, on one occasion, a member of the society gave an account of a ease in which the same effects were produced by an emanation from the body of the horse, so that the person was debarred from riding or driving on that account. I have recently heard of another case of this kind. Salter gives several examples in his work on asthma. Cases have been known in which asthmatic attacks were brought on by emanations from the bodies of other animals, as the cat, rabbit, etc.

In persons subject to asthma, the paroxysms are liable to be induced by a variety of exciting causes, but individual cases differ as regards a particular susceptibility to certain of these causes. In some cases the inhalation of dust is particularly apt to bring on an attack. I have known a patient especially susceptible to the dust of grain and that arising when carpeted rooms are swept. The fumes of burning sulphur are apt to produce asthmatic breathing in those subject to the affection. Indigestion, in some cases, proves an exciting cause oftener than anything else. Some can refer paroxysms frequently to constipation. Salter relates a case in which the application of cold to the instep was sure to provoke an attack. Mental emotions sometimes act as an exciting cause. Occasionally in females the paroxysins are habitually connected with the menstrual function.

In the great majority of the cases of asthma bronchitis coexists. In a certain proportion of cases the bronchitis is constant or habitual, and the frequency of the paroxysms depends in a measure on the bronchitis; for, if the latter affection be relieved or removed, the asthmatic attacks become less frequent. The susceptibility to the exciting causes of spasm is increased by the existence of bronehial inflammation. In other cases, the attacks of asthma occur whenever bronchitis, or a common cold, occurs. Finally, climatic influences are involved in the cansation. for it is a matter of frequent observation that the same persons are much more liable to 
recnrrences in some situations than in others. And it is also a matter of common observation with some asthmatics residing in a locality in which they are subject to frequent recurrences, that atmospherical changes are apt to induce an attack.

A sthma occurs more frequently in males than in females, the proportion being as two to one. As regards the periods of life when it may become developed, the following are the results of an analysis of 47 cases by Dr. Salter: It was developed during the first year of life in 9, and from one to ten in 10 cases; from ten to twenty years of age in 8 cases; from twenty to thirty years in 7 cases; from thirty to forty in 6 cases; from forty to fifty in 3 , and from fifty to sixty in 4 cases.

Diagnosis.-The diagnosis in well-marked cases is not difficult. The features of the asthmatic paroxysms are quite distinctive. First paroxysms occurring in young children may occasion some perplexity, but, with proper knowledge and care, the disease should not be confounded with other affections which involve embarrassment of respiration. Capillary bronchitis is distinguished by the frequency of the respirations, the acceleration of the pulse, and the diffusion over the chest of the subcrepitant rale. In asthma the respirations are not accelerated but labored, the pulse is not notably frequent, and the dry bronchial rales are abundant. Laryngeal affections involving obstruction may be excluded by the fact of the voice remaining unaffeeted. The dyspnoa incident to disease of the heart often goes by the name of asthma, but the breathing in this case is not labored, especially the expiratory acts, and accompanied by wheezing, as it is in asthma, and the dyspnœa is more or less habitual. Moreover, the existence of heart-lesions adequate to the production of dyspnoa is determinable by definite physical signs. Asthma and cardiac lesions, however, may be associated. If asthma have existed for some time, it is found usually associated with emphysema, and this association is diagnostic ; yet, it is to be borne in mind that emphysema, sufficient to occasion dyspnœa, which may become greatly increased with a fresh occurrence of bronchitis, may exist without asthma ; in other words, the dyspnœa due to emphysema and coexisting bronchitis is not properly called asthma. In addition to the abundance and diffusion of the dry bronchial rales, the physical signs, during a paroxysm, are enlargement of the chest with diminished respiratory movements, increased resonance on percus sion and suppression of the respiratory murmur.

Prognosis.-A paroxysm of asthma, however severe or protracted, involves no immediate danger to life. Formidable as the affection appears, when a patient is experiencing an attack of great severity, and intense as is the suffering, it is doubtful if a fatal termination ever took place. In view, however, of the suffering incidental to the affection, and its tendency to become confirmed, with an increasing liability to recurrences, its existence must be regarded as a great calamity. If the paroxysms be produced by a particular exciting cause, which, being known, may be avoided, the disease imposes simply more or less inconvenience; but when the paroxysms are liable to be produced by various canses which cannot be guarded against, it is truly a grievous affliction; and it is calamitous, of course, in proportion to the frequency with which the paroxysms recur, and their severity.

Asthmatics are not infrequently long-lived; yet, that it may contribute to shorten the duration of life, cannot be doubted. The labored efforts of breathing in the paroxysms contribute to the development and increase of 
emphysema. During the paroxysms, also, the circulation through the lungs being impeded, the right ventricle and auricle must be unduly distended with blood returned by the systemic veins, and hence the affeetion contributes to dilatation of the right side of the heart. 'The ehances of long life are lessened by these lesions. Moreover, if the paroxysms be frequent and severe, they can hardly fail to impair the powers of life, and diminish the ability to resist other diseases. If asthma secure, to a certain extent, exemption from pulmonary tuberculosis and pneumonitis, this advantage is perhaps overbalanced by the fact that pneumonitis, when it does occur, is apt to prove fatal, and that the supervention of a severe attack of bronchitis is apt to destroy life.

Treatment.-The management of asthma embraces, first, the treatment of the paroxysms, and, second, the treatment in the intervals.

During the paroxysm the objects of treatment are to lessen the suffering and bring the paroxysm to an end as speedily as possible. The measures for these objects have reference to spasm as the essential pathological condition. The measures to relieve spasm are various, each of which proves efficacious in some cases and not in others. Frequently the past experience of the patient is the best guide as to the particular measure which will be most likely to afford relief. In eases of asthma unaccompanied by bronchitis, I have known a full opiate quickly and completely successful; but, in the larger proportion of cases, it will not succeed in cutting short the paroxysm, nor afford marked relief. Of other narcotics, stramonium is best suited to this affection. The usual mode of administration is to smoke the dried leaves or fibres of the root either in a pipe or prepared as a cigarette. It is undoubtedly true that this measure in some persons acts like a charm, and may be confidently relied upon as a prompt and effectual mode of obtaining relief; but, in the great majority of cases, it either produces no effeet, or merely mitigates the severity of the paroxysm. Assafoetida, draeontium, or skunk-cabbage, and the Indian hemp have been found to be sometimes efficacious. The ethers, given internally, are to some extent useful as palliatives, and oceasionally produce complete relief. Belladonna is sometimes signally efficacions, given as recommended by Salter and Ringer, namely, 10 minims of the tincture repeated every two or three hours unless disturbance of vision be produced. The bromides in full doses sometimes afford marked relief.

Marked relief is frequently obtained, and the paroxysm is sometimes cut short, by nauseant remedies, viz., antimony, ipecacuanha, lobelia inflata, and common tobacco. The two remedies last named are especially efficacious in a certain proportion of cases. The common tobacco will be more likely to be successful if the patient be not accustomed to its use. With a view ta the relief of the spasm, these remedies need not be carried to the extent of producing vomiting; if not effectual when nausea is induced, it will be useless, if not injurious, to push them further. I have known a paroxysm to be arrested at once by bloodletting, but this is a measure too potent to be employed except occasionally in plethoric persons.

The inhalation of the vapor of chloroform or ether is a measure of great value in the treatment of asthma. Not infrequently the paroxysms are completely controlled by it, the patient passing, in the space of a few moments, from a condition of great suffering into one of ease and comfort. These cases are among those which afford the most striking examples of the resourees of practical medicine. The dry bronchial rales which, before the inhalation, were loud and universally diffused over the chest, 
sometimes disappear as soon as complete relief is procured by the inhalation, a fact proving conclusively the existence and the cessation of spasm. Unhappily this measure, like the others, is only efficacious in a certain proportion of eases. It should be tried always, provided there be no circumstances to contraindicate it. Its employment should never be intrusted to the hands of the patient. Dr. O. S. Monell, of New York, states that he has succeeded in obtaining relief in an attack of asthma by prolonging as far as practicable the expiratory act ; then waiting as long as possible before inspiring, and retaining the air at the end of the inspiration as long as possible, this plan being continued for fifteen minutes. $\mathrm{He}$ states that he has repeatedly obtained immediate relief in this way, and that he knows of several cases in which the plan has proved successful.'

It is hardly necessary to say that if there be ground to suppose the attack to have been brought on by overloading the stomach or by constipation, an emetic or cathartic is indicated. And, of course, the patient is to be removed from the action of any known exciting cause, such as the emanations from hay, feathers, etc. 'The apartment should be large, high, and airy. Warm and stimulating pediluvia are useful as palliatives. Strong coffee, taken hot, is generally highly useful as a palliative. A nother palliative measure, whieh sometimes proves to be enrative, is to be added. This consists in diffusing throughout the apartment the fumes of burning nitre-paper, that is, bibulous paper clipped in a saturated solution of the nitrate of potassa, and dried. Some patients find great relief from this measure, and oceasionally the paroxysms are arrested by it.

The treatment in the intervals has for its object prevention of the paroxysms. The removal of the predisposition would be the most effectual mode of aecomplishing this object. This, however, is not to be expected, and the object is to be attained by measures having reference to circumstanees which increase or act upon that susceptibility of the bronchial museular fibres constituting the predisposition to the affection.

When, owing to a peculiar idiosynerasy, paroxysms are excited by a particular cause, such as emanations from hay, feathers, etc., avoidance of exposure to the cause, if possible, is obviously indicated. It is doubtful if any remedy will produce insusceptibility to the action of the particular cause, or secure exemption so long as the operation of the cause continues. In the cases of summer asthma which have come under my observation, a great variety of remedies have been tried, but all without avail. The patient must either endure the continuance of the affection for several weeks, or he must seek a situation where he is not exposed to the exciting cause, whatever it may be.

In a large proportion of cases, the liability to paroxysms is more or less favored by the existence of habitual or chronic bronchitis. 'The relief or cure of the latter affection, in these cases, is the immediate object of treatment. The iodide of potassium should always be tried. I have known patients who were rendered comparatively comfortable, as regards the recurrences of asthma, by the use of this remedy. In small or moderate doses it may be continued indefinitely without any apparent injury. I have known from 5 to 10 grs. to be taken most of the time during four or five years, the patient finding that thereby he was protected against a recurrence of the paroxysms. The remedy will be useful in proportion to its curative effect on the bronchitis ; it is, however, useful in some eases in which the coexisting bronchitis is slight, and when no apparent effect upon the latter is produced. The bromides are serviceable in certain

1 New York Med. Record, Ang. 15, 1866. 
cases, and they are well borne by some who cannot take the iodide of potassium without unpleasant effects. The chlorate of potassa is sometimes useful, and the other measures indicated in cases of chronic bronchitis may be employed. 'The arsenical medication, that is, arsenic in small doses continued for a considerable period, has been found in some cases highly useful.

If measures addressed to the bronchitis prove ineffectual, or if the paroxysms recur when bronchitis does not coexist, and the paroxysms be not referable to particular causes which may be avoided, nothing is to be depended on but change of locality. And it is probable that most asthmatics may find some situation in which they will be comparatively, if not entircly, free from the affection. Facts showing the immunity obtained by change of residence are remarkable, as illustrative of the apparent capriciousness of this affection. Persons residing in the country often find relief by living in eities or large towns. A change from the most salubrious part of a city to a part where the atmosphere is smoky and insalubrious as regards the general health, sometimes secures exemption from this affection. A change of apartments from one story to another, or to a different exposure, has been known to prove effectual. I have known a patient to suffer nightly from a severe attack, directly on the seashore, whereas the nights were passsed comfortably a mile inland. There are no fixed laws with respect to the best climate or situation for asthmatics; each case has its own law, which is only to be ascertained by experience. In commencing trials of change of locality, a situation should be selected in which the climatic influences are the opposite of those belonging to the situation in which the patient resides; that is, if he live in the country, the city may be tried, and vice versa; if on the seashore, an inland situation, and vice versa; and so with regard to temperature, moisture, elevation, etc. The principle to be acted on is, that there is a locality in which each martyr to this complaint will suffer less, and perhaps be entirely free from it, and the plan should be to make repeated trials until the desired spot is found. I could eite from the cases which have come under my observation several in which this plan has proved successful. It is to be enjoined, wherever practicable, as soon as it is evident that other measures will not secure the patient against the suffering incident to the frequent recurrence of the atfection.

\section{Pertassis-Whooping-Cough.}

This affection belongs among the diseases of children, and, for this reason, it was omitted in the first edition of this work. Although in the vast majority of cases the patients are children, it occasionally occurs after childhood and at all ages. In the cases in which it occurs after adolescence, it is important that the physician be preparcd to recognize the affection. Moreover, the affection has much pathological interest. I shall, therefore, introduce a succinct account of it in the present edition of this work. The affection has no anatomical characters except those of ordinary bronchitis. Other morbid appearances found after death are due to complications which will be noticed under the head of the clinical history.

Chinicar. History.-The names of this affection derive their significance from certain characteristics pertaining to the congh which will presently be described. The first or the forming stage embraces a period prior to the appearance of these characteristics. The primary symptoms are those of simple coryza and bronchitis. Frequently during this period there is 
nothiug which denotes the affection to be other than a common cold. But, in the majority of cases, the cough is more violent than in an attack of ordinary bronchitis ; the acts of coughing persist for a longer time, and they progressively increase in duration and violence. There is also more or less febrile movement, which is more marked and continues. longer than in ordinary bronchitis. At length the cough becomes distinctly, and in a marked degree, paroxysmal, and the distinctive features of the affection relate especially to the paroxysms. The duration of this first stage varies from two to three days to two to three weeks. After the affection has advanced to the second stage, the patient is generally aware for a few moments previous to a paroxysm that it is impending. A child engaged in play suddenly is quiet, and the countenance expresses appreheusion and distress. The morbid sensations are a sense of constriction, and an irritation in the laryux and trachea. These premonitions are sufficient to arouse the patient when asleep. The paroxysm is denoted by cough which is characterized by a series of violent expiratory acts succeding each other so quickly that the patient is unable to take an inspiration between them. 'The number of expiratory coughing efforts which thus follow without an iuspiration, varies, according to the severity of the paroxysm, from six to twenty. A long and labored inspiration then takes place, giving rise to a crowing somnd evidently due to spasm of the glottis; this is the whoop which enters into the name of the affection. Another series of coughing expiratory acts succeeds, followed again by the sonorous inspiration or whoop; and these alternate acts of coughing and whooping are repeated until the paroxysm ends. The contraction of the lungs by the spasmodic acts of coughing interrupts not only inspiration, but the pulmonary circulation, so that an accumulation of blood takes place in the right cavities of the heart. These effects are shown by notable congestion and lividity of the face and turgescence of the cervical veins. Tear's flow in abundance. The suffering from dyspnca is in proportion to the violence and length of the paroxysm. 'The contents of the stomach are frequently expelled; and, at the close of the paroxysm, more or less mucous secretion is expectorated. In proportion to the violence and length of the paroxysm the patient is fatigued or exhansted. However severe the paroxysm, there is scarcely any immediate danger either from apnoa or syncope. I have never known of an instance of death in a paroxysm. A medical friend, however, has related to me a case in which the breath was lost, and restored by resorting to the procedure known as Marshall Hall's ready method.

The paroxysms, in different cases, differ widely in severity, duration, the degree in which the characteristics are marked, and in frequency of recurrence. When extremely severe, they occasion great distress, and serious incidental events are liable to occur which will be presently noticed. On the other hand, when quite mild they are comparatively trivial. Only one or two of the spasmodic expiratory acts may occur, or they may be repeated so that the paroxysm lasts for several minutes. The whoop is more or less lond and long in proportion to the amount of spasm of the glottis. Although generally present and marked, being, as the name implies, the most characteristic feature of the paroxysm, it is sometimes slight and sometimes wanting. In the latter case, the character of the disease las to be determined by other diagnostic features. A few paroxysms only may occur in the twenty-fours hours, or they may recur during night and day at short intervals. 'They may amount to even a hundred within the twenty-four hours. In almost all cases they occur in greater number in the night than during the daytime, and in some cases they occur 
only at night. Exceptionally they are more frequent during the daytime. The paroxy'sins which occur in sleep are, of course, not produced by any obvious exciting cause. This is also true of paroxysms occurring in the waking hours. Physical exertion and mental excitement, especially the latter, appear not unfrequently to determine the occurrence of a paroxysm. Children are apt to experience a paroxysm whenever a fit of crying takes place. A patient is apt to have a paroxysm on witnessing a paroxysm in another patient, this fact illustrating the power of involuntary imitation.

Various events are incidental to the paroxysms, especially when the latter are severe. One of the most frequent of the incidental events is hemorrhage. Epistaxes is the most common form of hemorrbage. Not infrequently blood flows from the nostrils, in more or less abundance, with every paroxysm; and the loss of blood indueing notable anæmia, this favors the persistence of the hemorrhage. Hæmoptysis is an occasional form of hemorrhage. Blood sometimes escapes from the conjuctiva, or accumulates beneath this memurane. Trousseau cites a case in which a nævus situated on the face was the seat of hemorrbage with each paroxysm. Bluod has been known to be forced from the ears. 'The primary and chief' cause of the hemorrhage is the venous congestion arising from accumulation of blood within the right eavities of the heart. The urine and fieces, or both, are sometimes expelled involuntarily during the paroxysm. Intestinal hernia is sometimes produced. Rupture of air-vesicles is an accident which sometimes occurs, giving rise to interlobular emplyysema; and the air, finding its way along the areolar tissue connected with the primary bronchi and trachea, may produce emphysema of the neck and even extend over the entire body. Dilatation of the air-cells, or vesicular emphysema, is another inciclental event. It is questionable whether this be not due to the bronchitis associated with whooping-cough rather than to the violent acts of coughing; but the latter, at all events, contribute to its production. When the paroxysms recur frequently, and are generally accompanied by vomiting, the system suffers from innutrition. Here is a source of anæmia in addition to the hemorrhages. Convulsions occur in some cases, especially in children, being attributable to the cerebral congestion occasioned by the venous obstruction of the right side of the heart.

In the intervals between the paroxysms, aside from complications or intercurrent affections, the general condition will depend on the frequency of the paroxysms, their severity, and the incidental events. The patient is enfeebled in proportion as the paroxysms are long, violent, and frequent, and in proportion to the amount of hemorrhage, and the interference with nutrition by vomiting. The face is pallid in proportion to the anæmia. The veins of the neck furnish the venous hum, and the arteries a bellows murmur. Cephalalgia is a prominent symptom in some cases, arising from cerebral congestion. The febrile movement, which generally exists, in a greater or less degree, during the first or forming stage, as a rule, disappears when the characteristic paroxysms are established, that is, in the second stage. If it continue into this stage, or if it be reproduced, it is due to an unusual intensity of the bronchitis, or to some inflammatory complication. The face is swollen or puffed, and this, with the pallor, renders the appearance quite characteristic. Certain complications are liable to occur, which add much to the gravity of the affection. Mild bronchitis is a part of the affection. It is almost invariably present.

If the affection be uncomplicated, physical exploration of the chest elicits good resonance on percussion, with. perhaps, the dry or moist bronchial rales. The bronchitis in some cases is unusually acute, giving 
rise to febrile movement and abundant mucous secretions. Collapse of pulmonary lobules is liable to occur in young children. The occurrence of vesicular emphysema (probably dependent on collapse of lobules) has been already stated. The inflammation may extend into the minute bronchial tubes, giving rise to capillary bronchitis. This will be denoted by the subcrepitant rale diffused over the chest, in conjunction with great frequency of the respirations, notable acceleration of the pulse and lividity, the resonance of the chest on percussion not being diminished. Pneumonitis is developed in some cases. Pleuritis with effusion is another complication, which is more likely to occur in adults than in children. 'These siveral pulmonary complications are rendered severe and dangerous by the recurrence of the paroxysms of whooping-congh. Frequently the latter become less violent and frequent when any of the complications just named are developed; so that a premature improvement as regards the paroxysms of whooping-cough may be an unfavorable omen. When whooping-cough is acciclentally associated with some acute disease, such as measles or scarlet-fever, the paroxysins of cough, as a rule, become less frequent and violent; these intercurrent diseases interfere with the natural course of the affection. Finally, whooping-cough appears to determine the development of phthisis in some cases in which the tuberculous diathesis exists.

The duration of the affection is subject to considerable variation. Exceptionally the affection ends, of its own accord, in a few days. These cases are infrequent; but the fact that the affection is occasionally of short duration, when let alone, is to be borne in mind with reference to the supposed efficacy of remedies in abridging or arresting it. It is rare for the affection to end within a period of six weeks. In the majority of cases it continues for a longer period than this, and it sometimes persists for many months. As a rule, it gradually declines before it disappears, the paroxysms by degrees becoming less frequent and severe. Frequently, for a considerable period after the affection has ended, the characteristies are manifested, to a greater or less extent, whenever a bronchitis or common cold is contracted, or congh is excited by any cause. 'Troussean states that the affection continues for a long or short period in proportion to the cluration of the symptoms prior to the characteristic paroxysms; that is, if the latter are delayed the affection will be protracted, but if they are quickly developed the affection is not likely to last long.

Pathological Character.-This remarkable affection consists of three pathological elements. Bronchitis is one of these. 'This is the initial element. It is stated that this element is sometimes wanting, but examples must be exceedingly rare. Another element is fever. 'This is mole or less marked in different cases, but is seldom wanting during the first or forming stage. The fever, as a rule, is out of proportion to the bronchitis, and is, therefore, to be regarded as not symptomatic, but idiopathic or essential. The most prominent of the three elements relates to the nervous system. 'This is manifested by the spasmodic expiratory movements in the paroxysms of cough, and by spasm of the glottis, giving rise to the whoop. Some have considered the affection as belonging among the neuroses, others have considered it to be an essential fever, and by others it has been considered as a peculiar variety of bronchitis. It is neither of these separately, but collectively they constitute the affection. Depenclent, as will be seen presently, on a special cause, the primary, essential pathological condition is general or constitutional, of which the bronchitis and cough are the local expressions. 
CaUsation.-Whooping-congh is an infectious disease; that is, it is communicated by a miasm generated in the bodies of those affected with it. It probably originates in no other way. It is highly infectious, and the number of those who pass through childhood without contracting it are few. Young infants are liable to contract it. No period of life is exempt from susceptibility to the infectious miasm. The reason for the infrequeney of the affection after elilllhood is, the great majority of persons experience it before adolescence. Having been once experiencerl, the susceptibility thereafter censes. This is the rule, but exceptionally the affection is experienced more than onee. 'The exceptions to the rule as applied to this aflection are not more numerous than to the rule as applied to other affections in the same category, for example, the ernptive fevers. The average period of ineubation is not well ascertained. It is variable, the limits being one and two weeks.

Diagnosis.- When whooping-cough has advanced beyond the first or forming stage, and the charneteristies pertaining to, the paroxysms are well marked, it is recogrrized without difficulty. Its diagnostic features are sufficiently evident to those non-medical persons who have seen cases of it. The whoop is a diagnostic eriterion, but this is sometimes imperfeet, and even wanting. In these cases the diagnosis is to be based on the oceurrenee of paroxysms presenting the characters of well-marked whooping-cough, minus the whoop, on the duration of the affection, and on the fact of known exposure to infeetion, together with knowlerlge of the fact that the patient has not already experienced the affeetion. The affection may be so mild and short that there is considerable doubt whether it has really oceurred; but sueh cases are very rare. It is desirable to make a probable diagnosis during the first or forming stage; that is, before the disease is fully declared by the characteristic paroxysms. It should be strongly suspeeterl when eough and febrile movement are out of proportion to the bronchitis, and eontinue unduly. Of course, the prevalence of the affeetion and known exposure are to be taken into account. There is no known method of preventing the disease, other than by isolation as regards exposure.

Prognosis.-Whooping-cough is rarely fatal per se; yet, indirectly, it leads to a considerable loss of life. Occurring in the course of other diseases-for example, measles, or during the ailments inciclent to dentition-it is apt to prove a serious affeetion. $\boldsymbol{A}$ fatal result is generally clue to complications, the more frequent and important of which have been stated, viz., capillary bronchitis, pleuritis, pneumonitis, vesicular and interlobular emphysema, and phthisis. Young children, especially during dentition, are sometimes carried off by convulsions. As regards the affeetion itself, the gravity and danger are proportionate to the frequency and severity of the paroxysms, the duration of the affection. the degree of anæmia and innutrition induced by hemorrhage and romiting.

Treatuent. - The treatment of whooping-cough embraces curative and palliative measures. It must be admitted that there are no known means by which the affection may be arrested; that is, abortive means. Measures are curative if they abridge the duration of the affection, or diminish notably its severity, and there are various remedies which possess more or less curative power.

Emeties were formerly considered as curative, given every other day for the space of a week or longer, at the commencement of the affection. 
They were much extolled by Laennec, who preferred, as the emetic drug, ipecacuanha. Others have preferred the sulphate of zinc. 'T'rousseau advocates, instead of the zinc or ipecacuanha, the sulphate of copper, given in solution, in small doses, according to the age of the patient, the doses being repeated at short intervals until vomiting is produced. In view of the testimony in behalf of the treatment with emetics, its efficacy. in some cases cannot be doubted; but, owing to its severity, it is nearly or quite obsolete in this country. 'I'his method of treatment, it is to be borne in mind, is not admissible except early in the career of the affection.

of other curative remedies, some are addressed to the bronchitis, and others to the neuropathic element. A combination of cochineal and the carbonate of potassa, commonly known as the cochineal mixture, has been much employed in this country ; this mixture consists of cochineal, half a scruple; of carbonate of potassa, a scruple; of white sugar, a drachm, in four ounces of water. A dessert-spoonful is to be given three times daily to a child a year old, and the dose increased in proportion as the age is greater. The affection is undoubtedly in some cases favorably modified by this preparation, as shown by diminution of the violence of the paroxysms, and of the frequency of their recurrence; these effects' of remedies can generally be appreciated when the affection has not already continued sufficiently long to attribute the improvement to a spontaneous decline. It is not so easy to appreciate the fact of the duration being abridged, as the natural duration varies within wide limits. This remedy probably acts upon the brouchitis. Meigs considers the cochineal as inert, and states that he has found tine same benefit from the carbonate of potassa alone, dissolved in syrup of gum and water. ${ }^{1}$

Alum, which was recommencled highly by Golding Bird, is considered by Meigs as giving more decided and satisfactory results than any other remedy which he has employed. From one to six grains may be given every fotir hours, the dose being graduated to the age. Dissolved in some form of syrup and water, it is not an unpleasant remedy. This remedy probably exerts its eurative effect by acting upon the bronchitis.

Belladomna has been recommended as a valuable curative remedy by many physicians in different countries. Trousseau lays down certain rules with regard to its administration, which he deems essential in order to secure its curative efficacy. The dose at first should be quite smallone-tenth of a grain for a child a year old, and one-fifth of a grain for a patient four years, or more, of age. The quantity to be taken in the twenty-four hours is to be given in a single dose. The dose is to be gradnally inereased until its effect upon the severity or frequency of the paroxysms is apparent. It should then be continued steadily without further increase. The action of this remedy is doubtless upon the neuropathic element of the affection. Some have regarded conium, stramonium, and hyoscyamus as not inferior to belladonna in this affection. In some remarks on the treatment of certain functional and organic affections of the nervous system, before the American Medical Association, in May, 1866, Brown-Séquard states that whooping-cough may be cured in three days by giving atropia in doses large enongh to produce delirium, and continued so as to keep up that condition for three days, except at night, when the patient is to be quieted by morphia or codeia. Brown-Séquard adds that it will be hard to get the consent of the parents to this method of treatment if they are told beforehand how the medicine will act. The method is precisely the opposite of that pursued by Tronsseau. Brown-

I A Practical Treatise on Diseases of Children. By J. Forsyth Meigs, M.D. 
Séquard elaims that his method arrests the neuropathic symptoms, but the bronchitis remains for some time after the treatment is discontinued.

Strong testimony has been borne by not a few physicians to the curative efficacy of nitric acid. Dr. A rnoldi, of Montreal, claims for this remedy much power in abridging the duration of the affection, as well as in diminishing its severity. His mode of administration is as follows : To a tumblerful of well sweetened water the acid is to be added until the acidity is of the strength of pure lemon-juice; of this a dessert-spoonful is to be given to a child a year old every hour, and a larger quantity above that age.

Since the bromides were introduced into medical practice, they have been employed considerably in this affection, and with apparent benefit. These remedies are probably useful as pharyngeal and laryngcal anæsthetics; hence, they are addressed to the neuropathic element of the affection.

Other remerlies which have been recommended, and which I shall simply name, are, tannic acid, assafœtida, arsenic, quinia, chloroform, the oxide of zinc and ergot. A measure which seems to claim something more than to be merely named was recently brought before the French Academy of Medicine, viz., inhalation of the fumes disengaged in the purification of coal-gas. It having been observed that children living in the vicinity of gas works suffered but little from whooping-cough, and reeovered after a short eareer of the affection, the effects were tried upon a large scale, and, as stated by Commerege and Bertholles, in their reports to the Freneh Academy, with signal benefit to a large proportion. 'To secure the arlvantage of this measure, patients should inhale the fumes at the place where the gas is purified, for the space of two hours at a time, for twelve consecutive days.' It would appear from the statements by Blache, Barthez, and Roger that this measure is often inefficacious. I have known of a single case in which it appeared to be promptly curative.

Of the different remedies which have been noticed, all are doubtless more or less curative in a certain proportion of cases. It may be doubted if any of them ever have a specific effect. 'They are, severally, useful in some, and not in other cases; the degree of the usefulness of each varies in different cases, and, with our present knowledge, the physician cannot judge beforehand what particular remedy in any individual ease will be most useful. Under these eircumstanees, if those first selected prove inefficacious, others are to be suceessively tried.

Palliative treatment is important. Relief may be afforded by opiates in small doses, if they be well borne, by ethers and the hydrocyanic acid. Strong coffee has been found to be sometimes useful as a palliative.

Hygienic measures form an important part of the treatment. If there be no complications which interfere with exposure to the open air, this should by all means be advised and enforced. Under proper prudential restrictions, the more out-of-door life the better. The diet should be nutritious. If, from the frequency of vomiting, the system suffer from innutrition, alimentation becomes an important object of treatment. The patient should take food often, and as soon after a paroxysm as possible. 'The times for giving food should be chosen as far as practicable remote from the paroxysms, and eating at the most favorable times should be insisted upon despite the absence of appetite. Solid is to be preferred to liquid food, as less likely to be rejected by vomiting. Troussean states that in some cases patients vomit with the paroxysms which occur in the

1 Vide American Journal of the Mcdical Sciences, No. for April, 1865. 
daytime, and not with those occurring during the night ; and in such cases food should be taken freely during the night. The stomach is sometimes made to tolerate food by minute doses of opium. Change of air is sometimes of signal efficacy in modifying the severity of the affection and bringing it to a close.

Hemorrhages, if profuse or recurring frequently, claim hæmostatic measures of treatment.

Complications call for the therapeutical measures appropriate under other circumstances, making due allowance for the effects of this affection on the circulation, the blood, nutrition, and the general strength of the system.

\section{CHA PTER VIII.}

Pulmonary Hemorrhage. Bronchorrhagia. Pneumorrhagia. Pulnonary Gangrene. Pulmonary CEdema. Carcinoma within the Chest. Hydatids.

DULMONARY hemorrhage occurs under the following different circumstances: First, and most frequently, the lemorrhage is into the bronchial tubes, giving rise to the spitting of blood, or hæmoptysis. Adopting the suffix expressive of hemorrhage, with the name of the anatomical situation, this variety of pulmonary hemorrhage should be called Bronchorrhagia. Second, it occurs from the rupture or ulceration of bands of pulmonary substance which traverse tuberculous excarations, these bands sometimes containing vessels of sufficient size to occasion a copious hemorrhage. It is then an accident occurring in the course of pulmon:ry tuberculosis, and gives rise almost invariably, if not always, to hæmoptysis. Third, the blood is contained within the air-cells, and may escape into the interstitial areolar tissue, and coagulation takes place in these situations : that is, the blood is extravasated, constituting what is commonly called pulmonary apoplexy, and usually under these circumstances there is hæmoptysis. The latter form of pulmonary hemorrhage may be distinguished as pneumorrhagia. In the second of these three varieties, the hemorrhage is so uniformly and plainly incidental to one affection, viz., pulmonary tuberculosis, that it will suffice to simply refer to it here. Bronchorrhagia and Pneumorrhagia, however, not being so constantly symptomatic, each of a particular affection, and sometimes occurring when the pathological connection of the hemorrhage is not obvious, claim some consideration independently of the affections in which they are liable to occur.

Bronchorrhagia, or bronchial hemorrhage, exists in the great majority of the cases in which hæmoptysis occurs. The latter term shonld be limited to the spitting of blood; and it should be applied only to the cases in which pure or unmixed blood is expectorated. It is not properly applicable to the sputa streaked with blood which belong to the history of bronchitis, nor to the blood intimately mixed with mucus in the rusty expectoration of pneumonitis. A true hæmoptysis is the raising of blood, and blood only. In bronchorrhagia the bronchial mncous membrane is the seat of the hemorrhage. A solution of continuity is probably requi- 
site, as in all hemorrhages wherever seated. In the rare cases, however, in which the loss of blood has occasioned death, ulcerations have not been found on post-mortem examination ; the hemorrhage is of the kind called capillary, the orifices throngh which the blood escapes being too minute to be discoverable with the naked eye.

lt is important to determine, when blood is ejected from the montl, whether it come from the air-passages. It may come from the stomach, from the posterior nares, and from the mouth or fances. If it come from the stomach, it is ejected by acts of volniting; it is likely to be commingled with other contents of the stomach; it has the characteristic acid odor of the latter, and gives an acid reaction, whereas pure blood is alkaline. Coming from the stomach, it usually has a black grumous appearance due to the action of the gastric acids. If it come from the posterior nares, it is in the form of dark solid sputa, which are removed by acts of hawking. If it come from the mouth or fauces, the fact may generally be ascertained by a close inspection of these parts. When it comes from the air-passages, it is raised by acts of coughing, which are generally not riolent; the blood rises into the trachea and larynx, and is expelled with slight efforts. In the majority of cases, the blood is liquid, of a bright arterial hue, and contains air-bubbles in more or less abundance. If, however, the hemorrhage have taken place slowly into the bronchial tubes, and remained there for some time before being expectorated, it undergoes coagulation, and acquires a dark or almost black appearance.

The amount of hæmoptysis varies much in different cases. It is sometimes quite small, a drachm or so of blood only being raised ; usually, however, when the amount is relatively small or moderate, several drachms or a few ounces are expectorated. Not infrequently the amount is considerable. It is not rare for patients to lose, during an attack of hæmoptysis, from half a pint to a pint of blood, and sometimes a much larger quantity. The blood is expectorated with more or less rapidity. The continuous cluration of an attack of hæmoptysis may vary from a few minutes to several hours and even many days; in the latter case, of course, the blood escaping slowly. Occasionally the flow of blood is so rapid that it escapes simultaneously from the nose and mouth; and death by suffocation may result from the accumulation in the air-passages. A single attack only may occur, or attacks may be repeated at intervals varying greatly in cluration in different cases. In some cases attacks recur daily, or repeaterlly during the day, for a variable period. In other cases the attacks recur after days, weeks, months, or years. After an attack it is common for the expectoration to be more or less colored with hæmatin for several days. In proportion as it is rapidly dissharged it is less frothy than when the quantity expectorated with each act of coughing is small. When the hemoptysis is rapid and abundant, if the patient be not in advanced phthisis, the bursting of an aneurism should be suspected. It is to be borne in mind that this is a source of hemoptysis, an aneurismal tumor sometimes opening into a bronclus or the trachea. The hemorrhage from this source, however, is not always at first abundant and rapid, the opening being, for a time, too small for the free escape of blood. In eases of phthisis advanced to the cavernous stage, the hemoptysis may be rapid and abundant, leading sometimes to fatal syncope, the hemorrhage being due to the opening of a vessel contained in one of the bands of pulmonary tissue which frequently traverse tuberculous cavities.

In the majority of cases, bronchial hemorrhage is, in some way, pathologically connected with pulmonary tubereulosis, and, from the frequency of this connection, it is important as a diagnostic symptom of that disease. 
Not infrequently it is the first event which awakens the attention of patients to the existenee of pulmonary discase. In a large proporti.n of cases, it occurs more or less frequently during the progress of tuberculosis. In a certain proportion of cases it precedes the deposit of tubercle; or, at all events, it oecurs when coexisting physical signs do not afford evidence of a tuberculous deposit, these signs becoming developed, sooner or later, after its occurrence. Hæmoptysis should always excite a strong suspicion of either existing or impending tubereulous disease; yet, its significance in this respect was undoubtedly over-estimated by Louis in his treatise on phthisis. Louis, having questioned a large number of patients affected with various diseases other than tuberculosis, and finding that spitting of blood had taken place in no instance save after injury of the chest or when the catamenia were suppressed, concluded that this symptom is almost pathognomonic of tuberculosis. Since the publication of that work, however, it has been found that cases are not very infrequent in which hæmoptysis is not accompanied nor followed by either the symptoms or signs of tuberculous disease. My own clinical records furnish a considerable number of such cases. But a paper communicated by the late Prof.' John Ware contains statistical information of special value with reference to this point. ${ }^{2}$ Prof. Ware, in this paper, gave the results of an analysis of $\mathbf{3 8 6}$ cases of hæmoptysis, noted in private practice during a period of about forty years. Of these cases, in 62 recovery from the bronchial hemorrhage took place, and the patients afterward were citler known to be living in ordinary health, or to have died of other diseases having no conncetion with the existence of tubercles. The length of time during which this immunity continued varied from two to thirty-seven years. In addition to these cases, in 52 a similar complete recovery took place, and, so far as known, there was no development of tuberculous disease, but the entire subsequent history of these cases had not been obtained. Making allowance for the probable occurrence of a small deposit of tubercle which underwent arrest and did not return, in a certain proportion of the cases, these facts show that neither the existence of tuberculosis nor a strong proclivity thereto is to be positively predicated on the occurrence of bronchorrhagia. They show, moreover, that, as regards any immediate or remote evils, bronchial hemorrhage may be quite innocuous.

Bronchorrhagia is incidental to certain cardiac lesions, especially those involving obstruction at the mitral orifice. These lesions lead to bronchial hemorrhage by inducing pulmonary congestion. It may oecur in connection with morbid conditions of the blood and tissues, which lead to hemorrhages from the mucous membrane in different situations, as in purpura hemorrhagica and scorbutus. It oceurs oecasionally in astlima. It may be produced by violent and prolonged muscular exertions, without the coexistence of any pulmonary or cardiac affection, and it has been olserved to occur from muscular exertions, not excessive, if conjoined with diminished pressure of the atmosphere in elcvated situations, as in ascending high mountains. It may be produced traumatically by injuries and wounds of the chest. It may occur as a secondary hemorrhage when the menses are suppressed. Well authenticated cases of this kind have been reported, but they must be exceedingly rare. I have met with a striking example, the hæmoptysis occurring regularly for the four years following the suspension of the menses, and no other pulmonary affection either accompanying or following the hemorrhage. The 386 cases analyzed by

' On Hæmoptysis as a Symptom. hy John Ware, M.D., etc. Publications of the Massachusetts Medical Society, 1860. 
Prof. Ware did not include a single case in which the hemorrhage could be considered as supplementary to the menses. Exertion and mental excitement, by their effect on the circulation, may act as exciting causes, if, from pulmonary disease or other circumstances, a predisposition exists ; but clinical observation shows that, in the larger proportion of cases, the hemorrhage takes place without any other exciting cause. It takes place frequently during the night-time.

The occurrence of hæmoptysis generally occasions much alarm and anxiety. If patients are seen at the time, the nervous agitation and disturbance of the circulation, which are often present, are attributable, in a great measure, if not altogether, to the mental condition induced by the attack. The first duty of the physician is to endeavor to dispossess the mind of needless apprehensions. Hæmoptysis, proceeding from bronchial hemorrhage, may destroy life, either by suffocation or by exhaustion from the loss of blood; but the cases are so very rare that the danger of fatal consequences is scarcely to be considered. The physician may assure the patient of the absence of any immediate danger. Nor, in general, does the hemorrhage lead to any evil results. It is suggestive of the existence of a grave affection, especially tubercle, but, aside from its symptomatic sig. nificanee, it affords little occasion for alarm. So far from favoring a tendency to tuberculous discase, there is ground for the suspicion that it sometimes takes the place of a tuberculous deposit. And when it occurs in connection with tuberculosis, elinical observation shows that it exerts no unfavorable influence on the progress of that discase, but, on the contrary, its influence seems, as a rule, to be favorable.

In exceptional cases, bronchial hemorrhage apparently leads to either the development or an increase of pulmonary symptoms, together with more or less fever; and the immediate local and constitutional sequences are sometimes exceedingly grave. I have met with a case in which a young man, apparently in good health, was seized with hremoptysis while making an evening visit at the house of a friend. The hemorrhage was profuse, and it was not considered prudent for him to return home. When I saw him, a few days afterward, he had a very frequent pulse, great frequency of respirations with dyspncea, a high temperature, and extreme prostriltion. Death took place within a fortnight from the date of the attack. I have met with several cases in which, dating from an liæmoptysis, the same symptoms oceurred in a lesser degree, the cases ending fatally after a ccrtain number of weeks or months. Such cases, however, are rare. Niemeyer attributes these symptoms to the retention of coagula in the bronchial tubes, and the inflammation which their presence excites. He states that he has verified this by the post-mortem appearances. In the striking instance just cited, which came under my observation, an antopsical examination was not practicable. According to Niemeyer, in many cases, after an attack of hemoptysis, close observation shows sorne elevition of temperature, with frequency of the pulse, and, not infrequently, the signs of eircunscribed pneumonitis and pleuritis are discoverable. These evidences of local and general disturbance, in general, disappear within a short time. In cases of death directly following a profuse hemorrhage, the bronchial tubes have been found, post-mortem, filled with clots; and the obstruction of respiration caused by these, doubtless, was the immediate cause of death. Blood-casts of the bronchial tubes are sometimes expectorated.

The measures of treatment directed to the hæmoptysis must have referenee to the profuseness, or otherwise, of the hemorrhage and the associated symptoms. Venesection, heretofore, has been frequently employed. This, 
however, is indicated only in eases in which the patient is plethoric, or the pulse is abnomally strong, and such cases are comparatively few. Even in these cases, unless the escape of blood be unusually rapid and abundant, a saline purgative and sedative remedies are to be preferred to bloodletting. If the hemorrhage be profuse and persisting, it has been proposed to apply cold to the chest, as in hemorrhages in other situations. This may be done by means of compresses wet with iced water, or with ether which refrigerates by its rapid evaporation. Even the application of ice to the chest has been recommended, and $I$ have resorted to this mode with apparent success, and without any untoward consequences. Revulsive measures may be employed, consisting of stimulating peliluvia, sinapisms, and dry cupping. To diminish the amount of blood returned to the heart, temporary ligation of the extremities may be resorted to. I have witnessed a prompt arrest of the hemorrhage by this measure. A popular remedy is common salt, which usually has been freely given before patients come under the eare of the practitioner. Its efficacy is doubtful, but its use serves to occupy the attention of the patient and friends until medical advice be obtained.

In all cases, quietude of body is to be enjoined, the use of the voice is to be restrained, the apartment should be kept cool, the head and shoulders shonld be raised, the diet should be bland or unstimulating, drinks should be cold, and small pieces of ice may frequently be taken into the mouth. These measures are, doubtless, of more or less importance, yet I have repeatedly known patients who, having become accustomed to attacks of hæmoptysis, paid little or no attention to it, and kept about their ordinary pursuits, as usual, without apparently increasing or protracting the hemorrhage by this conrse. I have known a lecturer continue the daily use of the voice in public speaking, without any apparent injury. The measures just enumerated are apt to be continued too long, patients being anxious to prevent a recurrence of the hemorrhage after.it had ceased. They may be assured that moderate exercise out of doors, a nutritious diet, etc., will not be likely to reproduce an attack.

Cough, if present, should be quieted by some form of anodyne. Anodyne remedies are also generally indicated by the excited condition of the nervons system. If the hemorrhage be slight or moderate, as a rule, nothing is required in addition to these remedies and such hygienic regulations as may be deemed prudent. If, however, the hemorrhage be considerable and prolonged, astringent remedies may be employed. Of the vegetable astringents, krameria and tamic or gallic acid may be mentioned as the most efficient. Ergot appears to be a valuable hemostatic in this as in other hemorrhages. Of the astringents belonging to the mineral kingdom, the acetate of lead has long been considered valuable in this application. The most efficient preparations of this class, however, are the persulphate and pernitrate of iron. The inhalation of astringent remedies in the form of atomized liquids or spray has been found effective for the arrest of hemorrhage. For this purpose solutions of alum, tannin, and the perchloride of iron may be employed.

I have met with a few cases of persistent bronchial hemorrhage which claim a separate notiee. The hemorrhage, in these cases, was slight, but it continued for a long period, without other evidence of pulmonary disease. It was not referable to disease of the heart, to suppression of the menses, or to any appreeiable canse. Moreover, the hemorrhage was confined to the bronchial mucons membrane; and the blood did not come from either the month, throat, or hard passages. The hemorrhagic expectoration was not pure blood, but a thin, bloody liquid. I have notes 
of three cases. In two of these cases, after expectorating almost daily more or less of a sero-sanguinolent liquid rluring several munths, recovery took place under the use of tonies and invigorating hygienic measures. In the third case, the hemorrhagie expectoration has continued for six years, during which period it has from time to time come under my observation. The patient is a young woman, and repeated examinations of the chest by others, as well as myself, have failed to discover any positive signs of pulmonary disease. During the greater part of the long period just named, there has been daily more or less expectoration of liquid containing hæmatin, the quantity being always small. Associated with this symptom are anæemia and hysterical ailments. Aside from these, nothing is discoverable.

Pneumorrrhagia, or extravasation of blood into the air-cells, and in certain cases, also, infiltration of the interstitial tissue, is commonly known as pulmonary apoplexy. 'There would be no impropriety in this use of the term apoplexy if we adopted the custom heretofore of French writers of expressing by it extravasation of blood into any situation, and nothing more; but with us, when applied to a cerebral affection, it inclucles cases in which there is no extravasation of blood, and it is not customary to apply it to extravasations elsewhere than in the brain and lungs. The continued use of the term, as applied to pulmonary extrarasation, is therefore objectionable, and, agreeably to the nomenclature now in vogue, pnenmorrhagia expresses the morbid condition.

Pulmonary extravasation is sometimes ineidental to bronchial hemorrhage, the blood being forced backward from the bronchial tubes into the air-cells by violent aets of inspiration. To this variety of pneumorrhagia, Laennec applied the term hemoptoic infarctus. Watson relates a case in which this took place from ulceration of the lingual artery, blood passing into the laryn $x$, and being drawn thence into the air-cells. It has been known to take place in cases of suicide by cutting the throat. It therefore probably sometimes happens in cases of bronchial hemorrhage when associated with affections giving rise to spasmodic inspirations, or when the flow of blood is so rapid and abundant as to be with difliculty expectorated. This, however, is not an adequate explanation of all cases of pulmonary extravasation. In the cases in which the extravasated blood occupies a considerable or large area, and when it is contained in the interlobular spaces as well as in the air-cells, the primary situation is probably within the latter, the hemorrhage being from the terminal branches of the pulmonary artery or the radicles of the pulmonary veins, whereas in bronchial hemorrhage the blood comes from the venous or arterial ramifications connected with the bronchial artery.

The morbid appearances in fatal cases of pneumorrhagia are of two kinds. In one form, solid masses or nodules, varying in size from that of a pea to an English walnut, and more or less numerous, are found in either one lung or in both lungs. On section they present a dark or even black color, and grumous blood can be scraped from the cut surfaces. The solidification is due to blood coagulated within the cells. The limits of the nodules are sharply defined by the lobular divisions, and the nodules feel like hard tumors, which project above the level of the pleural surface if they be situated near the superficies; they are, however, oftener seated in the centre of the lobe than near the surface, and they are oftener found in the lower than in the upper Jobes. In the other form, the extravasation gives rise to, or is connected with, laceration of pulmonary tissue, and there is a wider diffusion of the coagulated blood. In this form, larger 
portions are solidified by the coagulated blood and they are not bounded by the lobular divisions. 'The blood is contained in the areolar tissue as well as in the cells. There is sometimes considerable breaking down of the pulmonary tissues, forming spaces which are filled chiefly with coagula. Rupture of the pleura has been known to occur, followed by a discharge of blood into the pleural sac, and giving rise to pleuritis. The two forms of extravasation may be distinguished as circumscribed and diffused. 'The latter is the more serious, and, as already stated, probably the hemorrhage takes place within the cells, the source being the vessels connected with the pulmonary artery. Gangrene may occur as a result of either of the two forms of extravasation.

The disseminated nodules first described as characterizing circumscribed pneumorrhagia, are identical with those which have been already noticed as occurring in certain cases of pulmonary embolism. (Vide page 78.) The name hemorrhagic infarctus is applied to them by late writers. Pulmonary embolism, thus, may be the immediate occasion of circumscribed pneumorrhagia, as, also, of circumscribed gangrene of lung. The question then arises whether embolism be not the cause of hemorrhagic extravasation in all cases, exclusive of those in which the hemorrhage is diffused, with laceration of tissue, and, also, of those in which blood is inhaled from the bronchial tubes into the air-cells. Accepting the current doctrine of embolism and hemorrhagic infarctus, it is consistent therewith to answer the foregoing question in the affirmative. Doing so requires a modification of the rationale, heretofore accepted, with respect to the etiological relation of disseminated extravasations to disease of the heart. This form of pneumorrhagia and disease of the heart are associated often enough to show a causative relation of the former to the latter. The explanation which has heretofore seemed adequate is that certain cardiac lesions, namely, those giving mse to mitral obstruction, cause disseminated extravasations by inducing pulmonary congestion. It seems, however, more rational to consider that mitral obstructive lesions are causative by favoring the occurrence of thrombosis in the right side of the heart. This is liable to occur when as a consequence of mitral lesions the right ventricle becomes weakened by dilatation; and thrombi here may furnish emboli, which give rise to hemorrhagic infarctus in disseminated circumscribed portions of the lungs. This explanation, however, of course, does not apply to the hemorrhagic infiltration of air-cells which results from bronchorrhagia, Lænnec's hemoptoic infarctus; nor is it applicable to hemorrhagic infiltration of the areolar tissue, or diffuse pneumorrhagia. The latter may sometimes, although very rarely, be produced by violent contusions of the chest. In general, it is an effect of distension of the pulmonary vessels, that is, passive congestion incident to mitral obstructive lesions. It is intelligible that, under these circumstances, the giving way of the vascular coats will be favored by atheromatous or other changes which diminish their resistance to the mechanical pressure produced by the distension from an over-accumulation of blood, and, also, by causes which, aside from the cardiac lesions, promote pulmonary congestion.

The diagnosis of either form of pneumorrhagia is, by no means, always easy.

Hæmoptysis is, of course, present when the source of the hemorrhage is bronchial, and the extravasation is due to the inhalation of blood into the cells. This symptom is also present in the majority of cases in which the hemorrhage takes place within the cells; but it may be wanting. When present, the hxmoptysis may be either small or abundant; the 
amount of extravasation is by no means in proportion to the quantity of blood expectorated. The respirations are hurried and labored in proportion to the extent of lung soliditied by the extravasated blood. Dyspncen and a sense of oppression are also proportionate to the degree in which the respiratory function is compromised. These symptoms, llowever, are measurably due to the coexisting cardiac lesions when the latter stand in a causative relation to the hemorrhage. Dulness on percussion will be found over a space, or over spaces, corresponding to the situation and extent of solidification. This may not be appreciable if the extravasation be in the form of small disseminated nodules. But if the extravasation be diffusel over a considerable space, the dulness becomes a very significant sign, provided tuberculosis, pneumonitis, and other affections involving solidification can be excluded. A feeble bronchial respiration may be associated with the dulness on percussion, or the respiratory sound may be suppressed over the extravasation. The presence of blood in the cells and smaller air-tubes will be likely to give rise to the subcrepitant and perhaps to a well-marked crepitant rale within a limited space. These signs, suddenly developed in connection with hæmoptysis, and with mitral cardiac lesions, may lead to a diagnosis; but in the cases in which no blood is expectorated, a positive diagnosis is extremely difficult if not impracticable.

Circumscribed extravasation, incidental to bronchial hemorrhage, is not necessarily serious; and perhaps it occurs, to a limited extent, not infrequently in cases of abundant hamoptysis, followed by recovery. If there be no injury of the pulmonary structures, and the blood simply infiltrate the air-cells, it may be gradually removed by absorption and expectoration, the normal condition being restored, as after solidification from pneumonitis. If due to embolism the hemorrhage may lead to gangrene; or the infarcted portions may become the sites of embolic pneumonia, and the so-called metastatic abscesses. If these results do not follow, and the number and size of the spaces in which the extravasation has taken place be not large, recovery may take place. When, however, the hemorrhage is into the cells, leading to infiltration of the areolar tissue, and the extravasation is considerably diffused, the termination is usually fatal, and death may take place speedily. Other things being equal, the gravity of the symptoms, and the immediate danger, are in proportion to the amount of extravasation.

The treatment of different forms of pneumorrhagia must have reference to the symptomatic indications in particular cases, in addition to analeptic and supporting measures.

\section{Pulmonary Gangrene.}

Pulmonary gangrene has already been referred to as occurring in cases of pneumorrhagia and of pneumonitis. It is, however, one of the most infrequent of pulmonary affections. Its infrequency is shown by the following statistics : of the records of 1069 antopsies analyzed by Dr. Lauthna, of Vienna, there were only 5 cases. Of 3437 autopsical records examined by Dr. Fischel, of Prague, there were 75 cases. Fuller states that in the post-mortem records in St. George's Hospital, London, for ten years, there were 19 cases. I had met with about 15 cases at the date of the first edition of this work, namely, in 1866.

It is presented in two forms, distingnished as diffused and circumscribed. In the diffused form, the gangrene extends over a considerable space, sometimes involving the greater part or the whole of a lobe. This is the 
graver form, and almost of necessity proves fatal. The circumscribed form is that which usually occurs. In this form, the gangrene is limited to a space varying in size from that of a bean to a hen's egg, the limits being sharply defined. It is an extremely grave affection when circumscribed, but recovery takes place in a certain proportion of cases.

The appearances after death differ according to the period when the fatal ending takes place. If the patient die before the sloughing is completed, the gangrenous portion is of a dark, greenish color, friable or diffluent, emitting a characteristic odor, bloodvessels and other traces of organization being destroyed-in short, the portion is dead and more or less decomposed. If the slonghing have been completed, and the decomposed mass removed by expectoration, there remains a cavity corresponding in size to the portion of lung which has been lost. When recovery takes place, restoration is effected as follows: lymph is exuded around the gangrenous mass, and the latter is in this way isolated. 'The cavity left after the completion of the sloughing is lined by a kind of cyst. Gradual contraction of the cavity ensues, ending, finally, in closure and union, leaving a white line with more or less depression and puekering. 'The recovery is then complete, with, of course, some diminution of the volume of lung. In some cases cicatrization does not take place, but the cavity remains for an indefinite period. The posterior aspect of the upper portion of the lower lobe is the most frequent site of gangrene.

The occurrence of gangrene as incidental to pneumonitis, and to circumscribed extravasation of blood or pulmonary infarctions, does not suffice to account for it in all cases. It is, probably, sometimes an effect of embolism of a branch of the bronchial artery, the embolus being derived either from the left ventricle or the aorta. Embolism of a branch of this nutrient artery may perhaps occasion such a rlegree of local anæmia or ischemia that the part supplied by the obstructed vessel loses its vitality; whereas, when the gangrene is an effect of a hemorrhagic infarction, it is caused by the interruption of the capillary circulation by the pressure of the extravasated blood. The latter explanation is. the same as when the gangrene results from pneumonitis. The interruption of the circulation in the seconcl stage of pneumonitis, arising from the pressure of the deposit within the cells, is such that the infrequency of a loss of vitality is surprising. In some cases of embolism of a branch or branches of the pulmonary artery, the occurrence of gangrene is attributed to a specific effect of the emboli; that is, the emboli being derived from a part in which a gangrenous process is going on, this process is reproduced in the parts in which they become fixed. Putrescent morbid products remaining in cavities formed by dilated bronchial tubes (bronchicctasis), are supposed sometimes to lead to gangrene of the proximate pulmonary structures. Gangrene is sometimes attributable to the presence of pus derived from an abscess of the liver, spleen, or kidneys, and from the contents of the stomach involved in the act of vomiting. It may be caused by the pressure of an aneurismal or other interthoracic tumor. It appears sometimes to be an effect of the inspiration of irritating gases.

Causes, however, more general in their operation, are evidently involved in a certain proportion of cases. It occurs oftener in males than in females. It sometimes follows typhus and measles. Intemperance and diabetes involve a predisposition to it. It occurs in a larger ratio among the insane. These facts show that a morbid constitutional state, or a special impairment of the condition of the blood, or a cachexia, enters into the etiology.

These symptoms, in cases of gangrene, will relate measurably to the 
general condition or to associated affections. The pulmonary symptoms due to the gangrenous condition, at first, are those which belong to circumscribed pneumonitis, consisting of cough, some pain, and accelerated breathing, and, after a time, an expectoration, more or less abundant, takes place, which is of a dark or greenish color, and emits an intensely fetid odor. This expectoration consists of the debris of decomposed pulmonary substance. 'The gangrenous sputa may contain elastic fibres visible under the microscope. Vegetable parasites have also been observed. The characteristic expectoration continues for a certain time, and, if the case do not end fatally, is accompanied and followed by a muco-purulent expectoration, more or less copious, which, at length, loses the gangrenous odor, and, gradually lessening, ceases when recovery takes place. While the decomposition and sloughing are going on, the pulse is frequent and feeble, and the patient is considerably or greatly prostrated. Of course, if the gangrene be incidental to pneumonitis, extravasation of blood, tuberculosis, or other affections of the lungs, pulmonary symptoms proceeding from these affections, will precede and accompany the symptoms due to the gangrene.

The diagnosis is to be based chiefly on the characteristic appearance and odor of the expectoration. Prior to the oceurrence of this expectoration the condition is not determinable. A gangrenous odor of the breath, without the presence of decomposed pulmonary tissue in the expectoration, is not adequate proof of the existence of the affection, ancl this fact not being always sufficiently considered, the diagnosis is sometimes made on insufficient ground. In cases of tuberculosis advanced to the cavernous stage, the odor of gangrene in the matter expectorated and in the breath may be produced by the sloughing of pulmonary tissuc within the cavities. This is not very uncommon. A superficial slough of a portion of the bronchial mucous membrane may give rise to great fetor of the breath in cases of bronchitis. The latter is the explanation in some cases in which the fetor is of brief duration, and when recovery takes place without phenomena which denote any grave affection. I have met with several cases of this deseription.

'I'he gangrenous portion of lung, together with the solidification from the exudation of lymph around this portion, furnishes dulness or flatness on percussion within a circumscribed space, which, in view of the most frequent situation of gangrene, will generally be over the scapula below the spinous ridge. Within this space either the respiratory murmur is wanting, or there is a feeble bronchial respiration, with, perhaps, either exaggerated vocal resonance or weak bronchophony. Moist bronchial or bubbling rales are likely to be heard within and around this space. These signs are not available if the gangrene occur in connection with pneumonitis or tuberculosis; but they serve to establish the diagnosis, taken in connection with the characteristic expectoration, if the affections just named be not associated. If the gangrene be circumscribed, and the putient survive the sloughing and removal by expectoration of the decomposed mass, the cavernous signs may be discovered. I have repeatedly found a well-marked cavernous respiration; and I have observed a circumscribed depression in front corresponding to the situation and size of the gangrenous cavity.

As regards prognosis, if the gangrene be circumscriber, and it do not occur under circumstances which are dangerous, irrespective of the gangrene, recovery may take place. The chances of recovery are differently estimated by different writers, which, perhaps, may be accounted for by supposing that the diagnosis of gangrene is not infrequently based on 
insufficient grounds. It can hardly be doubted that the result is fatal in a large majority of cases. Hæmoptysis sometimes takes place in connec. tion with the separation of the slough or eschar. I have known the hemorrhage to be so abundant as to prove the immediate canse of death. If the gangrene involve the pleura, perforation of the lung takes place, and pleuritis with pneumothorax is developed. These cases probably always end fatally. I have met with several examples.

The treatment of pulmonary gangrene is to be direeted more to the system than to the local affection. The affection never occurs under circumstances which render depletion or other debilitating mensures appropriate; on the contrary, such measures can hardly fail to be pernicious. 'Tonic remedies are indicated, and the sustaining treatment. The diet should be as nutritious as possible, and alcoholic stimulants are to be given with a freedom proportionate to the tendency to failure of the vital powers. The employment of opium, in some form, is important, in order to palliate pain or undue congh, and to allay constitutional irritation. The chlorate of potassa has been suggested as likely to be useful, from its apparent usefulness in gangrenous affections of the mouth and throat. The tincture of the chloride of iron is suggested by the same analogy. The inhalation of the vapor of turpentine poured upon boiling water is extolled by Skoda as exerting a favorable local effect. Others have attributed a curative influence to the inhalation of tar vapor, creosote, and chlorine. These remedies are administered by inhalation much more efficiently in the form of atomized liquids or spray than by means of vapor.

'To diminish the offensive odor of the breath, chlorinated water, or a solution of the permanganate of potassa, may be used as a collutory.

\section{Pulmonary CEdema.}

Pulmonary œdema is always dependent on other pathological conditions, and is not entitled to be considered as an individual affection. It is, however, an event of not infrequent occurrence, and is important as interfering with the function of respiration, and, in not a few instances, proving the immediate cause of death. The term adema denotes, in other situations, a dropsical effusion into the areolar tissue; but in œdema of the lungs the transudation is primarily and chiefly within the air-cells, the serum also infiltrating the interlobular structure.

An œdematous lobe or lung is increased in volume and weight; it pits on pressure like the integument in ordinary œdema; on section, a purely serous or sero-sanguinolent liquid escapes in abundance, containing but few air-bubbles, and, on pressing out the liquid, the mass of the lobe, or. lung, and its weight are found not to be greater than in health, showing the absence of any solid deposit. Microscopical examination shows the pulmonary structure to be intact. More or less œdema of the lungs is frequently found in post-mortem examinations. It may extend over portions of both lungs, generally, under these circumstances, being situated in the posterior portions, or it may be limited to one lung, in the latter case extending over the whole lung, or confined to one lobe.

The pathological conditions on which it is dependent are various. It is one of the sitnations of dropsy in cases of degenerative lesions of the kidneys and of acnte albuminuria. It is incidental to the pulmonary congestion necessarily induced by cardiac lesions, more especially those which involve mitral obstruction or regurgitation, or both. It occurs in cases in which congestion of the lungs is produced by obstruction of the pulmo- 
nary veins from the pressure of an aneurismal tumor. The hypostatic congestion which occurs in low fevers and other diseases in which the blood is impaired, and when patients maintain, for a long time, recumbency on the back, gives rise to it, and under these circumstances it is not infrequently the immediate cause of death. It is generally associated with more or less hydrothorax. It may take the place of hydrothorax if the pleural surfaces are united by old adhesions. In a case of general dropsy succeeding scarlatina, the patient dying from interruption of the respiratory function, or apnca, hydrothorax existed on one side, and the lung of the other side was highly cdematous, the pleural sac, in the latter side, being abolished by universal close adhesions due to an old pleurisy.

The symptoms of œdema are increased frequency of the respirations and dyspnoa, in proportion to the extent of lung affected, together with more or less cough and serous expectoration, or bronchorrhoa. The displacement of air in the air-cells by liquid, gives rise to dulness or flatness on pereussion over a space corresponding to the oedematous portion of lung, and within this space, the respiratory murmur is lost, or it is feebly bronchial or broncho-vesicular. The vocal resonance may be increased. 'The presence of liquid in the smaller bronchial tubes is denoted by fine mucous or subcrepitant rales. It is stated that a true crepitant rale may be produced, but this must be extremely rare. The diagnosis is to be based on duluess or flatness on both sides of the chest, associated with moist bronchial rales, pneumonitis being excluded, and the existence of Bright's disease, cardiac lesions, and other causative conditions, being considered.

The treatment must have reference to the circumstances under which it occurs. Occurring in connection with disease of the kidneys, those measures are indicated which are applicable to dropsical effusion in other situations, viz., hydragogue cathartics, and suclorifics, selected and regulated according to the circumstances belonging to individual cases. When incidental to disense of the heart, the main reliance is upon revulsive measures, in connection with such remedies as the condition of the heart may claim irrespective of this result. In the cases in which it is dependent on adynamia and an impaired condition of the blood, tonic and sustaining measures are called for. The liability of its occurrence in connection with hypostatic congestion, in fevers and other protracted diseases, accompanied by feebleness of the circulation and depression of the vital powers, renders it an important part of the treatment of these diseases to see that the patient be not allowed to lie constantly in one position. The position of the body should be frequently changed, in order to obviate the gravitation of blood to the dependent portions of the lungs.

The name acute pulmonary odema may be applied to certain cases in which the odema occurs suddenly and to such an extent as to give rise to great dyspnœa. It may prove quickly fatal by apnœe. In such cases, prompt and efficient measures of treatment are called for, namely, an active hydragogue purgative, with dry cups to the chest, followed by sinapisms. The hot-air bath may be employed if hydragogues are contraindieated by the fecbleness of the patient. I have known the inhalation of oxygen to afford marked relief. In my experience the acute form has occurred only in connection with renal disease.

\section{Carcinoma within the Chest.}

Intra-thorncic carcinoma is rare, and is almost always secondary to carcinoma in other situations. Walshe states that it is especially apt to follow cancer of the testicle. In a considerable proportion of cases it 
follows the removal of a cancerous breast. The medullary or encephaloid is the variety usually occurring within the chest. Scirrhus in this situation very seldom occur's, and the colloid variety has been known to occur in only a few instances.

Pulmonary carcinoma is said to be infiltrated when it extends orer a considerable, or, sometimes, a large, space, the substance of the lung being supplanted by the morbid growth. Oftener it is in the form of nodules, varying in size from that of a pea to an orange, the boundaries of which are sharply defined, and which are more or less numerons, being usually disseminated throughout both lungs. In either form, the physical condition of the affected part or parts is solidification or induration. After a time, which is very variable in duration, softening and ulceration ensue. Pulmonary cavities may be produced. Circumscribed pleurisy is usually induced. In some cases, perforation of the pleura takes place, and pneumo-hydrothorax is the result. The lymphatic glands within the chest, and also those of the neck and axilla, are apt to become involved. If the carcinoma be unilateral and diffused over the greater part or the whole of one lung, it leads to notable contraction of the affected side. Hemorrhage and gangrene are incidental events occurring in some cases. The symptoms are pain, cough, and expectoration, the latter becoming muco-purulent, and sometimes presenting an appearance of currant jelly. This appearance is somewhat diagnostic.

The diagnosis is to be based on the symptoms, taken in connection with the signs of solidification, and sometimes the cavernous signs. The physical evidence is not very distinctive of this affection. Pulmonary tuberculosis and chronic pneumonitis are to be excluded by a careful investigation with reference to the history, as well as the existing symptomatic phenomena. The coexistence of carcinoma in some other situation, or its previous existence, is to be taken into account in arriving at a diagnosis.

When the primary seat is exterior to the lungs, it may be presented in the form of nodules, more or less numerous, varying in size from that of a pea to that of an orange, which may be confined to one side, but usually are in both lungs; the pleural surfaces are sometimes thickly studded with these. Or, it may be in the form of one or more tumors, which attain, in some instances, to a great size, occupying the greater part or the whole of one side (oftener the right), and sometimes encroaching more or less on the other side of the chest. The lungs are displaced, and undergo compression in proportion to the extent of the carcinomatous growth, and the affected side may be more or less dilated. The dilatation, in some cases, is increased by liquid effusion due to coexisting pleuritis. Under these circumstances the affection is liable to be mistaken for empyema or chronic pleuritis. This error has happened to physicians of skill and experience in the physical exploration of the chest.

The presence of tumors, great or small, gives rise to dulness or flatness on percussion, with either suppression of the respiratory murmur, or the modifications which denote solidification. The tumors may be so situated as to give rise to symptoms and signs proceeding from pressure on important parts other than the lungs. The heart may be displaced. The calibre of the trachea or bronchi may be diminished. Aphonia or laryngeal spasm may be induced, if the recurrent nerve be involved. Congestion limited to the upper extremities, head and neck, and subcutaneous œdema, denote obstruction of the superior vena cava. Pressure on the pulmonary veins may give rise to bronchorrhagia and œedema of the lungs. Compression of the œsophagus occasions difficulty in the ingestion of food and drinks. These results of pressure on adjacent parts, however, are common to other 
than carcinomatous tumors, for example, aortic aneurisms. A cancerous tumor, circumscribed and situated in the tract of the aorta, may simulate the physicil signs of aneurism. It may pulsate and give rise to a murmur. I am satisfied, by my own observations, that it may occasion a double murmur, that is, a systolic and a diastolic murmur.

I shall content myself with this brief reference to carcinoma within the chest. Fol a full consideration of the points involved in the diagnosis, the reader is referred to works which treat in extenso of thoracic affections. In a practical view, only the diagnosis of carcinoma claims much consideration. 'The affection is hopeless, and the measures of treatment have reference only to the palliation of symptoms, and measures to prolong life. According to Lebert, the duration of life varies from one to two years, and, in some cases, death does not take place for several years.

\section{Hydatids.}

Among the extremely rare affections of the pulmonary organs belong hydatid productions. These may be developed within the lungs, or they have been developed in the liver and made their way through the diaphragm into the pulmonary organs, in the manner in which hepatic abscesses are sometimes evacuated into the bronchial tubes, circumscribed peritonitis and pleuritis taking place, and learling to adhesions which prevent the evacuation from taking place into either the peritoneal or pleural sac.

Acephalocysts within the lungs may remain for a variable period latent, that is, giving rise to few or no pulmonary symptoms. Sooner or later, however, they excite inflammation of the surrounding parenchyma and bronchial tubes, and then occasion pain, cough, expectoration, febrile movement, ete. They may be discharged by ulceration into the bronchial tubes, leaving pulmonary cavities. The affection is likely to be mistaken for pulmonary tuberculosis. Mieroseopieal examination of the matter expectorated may show the hooklets of echinococci, and without this demonstration the diagnosis is impracticable. The question as to the primary seat of the cysts is to be settled by the previous history, which, if they come from the liver, will show the existence of hepatic trouble prior to the occurrence of pulmonary symptoms.

The presence of hydatids in the lungs always involves great danger; the danger is greater when they are derived from the liver than when they are developed within the lungs. Fuller estimates that of cases of the latter recovery takes place in the proportion of one-half. There is no special plan of treatment. Palliative and sustaining measures are to be adapted to the circumstances of individual cases.

\section{Hypertrophy and Atrophy of the Lungs.}

Pulmonary hypertrophy can hardly be said to exist under circumstances renclering it of much importance in a practical point of view. Increase of the volume of the lungs from distension or dilatation of the air-cells is not hypertrophy, but, on the contrary, it is associated, in eases of emphysema, with atrophy. True hypertrophy, that is, an actual increase of the tissues forming the pulmonary organs, is stated by Rokitansky and others to take place when, owing to abolition of the respiratory function of one lung, the other lung takes on an increased functional activity, the latter becomes hypertrophied. Hypertrophy is also described by Skoda and Virchow as occurring in cases of prolonged pulmonary congestion eaused by mitral 
cardiac lesions or by dilatation of the right ventricle. Under these circumstances, the increase of the pulmonary tissues is at the expense of the capacity of the air-cells; the tissues present a brownish color, and the pigmentary deposits are unusually numerous and large.

Atrophy of lung is a lesion involved in the form of emphysema distinguished as atrophous or senile, and has been already noticed in that connection.

\section{CH A P T E R I X.}

Affections of the Larynx and Trachea. Points relating to the Anatomy and Physiology of the Larynx which are involved in the consideration of Diseases in this situation. Acute Simple Laryngitis-Anatomical Characters-Clinical History-Pathological Character-CausationDiagnosis-Prognosis-Treatment. Sabacute Laryngitis. Chronic Laryngitis. Laryngitis with Exudation of Lymph-Clinical History-Pathological Character-Causation-Diagnosis -Prognosis-Treatment. Edema of the Glottis. Spasm of the Glottis. Nervous Aphonia. Morbid Growths.

TMPORTANT diseases affecting the respiratory apparatus are seated above the chest, viz., in the larynx and trachea. Diseases of the larynx frequently involve the trachea, but it is rare for the latter to be affected without the former, and it will suffice to consider tracheal affections incidentally in treating of those of the larynx.

Certain anatomical and physiological points pertaining to the larynx are to be kept in mind with a view to a clear apprehension of the diseases in this situation. The larynx is composed of a collection of cartilages, viz., the cricoid, thyroid, arytenoid, and epiglottis. 'The latter was formerly supposed to be essential as a protection against the entrance of food and drink into the laryngeal cavity during the act of deglutition; the removal of this appendage, howerer, in inferior animals, has shown that its loss does not occasion serious inconvenience. It has occurred to me to meet with a hospital case in which this part was completely destroyed by syphilitic ulceration, as determined by the touch and the laryngoscope. The patient, a young female, was for some time under my observation at Bellevue Hospital. So long as ulceration existed, there was considerable difficulty arising from spasm of the glottis, excited by contact with food and drink, and deglutition was performed with the least annoyance while the patient was lying on the back. But, after the ulceration had healed, the difficulty nearly ceased, liquids and solids being swallowed without much inconvenience. The quality of the voice underwent some change, and the patient stated that she had not the power of producing notes in singing as before.

The small size of the rima glottidis is an important point in connection with laryngeal diseases. In the adult male, after death, it is a triangular space an inch in length, and, at the base, about a quarter of an inch in width, the size being still smaller in the female and child. The dimensions, however, vary with the two respiratory acts. When examined in a living animal, the vocal chords are found to separate widely in inspiration and return in expiration, forming the respiratory movements of the glottis. The respiratory movements take place from a reflex influence communi- 
cated through the recurrent nerves. These points are of interest and importance in their practical applications.

Other movements of the muscles of the larynx are produced by volition in the acts of speaking. The larynx being the seat of the voice, modifications of vocal sound constitute important symptomatic phenomena of disease. The experiments of Bernard have shown that in producing the movements concerned in phonation the will acts through branches of the spinal accessory contained in the recurrent laryngeal nerves. The laryngeal museles are subject to spasm and paralysis.

The solidity of the walls of the larynx is an important point in connection with certain affections. The cricoid and thyroid cartilages do not readily yield to internal pressure, and hence arises obstruction from the presence of morbid products which encroach upon the small space between the vocal chords. Another point to which reference will be made is the abundance of loose areolar tissue beneath the mucous membrane, especially above the vocal chords; this is much more marked in the adult than in the child, a fact which will serve to account for certain differences as regards the effects of disease during and after infantile life.

Of the affections of the larynx, those involving inflammation will be first considered. Inflammation here may be simple in character, that is, not distinguished by any unusual features save those which are incident to the situation of the affected mucous membrane. On the other hand, it may be of an unusual character, viz., accompanied by an exudation of lymph. The latter will be considered under a separate head. Simple inflammation may be either acute, subacute, or chronic. 'These three varieties are to be considered separately. The appropriate name for inflammation affecting the larynx is laryngitis ; this name should take the place of the terms angina and cynanche, which were formerly in vogue, but now rarely used. The term croup is applied to laryngitis with the exudation of lymph, and also to cases of simple laryngitis and of a non-inflammatory affection, viz., spasm of the glottis, occurring in children. The first of these affections embraced by the term, is sometimes distinguished as true croup, while the others are called, by way of distinction, false croup. The term is an unfortunate one, and tends to produce confusion. I shall consider the several affections which the term embraces, not under the head of croup, but separately under their appropriate names.

\section{Acute Simple Laryngitis.}

Anatomical Characters.-The anatomical characters are essentially the same as in bronchitis, so far as they relate to the mucous membrane. The morbid changes may be limited to this membrane, or they may extend to the submucous areolar tissue. In the former case, the inflammation has been called erythematic, from its analogy to erythema of the skin. In the latter case the products of inflammation, lymph, and perhaps pus, are situated beneath the membrane. In the liability to this extension of inflammation laryngitis differs from bronchitis, the difference being probably due to the greater abundance of areolar tissue beneath the laryngeal mucous membrane.

Cases of acute laryngitis differ as regards the point just stated, and the distinction is practically important. Some writers, following Cruveilhier, have based thereupon a division of the disease into two varieties, viz., mucous or erythematic laryngitis, when the changes are confined to the membrane, and submucous or œdematous laryngitis, when inflammatory products are deposited beneath the membrane. This formal division is hardly ne- 
cessary, but the gravity of the disease depends, in a great measure, on the presence of submucous infiltration. The latter occur's to a much less extent in children than in adults, owing, probably, to the fact that the submucous areolar tissue is less abundant in early life. The epiglottis frequently participates in the inflammation, and may be seen during life to be enlarged and reddened.

Chinical History.-The symptoms of acute laryngitis are intelligible when the morbid changes are considered in connection with the small size of the rima glottidis and the unyielding walls of the larynx. Simple swelling of the mucous membrane may produce more or less distressing symptoms and danger due to narrowing of the aperture of the glottis. But, with submucous infiltration added, the obstruction is increased, and will be likely to prove fatal by strangulation. The obstructive effect of the morbid changes explains the symptomatology and danger. 'The chief element of danger is the submucous infiltration. This takes place in some cases and not in others; and it ocenrs in varying degrees, the severity of the symptoms and the danger being in proportion to its amount. The submucous areolar tissue, however, may be infiltrated with serum without an extension of inflammation beneath the membrane, and the presence of lymph or pus in the latter situation, in cases of acnte laryngitis, is infrequent.

The development of the disease may be preceded by subacute laryngitis, or a common cold. The voice is at first hoarse; and, with the development of acute inflammation, is lost. The aphonia is accompanied with a stridulous congh, and a small expectoration of glairy mucus which may be streaked with blood. Febrile movement is more or less intense or it may be wanting. These symptoms may exist without notable difficulty of breathing, provided the changes are limited to the membrane, and the swelling be not great.

With a greater amount of swelling, together with submucous infiltration, labored respiration and dyspnœa are added. The obstruction affects both inspiration and expiration, but more especially the former, owing to the fact that the infiltration is greatest above the rima where the areolar tissue is most abundant. The respiration is stridulous or noisy. The patient complains of a sense of constriction at the larynx, or feels as if the cavity were filled with a foreign substance. There is frequently more or less difficulty in deglutition. Fluids, by coming into contact with the inflamed epiglottis, excite cough and add to the distress. The labor of breathing and dyspnoea are increased by the occurrence of spasm of the arytenoid muscles. The exacerbations of distress from obstruction are dne chiefly to a spasmodic element.

In cases of acute laryngitis with great obstruction, the suffering is intense. The face and eyes are swollen and congested, the countenance denotes anguish, and, after a time, lividity occurs. The distress is much increased during the exacerbations caused by spasm, and in the intervals of comparative ease the patient manifests drowsiness, but is rarely able to obtain refreshing sleep. "With the muscular efforts to enlarge the aperture of the glottis, the larynx rises and falls in the acts of inspiration and expiration. There are frequent efforts to cough and expectorate, as if to get rid of an accumulation within the larynx. The cough may be dry and croup-like, but is oftener husky or abortive. The larynx is sensitive to pressure. Pitting over the pomum adami has been observed. The respiratory acts are less frequent than in health. In a case which I have recorded they were twelve per minute. 
With these symptoms, in a large proportion of eases the disease has been found to end fatally. 'The mode of dying is by apnoa, and it may take place gradually, or suddenly in an exacerbation of unusual violence.

Assuming that other affections do not coexist, the symptoms, in arldition to those which relate directly to the laryngeal obstruction, are incidental to febrile movement, deficient oxygenation of the blood, and the suffering which belongs to the disease. Slight delirium may occur toward the close of the disease if it be prolonged, but, in general, the mental faculties are unaffiected.

Pathological Character.-In simple acute laryngitis, the inflammation has no special eharacteristies. The severity and danger are not owing to the intensity or extent of the inflammation. The same amount of inflammation in other situations would constitute a trivial affection. The gravity is due to the fitet that the usual results of inflammation in this situation obstruct the passage of air through the larynx, in consequenee of the small size of the rima glottidis and the unyielding laryngeal walls. In children, simple acute laryngitis gives rise to croupy cough and respiration, and hence, this is one of the different affections commonly embraced under the name croup. Ware, in his admirable papers on the subject of croup, distinguished this variety as inflammatory croup.

Causation.-Acute laryngitis in the adult is so rare that practitioners of considerable experience may have never met with an example of it. It is less rare in infants and children than after adult age. Its infrequeney is remarkable in view of the frequent occurrence of inflammation in the pharynx; and the fact exemplifies the principle of eonservatison involved in the law by which inflammation does not tend to extend from one part to other parts, although in immediate proximity. I have seen a case in which the pharynx was laid open by a gunshot wound in the neck, so that the top of the larynx could be seen through the wound, and yet, during the progress of reeovery, there was no evidence of the slightest laryngeal inflamination.

Occasionally, however, laryngitis is developed in connection with pharyngitis. 'This was observed in some eases of the so-ealled epidemic erysipelas, or " black tongue," which formerly prevailed in many parts of this country. I have known it to occur, in comnection with the pharyngeal affection, in scarlatina. The laryngitis which, in a subacute form, is common in rubeola, sometimes becomes acute. Acute inflammation may be excited in variola by the oceurrence of the eruption in this situation. Under the several eircunstances just named, it is a seeondary affection. It may oceur as a primary affection after exposure to cold and wet, as in the case of Washington.

It might be conjectured that excessive use of the voice would be likely to predispose to this affeetion, but clinical observation furnishes no proof that such is the fact. It may be produced, traumatically, by the inlaalation of irritating vapors. A distinguished professor of chemistry, some years ago, lost his life by laryngitis caused by aecidentally clrawing into the larynx a corrosive acid through a pipette, the extremity of which had been broken.' $A$ number of cases have been reported in which young ehildren have suffered and died with this disease-produced by sucking boiling liquid and stean from the spout of a water- or teapot.

1 The late Prof. Palmer, of Woodstock, Vt. 
Diagnosis.-The diagnostic characters of acute laryngeal inflammation are the loss of voice and the husky, stridulous cough, in connection with the febrile movement and other symptoms. That the labor of breathing and dyspnoea are due to the obstruction of the larynx, is shown by the sensations of the patient, the diminished frequency of the respiratory acts, and the absence of physical signs denoting pulmonary disease. In proportion as the aperture of the glottis is narrowed, the respiratory murmur will be weakened, and this affords a better criterion of the amount of obstruction than the sufferings of the patient. The only difficulties in diagnosis relate to the discrimination of simple acute laryngitis from laryngitis with the exudation of lymph, from œdema of the glottis, and from spasm of the laryngeal muscles. In arriving at the conclusion that simple acute laryngitis exists, the affections just named are excluded by the absence of distinctive circumstances belonging to the clinical history of each. Suffice it to say here that the presence or absence of the exudation of lymph in cases of laryngitis is generally determinable; that odema of the glottis may be demonstratively ascertained; and that spasm without inflammation, or with only subacute inflammation, is wanting in characters which are essential to the diagnosis of acute laryngitis.

Prognosis.-Acute laryngitis in the adult is a grave affection. The gravity, as already stated, depends mainly on the occurrence of submucous infiltration. Cases without this contingency occur and may end favorably without very alarming symptoms. The suffering in these cases arises chicfly from spasm. If the constant obstruction-i.e., obstruction exclusive of that due to spasm-be sufficient to interfere greatly with respiration, the tendency is to a fatal result. A proportion of such cases will end fatally if life be not saved by timely surgical interference. The course of the disease in fatal cases is rapid. It has been known to prove fatal in seven hours. The duration rarely exceeds a week.

In young subjects this disease is less severe and less likely to prove fatal than in adults, notwithstanding the smaller size of the rima glottidis in early life, the difference being probably due to the fact that submucous infiltration occurs to a less extent in children. Of eighteen cases, included among the cases of so-called croup analyzed by Ware, in not one did the disease end fatally.

Treatment.-The treatment of acute laryngitis relates to gencral and local measures. The general measures embrace those designed to lessen the intensity of inflammation, limit exudation, promote resolution, and the absorption of exuded products. The measures which may be employed for these ends are the same as in acute inflammation in other situations. It may be doubtful how far the ends are attainable, still, the measures which are supposed to be in any degree operative are not to be withheld in a disease involving so much danger as this. 'The employment of bloodletting is to be guided by the same principles as in other inflammations, but the evils or danger of abstracting blood freely are not as great as in certain other inflammations, for example, pneumonitis or pleuritis, because there is not the amount of morbid effects to be recovered from, and it is therefore not so important to spare the vital powers for the processes of restoration. The danger in acute laryngitis, it is to be borne in mind, is not from the extent or degree of the inflammation, nor from the lesions which it may induce per se, but it is incidental to the seat of the inflammation. And if a fatal result take place, the dying is by apnœa, not by asthenia. But in the use of bloodletting and other depressing measures, 
this consideration must be kept in view : the patient's strength is not to be so far diminished as to incapacitate for the prolonged muscular exertions which the labor of breathing may require. Bloodletting should never be practised atter lividity of the prolabia and face gives evidence of deficient oxygenation of the blood. Under these circumstances, a fatal result would be hastened by abstracting blood.

The bowels should be moved efficiently by cathartics of the saline elass. Nauseant or the direct cardiac sedatives are indicated as in other inflammations. Nauseant remedies should not be carried so far as to produce vomiting, which will add greatly to the distress, and tend to aggravate the inflammation. If antimony be used, it should be given largely diluted, to avoid its local action on the throat.

Mereurialization may be advocated in this, as in some otler affections, on the following ground: Although the antiphlogistic and sorbefacient powers of mercury have doubtless been greatly orer-estimated, it is not certain that it exerts no remedial influence in these clirections. So long, therefore, as there is room to suppose that it may be useful in any measure, it should be employed in an affection like this, which frequently tends rapidly to destroy life by apnœa. The general depressing effect and other evils of mercurialization are of little comparative account, provided the remedy will prove in any degree useful in its influence on the local affection. If mereurialization be determined on, it should be induced early and rapidly, either by large doses guarded with opium, or by small doses repeated at short intervals, mercurial inunetion being added.

Remedies to palliate spasm and cough are indicated. The spasmodic element may be more or less prominent. Its degree of prominence is shown by the frequency and severity of the paroxysmal distress; whereas, the amount of obstruction due to the swelling and infiltration is shown by the labor of breathing in the intervals between the paroxysms or exacerbations. Opium and antispasmodic remedies are important in proportion to the amount of suffering from spasm, but opiates should not be carried so far as to blunt the perception of the want of breath.

In the foregoing remarks on the general measures of treatment, reference has been had especially to icliopathic.or primary laryngitis. When the affection occurs as a complication of scarlatina, rubeola, or variola, and if it be associated with pulmonary or any other important disease, the same measures may not be admissible. This statement applies to bloodletting, mercurialization, and other depressing measures. Inflammation of the trachea frequently, if not generally, coexists, and bronchitis may be developed in accordance with the law respecting the extension of inflammation in the air-passages, viz., that it tends to travel clownward, and but rarely upwards. Of course, the general symptoms, febrile movement, etc., will be increased in proportion to the extent of mucous surface inflamed.

The local measures of treatment embrace fomentations or poultices applied to the neck, with a blister, perhaps, applied over the sternum; and either the atmosphere of the apartment should be charged with steam, or the vapor of hot water inhaled by means of a convenient apparatus. The application to the throat of compresses dipped in ice-water, and renewed at intervals of a few minntes, is a measure which may be tried with safety; and, if it afford relief, it may be often repeated, being continued as long as it is agreeable to the patient. Early in the disease it may act favorably as an antiphlogistic; and, afterward, it may afford relief by diminishing spasm.

Topical applications to the larynx may be made by means of a probang 
suited for that purpose. I have been assured by an intelligent medical student, who had experienced the disease, that he derived much temporary relief from the frequent introduction of a sponge saturated with a strong solution of the nitrate of silver. 'The application was repeated every three or four hours. The relief was probably due to the morbicl sensibility of the part being blunted by the topical application, and the liability to spasm thereby lessened.

Tracheotomy is to be employed in this disease, provided the obstruction becomes so great as to render the danger of death imminent. Patients should nerer be allowed to die from strangulation for want of timely surgical interference. If the danger be purely or chiefly from apnoa, this operation will often prove the means of saving life. 'The important question is-when is it to be performed? It should not be delayed after permanent lividity occurs, that is, lividity due to the obstruction eaused by the swelling and infiltration irrespective of spasm. Persisting lividity denotes imminent danger, and subsequent delay impairs the probability of success from the operation.

After convalescence is established, the recovery of the voice is slow, the pitch and quality remaining altered for some time.

'The treatment of aente laryngitis in children, owing to its being a less severe and grave affection than in the adult, claims less vigorous measures. Cases frequently, if not generally, do well with simple palliative measures. Emetics may be important in order to effect the removal of viscid mucus from the larynx. Death may be produced solcly by obstruction due to the accumulation of mucus in this situation. It would hardly be supposed that the larynx may be so tolerant of obstruction from this source, that, occurring in a bealthy child, efficient efforts of coughing would not be excited, but life be destroyed by slow apnœa; yet a case exemplifying the fact has fallen under my observation.

\section{Subacute Laryngitis.}

Subacute inflammation of the larynx is of frequent occurrence, either as preceding the development of bronchitis, or the inflammation not extending to the bronchial tubes. It characterizes certain cases of a common cold. The inflammation is of a low grade of intensity, or erythematic. There is either hoarseness or aphonia, with cough, and an expectoration at first transparent and viscid, and, afterward, opaque, thick, and loose. There is no obstruction to respiration. Laryngeal spasm in the adult is rarely excited. There is little or no febrile movement.

It is only important as involving some liability to an increase in the intensity of the inflammation or the development of the acute affection. But the liability to this is small. The measures of treatment are those inclicated in cases of bronchitis, or a cold.

Subacute laryngitis exists in a certain proportion of the cases of socalled croup. Ware distinguished these as cases of catarrhal croup. The croup-phenomena are due to spasm, the laryngitis being an unimportant element. This variety of so-called croup is unattended by danger, however violent may be the symptoms referable to spasm.

\section{Chronic Laryngitis.}

Chronic laryngitis occurs much more frequently than the acute form; it occurs, therefore, without having been preceded by acute laryngitis. Acute laryngitis, in fact, if it do not destroy life, ends in recovery, with- 
out eventuating in the chronic affection, and, on the other hand, in chronic laryngitis, the inflammation rarely, if ever, becomes acute.

Chronic inflammation leads to thickening of the mucous membrane, and ulcerations more or less deep and extensive. 'The rocal chords are sometimes partially or completely destroyed by the uleerative process, and sometimes the destruction extends to the muscles, and even cartilages. The epiglottis may be involved in the inflammation and ulceration. In some cases suppurative inflammation is developed primarily between the perichondrium and cartilage. Niemeyer describes this under the name laryngeal perichondritis. Owing to the detachment of the perichondrium, neerosis of the cartilage may take place.

In the vast majority of cases, chronic inflammation in this situation occurs in connection with either syphilis or pulmonary tuberculosis. The localization of syphilis in this part is rare, so that, as a rule, chronic laryngitis is associated with tuberculous disease of, the lungs. The exceptions to this rule are so infrequent that the existence of chronic laryngitis constitutes presumptive evidence of the existence of plithisis. Moreover, syphilitic laryngitis, in general, follows a characteristic affection of the pharynx; the disease, in fact, extends from the latter situation into the larynx, and involves the epiglottis. In eases of pulmonary tuberculosis, with ehronic laryngitis, it was formerly supposed that the latter preceded the former, and that the disease extended from the larynx to the lungs. The term laryngeal phthisis was used to distinguish the affection in these cases. Improrement in the means of detecting a tubereulous deposit in the lungs, however, has led to a knowledge of the fact that the laryngitis in these cases is secondary, the pulmonary disease always being first developed.

The most characteristic of the symptoms relate to the voice. It becomes either loarse, husky, or stridulous, and it may be lost, that is, there is difficulty of speech (dysphonia) or extinction (aphonia). If aphonia exist, the patient speaks in a liusky whisper. The extent to which the roice is affected is not a criterion of the extent to which the vocal organs are damaged by ulecration. Slight uleerations and thickening will lead to great huskiness and even extinetion of the voice. If notable hoarseness exist, more or less destruction of the vocal chords, enlarging the rima glottidis, may be suspeeted.

$\Lambda$ s regards congh, its prominence, as a symptom, varies in different cases. It is modified like the voice, that is, it is either hoarse, husky, or stridulous. 'The expectoration, also, varies. It may be slight, or more or less abundant. Its characters may be those of mucus, muco-purulent matter, or pus. Not infrequently it presents bloody streaks, and it may be fetid. 'The bloody streaks and pus point to uleeration ; the fetor points to caries of the cartilages. Cough and expectoration, however, it is to be borne in mind, are measurably due to the tuberculous affections of the lungs, with which the laryngitis is generally associated. Some cases of tuberculous laryngitis are characterized by violent paroxysms of cough, resembling those of whooping-cough, and vomiting is apt to oceur during the paroxysms.

$\Lambda$ marked difference in different cases relates to deglutition. In some cases the act of swallowing is attended with no inconvenience; but in a certain proportion of cases it is a source of great distress. The passage of food and drink causes pain, excites spasms of the glottis, thus occasioning distressing dyspnœa, and frequently liqnids are returned through the nostrils. Patients are obliged to confine themselves to the blandest articles of diet, and even these are sometimes ingested with so much difficulty 
that death is hastened by innutrition. This difference, in different cases, depends on the situation of the uleerations, these being situated sometimes above the vocal chords and upon the epiglottis, where the ulcerated surface eomes in contact with the food and drink in the act of swallowing, and sometimes sufficiently below the top of the larynx to be secure from this souree of irritation.

The diagnosis of chronic laryngitis is sufficiently easy; the morbid characters of the voice at once indicate the affection. Its connection with pulmonary tuberculosis is to be determined by a physical exploration of the chest, together with an examination into the previous history and symptoms. If pulmonary disease be excluded, the syphilitic source of the affection is to be investigated. The affection may be regarded as idiopathic in cases in which both syphilis and phthisis can be excluded; but, as already stated, such cases are extremely rare.

The prognosis in cases of chronic laryngitis will, of course, have reference to its pathologieal associations. Its conneetion with phthisis does not render the latter disease more rapidly progressive; on the contrary, the progress of the tuberculous affeetion of the lungs is, as a rule, more slow with, than without, this complication. Oeeurring in this connection, however, the laryngitis is, I believe, never fully recovered from. The inflammation and ulcerations usually continue, but, if not, the voice remains permanently more or less impaired. Considerable improvement may take place in cases of tubereulous laryngitis, although the affection continues. Chronic laryngitis, dependent on syphilis, offers a better prospect of recovery. The voice, however, after recovery, will be likely to remain permanently affected.

In my experience, cases of tubereulous laryngitis hare never presented notable embarrassment of breathing from obstruetion. In not a few of the cases, however, of syphilitic laryngitis which I have seen, there has been sufficient stenosis to render respiration more or less labored; and in some eases, there supervened a sufficient amount of obstruetion to threaten life by apnœa. In a considerable number of cases which have come under my observation at Bellevue Hospital, the operation of tracheotomy has been resorted to, and, apparently, the lives of patients thereby saved. It has sometimes been resorted to more than onee in the same case.

The treatment of chronic laryngitis, as regards general measures, will have reference to its pathological relations. In the great majority of cases, the treatment must be that indicated by the coexistence of pulmonary tuberculosis. If the affection be traceable to syphilis, the anti-syphilitic remedies are called for, viz., mercury and the iodide of potassium.

As regards loeal measures, counter-irritation over the neck by means of the croton oil or small blisters is sometimes useful, even in cases of tuberculosis. The local treatment, however, relates more especially to applications to the affected part: that is, within the larynx. The injection of, medicated liquids and the insufflation of remedies in the form of a dry powder have been employed for this purpose. Both these means are unsatisfactory, owing to the difficulty of limiting the application to the diseased parts, and to the cough and spasm which they are liable to excite. A more satisfactory method is the introduction of a sponge attached to a probang, properly curved, as practised first by Dr. Horace Green.

Heretofore there has been much discussion respecting the feasibility of passing the sponge within the larynx, and there are those who still maintain that it is impracticable. It is true that the operation is extremely difficult, if, indeed, it be practicable, on the cadaver, and the scepticism 
of some as to the feasibility of the operation on the living body is based on this fact. But the enlargement of the glottis, which takes place in the act of inspiration, divests the operation of the difliculty which attends its performance after death. It is during the act of inspiration that the introduction must be made. During this act, as is well known, foreign bodies of large size are sometimes drawn with the inspired breath into the air-passages. I do not doubt that the sponge is readily passed into the larynx with proper knowledge and skill, but doubtless practitioners often deceive themselves, pushing the instrument into the osophagus instead of the larynx.

The topical remedy most frequently applied by means of the sponge is the nitrate of silver. A strong solution is commonly used, viz., from one to two seruples to the ounce of distilled water. The sponge wet in this solution is carried between and below the vocal cliords. Spasm of the glottis occurs, and the liquid, being expressed from the sponge, comes into contact with the whole of the interior surface of the larynx. 'This application is sometimes beneficial, affording immediate and marked relief. It may then be repeated from time to time. In many cases, however, no relief is afforded, and in these cases, after a fair trial, it is useless to persevere in it.

Trachcotomy is to be resorted to in eases of syphilitic laryngitis whenever obstruction occurs sufficiently to endanger life.

The recent introduction of the laryngoscope has proved highly serviceable in the diagnosis and treatment of laryngeal affections. By means of this instrument the nature and seat of lesions within the larynx may be ascertained, and the knowledge thus obtained affords aid in judging as to the propricty of topical applications, and in showing the particular points at which they are to be made. The laryngoscope, in fact, may be said to have, in a great measure, transferred the interior of the larynx from the domain of medicine to that of surgery, by rendering this part open to inspection, and making the information thus obtained available for surgical operations. By inspection of the parts within the larynx, lesions may be excluded, and in this way laryngoscopy is sometimes of practical advantage. The presence or absence of forcign bodics may by this means be ascertained. Inspection of the glottis is useful in order to determine whether paralysis of the laryngeal muscles exists or not. Moreover, the characteristic appearances on inspection enable the experienced observer to differentiate thereby syphilitic disease. For a description of these the reader is referred to other works.

\section{Laryngitis with Exudation.}

Laryngitis attended with an exudation, occurring for the most part in children, is commonly known as true croup. It occurs, also, as an occasional complication in diphtheria, an epidemic disease characterized by inflammation, with exudation, of the pharynx, and of the mucous membrane in other situations. As belonging to the clinical history of diphtheria, it will be considered in treating of this discase in another section of this work. Suffice it to say here that, as concerns the laryngitis, it is essentially the same in diphtheria and in the affection called croup; yet, taking other circumstances into account, diphtheria and croup are quite different diseases, and the former is more appropriately placed elsewhere than among diseases affecting the respiratory apparatus. In treating, therefore, now of laryngitis with exudation, reference is had to the affection commonly known as true croup. The exudation is generally regarded 
as identical with fibrin, and it is often ealled coagulable lymph. E. Wagner, and Cornil and Lianvier, however, affirm that it consists of epithelial cells infiltrated by an albuminoid substance. Other pathologists do not coneur in this statement. I'ractically the question is not of great importance.

The term croup, as already stated, embraeing affections differing widely in pathological eharacter and importanee, tends to produce confusion, and it is clesirable that as the name of a disease, the term should become obsolete. The term denotes any affection with a peculiar cough called the croupal congh; a cough shrill, barking, crowing, as if the sound were produced within a metallic tube, the tussis clangosa of Cullen, and aecompanied frequently by sonorous or striclulous breathing. Whenever the cough and respiration in ehildren present the croupal character, they are said to have croup. This eroupal cough and respiration aecompany the affection under present consideration ; but it may also accompany, in children, ordinary acute laryngitis, subacute (catarrhal) laryngitis, and spasm of the glottis without any laryngeal inflammation. Ware, in his valuable papers on croup, using this term in its comprehensive sense, recognized four varicties corresponding to the four different pathologieal conditions just stated. He ealled these varieties membranous, inflammatory, catarthal, and spasmodic croup. The first of the four varieties is true croup, and the other varieties belong under the head of false eroup. This division is true to nature, were we to continue to use the term croup in the comprehensive sense in which it has becn and is still eommonly used.

Laryngitis with an exudation has been ealled by various names other than true eroup, such as membranous, diphtheritic, exudative, fibrinous. Each of these names denotes the characteristic feature of the disease. I prefer the simple expression which I have adopted. The characteristie feature referred to is the exudation on the inflamed mucous surface, forming what is commonly called a false membrane. This is diffused over the inflamed surface, and, for a time, adheres pretty closely to the mucous membrane; but after several days, it becomes loosened, and is finally thrown off. It is sometimes detached and reproduced. The exudation may not extend below the larynx, but, as a rule, it takes plaee in the trachea; in other words, the laryngitis is accompanied by tracheitis. Not infrequently bronchitis eoexists, and the exudation may form within the bronchial tubes. In the vast majority of eases, the exudation exists on the epiglottis, and, to a greater or less extent, within the pharynx. 'This faet is of mueh practical importance with reference to diagnosis, and will presently be referred to in that connection.

Certain faets relating to the exfoliation of the false membrane will be found to have important practical bearings. Sooner or later the membrane is thrown off, if the life of the patient be suffieiently prolonged. But before it ean be thrown off it must become loosened. The loosening is effected by a suppurative proeess beneath the false membrane. This process requires a eertain period which varies in different eases, but several days must elapse before it is possible for the membrane to be exfoliated. When detached, it is ejeeted by aets of coughing from the air-passages, and, if the patient be old enough to expectorate, membraniform patches, in greater or less abundance, are found in the expectorated matter, and sometimes branching eylinders are discovered, whieh are fibrinous casts of the bronchial tubes.

Clinicat History.-The symptomatic phenomena in this variety of inflammation, at the outset, are the same as in ordinary subacute laryngitis. 
The local and general symptoms are not as marked as in cases of simple acute laryngitis. It is often supposed to be nothing more than a common cold. There is more or less cough, and the cough is hoarse or barking; hoarseness or huskiness of the voice exists, and moderate febrile movement. 'The symptoms denoting gravity of disease arise from the obstruction due to exudation, in connection with swelling of the glottis and the occurrence of either spasm or paralysis of the laryngeal muscles. One, two, or three days may elapse before the disturbance of respiration is suflicient to create any apprehension. Not infrequently the general symptoms are not sufficient to prevent the child from being up and engaging in play, even after a certain amount of obstruction has taken place. The obstruction due to the exudation and swelling is shown by labored breathing in both acts, and by dilatation of the nostrils. These evidences may be apparent before the obstruction is suflicient to occasion suffering from dyspnoa. The additional obstruction clue to laryngeal spasm is shown by the difficulty which occurs in paroxysms or exacerbations. Spasm may be more or less prominent as an element of this form of disease; as a rule, it is far less prominent than in the affections known as false croup. IIence, of all the varieties of so-called eroup, this, in its development and early progress, is the least likely to excite alarm.

As the disease advances, the obstruction is increased. The labor of breathing becomes greater, and is attended with manifestations of distressing dyspnœe. The current of inspired air is insufficient for the frec expansion of the lungs, as denoted by contraction of the lower part of the chest, with sinking in of the soft parts above the clavicles and sternum in the act of inspiration. 'The cough becomes stridulous and abortive. The roice or cry is reduced to a husky whisper. T'the face is congested ; an expression of anxiety and distress is marked. More or less febrile movement continues. The sufferings are increased at variable intervals in proportion as the spasmodic element is prominent. If life be prolonged, the cough at length becomes loose, and portions of the false membrane are expectorated from time to time. The obstruction may then gradually decrease and recovery take place. If the disease prove fatal, the mode of dying is by slow apnœa; but not infrequently the suffering from dyspmœa diminishes toward the close of life, and the labor of breathing may be so far lessened as to excite delusive hopes of improvement. Convulsions occasionally occur toward the close of life.

Niemeyer considers as a cause of obstruction, in certain cases, paralysis of the laryngeal muscles, and especially of the posterior crico-arytenoid muscles. As a result of paralysis of these muscles, dilatation of the glottis does not take place in inspiration, and obstruction during the inspiratory act occurs as after section of the recurrent laryngeal nerves in the experiments to illustrate the respiratory movements of the glottis. The author just named states that he has ascertained the fact of immobility of the glottis in cases of the disease by laryngoscopic examination. In so far as the obstruction is due to this cause, it will be manifested in the inspiratory act. A case recently under my observation seemed to exemplify obstruction from this causc. The obstruction was limited chiefly to inspiration, and was accompanied by a laryngeal sound suggestive of a sucking inward of the glottis. In this case the obstruetion occurred after the laryngitis had existed for six days, no obstruction having previously existed ; and after its occurrence it increased so rapidly that in six or eight hours tracheotomy was required. The age of the patient, three years, rendered the occurrence of œedema of the glottis improbable. Dr. J. Isewis Smith has indicated another important, incidental circumstance which increases 
the interference with respiration, namely, the accumulation of muco-pus in the trachea and bronchial tubes, the presence of the false membrane in the larynx, and the feeble expiratory efforts, preventing expectoration. He has observed a case in which this seemed to be the cause of death. The danger from accumulation below the larynx is, of course, incidental to the cases in which bronchitis coexists. ${ }^{1}$

Pathological Cinaracter.-The exudation occurs exceptionally in inflammation of a mucous structure. It denotes a peculiar kind of inflammation, and, probably, represents an underlying, special, constitutional, morbid condition. This peculiarity of the inflammation, thus, belongs to it from the beginning. It is not evidence of intensity of the inflammation; on the contrary, the inflammation in this variety of laryngitis is less intense than in the simple acute variety. Simple acute laryngitis, therefore, has no tendency to eventuate in exudation. And in the other affections of the larynx which are commonly embraced under the name croup, the form of disease under consideration, distinguished as true croup, is not liable to supervene.

Causatron.-This form of disease is generally met with in young children, but rarely in infantile life. The age, in the great majority of cases, is between two and seven years. The disease, however, may occur at any period from birth to the age of two years, and at any period after seren years. Boys are oftener affected than girls. It occurs oftener in some situations than in others; a cold, humid, changeable climate contributes to its occurrence. Cases oceur oftener in the spring and autumnal months than at other portions of the year. Happily, it is everywhere a rare disease, excluding, it will be borne in mind, its oecurrence as a complication of diphtheria. It may occur as a primary or a secondary affection. Exclusive of its occurrence in diphtheria, it is sometimes developed in connection with the affection of the pharynx in scarlatina. It occasionally follows measles. The inflammation and exudation, as already stated, very frequently, if not generally, involve more or less of the pharynx. It would seem that the point of departure is, in some cases, the larynx, and in other cases, the pharynx, oftener the latter. ${ }^{\text {D }}$

Dingnosis_-Considering the great fatality of this disease, the diagnosis is of great importance with reference to the prognosis and treatment. It is to be discriminated from simple acute laryngitis, from subacute laryngitis with spasm, and from a purely spasmodic laryngeal affection which is presently to be considered. In each of these affections laryngeal spasm is more or less involved, and the character of cough and respiration distinguished as croupal, is chiefly attributable to this element. The spasmodic element is more likely to be prominent, especially at the outset, in the other affections just named, than in this affection; in other words, this, the so-called true croup, presents the croupal phenomena less marked than either of the forms of the so-called false croup. The insidious development of this, the only one of the affections embraced under the name croup which is attended with danger, renders it the only one liable to be overlooked until the disease has progressed so far as to give rise to serious obstruction.

As contrasted with the other affections just named, this slow, insidious

1 Vide Diseases of Infancy and Childhood, by J. Lewis Smith, M.D., etc., 1872.

2 Vide Meigs on Diseases of Children, 3d edition. 
development is one of the points involved in the differential diagnosis. In the so-called catarrhal and the spasmodic variety of croup, the croupal phenomena occur suddenly and violently, the attack almost always being in the night. When such an attack oceurs without having been preceded by cough, febrile movement, and alteration of the voice, it is almost certain that the affection is not true croup. Alteration of the voice is an important diagnostic feature. This will distinguish it from a purely spasmodic affection, but not from simple laryngitis, either acute or chronic; the voice, however, is affected in a more marked degree in laryngitis with exudation.

The presence of exudation about the epiglottis, and more or less within the pharynx, is a point of great importance in the differential diagnosis. With reference to this point, the observations of Ware are valuable. In this analysis of cases of the several varieties of croup, ont of 33 cases of true croup, i.e., laryngitis with exudation, a pharyngeal exudation was observed in all save a single case. On the other hand, of 45 cases of the affections included under the head of false croup, an exudation within the pharynx was sought for and found to be wanting in every case. Of 19 cases observed by Meigs with respect to this point, in all but three an exudation occurred in the pharynx either before or after the devclopment of the laryngitis. In view of these facts, the presence of an exuclation within the pharynx, taken in connection with the symptoms which denote laryngitis, renders it extremely probable that exudation exists within the larynx. It is to be borne in mind, however, that the pharyngeal exudation is sometimes wanting, and that its absence in the larynx cannot be inferred from its non-existence in the pharynx, with as much positiveness as its presence in the larynx is to be inferred from its existence in the pharynx.

In the progress of the disease, if it do not destroy life, the discovery of patches of false membrane in the matters expectorated should be considered as the only reliable test of the correctness of the diagnosis. The non-observance of this test has led not infrequently to cases of simple laryngitis being regarded as cases of laryngitis with exudation successfully treated.

With capillary bronchitis this affection need never be confounded. In capillary bronchitis the respirations are very frequent, panting, gasping; in laryngitis with exudation the acts are labored and difficult, and, as a rule, not notably frequent. The embarrassed, noisy breathing, especially during sleep, in cases of enlargement of the tonsils, may, at first, suggest the affection under consideration; but it is readily excluded by the absence of the croupal cough, and of hoarseness.

Prognosis.-The tendency of this disease is to a fatal result. Of twenty-two cases analyzed by Ware, nineteen proved fatal. Even if the affection be limited to the larynx, and uncomplicated, it will destroy life in the great majority of cases, if patients be not saved by surgical interference. The danger is, of course, increased, if the affection extend to the bronchial tubes, and in proportion as it extends towards the smaller tubes. Not unfrequently pneumonitis exists as a eomplication, and this will, of course, augment the danger. The duration of the disease, in fatal cases, is usually from four to six days. In a small proportion of cases, it kills in less than four days, or life is prolonged beyond six days. If recovery take place, convalescence is reached after a gradual improvement, and is generally slow, the voice remaining more or less affected for a considerable period. 
Treatment.-Under the measures of treatment heretofore relied upon, this disease has proved fatal in the vast majority of cases. These measures have been based on the supposition that the exudation depends on the intensity of the inflammation, and, with a view to reduce this intensity, the measures known as antiphlogistic have been vigorously employed. These measures have been employed sufficiently to show that they are not successful; and, if they do not do good, they can hardly fail to do harm. With a view to the proper objects of treatment, it is to be considered that cases are rarely seen prior to the occurrence of exudation, and that the prevention of exudation, therefore, is not the end to which therapeutical measures are to be directed. It is also to be considered that, exudation having taken place, recovery is effected by the separation of the false membrane, and that its separation takes place as a result of a suppurative process beneath it, this process requiring several days. These facts being considered, the rational objects of treatment are to prolong life until the separation of the false membrane can be effected, and to promote the process by which this effected.

In accordance with these objects, depletion by bloodletting or other means is of more than doubtful propriety. By reducing the strength of the patient, it conflicts with the first object, viz., the prolongation of life. On the contrary, the strength is to be supported, as far as practicable, by concentrated nourishment, and the judicious use of alcoholic stimulants. Emetics, which have heretofore been used excessively in this disease, are to be employed with circumspection in order not to conflict with the first object. They have been given, and frequently repeated, in order to relieve spasm, and it is undoubtedly true, that, for a short period after their operation, the breathing is comparatively easy, but this end may be attained by other measures less objectionable on the score of depressing the powers of life. Another purpose of emetics is to aid in detaching the false membrane. But it is useless to give emetics for this end until the exudation has been loosened by the process of suppuration. Emetics, however, are not to be entirely withheld. In children they compensate for the want of voluntary efforts of expectoration. They may aid in the expulsion of the false membrane when it becomes detached. A fact stated under another head in this chapter is not to be lost sight of, viz., the tolerance of the larynx in young children of an accumulation of mucus sufficient to occasion fatal obstruction. Emetics, then, may be given from time to time, but not too frequently, and the emetic substances used should be those which excite prompt and efficient vomiting without producing prolonged nausea and depression. This will exclude antimony. Ipecacuanha, powdered alum, turpeth mineral, and the sulphate of zinc, are among the articles which may be selected. Of these articles, the turpeth mineral acts promptly and efficiently, without much depression or nausea. My colleague, Prof. Barker, advocates very strongly the employment of this emetic in cases of so-called croup. He considers that, administered at an early period, it modifies the disease, preventing or limiting the production of false membrane. It is to be borne in mincl that the accumulation of muco-pus in the trachea and bronchial tubes, in certain cases, furnishes an indication for the use of emetics.

Anodyne remedies are indicated to allay spasm. Opium, however, is to be given with great circumspection. Mercury is admissible on the ground stated under the head of simple acute laryngitis, viz., the objections to its use are not to be taken into account in so dangerous a disease as this, so long as there is any ground to suppose that it will do any good.

In the early part of the disease, cold applications to the neck may be 
tried, as in the treatment of simple acute laryngitis. This measure, as in cases of the latter form of laryngeal inflammation, has reference, first, to lessening the intensity of the inflummation, that is, to an antiphlogistic effect, and, second, to a sedative effect as regards spasm of the laryngeal muscles. 'To employ cold efficiently, compresses dipped in ice-water may be applied and removed at intervals of a few moments. 'The continuance of these applications and their repetition are to be determined by the relief which they appear to afford. They should not be persisted in if the patient resolutely resist them, or if they do not seem to give relief. Nemeyer advocates cold applications in this disease; their value was, however, inculcated by Prof.' Peaslee, of this city, in 1854.' Prof. Peaslee considers the continuous application of cold essential in order to secure its full effect. Prof. J. Lewis Smith employs, instead of compresses dipped in cold water, crushed ice surrounded by oiled silk.

Topical measures of an opposite character have reference to the second object, viz., promotion of the process by which the false membrane is separated. 'The neck may be enveloped in a poultice or the water dressing, for the same reason that these are deemed useful in phlegmonons inflammation, viz., to hasten suppuration. But the most efficient topical application for this object is the inhalation of steam, and the best method of making this topical application is to charge the atmosphere of the room with as much rapor from boiling water as possible. My colleague, Prof. Sayre, who has been remarkably successful in the management of this disease, attributes more importance to this than to any other remedial measure. He directs the steam to be generated by means of a stove, on which a caldron of boiling water is placed, and the room to be kept at a temperature of $90^{\circ}$ Fahr. In a room at this temperature, filled with steam, patients are free from spasm, and breathe with comparative ease. I have observed a case in which the patient passed safely through the disease, the inhalation of steam, after Prof. Sayre's method, being the chief measure relied upon.

Küchenmeister, of Iresden, and Biermer, professor of clinical medicine in the University of Berne, having ascertained that pseudo-membranous exudations are rapidly dissolved in lime-water, considerable masses disappearing in from ten to fifteen minutes, were led to resort to the inhalation of spray from lime-water as a means of removing the false membrane from the larynx and trachea, and they have reported cases in which this treatment was successful. ${ }^{2}$ A case has been reported by Dr. H. Ernest Schmid, in which prompt relief was obtained by this measure. ${ }^{3}$ Slacking quick-lime is an effective mode of sccuring this object, and, at the same time, furnishing a warm, pleasant vapor. Breathing pure oxygen from time to time, and cliffusing it in abundance in the atmosphere of the room, affords marked relief in dyspnœa. In the case of a child of my late collengue, Prof. Elliot, steam from boiling water, the lime vapor produced by slacking a barrel or more daily of quick-lime in the room, the inhalation of oxygen which the child soon desired from the relief derived from it, and keeping the atmosphere of the room charged with oxygen from a continuous stream of the gas, were the only measures employed, in addition to alimentation and a little alcoholic stimulant, excepting an emetic of turpeth mineral when the symptoms and signs denoted loosening of the laryngeal false membrane. The child passed through the disease not only safely, but without great suffering.

1 Vide American Mediral Montbly, 1854.

2 Vide Am. Jour. of Med. Sciences, Oct. 1865, p. 518.

3 Vide N. Y. Med. Record, April 15, 1867. 
Dr. R. R. Livingston, of Plattsmouth, Nebraska, has reported a case in which the inhalation of ether appeared to effect a speedy separation of the false membrane.' 'The inhalation of vapor from lime-water containing lactic acid lias been employed with success as a solvent of the false membrane."

The topical application of a strong solution of the nitrate of silver was advocated by Dr. Green. The introduction of the sponge probang into the larynx of the child is difficult, owing to the small size of the glottis, and, frequently, the want of co-operation on the part of the patient; yet it may be effected. I have witnessed a recovery in a case in which this was the chief measure relied upon. The applicatiops were made twice daily. When the false membrane is loosened, the introduction of the sponge may be useful mechanically, detaching the membrane and pushing it below the glottis, thus leading to its expectoration.

Lastly, the propriety of tracheotomy, in cases of this affection, is to be considered. 'The subject has been much discussed of late ycars, and opinions in Great Britain and in this country are divided. Introduced by Bretonneau, and finding an ardent advocate both by practice and precept in Trousseau, the propriety of the operation appears to be generally conceded in France. For statistics bearing on this subject the reader is referred to treatises on the diseases of children, and articles in medical periodicals. ${ }^{3}$ The propriety of the operation hinges, not on the number of cases in which it has proved successful, but on the answer to the question whether any lives are saved by it. Now, it cannot be doubted that patients with this disease have been snatched from impending death by this operation. It is, therefore, not only justifiable, but the practitioner is bound, if possible, not to allow 'patients to die from suffocation for the want of its performance. Of course, the duty of resorting to the operation is the more imperative the greater the probability of its success; and a fair examination of statisties will show that not a small proportion of those who would otherwise die may be reseued by it.

The suecess of tracheotomy must depend much on its timely performance. Its failure in many cases is attributable to its having becn too long deferred, but it has proved successful repeatedly as a dernier ressort under the most unpromising circumstances. So soon as the persisting obstruction, that is, the obstruction due to the exudation, associated perhaps with paralysis of the dilating muscles of the glottis, compromises respiration sufficiently to render it probable that the patient's life will not be prolonged until the separation of the false membrane can take place, the operation is called for. Its success, doubtless, depends much on the manner of its performance, and the subsequent management. On these points the reader will do well to study the instructions of Trousseau. My colleague, Professor Sayre, attaches great importance, after the operation, to an atmosphere heated to $90^{\circ}$ and charged with steam.

Dr. William Jenner has pointed out an objection to a premature performance of tracheotomy, namely, the effect of the operation on the ability to congh. In an efficient act of coughing, first, a deep inspiration is taken and the glottis closes; then by means of forcibly expiratory efforts the

1 Am. Jour. of Med. Sciences, April, 1867, p. 376.

2 For review of experiments by Brichteau and Adrian, vide Am. Jour. of Med. Sciences, Oct. 1868.

${ }^{3}$ Vide article in the New York Journal of Medicine, Jan. 1860, by A. L. Voss, I.D., in which the writer gives the results in 1249 cases, the recoveries being 294 . Also, valuable statistics, etc., by Prof. Jacobi, who has had a large experience. Am. Journ. of Obstetrics, New York, May, 1868. 
current of expired air, dislodging from the bronchial tubes their contents, carries them into the trachea, and, the glottis suddenly opening, expectoration is effected. Now, with a tube in the trachea an eflicient act of coughing camnot take place; hence, there is a greater liability to accumulation in the bronchial tubes than if tracheotomy had not been performed. The danger from this effect of the operation thus relates to the bronchial tubes in the cases in which bronchitis is associated with the laryngitis.

If the operation prove unsuccessful, it diminishes greatly the suffering from obstruction, and is fully justifiable with a view to euthanasia.

\section{Edema of the Glottis.}

This name denotes a serous or dropsical effusion in the areolar tissue beneath the mucous membrane above the vocal cords. It is for convenience used, however, in a more comprehensive sense, embracing cases in which inflammatory products are deposited in the same situation. In the latter case, the condition is incidental to laryngitis. As a true odema, it is an occasional accident, in cases of pharyngitis and tonsillitis. I have met with an instance in each of these comnections. Its occurrence is due to the inflammation of the mucous membrane in proximity to the seat of the effusion, and it is analogous to the subcutaneous odema which accompanies inflammation of the skin in situations where the areolar tissue beneath is abundant and lax, as, for examples, around the eyes and on the penis-the collateral celema of Virchow. The affection occurs in cases of typhus and typhoid fever, in epidemic erysipelas, in albuminuria, and various other diseases. Its occurrence in these pathological connections is extremely rare. ${ }^{1}$ As an idiopathic affection it is certainly one of the rarest diseases. It is important to add that it may occur as the first and only dropsical affection connected with disease of the kidneys. Its gravity, and the necessity of prompt and efficient interference, render it vastly important to appreciate the pathological condition, and recognize its existence.

The situation of the effusion is to be borne in mind. It takes place in the areolar tissue, which, in the adult, is abundant and loose between the vocal cords and epiglottis on the top of the glottis. The accumulation of liquid in this situation may give rise to tumors as large as, or even larger than, a pigeon's egg, which, lying upon the glottis, are sucked inward with the inspiratory act, and thus occasion obstruction in this act. The current of air in expiration separates and pushes the tumors upward, so that in cases of odema without laryngitis there is little or no obstruction in the expiratory act. The œdema usually exists on both sides, but it is sometimes linited to one side. The oceurrence of the arlema may be sudden, and life be destroyed in a few hours, or even minutes. ${ }^{3}$ In a case occurring in connection with mild pharyngitis, the suffering from obstruction was developed during the night, and the patient, leaving his apartment to seek for aid, fell upon the floor and clied before any assistance conld be rendered. In the case in which it occurred in conneetion with tonsillitis, the patient was left to sleep in an apartment by himself, there

1 In an article on this subject by Dr. Thomas Addis Emmet (American .Journal of the Medical Sciences, July, 1856), Dr. E. states that only seven cases of simple oedema occurred in one thousand nine hundred and thirty-one cases of typhus, during a service of five years in the New York State Emigrant Hospital at Ward's Island.

${ }^{2} \Lambda$ case is reported in Guy's Hospital Reports, April, 1855, which terminated fataliy within five minutes after difficulty from obstruction was experienced. 
being no difficulty of breathing, and, in the night-time, a relative in an adjoining room was awakened by his noisy breathing. He was found to be unconscious, and, although medical aid was promptly called, and the trachea opened without delay, the operation was too late to prevent a fatal resuit.

Diffieulty of breathing, due to obstruction at the glottis, is a prominent symptom. This is associated with a sense of a foreign substance at the seat of the obstruction, and a strong disposition to hawk and swallow. The diagnostie point relating to the difficulty of breathing is, the inspiratory aet is alone, or chiefly, obstrueted, the act of expiration being free. The inspiration is arrested before it is completed, being, as it were, suddenly cut short, and both the inspiration and expiration are accompanied by marked stridor. If laryngitis do not coexist, the voice is devoid of hoarseness or huskiness. This will serve at onee to exclude laryngitis. I have met with a case, ending fatally, in which there was no hoarseness nor huskiness of the voice, and no appearance of laryngitis after death. But the diagnosis may frequently be made positive by means of the touch. With the forefinger introduced into the mouth, carried to the base of the tongue, and applied below the epiglottis, the codematous tumors may be distinctly felt. This diagnostic test, enunciated by Tullier in a thesis submitted to the Faculty of Medicine, in Paris, in 1815, appears to have been lost sight of until attention was called to its appearance a few years since, by Dr. Gurdon Buck, of New York.

Statistics show a large fatality in cases of œdema of the glottis. Bayle found that in 17 cases only 1 recovery took place. Of 40 cases collected by Valleix, 31 were fatal. of 168 cases collected by Sestier, 127 died. With a correct appreciation of the pathological condition, a prompt recognition of it, and timely surgical interference, many, if not most, patients may be saved. It is fair to attribute the large proportion of fatal cases heretofore either to an imperfect knowledge of the affection, error or doubt in the diagnosis, or want of promptness in resorting to efficient interference.

The affection tends to destroy life quickly by apnœa. The object of treatment is to prevent suffocation, and if the obstruction be sufficient to occasion great labor of breathing, lividity, etc., there is no time to be lost in fruitless efforts to obtain relief by medication. The larynx or trachea must be opened, or the method of scarifieation, as practised by Dr. Gurdon Buck, must be resorted to. Dr. Buck's method consists in introducing a curved bistoury, properly guarded, into the mouth, carrying it below the epiglottis guided by the left index-finger, and scarifying on both sides, so as to give exit to the effused liquid. The scarification may be repeated if required. ${ }^{+}$

My personal observation of Dr. Buck's operation is based on a single case, in which it was employed by a surgical colleague at a moment's notice, and without the advantage of a prior acquaintance with it. The difficulty in the way of its performance is greater than might be supposed without a trial of it. The increase of the suffering for want of breath caused by introducing the fingers into the mouth, the morements of the larynx incident to the labored breathing, and the acts of romiting which are excited, render it not easy to deliberately scarify the œdematous parts. In the case referred to, the result was unfortunate. The tumors were not sufficiently scarified, but wounds were made which led to hemorrhage and the flow of blood into the air-tubes. Tracheotomy was subsequently per-

1 Fido Trans. Am. Med. Association, vols. i. and iv. Several cases successfully treated by this method are reported by Dr. Buck. 
formed, but too late to save the patient. The case is referred to in order to enforec the importance of being prepared to meet with more diflieulty than might be anticipated. Without assuming to adjudicate in a matter which is purely surgieal, I an disposed to think that the simpler, as well as the more effectual, operation is to open the larynx or trachea. Niemeyer states that in some cases the finger-nail will suflice to open the cedematous tumors.

$A$ case at Bellevue IIospital exemplified the importance of opening the trachea before the obstruction threatens immediate deatll. A patient was suffering considerably from diffieulty of breathing, but it was decided not sufficiently to require tracheotomy. 'The House Physician, however, made every preparation to perform the operation at an instant's notice. Suddenly the diflienlty of breathing inereased, and the physieian was in a few monents at the bedside, but too late to save the patient. The liability to a very rapid increase of obstruetion proving quickly fatal, is to be considered in deeiding upon the propriety of opening the trachea. The operation may be advisable, as a precautionary measure, even if life be not at the moment threatened, when the practitioner cannot remain with or near the patient until danger is passed.

Edlema of the glottis very rarely, if ever, occurs in infaney or elildhood, the reason probably being that the areolar tissue at the situation where the odema oecurs is less abundant and loose in young subjects than after puberty.

\section{Spasm of the Glottis.}

Spasm of the glottis is an important element in most of the affections of the larynx which have been considered. To this element are attributable the croupal cough and the paroxysms or exacerbations of dyspnoa in simple acute, and subaente laryngitis in ehildren, and in larygitis with exudation. But spasm of the glottis oceurs as a functional affeetion, that is, irrespective of inflammation or lesions within the larynx. It is an element of several of the functional affections of the nervous system (neuroses) to be hereafter considered, namely, in hysteria, epilepsy and epileptoid convulsions and tetanus. Occurring independently of these conneetions, it may be reckoned among the functional affections of the larynx.

Spasm oecurs in young children, affecting the respiration without inducing cough. 'This is the pathological condition when infants are said to have fits of "holding the breatl." Respiration is arrested until the face becomes congested and livid, and, when the spasm relaxes, the inspiration may be sonorous, as in pertussis. In some children these paroxysms oceur more or less frequently. They are sometimes accompanied by carpo-pedal spasm. Convulsions may oceur. Death may take place in a prolonged paroxysm, or in consequence of repeated paroxysms in rapid suecession. I have known an instance of this kind. The affection is commonly known as laryngismus stridulus. From a supposed connection with persisting enlargement of the thymus gland after birth, it has been called thymic asthma.

The affection is purely neurotic. It involves a peculiar suseeptibility to spasm in the laryngeal muscles. This susceptibility may be inciclent to a certain period, as during weaning or clentition, but, in some cases, it appears to be an idiosyncrasy of infantile life. When the predisposition exists, paroxysms are liable to be excited by various causes, acting physically and mentally. This susceptibility is sometimes observed in the different children of the same family, a fact in confirmation of its innateness. 
During a paroxysm, it is customary to employ means to produce a strong impression on the surface, as slapping the back or sprinkling cold water on the face. The treatment otherwise involves the removal of all local causes of irritation, such as the distension of the gums and the retention of fecal matter, avoidance of causes inducing mental excitement, and measures to invigorate the system.

Spasms of the glottis occurring in children over two years of age, accompanied by laryngeal irritation and cough, is a frequent affection, known as spasmodic croup. A ehild is suddenly attacked during the night, having, perhaps, gone to bed apparently in perfect health. The breathing is labored and sonorous, the cough presents, in a marked degree, the shrill, ringing, croupal character. Great alarm is produced, and the physician is sent for in urgent haste. The surface of the body is cool; the pulse is small and perhaps not accelerated; the voice or ery is not hoarse nor husky as in laryngitis : the affection, in short, is purely spasmodic. It is unattended with danger. When the violent symptoms are relieved, the respiration is found to be unobstructed. There is no liability to its eventuation in so-called true croup, that is, to laryngitis with exudation. It is to be discriminated from the latter affection. The points involved in this discrimination are as follows : the abruptness of the attack, which is not preceded by symptoms denoting laryngitis; the violence of the attack, in this respeet differing from so-ealled true croup; the absence of hoarseness or huskiness of the voice, of febrile movement, and of exudation in the larynx; and, finally, the speedy and complete relief. The paroxysms may return for two or more successive nights.

The treatment consists of a mild emetic, if there be reason to suppose that the stomach is overloaded, a hot foot-bath, and warm fomentations to the neek. 'These measures are uniformly successful. The treatment of these cases is sometimes needlessly active, under the impression that a serious affection is threatened; and practitioners are apt to congratulate themselves and the friends of the patient on their success in preventing true croup.

Spasm of the glottis may occur as a functional affection in the adult. It is occasionally incidental to hysteria, and may simulate, as regards some of the symptoms, laryngitis, or œdema of the glottis. The diagnostic points are as follows: 'The coexistenee of hysterical phenomena; the absence of huskiness or hoarseness of the voice; intermittency of the difficulty of breathing; the speedy relief obtained by anodyne remedies, and the sudden development of the affection. I have bcen eonsulted in several cases in which spasm of the glottis occurred in male adults without any evidence of disease within the larynx or intra-thoracie disease.

The measures of treatment are anodyne or antispasmodic remedies for immediate relief, and afterward those indicated by the general condition, and which are employed in analogous nervous 'affections.

Spasm of the glottis may be occasioned by irritation of the recurrent laryngeal nerve from the pressure of a tumor or other causes. It is one of the symptoms of aortic aneurism, and, occurring in a person over forty years of age, should always excite a suspicion of aneurism. I have reported a case of aortic aneurism in which the life of the patient was destroyed by frequently recurring paroxysms of laryngeal spasm.'

1 American Medical Times, 1864. 


\section{Nervous Cough.}

The term nervous cough is used to designate a neuropathic disorder characterized by a dry, peculiar cough occurring without laryngitis, bronchitis, or any pulmonary affection. It may be noticed with propriety among laryngeal affections, inasmuch as the distinctive character of the cough relates to the larynx. In most of the cases which I have seen, the cough has had a peculiar barking tone; and the pitch has been low, showing that the glottis was dilated at the instant of coughing. In some cases, however, the tone is shrill, and the quality of sound croupal, showing spasm of the glottis. In a case recently under observation, the cough consisted of a single, short, hoarse bark, often repeated several times in a minute. 'The congh is sometimes in paroxysms, having a resemblance to those of whooping-cough. The peculiar sound of the cough, together with its frequent recurrence, and sometimes its violence, renders it distressing to those whose sympathies are excited, and annoying to others.

This disorder rarely, if ever, occurs in males. It is often associated with more or less of the symptoms embraced under the name hysteria. The cough has been called tussis hysterica, and it is usually included, by medical writers, among the diversified hysterical manifestations. It occurs chiefly in young chlorotic females. I have known it to occur before the age of puberty. It is apt to persist for a considerable period.

The diagnosis is to be based on the peculiarity of the cough; its dryness, except that the acts of coughing, if frequent and violent, may occasion some mucous expectoration, and the negative result of a careful physical cxploration of the ehest. The diagnosis is strengthened, on the one hand, by the coexistence of anæmia, and of hysterical phenomena, and, on the other hand, by the absence of rational symptoms pointing to tuberculosis.

The indications for treatment relate to anæmia, if it exist, and to other morbid conditions affecting the nervous system. Tonic remedies, a nutritious diet, out-of-door life, and, in a word, invigorating measures are indieated. In addition to chalybeates, quinia, small doses of strychnia, and the preparations of zinc may be mentioned as appropriate remedies. Hygienic measures, however, are of the first importance. A change of climate is sometimes advisable, if other measures fail. A sea-voyage proved effective, in a case under my observation, after a long persistence of the cough in spite of various measures of treatment. As palliatives for the cough, antispasmodic remedies may be prescribed, but opiates are not advisable.

\section{Nervous Aphonia.}

Loss of voice, or aphonia, is incidental to the several varieties of laryngitis which have been considered. Iut it occurs when not dependent either on inflammation or lesions within the larynx; in other words, as a functional affection. It is then known as nervous aphonia. The loss of voice is due to paralysis affecting the nerve of phonation, viz., the spinal accessory. The affection is limited to the voluntary movements of the laryngeal muscles, the reflex movements involved in respiration being unaffected.

This form of aphonia is met with not infrequently in females, and is nsually associated with more or less of the phenomena embraeed under the name hysteria. As a purely neurotic affection, that is, not dependent on any lesion of the nervous system, it must be extremely rare in the male sex. A distinction is to be made between loss of voice and loss of speech, 
the latter occurring in a certain proportion of the cases of hemiplegia, and dependent, not on the loss of power to produce vocal sounds, but on either a defect in articulation or the loss of memory of words. A phonia occurs from pressure on the recurrent laryngeal nerve of an aneurismal or other tumor, and the loss of voice should direct attention to this as a possible or probable cause.

'I'he diagnosis of nervous aphonia may be made by attention to the character of the whispered voice; the patient speaks in a pure, soft whisper, without effort. On the contrary, if the aphonia be due to laryngitis, the whisper is stridulous or husky and labored. Moreover, in aphonia due to laryngitis there is more or less cough and expectoration, symptoms generally absent in nervous aphonia. As the question is usually to decide between nervous aphonia and chronic laryngitis, and as the latter affection is gencrally associated with pulmonary tuberculosis, the absence of the symptoms and signs of the latter disease will serve to confirm the diagnosis. Examination with the laryngoscope will corroborate the diagnosis by showing that the larynx is free from lesions; and the absence or the incompleteness of movements of the glottis when an effort to speak is made may be ascertained by inspection.

Inspection with the laryngoscope shows that the paralysis may be bilateral or unilateral ; and that the different muscles, namely, the adductors and abductors, the tensors and the laxors, may be affected separately. Unilateral paralysis, of course, points to some cause affecting the accessory nerve on one side only, and it is especially suggestive of an aneurismal tumor pressing upon one of the recurrent laryngeal nerves.

If the aphonia be purely neurotic, in other words, involving no lesion of the nervous centres, nor pressure on either the par vagum or the recurrent laryngeal nerve, recovery may be expected after a duration of the affection varying much in different cases. The voice is sometimes restored instantaneously and unexpectedly. I have met with cases in which repeated attacks had occurred.

Treatment in these cases should be directed to the general condition; tonic remedies and invigorating hygienic measures are indicated. Moderate counter-irritation may be useful. Electrization of the laryngeal muscles has been found signally useful. Both the induced and the direct current are used with success. The recovery of the voice sometimes follows a single application, or even a single electrical shock; but, in some cases, the treatment, to be successful, must be continued for several weeks. In some of the cases in which clectricity at once effects a cure, there is reason to think that the success is due to a moral influence. Dr. Henry K. Oliver has found the following method of manipulation successful in several cases: The wings of the thyroid cartilage, in their posterior and upper part, are to be compressed by the thumb and forefinger, the patient being instructed, at the same time, to make an effort to speak. In his cases a feeble voice sound, after a little time, was produced, and this was followed shortly by the full restoration of the voice. He explains the effect by supposing that the approximation of the vocal cords from the pressure, as it were, "starts the machinery of the vocal apparatus." This method is suited only to cases of bilateral paralysis of the adductors. It may reasonably be conjectured that the efficacy of the method is measurably, at least, dependent on a psychical effect.

After a partial restoration of the voice, occurring either under general treatment only, or following local measures, complete recovery may be

1 Vide Am. Jour. of Med. Sciences, No. for April, 1870. 
expected by systematic efforts of speech, in the same way as the control of the will over other paralyzed muscles is effected by means of persistent voluntary exercise. Commencing with the vowels most easily spoken, the practice should extend to those more difficult of utterance, then to consonants and words of one syllable, and finally to sentences. A case has been related to me in which aphonia had existed for sereral years, and the patient, a female, was promptly cured by taking a quantity of spirit sufficient to produce inebriation. The patient's habits were temperate, and this was adviscd as a curative measure by her physician, who had heard of a cure having been thus effected in another case.

\section{Morbid Growths.}

The laryngeal mucous membrane, especially at the vocal cords, is sometimes the seat of morbid growths in the form of warty vegetations, or tumors, which are generally epithelial, but sometimes fibro-cellular, either sessile or pedunculated, the latter called polypi ; these occasion inconvenience, suffering, and death, by producing obstruction. Surgical interference becomes necessary when the obstruction is sufficient to endanger life. A tube may be worn in the trachea for an indefinite period. In a case in which a permanent artificial opening becomes necessary, it may be a question whether a large fistulous orifice might not be established, rendering the tracheal tube unnecessary. As bearing on this question, the following case is of interest. A patient, aged about forty, admitted into one of my wards in Bellevue Hospital, suffering from the effects of a debauch, had a fistulous orifice in the anterior portion of the trachea large enough to admit the end of the forefinger. This fistula followed a wound received in a fracas, and had existed for seventeen years. It occasioned little or no inconvenience in respiration. 'There were no symptoms of inflammation or irritation of the trachea or bronchial tubes, and he was entirely free from pulmonary disease; the only inconvenience which it occasioned was in speaking. In order to direct a current of air through the glottis sufficiently for the production of the voice, he was accustomed to approximate the chin to the sternum, and in this way he was able to close the fistula. The patient died in hospital of delirium tremens, and the larynx is preserved in the hospital museum. Cancer seated primarily in the larynx is rare; the disease, in the cases in which this part becomes affected by it, generally originating in the cesophagus or in the neck exterior to the larynx. The laryngoscope has proved to be of great practical value in determining the existence, situation, size, and character of morbid growths within the larynx, and in facilitating surgical operations for their remoral through the laryngeal aperture. 


\section{CHA P TER $X$.}

\section{PULMIONARY TUBERCULOSIS.}

Definition and Scope of the Term Pulmonary Tuberculosis-Anatomical Characters-Clinical History-Pathologieal Cliaracter-Causation.

CERTAIN changes in nomenclature recently proposed, and to some ex' tent adopted, render necessary an explanation of what is meant by the term pulmonary tuberculosis. As stated in the first part of this work, some pathologists, taking as a point of departure histological observations, propose to restrict the name tubercle to the bodies known as gray tubercles, semi-transparent granulations, etc. (vide page 36 ) ; and to apply the term pneumonia to the affection characterized by the presence of the product commonly known as yellow or infiltrated tubercle; and in order to distinguish the latter from other forms of pneumonitis, varions adjectives liave been proposed, such as tuberculous, lobular, disseminated, cheesy, catarrhal, etc. The term pulmonary tuberculosis has hitherto embraced the affection to which has been of late applied the name pneumonia with these various prefixes. I shall continue in the fourth edition of this work, as in the previous editions, to use the term in the comprehensive sense just stated. Without any qualifying prefix, it is considered as denoting a chronic affection. A rare variety of tuberculous disease is distinguished as acule pulmonary tuberculosis. This will be notieed after having considered the common form of tubereulous disease. Popularly, the affection is known as consumption, or, sometimes, the decline, names which are significant in view of the wasting of the body which attends its march, but yet not distinctive, inasmuch as progressive emaciation belongs to the history of other affections. The name phthisis, or phthisis pulmonalis, has the same significance, that is, expressing wasting or emaciation, and is open to the same objection. Of the diseases which afflict the human family, this is one of the most important, prevailing, more or less, in every quarter of the globe, and occasioning, exclusive of those which prevail epidemically or endemically, a larger proportion of deaths than any other disease. Statistics show that of the population of England, France, Germany, and Russia, amounting to two hundred and thirty millions, the annual deaths from this disease are eight hundred and sixty-nine thousand; and that of the people inhabiting the globe, amounting to nine hundred and sixty-eight millions, three millions die each year with this disease."

Anatomical Characters.-For an account of the morbid products known as the gray and the yellow tubercle, the reader is referred to Part First of this work (vide p. 36). The differential characters belonging to these two products are there given, and certain questions, concerning the relationship existing between them, briefly considered. The pathological interest of these questions is greater than their apparent practical importance. In general, gray tubercles, and the so-called yellow tuberculous infiltration (tuberculous pneumonia), are associated, the relative proportion

Dr. Schnepp, in Archives de Médicine. Juin, 1865. 
of each, as regards quantity, varying much in different cases. In chronic pulmonary tuberculosis, the latter product, that is, the tuberculous infiltration, is especially important, for it is this which, from its amount, the changes which it undergoes, and the destruction of pulmonary tissue to which it leads, is the chief source of danger so far as concerns the local pulmonary conditions.

As regards the amount of morbid products, pulmonary tuberculosis varies much at the outset, in different cases. Different cases also differ much as regards their progressive accumulation. In some cases an increase goes on steadily, that is, without intermission; in other cases, after a certain amount, there is no further increase for an indefinite period. Not infrequently products occur, as it werc, in successive crops, at epochs more or less remote. In some cases they are disseminated in nodules, or tubercles, of small or moderate size. In other cases the nodules, at first isolated, become aggregated by accretion, multiplication, and coalescence, forming irregular masses of considerable size.

They are situated first at or near the apex of one lung, in the vast majority of cases, and in the progress of the affection they extend downwards, more or less, towards the base; but, sooner or later, they occur at or near the apex of the other lung, thence extending downward. Different collections of cases analyzed in order to determine whether the right or left lung is most liable to be first affected, have furnished discrepant results, showing that there is no fixed law with respect to this point. Exceptionally, the affection commences at the base and extends upward. These exceptional cases are exceedingly rare; of 4,530 cases analyzed with reference to this point by Pollock, the affection commenced at the base in sixy-four.

In the early period of the disease, there is a marked difference in different cases in respect of the latency of the affection. In some cases the tuberculous products, although considerable, occasion but little local disturbance; in other cases they cause irritation and inflammation in the surrounding tissues. Circumscribed bronchitis occurs sooner or later, and is more or less marked. Inflammation of the parenchyma, extending over a limited number of lobules (circumscribed pneumonitis), is incidental to the deposit in some cases, increasing, for a time, the degrec and extent of solidification, and sometimes contributing to the breaking down of the pulmonary struetures. The parenchymatous inflammation now referred to does not differ from ordinary pneumonitis. As in the latter, the exudation within the air-cells may be absorbed and sometimes disappears rapidly. It is, of course, to be understood that the yellow tuberculous product to which some pathologists of late apply the name chronic pneumonia is not now referred to. Chronic or interstitial pneumonitis is also a frequent contingent affection, increasing, more or less in degree and extent, the solidification of lung. Tuberculous collections are not infrequently surrounded with dense tissue, or, as it were, encysted, as a result of interstitial inflammation. A result of the latter is sometimes the production of cavities by dilatation of the bronchial tubes. Attacks of circumscribed pleuritis occur almost invariably. They may occur early in the history of the affection. They may recur at variable periods, leading to the exudation of lymph, without liquid effusion, and to adhesion of the pleural surfaces, by means of intervening organized membrane. The occurrence of successive attacks of dry circumscribed pleuritis explains the firm atachment, which is generally found in examinations after death, of the pleuritic surfaces over portions of the lungs corresponding to the sites of the tuberculous collections. Diminished volume of the affected portions of the lungs due to collapse of pulmonary lobules and interstitial pneumonitis are 
incidental to the affection, even before softening of the tuberculous collections has taken place. Emphysema of more or less of the lobules in proximity to the tuberculous collections, is another morbid condition not infrequently observed.

Softening, the discharge of liquefied tubercle by ulceration into the bronchial tubes, and its expectoration, take place after a period which varies much in different cases. The affection has now advanced to the formation of cavities. When the tuberculous collections first formed have in this way been removed, those of a subsequent date are undergoing softening, and at length are, in like manner, removed. Hence, as the affection progresses, new cavities are formed. In examinations after death, cases differ much as regards the number and size of the tuberculous cavities. The greater part of the upper lobe of one lung, and sometimes of both lungs, may be converted into a single cavity or a series of cavities. These cavities are generally irregular in form, with ridges of pulmonary tissue and depressions, and hence they are distinguished as anfractuous. Not infrequently they are traversed by bands of pulmonary tissue, containing bloodvessels, and the rupture of these is the source of the hemorrhage which occurs in certain cases. The cavities become lined with a membrane which is thick and dense in proportion to their age. The destruction of lung is, of course, in proportion to the number and size of the cavities, and their formation leads to a still further reluction of the volume of the portions of the lung in which they are seated. Exceptionally, the liquefied tubercle is evacuated by ulceration through the pleura, into the pleural sac, giving rise to pleuritis with effusion, and allowing the entrance of air, thus causing pneumo-hychothorax. The adhesions resulting from the successive attacks of dry circumscribed pleuritis are conservative as regards protecting against this accident which occurs in only a small proportion of cases.

$A$ simple and convenient division of this affection into stages is based on the marked difference, as regards the anatomical characters, before and after the formation of cavities. The first stage embraces the period during which the tuberculous products accumulate and soften, up to the evacuation in a liquefied state. The second stage extends from the time when cavities are formed to the termination of the affection. A stage of softening, distinct, on the one hand, from the period during which the products are solid or semi-solid, formerly called crude tubercle, and, on the other hand, from the cavernous stage, is superfluous, for the existence of such a stage cannot be predicated with any degree of certainty on either symptoms or signs. As regards the two stages, viz., before and after excavation, it is to be borne in mind that frequently, if not generally, both are represented at the same time in different portions of the lungs. After cavities have been formed in certain portions, more recent collections in other portions are still either crude or undergoing the process of softening.

In this division into two stages, it is assumed that the affection goes on to the formation of cavities. This, unhappily, is the rule; yet, exceptions to the rule are not very infrequent. If the tuberculous products be disposed in small, disseminated nodules or tubercles, they may be absorbed. In some cases the animal constituents of the deposit are absorbed, leaving the mineral portion, and the latter become hard calcareous bodies which may remain imbedded in the lung, or, ulcerating into the bronchial tubes, they are expectorated. In these modes a cure of the local affection takes place in a certain proportion of cases. But a cure may also take place after the affection has gone on to the second stage. It is well ascertained that cavities, even of considerable size, may gradually contract, and, at length, perfectly cicatrize. In some cases in which cicatrization does not 
take place, cavities become lined with a non-secreting membrane, and they may remain nearly innocuous for an indefinite period.

Some writers have reeognized, as a stage of the disease, a period anterior to the tuberculous deposit, which has been called the pre-tuberculous stage. It has been supposed that such a stage may be determinable by symptoms and signs. That both a general and local morbid condition precede the deposit can hardly be doubted, but that anatomical changes take place, which are represented by symptomatic phenomena, and which may be appreciated by physical exploration, cannot be considered as established by elinical observation.

Clinical History.-In sketching the clinical history of this affection, the symptoms attending the development and the first stage, will, in the first place, claim attention.

The affection is often remarkably insidious in its development. In a certain proportion of cases the pulmonary symptoms are preceded, for several weeks or months, by deteriorated general health, as denoted by loss of weight, impaired muscular strength, and a pallid aspect. These premonitions, however, are not constant, and, if they exist, are rarely sufficiently marked to excite the attention of the patient or others. It has been stated by James Clark, Bennett, 'Tweedy, Todd, and others, that certain dyspeptic ailments are apt to precede the development of the affection. If this be so, the ailments are not, as a rule, of a striking character. In a pretty large proportion of cases, the pulmonary symptoms which may be considered as denoting the tuberculous affection, are neither preceded nor accompanied by ailments of any description which lead the patient to suspect the existence of any disease. In the majority of cases, when the patient first comes under the cognizance of the physician, the previous history renders it probable that the affection has existed for several weeks, or, perhaps, months.

Of the pulmonary symptoms, the first which occurs is cough. Unless the affection be developed coincidently with an attack of bronchitis, which is not common, the cough is, at first and for some time, slight, dry, hacking, and perhaps excites little or no attention. Occasionally, however, it is, from the outset, a prominent symptom. The expectoration, for some time wanting, is at first small, and consists of foamy or glairy mucus. It progressively increases, becoming gradually more or less abundant, and at length solid and opaque. These symptoms proceed from irritation and circumscribed bronchitis. Hæmoptysis frequently takes place shortly after the occurrence of cough and expectoration. In some cases it precedes these symptoms. It is often the event which first excites any apprehension of serious disease, and leads the patient to seek medical advice. If cough and expectoration have existed for some time prior to this event, the patient had not heeded them, thinking they were due to a slight cold, and that they would shortly disappear.

Pain in the chest does not belong to the tuberculous affection per se. Pain, however, is apt to occur, at times, more or less frequently, during the first stage of the disease, being due to the circumscribed pleuritis to which reference has been made. 'The pain is sharp, lancinating or stitchlike, referred to the summit of the chest, and frequently shooting beneath the scapula. It varies at different times and in different cases in severity, being rarely sufficient to keep the patient in bed or even within doors, and it may continue for one, two, or three days. In the progress of the disease, the pain in these attacks is referred successively to both sides of the chest, and it may be most severe and frequent on the side in which the 
amount of the tnberculous affection is least. These attacks are frequently considered by patients to be rheumatic or neuralgic. They possess considerable diagnostic significance, but they are of very little importance as indicating gravity or progress of the disease. Intercostal neuralgia occasionally occur's in tuberculous patients, and is to be distinguished by the diagnostic points which have been presented in treating of that affection." The respirations are usually increased in frequency, and the increase, other things being equal, is in proportion to the amount of the tuberculous products. This symptom may not attract the notice of the patient. It is apparent especially on exercise. It is rarely the case that suffering from dyspnœa is experienced, and to ascertain the existence of this symptom, it may be necessary for the physician to observe for himself, and not trust to the statement of the patient. Even when, owing to the amount of tuberculous products, and the rapidity with which they have been developed, lividity is marked, dyspnœa is not always complained of.

Among other than pulmonary symptoms, the condition of the circulation, as represented by the pulse, holds an important place. During the first stage, more or less acceleration of the pulse is the rule, and this acceleration, although not great, is an important symptom in a diagnostic point of view. The amount of acceleration differs much in different cases. In general, the frequency of the pulse is in proportion to the activity of the tuberculous affection. If the pulse be habitually frequent, the disease will be likely to be rapidly progressive. A certain proportion of cases are characterized by frequency of the pulse, which is also quick, vibiatory, and compressible, qualities denoting morbid activity, but diminished power, of the contractions of the left ventricle. In these cases the career of the disease is apt to be short, the products progressively accumulating and running rapidly through their changes.

The temperature, as determined by the thermoneter in the axilla, as a rule, is more or less raised in proportion as the tuberculosis is progressive. The thermometer is thus useful by aiding in the diagnosis of tuberculous disease, and if the diagnosis be not doubtful, by affording evidence of the disease being progressive or otherwise. When it is a question as to the diagnosis, inerease of animal heat is evidence for, and absence of any increase of heat is evidence against, the existence of tuberculosis. In well-marked cases of tuberculosis the amount of increase of heat is a measure of the activity of the disease, whereas a normal temperature denotes that the disease is non-progressive.

Daily febrile exacerbations, commonly called hectic fever, may occur in the first stage. Toward evening the acceleration of the pulse is increased, with increased heat of skin, and sometimes a marked sensation of burning in the soles and palms; the cheeks may present a circumseribed flush, and after some hours the exacerbation goes off with perspiration. The exacerbation is sometimes preceded by a chill. Chills also occur without being followed by notable febrile movement. They may be pronounced and accompanied by some rigor, or they are slight and transient, consisting, in some cases, of occasional shiverings or a sensation as if water were trickling down the back. Well-marked chills, followed by increased heat and perspiration, may occur with such regularity that the patient is supposed to be affected with intermittent fever, and, under these circumstances, if the pulmonary symptoms be slight, the affection of the lungs may be for some time overlooked. I suppose there are few physicians of much experience in situations where they are liable to meet with intermittent fever,

1 Vide page 202. 
who cannot recall cases in which this error of diagnosis has bcen made. Febrile movement of limited duration may be due to an intercurrent circumscribed pneumonitis which is simple, that is, not tuberculous in character.

As regards appetite and digestion, there is much diversity in different cases. In general, the desire for food is diminished, and the ability to digest food impaired. In some cases there is almost entire loss of appetite and even a repugnance to food. This is an unfavorable feature of the disease. Disorder of digestion is sometimes so prominent as to appear to be the chief malady. The physician should be on his guard against considering the cough in such cases as merely dyspeptic or sympathetic. Antipathy to fat is by no means a constant feature of tuberculous cases; some patients take fatty articles of food freely without repugnance and with relish. And, on the other hand, not a few patients who are not tuberculous have an antipathy to fat.

Diarrhoa occurs, in the first stage, in a certain proportion of cases. If occasional only, it may proceed simply from incomplete digestion; but if frequently recurring or persisting, it points to the existence of a tuberculous affection within the intestinal canal, usually seated in the small intestine. Persisting or frequently recurring diarrhœa, accompanied by cough, if the latter be never so slight, should excite suspieion of pulmonary tuberculosis, and the existence of this affection being ascertained, a tuberculous affection of the intestinal camal, as a secondary affection, is to be inferred. Diarrhœa proceeding from tuberculous enteritis in an unfavorable symptom, diminishing the prospect of improvement. The seat of the affection is usually the Peyerian and solitary glands. It leads to ulcerations differing from those which are characteristic of typhoid fever in a tendency to extend in a transverse direction and to occupy only portions of the elliptical patches; also, in having hardened and raised margins. Tuberculous uleerations are sometimes found in the large, as well as in the small, intestine, and they may be confined to the former. The ulcerations may lead to circumseribed peritonitis, and the peritoneal inflammation sometimes becomes general. Perforation of the intestine occasionally takes place, giving rise to general peritonitis, provided the peritoneal surfaces over the ulecrated space within which the perforation oceurs have not become adherent from previous circumscribed inflammation.

Impaired nutrition, denoted by diminished weight or emaciation, is frequently one of the earliest symptoms. As a rule, reduction in weight continues so long as the disease is progressive; on the other hand, the disease may generally be considered as non-progressive if the patient be not losing weight, and, still more, if he be gaining inflesh. Defect in the processes of assimilation is shown also by anæmia, which, in most cases of pulmonary tuberculosis, is an early symptom, and frequently exists in a marked degree.

The muscular strength progressively diminishes if the disease be progressive, but here there is much variation in different cases. Patients are rarely compelled to keep the bed in the first stage, and they are generally able to be out of doors. Deficiency of breath, in some cases, precludes an amount of active exercise or labor for which muscular strength is suffciently retained.

In females, suppression of the menses is a symptom significant of the progress of the disease; the menstrual discharge climinishes in quantity, and generally becomes suppressed. They are apt to attribute importance to the suppression as a cause of their other ailments. It is, however, an event incidental to the tuberculous disease, and cloes not claim special 
measures of treatment. Facts do not show that suppression of the menses from other causes exerts an influence in the development of tuberculosis.

This disease does not tend directly to impair the intellectual faculties. The intellect frequently remains bright and active notwithstanding the progress of the disease. 'The feelings are usually cheerful and buoyant. Patients are hopeful respecting their condition; they depreciate their symptoms, and are reluctant to admit that the malady is serious. This state of mind sometimes amounts to an infatuation which renders it difficult to obtain from the patient a fair account of the symptoms, and it is necessary for the physician to rely chiefly on the statements of others and his own observations. To such an extent is the absence of anxiety characteristic of the disease, that an opposite frame of mind militates somewhat against the supposition of the existence of tuberculosis. 'Tuberculous patients are readily persuaded that they are improving, and hence they fall an easy prey to quacks. They sometimes dislike to be told the truth, and take offence at an intimation that they are consumptive.

Proceeding to notice the points in the clinical history which distinguish the second stage, it is to be borne in mind that it is not easy, nor is it practically important, to draw an exact line of demarcation between the two stages. Successive tubereulous irruptions are apt to take place, or the accumulation may be going on steadily, so that, while the local affection has advanced to the second stage in one part, usually near the apex, the first stage may be represented in other parts. The disease is to be considered as having advanced to the second stage when it has led to the formation of a cavity or of cavities of sufficient size to give rise to certain distinctive signs and symptoms. The symptoms of the first stage continue into the second, in most cases, being increased and modified by the existence of cavities.

The cough in the second stage usually becomes more prominent. It is less irritable, and more exclusively for expectoration. It is troublesome and difficult in proportion as the contents of the cavities are not easily evacuated, owing to their size, want of free communication with the bronchial tubes, etc. 'The expectoration consists of liquefied tubercle, matter furnished by the lining membrane of the cavities, and products of secretion from the bronchial mucous membrane. The quantity of expectoration is very variable, amounting, in some cases, to only a few ounces, and, in other cases, to a pint or more daily.

The appearances of the expectoration are various. It is oftenest mucopurulent with more or less predominance of the characters of pus. In proportion as it has the character's of pus, the sputa do not remain distinct, but run together to form a homogeneous mass. Sometimes a collection of the liquid expectorated presents a thick, opaque appearance not unlike that of pea-soup or gruel. The sputa are sometimes solid, remaining distinct, and, in falling upon the bottom of a vessel, assume a flat, round appearance like that of a coin, and hence are called nummular sputa. The sputa occasionally present yellow striæ, which consist apparently of pus. The edges of the sputa are sometimes ragged; another appearance is that of small particles, resembling boiled rice, contained in the matter expectorated. It is very rare for a semi-softened, cheesy, morbid product to be expectorated; but, in one case under my observation, several masses of this description were raised, evidently due to an unusually large ulcerated orifice being established between a tuberculous collection and a bronchial tube of considerable size. In this case, the signs of a cavity were developed directly after the expectoration of a large amount of matter which contained the partially softened masses. A sudden expectoration of mat- 
ter, in considerable quantity, presenting a purulent or puruloid appearance, denotes the opening by ulceration of a so-called tuberculous abscess. Not infrequently this occurs repeatedly during the progress of the disease ; but in many cases the ulcerated opening is at first small, and the liquefied matter escapes gradually into the bronchial tubes. 'The constituents, as shown by the microscope, are pus-globules, epithelium, fatty granules, blood-clisks; occasionally the yellow elastic fibre, and sometimes vegetable sporules and spores.

'The quantity of matter expectorated is, in general, in proportion to the number and size of cavities. It is usually more abundant in the morning than at any other period, owing to its having accumulated during sleep. 'Toward the elose of life the act of expectoration becomes difficult on account of muscular feebleness, and the consequent accumulation of morbid products in the air-passages not infrequently hastens death. Occasionally the matter expectorated, and the breath of the patient, emit considerable fetor, due to sloughing of small masses of pulmonary structure within the cavities.

Hæmoptysis occurs less frequently in the second than in the first stage ; and, occurring in the second stage, it may be due to the rupture of the bands of pulmonary tissue which so often traverse tuberculous eavities, these bands sometimes containing vessels of sufficient size to furnish an abundant hemorrhage. It is possible for the loss of blood in this way to be suffieient to destroy life, but instances are extremely rare. The hemorrhage, however, may contribute to a fatal result. I have known a large cavity to become filled with coagnlated blood.

Hoarseness, or huskiness, and sometimes extinction of the voice, denote laryngitis, which may be developed in the first, as well as in the second, stage. This affection has been considered in the preceding chapter. It is to be borne in mind that its existence constitutes presumptive proof of pulmonary tuberculosis, the latter existing prior to the laryngitis.

Lancinating pains incident to circumseribed pleuritis reeur in the second as well as the first stage, from time to time. Occasionally, both in the first and second stage, the pleuritis becomes general, and is attended with liquid effusion. So far as my observations go, they confirm the statement of Louis, that double pleuritis with effusion always denotes the pre-existence of tubercle. Acute pain, suddenly developed, with embarrassed breathing, acceleration of pulse, and prostration, point to perforation of the lung, and the signs of pneumo-hydrothorax are to be sought for.

The pulse, as a rule, is more accelerated in the second than in the first stage. Febrile exacerbations are oftener present and more marked, witl burning of the soles and palms. The night perspirations are often copious, being a source of debility and great discomfort.

The appetite and digestion are more or less impaired, cases differing greatly as regards the symptoms connected with the ingestion of food. Diarrhea is more frequently present and is a more prominent symptom in the second stage, being connected often with intestinal ulcerations. If profuse and persisting, it has been distinguished as colliquative diarrhcea. Colliquative diarrhœa occurs at an advanced period of the disease when tuberculosis of the intestines does not exist. It is then due to impairment of intestinal digestion, and to transudation from impoverishment of the blood. With the progress of the disease the body progressively diminishes in weight, and the patient is often reduced to an extreme degree of emaciation. The strength varies greatly in this stage. The patient may be confined to the bed for a greater or less period before death, but not infrequently strength is preserved sufficiently to be up much of the time, 
and even to go out of doors, within a few days or even a few hours of death.

Bulbous enlargement of the ends of the fingers, with incurvation of the nails, forming what are commonly called clubbed fingers, is occasionally observed. 'I'his peculiar appearance is characteristic of tuberculous disease, but it is not a pathognomonic symptom. I have known it to be strikingly marked in connection with organic disease of the heart withont tuberculosis, and also in chronic pneumonitis. The toes present a similar appearance, but in a less marked degree. This symptom occurs only in cases in which the disease has existed for a considerable period. Of 1776 cases analyzed by Pollock, the fingers were more or less clubbed in 654, being a fraction under 27 per cent.

The mental condition in the second stage is frequently not less characteristic than in the first. The expectation of improvement, prolonged life, or even recovery, amounts, in some cases, to an insane delusion. Patients are sometimes occupied in forming plans for the future, when it is obvious to any observer that they are on the verge of the grave. They may insist to the last that there is no danger. Over and over again, in the hospital pulmonary wards, I am asked by patients reduced almost to skeletons, and too feeble to sit up for a moment, if there be any danger, or if I do not think they will recover. But whenever patients affeeted with this disease are satisfied that there is little or no hope of recovery, they generally become quickly and completely resigned.

Edema of the feet and ankles is a symptom of the second stage, denoting notable failure of the circulation. For a time this may occur only after sitting or standing, disappearing after the recumbent posture has been maintained for some hours; but, at length, it is permanent, and may increase and extend over the whole of the lower extremities, rendering them unwieldy or powerless. When the oedema becomes thus marked, extensive, and permanent, thrombosis, with, as a consequence, an obstruction of the crural veins, is to be suspected, an event which is liable to occul in this disease. Coagulation in the crural vein on one side will account for the cedema in some cases in which it is either limited to, or is much greater in, one of the lower limbs. If the codema become diffused over the body, constituting anasarea, coexisting disease of the kidneys is to be inferred ; but degenerative disease of the kidneys is a rare complication of pulmonary tuberculosis.

The clinical history, in cases of pulmonary tuberculosis, is affected, in no small degree, by complications and coexisting affections. The occurrence of tuberenlous disease of the intestines, tubercnlons laryngitis, pneumo-hydrothorax, and disease of the kidneys, has been already referred to. Chronic peritonitis is an occasional complication. The researches of Louis have shown that chronic peritonitis, not traumatic, is almost invariably dependent on tuberculosis in this situation, and hence that, from the existence of the peritoneal affection, pulmonary tuberculosis may be inferred. Acute peritonitis, from perforation, is sometimes incident to the ulcerations which proceed from the tubereulous affection within the intestinal canal. Meningitis occurs as a rare concomitant in the adult, but not infrequently in early life. This coexisting affection accounts for the development of delirium and coma in certain cases. Perineal abscess, leading to fistula, is another complication occurring in a certain proportion of cases, proceeding probably from tubercle in this situation. Clinical observation shows that, as a rule, when this complication occurs, the amount of tubercle in the lungs is small or moderate, and the progress of the pulmonary affection is slow. An accumulation of fat within the liver 
cells is a morbid condition observed in a small proportion of cases, occurring oftener in females than in, males.

The duration of this disease is extremely variable. In the vast majority of cases it is essentially a chronic affection, but sometimes it runs a rapid course, passing through its changes and destroying life in a few weeks. The disease in such cases has been known as "galloping cousumption." The disease, in these cases, is quite different from that known as acute phlhisis, which will be noticed briefly under a distinct heading.' Distributing fatal cases into groups according to the duration of the disease, exclusive of the rapid cases just referred to, one groul will consist of cases in which the disease continues from three to six montlis; in another group, death takes place between six months and a year; in a third group the career extends from one to two years; and in another group the fatal termination is held in abeyance for many years; I have met with a number of persons who have been tuberculous for more than twenty years, and a patient was for a long time under my observation in whom there is reason to believe the disease existed for forty years.

Limiting attention to the eases in which, sooner or later, the disease proves fatal, its march is extremely irregular. It is not uncommon for little or no progress to be apparent for several successive weeks, months, or even years, and then the disease to advance with more or less rapidity. Distinct tuberculous irruptions occurring at successive periods are frequently points of departure for the onward march of the disease; but cases differ much as regards the local destruction, changes incident to the collections of tubercle, and the rapidity with which softening, ulceration, and the formation of cavities take place. Further remarks on the mareh of the disease will be offered under the head of prognosis.

Pathologrcal Cinaracter.-The pathological character of this affection has already been considered in the first part of this work. In a practical point of view, the fact to be especially borne in mind is that pulmonary tuberenlosis is not, primarily and essentially, an affection of the lungs. The tuberculous products proceed from a prior morbid condition of the system; in other words, the pulmonary disease is the local expression of a special constitutional affection called the tubereulous cachexia or dyscrasia. It is a rational inference that a vice of assimilation is involved in the existence of the tuberculous cachexia, but the nature of the latter and the mode of its production are problems remaining to be solved by future researches. Grounds for the foregoing statement as regards the constitutional origin of pulmonary tuberculosis will appear in proceeding to consider the causation.

Causation.-The causes of pulmonary tuberculosis may be divided into those which act, first, locally, upon the lungs; and, second, generally, or upen the system, in producing the constitutional condition or cachexia which constitutes the disease. With respect to local causes, it may be doubted whether they are ever sufficient in themselves to produce the disease. It is probable that they only act as exciting causes. 'The tuberculous affection is sometimes either preceded by, or occurs in connection with, some other pulmonary affection, viz., bronchitis, pleuritis, or pneumonitis. If this association be more than a coincidence, the latter affections only serve to determine the local manifestation, the cachexia already existing;

'By some writers the term "galloping consumption" is applied to the latter form of disease. 
that is, they act the part of the match when everything is ready for the explosion. These affections occur so frequently without being either accompanied or followed by tuberculosis, that it is certain they have no intrinsic tendency to that result; and, on the other hand, in the great majority of cases, pulmonary tuberculosis is devcloped irrespective of these affections. It has been supposed that the inhalation of irritating substances, such as stone-dust, fine particles of metal, etc., necessarily incidental to certain occupations, is sufficient to give rise to tuberculous disease. Even this may be doubted. When the disease attacks needlegrinders, stone-cutters, etc., causes are probably involved other than those acting locally, the latter playing only a subordinate part in the etiology. The inhalation of irritating substances gives rise not infrequently to chronic interstitial pneumonitis accompanied by signs and symptoms which render it not easy to differentiate the affection from tuberculosis.

The statements in the foregoing paragraph are not in accordance with views held by some late writers, namely, Niemeyer and others. According to these views, the development of pulmonary tuberculosis, that is, of the yellow tuberculous product, is often dependent on bronchitis. Hence, the name adopted by Niemeyer, chronic catarrhal pneumonia. Niemęyer and others, indeed, go so far as to attribute the development of this tuberculous affection chiefly, or entirely, to a bronchitis or bronchial catarrh, attributing little or no importance to a diathetic or constitutional agency. $\Lambda$ question of this kind is settled not by a theory based on histological observations, but by clinical facts. Now the early clinical history, in cases of tuberculous disease, shows, as already stated, that a bronchitis, or bronchial catarrh, rarely precedes the symptoms and signs which denote tuberculosis. I make this positive assertion with the utmost confidence in its correctness, inasmuch as it is based on the study of a large number of cases with reference to the earliest symptoms and signs. Moreover, it accords with the clinical researches of Louis, which, so far as they go, are as reliable and as valuable to-day as when they were made, now nearly half a century ago. A side from clinical facts, it is certainly irrational to suppose that a bronchitis, which is a bilateral disease, should give rise to an affection almost uniformly limited, at first, to the apex of one lung. The doctrine that the development of pulmonary tuberculosis is dependent on bronchitis, or other local causes, rather than on an unknown constitutional agency, called a cachexia, is the more to be protested against, since, upon this doctrine are based prophylatic and therapeutical measures which are opposed by clinical experience.

Niemeyer attributed the production of phthisis, in some cases, to bronchorrhagia, the presence of blood remaining in the bronchial tubes being supposed to prove a local cause. As already stated, clinical observation, so far from affording ground for this opinion, goes to show that hæmoptysis is oftener a salutary event than otherwise, if tuberculous disease already exist; and there is reason to think that it is sometimes a substitute for, and, therefore, preventive of, tuberculosis. But in some rare, exceptional cases an attack of hæmoptysis appears to be the point of departure for either the development or the active progress of tuberculous disease.

To the conformation of the thorax was formerly attributed consirlerable causative agency. This doubtless arose in part from observing that tuberculous patients often present contraction of the chest, occurring as an effect of the disease. Persons with great deformity of the chest are not specially prone to tuberculosis. So, also, the idea that the exercise of the voice may be a predisposing or exciting cause, has become obsolete; and 
observation shows that, if any influence be derived from this source, it is conservative rather than eausutive.

The opinion appears to be entertained by some writers, that chronic pharyngitis, which is an exceedingly common affection in this part of the world, has a tendency to eventuate in pulmonary tuberculosis. I am satisfied that this opinion is erroneous. After a large opportunity of observing cases of chronic pharyngitis, I have come to the conclusion that it generally denotes a condition of the system unfavorable to tuberculous disease. The sebaceous collections which are liable to accumulate in the glands of the tonsils, and to be ejected by coughing in the form of round, hard pellets, are sometimes mistaken for tubercles. These may be distinguished from obsolete tubercles or pulmonary calculi by their being unctuous to the touch, and emitting, when crushed, a fetid odor.

'Tlue efficient eauses of pulmonary tuberculosis must be sought for among those which affect the system. Were the essential nature of the disease understood, we might hope to reach, synthetically, the knowledge of its causation. A rational pathology and etiology must of necessity go hand in hand; but our present knowledge of both is confined within narrow limits. 'The actual knowledge which we possess is derived from clinical observation and statisties, and there is much room for further information to be derived from these sources. I shall state certain conclusions which appear to be well grounded, referring the reader, for a fuller exposition of the facts on which they are based, to works treating at greater length of this most important branch of the subject.

Facts show the tuberculous diathesis in a certain proportion of cases to be congenital and inherited. Why a peculiarity of constitution rendering a person specially liable, at a certain age, to the development of this disease should be transmitted from parent to child, camnot be explained more than the fact that peculiar traits of physiognomy or mental character are inherited. The conelusion rests on obserration. It is a matter of common remark that the offspring of cousumptive parents are apt to become tuberculous. But it is to be borne in mind that, owing to the frequency of the disease, a tolerably large proportion of tuberculous parents must have a consumptive parentage from mere coincidence; and it may be true that, sufficient consideration not having been aceorded to this fact, hereditary influence has been overrated. Walshe's statistical researches appear to give support to this supposition. Interrogating a considerable number of hospital patients affected with the disease, viz., 162 , he found that of this number in 26 per eent. either the father or mother, or both parents, were tubereulous." He argues that this proportion is not much above that which would be expected from the prevalence of the disease irrespective of any lereditary influenee. These statisties, however, are open to the eriticism that the statements of hospital patients are not very reliable, and that, in a certain proportion of cases, the parents might subsequently become tuberculous. Moreover, the number of patients interrogated, although considerable, was not very large. The plan of investiuration might be pursued farther with perhaps a different result; and it would be more satisfactory to analyze cases in private practice with respect to this point.

A congenital and hereditary influence is most strikingly shown by facts relating to certain families. In another work I have eited the following instance, occurring under my own observation : $A$ ll the children of $a$

1 British and Foreign Med.-Chir. Rev., Jan. 1849.

2 Physical Exploration of the Cliest, and the Diagnosis of Diseases affecting the Respiratory Organs. 1866. 
family, seven in number, were victims of this disease in five years-the ages being between 18 and 23 . The mother died of the disease shortly before the death of the first of the seven children who died. 'The father was a man of robust health. Another instance within my knowledge is as follows: $A$ tuberculous patient was under my care in January, 1862, and subsequently died. This patient had lost eight brothers or sisters from the disease, and both parents. Two sisters, then living, were all that remained of the family. Another striking illustration is afforded by the following facts : Or a family comprising eight children, one died in childhood with convulsions, three daughters and one son have died with phthisis, another daughter and a son are now tuberculous, and the only remaining child, the eldest daughter, presents the history and signs of arrested tuberculosis. Both parents died tuberculous. 'The following statement, communicated to me by Dr. Henry E. Paine, of Dixon, Illinois, illustrates a congenital tendency without the evidence of inleritance: Mr. S. is 57 year's of age, and in good lealth. His wife is 52, and well. No progenitors have been known to die with tuberculosis. All the children, five in number, died with this disease between 1853 and 1861 -their ages respectively being, at the time of death, 23, 25, 24, 22, and 23. The following facts are interesting as showing a remarkable limitation of the disease to one branch of a family: 'Two brothers married sisters. In neither family was there evidence of hereditary predisposition to tuberculous disease. One of the brothers and his wife died of the disease. Of two sons and two danghters, both the latter and one of the sons have died with the disease ; and the remaining son is probably tuberculous. The other brother and his wife are living in good health, and of several children, none have been affected with tuberculous disease.

Facts, such as have just been cited, certainly show that the tuberculous diathesis is sometimes inherent in the constitution and inherited. 'To what extent a congenital influence is involved in cases in which circumstances clo not render it evident, must be left for conjecture. It is not irrational to suspect its existence in a large proportion of the cases in which proof is impossible.

The predisposition has relation to age. The disease is most liable to be developed between 20 and 30. The other decennial periods of life are arranged in the following series, according to the rate of predisposition: 30 and 40,10 and 20,40 and 50,50 and 60,0 and 10,60 and 70, 70 and 80,80 and 90,90 and 100 . No age is exempt from a liability to the disease, and it may affect the fotus in utero.

Climate appears to exert an influence either for or against the development of the disease. It may be stated that the prevalence of the disease is less in climates either uniformly warm and dry, or uniformly cold and dry, than in those which are moist and subject to frequent alternations of cold and warmth. A high altitude appears to afford protection against the disease. Jaccoud states that observations for fifteen consecutive years warrant him in asserting that in Alpine situations elevated 4000 feet, tuberculosis is almost unknown; and especially is this true of villages at an elevation of 5500 feet, where, in the language of the country, there are " nine months of winter and three months of cold weather." According to Dr. Hjaltelin, who resides in Iceland, the inhabitants of that country enjoy exemption from phthisis. The disease prevails to a much greater extent in cities than in the country, showing, doubtless, the operation of auxiliary causes in the former. It prevails more on the seaboard than in inland situations. The elaborate researches of Prof. Bowditch go to show that, in the State of Massachusetts, cases occur more frequently in localities in 
which the atmosphere is rendered humid by streams or marshes. Dr. Buchanan's observations show that, in England, cases are comparatively rare in dry, elevated situations. 'There is no foundation for the opinion that the terrestrial emanations known as malarial afford any protection against the disease. Newly-settled places are firequently exempt, for some time, from its prevalence to much extent; but the explanation of this fact is, the pioneers who compose the early population in these places are persons, generally, of sturdy, vigorous health, and the habits of life, in such a population, are protective against this discase. $\Lambda$ s regards season, the disease is oftener developed during the spring months and the hot montlis of summer than at other portions of the year.

The disease prevails much more among those whose pursuits are seclentary than among those whose occupations involve out-door life. Want of exercise, defective ventilation, deficiency of light, and the depressing emotions undeubtedly contribute to the production of the tuberculous cachexia. With reference to the influence of ventilation, the following researches of Dr. Guy, of London, are interesting: 'Taking hremoptysis as a criterion of either tuberculosis or the tuberculous cachexia, Dr. G. interrogated 104 compositors who worked in apartments having less than 500 cubic feet for each person. Of this number thus situated, 12.50 per cent. had had hæmoptysis. He then interrogated 115 compositors who worked in apartments having firom 500 to 600 cubic feet, and of this number 4.35 had had liæmoptysis. Of 101 compositors working in apartments with more than 600 feet only 1.98 had had hamoptysis. The influence of the several causes just mentioned is seen in the greater liability of domesticated animals to tuberculous disease than of the same animals in a wild state. "The stabled cow, the penned sheep, the tame rabbit, the monkey, the caged lion, tiger, or eleplant, are almost invariably cut off' by tuberculous affection."

The infrequency with which the disease is found in the post-mortem examinations of those who have died from intemperance, has led to the supposition that the use of alcohol antagonizes the tubereulous cachexia. $\Lambda$ ssuming that there is ground for this supposition, it would be irrational to reject it because it may be made to sanction the unnecessary use of alcohol; but the moral, as well as physical considerations which this application involves are, of course, to have due weight in affecting the conduct of practitioners. The following statistical facts relating to the prophylactic influence of alcoholism have been communicated by Leudet :Of 121 persons addicted to the use of alcohol, twenty were tuberculous, and these twenty were the only intemperate persons among 600 tuberculous patients. ${ }^{2}$ There is reason to believe that habits of diet unduly restricted as regards variety and quantity, either from choice or necessity, may contribute to the development of the disease.

Pregnancy has been supposed to antagonize the tuberculous cachexia, and marriage has sometimes been advised as a prophylactic and even curative measure. Facts, however, do not afford support to this supposition. The researches of Grisolle and Dubreuilh show that, so far from this being true, the development of the disease not infrequently takes place during pregnancy, and when tuberculous patients become pregnant, the progress of the disease is hastened rather than retarded. Of 155 cases in patients who had recently been confined, analyzed by Pollock, in 48 the disease was developed during pregnancy, and in 51 it began at confinement. ${ }^{3}$ An

' Aitken, Science and Practice of Medicine.

2 Hérard and Cornil, op. cit.

The Elements of Prognosis in Consumption, by James Edward Pollock, M.D. London, 1865. 
analysis, by the same author, of 94 cases in which the disease was developed after confinement, showed that it began during lactation in 54 . Clinical facts are opposed to the notion, that the progress of the disease is retarded by lactation.

Certain diseases exert an influence, on the one hand, to promote, and, on the other hand, to prevent the development of tuberculosis. It rarely occur's in persons affected with pulmonary emphysema. Persons affected with cardiac lesions which interfere with hæmatosis, rarelyo become tuberculous. On the other hand, measles and typhoid fever leave the system in a condition favorable for the development of the tuberculous cachexia. Are they who suffer in early life from a scrofulous affection of the lymphatic glands of the neck especially prone to pulmonary tuberculosis in after-life? I have been led to think that this question may be answered in the negative. I have collected a number of cases in which young and middle-aged persons presenting the characteristic cicatrices on the neck were free from tuberculous disease of the lungs; and, on the other hand, it is extremely rare to find these cicatrices in persons who are affected with pulmonary tuberculosis. A ssuming the tuberculous and the scrofulous cachexia to be the same, it would seem that it is, as it were, exhausted by the deposit in the glands of the neck, and that afterward it is not likely to recur. Contrary to what might be expected à priori, anamic persons rarely become tuberculous. Pollock states that of 125 carefully noted cases of anæmia, with murmurs, in none was there any sign of tubercle. The association of anæmia with pulmonary tuberculosis, except as a complication or an effect, is certainly rare. It is rare for tuberculosis to follow an attack of lobar pneumonitis.

In conclusion, of the various agencies which are involved, more or less, in the causation of pulmonary tuberculosis, it is difficult to assign to each its proper relative importance. It is probable that this is pre-eminentiy a diathetic disease; but it is impossible to determine whether the diathesis be always innate, or whether, in a certain proportion of cases, it be acquired. Do the causes which contribute to the development of the disease produce the diathesis, or do they act solely by producing the cachexia, the diathesis already existing? Our present knowledge does not enable us to answer this question. Yet our limited knowledge of the causation is of immense value in its practical application to prophylaxis and treatment.

The production of tuberculous disease in certain inferior animals by inoculating them with matter obtained from the tuberculous products in man has been already referred to (vide Part I., p. 40). The discovery of this interesting and important fact naturally leads to the inquiry whether pulmonary tuberculosis may not sometimes be caused in man by the inhalation of a virus contained in the breath or exhalations from the body of tuberculous patients. The doctrine of the contagiousness of the disease has now, as hitherto, its advocates, but the general belief of the profession is in its non-communicability. The advocates for the doctrine have relied for its support especially on the number of instances in which husbands and wives have been affected in succession. If the communicability of the clisease by inoculation establish the existence of a specific virus, this fact would lend considerable support to the doctrine of contagiousness. 


\section{CH A P T E R XI. PULMONARY TUBERCULOSIS-(CONCLUDED.)}

Diagnosis-Prognosis-Treatment. Acute Pulmonary Tuberculosis.

$\mathrm{D}^{\mathrm{T}}$ IA GNOSIS.-A positive, and, especially, an carly diagnosis, in cases of pulmonary tubereulosis, must rest mainly on physical signs, in connection with the history and symptoms. A full consideration of the physical diagnosis does not fall within the scope of this work. For this, the reader is referred to treatises devoted to physical exploration, or to diseases of the chest.' I shall content myself with giving an enumeration of the important signs involved in the discrimination of the disease, and, afterward, recapitulate the diagnostic symptoms embraeed in the clinical history.

The physical conditions, when the amount of tubercle is abundant or when the disease is advanced to the second stage, are represented by wellmarked signs, rendering the diagnosis sufficiently easy and positive. 'The diminished volume of lung at the apex, whieh frequently occurs, causes a depression at the summit of the chest on the side most affeeted, and, owing to pleuritic adhesions, the expansion, at the summit, is less than on the opposite side. 'The scapula, on the side most affeeted, is often restrained in its movement upward in inspiration, contrasting, in this respect, with the opposite scapula. These signs, obtained by inspection, are available in a certain proportion of cases. Percussion clicits relative dulness, and sometimes even flatness, over the tuberculous collections. Exceptionally, over the solidified portion of lung, the resonance is tympanitic. A tympanitic resonance over a cireumscribed space, and the varieties of this resonance ealled cracked metal and amphorie, point to the existenee and situation of eavities. 'The respiratory sound, obtained by auseultation, is either bronchial, broncho-vesicular, or cavernous, and, not infrequently, these different signs are found in different situations, in the same case. The first represents considerable or complete solidification, the second moderate or slight solidification, and the third a cavity or cavities. 'The cavernous respiration, in some cases, has an amphorie quality. 'The correlative voeal signs are likely to be present, namely, either bronchophony with the loud and whispered voice, or exaggerated vocal resonance, and, in rare cases, pectoriloquy. More or less of these auseultatory signs are available in the great majority of eases. And these, taken in connection with the cough and expectoration, the emaciation, and other symptoms belonging to the history of the disease, render the diagnosis almost aiways an easy problem.

It is only in cases in which the quantity of tubercle is morlerate or small, the symptoms being, at the same time, less marked, that the liagnosis is difficult; and the difficulty, in such cases, is rarely great, provided

1 The author has considered at length the physical diagnosis of this as well as other pulmonary affections, in his work entitled Physical Exploration of the Chest and the Diagnosis of Diseases affecting the Respiratory Organs. Second cdition, 1866. 
the physician have a good practical knowledge of physical exploration. Inspection may, in these cases, furnish the same signs, but less marked than in cases of abundant tubercle. As a rule, slight or moderate, but distinct, dulness on percussion may be ascertained. The exception to this rule is when more or less of the pulmonary lobules become emphysematous, and then the resonance is altered, although not diminished-it is vesiculo-tympanitic. A comparison of the respiratory murmur on the two sides at the summit, will be likely to show abnormal changes, viz., the characters of the broncho-vesicular (rucle) respiration. So, also, on a comparison as regards vocal signs, the vocal resonance and the bronchial whisper may be found to be exaggerated. The same is truc of vocal fremitus. A careful exploration with reference to the foregoing signs, will furnish direct evidence of the disease.

In addition to these signs, there are several, occasionally present, which are inferentially evidence of tuberculosis. These may be distinguished from those already enumerated, as the accessory signs of the disease. Fine inucous or the subcrepitant rales, heard within a circumscribed space at the summit of the chest on one side, are highly significant of tubercle. They proceed from circumscribed bronchitis excited by the presence of a tuberculous product. A crepitant rale, in like manner limited to a small space at the summit on one side, has the same significance, being due to circumscribed pneumonitis excited by the tuberculous product. Crumpling and crackling sounds, also, are significant, provided they be limited to the summit on one side. A pleural friction sound limited to the summit on one side denotes a circumscribed pleuritis which is probably secondary to a tuberculous affection. Other accessory signs are, an abnormal transmission of the heart-sounds, and an interrupted or jerking respiration. The diagnostic significance of all these signs, it will be observed, depends on their situation at the summit of the chest on one side. Separately they are not sufficient for the diagnosis, but, coexisting with the direct signs, viz., dulness on percussion, broncho-vesicular respiration, exaggerated vocal resonance, and increased bronchial whisper, they serve to confirm these signs and render the diagnostic evidence positive.

The diagnostic points pertaining to symptoms, to which attention is to be directed in determining whether the disease be or be not present, when the signs are not so well marked as to render the diagnosis easy, are as follows: (a.) Cough and expector'ation, not succeeding an attack of acute bronchitis, and not connected with chronic pharyngitis; the cough at first dry, and, afterward, an expectoration, at first small and transparent, and becoming gradually more abundant and opaque. (b.) Stitch-pains at the summit, not connected with intercostal neuralgia. (c.) Chills not referable to malaria. (d.) Hæmoptysis. This is always a symptom of great significance, but the fact is to be borne in mind that it occurs, in a certain proportion of cases, without being associated with tubercle. (e.) Accelerated breathing. (f.) Loss of weight. (g.) Pallor or anæmia, not otherwise explicable. (h.) Hoarseness or huskiness of voice, proceeding from chronic laryngitis. (i.) Chronic peritonitis, not traumatic. (j.) Suppression of the menses. (k.) Buoyancy of mind, instead of despondency.

Facts which render the existence of a hereditary predisposition probable, are to be taken into account in the diagnosis. The age of the patient is another point. As the discrimination frequently lies between tuberculosis and chronic bronchitis, it is to be considered that, except in aged persons and those affected with disease of the heart, chronic bronchitis is comparatively so rare that the chances are in favor of the existence of tu- 
bereulosis. The chances are still greater, if, in addition, are exeluded the cases in which chronic bronchitis is associated with asthma and emphysema, affections which rarely coexist with tuberculosis.

In certain cases of pulmonary tuberculosis the soliclification represented by physical signs at the time of an examination of the chest depends, to a greater or less extent, on an intereurrent, transient, circumscribed pneumonitis. This can only be aseertained by repeated examinations of the ehest after certain intervals of time. The solidification, so far as due to superadded simple or ordinary pneumonic inflammation, will be found to disappear, leaving that which is caused exclusively by the tubereulous affection. If the solidification, as ascertained by physical signs, be unreservedly attributed in all cases to the tubereulous products, the practitioner will sometimes be led to over-estimate the amount of the latter. It is important, therefore, to bear in mind the liability to a temporary increase of solidification of lung, in cases of tuberculosis, from an intercurrent pneumonitis which may not reveal itself by any distinctive symptoms.

Prognosis.-The prognosis, in cases of pulmonary tuberculosis, is extremely unfavorable, in view of the fact, that, in many, if not most, parts of the world, the mortality from this disease exceeds that from any other, exclusive of fevers and affections which prevail epidemically or endemieally. Yet the mortality from the disease has undoubtedly diminished within the past ten or fifteen years. This must be obvious to medical observers whose professional experience extends backward a quarter of a century or more. The faet is also shown by the statistics published by the registrar-general of Great Britain, and by the mortuary reports of the large cities in this country. The explanation of the fact is probably to be found in improved views as regards the management of the disease. The diminution which has already taken place in the cleath-rate from this disease, affords ground for the hope that its formidable character may be still further mitigated.

The different modes in which the disease may pursue a favoralle course have been alrealy stated. 'The entire deposit may be absorber, or the animal portions may be absorbed, the mineral constituents remaining in the form of obsolete tubercles or calculi, and these may remain quiescent, or they may find their way into the bronchial tubes and be expectorated. Cavities may completely cicatrize. In these modes complete recovery may take place. 'This, of course, is the most satisfactory termination. And next to this is the persistence of cavities withont any fresh products, the cavities giving but little inconvenience for an indefinite period, and even through a long life. A ceording to these different modes in which the course of the disease is favorable, cases may be divided into those in which an arrest takes place without recovery, and those in which the arrest is followed by recovery. Whether recovery follows, or not, the arrest will depend on the amount of tubercle, and other circumstances. Not infrequently an arrest takes place, with or without recovery, and, after the lapse of several months or years, a fresh irruption of tubercle occurs. The latter, then, is not properly a continuanee of the disease, but a relapse. These distinctions seem to be pathologically just, and they are practically important.

Pulmonary tuberculosis may end in recovery from an intrinsic tendency, that is, without any therapeutic or hygienic measures having been employed. Of sixty-two recorded cases in which an arrest of disease took place, in seven this arrest was irrespective of any extrinsic influences, and 
in four of these seven cases the recovery was complete. 'To what an extent an intrinsic tendency to arrest is involved in cases is which measures of management are employed, it is difficult to say, but it is fair to conclude that this tendency enters more or less into many, if not most, of the cases which pursue a favorable course. Assuming this conclusion to be correct, it becomes all important question, what circumstances denote an intrinsic tendency to arrest, or, in other words, what circumstances encourage a favorable prognosis in this disease? The answer to these questions should be based on a comparison of the results of the analyses of collections of cases ending, on the one hand favorably, and, on the other hand, unfavorably. Such a comparison, as an object of clinical research, claims more attention than it has, as yet, received. I shall give certain conclusions, based chiefly on the study of a considerable number of cases pursuing a favorable course under my own observation.

A small amount of tuberele, and a stationary condition as regards the amount, are favorable circumstances. An arrest may take place although the amount of tubercle be large, but it is obvious that, inasmuch as the local damage is proportionate to the amount, the prospect of recovery is better the less the quantity of tubercle. A nother favorable circumstance is the quiescence of the disease, as denoted by the local and general symptoms. Tolerance of the affection is shown by the cough and expectoration being slight, whereas, the prominence of these symptoms indicates a proportionate local disturbance. The symptoms denoting general disturbance are, acceleration of pulse, chills, sweating, and debility. The prospect is favorable in proportion as these symptoms are wanting or without prominence. It may be here stated that the local and general disturbance are by no means uniformly in proportion to the amount of the tuberculous affection; they are sometimes marked when the amount of tubercle, as determined by physical signs, is small, and, on the other hand, they are slight in cases in which the amount of tubercle is large. It is evident that the constitutional symptoms are not exclusively symptomatic of the local affection. The import of hæmoptysis has been already considered. The observations of $W$ are and $W$ alshe have shown that, as a rule, the prospect is more favorable in the cases characterized by the repeated occurrence of hæmoptysis, and my own observations have led me to the same conclusion.

The condition of the appetite and digestion affects the prognosis. The probability of arrest is greater in proportion as the appetite is good and the digestive organs free from disorder. It is a farorable circumstance if fatty articles of food can be taken without repugnance or disturbance of digestion, and if alcoholic stimulants be well borne. Diarrhœa is always an unfavorable symptom, whether functional or proceeding from tuberculous disease of the intestine. The condition as regards weight furnishes, on the one hand, one of the most favorable of prognostics, and, on the other hand, the most striking evidence of the unfavorable progress of the disease. The prospect is favorable if the loss of weight have been small and if it be not progressive, and, per contra, the prospect is unfavorable in proportion to the degree of emaciation and the rapidity with which it is going on.

The occurrence of perineal fistula is favorable, and so I have been led to regard laryngitis, excepting the cases in which the epiglottis and top of the larynx are involved, so as to interfere with deglutition. Facts

1 Clinical Report on the Management of Pulmonary Tuberculosis. By Austin Flint, M.D. Trans. N. Y. Acad. of Med., 1863. 
which go to show a strong hereditary or congenital tendency to the disease are unfavorable. Finally, energy of character and a determined will are highly favorable, by seeuring a faithful and persevering employment of the hygienic measures upon which the suceessful management of the disease mainly depends.

The disease proves fatal generally by asthenia. It is rare for tuberele to be so abundant, or the destruetion of lung to be so great, as to destroy life by apnoa. The patient is gradually worn out by the protracted disturbance oecasioned by the disease, conjoined with progressive emaciation and exhaustion. The development of pneumo-hydrothorax from perforation is apt to prove fatal in a short time, and sometimes by apnoa. Acute peritonitis from perforation has been known to oceur, and this affection is likely to prove rapidly fatal. Meningitis is another eomplieation which hastens the fatal termination.

Hamoptysis is very rarely an immediate eause of cleath. A case has, however, fallen under my observation in which profuse hemorrhage oecurred day after day in connection with a small amount of tubercle, and the patient died apparently from the loss of blood. A profuse and rapid flow of blood may occasion death by suffocation, the blood filling the bronchial tubes. As a rule, hæmoptysis, so far from denoting immediate danger, is a favorable event as regards the prognosis.

Thentment.-The point of departure for the consideration of the management of this affection is the pathological fact that the local affection is the expression of a general or constitutional morbid condition, the latter being the essential disease. The great object of treatment, therefore, is the removal of this constitutional morbid condition or the tuberculous eachexia. Measures addressed to the pulmonary affection are of secondary importance. The chief end to be kept in view is the prevention of the further production of tuberele, or, in other worls, an arrest of the disease. It is immensely desirable for the arrest to take place as quickly as possible, while the amount of tubercle is limited and the pulmonary clamage proportionably small; and with a view to a speedy arrest, the importance of an early diagnosis can hardly be overrated.

Is the tubereulous cachexia removable by any known special remedy or remedies? This question is to be answered clistinctly in the negatire. Various remedies at different times have been supposed to exert a specific curative influenec orer this disease. Thus, going back only a quarter of a century, during this period wood-naphtha, cod-liver oil, phosphate of lime, the liypophosphites, and the chlorate of potassa have successively been advocated as effecting a cure in cases of pulmonary tubereulosis. Few, if any, at the present moment consider either of these remedies, or any other remedy, as specifically curative. This, however, is by no means saying that these remedies are not useful in cases of pulmonary tubereulosis. Important indications are to be fulfilled by remedies, but it shonld be clearly understood that far more reliance is to be placed on hygienic than on medicinal measures of treatment.

Certain therapeutic measures which have heretofore been employed, to a greater or less extent, under the guidance of false pathological views, undoubtedly do harm rather than good. In this category belong bloodletting, either general or local, blisters and other severe modes of eounterirritation, mereurialization, antimonial and other nauseating preparations, emetics, and cathartics. These are contra-inclicated. In general terms, everything is to be avoided which tends to impair the appetite, disorder digestion, and lower the vital powers. 
The measures indicated are those which tend to strengthen and invigorate. These terms, although lacking scientific precision, have a practical significance which is sufficiently intelligible. Directing attention to the measures which fall under the head of hygiene, these relate to diet, exercise in the open air, clothing, mental encouragement, and change of climate.

The diet of tuberculous patients should be generous as regards quantity, quality, and variety. The articles should be highly nutritious and adapted to the digestive powers. All the varieties of wholesome food which the patient is able to take with relish should be allowed, and the appetite should be encouraged as much as possible. With respcet to the kinds of food to be particularly recommended, there are no general rules which are applicable to all patients. Meats, milk, farinaceous articles, and the different vegetables are to be combined in relative proportions according to the choice, habits, and experience in individual cases. It is desirable that cream, butter, and other fatty articles should enter into the diet as largely as possible without indncing disgust and disorder of digestion. The same remark applies to sugar. The intervals between taking food should be such as will secure the largest amount of nutriment which can be taken and digested during the twenty-four hours. In short, it is far from desirable to arrange any fixed system embracing details of dieteties to which all patients are expected to conform. The object is to nourish the body to the fullest possible extent, and this requires, in every case, the co-operation of the physician and patient. Deficiency of appetite, especially for nutritions articles of diet, and weak and disorganized digestion, are apt to prove serious difficulties in the way of the successful management of the disease. Much may be done by persevering efforts on the part of the patient, and he should, therefore, be made to understand the object which it is desired to effect:

A diet consisting largely, or eren chiefly, of fresh milk has undoubtedly proved sometimes successful, and a trial of it may be advised when the appetite is deficient, or when other articles of food oecasion dyspeptic disorders. Goats' milk and asses' milk are rarely used in this country. They have heretofore been, and are still, to some extent, in European countries, considered as preferable to the milk of the cow. In order to carry out effectually the "milk cure," as it is sometimes called, milk should not only constitute the chief article of diet, but it should be taken largely.

Exercise in the open air, or, as I would prefer to say, ont-of-door life, is, of all measures, the most important. In a paper already alluded to, on the management of pulmonary tuberculosis, ${ }^{1} \mathrm{I}$ have analyzed the recorded histories of sixty-two cases in which an arrest took place, with a view to the points in the treatment which were common to all or a greater or less number. In twenty of these cases the arrest took place under hygienic measures without medicinal treatment. In these cases the most important point of agreement related to change of habits as regards exercise and out-of-door life. In respect to this point, there was also an agreement in the cases in which medicinal measures were employed. The following extract from the paper contains a summary of the facts, with the general conclusions: "The most striking and valuable of the results of the analytical study of these cases is their almost uniform agreement as regards change of habits with respect to exercise and out-of-door life at the time of the arrest. Excluding the seven cases in which the arrest was attributable solely to an intrinsic tendency, and two cases in which the facts with respect to this point were not noted, of the remaining fifty-

1 Trans. N. Y. Acad. of Med., 1863. 
three cases, in all, save three, the histories show a greater or less change of habits to have been made; and in many cases the change consisted in relinquishing sedentary callings for other pursuits, in order to carry out more effectually the desired reformation. I am well satisfied that here is the foundation for the successful management of pulmonary tuberculosis. I would rank exercise and out-of-door life far above any known remedies for the cure of the disease. There are grounds for believing that the advantage of a change of climate mainly consists in its being subsidiary to a change of habits as regards exercise and out-of-loor life. So deeply impressed am I with the correctness of this view of the regimenal management of the clisease, that I cannot express myself too emphatically in trying to enforce its practical importance."

Tubereulous patients should sleep in well ventilated rooms. In conformity with popular notions, they are apt to fear the cool or cold air of night. A fire may be kept in sleeping-rooms in the winter season, but the windows should be kept open. Dr. James Blake, of San Francisco, California, has reported several cases in which most favorable results followed living in the open air during the summer months, at an elevation of from three to five thousand feet above the sea, in the coast range of the mountains of California where the temperature is very equable, and no rain falls for five or six months. The patients did not even sleep in tents, but were in the open air during night and day.

Exercise in the open air should be accompanied by either mental reereation or occupations which interest the mind. It should, as far as possible, be incidental to pursuits which engage the attention. Adopted simply as a hygienic measure, it will rarely be persisted in. It is often essential, therefore, for patients to make a radical change in business, or, if they be able to devote their time to the restoration of health, hunting, sporting, travelling, ete., are to be resorted to as means of securing the union of out-of-door life with an agreeable exercise of the mental faculties. Overexercise of the muscles is to be avoided. Patients sometimes, supposing that the benefit from muscular exercise is proportionate to its amount, carry it to the extent of producing a degree of fatigne and exhaustion which is injurious. It is not from the amount of physical exertion, but of out-of-door life with such exercise as is well borne, that benefit is to be expected.

The body should be protected against atmospherical changes, the temperature and functions of the skin being maintained by warm elothing. The garments worn next the surface should be made of a material which is a good non-conductor of heat, viz., either wool or silk. But a superfluity of clothing is to be avoided. The olject is to provide adequate protection, without an undue accumulation of heat and perspiration. In a cold or temperate latitude, during the winter season, a waisteoat of buckskin or chamois leather, worn, during the daytime, over a light woollen or silk shirt, is to be recommended to patients of either sex, as securing the proper protection without the ineonvenience of being bundled with an overplus of garments. The feet should be well guarded against cold and wet. Properly elothed, the patient, if he be strong enough to go out of doors, should rarely be kept within doors by the state of the weather, but should resolutely keep up habits of out-of-door life, despite the ordinary changes of temperature and winds, remaining in the house only on inclement days.

I have frequently known the sponge bath to be taken daily by tubereulous patients, with apparent benefit. It may be safely tried and continued, provided it be followed by an agreeable glow. The temperature of the 
water used should be from $64^{\circ}$ to $68^{\circ} \mathrm{Fahr}$. It is to be borne in mind, with reference to this measure, as well as exercise in the open air, that the susceptibility to attacks of bronchitis is less, the more the exposure, and, also, that an attack of bronchitis does not usually exert any unfavorable influence on the tuberculous disease. Many patients are deterred from going out of doors, when the weather seems unfavorable, by needless apprehension of taking cold. The danger from this source is certainly less than from a deficiency of out-of-door life.

The proportion of cases in which an arrest of the disease is effected is sufficient to authorize the physician to encourage patients strongly to employ the hygienic measures which have been briefly considered. The influence of the disease on the mind is to induce either an expectation of recovery without effort, or resignation to death. It is important to make patients understand that very much depends on their own exertions. One of the great difficulties in the way of successful management is a passive, patient, tranquil frame of mind, which cannot be aroused to vigorous efforts. Persons endowed with resolution, energy, and perseverance, other things being equal, are more likely to struggle successfully with the disease than they who are deficient in these mental qualities. The measures which have been considered, if not successful in effecting an arrest of the disease, tend to retard its progress, and render the condition of patients most comfortable while life lasts.

Change of climate, as a means of effecting a cure of pulmonary tuberculosis, has been a fruitful topic for discussion. It does not fall within the scope of this work to enter into a discussion of this topic. I shall present bricfly certain considerations which may be of some practical utility in connection with the question in individual cases, "Shall I try change of climate, and, if so, where shall I go?"

It may be assumed that a change of climate is frequently useful. Granting that in the majority of cases it does not prove successful, there is ample ground for the belief that in not a few cases it is highly beneficial, and it appears sometimes to effect an arrest of the disease. 'The judgment of patients or their friends, in the matter, is not of much value, because it is usually based on the knowledge of a few cases, or perhaps of a single case, in which benefit may, or may not, have been apparently obtained. Change of climate, therefore, is to be advised under certain circumstances.

It may be taken for granted that there is no specific influence in any climate. It would be therefore quite needless to inquire if there be one particular spot which is to be preferred above all others. Observation shows that different climates are suited to different cases. The qualities which render a climate favorable are elevation, uniformity, and dryness. As regards the latter, the rule is perhaps not without exceptions; some patients appear to do best in situations in which the atmosphere is warm and humid. With respect to temperature, a uniform cold climate is best for some cases, and a uniform warm climate for other cases. Of late years, the custom has been gaining ground of sending patients to a cold latitude, and $I$ have known of a number of instances in which the climate of Minnesota has proved highly serviceable. Were it consistent with the limits of this work, I could cite some cases which are strikingly illustrative of the salutary influence of the uniform, dry, cold atmosphere of that northern region. On the other hand, I have known of numerous instances in which a change to a warm climate has appeared to accomplish all that could be desired. What circumstances, then, are to guide us in deciding whether a cold or warm climate will be likely to prove most advantageous? The feelings and choice of the patient are to have considerable weight. If, 
when in health, more vigor and enjoyment are habitually experienced in summer than in winter, a warm climate will probably be best, and, if the reverse be true, a cold elimate is to be preferred. The condition of the patient as regards feebleness is an essential point. If he be so feeble as not to be able to live out-of-doors in eold weather, or if the reaction from the impression of cold be slow and imperfect, a warm climate is more suitable. It is rarely proper to send patients to a cold climate if the disease be considerably advanced, and, as a rule, a cold climate is better suited to male than to female patients.

With reference to the particular situation to be selected, it should contain resources for oceupation and mental interest. There must be inducements for out-of-door life. I have known patients who had been aceustomed to active habits, suffer intolerably from emuni in going to jlaces where the supposed excellence of climate was the only attraction. It is frequently better to move abont from one place to another than to remain stationary; as soon as a place becomes tiresome, it is best to leave it. Travelling in foreign countries, without any special regard for climate, is often the best plan, the advantage consisting in the interest and inducements to out-of-door exereise derived from a succession of new scenes. A sea-voyage in warm latitudes is generally useful, and if patients are fond of the sea, a long voyage, if practicable, may be advised. During the past few years many patients under my observation have resorted to sea-voyages; and I can affirm that, while in not a few instances the benefit has been marked, I have never known any harm to ensue. I have known several instances in which patients, in an advanced stage of the disease, the strength being much reduced, have taken long voyages with notable improvement. Places which are especially the resort of patients are to be avoided; the moral effect of seeing daily examples of different stages of the disease is unfavorable. A change is often useful when there is no superiority on the score of climate, because it is in this way only, in certain cases, that relief from the eares and anxieties of business can be seeured.

The habits and taste of the patient are to be considered. Persons who are dependent on the associations and comforts of home and friends for their happiness will not be likely to be benefited by being sent away, especially if alone and among strangers. The stage of the clisease and the rapidity of its progress are points of great importance. It is truly a crucl act to send to a distance patients who are in a condition admitting of but little prospect of improvement, and who will probably not live to return. The amount of damage which the lungs have sustained, as determined by physical signs, as well as the symptoms, is to be taken into account, before deciding with regard to change of climate. And if a change be made and prove effectual, it becomes an important question whether, if practicable, the change should not be permanent. An arrest of the disease does not extinguish the diathesis, and the safest course, undoubtedly, is to take up a residence in the climate in which the cachexia is less liable to be reproduced than in the climate in which it has been already onee developed. Finally, in forming an opinion of the importance of change of climate in the management of this disease, it is to be considered that the progress of the disease will be unfavorable in a large proportion of cases, whatever be the change or changes made; an arrest of the disease is not to be expected save in a small minority of cases. The fact that, of the persons who make trial of change of climate, the number who are not apparently benefited greatly exceeds the number who find this measure either successful or beneficial, should not discourage resorting to the measure so long as in a small proportion of eases it proves effective. Another 
consideration is to be added, namely, a change of climate not infrequently appears to retard the progress of the disease, rendering the patient more comfortable and prolonging life, although an arrest of the disease be not effected.

Passing now to consider the remedies which enter into the management, I place under this head alcoholic stimulants, without discussing the question whether they are properly so regarded, or whether, as was claimed by Todd, they are alimentary rather than medicinal. The remedial agency of alcoholics in this disease has given rise, of late, to much discussion, and, on this point, the views of physicians are somewhat divided. Basing my opinion on clinical experience, I do not hesitate to express the belief that, in a certain proportion of cases, alcohol exerts a curative influence. Of the sixty-two cases of arrested tuberculosis which I have analyzed, in fourteen the treatment, irrespective of hygienic measures, consisted exclusively in the use of alcoholic stimulants. In nine of these cases the recovery was apparently complete, the patients remaining well after periods, dating from the time of the arrest, varying between four months and eleven years. Some cases which I have observed scem to exemplify in a striking manner the curative effects of alcoholic stimulants.

Alcoholic stimulants, however, by no means exert a curative influence in all cases. I have known them to be tried and sometimes tried repeatedly in the same case, and their use abandoned because they were found to produce unpleasant effects. The determination of their appropriateness and importance, or otherwise, is thus to be made in each individual case, and based, of course, upon a fair trial. And the question at once arises, what are the circumstances which experience shows to denote their usefulness on the one hand, or their want of usefulness, if not hurtfulness, on the other hand? If the immediate effect be that of a cordial, that is, if they produce a sense of comfort; if they be followed by a feeling of increased strength and a greater disposition to exercise; if they do not excite unduly the circulation or nervous system, benefit may be expected from their use. Per contra, if their immediate effect be discomfort; if they be followed by a feeling of increased weakness and less disposition to exercise, and if they excite unduly the cireulation or nervous system, they will not do good, and they may do harm.

The quantity to be taken is an important matter. Some physicians, overconfident in the curative power of alcohol, advise their patients, in general terms, to drink as freely as possible. This is to be deprecated as well as the indiscriminate use of alcoholic stimulants. They are to be given sometimes largely, sometimes moderately, and sometimes sparingly. In the previous editions of this work I gave the synopsis of the case of a young girl who took a pint of whiskey daily for two years, an arrest of the disease being apparently effected by this free use of spirit. It would be an erroneous inference from the curative power manifested in such cases, that a large quantity is to be given in all the eases in which this remedy is found to be useful. How are we to be guided in this matter? I will state the rule which experience has led me to adopt. Under the conditions which indicate the use of alcoholic stimulants, they may be given, with reference to a curative influence, as freely as they can be taken without discomfort, without a sense of weakness or indisposition to exertion, and without undue excitement of the circulation or nervous system. The limitation in quantity is to be determined by the same circumstances which indicate their use, or, in other words, by their immediate effects. And, guided by these circumstances, it will be found that some patients will bear a large, some a moderate, and some only a small quantity. This 
disease is one of those which, in certain cases, induce a remarkable tolerance of alcoholic stimulants.

The form of alcoholic stimulants to be used is another practical point. In the cases which I have observed, whiskey has been oftenest used. Some have thought that this form of spirit is especially adapted to cases of tuberculosis. I do not join in this opinion. Whatever be the form used, I believe the remedial principle to be alcohol. But no form is equally applicable to all cases. Some patients are benefited especially by spirits, some by wine, and some by malt liquors. Of the different varieties of spirits, wine, and malt liquors, each is best suited to particular cases. The indication for the selection of the form to be used in individual cases must be derived from the preference of the patient and the results of experimental trials.

In conclusion there is an important matter connected with the employment of alcoholic stimulants which, although not pertaining to the consideration of their therapeutical influence, should not be ignored. I refer to a risk of forming habits of intemperance. If their remedial use involve this risk, it becomes a question how far we are warranted in incurring it by their curative power. But in no ease in which $I$ have employed alcoholic stimulants has there been dereloped, so far as I know, a desire for them or a reliance upon them, rendering it difficult to relinquish their use. I have directed my attention particularly to this point, and I have not yet found an instance in which there was any reluctance to discontinue alcoholic stimulants whenever it was deemed advisable.' I am not aware that in a single case among the great number in which $I$ have advised the use of alcoholic stimulants has a patient fallen into intemperate habits. I certainly am not prepared to advocate the use of alcoholic stimulants as a prophylactic ; that is, to sanction indulgence anong those who balieve or fancy that they are in danger of becoming tuberculous. I would not advise their use in donbtful cases; it shonld follow a clear diagnosis based on signs and symptoms. In persons with the unfortunate idiosyncrasy which leads to an irresistible craving on the slightest indulgence, the immediate effects would always contra-indicate their use in conformity with the rules which should govern our practice in eases of tuberculosis. And finally, when employed as a remedy, they are not to be taken as a means of conviviality, or for any other than a curative influence. Observing these precautions, we may expect to secure their remedial agency without exposing our patients to a calamity which, in a moral point of view, would be far greater than a failure to effect an arrest of pulmonary tuberculosis.'

With regard to cod-liver oil, the same inquiry arises as with respect to alcoholic stimuiants, viz., whether it be proper to regard it as a food or a medicine. Not stopping to discuss the propriety of classing it among remedies, I proceed briefly to consider the practical points connected with its employment in eases of pulmonary tuberculosis. All clinical observers unite in according to it more or less value. Its usefulness in a certain proportion of cases can hardly be doubted. Of the cases of arrested tuberenlosis which I have analyzed, in five the treatment consisted exclusively in the use of cod-liver oil and hygienic measures. How much importance is to be attached to an intrinsic tendency, and how much to

1 These remarks on the use of alcoholic stimulants, and the remarks which follow on the use of cod-liver oil, are, in a great measure, taken from the author's clinical report to the New York Academy of Medicine on the management of pulmonary tuberculosis. 
hygiene, in these cases, cannot be determined, but an examination of the circumstances belonging to the individual histories goes to show that a certain amount of curative influence belongs to the oil.

The amount of curative influence which cod-liver oil, or any remedial agent, exerts, cannot be accurately defined, because it is impossible to determine the proportionate amount of influence that may be exerted either by self-limitation of the disease or by hygienic measures, but, practically, this is of less importance than to ascertain whether a remedy possess curative power, be the degree greater or less. If a remedy have considerable influence, it is, of course, more desirable to employ it than if the amount of influence were small; but let the influence be never so small, we are not, on that account, warranted in withholding it in a disease of such gravity as this. Praetically, the question of the use or nonuse of the oil relates to individual cases. Clinical observation shows that it is not useful in all cases. I shall give, briefly, the rules which my own experience has led me to adopt respecting its use and non-use.

If it be taken without great repugnance, if it do not impair the appetite or digestion, nor occasion derangement of the bowels, it may be expected. to do good. If it produce any of the effects just named, its propriety is questionable. It is best to begin with a small quantity and increase to the maximum. quantity, which is from half an ounce to an ounce three times daily, taken near the time of meals, either before or after, as choice or experience may dictate.

The hypophosphites were introduced, some years since, by Dr. Churchill as a specific remedy, the pathology of the disease being supposed to involve a deficiency in the system of phosphorus, and this element existing in the hypophosphites in a form readily assimilable and in a low state of oxidation. Experience has abundantly shown that the disease is not arrested by the introduction of phosphorus into the system, in other words, that this has no claim to be considered as a specific remedy; but it appears in some cases to be highly useful as a tonic remedy.

The fusel oil, introdueed as a remedy in this disease by Dr. Morrell Wyman, has been employed to a considerable extent in this country, more especially in the New England States, not as a remedy supposed to exert any specific influence, but as affeeting favorably the pulmonary symptoms. During a service of three months in the heart and lungs department of the Demilt Dispensary of New York, I preseribed this remedy in a large number of tuberculous cases. In the majority of cases no apparent benefit was produced, but in some instances the patients reported relief as regards the cough and expectoration.

The ehlorate of potassa was supposed, by the late Dr. Fountain, to have a special efficacy in this disease. I made trial of this remedy in all the hospital cases received into my wards at the New Orleans Charity Hospital, in the winter of 1860 . The cases were fourteen in number. They were at once placed on the use of this remedy, from three to four drachms being taken daily, with no other remedy than a palliative for cough, alcoholic stimulants being generally withheld; and this treatment was continued in each case so long as the patient did not seem to be losing ground. A elinical report of these cases was published in the American Journal of the Medical Sciences, October, 1861. The conclusion drawn from these cases, as regards the curative influence of this remedy, is stated in the report as follows: "Of fourteen reeorded cases of phthisis in which the chlorate of potassa was given in suffieient doses and for a sufficient period to test its remedial power, in nine the histories afford no evidence of any salutary effect from the remedy; in four cases the circumstances render it 
doubtful whether much, if any, influence was fairly attributable to the remedy, and in one case only is there room for the supposition that the remedy was highly beneficial. These cases, therefore, fail to furnish proof of any special efficacy in this remedy to arrest or retard the progress of the discase." This conclusion is not at variance with the opinion that, as a tonic remedy, the chlorate of potassa may be uscful in certain cases of the disease.

The different tonic remedies are indicated in this disease. Quinia, the bitter infusions, the infusion of the wild cherry bark, the citrate of iron and quinia, and the different chalybeates, are to be preseribed according to the eireumstances belonging to different cases, and frequently in succession in the same case. It has been conjectured that the continued use of iron may favor hæmoptysis. I have never seen any clinical evidence of this, but, if there be ground for the conjecture, the inappropriateness of the remedy does not follow. The fact that tuberculous disease is not often developed in patients affected with anæmia has been supposed to militate against the propriety of chalybeate remedies; but it is manifestly illogical to infer from this fact that the anæmia which is secondary to, and probably an effect of, the tuberculous disease exerts any retarding influence upon the latter. Some eminent French authors oppose the use of iron in cases of pulmonary tuberculosis; but the majority of experienced physicians hold the opinion that chalybeates are often useful, and this opinion is in accordance with my own observations. Other remedies, the value of which is attested by experience, although they have no claim to be considered as excrting a special anti-tuberculous effect, are sulphur, arsenic, and iodine. Sulphur is to be given in small doses, or a trial may be made of the mineral waters containing this element. Arsenic, in minute doses, long continued, is extolled in strong terms by two French writers, M. Isnard and M. Moutard-Martin. Of late, I have prescribed this remedy in a considerable number of cases with manifest advantage. Preparations of iodine have been thought by many to be useful in eases of tuberculosis, as they are undoubtedly in cases of scrofulosis. The chloride of sodium is to be added to the remedies just named. Still another remely to be enumerated is a solution of caoutchouc in the spirits of turpentine and prepared as a confection. Dr. Theodore R. Varick, of Jersey City, has used this last-named remedy in many cases, as he thinks, with notable benefit. Having at his suggestion made trial of it in a number of patients at Bellevue Hospital, I can bear testimony to its apparent utility in certain cases. It is not a disagreeable remedy, but some patients after a time acquire a repugnance to it.

Certain pulmonary symptoins claim -attention. Expectorant remedies, as a rule, are not called for. If they diminish appetite, occasion nausea, or distnrb digestion, they do more harm than good. But remedies to allay superfluous cough, that is, cough not required for expectoration, are useful. For this end the succedanea of opium, viz., hyoscyamus, conium, belladonna, and hydrocyanic acid may be prescribed, but, if these prove incfficient, opium in some form is required. Patients should be instructed not to yield to the disposition to cough, but to resist it whenerer it is not. effective. The habit of coughing unnecessarily is, in a great measure, under voluntary control. Soothing inhalations are sometimes useful in allaying the irritation which excites cough. The vapor of water, or spray, impregnated with conium or opium, may be inhaled. I lave known the vapor of chloric ether to be highly useful as a palliative.

I can say nothing of the attempt to inject tuberculous cavities with a solution of the nitrate of silver or other medicated liquids through the 
larynx and trachea. $\Lambda$ side from the difficulty of the operation, and the impossibility of limiting the application of the injected liquid to cavities, nothing beyond a palliative effect could be expected from this measure were it ever so successfully executed.

Certain complications furnish indications for treatment. Attacks of dry, circumscribed pleurisy, call for mild revulsive and soothing applications, such as sinapisms, liniments, or the belladonna plaster. Local bleeding by leeches or cups is never required. The pain incidental to these attacks generally passes off in a few days at furthest. The treatment of coexisting laryngitis has already been considered. Diarrhœa, dependent on tuberculous enteritis, is to be kept in check by astringents and opiates, the latter being used with as much reserve as is consistent with the object to be attained. Chronic peritonitis claims only palliative measures. Perineal fistulas should be allowed to continue without surgical interference.

In the progress of the disease, symptoms other than those relating to the chest, require palliative measures. 'The night perspirations are frequently the source of great discomfort. The mineral acids, especially the dilute sulphuric acid and gallic or tannic acid, frequently afford relief. The external application of an astringent, such as alum dissolved in spirit, is sometimes effective. Dyspnœa may be mitigated by ethereal preparations.

A comparison of the measures of management now generally pursued in this disease with the measures in vogue a quarter of a century ago, shows a complete and radical change. Formerly, general and local bloodletting, cathartics, emetics, mercurials, counter-irritation, low diet, and confinement within doors were sanctioned by teachers and writers, and entered, more or less, into the treatment usually adopted. At the present time, the measures which are most approved are, in all points, the reverse. The latter are based on more correct pathological views, and the results of clinical observation. It is not unreasonable to hope that, at some future time, a remedy or remedies may be discovered, capable of exerting a special influence over the disease, but, in the mean time, reliance is to be placed on an early diagnosis, the employment of remedies which, directly or indirectly, may exert, to some extent, a curative influence, and, above all, the bygienic measures which tend to invigorate and streugthen the system.

\section{Acute Pulmonary Tuberculosis.}

The distinction between rapid and acute tuberculosis has already been stated. In rapid tuberculosis the disease runs its course speedily, the accumulation of the product, softening, ulceration, and the formation of cavities taking place within a few weeks. Cases of the acute variety differ in this: the tuberculous product is in the form of small, distinct, round tubercles, called from their size miliary, which accumulate in immense number, and life is quickly destroyed by the interference with the function of respiration, and a high degree of constitutional disturbance. The miliary tubercles, in cases of so-called acute phthisis, may consist of the yellow tuberculous product; or they are the semi-transparent bodies distinguished as the gray tuberculous product. The accumulation of these bodies in immense number characterizes cases of so-called acute phthisis. It is a question whether these two products are pathologically distinct, or whether the yellow are the gray tubercles which have undergone a cheesy metamorphosis. However this may be, the distinctive fea- 
ture of the form of pulmonary tuberculosis now under consideration is the rapid accumulation of miliary tubercles, and a fatal termination without tuberculous infiltration or the conlescence of the tubercles forming masses which liquefy and lead to the formation of cavities.

Cases of acute pulmonary tuberculosis present striking points of difference, as compared with the ustual chronic form of tuberculosis, not only in respect of duration and the appearances found after deatl, but as regards the physical signs, and the symptoms belonging to the clinical history. The readiest way of presenting a general view of this rare form of disease will be to give a synopsis of a few illustrative cases.

CASE 1. Miss C., aged 32, daughter of an eminent physician, while away from home on a visit of pleasure, considering herself well, was attacked in the night with hæmoptysis. It was at first slight, bit recurred twice during the same night, and was considerably profuse. I saw her ten days afterward. In the mean time she had kept the bed, the respirations had been accelerated, the prolabia had been livid, the pulse frequent, and she was greatly prostrated. When I saw her, these symptoms continued, the respirations being 36 per minute, the pulse varying from 120 to 130 , the cough and expectoration slight. She died the following night, eleven days after the occurrence of hæmoptysis. She had had a slight hamoptysis six years before, and subsequently had been subject to a slight cough, but there liad been no notable diminution in weight, and she had always considered herself well.

An examination after death was made by the attending physician, Dr. Walter Carrey, of Buffalo, who stated that he found old pleuritic adhesions on both sides, and both lungs stuffed with miliary tubercles, which he described as hard and semi-transparent; there was no aggregation of tuberculous matter into masses, and there were no cavities.

It is fair to conclude that in this case either the formation of miliary tubercles, or gramulations, commenced at the time of the hremoptysis, or, if they existed previously, they were comparatively few in number, but rapidly accumulated from that time, destroying life in so short a period as eleven days.

CASE 2. In November, 1860, I examined a slave-girl, aged 18, in an infirmary, at New Orleans, under the charge of my colleague, Prof. Beard. She was at that time apparently convalescing from typhoid fever, and a careful exploration of the chest disclosed no signs of pulmonary disease. She secmed to be slowly convalescing, when, about the first of Jamuary, 1861 , a violent cough occurred, the pulse became frequent, she lost weight and strength rapidly, and died in about six weeks. On examination after death, the lungs were free from adhesions, but crammed with small, hard bodies, not larger than pins' heads. Both lungs were about equally crammed. The spleen and liver contained the same bodies in great numbers, and the peritoneum was studded with them. Hæmoptysis did not occur in this case, and the expectoration was slight.

CASE 3. A male patient, the age not noted, was admitted into the Charity Hospital of New Orleans, in the service of my colleague, Prof. Fenner, February, 1861, and died on the third day after his admission. When admitted, he declared he was perfectly well at the time he was attacked with his existing disease, which was five weeks before his almission. His symptoms were congh without expectoration, accelerated breathing and dyspnoca, lividity of prolabia, and frequency of the pulse. He was examined by one of my private pupils, who found everywhere a clear resonance on percussion, and abundant subcrepitant rales.

After death, the lungs, which were placed at my disposal, were found 
to contain an immense number of miliary tubercles, of about the size of a pin's head, not hard and semi-transparent, but opaque and cheesy. At the apex of the right lung was a cavity of about the size of an English walnut, anfractuous, empty, and lined by a smooth serous-like membrane. Another small eavity existed near the apex of this lung. Near the apex of the left lung was a cavity of the size of a common American walnut, not anfractuous, but also empty, and lined by a serous-like membrane. The miliary tubercles were less abundant at the summit than in the middle and inferior portions of the lungs.

This case affords an illustration of one of the modes in which pulmonary tubereulosis ends favorably, viz., in dry, innocuous cavities. The miliary deposits were undoubtedly of recent date, and were sufficient to destroy life without the processes of aggregation, softening, and excavation.

The diagnosis in cases of acute pulmonary tubereulosis may be attended with difficulty. If hæmoptysis do not occur, and the pulmonary symptoms be not marked, the existence of thoracic disease is liable to be overlooked, and the patient may be supposed to have typhoid fever. This error in diagnosis has been repeatedly made. Delirium sometimes occurs, and this tends to obscure the diagnosis. A careful examination, however, should lead to the conclusion that there is some grave affection within the chest, and typhoid fever is to be excluded by the absence of the characteristic symptoms which belong to the clinical history of that discase. But having decided that the disease is seated within the chest, the diagnosis is still not altogether easy, because physical signs do not point directly to the nature of the affection, and analogous symptoms belong to several thoracic affections, viz., capillary bronchitis, pneumonitis, pleuritis, and certain affections of the heart. The signs of the disease are not positive, for, the tubercles being minute and discrete, and, moreover, existing equally in both lungs, exploration of the chest may furnish no marked disparity in the resonance on percussion, and none of those modifications of the respiration and voice which denote pulmonary solidification. The diagnosis is to be made, so far as physical signs are concerned, mainly by exclusion. Pneumonitis, pleuritis, and diseases of the heart, may be readily excluded by the absence of the signs of those affections. Capillary bronchitis is not so readily excluded, but this affection generally supervenes on an attack of ordinary acute bronchitis; it runs a more rapid course than acute tuberculosis, and, if it end fatally, presents a much greater viclence of pulmonary symptoms, especially dyspnœa.

Unhappily, the treatment of acute pulmonary tuberculosis may be dismissed with a very few words. The condition of the patient is hopcless, and all that the physician can do is to palliate symptoms and support the powers of life. 


\section{SECTION SECOND.}

\section{DISEASES AFFECTING THE CIRCULATORY SYSTEM.}

\section{CH A P T E R I.}

Introductory Remarks. Pericarditis-Anatomical Characters-Cinical History-Pathological Character-Causation-Diagnosis-Prognosis-Treatment. Chronic Pericarditis. Pneumo. Pericarditis.

$\mathrm{D}$ ISEASES affecting the circulatory system are seated in the blood, the bloodvessels, and the central organ of the circulation-the heart. The morbid conditions of the blood, so fir as they are at present known, are, in general, either common to different individual diseases, or they are involved in affections which are distributed in other nosologieal divisions. The consideration of these conditions, therefore, belongs to general, rather than special, pathology, and they have been considered in the first part of this work. Diseases of the vessels-the arteries and veins-are fully considered by surgieal writers, entering, as they do, much more largely into maladies which belong to the surgeon, than into those which fall within the province of the physician. Of the affections of the arteries, aneurisms situated within the chest and abdomen will alone claim attention in this treatise. Thoracic aneurisms will be noticed in this section; those situated within the abdomen will be referred to in connection with abdominal tumors, in the next section. Inflammation of the veins (phlebitis) has been referred to in connection with purulent infection of the blood. This section will be devoted chiefly to diseases affecting the heart.

The clinical study of the diseases of the heart has been prosecuted with much success within the past few years, and, perhaps, in no other department of medicine is the recent advaneement of practical knowledge inore conspicuous than here. This is, in a great measure, due to the successful employment of physical exploration in the diagnosis of these diseases. The diserimination of these diseases by means of the signs derived from the application of percussion and anseultation, has been brought to great perfection by the researches of Bouillaud, Hope, Stokes, and others. It does not come within the scope of this work to treat fully of the signs involved in the diagnosis of cardiac affections. For this important information the student and practitioner are referred to treatises devoted specially either to diseases of the heart, or to the physieal exploration of the chest. I shall content myself here, as in treating of pulmonary affections, with a brief account of the phenomena determined by percussion and auscultation.

The diseases of the heart are conveniently arranged in the following groups: 1. Inflammatory affections. 2. Structural lesions. 3. Functional disorder. I shall consider them in this order, and the present chapter will be devoted to one of the inflammatory affections. Inflammation affecting the heart gives rise to different affections according to the 
structure in which it is seated. Inflammation of the serous membrane investing the heart, or the pericardium, is one affection, called pericarditis. Inflammation of the membrane lining the cavities of the heart, or the endocardium, is another affection, called endocarditis. Inflammation of the substance of the heart, or the muscular walls, is distinguished as myocarditis. These three inflammatory affections claim separate consideration.

\section{Acute Pericarditis.}

Periearditis occurs as an acute and a chronic affection. Acute pericarditis will be now considered, and, afterward, the chronic form of the discase.

Anatomical Ciraracters.-The morbid appearances resulting from acute inflammation in this situation are essentially the same as in other serous membranes when inflamed, for example, the pleura. Exudation of fibrin or lymph takes place in more or less abundance, commencing shortly after the development of the inflammation. The lymph is disposed in the form of a layer, or a series of layers, on the visceral and the parietal surface of the membrane. It is at first soft and easily removed, but becomes dense and closely adherent in proportion as it is of old date. The movements of the pericardial surfaces frequently cause the lymph to assume a reticulated or areolated appearance, or it is disposed in masses, and it is sometimes in the form of numerous filaments or villons projections giving to the surface a shaggy aspect. The vessels beneath the membrane may be congested, giving rise to arborescent redness, and spots of ecchymosis are sometimes observed. Different cases differ greatly as respeets the quantity of lymph, and the extent of surface covered by it, as well as the appearances caused by the different modes in which it is disposed. The presence of $1 y \mathrm{mph}$, here, as in other serous inflammations, is essential as proof of the existence of pericarditis.

More or less liquid effusion usually takes place, as in other serous inflammations. The effused liquid is serum, turbid from the admixture of lymph, and it contains, usually, flakes or shreds of lymph in greater or less abundance. The presence of a purely serous liquid within the pericardial sae, that is, without lymph, is not evidence of inflammation; it is a dropsical, not an inflammatory, effusion. 'T'he quantity of effusion in different cases of pericarditis varies greatly, amounting, in some cases, to a few ounces only, and in other cases to a pint or more. The liquid is sometimes, but very rarely, purulent. It is sometimes sanguinolent.

If the disease progress favorably, the effused liquid is reabsorbed, and the pericardial surfaces, then coming into contact, are agglutinated by means of the intervening lymph. If recovery take place, the lymph is slowly absorbed; adventitious tissue becomes developed, leading to permanent adhesion of the pericardial surfaces. The adhesion by means of the newly-formed tissue may be limited to portions of the heart, and then the surfaces may become connected by bridles or bands which are sometimes drawn out to a considerable length; or, the adhesion may extend over a quarter, a half, or three-fourths of the organ; or, the surfaces may be everywhere united, the sac being completely obliterated. The latter is the most frequent result of acute pericarditis.

The most convenient mode of dividing the disease into stages is the same as that adopted in pleuritis. The first stage extends to the time when effusion takes place to an extent sufficient to be appreciated by the 
physical signs. The second stage, or the stage of effusion, continues until the liquid is absorbed. The third stage extends from the absorption of the liquid to the recovery, and this is, generally, the stage of convalescence.

Chinical IIstony.-The symptoms present in cases of acute pericarditis are almost invariably made up, to a greater or less extent, of those arising from coexisting affections, for, in the great majority of eases, the disease is associated with either articular rheumatism, Bright's disease of the kidneys, or pleuritis with or without pneumonitis. Under these cireumstanecs, it is not always easy to determine to what extent certain symptomatic phenomena are due to the cardiac affection. $A \mathrm{~s}$ a rule, the development of the inflammation is attended with more or less pain which, in some eases, is acute and lancinating, like the pain in pleuritis, and increased by foreed breathing, so that the disease has not infrequently been supposed to be inflammation of the pleura. The intensity of the pain varies much in different eases; it is by no means always a prominent symptom, and it is sometimes slight or wanting. A dry, irritable, suppressed cough is generally present. Tenderness over the procordia is more or less marked, and pressure in the epigastrium upward in a direction toward the heart, sometimes occasions acute pain. The pain produced by a deep inspiration may cause the patient to shorten this act, and, henee, the number of respirations per minute is increased. The alre nasi may dilate in inspiration. Patients sometimes manifest suffering from an indefinite sense of distress, without acute, localized pain, and, in females, hysterical phenomena may be associated with the development of the disease. The action of the heart is increased, amounting, sometimes, to palpitation. The pulse is more or less accelerated, quick, and vibratory. The usual concomitants of febrile movement, viz., anorexia, debility, etc., are present.' These are symptoms belonging to the first stage.

The duration of the first stage is usually short. An appreciable amount of effusion may take place in a few hours, and it is rarcly delayed beyond one or two days. The acuteness of the local symptoms, viz., pain and soreness, then diminishes, and, if the amount of liquid effused be suffieient to fill or distend the periearlial sac, symptoms are added which proceed from the pressure of the liquid upon the heart. These symptoms are, a sense of oppression referable to the præcordia; a tendeney to syneope on exertion, which leads the patient to refrain as nuch as possible from movements of the body ; feebleness and irregularity of the pulse, with a notable increase on emotional excitement or any museular effort; dyspnœa, sometimes amounting to orthopnea, if the accumulation of liquid be lairge and rapid; feebleness of the voice, and dysphagia, in some eases, produced either by pressure of the distended sac on the cesophagus, or as a neuropathic concomitant. Vomiting is an oceasional symptom. Some cases are characterized by remarkable disturbance of the nervous system. Mental aberration, consisting in obstinate taciturnity and indifference, alternating with paroxysms of maniacal exeitement under the influence of delusions which excite terror, is occasionally observed. Coma and tetanic convulsions have been known to oceur. These symptoms are apt to mask the cardiac disease, and lead the practitioner to suspeet disease of the brain. A utopsical examinations show that, in these eases, neither inflamination nor any appreciable lesions of the nervous system exist, and, hence, the phenomena are to be referred to functional disturbance of the nervous system.

The scverity of the disease, as denoted by the symptoms, corresponds 
to the intensity of the inflammation and the amount of effusion. If the inflammation be slight or moderate and the quantity of effused liquid small, the disease may run its course without any symptoms denoting gravity, and the symptoms may not even point to the existence of any affection within the chest. In other cases it is one of the most distressing and formidable of diseases. The symptoms due to compression, of course, diminish as the liquid is absorbed. The absorption sometimes goes on very rapidly, and, in this respect, different cases present great variation. If, instead of being absorbed, the liquid continue to accumulate, and life be not rapidly destroyed, the pericardial sac may become greatly dilated, and the affection becomes chronic. 'The rapidity and completeness of recovery after absorption will depend on the amount of lymph which has exuded. This may be too abundant to be absorbed, and the disease may end fatally after continuing for a greater or less period in a chronic form. In cases which go on favorably toward recovery, the action of the heart is apt to be feeble and easily excited during the stage of convalescence.

Patiological Character.-Acute pericarditis does not differ essentially in charucter from other serous inflammations. The same series of processes which take place in the serous inflammation considered in the preceding section (pleuritis) occur in this inflammation, viz., exudation and liquid effusion, agglutination followed by permanent adhesion of the free surfaces of the membrane, and sometimes the formation of pus. Suppurative pericarditis, however, occurs in a smaller proportion of cases than suppurative pleuritis, or empyema. $A \mathrm{~s}$ in cases of pleuritis and other serous inflammations, so in different cases of pericarditis, both the actual and relative anount of lymph and serum differ greatly. In some cases, the quantity of lymph exuded is small and the effusion of liquid is abundant; in other eases, the lymph is abumdant with but little liquid, and, again, in other cases, both lymph and liquid are either abundant or small. Judging from the number of cases which I have seen, $d r y$ pericarditis, that is, the presence of lymph without serous effusion, is not extremely rare.

CaUsation.-Acute pericarditis may be produced traumatically by perforating wounds of the chest, or contusions. In one of the cases which I have observed, it was produced by a wound inflicted by a one-tined fork. Walshe refers to a case in which the pericardial sac was perforated in the juggler's trick of swallowing a sword, and fatal pericarditis induced. In the Museum of Bellevue Hospital is a remarkable specimen, in which a set of false teeth is contained within the pericardial sac. The teeth were swallowed during profound intoxication, and, lodging in the lower part of the œsophagus, produced ulceration through this tube and into the pericardium, giving rise to fatal pericarditis.

Exclusive of its traumatic origin, the disease is almost always secondary, and is developed in the course of acute aiticular rheumatism oftener than in connection with any other affection. Occurring in the course of rheumatism, it is distinguished as rheumatic pericarditis. It would appear, from statistical reports, to occur in cases of acute rheumatism in a proportion of about one to six. According to the statistical researches of Bamberger, of 100 cases of pericarditis in about 30 the disease is rheumatic. ${ }^{1}$ Generally it is developed in the early part of rheumatism, after more or less of the joints have been affected, but occasionally, the pericarditis precedes the articular affection. When it follows the affection of the joints, it is 
not due, as was formerly supposed, to a metastasis of the articular affection, but it proceeds from the same morbid condition which underlies the latter; in other words, both are effects of a common internal cause, supposed to be the presence of lactic acid in the blood. Rhenmatic pericarditis is almost always, if not invariably, associated with endocarditis.

Next in frequency to its development in the course of rheumatism, it occurs in connection with either acute or chronic disease of the kidneys. It is not very infrequent in cases of chronic Bright's disease, and is an occasional concomitant of the renal affection which follows scarlatina, and in other cases of so-called acute albuminuria. Under these circumstances, its causation is imputed to the accumulation of urea in the blood.

Pyæmia, scorbutus, the continued and eruptive fevers, erysipelas, and influenza, are other affections in connection with which this disease has been observed to occur, the pathological relation, probably, being the same as when it occurs in connection with rheumatism and renal affections, viz., a common causative condition pertaining to the blood being involved. It is a tolerably frequent concomitant of pleuritis and pneumonitis, affecting either the right or left lung-oftener the latter. And in this connection, it is generally attributed to an extension of the inflammation from the pleura to the pericardium in consequence of the proximity of these membranes. But here, too, it is more rational to refer the pleural and pericarclial in: flammation to a common internal causative condition, than to suppose the latter to be dependent on the former.

Idiopathic, spontaneous pericarditis is one of the rarest of diseases. I have, however, met with a few examples, or, at all events, cases in which a careful examination failed to discover any pre-existing or coexisting disease, nor was any other affection developed, so long as the patients remained under observation, the period extending only to the time when they had recovered suffieiently to leave the hospital. The extreme infra quency of such cases renders it almost certain that, whenever pericarditis exists, some one of the affections just named will be found associated with it.

Diagnosis.-The erents belonging to the clinical history of acute pericarditis are not sufficiently diagnostic for the disease to be discriminated by the symptoms alone, from pleuritis, pneumonitis, or pleurodynia. Its coexistence with the two former of these affections could not be ascertained, and its existence would surely be overlooked in the cases in which the symptoms are but little marked. The diagnosis must rest on physical signs. The presence of lymph gives rise to an adventitious sound or murmur, caused by the rubbing together of the pericardial surfaces in the movements of the heart. The development of this sign is proof of the existence of pericarditis; and, as the exudation of lymph takes place usually within a few hours after the commencement of inflammation, the sign becomes available for diagnosis at an early period of the clisease. The sign is called the pericardial or cardiac friction murmur, or sometimes, in contradistinction from the murmur produced by blood-eurrents within the heart, an exocardial murnur.

The friction murmur is to be distinguished from the murmur produced within the heart, called endocardial or bellows murnur, by attention to the following points: It is generally double, that is, consisting of two adventitious sounds for each bent of the lieart, one produced by the systolic and the other by the diastolic cardiac movements. The character of the murmur frequently denotes friction; that is, it is rubbing, grazing, or grating, and, at a later period in the disease, may be creaking, like the 
sound of new leather. It is generally limited to the præeordia, not propagated beyond the borders of the heart, and it may be limited to a portion of the precordial space. $A$ s regards its intensity or loudness, it differs much in different cases, and it is apt to fluctuate in the same case within the time the examination is continued, varying with the force of the leart's action. It has not the same fixed connection with the normal heart-sounds as the endocardial murmurs; in other words, it does not observe the same regularity in rhythm as the latter murmurs. This is a striking point of distinction, provided the auscultator be practically conversant with the endocardial murmurs. It is intensified and sometimes changed in character by firm pressure with the stethoscope.

$A$ friction murmur, if ever wanting in the first stage of pericarditis, is present so generally that we are warranted in basing an exelusion of the disease on its absence, provided sufficient time las elapsed for inflammatory exudation, and limiting the question of diagnosis to the first stage. The production of the murmur is almost pathognomonic, taken in connection with the symptoms and history; yet there is one source of fallacy, viz., the production of a friction murmur by the attrition of the outer surface of the pericardial sac with the pleural surface in cases of pleuritis. $A$ friction murmur produced by the heart's movements, continuing while the patient's breathing is voluntarily suspended, may be thus produced irrespective of pericarditis. Examples have been reported by Addison, Stokes, and others. I have met with several cases, the absence of pericarditis, notwithstanding a well-manked murmur with eardiac rhythm, being determined by examination after death. This variety of the sign is called a cardiac pleural friction nurmur. It is to be suspected, if pleuritis be known to exist, with or without pnemmonitis (oftener the latter), when the nurmur is limited to the border of the heart, the intensity of the murmur varying much more than the ordinary cardiac murmur during the periods of examination, it being limited, in some cases, to the end of inspiration, and generally affected more or less by the respiratory movements.

In the second stage, the occurrence of liquid effusion and its amount are determined by percussion. Abnormal dulness exists over an increased area in the præcordial region. The pericardial sac, if it be filled with liquid, forms a pyramidal tumor, the boundaries of which are readily found by percussion. The base of the triangular space is situated below the level of the apex-beat in health; the left border is without the left nipple, and the right border between the right margin of the sternum and the right nipple ; the apex is near the sternal notch. The situation, size, and shape of the area of dulness denote pericardial effusion. If the pericardial sac be partially filled with liquid, the area of dulness in the præcordia is abnormally widened at and below the apex of the heart, the widening extending more or less upward in proportion to the quantity of effusion; vocal resonance is diminished within the area of dulness, and also vocal fremitus. If the sac be distended with liquid, the præcordia may be abnormally prominent and the intercostal depressions abolished, as in pleuritis, within this region. Mensuration will show the increased size of the pracordia. The extent of dulness at different periods of the disease will show, on the one hand, progressive increase, or, on the other hand, diminution of the quantity of liquid, and its complete removal.

The friction murmur either diminishes or disappears after considerable effusion of liquid has taken place. Not infrequently it continues, althongh the pericardial sac be filled with liquid and even much dilated. Under these circumstances, it may be sometimes heard when the patient is raised 
to the sitting posture, and disappears when the patient lies on the back. If it have disappeared or been diminished during the stage of effusion, it will be likely to return, and may be more strongly marked, after the absorption of the liquid; and it then persists until agglutination of the pericardial surfaces takes place. Occasionally it continues during convalescence and after apparent recovery.

The apex-beat of the heart, which, in the first stage, is in most cases abnormally strong, is weakened and may be suppressed by the presence of liquid. If the accumulation of liquid be considerable, the situation of the beat is altered; it is raised to the fourth intercostal space and carried to the left as far as, or even beyond, the nipple. 'The sounds of the heart are weakened by the presence of liquid, especially the first sound, and this sound becomes short and valvular like the second sound.

By means of the signs which have been briefly considered, the diagnosis of pericarditis is generally made without difficulty. Bearing in mind the frequeney of its occurrence in articular rheumatism, the practitioner should not omit daily examinations of the chest in cases of rheumatism, with reference to the signs of this complication. The fact that it occurs pretty often in cases of Bright's disease should also be borne in mind; in fact, it is a good rule, in examining a patient with any disease, not to omit an explorition of the præcordia. Developed in connection with pleuritis or pneumonitis, it is apt to be overlooked, and the diagnosis involves more difficulty than when it occurs in other pathological connections. The determiuation of pericardial effinsion, if a large effusion also exist in the left pleural sac, is not always easy, and the chief reliance in such a case must be on the presence of a friction murmur which is believed to be produced, not without, but within the perieardial sac. The occnrrence of delirium, coma, etc., in some cases of pericarditis, masks the symptoms pointing to the latter. An examination for the signs of pericarditis should not be omitted in cases presenting notable disturbance of the nervous system.

Procnosis.-Acute pericarditis is in some cases a very distressing and dangerous discase, but in other cases comparatively mild, and, as regards immediate danger, trivial. 'The gravity depends much on the intensity of the inflammation, the amount of exudation of lymph, and more especially the quantity of liquid effusion, together with the rapidity with which it takes place. A large quantity of liquid, rapidly effused, may lead quickly to a fatal termination. Death, in such cases, is produced by paralysis of the heart from compression, and cases have occurred in which life was destroyed in this way after the lapse of a few hours only from the time of the attack. Generally, however, the duration of the disease, in fatal cases, is from one to two weeks, and death is produced by slow asthenia. In such cases, the immediate cause of death is either the prolonged compression of the heart by liquid, or weakening of the heart's action as a direct effect of the inflammation on the muscular fibres, or both causes combined. Sudden death, by syncope, is liable to be produced by muscular exertion, especially if there be much liquid effusion. In my work on diseases of the heart, I have cited a case in which a patient under my observation, affected with mild pericarditis, died instantly on getting out of bed to go to stool.

But the fatality in cases of acute pericarditis is due, not so much to the disease per se, as to coexisting affections. In fact, exclusive of the accidents just referred to, the disease in itself tends intrinsically to recovery. The danger varies greatly with its different pathological connections. Rheumatic pericarditis, not associated with pleuritis or pneumonitis, is 21 
rarely fatal. On the other hand, pericarditis developed in the course of Bright's disease proves fatal in a very large proportion of eases. This difference is explained by the difference as regards gravity between the latter and rheumatism, the ability of the system to sustain any important intercurrent affection being widely different in these two diseases. Cases in which the pericarditis occurs as a complieation of pleuritis or pneumonitis slow a large rate of fatality. Traumatic eases generally end in recovery, provided the leart be not injured. Occurring as an idiopathic affection and uncomplicated, the prognosis is favorable.

If the disease do not prove fatal during its acute eourse, it either ends in reeovery, or it becomes chronie. Chronic pericarditis will elaim separate consideration. In the cases which end in recovery, the usual result is adhesion, by the intervention of newly-formed tissue, of the pericardial surfaces over the whole or the greater part of the organ. In a small proportion of eases, the adhesion is limited to a few small spaees. Occasionally, patehes of either false or true membrane, without adhesion of the two surfaces, are the only permanent effects of the disease. It is doubtful if eomplete absorption of the effused lymph ever take place, leaving no adventitious tissue nor any traces of the disease.

Treatment.-The widely different eircumstances under which acute periearditis occurs must, of course, be taken into aceount in considering the treatment. The question as to the propriety of bloodletting and other measures heretofore distinguished as antiphlogistic, can only be entertained in the first stage of the disease, and when it is idiopathic, or traumatie, or connected with rheumatism. These measures are undoubtedly inappropriate when the disease is developed in the progress of Bright's disease, or when it is secondary to pleuritis or pneumonitis; in general terms, the propriety of these measures admits of consideration only when the periearditis is either primary, or seeondary to affections which do not in themselves impair considerably the ability of the system to sustain an additional disease. And when not associated with other diseases which are in themselves dangerous, it is to be borne in mind that periearditis intrinsically tends to recovery.

In the eases to which bloodletting must be limited, the same general principles are to govern its employment, as in other acute inflammations. These prineiples have been considered.' Some importance, perhaps, belongs to this consideration, viz., by diminishing the mass of blood, the heart is less excited, and its labor diminished. On the other hand, it is to be considered that the danger incident to the disease is mainly from paralysis of the heart or impairment of its muscular power. Giving due weight to the latter consideration, bloodletting should be employed with great circumspection, even in the few eases in which the symptoms relating to the circulation, in the first stage of the disease, may appear to indicate it. It is never indieated after considerable effusion has taken place.

Depletion by means of saline purgatives, with restricted diet, is indieated, in the first stage, by the same symptoms as in other acute inflammations. This remark is alike applicable to nauseant and other sedatives. These should be limited to the first stage. They might be pernicious if con.tinued after effusion has occurred, and, hence, the great importance of determining the presence of liquid and its amount, by physical signs.

Shall the system be brought under the influence of mereury in the first stage? This is a question which different writers and practitioners answer

1 Vide page 148. 
quite differently, some advocating rapid mercurialization as highly important, others regarding it as needless and hurtful. In the cases in which mercury is by some considered important, and by many admissible, my experience leads me to be satisfied to forego its use.

Opium is invaluable in this, as in other acute inflammations. It is indicated by pain and constitutional disturbance, and is to be employed sufficiently to relieve the general and local symptoms. 'The form of opiate and the mode of administration are to be determined by the cirenmstances in individual eases. The hypodermic method in this, as in various other affections, is eligible and efficient.

Locally, mild, revulsive, and soothing applications are useful in the first stage. Sinapisms, a poultice, the water-dressing, or the spongiopiline saturated with a warm anodyne infusion, may be employed. Blisters in this stage should not be employed. Aside from their interference with the daily physieal explorations, which are vastly important, they do more harm, by addling to the pain and constitutional disturbance, than good by way of revulsion. By some German writers cold applied to the præcordia, as in the treatment of acute pleuritis and pneumonitis, is said to be useful. 'The evidence of its usefulness is the relief of pain, together with diminished frequency of the heart's action.

The indications in the second stage relate, first, to the liquid effusion. If the amount of effusion be sufficient to enfeeble the heart by compression, it is highly inportant to effeet the removal of the liquid as speedily as possible. For this end, the precordia may be painted daily with the tincture of iodine, and small blisters may be applied, removing them as soon as vesication begins, and allowing the blistered surface to dry up rapidly. Hydragogue eatharties and diureties may be employed, as in pleuritis, taking care not to earry these measures so far as to depress the vital powers and weaken the heart's action. This causation is of great importance, and it suggests a second indieation which may be present in the second stage, viz., to strengthen the heart's action, and support the powers of life, by the timely and judicious use of alcoholic stimulants, tonics, and nourishment. Keeping in view the fact that the disease, if it end fatally, destroys life by affecting directly the heart's action, the measures just named are indicated with an urgency proportionate to the degree of feebleness of the circulation. All measures which tend direetly or indirectly to weaken the cireulation or depress the vital powers, are contraindicated in the seeond stage. The liability to fatal syncope on exertion is to be borne in mind, and quietude should be rigidly enjoined. Feebleness and irregularity of the pulse in the second, and sometimes in the first, stage, constitute an indication for digitalis, which, however, should not be given sufficiently to retard unduly the heart's action.

During the stage of convalescence, precautions against undue excitement of the action of the heart by exercise, mental emotions, over-repletion of the stomach, etc., are more important than during reeovery from most other acute inflammations. At the same time, convaleseenee will be expedited and complete recovery secured, by a nutritious diet, tonic remedies, gentle gestation out of doors, and other hygienic measures to promote speedy recuperation.

In the treatment of pericarditis developed in the course of either rhenmatism or renal disease, it may be important to take into aceount the supposed presence in the blood of a morbid material upon which the pericardial inflammation depends. The indication derived from this view of the causation is, in general terms, to remove, if possible, the continued operation of the cause, by measures addressed to the morbid conditions 
of the blood belonging to those affections. This part of the treatment, however, will be more appropriately considered in connection with rheumatism and renal disease hereafter. The importance of measures to prevent the development of pericarditis, in the course of these diseases, will also be then considered.

If the disease end in recovery, without becoming chronic, adhesion of the pericardial surfaces, to a greater or less extent, may be expected to take place. This result was formerly supposed, by Hope and others, to lead to progressive enlargement of the heart, an $l$ therefore pericarditis was regarded as a highly serious disease with reference to its remote effects. This is an error which arose from imputing to pericardial adhesions the consequences of the valvular lesions which often coexist. It may be doubted if adhesions alone tend to produce enlargement, and there is reason to believe that, if not associated with valvular lesions, they may give rise to little or no inconvenience. The pericardial surfaces are not infrequently found, in autopsical examinations, universally adherent, as a result of ancient pericarditis, without any other evidence of eardiac disease, and when no symptoms referable to the heart had existed during life.

\section{Chronic Pericarditis.}

Chronic pericarditis may be subacute from the beginning, or it may follow the acute form of the disease. If acute pericarditis do not end in convalescence after the lapse of from two to three weeks, it becomes a chronic affection. As regards anatomical characters, cases differ. In some cases of chronic pericarditis, there is no liquid effusion, but the pericardial surfaces are agglutinated by several layers of lymph, which collectively may be half an inch or more in thickness. Under these circumstances, the lymph is not absorbed, a low grade of inflammation continues, and the disease ends fatally after a variable duration.

In other cases, liquid effusion remains and progressively accumulates, the pericardial sac becomes more or less dilated, and it is sometimes so much enlarged as to depress the diaphragm and oceupy the greater part of the thoracic space. The amount of liquid which has been known to accumulate in the pericardium is enormous, amounting to eight or ten pints.

In chronic pericarditis without liquid effusion, pain is rarely prominent. The symptoms are those dependent on feebleness of the circulation, due to the impaired muscular power of the heart, and on constitutional irritation. In the cases in which large effusion exists, the heart is weakened by compression, and the patient suffers from dyspnca caused by interference with the respiratory function. The dyspnoea may amount to orthopnœa if the accumulation of liquid be very large. Pain under these circumstances is rarely a prominent symptom.

The diagnosis, if liquid effusion be not present, must often be inferential, being based mainly on the knowledge of the existence of antecedent acute pericarditis. Creaking friction-murmur exists in some cases, notwithstanding the agglutination of the pericardial surfaces. If frictionmurmur be not present, and acute pericarditis be not known to have existed, a positive diagnosis is extremely difficult, if not impossible. The heart is found to be more or less enlarged, but there are no means of determining that the enlargement proceeds from the deposit of lymph. If liquid effusion be present, the physical signs render the diagnosis easy and positive. Flatness on percussion exists on the anterior surface of the chest, extending on either side of the sternum toward the axillary 
region, in proportion as the pericardial sac is dilated. Obliteration of the intercostal depressions and cren bulging may be observed. Fluctuation in the interoostal spaces is sometimes felt. Laterally, beyond the limit of the flatness on each side, resonance on percussion and the respiratory murmur show the presence of lung. The heart's impulse is suppressed, but a shock communicated by the action of the heart may be felt. A friction-murmur is sometimes heard, even when the quantity of effused liquid is very large. 'The sounds of the heart are feeble, the first sound being more weakened than the second, and short and valvular like the second sound. These signs render the diagnosis sufficiently clear.

The prognosis in cases of chronic pericarditis is always extremely unfavorable. The disease ends fatally after a duration varying much in different cases.

The indications for treatment are to improve the tone of the system and impart vigor to the heart by tonic remedies, together with nutritious diet and other hygienic measures. The propriety of much counter-irritation is doubtful. Everything which tends, directly or indirectly, to weaken the heart or the vital powers is to be avoided. In this point of view, mercurialization is objectionable. If there be much liquid effusion, diuretic remedies may be tried, and small blisters. Hydragogue catharties, in these cases, if the patient be feeble, are to be employed with great circumspection. In a case under my observation, elaterium given in small doses and producing but a moderate effect, appeared to hasten the fatal termination. Iodine may be employed as a sorbefacient, externally and internally. Puncture of the pericardial sac is admissible, if the effusion be large and other measures fail to diminish it. Clinical data for determining the value of the operation of paracentesis are wanting.

\section{Pneumo-Pericarditis.}

This name denotes the presence of air or gas within the periciardial sac in cases of pericarditis. Air or gas may find its way into this situation through a wound of the thoracic walls, or of the œsophagus, and through a fistulous communication between the lungs or the stomach and the pericardial cavity. Air may get access into this cavity through a wound of the chest, and pericarditis not be dereloped. The condition, then, is expressed by the term pueumo-pericardium. It is possible that gas may be generated by decomposition of the products of inflammation within this cavity, but examples must be exceedingly rare.

The presence of air and liquid gives rise to splashing and sometimes metallic sounds, with the movements of the heart, which are heard on auscultation; and percussion over the precordia elicits a tympanitic resonance. These cases belong among the curiosities of clinical experience. The treatment of pneumo-pericarditis involves the same principles as the treatment of pericarditis without the presence of air or gas. 


\section{CH A P T ER II.}

Endocarditis-Anatomical Characters-Clinical History-Pathologicai Character-CausationDiagnosis-Prognosis-Treatment. Myocarditis.

TNFLAMMATION of the membrane lining the cavities of the heart, or 1 the endocardium, is called endocarditis. Our knowledge of the existence of this disease is one of the developments of modern pathological research. It is a highly important disease considered more especially in its . relation to structural lesions of the valves and orifices of the heart.

The inflammation is doubtless in some cases acnte, and in other cases subacute or chronic, but, as the symptoms and signs do not enable us to determine clinically the grade of inflammatory actinn. a division of the disease into the acute and chronic form is umnecessary.

Anatomical Characters. - The disease very rareiy proves fatal during its progress, and, therefore, the opportunity of observing the morbid appearances is seldom offered. On this account the observations of Dr. Richardson in cases of endocarditis produced, artificially, in inferior animals, by the introduction into the blood of lactic acid, injected into the peritoneal cavity, are valuable. 'These observations show arborescent redness from congestion of the vessels beneath the membrane, and considerable swelling of the valves, especially the anricular valves, from the exudation, beneath the membrane, of lymph with serum. More or less exudation also takes place on the free surface of the membrane covering the valves and tendinous cords. Much of this exudation must be washed away with currents of blood; that which remains sometimes assumes the form of patches of false membrane, but it is oftener disposed in the form of small masses, or beads, situated especially at the base or near the extremities of the valvular curtains and segments. These small masses are often observed, in greater or less number, varying in size from that of a pin's head to a millet-seed, oftenest in the auricular aspect of the mitral curtains, and the ventricular aspect of the aortic segments. According to Virchow they are due not to fibrinous exudation, but to morbid growth or proliferation of the connective tissue of the endocardium. Roughening the surface of the membrane, they become the nuclei of the so-called warty excrescences or vegetations, their size being increased by the deposit of fibrin from the blood within the cavities of the heart. In this way they may attain to a considerable size. It is not uncommon to find, attached to the valves, vegetations as large as a pea, a bean, a filbert, and even still larger. They may be loosely attached to the endocardium, so that but little force is required to detach them, or they may adhere with considerable firmness. They are liable to be separated during life, giving rise to emboli, to which reference has already been made, and which will be again referred to in other connections.

Aside from these products of inflammation, the membrane in certain situations, especially over the valves, loses its smooth, polished appearance, becoming rough and velvety. It is more easily separated than in

1 Vide Prize Essay on Coagulation of the Blood, by W. B. Richardson, M. D. 
health, owing to brittleness of the areolar tissue beneath it. Perforation of the valves, laceration and morbid adhesions of the valvular curtains or segments, are occasionally incident to the progress of endocarditis. These and other changes, however, are oftener remote than immediate effects of inflammation, and they will be considered under the head of valvular lesions, in another chapter. Ulcerations of the membrane charaeterize a small proportion of cases; the effects of so-called ulcerative endocarditis will be noticed in connection with the prognosis. In examinations of the heart after. death, redness of the endocardial membrane and of the lintng membrane of the vessels near the heart, is frequently foumd, which is due to staining with hematine dissolved out of the red corpuscles, and occurs after death. This appearance is no evidence of diseasc of the endocardium.

The anatomical characters of endocarditis are generally limited to the left side of the heart, that is, the left ventricle and auricle. 'The right cavities are very rarely the seat of endocardial inflammation, and never withont inflammation of the left cavities. This statement, os course, applies to the disease as occurring after birtl. There is reason to believe that endocarditis may oceur during fotal life, and that the reverse of the statement just made then takes place, viz., the right cavities, and not the left, are the seat of the inflammation. The inflammation is either limited to, or is especially marked in, the valvular portions of the endocardial membrane. This is probably owing partly to the presence of fibrinous tissue beneath the endocardium in these situations, and in part to the strain which falls upon the valves in the performance of their functions.

Chinical History.-Authenticated cases of endocarditis disconnected from other diseases which serve to mask its symptoms to a greater or less extent are wanting. The clinical history of the disease, therefore, so far as it is at present known, will require but a bricf space. Our knowledge of the disease is confined chiefly to the cases in which it oceurs in connection with acute articular rheumatism, and in a certain proportion of these cases it is associated with pericarditis. Pain referable to the præcordia is not a constant, and is rarely a prominent symptom. The pain, when present, is dull, not sharp or lancinating like the pain in pericarditis or pleuritis. The patient may complain of an obscure sense of distress in the pracordia, not amounting to pain. The action of the heart is morbidly excited. The organ beats with abnormal quickness and frequency, and its action may be irregular. 'The action of the heart may be found to be out of proportion to the force of the pulse. The local symptoms, in short, as regards the action of the heart, are those of palpitation. In proportion to the acuteness of the inflammation, doubtless febrile movement is induced, together with the symptoms of constitutional disturbance which accompany fever symptomatic of inflammation in other situations. The local and general symptoms, however, are often not prominent, and, so far as these are concerned, the disease is not infrequently latent. Hence the existence of the disease was unknown prior to the early part of the present century.

Pathological Character.-The morbid changes which belong to endocarditis are, in many respects, the same as in serous inflammations. But the inflammatory products in this situation are not retained to be removed by absorption, but are, in a great measure, carried by the currents of blood directly into the circulation.

It may be doubted whether suppuration often takes place upon the endo- 
cardial surface. Newly-formed tissue, giving rise to morbid growths, is a result of inflammation in this situation. 'The warty vegetations which have been referred to are sometimes in part organized. Adhesion of parts in contact, viz., of the rnitral curtains to each other, and the aortic segments to the walls of the aorta, is an occasional result of inflammation. It is stated by Bouillaud that the valves may become gangrenous as a result of inflammation; this must be exceedingly rare. As already stated, ulcerations sometimes occur, characterizing a form of the disease which is called ulcerative endocarditis.

Causation.-Endocarditis, in the great majority of eases, is not a primary disease. There is reason, however, to believe that it oceurs as an idiopathic affection not very infrequently, and is overlooked in consequence of its latency as regards cardiac symptoms. This may be inferred from the number of eases in which valvular lesions referable to inflammation are found in persons who have never experienced the disease in the course of which endocarditis is apt to be developed, viz., articular rheumatism. As met with in practice, it is generally incidental to the disease just named. The proportion of eases of rheumatism in which it occurs is not settled. The statistics of different observers show a variation from two-thirds to nine per cent. A reason for this variation is that the existence of the disease has been often assumed on insufficient evidence. The ground for this statement will appear in colnection with the consideration of the diagnosis. It is not to be doubted that the disease is frequently developed in the course of rheunatism. Endocarditis, like pericurditis, may perhaps in some cases precede the affection of the joints. It may occur at any time in the course of a rheumatic attack, but it is usually developed in the early or middle period.

When developed in the course of rheumatism, it is not from the transference of the affection of the joints to the endocardial membrane; that is, not by a metastasis, but, like pericarditis developed in the same connection, it is produced by the internal causative condition which gives rise to the articular affection. That this cansative condition consists of a morbid material in the blood is probable, and the fact of the endocarditis being generally limited to the left side of the heart would seem to render it probable that the morbific agent is generated within the blood during its passage through the pulmonary organs. If the agent in the blood exerted its effect upon the endocardium by means of the vessels distributed to the heart, there is no apparent reason why the right side should not be affected as well as the left; but, assuming that the agent in the blood acts by being bronght into direct contact with the endocardium, it is intelligible that this agent existing in the blood received into the left cavities from the pulmonary circuit should act upon the membrane lining these cavities, and that it should be eliminated, neutralized, or decomposed before the blood has passed through the systemic circuit, and reached the right eavities of the heart. This is the view taken by Richardson, and it is sustained by the fact that in his experiments of injecting lactic acid into the peritoneal cavity, endocarditis was produced not in the left, but in the right cavities of the heart, the acid being destroyed in these experiments before the blood passes through the pulmonary circuit and reaches the left cavities. Moreover, these experıments render it probable that the morbific agent which gives rise to the affections of the heart and joints in rheumatism is lactic acid produced in the blood during its passage through the lungs.

Endocarditis and pericarditis are not infrequently associated in cases 
of rheumatism, and this compound affection is denominated endo-pericardilis. Rheumatic pericarditis very rarely, if ever, exists without endocarditis. The reverse of this, however, does not hold good; endocarditis is not infrequently developed without pericarditis.

Endocarditis, either with or without pericarditis, is developed, in a certain proportion of cases, in the course of Bright's disease. It is stated, also, to occur occasionally in the eruptive and continued fevers, and in cases of pyæmia. It may possibly be produced by contusions of the chest. It may be associated with pleuritis or pneumonitis, when it occurs in rheumatism or in other pathological connections. The most rational view in these cases is to attribute the coexisting affections to a common causative condition.

Diagnosis.-The symptoms in cases of endocarditis are insufficient for a positive diagnosis. The symptoms are even less diagnostic than those which belong to the clinical history of pericarditis, and the disease is oftener latent. 'The diagnosis of this disease, as well as of pericarditis, must rest on physical evidence. The exudation of lymph, or the deposit of fibrin upon the endocardium, gives rise to an endocardial murmur, and the diagnosis is to be based on the development of this murmur, taken in connection with the symptoms. The murmur is due to the roughening of the endocardial surface, and, as the anatomical changes are especially situated upon the eurtains of the mitral valve, at least in cases of rheumatic endocarditis, the murmur is usually of mitral origin. The murmur is of a soft or bellows character. It accompanies the first sound of the heart; this is, it is systolic. It is heard loudest at or near the apex of the heart, and may be limited to this situation. As a rule, if, prior to the endocarditis, the patient were free from valvular lesions, the systolic murmur is not propagated far without the left border of the heart. It is probable that the anatomical changes which generally occur during the progress of the endocarditis rarely. in volve immediate valvular insufficiency, either at the mitral or aortic orifice. Hence, the mitral murmur may not proceed from an actual regurgitant current from the ventricle to the auricle. It is not, then, strictly correct to call it a mitral regurgitant murmur. It is an intra-ventrieular murmur, or it may be called a mitral systolic murmur, this term not implying the occurrence of regurgitation.

The physical evidence of endocarditis is not afforded by the existence of the murmur just named. A mitral murmur exists suffieiently often without clenoting existing inflammation. It may proceed from valvular lesions which are to be presently considered. To be evidence of endocarditis, the murmur must be developed under observation; in other words, the practitioner must be satisfied that the murmur which he discovers did not exist on a previous examination. As it is generally in cases of rheumatism that endocarditis occurs, let it be assumed that, on the first examination of a patient affected with rheumatism, a mitral murmur is found. The murmur is not proof of endocarditis, for it is not known that the murmur did not exist prior to the rheumatic attack. The existence of endocarditis is only probable, and the probability of the disease is greater if this be the first attack of rheumatism. But if, on a first examination, no murmur be found, and subsequently, a mitral murmur become developed, it is evidence of endocarditis. The evidence is strengthened, if, at the same time, pain or precordial distress and excited action of the heart point to the occurrence of a cardiac complication.

Reference has been made, thus far, to a mitral murmur only as evidence of endocarditis. The exudation of lymph, and the deposit of fibrin at the 
aortic orifice, may occur in this disease, giving rise to an aortic murmur. I have been led, however, to think that an aortic and a pulmonic murmur are not infrequently devcloped in cases of rheumatism without endocarditis, more especially in females, being due to the condition of the blood; in other words, being inorganic murmurs. Hence, I would not base a positive diagnosis of endocarditis on the development of an aortic murmur alone, unless the symptoms, at the same time, denoted a cardiac complication, and pericarditis did not exist. Irrespective of cases of rhcumatism, it is well known that an aortic murmur with the first sound $i . e .$, systolic, is sufficiently common as an inorganic murmur, and, alone, is nerer proof of endocarditis. It may be cloubted if insufficiency of the aortic valve be ever an immediate result of endocarditis; at all events, I have never met with an aortic regurgitant murmur developed in the progress of endocarditis.

In view of the fact that in the great majority of the cases of endocarditis it is a complication of articular rheumatism, not only do we seek for the evidence of its development in the course of the latter disease, but the existence of articular rheumatism renders the diagnosis more complete than if this clisease did not exist.

An endocardial murmur produced by endocarditis is generally permanent, but $I$ bave repeatedly known it to disappear after recovery from rheumatism. I have known an aortic and a mitral murmur to continue for several years after recovery and at length to disappear entirely. In cases of endo-pericarditis, the endocardial exists with an exocardial or friction murmur. In general, the two murmurs may be readily distinguished, and each referred to its source.

Prognosis.-In the great majority of cases, endocarditis in rolves no immediate danger. 'The danger is, for the most part, remote, relating to the valvular lesions which are apt to follow. (Vide Chapter III.) The persistence of inflammation in a chronic form is not determinable. The continuance of endocardial murmur is not proof that inflammation persists; ronghening of the membrane from vegetations or lymph which remains will suffice to produce murmur. The chief source of important morbid effects and of danger, exclusive of valvular lesions, is embolism. Vegetations, or masses of fibrin of greater or less size, are liable to be detached, and, forming emboli, occasion obstruction (infarction) of arteries in different organs. Passing, of course, into the systemic circulation, they are especially apt to become lodged in the spleen, where they give rise to characteristic lesions. (Vide page 78.) Occasionally they give rise to analogous lesions in the kidneys, and sometimes, although very rarely, the emboli are carried to the liver. The affection hereafter to be considered under the name acute or yellow atrophy of the liver, is supposed to be an effect of embolism of the hepatic artery. A gain, cerebral embolism may occur, and cases have occurred in which gangrene of the lower limbs has resulted from embolic obstruction of their distributing arteries. Moreover, the washing away of lymph or fibrin in small particles may occasion capillary embolism. For a general account of embolism, the reader is referred to the first part of this work. (Vide page 71 , et seq.) These are accidents incidental to endocarditis in some cases. In general, as occurring in the pathological connection in which it usually occurs, viz., in the course of acute articular rheumatism, and disconnected from pericarditis, endocarditis involves no immediate danger, gives rise to no symptoms denoting gravity of disease, is frequently completely latent, and is an important complication chiefly because it may be the foundation for valvular lesions 
which, after the lapse of many months, or it may be many years, occasion serious consequences.

The so-called ulcerative endocarditis is characterized by small ulcerations limited, in the majority of. cases, to the valves, but sometimes occurring in portions of the endocardium which are in contact with the muscular walls of the heart. These ulcerations may lead to perforation of the valves, and, also, to aneurismal dilatations. The immediate pathological effects, however, which belong to certain cases of ulcerative endocarditis, relate to embolism and an infection of the blood. The decomposed tissue and morbid products which are thrown off in the formation of the ulcers, and from the ulcerated surfaces, although the quantity is small, may be sufficient to infect the blood. The solid particles in the detritus, at the situations where they become lodged, not only cause mechanical obstruction, or infarctus, but they excite suppurative inflammation, and, perhaps, ganglene; and these may prove foci whence a further infection of the blood is produced. In short, septicæmia and pyremia are sometimes attributable, thus, to ulcerative endocarditis; and, bearing this fact in mind, the existence of these blood-conditions, without other obvious causation, should lead us to examine for the signs of endocardial clisease. Among the systemic arteries which are liable to receive the detritus in cases of ulcerative endocarditis, are the mesenteric; and embolism here leading to intestinal ulcerations, the cases may simulate closely typhoid fever. Happily, pyæmic and septic conditions attributable to endocarditis are extremely rare. In ulcerative endocarditis the ulcerations are generally limited to the left sidic of the heart. Exceptionally they oecur in the right side, and the local embolic effects then, of course, talie place in the lungs. ${ }^{1}$ The prognosis in cases of embolism, pyrmi:1, and septicæmia attributable to ulcerative endocarditis, is the same as when these conditions occur in other pathologieal connections. From the two last named conditions recovery is to be expected in only a small proportion of cases.

Treatuent.-The inflammation in endocarditis being confined, generally, to one side of the heart, and limited chiefly to the valvular portion of the endocardium, does not occasion great constitutional disturbance. Following the general principles which are to govern the employment of bloodletting, it is never called for. Depletion by means of saline purgatives or laxatives may be advisable.

Pracordial pain and clisturbed action of the heart indicate anodyne remedies and soothing applications to the chest. Blisters, with reference to these symptoms, are of doubtful propricty. Sinapisms and stimulating liniments will secure all the advantages to be derived from counter-irritation.

Measures addressed to the supposed causative condition of the blood are rationally indicated. Occurring generally in connection with rheumatism, if the local affections, this of coursc included, depend on the presence of a morbid material in the blood, it is plsinly an object either to neutralize or eliminate this material. The means to be employed for this object will be hereafter considered in connection with the subject of rhemmatism.

The remote evil consequences of endocarditis proceed from the thickening, morbid growths and the deposit of fibrin upon the valvular portion of the endocardium. It is, therefore, undonbtedly desirable to limit and remove these immediate effects of the inflammation. The fibrin of the

1 Fide "Case of Ulcerative Endocarditis of Right Heart with Sloughing of Lungs, etc., by W. Moxon," Trans. Path. Soc., of London, 1871, p. 107. 
blood is increased in acute rheumatism more than in any other disease, and for this reason, perhaps, fibrinous deposits are apt to take place. With this view, measures to reduce the excess of fibrin, and to favor its liquid state, are rationally indicated. Alkaline remedies and ammonia, there is reason to believe, contribute to the latter of these two ends. Mercury has been supposed to diminish the amount of fibrin in the blood. That it possesses this power, however, has been inferred rather than demonstrated, and the correctness of the inference admits of doubt. It may be doubted whether the fibrin deposited upon the membrane is ever absorbed ; it either remains or is washed away, gradually or in mass, by the current of blood.

The treatment in the cases in which ulcerative endocarditis exists and causes the effects noticed in counection with the prognosis, is not embraced in the foregoing remarks. The indications, however, in these cases are those which relate to embolism in different situations, and to pyæmia and septicæmia. These indications need not be here considered.

The practitioner should bear in mind that the persistence of an endocardial murmur is not sufficient ground for sorbefacient or other remedies. The persistence of the murmur is not evidence that the inflammation continues. The treatment of endocarditis should cease when the local and general symptoms of the disease are no longer present.

\section{Myocarditis.}

Inflammation of the muscular structure of the heart is rare. When it occurs it is generally in connection witi endocarditis or pericarditis, singly or combined. It may occur, however, irrespective of either of these affections. Suppuration may take place as a result of myocarditis, the pus being either collected in abscesses or infiltrated. The pus may be discharged into the pericardial sac, giving rise to pericarditis, or into one of the cavities of the heart, causing purulent infection of the blood. Rupture of the heart may be a consequence of inflammation and suppuration. An abscess in the inter-ventricular septum may lead to perforation. Induration or sclerosis is a result of interstitial inflammation. Aneurismal dilatation is still another. The inflammation is oftener seated in the walls of the left, than of the right, ventricle. Perhaps the most frequent of the causes, exclusive of endocarditis and pericarditis, is embolism of a branch of the coronary artery. It may sometimes be imputed to syphilis. It is a serious affection, as impairing, in proportion to its extent, the muscular power of the heart, and as involving a liability to the accidents just stated. Associated with either endocarditis or pericarditis, or both, it adds, of course, to the gravity and danger. Its coexistence with these affections, and its existence without them, cannot be determined during life. 


\section{CHA P T ER III.}

\section{VALVULAR LESIONS WITH ENLARGEMENT OF THE HEART.}

Anatomical Characters - Clinical History-Pathological Character-Causation-DiagnosisPrognosis-Treatment.

$\mathrm{H}$ A VING considered, in the two preceding chapters, the inflammatory affections of the heart, the structural lesions of this organ next claim attention. The structural lesions relate, in the first place, to the valves and orifices of the heart. These are known commonly as valvular lesions. Other lesions relate to the walls and cavities of the heart. Enlargement of the heart, in the great majority of cases, proceeds from valvular lesions, and the latter, sooner or later, in the great majority of cases, give rise to cardiac enlargement. In considering, therefore, valvular lesions, I shall also consider enlargement as dependent thereon, and afterward notice enlargement occurring without valvular lesions.

Anatomical Cinaracters. - Valvular lesions are situated, in the great majority of cases, in the left side of the heart, that is, at the mitral and the aortic orifice. Tricuspid and pulmonic lesions are comparatively rare. The morbid appearances in different cases are extremely diversified. The valvular curtains and segments are frequently thickened and contracted; or, they may be simply encumbered with vegetations of greater or less size, without being incapacitated for the performance of their function. They are sometimes rendered more or less rigid by calcareous deposit. The mitral curtains are not infrequently united at their sides so as to form a fumnel-shaped canal leading from the auricle to the ventricle, the opening consisting of a small slit, called the button-hole contraction of the mitral orifice. The aortic.segments, expanded and rigid, in some cases diminish the size of this orifice to that of a crow's quill, or even much smaller. The valves may become atrophied, and are liable to rupture or perforations. Rupture of one of the valvès is an occasional accident without prior disease, when an excessive strain is induced by the violent action of the heart in severe muscular exertions; under these circumstances the tendinous cords which unite the extremities of the mitral curtains to the papillary muscles are especially apt to give way. For a full description of the varied clianges which are observed in difterent cases of valvular lesions, the reader is referred to works on morbid anatomy.

In a clinical point of view, all the diverse alterations of structure embraced under the name valvular lesions may be arranged, according to their effects upon the circulation, into the following groups : First, obstructive lesions, that is, lesions which impede the flow of blood by producing contraction of the orifices; second, regurgitant lesions, that is, lesions which interfere with the function of the valves, rendering them incompetent or insufficient, and consequently allowing backwarl or regurgitant currents ; third, lesions which involve both obstruction and regurgitation; and, fourth, lesions which involve neither obstruction nor regurgitation, but give rise to morbid sounds by roughening the surface over which the blood flows. The lesions belonging to the last group are of 
little or no immediate importance, and, although giving rise to abnormal sounds known as endocardial or bellows murmurs, they may be quite innocuous.

The lesions which involve either obstruction or regurgitation, or both, lead to enlargement of the heart. They produce this result by giving rise to over-repletion of certain of the cavities of the organ. Limiting attention to the lesions situated at the mitral and aortic orifice, the primary effect differs according to the situation. Mitral obstructive and regurgitant lesions, as a rule, produce, first, dilatation of the left auricle, because these lesions give rise, as the primary effect, to over-repletion of this cavity. Dilatation of the left auricle occasions pulmonary obstruction, because this cavity, being already distended, does not offer space for the free admission of the blood returned to the heart from the lungs. As a consequence of pulmonary obstruction, blood accumulates in the right ventricle, and lience occurs enlargement of this portion of the heart as the next effect. Over-repletion of the right auricle is the next effect, leading to dilatation of this cavity; and finally, as a consequence of obstruction of the systemic venous system due to the right auricle being constantly filled, the left ventriele may become enlarged. 'This is the series of effects caused by mitral lesions which involve either obstruction or regurgitation, or both.

Lesions of the aortic orifice, either obstructive or regurgitant, or both, produce a similar series of effeets with a different point of departure. The over-accumulation is first in the left ventricle, and this becomes enlarged. Dilatation of the left auricle follows, and successively the right ventricle and right auricle are enlarged, if life be sufficiently prolonged. Cases are not infrequent in which obstructive or regurgitant lesions are situated at both the mitral and aortic orifice. The effects of aortic and mitral lesions are combined in these cases.

Enlargement of the heart, with or without valvular lesions, is of two kinds, viz., first, enlargement due to abnormal growth or hypertrophy, and, second, enlargement due to dilatation. In examinations after death from valvular lesions with enlargement, the enlargement is not in general found to be exclusively by either hypertrophy or dilatation, but the two kinds of enlargement are combined. A nuarked difference exists in different cases, as regards the symptoms, signs, and the danger, according to the predominance of either hypertrophy or dilatation. As a rule, hypertrophy of the ventricles takes place before dilatation. The overrepletion of the ventricular cavities induces augmented power of muscular action, and this causes hyper-nutrition or abnormal growth. The ventricles grow in consequence of long-continued increased power of muscular action, precisely as the voluntary muscles become enlarged as a consequence of exercise.

The hypertrophic growth of the ventricles has its limit, as the enlargement of voluntary muscles is limited. The voluntary muscles cannot increase indefinitely, but, after a certain amount of increased growth, it ceases, notwithstanding the exercise of the muscle is continued. So with the heart-the hypertrophy at length reaches a point beyond which no further growth takes place. The extent to which the heart can increase by hypertrophy varies in different persons, as the point at which the increase of the voluntary muscles varies. The enlargement by hypertrophy is represented by the increased weight of the heart. In cases in which a very great amount of liypertrophy takes place, the weight of the heart is found to be four or five times greater than in health.

Dilatation, sooner or later, follows hypertrophy. When nutrition can 
no longer be excited to cause progressive growtl, the continued overrepletion of the cavities causes the walls to yield and dilatation takes place. The dilatation goes on, and at length it predominates over the hypertrophy; that is, in relative amount the former exceeds the latter. The enlargement by hypertrophy is due to a vital process, viz., hypernutrition; but the enlargement by dilatation is produced mechanically. Hypertropliy involves augmented power of the muscular walls ; dilatation involves diminished power, or weakness, in proportion as the cavities are enlarged. In proportion as dilatation predominates over hypertrophy, the volume of the heart, rather than its weight, is increased.

Enlargement may be much more marked in one portion of the heart than in another portion, or it may be limited to a part of the organ. 'The right ventricle, for example, may be chiefly or exclusively the seat of enlargement in connection with mitral valvular lesions, and this may hold true of the left ventricle in connection with aortic valvular lesions. The left ventricle may be enlarged by predominant dilatation while the right ventricle is enlarged by predominant hypertrophy, and vice versâ.

Chinical History.-Valvular lesions, so long as they involve neither obstruction nor regurgitation, give rise to no symptoms of disease, and their existence is only ascertained by means of physical signs. Lesions involving obstruction or regurgitation, or both, as a rule; do not occasion much inconvenience until they have led to enlargement of the heart. Moreover, as a rule, lesions occasion suffering, and are attended with danger, in proportion to the enlargement which they have produced, and chiefly to the enlargement due to dilatation.

The first symptoms proceeding from mitral obstructive or regurgitant lesions pertain to the respiratory system. Deficiency of breath on exercise is, for some time, the chief inconvenience. This progressively increases, in proportion as the obstruction to the pulmonary circulation increases. The patient is at length obliged to discontinue any active muscular exertion, but may be comfortable while remaining quiet. Dyspnoa finally becomes habitual, and is more or less prominent as a symptom after dilatation of the right ventricle has taken place. A still further development of this symptom renders the patient unable to lie down, constituting, then, orthopnca. This may be due, in a measure, to coexisting pulmonary accidents. Cough and more or less muco-serous expectoration are incidental to congestion or a low grade of inflammation of the bronchial mucous membrane. An abundant serous expectoration sometimes occurs, constituting bronchorrhœa. Hxmoptysis is not infrequent, the hemorrhage being usually small. This event generally denotes simply bronchorrhagia, but occasionally blood is extravasated into the air-cells, constituting what is commonly called pulmonary apoplexy. Pulmonary œdema is another intercurrent event which is liable to oceur, increasing the suffering from dyspnoa. Hydrothorax is still another source of embarrassment of respiration. "Pigment induration" of the lungs dependent on persistent congestion, caused by regurgitant and obstructive mitral lesions, has been noticed in a preceding chapter (vide page 196). This enters into the rationale of dyspnoea in some cases.

After enlargement of the heart takes place, its action, so long as the hypertrophy predominates, is abnormally strong. 'This may be perceived by the hand placed over the pracordia. The patient, however, rarely complains much of palpitation, having become accustomed to the gradual increase of the power of the heart's action. The pulse is small and weak in proportion to the amount of obstruction or regurgitation, 
each of these immediate effects of the lesions lessening the quantity of blood propelled into the aorta and its branches by the systole of the left ventricle. The quantity of blood contained in this ventricle at the time of the systole may sometimes be so much lessened that the radial pulse is lost; hence one source of intermission of the pulse; another source is an actual intermission of the ventricular systole. Inequality of the successive beats of the pulse, as regards volume and force, represents unequal supplies of blood from the left auricle to the left ventricle. Irregularity in the action of the heart is not infrequent. Intermittency of the pulse and inequality of successive beats, due to deficiency of the supply of blood to the left ventricle, are characters which denote mitral contraction rather than regurgitation.

The characters pertaining to the form of the pulse, as ascertained by the sphygmograph, are notable variations, in different pulsations, in the length of the line of ascent, shortness of this line in a greater or less number of pulsations, its vertical direction, and marked dicrotism in the line of descent. In cases of mitral obstruction, according to Marey's observations, the line of ascent is longer and the variations in different pulsations are less, the direction of this line being also vertical, and the line of descent showing dicrotism. These characters denote quickness of the ventricular systole, feeble arterial tension, and want of uniformity as regards the quantity of blood sent into the aorta in different beats.

Pain is rarely a prominent symptom during the progress of mitral lesions, but uneasiness and an indefinite sense of distress, referred to the præcordia, may be complained of. Nor are there any notable symptoms referable to the nervous system. There is a marked contrast, as regards mental anxiety and apprehension, between the cases in which the action of the heart is disturbed by lesions and those in which the disturbance is purely functional. In the former, mental depression is proportionate to the physical suffering, and patients are apt to be apathetic with respect to danger; in the latter, the mind is depressed out of proportion to the actual ailment, and patients are harassed by imaginary fears. If dyspnoa be a prominent symptom, the loss of sleep adds to the sufferings of the patient. The sleep which is obtained is apt to be imperfect and disturbed by frightful dreams. It is not uncommon for patients to be unable to lie down for many weeks before death, short periods of sleep being obtained by inclining the body forward and resting the elbows on the knees or on some solid support.

Sooner or later, if life be not cut off by some intercurrent affection, dropsical effusion takes place into the areolar tissue and serous cavities. The lower limbs generally first become œdematous, afterward the face and boly, and more or less liquid accumulates within the peritoneal and pleural sac. The limbs and body sometimes become greatly swollen, and the scrotum acquires an enormous size. The skin on the lower limbs, under these circumstances, may become erythematous; ulcerations or fissures are apt to occur, with abundant draining away of serum. The face is congested, and not infrequently the lips are livid. The dusky hue, due to congestion or cyanosis, combined with œdema, gives to the face an appearance which contrasts with the pallid aspect characterizing general dropsy from renal disease. General dropsy arising from mitral lesions occurs after enlargement by dilatation of the right side of the heart has taken place, and is dependent mainly on the venous obstruction which the latter condition involves. Tricuspid regurgitation, which exists to some extent normally, is increased in this condition, and adds to the renous obstruction. This condition of the right cavities gives rise to turgescence 
of the cervical veins, especially when the patient is sitting or standing, and this turgescence is sometimes very great. Under these circumstances, pulsation of the superficial veins of the neck, occurring synehronously with the auricular or ventricular systole, or with both, is not uncommon. Jugular pulsation is, however, observed in some cases without venous turgeseence, and when dropsy has not taken place. Other circumstances than dilatation of the right side of the heart may contribute to the oceurrence of dropsy, for example, anæmia.

The organs composing the digestive system suffer from the congestion arising from dilatation of the right side of the heart. The appetite and digestion become impaired; enlargement of the liver is sometimes observed. Prior to the occurrence of dilatation, however, the appetite and digestion may be but little impaired. Nutrition may be but little affected even after the lesions have led to considerable suffering. Emaciation is not an early, nor, at any time, a marked effect of valvular lesions of the heart. 'The kidneys participate in the congestion, and the secretion of urine is apt to be scanty. Albumen in small quantity may be present in the urine, as an effect of renal congestion, without denoting degenerative disease of the kidneys. Hemorrhage from the stomach or intestines is an occasional effect of the congestion of the mucous membrane in these situations. Hemorrhoids and epistaxis belong in the same category.

A ortic lesions involving obstruction or regurgitation. usually give rise, as the first symptom, to inconvenience from the increased power of the hear't's action, the primary effect of these lesions being hypertrophy of the left ventricle. The patient complains of palpitation, especially on exercise or under the influence of mental emotions. The action of the heart is felt, by the hand over the pracordia, to be inordinately strong, and the dress or portions of the body may present visible movements with each ventricular systole. Pain is more apt to be present than in cases of mitral lesions, and, irrespective of the painful affection called angina pectoris, is not infrequently a prominent symptom. Cough, expectoration, dyspnon, and hæmoptysis, dependent on pulmonary congestion, and the accidents incident thereto, occur less frequently in connection with aortic than with mitral lesions. Pulmonary congestion, dependent on dilatation of the left auricle, does not occur until the left ventricle becomes sufficiently dilated to render the mitral valve insufficient, and the disease may end fatally without this result taking place.

If the lesions produce contraction, with but little or no regurgitation, the pulse is simply weakened, but not in a notable degree, even when the contraction is considerable. Under these circumstances, however, the pulse is frequently weak in proportion to the power of the heart's action; the force of the stroke felt by the finger over the artery is in striking contrast to the impulse felt by the hand over the pracordia. If the lesions impair the aortic valve so as to involve considerable regurgitation, the pulse is somewhat distinctive of this fact. The artery strikes against the finger with quickness, and appears instantly to recede; the stroke is not sustained, and the pulse is said to be jerking or collapsing, giving a sensation as if a "ball of blood were shot through the artery." This is due to the fact that, directly after the ventricular systole, the aorta is emptied by the regurgitant current, and this current and the lirect current come into collision when the ventricle contracts. Another symptom pertaining to the arteries, in some cases, is distinctive of free aortic regurgitation, viz., visible movements of the arterial truuks which are superficially situated, such as the carotid, temporal, subelavian, brachial, etc. The appearance caused by the movements of these vessels is some- 
times very striking, and is so distinctive that aortic regurgitation is rendered highly probable by this symptom alone.

The tracings by the sphygmograph in cases of aortic obstructive lesions give an oblique and curved line of ascent, denoting the slowness with which the blood is driven into the aorta ; and in the line of descent, dicrotism is either slight or wanting. In cases of aortic regurgitation, the line of ascent is vertical, and dicrotism in the line of descent is marked. If, as is not infrequent, senile induration of the arteries coexist, the summit presents a plane of greater or less length; if this condition of the arteries do not exist, the upper part of the diagram representing a pulsation is pointed, and the line of ascent may be notehed near its upper extremity.

General dropsy occur's in only a small proportion of cases of aortic lesions, that is, dropsy arising from the cardiae affection. 'This is owing to the fact that life is generally destroyed before the lesions have led to dilatation of the right cavities of the heart. Hence, turgescence of the cervical veins and jugulai pulsation do not belong to the clinical history of aortic lesions. 'This statement also holds good with respect to congestion of the abdowinal viscera and the kidneys.

Aortic regurgitant lesions are apt to give rise to symptoms proceeding from a sudden increase of the accumulation of blood within the left ventricle sufficiently to embarrass the action of this rentricle by distention. This ventricle becoming overloaded by the regurgitant current in addition to the direet mitral current, the ventricular walls are distended so as to weaken their power of contraction. This is a rational explanation of paroxysms of distress to which these lesions give rise. The action of the heart is irregular and spasmodic, and the patient experiences a sense of great oppression at the præcordia, with a feeling of impending death. These attacks are excited, at first, by muscular exercise or mental emotions, but, after a time, they may occur without any obvious exeiting cause. They occasion great distress, and sudden death may take place in an attack of unusual violence, the left ventricle being paralyzed by over-distension. They are more frequent and serious when dilatation of the left ventricle predominates over hypertrophy, yet they may be more or less frequent and violent, and may prove fatal, while hypertrophy is predominant. The occurrence of these attacks renders patients affected with aortic lesions more anxious and apprehensive than those affected with mitral lesions. As conducive to these paroxysms and the liability to sudden death, more or less importance belongs to defective circulation through the coronary arteries, which is incident to free aortic regurgitation. 'This defective circulation arises from the fact, as explained by Manriac, ${ }^{1}$ that the blood is forced into these arteries, not by the rentricular systole, but by the recoil of the aorta after the systole. In order that this recoil force shall affect the circulation in the coronary arteries, the aortic valves must be sulficient; if these valves be insufficient, the recoil foree is expended on the aortic regurgitant current, and the heart suffers from a defective supply of blood. The weakness of the ventricles from this cause, and, also, from venous congestion, due to the fact that, when the heart contracts feebly, the blood within the veins of the ventricles is imperfectly expelled, conduces to paralysis, from over-distension, of the left ventricle.

Aortic and mitral lesions, involving either obstruction or regurgitation, or both, in each situation, are not infrequently associated in the same case. Certain of the symptomatic phenomena arising from lesions at the

1 Nouveau Dictionnaire, Paris, 1868, Art. Cœur. 
mitral and aortic orifice are then combined. Mitral obstruction and regurgitation, however afford protection against the paroxysmis of distress and the liability to sudden death incident to aortic regurgitation, by preventing over-distension and paralysis of the left ventricle. In this point of view, the former are compensatory, or conservative.

Tricuspid and pulmonic lesions, as already stated, are comparatively rare. In the majority of the cases in which they exist, they are congenital. They are, therefore, most liable to be met with in young subjects. Tricuspicl lesions, in proportion as they involve obstruction or regurgitation, lead, primarily, to dilatation of the right auricle, and thence to systemic congestion. General dropsy, uncler these circumstances, is likely to occur early, without having been preceded by the symptoms denoting pulmonary congestion. Turgescence of the cervical veins and venous pulsation are also early symptoms in such cases. Lesions at the pulmonic orifice, involving contraction or regurgitation, in like manner, soon lead to dilatation of the right auricle and the symptomatic phenomena dependent thereon.

Patiological Cinaracter.-The primary evils of valuular lesions are mechanical. They do harm by obstructing the direct or normal currents of blood, and by allowing abnormal or regurgitant currents to take place. They induce hypertrophy or morbid growth of the walls of the heart, by increasing the force of the heart's action, and thereby inducing hypemutrition. This result, it is to be borne in mind, is not an evil. The hypertrophy resulting from valvular lesions is conservative. The angmented power of action which the heart acquires with its increased muscular growth enables it better to carry on the circulation despite the obstacles afforded by obstructive and regurgitant lesions. The lypertrophy is, to a certain extent, compensatory for the obstacles which these lesions afford. This fact has an important practical bearing on the management. Dilatation of the cavities is another result of valvular lesions. This result is an evil. The heart is weakened in proportion as it becomes dilated. The circulation then suffers, not only from the obstacles afforded by the valvular lesions, but from the diminished power of the lieart's action. As a rule, it is not until dilatation predominates over the pre-existing hypertrophy, that distressing and grave symptoms of disease of the heart are developed; and the suffering and danger increase as the dilatation progresses.

It is important to bear in mind that valvular lesions do not involve existing inflammation. The atrophy and calcareous degeneration which lead to contraction of the valves, rigidity, rupture, etc., are not inflammatory processes, although they may be, and, in a large proportion of cases, probably are, the after-effects of inflammation.

Causation.-In the majority of cases, valvular lesions originate in the endocarditis which complicates acute articular rheumatism. Of sixty-one cases which I analyzed, some years ago, with reference to this point, rheumatism had existed at a former period of life in forty-three. And of these forty-three cases, mitral lesions alone existed in twenty, aortic lesions alone existed in seven, and mitral with aortic lesions existed in sixteen. The degenerative changes which follow endocarditis lead to obstruction or regurgitation after a period more or less remote. When the evils or inconvenience resulting from valvular lesions are sufficient to lead the patient to seek for medical aid, it may be found that one or more attacks of rheumatism were experienced five, ten, fifteen, or twenty years before. 
And when cases first come under observation, more or less enlargement of the heart has already taken place. Enlargement, indeed, has probably existed for a long time, and it may not be until the heart becomes weakened by dilatation, that the patient applies to the physician. In some of the eases in which valvular lesions have not been preceded by rheumatism, their point of departure may have been in an endocarditis not rheumatic, for it is probable that non-rheumatic endocarditis oceurs not very infrequently when its existence is overlooked. Lesions arise from the atheromatous and calcareous deposit and atrophy of the valves, leading to their perforation and rupture. Moreover, rupture of the valves or tendinous chords sometimes results from the violence of the heart's action, without any previous structural change. When valvular lesions have not originated in rheumatic endocarditis, there is reason to believe they are often due to syphilis. My colleague, Prof. Janeway, one of the Curators of Bellevue Hospital, has been led to this conclusion by finding that syphilis enters into the previous history of a large proportion of the cases in which valvular lesions occur in those who have never had rheumatism. This statement applies especially to cases of valvular lesions occurring prior to middle or advanced age.

Enlargement of the heart, as alrearly stated, is an effect of the overaccumulation of blood in the cavities, in consequence of obstruction and regurgitation. And of the two forms of enlargement, lypertrophy and dilatation, the former precedes the latter.

Dragnosis.- The existence of valvular lesions can be determined with positiveness, only by means of auscultatory signs. By means of signs, the existence of lesions, their situation, and their character as regards being obstruetive or regurgitant, may generally be ascertained. Lesions situated at the different valves and orifices are represented by endocardial murmurs, and the presence of these is evidence of the existence and situation of the lesions. It will suffice here to enumerate the several organic murmurs produced within the heart, together witl the distinctive characters of each and its significance. They are naturally arranged into mitral, aortie, tricuspid, and pulmonie murmurs.

Mitral murmurs.-A murmur heard with the first sound of the heart, i.e., systolic, having its maxmum of intensity at, or near, the situation of the apex-beat, or perhaps limited to that situation, represents mitral lesions. These lesions may or may not inrolve insufficiency of the valve and consequent regurgitation. If a regurgitant current exist, the murmur is properly called a mitral regurgitant nurmur. But as a mere ronghness of the valve may give rise to a murmur with the charaeters just mentioned, without regurgitation, the name just given is not always strictly correct. A better name is mitral systolic murmur, with or without regurgitation. If the murmur be diffused beyond the apex, around the left lateral surface of the chest, and heard on the back near the lower angle of the scapula on the left and sometimes on the right side, mitral regurgitation may be inferred. If the murmur be heard only around the apex and over the body of the heart, it does not afford proof of regurgitation; it is then, simply, a mitral systolic murmur. A mitral systolic murmur, regurgitant or non-regurgitant, is not transmitted above the heart, and is not heard over the carotid arteries. This murmur may be soft (bellows-like) or rough, and sometimes it is musical.

A murmur heard after the second, and just before the first sound of the heart, i.e., presystolic, continuing up to the first sound and instantly arrested when this sound occurs, also represents, generally, mitral lesions. 
This murmur is heard at and near the apex, and is usually confined within a circumseribed area around the apex. It is almost always rough, resembling a sound produced by throwing the lips or tongue into vibration with the expired breath. This murmur is produced especially by contraction of the mitral orifice in consequence of the union, at their sides, of the mitral curtains, forming a buttonhole-like slit. The murmur represents mitral contraction witl occasional exceptions. In some cases in which free aortic regurgitation exists, the left ventricle becoming filled before the auricles contract, the mitral curtains are floated out, and the valve elosed when the mitral eurrent takes place, and, under these circumstances, this murmur may be produced by the current just named, although no mitral lesion exist.' 'This murmur is called the mitral direct murmur. Dr. Gairdner has proposed, as a better name, the auricular systolic murmur.

The two mitral murmurs not infrequently exist together; but either may exist without the other.

Aortic murmurs.-A murmur with the first sound, or a systolic murmur, heard with its maximum of intensity at or above the base of the heart, and generally propagated into the carotids, is called an aortic direct murmur. 'This murmur may represent aortic lesions, and it is then called organic, or it may be due to a morbid condition of the blood, without aortic lesions, and it is then called an inorganic or an anæmic murmur. The following circumstances render it probable that the murmur is inorganic or anemic. The evident existenee of anemia ; the absence of other signs of cardiac lesions; the variableness of the murmur, being sometimes present and sometimes wanting, and differing much at different times in intensity; the presence of murmur in the larger arteries, such as the caroticl, subclavian, etc., and a continuous murmur in the veins of the neck commonly known as the venous hum. Attention to these points will generally suffice for determining whether an aortic direct nurmur be organic or inorganic. The organic murmur denotes lesion of some kind, but not necessarily obstructive; that is, the lesion may simply render the membrane at or near the aortic orifice rough, without involving obstruction to the current. The murmur may be soft or rough, and it is sometimes musical.

An aortic murmur with the second sound of the heart, i.e., diastolic, heard usually at the base, but, in most cases, having its maximum a little below the base, on or near the left margin of the sternum, is called an aortic regurgitant murmur. It represents insufficiency of the aortic valve and consequent regurgitation from the aorta into the left ventricle. This murmur may be more or less loud and prolonged; it is generally soft, but sometimes rough, and it may be musical.

The aortic direct and aortic regurgitant murmur not infrequently exist in combination, but either may be present without the other. One or both of the aortic murmurs coexist with either or both of the mitral murmurs in the cases in which mitral and aortic lesions are associated.

Tricuspid murmurs.-Tricuspid lesions are comparatively rare, and hence a murmur referable to this orifice is infrequent. A trieuspid regur. gitant murmur occasionally exists, representing an unusual amount of regurgitation from the right ventricle to the right auricle, with, perhaps, hypertrophy of the ventricle. It occurs with the first sound, and is to be distinguished from a mitral regurgitant murmur by its being either

- Vide article on cardiac murmurs, by the Author, in the American Journal of the Medical Sciences, No. for July, 1862. 
limited to, or having its maximum of intensity at, the right inferior border of the heart, viz., near the ensiform cartilage. It is apt, without care, to be confounded with a mitral regurgitant murmur. A tricuspid direct murmur is exceedingly rare, owing to the fact that lesions analogous to those which give rise to a mitral direct murmur, are so infrequent at the tricuspid orifice.

Pulmonic murmurs.-A pulmonic direct murmur is not of very infrequent occurrence. It is with the first sound of the heart, and is heard at, or above, the base, over the pulmonary artery, viz., in the second intercostal space near the left margin of the sternum. It is to be distinguished from an aortie direct murmur by its situation as just stated, coupled with its nontransmission into the earotids. It represents pulmonic lesions, when it is not inorganic or anemic, as it not unfrequently is. The circumstances which render it probable that it is inorganic are the same as when the question is as to the organic or inorganic character of an aortic direct murmur.

By means of the foregoing murmurs, the diagnosis of valvular lesions is made, but it is important to bear in mind that neither the extent nor gravity of lesions is determinable by the murmurs. Whether the direct murmurs are produced by lesions which involve obstruction, or not, cannot be determined by the study of the murmurs alone; and whether the regurgitant murmurs proceed from much or little regurgitation, must be ascertained by other evidence than that which the murmurs afford. The intensity of the murmurs is no proof of the gravity of the lesions; slight or innoeuous lesions may give rise to loud murmurs, and grave lesions may be represented by feeble murmurs. Nor does the character of the sound as regards softness, roughmess, or a musical intonation, furnish any definite information respecting the extent or gravity of the lesions. With reference to this important point of investigation, therefore, evidence is to be sought for elsewhere. Synptoms relating to the cireulation, which have been considered under the. head of the clinical history, shed light upon this point, but, in addition, other physical signs are to be taken into account.

A comparison of the second sound of the heart as produced, separately, at the aortic and pulmonic orifice, is one source of information. This comparison is made by listening suceessively to the second sound as heard in the right and left intercostal space near the margin of the sternum, the sound in the right side emanating from the aortic, and the sound of the left side from the pulmonic orifice. If aortic lesions be indicated by the presence of the aortic direct or regurgitant murmur, and the lesions be of such a character that the aortic valve is impaired, the second sound, as produced by this valve, will be more or less weakened, or perhaps cxtinguished, and the alteration is ascertained by a comparison with the pulmonic second sound. If, on the contrary, the aortic sound be unimpaired, it may be inferred that the aortic valve is intact. A comparison of the aortic and pulmonic second sound is not less usefnl in the cases in which mitral lesions are shown to exist by the mitral direct or the mitral regurgitant murmur. If there be much obstruction or regurgitation, or both, at the mitral orifice, the aortic second sonnd will be weakened by the diminished amount of blood propelled into the aorta with each ventricular systole; and if the mitral obstruction or regurgitation, or both, have led to hypertrophy of the right ventricle, the pulmonic second sound will be intensified by the greater force with which the blood is propelled into the pulmonary artery by the systole of the right ventricle. By means of this beantiful 
application of auscultation, thus, useful information is obtained respecting the extent or gravity of the lesions, the existence and situation of which are revealed by the endocardial murmurs.

Further evidence of the extent or gravity of valvular lesions relates to enlargement of the heart. Enlargement of the heart is an effect of valvular lesions, and is proportionate to the amount and duration of the obstruction and regurgitation which the valvular lesions occasion. Hence, the degree of enlargement is a criterion of the extent and gravity of the valvular lesions, and, moreover, the enlargement is a lesion of importance, as has been alrealy seen. 'The question then arises, How are the existence and degree of enlargement of the lieart to be aseertained? The altered situation of the apex, if not attributable to extrinsic eauses, is evidence both of the existence and the degree of enlargement. The first eflect of enlargement is to carry the apex to the left of its normal situation within the linea mammalis. 'The next effect is to lower its sitnation to the sixth, seventh, or eighth intereostal space. In proportion as the apex is removed without the left nipple, and lowered, is the amount of enlargement. The situation of the apex is to be determined by the eye or touch, or if it can neither be seen nor felt, by finding the point where the first sound has its maximum of intensity. Other evidence of enlargement is obtained by percussion. The dulness within the area known as the superficial cardiac space, or the space in which the leart is uncovered of lung, is increased in degree and in extent, in proportion as the heart is enlargerl. The left border of the heart is found by perenssion to fall one, two, or three inches without the left nipple, the situation of the right border, and the base of the heart being, in most cases, but little changed.

Having determined the existence of enlargement and its extent, it remains to ascertain the kind of enlargement, that is, whether liypertrophy or dilatation predominate. The most important signs in this discrimination are furnished by palpation. If either the apex-beat, or additional impulses in the intercostal spaces above the apex, be felt by the hand to be abnormally strong, and especially if there be a heaving elevation of the precordia with the ventricular systole, hypertrophy predominates. 'The apex-beat, under these circumstances, may be weak, owing to the globular form which the heart may assume when enlarged; the augmented power of the heart's action is then to be appreciated by the impulses above the apex or by the prrecordiai heaving. On the other hand, if dilatation predominate, the cardiac impulses, if felt at all, are feeble. Auscultation also furnishes signs of importance in this discrimination. The first sound of the heart over the apex is loud, prolonged, and booming, in proportion as hypertrophy predominates; and, on the contrary, weak, short, and valvular if dilatation predominate. Attention to these points, in conneetion with the symptoms, will enable the pliysician to judge of the kind of enlargement to which the valvular lesions have given rise.

Prognosis.- Talvular lesions, not involving either obstruction or regurgitation, may remain for an indefinite period,innocuous. 'The physician should be careful not to attach undue importance to the presence of one or more of the organic murmurs. These are frequently discovered in examinations of the chest when patients complain of no symptoms referable to the heart, and in persons who suppose themselves to be in perfect health. If the lesions be accompanied by enlargement of the heart, obstruction or regurgitation, or both, may be inferred, and the lesions are not innocuous; yet, so long as the enlargement is exclusively or mainly hypertrophic, serious evils directly attributable to the cardiac lesions 
rarely occur. The patient, under these circumstances, as a rule, simply suffers mole or less inconvenience. The suffering and danger, as already stated, depend chiefly on the weakness arising from dilatation of one or more of the cavities of the heart. The progress of enlargement is generally slow, and it is not uncommon for patients affected with valvular lesions, together with more or less hypertrophy, to live many years, and even to old age.

The symptoms which denote danger, immediate or not remote, differ according to the seat of the valvular lesions. In connection with mitral obstruction and regurgitant lesions, habitual and considerable dyspnoa, if referable to the cardiac affection, that is, not dependent on a coexisting affection such as asthma, is evidence that the fatal termination is not very far distant. The supervention of gencral dropsy generally shows that the end is near at hand. Yet, sometimes, under judicious management, the dropsy diminishes or disappears, and life is prolonged for a considerable period. Death takes place suddenly in some cases in which the valvular lesions are exclusively mitral, but, as a rule, if life be not cut off by some intercurrent affection, the patient dies after a period of suffering more or less prolonged. In cases of aortic lesions involving obstruction and regurgitation, espeeially the latter, danger is indicated by the occurrence of paroxysms characterized by great præcordial distress, a sense of impending death, the action of the heart being irregular or tumultuous. Sudden death is liable to occur in paroxysms of this kind, the heart being paralyzed by over-distension of the left ventricle. Sudden death sometimes occurs in cases of aortic lesions when little or no habitual ineonvenience from the cardiac affection had been experienced, and the existence of any, affection of the heart was perhaps unknown. It is rare for lesions exclusirely aortic to lead to general dropsy. The immediate or proximate danger is to be determined by the amount of obstruction or regurgitation, and more especially the latter, in connection with the degree of enlargement, and particularly the enlargement by dilatation.

In cases of tricuspid and pulmonie lesions, general dropsy is an event denoting a degree of weakness of the right side of the heart from dilatation, which will be likely to lead, before long, to a fatal result.

In all cases of valvular lesions, associated with much enlargement, especially if the enlargement 'be by dilatation, the prognosis is unfavorable; the patient will die sooner or later with the cardiac affection, if life be not destroyed by some other disease, the occurrence of a fatal result being only a question of time, and the duration varying greatly in different cases.

Death may occur in consequence ofcertain local affections or aecidents incidental to the cardiac affection. Thus, pulmonary odema and pneumorrhagia, due to mitral lesions, may be the immediate cause of a fatal result. Extravasation of blood within the skull may be favored by dilatation of the right side of the heart, and perhaps by hypertrophy of the left ventricle. The loss of blood by hemorrhage from mucous surfaces may, in like manner, lead to a fatal termination. The effects of emboli derived from cavities of the heart are to be included in this category.

Treatment.-The treatment, in cases of valvular lesions, will have referenee, first, to the condition of the heart as respects these lesions and coexisting enlargement, and, second, to the symptoms or events which are incidental to the cardiae affection.

Valvular lesions, so long as they are either innocuous or have not led to enlargement of the heart, claim attention only with regard to prevent- 
ing or retarding their progress. And, for this end, the strain upon the valves occasioned by the excessive action of the heart, is, as far as possible, to be avoided. Very active muscular exertions, the excitement of the eirculation produced by the abuse of alcoholic stimulants, and violent mental emotions, will be likely to hasten or increase the damaging effects of the lesions. It is, however, by no means necessary to enjoin great restriction in the manner of living in these cases; it is sufficient that the habits of life are governed, in all respects, by prudence and noderation. It is not advisable, under these cireumstances, for persons to consider themselves as invalids. If, as is frequently the case, the existence of the lesions be discovered accidentally, or casually, it may not be always judicious to communicate the fact to the patient. If a medical opinion be not requested, and proper hygienic management can be secured without directing the patient's attention to the heart, much needless anxiety is sometimes spared. If, however, information be desired, it should not be withheld, but explanations made which will tend to prevent an over-estimate of immediate danger.

If cardiac symptoms have led the patient to apply to the physician, more or less enlargement will probably be found associated with the lesions. Let it be supposed that the enlargement which exists is mainly due to hypertrophy; it is not desirable to encleavor to diminish this lypertrophy. Inasmuch as whatever obstruction or regurgitation the lesions oceasion must continue, the abnormal growth of the walls of the heart is conservative; the comfort and safety of the patient depend upon it, and measures which tend to rreaken the heart will do positive harm. On the contrary, measures to maintain the muscular tone and vigor of the heart are indicated. 'The diet should be nutritious. The appetite and digestion, if impaired, should be improved by tonic remedies. Moderate open-air exercise is to be encouraged, very active exertions being, of course, interdicted. In short, the object is in every way to invigorate and strengthen the system.

If dilatation have taken place, the measures indicated are still those which tend to give, as far as possible, tone and vigor to the heart. The labor of the circulation may be in some degree diminished by restricting the quantity of liquids ingested, redundancy of the mass of blood being in this way prevented. Especial care, however, is to be taken not to impoverish the blood. The diet is to be highly nutritious, although as dry as compatible with comfort; tonic remedies are to be employed, and moderate exercise out-of-doors is still advisable. In proportion as the powers of the system are lowered, the heart is weakened, and dilatation favored. The object of management is, by strengthening and invigorating the system, to retard the progress of dilatation.

Thus far the treatment has reference to the condition of the heart. of the symptoms incidental to the cardiac affection, irregularity of the heart's action is one which may call for treatment. 'The appropriate remedies are those which regulate and tranquillize, without weakening, the action of the heart. Digitalis is a valuable remedy, appearing to relieve irregularity of the heart's aetion without diminishing, but, on the contrary, increasing the power of the ventricular contractions. 'The conclusion from $\mathrm{my}$ own experience accords with that of many observers of late years with respect to this remedy, viz., that, as regards its effect upon the heart, it may properly be called a cardiac tonic. 'The remedy is, therefore, particularly suited to cases in which the action of the heart is rendered irregular and feeble by dilatation, and it is not suited to cases in which the symptoms and signs denote hypertrophy. Cafeine, or the infusion of coflee, has 
measurably a similar effect. Hyoseyamus, belladonna, hydrocyanic acid, and other remedies of the same class, are useful. A belladonma plaster over the præcordia seems to have a good effect. 'The nauseant sedatives are not to be employed, and the veratrum viride is not appropriate.

Dyspnœa and other pulmonary symptoms are sources of annoyance and suffering in certain cases. So far as these symptoms are dependent on the cardiac affection, all that is to be expected from treatment is palliation. The ethers and mild revulsive applieations to the chest or dry cupping may mitigate the dyspnœa. Opiates to check expectoration will be likely to increase the distress instead of affording relief.

General dropsy, in eertain eases, is an event requiring treatment. Diuretic remedies should be first tried, and if these prove inoperative, hydragogue catharties are to be resorted to. Of these, elaterium is the most efficient. Given in small doses, this remerly may often be continued for a long period without producing depression. The bitartrate of potassa with jalap, and podophyllin are valuable hydragogues. . In the majority of cases only partial and temporary relief of the dropsy is procured, but in some cases it is completely removed, and it may not return for a considerable period. Diuretic remedies sometimes act very efficiently. They are more likely to effect the absorption of the effused liquid in cases of dropsy dependent on diseases of the heart than in eases of renal dropsy ; in the latter cases, the kidneys, being diseased, rarely respond to diureties. I have known the infusion of parsley-root to act with wonderful power as a diuretic remedy. If the anasarea be extremely great, so that the limbs become enormonsly distended, numerous punctures may be made with the point of a needle, the punctures being so slight as not to draw blood, and through them a large amount of liquid will sometimes escape, affording, for a time, marked relief. Vesications or cracks in the integument of the lower limbs, in some cases, oceur as a consequence of the distension, giving exit to an abundant flow of serum, with relief. Incisions or deep punctures are attended with a risk of being followed by gangrene. In the treatment of the dropsy, restriction of the amount of ingested liquid, as far as is compatible with comfort, is highly important.

$A$ highly important point in the treatment. of all cardiac lesions relates to coexisting anæmia. Anæmia is not infrequently associated with the lesions under consideration, and, as a consequence, functional disorder of the heart is superadded to the disturbance caused by the lesions. Irregular action of the heart, or excessive action, dyspnœa, and even dropsy, may be due to the superadded functional disorder. 'The error is not infrequently committed of attributing the symptoms in such cases exclusively to the lesions, and forming a prognosis more unfavorable than the lesions actually warrant. By removing the anæmia the symptoms are relieved, and the patient may even seem to recover completely from the cardiac affection. Anæmia existing in connection with valvular lesions claims appropriate treatment, viz., removal of the cause if it be apparent, for example, lactation, the employment of chalybeate tonies, with nutritious diet, and other hygienic measures. And such cases call for a certain amount of reserve in referring the symptomatic phenomena to the lesions. 


\section{CH A PTER I V.}

Enlargement of the Heart withont Valvular Lesions. Hypertroply. Dilatation. Atropiy of the Ileart. Fatly Degencration and Softening of the IIcart. Rupture of the IIeart. Functional Disorder of the Heart. Enlargement of the Thyrold Body and Iromineuce of the Eyeballs assoclated with Functional Disorder of the Heart. Angina I'ectoris. Thoracic Aneurisin.

$\mathrm{I}^{\mathrm{N}}$ the small proportion of cases in which enlargement of the heart exists without valvular lesions, hypertrophy and dilatation may be combined, either the one or the other predominating, as in cases in which enlargement proceeds from valvular lesions, or in certain eases, hypertrophy exists without dilatation, and dilatation without hypertiophy. The latter are distinguished as cases of simple hypertrophy and of simple dilatation. These cases do not claim separate consideration; it will suffice to notice, on the one hand, enlargement in which hypertrophy either exists alone or is predominant, and, on the other hand, enlargement in which dilatation predominates.

Hypertrophy is sometimes referable to an obstruction to the cireulation, either in the capillaries or at some point in the arterial system of vessels. Thus, hypertrophy of the left ventricle may result from contraction of the aorta and aortic aneurism. IIypertroply of the right ventricle is a consequence of obstruction in the pulmonary circuit in cases of resicular emphysema and chronic pleuritis. Hypertrophy of the left ventricle has been supposed to be sometimes due to obstruction to the capillary circulation, incident to certain eases of Bright's disease, and hence the rationale of its occasional association with the latter. In like manner the degenerative changes in the arteries which are liable to oecur in advancing age, and the enlarged area of the arterial branches occasioned by corpulency, are supposed to explain the occurrence of hypertrophy. In ail these instances the mechanism of the production of the hypertrophy is the same; it is due to long-continued, angmented power of the heart's action.

Mere functional disorder of the heart, under ordinary circumstances, very rarely leads to hypertrophy. Dr. J. M. Dacosta has observed some cases among soldiers during the late civil war, in which hypertrophy, occurring without valvular lesions, seemed to him to be attributable to continued functional disturbance in connection with heary marching, and especially in soldiers who had recently reeovered from fever.' I have known hypertrophy to become developed in connection. with excessive action of the heart during several years, there being no evidenee of valvular lesion, the patient being affected with exophthalmic goitre. Hypertrophy is sometimes found where it cannot be referred to any cause, and it is probably then either congenital or due to an intrinsic tendeney in the ventricular walls to an abnormal growth.

The abnormal growth in cases of hypertrophy without, as well as with, valvular lesions varies much in different eases. The walls of the left ventricle at its thickest part, in health, measure in the male about half

1 Vide Sanitary Memoirs of the War of the Rebellion, collected and pullished hy the United States Sanitary Commission, medical volume, edited by Austin Flint, M.D chap. x., p. 360. 
an inch, and in the female a fraction less. The walls of the right ventricle are about one-sixth of an inch in thickness at the base. A certain amount of increase is within the limits of health; but the left ventricle, in different cases of hypertrophy, is found to measure from one to two inches, and the right ventricle has a corresponding range of increase. A remarkable specimen of simple hypertrophy, not referable to any obvious cause, and supposed to have been congenital, was exhibited by me to the New York Pathological Society about two years ago. I was indebted for the specimen to Prof. Gilfillan, of the Long Island College Hospital. The left ventricle measured, at its thickest part, $1 \frac{5}{8}$ inch, the right ventricle $\frac{3}{8}$ inch. The cavities were not dilated, and the valves were found, by the water test, to be competent. The weight of the heart was $15 \frac{1}{2}$ ounces; the average normal weight in the male being 9 , and in the female 8 , ounces. The subject was a young man aged 23. Death occurred suddenly from congestive apoplexy, the existence of cardiac disease never having been suspected.

'The symptoms of hypertrophy are referable to the increased power of the heart's action. Hypertrophic enlargement of the left ventricle leads to an abnormal force of the current in the systemic arteries; the pulse is strong and full, the face flushed, active cerebral congestion is favored, and certain symptoms, such as pain, vertigo, tinnitus aurium, are attributable to the latter. 'The patient feels the powerful action of the heart, but he becomes aceustomed to it, and it may oceasion but little annoyance. As in the ease just referred to, a considerable amount of hypertrophy may exist withont symptoms which lead the patient, or others, to suspect the existence of any disease.

If the hypertrophy have been induced by an impediment to the circulation, it is conservative, as when it proceeds from valvular lesions, and is not, therefore, to be regarded as an evil. It may, however, lead or contribute to serions accidents. In the case just referred to, the congestive apoplexy, which proved suddenly fatal, was attributable to no other obvious cause, the organs of the body, with exception of the heart, being free from disease. It is intelligible that hypertrophy of the left ventricle, withont valvular lesions (these being, in a measure at least, protective), may favor cerebral hemorrhage in cases in which the arteries of the brain have become weakened from degenerative changes. In like manner, it is not improbable that hypertrophy of the right ventricle may sometimes contribute to pulmonary hemorrhage.

The diagnosis of hypertrophy must rest on physical signs, which are the same as when the hypertrophy is associated with valvular lesions. The fact of enlargement and its degree are determined by the altered situation of the apex-bent, together with the enlarged area of the superficial and the deep cardiac space. That the enlargement is due, either exclusively or mainly, to hypertrophy, is determined by the abnormal force of the impulses of the heart, and by the prolongation, intensity, and booming character of the first sound. ${ }^{2}$ The absence of endocardial murmurs is the basis of the conclusion that the enlargement is not connected with valvular lesions.

Hypertrophy, when conservative, or compensatory, of course does not claim treatment. If it be congenital, or there be sufficient grounds for considering it otherwise than conservative, measures to prevent further growth and to obviate accidents are called for. Bloodletting and other measures which impoverish the blood are not indicated. Anæmia, by

1 Vide preceding chapter. 
rendering the heart unduly excitable, involves additional inconvenience and danger. If plethora exist, it should be removel by dietetic means and depletion by saline laxatives. Excessive action of the heart may be moderated by tranquillizing remedies, such as aconite, hydrocyanic acid, and belladonna. In the cases of hypertrophy diseonnected from valvular lesions observed by Dacosta among soldiers, marked benefit was olotained by the use of aconite, one or two drops of the tincture being given twice or thrice daily, and continued, without any increase of the dose, for months. The diet should be suffieiently nutritious, but unstimulating. Alcoholic stimulants are to be interdicted. Liquids should be taken sparingly. Active muscular exercise and emotional excitement are to be avoided.

Dilatation of the cavities of the heart may follow hypertrophy without, as well as with, valvular lesions. If there be causes giving rise to hypertrophy, which continue after the morbid growth has reached its limit, dilatation of necessity follows, and, in time, will become predominant. But, in most eases, dilatation proceeds from weakness of the museular walls, arising from fatty degeneration or other causes. The museular walls may yield to distension, and dilatation take place when there is no abnormal impediment to the cireulation; and dilatation, however induced, tends progressively to increase.

The symptoms of dilatation are referable to weakness of the heart's action. This weakness is shown by feebleness of the pulse, coolness and congestive redness of 'the surface; and, if' the right cavities become considerably dilated, general dropsy ensues. Fatal syncope may occur from an over-accumulation of blood in the cavities in cases in which the organ is greatly enfeebled by dilatation. 'The physical signs show enlargement of the heart; and that the enlargement is due to predominant dilatation is shown by the feebleness of the cardiac impulses, and by the weakness, shortness, and valvular quality of the first sound as heard over the apex. The absence of valvular lesions is inferred from the absence of endocardial murmur.

Of the two lesions, hypertrophy and dilatation, the latter is by far the more serious. The indications for treatment are the same as when the dilatation is in association with valvular lesions, the object, in general terms, being to endeavor to improve the tone and vigor of the museular walls.

\section{ATROPIY OF THE HEART.}

Atroplyy of the heart calls only for a passing notice. Reference is had here to wasting of the museular walls, irrespective of degenerative eliange, represented, anatomically, by a diminution in weight and volume proportionate to the degree of atrophy. The heart climinishes in size in the progress of chronic diseases attender with great emaeiation, for example, tubereulosis. I have met with a speeimen, taken from the body of a female dead with chronic pleuritis, in which the weight was reduced to $4 \frac{1}{2} \mathrm{oz}$. Atrophy may result from the compression arising from the adhesions following periearditis, and from the abundant deposit of fat upon the exterior surface. Of course, the muscular power of the organ is diminished in proportion to the degree of atrophy. In a clinical point of view, this lesion is unimportant. As a general remark, it oceurs under circumstances which divest it of evil consequences, if, indeed, it be not a conservative provision. 


\section{FATTY DEGENERATION AND SOFTENING.}

Fatty degeneration is the deposit of oil-drops or granules of fat within the sarcolemma, and the substitution of these for the proper muscular tis: sue. The pathological character of the lesion has been considered in the first part of this work.' An abnormal accumulation upon the surface of the heart of adipose matter and its extension between the muscular fibres, constitute, not fatty degeneration, but fatty growth or obesity of the heart. The accumulation of fat exterior to the sarcolemma may embarrass the heart by its weiglit, and produce atrophy of the muscular structure by compression; and it may, or may not, be associated with fatty degeneration. Fatty degeneration, if considerable, produces marked changes in the gross characters. The muscular walls present a yellowish or fawn color; the striated or fibrous aspect is obscured; the organ is soft and flabby, and it is easily penetrated or broken down by pressure with the finger. But the microscope furnishes the most conclusive evidence, in the deficiency or absence of the transverse strix, and in the presence of oildrops or fatty granules in abundance within the sarcolemma. This degenerative change may be general or limited. It may be confined to one ventricle, oftener the left, or to circumseribed patches varying in situation, size, form, and number. 'The latter, if situated at the exterior, give to the surface a mottled appearance. It may affect the inner layers of fibres, the outer layer being unaffecterl. The papillary muscles may be particularly affected. Dilatation is often associated with fatty degeneration, the muscular walls either preserving their normal thickness, or the thickness somewhat increased, or again, the walls more or less abnormally thin. Fatty degeneration of the walls of the heart is not infrequently associated with valvular lesions.

In proportion to the degree of fatty degeneration, the heart is, of course, enfeebled, and the symptoms which make up the clinical history of the affection are due to a deficiency of power in the heart to carry on effectively the circulation. Degeneration of the left ventricle occasions feebleness of the pulse. The pulse is apt to be irregular. It has been observed to be notably slow, probably owing to many of the ventricular systoles being too feeble to propel the blood with sufficient momentum to be felt at the radial artery. Dyspnca, especially on exercise, is more or less prominent as a symptom, according to the feebleness with which the circulation through the pulmonary circuit takes place; this symptom is more marked when the right ventricle is affected. Paroxysms of syncope are liable to occur, accompanied by a sense of præcordial oppression and distress. These denote distension of the cavities from an accumulation of blood. Seizures resembling apoplexy, characterized by temporary loss of consciousness, withont paralysis, the surface being pallid and cool and the circulation extremely feeble, have been observed in persons who have subsequently died of this affection. These pseudo-apoplectic seizures, in some cases, have been of frequent recurrence.

Feebleness of the circulation, however, in an equal degree, may proceed from dilatation either with, or without, valvular lesions. The existence of fatty degeneration, therefore, cannot be ascertained positively by means of the symptoms alone. Other circumstances and physical signs are to be taken into account in order to arrive at a diagnosis. The signs which accompany this lesion are, feebleness or absence of cardiac impulse, weakness of the heart-sounds, more especially the first sound, this sound some- 
times suppressed over the apex, and, if heard, short and valvular like the second sound. These signs are equally present when the heart is considerably or greatly dilated, and they clenote fatty degeneration only when the organ is found not to be sufficiently enlarged to refer them to dilatation. 'I'ie age of the patient is to be considered. Fatty degeneration very rarely occurs in the male under fifty, and in the female under forty years of age. 'The evident tendency to fatty deposit is another diagnostic point. Fatty degeneration of the heart, it is true, occurs in lean subjects, but it is more apt to oceur in those prone to corpuleney. 'The presence of the arcus senilis possesses some diagnostic importance, although clinical observation has abundantly shown that this fatty change in the cornea occurs without degeneration of the heart and vice versâ. 'The presence of the arcus shows a constitutional tendency to fatty change, and it is significant if signs and symptoms referable to the leart point to degeneration of this organ. The habits of the patient are to be considered with reference to diagnosis. This lesion oceurs especially in persons of sedentary or indolent habits as regards muscular exertion, and addicted to the pleasures of the table. The habitual use of alcoholic liquors favors its oceurrence. Giving due attention to all the foregoing points, the existence of fatty degeneration may often be determined with much positiveness. If valvular lesions and dilatation be present, its existence is ascertained with less positiveness. Under these eircumstances, it is to be suspected when, in addition to the diagnostic points just named, the valvular lesions and enlargement appear to be inadequate to account for the feebleness of the heart's action.

To give rise to symptoms which point to the heart as the seat of disease, the amount of fatty degeneration must be considerable. A small or moderate amount may not oceasion sufficient weakness to give rise to any notable symptoms. Nore or less degenerative change is not infrequently found after death in cases in which it had not been suspected during life. Existing in a small or moderate amount, however, it may constitute an important element in various cliseases, rendering the system less able to resist them, and also less able to bear bloodletting and other therapeutical measures which tend to enfeeble the circulation. Existing in a considerable or great amount, it is a grave lesion. In such cases it involves a liability to sudden death. Death may take place from rupture of the ventricular walls. 'This occurs oftenest in the left ventricle near the apex. In the great majority of the cases of this accirlent, it arises from softening incident to fatty degeneration. Of 29 cases of sudden death, in persons affecterl with fatty degeneration, analyzed by Aitken, in 9 the immediate cause of death was rupture. 'The occurrence of sudden death, when rupture does not take place, is accounted for by over-accumulation of blood within the eavities, and consequent paralysis of the muscular walls from distension.

The objects of treatment, in cases of fatty degeneration, do not embrace recovery from the lesion. T'he change which has already taken place must continue. The lesion is irremediable. The objects of treatment are to prevent further progress of the degenerative change, and to develop the muscular vigor of the heart to the extent of its impaired capacity for improvement in this respect. The diet should be nutritious, consisting of animal food in as large proportion as is compatible with the appetite and digestion. Fatty food is to be interdicterl. Farinaceous and saccharine articles should form a small proportion of the diet. Liquids should be restricted as much as possible. Alcoholic stimulants should be taken in proper moderation. Moderate exercise out of doors is highly 
important; it should not be carried, of course, to the extent of overtasking the leart, but taken within the limits of comfort, that is, so as not to occasion hurried circulation, præcordial oppression, or dyspnœa.

'l'he liygienic is far more important than the medicinal management. Yet remedies may be highly useful. Tonic remedies are generally indicated. 'The appetite and digestion, if impaired, are to be improved as much as possible. If the patient be anæmic, this condition claims appropriate treatment. Coexisting disorders of any kind are to be relieved. The symptomatic effects of weakness of the heart will claim palliative measures. Attacks of syneope and pseudo-apoplexy require ethereal and alcoholie stimulants. Irregular action of the heart may be relieved by hydrocyanic acid and digitalis. The latter appears to act as a direct tonic upon the heart. Dyspnca, general dropsy, and other effects call for the same palliative measures as when they proceed from dilatation of the heart.

By judicious management, life and comfortable health may be maintained for a long period, notwithstanding the existence of this lesion in a considerable amount.

Softening of the muscular walls of the heart, without fatty or any degenerative cliange, has been found to occur in the course of typhus and typhoid fever, the eruptive fevers, pyamia, scorbutus, and other affections. Attention has been directed to its occurrence in typhus and typhoid fever, especially by Louis and Stokes. It may explain the weakness of the circulation disproportionately to the adynamia or general debility in certain cases of these and other diseases. The symptoms and signs are those which represent notable feebleness of the heart's action. As regards the signs, diminished intensity of both somnds, but more especially the first sound, characterizes the feebleness from this as well as other lesions. But a point which is distinetive of softening in the continued fevers, according to Stokes, is derived from the fact that the softening is ehiefly of the left ventricle. The first sound, as heard over the apex, is less intense than orer the right border of the heart, the sound representing, in the former situation, the force of the systole of the left, and, in the latter situation, of the right ventricle.

Softening incidental to the affections named is due to the disturbance of nutrition which these affections involve. Doubtless, it adds to the danger, but it is not a permanent lesion, the normal condition of the heart being regained if the patient recover from the affection to which the softening is incidental. The existence of softening contra-indicates depletory or debilitating measures of treatment, and, on the contrary, furnishes a reason for supporting treatment, in addition to the indications derived from the condition of the system under which the softening occurs. Without due attention the condition of the heart arising from decomposition after death may be attributed to morbid softening. This error is to be avoided by observing the odor of putrefaction, and by comparing the condition of the heart with that of other organs.

Lesions of the walls of the heart, extremely rare, are due to fibroid formations, extravasation of blood, carcinomatous disease, tuberculous deposit, and acephalocysts. Aneurism of the ventricular walls is another rare lesion. Happily, these belong among the curiosities of clinical experience. They are, of course, grave, but the symptoms and signs to which they give rise are not distinctive; they cannot be made out during life, and hence, their consideration in this work would be ont of place. Important lesions of the walls and valves are embraced under the head of 
congenital malformations, and connected with these is the affection known as morbus cæruleus, the blue disease, or cyanosis. This affection is to be included among the diseases of infantile life, and is treated of fully in works devoted to these diseases, as well as in treatises on the diseases of the heart. 'To the latter, and to works on morbid anatomy, the reader is referred for information concerning congenital malformations of the heart.

\section{RUPTURE OF THE HEART.}

As already stated (vide page 351), this accident is generally incident to fatty degeneration. It occurs, however, as an effect of eardiac aneurism, or myocardiac abscess. It very rarely happens in persons under 70 years of age. Usually it causes instant or speedy death; but, if the perforation be small, it may be temporarily plugged by a elot, and life continue for hours and even days. I have known death to be delayed for six hours after the symptoms denoted the occurrence of the rupture. The symptoms are those which sudden compression of the heart by the accumulation of blood in the perieardium would be expected to produce, together with the loss of blood. In most eases, however, there is no time, before death, for the observation of symptoms. Rupture has been repeatedly observed to occur in connection with a paroxysm of precordial pains resembling angina pectoris.'

\section{FUNCTIONAL DISORDER.}

By the term functional disorder is meant disturbed action of the heart, not dependent on either inflammation or structural lesions. The beating of the heart is sometimes morbidly intense. 'The patient is conscions of its violent action, as when it is temporarily excited by fear or some other powerful mental emotion. This is commonly known as palpitation. The sense of violence may be a subjective symptom only, the force of the heart's action not being actually increased. Generally the action is, at the same time, accelerated and irregular. The irregularity is represented by the pulse, and felt when the hand is placed over the pracordia. The patient is also painfully conscious of it; the organ appears to perform a rolling or tumbling movement, and a sensation is sometimes described as if the heart were in the throat. Intermittency of the heart's action is another feature in certain cases. The action of the heart is, for an instant, arrested; one, two, or three beats are lost, and generally irregularity of action precedes and follows the intermissions. Of these the patient is vividly sensible, and the feeling is that fatal suspension of the licart's action may be liable to take place. These rhythmical aberrations may be accompanied with either violent or feeble action of the heart. The different varieties of functional disorder occur in paroxysms which are very variable as regards severity, frequency of occurrence, and duration. Excessive action of the heart, however, sometimes persists steadily for successive days, weeks, and montlis. An oceasional form of disorder is characterized by every alternate ventricular systole being so feeble as not to be appreciable by a radial pulse, the action of the heart being more or less rapid. On auscultation the two heart-sounds are alternately loud and feeble. I have known this form of disorder to persist for several weeks.

Paroxysmal disorder is quite common. It usually gives rise to great

1 For an analysis of 24 cases of spontaneous rupture of the heart, by Barth, vide Archives Générale de Médecine, Paris, 1871. 
apprehension. The patient has a firm conviction of the existence of organic disease, and is in fear of sudden death. It is sometimes difficult to remove this conviction by the most positive assurances to the contrary. Cases of functional disorder are characterized, as a rule, by mucl mental anxiety and depression, in this respect differing from cases in which disturbed action is dne to structural lesions.

The causes of functional disorder are varions. It is sometimes attributable to an overplus of red corpuscles of the blood or pletlora. As incident to this condition, it occurs in persons addicted to the pleasures of the table, digesting and assimilating actively, and taking bnt little active exercise. It is much oftener, however, associated with the opposite condition, viz., anæmia. Anæmic persons are rarely exempt from more or less disturbance of the heart's action. It may be prodnced by longcontinued mental anxiety or depression. It is apt to occur in persons of an anxions, worrying disposition, and in those who are constitutionally disposed to melancholy. The effect which it produces on the mind reacts on the disorder, and tends to perpetuate it. It appears, in some cases, to be dependent on indigestion or dyspepsia, and paroxysms are apt to be excited by dietetic errors. The use of tobacco gives rise to it in some persons, so, also, strong tea, or coffee. It is incidental to the gouty diathesis. The exhaustion following prolonged mental excitement and physical exertions may be accompanied by it. It prevailed to a great extent among soldiers during the late civil war, excessive and persistent palpitation often leading to discharge on the ground of disability from supposed organic disease of the heart. Excessive venery and habits of self-abuse are to be included among the causes. The associated symptoms will, of course, vary according to the different circumstances under which it occurs.

It is extremely desirable, in view of the comfort and welfare of the patient, to deternine with positiveness, in cases of functional disorder, that structural lesions do not exist. Several points, connected with the history and symptoms, have a bearing on the diagnosis. The occurrence of the disturbance in paroxysms, the action at other times being regular; the paroxysms occurring at night rather than in the daytime, and frequently not being occasioned by any obvious cause, such as muscular exertion or mental excitement; the ability of the patient to take active exercise without palpitation or dyspnœea when not suffering from the disorder, and the intensity of mental anxiety and apprehension, are points which render it probable that the difficulty is purely functional. These points, however, are not conclusive. A positive diagnosis is to be based on the exclusion of lesions of structure by the absence of the plysical signs of the latter. If, on a careful examination of the chest, the heart be not found to be enlarged; if there be no murmur present, or if an existing murmur be inorganic, and the heart-sounds be normal, the affection may be confidently pronounced functional. Without the negative proof . afforded by physical exploration, the mind of the practitioner must be in doubt as to the diagnosis. If he give a decided opinion, it is a guess which may prove to be either right or wrong. If he avoid giving a decided opinion, the inference which the patient usually draws is, that organic disease exists, and that the physician is reluctant to tell him the truth. I could cite, from the cases which have come under my observation, not a few in which patients were for many years rendered unhappy and deterred from engaging in the active duties of life, by either an erroneous medical opinion that they had organic disease of the heart, or by a fixed belief that such was the fact, based on the indecision of their physicians. 
The curative treatment of functional disorder must have reference to the circumstances with which it is connected. Occurring in connection with plethora, the measures indicated are, restriction of diet, change of habits as regards exercise, depletion by saline laxatives and perhaps by small bloodlettings. Occurring in eonnection with anæmia, measures of precisely an opposite character are indicated, viz., those designed to restore the normal condition of the blood. An investigation with reference to the source of the disorder is to be made in all cases, and the causes, if apparent, are to be obviated. If connected with dyspeptic ailments, these are to be remedied by appropriate treatment; and so if dependent on the gonty diathesis, this will claim suitable remedies. In the great majority of cases, the proper treatment consists of tonie remedies, a nutritious, regular diet, regularity of habits as regards excesses of all kinds and a proper amount of sleep, the interdiction of tobacco, strong coffee and tea, and out-door exercise with mental recreation; in short, an invigorating system of hygiene. Positive assurances of the absence of organic disease, such as may be given if based on the absence of all the physical signs of lesions, will often go far toward effecting a cure, the disorder being frequently kept up by mental inquietude occasioned by the fear of sudden death.

During the paroxysms palliative measures are called for. These consist of ethereal stimulants, anti-spasmodics, and occasionally opiates, with sinapisms or stimulating liniments to the chest. If paroxysms occur frequently, a belladomna plaster may be constantly worn over the præcordia. Blisters or other severe counter-irritants are never requisite. Small doses of aconite are useful if the action of the heart be violent, and, on the other hand, if the action be feeble and irregular, digitalis may prove of use.

Some persons are strongly predisposed to disturbed action of the heart, and suffer, more or less, from functional disorder for many years, or during their whole lives. The mental anxiety in such cases after a time ceases, and patients become reconciled to this as to other physical evils. The frequent recurrence of functional disorder for an indefinite period does not lead to the development of organic disease. The physician is fully warranted in giving positive assurance to the patients on this point.

In the foregoing remarks on functional disorder, it has been assumed that structural lesions are not present. But functional disorder and lesions may be associated when the former is not dependent on the latter; in other words, the canses which give rise to functional disorder when the heart is free from lesions will produce the same effect when cardiac lesions exist. It is highly important for the practitioner to appreciate this fact. Not infrequently the disturbance of the heart's action, in cases in which organic murmurs are found, is mainly or exclusively functional. It is not uncommon for practitioners to err in considering all the symptoms referable to the heart as arising from organic lesions if these exist in/ever so small an amount. Anæmia, coexisting with trivial lesions, may occasion greatly disturbed action of the heart, with dyspnœa and even general dropsy, so that the patient may seem to be in the last stage of cardiac disease, and, if the anæmia be cured, the recovery appears to be complete. It should always, therefore, be a question, in cases of organic lesions, whether more or less of the symptomatic phenomena may not be due to functional disorder arising from anæmia, deranged digestion, or a morbid condition of the nervous system produced by tobacco, coffee or tea, mental depression, excessive venery, or gout. This question is to be decided in the affirmative when causes of functional disorder are discoverable, and 
when the amount of the organic affection, as determined by the physical signs, appears to be insufficient to account for the symptomatic phenomena. In proportion as the latter are fairly attributable to functional disorder, the prognosis is, of course, less grave, and improvement may be expected from appropriate treatment.

\section{ENLARGEMENT OF THE THYROID BODY AND PROMI- NENCE OF' THE EYEBALLS (EXOPHTHALMIC GOITRE) ASSOCIATED WITH FUNCTIONAL DISORDER OF THE HEART}

Within the last few years, the attention of clinical observers has been directed to an affection characterized by enlargement of the thyroid body and prominence of the eyeballs, conjoined with inordinate action of the heart. These three pathological events form a striking combination, giving to the affection a well-marked individuality. In the absence of a name expressive of its eharacter, a German author, Hirsh, applied to it the name Basedow's disease, after a German observer, who was among the first to describe it fully; and the distinguished clinical teaeher, Trousseau, in view of the prior claim of the late Dr. Graves, proposed to call it Graves' disease. The three erents just named evidently have some pathological connection, the nature of which is not yet fully understood.

The prominence of the eyes is the most remarkable of the triple events. Existing, in some eases, in a marked degree, it gives to the countenanee a peculiar expression. The projection of the globes displays more or less of the tuniea albuginea, and the patient has a ferocious, staring look which, existing in both eyes, is at once diagnostic. The projeetion is sometimes so great as to prevent closure of the lids, and the eyeballs are partially exposed during sleep. Vision is generally not impaired, and the appearance of the eyes, aside from the prominence, is natural. The condition of the eyes does not involve pain, but the patient sometimes feels as if the eyeballs were being squeezed out of the soekets. The tliyroid body is more or less enlarged, the enlargement being usually greater on the right side. The increase does not go on indefinitely, forming the immense tumors which are sometimes seen in eases of ordinary bronchocele or goitre, but eeases after a moderate or considerable angmentation of bulk has been attained. The swelling does not give rise to pain, but it may occasion some obstruetion to respiration from pressure on the trachea, and may lead to embarrassed breathing and modification of the roice by interfering with the funetion of the recurrent laryngeal nerve. The thyroid arteries are enlarged, and a strong pulsation is felt over these and the carotids, frequently accompanied by marked thrill. Arterial and venous murmurs, frequently loud and sometimes musical, are heard when the stethoscope is placed over and near the enlarged thyroid body.

A morbidly increased activity of the heart is a constant and notable feature. In some cases this amounts to a persisting palpitation. This functional activity is increased in paroxysms. The beats are morbidly accelerated, the pulse varying from 100 to 140 beats per minute. Valvular lesions and enlargement of the heart exist in some, but not in all, cases. When they are present they do not account for the functional disorder. Loud inorganie murmurs at the arterial orifices of the heart are not infrequent. The inordinate action of the heart may continue persistingly for months and even years.

More or less anæmia coexists generally, but not invariably. This has been by some considered as an essential pathological element of the affec- 
tion. The anæmia is usually marked. Other symptoms which have been noted are, mental depression and irritability, inability to sleep, amenorrhœa, abnormal increase of appetite followed by anorexia, sometimes hysterical phenomena, and emaciation. The affection is essentially chronic. In the majority of cases it continues for months and years. It does not seem to tend intrinsically to a fatal result, but patients are apt to be eut off during its eontinuance by some intereurrent affeetion. Recovery takes place in a certain proportion of cases, the improvement being slow, and some degree of enlargement of the thyroid body and prominence of the eyes remaining permanently.

The affection is rare, and, sinee the attention of clinical observers has been directed to it only within the few past years, further materials for its clinieal history are yet to be gathered. I have notes of only seven cases. In three of these the patients were only temporarily under my observation. Of the four remaining cases the facts relating to the progress and termination were briefly these.' In one case, that of a girl 16 years of age, the affeetion, although distinet, was slight as regards the enlargement of the thyroid bodies and the prominence of the eyes. It nearly disappeared, and she was married at the age of 22 . She died three years after her marriage, having been reeently eonfined. The only faets learned respecting her last illness were that she was dropsical; that there was difficulty of breathing, and that she was said to have disease of the heart. In another case the patient was under observation, from time to time, at the Bellevue and the Charity Hospitals, during eight years. At first there was no evidence of organic disease of the heart. Subsequently, considerable hypertrophy became developed. The prominence of the eyes and the enlargement of the thyroid body became much less than at first, but she very gradually declined in strength, became greatly emaciated, and died by exhaustion. 'The heart weighed $20 \mathrm{oz}$., and there was no valvular lesion. The lungs were emphysematous, and the kidneys presented fibroid degeneration. In a third ease the patient, a female aged 29, was under my observation for three years. 'The prominence of the eyes, which was very marked, continued, but the enlargement of the thyroid bodies had disappeared. The pulse was 140 . 'There was no material change during the time she was under my observation. In the fourth ease, a female aged about 40, the affection had existed for ten years, and she had notably improved as regards the prominence of the eyes, the thyroid enlargement, and the rapidity of the pulse. She came under my observation in an attack of pneumonia which proved fatal apparently from heart-clot.

The pathological character of the affection is not established. That the three pathological events are due exelusively to anæmia is hardly probable, since anæmia is sufficiently common without these events, and, moreover, there is not always coexisting anæmia. A ceording to 'Trousseau, the anæmia in some cases does not oecur until after the development of the affection. Trousseau and others have considered it as one of the functional affections of the nervous system or the neuroses. The enlargement of the thyroid body seems to be due to abnormal vascularity and hypernutrition. The prominence of the eyes is not due to enlargement of the globes. In some of the few autopsical examinations which have been reported, an abnormal amount of areolar and adipose tissue has been found at the bottom of the orbit. But that the protrusion is not always due to this cause is shown by the fact that it has been observed to cease

I For a fuller account of three of these cases, vide my work on the Diseases of the Heart, second edition, 1869. 
after death; and during life, in certain cases, moderate pressure suffices to restore the globes to their normal situation. It has been attributed to a relaxed condition of the recti muscles, and to congestion of the vessels behind the eyeballs. The prominence of the eyes and enlargement of the thyroid body are consecutive to the cardiac disorder. ${ }^{1}$ Cases are observed in which enlargement of the thyroid body is conjoined with inordinate functional activity of the heart, without prominence of the eyes, and 'Trousseau cites a case in which the two latter' were associated without enlargement of the thyroid body. An example of the latter has fallen under my observation. In my case the prominence of the eyes nearly disappeared in a year after the patient first consulted me. Mitral stenosis existed in this case. The affection, in the great majority of cases, occurs in females. Of fifty cases collected by Wilthuisen, a Danish author, only eight were male subjects. ${ }^{2}$ Of my seven cases, in all, save one, the patients were females.

The indications for treatment relate to the functional disorder of the heart, together with the morbid state of the nervous system and the coexisting anæmia. Sedative remedies which tranquillize the action of the heart without producing depression or interfering with the appetite and digestion, are indicated. For this end, hydrocyanic acid, aconite, belladomna, and digitalis may. be employed. The anæmia calls for tonic remedies, and especially ferruginous tonies; the latter have seemed to be beneficial in the cases which I have observed. Arsenic has been found useful. Iodine, with reference to the affection of the thyroid body, is of doubtful utility. Hygienic measures are probably of greater importance than remedies. Freedom from mental depression is to be secured as far as possible, and, whenever practicable, change of scene and other means of mental recreation are advisable. The diet should be nutritious, and ontdoor life is desirable. Trousseau advocates the employment of cold water after the hydropathic method.

\section{ANGINA PECTORIS.}

The affection known as angina pectoris is essentially neuralgic, and might, therefore, be included among the diseases of the nervous system. It is considered here on account of its frequent connection with cardiac lesions and with notable disorder of the heart's action. In the previous editions of this work a distinction was made between true angina and pseudo-angina, the former being the neuralgic affection associated with cardiac lesions, and the latter a neuralgia occurring independently of any disease of the heart. This distinction, although based on a point of difference of great practical importance, seems to me pathologically incorrect, and, therefore, in the present edition I shall cease to use the term pseudo-angina, embracing this purely neuralgic affection under the name angina pectoris, and expressing the distinction by saying angina with, and angina withont, cardiac clisease. The following description will relate to typical cases in which the angina is associated with discase of the heart.

The affection is characterized by pain, usually intense, burning, tearing, or lanciuating in character, emanating from the præcordia or beneath the sternum, and radiating thence in different directions, viz., into both sides

1 For the facts bearing on the theory that this affection depends on a lesion (hypertrophy of connective tissue and atrophy of nerrous sulstance) of the lower coni. cal ganglia of the sympathetic nerve, vide prize essay on the ganglionic nervous system, by Robert T. Edes, M.D. Trins. N. Y. Acad. of MIed., 1869.

2 'Troussean, Clinique Médicale, tome ii. 
of the chest, the back, and frequently the left shoulder; extending down the arm and sometimes to the forearm; occasionally felt in the forearm and hand and not in the shoulder and arm; in some eases extending more or less into both upper extremities, and it has been known to extend into one and botls of the lower extremities; and, finally, it may shoot upward into the neck, temples, and jaws. Different cases differ much as regards the intensity of the pain and the situations, among those just named, in which it is felt. The pain in some cases is so intense as to be truly excruciating, and is accompanied by a feeling of constriction of the chest; in other eases it is moderate, or even comparatively slight.

The affection is paroxysmal, and the pain is but one of the elements which enter into the paroxysm. The action of the heart is generally more or less disturbed. The beats are irregular, sometimes violeut or tumultuous, and sometimes feeble. In some cases the pulse is notably slow. An indeseribable anguish or "heart-pang" is another element. A vivid sense of impending death is felt. 'T'he paroxysm is usually developed snddenly, and the patient is compelled instantly to remain perfectly still; he is apprehensive of making the slightest movement, and seizes hold of some firn support in order to maintain a fixed position. IIe is afraid to breatle freely, although the power of doing so is not lost. Dyspnoea does not belong to the paroxysms save in so far as it may depend on existing cardiac lesions, and is due to voluntary restraint of the respiratory movements. He is perhaps afraid to speak. A sense ot numbuess is felt in the parts into which the pain extends. The comntenance denotes anxiety and suffering. The surface is generally pallid, cool or cold, frequently bathed in perspiration, and sometimes cyamosed or livid.

Cases differ greatly as regards, not only the severity of the paroxysms, but their duration and the frequency of their recurrence. They usually last for a few moments only, but in some cases they continue for several hours. They may recur at wide intervals or frequently. $\Lambda$ s a rule, to which there are a few exeeptions, if a paroxysm have been once experienced, other paroxysms will follow sooner or later. Generally they become progressively more and more frequent, if the life of the patient continue. At first, and for some time, they appear to be excited by some notable cause, such as unusual muscular exertion or a powerful mental emotion, but, after a time, they are liable to ocenr from trivial causes and without any apparent cause. They are sometimes produced during sleep apparently by distressing dreams.

The affection, as thus described, is incidental generally to organic lisease of the heart or aorta, but, so far as at present known, not with any one form of lesion. It is much oftener associated with lesions at the aortic than at the mitral orifice, yet it oceurs in only a very small proportion of the cuses of aortic lesions. It oceurs in some cases of fatty degeneration of the heart without any valvular lesions. It is sometimes associated with aneurism of the aorta. It has been observed in cases in which the coronary arteries are obstructed by calcification or other lesions, but this is not essential to its occurrence, as was at one time supposed. It is an affection of rare oceurrence. Of over 150 cases of organic disease of the heart which I analyzed in 1859, it occurred in only 7 ; and it occurred in 8 ont of 189 additional cases analyzed in 1869. That it has a pathological connection with organic disease of the heart is certain, but upon what particular condition or circumstance common to different forms of organic disease it depends is not aseertained. This statement applies more particularly to the pain or neuralgic element of the paroxysms. The other elements may perhaps be satisfactorily explained. 
Angina pectoris, when associated with diseases of the heart, involves liability to sudden death. Death may take place suddenly, in a paroxysm. The most reasonable way of accounting for the sudden death, as it seems to me, is to attribute it to over-accumulation of blood within the ventricular cavities, and arrest of the heart's action as a result of paralysis from distension. The explanation, in fact, is the same as of sudden death in cases of free aortic regurgitation, and fatty degeneration. The liability to sudden death, if this be the correct explanation, charaeterizes cases in which the coexisting organic disease is of a kind to favor sudden over-accumulation of blood in the cavities. Aortic lesions and fatty degeneration have this effect. If the lesions do not lead to this effect, patients rarely die suddenly, but linger on for an indefinite period with the recurrence of the paroxysms more or less frequently. Moreover, the overloading of the cavities of the heart will account for elements of the paroxysms other than the pain, viz., the disturbed action of the heart, the indescribable anguish, and the sense of impending death. If the lesions to which the angina is incidental be not of a character to lead to overloading and consequent distension of the ventricular walls, the suffering is mainly from the pain. Some cases of angina are of this description, very little disturbance of the heart's action accompanying the paroxysm. On the other hand, all the elements of a paroxysm of angina, except the pain, are observed, not infrequently, in cases of acrtic lesions with regurgitation; and sudden death is liable to occur in the paroxysms of distress to which patients affected with these lesions are subject, the paroxysm lacking only pain to constitute angina pectoris. The prognosis in cases of angina is always grave if physical signs show the existence of serious eardiac lesions ; and sudden death is to be expected if there be much aortic regurgitation, or the habitual weakness of the heart render probable fatty degeneration or impaired nutrition of the organ. The danger, it is to be observed, is not to be measured by the intensity of the pain : it is not the pain which kills, but the coexisting organic disease.

Angina pectoris, in so far as the neuralgic element is concerned, undoubtedly occurs without any evidence of coexisting disease of the heart or the aorta. Several examples have fallen under my observation. In these cases the paroxysms generally lack those elements which are derived from cardiac lesions. The necessity of keeping a fixed posture, while the paroxysm lasts, is not felt in the same degree; the patient may writhe with pain, and try frequent changes of posture with the hope of obtaining relief. The sense of impending death is less, or wanting. There is little danger of sudden death, and the prognosis, as regards recovery, is favorable. These points are to be considered in the differentiation of these cases from those in which the angina is connected with disease of the heart; but the absence of cardiac lesions is determined by the negative result of a careful physical exploration of the chest.

1 For a fuller consideration of angina pectoris, see my work on Diseases of the Heart, second edition, 1869. The knowledge of the functions of the pneumogastric nerve, acquired by means of late experimental researches, explains the disturbance of the heart's action in certain cases of angina. The effect is analogous to that of galvanism transmitted through this nerve. Through this effect sudden death may take place irrespective of cardiac lesions. This statement is not in accordance with what was stated in the previous editions of this work. But $I$ have met a case in which sudden death occurred in a paroxysm, and cardiac lesions sufficient to account for the death were not found on post-nortem examination. The danger of death during a paroxysm may be estimated by symptoms denoting the degrec of the disturbance of the heart's action, namely, by the slowness, feebleness, and irregularity of the pulse, taken in connection with the feeling of impend- 
The treatment embraces measures to afford relief in the paroxysm, and those indicated in the intervals. The palliative measures embrace opium, given promptly and sufficiently to relieve the pain. It should be given in a form to act as speedily as possible. The hypodermic method, if the physician happen to be at hand, is to be preferred. Alcoholic, ethereal, and other stimulants are indieated in proportion as the action of the heart is irregular and feeble. Revulsive applications to the chest and extremities are appropriate. Swallowing pieces of ice has been fomd effective. Recently the nitrite of amyl has been introduced as a remedy for arresting the paroxysms. Cases have been reported by trustworthy observers in which it lias proved very promptly and completely efficacious. It is to be given by inhalation. Great cantion is requisite in its use. Five drops may be inhaled from a handkerchief held over the nostrils. In two eases under my observation the relief has been immediate and complete whenever a paroxysm has occurred. It produces a sense of fulness in the head, with flushing of the face, increased frequency of the heart's action, and a sense of general relaxation. 'These effects are of transient duration. The remerly may be repeated at intervals during the same day, should there be recurrence of the paroxysms.'

The inhalation of ether or of chloroform is to be enumerated among the resources for arresting the pain, or mitigating its severity. The inhalation of chloroform should not be resorted to if the action of the heart be feeble. In the intervals, the first point is to avoid, as far as possible, everything which will be likely to provoke a paroxysm, such as active exercise, mental excitement, over-ingestion of food, and the abuse of alcohol. Aside from prophylaxis, the measures indicated are those which have reference to the causes of neuralgia, to the general health, and, in the majority of cases, to coexisting organic clisease of the heart or aorta. Anstie has found arsenic, in small doses long continued, extremely useful in preventing the paroxysms." 'Trousseau, who regarded the affection as analogous to epileptic seizures, and called it cardiac epilepsy, advised the long-continued employment of belladomna. Electrization of the surface within the pracordia has been found useful both in arresting the paroxysms and postponing their occurrence.

\section{THORACIC ANEURISM.}

Referring the reader to works which treat especially of cliseases of the heart and aorta, and to surgical treatises, for an account of the different varieties of aneurismal tumors, of the modes in which they are produced, etc., I shall simply enumerate the diagnostic symptoms and signs of aneurism of the thoracic aorta, with the prognosis and treatment, limiting the term aneurism to a circumscribed dilatation of the vessel, forming a tumor of greater or less size. Aneurismal tumors may spring from the thoracic aorta at different points. Not infrequently they originate within the pericardium, in the sinuses of Valsalva, and, in this situation, rupture takes place before the tumor attains to great size, the hemorrhage taking place into the pericardial sac, and, of course, causing instant death. A fatal

ing danger. These symptoms, of course, denote a far greater amount of danger when cardiac lesions coexist which in themselves involve more or less danger of sudden death, namely, free nortic regulation, with dilatation of the left ventricle, and fatty degeneration. Note to fourth edition.

I Vide articles by Prof. H. C. Wood in Am. Jour. of Med. Scicnces, July and Oetober, 1871.

2 On Neuralgia, etc., 1872. 
result from aneurism in this situation generally oceurs without any diagnostic symptoms, and without any symptoms denoting a grave disease. The diagnosis is very diffieult and perhaps impossible. Of 703 cases analyzed by Dr. Sibson (Med. Anatomy, fasc. v.), 87 were within the pericardium. The ascending portion of the arch without the pericardium is the most frequent site; in 193 of the 703 cases analyzed by Dr. Sibson, the situation was in this portion. The ascending and transverse portions are not infrequently both involved in the dilatation. This was the case in 140 of 703 eases. The transverse portion was affected alone in 120 cases, and, conjointly with the descending aorta, in 20 cases. The descending portion of the arch was the seat in 72, and the aorta below the arch in 71 cases.' The aneurismal tumors in the larger proportion of cases spring from the outer aspect of the artery, but they may spring from either the inner, posterior, or anterior aspeet.

Aneurismal tumors, according to their situation and size, give rise to certain symptoms by pressing upon the different surrounding parts. Unless referred to their true source, these symptoms may mislead the physician by appearing to indicate other affections. And by means of these symptoms, not only may aneurism be suspected, but, assuming its existenee, its situation may be inferred.

Interruption of the circulation through the innominata and the left carotid and subclavian artery may arise either from the pressure of the tumor on these vessels or obstruction by fibrinous plugs. Feebleness or extinction of the pulse in the radial, brachial, or carotid artery on one side is one of the symptoms of aneurism, and indicates its situation to be at the right or left extremity of the transverse portion of the arch. When the radial pulse is not abolished, but more or less weakened, the contrast between the pulse of the two arms is well shown by a comparison of the visible characters as traced by the sphygmograph. Niemeyer has called attention to a notable retardation of arterial pulsations beyond the seat of the aneurism. Thus, when the aneurism is seated between the points of origin of the great vessels of the arch of the aorta, the radial pulse is felt later on one side than on the other; and, if the aneurism be seated in the clescending aorta, the pulsations of the arteries below ocenr later than the pulse at the wrist. An aneurismal tumor springing from the ascending or transverse portion of the arch may press upon the superior vena cava or the venre innominatr, and induee venons eongestion limited to the head and upper extremities, or to one upper extremity, accompanied, perhaps, by more or less œdema. These symptoms point to the existence and situation of aneurism.

An aneurism seated in the transverse portion of the arch may press upon the trachea or one of the primary bronchi, and interfere with respiration. If the obstruction be considerable, the breathing is labored, and may be aecompanied with a noise, heard at a distance, called stridor. Uncler these circumstances, auscultation will show feebleness of the respiratory murmur on both sides of the chest if the trachea be pressed upon, but feebleness limited to one side, and the murmur exaggerated on the other side, if only one of the bronchi be obstructed. Embarrassment of respiration may proceed from pressure upon another part, viz., the recurrent laryngeal nerve. If the pressure be sufficient to interrupt the function of the nerve on one side, laryngeal obstruction arises from an arrest of the respiratory movements of the glottis on that side. 'The embarrassment from this cause, however, is not very great. The laryngoscope in

1 Fuller, on Diseases of the Chest. 
these cases shows the vocal chord on one side to be motionless, that is, paralyzed, while the respiratory movements of the other chord continue. Spasm of the glottis may be indueed if the relation of the nerve to the tumor be such that it is irritated instead of its function being annulled. I have reported a case in which, the left recurrent nerve being situated between a calcareous deposit and the aneurismal tumor, spasin of the glottis occurred so frequently and to such an extent as to prove fatal. ${ }^{1}$ Modification of the voice and aphonia are effects of either incomplete or complete paralysis from pressure on the recurrent laryngeal nerve, and the affection of the voice is found to vary at different times according to the varying amount of pressure. These laryngeal symptoms are apt to mislead by directing attention to the larynx as the seat of disease. Tracheotomy has been repeatedly performed under the belief that the obstruction of respiration was due to an affection of the larynx. My colleague, Prof: McCready, has given me the account of a case in which the primary and most marked effect was manifested by romiting and pyrosis, an examination after death showing that the tumor pressed upon the pneumogastric nerve.

Pressure on the asophagus, when the tumor is situated in the transverse or descending aorta, may occur so as to occasion obstruetion to the passage of food. 'This symptom should excite suspicion of aneurism, and such a suspicion should enforce caution in the use of bougies to explore and dilate the csophagus. Pressure on the thoracic duct has been known to occur, inducing marked emaciation. Pressure on the sympathetic nerve is another effect of an aneurismal tumor, leading to contraction of the pupil of the eye on the affected side. If the tumor be seated in the descending aorta, it is likely to lead to erosion of the vertebral column, and may thus occasion paralysis by affecting the spinal cord. 'These cases are generally characterized by considerable pain referred to the back. Neuralgic pains and well-marked angina pectoris sometimes accompany ancurism seated in the ascending aorta.

The diagnosis of thoracic aneurism is to be based on the presence of more or less of the foregoing symptomatic phenomena, taken in connection with the physical signs of a tumor within the chest, situated at some point in the tract of the aorta. 'The signs are very obvious when the anenrism has led to erosion of the ribs and an external tumor, pulsating, perhaps presenting a thrill, the heart-sounds heard with more or less intensity when the stethoscope is placed over it, with a single or double bellows murmur. The signs are less marked before the tumor makes its way through the thoracic walls, but they may be sufficient to render the diagnosis quite positive. Dulness on percussion over a circumseribed space in the tract of the aorta is an important diagnostic point. This space will be on the anterior surface of the chest, if the aneurism spring from the arch, and posteriorly if it spring from the descending aorta, below the arch. Careful inspection and palpation may disclose pulsation and perhaps thrill before any external tumor is apparent. Undue audibleness of the heart-sounds within the space found to be dull on percussion, and a single or double murmur not transmitted from the aortic orifice, are highly significant signs. They are, however, by no means uniformly present, and, the efore, while their presence contributes to the diagnosis, their absence is not proof that aneurism does not exist.

The prognosis, in cases of thoracic aneurism, relates chiefly to the duration of life. The instances of recovery are so few that practically they

1 American Medical Times, 1864. 
are to be disregarded. In the majority of cases, death is the result of rupture of the aneurismal sac. 'The rupture nay take place in various directions, viz., externally, or into the osophagus, trachea, bronchi, pleural cavity, heart, venæ cava, spinal canal, pericardial cavity, etc. The rupture may be at first small, so that death takes place more or less gradually; or it may be large enough to cause a sufheient hemorrhage to destroy life within a few moments. The progress of the aneurism varies much in different cases, the duration of life being in some cases but a few months, and in some cases several years. In a considerable number of cases life is destroyed before rupture takes place. Pressure on the trachea, œsophagus, vena cava, thoracic duct, or irritation of the recurrent laryngeal nerve, may lead to a fatal result, and tle patient may be cut off by some intercurrent affection. Organic lesions of the heart coexist in a certain proportion of cases. These, of course, involve distress and danger in proportion to their nature and extent, and may prove the immediate cause of death. Clinical observation shows that patients affected with thoracic aneurism rarely have pulmonary tubereulosis.

The treatment relates to the palliation of suffering and the prolongation of life. All active exertions are to be avoided, and, as far as possible, emotional excitement. The body is to be well nourished, if possible, but the diet should be unstimulating, and alcoholic stimulants interdicted. Liquids should be taken sparingly. The action of the heart, if abnormally strong, is to be diminished by sedative remedies, and perhaps, if the patient be plethoric, in some cases, by small abstractions of blood. It is highly important not to impoverish the blood, and anæmia, if it exist, calls for the treatment appropriate to that condition. Pain and other incidental symptoms are to be palliated by appropriate remedies.

It is well to bear in mind that the progress of the aneurism is retarded by the deposit of successive layers of fibrin within the aneurismal sac. It is in this mode that a cure sometimes takes place. It is desirable, therefore, that the blood slould be rich in fibrin, and it is probable that the deposit is more likely to take place in proportion as the action of the heart is slow. Cardiac sedatives, namely, aconite, digitalis, and the veratrum viride, may in this way be useful. Certain remedies have been supposed to have a more direct curative effect in some cases of aneurism; and experience has seemed to fumish evidence of such an effect. 'The acetate of lead and the iodide of potassium, more especially the latter, are remedies which have lately been so considered. Roberts, of Manchester, England, Balforr, of Edinburgh, Bouillaud, and others, have reported cases in which the iodide of potassium, given in doses of from 15 to 95 grains daily, appeared often to be useful, and sometimes to effect a cure. It is difficult to understand how these or other remedies can exert a direct curative influence in any other way than by promoting the deposit of fibrin in stratified layers which adhere to each other and the inner surface of the aneurismal sac. It is claimed that the remedies first named do exert a curative influence in that way; their therapentic value remains to be determined. The longcontinued use of the acetate of lead is objectionable; but the iodide of potassium may be tried sufficiently without risk of evil consequences. In a case of aortic aneurism which has fallen under my observation, diminution of the size of the sac, with notable relief of pain, has followed the use of the iodide of potassium in doses of grs. $\mathbf{x x x}$, three times daily.

1 Since this sentence was written I have witnessed apparent benefit from this remedy in several bospital cases, although in no instance has a cure been effected. - Note to 4th Edition. 
If spasm of the glottis be induced, through the recurrent laryngeal nerve, to such an extent as to endanger life, laryngotomy is warrantable, if not advisable, with a view to palliation and the prolongation of life. This operation will, of course, be of no avail if the obstruction be due to pressure of the aneurismal tumor upon the trachea.

A half century or more ago a plan of treatment was devised by two Italian physicians, Albertini and Valsalva, for the cure of ancurisms. This plan embraced entire physical rest, frequent and copious venesections, and reduction of food to the lowest point compatible with life. Although sanetioned by Laennee, Bouillaud, and Hope, this course was never generally adopted, and for several years has been obsolete. A reproduction, in part, of this plan was proposed by the late Dr. Bellingham, and Joliffe 'Tuffinell, of Dublin, has reported a series of cases which show a remarkable success from it. 'Tuffinell does not employ bleedings, but he requires perfect rest in a recumbent position for two or three months, the patient during this time not raising himself even to the semi-recumbent posture, but he is allowed to turn carefully from side to side, and at times to lie on the face to relieve the back. He is to be in a sunny room, where he can have some range of observation, and is to be amused as far as practicable. The diet is to consist of two ounces of white bread and butter for breakfast; the same for supper, with two ounces of milk, or cocoa or tea, and for dinner three ounces of boiled or broiled meat, with three ounces of potatoes or bread, and four ounces of water or claret. The diet is to be rigidly restricted to the articles and quantities just stated. Under this plan of treatment Tuffnell elaims to have cured five or six cases of aortic aneurisin, in one, the tumor having projected through the sternum.

Dr. Henry J. Bowditch suggests, as a modification of Tuffnell's plan, venesection in some cases, and less reduction of the daily allowance of food, the chief reliance being placed on absolute rest in the horizontai position. ${ }^{1}$

I have had under observation four cases in which "Tuffnell's plan" was employed. In none of these cases was the plan successful, although in all it seemed to be more or less useful. ${ }^{2}$ I should have resorted to the plan in a larger number of eases, were it not that it is diffienlt to secure the complete and continued co-operation of the patient. The merits of the plan are, of course, to be determined by further experimental observation; and, for this end, reports of cases in which it has been faithfully carried out under the direction of competent and trustworthy observers, are desirable.

Measures desired to produce directly coagulation within the aneurismal sac have been to some extent employed, namely, the injection of astringent liquids, the introdnction of solid substances, and the application of the electrical current by means of needles introduced within the sac. These measures have not proved successful. If they produce within the sac simple coagulation, that is, either clots of blood or loose masses of fibrin, they not only fail to effect a cure, but the patient is exposed to the dangers incident to embolism.

1 Vide paper by Dr. Bowditch, Boston Medical and Surgical Journal, 1866.

2 For a report of these cases, and also an account of Tuffinell's cases, vide my work on Diseases of the Heart, second edition, 1869. 


\section{SECTION THIRD.}

\section{DISEASES AFFECTING THE DIGESTIVE SYSTEM.}

\section{CHA PTER I.}

Introductory Renuarks. Sporadic Dysentery-Anatomical Characters-Clinical HistoryPathological Character-Causation-Diagnosis-Prognosis-Treatment. Epidemic Dysentery. Chronic Dysentery. Inflammation of the Cacum. Perforation. Fecal Abscess. Palnful Tumor near the Cacum.

THE important organs composing the digestive system are, the mouth 1 with the parotid, submaxillary, and sublingual glands, the pharynx and osophagus, the stomach and duodenum, the small and large intestine with the mesenteric glands, and the solid abdominal viscera, viz., the liver, pancreas, and spleen. Affections of the two outlets of the alimentary canal, viz., the mouth, pharymx, and csophagus, with the appended glands, and the rectum and anus, properly belong to the department of surgery, being either open to view or accessible by manual exploration, and often requiring surgical operations. These affections, for the most part, will not be considered in this work. 'This section will, therefore, be devoted mainly to the diseases affecting the hollow viscera and the solid organs within the abdomen. The affections of the digestive system, as of other anatomical systems, consist of, first, inflammations; second, structural lesions; and, third, functional disorders. The inflammatory affections of the hollow viscera will be first considered. Inflammation affecting these viscera may be seated in the mucous membrane lining the alimentary canal, or in the investing serous membrane, the peritoneum. Inflammation seated in the mucous membrane is generally limited to a certain section of the alimentary canal, viz., the portion lining either the stomach and duodenum, the small intestine, exclusive of the duodenum, or more or less of the large intestine. This rule will also be found to apply to functional disorders, that is, the sections just named are apt to be disordered separately. Inflammation affecting these sections gives rise to separate diseases. I shall proceed to consider inflammation of the large intestine, constituting the affection commonly known as dysentery. Inflammation, in this situation, may be either acute, subacute, or chronic. Chronic dysentery will claim consideration under a distinct head. A capital distinction relates to dysentery occurring as a sporadic and as an epidemic affection. Sporadic and epidemic dysentery will claim separate consideration.

\section{Sporadic Dysentery.}

The term dysentery has long been in use to designate inflammation of the large intestine, attended with mucous and bloody dejections. As a name for the disease, the term is not very distinctive, its etymology (3tili) 
simply expressing intestinal difficulty; bnt it is not easy to substitute a term conformable to the nomenclature of inflammatory affections, sinee there is no anatomieal name for the large intestine as a whole. The term colonitis, or colitis, is sometimes used in the place of dysentery, but this implies that the inflammation is limited to the colon, whereas the reetum is generally, if not always, involved, and, in fact, in some eases of dysentery the affection may not extend above the rectum. The term dysentery has the recommendation of not expressing anything erroneous or doubtful in regard to the pathologieal eharieter of the clisease. Sporadic dysentery is presented in practice as an aente and a subacnte affection; that is, the local and general symptoms frequently denote more or less gravity of disease, compelling the patient to keep the bed, and in some eases it is an extremely mild and almost trivial affection. Different eases of the sporadie form present every gradation as regards severity, and there are few diseases in the nosology which offer a wider contrast than the mildest cases of sporadic, and the gravest cases of epidemic dysentery.

Anatomicar Characters.-In subacute or mild cases, the inflammation has but little intensity, and may be limited in extent, being confined perhaps to the rectum. As in these cases the disease involves no danger, the opportunity of inspecting the parts after cleath is not offered. 'The mildness of the symptoms and the short career of the disease suffice to show that ulcerations or other lesions of importance do not take place in these eases. In acnte or severe cases, the inflammation is not only more intense, but more extensive, involving, not only the rectum, but the greater part or the whole of the colon. These cases are liable to end fatally, and the affected membrane is found, on examination after death, to be redilened from active congestion, swollen, softened, pulpy, presenting, in different cases, ecchymoses, excoriations from desquamation of the epithelium, abrasion and ulcerations, in greater or less number, the latter being sometimes small and sometimes of considerable size. The uleers may or may not be seated in the intestinal glands and follieles. The redness and swelling are frequently not uniform over the extent of intestine affected, but are either limited to, or more marked in certain portions, more especially the projecting folds of the membrane. Small vesicles are sometimes observed. The swelling of the membrane is due, in part, to submucons infiltration, and the latter is sometimes so great, at certain points, as to give rise to protuberances resembling warty growths or fungoid exerescences. These protuberances may be more or less numcrous, and sometimes coaleseing give to the surface a mammelonated aspect. Patehes of exuded fibrin are frequently adherent to the inflamed membrane, presenting a greenish or brownish color. The intestine contains more or less morbid matter corresponding to the dejections during life, consisting of mucus, pus, fibrinous flakes, desquamated epithelium, and sero-sanguinolent liquid. 'The intestine may present a dark and almost black appearance from congestion; but sphacelation, beyond the circumscribed sloughing which precedes ulcerations, is rare. As a rule, the appearances denote progressively a greater amount of disease in passing from the upper part of the large intestine downward to the anus, the greatest amount being at the rectum and sigmoid flexure. There has been much diseussion whether the morbid appearances in dysentery denote ordinary inflammation varying in different cases in intensity, or whether they are due to a peculiar morbid process; also, whether the intestinal glands and follicles are generally and primarily involved. It does not 
fall within the scope of this work to consider the morbid anatomy with reference to these points of inquiry.

The mesenteric glands are sometimes found to be considerably enlarged, and in some instances to contain pus.

The cicatrization of ulcers in some cases leads to stricture of the intestine.

Cininical History.-The disease is generally preceded by an ordinary diarrhœa ; that is, more or less frequent dejeetions of a feculent character. Of thirty-three recorded cases which I have analyzed with reference to this point, these dejections preceded the characteristic dysenteric evacuations in thirty. The latter appeared after the former had existed for a period varying from twenty-four hours to seven days.' With this prodromic diarrhoa there are colic pains, the appetite is impaired, and there is a general feeling of malaise. A pronounced chill rarely occurs, and even chilly sensations are not eommon.

The development of the disease is denoted by characteristic evacuations consisting of mucus, with which more or less blood is usually commingled. The quantity passed at each act of defecation is generally small, but the act is frequently repeated, slight evacuations often taking place every hour or two, and sometimes after intervals of a few moments only. The quantity of mucus expelled in some eases is abundant, and forms a jellylike mass, not unlike a collection of the rusty sputa of pneumonitis. The popular term applied to the matter of these evacuations is slime, and by those who are familiar with the preparation of intestines for sausages, they are often compared to the "scrapings of hogs' guts." Rarely in sporadic, but not infrequently in epidemic dysentery, the evacuations contain fibrinous laminze or flakes in greater or less quantity. A scrosanguinolent liquid, either small in quantity or abundant, also occurs occasionally in sporadic, but much oftener in cases of epidemic dysentery. The latter has been compared to water in which meat has been washed, and was called by the older writers lotura carnium. Most of the evacuations are exclusively dysenteric, that is, consisting of mucus, epithelium, lymph, and blood, but with some of the evacuations fecal matter is discharged. Frequently this presents a green color. If the cliaracteristic dejections be filtered, the liquid which is obtained is found to be notably rich in albumen, the greater part solidifying on the addition of nitric acid. Occasionally round hardened lumps of feces are expelled; these are called scybala. The amount of morbid matter evacuated, together with the absence of fecal matter, constitutes, measurably, a criterion of the extent of intestinal surface affected. In the course of the disease the evacuations may contain more or less purtuent matter, but this belongs more especially to chronic dysentery. In lieu of the fecal odor, they frequently emit a putrid or cadaveric fetor.

The inflammation of the rectum occasions a sensation as if this portion of the bowel were filled, and leads to the frequent desire to defecate, with as much straining as the soreness of the parts will allow. This desire to strain ineffectually is called tenesmus. Sometimes the straining efforts produce prolapsus of the intestine. The evacuations are frequently preceded by griping or colic pains, which have been called tormina. The tormina and tenesmus are not proportionate to the gravity of the disease; they are sometimes wanting in fatal cases, and are prominent symptoms in some

${ }^{1}$ Clinical Report on Dysentery, based on an analysis of forty-nine cases, by the author. 1853 . 
mild cases. They are, however, the chief sourees of suffering. Tenderness on pressure is in some cases more or less marked over the descending colon, and sometimes over the whole tract of the large intestine. It is rarely great, and not infrequently wanting. Meteorison or tympanitic distension is rarely. present; the abdomen is usually depressed. Strangury and retention of urine are occasional symptoms, and, in the female, leucorrhoe may be produced.

The pulse, in the majority of cases, is but little, and sometimes not at all, accelerated. Exceptionally, high febrile movenent exists ; this is in robust persons, the inflammation being more intense and diffused than usual. Great frequency of the pulse always denotes gravity and danger, but the converse does not hold good; in some fatal cases the pulse is never much accelerated. The skin is generally either cool or moderately hot, but, exeeptionally, with high febrile movement, there is much heat of the surface. The tongue may be frosted, or more or less coated, or it may present a natural appearance. Iuring the course of the disease, the appetite is more or less diminished, or lost. Thirst is usually present, and is sometimes urgent. Vomiting occasionally oceurs, and may be prominent as a symptom, the matter vomited being of a greenish color. The intelleet is unaffected as a rule, delirium occurring with great infrequeney in cases which end in recovery, but occasionally, in cases which prove fatal, toward the close of life. The loss of strength varies greatly in different cases according to the intensity and extent of the local affection, depending, also, on the condition of the patient when attacked.

The duration of the discase, from the date of the attack to convalescence, varies from four to twenty-one days. These figures represent the minimum and maximum duration in thirty recorded cases.

Pathological Cinaracter.-Our knowledge of the pathology of sporadic dysentery embraces only the intestinal affection, the local and general phenomena which make up the clinical history being symptomatic of this affection. The disease is a spontaneous inflammation of the large intestine, using the term spontaneous in the sense already defined; it is altogether probable that the inflammation is developed as a result, or a loeal expression, of a prior internal morbid condition of which we have at present no knowledge. This view of the pathologieal charaeter is consistent with what will be presented under the head of causation. The view, however, is by no means peculiar to this disease, but is applicable to most of the so-called spontaneous local affections.

The question arises in this connection-Is there more than one species of sporadic dysentery? Writers have described different kinds of the disease, which they liave distinguished as typhous, bilious, intermittent, and remittent, rheumatic, febrile and non-febrile, etc. These terms denote either variations in the phenomena of the disease or the coexistence of other diseases. Dysentery may oecur as a complication of the continued and periodical fevers. So rheumatism and dysentery are occasionally associated. These combinations are important in their practical relations, but it does not follow that the disease, under these circumstances, is specifically different from its ordinary form. Variations as regards febrile movement, supposed disorder of the liver, ete., do not affect the special character of the disease. Paralysis affecting eertain muscles, especially the muscles of the lower limbs (paraplegia), has been observed, in some cases, to either accompany or follow dysentery; and different theories have been proposed to explain the pathological connection between the paralytic and the dysenteric affection. The truth is, the 
affections are so rarely connected, that perhaps the most rational conchlsion is, their association is a mere coincidence. At all events, paralysis, either as a complication or sequel, does not occur often enough to show any intrinsic tendency thereto in dysentery.

Causation.-No age is exempt from liability to this disease, but, in the majority of cases, the patients are adults and under thirty-fire. This was true of 30 out of 44 cases analyzed with reference to this point. A larger number of males than females are attacked, but the preponderance is not sufficient to show that a marked predisposition relates to sex. The causation has a very obvious relation to climate. The disease is vastly more frequent, in the sporadic as well as epidemic form, in tropical than in cold and temperate climates. The causation has also a striking relation to season. Of forty-four cases which I recorded during fourteen years, all save one case occurred in July, August, September, and October. The constitution and previous health were good in one-half of the cases which I have analyzed, and in the remaining half there was no uniformity in character as regards the antecedent affections or disordered health.

Writers have been accustomed to state that the disease may be produced by various obvious causes, such as atmospherical changes, excesses in eating and drinking, indulgenec in acid, unripe fruits, crude vegetables, etc. But in the majority of cases, it is not easy to trace its origin to these causes, nor to any obvious cause. This renders it probable that a special cause is involved in sporadic, as well as in epidemic, dysentery. The limitation of the occurrence of the disease to the summer and autumnal season, and its more frequent occurrence in tropical climates, point to this conclusion, and to the agency of a high temperature in the evolution of the special cause. Various circumstances stand in a cansative relation to the disease in an epidemic form, which may also be more or less frequently involved in its production when it is sporadic. These causative circumstances will be noticed in treating of epidemic dysentery. There are no grounds for considering sporadic dysentery as contagious.

Diagnosis.-The diagnosis of dysentery involves no difficulty. The characteristic evacuations are pathognomonic. It is to be discriminated from cases of bloody evacuations and irritation of the rectum incidental to hæmorrhoids, of intestinal hemorrhage or melæna, and of the clischarge of pus from an abscess opening into the intestine. The points of contrast with dysentery which these cases offer are sufficiently apparent.

Prognosis.-Sporadic dysentery, in temperate climates, intrinsically tends to recovery. It is a distressing disease, but attencled with very little danger to life. A fatal result is due either to an unusual intensity and extent of the local affection, or to an inability of the system to resist the disease, from feebleness of the constitution, or to some coexisting affection. The prognosis in tropical climates is undoubtedly less favorable. The recovery from the disease in temperate climates is almost always complete; that is, it rarely eventuates in chronic dysentery. Nor does it leave a predisposition to any other disease. In tropical climates, abscess of the liver is not an infrequent sequel. This very rarely, if ever, follows in temperate climates. Its occurrence is attributed either to the absorption of septic matter from the intestinal canal, or to the detritus of thrombi formed in the veins of the intestine. So far as my experience goes, immediate relapses are not apt to take place, and the occurrence of the disease does not induce a greater liability to its recurrence at a subsequent period. 
With reference to the latter point, my analysis of cases, although the number was not large, developed an interesting result. Of all the eases occurring during fourteen years, in not a single instance was the disease known to recur. And in sixteen of these eases the patients were under observation after recovery during periods varying from one year to thirteen years. This result was the more striking from the fact that the patients for several years were within the sphere of an epidemic influence, which, in some instances, affected other members of the same families.

Treatuent.-In the great majority of cases, sporadic dysentery would doubtless end in recovery without medicinal treatment. But there is reason to believe that the disease is sometimes arrested, that its duration may be frequently abridged, and the distressing symptoms may be greatly relieved, by judicious therapentical measures.

It is desirable that, as early as possible, the fecal contents of the large intestine should be effectually removed, in order to prevent their continued passage over the inflamed surface, and to secure, so far as may be, that important end in the treatment of all inflammations, viz., quietude of the inflamed part. It would seem that nature encleavors to relieve the bowels of their contents by the diarrhoa which precedes the dysenteric evacuations. The first point in entering on the treatment is to ascertain if the bowels have been spontaneously relieved. With reference to this point, the size and eharacter of the stools are to be ascertained, and the abdomen examined by palpation. In general, it is advisable to.render the effort of nature more complete by giving an effective purgative. Castoroil has been in much repute as an appropriate remedy, but, aside from the objection on the seore of the disgust which many persons have for this remedy, the saline cathartics are to be preferred. 'The sulphate of magnesia or soda, the Rochelle salts, or the citrate of magnesia, may be employed. The saline sclected shonld be given in suffieient doses to produce promptly abundant dejections, and it is then to be discontinued. It will sometimes happen that after free purgation, the dysenteric evacuations do not return, and recovery at once ensues. This abortive effect takes place only in some cases in which the inflammation is not intense, and is limited to the lower part of the intestine. Notable diminution of the frequency of the dysenteric evacuations, and relief of the tormina and the tenesmus, not infrequently follow the operation of the saline purgative. This treatment may be pursued except in cases in which it is contra-indicated by great feebleness of the constitution of the patient.

After purgation, opium, in some form, is the remely to be most relied upon. A full dose of opium administered by enema, or as a suppository, will sometimes succeed in arresting the disease. It often mitigates the severity of the disease and affords marked relief. Administered by the rectum, however, it is not always retained, and then reliance must be had upon its administration per orem. It may be given in full doses repeated every four, six, or eight hours, or in smaller doses repeated at shorter intervals. As regards the form of opium selected, I believe this is chiefly important in respect of the preference or peculiarity of the patient. I have seen the opium in gum or powder, the various liquid preparations, and the salts of morphia equally effectual. The form found to be preferable is to be continued during tbe progress of the disease, the doses being regulated by the frequency of the evacuations and the suffering. Experience in each case is to be the guide as to the mode of administration. Generally the administration by the mouth and rectum alternately is advisable. The opiate remedies are to be gradually diminished as the dysenteric 
evacuations become less frequent and the suffering from tormina and tenesmus is less. After two or three days, if the disease continue, the saline purgative may be repeated if not contra-indicated by the feebleness of the patient, and afterwards the use of opium resumed. Skoda has suggested that opium in dysentery, and in certain cases of diarrhoa, is efficacious by keeping the sphincter ani in a state of contraction, and by causing permanent contraction of the small intestine so that the contents of the latter are not propelled far enough to induce the irritation which eauses their expulsion; and the contents thus retained in the small intestine may be considerably diminished by absorption.

In this plan of treatment the reliance is upon free purgation and opium. The chief reliance, I believe, should be upon these measures; but other measures may be conjoined. 'The subnitrate or the subcarbonate of bismuth in scruple or lialf drachm doses, the acetate of lead, the sulphate of copper, gallic acid, and the various vegetable astringents, such as rhatania, kino, catechu, hæmatoxylon, etc., are useful as adjuvants. These are to be relied upon, to the exclusion of opium, in those cases only in which, from a constitutional peculiarity, the latter produces unpleasant effects sufficient to preclude its employment. It is undoubtedly true that some persons are affected so unpleasantly by opium that its use is, if possible, to be avoided; but it is often the case that it is well borne in this and some other cliseases, although it may not be in trivial affections. Certain preparations may be well borne, although others occasion unpleasant effects. An aqueons solution or extract is frequently taken without difficulty by those who cannot comfortably take other preparations. Codeia sometimes answers a good purpose as a substitute for the preparations of opium and morphia.

Ipecacuanha has been considered a valuable remedy in dysentery. Some have attributed to it a special eurative influence, and have advocated its employment in as large closes as can be borne. Dr. Maclean, professor of clinical and military medicine in the army medieal sehool, Netley, states that the system of treating acute dysentery, now generally followed in India, is as follows: Thirty drops of the tincture of opium may be at first given, but this may be .sometimes omitted and the ipecacuanha given at once. If the opiate be given, in half an hour it is to be followed ly from 25 to $30 \mathrm{grs}$. of ipecacuanha. The latter remedy should be given in as small a quantity of fluid as possible, and a little syrup of orange-peel covers the taste of the medicine. The patient, after this dose, should keep perfeetly quiet and abstain from fluid for, at least, three hours. With these precautions, nausea and vomiting are rarely troublesome. After eight or ten hours, the ipeeacuanha may be repeated in a reduced dose, observing the same precautions. Dr. Maclean states that the effects of one or two doses of the ipeeacuanha, given in this manner, are often surprising; "the tormina and tenesmus subside, the motions quickly become feculent, blood and slime disappear, and often, after profuse action of the skin, the patient falls into a tranquil sleep and awakens refreshed." If required, the treatment is to be continued for several days, the remedy being given in diminished doses, with intervals sufficient to allow mild nourishment to be taken. He eites statistics showing the mortality in India, under the use of ipecacuanha, to be 28.87 in a thousand ; whereas, under the old system the mortality was 88.2 in a thousand. It is to be borne in mind, however, that the old system of treatment embraced bloodletting and the free use of mercury. I have resorted to this

1 Vide article in "A System of Medicine," edited by Reynolds, vol. i. 1866. 
plan of treatment in a considerable number of cases at Bellevue Hospital, but without a very satisfactory degree of success. In a small proportion of cases the clisease was either arrested or favorably modified by it; but in the majority of cases it had no apparent influence on the disease.

Mercury has been, heretofore, much relied upon in the treatment of this disease, given in either large doses, united with opium in order to prevent a cathartic effect, or in small doses repeated at short intervals. This remedy has been thought to be useful in dysentery, as in all inflammations, by reason of a supposed antiphlogistic influence, but, in addition, a special efficacy has been attributed to it from its supposed action on the liver. Defieient hepatic secretion and consequent portal congestion have been regarded as important elements in the pathology of dysentery, and mercury has been looked upon as a remedy having reference specially to these elements. The pathologieal view just stated, however, is purely conjectural, and clinical observation fails to furnish evidence of any special curative influence to be derived from mercury. The recovery, when treated with mercury, is, of course, no proof of its value in any disease which, like sporadic dysentery, tends intrinsically to recorery. T'Te duration of the disease, the relief of the symptoms, the rapidity of convalescenee, and the subsequent condition of health, are the points to be eonsidered in estimating the influence of remedies in this disease. With reference to these points, a fair estimate of the effects of mercury, especially when pushed to ptyalism, it is believed will lead to its rejection as a remedy for dysentery ; and, indeed, it has already, in a great measure, in this country, fallen into disuse.

During the early part of the disease, the diet should be restricted to a small quantity of the blandest articles of food. It is an object throughout the disease to keep the patient on a diet which will leave but little residuary matter after digestion to pass into the large intestine; in other words, a diet as purely nutritious as possible. After the first few lays, however, it is important to provide for proper support of the system by nutritive supplies, and for this purpose milk and animal broths are best suited. If the symptoms denote failure of the vital powers, concentrated nourishment and alcoholic stimulants are to be given freely, as in all diseases which destroy life by asthenia. Tonic remedies may be given with advantage during convalescence.

Patients should be instructed to refrain as much as possible from repeated acts of defecation and straining efforts. They will be induced to do so if it be explained to them that the sensation of fulness of the rectum is delusive, being dependent on the inflamed condition of the membrane. Cold applications to the anus sometimes relieve the tenesmus. A piece of ice may be wrapped in cloth and applied frequently in this situation. Enemas of cold water are often grateful. Relief, in some cases, is afforded by injecting astringent liquicls. The nitrate of silver, a solution of tamnin, acetate of lead, or the sulphate of zine, may be thus used. Dr. Gairdner recommends highly large emollient injections, each containing from five to ten drops of creasote. Dr. Thomas F. Cock, of this city, has found that notable relief of the tenesmus is afforded by the introduction within the rectum of the vapor of chloroform. It is introdnced by pouring a little chloroform (about 20 drops) upon a piece of sponge, which is placed in a common syringe, the tube passed into the rectum and kept there until enough of the vapor has been introduced to afford relief. It is not necessary to use any means of forcing the vapor into the rectum, but the vaporization is rendered more rapid by warming the syringe. Care is to be taken that the chloroform does not come in contact with the anus. 
Fomentations and mild revulsive applications over the abdomen are useful as palliatives in proportion to the amount of tormina and abdominal tenderness.

\section{EPIDEMIC DYSENTERY.}

Epidemic dysentery is essentially the same disease as sporadic dysentery. Certain events occur much oftener in the former than in the latter, yet there is nothing pertaining to the epidemic which is not occasionally seen in the sporadic form of the disease. Epidemic dysentery is often very fatal, yet, in the epidemics attended with the largest fatality, cases occur which are as mild as the mildest sporadic cases, and, on the other hand, occasionally in sporadic cases life is quickly destroyed. Like other diseases which prevail epidemically, this differs greatly at different times and places as regards gravity, and at certain times and places it presents features which it does not present at other times and places. The differences between different epidemics of this disease are in a measure to be explained hy the coexistence of other diseases, and by the conjoint operation of morbific causes not standing in a special relation to the production of this affection. In treating of epidemic dysentery, the main object will be to point out the circumstances in which it is apt to differ from sporadic dysentery.

With respect to the anatomical characters, they are essentially the same as in cases of sporadic dysentery. In fatal cases of either form, the varied appearances which have been stated may be found. Extensive and disorganizing changes oftener occur in epidemic dysentery. As a rule, in the latter the inflammation extends over a larger portion of the large intestine, and it sometimes extends into the ileum. Ulcerations and diphtheritic exudation are much more apt to occur in epiclemic dysentery. Perforation of the intestine is sometimes a result of ulceration, giving rise to peritonitis. Perforation of the cæcum may take place in this disease, leading to abscess in the areolar tissue beneath the peritoneum, or fecal abscess. Perforation of the rectum and fecal abscess in that situation have been known to occur.

The clinical history of epidemic differs from that of sporadic dysentery according to differences as regards the extent and severity of the local affection, and also according to various morbid conditions due to the action of accessory causes, the latter oftener co-operating in epidemic than in sporadic cases. Severe cases of epidemic dysentery are characterized by the occurrence of sero-sanguinolent dejections, the lotura carnium of the older writers, containing usually flakes of lymph in more or less abundance, and holding in solution albumen in abundance. Hence, epidemics of dysentery are popularly known as bloody flux. Dejections of this character always denote gravity of disease. Tormina and tenesmus may be less prominent as symptoms in severe cases of epidemic than in most cases of sporadic dysentery. In some fatal cases these symptoms are entirely wanting. Tenderness over the tract of the colon may, or may not, be marked. The local symptoms in some cases of epidemic dysentery are comparatively slight, and in general, so far as my observation goes, patients suffer far less in fatal cases than in sporadic cases wholly devoid of danger.

The differences, as regards the general symptoms, in different epidemics and different cases, are marked. Severe cases are characterized by prostration, feebleness of the circulation, coldness of the surface, in short, by symptoms denoting adynamia. The pulse may become frequent, small, 
soft, vibratory but compressible, or it may be small, weak, thready, with but little, and sometimes not any, increase of frequency. These characters of the pulse; denoting feebleness of the action of the heart, together with other adynamic symptoms, are developed in some cases early in the disease, without having been preceded by active febrile movement, but in other cases the pulse for a time is full and strong, the skin hot, the adynamia succeding these symptoms of high symptomatic fever. In fital cases, a condition analogous to the stage of collapse in epidemic cholera may precede, for a varialble period, the termination of the disease. In this condition the pulse is scarcely appreciable, or it is extinct, the surface is colrl and either dry or bathed in perspiration, the teeth are sometimes covered with sordes, the quantity of urine is greatly diminished, cramps of the muscles of the lower extremities sometimes occur, and this collapsed condition may exist for one, two, or three days before death. The mode of dying is by slow asthenia.

'The intellect generally remains intact, or slight mental aberration only occurs in the latter part of life. But some cases are characterized by delirium. 'The patient talks incoherently mider the influence of transient delusions; he may attempt to get out of bed, and require to be restrained. In a case which came under my observation some years ago, the delirium was of a boisterous, mirthful character, the patient singing snatches of humorous songs, shouting, and attempting to get out of bed, after the pulse had become extinct, and the surface of the body was cold like that of a cadaver. In this case, the manifestations of delirium were so painfully incongruous that the patient was kept sufficiently under the influence of chloroform to repress them for several hours before his death.

'The rlelirium and other symptoms, in some cases, are analogous to those belonging to typhus and typhoid fever. The typhoid state may exist in cases of this disease as of various other affections. But is to be borne in mind that dysentery sometimes occurs as a local complication of typhus and typhoid fever. Certain epidemics of these fevers are characterized by the oecurrence of this eomplication. The distinetion between dysentery with typhoirl symptoms, or typhoid dysentery, and typhus or typhoid fever with a dysenteric affection developed secondarily, is the same as between typhoid pneumonitis and typhus or typhoid fever complicated with pueumonitis. The symptoms of scorbutus are associated with those of dysentery when, as is sometimes the case, the latter prevails among persons who are suffering from scorbutic deterioration of the blood. So, in districts where periodical fevers prevail, epidemics of dysentery may be characterized by periorlicity as regards febrile movement, etc., due to the union of dysentery with intermittent or remittent fever, or the conjoined operation of the special cause producing the latter.

The duration of the disease in cases of epidemic dysentery is very variable. In malignant epidemics, the disease sometimes runs very rapidly to a fatal issue, the collapsed condition occurring perhaps in the course of a few hours. On the other hand, cases may be protracted to the third and fourth week before either convaleseence or death takes place. As already stated, different epidemies differ widely as regards the severity of the disease. In some epidemies, the majority of cases are mild and the rate of mortality is very small; but in other epidemics the proportion of deaths is very large. The latter are justly distinguished as malignant. There are few epidemics more to be dreaded than those of malignant dysentery. They occur much oftener in tropieal than in temperate climates, but there is no part of the world exempt from their occasional occurrence. It is one of the scourges of camps in time of war, especially if, to causes operating 
particularly on armies, tropical influences be added. It prevailed to a very great extent in the late civil contest in this country, among the national and the insurgent forees, especially in the Southern States, and it was often associated with phenomena referable to malaria.

The causation of epidemic dysentery doubtless al ways involves a special cause which is undoubtedly derived from without the body, or external. The special cause is probably essentially the same in epidemic as in sporadic dysentery, differing, at different times and places, either in quantity or in morbific intensity. The various agencies which may appear to give rise to the disease, such as the exposure to atmospheric vicissitudes, fatigne, and other hardships incident to army life, insufficiency of diet, over-erowding, marsh miasmata, etc., probably act only as co-operating causes, rendering the system more susceptible to the dysenterie poison and giving rise to other morbid phenomena than those which the special cause would alone produce. Epiclemies would probably not often occur were it not for the operation of aceessory causes ; hence the rationale of their occurrence in camps, in prisons, and in situations where numbers are exposed to hygienic influences farorable for the development of any disease. With respect to the source of the special cause of dysentery, or the conditions under which it is generated and diffused, we have at present no positive knowledge. The contagionsness of epidemic dysentery has been a much mooted question. Without entering into any discussion of this question, I shall content myself with saying that this is probably one of a group of diseases, the evidence of the communicability of which rests mainly on the successive occurrence of a greater or less number of cases among members of one household, or persons who, from proximity of residence, are brought into eontact with each other; and the diffusion of the disease, under these circumstances, may be satisfactorily accounted for by the fact that the persons attacked are alike exposed to the special cause, together with the same co-operating canses. It is, however, proper to state that some distinguished anthors suppose that the intestinal excreta contain a virus by means of which the disease may be communicated. ${ }^{1}$

The opinions of different writers and practitioners concerning the treatment of dysentery, especially as an epidemic, show great diversity, and there is abundant testimony of the success of measures quite opposite in character. The fact just stated shows, either that the recoveries were due mainly to an intrinsic tendency of the disease, or that the disease is presented at different times and places under different pathological phases, and the measures of treatment require to be correspondingly varied. Both explanations are doubtless measurably applicable. Sporadic dysentery, as a rule, and not unfrequently epidemic dysentery, tend intrinsically to recovery. Recovery, under these circumstances, takes place, whatever be the treatment, provided it be not destructive. The success of treatment in such cases is shown by the short duration of the disease, its diminished severity, etc. On the other hand, in a certain proportion of cases of epidemic dysentery, the disease must, of necessity, end fatally. The extent of mucous surface affected, the loss of fluids by hemorrhage and transudation, and the destructive lesions which take place, preclude recovery under any treatment. But it is also true that the different features which the disease assumes in different epidemics, and the various morbid conditions with which it may be associated, must influence, more or less, therapeutical measures.

Purgatives, especially of the saline class, may be as appropriate in epi-

1 Vide Niemeyer, op. cit. 
demic as in sparodic dysentery; but they should be given eautiously or omitted if sero-sanguinolent dejections occur or if the symptoms denote much tendency to adynamia. It is immensely desirable to arrest or moderate the dejections just named, for these doubtless conduce to the condition of collapse into which patients are apt to fall. For this purpose, opium is to be given in as large doses as are required or as are well borne, in conjunction with astringent remedies, such as gallic acid, acetate of lead, the persulphate or pernitrate of iron, rhatania, ete. I believe the main reliance should be placed on opium, in some form, in these cases, other remedies being considered as auxiliary. The latter, therefore, are not to be employed as substitutes for opium except in cases in which, from a constitutional peculiarity, this remedy, in any form, produces such distressing effects as to preclude its use. It should not be decided that opium cannot be tolerated on the assertion of the patient, but only after a fair trial.

In this disease, as in some others, there is frequently a wonderful tolerance of opium, a tolerance not to be explained by the antagonizing influence of pain, since it is manifested in cases in which pain is not a prominent symptom. Christison, in stating this fact, adds that he has known from 24 to 30 grains of the gum of opium to be taken in the course of 24 hours. I have given 2 grains hourly, in the case of a female affected with dysentery charaeterized by abundant sero-sanguinolent dejections for the space of a week, and without, at any time, symptoms denoting nareotism. I have met with a case exemplifying a still greater tolerance of this remedy. In this case, the patient not being habituated to the use of opinm before his illness, the quantity of the sulphate of morphia, given hourly, was gradually increased to one grain, making 24 grains in the 24 hours, and this quantity was given for several consecutive days without any nareotism. Opium is not to be given as a matter of course in doses approaching these, but it is to be given with a view to the desired end, viz., the arrest of the sero-sanguinolent dejections; and if this effect be not obtained, the doses are to be increased to the amount which will be borne without nareotism, be the amount never so large. It is hardly necessary to say that, in increasing the dose, the condition of the patient is to be carefully watehed; attention is to be directed to the pupils, and to the number of respirations, as well as to the soporific state, in order to aroid a cumulation which may result, unexpectedly, in narcotism. The quantity of opium which may with prudence and propriety be given in dysentery, will vary greatly according to the varying tolerance in different eases.

In proportion as the symptoms denote adynamia, or, in other words, a tendency to death by asthenia, the powers of life are to be supported by alcoholic stimulants and nourishment. The general prineiples which should govern the employment of supporting measures are the same as in other affections which, in like manner, tend to destroy life by exhaustion. Aleoholic stimulants are sometimes tolerated in large quantity in severe cases of this disease. I have known over 40 ounces of brandy to be taken in 24 hours by a patient who, in health, was not accustomed to the use of ardent spirits, and this large quantity produced no excitation of the circulation or nervous system. This patient was apparently saved by the persevering use of stimulants with large doses of the sulphate of morphia. I have repeatedly seen patients recover under the vigorous employment of supporting measures, conjoined with opium in large doses, when, as judged by the symptoms, the condition appeared to be truly desperate. Yet, of the cases properly distinguished as malignant, many must die, despite the faithful employment of the measures on which the chief reliance is to be placed.

Dysentery associated with phenomena denoting the operation of the 
special cause of periodical fever, calls for treatment having reference to the latter, in addition to the measures addressed to the dysenteric affection. Quinia is a highly important remedy in these cases. Associated with scorbutus, the dietetic and remedial measures appropriate to this condition are indicated. Occurring as a complication of the continued fevers, it furnishes an additional indieation for the supporting measures which these fevers claim irrespective of the dysenteric complication. Coexisting rheumatism calls for the addition of the remedies which are deemed appropriate for this disease.

\section{CHRONIC DYSENTERY.}

The term chronic dysentery should be restricted to cases either of subacute and persisting inflammation of the large intestine, or of ulcerative and other lesions resulting from inflammation in this situation. 'The term, however, is sometimes incorrectly applied to cases of functional diarrhoe, and, on the other hand, the term chronic diarrhoea is often applied to cases of chronic dysentery.

The large intestine is the seat of ulcers in some cases of tuberculosis, the ulcers resulting from a tuberculous affection here; they may also be connected with carcinomatous disease. They have been found in cases of poisoning by a corrosive substance such as the bichloride of murcury, when the small intestine had escaped. They occasionally occur when their occurrence cannot be explained, and they are sometimes found after death when their existeuce had not been suspected during life.

The affection properly called chronic dysentery very rarely occurs save as a sequel of acute dysentery; recovery from the latter does not take place, but the inflammation becomes chronic, or, more commonly, ulcerations which take place during the acute affection do not cicatrize, but continue for an indefinite period. Acute dysentery, however, very rarely eventuates in the chronic form of the disease in temperate climates, and, hence, chronic dysentery is extremely rare in these climates. It is otherwise in warm climates. Here the chronic often follows the acute affection. Chronic dysentery thus prevails chiefly in tropical countries, or among those who have contracted acute dysentery in these countries. In the Northern States of the Union, the cases observed are, for the most part, imported from the South. Of the soldiers who returned from the war in Mexico, large numbers were affected with this disease; and, recently, during the civil war, cases were very numerous among the troops sent to the northern military hospitals from different points in the insurgent States.

In chronic dysentery, the evacuations, either constantly or more or less frequently, contain morbid products denoting inflammation or ulceration, viz., mucus, patches or flakes of lymph, pus, together with blood. Cases differ much in the frequency and abundance of these constituents of the evacuations. In severe cases, the stools are always loose and thin, the excrementitious contents of the intestine being liquefied by serous transudation; the function of fecation is suspended. In these cases it is to be inferred that the inflammation or lesions extend over the whole, or the greater part, of the large intestine. In other cases, dysenteric discharges occur more or less frequently in alternation with fecal dejections. In these cases, the inflammation or lesions are less diffused. If the evacuations be generally or frequently solid or moulded, the dysenteric evacuations being superadded, the inflammation or lesions are circumscribed and situated near the anus. In these cases, ulcerations may sometimes be seen, if examination be made with the speculum. 
Chronic dysentery is one of the most intractable and hopeless of diseases. For a time, if the evacuations be held in check by palliative measures, the appetite and digestion not being greatly impaired, the general aspect and strength may not show much deterioration, but at length the appetite and digestion fail, and the continued irritation and loss of fluids induce progressive emaciation and debility. The duration of the disease embraces usually several months, and sometimes years. If not destroyed by some intercurrent affection, the patient becomes extremely emaciated, reduced almost to a skeleton, the surface is usually dry, cool or cold, the pulse becomes more and more feeble; the mental faculties are weakened, delilium rarely occurring, but the mind, in certain cases, falls into an apathetic state, the patient being indifferent to, and taking but little notice of, persons and things around him. Anorexia becomes complete, and vomiting, in some cases, is a prominent symptom; œedema of the lower limbs sometimes occurs; ulceration of the cornea is an occasional event, and I have known the colnea to be perforated, with loss of the humors of both eyes; the mode in which a fatal termination takes place is generally typical of dying by slow asthenia.

The observations of Prof. A. Clark, at Bellevue Hospital, in the winter of 1862-63, when this hospital was thrown open to sick soldiers from the peniusular campaign in Virginia, go to show the frequent oceurrence of disease of the kidneys in cases of chronic dysentery. In seventeen cases reported by the late Dr. Henry W. Cooke, house physician, ${ }^{1}$ in which antopsies were made under the direction of Prof. Clark, in all the kidneys were found to be more or less diseased. They were moderately increased in weight, the secreting or cortical portion lighter in color than natural, and having a gramular appearance. On microscopical examination, the tubuli uriniferi were found filled with granules and detached epithelium. In these and numerous other cases, the urine, examined during life, was found to contain casts of the uriniferous tubes, but no albunen. It remains to be ascertained whether these evidences of renal disease were peculiar to this group of cases of chronic dysentery, or whether they are to be found in cases of the disease occurring at all times and places; and, with respect to the cases in which the kidncys become affected, it is an interesting question, how far the renal affection contributes to the symptomatic phenomella of the disease. Urmic poisoning was not evidenced, in any of the cases observed by Prof. Clirk and others, by convulsions or coma, perhaps because the excessive aecummulation of urea in the blood was prevented by the abundant draining away of serum through the intestinal canal. The mental apathy, anorexia, vomiting, and the oceasional oclema, may be fairly attributed, in part at least, to diminished elimination of urea by the kidneys.

The diagnosis of chronic dysentery is not altogether easy. The disease is to be discriminated from functional diarrhoea, and this is not always readily done. The presence of pus, lymph, mucus, and blood at times, if not frequently or constantly, is the important point in the differential diagnosis. It is also to be discriminated from ulceration of the large intestine due to tuberculosis, or carcinoma, or other causes. Tuberculosis may be excluded if the lungs be free from the deposit of tubercle. In carcinomatous disease, frequently a tumor is to be felt throngh the abdominal walls, or if seated in the rectum it is accessible to ex:mination by means of the touch and speculum. The fact that chronic, is, in the great majority of cases, preceded by acute dysentery, is highly important in the diagnosis.

1 American Medical Times, Feb. 20, 1863. 
We may conclude at once that the disease is chronic dysentery if it have followed the acute disease, and the latter fact may generally be without difficulty ascertained, owing to the well-marked diagnostic symptoms of acute dysentery. In temperate climates the affections with which chronic dysentery is liable to be confounded are most likely to exist in cases of doubt, owing to the great infrequency of the latter.

'The treatment of chronic dysentery relates, first, to the local affection. Remedies to allay irritation and to promote the healing of ulcerations are indicated. But, unhappily, in the great majority of eases, there is very little probability that a cure will be effected, and all that can be hoped for from judicious treatment are palliation of symptoms and prolongation of life.

Certain remedies have been employed with a view to their direct action upon the affected portions of the intestine. The nitrate of silver is one of these; but it is absurd to suppose that this remedy, given as largely as prudence will allow, passes through the stomach and small intestines unchanged. Whatever benefit may be derived from it, therefore, is not to be explained on the ground of its topical applieation to the diseased surface. The balsamic medicines, particularly the balsam of copaiba, have been given for the same purpose, and with more reason ; but clinical observation shows not much benefit to be derived from them. The subnitrate or carbonate of bismuth may be included among the remedies supposed to act by coming in contact with the affected parts. This remedy is often beneficial as a palliative, the dejections becoming less frequent, with relief of pain, etc., under its use. It should be given in doses of from 20 to 30 grains three or four times daily. Some have recommended much larger doses, and, given almost without limit, it prorlnces no deleterious effects ; but probably all the benefit to be derired from it is secured by the doses just stated. Its administration in enemas has been advocated in this disease. The various astringent remedies are to be tried in succession. Of those most likely to be useful, the following may be mentioned: tannic or gallic acid, alumen, rhatania, rubus villosus (blackberry-root), and the persulphate or pernitrate of iron. Dr. Beck, of Cincinnati, recommends, as a valuable remedy in both acute and chronic dysentery, but especially the latter, common resin, given in doses of a scruple or more every four hours.' He states, in a letter to the author, that he has used this remedy in his practice, with much success, for the last twelre years. He attributes its efficacy to its local action on the diseased membrane.

In so far as the dejections are controllable by the foregoing remedies, they shonld be relied upon to the exclusion of opium, because they will interfere less with the appetite and digestion. Opiates, however, will be required, to a greater or less extent, in conjunction with other remedies. So far as practicable they should be administered by the rectum, either in enemas (a salt of morphia, or some liquid preparation, contained in an ounce of mucilage or thin starch-water), or in suppositories. The lower part of the rectum should be examined with the speculum, and appropriate topical applications made if uleers in this situation be discovered.

The treatment relates, secondly, to the system. In relative importance, the general take precedence of the local measures. The surface of the body should be well protected against atmospheric changes. The diet should be nutritious but bland, articles of food being selected which do not leave much indigestible matter to pass into the large intestine. Milk, eggs, and farinaceous food are generally best suited, but in some cases animal food

${ }^{1}$ Cincinnati Mredical and Surgical News, edited by J. C. Beck, M.D., July, 1863. 
is found to agree. The plan of diet is to be governed by experimental trials. Tonic remedies and alcoholic stimulants are indicated by defeetive appetite and digestion. Of tonic remedies, quinia, the chalybeate preparations, the mineral acids, and the bitter infusions are generally to be employed in succession. The use of alcoholic stimulants is to be regulated by their effects in individual eases. Moderate out-of-cloor exercise and mental recreation are important. Change of climate is sometimes effectual when other measures prove unavailing. 'The experience of the late war has shown the happiest effect of transferring soldiers to nortlem hospitals in salubrious rural situations.

\section{Inflammation and Perforation of the Cæcum. Fecal Abscess.}

Inflammation of the cxcum, the inflammation limited to this portion of the large intestine, constitutes an affection called typhlitis, tuphlo-enterilis, or ceccilis. Acute inflammation confined to the cxecum is not of frequent occurrence. 'The symptoms are, pain and tenderness within a cireumscribed space corresponding to the situation of the exeum, with vomiting, diarrhoa, and febrile movement. It is liable to be confounded with phlegmonous inflammation comected with disease of the vertebra, renal calculus, circumseribed peritonitis from perforation of the vermiform appendix, and, in the female, inflammation of the right ovary. Peritoneal inflammation, limited to the portion of peritoneum covering the cacum, may accompany the affection. The pain and tenderness are then more marked, and the cecum is distended by an accumulation of gas, the peritoneal inflammation producing paralysis of the muscular tunic. The distension sometimes is so great that this portion of the large intestine is distinetly seen and felt through the abdominal walls. Diarrhea is not uniformly present. Extension of the limbs may occasion pain, as in cases of general peritonitis. The cases which I have seen have terminated in recovery. I have not met with perforation followed by either general peritonitis or feeal abscess in a case of acute inflummation of the cacum. Subacute or chronic inflammation gives rise to symptoms less distinctive. It may oceur, and recovery take place without the development of symptoms denoting an affection of importance; but it becomes a very important affection in certain cases in which it leads to ulceration and perforation of the intestinal coats. If the perforation take place in that portion of the creum not invested with the peritoneum, the gaseous and other contents of the intestine, escaping into the areolar tissue, give rise to inflammation which may be either diffused or circumseribed, in the latter case forming an abscess called a fecal abscess. If diffused, the inflammation may extend more or less over the abdominal walls, which become swelled, pitting on pressure as in odema, with a feeling of erepitation due to the permeation of intestinal gas. If an incision be made, a dark, sanious liquid escapes with gas emitting an intestinal odor. Under these circumstances, a fatal result is inevitable. If, however, the perforation lead to a circumscribed abseess which opens externally, gas and fecal matter, with pus, escape, a fistulous communication with the intestine is established, and this may remain permanently, or it may gradually close and recovery take place. Some years since I met with a medical gentleman who, several years before, had crecitis with perforation and fecal abscess, resulting in a fistula which had gradually diminished, and, at that time, had nearly closed, intestinal gas having ceased to escape for many months. The recovery, in this ease, seemed to be nearly complete. Perforation taking place tlirough the portion of the 
intestine invested with the peritoneum, occasions the development of peritonitis. Perforation of the appendix veriformis, occurring irrespective of cacitis, is of not very infrequent occurrence, and will be noticed in treating of peritonitis.

From an analysis of thirty-two cases of fecal abscess, Prof. Roberts Bartholow derives the following conclusions :' In the majority of cases, perforating ulcer occurs irrespective of the presence of extraneous bodies; it was attributed to the latter in only six of thirty-two cases. In a large majority of cases, the perforating ulcer selects the posterior part of the gut. Of fourteen cases, in two only did the ulcer involve the portion of the bowel covered by peritoneum. Perforation of the vermiform appendix is not embraced in this statement. If the perforating ulcer be situated in the part covered by peritoneum, general peritonitis, with a fatal termination, is the rule; but circumscribed peritonitis, with adhesions, sometimes occurs, and fecal abscess is the result. Pcrforation of the appendix usually leads to general peritonitis. Adhesions, however, sometimes occur, perhaps before the perforation takes place, and a fecal abscess, without general peritonitis, may be a result. It is probable that suppurative inflammation of the sub-creal connective tissue, irrespective of either perforation or of prior disease of the cæcum, is very infrequent, if indeed it ever occul's. In cases of perforation of a portion of the cæcum not covered by peritoneum, cansed by a foreign body, the prognosis is favorable. Recovery takes place in one-half of the cases of perforation of a part not covered by peritoneum, by a perforating ulcer. The prognosis is always unfavorable in cases of perforation of the appendix. As regards diagnosis, feeal abscess is to be discriminated from psoas abscess, abscess with caries of pelvic bones, pelvic cellulitis, abscess in abdominal wall, and disease of the hip-joint. Tympanitic distension and crepitation on pressure due to the presence of intestinal gas are diagnostic points.

A specimen was shown to the New York Pathological Society, by Dr. Krackowitzer, March, 1867, in which the vermiform appendix had become attached to the bladder and perforation had followed, causing a vesicointestinal fistula. The first evidence of this was the appearance of a lumbricoid worm at the end of the penis, the patient then being eight years of age. Subsequently other worms were discharged, and repeatedly the seeds of fruit. Most of the urine was discharged by the bowels. The fistula for many years gave but little inconvenience. The patient served as a soldier in the late civil war. He was operated upon for stone in the bladder when 28 years of age, and died five days after the operation, the fistula thus having existed for twenty years. A similar case is given by Davaine in his work on Entozoa.

The late James Jackson, of Boston, in his admirable work entitled "Letters to a Young Physician," describes an affection under-the name, "a painful tumor near the cæcum." As this name implies, pain is felt in a situation near the cxcum, and a tumor is found in this situation. The pain and tumor are situated on a horizontal line connecting the two anterior superior spinous processes of the ileum, at the point where this line intersects the right margin of the rectus muscle on the right side. The pain is sometimes marked, and felt especially on movements of the body. The tumor is oval, and is quite small. It is not superficial, but is felt on deep pressure through the abdominal wall. Marked tenderness on pressure exists within a circumscribed space over the tumor. In the cases detailed by Dr. Jackson, the pain and tenderness, together with the tumor, slowly

Vide American Journal of Medical Sciences, October, 1866. 
disappeared under treatment with catharties, leeches, a blister to the part, and opium to relieve pain. Dr. Jackson confesses his inability to form an opinion as to the precise seat and character of this affection. I will venture the conjecture that it is inflammation of the mucous membrane lining the appendix vermiformis, with clilatation, either from the products of inflammation or fecal matter, the inflammation extending, perhaps, in some eases, more or less, over the lining membrane of the crecum. Several cases presenting the features described by Jackson have fallen under my observation. In none of the cases which I have observed has perforation taken place, and none have ended fatally.

\section{CH A P TER II.}

Acute Enteritis-Anatomical Characters-Clinical History-Pathologicai Character-Causation - Diagnosis - Prognosis-Treatment. Subacute and Clironic Enteritis. Acute GastritisAnatomical Characters-Clinical History-Pathological Character-Causation-DiagnosisPrognosis-Treatment. Subacute and Cironic Gastritis. Duodenitis. Pharyngitis. Parotiditis.

\section{Acute Enteritis.}

TNFLAMMATION of the mucous membrane of the small intestines is designated enteritis. This name, signifying inflammation of the intestine, is conventionally restrieted in its application to inflammation of the small intestine. As a rule, inflammation is limited to either the small or large intestine, but as, exceptionally, in cases of epidemic dysentery the inflammation extends from the cxeum into the ileum, so, in some cases of enteritis the inflammation descends into the colon. Are the several portions of the small intestines liable to be inflamed separately; in other words, may ileitis, jejunitis, and duodenitis occur as separate affections? There is ground to suppose that the duodenum may be inflamed by itself, or, at all events, duodenitis has certain pathological relations which render it desirable to consider it as a distinct affection, but it is impossible to distinguish clinically jejunitis and ileitis.

The small intestine is the seat of a highly important local affection, seated in the Peyerian and solitary glands, which is characteristic of typhoid fever. It is also the seat of the deposit of tubercle in a certain proportion of the cases of tuberculosis. Exclusive of these affections, which are elsewhere considered, acute enteritis is a rare form of disease after infantile life. It belongs among the affections to which young children are especially prone. Yet examples are occasionally met with in practice, occurring in the middle-aged and the aged, as well as in the young. Referring the reader to works which treat of the diseases of children for an account of this discase as occurring in infantile life, it will require but a brief consideration.

Anatomical Characters.-As the disease, in itself, rarely proves fatal, the opportunity of inspecting the appearances after death is not often offered. This fact shows that lesions corresponding to those in fatal cases 
of dysentery-ulcerations, sloughing, sphacelus-do not occur in enteritis. The mucous membrane is usually more or less reddened; the redness is arborescent, and is best shown by an examination of dried specimens; the membrane is softened and thickened; softening of the submucous tissue from infiltration also occurs, rendering the detaehment of the membrane easier than in health; ecchymoses within and beneath the membrane are sometimes observed. The Peyerian and solitary glands remain unaffected. The affected surface is more or less abundantly coated with mucus. The morbid changes are either limited to the ileum or more marked in this portion of the small intestine, and the lower part of the ileum is usually most affected.

Clinicar Historx.-The local symptoms, in cases of acute enteritis, are pain, tenderness over the abdomen on pressure, and diarrhœa, with nausea and romiting. 'The pain is not intense, and is of a dull, aching character, with exacerbations in which it resembles the pain of colie. The pain is referred to the umbilical region. 'The tenderness is not great. Slight pressure is well borne, but deep, firm pressure oecasions more or less suffering. The tenderness is over the lower part of the ileum and around the umbilieus. Diarrhea is generally, if not always,.present. It is stated by Habershon that the bowels may remain eonstipated through the disease, but examples are probably very rare. The stools are more or less frequent, and in eharacter they are loose or watery, and gelatinous from mucus intimately mixed. Tenesmus does not exist unless inflammation of the lower part of the larger intestine be added. The dejections are frequently acrid, producing a painful, burning sensation in their passage. Dysentery is sometimes developed, perhaps, by the irritation caused by the acrid matter contained in the dejections. Nausea and vomiting are excited sympathetically, or, in some cases, perhaps, from an extension of the inflammation to the stomach:

The general symptoms usually do not denote great constitutional disturbance. The febrile movement is slight or moderate. The debility is not great, but sufficient to keep the patient in bed. The intellect is unaffected except in severe cases, when delirium may oceur, together with tympanites, hiceough, and symptoms denoting adynamia.

The duration, in favorable cases, is brief, convalescence being declared in a week or ten days.

Pathological Character.-Exeluding enteritis occurring in connection with typhoid fever, tubereulosis, and the diseases of infancy, the disease has no speeial pathological character. It is a simple inflammation of a mucous membrane. It very rarely occurs save as a sporadic disease, in this respect differing from dysentery. It differs also from dysentery in not leading to the destructive lesions of the affected membrane like those which occur especially in the epidemic form of that disease.

Causation.-The susceptibility of the mucous membrane of the small intestine to acute inflammation, after infancy, is slight. It may be produced by excesses in the use of stimulating food and alcoholic drinks, by poisoning with aerid substances, and by the use of drastic purgatives. It is sometimes attributable to the action of eold upon the body when heated and perspiring. Fatigue and debility will co-operate with these causes. But, like inflammation seated in other parts, it may be developed without any appreciable causation. 
Diagnosis.-Acute enteritis is to be discriminated from dysentery, gastritis, colic, peritonitis, and typhoid ferer. The diserimination from dysentery is sufficiently easy, if the inflammation be limited to the small intestime. Assuming that the local symptoms are sufficient to denote inflammation somewhere within the abdomen, the absence of the characteristic dysenteric evacuations, of tenesmus, and of tenderness in the tract of the colon, suffices to exclude dysentery. The only room for doubt is when more or less inflammation of the large intestine is superadded to the enteritis. In such cases, the diagnosis of enteritis is to be based on the existence of the symptoms of this disease for some days prior to the occurrence of the symptoms of dysentery, and on the predominance of the enteritic symptoms. The cases in which the supervention of dysentery renders this differential diagnosis in any degree difficult are extremely rare.

Nausea and vomiting, if unusually prominent, may suggest acute gastritis. But the symptoms of acute gastritis are of a much graver character than those of acute enteritis, and they point distinctly to the stomach as the seat of the disease. This disease may generally be excluded without difficulty. The two diseases, however, may be combined. The inflanmation, in some cases, perhaps, extends from the small intestine to the stomach ; and, in cases or poisoning with acrid substances, the local effects of the latter may be produced within the small intestine as well as in the stomach.

Colic is a functional affection characterized by intense pain, without tenderness or febrile movement, and is oftener accompanied by constipation than diarrhœa. These points are sufficient for its exclusion.

As regards the local symptoms, acute enteritis approximates to acute peritonitis more closely than to any other affection. But, as a rule, there is a marked disparity in the intensity of the symptoms in these two affections. The pain, tendemess, and tympanites common to both, are, in the great majority of cases, notably greater in peritonitis. Diarrhœe, which is the rule in enteritis, ocenrs exceptionally in peritonitis. Rigidity of the abdominal muscles, which is one of the diagnostic symptoms of peritonitis, is wanting in enteritis. Moreover, the constitutional disturbance, as denoted by frequency of the pulse, prostration, etc., is mueh greater in peritonitis. $\Lambda$ ttention to these points renders the differential diagnosis, in most cases, sufficiently easy.

The abdominal symptoms in typhoid fever may lead the physician to suppose that disease to be simply enteritis; and, on the other hand, a primary enteritis may be supposed to be typhoid fever. Typhoid fever is to be excluded by the absence of the diagnostic symptoms which attend its slow development; and its progress, viz., cephalalgia, epistaxis, bronchitis, the rose eruption, and mental aberration. Moreover, the adynamic symptoms in typhoid fever are generally greater than in simple acute enteritis, while the enteric symptoms are less marked.

Proc nosis.-Acute enteritis, after infancy, in the majority of cases, is not a disease of great gravity. If it be not associated with any other important affection, and the patient be not of feeble constitution, a favorable prognosis may generally be entertained. The disease, however, sometimes ends fatally. This is to be apprehended when the pulse becomes feeble and fiequent, the prostration marked and delirium is developed, the local symptoms persisting. The mode of dying is by slow asthenia, as in cases of dysentery.

Treatment. - The indications for treatment may be embraced in a few 
words. An efficient purgative is generally, at first, advisable. Afterward, opium in some form is to be given sufficiently to relieve pain and diarrhœa. It may be administered by either the mouth or rectum, or, alternately, in both modes. It is to be given in doses proportionate to the urgency of the symptoms, and graduated accorcling to its effects. Fomentations over the abdomen by means of poultices, the water dressing, or spongio-piline, are useful. Moderate stimulation of the surface of the abdomen by sinapisms or liniments is appropriate. General or local depletion by bloodletting is very rarely, if ever, called for. Mercury, as an alterative remedy, or given to "improve the secretions," is not to be recommended. The supporting treatment is indicated in proportion to the duration of the disease and the tendency to failure of the vital powers. The diet at first should be restricted and bland ; but alimentation is, of course, important if supporting measures be indicated.

\section{Subacute and Chronic Enteritis.}

Chronic enteritis, save as connected with tuberculosis, is much more infrequent after infancy than the acute form of the disease. The symptoms are the same as in acute enteritis, but less marked. Anodyne and astringent remedies are indicated, with moderately revulsire applications to the abdomen, tonic remedies, and a nutritious but carefully regulated diet.

Subacute enteritis, not becoming chronic, is of frequent occurrence, arising either from dietetic errors or an arrest of digestion by exposure to cold, over-exertion, ctc. It is not easy to distinguish, practically, cases of transient subacute enteritis from cases of functional diarrhoa; nor is it of much practical consequence to make this discrimination, since the indications for treatment in either case do not materially differ, embracing mild purgation, followed by anodyne remedies and regulation of the ingesta. Enteritis, acute, subacute, and chronic, is described by some authors under the name intestinal catarrh. Here, as in other connections, the term catarrh is not adopted, inasmuch as it seems to be superfluous, and its use leads to confusion. Authors who treat at length of intestinal catarrh, embrace under this head cases which I shall notice in connection with diarrhoa and intestinal dyspepsia.

\section{ACUTE GASTRITIS.}

A remarkable change has taken place within the last quarter of a century in the opinions of physicians respecting the occurrence of acute inflammation of the stomach. It was formerly supposed to occur frequently. According to Broussais, whose doctrines, for a time, were accepted by many, inflammation of the mucous membrane of the stomach and small intestine (gastro-enteritis), constitutes the disease in all the essential fevers, and exists in most, if not all, cases in which symptomatic fever becomes developed. Reasoning à priori, it would seem that gastritis must be a frequent disease. This would be inferred from the quantity of ingesta which this organ daily receives, the various stimulating and irritating substances which enter into the alimentary supplies, its great functional activity, the alternations during and between the acts of digestion as regards the quantity of blood which it contains, and its extensive glandular arrangement. But late pathological and clinical researches have shown that acute inflammation in this situation is one of the rarest of diseases. Its existence as a spontaneous discase has even been denied. It is developed spontaneously, but so infrequently that many physicians of long experi- 
ence have never met with an example of it. Valleix was able to collect only seventeen authenticated cases for analysis. ${ }^{1}$ What is known of the disease is chiefly derived from cases in which it has been produced traumatically, that is, by the direct contact of acrid or corrosive poisons. It should be added that the term acute gastritis is understood to denote gastric inflammation of such intensity as to place life in imminent danger. $\Lambda \mathrm{s}$ will be seen in treating of subacute gastritis, the inflammation in some cases of the latter affection approximates closely to acuteness, and, in fact, the line of demareation between acute and subacute gastritis is fixed somewhat arbitrarily. Acute gastritis, as here considered, embraces only the most severe of the cases included by recent French and German authors, e.g., Niemeyer and Jaccoud, under the name acute gastric catarrh.

Anatomical Characters.-If the disease destroy life quickly, as it sometimes does, marked redness, arborescent and punctiform, may constitute the chief morbid appearance. 'I'his appearance, however, may be produced by hyperamia incident to venous obstruction arising from lesions of the liver or heart, or of both organs. It is to be taken as evidence of inflammation when the hyperamia is limited to, or especially marked in, the stomach; when it is not associated with hepatic or cardiac lesions, and when the symptoms of gastritis lad existed during life. If, however, life be not quickly destroyed, in conjunction with the redness, the mucoins membrane and submucous tissue are notably softened, this change being observed in situations where it is not attributable to the action of the gastric juice. The mucous membrane is thickened, and the surface is usually covered with an abundance of mucus. The organ is contracted. The rugæ are few or wanting. If the disease have been produced by a caustic poison, such as some one of the corrosive acids, cyanide of mercury, etc., portions of the membrane which have come into contact with the poison present either eschars or ulcerations following the separation of slouglis. The destruction of tissue may not be limited to the mucous membrane, but may extend to all the coats, leading sometimes to perforation. The inflammation in cases of poisoning by the essential oils, or by any of the acrid poisons, is sometimes accompanied by diphtheritic exudation. In cases of poisoning, the action of the irritant or caustic substance ingested occasions more or less inflammation and perhaps sphacelation within the pharynx and asophagus. The inflammation in cases of acute gastritis may extend into the duodenum.

Chinicar History.-Acute gastritis gives rise to intense pain of a burning character, referred to the epigastrium, together with a painful sense of constriction, the pain shooting into the chest. The pressure of the diaphragm upon the stomach in inspiration increases the pain, and hence the breathing may be costal, and the number of respirations per minute is increased. Nausea and vomiting are prominent symptoms. In some cases the stomach is intolerant of water or the blandest liquids, even in very small quantities. The vomited matter is muco-serous, of a greenish color, the presence of bile being evident from the bitter taste, and not infrequently it is sanguinolent. The acts of vomiting occasion great suffering. The thirst is frequently, but not always, intense. The desire for cold water is sometimes so irresistible, that the patient is not deterred from drinking it by the painful acts of vomiting which it provokes. Tenderness on pressure over the epigastrium is marked. The pulse is more or

1 Guide du Médecin Practicien. 
less frequent and small. The temperature of the skin is moderately, and sometimes considerably, raised. The bowels are constipated except in cases of poisoning. Dysphagia and aphonia are occasional symptoms. The tongue presents no special morbid appearances. The mind is depressed, and the expression is anxious and haggard. If life be not speedily destroyed, and the disease rum on to a fatal termination, vomiting of black, grumons, coffee-ground matter is likely to occur. Toward the close of life the contents of the stomach are ejected by acts of regurgitation rather than by vomiting. The prostration becomes extreme. Hiccough occurs. The pulse becomes very frequent and thready. Coldness of the surface is marked. The mode of dying is by asthenia. The career of the disease is rapid. Data are not available for determining the average duration. In fatal cases, death takes place usually within a few days; and it takes place in some cases within twenty-four hours, being produced in such cases apparently by shock. In a case reported by Dr. T. C. Finnell, at a meeting of the New York Pathological Society, in which a young girl took for self-destruction an infusion made by steeping in warm water a box of lucifer matches, death took place in six hours. If the disease end in recovery, improvement in all the symptoms takes place slowly; convalescence is gradual, and the inflammation may persist in a chronic form. In cases of gastritis produced by the corrosive poisons, if perforation of the stomach occur, the symptoms of peritonitis are added. In a case of spontaneous acute gastritis which came under my observation, slight jaundice occurred toward the close of life. The examination, after death, in this case, showed that the inflammation extended into the duodenum.

Pathological Character.-The stomach, aside from its office as a receptacle of the ingesta, is to be regarcled as a glandular organ, furnishing daily a large quantity of an important secretion--the gastric juice. In its reluctance, so to speak, to take on acute inflammation from the internal and inappreciable causes which give rise to the so-called spontaneous inflammations in other situations, it resembles other glands, such as the liver, pancreas, kidneys. In the extremely rare instances in which acute inflammation is spontaneously developed in this situation, it does not differ in pathological character from acute inflammation seated elsewhere in a mucous structure. The gravity of the disease depends on the physiological relations of the stomach. Acute gastritis produced, traumatically, by the action of caustic or irritant poisons, is developed like inflammation from the action of the same local causes in other situations.

Causation.-Exclusive of the action of irritant and corrosive poisons, it is difficult to say what causes may give rise to acute gastritis. It may be produced by excessive indulgence in alcoholic drinks, especially when taken without food, but the alcohol in these cases doubtless acts as a local irritant. The same remark will apply to the over-ingestion of highly stimulating food. Various causes mentioned by writers, such as copious libations of cold water, intense moral emotions, were supposed to produce the disease in eases in which the diagnosis was based upon insufficient ground. In a well-marked case which has fallen under my observation, the patient was an habitual drinker, but the attack was not referable to a debauch. The patient attributed the disease to lying in a cold cellar. In this case, the evidence of the disease was based on the appearance after death, as well as the clinical history. I have known the disease to follow a debauch, and prove rapidly fatal. 
Dinanosis.-The symptoms of acute gastritis are strongly diagnostic. There is greater probability of the disease being incorrectly supposed to exist, than of its being mistaken, when existing, for other diseases. Vomiting, however frequent and persisting, is never alone sufficient evidence of gastritis. I have known a case of elironic meningitis with few symptoms referable to the head, but with persistent vomiting, to be treated for acute gastritis, and the error not discovered prior to the autopsy. Peritonitis, if accompanied with romiting, may assume an appearance of gastritis, but the diffusion of tenderness over the abdomen, the tympanitic distension, and the rigidity of the abdominal muscles, generally suffice for this differential diagnosis. Dr. Stokes has known gastritis to be mistaken for pneumonitis, but plyysical exploration of the chest should enable the physician to detect or exclude the latter affection. The author just named enforces the danger of overlooking gastritis developed after a debanch. I can testify, from my own experience, to this liability to error. Many years ago, in hospital practice, my attention was called to a patient who had been drinking to excess, and who was supposed, without close examination, to be affected with incipient delirium tremens. On my visit the following day, the ease had ended fatally, and an examination after death revealed the existence of acute gastritis.

With a view to appropriate treatment, and, also, in a medico-legal point of view in certain cases, it is desirable to determine from the symptoms, whether acute gastritis be attributable to poison. This is always to be suspected, owing to the great infrequency of the disease as otherwise produced. Burning in the throat is complained of, if a caustic or corrosive poison lave been taken, and the local action of the poison may be seen in the mouth and fauces. The matters vomited contain blood earlier and in larger quantity, as a rule, if the gastritis be due to a poison. Diarrhœa is more apt to occur in cases of poisoning. If caused by a poison, the disease is developed suddenly, and inquiry is to be made to ascertain if the sudden development were not immediately preceded by a meal or the ingestion of something in the way of food or drink.

Gastralgia was formerly confounded with gastritis. The paroxysmal occurrence of pain, or its increase forming marked exacerbations, the absence of febrile movement, the occurrence of vomiting in some cases only, the absence of tenderness on pressure, and the ability to take food sometimes with relief of the pain, sufficiently characterize the former affection as neuralgic.

Prognosis.-The opinions of different writers differ respecting the gravity of this disease when it is idliopathic. $\Delta \mathrm{s}$ it is only within a few years that the diagnosis lias been placed on a correct basis, and the dise ease being so infrequent, the number of reported cases is not sufficient for determining the rate of mortality. It is undoubtedly a disease of great gravity. It may destroy life quickly, within a few hours, the mode of dying being by rapid asthenia or shock. These eases, however, are exceptional. The mode of dying is generally by slow asthenia.

When the disease is due to the action of an irritant or eorrosive poison, the danger will depend, other things being equal, on the amount of local injury. Death in these cases sometimes takes place within a few hours by rapid asthenia or shock, dependent, not so much on the amount of local injury, as on the inability of the system to tolerate it. In some instances life is prolonged for several days, although the local injury be very great. A striking illustration of this fact is afforded by the case of a man who, at the burning of the Eddystone Lighthouse in 1755, swallowed a 
quantity of molten lead, which accidentally dropped into his mouth when looking upwards to observe the progress of the fire. This patient lived for several days, and, after his death, a lump of lead, weighing 7 ounces, 5 drachms, and 8 grains, was taken from the stomach.'

Treatment.-The indications for bloodletting are rarely, if ever, present in this disease. Bearing in mind that the danger is from asthenia, and that life is sometimes quickly destroyed, it may fairly be doubted if the abstraction of blood is in any case admissible. The first and most. important object in the treatment is to seeure for the inflamed organ as much rest as possible. With reference to this object, the less of anything introduced into the organ the better. To allay intense thirst, small pieces of ice may be swallowed, or iced water taken often, in a very small quantity at a time. The physician should resist the temptation to try in succession the host of remedies to allay romiting, all of which will be likely to aggravate this distressing symptom. It is desirable to administer opiates in order to quiet the stomach and render the system more tolerant of the disease. The salts of morphia placed dry upon the tongue may be tried, and, if romiting be provoked by this mode of administration, they may be sprinkled over a vesicated surface, or administered hypodermically, or some form of opiate given per enema. Fomentations or a small blister may be applied over the epigastrium. Cathartics are inadmissible for the same reason that Cayenne pepper would be an inappropriate topical application to an inflamed eye. The bowels may be moved by stimulating enemas. Mercury, either in large or small closes, is not indicated more than in a case of an extensive burn or scald of the integument. The importunities of the patient for an emetic are to be resisted. He may have a feeling as if the stomach were distended, or as if there were an accumulation there aggravating his distress. This feeling arises from the inflamed state of the membrane, as tenesmus is incident to inflammation of the rectum.

If the symptoms denote a tendency to death from asthenia, the powers of the system are to be supported. The stomach, however, will not bear alcoholic stimulants and concentrated nourishment. These are to be introduced into the rectum in quantities proportionate to the urgency of the symptoms. If, on the other hand, the progress of the disease be favorable, and supporting treatment be not urgently called for, bland nourishment may be cautiously ingested when the improvement in the symptoms renders it probable that food will be retained. Milk, with the addition of lime water, will be likely at first to be best borne, given in small quantities at short intervals. Other forms of food may be gradually added. After the inflammation has disappeared, the stomach will be left in an atonic condition, and remedies of a tonic character may be indicated during convalescence. If the acute end in the chronic form of inflammation, the treatment due to chronic gastritis is to be employed.

Acute gastritis caused by the acrid or corrosive poisons is to be treated after the plan just stated, with the addition of measures having reference to the evacuation and neutralization of the poisonous substance. Emetics are to be promptly given, and if they fail to act efficiently, the stomachpump is to be resorted to, provided the poison be one which can be ejected. Arsenic, corrosive sublimate, alcohol, antimony, cantharides, copper, phosphorus, together with a great number of vegetable irritants, such as capsicum, gamboge, croton oil, etc., may be expelled from the 
stomach; but, if any of the corrosive acids have been taken, reliance must be placed on the prompt administration of antidotes. Antidotes are also to be given after the employment of emetics or the stomach-pump to effect the removal of acrid poisons. For details respecting the management of cases of poisoning, the reader is referred to works which treat of toxico$\log y^{1} \quad$ I shall simply subjoin, for the convenience of the practitioner, an enumeration of the antidotes for the more important of the poisons which act locally upon the stomach.

Arsenical Preparations: 'The hydrated sesquioxide of iron is the special antidote. It should be recently prepared, and given in large quantity. If this preparation be not immediately available, the sesquioxide or common red oxide of iron (crocus martis) may be given in its stead. Bouchardat advises to combine with the preparation of iron, magnesia, in orcler to promote the passage of the iron from the stomach into the intestines.

Corrosive Sublimate and other Salts of Mercury: The most reliable antidote is albumen, and the white of eggs is the best and most available article.

Salts of Copper: Albumen is an antidote. Iron reduced by hydrogen and the hydrated persulphate of iron are said to be antidotal, by Bouchardat, both to the salts of mercury and copper.

Tartar Emetic: Substances containing tannin in abundance, and magnesia.

Nitrate of Silver : Chloride of sodium.

Mineral Acids : Magnesia or chalk mixed with water or milk; the alkaline carbonates or soap.

Oxalic Acid : Magnesia, chalk; whiting or plaster scraped from the wall of an apartment may be given, mixed with water. Alkalies are not to be given.

Ammonia: Vinegar or any of the vegetable acids.

Potassa and Soda: The same.

\section{SUBACUTE GASTRITIS.}

Subacute gastritis, not becoming chronic, but of transient duration, occurs not infrequently. It is not easy to say with positiveness how frequently it occurs, because practically it is difficult to fix the line of demarcation between slight inflammation and merely functional clisturbance in this situation. Subacute gastritis doubtless enters into certain cases of the affection which will be noticed in connection with functional disorder of the stomach, under the name acute dyspepsia. Subacute as well as acute inflammation, when not involving diphtheritic exudation, is called by some authors catarrh or catarrhal inflammation of the stomach. The term catarrh, however, is superfluous here as in other applications. It is to be noted that the secretion of mucus, in more or less abundance, is a physiological event in connection with hyperæmia during stomach digestion. In this respect the gastric mucous membrane differs from mucous membranes in other situations; the latter, as a rule, do not secrete mncus in abundance except in abnormal conditions. Subacute inflammation of the stomach is much more frequent in infants and young children than in the after periods of life. As here considered, reference will be had chiefly to the affection occurring subsequently to the period of infancy.

The degree of inflammation in different cases of subacute gastritis varies,

I Taylor on Poisons may be consulted for this purpose. 
being either slight, moderate, or considerable, as evidenced by the local and general symptoms. Inasmuch as it never proves fatal per se, the opportunity of observing the morbid appearances post mortem is rarely offered. There is doubtless more or less redness from hyperæmia or congestion of the mucous membrane, but this may measurably or entirely disappear after death. The quantity of mucus is morbidly increased, and the contents of the stomach may, in consequence, have an alkaline reaction. The membrane is more or less thickened and softened, but it is diffieult to determine to what extent the latter may be due to cadaveric change.

The local and general symptoms are marked according to the intensity of the inflammation. In cases in which the inflammation is slight or moderate, the appetite is impaired but not lost. The ingestion of food is followed by uneasiness or distress referred to the epigastrium, and by symptoms denoting indigestion, namely, flatulency, eructations, etc. 'Thirst is a symptom which is constant and is more or less frequent. Tenderness over the epigastrium and a furred or coated tongue are other symptoms. The urine is high-colored and loaded with the urates. In some cases there is constipation, and in other cases diarrhœe, the latter being due to intestiual indigestion. Pain in the head is a frequent, and sometimes a prominent symptom; and when connected, as it frequently is, with nausea, it is commonly known as "sick headache." There may be slight febrile movement, but it is frequently or generally wanting, except after the ingestion of food or stimulants.

When transient febrile movement is not thus produced, the pulse is usually feeble, and the extremities are apt to be cold. There is more or less general debility, but the sense of weakness exceeds the actual reduction of strength. The spirits are depressed, and the suffering may be considerable from malaise withont any definite location of pain or distress.

This is a sketch of a class of cases which the practitioner meets with frequently, and which, together with cases of the affection to be considered in another chapter under the name acute dyspepsia, is popularly known as a "bilious attack."

If the gastric inflammation be greater, approximating in intensity to acute gastritis, the local and general symptoms are much more marked. There is suffering from gastric distress, and sometimes from a dull, obtuse pain in the region of the stomach. The epigastrie tenderness is often considerable, anorexia is complete, with loathing of food and nausea. Vomiting is apt to be a prominent symptom. Mucus is vomited in abundance; and, if the efforts of vomiting be prolonged, bile is rejected. The patient experiences a temporary sense of relief after vomiting. He has a feeling as if the stomach were distended, and he is led to provoke and assist acts of vomiting by voluntary efforts with the idea that if the contents of the stomach were effectually discharged the relief would be permanent. This feeling arises from the inflamed condition of the membrane, and is analogous to the sensation connected with tenesmus in dysentery. Thirst is apt to be urgent, and cold drinks are craved. The tongne is coated at first, but, in the course of the affection, it may become clean and reddened. Either constipation or diarrhœa may exist. There is more or less febrile movement, with evening exacerbations, as shown by the pulse and axillary temperature; the latter, however, rarely exceeds $100^{\circ}$ Fahr. Chilly sensations and rigors are common, continuing for a few seconds and recurring irregularly. Cephalalgia is generally marked, and in some cases is accompanied by a sense of tension, or a feeling as if the head would break. The debility is such that the patient keeps the bed. 
There is often a sense of great prostration, with depression of spirits and sometimes a notable degree of mental apathy. In cases of maximum severity, subacute gastritis so closely approximates to acute gastritis that there is scarcely a well-marked line of division between the two forms of the disease. It has been usual to embrace under the name acute gastritis, only cases in which the intensity of the disease is such that life is in imminent peril ; whereas, in the severest cases of subacute gastritis, the prognosis is always favorable. This distinction, it must be confessed, is somewhat arbitrary, and its strict pathological propriety is perhaps questionable.

In cases of subacute gastritis with considerable febrile movement, the disease is sometimes called "gastric fever." As the ferer is symptomatic, or secondary to the gastric inflammation, there is the same impropriety in using the term gastric fever as in calling pneumonitis "lung fever."

Subacute gastritis, if the inflammation be slight or moderate in degree, is usually of short duration, convalescence taking place in a few days. If the inflammation have considerable intensity, the duration of the disease is from a week to three weeks. If the inflammation persist much beyond the latter date, the affection is to be considered as having become chronic.

Subacute gastritis, in some cases, is evidently of dictetic origin. It is sometimes attributable to the decomposition of ingested aliment, owing to a deficiency of gastric juice, or to the quantity of food ingested being out of proportion to the digestive capability of the stomach, and to the ingestion of food difficult of digestion, or in which putrefaction had begun prior to the ingestion of it. These causes, however, much oftener give rise to merely functional disorders, namely, vomiting, acute dyspepsia, crapulous colic and diarrhœa than to a well-marked attack of gastritis. Persons who are habitually addicted to over-indulgence in eating, or to highly seasoned dishes and the frec use of condiments, are doubtless more liable to this affection than others, as a consequence of a gluttonous meal, or when, from any cause, stomach digestion is arrested. The affection sometimes follows a prolonged debauch. The habitual free use of spirits begets a liability to it. It occurs especially among drunkards. It may be caused by any of the acrid or irritant poisons not taken in sufficient quantity to produce acute gastritis. In a certain proportion of cases the causes, both remote and proximate, are not apparent.

An affection to which subacute gastritis is closely affiliated is that to be considered under the name acute dyspepsia. Gastric inflammation doubtless enters into certain cases of the latter affection, but the functional disturbance is disproportionately great as compared with the amount of inflammation, and is not to be accounted for by the latter; hence, the propriety of considering it a functional affection of the stomach. The differential diagnosis of these affections is not, practically, of great importance. The symptoms of gastritis are present in certain cases of remittent fever, and, perhaps, they proceed actually from subacute inflammation of the stomach in these cases. The symptomatic phenomena of remittent fercr, however, are sufficiently distinctive to render its discrimination from simple gastritis easy. Cases of ephemeral fever, or febricula, are to be discriminated by the want of proportion between the febrile phenomena and the gastric symptoms. Cases of continued fever, typhus and typhoid, very rarely present symptoms of gastritis with sufficient prominence for the differential diagnosis to be a matter of question. In general, the diagnosis of subacute gastrititis is not difficult. In making this statement it is to be borne in mind that reference is had to the disease as occurring after infancy and carly childhood. 
In the treatment of subacute gastritis, rest of the inflamed organ is of primary importance. If the inflammation be but slight or moderate, it will suffice to place the patient on a diet reduced in quantity, consisting of milk with lime water, and farinaceous articles. In cases of greater severity, complete abstinence for twó, three, or four days may be advisable; the vomiting and loathing for food will be likely to secure this end without any injunctions on the part of the physician.

In the mean time, if the symptoms denote exhaustion, the patient may be supported by nutritious enemas. The abstraction of blood, either locally or by venesection, is never indicated. Benefit may be derived from sinapisms or a blister over the epigastrium. Emetics and purgatives are contraindicated. The sensations of the patient may lead to a desire for both, especially the former, but they do not effect any desirable object, and their local action upon the inflamed membrane will do harm. If the bowels be constipated, simple enemas may be employed. Mercury is not indicated more than in cases of inflammation affecting the mucous membrane or other portions of the digestive system. On the other hand, remedies to soothe the inflamed membrane, and to allay vomiting, are indicated, namely, bismuth in a solid or in the liquid form, hydrocyanic acid, lyyoscyamus, and especially the salts of morphia. Small pieces of ice may be swallowed, or iced water given in a small quantity at a time and often repeated, if found to bo grateful to the patient. Carbonated water is not only taken with comfort, but appears to be useful. The alkaline carbonates are considered useful by Niemeyer, as diminishing the viscoscity of the gastric mucus, and thus favoring its removal. It is, however, not certain that the latter object is of great importance. The bicarbonate of soda is the alkaline carbonate to be preferred, and it is best giren in carbonated water.

It is important not to continue the abstinence from food or a great reduction of dict too long. As soon as nutritious articles of food are well tolerated by the stomach, they are admissible. The improvement of diet should be made gradually and tentatively. During convalescence the mineral acids are useful as tonics; and, after the inflammation has ceased, other tonics may be appropriate. Patients who are spirit-drinkers should be told of the connection of the disease with their habits.

\section{CHRONIC GASTRITIS.}

Chronic gastritis is not infrequent, although by no means so common an affection as was supposed some years ago. Chronic functional disorders were formerly considered as inflammatory. It is not always easy to discriminate clinically between chronic inflammation and the functional affections embraced under the name dyspepsia. The differential points involved in this discrimination will suffice for the clinical history and diagnosis of the affection.

Symptoms denoting indigestion or difficult digestion are present in cases of chronic gastritis, but these, alone, are in no wise diagnostic, since they alike exist in cases of dyspepsia. Pain may not be present, and, if present, is not distinctive. A burning sensation or a feeling of heat in the gastric region, is somewhat diagnostic. Tenderness over the epigastrium is somewhat diagnostic, provided it be limited to that region and constant, that is, not present merely during the process of digestion or when the stomach is distended. Thirst is diagnostic, if habitural, that is, present, not, only cluring the process of digestion, but at other times. The appetite is much oftener impaired in connection with chronic gastritis than in cases of dys- 
pepsia; the appetite is generally preserved, and often craving, in the latter. The nutrition is oftener defective in cases of chronic gastritis; dyspeptics often preserve their weight unimpaired. In chronic gastritis, stimulating articles of food, such as meat and condiments, are not as well borne as bland aliments, the reverse being the case in cases of dyspepsia. Alcoholic and other stimulants produce gastric distress in cases of chronic gastritis, whereas, they often relieve distress incident to merely functional disorder. Nausea and vomiting are more apt to occur in connection with chronic gastritis, excluding cases of so-called acute dyspepsia and certain cases of functional disorder characterized by vomiting; in ordinary cases of dyspepsia, nausca and vomiting are not common. A slight grade of febrile movement is sometimes observed in chronic gastritis, and very rarely in a purely functional disorder.

The diagnosis is to be based on the combination of more or less of the foregoing differential points. In making the diagnosis, certain struetural affections of the stomach are to be excluded, viz., carcinoma and ulcer. The diagnostic symptoms of these affections will be considered in the next chapter.

Chronic gastritis may follow an inflammation of the stomach developed spontaneously or produced by the acrid or corrosive poisons. It may proceed from excesses in cating or spirit-drinking, and, on the other hand, long fasting or too rigid dieting may give rise to it. Andral found the stomach inflamed, with ulcerations, in dogs destroyed by starvation. ${ }^{1}$ Dr. Stark, who fell a victim to experiments in dietetics in 1769 , had symptoms of gastritis developed during prolonged abstinence. Arsenic introdnced into the system by absorption from an external wound, gives rise to gastritis, as ascertained by Brodic and verified by the observations of others. The venous congestion of the stomach which is an effect of diseases of the liver involving portal obstruction, more especially cirrhosis, and of diseases of the heart, favors the production of gastritis if exciting causes be addecl. Chronic gastritis occurs in certain cases of renal disease involving uremia ; the probable explanation being that the carbonate of ammonia which is formed from the decomposition of the urea eliminated by the gastric mucous membrane, acts as a local irritant.

'The first point in the treatment is the removal of dietetic causes which may have originated or which tend to perpetuate the affection. The patient is to be placed on a bland and easily-digested diet, consisting of milk, eggs, stale bread, soft-boiled rice, and other farinaccous articles-articles which are digested in the small intestine. Meat and stimulants are to be interdicted. For a short time it may be advisal)le to reduce the quantity of food below the amount which the wants of the system require. Food should be taken in small quantities and at shorter intervals than in health. Moderate counter-irritation over the epigastrium appears to be useful. The remedies to be administered are few. Solicitations for emetics and cathartics are to be resisted. Patients are apt to declare that they are "bilious," or that there cxists "foulness of the stomach," and, hence, that they need evacuant medicines. But in place of medicines which, from their irritant action, will tend to increase or prolong the inflammation, remedies to soothe the inflamed membrane are indicated, viz., small doses of morphia, hyoscyamus, hydrocyanic acid, bismuth, etc. After the inflammation has ceased, functional debility or atony remains. 'Tonics

I Vide Budd on the Stomach. Inflammation of the stomach, howerer, is not an invariable effect of starvation. It was not found by Martigny in dogs starved to death. - Magendie, Journal de Physiologie, tome viii. 
and improved diet are then required. It is a point of delicacy to determine when to make this change in the treatment. It is like resorting to "passive motion" in cases of fracture or dislocation. The change is to be commenced tentatively, and continued, or not, according to the effect.

\section{DUODENITIS.}

In acute gastritis the inflammation, as already stated, may extend into the duodenum. This is also true of subacute and chronic gastritis. But it would appear, from the symptoms, that subacute duodenitis may occur as a separate affection, not comnected, on the one hand, with gastritis, nor, on the other hand, with inflammation of the lower portion of the small intestine. The diagnostic symptoms are, obscure pain, uneasiness or distress occurring some hours after the ingestion of food, that is, after it passes from the stomach into the duodenum, and tenderness below the epigastrium over the site of the affected organ. Duodenitis, occurring either separately, or in connection with gastritis and enteritis, is interesting and important as standing in a causative relation frequently to jaundiee. The jaundice is to be explained by obstruction either from swelling of the mucous membrane of the duodenum at the opening of the ductus choledochus, or from an extension of the inflammation into the duct. The latter is the more probable explanation. The treatment is the same as that of subaente gastritis. This affection will again be referred to in the consideration of jaundice.

It is a curious fact, ascertained by Curling, that a sloughing ulcer is apt to take place at the upper part of the duodenum, within a few days after the occurrence of a serere burn on the skin. Perforation may occur under these circumstances. Ulceration of the duodenum is not a constant effect of an extensive burn. Wilkes states that he failed to find it in several cases which had come under his observation.

\section{PHARYNGITIS.}

Affections of the buccal and guttural portion of the alimentary canal, namely, glossitis, the several varieties of stomatitis, etc., belong to surgery. I shall accordingly pass them by with the exception of a brief notice of pharyngitis. Certain affections of the mouth, prevailing especially in early life, are embraced among the diseases of children, and are considered in treatises devoted to these diseases.

Acute inflammation of the pharynx may be limited to the mueous membrane, or associated with inflammation of the tonsils or of the submucous areolar tissue. Associated with inflammation of the tonsils, it constitutes the affeetion called tonsillitis and commonly known as quinsy. The tonsil on one side only or both tonsils may be affected. Acute tonsillitis generally ends in suppuration; an abscess forms, and purulent matter, sometimes fetid, and nauseous to the taste, is discharged after a period varying in different cases from two to ten days. It is a very distressing affection in consequence of the pain and difficulty of deglutition. The enlargement of the tonsils, when both are affected, is such that the pharynx appears to be closed. The mucous membrane covering the tonsils and the arches of the palate is reddened and swelled. The affection, however, is attended with danger only from the very slight liability to the occurrence of œdema of the glottis. Of this I have met with a single example. An attack of tonsillitis is sometimes attributable to exposure to cold, but it often occurs without any obvious causation. The causation involves a 
predisposition to the affection, and persons predisposed are apt to experience repeated attacks. The treatment consists of poultices to the neck, or the water-dressing, the inhalation of the warm vapor, and anodynes to render the condition of the patient less uncomfortable. Milk is the form of nourishment best taken. When the abscesses point they may be opened, and the patient is thus relieved sooner than if they were left to break.

Acute pharyngitis, giving rise to inflammation and suppuration in the areolar tissue between the mucous membrane of the pharynx and the vertebral column, constitutes the affection called retro-pharyngeal abscess. The point of departure in the development of this grave affection, however, is rarely, if ever, the unucous membrane ; it may occur, traumatically, from the penetration of a sharp piece of bone contained in food, or as secondary to caries of the vertebra."

Inflammation of the pharynx with an exudation of lymph, constituting what is called diphtheritic, pseudo-membranous, or exudative inflammation, occurs in connection with two important diseases to be considered in another section, viz., diphtheria and scarlatina.

Ordinary or simple acute pharyngitis, that is, the inflammation limited to the mucous membrane, or erythematic, and unattended by the exudation of lympl, is an affection of frequent occurrence. It is one of the forms of "a cold." The inflammation may extend over more or less of the pharyngeal space and fauces. Its extent and intensity are to be ascertained by inspection of the throat, but it sometimes extends upward into the posterior nares, and lower in the pharynx than the eye can reach. It occasions more or less pain, especially in the act of swallowing, and the patient is annoyed by a constant disposition to swallow. At first the inflamed parts are dry, but, after a time, a secretion of mucus takes place, and this provokes efforts at hawking and expuition. If the inflammation extend low in the pharynx, coughing is exciter. The cough is of a peculiar character, easily recognized and distinguished as a throat-cough, the forcible current of expired air being bronght to bear on the pharynx. The limitation of the inflammation to the pliarynx, or its non-extension into the larynx, is shown by the absence of huskiness or hoarseness of the voice. The tonsils may present a cleposit of a white material in patches of greater or less size, which is a sebaceous secretion from the follicles in this situation. These white patches have a resemblance to diphtheritic exudation, and it is highly important to discriminate between the two. The follicular secretion forms a pultaceous deposit, not a membraniform layer like the deposit of lymph, and cannot be removed in strips like the latter. The patches are more prominent than those formed by a layer of lymph, and their borders are more abruptly defined. They are more easily removed than patches of lymph recently exuded, and they disappear spontaneously more quickly. Finally, on close inspection, the secretion will be seen to dip into the follicular depressions. The follicular deposit occurs without any abrasion of the mucous surface, but it is common for patients to consider the patches as ulcers.

Simple acute pharyngitis occurs as an epidemic affection, sometimes prevailing greatly and widely. It prevailed to a considerable extent in this city (New York) during the spring of 1864 . In many if not most epidemics, the clinical history shows the affection to be a form of fever rather than simply a local inflammation. This was manifestly true of an epi-

1 For an excellent article on retro-pharyngeal abscess, with a statistical table of 58 cases, by Charles M. Allen, M.D., vide N. Y. Journal of Med., Nov. 18:j1. 
demic which I studied in the western part of this State in 1857. From the results of an analysis of twenty-three recorded cases of that epidemic, the following conclusions were deduced: "The disease was an epidemic fever characterized by mild erythematic inflammation of the fauces as a constant local complication. Its character as essentially a fever is established by the febrile movement being in a marked degree out of proportion to the local affection; in other words, evidently not being symptomatic of the latter, and by its rumning a definite although a brief career. It was a fever of from three to five days' duration. Its epidemic character is sufficiently apparent. It prevailed extensively for about two months, reaching its acme gradually, declining gradually, and at length disappearing, affecting both sexes and different ages without notable cliscrimination. As an epidemic fever, its symptomatic features were very uniform. The affection of the fauces constituted the only positive character aside from the brief duration of the febrile career. 'The other symptoms uniformly present were only those incident to febrile movement, and the symptoms observed in a few cases only were incidental events, not intrinsic elements of the disease. The small white patches in the throat observed in a few eases do not establish any relation to the affection known as diphtheria. These were evidently due to follicular secretion. The occurrence of several cases repeatedly in the same family does not suffice to prove that the disease was propagated by contagion, since this fact is explicable on the supposition of the patients being equally exposed to an epidemic influence, and there being a marked discrepancy in the intervals separating the cases successively occurring in the same family."'

In the report from which the foregoing conclusions are quoted, facts are given which show conclusively that the affection was not scarlatina. It resembled influenza, save that the pharyngeal, and not the bronchial mucous membrane, was the seat of the local manifestation. It may be a question whether an epidemic fever with simple pharyngitis be not a form of influenza, or a variety of the disease characterized by pharyngitis with the exudation of lymph (diphtheria), or of the affection called epidemic erysipelas, popularly black tongue.

Simple pharyngitis, occurring as a sporadic disease, or as the anatomical characteristic of an epidemic fever, is a mild disease, having little or no tendency to end fatally. Edema of the glottis is an accident which may occur, but it is extremely infrequent. I have met with one example of its occurrence in a case of sporadic pharyngitis. The therapeutical measures indicated are, mild purgation, followed by anodyne remedies, and quinia or the chlorate of potassa. Gargles of the chlorate or the nitrate of potassa may be employed, and to allay the dryness and irritation of the throat, a mucilaginous liquid, or glycerine, may be taken frequently in small quantity, or small pieces of gum allowed to dissolve in the mouth. Caustic or stimulating applications to the inflamed membrane are not called for.

Subacute pharyngitis, not becoming chronic, is very common, and is generally treated without the advice of a physician. It is generally sufficient to supply the absence of mucus, before secretion takes place, by mucilage or glycerine. Lumps of sugar soaked in salad-oil and allowed to dissolve in the mouth is a simple mode of palliating the local irritation. If other measures appear to be called for, those appropriate to the acute affection may be employed.

Chronic pharyngitis is, in my experience; an extremely common affection. It is generally a subacute affection at the beginning, and is de-

'Buffalo Medical Journal, rol. xii. p. 718. 
veloped imperceptibly, so that, in most cases, when they first come under medical observation, there is reason to suppose it las existed for a considerable period. 'The membr'me is more or less reddened, and irregularly thickenel so as to present a granulated or mammillated appearance, especially marked on the posterior wall of the pharynx. Adhesive mucus is sometimes seen sticking to the part, when the throat is exanined. 'This inflammation sometimes extends to the posterior nares, and the patient is then said to have catarrh. It may extend around the top of the larynx, but it has no tendency to pass into the laryngeal eavity. Occasionally, the mucous follicles are distended with sebaceous matter. The appearance is not sufficiently common to render the name follicular pharyngitis appropriate except as applied to certain cases.

The tonsils in some cases present white masses varying in size from that of a pin's head to a pea, either wholly imbedded in, or in part projecting from, the mouths of the follicles. I have met with cases in which the tonsils were very thickly studded with these masses. If removed mechanieally, as may easily be done, they are sometimes quickly renewed. If not understood, the striking appearance of the throat in these cases may lead to the apprehension that the affection is more important than it really is.

Chronic granular pharyngitis in some persons occasions but little inconvenience. It is often found when the patient makes no complaint of any trouble in the throat. In other cases, it occasions more or less annoyance. An uncomfortable sensation is felt in the throat. The presence of adhesive mucus exeites efforts of hawking and conghing. Partial obstruction of the Eustachian tubes may occur. The proximity of the inflammation to the larynx may give rise to a dry, hacking cough. The voice becomes slightly hoarse after considerable use in speaking or reading. The use of the voice is followed by a sense of fatigue in the vocal organs.

The affection is much more frequent in males than in females. It occurs most frequently after puberty, and in middle life. From its frequent occurrence among the clergy, it has been called clergyman's sore throat; but it is by no means peculiar to the clergy, oceurring perhaps quite as often in persons of other callings. It occasions more inconvenience to clergymen than to others, from the necessity which they are under of using the voice in public speaking, and they are apt to be apprehensive lest it may incapacitate them from preaching. It may be doubted whether the use of the voice leads to the affection. It occurs especially anong persons of sedentary habits who suffer from the wear and tear of continued application, without physical and mental relaxation. The number of persons in this country who overtask the powers of the system by steady labor in the oflice, the counting-house, or the work-shop, continued without intermission for many year's, giving no time whatever to recreation, is very large. It is among those who in this way violate the laws of health that this affection prevails. It is accompanied with symptoms denoting impairment of the general health. Patients complain of debility, and a want of their accustomed energy ; they are generally depressed in spirits, and have forcbodings of loss of health; they are very apt to fancy the existence of some serious disease, especially pulmonary consumption, and it is sometimes difficult to convince them that the latter disease does not exist. Dyspeptic ailments frequently coexist. Palpitation is not uncommon.

The affection tends to continue. It is usually long persisting. In many of the cases which I have seen it had existed for many years. But there is no tendency to eventuate in an affection of the air-passages, or in 
tuberculous disease. Patients with this affection rarely become tubercu. lous; its existence is, to some extent, évidence of the non-existence of tuberculosis. It is a popular notion that the affection does denote a consumptive tendency, and this notion is encouraged by the host of irregular practitioners who, within late years, in this comtry, have made diseases of the throat a specialty. The mental condition of patients with this affection renders them an easy prey to quacks.

The treatment, to be effective, must have reference to the system. After considerable experience I have come to regard topical applications as generally of little or no curative value. In some cases, however, a solution of the nitrate of silver, of tannin, or of iodine, appears to be useful in the way of palliation. As regards the mode of employing remedies topically, inhalations carry the medicated application too far, while gargles do not carry it far enongh. Projecting a spray of medicated solutions into the throat is an efficient mode, but a simple and satisfactory plan is to use a small mop made of soft linen, which is to be dipped in the medicated liquid and carried into the throat. The application by the latter mode can be easily made by the patient. The remedies which exert a curative influence in certain cases of bronchitis are sometimes useful, namely, the iodide of potassium, the bromide of potassium, the chlorate of potassa, and the hydrochlorate of ammonia. Alteration of the habits of life is first in importance. Relaxation, recreation, and outof-door life are far more effieacious than medieines, and the latter are of little use withont the former. When circumstances permit, relinquishment of business for a time for travel or rural occupations is of signal benefit. Tonic remedies may often be advantageously conjoined with proper hygienic management. Tonies, to be efficacious, should be continued for a long period, and the form of tonic changed from time to time. The diet should be nutritious. The object of trcatment, in short, is to restore the general health.

The diagnosis of chronic pharyngitis is, of course, sufficiently easy. Inspection is alone necessary. But it is important to discrininate simple chronic pharyngitis from that dependent on syphilis. The latter is to be suspected from the local appearances (ulcerations and the so-called mucous patches), and discriminated by these appearances in conjunction with other secondary syphilitic phenomena, together with a knowledge of the fact of syphilis having existed.

In the follicular variety of chronic pharyngitis, the matter which accumulates in the follicles may be ejected by coughing, in the form of round, solid pellets which are sometimes supposed to be tubercles or pulmonary calculi. They are to be distinguished from the latter by being easily broken down with pressure, by being unctuous to the touch, and emitting, when crushed, a fetid odor.

\section{PAROTIDITIS. MUMPS.}

The affection commonly known as mumps may be appropriately noticed in this connection, as seated in an organ accessory to the buccal cavity. The parotid gland appears to be the seat of the disease, but it evidently involves a constitutional morbid condition and a special causation. The local affection consists of enlargement of the gland together with swelling of the surrounding parts, soreness to the touch, pain especially in mastication, the pain frequently extending to the ear and in other neighboring situations. The submaxillary gland is sometimes involved. The swelling in the site of the parotid pushes outward the lower part of the ear. 
CEdema of the face is sometimes associated. The amount of swelling of these several parts varies in different cases; if considerable or great, it causes a notable change in the physiognomy. 'The skin generally is not discolored. The affection may be limited to one side, or both sides may be affected. In the latter case, usually the two sides are affected successively, with an interval of a day or two, but in some cases both sides are simultaneously seized.

The disease is frequently ushered in by a light chill, or by chilly sensations, and in its progress it may be aecompanied with moderate febrile movement, diminished appetite, pain in the head, and general malaise. The disease continues for four or five days. The swelling, pain, and soreness gradually subside and disappear. The inflammation has no tendency to suppuration nor to continue in a chronic form. An abundant perspiration sometimes takes place at the time of convalescence. Occasionally, during the progress of the disease, or when the affection of the parotid is about to disappear, swelling, pain, and soreness of one or both of the testes occur. This must be extremely rare. I have met with but a single example. In the female, it is stated the mammary gland and the labia majora are liable to become affected. I have never net with an example. When parts, other than the parotid, are involved, it is not from a metastasis, but from the operation of the same internal morbid condition which occasions the parotiditis.

The communicability of this disease is generally admitted, but it has been denied by some writers of high authority, for example, Valleix. It prevails, at certain times and places, to such an extent as to be an endemic disease. But this is true of diseases which are exclusively propagated by contagion, e.g., smallpox. Persons between twenty and thirty years of age are most susceptible to the infectious miasm, but it occurs not infrequently under puberty. Males are more susceptible than females. It is one of the diseases which affect the same person but once.

The prognosis is always favorable. The disease, exeept that it occasions considerable discomfort, is trivial. In the way of treatment, it claims only soothing embrocations, and the application of flannel, eotton batting, or wool to the neck, with some anodyne remedy if the pain be considerable. Bloodletting, purgation, or other of the so-called antiphlogistic measures are not indicated. 'The popular apprehension of danger from "taking cold" in this disease, is based on the idea that, if the affection of the parotid be arrested, a metastasis is apt to take place. There is little ground for this idea, but a patient probably passes through the disease more comfortably by avoiding exertion and exposure.

Parotiditis occurs as an occasional complication of typhus and typhoid fever. Under these circumstances it is a widely different affection from mumps. Suppuration takes place in the great majority of eases, the abscesses evacuating either externally or into the meatus auditorius, and frequently there is considerable sloughing of the areolar tissue. As a complication of fever it is attended with much suffering and clanger. Virchow teaches that in the non-suppurating form, that is, in mumps, the inflammation has its point of departure in the duets, whereas, in suppurating parotiditis, the inflammation is seated in either the substance or the areolar tissue. 


\section{CHA P TER III.}

\section{STRUCTURAL AFFECTIONS OF THE STOMACH.}

Induration. Softening. Gastric Ulcer. Carcinoma of Stomach. Dilatation of Stomach. Degeneration of the Gastric Tubules.

$\mathrm{H}$ AVING considered the inflammatory affections of the mucous membrane lining the different sections of the alimentary canal, the structural affections of the stomach and intestines next present themselves for consideration. Directing attention, first, to the stomach, the following are the structural affections to be noticed: Induration, softening, ulceration, carcinoma, dilatation, and lesion of the gastric tubules. These several affections will be briefly considered in the order in which they have just been enumerated. For a fuller consideration of these affections than is consistent with the seope of this work, the reader is referred to works on morbid anatomy and treatises devoted specially to diseases of the stomach.

\section{INDURATION (SCLEROSIS).}

Induration, or sclerosis, denotes a structural change characterized by increased density and thickness not dependent on carcinomatous disease. The name has the merit of not implying anything respecting the pathological character of the lesion. The affection is apt to be confounded with scirrhus. It is due to a morbid product or growth of a fibroid character, seated primarily in the submucous areolar tissue. The firmness and weight of the stomach are increased. The firmness may be such that the organ does not collapse by its own weight. The thickness of the walls is sometimes greatly increased. The gross and microscopical appearances show the induration to depend on the addition of a substance analogous to that of fibrous tumors, or the structure of a cicatrix. The substance appears to be like that between the hepatic lobules in cirrhosis, and the affection has been called cirrhosis of the stomach. Brinton has proposed to call it linitis. Habershon calls it fibroid degeneration. It is supposed to be an effect of chronic inflammation of the submucous areolar tissue.

The lesion may be more or less extensive. The whole of the organ may be affected, or it may be limited to a small portion. In the latter case, the pyloric extremity is apt to be the seat. The danger connected with the affection depends much on its extent and situation. If the pylorus be involved, it induces stricture, and this renders it a serious lesion. If the lesion be extensive, the incapacity of the stomach for dilatation stands in the way of the ingestion of a sufficiency of food.

The existence of this lesion is not denoted by any distinctive symptoms. In a case under my observation, in which, after death, the walls of the organ were found to be from half an inch to an inch in thickness everywhere except at the pyloric third, the patient, up to a few weeks before death, supposed he was simply dyspeptic, his ailments not being sufficient to lead him to seek for medical advice. In this case, the organ formed a resisting movable tumor, felt through the abdominal walls, and the dis- 
ease was supposed to be carcinoma. If pyloric obstruction be produced, vomiting, from an aecumulation of the ingesta in the stomach, is a prominent symptom. Dilatation of the stomach may ensue. The immediate cause of death in such cases is inanition.

The affection is rare. Most writers concur in regarding it as occurring chiefly in spirit-clrinkers, and very rarely in persons under forty years of age. Habershon states that it is met with in temperate persons of either sex, and in early life. If seated at the pylorus and involving stricture, it may be suspected when the evidence of pyloric obstruction is afforded by vomiting of accumulated ingesta and dilatation of the stomach, provided carcinoma can be excluded. The absence of a tumor to be felt through the abdominal walls, and of hemorrhage from the stomach, renders it probable that carcinom: does not exist. If the affection do not involve pyloric obstruction, the diagnosis can hardly be made with any degree of certainty. If the thickened and hardened stomach can be felt through the abdominal walls, there are no means of determining positively that the affection is not carcinoma. The long duration of the affection, the absence of the cancerous complexion, and the non-existence of cancer in other situations, are the points involved in this differential diagnosis.

The treatment embraces remedies to palliate irritation of the stomach, abstinence from spirits and other stimulants, and careful regulation of the diet.

\section{SOFTENING. (GASTRO-MALAXIA. GASTRO-MALACIA.)}

Softening of the mucous tunic of the stomach has been already mentioned as one of the anatomical characters of gastritis. It is observed in cases in which the other appearances and the history during life show it to be non-inflammatory. The softening may be limited to the mucous membrane or extend to the other coats of the stomach. Limited to the mucous membrane, the walls of the organ are thinned; the affected coat cannot be removed in strips, but is easily scrapel away, and in some cases the membrane is converted into a pulpy layer, the disorganization being complete. This form of softening has been studied particularly by Louis, who calls it pullaceous soflening. Softening of the several coats has been clescribed very fully by Cruveilhier under the name gelatiniform softening; also by Billard. These and other French writers regard the latter as a special lesion characteristic of an individual disease affecting chiefly children and accompanied by symptoms analogous to those belonging to the affection known with us as cholera infantum.

It is probable that non-inflammatory softening may sometimes, during life, be the result either of a defective nutrition of the membrane, or of the chemical action of the gastric juice. A deficient supply of arterial blood, from embolism or other causes, may lead to softening here as in other situations; and, according to Pavy, the gastric juice, under these circumstances, may act as a solvent, this action, in health, being prevented by the alkalinity of the blood. But in the great majority of cases, both forms of softening are to be regarded as not morbid, but due to the action of the gastric juice after death. The changes are post-mortem, resulting from self-digestion by the stomach. John Hunter was the first to establish the fact that, under certain circumstances, the stomach undergoes digestion after death. He observed several cases in which perforation was produced by this cause, the contents of the stomach escaping into the peritoneal cavity. This has since been abundantly shown by experimental observations on inferior animals. Perforation of the stomach from this 
cause in fishes is common. Post-mortem digestion is found especially in cases of sudden death occurring shortly after the ingestion of a meal, but it has also been observed in cases of sudden death when the stomach was empty, and even perforation has taken place under the latter circumstances. Softening, more or less in degree from this cause, is common after death from various diseases, varying very much in different cases according to a diversity of circumstances connected with the state of the stomach at the time of death, all of which are not as yet understood. In a case of death at Bellevue Hospital from fracture of a cervical vertebra, the stomach and the diaphragm were digested so that the contents of the stomach were found in the left pleural cavity. In this case the patient had taken only liquid nourishment for two days before his death. It is supposed that alcoholic stimulants, given shortly before death, may prevent the solvent action of the gastric juice. A high temperature farors post-mortem softening. It will not be likely to take place in cold weather, nor when bodies are placed in ice. The case just referred to, however, oceurred in the month of October, and the body was in a cold place. It is met with oftener in the bodies of children than of adults. According to Virchow and others, softening, whether occurring during life or after death, may be due to the prodnets of the fermentation of the ingesta, the action being purely chemical and independent of the gastric juice. 'The ingesta in which fermentative processes may take place and lead to this post-mortem effect, are milk and the amylaceous articles of diet. The presence of an abundance of muens in the stomach is supposed to favor, by catalytic influence, these processes.

Post-mortem softening is more likely to occur at the cardiac than at the pyloric portion of the stomach, but it may extend to the latter and even to the duodenum. The gastric juice may regurgitate into the osophagus and lead to perforation of this tube, and the escape of the contents of the stomach into the cavity of the chest has been repeatedly observed. The softened portions of the stomach are white, or of a bluish-white color, if the bloodvessels are empty, but if these contain blood it is rendered dark by the action of the gastric juice, and, escaping through the coats of the vessels, it gives to the tissues a darkish color. The softened parts have an acid reaction, and are free from the odor of putrefaction, the gastric juice acting as an antiseptic. 'This latter point distinguishes from softening due to putrefactive change. If perforation take place, the aperture is a " ragged hole," with pulpy edges. The organs with which the gastric juice comes into contact after escaping from the stomach, viz., colon, spleen, liver, etc., may present softening. Perforation of the stomach from selfdigestion is not to be confounded with the destructive effects of corrosive poisons, and the knowledge of the former is highly important to be borne in mind in making autopsies after sudden death, in cases involving a suspicion of poisoning.

\section{GASTRIC ULCER.}

Ulceration of the stomach is among the anatomical characters of acute gastritis, especially when the latter is produced by corrosive poison; but it occurs independently of acnte gastritis, the inflammation, if it exist, being limited to the ulcerated space or spaces. The ulcer varies in size in different cases, being sometimes so small as to be discerned with difficulty, and sometimes of the size of a half-dollar, a dollar, or even considerably larger. If small in size, it has generally a round or oval form, and presents an appearance, in some cases, as if a portion of the tissues had 
been punched out. This kind of ulcer, especially, is apt to eat through the several coats of the stomach, and is distinguished as the round, eroding or perforating ulcer. The uleerated space is largest in the mucous membrane, next in the muscular tunic, the ulcer being funnel-shaped. The liability to perforation followed by fatal peritonitis, and to another accident, viz., erosion of a vessel of the stomach, giving rise to a lemorrhage which may prove fatal, the difliculty of cicatrization, if these accidents do not oceur, and the interference with the functions of the stomach which the uleer occasions, render the affection one of much gravity.

Gastric ulcer is not an extremely infrequent affection. Brinton's researches show that it is found after death in an average of 5 per cent. of persons dying from all causes.' Jaksch, quoted by Niemeyer, found in 2330 autopsies, ulcer in 57 , and cicatrices denoting ulcers which had healed in 56 ; making the ratio of an ulcer, either existing or having existed, as one to every 20 subjects.

In the greater proportion of cases a single ulcer exists, but in the proportion of one to every 5 cases, two or more ulcers are found after death. The situation, in the great majority of cases, is the posterior surface of the stomach towards the pyloric extremity. Brinton found the situation to be as just stated in the proportion of 86 in 100 cases. Aclhesion of the stomach, at the site of the ulcer, to adjacent parts, the pancreas, left lobe of the liver, etc., is frequently found, dele to circumscribed peritonitis excited by the proximity of the ulcer. This is conservative, rendering perforation less frequent than it would otherwise be.

The clinical history embraces, as the most prominent symptoms, pain, tenderness on pressure, voniting, and hemorrhage from the stomach. These are the important symptoms in a diagnostic point of view. Pain is very rarely wanting. It is burning or gllawing in claracter, coming on directly, or soon after, the ingestion of food, and continuing until the contents of the stomach have either passed into the duodenum or been ejected by vomiting. The quickness with which the pain follows the ingestion of food is a diagnostic point; if due to simple indigestion or neuralgia, it generally occurs after a greater or less interval from the time of taking food. It is situated at, or near, the epigastrium, and is generally confined to a small space. A similar gnawing pain in the back, between the shoulders, is not uncommon. The probable cause of the pain is irritation of the ulcerated space by the contact of the ingesta and the gastric juice. The pain from this cause is especially marked after the ingestion of stimulating articles of food or drink, and when, from indigestion, the contents of the stomach undergo chemical changes. Pain may also be caused by circumscribed peritonitis, and by the traction of adhesions which have resulted from peritoneal inflammation. 'The latter causes may account for the exceptional occurrence of pain irrespective of the ingestion of food. The pain caused by the action of the gastric juice is felt directly after eating, because the secretion of the juice is excited by the presence of food in the stomach. Tenderness on pressure over the epigastrium is almost invariably present, confined, like the pain, within a small area, and varying considerably in degree in different cases. Vomiting is a very constant symptom, occurring after a period from the time of taking foocl, varying in the different cases, the act of vomiting usually not violent but complete, and the ejection of the contents of the stomach followed by notable relief. Mucus may be expelled, in more or less abundance, with the ingesta, show-

1 Brinton's work on diseases of the stomach contains elaborate statistical researches on this subject; see, also, a monograph on this subject by the same author. 
ing inflammation of the mucous membrane surrounding the ulcer. As respects vomiting, much will depend on the quantity and kind of food ingested. Indigestible and stimulating articles are more likely to provoke it, as well as to increase the pain, than aliments bland and easy of digestion. Hot substanees are not as well borne as cold. Hemorrhage, as shown by the vomiting of blood in greater or less quantity, is a symptom of frequent occurrence. The vomiting of blood generally occurs after a meal. The hemorrhage is sometimes so abundant that life is lost from this cause. Cases have been reported in which life was lost from hemorrhage, although no blood was vomited, the stomach being found after death filled with elots. The blood vomited is dark or grumous from the action of the gastric juice, provided the vomiting does not immediately follow the hemorrhage. In view of the diagnostic value of hemorrhage as a symptom, Brinton enforces the importance of an examination of the matters vomited with the microscope, although the gross appearance may not denote hemorrhage, and he states that blood-globules may be found in the sediment of a comparatively clear liquicl. The stools also should be examined for the gross and microscopical appearance of blood, for hemorrhage may take place without romiting, the blood passing into the intestinal canal. Profuse hemorrhage occurs especially in connection with the small perforating ulcer.

The bowels, in cases of gastric uleer, are generally constipated, a symptom easily understood in the cases in which vomiting is a prominent symptom. Amenorrhœa, in the female, is common, but, if there have been much hemorrhage, this symptom is a result of the loss of blood. There is little foundation for the idea that hemorrhage from the ulcer occurs vicariously in the place of menstruation, but the latter ceases, or is deficient, as in cases of chlorosis, or anæmia produced by hemorrhages elsewhere, or from other causes.

The pathological character of gastric ulcer is not fully settled. Whether it be a result simply of a circumscribed inflammation, or whether it be due to a non-inflammatory sloughing process, as held by Rokitansky, is still an open question. Nor is it certain whether it commences on the mucous surface, or has its point of departure within the tubules. It has been conjectured that small ecchymoses within and beneath the mucous membrane may precede and give rise to ulceration. Virchow attributes the perforating ulcer to obstruction of an arterial branch and consequent mortification of the walls within the space nourished by the branch which has become obstructed, the action of the gastric juice upon the mortified part co-operating to effect the separation, leaving the ulcer. The arterial obstruction may be due either to embolism, thrombosis, or calcareous degeneration of a branch of the gastric artery.

Exclusive of the form which is especially distinguished as perforating, gastric ulcer occurs chiefly in persons of middle and advancing life. The iruly perforating ulcer, on the other hand, occurs in young girls, and particularly in young maid-servants. Statistics show that females are more liable to ulcer, the proportion being as 2 to 1 . Clinical observation fails to show any dependence on particular habits of diet, or other causes, nor has it any known relationship with other affections. It may be stated, however, that persons of feeble or impaired constitution are more liable to it than the robust.

The diagnosis of gastric ulcer may be made with great certaint5, provided the diagnostic symptoms are present, viz., pain of a burning or gnawing character limited to a circumscribed space in the epigastrium, with tenderness also circumscribed, the pain especially felt after the inges- 
tion of food, and especially after certain kinds of food, romiting occurring during the process of stomach-digestion, and followed by relicf, and, finally, gastrorrhagia or hæmatemesis. Analogous synutoms, it is true, may be present in cases of eancer of the stomach, but there are points of contrast involved in this differential diagnosis, which will be noticed presently in considering cancer. Moreover, the absence of a tumor appreciable through the abdominal walls goes far toward the exclusion of cancer. Chronic gastritis and ulcer have symptoms to some extent in common. But in chronic gastritis pain is less prominent, vomiting does not oceur so frequently, and hemorrhage is wanting. In cases of gastralgia, the pain might suggest the idea of ulcer. But the pain in gastralgia is not aggravated, and, on the contrary, often relieved by the ingestion of food; tenderness over the epigastrium is not present, but the pain is often relieved by pressure; vomiting is an oceasional symptom only, and hematemesis does not occur. In cases of merely funetional disorder, pain, vomiting, and hrmatemesis are all wanting. Hrmatemesis, it is to be borne in mind, is not alone evidence of ulcer. It occurs, irrespective of disease of the stomach, in certain cases of cirrhosis of the liver. It is the union of the several symptoms just named that renders the diagnosis eomplete. These diagnostic symptoms, however, are not united in all cases of gastric uleer. Hemorrhage is not invariably present; romiting is not always a prominent symptom and may be wanting; pain and tenderness are not in all cases marked-the affection, in short, is sometimes latent, more or less difficulty or disturbance of digestion only being manifested. Under these circumstances, a positive diagnosis cannot be made. A strong suspicion of the existence of the affection may, in some eases, be reasonably entertained, when the symptoms do not warrant a positive diagnosis.

Gastric ulcer proves fatal in different modes. One of these is by perforation, fatal peritonitis being indueed by the eseape of the contents of the stomach. This accident would take place oftener than it does, were it not for the adhesion of the stomach to the arljacent organs as a result of eircumscribed peritonitis. Perforation is most apt to occur when the nlcer is situated on the anterior surface of the stomach. According to Brinton, it occurs in the proportion of 1 to 7 or 8 cases. It is most likely to occur in the uleer distinguished as perforating, i.e., the small, round, punched uleer, although not limited to this kind of uleer; hence, it occurs oftenest in females between sixteen and thirty years of age. The perforation generally takes place after the ingestion of a meal, or in the act of straining or sneezing, or in some sudden and forcible movement of the body. In some cases peritonitis, following perforation, oceurs without having been preceded by any symptoms pointing to the existence of ulceration of the stomach.

Another mode in which it proves fatal is by hemorrhage. Life may be slowly destroyed by the continued escape of blood, or repeated attacks of hemorrhage, or the loss of blood may be so rapid as to destroy life quickly. Aceording to Brinton, death from hemorrhage occurs in the proportion of 1 to 20 cases. Fatal hemorrhage oecurs much oftener in the male thin female. As an example of fatal hemorrhage arising from an extremely minute perforating uleer, the following case, communicated to me by the late Dr. W. W. Reid, is of interest:-

A young woman, a chambermaid, æet. 25, for a year or more had had pain in the stomach, at times so severe as to disable her from her work for a few days. During the week previous to the 12th ult. she had more pain than for some months. There was marked tenderness over the epigastrium at all times. On the 12th ult., she went to bed more unwell 
than usual, and before falling asleep became sick at the stomach and vomited. Un the morning of the 13th, she arose and dressed herself, but was faint and obliged to go to bed. At this time she discovered that what she had thrown up in the night was blood, and the quantity was reported to be abont three pints. In the afternoon she again romited a pint or more of blood. On the 14 th and 15 th she again vomited blood. From the 19th to the $22 \mathrm{~d}$, she was considered better and improving, but was unable to be raised up without fainting. On the $22 \mathrm{~d}$ she had something like a convulsion, and became comatose. She continued in this conclition until the morning of the $23 \mathrm{~d}$, when she had another convulsion, and died during the afternoon of this day. Shortly before death she threw up an inky liquid. On examination after death, the only morbid appearance in the stomach which was at first observed was a small patch three or four inches square, in which the mucous membrane was injected. This was situated about three inches from the cardiac orifice. On close inspection in the centre of this patch, a small ulcer was discovered resembling the puncta lachrymalis when large. The blunt end of a common silver probe readily entered the opening and passed directly into a branch of the gastric artery. Dr. Reid, in his remarks on the case, says that the ulcer was detected almost by accident, on account of its minuteness, and in view of the general healthy appearance of the gastric mucous membrane: he suggests that in similar cases the existence of a minute ulcer may have been overlooked.' The occurrence of gastrorrhagia is sometimes, like perforation of the stomach, not preceded by symptoms denoting the existence of gastric ulcer, the latter being latent as regards the diagnostic points which have been named.?

Another mode in which it proves fatal, is by inanition. Death takes place by slow asthenia when the stomach is so intolerant of ingesta that aliment sufficient for the support of life is not retained. In other words, patients are gradually starved to death, provided life be not cut off by some other disease which the system may be rendered unable to resist in consequence of the debility incident to the stomach affection.

The duration of the affection is very variable. Death sometimes takes place from perforation or hemorrhage within a few weeks, or even a few days, after the time when the symptoms first denoted gastric trouble; or, as already stated, perforation and hemorrhage may occur when previous symptoms denoting gastric ulcer were wanting. But if these accidents do not prove the immediate cause of death, life is usually prolonged for many months, and it may be for very many years. Brinton states that he has notes of one case in which probably a continuous open ulcer existed for 35 years, and of two cases in which the duration was 30 years, together with three or four of 20 , four or five of 15 , and several of $10,7,5$, and 4 years' duration. It is not uncommon in the course of this affection for the symptoms, from time to time, to subside, or even to disappear, and subsequently to return. These remissions are probably due to healing of the ulcer which afterward again opens.

Gastric ulcer, although a serious affection, is by no means incurable. Brinton estimates that a cure takes place in about one-half of the cases which occur. This estimate is based on the number of instances of cicatrized ulcer found in autopsical examinations. The cicatrices are generally small or of moderate size, but in some instances they occupy considerable space; in the latter case, they may induce contraction and

I This case is given more fully in the Buffalo Medical Journal, rol. ii. p. 641, 1846.

2 Vide case reported by Dr. C. Murchison in Trans. Patholog. Society of London, 1871 . 
considerable deformity of the stomach. Situated at the pyloric orifice, they sometimes lead to stricture.

The treatment of gastric ulcer embraces the following objects: First, and most important, sccuring for the stomach as much rest as is compatible with a proper amount of nutrition; second, the arrest of hemorrhage and the palliation of suffering; and, third, the cmployment of remedies to promote cicatrization.

The first of these objects is to be seenred mainly by regulation of the diet. The patient is to be nourished by those nutritious articles of food which will be best retained by the stomach, and give rise to the least inconvenience. Milk and farinaceous substances will be best borne. As remarked by Budd, the articles which, combined ont of the body, would make a soothing poultice for an ulcer on the skin, are the most likely to be tolerated by the stomach in cases of this affection. Condiments, alcoholic stimulants, solid and indigestible food of any description, meat, and, according to Chambers, sugar, should enter as little as possible, or not at all, into the diet. The quantity of food taken at a time is a point of importance. 'The rule is, a small quantity at a time, the intervals regulated according to circumstances. Experience in each case is, of course, to guide in this regard, and also in the choice of articles. Milk and other liquids should be taken cold. Limewater added to milk renders it less likely to occasion disturbance. Quietude of the body is to be enjoined, more especially during the process of digestion. It is desirable to limit, by habits of quietude, the waste of the tissues, in order that the demand for nutritions supplies may be proportionably limited.

In some cases the most judicious and careful regulation of the diet proves unsuccessful. Vomiting is constant, and the pain is severe so long as the stomach contains food. It is evident that the ulcer is not healing, and the patient may be in danger from inanition. Under these circumstances, an attempt may be made to secure for the stomach complete rest, as regards the ingestion of food, by the introduction of nourishment exelusively by the rectum. Brinton advises this procedure for a few days, from time to time, in severe cases. It may sometimes be continued for weeks, and eren months. In a case of supposed ulcer at Bellevue Hospital, I pursued this course for three weeks-enemas of the essence of beef and milk being given every four hours throngh the day, and nothing taken into the stomach but small pieces of ice. The pain and romiting were greatly diminished during this period, and the patient lost but little gronnd as regards strength. She did not suffer from hunger, but thirst was a sonrce of some inconvenience. The course would have been longer continued, but for the irritability of the rectum, in consequence of which, at length, the nutritious enemas were immediately returned. On returning to alimentation by the stomach, she bore food better than before, and after some months she improved sufficiently to leave the hospital. I saw her several months after she had left the hospital, and she reported herself quite well.: My friend and former colleague, Prof. Rogers, of Louisville, has related to me a case in which a child was supported by enemas for a month, the case being one of poisoning with caustic potash, death taking place at the end of the month. In the American Journal of the Medical Sciences, in 1852 , Dr. D. L. Pierce, of Pennsylvania, has reported a case of supposed gastric ulcer, the patient being sustained wholly by enemas

1 Eight years afterward this patient re-entered hospital, with a renewal of the diagnostic symptoms of gastric ulcer, having had good health up to several months before her readmission. 
for threc consecutive months. The following statements, taken from the report, contain the material points in this remarkable case:-

The patient was a female, aged 26 . She appeared to vomit everything ingested a few moments after eating. Circumscribed tenderness existed over the epigastrium. She was emaciated, feeble, and had been confined to the bed for three months. Purulent matter was ejected from the stomach with the food, and sometimes by itself. Severe pains were referred to the epigastrium. Remedies, as well as food, being quickly vomited, Dr. Pierce proposed to her to submit for a month to entire abstinence as regards the ingestion of food by the stomach, and to try the plan of taking nourishment by enemas, to which she assented. A halfpint of good lamb or mutton broth was injected every three hours. For the first week she took by the mouth several times a day a teaspoonful of gum-arabic or pure water, but after the first week this was discontinued. Dr. Pierce says that he has no doubt of the strict compliance, on her part, with the plan of treatment adopted. The epigastric region was vesicated, and the surface sprinkled with morphia. During the first week vomiting of purulent matter occurred several times daily, but during the three following weeks it diminished, and she was in all respects more comfortable. It was resolved to continue the plan for another month. The vomiting of purulent matter during this month occurred only occasionally, and the improvement continued. During this month Dr. $\mathbf{P}$. believes not a morsel or drop of anything passed her lips. It was agreed to continue the plan for still another month. The improvement progressed rapidly, and by the middle of the month all evidence of gastric disease had disappeared. She began before the month ended to take a little water and mucilage in small quantity. She had gained in flesh and strength during this plan of treatment. On returning to the introduction of food by the stomach, the symptoms did not return, and two years afterward the patient was in the enjoyment of excellent health.

Hemorrhage is to be arrested by small pieces of ice swallowed, and by the application of cold to the epigastrium if the loss of blood be considerable. Astringent remedies may be given if the stomach will retain them. Pain will require the use of opiates, the form being selected which is found on trial to be the least objectionable. It may be administered per enema, epidermically, or hypodermically, if not tolerated by the stomach. Moderate counter-irritation over the epigastrium by liniments, or by dry cupping, is useful. Opiates may also serve, in some cases, to alleviate the vomiting. The subcarbonate of bismuth is a very useful remedy in some cases, given in scruple doses three or four times daily.

It is doubtful whether any remedies exert a direct effect to promote cicatrization. The nitrate of silver and other remedies have been given with the expectation that they will exert a topical effect upon the ulcer. Clinical observation appears not to furnish evidence of their utility in this way. Cicatrization is promoted indirectly by remedies which arrest the peristaltic movements of the stomach and quiet the irritability of the organ, in other words, remedies which have reference to the first object of treatment, viz., securing as much rest as possible; and for this end opium in some form is the most effective remedy. Cicatrization is also. promoted indirectly by tonic remedies if these be borne. Local bloodletting is injurious by lowering the vital forces. Mercurialization is objectionable on the same ground. Cathartics are hurtful on account of their direct action. Constipation, if it exist, is to be relieved by enemas.

When an improvement in the symptoms renders it probable that the ulcer is healing, great care in diet is important to prevent a relapse, and 
it is to be borne in mind that remissions are apt to take place, followed by a renewal of all the symptoms. Care is neessary for some time after the healing appears to have been completed ; the patient is not to be considered as safe for some time after apparent recovery.

\section{CARCINOMA OF STOMACH.}

Cancer of the stomach is far more infrequent than gastric ulcer. According to Brinton's researches, primary cancer of the stomach was found in only 81 of 8468 autopsies, or in the proportion of about one per cent. Yet cancer in this situation appears to be as frequent as elsewhere. Brinton deduces from his own researches and those of others, that, of all primary cancers, one-third are seated in the stomach. The same author estimates the liability to ulcer as four times greater than the liability to cancel. As a rule, eancer when seated in the stomach is primary; that is, it is not preceded by caneer in some other situation. The pylorus is the part most frequently affected, viz., in the proportion of 3-5. The cardiac orifice is the seat in the proportion of ten per cent. As exceptions to the rule, cancer may be seated at a point between the two orifices, oftener in the line of the small thai the great curvature. The point of departure for the morbid deposit or growth is usually the submucous areolar tissue. As a rule, to which there are very few exceptions, cancer of the pylorus is limited to the stomach, not extending to the duodenum, but cancer of the cardiac orifice extends more or less into the osophagus. The cancer in the great majority of cases is of the scirrhus variety. Brinton's statistics show the proportion to be seventy-two per eent., whereas, the proportion in which the cancer is medullary is eighteen per cent., and the proportion of colloid cancers about ten per cent. In the progless of the cancerous affection, ulceration of the mucous coat is apt to take place, so that symptoms of ulcer and cancer are combined. Sloughing away of portions of the cancerous mass is not uneommon. Perforation of the stomach sometimes occurs. It occurred in 21 of 507 cases analyzed by Brinton. The cancerous affection leads to adhesion of the stomach to adjacent organs. Stenosis or strieture at the pyloric or cardiac orifice is another effect, playing an important part in the production of symptoms. The opening of ressels is still another effeet, giving rise to hemorrhage. The carcinomatous growth, especially when seated at the pyloric orifice, forms a tumor of greater or less size. Obstruction at this orifice induces dilatation of the stomach and hypertrophy of its muscular tunic. Commonly, obstruction at the cardiac orifice leads to contraction of the organ.

The symptoms which make up the clinical history of this affection are as follows: Impaired appetite, or anorexia, is gencrally more or less marked, but in some eases the desire for food is maintained. Pain is usually more or less prominent, and the character of the pain is frequently lancinating. 'The pain is sometimes gnawing, as in ulcer, and, in these cases, ulcer may have occurred as secondary to the cancer. Tenderness over the epigastrium may, or may not, exist. Vomiting is a frequent symptom, but it occurs less frequently than in cases of simple ulcer, and at a later period after taking food, provided the affection be not seated at the cardiac orifice. The vomiting, if the cancer be at the pylorus, may be chiefly due to obstruction at this orifice, and consequent accumulation. The cryptogamous plants, sarcinze and torulæ, may be found in the vomited matters when these have undergone fermentation within the stomach. Blood is not infrequently found in the vomited matters, gene- 
rally in small quantity, and having the coffee-grounds appearance. Purulent or sanious matter is frequently vomited. With these symptoms referable to the stomach are assoeiated progressive loss of weight and strength, anæmia, not infrequently febrile movement, usually eonstipation but occasionally diarrhœa, and in some cases jaundice. The last-named symptom points to the probable oceurrenee of secondary cancer affeeting the liver.

With respeet to the pathology and eausation, nothing is to be added here to what has been stated already in the first part of this work. Cancer in the stomaeh, as elsewhere, involves a constitutional predisposition or diathesis, and a morbid change preceding the local affection, or a cachexia. Statistical researches do not appear to show that the development of the affection is favored by particular habits of life or any appreciable causes. The causation has reference to age. The liability to the affeetion prior to the age of forty is small. In the great majority of cases it oecurs between fifty and sixty. The liability to it diminishes after sixty, and is small after seventy. Males are affected more frequently than females, the proportion being as 2 to 1 . The occurrence of cancer of the uterus in the female compensates for this difference. The transmission of the cancerous predisposition or diathesis by inheritance is well established. Faets, also, appear to show that a predisposition to eancer seated in the stomach may be inherited. A well-known example is the case of Napoleon Bonaparte, whose father, and a sister also, died of eaneer of the stomach.

Cancer of the stomach is to be diseriminated from merely functional disorder, or dyspepsia, and chronic gastritis. In merely functional disorder, pain and vomiting are symptoms rarely as prominent as they are generally in eancer. The ailments in dyspepsia are seldom so protracted; they do not lead to the same amount of emaciation, feebleness, and pallor; hæmatemesis and the vomiting of purulent matter are wanting. The same is true of ehronic gastritis. Persistent disturbance of digestion, especially if aceompanied by pain and vomiting, leading to considerable loss in weight, diminution of strength, and anæemia, in a person between forty and sixty, should always excite strong suspieion of eancer, although blood and pus have not been vomited. Cases of gastralgia lack all the diagnostic features of cancer except pain. Gastric uleer is liable to be confounded with eaneer, and in view of the great difference as regards prognosis, it is desirable to discriminate between these affections. They present certain points in contrast as regards the symptoms. In cancer the appetite is more impaired than in ulcer. Vomiting is more uniformly present in ulcer; it oecurs earlier after the ingestion of food, and, in general, is evidently due to the irritation caused by the presence of food. In eancer it is present in only a eertain proportion of eases ; it does not follow so soon after taking food, if the cardiac orifice be not obstructed, and is frequently due to the mechanieal distension of the stomach owing to the pyloric obstruction. Hemorrhage is more constant, and is apt to be more profuse in uleer. If the patient be a young girl, the chances are much in favor of ulcer: and, at any age, if the patient be a female, uleer is more probable than cancer. The pain in eancer is apt to be lancinating, in ulcer burning or gnawing. The duration of the local symptoms is of importance in this differential diagnosis; if the symptoms have existed for a long period, the chances are in favor of ulcer rather than cancer. Age is to be considered in the diagnosis, cancer occurring rarely before forty.

Considerable importance has been attached to a peculiarity of complexion which has been supposed to denote the caneerous cachexia, consisting of pallor with a faint tint of yellow or green, the conjunctiva 
remaining unaffected. It has been called a waxy complexion. This is sometimes well marked in cases of cancer, but it is by no means always present, nor is it pathognomonic when marked. If members of the family have had cancer, this fact is entitled to some weight in the cliagnosis. So, also, is the coexistence of cancer in some other situation.

In some cases of cancer there are few or no symptoms denoting any serious affection up to a short time before death. Like gastric ulcer, it is sometimes remarkably latent, the patient appearing to suffer only from moderate or slight dyspeptic ailments, the appetite remaining unimpaired, the strength not failing, and the aspect indicating health. Watson eites several examples of this kind. Some time ago I was accustomed to meet frequently a gentleman supposed to be simply delicate and a dyspeptic, who, at length, was seized with severe gastric symptoms and died after a short illness. At the post-mortem examination, the pylorus was found to be the seat of a large cancerous tumor which involved a large portion of the stomach. As another example, I visited in consultation an aged gentleman who died a few days afterward with unmistakable cancer of the stomach, and in this case it was only within a few days that the patient had had a suspicion of anything more than a trivial disorder.

In the latent cases just referred to, and also when the differential diagnosis relates to ulcer and chronic gastritis, much importance belongs to the discovery, or otherwise, of an ablominal tumor in the site of the pylorus or stomach. Exclusive of the cases in which the cardiac orifice is the seat of the cancer, a tumor is discoverable by physical examination in the majority of cases. According to Brinton, it exists in 80 per cent. of all cases of cancer. The tumor is situated generally between the false ribs and the umbilieus, a little to the right of the median line. It may be movable or fixed. If movable, Valleix attaches considerable importance to its change of position, according as the stomach is distended or empty. 'The tumor varies in size from a pigeon's egg to the size of the closed hand. It is usually resisting, and the surface irregular or nodulated.

'The presence of a tumor, if ascertained to be seated in the stomach, renders the diagnosis quite positive. But it may not be always easy to decide positively that the tumor is seated in the stomach. A cancerous tumor may simulate aneurism of the abdominal aorta. The characters of an aneurismal tumor are expansile pulsation, thrill, and murmur. $\mathbf{A}$ cancerous tumor may present, more or less marked, the same characters. It may present not only a single, but a clouble, murmur. How are the two to be distinguished from each other? An aneurismal tumor is rarely movable; it has usually a smooth outline, and may be diminisher by pressure; the pulsation is felt laterally as well as on its anterior surface; by a cliange of position of the patient, as placing him on his hands and knees, the pulsation is not materially affected, whereas if the tumor be not aneurismal, its pressure on the artery is taken off and the pulsation may be found to cease. But aneurismal tumors do not always pulsate, and both thrill and murmur may be wanting. Under these circumstances, in this differential diagnosis, reliance must be had upon the diagnostic points relating to the history and symptoms. These are, of course, always to be carefully considered. Other tumors, from their situation are liable to be confounded with cancer of the stomach, viz., tumors connected with the left lobe of the liver and the pancreas, fecal tumors, etc. The distinctive characters of these will be considered in otber connections.

Cancerous affection of the cardiac orifice, leading to stenosis or stricture, gives rise to symptoms quite different from those which accompany cancer of the stomach otherwise situated. Food accumulates in the lower 
part of the œsophagns, and is regurgitated directly after deglutition. The obstruction, if considerable, interferes with vomiting as well as the ingestion of food. The food is regurgitated without change, and is devoid of the taste and odor of the gastric fluids. The patient is unable to take solid food, and is compelled to take liquids slowly and in small mouthfuls. If the stricture be great, death takes place gradually by inanition. A patient was admitted into Blackwell's Island Hospital, æet. 45, in a state of great exhaustion, and evidently very near death. He stated that for a long time he had been unable to take anything but liquids. The bowels were constipated, no dejection, according to the patient's statement, having taken place for a month. Death occurred twenty-four hours after admission. On post-mortem examination, the cardiac orifice was found to be the seat of cancerous disease which extended somewhat up the cesophagus. The carliac orifice was so completely occluded, that a fine silver probe was passed with difficulty through it, and water poured into the osophagus remained there. I met with a similar case, the inanition not so far advanced, at my elinic at the Long Island Hospital. Two additional cases have come under my observation since the first edition of this work appeared.

In these eases, a tumor is not discoverable. The symptoms are the same as in cases of stricture at the lower portion of the œsophagus from other than cancerous disease, and the differential diagnosis cannot be made with positiveness. Spasmodic stricture of the osophagus may simulate contraction from either cancer or some other organic affection. The passage of a probang into the stomach will enable the practitioner to exclucle the latter.

I have not referred to the microscopical examination of the matters vomited, in cases of suspected cancer, with a view to the discovery of the cancer cells. 'This test is only available at an advaneed period of the disease, and then only in a very small proportion of cases, owing to the action of the gastric juice and the admixture of the exfoliated cancerous material with the ingesta.

The prognosis in cases of cancer of the stomach is as unfavorable as possible. If a cure be within the range of possibility, the probabilities are so vastly against it that we are not warranted in entertaining the least expectation of such a result in any individual case. A fatal termination is merely a question of time. Statistics show the average duration to be about a year. The maximum duration is about three years, and the shortest period is a month. Death oecurs by slow asthenia. Perforation was the immediate cause of death in 21 of 507 cases analyzed by Brinton. Of 23 cases reported by Dr. Alfred L. Haskins, this was true of only five. ${ }^{2}$

The treatment has reference only to the prolongation of life and the palliation of symptoms. A diet, bland and nutritions, consisting of the articles of food which experimental trials, in each case, show to be best borne, taken in quantities and at intervals to be determined also by trial; remedies to relieve pain and quiet the irritability of the stomach; avoidance of cathartics and any measures which impair the vital potrersthese are the points to be kept in view in the management of this distressing and hopeless affection.

\section{DILATATION OF STOMACH.}

Dilatation of the stomach is, in general, a lesion dependent on obstruction at the pyloric orifice. It enters into the anatomical characters of

I Am. Journ. of Med. Sciences, January, 1869. 
certain cases of cancer, it is incidental to cicatrization of ulcers seated at the pylorus, it occurs in connection with pyloric thickening from fibroid growth, and it may be caused by the pressure of a tumor on the lower portion of the stomach. It takes place irrespective of obstruction at the pyloric orifice, but the instances are extremely rare. I have notes of two cases which liave fallen under my observation, in which the dilatation was sufficient to form a notable projection of the epigastrium and upper part of the abdomen, there being no evidence of obstruction at the pylorus, save the existence of the dilatation. Both these cases were under observation for a short time only. ' But cases have been observed in which the dilatation was so great that the boundaries of the stomach embraced the greater part of the abdominal surface, causing enlargement of the abdomen as great as in hydro-peritoneum or an advanced period of pregnancy. The capacity of the organ has been known to be increased so that it would contain several gallons of liquid. 'The coats of the stomach, in these cases, are atrophied, especially the muscular coat, and the walls are sometimes as thin as paper.

In these cases of excessive dilatation, vomiting occurs after intervals of a few days, an chormons amount of matter being expelled, the matter consisting, in a great measure, of imperfeetly digested aliment, which accumulates in consequence of the inability of the stomach to propel its contents into the duodenum. The vomited matter emits a putrescent odor, and frequently contains sarsinæ and torulæ cerevisiae. 'The appetite is good or even voracious, but, owing to the accumulation of food in the stomach and its ejection by vomiting, the assimilation is not proportionate to the ingestion, and the body wastes.

The production of this lesion is attributed to atrophy of the muscular fibres, to paralysis of the stomach, and to habits of immoderate eating. Chambers is of opinion that in most cases of clilatation not the effect of mechanical obstruction at the pylorus, it is due to a congenital deformity. This author cites examples of its existence in persons who were accustomed to eat sparingly. The number of cases which are to be collected is, as yet, too few for analysis with reference to the causation and other points of investigation.

Dilatation of the stomach occasions an abnormal prominence of the abdomen especially marked at the epigastrium, and extending more or less below the umbilicus, according to the augmented size of the organ. The projecting tumor yields, at the upper part, a tympanitic resonance on percussion, having a characteristic gastric quality of sound. At the lower portion, if the stomach contain ingesta, there is flatness on percussion, and the relative situation of the flatness and tympanitic resonance changes as the position of the patient is changed from the vertical to the recumbent. On succussion, a splashing sound may be produced, analogous to that in pneumo-hydrothorax. 'This was marked in one of the cases under my observation. In the same case, when the patient swallowed liquid, metallic tinkling was heard with Cammann's stethoscope placed over the stomach, above the level of its liquid contents. If the abdominal walls be thin, the form of the stomach may be apparent, and its peristaltic movements may be seen. The enlargement varies at different times according to the variation in the contents of the stomach; it diminishes greatly or disappears after the complete expulsion of the contents by vomiting. A sense of fluctuation may be felt when the stomach contains considerable liquid. This is noted in the history of one of my cases.

Valleix states that paracentesis has been practised in a case of enormously dilated stomach, under the belicf that the affection was hydro- 
peritoneum. Physical examination of the abdomen should enable the practitioner to avoid this error. In hydro-peritoneum, the enlargement, if not uniform, is greatest at the lower part of the abdomen, the reverse being true of dilatation of the stomach. In hydro-peritoneum the enlargement is proportionate anteriorly and laterally; in clilatation of the stomach the anterior projection is greater than the lateral. In hydro-peritoneun flatness on percussion exists at the lower part of the abdomen; in dilatation of the stomach, intestinal tympanitic resonance will be likely to be found below the space which yields flatuess, in the hypogastric and iliac regions. The variation in the enlargement, and its disappearance after complete vomiting, suffice for the diagnosis.

In the cases which $I$ have observed, the affection was not attended by notable inconvenience. Vomiting did not occur in these cases. In cases of excessive dilatation, nutrition suffers from the accumulation of aliment in the stomach and its ejection by vomiting. Yet, it is stated by Valleix that there is no well-attested instance of death produced by simple dilatation.

Regulation of the ingesta as regards quantity, and tonic remelies constitute the appropriate treatment. Indigestible food and articles which, from decomposition, develop gas in abundance, should be interdicted. .A diet of milk, containing grated ice, is recommended by Petrequin, who reported two of the cases collected by Dr. Haskins.

\section{DEGENERATION OF THE GASTRIC TUBULES.}

The degeneration of structure in different organs has been, within late year's, much elucidated by microscopical researches. Of this fact, the affections of the liver, of the kidney, of the arteries, of the muscular tissue, etc., furnish striking examples. By the aid of the microscope, the intimate normal structure of different parts has been unfolded, as a point of departure for the study of abnormal deviations. Our knowledge of the gastric tubules has been in this way recently acquired. The stomach has been shown to contain an immense secreting surface furnishing an enormous amount of secretion daily. The glandular arrangement is essentially the same as in the other glands, for example, the kidneys, the difference being that the secreting surface is disposed in innumerable short tubules instead of convoluted tubes. Now, analogy would lead to the supposition that the tubules of the stomach may be the seat of disease and degeneration, as well as the tubes of the kidneys, or the secretory structure of other glandular organs. As yet, however, very little attention has been given to the study of the morbid conditions of the gastric glands. The subject has been referred to by two German writers, Engel and Wendl.' But to Hanfield Jones is due the credit of having broken ground in this new field of research. In a paper entitled "Observations of Morbid Changes in the Mucous Membrane of the stomach," Dr. Jones has tabulated the appearances of the gastric tubules in 100 stomachs, the subjects not selected, but taken consecutively, and dead with a variety of diseases. ${ }^{2}$ The results go to show that here is a field the further cultivation of which may lead to highly important additions to our pathological knowledge.

1 Chambers on Digestion and its Derangement.

2 Medico-Chirurgical Transactions, vol. xxxvii. London, 1854. Further observations have been contributed by Dr. Wilson Fox, in ibid. 1857 and 1858; also, by Dr. Fenwick, in ibid. 1865. 
Of the 100 examinations, in 72 the appearances denoted more or less degenerative change. Of these 72 cases, in 47 the degeneration was limited to a portion of the stomach, usually the pyloric portion, the tubules in the remainder of the organ being healthy. In 11 cases the extent of the aflection was greater, but still moderate. In 14 eases the appearances denoted destructive changes extending over the greater part of the stomach. The appearances denoting degeneration within the tubules consisted of the deposit of black pigment and fatty granules, the presence of granular matter in the place of the normal epithelium, supposed to be the debris of the latter, and atrophy or loss of the epithelium. These changes are analogous to those oluserved in the degenerative changes which take place within the convoluted tubes of the kidmey in cases of the chronic affections of these organs embraced under the name Bright's disease.

In the 14 cases in which the gastric tubules were extensively degenerated, the patients died with a varicty of diseases, and it is not easy in so limited a number of cases to determine what symptoms were attributable to the affection of the stomach. It is not improbable that further researches will show these glands to be the seat of morbid conditions of great importance, as occurring alone or in association with other affections. There is room for the conjecture that the impairment of digestive power, the anæmia, debility, etc., which sometimes occur without being connected with any obvious disease, as in the so-called idiopathic anæmia described by Addison, and which are sometimes superadded to various diseases, may be due to morbid conditions seated in the gastric tubules. Further explorations in this new field of research may lead to developments of as much pathological importance as those which have resulted, within the past few years, from the study of the morbid conditions of the kidney.

The study of the secretory apparatus of the small intestine, or the follicles of Lieberkuhn, may afford a similar field for fruitful research, which as yet appears to have hardly been begun.

The points which it is desirable to settle by a sufficient number of observations are: First, the variations in appearances which occur within the limits of health; and the appearances which denote disease, and not cadaveric changes. Second, the different kinds of morbid change, together with their pathological character and import. Third, the gross appearances corresponding to the changes ascertained by the microscopc. Fourth, the symptoms associated with different anatomical changes.

The foregoing remarks on degeneration of the gastric tubules were contained in the previous editions of this work, and are retained in this (4th) edition without alteration. Further reflection has served to strengthen, in $1 \mathrm{ny}$ own mind, the conviction that important results may be expected from histological researches in this direction. For a long time it has seemed to me a rational supposition that lesions compromising the secretory function of the gastro-intestinal tubules exist in a class of cases characterized by progressive and fatal inanition taking place without the evidence of disease existing elsewhere sufficiently to explain the symptoms and the termination. In an essay written since the publication of the last (3d) edition of this work, I have given abstracts of several cases of this kind which have fallen under my observation. ${ }^{1}$ 'These cases represent a class with which all practising plyysicians of much experience are more or

1 The Pathological relations of the gastric and intestinal tubules, by Austin Flint, M.D., New York Med. Journal, March, 1871. 
less familiar. The marked characteristics are gradual and, at length, complete loss of appetite and digestive power, with progressive debility, and death by exhaustion, adequate morbid conditions seated elsewhere than in the glands of the alimentary canal being excluded by an investigation of the symptoms and signs. In the cases cited in the essay just referred to, the patients were somewhat advanced in years, and they were healthy prior to the illness which ended fatally; this illness was developed imperceptibly and advanced slowly ; the persons were singularly exempt from apparent morbitic influences, being temperate and their habits of life regular; they were in easy circumstances, and in a great measure withdrawn from active occupation; the intellectual faculties remained intact up to a few hours before death, and the mode of dying was typical of slow asthenia. The impairment of appetite was the first symptom.

My object in the essay was to show, from a clinical point of view, first, the existence of a well-defined class of cases characterized by anorexia, impaired digestion, progressive debility and death from inanition; and, second, in view of these elinieal charaeteristies, together with the absence of adequate lesions elsewhere, the probability that the essential disease is seated in the secretory glands of the alimentary canal.

In order that, in this class of cases, lesions of these glands shall be recognized as an established fact, the existence of the lesions must, of course, be demonstrated by microscopical examinations after death. This appears to have been done in a case reported by Dr. Samuel Fenwick, ${ }^{2}$ in which the diagnosis of disease of the gastrie tubules was made from the symptoms and history. In this case the existence of distinctive lesions here was verified by the microscope, and by estimates showing notably deficiency of the gastric juice. Probably, the whole extent of the pathologieal relations of the gastro-intestinal tubules is by no means embraced by supposing that the anatomical characteristics in the cases jnst referred to, are to be sought for in these glands. There are grounds for the belief that lesions here seated are often associated with varions diseases, and that functional disorder of these glands enters more largely into morbid phenomena than has hitherto been supposed.

From the histological researches of Jones, Fenwick, and Fox, lesions affecting the secretory function of the tubules correspond with those which occur in other glandular organs, c. g., the kidneys. The point of departure in some cases is within the tubules, the secretory cells undergoing granular fatty metamorphosis, and, finally, disappearing. In other cases, the primary seat appears to be in the connective tissue surrounding the tubules, the latter becoming atrophied by pressure, as in cases of cirrhosis of the kidneys.

${ }^{1}$ Vide London Lancet, July 16, 1870. I have quoted the case in full in my essay. 


\section{CH A P TER IV.}

Structural Affections of the Intestinal Canal involving Obstruction-Invagination-Strangulated Hernia within the Abdomen-Rotation or twisting of Intestine-Compression and Stricture of Intestine. Obstruction froin Impaction of Feces, Entcrolithes, and Foreign Bodies. Functional Obstruction.

QTRUCTURAL affections of the intestines are important, measurably $D$ or chiefly as giving rise to obstipation due to mechanical obstruction to the passage of the intestinal contents. Hence we may consider these affections under the head of obstruction of the bowels. And, having considered the important structural affections involving obstruction, it will be convenient to consider, in this chapter, obstipation (meaning by this term obstruction as distinguished from constipation) dependent on causes other than lesions of structure, viz., on the impaction of feces, the formation of concretions called enterolithes, the introduction of foreign bodies, and on merely functional disorcler. The term ileus or iliac passion, and other names, have been applied to cases of obstipation with the ejection from the month of the intestinal contents, or so-called stercoraccous romiting. As the latter symptom occurs in connection with obstruction from various causes, the names based upon it have no special pathological significance and tend to produce confusion.

Obstruction from structural affections is happily not of frequent occurrence. According to the statistical resources of Benjamin Phillips, it is met with in a proportion of one per cent. in post-mortem examinations.' Similar researches by Brinton, the whole number of promisenous necropsies being 12,000, showed obstructions from various causes, exclusive of hernia, in the proportion of 1 in 280 deaths. $^{2}$ 'The lesions involving obstruction are the following: Intussusception, invagination or volvulus; strangulation from hernia within the abdomen; rotation of a portion of intestine upon its own axis, or upon the mesentery, and, according to Rokitansky, twisting of a portion of intestine around another coil of intestine; compression of one portion of intestine by another portion, or by a tumor situated extcrior to the canal, and stricture produced by morbid growth within the canal or by the contraction following the cicatrization of ulcers. The symptoms attending these several affections are not, in all respects, the same, although obstipation is a common feature. They will therefore claim separate consideration. But it will be seen that it is not always easy to determine, by means of the symptoms in individual cases, the particular lesion which occasions the obstruction.

\section{INVAGINATION.}

Invagination, intussusception, or volvulus, is the reception of a portion of the intestines into another portion. Generally the invagination is from above downward, but sometimes the lower portion is received into the upper. The latter, called retrograde invagination, was found in 3 of 59 cascs ana- 
lyzed by Dr. S. Foster Haven. ${ }^{1}$ It is certain that invagination may occur transiently, giving rise to no symptoms. In post-mortem examinations, especially in children, innocuous invaginations of the small intestine are not infrequently found ; the invaginated portion is restored without diffculty, and, aside from the displacement, there are no morbid appearances. In such instances, they have probably occurred in the last moments of life. I counted as many as fifteen of such invaginations in the body of a child dead with typhoid fever. It is not unlikely that they occur frequently in connection with various diseases and even in health. It is only when the invagination becomes fixed, giving rise to congestion, inflammation, etc., that it occasions obstruction and other symptoms. It is then a lesion of very great gravity, in the great majority of cases ending fatally. This is the most frequent of the lesions causing fatal obstruction. Of 169 cases of obstruction from various lesions collected by Phillips, 63 were of this class. An analysis of 600 cases by Brinton showed in 43 per cent. the obstruction to be from intussusception.

Invagination brings into apposition three layers of intestine, viz., an entering, a returning, and a receiving layer. In the relation of the entering and receiving layer, mucous surfaces are in contact, and these do not become adherent. But serous surfaces are in contact in the relation of the entering and returning layer, and inflammation, excited at the point of entrance, leads to adhesion of these surfaces, extending more or less beyond the point of entrance. In this way the invagination becomes fixed. The vessels of the portion of the mesentery connected with the invaginated intestine are obstructed by tension. Congestion and swelling of the invaginated intestine ensue, and, at length, it becomes gangrenons, as if strangulated, and sloughs away, if life be sufficiently prolonged. The obstruction is due to the swelling from congestion and the exudation of lymph. Usually the obstruction is complete, but exceptional cases have been reported in which the intestinal passage remained pervious but much contracted. Peritonitis is apt to extend more or less around the seat of the invagination, leading to morbid attachment to the adjacent parts.

The invagination may occur at any point in the intestinal tract. Its most frequent seat is at the junction of the ileum and crecum. This is shown in cases of children, by the valuable statistical researches of Dr. J. Lewis Smith. ${ }^{2}$ Dr. Smith's account of the mode in which it generally occurs is as follows: "The intussusception not infrequently begins in the prolapse of the ileum through the ileo-cæcal valve, in the same way that prolapse of the rectum occurs through the sphincter ani. If death take place early, only a small portion of the ileum may have passed the valve. If the case be protracted, the tenesmus brings down more and more of the ileum with its accompanying mesentery. The constriction of the valve, which acts as a ligature, prevents the further descent of the ileum, and, the tenesmus continuing, the next step is the inversion of the caput coli, which is drawn into the colon by the rlescending mass; and unless the case terminate by sloughing or death, the ascending and transverse portions of the colon are successively invaginated. Not infrequently, the cæcum is the part primarily inverted and invaginated, and, descending along the colon, it draws after it the ileum which sustains its natural re-

1 American Journal of Med. Sciences, October, 1855.

2 Statistical Researches relative to the Seat, Symptoms, Pathological Anatomy, etc., of Intussusception in Children. Am. Jour. of Med. Sciences, Jan. 1862. Vide, also, Smith on the Diseases of Children, second edition, 1872. 
lation to the ileo-creal valve. 'These two forms of invagination-that in which the ileum, passing through the ileo-cæeal valve, successively inverts and draws after it the caput coli and the divisions of the colon, and that in which the caput coli is primarily invaginated, and, descending along the large intestine, inverts the latter, and draws after it the ileum-constitute the vast majority of cases of this clisease in ehildhood." The invaginated portion may descend so low as to be felt and seen at the anus, and it may even protrude from the body. It is, lowever, to be borne in mind that the invagination may occur at any point in either the large or small intestine. In Dr. Haven's analysis of 59 cases it was seated in the small intestine in 23. Of the cases analyzed by Brinton, the intussusception was ileo-creal in 56 per cent.; iliac in 28 per cent.; jejunal in 4 per cent., and in the colon in 12 per eent.

Inragination causing obstruction, as alrealy stated, proves fatal in the great majority of cases; yet the affection is by no means absolutely hopeless. The manner in which recovery usually takes plaee is of importance in its bearing on the treatment. 'The invaginated portion of intestine sloughs awny and is evacuated, the entering and receiving portion at the point of entrance remaining adherent, and the perviousness of the canal being restored. Cases have been reported in which a large portion of invaginated intestine was thrown off, and reeovery followed. Prof. Yan Buren reported a case to the New York Pathological Society, in which five feet of intestine were passed per anum, the patient recovering. Prof. Peaslee exhibited at a meeting of the New York Academy of Medicine, 1865, five feet of intestine which had been passed per anum four months before the death of the patient. He also exhibited the intestines removed from, the body of this patient after death. The small intestine was only 16 feet in length, the length of the large intestine being 5 feet and 10 inches. The invagination and sloughing had taken place in the small intestine at a distance of six feet from the duodenum. A stricture existed at this point; the intestine above was much clilated, and that below greatly reduced in size. The patient died from inanition dependent on the stricture of the intestine.' Dr. William Thompson, of Edinburgh, collected 43 cases ending in recovery." Of Dr. Haven's 59 cases, in 12 the invaginated portion was passed per anum, and of these 12 eases 10 ended in recovery. This is the mode of recovery which is to be hoped for after permanent incarceration of the invaginated intestine has taken place.

The prominent local symptoms which enter into the elinical history of invagination are as follows: Pain is more or less prominent, at first paroxysmal as in colic, and after a time becoming constant, with frequent' exacerbations. The pain, if the patient be old enough to describe it, appears to emanate from a certain fixed point. Tenderness at first may not be marked, but becomes developed, being either limited to, or greatest at, the point whence the pain emanates. The tenderness and constant pain denote the occurrence of peritonitis at the seat of the invagination. Vomiting soon becomes a prominent and persisting symptom, with very. few exceptions. The vomited matter, after a time, may have the odor of feces, and is then said to be stercoraceous. A truly stereoraceous vomiting, however, very rarely, if ever, takes place-that is, the contents of the large intestine are not ejected. This could, of course, only happen when the obstruction commences below the cæcum, which is extremely rare;

1 Vide Bulletin of the New York Academy of Medicine, rol. ii., Nos. 25-29.

2 Vide article on Internal Strangulated Intestine, by Elisha Harris, M. D., in New York Journal of Medicine, 1853. 
but, even if the cæcum be above the seat of the invagination, the ileo-cæcal valve, certainly in the great majority of eases, effectually prevents regurgitation of the contents of the large into the small intestine. Experiments and clinical observation show that the ileo-cecal valve will sustain an amount of pressure sufficient to cause rupture of the intestinal walls. It is not, however, improbable, as has been conjectured, that regurgitation may sometimes take place to some extent, provided the ileum as well as the excum at the point of junction be distended. The accumulation of ingesta and gas above the obstruction occasions more or less abdominal distension, provided the invagination be not situated in the upper portion of the small intestine. Frequently the accumulation of intestinal contents at the seat of the invagination gives rise to a tumor appreciable by the eye and touch, and to dulness on percussion. After the contents of the intestinal canal below the point of obstruction have been evacuated, there is persisting obstipation, save in a very few exceptional cases. If the invaginated portion of intestine descend to the rectum, tenesmus is felt, and the patient is led to make straining efforts. Under these circumstances bloody mucus is expelled, as in dysentery.

The general symptoms at first may not be marked, but soon they denote a grave affection. The pulse becomes accelerated, its frequency progressively increases and it is proportionally feeble or compressible. Progressive prostration, hiccough, coolness or coldness of the surface, an anxious or haggard expression, denote the progress toward a fatal termination. The mode of dying is by asthenia, the pain and tenderness frequently diminishing or ceasing for some time before death.

A favorable progress is denoted by the occurrence of free evacuations from the bowels, the diseharge of the invaginated portion of intestine, together with improvement in all the local and in the general symptoms.

Invagination is to be discriminated from functional colie, acute peritonitis, and obstruction from other causes. The symptoms at first may denote nothing more than colic. The persistency of pain, the development of tenderness, the acceleration of the pulse, the vomiting, ete., however, soon point to an affection of greater gravity than colic. Acute peritonitis is to be excluded by the gradual development of the local and general symptoms ; by the absence of diffused tenderness over the abdomen, and of rigidity of the abdominal museles; by the localization of pain and tenderness within a circumscribed space, and by the signs of a tumor in some cases. A cute peritonitis sometimes supervenes, in cases of invagination, from rupture of the intestine above the obstruction. From obstruction caused by hernia within the abdomen, and rotation and twisting of the intestine, the discrimination is more difficult, and, indeed, cannot always be made with positiveness. The age of the patient has a bearing on the diagnosis. Invagination occurs most frequently in infancy. According to Dr. Smith's statistics, it is most apt to occur between the second and third month of infantile life. Of 47 eases only 18 occurred between the ages of one year and twelve years. In Dr. Haven's cases the mean age was 18 years; the youngest age was 3 montls, and the oldest 65 years. Male children, more than female, are liable to it, the relative proportion, in Dr. Smith's cases, being thirty-two to twentytwo, and in Dr. IIaven's 59 cases 34 were males. On the other hand, hernial obstruction occurs oftener after infancy and childhood, and eases oceur oftener in females than in males. The discharge of blood and mucus is distinctive of invagination involving the large intestine.

A point in diagnosis is to determine the probable seat of the invagination. The chances are that it is seated at the junction of the large and 
small intestine. The probability of this being the seat is increased, if the pain, tenderness, and swelling are situated in the neighborhood of the right iliac fossa. Tenesmus, with the discharge of blood and mucus, shows that the large intestine is involved. The invaginated portion may sometimes be felt and seen within the rectum. If seated in the small intestine, the constitutional disturbance is greater, and, if the point of obstruction be toward the upper portion of the small intestine, there will be little or no abdominal distension. Barlow considers the quantity of urine as furnishing a guide to the situation of the obstruction. If the small intestine be unobstructed, liquids ingested are abundantly absorbed, and the urine is proportionately abundant, but if the obstruction be situated high $u p$ in the sinall intestine, the diminished absorbing surface involves a seanty secretion of urine.' It is doubtful if much reliance is to be placed on this test.

The prognosis is extremely unfavorable. The usual mode of recovery in the exceptional cases in which the affection does not end fatally, has been stated, viz., by sloughing away of the invaginated portion of intestine, the adhesions at the point of entrance being permanent. It is possible that the invaginated portion may be restored, in some cases, after symptoms denoting the affection are developed, but such instances must be exceedingly rare. And it must be equally rare for the invaginated portion of intestine to remain, the camal becoming or continuing pervious. Of the 50 cases analyzed by Dr. Smith, in 7 recovery took place, and in each case by sloughing. In all these 7 cases the ages were between 5 and 12 years, Dr. Smith's researches being limited to cases occurring in childhood. The constitutional powers in infancy do not seem to be adequate to support the affection for a suflicient period for the sloughing process to be completed. The separation of the invaginated portion of intestine takes place between the sixth and twelfth day. In fatal cases, the duration rarely extends beyond eight days. Death may take place within twenty-four hours. In the majority of eases, life is not prolonged beyond the third day. Dr. Smith eites a case in which the symptoms of invagination had existed for six weeks, and in another case, for three months before death, the obstruetion in both cases not being complete, and the invaginated portion of intestine not completely strangulated. Death sometimes is hastened by peritonitis caused by rupture of the intestine above the seat of the obstruction. In young ehildren the duration is sometimes shortened by the oecurrence of convulsions. Generally the mode of dying is by slow asthenia.

If the existence of invagination could be ascertained prior to the development of symptoms denoting incarceration, it is probable that measures for restoration might be successfully employed. But generally a day or two elapses before the character of the affection is even suspected. In the mean time, cathartics will be likely to increase the invagination and diminish the practicability of restoration. The liability to the existence of this affection, especially in children, should be borne in mind in cases of apparent colie, and should dictate reserve in the employment of cathartics. So soon as the character of the affection is rendered probable by the symptoms, measures to effect restoration may be tried. These measures are the injection of water or of air in considerable quantity into the rectum. If practicable, the injection should be made through a long flexible tube carried into the intestine as far as it can be made to pass without undue force. The object is to effect the restoration by the upward pressure of 
the air or water, the invagination being, in the great majority of cases, in a downward direction. The injections are not to be pushed beyond the point at which they are borne without much suffering, and, if they do not succeed after a fair trial, they are not to be persisted in. They will very rarely succeed after the invaginated portion of intestine has become swollen by congestion and the peritoneal surfaces in contact have become adherent. If pushed too far, rupture of the intestine below the seat of the obstruction may be prodnced. I have known rupture to result from the injection suceessively of an acid and alkaline liquid, giving rise to the evolution of gas by combination within the intestine. This method of employing pressure is highly objectionable, beeause the amount of pressure cannot be regulated. Cases have been repeatedly reported in which the injection of air or water has apparently effected restoration in cases of supposed invagination, even after obstruction had existed for several days. But it is probable that in a certain proportion of these cases invagination did not really exist. 'These measures for reduction are of no avail if the seat of the invagination be above the ileo-eæcal valve.

Exclusive of measures for reduction, the objects of treatment are to secure as much quietude of the intestinal canal as possible, to palliate suffering, and support the powers of life, under the hope that sloughing and recovery may take place. The avoidance of cathartics is essential. The practitioner is not to be tempted to employ them with the hope of overcoming the obstruction. They interfere with the objects just named, and are clestructive. Not only the active cathartics, but even mild purgatives and laxatives are contra-indicated. To quiet the peristaltic movements, opium is to be given in sufficient doses to secure relief of pain without inducing narcotism. Fomentations over the abdomen are useful. The strength of the patient is to be supported by concentrated and purely nutritious food given in small quantities at a time, together with alcoholic stimulants. It is to be borne in mind that the recovery will depend on life being sufficiently prolonged for the slonghing away of the invaginated portion of intestine. Bloodletting, counter-irritation, or any measures which tend to impair the vital forces, are never indicated, and cannot fail to do harm. After sloughing has taken place and the obstruction is removed, purgatives are not to be employed, lest, by exciting unduly peristaltic movements, the adhesions at the point of the invagination may be broken up and peritonitis ensue from the escape of the contents of the intestine into the peritoneal sac.

Gastrotomy has been resorted to as a last resource in cases of invagination. Phillips collected 27 cases in which this operation was performed, and in 2 of these cases it proved successful. One was the case of a negro reported by Dr. Wilson, in the Anerican Journal of Medicine, vol. x. The first object of the operation is to restore the parts if practicable, and a second object to perform enterotomy and establish an artificial anus. Life may be prolonged if the latter object be accomplished, although recovery does not take place. The chances for restoration would be greater in proportion as the operation was resorted to early, but few surgeons would be willing to make so formidable an operation at a period when the diagnosis might hardly be considered as settled. After strangulation has existed for some time, it would not be advisable to restore the parts even were it practicable. In short, at the time when the operation, if employed at all, would be advisable, the chances of recovery after it would be less than if reliance were placed on a spontaneous cure. Even with a view to artificial anus, the operation would be likely to lessen the chances of a 
spontaneous cure. The propriety of surgical interference has therefore justly not many advocates.

\section{STRANGULATED HERNIA WITHIN THE ABDOMEN. ROTA- TION OR 'TWISTING OF INTESTINE.}

Several abnormal conditions lead to hernia within the abdomen. Fissures sometimes exist in the mesentery, omentum, and diaphragm, into which a portion of intestine passes and is liable to become incarcerated and strangulated. The vermiform appendix of the cæecum sometimes becomes adherent to an adjacent part, leaving a loop through which intestine may pass and become ligated. 'The same may happen in connection with diverticula of the intestimal tube, which are not very infrequent. Bands of adventitious membrane, resulting from peritonitis, may leare spaces for hernial protrusions. Whatever be the mode in which the strangulation is produced, the symptoms are the same. They are, in fact, identical with the symptoms of ordinary strangulated hernia occurring at the femoral or the inguinal outlet, and they are, for the most part, the same as in cases of invagination. The existence of tenesmus with bloody and mucous evacuations, in certain cases of the latter, constitute the chief point of distinction. Irrespective of the symptoms just named, the differential diagnosis can hardly be made with positiveness, but the chances are that in the young child invagination exists, whereas, in after life, obstruction from other causes is more liable to occur. The small intestine is far more likely to be the scat of hernia within the abdomen than the large intestine. Sloughing of the strangulated portion of the intestine is destructive, whereas, in cases of invagination, it is the mode of cure. The only hope of recovery is in the spontaneous reduction of the hernia, bcfore gangrene takes place. The practitioner can do nothing directly towards reduction. Distending the large intestine with air or water will be useless if the seat of the hernia be in the small intestine. Cathartics in these cases are more likely to do harm than good. Opiates to palliate pain, fomentations to the abdomen, and supporting measures constitute the appropriate treatment. The prognosis is as unfavorable as possible.

Rotation or twisting of a portion of intestine, so as to induce strangulation, is most apt to occur at the sigmoid flexure of the colon. Rotation of the sigmoid flexure twice upon itself, causing fatal obstruction, occurred in the person of a distinguished member of the cabinet of the United States many years ago." Habershon gives a case in which the rotation occurred at the cæcum, this portion of the large intestine being unusually mobile. Twisting of a portion of intestine round an axis formed of mesentery, or around another coil of intestine, occurs in the small intestine. These accidents are happily to be reckoned among the rare curiosities of clinical experience. Their symptoms are those of strangulation, and it is impossible to discriminate them from invagination or hernia within the abdomen. In the analysis of 600 fatal cases of obstruction from various causes, exclusive of hernia, rotation or twisting of intestine occurred in 8 per cent.

A highly important injunction is to be added to the consideration of obstruction dependent on the foregoing structural affections. It is for the practitioner, in all cases which present the symptoms of these affections, to examine carefully for inguinal or femoral hernia. The hernial

I Hugh S. Legaré. Case reported by Prof. Jacob Bigelow, Am. Jour. of Med. Scicnces (new series), vol. vii., 1843. 
tumor may be so small as not to excite the attention of the patient, and, in females, motives of delicacy may prevent the patient from suggesting an examination, even when the presence of a tumor is known to her. Instances have repeatedly occurred of patients dying with strangulated femoral or inguinal hernia which was overlooked, the symptoms being attributed to some inaccessible lesion, or even to functional disorder.

\section{COMPRESSION AND STRICTURE OF INTESTINE.}

Obstruction due to closure or diminished calibre of the intestinal tube by morbid growths within the tube, the pressure of tumors situated exterior to the tube, or constriction following the cicatrization of ulcers, differs from obstruction caused by the structural affections already noticed, in not involving strangulation. The obstruction, under these circumstanees, is developer gradually, having been preeeded by progressively increasing constipation. The obstruction is rarely complete. The local and constitutional symptoms incident to sphrcelation and sloughing of the intestine are wanting. The symptoms are those arising from the gradual accumulation of the contents of the intestine above the point of obstruction. The affection progresses more slowly toward a fatal termination, after the obstruction becomes nearly or quite complete.

Cases of obstruction falling under this head form a considerable proportion of the cases of obstruction from all eauses. In an analysis of 127 cases, including cases of obstruction from fecal aceumulation and functional disorder as well as struetural lesions, by George Pollock, 77 were of this class. ${ }^{1}$ And in the great majority of the cases of this class, the seat of the obstruction is in the lower part of the large intestine. Of the 77 cases just referred to, in 60 the seat was either at the sigmoid flexure of the colon or in the rectum. In about one-half of these 77 cases the obstruction was due to cancer. Cancer is much more infrequently seated in the intestinal canal than in the stomach. It is oftener seated in the large than in the small intestine, and the portions of the large intestine in which it is most apt to be seated are the rectum and the sigmoid flexure of the colon.

The discrimination of the cases of obstruction caused by compression or stricture from the cases involving strangulation can generally be made, clinically, by means of the differential points which have just been stated.

The fact of the obstruction being seated in the lower part of the large intestine can generally be ascertained. If seated in the rectum, it is accessible to direct exploration. If seated at the sigmoid flexure, the fact is shown by the evidence, afforded by manual exploration, of distension of the transverse and ascending colon, and by the inability to inject liquid beyond the rectum. Per contra, the situation at the upper part of the large intestine or in the small intestine is shown by the absence of distension of the large intestine with intestinal contents, and by the inability to fill the colon with injected liquid. The obstruction may be complete, but, in general, a certain quantity of liquefied fecal matter passes the point of obstruction and is evacuated.

The distension from accumulation of the intestinal contents may lead to rupture of the intestine. And the distension may gradually go on and lead to this accident without sufficient pain or other symptoms to direct attention to the existence of any serious obstruction. This fact was illustrated by a case under my observation at Bellevue Hospital, in which

1 Vide Medico-Chirurg. Review, 1853, vol. xii. p. 243. 
nothing more than ordinary constipation had been suspected, when peritonitis suddenly became developed, ending fatally in a short time. $\mathrm{Ob}$ struction was produced in this case by a cancerous tumor at the sigmoid flexure; the colon and cxcum were enormously distended with feces, and the peritonitis was due to rupture of the caecum. This case illustrates the ability of the ileo-cæcal valve to resist an amount of pressure sufficient to lead to rupture of the creal walls.

In the treatment of obstruction from compression or stricture, active purgatives are to be aroided, but the trial of saline laxatives in small doses, in order to liquefy the intestinal contents, is admissible. Colic pains are to be relieved by opiates. The diet should be nutritious and concentrated, in order to leave as little fecal residue as possible. If the obstruction be situated at the lower part of the large intestine, carcful efforts to pass a flexible tube beyond the constricted portion may be employed, and, if snecessful, liquid may be injected above the obstruction.

In cases of obstruction from compression or stricture at the lower part of the large intestine, enterotomy with a view to the establishment of an altificial anus is warrantable, provicled other measures to free the intestine from an amount of an accumulation likely to lead to rupture be not successful. Cases have been reported in which life has been prolonged and comfortable health obtained by means of surgical interference.' Cxsar Hawkins reported the results of the operation for artificial anus in 44 cases. $^{2}$ In 10 of these cases death took place within 48 hours, in 21 within five weeks, and in 13 the operation was successful. Of these 13 cases, 6 died in six months, and 7 survived more than a year.

\section{OBSTRUCTION FROM IMPACTION OF FECES, ENTERO- LITHES, AND FOREIGN BODIES.}

Obstipation may be produced by the causes named in the above heading, but in general they induce only more or less constipation, and, in treating of the latter affection, they will again be referred to. An accumulation of a mass or of masses of hardened feces, suflicient to produce obstruetion, may take place in different parts of the large intestine. The rectum in aged persons not infrequently is the seat of such an accumulation. It is to be suspected when enemas camnot be given, and perhaps the pipe of the syringe is found to penetrate a fecal mass. Examination shows the seat and the character of the obstruction. It may be necessary to break down and remove the accumulation by a scoop or the handle of a spoon. The accumulation may be at the sigmoid flexure, and then resistance to the passage of liquid injected and to the introduction of a flexible tube is found to be at that point. The aceumulation may be at any point abore the sigmoid flexure, but it is oftenest in the cæcum or ascending colon.

Masses of feces above the sigmoid flexure may give rise to a tumor or to tumors appreciable by manual exploration through the abdominal walls. These fecal tumors are liable to be mistaken for others of a different character. $\Lambda$ female in the comntry presented an abdominal tumor of the character of which her attending physician was not satisfied, and an eminent professor of anatomy, now deceased, was requested to see the case. He was led to regard the tumor as malignant, and, of course, to form a very unfavorable prognosis. Shortly after the consultation a profuse

1 For a striking case, see Medico-Chirurg. Trans. of Royal Med. and Surg. Society of London, vol. x. 2d series, 1845.

2 See ibid., vol. xxxv., quoted in Habershon's work. 
diarrhœa set in, and, much to the astonishment of the patient and her physician, the tumor suddenly disappeared. It was a fecal tumor. In view of the liability to error of diagnosis, and in order that appropriate measures of treatment may be pursued, the distinctive features of these tumors are to be borne in mind. One point in the diagnosis is their situation in the tract of the colon. They are not tender on pressure, but may be handled freely without giving pain. By firm and continued pressure, perhaps, they may be moved backward or forward in the direction of the colon. By firm and continued pressure, also, the form of the tumor may be altered.

The absence of pain, tenderness, vomiting, and the constitutional symptoms accompanying invagination, internal hernia, and twisting of the intestine, suffices to exclude these affections; but it cannot be at once determined that the obstruction is not due to compression or stricture. The effect of measures of treatment will be likely to settle the diagnosis. The measures to be employed are purgatives, especially salines, and stimulating enemas. The latter will be more effective if administered through a long flexible tube introduced into the bowels. These measures, judiciously employed, will generally prove successful, without much delay, in relieving the obstipation and causing the fecal tumors to disappear. Mild purgatives may be required for some time after the obstruction is removed; the treatment, then, will resolve itself into that adapted to habitual constipation.

Obstruction may be due to the presence of intestinal concretions, or enterolithes. These are composed of different substances, such as magnesia or the sesquioxide of iron taken as a remedy, the husk of oatmeal in countries in which this article of food is largely used, cholesterine, the flour of sulphur, cubebs, white mustard-seed, chalk, etc. The seeds or stones of fruit and other indigestible constituents of food may form a conglomerate mass of sufficient size to give rise to obstipation. In a case related to me by Dr. Sands, of Newport, R. I., obstipation resulted from an accumulation of grape seeds, the patient having eaten, for the space of three months, two or three pounds of grapes daily; the patient, for several weeks, discharged grape seeds at his stools, and, also, with flatus. I have known a smooth, oval body, as large as a hen's egg, to be passed from the bowels, which appeared to be composed of pure cholesterine. A gall-stone, or some solid body contained in the aliment, may serve as a nucleus on which earthy matter in the intestinal contents is deposited, forming a concretion of greater or less size. These obstructing bodies may be situated within the cæcum, at the sigmoid flexure, or in the rectum. They may sometimes be felt through the abdominal walls. The nature of the obstruction in these cases can be determined only after the concretions have been evacuated, or by means of a post-mortem examination. They may lead to ulceration and perforation of the intestine, or, if not discharged and the obstruction be complete, vomiting of so-called stercoraceous matter ensues, and death may take place from exhaustion.' An intestinal concretion, cuboid in form, the vertical and transverse diameter one and a half inches, its weight fifteen drachms, was sent to me by Dr. O. T. Jameson, of South Otselic, Chenango County, New York, with a report of the case, which I communicated to the New York Pathological Society, February, 1867. This concretion was found after

1 For statistical details relating to the different lesions occasioning obstruction, vide Dr. Haven's article, before referred to. The article gives the results of the analysis of 258 cases. 
death in the ileum just above the caeum. It was distinetly felt through the abdominal walls during life, and had existed for over twenty years. The patient was a shoemaker, and experienced notable inconvenience from the eoncretion only at times after overwork, or after violent muscular exertion. He died of pneumonia, at the age of 47 . Portions of the concretion were examined for cholesterine with a negative result; the composition appeared to be fecal.

Finally, foreign bodies, that is, by way of distinetion, bodies not contained in the aliment nor derived from the body, may pass into the intestinal canal from the stomach, or be introduced into the rectum. In the latter case their presence is readily ascertained by exploration, and they are removed by appropriate surgical appliances. When iftroduced into the stomach, they have been swallowed either deliberately, as is done by jugglers, or inadvertently. Prof. Gross, in his comprehensive work on Surgery, cites the case. of a man in Iowa, who swallowed a bar of lead weighing a pound, which was removed from the stomach by an opening made through the abdominal walls, and the patient recovered. The same author refer's to another case in which a large teaspoon was swallowed in a fit of delirium, and this was extracted from the ileum by the operation of enterotomy.'

Foreign bodies, not suffieient in number, or not large enough, to occasion obstruction, are often swallowed, especially by children. The practitioner is frequently summoned in haste, and great anxiety is felt when bodies such as pins, coins, portions of glass, etc., are known to have been swallowed. They may give rise to more or less irritation of the mucons membrane; they may even occasion ulceration and perforation of the stomach or intestines. In general, however, they pass through the alimentary canal without any appreciable inconvenience; and the physician is warranted in giving assurances of the absence of danger. It is not judicious, in such cases, to give catharties or laxatives. The mucons membrane is best protected against injury, if a slarp-pointed or irritating body be contained in the solid contents of the bowels. Moreover, the increased peristaltic movements caused by a purgative increase the risk of injury. The desire to have anxiety relieved as quickly as possible, by aseertaining that the foreign body has passed, is an inducement to resort to purgation, which is to be resisted.

\section{FUNCTIONAL OBSTRUCTION.}

Persisting obstipation accompanied with vomiting of so-called stereoraceous matter, colic pains, etc., may occur, there is reason to believe, as a purely functional disorder, constituting an affection which has been called idiopathic ileus. A person is seized with abdominal pain, increased paroxysmally; vomiting soon takes place, and, after a time, the matter vomited has the odor of feees; measures to procure evacuations from the bowels are ineffectual, the pulse becomes frequent, and there is more or less prostration. After the lapse of a week or more, these symptoms subside, free evacuations from the bowels ensue, and the patient recovers. These are the prominent features of a case which I reported some years ago. ${ }^{2}$ The patient was a female, aged twenty-five; hysterical symptoms

1 For a collection of cases of foreign bodies in the stomach and the intestines, by Alfred Poland, vide Guy's Hospital Reports, vol. ix. p. 1063.

2 Buffalo Med. Journal, vii. p. ฮ30, 1851. 
were associated; the treatment consisted of the free use of opiates, and enemas administered through the long flexible tube. In this case, the patient declared that she tasted castor-oil which was given exclusively per enema, and it was said that oil was observed to float on the matters vomited. Cases have been reported in which diverse substances injected into the rectum were supposed to be ejected from the stomach. In most of such instances the accuracy of the observation may fairly be questioned, and I do not suppose that in the case reported by me the oil passed above the ileo-creal valve. The taste of oil may have been real, and may be explained on the supposition that enough was absorbed from the large intestine to be appreciated by the gustatory nerve.

Dr. S. B. Hunt subsequently reported a case in which obstipation existed for fifteen days, and during this period the patient vomited matter described as "horribly fetid and stercoraceous." This case was treated for the first five days by giving active cathartics. At the end of this time the condition was so unpromising that the cathartics were discontinued and a soothing treatment adopted with a view to euthanasia. Under the latter treatment the symptoms denoted gradnal improvement, evacuations from the bowels occurred spontaneonsly, and recovery took place. This patient was a female, aged thirty-five, and a sufferer from symptoms connected with spinal irritation. ${ }^{1}$

Such cases simulate those of obstruction from the structural affections which have been considered, and there is ground for the belief that in a certain proportion of the reported cases of the successful treatment of invagination or strangulated hernia within the abdomen, the affection was purely functional. The regurgitation of the contents of the small intestine, which have a stercoraceous odor from the diffusion of the gases contained in the large intestine, in these as in other cases of obstruction, is generally attributed to an inversion of the peristaltic movement. Brinton explains it by supposing that in the centre of the intestinal tube a retrograde current is produced by the normal peristaltic movements, if there be an obstruction. The obstruetion, when finctional, may be caused by spasmodic contraction of the tube, or it may be due to paralysis of a portion of the tube, and consequent failure of this portion to propel its contents.

The elinical discrimination of functional obstipation from obstruction caused by structural affections, cannot always be made at once with confidence. The absence of tumor and of pain and tenderness localized at a particular point, tends to the exclusion of the latter. The functional character of the affection may be surmised if the patient be a female subject to hysteria, and suffering from the nervous symptoms associated with socalled spinal irritation. The probability of the affection being functional is increased in proportion as the obstipation, with vomiting, etc., continues, without the supervention of the symptoms which occur after sphacelation of intestine has taken place, viz., frequency and feebleness of the pulse, coldness of the surface, extreme prostration, great tympanites, in short, the symptoms denoting impending death by asthenia.

In the treatment of functional obstipation, opium and other measures to relieve pain and allay spasmodic action are indicated. Cathartics may be cautiously tried, but, if ineffectual, should not be persisted in. Enemas administered by means of a long flexible tube are to be employed.

The importance of examining for the evidence of femoral and inguinal hernia is to be enforced in connection with the subject of functional obstipation.

Ibid. vol. vii. p. 383, 1852. 


\section{CHA PTER V.}

Functional Affectlons of the Stomach and Intestines-Dysjepsia-Polyphagla, Malacia or Pica, Polydipsia, Dipsomania. Gastrorriagia.

$\mathrm{F}$ the functional disorders of the stomach and intestines, a large proportion relate to digestion, that is, to the processes involved in the changes which the ingesta undergo prior to the function of absorption. The processes of digestion take place chiefly within the stomach and small intestine. Clinieal observation shows, it is true, that, under certain circumstances, aliment introduced into the large intestine is digested. But it would appear that digestion in the large intestine is performed vicariously, and hence, disorders of this function are hardly applicable to this portion of the alimentary canal. The greater part of the functional disorders of digestion are commonly embraced under the name dyspepsia. This name signifies simply difficulty of digestion, but, conventionally, it is applied to cases in which the digestive processes are disturbed in varions ways, as well as to the cases in which their performanee appears only to be difficult. Gencrally, dyspepsia denotes ailments which are sufficiently persisting to be considered as chronic. The term acute dyspepsia, however, is applied to disorder of the digestive organs, attended with comparatively violent symptoms, and having a short duration. The disorder so called claims a brief notice.

The term acute dyspepsia denotes an affection called by French writers embarras gastrique, and popularly known with us as a bilious attack. When accompanied with more or less febrile movement, it is sometimes called gastric fever. The latter term is objectionable, because it implies that the affection is essentially a fever, whereas the febrile movement is symptomatic. The term bilious attack is also objectionable, because it is by no means clear that the pathology of the affection involves any special disturbance of the liver. In the absence of precise knowledge of the pathological character of the affection, the term acute dyspepsia is to be preferred, inasmuch as, pathologically, it expresses no more than a functional disorder pertaining to digestion. The term, in fact, is applied to cases embracing, probably, different morbid conditions.

In certain cases of acute dyspepsia, the difficulty is expressed by calling it a fit of indigestion. Gastric disorder is occasioned by over-repletion of the stomach, the ingestion of indigestible articles of food, or an arrest of digestion by strong emotions, fatigue, or other causes. The disorder is manifested by a sense of weight, fulness, or pain in the epigastrium ; nausea and perhaps vomiting may occur, or, after a time, looseness of the bowels ensues; some febrile movement may be induced, with pain in the head and general malaise. Succeeding these symptoms are loss of appetite, coating of the tongue, an unpleasant taste, with more or less uneasiness referable to the digestive organs for several days. Such cases are sufficiently common. In the way of treatment, they claim only a mild evacuant remedy, and regulated diet for a few days, with, perhaps, some tonic remedy.

The practitioner meets often with cases which differ from the preceding 
in the disorder not being distinctly referable to a fit of indigestion. There is impairment or total loss of appetite ; nausea and slight vomiting may occur, but oftener a liquid containing bile is regurgitated ; uneasiness or undefined distress is felt in the neighborhood of the stomach; the bowels are sometimes constipated and sometimes loose ; the patient complains of a bitter taste, and the breath is frequently offensive; the tongue becomes furred or coated: pain in the frontal region of the head is a frequent symptom, which, if accompanied by nausea, goes by the name sick headache; the urine deposits in abundance the lithates; in some cases there is moderate febrile movement; the countenance frequently presents a sallow appearanee. These are the symptoms belonging to an affection which may be called acute dyspepsia. Patients imagine that their malady is very clearly defined when they declare that they are bilious. It is frequently, if not generally, difficult to trace the affection to any cause. It occurs undoubtedly oftener in the spring than in other seasons of the year. It continues a few days, and gradually the normal condition of the stomach and system is regained. It is probable that in many, if not most, cases of acute dyspepsia, there is subacute gastritis. In certain cases the affection is that called by some authors catarrh of the stomach, to which reference has been already made.

Patients with acute dyspepsia are apt to desire active treatment. They fancy they need cleansing remedies, and in this light they regard emetics and eatharties; or they solicit medicines to act upon the liver. Readjustment of digestion, cte., takes place withont medication, but a mercurial cathartic often appears to afford prompt relief. A few grains of calomel or blue mass, followed by a saline purgative, and, afterward, a mild tonic such as a grain or two of quinia two or three times daily, with regulated diet, are appropriate measures of treatment.

The term dyspepsia, without the prefix acute, is applied to a more persisting affection than that just considered. As the name of a functional affection, it is not correctly used when applied to disorders of digestion connected with inflammation or structural lesions of the digestive organs, nor to disorders incident to ferer and other general diseases. The term is much less frequently used now than formerly. One reason for this is, formerly the term had a wider latitude than now, embracing affections which progress in pathological knowledge has placed nosologically elsewhere. But the affection is undoubtedly less prevalent than it was a quarter of a century ago in this country. Of course, as a functional affection, it has no anatomical characters or morbid anatomy.

The symptoms of dyspepsia are, in the first place, local, that is, referable directly to the stomach or small intestine, and, in the second place, manifested elsewhere, in other words, sympathetic or consecutive. The local symptoms, which will be first noticed, are varied in character, and might serve as a basis for the subdivision of dyspepsia into several varieties.

These symptoms are conveniently classed as follows : First, symptoms denoting labored or difficult digestion ; and, second, symptoms proceeding from imperfect or disturbed digestion.

In certain cases of dyspepsia, the processes of digestion appear to be fully accomplished, but they are accompanied with uncomfortable sensations. In healthy persons, as is well known, a good digestion is productive of a sense of comfort. It is quite the reverse in some dyspeptics. They are often uneasy or miserable while the digestive processes are going on; they complain of uneasiness in the stomach or intestines, of a sense 
of distension, and of a general sense of discomfort. And these symptoms may attord the only evidence of disorder. The food appears to be digested, and the body may be well nourished, whereas, in other cases, these symptoms are associated with those denoting imperfection or disturbance of the digestive processes. A morbid sensitiveness of the digestive organs, and morbid sympathetic influences, appear to be the sources of the suffering in the cases referred to. On the other hand, when digestion is ineomplete or disturbed, various symptoms arise in different cases, and with these there is sometimes comparatively little suffering. It is a curious apparent inconsistency that some persons manifest habitually more or less derangement of digestion, without much annoyance, while other persons habitually digest perfectly, but pass a wretched existence. The more prominent of the local symptoms in cases of dyspepsia may be arranged under the following heads: Regurgitations including pyrosis, cardialgia, tympanites, and vomiting.

Certain cases are characterized by regurgitations from the stomach. The liquid regurgitated is sometimes intensely sour from the presence of either the lactic, hydrochloric, or acetic acid. If the regurgitations occur, as is usual, during the progress of stomach-digestion, the acidity is probably due to chemical changes in the ingesta, but occurring, as is sometimes the case, after long fasting, when the stomach contains no food, it must come from the gastric glands. The regurgitated liquid is sometimes acrid, appearing to scald the throat; it may be greasy, or nauseous, having the odor and taste of rotten eggs, the latter denoting putrefactive changes. Some persons possess or acquire the faculty of voluntary regurgitation, and are able to expel, at will, the contents of the stomach-a faculty analogous to that of rumination in herbivorous animals.

The regurgitation of a considerable quantity of a liquid which is either insipid, or saltish or brackish, and sometimes acid, when the stomach is empty of food, and usually in the morning, is called pyrosis, or water-brash. Chambers supposes that the liquid is derived chiefly from the salivary glands, and that the collection is not within the stomach but at the lower part of the cesophagus. ${ }^{2}$

Cardialgia signifies pain at the cardiac orifice of the stomach, of a burning eharaeter, shooting into the chest, and up the osophagus into the throat. It is vulgarly called heart-burn. Every one has occasionally experienced this symptom. It characterizes certain cases of dyspepsia, and is evidently dependent on the presence of an acid in the stomach, as it is quickly and completely relieved, for a time, by an alkaline remedy.

A painful sense of fulness after eating in dyspepsia is common, without any abnormal distension; but more or less distension from air or gas is a prominent symptom in certain cases, constituting tympanites. The tympanites may be situated in the stomach or small intestine. In most eases the gas is derived from putrefactive or formentative changes in the ingesta. The nature of the gas will depend on the kind of food ingested. In the decompositon of albuminoid substances sulphuretted hydrogen is produced, and the gas expelled from the stomach has a characteristic fetor; the butyric fermentation furnishes hydrogen and carbonic acid gas, and the latter is also furnished by the acetic fermentation. The formation of lactic acid is unattended by the production of gas. The accumulation of gas within the stomach, if considerable, occasions painful distension, embarrasses respiration by interference with the descent of the diaphragm, disturbs the action of the heart, and interferes with the gastric peristaltic move-

1 "The Indigestions," by Thomas King Chambers, M.D., etc. 
ments. The formation of gas resulting from over-repletion of the stomach, may distend the stomach to such an extent as to eause sudden death. Two instanees of this kind have fallen under my observation.'

Another source of flatulent distension is from gas secreted or evolved in some way independently of ehemical changes in the ingesta. The accumulation is especially in the small intestine. Some persons suffer from an habitual tympanites. Females are more liable to it than males. Cases are occasionally met with in which the rapid development of tympanites oecasious great suffering, the abdomen becoming enormonsly distended, the respiration embarrassed by interference with the descent of the diaphragm, and, in females, the suffering being inereased by pressure upon the uterus and bladder. A case has recently been under my observation in which paroxysms of intestinal tympanites, oceasioning intense distress, oceurred daily for sereral successive months, an inodorous gas sometimes passing off by the rectum, and sometimes the tympanites disappearing without any eseape of gas. A host of remedies were employed in this case unsuccessfully, and at length improvement has slowly taken place under simple palliative treatment. In a case of this deseription which I saw with my friend and eolleague, Prof. Isaac E. Taylor, the appearance of the abdomen during an attack of tympanites was extraordinary. The convolutions of the small intestine and their peristaltie movements were distinetly visible through the distended abdominal walls. So far as my observation goes, such cases occur only among females, and the tympanites is associated with more or less neuropathic disorder. Hysterical phenomena are sometimes associated with it.

There is still another source of flatulent distension, the seat of the tympanites being the stomach, viz., the ingestion of air. More or less air is habitually commingled with food and drink, but the quantity received in this way is never sufficient to distend the stomach. A habit of swallowing air is sometimes acquired, resembling the trick which, in horses, is known as erib-biting or wind-sucking. Some writers refer to this habit as not uneommon, but the effort required in the deglutition of air is such that the act could not escape detection; and as I have met with only a single example, I must think that, in this country, cases are by no means frequent. The case just referred to I saw with my friend and colleague, Prof. Sayre. The patient was about fifty years of age, and had suffered from a disorder of the digestive organs for twenty-five years. In order to direct my attention to the difficulty, Prof. Sayre requested him to drink a tumbler of water. He drank it precipitately, and appeared to make violent efforts to force it downward. During these efforts the face became flushed and the eyes watery. Shortly the efforts were discontinued, and at the same moment a loud gurgling sound was heard in the region of the stomach. I supposed, at first, that there was obstruction at or near the cardiae orifice of the stomach, but, to show that the passage was free, Prof. Sayre passed readily a probang into the stomach. I was then at a loss what to think of the case, but Prof. Sayre had already arrived at the conclusion that the patient had the habit of swallowing air. The patient stated that the habit had existed for twenty-five years. He was led to it gradually in order to prevent regurgitation, which he stated always occurred if he failed to ingest air in conjunction with his food and drink. He was accustomed to belch air frequently. Pereussion over the stomach showed considerable gastric tympanites. He declared that he could not

1 Reported in Boston Med. and Surg. Journal, March 10, 1841. 
overcome the habit, but promised to make the attempt. Nothing was heard of the case afterwards.

An habitual tympanites of the stomach oceasions, in some persons, after the ingestion of liquid, a succussion sound in walking, resembling the sound frequently heard in horses. This is a source of much annoyance and mortification, especially in females. It may be avoided by taking into the stomach only very small quantities of liquid at a time.

Vomiting is not common in cases of habitual dyspepsia. It is more frequent in occasional fits of indigestion or in acute dyspepsia. It is, however, characteristic of a form of chronic dyspepsia occasionally met with, occurring generally in young females. In this variety of disorder, vomiting almost constantly follows the ingestion of food or drink; the stomach seems to be intolerant of all forms of nourishment, although taken in small quantities. The vomiting occurs directly or soon after food is taken, and, in some eases, it is surprising that there is not more evidence of inanition, since everything taken into the stomach appears to be ejected; yet, although the disorder may continue for a long time, the patient does not become greatly emaciated nor notably enfeebled. The disorder is apt to resist all the usual remedies to relieve irritability of the stomach, such as bismuth, creasote, opiates, hydroeyanic acid, oxalate of cerium, etc. It is usually accompanied with mental depression, hyperasthesia of the surface, and marked tenderness over the spinal column. In determining that the affection is purely functional, gastritis, gastric ulcer, subacute meningitis (especially in children), pregnancy, and disease of the kidneys are to be excluded. The treatment adopted in a case of this description by William Hunter (which has been often quoted) will. sometimes prove effectual, viz., restricting the patient to a very small quantity of soine bland nourishment, such as milk or animal essence, taken at short intervals. But the plan of treatment which, in my experience, has proved most successful, consists in chinge of scene, and living as much as possible out of doors. I have known a sea-royage to prove efficacious.

The local symptoms just noticed are not peculiar to dyspepsia. They may occur in conneetion with subacute gastritis and structural lesions of the stomach. In arriving at the diagnosis of dyspepsia-that is, determining that a functional affection only exists-inflammation and the lesions which have been considered are to be excluded. They are to be excluted by the absence of their diagnostic symptoms, and for these the reader is referred to the preceding chapters. ${ }^{1}$

Of symptoms other than those referable direetly to the stomach and small intestine, mental disorder is one of the most noticeable. During the processes of digestion, dyspeptics are apt to experience an uncomfortable heaviness or dulness, rendering it diffieult to concentrate the attention upon any subjeet. 'I'he following extract from Chambers's work gives a trutliful representation of the condition in many cases: "There is great languor and incapacity for cxertion, coming on generally about an hour after food, and accompanied in some cases by an almost irresistible drowsiness, which lasts for several hours. This symptom is particularly marked in the afternoon, if the patients dine in the middle of the day and endeavor to continue their employment afterward. Yielding to the drowsiness and taking a siesta seem to make matters worse, for there is usually felt after such an indulgence an intense headache or giddiness, which continues longer than the

1 Diarrhca dependent on intestinal indigestion will be considered in the next chapter. 
symptoms would have done had the inclination to sleep been resisted. The depression of spirits is not so remarkable as the feeling of utter helplessness, both of mind and body, accompanied in persons naturally active with a sense of shame at their unwonted apathy." This condition may be connected with the process of digestion in the small intestine, and may be unaccompanied by symptoms denoting disorder of stomach-digestion.

In most cases of dyspepsia, patients suffer more or less from mental depression. 'They are devoid of buoyancy, gloomy, and disposed to look always on the dark side. Frequently this depression is increased by anxiety respecting health. 'They are apprehensive of the existence of some. grave disease, such as consumption or an affection of the heart. Impotency is sometimes fancied. Their attention becomes concentrated upon themselves. They are eonstantly watching the sensations connected with digestion, and fall into the habit of counting the pulse, feeling the heart's beat, examining the abdomen, urine, etc. This mental disorder, carried beyond a cortain point, eventuates in the forms of mental aberration known as melancholia and hypochondriasis.

In addition to these symptoms of mental disorder, various symptomatic phenomena belong to the clinical history of dyspepsia. Irregular action of the heart, or palpitation, is not uncommon. Constipation generally exists, sometimes alternating with diarrhoea. 'The skin is apt to be dry and rough; the extremities are often cold, showing deficient activity of the cirenlation; the tongue is usually furred or more or less coated. The urine deposits the lateritious sediment, and, on microscopic examination, erystals of oxalate of lime may be found in abundance-the latter, being incidental to various morbid conditions, and not uncommon in commection with trivial disorders, are incorrectly considered as denoting a special affection called oxaluria. Vigilance is a frequent source of complaint, and the sleep obtained is often incomplete and disturbed by dreams, the patient arising in the morning umrefreshed and miserable. ${ }^{2}$ The appetite in the morning is often poor, becoming, perhaps, during the day, morbidly craving. A dry hacking cough is reckoned among the sympathetic phenomena, but, in my experience, this is rare. The existence of cough should always excite suspieion of pulmonary diseasc or of chronic pharyngitis ; the latter is quite common.

With reference to the pathology of dyspepsia, it is to be considered that digestion is not a simple process. Including under this term all the changes which take place in the ingesta prior to absorption, there are certain changes in the stomach from admixture with the salivary fluids and the gastric juice, others in the duodenum from the addition of the bile and pancreatic secretion, and others in the small intestine, below the duodenum, firom the action of the intestinal juice. For the proper activity of the organs, and for the secreted liquids, the latter being vastly more abundant than was formerly supposed, a large supply of blood is required. The morements due to the muscular coat of the stomach and intestine are important. A certain influence derived from the nervous system, called innervation, is requisite for the secretion of the liquids and the peristaltic morements. An analysis of the symptomatic phenomena in different cases of dyspepsia shows that the different elements which enter into the function of degestion may be affected either separately or with different degrees of predominance.

1 Digestion and its Derangements. American edition, page 367.

2 The poet Cowper, who appears to have suffered from intense mental depression in connection with dyspepsia, describes his feeling in the morning in the following forcible language: "I awake like a toad out of Acheron, covered with the ooze and slime of melanclioly." 
Thus, vomiting and regurgitation show irritation and spasmodic action; pain or distress, morbid sensibility; prolonged digestion with chemical changes, deficiency in the digestive liquids, etc. It is of importance to take cognizance of these several elements in the management. The period which elapses after eating before the inconvenience is felt, and the absence of symptoms of disorder referable to the stomach, go to show intestinal rather than gastric dyspepsia.

The causation of dyspepsia involres agencies affecting the digestive processes either directly or indireetly. Excesses in eating and drinking act as direet eauses. The digestive powers may be overtasked by the quantity, or over-exeited by the quality, of food. Under these circumstances, dyspepsia has been humorously called "the remorse of a guilty stomach." 'The habit of spirit-drinking engenders dyspepsia ; drunkards after a time beeome dyspepties. Dyspepsia may follow subacute gastritis or cuteritis produced by clietetic errors or other causes; inflammation leaves the organs in a weakened state. 'These direct causes, however, are not so largely operative as is generally supposed.

'The indirect, more than the direct, ageneies are involved in the causation. These are various. Anremia is one of the direct eanses. It gives rise to dyspepsia by diminishing the functional activity of the gastrie and intestinal glands, the digestive liquids being lessened, or affeeted in regard of their quality. Clinical observation shows that, in cases of anæmia, the digestive processes are generally more or less disordered. On the other hand, disordered digestion may stand in a eausative relation to anæmia. A notable change in habits as regards physical exercise not infrequently gives rise to dyspepsia. The vigor of the digestive organs is in a measure commensurate with the demand for nutritive material, and the latter is dependent on the waste of the tissues. Now, certain dietetic habits are formed in accordance with occupations involving physical activity and a proportionate amount of disassimilation. Persistence in these dietetic habits after a change from an active to a sedentary life is apt to induce disorder. This is, in part at least, an explanation of the occurrence of dyspepsia among those who exchange the farm or workshop for the desk or study, and among those who retire from business to become men of leisure.

In a large proportion of cases, (lyspepsia originates and is perpetuated by inental eauses. It is induced and kept up by anxiety and depression. Disorder is often, in the first place, produced by mental causes, and then the dyspepsia reacts upon the mind, increasing its morbid condition. An examination into the previous history of cases of dyspepsia will frequently show the affection to have been preceded by mental inquietude of some sort. Persons of a certain mental constitution are especially prone to dyspepsia, viz., those so constituted as to be constantly anxious about something, such as acquiring success in life, getting out of debt, securing an independent position, or about imaginary troubles. The period of life when persons are most prone to dyspepsia is that in which anxieties are apt to be grcatest, viz., between adult age and middle life. After the latter period, the events of life are taken more calmly, and there are fewer sources of intense anxiety. Persons who have been dyspepties for many ycars are often free from this affection after having entered upon middle life. It may be doubted if the affection is often produced by overtasking of the intellect. When it occurs in those who are excessively devoted to intellectual pursuits, it arises generally from the sedentary habits connected with these pursuits or from mental anxiety ; on the other hand, deficient exercise of the intellectual and moral faculties is apt to lead to it. Conditions in life involving ennui and dissatisfaction predispose to it. Under 
these circumstances the attention is apt to become concentrated on the functions of the body, which go on best without close surveillance. These views are sustained by observing the classes of persons who suffer from dyspepsia. Epicures and gormandizers by no means of necessity become dyspeptics; they often eseape this affection when their indulgences lead to other consequences, such as gont, etc. The laboring classes are not prone to dyspepsia, notwithstanding the use, frequently, of the coarsest and most indigestible kinds of food, prepared without any regard to the art of cookery. But cases of dyspepsia are met with especially among retired rich persons, without occupation or mental resources, among those who have no usefinl or proper aims in life, and among those who are debarred from the interests of domestic life; and in these classes it may be developed irrespective of any dietetic errors.'

Treatment.-In the management of dyspepsia, as of other affections, the first point is to remove or obviate causes, in so far as this is practicable. The practitioner may be able to control causes relating to dietetic errors, but he may not be able to reach those connected with the mind, and hence a difficulty in effeeting a cure. Measures of treatment may be arranged in three divisions, viz., 1st, those relating to diet and regimen; $2 \mathrm{~d}$, measures addressed to the mind; and, $3 \mathrm{~d}$, medicinal remedies.

1. If the affection have been induced by dietetic excesses, it is sometimes useful to give the digestive organs a short period of rest, and, with this view, to reduce the diet, for a time, below the wants of the system. This, however, should be but temporary. A cure is not to be effected by persisting in this course. Not only the system is rednced, but the digestive functions are impaired by too great or too protracted abstemiousness. Dyspepsia is, in fact, perpetuated, if, indeed, it be not sometimes produced, by attempts to live on as little and as coarse food as possible. Persons who think it vulgar, unrefined, or worldly-minded to eat well are apt to be the subjects of dyspepsia, as well as of other affections. 'The object of treatment is to bring the digestive powers $u p$ to such a point of improvement that particular care in diet is not requisite.

As regards the diet for dyspeptics, there are no rules suited to all cases. Individnal experience, in each case, is to be a guide, but there is a liability to error in regard of this experience. Unusual difficulty or disturbance of digestion after a meal is often imputed by the patient to certain articles of food, when it was due to some other incidental eireumstance. Idiosyncrasies in relation to particular articles of food are far less common than the statements of patients would lead one to suppose. In general, articles which are wholesome to most persons are not unwholesome to any. It is rarely true that "what is one man's meat is another's poison." As a rule, when a patient says that he cannot take such and such articles which general experience shows to be digestible and nutritious, it is fair to presume that he is deceived, and of this the patient may generally be convinced if he be persuaded to persist in their use. At first, the expectation that an article will prove hurtful contributes to render it so ; but, after a time, the idea is overcome. It is often an object in the treatment of dyspepsia to do away with these fallacious ideas respecting various kinds of food. Some persons seem to be fond of encouraging the notion that their digestive organs are endowed with peculiar susceptibilities whieh prevent them from conforming to general rules of diet. In most cases of dyspepsia,

I For a fuller exposition of these views of the causation of dyspepsia, vide article by the author in the American Journal of Med. Sciences, January, 1841. 
animal food is best digested, especially old and tender meats plainly but well cooked; but, in some cases, a milk and farinaceous diet is found to agree best. An obvious reason why so many persons imagine they do not digest milk well is, it is generally taken as a beverage after or with solid food, the fact that it is, in effeet, a solid article of food not being appreciated. Bread, to be readily digestible, should not be new, nor is it desirable that it should be stale. Well-boiled rice and eorn-meal mush aro easy of digestion. Crude vegetables are apt to be digested with diflieulty in eases of dyspepsia, but not invariably. Some dyspeptics find even the much abused cucumber grateful to the stomach as well as to the palate. Pastries, rich puddings, and sweetmeats are generally to be eaten sparingly or discarded. Ripe fruits in moderation are useful. It is generally not advisable for the patient to limit himself to a restricted range, or to adopt any particular system of diet. On the contrary, he should persevere in attempting to digest all the varied forms of wholesome food, not confining himself to meat or a vegetable diet, but aining to eat like persons in health, without the need of particular care in the selection of food.

Different kinds of food are suited to different cases according as the functional disorder is chiefly gastric or intestinal. If gastric, farinaceous articles will be likely to be best suited, as these are cligested mainly in the small intestine. Fatty articles may be well cligested in these cascs. On the other hand, if the disorder be intestinal, farinaceous and fatty articles of fool will be likely to be diffieult of digestion, and albuminoid substanees or an animal diet will be best borne.

Regularity in meals is a point of importanee. Frequently more than three meals a day are advisable. If a patient have no appetite for breakfast, an early lunch should be taken. If he dine near the middle of the day, and have an early tea, some light food in the evening is advisable. Long intervals between the periods of taking food tend to weaken the powers of digestion. The quantity of food taken at a meal is to be graduated to the diminished digestive powers. The appetite is not always to be relied upon in dyspepsia. The appetite is often craving; the system ealls for nutritive supplies beyond the ability of the digestive organs to prepare food for assimilation. Food should be eaten slowly. Deliberate mastication prepares the food for the action of the gastric juice. Moreover, slowness in eating gives the stomach a chance to express satiety, and is, therefore, a protection against over-eating. A moderate quantity of liquid only should be taken at meals, in order not to dilute too much the gastric juice, but drink may be taken freely between the acts of digestion. Under erroneous notions, abstinence from liquid is sometimes carried to an extreme.

With regard to the use of wine or spirits, the danger of intemperance is always to be considered, especially as dyspepsia not infrequently leads to a morbid desire for stimulants, and in view of the fact that the mental state is favorable for the formation of intemperate habits. Yet an alcoholic stimulant taken with meals is often useful until the normal powers of the digestive organs are restored. If spirits be taken, they should be as little diluted as possible, the object being to stimulate the stomach. Stimulants should never be taken when the stomach is empty.

Tea and coffee are sometimes hurtful, the latter especially. 'Tobaceo is often injurious, from the waste of saliva and the depressing effect on the nervons system. If used at all, the quantity used should be quite small, and, in general, total abstinenee is easier than moderate indulgence.

Finally, with regard to diet, it may be well to remind the practitioner that it is unphilosophical to judge concerning his patients from his own 
personal experience. 'The physician who has his notions of what kinds of food agree with himself best, is apt to apply these notions indiscriminately to his patients. ${ }^{1}$

As regards regimen, the clothing of dyspeptics should be such as will secure unifsrmity of temperature and maintain the functions of the skin, withont an over-aceumulation of heat or exciting perspiration. Exercise is of great importance; but to be as useful as possible, it must be taken with a motive and end, aside from the sanitary object. Exercise, taken simply as exereise, tends to keep the mind of the patient on his disorder. and will not be likely to be persisted in. There is a risk of resorting at once to over-exercise, a too violent change of habits in this regard proving hurtful and discouraging the patient. The exercise should be in the open air. A change of business from one requiring sedentary habits to one involving out-of-door occupation is often advisable. 'Travelling, especially in foreign countries, where the attention is diverted with a succession of novel scenes, is highly useful. Sir James Johnston said that no case of purely functional dyspepsia could resist a perlestrian tour over the Alps.

2. The moral treatment consists first in establishing confidence by attention and sympathy. Inattention to the details into which dyspeptic patients are fond of entering, and ridicule of hypochondriacal fears prevent the physician from being of service to this unhappy class of patients, and lead them to seek for aid from quack remedies and quacks. If the confidence of the patient be secured, the assurances of the physician will often remove needless apprehensions, and afford an encouragement which, in itself, will contribute not a little toward improvement. If the patient be convinced that his mental state is in a measure due to a disordered condition of the body, he derives eonsolation and encouragement from this belief. 'The treatment relating to the mind will embrace, as far as possible, measures having reference to the removal of mental canses which may have produced or which perpetuate the affection. It is important to incite to occupations which divert the attention from the bodily functions, espeeially during the process of digestion. It is desirable that the mind sloonld be pleasantly oceupied. It is often observed by dyspeptics that if they dine alone, and are left by themselves to wateh the sensations connected with digestion, they are sure to suffer, whereas they experience no inconvenience from a greater amount of indulgence in eating if they be in agreeable company during and after dinner. Popular works on indigestion do harm by directing to the subject the attention of those prone to dyspepsia. The benefit of travel is, in a great measure, moral, involving recreation as well as exercise, and keeping the mind occupied with extrinsic objects.

3. The division of the treatment which relates to medieinal remedies is, relatively, least important, but by no means unimportant. If the symptoms denote morbid sensibility of the stomach, counter-irritation on the epigastrium is often useful. Small blisters, the strong aqua ammoniæ, or the croton oil, may be used for this purpose. The benefit is, probably, in part, due to a moral effect. The nitrate of silver may be tried in these cases, care being taken not to continue it too long. If anæmia be associated, the chalybeate tonies are indicated, of whieh, in cases of dyspepsia, the tincture of the chloride of iron is one of the best. The citrate of iron and quinia is an excellent form of tonic in dyspepsia associated with anæmia. The powder of iron redueed by hydrogen is another eligible form.

1 It is perhaps hardly necessary to state that these few remarks on diet in cases of dyspepsia are designed only as hints for the practitioner. To consider the subject of dietetics fully would be incompatible with the scope of this work. 
If there be constipation, the bowels are to be regulated by appropriate measures, but purgation is to be avoided. Constipation will be considered as a distinet functional affeetion. There are no special indieations for mercurial remedies exeept as an oecasional eathartic or laxative. The success of the Abernethean plan of giving frequently blue mass followed by the infusion of senna, was doubtless due to a moral effeet, in conjunction with advice as regards diet and regimen. In general, the various vegetable tonies are useful, the form of tonic being varied from time to time. A grain or two of quinia twice or thriee daily, continued steadily for several weeks, appear's to be often useful. $\mathrm{A}$ few drops of hydrochloric acid just before or after eating, may frequently be prescribed with advantage. The benefit of the tincture of the chloricle of iron is, probably, in part, due to the hydrochloric aeid. Of late, pepsin has been somewhat in vogue as a remedy in dyspepsia. Experience does not appear to offer much testimony to its value. The principle on whieh it is employed, viz., to supply a deficient element in the gastric juice, entitles it to be considered only a palliative, not a curative, remedy. A convenient and eligible form in which pepsin may be given is the preparation commonly known as rennet-wine. 'This is prepared by putting a fresh rennet, cut up into small pieces, into a pint of sherry wine, which, after maceration for two weeks, is to be strained, and it is then ready for use. Of this preparation, from half a teaspoonful to a teaspoonful is to be given at the time of, or shortly after, taking food. This remedy is useful, not only in eases of dyspepsia, but in various diseases when it is desirable temporarily to resort to artifieial digestion.

Symptoms characterizing ecrtain eases of dyspepsia claim particular remedies. Pyrosis is generally relieved by bismuth in full closes, in conjunction with measures relating to diet, regimen, etc., and followed by tonic remedies. The purified oxide of manganese is stated by Dr. Leared, of Dublin, to be equally, if not more, efficacions in relieving this form of disorder. The dose is from ten to thirty grains, given three times claily. Cardialgia calls for alkaline remedies, viz., lime-water, liquor potassæe, subcarb. of soda, magnesia, the two latter especially if there be constipation. Flatuleney and tympanites are to be relieved by carminative stimulants, the tincture of the essential oils, the aromatic powder, and charcoal powder, the latter being useful on account of its faculty of absorbing gases. All these remedies, howerer, are merely palliatives. The conditions on which depend the several symptoms just named, are, if possible, to be removed by tonie remedies, such as quinia, and the mineral acids. Tympanites occurring in paroxysms, from the secretion of gas, is apt to be an extremely obstinate form of disorder. Purgatives are not useful. Opiates to relieve present suffering are required. Carminative and alcoholic stimulants will sometimes afford relief. $\Lambda$ ssafotida giren by the mouth or rectum is lighly useful, espeeially when hysterieal symptoms are associated. In the intervals between the paroxysms, measures, medieinal and hygienie, to invigorate the system are indicated, together with the use of antispasmodic remedies, such as bellaclonna, valerianate of zinc, ete. My friend, Prof. Isaac E. Taylor, has found small doses of strychnia useful in these eases. 'This remedy, or the nux vomica in the form of tineture or extract, is highly reconmended by many in other forms of dyspepsia. The closes should be sufficiently small to avoid any possibility of indueing poisonous effects.

The management in eertain cases of dyspepsia charneterized by persisting volniting after taking food, has been already alluded to. Change of scene and out-of-door life are the most eflicient measures. A sea-voyage 
will be likely to prove effectual. But various remedies may be employed in succession, each of which will sometimes be found useful, if not successful. The salts of morphia or codeia may succeed if the patient be not unpleasantly affected by opiates of any kind. If immediately rejected from the stomach, these remedies may be given by means of hypodermic injections. The hydrocyanic acid, in some cases, is an efficicit remedy. Creasote sometimes acts efficiently, given in doses of one or two minims, repeated after each act of vomiting. In young children this remedy, given in doses of one-eighth or one-sixth of a drop, frequently acts like a charm in arresting vomiting. Bismuth in large closes is a valuable remedy in some cases. Strychnia or the nux vomica has been found useful. The oxalate of cerium, from one to two grains at a dose, is another useful remedy. Counter-irritation on the epigastrium, by means of blisters or dry cupping, is frequently followed by relief. Milk with lime-water added is sometimes retained when other articles of food are rejected.

Vomiting is symptomatic of a great variety of affections, exclusive of inflammation or structural lesions of the stomach. Occurring as a form of functional disorder of the stomach, it is sometimes considered as an individual affection; but it may with propriety be included under the head of dyspepsia. There is a variety of vomiting which claims a brief separate notice. Reference is had to eases in which a liquid is thrown from the stomach in greater or less abundance, emitting an odor of fermenting wort, and, after standing a few hours, becoming covered with a mass of brownish froth resembling yeast. Goodsir, in 1842, on subjecting this liquid to microscopical examination, discovered in great abundance a vegetable fungus, which is now commonly known as the sarcina ventriculi, called by Robin merismopodia ventriculi. The sarcinæe are distinguished by their oblong or square form, and by their being clivided by lines into four equal squares, so that they resemble a package tied with a cord, as the name sarcina denotes. The vomited liquid containing sarcinæ may also contain the yeast fungus in abundance. The liquid is sour, due, according to Budd, to acetic acid, and the vegetable productions are incidental to a process of fermentation, attended with the formation of this acid, together with the evolution of carbonic acid.

There does not appear to be ground for the belief that the presence of the vegetable productions is the source of disorder. They are effects, rather than the cause, of disorder. The sarcinæ are not produced exclusively within the stomach ; they have been found in the urine, in the purulent discharges from a gangrenous abscess, and in the humors of the eye. Their production within the stomach appears to be owing to an undue retention of its contents, from pyloric obstruction or other causes. The treatment, therefore, relates, not alone to the circumstances immediately involved in their production, but to the morbid conditions giving rise to these circumstances. Remedies, however, designed to arrest the fermentative process are indicated. And, of these, the most efficient is the bisulphite of soda, first suggested as a remedy in this form of disorder by Jenner. From fifteen grains to a drachm may be given, dissolved in water, two or three times a day. Budd's method of employing this remedy is to prescribe a solution of the strength of two drachms of the salt in an ounce of water, and to direct a teaspoonful to be taken in a wineglass of water soon after meals, or when the fermentative process in the stomach is about to commence. According to the anthor just named, the remedy owes its efficacy to the fact that it is easily decomposed, and its decomposition liberates sulphurous acid, which prevents alcoholic and acetous fermentation. The sulphite of soda, and the other sulphites, are valuable as palliatives when- 
ever, from defective power of digestion, fermentative processes in the ingesta take place. 'The value of these remedies, in cases of dyspepsia, depends on their power in arresting fermentation.

Dyspeptics frequently derive advantage from the use of mineral spring waters, especially if connected with a sojourn, under agreeable circumstances, at a pleasant watering-place. Much of the advantage, doubtless, is dne to the change of habits, relief from care, together with the moral influence of the expectation of improvement. Of the various mineral waters, those from the gaseous and chalybeate springs are best suited to cases of dyspepsia. Cold sea-bathing, and the invigorating influence of a bracing sea-air, are frequently useful. The hygienic discipline of the hydropathic system is undoubtedly useful in some cases, the benefit being due, probably, in part, to a physical, but more to a moral effect. There is, perhaps, no affeetion in which the anticipation of improvement from the employment of certain measures contributes to thcir efficacy more than in dyspepsia.

\section{POLYPHAGIA, MALACIA OR PICA, POLYDIPSIA, AND DIPSOMANIA.}

These names express morbid conditions which relate to the ingestion of solids or liquids ; conditions which, severally, do not eonstitute inclividual affections, but which may be the most prominent, or perhaps the ehief, ailments, ancl, therefore, they claim a passing notice.

Polyphagia, or, as it is more commonly called, bulimia, denotes a craving for food beyond the wants of the system. $\Lambda$ s thus defined, these terms are not correctly applied to the greatly angmented appetite felt frequently during convalescence from fevers and other acute diseases, or when, from any cause, the system for a time lias been deprived of alinentary supplies adequate to its wants. The inordinate desire for foorl, under these circumstances, is an expression of the requirements of the system, and the ability to digest food may be proportionately augmented-a fact which illustrates, in a striking manner, the connection existing between nutrition and the function of digestion. True bulimia does not exemplify this physiological connection; the amount of food craved far exceeds the requirements for nutrition. Bulimia may be another name for gluttony. The love of eating may be cultivated to such an extent that little else is thought of, and persons who fall into this habit may be said to live to eat, rather than eat to live. The observation of every one will furnish examples of this kind. Habits of gluttony may lead to dyspepsia, which, by interrupting further indulgence, proves conservative, or, if the digestive function continue active, they may lead to obesity, fatty degeneration of the heart or other organs, and to various affections. Voracious eating characterizes certain cases of mental derangement. It may be a symptom of disease attended with progressive waste, for example diabetes. In a more marked and rare form, the craving for food greatly exceeds that under the circumstances just named. Curious instances have been reported, in which the morbid appetite appeared to be insatiable, all kinds of foorl, raw meat, candles, etc., being eaten, in some cases, with avidity, and in enormons quantity. It is difficult to explain the morbid appetite in these instances. Recovery from this condition may be expected. The inclications are to regulate the diet as far as possible, to establish the general health by hygienic measures, to correct any disorder of digestion which may exist, and to palliate the excessive craving for food by opium or, sometimes, by 
nauseant remedies. Swallowing pieces of ice has been found effective as a palliative measure.

Malacia and pica are terms applied to perversions of appetite, that is, to a morbid craving for particular substances. The terms are commonly used as synonyms, but, strictly, they denote different kinds of perversion: Malacia denotes a morbid claving for certain articles of food or articles which are not devoid of nutrition, whereas, pica denotes a desire for innutritious substances. 'The craving for strange kinds of food, sometimes attending pregnancy, and commonly known as longings, is sufficiently familiar. Similar eccentricities of the appetite are sometimes observed in connection with hysteria, and they may be due, in some cases, to that desire to become objects of wonder or interest which weak-minded hysterical women sometimes evince. Unnatural and disgusting perversions of the appetite are among the symptoms occasionally met with in cases of insanity. Perversions falling under the head of pica are sufficiently common. Innutritions substances frequently craved are charcoal, chalk, slate, and certain kinds of earth. The habit of eating these substances is sometimes carried to a great extent. In the case of a young lady seized, shortly after marriage, with epidemic dysentery which proved fatal, the dejections contained an enormous amount of a black substance which, on investigation, was found to be from a species of slate-stone which she was accustomed to eat daily in excessive quantity. In some cases of pica, the articles are at first taken with the idea of improving the complexion, and in this way the habit is formed. But, in other cases, a morbid uneasiness in the stomach leads to their use. The appetite is mostly confined to females, and is generally associated with spontancous anæemia or chlorosis. The measures of treatment are to be addressed to the associated disorders, the continued indulgence of the perverted appetite being interclicted.

Dirt-cating, as vulgarly called, or chthonophagia, is a morbid habit which has heretofore prevailed, to a considerable extent, among the plantation negroes of the Southern States. It was described by Joln Hunter as prevailing among the negroes of the Wrest Indies. The kind of earth selected is a loam or clay. Dr. James B. Duncan, of Louisiana, in a paper contained in Fenner's Southern Reports, vol. i. 1850, gives the following account of this form of pica: " $A$ very common disease among negroes on plantations in this part of the county, is a state of anæmia very often attributed, and perhaps justly, to the pernicious habit of dirt-eating. On examining negroes on a plantation, a medical man is surprised to meet with so many of these cases. Almost every large plantation has three or four, and sometimes more of them. Until the vital powers of the system are beginning to be undermined, no marked symptons of disease being visible to the eye of the planter, they are generally suspected of laziness or malingering: After this condition has existed for some time, the skin presents a paler hue than natural; or if the subject is a mulatto, an ashy white; the lips, tongue, lining membrane of the mouth, the palms of the hands, white, lacking the reddish tinge of health; the legs odematous, abdomen distended, pulse full, soft, and frequent; action of the heart violent; if blood is drawn, it is pale and watery; respiration on the slightest exertion hurried; in fine, all the symptoms which characterize chlorosis in females. We find this condition of things in subjects of both sexes. Many of these cases are, doubtless, produced and aggravated by the deleterious habit of dirt-eating. But I never heard a negro admit that he was addicted to the habit. Some admit that formerly, years ago, they ate dirt, but not now; and others, trusty, truth-telling negroes on 
other subjects, on this will lie most pertinaciously to the last, unless detected in the act. With many, no doubt, dirt-eating is a symptom only of a diseased condition of the digestive organs, and of the system generally. With them dirt-eating proceeds from the same propensity which leads white females to resort to chalk, magnesia, etc., in order to relieve a disordered acid condition of the stomach. This condition of system is often, in my opinion, produced by a deficiency of suitable nutriment. The diet of negroes on most plantations being salt pork, corn bread, and molasses-rarely fresh meat and regetables-a condition of the system is thus prodnced closely allied to scurvy. In addition to the symptoms above described, I have occasionally seen a spongy statc of the gums.

"The restoration of these cases to health, whether addieted to dirteating or not, is troublesome and tedious. The moral has to be preseribed for, as well as the physical, symptoms. To restore the healthy condition of the bolly, nutritious food, fresh meats, vegetables and greens, porter or wine, are necessary; and, as to medicinal agents, preparations of iron in any or all forms, combined with stimulating stomachics and bitter tonics, laxatives when indicated, and out-door exereise. Confinement within-dloors aggravates the disease. To cure the habit of dirt-eating, many resort to cruel methorls of preventing the indulgence of it, by tin masks for the face, iron gags, chaining on plank floors, etc. By using these means, it is true, the habit camot be indulged in, but the cause that produced the propensity still exists, and the disease camnot be cured so long as tlrese depressing agents are used. Restore the healthy tone of the system, invigorate the subject, put rich blood into his veins, clothe him well, feed him well, and do not overtask him; arouse his feelings of pride, teach him to feel that he is a reasonable and rational being, and, in a majority of eases, suceess will attend our efforts, and we shall have the satisfaction of reseuing a valuable servant from the grave."

Polydipsia is a term applied to denote a condition characterized by an excessive eraving for liquids. Water is drunk in a very large or enornous quantity, amounting sometimes to several gallons in the twenty-four hours. In some cases the patient experiences constant or frequently recurring thirst, associated with dryness of the tongue and fances. He is unable to sleep except for short periods, in eonsequence of the urgency of the desire for drink. The quantity of urine is proportionately great, so that this condition is also denoted by a term expressing the latter fact, viz., diabetes insipidus. The urine voided has but little specific gravity, presenting a clear limpid appearance like pure water, and containing neither sugar nor any abnormal constituent. In other respects, the health may not be notably disordered. The appetite and digestion may be unimpaired, the functions generally are well performed, and the body does not waste. This condition at filst suggests to the physician the probable existence of cliabetes mellitus, but an examination of the urine leads to the exclusion of the latter clisease. The condition may occur in early life or becomes developed at any age. It has been observed to occur as an intermittent malady, but, in general, it continues for an inclefinite period or during life. It does not appear to lead to any disease, and is serious, in itself, only on account of the annoyance and debility which it occasions. Its pathological character, with our present knowledge, cannot be satisfactorily explained. Existing to the extent just described, it is so rare as to belong among the curiosities of clinical experience. In our ignorance of the pathology, there are no rational indications for treatment, and experience has not furnished any reliable means of cure. Opium, valerian, camphor, and other antispasmodics have been found to diminish 
the thirst temporarily, and some benefit has been derived from the use of various tonic remedies.

Dipsomania is a term sometimes used to denote the peculiar delirium arising from the abuse of alcohol, but it is commonly applied to an insatiable desire for alcoholic drinks, and, used in this sense, it expresses a morbid condition which may be noticed in the present connection. A craving for this class of stimulants is sometimes developed in cases of dyspepsia, and may be the source of intemperate habits. This fact has been already referred to. It is not to be lost sight of in judging of the propriety of prescribing or sanctioning the use of alcoholic stimulants in dyspeptic cases. A morbid craving for aleohol in some form is an element of intemperate habits, whatever may have been their sourec. The habitual drunkard feels a desire for continued indulgence, which, with an impaired power of self-control and lessened self-respect, resulting from intemperance, he is, after a time, wholly mable to resist. It is useless, under these circumstances, to attempt to reason him into temperance; he may appreeiate the force of all the arguments which are presented, but he lacks moral strength enough to govern an acquired appetite which represents a morbid condition as much as the delusions of insanity. Reason and persuasion, however, may be effective before this condition of dipsomania is reached; that is, they may prevent this condition by leading to abstinence from alcoholic stimulants. Dipsomania is to be treated as a disease of body and mind. The cure is to be effected by withdrawing the patient from the use of alcohol in any form, and the employment of medicinal and liygienic measures to invigorate the physical and mental faculties. The treatment must be continued long enough for the cessation of the morbid craving for stimulants, and for the development of moral strength sufficient to enable the patient to aclhere to a course of total abstinence, in which consists his only safety. It is evident that successful management is rarely practicable, except by means of institutions in which the patient is under the same restrictions as in insane asylums. In fact, dipsomania is to be treated as a form of mental derangement.

There is a species of clipsomania which is constitutional and congenital. The desire for stimulants is paroxysmal, and an irresistible craving is developed by ever so small an indulgence. Persons with this unfortunate idiosyncrasy lose their power of self-control as soon as they feel the influence of alcohol. The only protection against inebriation, in such cases, is rigid abstinence.

Connected with the subject of dipsomania are questions pertaining to the causes of intemperance, and the means of its prevention, which are of the utmost importance to the welfare of mankind, but which do not properly fall within the scope of this work. The morbid effects of alcohol upon the system, aside from the development of dipsomania, will be referred to in connection with different individual diseases, and especially those affecting the nervous system.

\section{GASTRORRHAGIA.}

Hemorrhage into the stomaeh, and vomiting of blood, are denoted by the term Hæmatemesis. The term Gastrorrhagia is in uniformity with the rule of nomenclature by which a hemorrhage and its situation are expressed, and is, therefore, to be preferred. Moreover, the term gastrorrhagia applies to all cases of gastric hemorrhage, whether vomiting take place or not, the blood sometimes not being expelled by the mouth, but passing into the intestinal canal and being evacuated from the bowels. 
In the great majority of the cases in which hemorrhage takes place into the stomach, blood is vomited; and, more or less blood also passing into the intestinal eanal, the stools present appearances to be described under the head of enterorrhagia.

When blood is expelled from the mouth, the first point is to ascertain the source of the hemorrhage. It may be from the mouth or posterior nares. Coming directly from either of these sources, it is traced without difficulty; but if blood be swallowed and romiting occur, it may sometimes be a question whether gastric hemorrhage exists or not. In eases of bronchorrhagia, blood may be swallowed and vomited. In a child too young to expectorate, bronchial hemorrhage might thus be supposed to be gastric. Examples have occurred, but both bronchorrhagia and gastrorrhagia in young children are extremely rare. The rupture of an aneurism into the pharynx or osophagus is another source of hemorrhage which may appear to be gastric. But, in general, the question is, whether the blood come directly from the stomach or air-passages. The points involved in this differential diagnosis have been already presented in treating of bronchorrhagia, and need not be here repeated." ${ }^{2}$ Blood which has been vomited, in most cases presents a dark grumous appearance. Exceptionally, it is florid, like arterial blood. It then comes from an artery, and has been vomited directly it accumulates within the stomach.

Gastric hemorrhage, in the vast majority of eases, has no claim to be regarded as an individual affection. It may be a symptom of carcinoma of the stomach, or of gastric ulcer, and, as such, has been already considered. It is an occasional effect of portal congestion in cases of cirrhosis of the liver. It coexists with hemorrhage in other situations in cases of purpura hemorrhagica and scorbutus. It occurs in cases of acute gastritis. It is a prominent event in the natural history of yellow fever; the so-called black vomit, in this disease, denoting neither more nor less than gastrorrhagia. Exclusive of these pathological associations, it may occur in place of the menses in eases of amenorrhcea. Instances in which gastrorrhagia is to be regarded as vicarious menstruation are by no means common. In the majority of the cases in which it follows suppression of the menses, it proceeds from clisease of the stomach. Yet, that it may take the place of the menses, must be admitted. Of 27 cases of gastrorrhagia analyzed by Valleix, in 5 the hemorrhage could not otherwise be accounted for; and in all these cases recovery took place. The cessation of habitual hemorrhage in some other situation, the arrest of purulent discharges of long standing, and the healing of old ulcers, have been supposed to stand, occasionally, in a causative relation to gastrorrhagia, but this supposition does not rest on adequate clinical proof. Hemorrhage sometimes occurs from the stomach, as from the bronchial tubes, the Schneiderian membrane, and in other situations, without any apparent pathological connections, neither following nor preceding any appreciable morbid conditions. It is then to be considered as idiopathic, or as constituting an individual affection. Such cases are much more infrequent than cases of idiopathic bronchorrhagia. Hemorrhage from the stomach is an occasional event in pregnancy. Two of the 27 eases analyzed by Valleix exemplified this fact. It may be caused by a contusion received on the epigastrium. Irrespective of its occurrence as a symptom of gastritis, yellow fever, purpura hemorrhagica, or scorbutus, gastrorrhagia occurs oftener in females than in males. Of 72 cases analyzed by Dr. $\mathbf{H}$. Bence Jones, 53 were females and 19 males, a proportion of nearly 3 to 1. 
In 40 of these cases the existence of gastric ulceration was more or less certain, and in 32 cases the existence of gastric ulceration was more or less doubtful. ${ }^{1}$ It is a rare event in newly-born children, occurring a few hours after birth, the blood being. vomited and also passed from the bowels. It proves fatal in some of these cases.

The prognosis, in cases of gastrorrhagia, will depend upon its pathological import. The prognosis is, of course, unfavorable if it be associated with carcinoma, ulcer, or cirrhosis of the liver. If, however, the hemorrhage be vicarious, or not connected with any appreciable morbid conditions, the prognosis, as a rule, is favorable. 'The loss of blood, in piroportion as it is considerable or large, induces debility, anæmia, and it may prove the direct cause of death. In a case which came under my observation in consultation with my colleague, Prof. McCready, the patient, a young married female, was seized with gastric hemorrhage when apparently in good health, and the fatal result, which took place in two or three days, seemed to be attributable to the loss of blood. The amount and rapidity of the hemorrhage may be such as to induce sudden death from syncope. On the other hand, a supplementary hemorrhage, if moderate, is not only without danger, but may conduce to the welfare of the system, so long as the menstrual discharge remains suppressed. In determining the amount of hemorrhage, it is to be borne in mind that the quantity of blood vomited is not always a criterion. 'The blood may accumulate and coagulate within the stomach without being vomited. The hemorrhage may be sufficient to destroy life, althongh the quantity vomited be not large. Deatl may take place in such cases as in cases of concealed uterine hemorrhage. The evidence of hemorrhage out of proportion to the amount of blood vomited, is afforded by dulness of perenssion over the distended stomach, and symptoms denoting loss of blood, viz., feebleness of the pulse, coldness of the surface, pallor, faintness, etc. The blood thus accumulating within the stomach, if not vomited, passes into the intestines and is discharged by stool. Hemorrhage into the stomach has been known to occur and death take place from the loss of blood without any vomiting. Grisolle cites three examples, one of which was under his own observation. ${ }^{2}$ The stomach in this case was distended with an enormous clot. The diagnosis, when vomiting does not occur, must rest exclusively on the physical evidence of distension of the stomach by a liquid or solid, conjoined with the general symptoms denoting internal hemorrhage.

The treatment of gastrorrhagia has been already referred to in connection with gastric ulcer. The general principles of treatment having reference to the arrest of the hemorrhage, or the prevention of its recurrence, are the same under whatever circumstances it occurs. With reference to these objects, quietude of body and mind is important. Repose of the stomach should be secured by the introduction of no more aliment than the wants of the system require, and the food taken should be bland and easy of digestion. In cases in which there is danger from the amount of hemorrhage, entire rest of the stomach is essential, the system being supported by nutriment and stimulants administered per enema. Opium is useful by quieting the peristaltic movements of the stomach. The direct refrigerant effect of iced water taken in small quantities at a time, or of swallowing small pieces of ice, will be likely to be useful. In urgent cases,

1 Vide Med.-Chir. Transactions, published by the Medical and Chirurgical Society of London, vol. $\mathrm{xxv}_{\text {., }} 1860$.

2 Pathologie Interne, tom. ii. 
as a temporary means of arresting the hemorrhage, ice may be applied to the epigastrium. In less urgent cases, revulsive measures may be relied upon, viz., sinapisms and dry cups, applied in different situations over the abdomen. Hot, stimulating footbaths are useful by way of revulsion. Hæmostatic remedies are to be given, taking care not to provoke vomiting by their use. The acetate of lead, tannic or gallic aeid, and the astringent preparations of iron (the persulphate or pernitrate) are the most efficient of the remedies of this elass. The ergot has been supposed to be useful as a styptic in cases of gastric hemorrhage. Emetics are certainly not rationally indicated, except in cases in which percussion furnishes evidence of the stomach being filled with a coagulum, and their propriety may be doubted even under these circumstances. Purgatives are contraindicated so long as measures to arrest or prevent the hemorrhage are required.

These measures are to be graduated, in individual cases, by the urgency with which the arrest or prevention of hemorrhage is indicated. Mild, simple measures are sufficient if the loss of blood be small or moderate. If there be ground for regarding the hemorrhage as vicarious, interference will be required only to keep it within proper limits. This statement will also apply to hemorrhage occurring in pregnancy.

The treatment in most cases of gastrorrhagia, of course, embraces other measures than those having reference to the arrest and prevention of the hemorrhage, viz., meassures addressed to the morbid conditions with which the hemorrhage is associated. The latter are considered under other heads. So, also, measures called for after the occurrence of gastric hemorrhage, having reference to debility and anæmia dependent on the loss of blood, need not be here considered. After a considerable hemorrhage, the stomach, assuming the non-existence of cancer or ulcer, is enfecbled, and the liability to a recurrence of the hemorrhage is to be kept in view. Careful regulation of the diet, quietude of the body, and the avoidance of all disturbing agencies, are important during convalescence.

Gastric hemorrhage is sometimes simulated by hysterical females, and by malingerers among soldiers and prisoners. Blood obtained from animals, and even human blood, may be taken into the stomach, and vomiting excited by various means. This deception may be suspected in the case of a young female who manifests a morbid disposition to impose on the credulity of those around her, as regards her ailments, when the symptoms do not denote loss of blood, when there are no grounds, aside from hemorrhage, to suspect gastric ulcer, and when the menses are not suppressed. Among soldiers and prisoners, it is to be suspected when the usual effects of hemorrhage are not apparent, and when the morbid conditions with which gastric hemorrhage is usually associated are wanting. The microscope may be of use in the detection of this kind of deception. Bennett cites a case in which a specimen of blood, which it was pretended had been vomited, was found to present the characteristic red corpuscles of the blood of a bird. 


\section{CHA P TER VI.}

\section{FUNCTIONAL AFFECTIONS OF THE STOMACH AND INTESTINES.-}

(Continued.)

Inanition. Gastralgia. Passage of Gall-Stones. Diarrhœa. Enterorrhagla.

$\mathrm{T}_{\mathrm{N}}$ addition to the ailments embraced under the head of dyspepsia are various functional affections of the stomach and intestines. 'These will be considered in the following order: Gastralgia, including gastric spasm, and, in this connection, the passage of gall-stones, or hepatic colic, may be most conveniently considered; diarrhœa, and hemorrhage from the bowels, or enterorrliagia ; enteralgia and the affections known as saturnine or lead eolic; sporadic and epidemic cholera.

Before entering upon the consideration of the affections just enumerated, I will offer a few remarks on a pathological condition which is not, perhaps, entitled to rank in the nosological catalogue as an individual disease, but which is an element of all diseases compromising alimentation and digestion. 'The pathological condition referred to is inanition. The importance of a due appreciation of this condition ${ }^{\circ}$ in medical practice renders it desirable to devote to it some attention, and it may with propriety be noticed in connection with the functional affections of the alimentary canal.

\section{INANITION.}

Health requires the assimilation of nutriment adequate, in quantity and kind, to the wants of nutrition. Every one is familiar with the mental and physical exhaustion felt when abstinence is protracted for a few hours only after the sense of hunger indicates the need of food. Every one knows that the deprivation of aliment, for a period varying according to circumstances, proves fatal. During the progress of starvation, the body loses rapidly in weight, the fat disappears, the muscles diminish in size, exhaustion progressively increases, the heat of the body is notably lowered, vomiting and diarrhoea not infrequently occur, the mental powers are weakened, listlessness and hebetude are sometimes followed by delirium and coma, and, according to Chossat, if the deprivation of aliment be complete, death takes place when one-fourth of the weight of the body at the time aliment was withheld has been lost. The mode of dying is a type of slow asthenia. It is worthy of note that, during the progress of starvation, hunger is not usually a prominent symptom. If felt for a time, it is apt to disappear, and may be followed by a loathing of food. Our knowledge of the natural history of inanition in man is derived chiefly from cases in which starvation is unavoidable, as in times of famine, in shipwreck, or when it is resorted to for self-destruction. Under these circumstances, however, the phenomena due to the want of nutriment are apt to be more or less modified by extrinsic circumstances. Experiments made on inferior animals are unsatisfactory because the symptoms can be but imperfectly appreciated. The best source of information is voluntary abstinence for the purpose of scientific observation. As a near, approach to experiments of this kind, those made by Hammond to test the nutritive 
value of albumen, starch, and gum, may be cited.' Submitting himself to the trial of restricting his diet to these alimentary principles singly, Hammond found that each is incapable alone of supplying the wants of the system, and that the two latter are absolutely innutritious. Now, the morbid phenomena produced during these experiments are to be considered as resulting from the absence of aliment; in other words, they were due to starvation. So far as they go, therefore, these experiments serve to exemplify the symptomatology of inanition. Referring the reader to the work by Hammond for fuller details, I will simply enumerate here the more important of the symptoms which he experienced.

1. Under a diet consisting of pure albumen for ten days : Febrile movement, heat and dryness of skin, headache, loss of appetite, nausea, abdominal pains, progressively increasing debility, serious diarrhœea, vigilance.

2. Under a diet of pure starch for ten days: Debility, disturbed sleep, sense of oppression of ehest, palpitation, headache, slight scratches of the skin showing tendency to inflammation and suppuration, febrile movement, abdominal pains.

3. Under a diet of gum, which he was able to continue only for four days: Abdominal pains, disturbed sleep, headache, febrile movement, diarrhoa, exhaustion.

Inanition is a pathological condition entering into all diseases which interfere witl the ingestion or the assimilation of aliment. The phenomena of inanition in cases of these diseases are combined with the symptoms belonging to the particular disease which exists. Febrile movement, headache, anorexia, vigilance, delirium, debility, etc., occurring in the progress of a disease, arise, not exclusively from the disease per se, but are more or less clue to inanition. The inability of the system to support, resist, and overcome disease proceeds, in a greater or less degree, from this element. The immediate cause of death in many cases of disease proving fatal by slow asthenia is inanition. 'These are facts of very great practical importance, and they are not sufficiently appreciated by many practitioners of medicine.

An important practical bearing of these facts relates to alimentation in the management of diseases. Until recently, for many years past, not only has the importance of alimentation not been fully recognized in praetice, but in most diseases the dietetie treatment has contributed to inanition. The writings of Graves led to the employment of alimentation in fevers. It is not less important in all other diseases which tend to destroy life by asthenia. Patients may be starved to death in other diseases as well as in fevers. As a practical rule, it is an object in any disease in which the danger lies in the direction of asthenia to meet, as far as practieable, by alimentary supplies, the wants of nutrition. Harm may arise from the ingestion of food beyond the powers of digestion, but, in these diseases, no harm can arise from an undue assimilation of food; on the contrary, the greater the assimilation the less the danger from inanition superadded to the disease. Let it be borne in mind that, in all diseases tending to destroy life by asthenia, there is danger from inanition, but never from hyper-nutrition. In the language of Chossat, "inanition is a cause of death which marches silently in front with every disease in whieh alimentation falls below the normal standard. It reaches its natural termination sometimes sooner and sometimes later than the disease which it covertly

'Vide Physiological Memoirs, by Wm. A Hammond, M.D., Surgeon.General U. S. Army, etc., 1863. 
accompanies, and it may supersede the disease of which at first it was merely an incidental element."

Alimentation is important in diseases not attended with immediate danger. In proportion as the assimilation of food can be made to approximate to the normal standard, the phenomena of inanition are forestalled, the vital powers are better enabled to tolerate and overcome the disease, the cluration of the disease may be shortened, convalescence is hastened, and the recovery is more apt to be complete. As regards the selection of food and the modes of its preparation in different diseases, in addition to dietetic rules based on general experience, our knowledge of alimentary principles, etc., much reliance may generally be placed upon the desires and tastes of the patient; but the absence of appetite is not to be a guide as regards alimentation. Loss of appetite is one of the symptoms of inanition. The wants of the system are not in disease, as in health, always expressed by hunger. The state of the mind dependent on the morbid condition of the nervous system, the coatings on the tongue, etc., interfere with the desire for food and the sense of taste. The practitioner is to exercise his judgment and tact in securing, as far as practicable, the assimilation of an adequate amount of nutriment either with or without the cooperation of desire and taste on the part of the patient, and it is often better to incur risk of exceeding rather than falling below the amount of food which the wants of the system require. It may be laid down as a prineiple applicable to the management of most acute diseases, that the assimilation of food is important to the fullest practicable extent; and it is a maxim of conservative medicine that, under all circumstances, a chronic affection is less likely to be prolongerl, serious lesions of structure are less liable to take place, and a fatal termination is postponed, in proportion as the vital powers are maintained by a nutritious diet conjoined with other hygienic measures. ${ }^{2}$

Innutrition is not infrequently involved in the causation of diseases. The connection between scarcity of food and the prevalence of typhus has been repeatedly observed in epidemics in Ireland. The same connection in individual cases and families is observed frequently everywhere. Scor: butus is caused by a deficiency of certain alimentary principles, or the want of a sufficient variety in food. Insufficient nourishment, which is sometimes involuntary and sometimes voluntary, favors the development of tuberculosis in persons having the tuberculous diathesis. This cause may co-operate with other causes in giving rise to various diseases, whether epidemic or sporadic; and the ability of the system to bear up under disease is impaired in proportion as the powers of life are enfeebled by previous defeetive alimentation.

Want of success in the.treatment of diseases in hospitals and often in private practice, especially among the poorer classes of society, is attributable, in no small measure, to the want of effective alimentation.

\section{GASTRALGIA.}

Gastralgia, called frequently gastrodynia, is a neuralgic affection characterized by pain, more or less intense, referred to the region of the stomach. Pain is a symptom of gastritis, carcinoma of the stomach, and gastric ulcer;

' Recherches Expérimentales sur l'Inanition, Mém. de l.Acad. des Sciences, 1843.

8 Vide essays on Conservative Medicine, hy the Author, American Journal of the Medical Sciences, January and October, 1863. Vide, also, essay on alimentation in disease, New York Med. Journal, 1867. 
it also enters into the clinical history of dyspepsia, especially the variety called acute dyspepsia, and a peculiar character of pain constitutes the dyspeptic symptom called cardialgia, or heartburn. But, considered as characterizing a distinet functional affection, the pain is not incidental to inflammation or any appreciable lesion, nor is it always, although frequently, associated with dyspeptic disorder; in short, gastralgia exists when pain and other cireumstanees denote an affection of the same pathological character as neuralgia in other situations.

Gastralgia is presented in practice in two forms; in one form a severe attack of pain oecurs, and, if not relieved by remedies, continues for several hours or even days. The pain is often exerueiating, subduing the strongest resolution; the patient writhes and groans with intense suffering. A sense of constriction frequently accompanies the pain; vomiting may, or may not, occnr. Generally, tenderness over the epigastrium is wanting, and some relief may be afforded by pressure. The paroxysm is not attended with febrile movement, and the skin may be bathed in perspiration from the intensity of pain. These attaeks are generally attributed to spasm or cramp, and the affection has been called colic of the stomach. It is difficult to say how much of the suffering is due to spasmodic contraction, and how much to neuralgic pain. Practically, it is not important to make this discrimination.

Such attacks in some persons are produced by certain ingesta. Strawberries, for exanuple, have been known to prove in this way poisonous, and I have met with a patient who had always an attack after eating honey ; he came under my observation when suffering from an attack thus produced. These, like other idiosyncrasies, are inexplicable. Attacks appear to be sometimes attributable to exposure to cold, and fatigue from overexertion.

This form of gastralgia is readily diseriminated from acute gastritis. The pain may be much more intense than in cases of the latter disease. As a rule, the tenderness over the epigastrium which belongs to the history of gastritis, is wanting. Vomiting may be wanting, and, if present, is not as prominent a symptom as in gastritis. The absence of febrile movement is an important point in the exclusion of gastritis. The passage of gall-stones, or hepatic colic, offers many symptoms in common, and this discrimination is not at once readily made. The points involved in the differential diagnosis will be considered presently, in treating of the passage of gall-stones.

This form of gastralgia is to be treated by opiates carried to the extent of procuring relief. A lialf grain of a salt of morphia may be sprinkled on the tongue, and this dose repeated, if necessary, after the lapse of from half an hour to an hour, until the pain is relieved; or the same eourse may be pursued with other forms of opiate. If the opiate be rejeeted from the stomach, it may be given per enema. The hypodermic mode of administration is well suited to this, as also to other affections in which it is desired to produee quickly and efficiently an anodyne effect. This plan of treatment is not to be deferred for the operation of a cathartic, or an emetic, even when the attack is attributable to a dictetic cause. A sinapism, and fomentations to the epigastrium are useful as auxiliary measures. An alcoholic stimulant may be added with advantage, if not contra-indicated by vomiting, especially if the pulse be feeble and the surface cold. Rest, regulated diet, and a mild eathartic or laxative, provided the bowels do not act spontaneously, will eonstitute the subsequent treatment.

Although the suffering is as great as in any other affection, there is little or no danger. It may be doubted whether this affeetion, alone, ever proves 
fatal ; and the practitioner may count with confidence on procuring speedy relief by means of the plan of treatment just stated. The affection, therefore, is one of those which exemplify, in a striking manner, the resources of medicine.

The foregoing form of gastralgia may be distinguished as acute. In the other form, the affection becomes chronic. The pain, in the latter, is either limited to paroxysms which recur more or less frequently, or it is labitual, with exacerbations at variable intervals. The pain varies much in different cases, as regards intensity, and also in different paroxysins or exacerbations in the same case. It may have so little intensity as to occasion only annoyance, or it may be so severe as to give rise to extreme suffering. The character of the pain is deseribed by patients as burning, lancinating, or gnawing. Frequently the pain extends from the epigastrium in different directions, viz., to the back, into the chest, and, laterally, into the right and left hypochondrium.

With the affection, in some cases, are associated dyspeptic ailments, and in other cases, digestion is not disturbed. Paroxysms or exacerbations sometimes appear to be provoked by the ingestion of food, but as a rule, relief of pain is felt after eating. The desire for food is oftener increased than diminished. Generally, tenderness over the epigastrium is wanting, and pressure frequently affords relief. The decubitus during suffering from pain is sometimes upon the belly. Constipation exists much oftener than looseness of the bowels. Flatulent distension of the stomach or intestines is sometimes associated. 'The pulse preserves its normal frequency, and the temperature of the body is not increased. Patients who have suffered from the malarly for some time are apt to become melancholy and hypochondriacal. I have known it to be associated with a tendency to suicide.

Gastralgia rarely, if ever, occurs inder the age of puberty, or in old age. The average age, according to Barras and Valleix, is about thirty-two. It is generally considered that females, much more than males, are liable to it, but of 39 cases analyzed by Valleix, only 20 were females. It affects those of good constitution and the robust, as well as the feeble and delicate. Persons of sedentary habits appear to be more likely to be affected than those engaged in active pursuits. Prolonged mental depression has been supposed to favor its occurrence. Prof. Alfred Stille states that chewing tobacco is one of the most frequent of causes. It appears in some cases to depend on the gouty diathesis. Like neuralgia in other situations, it may be an effect of malaria; and attacks of gastralgia may recur with the regularity of the paroxysms of intermittent fever. I have known gastralgia to take the place of the cold stage of paroxysm, being followed by the lot and the sweating stage. Finally, causes which induce anæmia or lead to debility, may be more or less involved in its prodnction.

The diagnosis of the chronic form of gastralgia is to be based on certain distinctive characters, viz., the occurrence of pain either in paroxysms or marked exacerbations; the relief frequently afforded by pressure and the ingestion of food; the persistence of appetite in many cases, and, not infrequently, the absence of disorder of digestion, and the absence of febrile movement. These points in the history are involved especially in the discrimination of gastralgia from subacute or chronic gastritis. The diagnosis involves the exclusion of gastric ulcer and cancer. These affections are to be excluded by the absence of their diagnostic symptoms, irrespective of pain. In some cases of intercostal neuralgia, pain is referred to the epigastrium. 'This affection is excluded by the absence of its diag- 
nostic criterion, viz., tenderness on pressure over three points-posteriorly, laterally, and anteriorly.'

Chronic gastralgia is apt to be persisting. Its duration, in different cases, is extremely variable. In this respect it resembles other neuralgic affections. Of thirty-nine cases analyzed by Valleix, in one case the affection lasted for twenty-six years, and the patient, at the end of this period, was carried off by another disease. In the remainder of the cases, the duration varied from two months to twelve years. As regards prognosis, the worst to be apprehended is the indefinite protractedness of the affection. It cloes not tend to destroy life either directly or by eventuating in some other more serious discase. It is not prudent for the practitioner to predict that the affection will end speedily, or within any definite period; yet, not infrequently, it does not continue. In certain cases it is one of the affections most rebellious to therapentical measures, but in other cases it is readily amenable to treatment.

In the treatment of gastralgia, clinical experience has abundantly shown certain measures formerly in rogue to be not only ineffectual but prejudicial. 'This remark has reference to general and local bloodletting, emetics, mercurials, purgative remedies, and perhaps also vesication or other modes of active counter-irritation. Anodyne remedies are frequently called for to relieve pain. If the pain be severe, opium can hardly be dispensed with; but in this, as in other painful affections which are apt to be persisting, the liability to the formation of a habit of using opium is to be consiclered. Moreover, the use of opium, aside from the palliation of pain, is objectionable on account of its interference with the appetite and digestion, and, in this way, it may contribute to perpetuate the affection. Opium is, therefore, to be used with circumspection, and, when practicable, other palliative measures are to be substituted, such as belladonna, hyoscyamus, etc. Fomentations and embrocations containing opium, chloroform, aconite, etc., will sometimes succeed in affording relief. Cold water applied to the epigastrium has been found effectual. Certain remedies appear, in some cases, to exert a curative influence. Bismuth is one of these. I have known large doses of this remedy to be promptly curative. Monneret advises this remedy to be given to the extent of one, two, or three ounces daily. 'These enormous doses are not required. Whatever curative power the remerly has will be exerted in doses of from a scruple to a drachm three or four times daily. Alumen has been recommended by Dr. Griffin in doses of from ten to twenty grains three or four times daily." Strychnia and the nux vomica have been found useful, especially if the affection be associated with the formation of gas. A quarter of a grain of the watery extract of nux vomica may be given three or four times daily. Other remedies which may be employed with a view to a curative effect are the nitrate of silver, quinia in full doses, the subcarbonate of iron (precipitated carbonate or sesqui-oxide) given in drachm doses, the iodide of potassium, and arsenic, as in other neuralgic affections. Dr. Leared, of Dublin, has employed with.much success the purified oxide of manganese in doses of from five grains to half a drachm three times daily. ${ }^{3}$

Measures of treatment, other than the employment of direct curative remedies, are of greater importance than these. Change of habits fiom those of sedentary to active life is of the first importance in certain cases. The moral influence of recreation, change of scene, etc., is often of more

I Vide page 202.

Dublin Medical Press, 1865.

Stillé's Therapeutics, vol. i. page 191. 
value than medicines. The diet should be nutritious. Alcoholic stimnlants in moderation with meals may be advișed for a time, exercising that reserve dictated by the danger of patients becoming addicted to their habitual use. Tonic remedies, in most cases, continued steadily for a long period, and varied from time to time, are advisable. The valerianate of zinc may be mentioned as a tonic remedy likely to prove useful. Preparations of iron are especially indicated if anæmia be present. Dyspeptic ailments, which may be associated, are to receive appropriate treatment. In short, the object, aside from the employment of palliative and curative remedies, is to endeavor to place the digestive organs and the system in the best possible condition, and, for this end, hygienic and medicinal measures are to be adapted to the circumstances peculiar to individual cases.

\section{PASSAGE OF GALL-STONES.}

The passage of biliary calculi along the cystic and common duct may give rise to symptoms resembling those of acute gastralgia. The discrimination, at once, is not always easy. Moreover, a paroxysm of pain from the passage of a gall-stone, or hepatic colic, as it has been called, is to be discriminated from affections other than gastralgia, viz., peritonitis, ordinary colic, colic with intestinal obstruction, acute gastritis, and the passage of calculi from the kidney to the bladder. Biliary calculi usually form in the gall-bladder, but cases have been reported in which they have been found in the hepatic duct and its branches within the liver. They vary in size firom that of a pin's head to that of a hen's egg. They are frequently found in the gall-bladder after death, and sometimes in great number. A collection of several hundred is not uncommon. Bennett relates a case in which the number amounted to two thousand, and cases lave been reported in which the number was still greater. They are usually of soft consistence, and of a low specific gravity. They are sometimes white, but oftener either of a yellow, brown, or greenish color, from the imbibition of bile. On section they are often found to consist of successive layers which differ in consistence and cclor. In general they are composed chiefly of cholesterin, but they frequently contain the salts of hime. Remaining within the gall-bladder, they may occasion no inconvenience; but, if very numerous or large, they may give trouble by distending the gall-bladder, and they may lead to ulceration and perforation. If the perforation be into the peritoneal sac, peritonitis follows, and will be likely to prove fatal. If the distended gall-bladder become adherent to the abdominal walls, the perforation may lead to a circumscribed abscess which opens externally, and numerous calculi may be discharged through the opening. Prof. James P. White has reported an interesting case of this kind. ${ }^{3}$ They are also sometimes discharged by an ulcerated opening into the intestinal canal. The presence of gall-stones within the gallbladder has been determined by means of a characteristic sound, resembling the collision of nuts or marbles in a bag when shaken, produced by forcible pressure or strong percussion over the distended bladder. But. in general, their existence is not suspected until, having entered the cystic or common duct, they occasion, by their passage toward the intestine, paroxysms of so-called hepatic colic. It is evident that the time occupied. in the passage of a gall-stone, and the suffering, will depend, other things being equal, on its size and the irregularity of its shape.

1 Buffalo Medical and Surgical Journal, June, 1864. 
The pain occasioned by the passage of a gall-stone is frequently $a b$ ruptly developed without any obvious cause, but in some cases it is preceded by a sense of uneasiness referred to the right hypochondrium. In the majority of eases the attack occurs three or four hours after a meal. The suflering is often extreme, causing the patient to writhe, and try a variety of positions with the hope of obtaining relief. The pain is referred to the epigastrium or the right hypochondrium. It may radiate thence throughout the abdomen, often extending into the chest, and to the right shoulder. Usually there is more or less tenderness over the seat of the pain, but in some cases pressure affords a certain measure of relief. Vomiting speedily occurs in the majority of cases, and is repeated more or less frequently during the continuance of the pain. The bowels are constipated. The pulse, as a rule, is not accelerated, and it may be retarded; not infrequently it becomes small and weak. The retardation of the pulse is sometimes notably great; I have known it to fall to 30 and 20 beats per minute. Pallor of the face and coldness of the surface are marked in some cases. Chilly sensations, with rigor, are not uncommon. 'These symptoms are sometimes so marked as to suggest the existence of malarial disease. The duration of the paroxysm is variable; it may last for a few moments only, or for many hours, depending, of course, on the period occupied by the passage of the stone either onward into the intes. tine or backward into the gall-bladder. $\Lambda$ t length, the pain and other symptoms suddenly cease, and nothing remains but more or less soreness and the fatigue occasioned by the suffering. If the paroxysm have been of considerable duration, the urine may show the presence of bile, and jaundice is frequently produced. These symptoms disappear after the paroxysm is ended. If dejections occur, they may show deficiency or absence of bile. The resorption of bile, and its absence in the stools, show that the gall-stone is impacted in the common, not the cystic, duct, and that its form and size are sufficient to cause obstruction more or less com. plete. Under these circumstances, the bile may accumulate within the gall-bladder, and distend it so that it can be perceived by the touch below the lower margin of the liver. Finally, after the paroxysm is ended, one or more gall-stones may be discovered in the dejections if these be carefully examined. In some cases, however, careful examinations of the feces fail to show the presence of a calculus, or of calculi. The failure to discover them is not proof that an attack of hepatic colic has not been experienced, for the gall-stones, in these cases, alter remaining for a time in the cystic duct, may have passed backward into the gall-bladder.

When one attack has been experienced, recurrences are to be expected, but, as regards the intervals, cases differ greatly. Successive attacks may oceur after days, weeks, months, or years. Not infrequently, numerous attacks are experienced in quick succession, several, perhaps, occurring in the same day, and for several days in suecession. This is probably owing to the temporary dilatation of the cystic and common duct produced by the passage of calculi of considerable size, in consequence of which other stones within the gall-bladder more readily escape. The patient, under these circumstances, may be consoled by the reflection that the more frequent the passage of calculi, the less are the number remaining to give rise to subsequent attacks; provided, of course, they pass through the common duct into the duodenal cavity. The frequent repetition of attacks, if severe, may occasion considerable disturbance of the digestive system, and general prostration.

The differential diagnosis of the passage of gall-stones from acnte gastralgia is to be based on the following points: The occurrence of pain, 
not excited by any obvious dietetic or other cause; the prominence of vomiting as a symptom; the presence, generally, of more or less tenderness; the existence of bile in the urine, and of icterus or an icterode hue of the conjunctiva and skin; retardation of the pulse; the persistence of pain, notwithstanding opiates are given in full doses; the sudden relief occurring irrespective of remedies; and, after the attack, the discovery of biliary calculi in the evacuations.

The examination of the evacuations for gall-stones not only serves to establish the diagnosis, but, on the appearance of the stone, or stones, may be predicated an opinion that the gall-bladder does, or does not, contain calculi which have not escaped. If a single round or oval stone only be cliscorered, it is probable that this was a solitary calculus in the gallbladder, and other attacks need not be expected; but if several stones are discovered, polyhedral in form, with smooth or polished facets, which may be plane, convex, or concave, it is highly probable that the gall-bladder contains others, and the patient cannot count on being exempt from repetitions of the paroxysms produced by their passage. The most effectual morle of examining for the presence of stones is to dilute the feces and strain through a sieve.

Acnte peritonitis may be accompanied by pain, resembling in intensity that of hepatic colic. But, in determining the existence of the latter, peritonitis is to be excluded by the absence of tenderness diffused over the abdomen, of rigidity of the abdominal walls, of tympanitic enlargement, of the notable acceleration of the pulse, together with the general prostration which belongs to the clinical history of peritoneal inflammation. Peritonitis, however, may proceed from perforation of the biliary canal, caused by persisting impaction of a gall-stone. In cases of ordinary colic, the pain is more paroxysmal, with intervals of freedom from pain. Invagination and internal strangulation of the intestine are to be excluded by the absence of a tumor caused by the accumulation of the intestinal contents at the point of obstruction, by the absence of obstruction, and of acceleration of the pulse, together with the greater gravity of the general symptoms in those aflections. In acute gastritis, vomiting is a more prominent symptom than in hepatic colic, and the greater gravity of the affection is shown by the pulse and other general symptoms. In some eases of hepatic colic, the pain extends downward in the direction of the right ureter, so that the passage of a calculus along this duct may be suspected. The absence of certain symptoms belonging to the history of so-caller nephritic colic, viz., retraction of the testicle, deficiency of the urine, hrematuria, etc., will serve to exclude this affection. Knowledge of the occurrence of one or more prior attacks which have been ascertained to be due to the passage of gall-stones, will aid in determining the diagnosis. The age of the patient is of some account in the diagnosis. Gall-stones may form at any age, but they are most apt to form in middle or advanced life, and in females oftener than in males.

In the great majority of cases, gall-stones, escaping from the gall-bladder, find their way into the intestine, giving rise to no evil results beyond the pain, etc., which their passage occasions. But occasionally a stone becomes permanently impacted in the biliary canal, and, if situated in the common or hepatic duct, retention of bile ensues, giving rise to persisting cholæmia, and softening of the liver from the distension of the bile-ducts. Under these circumstances, ulceration and perforation of the duct are liable to take place, leading to peritonitis; or adhesive circumscribed peritonitis may have preceded the perforation, and, the latter extending through the intestinal coats, the stone may be discharged into the intes- 
tine or through the abdominal walls. Obliteration of the canal of the common duct, as a result of inflammation and ulceration exeited by the presence of the stone, sometimes occurs.

The rationale of the formation of gall-stones is not understood. Probably the deposit in a solid form of eertain of the constituents of bile takes place in consequence of the presenee in the biliary passages or gall-blaclder of mueus, collections of which are the nuelei for the formation of calculi. Hence gall-stones may be the result of intlammation of the gall-bladder or ducts. It has been eonjectured that cholesterin may be deposited in consequence of the bile losing the faeulty of holding this principle in solution from a diminution of the tauro-cholie aeid. It is not improbable that an exeess of the salts of lime or of bile pigment may lead to the production of nuclei.

The treatment of a paroxysm of hepatic colie consists of palliative measures. Opiates are ealled for in doses proportionate to the intensity of the pain, either administered by the mouth or reetum, or hypodermically. The degree of suffering, in some eases, is such that the inhalation of chloroform is warrantable as a palliative measure. Warm anodyne fomentations may be applied to the seat of pain. The hot bath may aid in procuring relief. The local application of eold by means of a bladder filled with pounded ice, has been found to be useful as a palliative measure. Remedies to palliate vomiting are frequently ealled for. Efferveseing draughts and the hydrocyanie aeid may be employed for this purpose. An alkaline remedy may be found useful, if, as is frequently the ease, the matters vomited show notable aeidity of the stomach. The foregoing palliative measures will alleviate the suffering, but it is not to be expected that they will procure eomplete relief.

Emeties and eathartics have been advocated uuder the notion that they aid in the passage of the stone. It is irrational to suppose that they have this effeet, and elinieal observation does not furnish evidence of their value. 'The passage of the stone is mainly due to the accumulation of bile behind it after it enters the common duct, conjoined with contraction of the muscular eoat of the gall-bladder. Exeiting the movements of the stomach and intestines by emeties and eatharties, if any effect be exerterl, is as likely to retard as to favor the onward passage of the stone. Nothing, in fact, ean be done by means of these or other remedies to expedite its passage. All that the physician can do is to palliate suffering until the stone is propelled into the intestinal canal, supporting the strength, if need be, by alcoholic stimulants. If, however, the distended gall-bladder form a tumor which is appreciable by the touch, an effort may be made, by pressure with the fingers, to propel the bile in the ducts, and thus aid in the removal of the obstruction.

It would be desirable, if possible, to effeet the solution of gall-stones within the gall-bladder. The alkaline carbonates have been given for this purpose; and the treatment for this end, known in France as Durande's method, consisting of the administration of sulphuric ether and the spirits of turpentine (3 parts of the former to 2 parts of the latter), was for some time in vogue. Dr. T. H. Buckler' has proposed the use of chloroform in view of the solvency of cholesterin in this liquid. He has reported a ease in which an indurated tumor felt through the walls of the abdomen directly over the inferior margin of the liver disappeared in five days under the use of chloroform given in teaspoonful doses after each meal. It was concluded that the tumor was the gall-bladder distended with calculi; but

1 American Journal of Medical Sciences, July 1867, p. 51. 
there is room for doubt as to the correctness of this diagnosis. It seems hardly probable that any remedies can be introduced into the system so as to enter into the composition of the bile largely enough to dissolve the cholesterin of which mainly biliary calculi are composed.

The occurrence of gall-stones cannot be anticipated, but, being known, it is, of course, desirable to prevent the formation of others. The circumstances, however, immediately concerned in their procluction, are not known; hence, there is no special course of treatment rationally indicated, and clinical experience is not to be relied upon, since the success of preventive measures cannot be proven. It would seem that sedentary habits predispose to them. A change, in this respect, is therefore advisable. Beyond this, a simple, wholesome diet, and measures having reference to the general health, constitute all that ean be done, with our present knowledge, in the way of prophylaxis. Dr. Buckler suggests the hydrated succinate of the peroxide of iron in cases in which gall-stones are known to have been passed, the object being to prevent their further formation; this remedy, as he supposes, controlling the fatty or cholesteric diathesis.'

Gall-stones of sufficient size to occasion intestinal obstruction, are sometimes found in the alimentary canal. These escape from the gall-bladder, not through the biliary passages, but by ulceration, the walls of the gallbladder becoming adherent to the duodenum, or some other portion of the intestine. Dr. Henry F. Walker has reported an interesting case in which a gall-stone presenting the form of an elongated gall-bladder, measuring in the long diameter $3 \frac{1}{2}$, and in the short diameter $1 \frac{1}{4}$ inches, was removed from the rectum. The previous history, in this case, showed peritonitis, which probably denoted the time when the stone was making its way from the gall-bladder into the intestinal cavity, and, afterward, intestinal obstruction with alarming symptoms. These symptoms suddenly ceased, the probable explanation being that the stone passed, at that time, from the small into the large intestine. ${ }^{8}$

A patieut who consulted me, having suffered much from the passage of gall-stones, at one time took, for a number of days, a pint or more of olive-oil daily, at the suggestion of some one who assured him that,in this way, he had gotten rid of a large number of stones withont pain. The patient stated that, under the use of this remedy, he discharged from the bowels an immense number of small hard bodies, which were supposed to be-biliary caleuli. These are not scybalæ, that is, masses of fecal matter, but they are composed of a concrete form of fatty matter like the bodies passed in some of the cases of fatty diarrhœa which have been reported. A case is quoted by Dunglison, in which olive-oil, taken abundantly for the relief of pains attributed to gall-stones, was followed by the discharge of fatty matter of a globular form, varying in size from that of a small pea to that of a moderate grape, of sufficient consistence to bear being cut with a knife like soft wax. ${ }^{\mathbf{s}}$

1 This remedy is given by Dr. Buckler as follows: Hydrated succinate of the

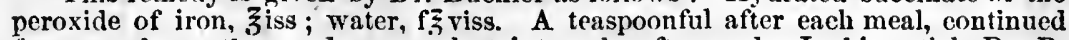
for several months; and resumed at intervals afterward. In his article Dr. B. stated that at that time (1867) the salt was prepared only by Dr. David Stewart and his brother, J. V. D. Stewart, of Baltimore.

2 Vide New York Med. Record, Nov. 15, 1871.

8 Dunglison's Practice of Medicine, vol. i. 135, 1842.

The student is referred to "A Treatise on Gall-Stones," by 'J. L. W. Thudicum, M.D., London, 1863, for a full consideration of their chemistry, pathology, and treatment. Vide, also, Murchison's Clinical Leetures on Diseases of the Liver, etc. 


\section{DIARRHEA.}

The term diarrhœa is used to denote morbid frequency of the dejections which are, also, liquid or morbidly soft, and often otherwise altered in character. Diarrhoea is a symptom of inflammation of the large and small intestine, and of certain structural lesions, such as those incident to tuberculosis of the intestine and to typhoid fever. It is a functional affection when it occurs independently of inflammation or any appreciable lesion of structure. It is an element of other functional affections, viz., sporadic cholera, dyspepsia, and certain cases of colic. As a functional affection, it may be either transient or chronic.

Writers have made numerous varieties of diarrhœa, based on diversities as regards the character of the dejections. It is sufficient to enumerate the more important of these diversities without considering each under a distinct head, as constituting a separate variety of the affection. The following division answers all practical purposes:-

A diarrhoea is said to be fecal, feculent, stercoraceous, or simple, when the dejections consist of the feces not much changed in character, but simply morbidly soft or liquid. This form of diarrhaea is represented by the operation of cathartics which increase the peristaltic movements and give rise to a certain amount of transudation. The dejections in this form of diarrhœa have the normal fecal odor. The dejections are called bilious when, from their yellow or green color, bile is supposed to be present in larger quantity than usual. Diarrhcea of this kind is represented by the operation of calomel in cathartic doses. 'The yellow color of the discharges, however, which characterizes the so-called bilious stools, is due to secretions from the mucous membrane of the large intestine, inasmuch as the contents of the small intestine do not present this color. The green color which has been considered so characteristic of the action of mercury upon the liver, according to Thudicum, is due to the presence of the subsulphide of mercury.' Robin, however, states that the microscope shows the coloring matter to be biliverdin. ${ }^{2}$ Diarrhoea is serous or watery, when there is an abundant discharge of liquid, that is, when there is copious transudation or enterorrhcea. This is represented by the operation of hydragogue cathartics, such as elaterium, jalap, and the bi-tartrate of potassa. The dejections are called lienteric when they contain undigested aliment. This form of diarrhoea is met with more especially in children. A diarrhoa is catarrhal or dysenteric, when mucus is apparent in the dejections. An abnormal amount of mucus may be secreted from irritation alone, without inflammation, but if the mucus be abundant, it denotes dysentery. This form of diarrhœa is represented by the operation of drastic cathartics, such as croton oil. Finally, in a curious and extremely rare form, called adipose or fatty diarrhoea, free fat or oil, in more or less abundance, is found in the evacuations. This last form claims some distinet consideration.

In cases of adipose or fatty diarrhœa, the fat is generally liquid when passed, resembling melted butter or grease, having, when cold, the consistence of butter, beef tallow, or wax. In some cases it is passed in the form of globular concretions, of a waxy consistency, varying in size, which melt with heat and burn like tallow. The fat is often passed by itself, and is apt to be passed involuntarily in a small quantity at a time. When passed with the feces, it separates and collects on the surface. It has usually an extremely offensive odor. The quantity passed per diem, 
in cases which have been reported, varies from two or three ounces to half a pound or more.

Assuming all the fat passed from the bowels to have been ingested as fat, the rationale involves simply the non-digestion of this alimentary constituent. But in some reported cases it is stated that the quantity of fat passed was not affected by the amount of fatty food. In a case reported by Dr. W. L. Wells, the patient abstained for several weeks from fatty food, and, as far as convenient, from articles convertible into fat, without any diminution in the quantily of fat passed, nor was the latter affected by indulging freely in fitty food. ${ }^{1}$ In two cases reported by Bright, no change was produced by excluding, as far as possible, from the diet fatty articles. Further and more precise observations with respect to this point are desirable. That the non-digestion of fat is, in a great measure, if not exclusively, involved in the pathological explanation, is not to be doubted. Late physiological researches have shown that the pancreatic secretion, together with the bile and intestinal juice, effects the digestion of fat, and hence we are to look to the sources of these fluids in tracing the causation of fatty diarrhœa. It is an interesting fact that Richard Bright, long before the late discovery of the particular function of the pancreas by Bernard, was led, by an analysis of three cases which came under his observation, to consider fatty diarrhoa as a symptom of disease of the pancreas. In these three cases, the only lesions found after death which were common to all were of the pancreas, duodenum, and liver. He excluded the lesions in the two latter situations because they occur in these organs so often withort fatty diarrhœa, but he admits that disease of the pancreas is not always accompanied by this symptom. ${ }^{2}$ The pancreas has been found diseased in a large majority of the reported fatal cases in which this organ was examined after death. Of twenty-five cases tabulated by Dr. John H. Griscom, ${ }^{3}$ in ten the bodies were examined after death; of these ten cases, in eight the pancreas was diseased, and in the remaining two cases the absence of disease of the pancreas is not stated. Yet fatty diarrhœa occurs in only a small proportion of the cases of disease of the pancreas. Thus, it was observed in only three of thirty-seven cases of cancer affecting this organ tabulated by Dr. Da Costa. ${ }^{4}$ Hence, it is to be inferred that, in connection with disease of the pancreas, lesions or morbid conditions affecting the liquids, other than the pancreatic secretion, concerned in the digestion of fat, are generally involved. Of the fatal cases among those tabulated by Griscom in which the morbid appearances were observed, the liver in several was either diseased or its ducts engorged; but in some of the cases this organ appeared to be healthy. The fact of complete recovery taking place in a certain proportion of cases, goes to show that the non-digestion of fat may occur as a functional disorder.

A larger number of cases than are as yet available are required in order to establish the clinical history, as well as the causation and pathological relations, of this form of diarrhoa. Jaundice was noted in several (six) of Griscom's cases. Diabetes mellitus co-existed in two cases. Hemorrhage

1 New York Medical Times, February, 1854.

2 Cases and Observations Connected with the Disease of the Pancreas and Duodenum, by Richard Bright, M.D., Medico Chirurg. Transactions, vol, xviii. Vide review in British and Foreign Medico-Chirurgical Review, No. for July, 1853.

3 Transactions of the American Medical Association for 1863.

- On the Morbid Anatomy and Symptoms of Cancer of the Pancreas, by J. Da Costa, M. D. Extracted from the Proceedings of the Pathological Society of Philadelphia, 1858. 
from the bowels, vomiting, pains in the abdomen sometimes resembling those caused by the passage of gall-stones, are among the symptoms noted in these cases. The duration of the affection varies much in different cases. In one case it had existed at frequent intervals for six years; in another case for two years; and in one case it lisappeared after a few weeks. It is evident that the prognosis is to be based on circumstances other than the discharge of fat, viz., enlargement of the pancreas and liver, persisting jaundice, progressive emaciation, etc.; in other words, the danger depends on the lesions or morbid conditions on which the discharge of fat may be dependent, or with which it is associated. Of the 25 cases colleeted by Griscom, 14 died, 8 recovered, and in 3 the termination was not noted.

The treatment inust have reference more to the circumstances connected with the fatty diarrhoea than to the latter. With our present knowledge, palliative measures according to the symptoms in individual cases, together with measures to improve digestion and invigorate the general health, will comprise the treatment. In one of Griscom's cases recovery took place under the use of olive oil in large quantity; and Watson states that a case was successfully treated by Elliotson in the same way. In a case observed by Griscom at the New York Hospital, the discharge of fat invariably ceased whenever the patient took from six to eiglit ounces of whiskey per diem. In the case reported by Dr. Wells, to which reference has been made, the patient, having had a daily discharge of three gills of fat for two years or more, recovered in a few weeks after giving up the occupation of a carpenter and adopting the life of a farmer.

Exclusive of the form just consiclered, diarrlıea involves different pathological clements, viz., increased peristaltic movements, increased transudation or flux, increased secretion from the mucous follicles, and perhaps, incre:sed flow of bile. The predominance of these elements, generally gives to the discharges the diversities which are observed in different cases. If the diarrhœa be purely functional, of course there is no inflammation of the mucous membrane. It is customary to express the morbid condition of the membrane by the term irritation. Practically, however, it must be confessed, it is not always easy to determine whether diarrhoa be purely functional or dependent on subacute inflammation. But, with reference to the treatment, to determine this point is not of great importance. It is not improbable that, of the cases considered as functional, in a eertain proportion there is a slight grade of inflammation, such as exists in coryza or a mild bronchitis. In making the diagnosis, acute inflammation is to be excluded by the absence of diagnostic symptoms, viz., pain, tenderness, febrile movement, anorexia. So, also, lesions of the mucous membrane are to be exclucled. In cases of chronic diarrhoa this is not always easy. Lesions are to be suspected if the diarrhoe follow an attack of dysentery or enteritis ; if it persist in spite of judicions management; if blood be sometimes present in the dejections, and if tubercles exist in the lungs. In the latter case, tuberculosis of the intestine is to be suspeeted; and, on the other hand, persistent or frequently recurring diarrhoe, conjoined with cough, be the latter never so slight, shouli excite suspicion of pulmonary tuberculosis.

Transient diarrhœa, due to arrested or defective intestinal digestion, is of frequent occurrence. With the diarrhœa which follows over-indulgence at the table, or the ingestion of certain articles of food, every one is practically familiar. This is the diarrhce crapulosa of the old writers. It may proceed from excesses in quantity, or from particular kinds of foor, the indigestibility of the latter, perhaps, showing an idiosynerasy of the person affected ; thus, ices, saluds, shell-fish, etc., in some persons always, 
and occasionally in all, give rise to transient diarrhœa. The undigested aliment, passing into the large intestine, having undergone chemical changes which the processes of digestion in health prevent, produces irritation, and acts like a cathartic remedy. Frequently the diarrhœa is preceded and accompanied by griping pains, and it may be, under these circumstances, an element of another functional affection, viz., colic. These attacks of diarrhœr cease spontaneously, as a rule, after the expulsion of the undigested matter, and they are rarely considered of sufficient importance to require medical advice.

An arrest of intestinal digestion may take place from causes other than errors of diet, and give rise to diarrhœea. A strong mental emotion may have this effect. I was present at an operation for hernia, when the surgeon, from the mental anxiety incident to his sense of responsibility, was obliged to relinquish the scalpel, and precipitately retire to evacuate the bowels. A gentleman in business receiving, suddenly, unexpected information which led him to know that he was a bankrupt, was immediately seized with diarrhœa. In like manner diarrhœa may arise from an arrest of digestion from an exposure to cold or over-exertion. In these cases the mechanism is the same as when the diarrhœa depends on dietetic errors, the undigested aliment in the large intestine acting like a cathartic.

Diarrhoa, not transient, but continuing more or less, perhaps becoming chronic or recurring at short intervals, also proceeds, in a large proportion of cases, from indigestion. Habitual or frequent indigestion, especially intestinal indigestion, leads to diarrhœa in the same way as when it is transient, viz., the undigested aliment undergoes chemical changes and causes either irritation or sub-acute inflammation of the large intestine. The dejections, as in transient diarrhœa, may be feculent, bilious, mucous, lienteric, or, more rarely, serous. The diarrhœa in these cases is, in fact, an element or symptom of dyspepsia, but not infrequently, the disorder of digestion being confined mainly to the small intestine, dyspeptic ailments, aside from the diarrhœa, are not marked. The frequent or habitual passage of undigested aliment into the large intestine leads to a persisting state of irritation in the latter, or perhaps, in some cases, to a low grade of inflammation. The indigestion thus giving rise to diarrhœa may be produced and kept up by various causes independent of excess or errors of diet; in fact, the causes are those of dyspepsia, mental anxiety often playing an important part in the causation.

It has been customary to attribute diarrhœa, in certain cases, to an excess or a vitiated quality of bile. This causation is inferred when the dejections are notably bilious. This view is not improbable, although not based on positive knowledge. The bile may not be reabsorbed from the alimentary canal, as in health, and hence accumulates in excess, without a morbid increase in the secretion; it may be secreted in undue quantity, or it may have a morbidly irritating property.

Diarrhœa is most apt to occur during the summer season. This may be, in part, explained by the larger proportion of fruit and vegetables in the ingesta during this season, and by the effect of heat in weakening digestion. It may also in part be due to a more abundant secretion, or to a lessened absorption, of bile. It is a much more frequent affection in tropical than in cold or temperate climates. It is apt to occur in travelling, especially in the summer season. Its occurrence is generally attributed to the water drank, but a more rational explanation is to refer it to indigestion caused by dietetic errors, over-exertion, and disturbance of the regular habits of life. Children are far more subject to diarrhœa than adults, especially during dentition, and, in children, the dejections 
are often manifestly lienteric, showing its dependence on indigestion. It is the most prominent element of the affection known as cholera infintum, or, popularly, in this country, as summer complaint. Among soldiers, especially in campaigns during the summer season, or in warn climates, diarrhoa is extremely common, attributable to irregular habits as regards diet, conjoined with the exposure and fatigue incident to active service. Diarrhcea occurs in persons exhausted from the want of food or other deprivations, and in females enfeebled and anæmic from protracted lacta- tion, due in these cases to intestinal indigestion. The same explanation, probably, applies to the diarrhœa which occurs at a late period in cases of pulmonary tuberculosis, and other chronic affections, when not depend. ent on intestinal lesions. Under these cireumstanees it has been called colliquative diarrhsea.

The aceumulation of urea in the blood, as a consequence of renal disense, occasions diarrhoea. 'The diarrhœa and vomiting which oceur under these circumstances, appear to be for the purpose of eliminating, vicariously, urea, and are, therefore, conservative. They oceur when the kidneys are removed in inferior animals, and, as shown by the experiments of Bernard and Barriswill, the liquid transudation into the alimentary canal contains either urea or the earbonate of ammonia. The dejections in uræmic diarrhnea are serous or watery.

Another pathological condition, occasionally giving rise to diarrhoe, is congestion of the portal vessels when the eireulation in this portion of the vascular system is obstructed by hepatic disease. It thus is a symptom, in some cases, of eirrhosis of the liver, and sometimes appears to limit the dropsical effusion into the peritoneal sac, which belongs to the history of that disease. The diarrhoea, under these circumstances, is serous, transudation taking place from the pressure of the blood within the congested veins.

Crapulous diarrhœa, or transient diarrhœa due to indigestion, in general calls for little or no treatment. If the contents of the large intestine be not spontaneously expelled, an evacuant remedy may be given. A saline purgative is best adapted to this end. The propriety of this measure is to be determined by ascertaining the quantity and character of the dejeetions which have occurred. If these have been abuntlant and feculent, a eathartic is not required. If, after the bowels have been freely evacuated, diarrhœa, pain, or uneasiness continue, the irritation may be quieted by a mild anodyne, for example, a drachm or two of the camphorated tineture of opium, for an adult, in chalk mixture, a grain of opium, a sixth or a quarter of a grain of a salt of morphia in mint water, or five grains of Dover's powder combined with two or three grains of aromatic powder. The remedy chosen is to be repeated after six or eight hours, if required. The diet for a day or two should be simple and somewhat restricted. Subsequently a laxative may be required if the bowels do not act spontaneously. The treatment of cases of diarrhoa tending to continuance, or frequently recurring and dependent on indigestion, embraces, in the first - place, remedies to relieve the irritation of the large intestine. Opium in some form may be used for this purpose, but only temporarily, because its continued use tends to impair still more digestion. A mild purgative may often be premised with advantage, for frequent and loose dejections are not incompatible with retention of hardened feces, in fact, with constipation. The carbonate or subnitrate of bismuth is an excellent remedy to relieve intestinal irritation. It is often effective, and is not open to the objections of opium. It should be given in doses of from a 
scruple to half a drachm to adult patients. If not alone effective, a small quantity of a salt of morphia may be added to it. Of a variety of remedies tried in cases of chronic diarrhoea among soldiers returning from the campaigns in Virginia in the summers of 1862 and 1863, bismuth, in my hands, proved the most effeetive, and this, I believe, was the result of the experience of others. Opiates are sometimes most effective, and least objectionable, when given per enema. Pepsin or the rennet-wine is sometimes useful temporarily as a means of artificial digestion, given as already recommended in cases of dyspepsia.

This part of the treatment, however, is merely palliative. The curative treatment embraces regulation of diet and measures to render digestion complete. The diet, in quantity and quality, should be adapted to the digestive powers. Chickens, eggs, and tender meats plainly cooked, are most likely to be digested, but in some cases a milk and farinaceous diet is found to be preferable. Experience in individual cases is to be the guide. Crude vegetables are to be interdicter, but ripe fruits, in moderation, may frequently be taken without incourenience and with advantage. A moderate quantity of food, taken at short intervals, is generally advisable, rather tlian a full meal once or twice daily. In short, the treatment, as regards diet, is essentially that of dyspepsia. This statement will apply alike to hygienic measures relating to exercise, clothing, and mental recreation. A sea-voyage, a visit or removal from the city to the country, or the change of a warm, variable, humid, relaxing climate for an atmosphere cool, uniform, dry, and bracing, will be likely to prove highly beneficial, if not curative, in eases which resist dietetic and medicinal treatment. The efficacy of these measures is strikingly illustrated in cases of the so-called summer-complaint of children.

The remedies, other th:in those merely palliative, which are useful in the cases of diarrhœa under consideration, belong among the tonies and astringents; the latter may be both palliative and curative. Of the various vegetable astringents, krameria, hæmatoxylon, kino, catechu, and rubus villosus are eligible articles. The tannic acid, if well borne by the stomach, is sometimes efficient. As purely tonic remedies, quinia or the non-officinal preparations of Calisaya bark, of late in vogue, and other bitter infusions, are often useful. Preparations of iron are especially useful when the patient is anæmic, as in cases of diarrhcea occurring in females during lactation. As a parely tonic remedy, the tincture of the chloride of iron is one of the best of the chalybeates. The persulphate or pernitrate of iron is sometimes highly efficacious as an astringent, as well as a tonic remedy. Of mineral tonics and astringents, the acetate of lead, nitrate of silver, and the sulphate of copper have been found useful. The carbonate or the subnitrate of bismuth is of great value in cbronic diarrhœa, not only as a palliative but a curative remedy, given in large doses, namely, from a scruple to a drachm, three or four times daily, sometimes in combination with small doses of a salt of morphia, as recommended above, in connection with palliative measures of treatment. The mineral acids are efficacious in some cases. The preparation known as Hope's mixture has been much in use. This mixture is composed of four drops of nitric acid and from forty to sixty drops of laudanum in four ounces of camphor water, a tablespoonful to be taken every two, three, or four hours. In rebellious cases of chronic diarrhœa, the numerous remedies which have been named are to be given in succession, and in various combinations. The curative and palliative remedies are to be combined to meet the indications in individual cases. 
Diarrhœa dependent on uremia, and on cirrhosis of the liver, does not admit of curative treatment, and, being conservative, is only to be kept within certain limits by palliative remedies.

\section{ENTERORRHAGIA.}

Hemorrhage into the intestinal canal is properly called Enterorrhagia. The term meliena has been used to denote dark-colored or black dejections consisting of blood which has passed from the stomach into the intestinal canal. Partial digestion of the blood, or the action upon it of the gastric and intestinal secretions, renders it tarry or pitch-like in appearance, and generally very offensive to the smell. Dejections of this character are apt to follow gastrorrhagia. The term melxena has been also applied to cases of gastrorrhagia in which the blood vomited presents a similis appearance. Moreover, the term has been loosely applied to denote dark-colored or black evacuations, not eonsisting of blood. 'The term is one of those which it is desirable should become obsolete. Cases in which blood evacuated from the bowels comes from the stomach, or from any of the abdominal viscera other than the intestinal canal, are not cases of enterorrhagia. Nor are eases of hemorrhoidal or other hemorrhages occurring near the anus, properly embraced under this head. The souree of the hemorrhage must be the nucous membrane between the stomach and rectum. The tarry or pitch-like appearance of blood in the stools is evidence of its gastric source. But this appearance is not positive proof that the matter evacuated is blood. It is probably blood if the stools have been preceded by the vomiting of blood, but, in some eases of gastrorrhagia, vomiting does not take place, all the blood passing into the intestinal canal. In doubtful cases, clilution in water of some of the matter evacuated, renders the appearanee of blood more evident, or recourse may be had to the microscope. When the source of hemorrhage is the rectum, the blood is fresh in appearance, and is passed alone, in greater or less abundance, either after a fecal evacuation, or with strsining efforts to procure a stool. Examination with the eye or touch, will show the existence generally of hemorrhoids, hut, in some cases, other local affections, such as ulcers, carcinoma, a polypus, or invagination.

In cases of enterorrhagia, the hemorrhage takes place in different situations along the tract of the sinall and large intestine. The blood is less changed in appearance the nearer the rectum its source, and the more quickly it is evacuated. If the hemorrhage be at the upper part of the intestinal tube, and the blood pass slowly along the tube, it becomes dark or blackish, its appearance approximating to that of the melienic stools in cases of gastrorrhagia, and there may be a smilar difficulty in determining whether the appearance be due to the presence of blood. 'The quantity of blood passed in different cases of enterorrhagia varies. It is frequently large, amounting, in some eases, to several pints and even quarts. Evacuitions may consist wholly of blood, or the blood is mixed with feeal matter. The blood may be entirely liquid, or the evacuations contain clots in more or less abundance.

Intestinal, like gastric hemorrhage, has rarely any claim to be regarded as an individual affection. Like gastric hemorihage, it is incidental to a variety of morbid conditions. It is an important occasional event in typhoid fever, and will be referred to in treating of that disease. It enters into the characteristic dejections of acute dysentery. It is an effect of ulceration of the bowels in chronic dysentery. It occurs in cases of carcinoma of the bowels. It is a rare occurrence in tuberculous uleeration of the 
small or large intestine. In may be a symptom in simple enteritis. It belongs to the natural history of scorbutus, purpura hemorihagica, and yellow fever. Like gastric hemorrhage, it may be vicarious of menstruation. It is not an uncommon effect of the portal congestion occasioned by cirrhosis of the liver. It occurs, however, when not connected with these or any other discoverable morbid conditions. This was exemplified in a case, under my observation, of an unmarried female aged about 40 , who was attacked, when apparently in perfect health, with griping pains in the abdomen, followed by loose evacuations containing blood in large quantity. These evacuations recurred for several days. There was no affection of the rectum, the menses were not suppressed, and the hemorrhage could not be traced to any of the affections of which it is usually a symptom. The enterorrhagia ceased after several days, and the patient recovered her usual health. This patient had several recurrences of the hemorrhage, in the intervals apparently being in perfect health. I have met with a few additional examples of enterorrhagia not connected with any of the several causative conditions which exist in the larger proportion of cases. While, therefore, the chances are that some of these conditions exist, the occurrence of hemorrhage is not proof of the existence of any of them. The discharge of blood in the cases just referred to is generally preceded and accompanied by abdominal pains, probably in part due to the congestion which it is to be presumed precedes the hemorrhage, but partly referable to the presence of the blood within the intestinal canal. Other symptoms are attributable to the loss of blood, and are marked in proportion to the amount of hemorrhage, such as debility, pallor, feebleness of the pulse, perspiration, sense of faintmess, etc. The loss of blood may be sufficiently great and rapid to occasion sudden death, but this is rare. The continuance of repetitions of the hemorrhage may lead to a degree of exhaustion and anæemia from which recovery is difficult, and a fatal result may take place by slow asthenia. In general, however, if the hemorrhage be not connected with affections or morbid conditions which involve danger, the prognosis is favorable. In cases of enterorrhagia, as in cases of gastrorrhagia, the amount of hemorrhage is not always represented by the quantity of blood contained in the evacuations. The general symptoms denoting loss of blood are to be taken into account. Hemorrhage sufficient to occasion fatal syncope may take place and no blood be discharged by stool. In such a case, the diagnosis of enterorrhagia is impracticable.

The treatment of the different affections or morbid conditions with which intcstinal hemorrhage is usually connected need not be here repeated. It will suffice to refer to measures having reference to the hemorrhage. These are essentially the same as in cases of gastric hemorrhage. Rest in the recumbent position and quietude of mind are important. The peristaltic movements are to be quieted by opium. Cold applications to the abdomen should be made if the arrest of the hemorrhage be urgently indicated. The most effective mode of applying cold is by means of a bladder or an India-rubber bag containing pounded ice. In cases in which this indication is less urgent, dry cupping, sinapisms, and warm stimulating pediluvia will suffice without the application of cold. Food and drinks should be taken cold. The diet should be restricted and bland. Purging is of doubtful propriety. The mineral acids are generally thought to be useful in cases of intestinal, as well as in gastric hemorrhage. The sulphuric acid lemonade has the advantage of being a grateful beverage. The various hemostatic remedies given in cases of gastrorrhagia, viz., gallic acid, acetate of lead, ergot, the astringent preparations of iron, etc., are to be resorted to in proportion to the importance of arresting the hemorrhage. 


\section{H A P T E R VII.}

FUNCTIONAL AFFE(TIONS OF TIIE STOMACH AND INTESTINES.-

(CoNTINUED.)

Constipation. Intestinal Colic. Enteralgia. Lead Colic.

CONTINUING to consider the functional affections of the alimentary $\checkmark$ canal, this chapter will be devoted to constipation, intestinal colic, enteralgia, and the so-called colic from lead.

\section{CONSTIPATION.}

The terms constipation and costiveness are commonly used as synonyms, denoting insufficiency of evacuations from the bowels. The latter term is sometimes used to denote a less degree of insufficiency than the former, the number of dejections being normal, but the quantity deficient, and the act of defecation labored. The term obstipation has been alrearly defined to denote a greater anount of difliculty than eonstipation, that is, obstruction of the bowels, either as a functional disorder or dependent on various lesions which have been considered in a preceding chapter. Constipation exists as a functional disorder, and it is incidental to various affections. As a functional disorder it is extremely frequent, and, although not a serious affection, it claims attention on account of the inconvenience which it occasions, its importance as leading to other ailments, and the difficulty of its removal unless it be correctly understood and managed.

'The affection is seated in the large intestine. The anatomical arrangements of this portion of the alimentary canal show it to be intended to serve as a temporary lepot for fecal matter, thus providing against the need of frequent acts of defecation. The contents of the alimentary canal are propelled more slowly along the large tlan in the small intestine, first, because the circumference of the former is larger in proportion to its muscular power; second, in the ascending colon the contents are propelled for a considerable space in opposition to gravitation, and, also, for a smaller space, at the sigmoid flexure; and, third, the liquid portion of the contents is absorbed in their passage through the small intestine. Experience shows that one free evacuation from the bowels daily is the rule in health. But this rule is not without exceptions. Some persons have, habitually, two or three evacuations daily, and, on the other hand, some have an evacuation regularly every second or third day, without any of the inconveniences of constipation; in fact, persons of the latter habit are apt to experience discomfort if, temporarily, evacuations take place daily. In determining, then, the existence of this affection, the habit in health is, of course, to be taken into account. In some cases, the act of defecation is delayed for one, two, or three days after the period when it should have taken place, but the evacuation, when it does occur, is ample. In other cases, the act occurs daily, but it is incomplete, and in these cases the act is usually labored and painful, the feces being dry, compact, and 
hard. The affection may be occasional and transient, or it may be habitual.

Constipation gives rise to various local morbid effects, such as a feeling of pressure or weight in the perinæum, a sense of abdominal distension or uneasiness, flatuleney, diarrhœa, and colic pains. Hæmorrhoids are often attributable to this affection. It gives rise, also, to pain in the head, dulness of the mind, flushing of the face, palpitation, and general malaise. If the act of defecation require violent straining efforts, these occasion, sometimes, hemorrhage into the brain, and hernial protrusions. The contractility of the museular tunic of the large intestine may be permanently impaired by long-coutinued distension, so that the aflection is necessarily permanent.

Occasional constipation is not generally considered of sufficient importance to require medical advice; persons resort, of their own aceord, to a purgative or an enema. It is when the affection has become habitnal or chronie, that cases come under the cognizance of the physician. And, in order to understand the pathological character of the affection in these cases, and its eausation, certain points pertaining to the function of defecation in health are to be borne in mind. In its normal condition, the rectum is, for the most part, empty, as shown by the researches of O'Beirne.' This portion of the large intestine is endowed with a peculiar sensibility which, in health, gives notice of the presence of feces, and occasions the desire to defecate. The ability to perform the act involves a certain contractile power in the large intestine, and also in the abdominal and other muscles which co-operate in the performance of the act. In habitual constipation, the contractile power of the intestine is impaired by distension. The musenlar tunic is more or less paralyzed from the accumnlation of the intestinal contents, in the same way as the bladder becomes paralyzed from over-distension. The accunulation giving rise to the distension may be owing to the large quantity of excrement, but it is generally a result of habitual negleet of the calls of nature. The desire to defecate is resisted, or, the mind being pre-occupied, the call is unheeded, and the act is postponed, until, at length, the sensibility no longer gives notice of fecal accumulation ; hence, the accumulation goes on, the rectum and other portions of the large intestine become distended, and paralysis follows. This is the manner in which constipation, as a chronic affection, is produced in a large majority of cases. The burried performance of the act of defecation, the evacuation being, in consequence, incomplete, has measurably the same result as the neglect of the calls of nature. Instead of receiving adequate attention as important to health, it is considered an annoyance to be escaped from as quickly as possible. The uneomfortable provisions for defecation, especially in the country, contribute to constipation by rendering it disagreeable to devote to the act more time than is absolutely necessary.

Varions circumstances may contribute to this affection. The abdominal museles play an important part in the act of defecation. These muscles become weakened by obesity, and, in females, as a result of pregnancy. The muscular tunic of the intestine, and the auxiliary voluntary muscles concerned in defecation, lose more or less of their contractile power, in common with the whole muscular system, in anæmia, and other enfeebling conditions. Deficiency of bile and the intestinal secretions may enter into the cansation in some cases, but probably the importance of this source of constipation has been over-estimated; constipation is not always present

1 New Views of Defecation, etc. By James O'Beirne. Am. ed., 1834. 
when no bile enters the alimentary canal in certain cases of jaundice. 'The ingestion of purely nutritious food, leaving but little exerementitious residue, contributes to constipation. Sedentary habits are supposed to fivor the affection, but it is probable that other causes are generally more operative, and especially inattention to the ealls of nature. On the other hand, active exercise induces constipation by rendering assimilation more active, the liquid contents of the small intestine being more entirely absorbed. Loss of fluids by abundant perspiration or by diuresis is another cause. The change of habits, with, generally, an increased assimilation, incident to a sea-royage, as is well known, induces, in most cases, constipation.

Occasional constipation, if slight, is relieved by a laxative pill repeated, if necessary, or by a small quantity of Epsom or Rochelle salts dissolved in a tumbler of simple or carbonated water, and taken upon an empty stomach. 'The Congress or Kissingen water may be taken in lieu of the salts just named. But a preferable method, which will generally suffice, is to excite the action of the large intestine by a simple enema of cold water. By means of Davidson's syringe, the patient may resort to this measure without any difficulty. If the constipation be more than slight, a few grains of blue mass or a purgative pill may be taken at bedtime, and followed, if necessary, by a saline draught or an enema in the morning. Persons are apt to resort, without consulting physicians, to purgatives, under an impression that they are always harmless, and generally useful aside from the relief of constipation. Hence, it is common to take active purgatives for slight constipation, and, often, when nothing is requirer. If the constipation give rise to little or no inconvenience, it is better to wait for spontaneous relief, and a little delay will often show that medicinal interference was unnecessary.

'The management of habitual constipation often requires much care and perseveranee on the part of the physician and patient. The object is to secure regularity and sufficiency in the evacuations. The means which may be employed are various. They may be arranged into dietetical, medicinal, and mechanical. The dietetical means consist in using freely articles which leave, after digestion, a bulky residuum, viz., eabbage, lettuce, and the various vegetables known in this country as greens; or articles having a laxative property, sueh as molasses, prumes, figs, ete.; or articles with indigestible constituents which stimulate or irritate the alimentary canal, viz., bran bread, corn meal, groats, or cracked wheat. A diet consisting, in part, of the foregoing articles, will sometimes succeed in obviating liabitual constipation. Bnt, with regard to the choiee of this class of means, the following practical rule is to be adopted : they are not to be preferred if they disorder digestion or occasion more disturbance than medicines. More harm sometimes results from overloading the digestive organs with articles of diet difficult of digestion, or subjecting the canal to the irritation of unbolted flour, than the continuance of constipation would occasion. A tumbler of simple water, or of carbonated water, taken in the morning before breakfast, is sometimes efficacious.

The medicinal means are laxative remedies. With regard to these, a general rule is, the remedy chosen should be mild, and the quantity as small as will suffice for the object. Purgation is to be avoided. Some patients fall into the custom of allowing the constipation to continue for several days, and then resorting to a free purgative. The constipation is, of course, relieved for the time, but the constipated habit becomes more and more fixed by this course. Another general rule may be stated: If more than one small dose of laxative be required, the remedy is to be 
given in small doses repeated twice or thrice daily, rather than given in a single dose. In this way the object is effected with a less amount of medicinal impression, and there is less risk of purgation.

As regards the particular remedy, aloes is especially suited to the desired object. It may generally with advantage be combined in a pill with a tonic, either the sulphate of quinia or a preparation of iron, and a small quantity of hyoscyamus or belladonna may be added. A very small proportion of aloes, often a small fraction of a grain in each pill, will sufficc. 'The old preparation known as the elixir proprietatis (tineture of aloes and myrrh) is well suited to this object, a drachm given at bedtime, and repeated, if necessary, once or twice during the day. This preparation loses, with age, its disagreeable bitterness, and is not generally repulsive given with a little water and an abundance of sugar. A small piece of rhubarb taken repeatedly during the day, effects sometimes the object satisfactorily, and this remedy to many persons is not disagreeable. I have known a few drops of the tincture of colchicum, repeated shortly after each meal, to answer admirably. There are various modes of rendering the daily use of a laxative remedy not umpleasant to the taste. Prunes stewed in an infusion of sennat answer well, and are not unpalatable. The confection of senna and medicated figs are also suited to persons who take a pill or potion with repugnance. Troussean claimed for his favorite medicine, belladonna, that it acts as an efficient laxative in cases of habitual constipation. It has failed to have this action in my experience, and I have frequently made trial of it. Here, as in other instances, experience in individual cases is to be the guide. In the majority of cases in which medicinal means are relied upon, some form of the so-called dinner-pills, or, as they have been aptly termed, "peristaltic persuaders," will be found most convenient and satisfactory; and, for the fastidious, they may be silvered or sugar-coated. The white mustard-seed was formerly a popular remedy much in vogue, but this is objectionable on account of a liability to accumulate and become impacted within the intestine. Laxative remedies are not to be continued for a longer period than is required to secure an habitual action of the bowels. $\AA$ minute dose of strychnia or nux vomica, added to a laxative, is thought by many to render the operation of the latter more successful.

The mechanical means consist in the use of enemas and suppositories. The regular use of an enema of cold water, at the same hour daily, is a simple, and sometimes an effective measure. This measure, however, is better suited to occasional than to habitual constipation. Habitually resorted to, it is apt to fail after a time ; the rectum becoming accustomed, as it were, to the stimulus of distension, it ceases to excite the peristaltic movements. In some cases a suppository of soap answers the purpose of provoking a regular and sufficient evacuation. The cocoa-nut butter may be used for this purpose. Common molasses candy answers equally well, an oval mass of the size of a pigeon's egg being introduced within the rectum. This, however, is only a temporary expedient, ceasing usually to be effective after a time.

The most important part of the management in cases of habitual constipation is not embraced in the foregoing measures. It is the adoption of a rule to solicit an evacuation at the same hour daily. The importance of this rule is to be enforced, but, of course, its success will depend on the perseverance of the patient. The time of the day most convenient for the act of defecation is to be selected, and, in general, the most favorable time is in the morning after breakfast. At the time fixed upon, the patient should devote a reasonable period to the function of defecation, but 
without persisting in violent fruitless efforts. This should be considered in the light of a duty, not to be omitted a single day except from necessity. It may be long before the desired object is accomplished, but, sooner or later, with the aid of some of the means which have been indicated, the desire will be felt at the appointed hour, and the ability to defecate at that time will be acquired in the great majority of cases. It is impossible to secure regularity and sufficiency of the evacuations without perseverance in this part of the management. The management also includes a rule never to neglect or postpone the call to defecate, when it occurs either after or before the time for the habitual performance of the act of defecation. The importance of waiting for a certain period after the contents of the rectum have been expelled is to be enforced. Often the expulsion of the contents of the rectum is immediately followed by the passage from the colon into the rectum of fecal matter which, remaining, blunts the sensibility of the bowel and renders it accustomed to distension. The objects in the management are to be understood and kept in view, viz., 1st, To preserve or restore the normal animal sensibility of the rectum, by which the presence of fecal matter is felt; and, $2 d$, To prevent paralysis from distension, and to regain the muscular power which has been lost from this cause.

If this plan were early inculcated and carried out in health, habitual constipation would be as rare as it is now frequent. The prevention is not less sure than simple. The function may be brought fully under the control of habit. This fact should be generally understood, more especially in the training of girls, most of whom become affected with habitual constipation, and suffer from it all their lives. Unfortunately the existence of the function of defecation is too often ignored from notions of false delicacy, and the affection becomes established because parents and teachers are either themselves ignorant of this simple method of prevention, or consider the subject as belonging exclusively to the physician.

\section{INTESTINAL COLIC.}

The term colic, in its etymology, relates to the colon, but it is often applied to paroxysmal, spasmodic pain in other parts. Thus, the phrase hepatic colic is used to denote the pain caused by the passage of gall-stones, and nephritic colic the pain caused by the passage of renal calculi along the ureter. A painful affection of the uterus is sometimes called uterine colic. In the present nomenclature of diseases, there is no suffix or prefix which, joined to the name of a part, expresses a painful spasinodic affection of that part. The affection of the alimentary canal known as colic may not be seated exclusively in the colon. It is probable that the small, as well as the large, intestine is affected in certain cases of intestinal colic. As applied to the intestine, colic denotes pain occurring in paroxysms or in marked exacerbations, the pain of a character supposed to indicate spasm, that is, twisting, constricting, or griping. Colic pains are symptomatic of different inflammatory affections, viz., dysentery, enteritis, peritonitis, and of the various lesions which give rise to intestinal obstruction. They enter, also, into the functional affection known as cholera. As constituting an individual malady, colic, of course, is independent of inflammation or structural lesions, and is disconnected from any other functional affection.

An attack of colic is characterized by paroxysms or exacerbations of pain, varying much in severity in different cases, the pain being usually in the region of the umbilicus. The pain is frequently extremely severe; 
the patient bends the body forward with the thighs flexed, writhes, and tries a variety of positions, groaning or erying aloud from the intensity of suffering. The severe pain continues for a few moments, and then either ceases completely or abates, to return again with the same severity after an interval usually of brief duration. If left to continue without measures for relief, an attack may embrace a few paroxysms only, or it may last for several hours. An attack lasting for a short time, and ending in one or more free evacuations from the bowels, is so common as to be familiar to every one.

The abdomen, in attacks of colic, is frequently retracted, but, in some cases, it is more or less tympanitic. Generally, there is absence of tenderness, and firm pressure with the hands, or lying upon the belly, affords relief; but in some cases there is more or less soreness and tenderness on pressure, especially over the site of the excum. During the paroxysins of pain, the abdominal muscles are sometimes spasmodically affected, especially the rectus muscle, the sections of which are felt as hard bunches. Usually there is constipation. Nausea is sometimes present and occasionally vomiting. The pulse-is but little, or not at all, accelerated. 'The skin is cool, and, during the severity of pain, frequently bathed in perspiration.

In a case presenting the characters just deseribed, it is important to determine, as soon as practicable, the existence simply of a functional affection, that is, to differentiate colic from affections in which pains like those of colic oceur. The diagnosis is to be made by excluding these affections. Dysentery is readily exeluded by the absence of the dejeetions characteristic of that disease. Enteritis is excluded by the absence of the symptoms denoting inflammation. The pain, in these two affections, is rarely as violent as in most eases of colic; but in eases of acute peritonitis, the exacerbations of pain may equal in violence those of a severe attack of colie, and the error of confounding these two affections has been committed in not a few instances. The exclusion of peritonitis is to be based on the absence of notable tenderness over the abdomen, of rigidity of the abdominal walls, of frequency of the pulse, and of other general symptoms denoting a graver affection than colic. Invagination, internal hernia, and other obstructive lesions eannot always be at once excluded. As a rule, the paroxysmal pain occurring in connection with these lesions at the outset, and, indeed, all along, is less violent than in colic, and, after a time, the existence of something more than colic is shown by persisting pain, tenderness, vomiting of the so-called stercoraceous matter, sometimes tenesmus, frequency of the pulse, prostration, etc. The absence of these symptoms, after a time, warrants the exelusion of obstruetive lesions. The possibility that some obstructive lesion may exist is not to be lost sight of in cases which at first have the appearance of simple colic. The possibility of inguinal or femoral hernia is also to be borne in mind, and a careful examination with reference to this point is not to be omitted. The resemblance of labor-pains to those of colic is to be recollected. Pregnancy is sometimes concealed, and it has happened that the physician, supposing his patient to be suffering from eolic, has been taken by surprise at the cry of a newly-born infant under the bedelothes.

The several varieties of colic which writers have generally recognized do not require separate consideration. Some of these varieties rest on an insufficient basis. The colic has been called crapulous when it proceeds from indigestion. Every one is familiar with the attacks which are apt to follow over-indulgence at the table, ending, frequently in crapulous diarrhœa. A colic is called flatulent when accompanied with tympanites, 
or the expulsion of gas with relief. This variety is most apt to occur in infancy. The term bilious colic has been used when the attection is supposed to depend on hepatic derangement. 'This dependence is merely conjectural, and there is no more reason for continuing to append the word bilious to this affection, than to various others in which the coexistence of disorder of the liver is equally hypothetical. Colic has been called stercoraceous when attributable to the retention of feces; but the latter is perhaps oftener an effect than a cause of colic. In another variety called verminous, the colic is supposed to depend on the presence of worms, but it may fairly be doubted whether colic ever proceeds from this source. The varicty ealled saturnine or lead colic is properly a distinct alfection, and will be considered as such.

The morbic condition in eolic is supposed to be spasm. Its seat is, therefore, the muscular tunic of the small or large intestine. 'That this is the pathologieal character of the affection, is shown by the kind of pain, the constipation, together with the local symptoms, and the therapeutical measures which are found to be successful. It is not improbable, as supposed by Abercrombie, that, in certain cases, the spasm may be preceded by paralysis of a section of the intestiual tube, and consequent obstruction to the passage of its contents. Like other spasmodic alfections, it may be produced by causes which act either directly-upon the part affected, or indireetly through its nervous communications. The late Prof. Charles Hooker, of Yale College, in an interesting paper on intestinal auscultation, published in 1849 , states that, during an attack of colic, bolborygmal sounds indicating the peristaltic movements of the intestinal tube are suspended, and that the termination of the attack is preceded by a return of these sounds for some time before the complete relief of pain.

Attacks of colic are frequently, if not generally, attributable to the local action of the ingesta. They may arise from indigestion caused either by excess in quantity, or the indigestible quality of food. They not infrequently follow exposure to cold or fatiguing exertions, but these causes probably act by occasioning indigestion. Certain articles of diet, for example shell-fish, in some persons, give rise to attacks of colic in consequence of an idiosynerasy which is inexplicable. The museular tunic of the intestine is especially susceptible to spasm in infumey, and colic oceurs much oftener in early than in midlle and advanced life. A susceptibility to intestinal spasm belongs to some persons, constituting a predisposition to this affection. In the practice of every physician there are those who are subject to attaeks of colic more or less frequently. This predisposition may continue for a certain number of years, and then disappear. Persons with this predisposition often have an uneomfortable sensation of weight, coldness, or numbness in the abromen, with general languor and irritability, preceding the development of colic, and by means of these premonitions they are able to predict an impending attack.

Although attended with great, and, if not efficiently treated, often protracted sulfering, colic is not a dangerous affection. It may be doubted if, alone, it ever prove fatal. It is one of those violent functional affections from which recovery takes place rapidly. It has no tendeney to eventuate in inflammation or any other disease, but, in proportion to its duration, it is followed by fatigue or weakness, and more or less abdoininal soreness may remain for some time.

The object of treatment is the relief of spasm as indicated by the cessation of pain. Measures are to be directed to this object without reference to the cause of attack, or the existence of constipation. If, as is gene- 
rally the case, an attack have been induced by the presence of irritating ingesta, measures directed to the spasm should take precedence of evacuants; and if, as is frequently the case, the bowels were constipated at the time of the attack, the removal of this condition is a seconclary object. So long as spasm continues, there is a resistance to the action of cathartics, and to defer measures for the relief of pain until the bowels have been evacuated, is to prolong needlessly the sufferings of the patient.

In mild attacks, warmth to the abdomen and extremities, a little spirit not largely diluted with hot water, some aromatic stimulant, such as anise, ginger, cloves, mint, etc., or a few drops of chloroform, will suffice to procure relief. 'Twenty drops of chloroform, repeated, if necessary, at short intervals, will sometimes afford prompt relief in severe cases. If not, opium in some form is to be given to the extent of procuring freedom from pain. Administration per enema is to be preferred. A drachm of laudanum with the same quantity of the tincture of assafotida, in a small quantity of mucilage or starch, may be injected, with injunctions to the patien' to resist the desire to expel it, and, usually, in a short time, this desire passes off. If complete relief do not follow in the space of an hour, the enema may be repeated. If the suffering be extremely intense, clouble this quantity of laudanum, in the case of an adult, may be given in the first or second enema. If necessary, the enema may be repeated a second or third time. A similar plan is to be pursued as regards the doses and repetitions, if the opiate be given by the mouth. Hot fomentations or dry heat should be applied to the abdomen. Speedy success may be counted upon by pursuing this plan of treatment.

'The subsequent treatment is to be determined by circumstances relating to the abdomen. If no inconvenience be felt, measures to effect a movement of the bowels need not be employed for twenty-four or even fortyeight hours. Not infrequently, if the physician be not in haste to give a cathartic, a sufficient evacuation occurs spontaneously. If measures to effect a movement be required, a large simple enema may suffice; if not, a mild saline purgative may be given in divided doses, or castor-oil if this remedy be not offensive to the patient. The diet for a day or two should be simple and restricted.

The certainty with which colic yields to an efficient opiate treatment renders it one of the affections which exemplify very palpably the resounces of medicine. Some patients, however, suffer considerably from the aftereffects of the opium given, and this in a measure compromises the mutual satisfaction of the physician and patient in the success of the treatment. Persons who are unfortunately affected unpleasantly by most forms of opium are sometimes able to take certain preparations with less inconvenience than others. The experience of the patient on this point should be consulted.

\section{ENTERALGIA.}

The term enteralgia denotes a neuralgic affection referable to the intestines. It has, to the latter, the same relation as gastralgia to the stomach. Gastralgia and enteralgia may be associated. Enteralgia and colic are not infrequently confounded, nor is it always easy to distinguish, clinically, the one from the other. Enteralgia is characterized by pain within the abdomen, with intermissions or remissions, the pain having more or less intensity, but lacking the twisting or griping character of the pain in colic. It may be so severe as to lead to contortions and oral expressions of suffering. In females, enteralgia is an element of attacks of tympanites 
due to the secretion of gas, and is apt to be associated with hysterical phenomena.

The diagnosis involves the exclusion of the various affections from which colic is to be discriminated and the exclusion of the latter affection. Rheumatism of the abdominal walls is also to be excluded. According to Valleix, lumbo-abdominal neuralgia bears a close resemblanee, as regards the seat, etc., of pain, to enteralgia, but it is to be discriminated by the existence of tenderness in front at the umbilical or hypogastric region, and also at the points in the lumbar and lateral portions of the body, whereas, in enteralgia, tenderness on pressure is generally wanting.

The treatment involves the same principles as the treatment of gastralgia and other neuralgic affections.

\section{LEAD COLIC.}

The affection now commonly known as saturnine or lead colic has been described by writcrs, at different times and places, under a variety of names. Painter's colic, plumber's colic, colic of Poitou or colica pictonum, Devonshire colic, colic of Madrid, vegetable colic, rachialgia, dry bellyache, and other names which might be added, all relate to the same affection, which is one of the varied forms under which lead-poisoning is manifested. For the evidences of the correctness of this statement, the reader is referred to trcatises entering into the consideration of the affection more largely than is consistent with the scope of this work. The affection differs from ordinary colic as regards not only its causation and clinical history, but its pathological character; it is not a spasmodic, but a neuralgic affection, that is, a variety of enteralgia.

The affection is developed usually in a gradual manner, and is preceded by prodromic phenomena which are the general effects of lead-poisoning, viz., pallor due to anæmia, and frequently an icterode hue of the skin, a peculiar fetor of the breath and a metallic taste in the mouth, loss of appetite, constipation, pain in the limbs, more or less emaciation, and muscular debility. Pain in the abdomen is at first comparatively slight and progressively increases, becoming, at length, the chief ailment. The pain is oftenest referred to the region of the umbilicus, but sometimes to the epigastrium or hypogastrium, and it may be limited to other situations, or it may extend over the whole abdomen, but it is usually confined within a small space. 'The pain frequently shoots into the back, the genital organs, and in other directions. The pain is sometimes dull or aching, and, at other times, acute and lancinating. It varies, in different cascs, within wide limits, as regards intensity. In a mild form it merely occasions annoyance, like the pain in certain cases of gastralgia, but in a severe form the suffering is extreme, causing the patient to assume a variety of unnatural and fantastie positions, and to utter loud groans or cries, as in severe cases of gastralgia and ordinary colic. The abdomen may be more or less meteorized or tympanitic, but it is oftener depressed and resisting to pressure. The resistance to pressure is caused by tonic contraction of the abdominal muscles, and this, in some eases, is a marked feature of the affection. Pressure on the abdomen, if made gradually and over a contsiderable space, is generally well borne, and often affords relief; patients are apt to lie upon the belly, with a folded pillow, or some other substance, and sometimes the closed hand, placed under them over the seat of pain. Pressure, however, with the ends of the fingers or percussion of the abdomen may not be well borne. The bowels are constipated, the exceptions to this rule being infrequent, and the dejections frequently 
consist of small hard lumps, or scybala. More or less nausea occurs in a majority of cases, and not infrequently vomiting. Hiccough and eructations of gas are not uncommon. The appetite is lost. The urine is scanty and micturition is sometimes difficult and painful. Circumscribed projections or tumors, changing their situation, and due, evidently, to an accumulation of gas in a portion of the intestinal tube, lave been observed. The pain may be exclusively in paroxysms, the patient, in the intermissions, being entirely free from it, but much oftener more or less constant pain is experienced with exacerbations. The paroxysms or exacerbations vary much in different cases in duration, as well as severity. They may last for a few moments only, or continue for several hours. The intermissions or remissions also vary in duration from a few moments to hours and even days.

In conjunction with the foregoing local symptoms, there is no febrile movement unless there be some accidental complication. The pulse is abnormally slow, and frequently irregular. It is generally fuller and harder than in health. The skin is cool. The mind is anxious and depressed. The strength fails in proportion to the severity of the pain and the duration of the affection. 'l'he affection may be complicated with other manifestations of lead-poisoning, viz., paralysis affecting certain of the voluntary muscles, and especially the extensors of the forearm, amaurosis, neuralgia in different situations, occasionally epileptiform convulsions, delirium, and coma. The latter have been embraced by Tanquerel and other writers under the name lead encephalopathy.

The affection has no definite duration, in this respect resembling neuralgic affections in general. When left to itself, it may end in a few days, or after the lapse of weeks, and, if the patient continne to be exposed to the cause, it may persist for an indefinite period. In itself, it involves little or no danger to life, nor is there ground to believe that it leads to any serious abdominal disease. But other effects of lead poisoning, which may complicate or follow this affection, especially the effects embraced under the name lead encephalopathy, are liable to end fatally. 'The affection frequently pursues an irregular course, varying in severity much at different periods; and relapses, after all the symptoms have ceased for days or weeks, without any fresh introduction of lead, are not uncommon.

The introduction of lead into the system in sufficient quantity to give rise to its morbid effects, may take place through the lungs, the stomach, the mucous membrane in other parts, and the skin. Certain occupations involve the inhalation of lead. Persons employed in the manufacture of lead-paints are most exposed, and, of those who suffer from lead-poisoning, the largest proportion are of this occupation. Next to this class, painters are most exposed. More or less exposure, by inhalation, belongs to a great number of occupations, such as paper-staining, grinding of colors, glazing carls, manufacturing earthen pottery, plumbing, shot-making, etc. Lead-poisoning has repeatedly occurred from sleeping in newly-painted rooms. Lead may be introduced through the stomach in various articles of food and drink. The colic of Poitou was due to wine, and that of Devonshire to cider adulterated with lead in order to give it sweetness. This kind of adulteration has been practised since the occurrence of the affection in the places just named, and it is perhaps still sometimes resorted to. Liquid and solid articles of diet, especially if they contain a free acid, become impregnated with lead by being in leaden vessels or earthen vesscls glazed with lead. Some years ago a large number of persons within a limited district in Ohio were affected with a disease which, for some time, was regarded as a novel epidemic, and received the name of dry 
cholera. It was found to have the diagnostic characters of lead colic, and was traced to the common use of a cheap kind of carthen-ware made in that vicinity, into the glazing of which lead entered.' Prof. Fenner, in the Southern Medical Reports, vol. i., describes an epidemie colic which he observed in New Orleans, and which he traced to lead contained in sodawater; and my colleague, Prof. Doremus, some years ago, demonstrated the presence of lead in considerable quantity in the soda-water drawn from fomtains in this city (New York). Water distributed in lead pipes is not infrequently the vehicle of its introduction into the system. ${ }^{2}$ Lead sometimes enters into the substances used to color confectionery, condiments, and other articles, in suflicient quantity to give rise to poisonous effects. Articles inclosed in lead foil may become contaminated. A uthentic instances of poisoning by the use of tobacco thus inclosed have been reported; and in France inclosing tobacco in lead is prohibited by law. The liabit of chewing shot and pieces of the lining of tea boxes has been known to give rise to various manifestations of lcad-poisoning lasting for four years before the cause of disease was discovered." During the spring of $181 ; 6$ two hundred and thirteen cases of lead-poisoning oecurred in the Walkill Valley, Orange Co., New York. After considerable research it was ascertained that lead was contained in the flour and meal used in that part of the country. The source of the lead was ascertained to be the stones used for grinding the meal and flour. The stones were old, constantly needing repair, large cavities frequently occurring, which instead of being filled up with cement were filled with common lead. The attrition of the grinding detaehed minute particles of lead, and the lead thus becoming mixed with the flour and meal was transformed into the carbonate by the proeesses of fermentation and baking." 'These are only a few of the diverse modes in which lead finds its way into the alimentary canal. Its use as a remedy has given rise to eolic and other morbid effects. It may be introduced through the skin, although doubtless with difficulty. Prof. I,ewis Rogers, of Louisville, has informed me that he has known the characteristic paralysis called wrist drop, fairly attributable to the long-continued application, to the hands and face, of a cosmetic containing lead; and my colleague, Prof. Sayre, has reported a oase in which this manifestation of lead poisoning followed the use of the article known as "bloom of youth." I have known a case in which phenomena denoting lead poisoning were attributable to the use, for a long period, of a clressing for the hair containing the acetate of lead. Finally, instances of lead-poisoning by means of collyria and vaginal injections have been reported.

The diagnosis of lead colic, in most cases, is not difficult. If the occupation of the patient involve obvious exposure to lead, a suspicion of the nature of the affection is, of course, at once aroused; but this, and other manifestations of lead-poisoning not infrequently occur in persons who are not aware of any such exposure, and it may require no small pains to discover the mode in which the poisoning has taken place, after the character of the affection has been ascertained. The diagnostic characters of lear colic as compared with those of ordinary colic, offer points of difference which enable the physician to discriminate it from the latter. The gradual development, the occurrence of remissions much more firequently than

' Article by E. C. Bidwell, M.D., in the Ohio Medical Jonrnal, 1821.

2 Vide appendix to translution of Tanquerel's treatise, and Report of Horatio Adams, M D., in Transactions of American Medical Association, vol. v.

Vide cass in Western Lancet, Cincinnati, communicated by Dr. Edward Murphy, of Indiana.

- Philadclphia Medical News and Library, July, 1866. 
intermissions, the persistency of the affection, the concurrence of pain in the back, limbs, and sometimes in the ehest, the occurrence of nausea and vomiting, the obstinacy of the constipation, and frequently dysuria, characterize this affection when contrasted with ordinary colic. Coexisting paralysis in some eases, especially if limited to the extensor muscles of the forearm, strengthens the diagnosis. Enteritis and peritonitis are excluded by the absence of symptoms denoting inflammation. Enteralgia, exclusive of its occurrence in connection with hysteria, is so rare, excepting when produced by lead, that its existence is strong presumptive evidence of this causation. A valuable sign was indicated by 'Tanquerel in his treatise on Lead Diseases, published in Paris in 1838, and its diagnostic importance enforced by Dr. Henry Brinton in a paper read before the London Medical and Chirurgical Society in 1840. This sign consists in a blue or slate diseoloration of the gums at their junction with the teeth. The blue line on the gums may be apparent on the upper or lower jaw or on both. It is wanting in sections where teeth have been lost. It is most marked in proportion as the teeth are incrusted with tartar, and in persons who neglect eleanliness in this situation. The diseoloration is attributed to the formation of the sulphuret of lead, sulphuretted hydrogen being evolved from the decomposition of particles of food remaining between the teeth and beneath the margin of the gums. The presence of the lead to combine with the sulphuretted hydrogen may be readily accounted for, if it be introdueed, as is usual, into the system through the mouth either with the inspired air or in food or drink; but there is ground for the belief that suflicient lead may be eliminated in this situation to account for the sign. The sign is not present in all cases. Its absence, therefore, is not proof that lead colic does not exist. Clinical observation, however, shows that the sign is present in a large majority of eases. It is found frequently to preeede the development of eolie and other affections dependent on leadpoisoning. It is not a transient sign, but is apt to persist, not only during the continuance of the affection, but for some time afterward. In a diagnostic point of view it is highly important, and the gums should be cxamined with reference to it whenever lead-poisoning is suspected. A chemical analysis of the urine may show the presence of lead in this fluid. In the ease reported by Prof. Sayre, just referred to, my colleague, Prof. Doremus, obtained from the urine sufficient lead in the metallic form for clinical illustration.

The treatment of lead colic embraces palliative and curative measures. The pain is to be palliated by anodynes given by the mouth or rectum, conjoined with soothing applications to the abdomen, as in cases of ordinary colic. Chloroform given by the mouth and applied over the abdomen is stated by Aran to act promptly and efficiently in relieving the pain. If this prove ineffectual, opium in some form will be required, the doses and their repetitions to be graduated by the intensity and persistence of the pain. Relief is afforded by free purgation, and, for this end, saline, or such other eathartics as the stomach will best support, may be given, and their operation aided by large purgative enemata. The obstinacy of the constipation often renders it difficult to proeure free evacuations from the bowels, and active cathartics may be required. Tanquerel advoeated the efficacy of croton oil, a drop to be given twice daily. He regarded active purgation as eurative, and this is the view generally held. Drastic purgatives entered largely into the complicated treatment instituted by monks in the hospital La Charite, in Paris, in the beginning of the 17th century, and, with various modifieations, continued ever sinee in that hospital. The employment of cathartics may be useful in removing lead contained in the 
contents of the alimentary canal. The warm bath is useful as a palliative, soothing measure.

Several remedies have been supposed to have a curative efficacy in this affection. Alumen is one of these. Its efficacy was adrocated especially by Brachet, in a prize essay on the subject of lead colic, who stated that he treated 150 patients with this remedy more successfully than by opium and purgatives. From one to two drachms of alumen were administered during the day in a ptisana to which 40 or 50 drops of laudanum were added, a mild aperient given on the third day, and the alumen continued for two or three days after the symptoms of the affection have disappeared. Other observers, Briquet, Tanquerel, and Grisolle, have not met with a similar success from the alum treatment. Sulphuric acid has been advocated especially by Gendrin, on the basis of remarkable suecess in a large number of cases. A drachm of dilute sulphuric acid in a quart of sweetened water forms a pleasant drink resembling lemonade, and this may be taken in the twenty-four hours. Tanquerel denied the efficacy of this remedy, giving, as the basis of his denial, the results of its trial in 53 cases. The success of Gendrin's method, however, is attested by $\mathrm{Dr}$. Bennet, who was a pupil and house physician of Gendrin for three years. ${ }^{1}$ The prophylactic efficacy of the sulphuric acid lemonade appears to have been satisfactorily proven in the lead works at Birmingham, England.a Strychnia or nux vomica has been recommended as a curative remely by Huss, Neligan, and, in this country, by Dr. Bulkley, and the late Dr. Swett. ${ }^{3}$

The remedy regarded in this country now, and of late years, as possessing most curative power, is the iodide of potassium. The use of this remedy as a curative agent, in the treatment of lead diseases, and also those due to the presence of mercury in the system, dates from the publication, in the Annales de Chimie et de Physique, in 1849, of a paper by Melsens. ${ }^{*}$ Assuming, as is generally believed, and as facts appear to prove, that the local manifestations of lead-poisoning are clue to the presenee of an insoluble compound of lead in the tissues of the affected parts, Melsens claims for the iodide of potassium the power of effecting the liberation of the lead and its elimination from the system. The iodine is supposed to eombine with the lead in the tissues, forming a soluble compound which passes out of the body in the urine. Chemical examination of the intestines, the paralyzed voluntary muscles, and other organs, in cases of lead-poisoning, has shown the presence of lead in larger quantity than is to be accounted for on the supposition that it may exist in the body in health. The examination of the urine in cases of lead-poisoning under treatment with the iodide of potassium, has shown the presence of lead, its absence in this liquid having been ascertained prior to the treatment. Finally, clinical observation appears to afford proof of the curative efficacy of this treatment. Further evidenee of the latter, consisting in the results of the treatment in a large number of recorded cases, is a desideratum. With reference to the therapeutics of this, as of other affections, knowledge of its natural history as regards termination when left to its own course, is important. Tanquerel, in his valuable treatise, gave the results

1 Appendix to Tanquerel's Treatise, by Dana.

- Stillé's Therapeutics, vol. ii. p. 247 .

- For a translation of this paper by William Budd, M. D., the render is referred to the British and Foreign Medico-Chirurgical Review, January, 1853.

5 For facts verifying this statement, see a report of the results of the treatment

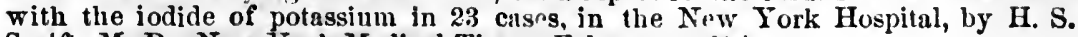
Swift, M. D., New York Medical Times, February, 1854. 
in 31 cases in which no treatment was pursued for 12 days after admission into hospital, the affection having existed for one or more days before admission. Of 8 of these cases in which the colic was violent, in 3 a spontaneous cure took place within the 12 days, viz., on the $3 d, 8$ th, and 11th day. Of 13 cases in which the colic was of moderate severity, in 5 a spontaneous cure took place on the 4 th, 7th, 8th, 9th, 11th day, and in 1 on the 13th day. Of 10 eases in which the colic was light, in 6 a spontaneous cure took place within the 12 days.

In the treatment of lead-poisoning by the iodide of potassium, Melsens enjoins to commence with moderate doses, and to inerease the doses gradually. He supposes that disturbance may arise from dissolving the lead out of the tissues rapidly. Five grains may be given three times daily at first, and the quantity increased to a drachm per diem, if the remedy occasion no inconvenience.

The utility of the warm bath as a palliative measure has been already referred to. It may perhaps be useful in promoting the elimination of lead through the skin. It is useful by removing from the skin lead which comes into contact with and adheres to the surface in persons engaged in certain occupations. The sulphurated or sulphurous bath may be employed for the latter object. This is made by adding four ounces of the sulphuret of potassium to thirty gallons of water in a wooden bathing-vessel. 'The use of this bath renders the presence of lead on the skin apparent by the production of a dark discoloration which is readily removed by a brush. 'The blackness is marked in various parts of the body in painters and workmen employed in the manufacture of the preparations of lead used as paints, especially if they have neglected cleanliness; but it may be due to the presence of lead eliminated from the body and remaining adherent to the surface. The bath renders the lead which may be in eontact with the skin inert, and it is useful as affording evidence that the surface of the body is entirely free from lead, whether derived from without or eliminated.

Prevention of the eontinued introduction of lead into the system is an essential part of the management. The oceupation is to be suspended if it involve exposure; and if the affection be renewed, or there be other manifestations of poisoning, on resuming the oecupation, it should be exchanged for some other. If the source of the poisoning be not apparent, it is to be sought for until found. This poison is one which gives no notice of its entrance into the body, and no manifestations of its presence until a certain amount of accumulation has taken place. The affection under consideration is but one, althongh the most frequent, of the forms of disease to which it gives rise. There is reason to think that obseure and indefinite ailments are not infrequently produced by it in cases in which suspicion of the cause is not awakened, and the liability to these, as well as the more marked effects, from the use of water conducted in lead-pipes and various other sources, some of which have been enumerated, is one of the important subjects pertaining to public health.

The protection of those necessarily exposed to an atmosphere more or less eharged with lead, or to contact with it, is also an important subject. Mueh may be done to prevent its introduction by attention to ventilation, clothing, baths, washing the mouth, and the occasional use of purgatives. Special means of protection, such as the application of moist sponges to the mouth and nostrils, or the use of masks, under certain circumstances, may be important.

After reeovery from the colic, an anæmic condition, with general dehility, will often claim appropriate medicinal and hygienic measures of treatment. 


\section{COLIC FROM COPPER.}

Writers have described a form of colic supposed to be produced by the introduction and accumulation of copper in the system, and the term metallic colic has becn used to embrace, not only the colic due to lead, but abiominal pains, with other symptoms, attributed to copper, mercury, and arsenic. 'The diagnostic features of so-ealled copper colic, as contrasted with lead colic, have been considered to be as follows : Limitation of pain to the abdomen, increase of pain by pressure, enlargement more frequently than retraction of the abdomen, diarrhoea with greenish stools, and infrequency of nausea or vomiting. An extended and careful examination of numerous establishments in which the workmen were exposed to emanations from and contact with copper in various modes, conducted by Chevallier and Boys de Loury, in Paris, led to the conclusion that such an affection as colic from copper does not exist. ${ }^{1}$ 'This conclusion, however, is by no means inconsistent with the fact that copper within the system exerts a deleterious effect. Arsenic and preparations of mercury, taken in quantities sufficient to produce the phenomena of a local acrid or irritant poison, give rise to pains which may resemble those of colic. Accumulating within the system, these minerals occasion certain toxical effects, but they do not, under these circumstances, lead to abdominal symptoms which may with propriety be considered as an individual affection under the name of colic.

\section{CHA P TER VIII.}

Sporadic Cholera-Clinical History-Pathological Character-Causatlon-Diagnosis-Prognosis -Treatment. Cholera Infantum. Epidemic Cholera-Anatomical Characters-Clinlcal History-Pathological Character-Causatlon-Diagnosis-Prognosis-Prevention-Treatment.

$\mathrm{F}^{\mathrm{F}}$ the functional affections of the stomach and intestines, enumerated (1) a preceding chapter, two remain to be considered, viz., sporadic and epidemic cholera. Cholera infantum, belonging among the diseases of children, does not come within the scope of this work. I shall, however, notice it briefly, referring the reader, for a fuller consideration of it, to works devoted to the diseases of children.

\section{Sporadic Cholera.}

The affection called cholera, in its ordinary form, is commonly known as cholera morbus. Aside from the incongruity of associating these two words, the one Latin and the other of Greek derivation, there is no good reason for appending the word morbus, which expresses no more than is implied in the name of any affection, viz., the existence of disease. The etymology of the term cholera is somewhat doubtful, but it is supposed to signify a flow of bile. The term is of ancient date, and was introduced when various liquids were embraced under the name bile. Not only is

1 Tide Nysten's Dictionaire de M'decine, etc., 1858. Also, Valleix, op. cit., tom. $v$. 
the term sanctioned by long usage, but it would be difficult, with our present knowledge, to substitute a better name for the affection under consideration. The term sporadic serves to distinguish this from another affection, known as cholera, which prevails as an epidemic. To distinguish it from the latter, which emanated from India, it is sometimes called by French writers, European, and by British writers, English cholera. Writers on this side of the Atlantic might, with equal propriety, call it American cholera, since it is sufficiently common, as an indigenous affection, in America. It is called by German writers cholera nostras.

Clinical History.-An attack of sporadic cholera is often abrupt, but, in some cases, is preceded, for a period usually brief, rarely longer than a few hours, by a sense of weight or uneasiness referred to the epigastrium, or extending more or less over the abdomen, with nausea, occasional colic pains, etc. The attack occurs much oftener in the night than in the daytime. It is ushered in by vomiting, which is speedily followed by purging. Vomiting and purging, occurring in quick succession, and recurring either simultaneously or in close alternation, constitute the prominent, distinctive, symptomatic features of this affection.

The evacuations from the stomach and bowels are generally abundant, and, at first, consist of the alimentary and fecal contents. Afterwards; liquid is vomited, sometimes in large quantity, often having the appearance and taste of bile, sometimes acid, and sometimes so acrid as to occasion a scalding sensation in the throat. In like manner, the dejections become liquid, and occasion frequently a burning sensation at the anus. The acts of vomiting and purging occur suddenly with but little premonition. They are often violent, the contents of the stomach and bowels being ejected with force. The acts of purging are preceded and accompanied by colic pains, often extremely severe, which are temporarily relieved by a free evacuation. Severe pains, like those of spasm or cramp, often attend the acts of vomiting. In the intervals between the acts of vomiting, the patient usually suffer's from dryness of the mouth and fauces with urgent thirst. The abdomen may be at first voluminous, but after repeated acts of vomiting and purging; it becomes contracted. Tenderness over the abdomen is either wanting or slight. Spasm of the abdominal muscles sometimes occurs, causing hard bunches or knots as in some cases of colic. Hiccough is an occasional symptom.

With the foregoing local symptoms are associated anxiety, restlessness, and a sense of exhaustion proportionate to the violence and duration of the attack. The pulse is more or less accelerated, small, and feeble. The skin is cool or even cold, and is frequently bathed in clammy sweat. The voice is feeble, sometimes altered in quality, and occasionally there is aphonia. In some severe cases cramps occur in the feet, the calves of the legs, and in other situations. These add greatly to the suffering of the patient.

The affection runs a rapid course. After continuing for several hours, the acts of purging and vomiting become less severe and occur at longer intervals, the pains, etc., progressively diminish, until, at length, all the symptoms disappear, leaving an amount of fatigue proportionate to the violence and duration of the attack. The patient usually rapidly recovers. It is remarkable that an affection involving so much disorder should leave the affected organs in a condition to resume so speedily the exercise of their functions. This is the rule as regards the progress and termination of the affection, even if its course be not arrested by remedial interference. Exceptionally, the vomiting and purging continue, the evacuations from 
the bowels occur involuntarily, the pulse becomes more and more feeble. coldness of the surface is more and more marked, contraction of the features takes place; in short, the patient falls into a state of collapse, and dies within a few hours from the date of the attack. This unfavorable course is extremely rare. I cannot recall an example that has fallen under my observation, exclusive of cases occurring in infancy.

Pathological Character.-Sporadic cholera appears to combine morbid conditions belonging to other functional affections of the alimentary canal. In a certain proportion of the cases considered as cases of cholera, the symptoms are attributable simply to indigestion. Vomiting and purging denote, in these cases, the coincidence of acute dyspepsia and crapulons diarrhœa. These cases are usually comparatively mild, and the vomiting and purging cease as soon as the offending contents of the stomach and bowels are expelled. In other cases something more than indigestion is involved in the pathology. The evacuation of liquid, often in great abundance, shows hyper-secretion or morbid transudation into the stomach and intestines. The affection then combines gastrorrhæa and enterorrhca. In some cases the secretion of bile would seem to be increased, but it is difficult to estimate, from the color and the bitter taste of the romited liquid, the relative proportion of bile. It had been common to suppose that in this, as in various other affections, disorder of the liver is in some way involved. There is no solid foundation for such a supposition; it rests on conjecture alone. It is only in a certain proportion of cases that the bile appears to be secreted in morbid quantity. The limit of our present knowledge of the pathological character of the affection is reached when the different morbid conditions into which it is resolvable are enumerated. These morbid conditions, or elements of the affection, are indigestion, irritation, spasm of the muscular coat of the stomach and intestines, enteralgia, morbid gastric and intestinal transudation, and, perhaps, in some cases, hyper-secretion of bile. By German authors the affection is considered as a variety of gastro-intestinal catarrh.

Causation.-uporadic cholera is not peculiar to any country, but is of more frequent occurrence in warm than cold climates. In cold and temperate latitudes it occurs very rarely except during the summer and autumnal months. An elevated temperature is thus involved in its causation, either by contributing to the development of causes, or by rendering the system more prone to be affected by them. The affection occurs oftener in childhood, youth, and middle age than in advanced life, and oftener in males than females. Frequently an attack seems to be attributable to the ingestion of particular articles of food or drink. Uncooked vegetables, fruits, ices, etc., are often supposed to stand in a causative relation to it. An attack may follow the arrest of digestion from various causes. In some cases, as already stated, the affection is apparently due to indigestion alone. It is probable that, exclusive of these cases, the affection involves a special cause, the nature and source of which are unknown.

Disarosis.-The diagnostic features of an attack of sporadic cholera are so strongly marked that little need be said under this head. Yet there is some liability to errors of diagnosis. I have known a case of acute peritonitis, accompanied by vomiting and purging, to be considered as a case of cholera, the nature of the disease not being discovered until the autopsy was made. It is extremely rare for vomiting and purging to 
have sufficient prominence, early in peritonitis, to lead to this error. The diagnostic marks of peritonitis, however, which will be considered in the next chapter, should always be sought after in cases of apparent cholera. The chief liability to error in diagnosis relates to cases of poisoning from the ingestion of acrid or corrosive substances. It is important to discriminate these cases from cholera, with a view to antidotal treatment, prognosis, and medico-legal considerations. 'The following are the differential points: 'The acrid or corrosive poisons occasion vomiting which continues for some time before diarrhœa occurs, whercas in cholera the purging is simultaneous with or follows quickly the vomiting. Moreover, the vomiting in cases of poisoning is out of proportion to the diarrhoa. T'he symptoms in cases of poisoning denote a condition of greater gravity; the pulse is more frequent and smaller, the expression is more haggard, etc., than in the majority of the cases of cholera. Redness or charring of the mouth and throat distinguishes certain cases of poisoning. There is marked tenderness over the stomach in cases of poisoning, and more constant, excruciating pain in this situation, in the intervals between the acts of vomiting-in short, the symptoms of gastritis are present. The matter vomited is more apt to be bloody. Finally, in cases of poisoning the symptoms are deveioped directly after a meal, or the ingestion of something containing the poison.

Prognosis.-As already stated under the head of the clinical history, this affection in the vast majority of cases intrinsically tends to recovery. A fatal termination is a rare exception to the rule; yet the fact that the termination is sometimes fatal is to be borne in mind and should enforce prompt and effective measures of treatment. Occurring in persons already affected with some important disease, the prognosis is, of course, not so favorable as when healthy subjects are attacked.

Treatment.-In the treatment of sporadic cholera, the first point is to ascertain whether the matters vomited have contained ingesta in more or less abundance. If not, and there be reason to suppose that the stomach contains undigested aliment, a mild emetic may be given. Almost invariably, however, the contents of the stomach are expelled with the first acts of vomiting, and, as an emetic is indicated for no other object, it is very rarely called for. In my own experience I have not prescriber an emetic in this affection for the last twenty or more years. The next object is to arrest the vomiting and purging, together with the pain, etc., by the employment of some form of opiate in doses sufficient to effect this object. In the vast majority of cases, this object may be speedily effected. The form of opiate should be chosen with reference to promptness of action and its being retained. Laudanum, the black drop, and an aqueous solution of opium are well suited to the management of this affection, but Majendie's solution, or a salt of morphia placed dry upon the tongue, is, in general, the best form. A full dose-half a grain of a salt of morphia, or an equivalent dose of any other preparation, to an adult_-should be given directly after an act of vomiting. The first dose will perhaps be immediately rejected; if so, a second should be at once given. If a second and third dose be instantly or quickly rejected, the administration by the mouth should be abandoned, and from one to two drachms of latldanum, or an equivalent dose of some other preparation, may be given in a little thin starch or mucilage per enema. The enema should be given directly after an evacuation, and the patient should be instructed to resist as long as possible the inclination to expel it. If the first enema be 
quickly rejected, a second and a third may be given. If the attempt to administer the opiate by the mouth and rectum fail, the hypodermic injection may be resorted to. In whatever way the remedy be introducel, it is to be repeated after an interval of from half an hour to an hour, until the vomiting and purging are arrested, the effects of each dose being carefully watched, and the intervals being sufficiently long to avoid any risk of inducing narcotism.

An important part of the treatment is withholding from the patient liquids. His intense thirst leads him to drink largely between the acts of romiting, and this tends to prolong the affection. He should be restricted to a tablespoonful of ice water at short intervals, or, what is better, to small pieces of ice which may be taken frequently and allowed to dissolve in the mouth. Strict compliance with injunctions on the score of drink is essential to the prompt success of the treatment. If there be great prostration, a little spirit and water may be taken, if retained, at short intervals.

In my experience this method of treating sporadic cholera has proved uniformly suceessful, and complete relief may generally be expected within an hour. No apprehension need be entertained with respect to the sudden cessation of the vomiting and purging; the more quickly the arrest is made, the better, after a free evacuation of the stomach and bowels. Mercury is, to say the least, superfluous. The success of treatment without it is all that could be desired.

Care as regards diet is alone required, in most cases, after the affection is arrested.

I do not deem it necessary to consider other methods of treatment, since the success of the plan just stated is, so far as my experience goes, uniform and immediate. The only objection to the plan is, that some persons are unpleasantly affected by opium, and, after the affection is arrested, the effects of the remedy may be more or less annoying for a time. This objection has not much weight, since the after-effects of opiates, however distressing, occasion far less suffering than the continuance of cholera. But they who frequently experience annoyance after the use of opium, under certain circumstances take it without inconvenience, and $I$ have been led to observe that the after-effects are apt to be slight or wanting when opium is given, in this affection, to patients who usually suffer from these effects.

\section{Cholera Infantum.}

The name cholera infantum, or the more popular phrase summer complaint, is supposed, by some, to denote an affection peculiar to this country. The morbid phenomena, however, considered by American writers as belonging to this affection are described in European works under other nanes. Cruveilhier enumerates many of the symptoms as occurring in cases in which gelatiniform softening of the stomach is found after death, a form of softening already referred to, which is probably due to the action of the gastric juice post-mortem. ${ }^{2}$ British writers on diseases of children generally describe the symptomatic phenomena under the head of diarrhœa. Weaning brash, watery gripes, and choleric fever of ehildren, are names under which these phenomena have been described by Cheyne, Armstrong, and Copland. By some French writers they have been described as belonging to colo-enteritis, follicular enteritis, choleriform diar- 
rhœa of children, and gastro-intestinal catarrh. German authors consider the affection as incidental to gastro-intestinal catarrh.

As commonly used in this country, the term cholera infantum, or summer complaint, embraces different pathologieal conditions, in fact, distinct affections, occurring in children under two years of age. These different conditions, however, may be developed at different periods in the same case. In certain cases the symptoms closely resemble those of the sporadic eholera of adults. A child is seized with vomiting and purging, the latter usually occurring first; the acts of vomiting and purging are violent and frequently repeated; after the contents of the stomach and bowels are expelled, the evacuations consist of secreted or transuded liquid in more or less abundance. The attack may cease or be arrested and recovery speedily ensue, as in cases of the sporadic cholera of adults; but this favorable course does not obtain so generally in children as in adults. If the course be unfavorable, the vomiting and purging continue; the child is tormented with thirst, but everything is rejected from the stomach; great prostration ensues; collapse follows, and death takes place in one, two, or three days. The fatal result may be preceded by convulsions and coma. In some cases the violent symptoms of cholera cease, and the attack eventuates in a chronic affection, accompanied by diarrhœa and occasional vomiting.

In other cases, the affection is gastro-intestinal indigestion. These are characterized by diarrhœa, the dejections being lienteric and watery. Vomiting occurs only occasionally, and is an accidental sympton. It may even not oceur at all. The diarrhoa is more or less persisting, the discharges being often green in color, an appearance which Golding Bird, and, more recently, Dr. J. Lewis Smith, have shown not to depend on vitiated bile $;^{1}$ the appetite is impaired or lost; colic pains are apt to be troublesome; the child progressively wastes, and death may take place from inanition. In another class of cases, the affection is either enteritis or entero-colitis. The dejections in these cases contain gelatinous or stringy mucus, which is not infrequently tinged or streaked with blood. Febrile movement, with remissions, occurs in these cases. There is more or less abdominal tenderness and pain. Vomiting may be prominent as a symptom, or it occurs only occasionally. Progressive emaciation and debility mark the unfavorable progress of this affection. Head symptoms are often developed in its course. The child becomes dull and somnolent, lies with the eyelids partially closed, and frequently rolling the head from side to side. Convulsions and coma may occur before death. A fter death, an examination reveals the evidences of inflammation of the mucons menbrane of the small, and, perhaps, also, of the large intestine, the follicles being especially involved. Enteritis or entero-colitis is liable to become developed in the cases which, at first, appear to be cases of diarrhoea dependent merely on indigestion. In still another class of cases the symptoms and the appearances after death denote the existence and limitațion of inflammation to the large intestine.

Under the name cholera infantum, then, are commonly embraced cases of sporadic cholera, diarrhœa from indigestion, enteritis, colo-enteritis, and dysentery. The head symptoms which are apt to be developed in.

I Vide article by Dr. Smith in the American Medical Times, Sept. 20, 1862. Dr. Smith in this article states, as a conclusion drawn from 37 autopsical examinations, that there is no evidence of congestion, torpidity, hyper-activity, or perverted secretoly action of the liver in cases of cholera infantum or summer complaint. Vide, also, Smith on the Diseases of Children. 
unfavorable cases of each of these affections in the young child, were formerly attributed to inflammation of the meninges of the brain. 'They were supposed to denote a metastasis to the head. The attention of the profession was called to the incorrectness of these pathological views by the writings of Gooch and Marshall Hall. It is not easy to say precisely what the morbid conclition is, but it is not inflammation, and it is dependent upon the exhaustion produeed by the abdominal affection. It is sometimes distinguished as the hydrencephaloid condition. Softening and injection of the cerebral tissue were found by Dr. Hallowell in eases in which coma and convulsions preceded death, but there was no evidence that these changes were due to inflammation.'

The affections embraced under the name cholera infantum are not, as alreudy stated, peculiar to America, but they doubtless prevail to a much greater extent in certain parts of this country than in Europe, owing, probably, in a great measure, to the heat in the summer months being greater here than abroarl. The eausation has relation to elevation of temperature; the affections prevail almost exclusively during the months of June, July, A ugust, and September. Something more than temperature is concerned in their prodnction, for they are almost limited to cities and large towns, and are more prevalent in the Northern and Niddle than in the Southern States. Children of the poorer classes, in insalubrious situations, living in erowded dwellings, are more liable to be affected, but the children of those in easy cireumstances, and of the wealthy, by no means escape. The irritation of clentition and the change of diet after weaniug, doubtless, render the system more liable to be affected. Infants brought up by hand are especially subject to these affections. The diet of the child, after weaning, has much to do, if not with the causation of these affections, at least with the ability of the system to resist and overcome them. Restriction to insufficient articles of nourishment, such as arrowroot and gelatin, is one source of innutrition in infancy, but there is reason to believe that infant mortality in eities is attributable, in no small measure, to the use of diluted, sophisticated, and artificial milk. The importance of undiluted milk from a pure source to the welfare of children is far from being generally appreciated. The quality of milk is comparatively of little consequence after childhood, because it then usually enters but little into the diet, but, as the chief reliance is upon milk in inf:uey, its purity is then of the utmost importance. ${ }^{2}$

The general principles which should govern the management of the same affections after the period of early childhood, are applicable to the diflerent forms of disease embraeed under the head of cholera infantum, with certain modifications which will be here referred to very briefly. The treatment, in the first place, will have reference to the form of cholera infantum which is presented. In the acute form, resembling the cholera of adults, in which there is a liability to a rapidly fatal termination by exhaustion from loss of fluids, the patient falling into a state of collapse, the indications are to arrest the vomiting and purging, to restore warmth

1 Vide paper in American Journal of Medical Sciences, July, 1847.

2 It is almost impossible to impress on the public mind certain important facts as a basis of sanitary reforms. The following quotation from the appendix to Pereira on Diet, written hy the American Editor, Prof. Charles A. Lee, twenty-five years ago, is as pertinent now as then: "We have inspectors of fiour, of leather, of tobaeco, of meat, fresh and salted, of fish, and almost every article of merchandise, but for milk, which is the chief source of sustenance to the young, no inspection is provided; and to judge from the apathy hitherto displayed in relation to it, we bave no good reason to expect such a salutary regulation." 
to the surface by the application of dry heat, mustard-water, and the warm bath, and to sustain the powers of tive system by stimulants and nourishment. Exclusive of this form, the affections do not occur with a sudden and violent attack, but, as a rule, are developed gradually and tend to become chronic. 'They occur when the system is reduced by dentition together with the change of diet incident to weaning. The ise of opium cannot be resorted to in any of the affections as efficiently as when they occur after childhood. It is a maxim applicable to all the diseases of infants, that opiates are to be given with great circumspection. Keeping in view these considerations, the treatment will relate, in the first place, to the relief of vomiting and diarrhoea. Measures for the relief of vomiting are a sinapism to the epigastrium, creasote, which, in doses of $\mathbf{a}^{\prime}$ sixth or an eighth of a clrop in mucilage, repeated after each aet of vomiting, sometimes acts like a charm; the snbcarbonate of bismuth in doses of from 10 to 30 grains ; small doses of calomel with chalk, chloro: form, and hydrocyanic acid. With reference to this symptom, a careful regulation of the ingesta is requisite, and milk with lime-water, given in small quantity at a time, will often be retained when other articles of nutriment are rejected.

With reference to the diarrhœa, occasionally a laxative is useful, and a few grains of calomel or blue mass followed by the syrup of rhubarb, are well adapted for this purpose. There are no indications for mereury except as a remedy for vomiting, and an occasional laxative remedy, the convenience of its administration being perhaps its chicf recommendation in cases of young children. Opium in some form, with due circumspection, is to be employed. It is most efficient when given by enema, provided it be administered with proper care and retained. If given by the mouth, the Dover's powder is an eligible preparation. If the dejections denote acidity, chalk, in powder or mixture, or lime-water, is indicated. The mineral and vegetable astringents are highly important. Of the mineral astringents, the most efficient are, bismuth, lead, and the persulpliate or pernitrate of iron. The nitrate of silver is highly recommended by Rillict and Trousseau. Of the vegetable astringents, krameria, tannic acid, kino, catechu, hamatoxylon, geranium, and rubus villosus, are articles from which a selection may be made, or which may be tried in succession.

Attention to diet and regimen is of prime importance. Many children, there is reason to believe, die with these affections from an insufficiency of nutriment. Pure milk is the most appropriate article of food. It may be rendered more nutritious by the addition of boiled flour, powdered biscuit, or other farinaceous preparations. Gelatin and arrowroot, or other amylaceous substances, are inadequately nutritious. The essence of meat and strong broths are often taken with avidity by very young children, and the desire for them should be indulged and encouraged. This remark is also applicable to broiled tender meats taken in a solid form. Within the past few years, the use of raw meat, introduced by a Russian physician, Weisse, has been highly recommended by Trousseau and others, as a form of diet adapted to the affections under consideration, and observation shows that it is frequently taken with relish. My late colleague, Prof. Elliot, advocated strongly this form of diet in these affections. Tender beef is to be preferred, but mutton and chicken are sometimes more acceptable to the patient. The meat is to be finely hashed, and rnay be given in this way, or it may be made into a kind of purée by being reduced to a pulp in a mortar and pressed through a fine sieve, so as to separate the vessels and areolar tissue. If the child mani- 
fest an aversion to it as thus prepared, the hashed meat, or pulp, may be rendered palatable by the addition of salt, sugar, or some kind of preserve, or it may be added to a broth made with sago or tapioca.' $\mathrm{My}$ own experience, as regards the use of raw meat, is too limited to serve as the basis of an opinion, but I have had considerable opportunity of observing the value of cooked solid animal food, and I cannot avoid the suspicion that the latter is generally more acceptable, equally digestible, and not less nutritious. The liability to trenia, as a result of eating uncooked meat, is not to be overlooked. Trousseatu states that this occurred twice in a case under his observation. Weisse, who introduced this mode of alimentation, states that the production of txnia has been repeatedly observed in St. Petersburg. ${ }^{2}$

As regards the administration of different kinds of food, the quantity given at a time is to be graduated by the ability of the stomach to retain and digest it; and this is the only rule of limitation. 'Trousseau states that for some days after commencing the use of raw meat, it may be found undigested in the stools, but, at length, the digestion of it becomes complete. It is of vast importance for the physician not to deem it too trivial to enter into full and minute clirections with respect to the cletails of nourishment. He should bear in mind that children often die from imutrition through the apprehensions and ignorance of nurses and mothers.

In conjunction with diet, alcoholic stimulants are highly useful. $\quad \boldsymbol{A}$ few drops of brandy, or some kind of spirit, may be given, at short intervals, with advantage. The preparation known as Calisaya cordial or elixir is well suited to children, combining a tonic and stimulant in a form agreeable to the taste. The need of a stimulant is shown frequently by the avidity with which children take it in these affections.

Finally, removal from the eity, either to a situation on the seaboard or to a salubrious point inland, is the most efficient of all curative means. 'This measure, if not deferred too long, rarely fails. If other measures be not speedily successful, this, whenever practieable, should be resorted to without delay. If it be not practicable, the child should be in the open air as much as possible, and should be carried daily as far from home as convenient, in order in this way to secure a change of air.

The head symptoms referred to in the brief notice of the clinical history claim sustaining measures_stimulants and nourishment-conjoined with mild revulsives.

In these affections, hope of recovery need not be relinquished under an assemblage of symptoms which may seem to render the prognosis as unfavorable as possible. Patients sometimes recover after lingering for a long time on the verge of the grave. The reason is, the affections, when they prove fatal, do not necessarily involve incurable lesions of strueture, but a fatal result is due to protracted irritation, exhaustion, and innutrition.

\section{Epidemic Cholera.}

The term epidemic cholera is selected as the one most frequently used and the most convenient to designate a disease which has received a great variety of names, such as Indian, Oriental, and A siatic cholera, cholera asphyxia, spasmodic cholera, malignant, pestilential cholera, etc. etc. The etymology of the word cholera, it is true, is singularly inappropriate,

- Vide Troussean : Clinique Mśdicale, tome second.

2 Traité des Entozodircs, par Daraine, Paris, 1860, page 91. 
accepting the derivation which signifies a flow of bile; but our present knowledge does not warrant a name expressive of the pathological character of the disease. Moreover, it is to be presumed that the disease is not essentially the same as sporadic cholera; the two are not merely different varicties of one disease, but they are distinct individual affections, having certain prominent symptoms in common, but differing in essential points relating to clinical history, pathological character, causation, and prognosis.

For an account of the prevalence of this remarkable epidemic in different countries at different epochs, the reader is referred to other works." Suffice it to say that the disease appears to be indigenous in India, and to have existed there, both as a sporadic and epidemic affection, for a long period. In other parts of the world it has made brief visitations solely as an epidemic. In 1817 it commenced its march from Bengal, and during the following 15 years it traversed nearly the whole of the known world. It prevailed in different parts of the American Continent for the first time in 1832, and again 1834. It began its march a second time from India in 1847 , and again traversed the greater portion of the globe, prevailing in the United States in 1849, '50, '51, and '52. Commeneing again its march over the globe in 1864 , it reached this country in $1866^{\circ}$ and during this and the following year it prevailed in many of the large towns in the different States of the Union. It may be proper to add that my opportunities for observing the disease in 1849 and 1852 were ample. During these years I treated, in hospital and private practice, about 200 cases, recording, at the bedside, the histories in about 90 cases. A few cases came under my observation in 1866 and 1867.

Anatomical Characters.-Epidemic cholera has no constant, appreciable, anatomical characters which appear to be commensurate with the gravity of the disease. The morbid appearances after death do not afford an adequate explanation of the symptomatic phenomena, nor do they elucidate the pathology of the disease.

The mucous membrane of the stomach and intestines present, frequently, more or less alteration in color, due to submucous congestion. The color is a rose or livid red. But in some cases it presents a white, blanched appearance. Ecchymoses are occasionally observed. 'The mucous surface is covered with a viscid albuminous layer containing disintegrated epithelium. The mucous membrane of the stomach, especially in the large cul-de-sac, is frequently softened. It gives an alkaline, instead of the normal acid, reaction. Microscopical examination shows the membrane of the stomach and intestines to be more or less denuded of epithelium. Of the lesions found, the absence of gastric and intestinal epithelium is most entitled to be considered in the light of an anatomical characteristic of the disease. This lesion is by some considered as occurring prior to the gastro-intestinal transudation and sustaining to the latter a causative relation; by others it is regarded as secondary to the transudation. The isolated glands or follicles are often abnormally large, and, in a certain proportion of cases, also the Peyerian patches. Small vesicles containing a serous-like liquid have been observed in the small intestine, by Horner and others. They are not constant, and hence have no claim to be considered an anatomical characteristic. They resemble sudamina or the miliary eruption on the skin. The contents of the stomach and intestines,

1 Vide The origin and travels of Asiatic Cholera, by John C. Peters, M.D., New York Medical Journal, August, 1871. 
existing in greater or less quantity, correspond to the discharges during life. The mesenteric glands and pancreas present nothing abnormal. The liver is sometimes congested; otherwise not altered. The gall-bladder usually contains bile, but sometimes a matter resembling glairy mucus. The spleen is contracted and sometimes softened. The kidueys are more or less congested. The urinary bladder is empty, and contracted to a small size resembling the virgin uterus. The ganglions and nerves of the sympathetic system present nothing abnormal.

The cavities of the heart contain a small or moderate quantity of dark blood, mostly liquid, with some soft black coagula, and sometimes small fibrinous clots. Analysis shows notable deficiency of albumen, fibrin, and all the constituents of the serum, while the coloring matter is angmented. The blood is less readily oxygenated on exposure to the atmosphere, than after death from other diseases. The lungs, in some cases, contain but little blood, and in other cases more or less hypostatic congestion is observed. Edema, hepatization, and splenization of portions of the pulmonary organs are occasionally observed. The pleura is covered with a viscid substance like glue, and this is true of other serous membranes.

The membranes of the brain, and the brain itself, are more or less congested. There are usually considerable subarachnoid infiltration and effusion into the ventricles. The effusion into the arachnoid cavity in a considerable portion of cases is sanguinolent. This appearance was noted in 15 of 33 autopsies made by Prof. John C. Dalton, in 1849: ${ }^{1}$ The spinal cord offers nothing abnormal.

Cadaveric rigidity is marked, and continues for a long period. In some cases, shortly after death, visible twitchings of the muscles occur, and occasionally considerable movements of the limbs. I have, in several instances, been called, under these circumstances, to determine whether life was actually extinct. Extensive movements, in these eases, may be produced by blows upon the muscles. The heat of the body becomes developed, in some cases, a few hours after death in a remarkable manner, so that the temperature is notably greater than before death. The temperature has been known to rise to $104^{\circ} \mathrm{Fahr}$. The muscles of the body are notably dry.

There is much variation, in different cases, as regards the morbid appearances just enumerated. Certain of the lesions, viz., enlargement of the intestinal follicles, pulmonary œdema, and hypostatic pneumonia, are veryrarely found if the career of the disease have been short; and when life is very quickly destroyed the tissues sometimes show little or no morbid alterations. That the disease does not necessarily involve any important lesions of the solid parts, is shown by the rapidity with which recovery takes place in some cases in which the disease is promptly arrested.

Clinical History.-In the great majority of cases, the development of the disease is preceded by simple diarrhoea, the dejections being more or less numerous and copious, and unattended with pain. The duration of this preliminary diarrhœa varies in different cases from a few hours to several days. With this diarrhœa in some cases occasional vomiting occurs. Aside from these symptoms, nothing denotes the tendency to the disease. The system otherwise is but little, or not at all, disturbed, and it is ditticult to persuade the patient, often, that the intestinal disorder is of any

1 For a full report of the autopsical appearances in these 33 cases, vide Report of the City Physician of Boston (Prof. Henry G. Clark), 1849. 
special importance. Of 45 cases in which the existence, or otherwise, of premonitory diarrhœa was noted in the histories which I have recorded, it existed in 34 cases and was wanting in 11. Statistics on a larger scale show a much smaller proportion of cases in which the premonitory diarrhœa is wanting. Thus, the report of the General Board of Health of London for 1848-49, contains the statement by Dr. MacLoughlin, one of the sanitary inspectors, that of 3902 cases the premonitory diarrhœa was not wanting in a single case. Of 142 cases treated in hospital, in Paris, by Michel Levy, the premonitory diarrhœa was wanting in only six. Of 274 cholera cases treated in the different Parisian hospitals in 1853, the existence of premonitory diarrhœa was ascertained in 740 , in the remainder of the cases it being absent or its existence not ascertained. By some the period during which the premonitory diarrbœa exists is reckoned as a stage of the disease. This is obviously improper, as there is nothing then distinctive of epidemic cholera, and during the prevalence of the epidemic very many have the same kind of diarrhoea without the development of cholera. The simple diarrhoen which prevails extensively during the prevalence of cholera, is called, in France, cholerine, a term adopted, to a considerable extent, in other countries. In a practical view, the importance of taking cognizance of the premonitory diarrhœa is very great, as will presently appear.

The development of cholera is generally first denoted by characters relating to the intestinal evacuations. If diarrhœa have existed, the discharges are suddenly increased in quantity, or sudden and copious liquid discharges, without previous diarrhœa, mark the onset of the disease. This constitutes the attack, which, in the majority of cases, occurs during the night. The characteristic stools, which follow the expulsion of the fecal contents of the large intestine, consist of a thin liquid resembling whey or rice-water, generally known as the rice-water or choleraic discharges. The appearance of the stools in different cases varies, but, in general, their positive or negative characters render them highly distinctive of the disease. The liquid may be clear, like water, semi-translucent, milky, muddy-looking like ditch-water, and occasionally resembling gruel. It contains, in more or less abundance, small, white, solid particles, like grains of rice in ricewater or flakes of curd in whey ; these are found, on microscopical examination, to be composed of epithelium, a few pus-globules or leucocytes, molecular granules, with an amorphous substance and mucus. It is devoid of bile, and has little or no fecal smell, but emits a nauseons spermatic oder, compared by Bouillaud to that of the alkaline chlorides. It usually contains no feces. The quantity expelled at each act, or in the aggregate, is very variable. A large quantity, enough to nearly fill an ordinary chamber-pot, sometimes escapes at a single dejection, or the quantity with each act may be small. 'The quantity is not always in proportion to other symptoms denoting gravity. It may exist in large quantity within the intestinal canal, when a small quantity only is discharged. The dejections are not accompanied by pain, but by a sensation of relief. There is no smarting at the anus. The patient is suddenly impelled, by a sense of distension, to evacuate the bowels, and, when the quantity is large, it escapes very rapidly. In some cases the evacuations, after several have occurred, cease ; in other cases, after a time the liquid flows array constantly, the patient being unable to prevent it. Occasionally the dejections are slight, and they may even be wanting. In these cases transudation occurs, but the effused liquid is retained within the intestinal canal. Borborygmus and gurgling are more or less marked.

Vomiting usually occurs. In a diagnostic and pathological view it is 
less important, as a symptom, than the characteristic diarrhœa. In some cases it is a prominent and persistent symptom, every thing taken into the stomach being rejected; in other cases it occurs occasionally, and sometimes it occurs at the onset and ceases. The matter vomited is a watery liquid, frequently consisting chicfly of water which has been drank, but sometimes evidently an effused liquid. The choleraic effused liquid has an alkaline reaction. The vomiting is not preceded nor accompanied by much nausea. The act of vomiting occurs suddenly, and appears to arise simply from distension of the stomach.

These symptoms mark the invasion of the disease. And these symptoms, conjoined with a sense of prostration, more or less feebleness, and, gencrally, frequency of the pulse, eoolness of the skin or perspiration, with, in some cases, cramps of the muscles of the limbs, may constitute the clinical history of the disease. In other words, the disease may either end spontaneously or be arrested, without advancing beyond the stage which may be distinguished as the stage of invasion. The vomiting and purging ecase, improvement as regards other symptoms follows, convaleseence is at once declared, and recovery is rapid. The duration, in these cases, of the disease is brief - a few hours only. But in a large proportion of cases the disease does not pursue such a favorable course. A group of striking symptoms becomes developed, and the disease passes into what has been called the algid or cyanosed stage, or, more commonly, the stage of collapse. It remains to sketeh the clinieal history when the disease does not end with the stage of invasion.

Important symptoms pertain to the circulation. The pulse is usually frequent and proportionately feeble, beating 120,130 , or 140 per minute, but in some cases it becomes mole and more feeble without aeceleration. I have observed it to fall below the normal frequency. When the state of eollapse is fully developed, the pulse at the wrist is extinct. Feebleness or absence of the apex-beat of the heart and of the heart-sounds also show greatly diminished power of the central organ of circulation. The blood stagnates in the veins, giving rise to lividity at the roots of the nails, and a dusky hue of the prolabia, face, and surface of the body generally. Hence, the significance of cholera cyanosis and cholera asphyxia as names of the disease. If venesection be practised, the blood trickles from the aperture, not flowing in a projected or continuous stream. Leechbites do not bleed as usual. The blood is manifestly thicker than in health.

The respiratory system presents some striking phenomena. The number of respirations is usually increased, but sometimes diminished. Dyspnœa is occasionally complained of, and sometimes exists in a distressing degree. The respiration is frequently suspirious or sighing, and irregular in rhythm. The expired atmosphere, when the collapse is complete, is low in temperature as compared with health $\left(76^{\circ}\right.$ or $80^{\circ} \mathrm{Fahr}$.), giving to the hand a sensition of cooluess. It contains more oxygen and less earbonic acid than in health, showing a notable deficiency of the ehanges incident to the function of respiration. The roice undergoes a marked change, becoming feeble, raised in pitch, husky, and, not infrequently, extinet, attributable in part to muscular debility, but chiefly to dryness and stiffness of the vocal cords. In the moribund state the traeheal rales which so often precede death from other diseases is wanting.

Very little pain is experienced except from muscular contractions or cramps. As regards the nervous system, the disease is charaeterized by mental indifference or apathy. The patient has no apprehensions, although prior to the attack there may have been intense dread of the disease. In 
some cases there is great restlessness and jactitation, and in other cases the patient lies quietly save when disturbed by evacuations and cramps. The latter give rise to most of the suffering. Cramps are usually, but not invariably, present. They are seated especially in the feet, calves, and abdominal walls, the muscles of the upper extremities, and even the face being somewhat affected. The prominence of this symptom renders the name spasmodic cholera significant. The muscular strength, in some cases, is retained in an extraordinary degree. Patients, if not prevented, will sometimes get up and walk about even a few moments before death.

The function of most of the secretory organs is nearly or quite suspended. No bile is contained in the matter vomited nor in the dejections; the urine diminishes and is at length suppressed. Patients do not weep. It is a remarkable fact, however, that in nursing women the lacteal secretion continues. 'The disease, also, does not interfere with the occurrence of menstruation, but the menstrual discharge is diminished. The skin, in addition to its cyanosed appearance, is cold and often covered with a copious, viscid perspiration; in other cases it is cold and dry. The coldness of the surface is like that of a cadaver. Of this, however, the patient is not conscious; on the contrary, he generally complains of a sense of heat, and often insists on being uncovered. The heat of the body is diminished, as shown by the thermometer in the rectum or vagina, the axilla, in this disease, not giving a fair representation of the internal temperature. The diminution ranges generally from two to four degrees below $98^{\circ}$; but it may fall to $90^{\circ}$, and even considerably lower; it has been known to fall to $73^{\circ}$. The skin is frequently shrivelled, and wrinkled like the arms of washerwomen. Its elasticity is diminished, as shown by its subsiding very slowly after being pinched $\mathrm{np}$. The tongue gives to the tonch a sensation of coldness.

Thirst is a prominent symptom; the patient craves constantly cold drinks, and iced-water or ice is taken with great avidity.

The general aspect in the collapsed stage is highly eharacteristic. The features are contracted, the eyes sunken, the whole body is diminished in bulk, the patient appears to have suddenly grown old, and the countenance is often so much changed that the person is scarcely recognizable by familiar friends.

Of the foregoing symptoms, most are present in all cases in which the disease is not speedily arrested. The differences in different cases relate chiefly to the degree of the severity of the disease. In stating the condition of patients, it is customary to say before or after collapse, and to express different degrees of the collapsed state by saying semi-collapsed or completely collapsed. The collapse is complete when the pulse at the wrist is extinct, the surface cold, etc. The collapse may ensue with more or less rapidity-from an hour to six or eight hours. It occurs almost invariably in fatal cases, the mode of dying being, of course, by asthenia; but I have known cases to end fatally without the stage of collapse, the patient becoming comatose and dying by apnoea.

If the patient emerge from the state of collapse, he does not, in general, pass at once into convalescence, but there follows a period of the disease commonly called the stage of reaction. In this stage there is more or less febrile movement, the temperature rising more or less above the maximum of health. Diarrhœa not infrequently continues, the dejections becoming green and gelatinous. Dysentery is sometimes a sequel. In other cases constipation, more or less obstinate, follows. Vomiting, in some cases, is a troublesome symptom in this stage, the vomited matter 
being either green or yellow. Capillary congestion of the surface, especially of the face, is frequently marked, as in cases of typhus. 'I'he secretion of urine shortly returns, and the urine is apt to be albuminous. This stage is not devoid of considerable danger. The patient may be worn out with continued vomiting or diarrhoa; somnolency and coma become developed in a certain proportion of cases, dependent, probably, on uræmia; delirium, of a passive kind, is not uncommon, together with subsultus, sordes, and other symptoms of the typhoid state. Pneumonia is sometimes developed. A fatal termination occurs in a pretty large proportion of the cases in which the stage of reaction takes place; and in the cases which recover, the powers of the system are generally impaired for a long period. Exceptionally, patients emerge from the collapsed state, and pass, either gradually or quickly, into convalescence without febrile movement and other symptoms belonging to the stage of reaction. The rapidity of the recovery in a certain proportion of cases shows that the gastric and intestinal epitheliun, if exfoliated, may be quickly reproducel.

Pathological Character.-The first appreciable important event in the clinical history of this disease is transudation into the alimentary canal. 'The characteristic dejections and the contents of the intestines found after death consist of water in great abundance holding in solution a little albumen, and, according to Mialhe and Becquerel, albuminose, together with several saline constituents, the most abundant being the chloride of sodium, and an abundance of desquamated epithelium. The white solid particles or flakes contained in the dejections consist, in a great measure, of epithelium, and this causes the opacity when it exists. The blood, of course, loses what is transuded into the alimentary canal. From the loss of water the blood becomes dense and viscid. The bloodglobules are relatively in excess, owing to the loss of water. The serum of the blood is deficient in the saline elements which have escaped with the trinsuded water. The salts of potassa and the phosphates, however, remain. The fibrin is not diminished. The albumen is relatively greater in proportion than in health, but actually diminished. The serum of the blood contains more or less urea.

Most of the striking phenomena of the disease are plainly attributable to the blood-lesions resulting from the loss of the constituents found in the choleraic dejections. The blood circulates with difficulty, and the changes incident to respiration take place imperfectly; hence, the fechleness and extinction of the pulse, the cyanosis, loss of temperature, thirst, mental apathy, spasm, unchanged expired air, defective secretion, etc. etc. The retention in the blood of excrementitious principles, doubtless, plays an important part in the production of morbid phenomena. The coma which occurs in some cases may be due to uramia. Cholesteramia probably exists, since the function of the liver is suspended.

The transudation, however, which is first in the catenation of appreciable events, involves an underlying and, at present, inappreciable pathological condition. The latter must be understood in order to know fully the pathological character of the disease. It is probable that the primary, essential change is in the blood; that, in consequence of this change, the water of the blood, holding in solution saline constituents, transudes into the alimentary canal. According to Robin, the essential pathological change is an isomeric modification of the coagulable constituents of the bloorl-plasma, in consequence of which they part with the water entering into their coniposition, and lose the power of appropriating water; hence, 
the transudation by exosmosis, and defective absorption or endosmosis. It is fair to infer that the primary essential change is not in the solid structures of the alimentary canal, from the rapidity with which recovery takes place when the disease is promptly arrested. This fact shows that the primary essential blood-change is transient and admits of speedy readjustment, provided the immediate effect of this change, viz., the transudation, be prevented. As already stated, by some pathologists the exfoliation of the gastric and intestinal epithelium has been considered to be the essential pathological condition, preceding and causing the transudation.

In opposition to the foregoing view of the pathological character of the disease, the theory of elimination has been revived and advocated especially by Prof. Geo. Johnson, of London. According to this theory, the choleraic, gastro-intestinal effusion contains morbid material which is to be eliminated from the system; hence the vomiting and purging are conservative, and are to be encouraged instead of being arrested. The theory is not sustained by positive evidence, that is, there is no direct proof of the existence of a morbid material in the gastro-intestinal effusion; and the commonly received doctrine which attributes the blood-lesions and the phenomena therewith connected, in a great measure, to the loss of water and other constituents of the hlood by means of the effusion into the alimentary canal, is believed to be in accordance with clinical facts. 'The necessary conclusion to which the theory of elimination leads as regards treatment, viz., that the choleraic effusion is to be encouraged rather than arrested, if the theory be not true, can hardly fail to prove pernicious. ${ }^{1}$

Prof. Johnson attributes the diminution of temperature, the cyanosis, and other symptomatic phenomena, to obstruction of the pulmonary circulation, and states that the lungs are found after death to be notably free from blood. The latter statement is by no means true of all cases; the lungs, in a certain proportion of cases, are congested. The cause of the pulmonary obstruction, according to Prof. Johnson, is spasm of the vessels of the lungs, and this spasm he supposes to be a direct effect of the choleraic poison in the blood. These views are purely conjectural.

Causation.-A special cause is undoubtedly essential to the production of epidemic cholera; ordinary causes of disease, alone, are incapable of producing it. 'This is a logical inference from the epidemic character of the disease, its remarkably distinctive features, and its great uniformity, as regards symptomatic phenomena, in all places and periods, although the nature and source of the special cause are unknown. Is the special cause a contagious or infectious material, that is, is the disease diffused by means of a virus or an infectious miasm derived from the bodies of those affected with it? To account for the diffusion of the disease on the supposition of its communicability, was the easiest mode, and certain facts favor this supposition. The epidemic, in its migrations, follows routes most travelled; it prevails in tloronghfares and cities ; frequently the first case in a new place is an imported case; different members of a household are successively attacked, etc. It is easy to gather isolated facts to support the doctrine of communicability. But surveying the facts on all sides, and weighing the evidence for and against contagion or infection, the con-

- The prediction concerning the perniciousness of the treatment has been verified. The use of castor oil in King's College Hospital, in the epidemic of 1866, proved extremely disastrons, and, in the hands of Dr. McNamara, in India, the mortality was fearful. Vide London Lancet, 1871, and Am. Juurn. of Med. Sciences, Jan. 1872, p. 252. 
clusion is to my mind irresistible that the spread of the disease is not due to its being cornmunicated from those affected to others. Without entering into a discussion of this important question, the following are the considerations on which the conelusion just stated rests :-

1. Of those who are brought into contact with, or elose proximity to, cholera patients, the proportion who become affected is not larger than that of those who are not thus exposed. This is true as a general statement. In the Report on Cholera in Paris, in 1831, published by order of the French government, it is stated that of over fifty-five thousand persons affected and over eighteen thousand deatlis, only one hundred and sixty-four were persons whose duties or profession called them to nurse or prescribe for the sick. These 164 cases were from over two thousand persons employed in the hospices or civil hospitals either constantly or temporarily.' Hospitals are not foci of infection. At St. Petersburg, of 58 persons employed in hospital, only one had the disease. At Moscow, of 123 persons attached to one hospital, 2 only; and of 253 persons connected with another lospital, only 4 were attacked. In the London Hospital, during the epidemic of 1866 , of 100 persons employed in the cholera wards, 5 had the disease. Three of these were nurses who slept outside the hospital. Of 11 laundry women, one had the disease. "No case ocenrred in the general wards of the hospital, although during the whole period of the epidemic they contained their full number of eases, most of whom came from cholera localities." 2 Facts like these could be multiplied to any extent. Contrast with these the faets with respect to the diffusion of typhus in hospitals!

2. Imported cases in situations where the disease had not previously existed do not lead to its diffusion. Some striking instances which appear to be exceptions to this statement might be cited, but in general it is true that the disease does not spread from isolated cases.

3. The epidemic breaks out in a place almost simultaneonsly in different situations, without any communieation of the cases with each other. ${ }^{3}$

4. It is diffused too rapidly to be propagated by contagion or infection. If contagious or infectious, it must be most highly so to account for the rapidity with which it extends, and the latter is not consistent with other facts.

5. It runs its career in places too soon, and leaves too suddenly and completely for a contagious or infectious disease. Compare its course as an epiclemic, in this respect, with typhus, smallpox, or scarlet fever !

6. Efforts to produce the disesse, experimentally, by the greatest amount. of exposure, by inoculation, tasting the exeretions, ete., have failed.

There can be little or no doubt that the special cause may be transported in clothing and other substances after the manner of fomites. Facts might be cited in proof of this opinion; in other words, the discase is portable, without being contagious or infectious.

Since the preceding remarks on the causation of cholera were written (1866), the repeated visitations of the disease in Europe and in this country, have given rise to renewed inquiries and speculations respecting the source of the special cause and the manner in which it is diffused.

I Vide Tardieu, op. cit.

- Am. Journ. of Med. Sciences, April, 1867.

3 Vide Report, by the author, of the Epidenic at Buffalo in 1849, in Buffalo Medical Journal, vol. v., 1850 ; also, Report of the New York Metropolitan Board of Health for 1866, page 166; also. Facts relative to the Rise and Spread of the Disease in London and other towns in England during 1848-49 and 1865-66, in Brit. and For. Medico-Chir. Rev., Oct. 1870, p. 483. 
The theory advocated by the late Dr. Snow, of London, has been accepted by many, and therefore claims notice. This theory is based on the supposition that cholera, pathologically, is a disease exclusively of the alimentary canal, and that the production of the disease is always attributable to the introduction of a specific virus within the canal. The disease being thus produced, the excreta from the alimentary canal, that is, the matters vomited and discharged from the bowels, contain the special virus, and it is exclusively by means of this virus that the disease is propagated. According to this theory the disease is communicable, and never produced otherwise than by a virus generated within the alimentary canal of those affected. Dr. Snow accounted for the diffusion of the disease by supposing that the virus existing in the excreta of cholera patients is especially liable to be contained in water which is drank, and that it may be contained in the air and thus get into the mouth or nostrils, and thence into the stomach. He supposed that the virus in a dried state may adhere to clothing, and that the disease is in this way portable." The theory of Dr. Snow has been accepted by some with certain modifications. Thus, Thiersch considers that the excreta do not at once contain a virus, but that a special poison is produced by a fermentation or decomposition of the excreta, the process requiring several days and being favored by a high temperature. Again, Pettenkofer supposes that the choleraic excreta only furnish germs which are developed into activity in the soil, various conditions pertaining to the moisture of the soil, the temperature, etc., being favorable, or otherwise, to the production of the poison. It is. supposed that the specific matter giving rise to the disease is produced within the alimentary canal, not only in the cases of fully developed or well-marked cholera, but in cases of so-called cholerine, the latter, as well as the former, affection being caused by the same virus. This supposition, of course, involves the conclusion that the diarrhœa which prevails during the prevalence of epidemic cholera, that is, cholerine, is essentially choleraic.

The theory of Snow, with as well as without these modifications, has an obvious bearing on the employment of preventive measures, as contrasted with the doctrine that the special cause is a miasm the nature and source of which are not ascertained, the miasm travelling through the atmosphere, or carried in clothing and other material substances, or diffused by both these modes, and giving rise to the disease either by the co-operation of other causes acting upon those who are attacked, or by finding in certain situations circumstances favorable for its development and increase. According to this doctrine, the special cause being neither of the nature of a virus, nor a miasm generated within the bodies of cholera patients, the disease is not contagions nor infectious. It is, however, true that, accepting this doctrine, the disease is in one sense communicable; the miasm, although not a morbid product, may be transported in clothing. The portability in the manner of fomites may serve to explain the well-known fact that the disease in its progress over the world follows the routes of travel, and seems in its causation to depend sometimes on human intercourse.

After a careful reconsideration of the question of the communicability of cholera, it seems to me that this view of the causation is rendered untenable by the considerations which have been presented. The occurrence

1 Vide Reports on the epidemic Cholera, by Baly \& Gull, London, 1854. Exposition of Dr. Snow's Theory by Dr. B. W. Richardson, in London Med. Times and Gaz. Oct., 1865; and in Am. Journ. of Med. Sciences, April, 1866. 
of.numerous cases either simultaneously or in quick succession, at points so far removed from each other that there could not possibly be any communication; the rapidity with which the epidemic reaches its elimax, and the brief duration of the epidemic, are facts entirely irreconcilable with the theory which attributes the diffusion of the disease exclusively to a virus contained in the excreta, or to an infectious miasm.

Rejecting the doctrine which attributes the causation of cholera to a contagious or infectious material, the nature and souree of the special eause of this disease are undetermined. This is a reiteration of a statement which, at the present time, is to be made with respect to many of the special causes of disease. The doctrine that cholera, as well as muncrous other diseases, is caused by the introduction into the body of certain cryptogamic or infusorial germs, has heretofore had able advocates. ${ }^{1}$ liecently this doctrine has been revired, and zealous pathologists in different countries have engaged in researches relating to it. It has been adrocated especially by Prof. E. Hallier, of Jena, who has studied with great zeal the choleraic emanations with reference to the presence of germs and fungi, their supposed sources, and their production by artificial eultivation. Without entering into details, suffice it to say that faets, so far as at present ascertained, do not appear to offer a demonstration of this doctrine, although, rationally considered, it is perhaps most consistent with what is known of the eausation of the disease.

A uxiliary causes appear to be essential to the efficiency of the special canse. Epidemics are chiefly confined to large towns, rarely extending into rural districts. It is not probable that the speeial eause is limited to eities. The prevalence often of mild diarrhoa or cholerine throughout the country shows the wide diffusion of an epidemic influence; but the various local canses of disease peculiar to cities give rise to cholera either by acting in conjunction with the special miasm, the latter of itself being insufficient, or by acting as localized conditions which promote the derelopment of the special eause. This view of the causation has an important practical bearing. If the special eause of eholera cannot be ciestroyed, it may be rendered to a great extent inoperative by means of sanitary regulations which remove auxiliary causes. The epidemic prevails mostly in districts in which the hygienic conditions are most unfavorable, in filthy streets, and among the poorer elasses, in overcrowded clwellings. Statistical researches by the Registrar-General of Great Britain slow the mortality in London from this disease to be in an inverse ratio to the elevations. ${ }^{2}$ Deprivations, intemperanee, depressing emotions, over-exertion, fear of the disease, etc., are auxiliary causes in certain cases, asicle from the causes which affect the inhabitants of a place or district in mass. The disease has prevailed in all climates and seasons. Feeble persons are more apt to be attacked than the robust. As regards age, no periorl of life is exempt, but the largest proportion of fatal eases is among persons adranced in life. Excluding old persons, the mortality is largest after adult age, next in early infaney, and last in ehildhood and youth. This statement is based on an analysis of 18,400 eases. $^{3}$

Draanosis.-During the prevalence of cholera, there is no difficulty in recognizing it when fully developed, that is, when symptoms denoting

I See Holland's Medieal Notes and Reflections, and a treatise on the Cryptogamic Origin of Diseases, by the late Prof. J. K. Mitchell. In a résumé of later firets and opinions, vide Aiken's Science and Practice of Medicine, sixth cdition, 1872.

2 British and Foreign Med-Clirurg. Review, July, 1852.

3 Valleix, op. cit., tome i. 
existing or impending collapse are present. There is scarcely any other disease in which the diagnostic features are so strongly marked. But it is vastly important to recognize it before it has advanced sufficiently to manifest its most obvious, striking characters. The diagnosis is then to be based mainly on the characteristic intestinal evacuations. As soon as the so-called rice-water or choleraic stools are apparent, the patient is to be considered as attacked with cholera. In a very small proportion of cases these evacuations do not occur at all, or not until other symptoms characteristic of the disease become developed. The diagnosis in these cases must be based upon the latter.

Cholera is to be discriminated from simple diarrhœa, or cholerine, which generally precedes it, and which, during the continuance of the epidemic, affects a vast number of persons without eventuating in cholera. It is probable that this premonitory and wide-spread diarrhoa proceeds from the epidemic influence, but cases are not to be considered as cases of cholera when the choleraic discharges and other characteristic symptoms do not occur. This discrimination is important with reference to determining the rate of mortality, and the value of therapentical measures. The apparent success attencling the practice of some physicians, or the employment of certain methods of treatment, may depend on cases of simple diarrhœe or cholerine being reckoned as cases of cholera.

This disease is one of the most insidious in its approaches. Aside from a mild diarrhœa, it gives no warning of the attack. So little are patients conscious of langer, that they often apologize for giving the physician the trouble of visiting them, when a glance suffices to show that they are on the verge of collapse. It hehooves the physician, in all cases of simple diarrhcea during an epidemic of cholera, to watch carefully the evacuations with reference to the diagnosis. On the other hand, during an epidemic, physicians are not infrequently called to see persons who, under nervous excitement, imagine that they are about to be attacked, when no symptoms whatever of the disease are present. These have been aptly called cases of choleraphobia.

Proanosis.-Of those attacked with cholera, a large proportion die. The average mortality in hospitals varies from one-half to one-third. When developed in hospital wards among patients admitted for other diseases, it proves fatal in the great majority of cases. In private practice, especially among the better classes of society, the mortality is considerably less. In individual cases the prognosis is widely different according to the period of the disease at which the patient is first seen. If seen immediately after the attack, before serious blood-lesions have occurred, the prospect of an arrest of the disease is good. But if the disease have advanced to the stage of collapse, the prognosis is always exceedingly unfarorable. After reaction from the collapsed state occurs, the danger is still considerable, but the cases in which recovery takes place preponderate. The statistical researches of Duchesne show the rate of mortality at different periods of life in the epidemic at Paris, in 1849.' The proportion in 1000 , in subjects under 5 years, was 148 ; from 5 to 15 years, 50 ; from 15 to 30 years, 177 ; from 30 to 45 years, 254 ; from 45 to 60 years, 206 ; and from 60 to 85 years, 162. The mode of dying is, in general, typical of asthenia. Accumulation of liquid in the air-tubes very rarely takes place. 


\section{Prevention of Cholera.}

The prophylaxis of cholera claims consideration under a distinct heading, and I give it precedence over the therapentical management, in view of its greater relative importance. In addition to the removal, as far as possible, of all the anxiliary causes of disease which contribute to render the special cause of cholera efficient, the prevention involves prompt attention to the diarrhœa, which, in the great majority of cases, precedes the attack. 'This premonitory diarrhœa is amenable to simple measures of treatment, and, if effectually treated, there is reason to believe the superrention of cholera is prevented. All physicians who have had much practical aequaintance with this disease will bear testimony, first, to the fact that an attack of cholers is generally preceded by diarrhœa, and, second, to the fact that an attack very rarely oecurs when this diarrhoa receives appropriate attention. Giving the results of my own experience with respect to the facts just stated, in 1849 , for the spaee of three months, I prescribed for as many private patients with the premonitory diarrhoea, or cholerine, as my physieal enduranee would permit, my praetice being chiefly among the prurlent elasses, and I had, during this epidemie, but 10 cases of cholera in private practice. In only one of these 10 eases did I prescribe for any premonitions, and, in the single case, the prescription was for a slight nausea only. In each of the 10 cases, either the premonitory diarrhcea did not exist, or it was negleeted. During another epidenic in 1852 , I had about the same number of eases of cholera in private practice; in not a single ease had I been ealled upon to prescribe for premonitory diarrhœa, and I preseribed for hundreds of persons with simple diarrhwea, not one of whom had an attack of cholera. The experience of others would doubtless furnish, in like manner, evidenee of the above stated facts, and from these facts the following eonclusion may be drawn: $\mathrm{Ex}-$ cepting the very small proportion of cases in which eholera is not precerded by diarrhœa, it may with almost absolute certainty be prevented. It needs but a little reflection to see the immense practical importance of this conclusion.

The treatment of diarrhœa during a cholera epidemic is very simple. An anodyne astringent remedy, with regulated diet, rest, and recumbency, suffiee. A few drops of landanum, with camphor, or the camphorated tineture of opium in conjunetion with the tineture of kino, catechn, or krameria, a salt of morphia or opium in pill or powder with tannic acirl, the acetate of lead, or bismuth and capsicum, may be given in doses sufficient to arrest at once, and repeated often enongh to prevent recurrence of, the diarrhøa. Cathartics are on no account to be given. If there be sufficient constipation to occasion inconvenience, the bowels should be relieved by simple enemas.

The great difficulty, as regards the prevention of cholera, is in the prompt applieation of simple but effectual treatment in all eases of diarrhoea during the epidemie. Very many pay no attention to the premonitory diarrhoea, throngh ignorance, and not a few of those better informed neglect it, either from reeklessness or because they cannot appreciate the fact that a disorder so slight and ordinary can be a precursor of a malady of such gravity as cholera. Public announcements by handbills, articles in newspapers, etc., of the importance of promptly resorting to treatment for diarrhœe, fail in accomplishing the object. The only effeetual plan is to organize a sanitary police, and provide for one or two domiciliary visits daily at every house within the limits of the epidemic, the purpose of the visits being to inquire if any one be affected with diarrhou, to impress 
the importance of immediate attention to it, and, when circumstances rencler it necessary, to supply at once appropriate remedies. This plan, faithfully carried out, would, I believe, demonstrate that cholera is in a great measure preventable. The prevention is effected by arresting at once diarrhœa, as often as it occurs and as soon as it occurs. A bundant proof of the successful operation of the system of house-to-house visitation was furnished by its practical results in London and other towns in Great Britain during the epidemic of 1849 .

Another mode of escaping the disease is to remove without the range of its prevalence. Persons not compelled to remain by necessity, or by a sense of duty, should go beyond the limits of the epidemic; and the removal of persons in districts, where, owing to the activity of auxiliary caluses, the disease is especially rife, should be enforced, as a sanitary metsure, by municipal authority.

The foregoing remarks relate to the prevention of cases of cholera during an epidemic visitation. To prevent the visitation of the epidemic is an object of still greater importance. In this connection the inquiry at once arises, Can the introduction of the disease be prevented by a rigid quarantine, and the suspension of intercourse with places in which the disease prevails? At the present time, neither the affirmative nor negative answer can be sustained by an appeal to facts sufficient to carry the weight of proof. The question is a mooted one, and it is not consistent with the plan of this work to enter into a discussion of it. In view of the fact that many hold to the doctrine which attributes the propagation of the disease to a virus, and of the fact that, assuming the special cause to be a miasm generated without the body, this miasm is probably portable, that is, liable to be transported from place to place in ships, clothing, merchandise, etc., the propriety of adopting certain measures of protection is hardly to be questioned. Ships with cases of cholera on board should be detained at quarantine, and proper disinfecting means resorted to. But the propriety of detaining well persons after thorough ablution, and a change or the disinfection of clothing, may fairly be doubted. Similar precautions, to be complete, should, of course, be extended to communications by land with places in which the disease prevails.

In the absence of positive knowledge, and of unanimity of opinion, respecting the nature and source of the special cause, the employment of disinfectants in the localities in which the disease breaks out is also to be advised. Of all measures first in importance are those which secure, to the fullest possible extent, ventilation and cleanliness. These are not to be supplanted by any or all of the disinfecting articles which are supposed to destroy the special cause by chemical action. Recent experimental observations, however, seem to show that the latter are of vast importance. The agency of disinfectants generally depends on their power to oxidize or deoxidize the substances with which they come into contact. Certain of them are supposed to act by destroying microscopic organisms, either animal or vegetable, and by arresting catalytic or fermentative processes. For disinfecting the atmosphere, chlorine has long been considered a most efficient agent. Ozone, eliminated by means of phosphor'us, is another efficient gaseous disinfectant. Bromine'belongs in this category. For the disinfection of clothing, the alvine excreta, etc., the compounds of chlorine, creasote, carbolic acid, the permanganates, the sulphite and hyposulphite of soda, the salts of iron and of lead, are to be named. These substances enter into various preparations which have been named after those introducing them, such as Condy's disinfectant, Darby's fluid, Le Doyen's disinfecting fluid, etc. It is generally 
agreed that dry heat is one of the most reliable of disinfecting agencies. A temperature of from $200^{\circ}$ to $260^{\circ} \mathrm{Fahr}$. is supposed to destroy the special cause of this and other epidemic diseases. For general disinfectant purposes on a large scale, charcoal and quicklime are sufficiently reliable, and, from their cheapness, readily available. ${ }^{1}$

of the measures to prevent the origin and diffusion of cholera, those relating to public health, irrespective of the destruction or arresting the introduction of the special cause, are of the first importance. There is reason to believe that sanitary measures which remove the ordinary causes of disease will prove effectual in preventing this epidemic. Whatever may be the nature and source of the special cause, auxiliary causes seem to be hardly less essential for the production of the disease. Whether the latter act by affording a pabulum for the development and increase of the special cause, or by impairing the ability of the system to resist the action of this cause, we cannot say ; but our ignorance of the true explanation does not render, practically, less important the fact that this epidemic, as a rule, prevails in localities where the ordinary causes of disease are rife. 'The causes which are most efficient as co-factors are those incident especially to cities or large towns, camps, and all places in which human beings are densely congregated. Deficient sewerage, cesspools, accumulations of filth of every description, overcrowded and unventilated dwellings, underground habitations-these are among the causes affecting public health, which, in some way co-operating with the special cause, lead to the rise and spread of cholera as of other epidemic diseases. 'These causes may be to a great extent removed by efficient sanitary measures, and epidemics in this way are either warded off or their prevalence greatly curtailed.

The efficacy of preventive measures in arresting the spread of cholera seemed to be strikingly exemplified in the history of the epidemic in New York in 1866 and 1867.

In anticipation of an epiclemic visitation, in 1866, the administration of affair's relating to public health was vested in five sanitary commissioners, four being distinguished members of the medical profession. Dr. E. Harris, widely known by his labors in behalf of sanitary science, was appointed Registrar of Vital Statisties; the late Dr. E. B. Dalton was selected as the chief executive officer, with the title of Sanitary Superintendent; and medical inspectors were employed for the different precincts.' The measures adopted with a view to arrest the spread of the disease were as follows: The removal, as fár as possible, of all localizing causes, the transference of patients to hospitals established in convenient situations, and a prompt resort to efficient disinfection wherever the disease might appear. Under the direction of the able and energetic superintendent, these measures were thoroughly carried out, and the citizens of New York have much occasion for gratitude in the result. As bearing on questions of vast importance in relation to the etiology and prevention of the disease, the ficts contained in the subjoined report of the sanitary superintendent, which

' Vide articles by Dr. Edward R. Squibb, in " The Medical Record," New York, May 1st and 15th, 1866, for details (which cannot be here introduced) respecting the relative value of different disinfectants, their mode of action, and the node of their employment ; and an article by Dr. C. A. Lee, in the same Journal, May 15, 1866. Vide, also, an able review, by Prof. Roberts Bartholow, in the American Journal of Medical Sciences, July, 1867; and articles by Dr. Elisha Harris, in "Military Medical and Surgical Essays," editcd by Dr. Hammond, and in Report of Metropolitan Board of Health, New York, for 1867. 
was kindly furnished at the author's request, are highly valuable. These facts show, in the first place, the development of cholera in different, widely separated situations, under circumstances which render untenable the doctrine of diffusion by means of a virus or miasm derived from the bodies of those affected with the disease; in other words, they show that the breaking out of the disease in different places was not due to the dissemination of a special material contained in the excreta, or to any other mode of communicability. 'This conclusion is not inconsistent with the portability of the special cause of the disease, nor is it incompatible with an admission of the possibility of the disease being in some cases communicated. The facts show the development of the special cause in connection with the operation of localizing causes, and, in this respect, the facts accord with those observed during the prevalence of the disease at other times and places.

In the second place, the facts contained in the subjoined report go to show that the special cause of the disease may be destroyed by disinfecting agencies. It is in this point of view that the facts are especially valuable. They warrant the belief that, by eflicient sanitary regulations as regards the ordinary causes of disease, and by resorting to means adequate to destroy the unknown special cause as soon as cases occur in any locality, the epidemic prevalence of cholera may be restricted within narrow limits.

The following report by Dr. Dalton embraces statistics of the disease in New York and Brooklyn :-

"The first case of cholera in New York occurred on the first of May, the second on the following day, and a third on the sixth (1866). Subsequently no case occurred until the fourth of June, since which time there have been more or less cases every day.

Number of cases in New York outside of public institutions, to Sept. 22d . 751

Number of cases in New York in public institutions, to Sept. 22d . . . . 421

Number of cases in Brooklyn outside public institutions, to Sept. 15th . $\quad$. 6.0

Number of cases in Brooklyn in public institutions, to Sept. 15th . $\quad . \quad 87$

"At first the cases occurred in localities widely separated, but, as the season advanced and the cases became more frequent, they occurred, not successively in the same localities, but, with comparatively few exceptions, within the limits of various circumscribed localities, which latter were themselves widely separate from each other, the result being, that individual cases were proximate, while the groups were widely separated.

"These localities, within the limits of which the mass of cases have occurred, were, without exception, insalubrious. A few isolated cases have occurred where such was not the fact.

"The original development of the disease was not distinctly traceable to any particular emigrant passenger, nor any particular lot of baggage or merchandise ; but it followed the arrival in our harbor of infected emigrant ships.

"There is no evidence that the disease was carried from one locality in the city to another, excepting that individuals have left an infected locality apparently well, and subsequently died of cholera in an uninfected locality, but without infecting other persons. It seems probable that the disease occurred in each locality independently.

"Experience here furnishes evidence that the dejections are one means of propagating the disease, instances having occurred where persons have been taken with it soon after having washed bedding, etc., soiled by a cholera-patient; but such persons had heen subjected to the same original causes as the one by whose dejections they appeared to become infected. 
The inass of evidence, however, is negative, depending upon the apparent controlling influence of disinfection of privies, vessels, and material used by cholera-patients.

"Every house in which the disease has been known to exist has been disinfected. Sulphate of iron in saturated solution, or scattered dry in wet places, has been used for privies and all vessels containing clejections. Solution of permanganate of potassa_one ounce to tive gallons-for bedding and elothing, the latter being boiled in such a solution for two hours and then reboiled and washed in pure water. For purifying the atmosphere, chloride of lime has been freely scattered about the floors, and often chlorine has been slowly set free in the room of the patient by adding sulphuric acid to a mixture of chloride of sodium and binoxide of manganese. Where entire fumigation of the house has been necessary, it has been done either by chlorine rapidly set free by adding sulphuric acid to chloride of lime, or by sulphurous acid set free by burning sulphur.

"Disinfection of the immediate surroundings of the patient has frequently been apparently sufficient, though in many instances a second and sometimes a third ease has oecurred in the same house.

"Fumigation, either with chlorine or sulphurous acid gas, has, with two exeeptions, been followed by complete immunity from the disease. In one of the exceptions a case occurred some five weeks subsequent to the fumigation, and in the other five days.

"No conficlence is felt in any particular line of treatment for the control of the disease. The ordinary means for alleviating the suffering of the patient have been used with suceess, and the subcutaneous injection of morphia has been efficacious in preventing the recurreuce of eramps."

The eflieacy of the preventive measures employed in the manner described in the foregoing report is confirmed by the experience of the subsequent year, viz., 1867. 'The facts relating to the disease in this year are concisely stated in the following extract from the Report of the Board of Health: "' Twenty-seven deaths from cholera have occurred during the past year (1867) in the city of New York, five in the city of Brooklyn, and eighteen at the military post in the harbor. The disease has not exeited public alarm, and never approached the character of a general epidemic. 'The means used to combat it were the same as those employed in the year' 1866. Wherever a case occurred, the Disinfecting Corps cleansed the buildings, or fumigated them with sulphur, and destroyed the clothing if it conld not be lisinfected by boiling water or the permanganate of potash. The experience of another year has confirmed the opinion that active interference, by means of disinfectants, is the surest way to control and arrest this terrible disease."

The effieacy of sanitary measures and disinfection in promptly arresting the prevalence of eholera was strikingly exemplified by the history of an outbreak of the disease at the workhouse on Blackwell's Island in 1866. The measures were instituted by my colleague, Prof. F. H. Hamilton; and the following are the facts contained in the history as given in a letter to the Registrar of vital statisties: The first case occurred in the workhouse July 28th, and the last case was on August 6th. During these nine days of about 800 inmates 123 died. The explanation of the rapid propagation and fatality of the disease was believed to be mainly continement and crowding. Prof. Hamilton undertook to drive out the disease in from three to five days, and sueceeded by means a summary of which is contained in the following extract:-

"T'The inmates were distributed as far as the vacant places in the build- 
ing would permit; the cell-doors were left open at night; the nightbuckets were supplied with disinfectants and left outside; the women's cooking-rooms were converted into hospital wards, and the women were kept out-of-doors from morning until night; corn-meal and molasses were taken from the diet-table; coffee, tea, and vegetables were added; at night each inmate was required to take, whiskey one ounce, water three ounces, tincture of capsicum fifteen drops. [These people are our eity vagrants, and probably are habitually intemperate.] A variety of disinfectants were employed freely and constantly in every vessel and closet which received the excreta; even the excreta from the stomach were disinfected immediately after they were received into a vessel or fell upon the floor; stoves were placed in each hospital ward to insure a draught; all windows were kept open day and night; the clothing taken from cholera patients was sent directly to the boilers; a ward was established for patients with the diarrhœa, and the value of this measure is shown by the fact that of the large number received into this ward only one died. It was difficult, however, to persuade these poor creatures to report themselves at this stage of the disease."

"Of fourteen house-physicians and surgeons employed in the several buildings, some of whom have been in constant attendance upon the sick, not one suffered from the epidemic."

Finally, with reference to the prevention of cholera, facts warrant the belief that, by disinfecting ressels with cholera patients, the prompt disinfection of the places in which successively the disease breaks out, the iminediate arrest, by simple measures, of diarrhœa, the accomplishment of the latter object, in all cases, being secured by house-to-house visitations, and the efficient carrying out of sanitary measures for the people at large, together with proper attention to personal hygiene, epidemics may be effectually controlled.

Treatment.-To consider the host of remedies and therapeutical measures which have been advocated as more or less efficacious in the treatment of this disease would require not a little space. There are but few articles in the materia medica which have not been tried, even including antimony and drastic purgatives. Much injury has doubtless been done by over-medication under the idea that the treatment, as regards activity, must be proportionate to the amount of danger from a disease. On the other hand, many of the remedies which have been employed exert little or no effect either for good or harm. During the epidemic of 1849 , a writer in one of our medical journals gravely announced as a remedy, hogs' bristles or the hair from a cow's tail burned to a cinder! It would be unprofitable to devote space to the consideration of the great variety of practice which the literature of cholera affords. There is no known remedy which is to be considered as a specific, yet there is reason to believe that the disease is frequently controlled by efficient treatment, and, when not arrested, the recovery may depend on the judicions employment of measures for that end. I shall limit myself to a brief statement of the principles of treatment which my own experience and reflections have led me to regard as most consistent with our present knowledge.

The treatment is to be considered as applicable to the different stages, viz., before collapse, during the collapsed stage, and after reaction. Prior to collapse, the paramount object is the arrest of the intestinal effusion. This effusion is the first appreciable link in the chain of morbid sequences, 
and, if promply arrested before it has proceeded so far as to affect seriously the blood and circulation, the patient is usually safe. The remedy on which most dependence is to be placed in effecting this object is opium. Some form of opiate is to be given promptly in doses sufficient to effect the object. The form of opiate is to be chosen with reference to promptness of action and the probability of its being retained. Opium in substance is unsuitable from the comparative slowness with which it is absorbed. Laudanum, the acetated tincture, or an aqueous preparation, is to be preferred. But the article which I have been led to regard as the most eligible is a salt of morphia, administered by placing it dry upon the tongue. In the endeavor to effect the object of treatment in this stage, moments are precious, for there is always danger that, if the object be not promptly effected, the patient will fall into the collapsed state. The opiate should, therefore, be given at once in a full dose. A grain of a salt of morphia is rarely, if ever, too large a dose for an adult. A physician should, if possible, remain with the patient. If the first dose be quickly rejected, a second should be instantly given. The doses are to be repeated at intervals of from half to three-fourths of an hour, until the dejections and borborygmi cease. If, owing to the occurrence of vomiting, the administration by the mouth be ineffectual, it should be given by the rectum; and in cases in which the symptoms are urgent, both modes of administration should be resorted to. The system, even in this stage of the disease, is not readily affected by opiates thus given. In view of the importance of the object, if it be necessary in order to effect it, some risk of inducing narcotism is justifiable; but if the administration be in the hands of the physician, and the effects of the closes watched with care, danger from this source may generally be avoided. The practical point is to employ the remedy freely and promptly so as to effect the object, bearing in mind the fact that the delay of half an hour or an hour is often fatal. Relying upon the opiate, it is best not to add other remedies, lest by increasing the bulk of the doses they will be more likely to be rejected. A full dose is preferable to small doses frequently repeated, because the effect within a short space of time is greater, and the remedy is more likely to be retained. Aside from the rejection of the remedy, vomiting is, if possible, to be prevented in view of its purtubatory effeets. The patient, in this stage, should be restricted to a very small quantity of water, or spirit and water, given at short intervals, or to small pieces of ice. Perfect quietude is important. He should not be permitted to get up to go to stool, and he should be urged to resist, as much as possible, the desire to evacuate the bowels. Frictions, the warm bath, sinapisms, etc., in this stage, are of doubtful expediency.

I have repeatedly succeeded in arresting the disease by this plan of treatment, and when arrested before proceeding to the stage of collapse, the recovery is usually speedy. Regulated diet, rest, with perhaps a tonic remedy, suffice for the cure. The bowels should be allowed to remain constipated for several days, and then, if movements do not spontaneously occur, simple enemas will probably be sufficient; if not, a little rhubarb or some other mild laxative may be given. I believe no other plan of treatment promises more than this, but it is not to be expected that it will always prove successful. It will fail, or rather it is not available, when, owing to the persistent romiting and frequent purging, the remedy is not retained sufficiently long to exert its effect; and it is not available when, owing to the great rapidity of the transudation, the state of collapse occurs so quickly that there is not time enough to obtain a remedial effect. These difficulties are equally in the way of success from any remedies. 
- The foregoing remarks on the treatment prior to the phenomena of collapse were written before the hypodermic method of administering opiates and other remedies had come into vogue, and the inquiry has arisen whether, by means of this method, the opiate plan of treatment of cholera, in the first stage, may not be carried out more efficiently than by giving opiates by the mouth or rectum. Considering the vast importance of the promptness of the effect of opiates, and the uncertainty attending their administration by the mouth or rectum, owing to their rejection by vomiting or purging, and the difficulty of absorption if they be retained in the alimentary canal, the hypodermic method seems to offer a great advantage as regards speediness and reliability of effect. The hypodermic injection of Majendie's solution of morphia was employed in a large proportion of the cases which came under my observation in 1866. The vomiting, purging, and cramps were generally arrested promptly by this measure; recovery, however, followed in a very small proportion of cases. So far as my experience goes, it affords evidence of the efficiency of the measure with regard to the immediate objects, viz., the arrest of the vomiting, purging, and cramps, but it does not afford proof of its curative efficacy. With regard to the latter, the character of the patients is to be borne in mind, nearly all who were seen in the first stage being inmates of the hospitals, and many being affected, when attacked with cholera, with some serions chronic disease such as phthisis, Bright's disease, etc. The value of the hypodermic method of employing the opiate plan of treatment, as a curative measure, remains to be ascertained by a more extended clinical experience embracing a more favorable class of cases. The hypodermic injection of a solution of morphia, in this disease, is to be employed with circumspection. Narcotism is sometimes induced by an amount much below that which might be given by the mouth without risk. I have known a little less than a grain, given in two injections, with an interval of two hours, to produce deep narcotism. The tolerance of opiates in large doses by the stomach or rectum is not a criterion of the quantity to be injected beneath the skin in epidemic cholera.

Injections of brandy and a strong tea-infusion were used considerably, with apparent efficacy in arresting the evacuations and preventing collapse. The proportions used were half an ounce of brandy and two ounces of the tea-infusion, the injections being repeated every half hour, every hour, or after longer intervals, according to circumstances.

In the stage of collapse, the plan of treatment indicated prior to this stage may prove not only ineffectual, but hurtful. . It is still an object to arrest intestinal transudation, if it continue; but to employ opiates very largely for this object may not be judicious with reference to the recuperative efforts of the system. 'The symptoms in this stage are due, mainly, to the damage which the blood has sustained in the loss of its constituents from the transudation which has already taken place. Opiates may be given, and, owing to the remarkable degree of tolerance under these circumstances, they may be given in considerable doses, but much care should be observed not to induce narcotism. They should be given by either the mouth or the rectum; never, in this stage, by the hypodermic method. Astringent remedies, if the stomach will retain them, may be added, such as tannic acid, the acetate of lead, bismuth, etc. If, however, these or other remedies provoke vomiting, they will be likely to do more harm than good. Remedies to allay vomiting may be tried, viz., the hydrocyanic acid, creasote, and chloroform. Niemeyer extols the application to the abdomen of compresses wet with cold water. 
In a large proportion of cases, after collapse has taken place little can be done with much hope of success. Even if the vomiting and purging cease, recovery may not follow. The blood may have been damaged irremediably. Under these circumstances it is plain that active treatment can effect nothing. Recovery, however, in a certain proportion of cases, takes place, and under a great variety of treatment. It may take place when no treatment is pursued. My first case of cholera, in 1849, illustrated the fact just stated. The patient was brought into hospital completely collapsed. I remained with him several hours, and resorted to various therapeutical measures. At length all measures were discontinued. He was allowed to drink abundantly of cold water, under the impression that the case was utierly hopeless, and, therefore, the indulgence could do no harm. Much to my surprise, after an absence of several hours, I found the vomiting and purging harl ceased and reaction was coming on. He recovered rapidly. I have been led to doubt whether, in general, active treatment effects much for the advantage of the patient in the collapsed stage of cholera, and I camnot donbt that it is often prejudicial. The object of treatment in this stage, aside from the arrest of vomiting and purging, is to exeite and aid the efforts of nature in restoring the cireulation, together with the functions dependent thereon. The measures to be employed for this object are external heat, stimulating applications to the surface, diffusible and other stimulants, and alimentation.

The application of heat may be made by means of warm blankets or bottles of hot water placed near the body. The more active modes of applying heat are of doubtful propriety. I have never seen benefit from the warm bath, or the application of steam or hot air. It is not desirable to excite perspiration, and, if perspiration occur, it should be wiped away with warm dry cloths. Violent friction does more harm than good. The surface may be gently stimulated with sinapisms or the tincture of capsicum. Diffusible stimulants, in the form of spirits and water, should be giver as freely as the stomach will bear, always reeollecting the risk and the evils of inducing vomiting. It will be most apt to be retained, if given in small quantities at a time, and often repeated. If vomiting be provoked by either drinks, remedies, or aliment, more or less injury is done. The ethers, stimulants such as capsicum, the essential oils, cardamom, ginger, etc., are appropriate if they be grateful to the stomach and retained. Concentrated nourishment-essence of meat, ehicken-broth, and milk-is to be given in small quantities at a time, provided the stomach will retain it. It is doubtless desirable to introduce liquid into the system as far as possible. 'The only objection to the free ingestion of water is the risk of provoking vomiting. Small lumps of ice should be freely allowed.

If the patient emerge from the collapsed state, the indications are to support the system by the moderate use of stimulants and by alimentation; to restore the function of the kidneys by diuretic remedies and mucilaginous drinks, bearing in mind that uræmia belongs among the dangers of this stage; to restrain diarrhœa, if it occur, by anodynes and astringents ; to strengthen by tonics, and to palliate, by appropriate remedies, the various symptoms which may arise. 


\section{CH A P T E R I X.}

\section{INTESTINAL WORMS.}

Ascaris Lumbricoldes. Ascaris or Oxyuris Vermicularis. Tricocephaius Dispar. Anchylostoma Duodenale. Ascaris Alata. Tæniæ. Trichina Spiralis.

$\mathrm{O}^{\mathrm{F}}$ the various entozoa, helminthi, or parasitic animals which dwell within (f the human body, several are developed in the intestines. They give rise to more or less disturbance, and are properly enough considered in connection with the functional disorders of the alimentary canal. I shall treat of intestinal worms only in so far as concerns the practical duties of the physician. The study of these and other parasites, as belonging to comparative anatomy and physiology, and as a branch of zoölogy, is highly interesting, and has led to valuable practical results, but other points than those which relate directly to them as morbid conditions would be here out of place.

The intestinal worms of frequent occurrence are the ascaris lumbricoides, lumbricoid or round worm, and the ascaris vermicularis, thread or seat worm. The worm known as tricocephalus dispar is probably sufficiently common, but of little or no practical importance. The different species of tænia, or tapeworm, are of rare occurrence in this country, but constitute an important affection. These different worms will be noticed under distinct heads. And, as appropriately classed among the intestinal worms, because they are received and developed within the alimentary canal, the trichina spiralis will be considered in this connection.

\section{Ascaris Lumbricoides.}

With this worm every young physician soon becomes familiar. It resembles the common earth-worm. It is the most common of all the intestinal worms. It varies in length from six to sixteen inches. It is of a whitish or yellowish color; the body is round, tapering toward each extremity, and the mouth is surrounded by three tubercles. The sexes are distinct.

The common lumbricoid worm inhabits the small intestine. It rarely exists in early infancy, and is most common between the ages of three and ten. It is not very uncommon in adults, but is extremely rare in old age. This species of worm is not usually solitary. In different cases the number of lumbrici varies greatly. Sometimes the number is very great, and in antopsical examinations they are occasionally coiled together so as to form balls or masses of considerable size. Cruveilhier cites a case in which over a thousand were found after death. They are not infrequently brought into the stomach by acts of vomiting and ejected from the mouth. They may find their way into the nares, and they have been known to pass into the Eustachian tube, frontal sinus, and larynx. They may migrate from the intestine into the biliary ducts and gall-bladder. In a subject dead with lumbar abscess connected with caries of the vertebræ, I found a great number within the hepatic ducts, some extending along the ducts, and others coiled up. 'They may possibly give rise to hepatic abscess. They may penetrate into the pancreatic duct. They have been found in 
the peritoneal cavity and in abscesses within the abdominal walls. In these cases they doubtless escape through perforations caused by ulceration or sloughing incidental to other morbid conditions; it is not probable that the worms ever perforate the intestines, as was formerly supposed. They have been observed to escape from the body through fistulas communicating with the intestinal canal.

The symptoms denoting the presence of these, or other worms, within the intestinal canal, are obscure. A host of symptoms having little or no signifieanee have been enumerated by writers. Nothing is more common than the passage of lumbriei from the stomach or bowels when there had been no symptoms of disorder. The morbid effects have heretofore been greatly exaggerated. These worms, it is probable, very rarely give rise to serious results except when they migrate into the biliary or pancreatic duets, the Eustachian tube, or the larynx, and these migrations are exceedingly infrequent. There is no foundation for the belief that they give rise to a form of fever, as implied by the term worm fever. They are not infrequently, in cases of fever, passed from the bowels, and fonnd in the intestines after death; but their presence is merely a coincidence. 'That they may give rise to convulsions, epileptic paroxysms, chorea, and other affections of the nervous sy.stem, as is generally supposed, must be considered as by no means established.

Symptoms generally regarded as denoting these worms are colic pains, tumefaction of the abdomen, impaired appetite, excessive flow of saliva, diarrhca, iteling of the nose, swelling of the face, offensive odor of the lireath, disturbed, dreamy sleep, and grinding of the teeth during sleep. It is never prudent for a physician to declare positively that a child between three and ten years of age has not worms, for he is liable to be convieted of error by the operation of a vermifuge given without his sanction; but the only readily available diagnostic proof is the presence of one or more in the stools. When more or less of the above-named symptoms are present, a brisk purgative may be given, and the stools examined, with referenee to diagnosis. If one or more worms be passed, it is proper to act as if there were others remaining. According to Davaine, a microscopical examination of feces will show the characteristic ova, if lumbrici be present. He states that their ova are readily recognized, and hence the diagnosis may be made positive in this way. Practitioners in general, however, will be satisfied to base the diagnosis upon other facts.

The lumbricoid worm is propagated by ova. 'The body of a female lumbricus contains a vast number of eggs, estimated by Eschricht to be sixty-four millions. These ova are discharged with the feces. The observations of Davaine and others show that their vitality is retained for many months. Their ingress into the alimentary canal is probably by means of water containing them; and, finding in the small intestines favorable conditions, the production of the worms takes place. That special conditions are required may be inferred from the fact that these worms are so common in certain periods of life, and so infrequent at other periods. As regards the nature of the conditions, we have no positive knowledge; mucus in abundance is supposed to be the nidus for their production.

The expulsion of lumbricoid worms is generally effected without difficulty. Premising a brisk purgative, which is sometimes alone sufficient,

1 Traite des Entozoaires et des Maladies Vermineuses de l'Homme et des Animaux Domestiques, 1860 . The reader is referred to this work as treating of entozos minutely and comprehensively. 
an anthelmintic remedy is to be given for several successive days. Of the various anthelmintics, those best suited for the destruction of these worms are chenopodium or wormseed, santonin, and spigelia or pinkroot. Santonin is most easily administered, and perhaps as effective as the other articles. The dose is from 3 to 6 grains, repeated three or four times daily, given either mixed with sugar, or made into dragées or troches. The oil of wormseed is the most convenient form of this remedy. From 5 to 10 drops may be given three times daily mixed with sugar or in an emulsion. The pinkroot has long been relied upon as an efficacious remedy. The infusion may be used, but the fluid extract of spigelia and semna is the most eligible form, the dose being from 1 to 4 drachms three times daily. Tenacetum or tansy, and absinthium or wormwood, are doubtless efficacious remedies. All these remedies act toxically, that is, by poisoning the worms. After two or three days, a brisk purgative should be given. If with this treatment no worms be discharged, their non-existence may be inferred. If worms be brought away by this treatment, it need not be continued or repeated unless, from the continuance or recurrence of symptoms denoting disorder, it be inferred that their expulsion is not complete. The absence of the ova in the stools, according to Davaine, may be relied upon as proof positive of the absence of the worms.

With a view to preventing the ingestion of the ova of lumbrici, attention should be directed to the purity of the water used as drink. Water from shallow wells in the neighborhood of dwellings and small rivulets should be avoided. Filtration would probably prove effectual in the way of prophylaxis. Davaine states that it is not common in Paris for ehildren and others to be affected with this worm, a fact which he attributes to the custom of using filtered water.

\section{Ascaris Vermicularis.}

This worm, called also oxyuris vermicularis and commonly known as the thread, pin, seat or maw-worm, inhabits the large intestine, and chiefly the rectum. It very rarely migrates into the small intestine. The size is very small, as indicated by certain of the names in common use; the length varies from two to five lines. The vermicular ascarides frequently exist in immense numbers, and they are sometimes voided in large masses or balls agglomerated by mucus. They occur chiefly in young children, but they are not extremely uncommon at all periods of life. I have met with several examples of their occurrence in aged persons.

These worms occasion pain in the rectum, tenesmus, and, more especially, pruritus at the anus. The latter symptom, which is often due to an herpetic eruption in this situation, should always excite suspicion of the presence of these parasites. The itching is apt to occur in paroxysms, and is particularly troublesome at night. Excitation of the sexual organs is apt to occur with seminal emissions, and it is stated that the habit of masturbation, in either sex, may originate from this source. In girls the worms sometimes migrate into the vagina, and occasion pruritus with leucorrhœa. The diagnosis is made without difficulty. On examination of the stools, the worms are seen, in greater or less numbers, adhering to the feces; and on an ocular inspection of the anus they may generally be discovered. It is doubtful if they ever lead to any serious disorders, but, owing to the irritation which they occasion, and loss of sleep, the general health may become more or less impaired. 
The treatment is simple and efficacious, but it may require a certain amount of perseverance. The same anthelmintics may be employed as for the destruction of the lumbricoid worms, and given in the same way. Purgative remedies are to be given from time to time. In addition, enemas of cold water, or salt and water, are to be administered once or twice daily. These are to be continued for a fortnight, or longer, until all the local symptoms disappear. The treatment by enemas will frequently alone prove effectual. Varions remedies in enemas have been advised, such as wormwood, vinegar, empyreumatic oil, assafœtida, camphor, sulphuret of potassa, etc., but it is very rarely the case that simple water or salt and water, in conjunction with the other measures, will not suffice. Injections of a weakly solution of earbolic aeid can hardly fail to prove efficacions. The irritation or slight inflammation about the anus, which is sometimes caused by the presence of these worms, may be relieved by the application of a soothing unguent, or the daily injection of a little almond or olive oil.

\section{Tricocephalus Dispar.}

This worm, as the name tricoeephalus denotes, is characterized by the hair-like appearance of the ceplialic extremity. It is from an inch and a half to two inches in length. The capillary portion forms about twothirds of its length. As in the lumbricoid and vermicular species, the two sexes are distinct. It inhabits the creum especially, but is sometimes found in the colon, and, very rarely, in the small intestine. The propagation is by ova, which, according to Davaine, are readily recognized by means of the microscope in the dejections. The parasite is found frequently, when sought after, in the bodies of subjects of all ages dead with different diseases. Some years ago, when engaged in examining bodies dead with typhoid fever, I was led to examine the cæcum for it, and I rarely failed to find one or more. It is not positively known that its presence gives rise to disorder. Barth, of Paris, has reported a case in which death took place with the symptoms of meningitis, and after death no lesions were found within the head, but the intestimes contained an enormous quantity of worms of this species.'

The foregoing species of intestinal worms belong in the class styled by helminthologists, helminthes nematoides, or cylindrical worms. Belonging to this class is a worm called anchylostoma, or sclerostoma duodenale, which has been observed in Milan and Egypt. Existing in the duodenum in great numbers, it has been supposed to give rise to the affection known as Egyptian chlorosis, by piereing the walls of the intestine and occasioning hemorrhage, from the great number of minute punctures, sufficient to produce anæmia. With our present knowledge, this parasite has no practical importance in this country. ${ }^{2}$ Another worm belonging to this class was deseribed by Bellingham under the name ascaris alata, ealled also ascaris mystax, and said to infest the domestic cat. ${ }^{3}$ This, as regards its practical importance, is in the category with the tricocephalus clispar.

1 Valleix, op. cit.

Tide Davaine, op. cit.

Aitken's Science and Practice of Medicine. 


\section{Tæniæ or Tapeworms.}

On the natural hisiory of tæniæ, constituting the class cestoidea, in helminthology, and commonly known as tapeworms, much light has recently been shed by the researches of Küchenmeister, Von Siebold, and others. The sources of these parasites seem to have been ascertained. Referring the reader to other works for a full account of these researches, suffice it to say, that different varieties of tænia have been shown to be the cystic entozoa known as cysticerci and echinococci, in a state of maturity or complete development. A cysticercus is an immature or undeveloped tapeworm. Different varieties of cysticerci are found in the solid parts of different animals, and each variety, received into the alimentary canal of another animal, and finding there favorable conditions, becomes a perfect worm or tænia, each kind of cysticereus becoming a particular kind of tænia. A cysticercus, on the other hand, is the product of the ovum of a tænia. The eggs of the tænia, passing out of the body, finding their way into the bodies of men and other animals, and probably entering into the circulation, are transported to, and fixed in, solid parts, where they become cysticerci. The latter, so long as they remain in solid parts, reach only a certain stage of development; if, however, the parts containing the cysticerci are eaten by another animal, they attain to their complete development in the alimentary canal of the latter. Thus a particular cysticercus found within the liver of the rat, if eaten by the cat, is developed into the kind of tapeworm frequently found in the intestine of the latter; and the ova from this tapeworm received into the borly of the rat gives rise to the kind of cysticercus found in the liver of this animal. The ova from the tænia of the dog, received into the body of the sheep, becomes the cystic entozoon, called cænurus cerebralis, found in the brain of the latter, which, if eaten by the dog, becomes the tænia found in the intestine of this animal. These highly interesting, and, in a practical view, important facts, appear to have been fully substantiated by accurate observation and experiments.

The tænia or tapeworm is distinguished, as these names import, by its ribbon-like form. It is composed of numerous segments or joints, each one of which, after it attains to its full maturity, is provided with male and female generative organs. Several varieties have been found in man, but, as regards medical practice, the discrimination is not of much importance, the same measures of treatment being alike applicable to all. In the vast majority of cases, the tænia developed in the human intestine is one of three kinds; it will suffice to notice these. In this country, of the three kinds of tænia, the one which is found oftenest is commonly known as the tænia solium, or solitary tænia, so called because it is supposed always to exist singly. This is true as a rule, with some exceptions; two or more tænia have been observed together, but instances are exceedingly rare. The tænia solium varies in length from four or five to thirty-five feet, the number of segments or joints varying from 800 to 1000. Toward the head it becomes attenuated, forming a thread-like neck of considerable length, and in this portion there are transverse markings or rugæ, but no visible joints. The joints succeeding the neck are about as long as they are broad, but lower the length is twice or thrice the breadth. From the resemblance of these joints to the seeds of a gourd, the worm has been called vermis cucurbitinus, or gourd worm. The generative foramina are placed near the middle of one of the margins of each joint, and are generally alternate. The head is very small, hemispherical or triangular in form, and, under the microscope with a low power, it is 
found to present four projections or suckers, and a double row of hooklets, numbering 12 or 15 in each row ; the latter are sometimes wanting." The joints of the inferior part are being constantly thrown off, and are passed almost daily by stool. These exfoliated joints contain an immense number of ova. Bennett quotes an estimate of the number of ova in the tapeworm of a cat to be 12,500,000. It is fortunate that worms are not developed from the ova within the alimentary canal !

The second variety, called tænia mediocannellata, has not until within late years been generally recognized as distinct from the trenia solium; and the relative frequency of its occurrence is not as yet ascertainerl. The generative foramina are situated alike in both varieties; but the joints are longer and thicker, and the head has four suckers withont hooklets.

The third variety is commonly known as the trenia lata, or broad tapeworm, reckoned by some helminthologists as a species distinct from the true trenia, and named Bothriocephalus latus. This worm is distinguished from the trenia solium by the breadth of the joints, which are broader than long, by the situation of the genital oritices, which are in the middle of the joints, and by the different conformation of the head. The head is unprovided with hooklets, and hence this and the second variety are distinguished as the unarmed tænia, while the tænia solium is said to be armed. The form of the head is elongated, and, instead of suckers, it has two lateral longitudinal depressions or fossæ; hence the name bothriocephalus, from $\beta$ ogp cov, a small pit. This worm may attain to even a greater length than the trenia solium. Like the latter, it is usually solitary. A tania called Bothriocephalus cordatus, distinguished by the form of the head, is common in dogs and has been found in man.

Of these varieties of tapeworm, the first is the kind commonly met with not only in this country but in most countries. It prevails in England, France, Spain, Austria, Prussia, Greece, and Italy, the trenia lata being almost unknown in these countries. Both occur in Holland, Finland, Norway, Poland, and Sweeden. The latter is the variety chiefly observed in Russia and Switzerland. It is observed that the tronia lata is apt to affect inluabitants living on the coasts of seas, lakes, and rivers, whence it has been inferred that it proceeds from a cystic entozoon existing in fish. The opinion, however, is entertained that the embryos of this worm may be taken into the system in lrinking water and developed in the intestines without the intermediate stage of cystic development in the solid parts. The trenia solium is attributer to the ingestion of the cysticercus cellulos $x$, which is common especially in swine and sheep. It is the presence of this entozoon in large numbers which renclers pork " measly." Animal food eaten raw or partially cooked is probably the source of the common tapeworm. Thorough cooking destroys the cysticercus, and the development into the tapeworm does not then take place. It is a matter of observation that pork-butchers and cooks are prone to tapeworm, and it is stated that in Abyssinia, where the custom of eating uncooked meat prevails, nearly all persons, of different ages, become affected with trenia. The production of trenia in children by the use of raw meat as food, in cases of so-called cholera infantum, has been already adverted to. The tænia mediocannellata has been observed in Austria, and some parts of Bavaria; but its geographical history has not been fully determined. The cysticercus, whence it is derived, has been found in veal and beef.

1 For fuller description, vide Owen, in Cyclopedia of Anatomy and Physiology ; Aitken, op.cit., 1872. Vide, also, Boylston Prize Essay on the Iluman Cestoids, by F. B. Sturgis, M.D., Cambridge, Mass., 1867. 
The habitat of a tapeworm is the small intestine. Then it attains to a great length, it extends into the large intestine, and has been found to reach nearly to the anus. No age is exempt from the liability to its occurrence, but, in the majority of cases, the ages are between twenty and forty years. It occurs oftener in females than in males. Prof. S. G. Armor has reported a case in which an infant five days old passed segments of tænia. Two months after the birth of the ehild the mother was treated for tænia. ${ }^{2}$ The frequeney of its oceurrence varies much in different countries and in different sections of the same country. The report of the military surgeons of the French army from 1840 to 1848 showed only seven cases among 250,000 soldiers. During a practice of nearly forty years I have not met with more than from thirty to forty cases.

The existence of a tapeworm is not denoted by well-marked diagnostic symptoms. The symptoms attributed to it are vertigo, tinnitus aurium, disturbance of vision sometimes amounting to temporary amaurosis, pruritus at the nose and anus, salivation, disordered appetite and digestion, colic or neuralgie pains in the abdomen, and emaciation. These are symptoms which may be due to other morbid conditions, nor are these symptoms uniformly, if generally, marked in cases of tapeworm. The existence of the worm is often discovered accidentally, and in persons who, prior to the discovery, considered themselves perfectly well. In the case of a young female affected with sciatic neuralgia, I was led to prescribe a full dose of turpentine, and the result was the expulsion of a large quantity of tapeworm, the existence of which had not been suspected. After the existence of the worm is known, disorders of all kinds are apt to be referred to it, and often the imagination creates varions morbid sensations. Convulsions, epilepsy, and chorea are supposed to be occasional effects, but clinical observation supplies but little data for this supposition. 'The pathological effects of the presence of this worm have doubtless been greatly exaggerated. Tapeworms are almost invariably found in the intestines of dogs destroyed for experimental purposes apjarently in perfect health.

The diagnosis must rest on the discovery of exfoliated joints or pieces of the worm in the evacuations. This mode of diagnosis is always available. Fragments of the worm are generally passed either daily or at short intervals, and hence it is usually not long before its existence is ascertained. If there be reason to suspect its existence before fragments have been seen, a brisk purgative may be given, and this will be likely to be followed by the expulsion of a greater or less quantity. If an examination of the stools for some time, and especially after the administration of purgatives, fail to discover any portions of the worm, the physician may be satisfied of its non-existence. Persons not infrequently fancy they have a tapeworm. This is one of the notions likely to be seized upon by hypochondriacs, and in some cases the notion becomes a fixed belief of which the mind cannot be dispossessed, becoming, in fact, an insane delusion. Several instances of this kind have fallen under my observation. A few instances are on record in which portions of the worm have been vomited.

With reference to treatment, a point of importance is the natural duration of the life of this parasite. It is undoubtedly long-lived. It is not uncommon for fragments to be passed at short intervals for many years. Cases have been reported in which this was observed for twelve, twentyfive, and even thirty-five years. If an interval of several months elapse

1 Detroit Review of Medicine, Jan. 1872. 
without any fragments being discharged, and subsequently they make their appearance, it is probable that a second worm has become developel. Sooner or later, the worm dies a natural death, and a cure takes place spontaneously. If successive remedies have been used in such a case, the last remedy, of course, gets the credit of having effected a cure. For a cure to be effected with certainty, the head must be expelled. The frequent exfoliation of joints is natural, and if, as an effeet of a remedy, or from any eause, a large portion, or even all the body be expelled, reproduction by growth may take place so long as the head remains; it is, however, a matter of observation that the parasite is apt to die when the separation takes place near the head. As positive evidence of a cure, the head is to be sought for, but it is not found in the larger proportion of the cases in which a cure is effected.

Of the remedies to be employed for the destruction of the tapeworm, one of the oldest, and, certainly, in many cases an effective remedy, is the oil of turpentine. It is most likely to be effective if given in large doses. A half ounee may be given in emulsion, with the addition of some aromatic tincture to prevent nausea, this dose being repeated every half hour until two or three ounces are taken, or, sometimes, the whole amount taken at once is well borne. Strangury is an occasional result, but is less likely to occur than when the remedy is given in small doses. After a large dose, the patient feels as if slightly inebriated. This remedy, thus given, may be repeated, if necessary, after intervals of sereral days. Given in the quantity just stated, it generally acts as a purgative. Chabert's empyreumatic oil, which has been much in vogue as a remedy for tænia, is probably efficacious chiefly from the turpentine which enters into its composition, and is much more disagreeable than the turpentine alone. It is stated by Bellingham and others that turpentine, given in moderate doses, and repeated for several suceessive days, is frequently suceessful.

The male fern (filix mos) is a trenifuge, the efficacy of whieh rests upon the testimony of many observers of large experience. In my own experience it has proved promptly efficacious. It may be administered in powder, from half a drachm to a drachm being given in divided doses in the course of a few hours ; but the oil and ethereal extract are more eligible preparations. The oil may be given in doses of a drachm or two in mucilage, or in gelatine capsules; the dose of the extract is from 20 to 30 grains. Dr. Alexander Fleming advises a drachm of the oil, with mucilage, to be given in an ounce and a half of sweet milk, at beltime, the dinner and evening meal of the day having been omitted. This mode of administration is advised on the supposition that milk being a favorite food of the worm, it is more effectually poisoned by imbibing the remedy in this vehicle. In the two last cases which have come under my observation (1872) the following course proved successful: No food was taken after dinner, but at night a full dose of castor oil. No breakfast the next morning, but a dessertspoonful of an equal mixture of the liquid extraet of male fern and mucilage, and repeated in four hours if the first dose be well tolerated and have no effect. In one of the cases a single dose was suffieient, and the head of the worm was expelled.

Another effeetive tænifuge is the kousso (brayera anthelmintica), the dried flowers of a tree in Abyssinia where tapeworm is exeeedingly common. This remedy is said to be relied upon in that country. Within the past few years it has been used with much success in Europe and in this country. Half an ounce of the powdered flowers mixed with water may be given in a dose, both the liquid and sediment being taken.

$\dot{A}$ remedy recently introduced as a trenifuge into this country is an 
emulsion of pumpkin-seeds (cucurbita pepo). The emulsion is prepared by pounding a couple of ounces of the seeds in a mortar with half a pint of water, and straining through cloth. This quantity will constitute a dose, which may be repeated for several days. This remedy has proved successful in the hands of a number of physicians in this country. It has the advantage of producing no unpleasant effects, being, aside from its action as a vermifuge, innocuous.

The bark of the pomegranate root (granatum) is another anthelmintic remedy which has been found destructive to the tapeworm. A decoction made by boiling two and a half ounces of fresh bark in a pint and a half of water until this quantity is redueed one-half, is the form in which this remedy is to be administered. The whole of the quantity just named may be given in the course of a few hours. It generally produces purging, and not infrequently vomiting. 'The Kamela, the powder and hairs from the eapsules of Rottlera tinetoria, is a powerful anthelmintic whieh has been found promptly effieacious in cases of tænia. The dose is from one to two drachms given in honey or thick gruel. It aets efficiently as a purgative. This remedy has been given with great suecess in combination with the ethereal extract of the male fern.

These are the most valuable of the athelmintics which experience has shown to be capable of destroying the tapeworm toxically. The powder of tin (pulvis stanni) given in doses of a scrmple or more, mixed with honey, has, in some cases, proved efficacious, destroying, probably, the worm by its mechanical action. It is far less reliable than the toxical remedies. Active catharties, such as ealomel and jalap, seammony, gamboge, and croton oil, occasionally suceed, their action, probably, being mechanical. They will generally bring away greater or less portions of the worm, but, alone, they are not to be relied npon for effecting a cure, that is, for destroying the worm. In conjunction with the toxical anthelmintics, they are generally useful.

The success of the treatment will depend much upon accessory measures. The worm is nourished, not by nutriment sncked from the intestinal walls, but from the alimentary contents of the intestine, which enter the body of the parasite by endosmosis. An important part of the treatment, therefore, is to weaken the parasite by starvation. The patient, by way of preparation for the administration of the tænifuge, should abstain from those articles of food which are digested in the small intestine, for at least a day or two. Beef-tea or chicken-soup may be allowed freely. The treatment should commence with a mild purgative, and the administration of the anthelmintic remedy should be followed; after a few hours, by a pretty aetive purgative. If the treatment prove unsuceessful, the strength of the patient should be restored by a return to a full diet and tonic remedies, and the same plan again repeated, employing the same remedy or selecting another. The treatment is to be repeated, allowing sufficient intervals for recruiting the strength, until a cure is effected. It is to be borne in mind that, if the greater part of the worm be expelled, although the head may not be found, the death of the worm is apt to follow, and the treatment, therefore, need not be repeated, until the discharge of tiragments is again observed.

The prophylaxis involves care never to eat meat not thoroughly cooked. In meat much underdone, the cysticerci which may be contained in it are, perhaps, not destroyed. Purity of the water used as a drink may be an important measure of prevention. The use of filtered water is to be recommended. The meat contained in the Bologna sausages is liable to contain living cysticerci. 


\section{Trichina spiralis.}

From the discovery of the trachina spiralis by Paget and its description by Owen, in 1335, up to a recent date, this parasite had been observed occasionally in the muscles of subjects in the dissecting room, but, in a pathological view, it was not considered to have any importance. Facts, however, accumulated within the past few years, have shown that the introduction and multiplication of trichinæe within the body of man may give rise to morbid effects of great gravity, which often prove futal. Much information has been obtained respecting the natural history of the parasite, the circumstances under which the human body becomes infested by it, and the morbid phenomena which characterize the disease. This information has added to the nosological catalogue a new and highly important affection known as the trichinal or trichinatous disease, trichinosis or trichiniasis.

Prof. Zenker, of Dresden, was the first to ascertain the nature and source of the trichinal disease. In a young girl who died after an illness of several weeks' duration, characterized by debility, fever, sleeplessness, abdominal tenderness, great pain in the museles, and œedema of the lower extremities, numerous trichinæ were found in the voluntary muscles, and in mucus from the small intestines. He traced the origin of the disease to the eating of ham and sansages, which, on microscopic examination, were found to contain in abundanee trichina. This was in January, 1860. Numerous examples of the newly discovered disease were soon afterward met with, especially in Germany. A large collection of cases occurred on a single occasion in Heldstädt, Prussia, in 1863. Of one hundred and three persons who participated in a hotel dinner, on a festive celebration, nearly all were attacked with the disease, and a large number died. The disease in these cases was traced to a kind of sausage called "Röstewurst," of which most, if not all the guests had partaken. On microseopic examination, the remnants of these sarisages, and the pork of which they were made, were found to be swarming with trichinse, and these parasites were found in abundance in the muscles of the persons affected. Several examples, shortly after this, fell under observation in this country. In February, 1864, several members of a family in the city of $\mathrm{New}$ York became seriously ill after eating raw smoked ham, and one of the children died. Dr. Joseph Sehnetter cxamined the ham, and found it filled with trichinse. Prof. Dalton also made an ex:mination and counted in a muscle $\frac{1}{2}$ inch square and $\frac{1}{50}$ inch thick, twelve trichinge, which would give, in round numbers, over 85,000 to the cubic inch. A short time afterward, a case of suspected disease from triehina came under the observation of Dr. Voss. In order to settle the diagnosis, Dr. V. cut down upon the deltoid muscle of the patient, and removed a portion of the tissue. It was examined microscopically by Dr. Voss and Prof. Dalton, and trichinxe were found in abundance, numbering over 7000 to the enbic inch. ${ }^{1}$ 'These were the fir st reported eases, in this country, in which the character of the discase was established. The next cases reported were observed by Dr. Krombein and Dr. Dingler, German plysicians practising in Erie County, N. Y. 'They were reported in the Buffalo Medical and Surgical Journal (1864), by. Dr. Krombein, with remarks by Dr. J. R. Lothrop. Subsequently, A pril, 1866, in a family consisting of ten persons, in Marion, Iowa, nine

1 Vide Observations on Triclinn Spiralis, hy John C. Dalton, M. D., Trans. New York A cad. of Med., vol. iii. 1864. Vide, also, lecture by Prof. Dalton published in the New York Med. Record, April 15th, 1869. 
were attacked with trichiniasis after eating raw ham. The member of the family who escaped did not partake of the raw ham, but ate of it freely after it had been thoroughly boiled. Of the nine who were attacked, five died. Portions of muscle from the body of one of those who died, and portions of the ham, sent to Prof. John C. Dalton, aud Prof. Austin Flint, Jr., of this city, were found to contain trichina in abundance. Prof. Dalton has kindly furnished me the following account of the trichinæ in the specimens examined by him :-

"The trichinæ in the specimen of human muscle from Iowa varied in size. The smallest were $\frac{1}{73}$ of an inch long by $\frac{1}{\sqrt{6} \sigma}$ of an inch thick; the largest were $\frac{1}{22}$ of an inch long by $\frac{1}{4} \sigma$ of an inch thick. The larger worms were much more numerous than the smaller ones. They were not completely encysted, but were all inclosed in smooth finsiform tubes, of indefinite length, with thin walls, containing a semifluid granulated matter. The worms were coiled up, with one or two turns, in the dilated portions of the tubes. In a piece of the muscle, ${ }_{12}^{1}$ of an inch square, and $\frac{1}{50}$ of an inch thick, I found 29 trichinæ. This would give a little over 208,000 to the cubic inch."

This specimen came from a boy 13 years of age, and showed the state of the infected muscles at about the twentieth day of illness.'

Since the date of these cases, several instances of the development of the disease in different parts of the country have been reported.

Repeated experiments have been made of giving to animals pieces of flesh containing trichinæ, from other animals, and the human subject. After the death of animals thus fed, trichinas have been found in great numbers within the small intestines, and in the muscles. These experiments have elucidated important points pertaining to the multiplication of these parasitic animals within the alimentary canal, and their migration to the muscles throughout the body. In short, the existence of a disease of great gravity, involving much danger to life, produced by the introduction into the system of trichinæ contained in food, may be considered as sufficiently established.

The trichina' spiralis belongs in the class nematoidea. As found in the muscles, it is coiled up in a cyst containing a granular substance at first, and afterward, calcareous matter. When expelled from the cyst, the parasites are seen to be round worms, $\frac{1}{28}$ of an inch long, and $6 \frac{1}{2} \pi$ of an inch in thickness. So long as they remain in the muscular tissue, the worms are quiescent. When, however, they are taken into the stomach, and set free by the action of the gastric liquids upon the muscular tissue and the cyst, they pass from the stomach into the intestinal canal, and inmediately begin to grow rapidly, attaining to three or four times their former size; they acquire fully developed generative organs, and, in the course of a week to ten days, the female parasites contain living young in great abundance. According to Virchow, each trichina gives birth to 200 young; Gerlach says 400, and Leuckart 1000 . The young trichinx, shortly after birth, penetrate the mucous membrane, and find their way rapidly to the different muscles throughout the body. They appear to select the voluntary muscles for their permanent dwelling-place. It is stated that they have not bcen found in the muscular walls of the heart. In other than muscular organs they are very rarely found.

Trichinæ are found especially in the muscles of swine; but they have been found in the muscles of various other animals, viz., eels, cats, rats

For a report of these eases by Dr. H. Ristine, of Marion, Iowa, vide The $\mathbf{N}$ w York Medical Record, August 1, 1866. 
and mice, dogs, badgers, hedgehogs and moles. They get into the bodies of swine from their feeding upon the flesh and excrement of other animals infested with these parasites, especially rats and mice. Hence, to prevent trichiniasis in swine, and in other animals which serve as food for man, it is highly important to cut off all the sources of the disease in the diet of these animals. They find their way into the alimentary canal of man chicfly from eating trichinous pork not subjected to processes of cooking sufficient to destroy the parasites. They are not destroyed by salting or smoking, and they may retain their vitality in roasted or boiled meat much underdone. To secure effectually their destruction, every portion of meat which is eaten should have been subjected in cooking to a temperature of $212^{\circ}$ Fahr. The custom which prevails among the Germans of eating sausages and smoked hams uncooked involves a liability to the disease. It has been traced to eating pork chops which were underdone in the centre, although sufficiently cooked on the outside. Meat abounding in trichinæ may present no evidence of the fact as regards the gross appearance, espccially if a calcareous deposit have not taken place in the eysts which contain them; hence, trichinous meat may be sold and bought without any suspicion of its being unwholesome. The only reliable evidence of the presence of trichinæ in the meat procured for food is afforded by microscopical examination. The trouble, however, of such an examination precludes its being generally resorted to as a means of protection. Moreover, a microscopical examination of a few specimens is not adequate to determine that trichinæ are not present. They may not be found in the few specimens examined, although present in other portions. Hence, this test is only reliable in a positive, not a negative, point of view.

It is of interest to know somewhat of the chances that the pork used for food may be trichinous. A committee of the Chicago Academy of Sciences appointed to make examinations with reference to this point, reported that of 1394 hogs examined in different packing-houses and butcher shops of Chicago, trichinæ were found in 28 . It was therefore estimated that, of the hogs brought to the Chicago market, one in fifty is affected by trichiniasis in a greater or less degree. In this report it is stated that in the city of Brunswick, Germany, of 19,747 hogs examined only two were found to contain trichinæ. ${ }^{1}$

After becoming encysted in the muscles the trichinæ retain their vitality for an indefinite time. In a case reported in Virchow's archives, trichina wele found encysted and alive in portions of muscle attached to a cancerous tumor removed from a patient. The previous history of this case showed conclusively that the patient was affected with trichiniasis twentyfour years before the operation. ${ }^{2}$ The flesh of animals, therefore, which have been affected with trichiniasis ever afterward will be likely to contain living trichinæ. Moreover, the flesh of a single trichinous animal may produce trichiniasis in a very large number of persons, so that the disease in its prevalence may present the character of an epidemic.

The disease in swine is manifested by symptoms analogous to those observed in man, viz., diarrhœa, stiffness of the limbs, debility, muscular atrophy, and frequently cough. The affection known in the Western States by the name "hog cholera" is supposed to be trichiniasis."

The elinical history of the disease caused by trichinæ is highly important with reference to an early diagnosis. The primary symptoms relate

1 Med. News and Library, Philadelphia, July, 1866.

2 Medical News and Library, Philadelphia, May, 1866.

- Vide report by Dr. H. Ristine, already referred to. 
to the alimentary canal, and are due chiefly to the perforation of the mucous membrane by the newly-produced worms. Abdominal pains, vomiting and diarrhoa characterize the first stage of the disease. These symptoms occur within a week or ten days after the ingestion of triehinous meat, that is, as soon as the young worms have been produced and become developed sufficiently to begin to migrate toward the muscles. It is not difficult to understand that the aggregated punctures of the mucous membrane by these parasites should occasion notable disturbance, when it is considered that the triching which have been found to be contained in a half a pound of meat may be suffieient to give birth, in a few days, to a brood numbering $30,000,000$. It is stated that peritonitis may be produced by the passage of worms into the peritoneal cavity.

The secondary symptoms relate to the muscles. Pains resembling those of muscular rheumatism are occasioned by the entrance of the trichinæ in the muscles. Certain of the muscles become contracted, in some cases, and their extension oeeasions great suffering. Constitutional disturbanee, more or less marked, accompanies both the primary and secondary symptoms. The general symptoms are not unlike those of typhoid fever, for which the disease is likely to be mistaken. The temperature in the axilla is raised to from $101^{\circ}$ to $106^{\circ}$. Edema of the face or lower extremities is apt to oceur, and sometimes anasarca. Sweating is generally prominent as a symptom. In some of the eases observed by Dr. Ristine, of Marion, Iowa, dyspepsia, cough, and a viscid, orangecolored expectoration, were prominent symptoms associated with physical signs denoting eapillary bronchitis. Death takes place in a certain proportion of eases, after a protracted period of suffering and exhaustion, being often preceded by coma. It is stated that life in some cases is destroyed by the impairment of the respiratory muscles from the presence of the parasites. Aphonia is a symptom which exists in some cases, attributable to the presence of trichinæ in the museles of phonation. The danger, ceteris paribus, is proportionate to the abundanee of trichinre generated within the alimentary canal. If the number be not suffieient to eause death from the amount of local and constitutional disturbance which they occasion, recovery takes place very slowly, the illness lasting for several weeks or even months. The trichinæ become eneapsulated in the muscles, thereafter remaining quiescent, leaving the muscles more or less impaired. An accumulation of a larger number of eases than is at present practicable, is necessary to furnish data for a complete clinical history of the disease, and for determining the relative proportion of deaths and recoveries.

Diarrhœa and abdominal pains, followed by muscular pains, together with more or less constitutional disturbance, should excite suspicion of this disease. Inquiries respecting food should be instituted, and, if any portion of the suspected meat remain, it should be examined microscopically. The diagnosis may be made still more complete by harpooning some one or more of the painful muscles and obtaining sufficient portions for examination with the microscope. Davaine suggests that the presence of trichinæ in the stools may be ascertained, and the diagnosis in this way renclered positive. If clinical observation should establish the availability of the latter method of arriving at a diagnosis, it would have this advantage, viz., the existence of the disease may be positively ascertained before the trichinæ migrate from the alimentary canal. The practical importance of making the diagnosis thus early, is sufficiently obvious.

It is evident that the treatment of this disease, to be effective, must be employed while the worms are in the alimentary canal. Hence, the impor- 
tance of an early diagnosis. It remains to be ascertained whether treatment ean be effeetively employed in this stage, and to determine the measures most likely to prove effeetive. 'The object is either the destruetion of the worms or their expulsion from the intestines. Cathartics are indicated for the latter object, but clinical experienee has yet to ascertain what toxieal anthelminties are best suited to destroy this parasite. Benzine has been found by Prof. Mosler, of Berlin, to destroy the trichina in the intestinal canal when given in doses whieh are well borne by the patient. Prof. Mosler's method of treatment with this remedy is to have a mixture consisting of two draehms of benzine, an ounee each of liquoricejuice and mucilage of gum-arabic, and four ounces of peppermint water. Of this mixture a tablespoonful is to be given every one or two hours. The effieaey of this remedy in man, however, remains to be established.' The carbolic aeid is a remerly which should be tried with a view to the destruction of the worms in the intestinal eanal. After the trichina have left the intestines, the opportunity of destroying or expelling them has - passed. Reeovery now depends on the ability of the system to endure them until they become eneysted. Measures to palliate pain, to restore disordered funetions, and to support the powers of life, are indicated, and the suceess of the treatment will depend on the judieious employment of measures for these ends.

The prevention of this disease is the great practieal benefit to be derived from its diseovery. The mode of prevention is sufficiently simple, viz., abstaining from meat, more especially the flesh of swine, not thoroughly cooked. The process of smoking, pickling, or salting, camnot be relied upon for the destruetion of this worm. Uneooked sausages cannot be eaten without danger. 'The only security is in the fact that every portion of the meat has been subjected to a temperature of $212^{\circ}$ Fahr.?

Animals not entozoötie, such as snakes, slugs, and lizards, are not infrequently exhibited to physieians as having passed from the alimentary canal. It is a popular notion that they may live and grow indefinitely within the stomach and intestines, and give rise to a variety of morbid symptoms. In the great majority of cases the statements with respeet to the passage of these animals are simply falsehoods, proceeding generally from a morbid exaggeration of that craving to beeome objeets of curiosity and interest, which enters largely into the mental constitution of many persons. In some eases, however, the statements are honestly made, persons being deeeived. Prof. Dalton has recently performed a series of experiments to ascertain the duration of life of the common garden slug (Limax agrestis) and the water-lizard (Triton millepunctatus) after having been introdueed within the stomach of a dog. The former were found eompletely dead at the end of nine and a half minutes, and no traces of them were discoverable when the dog was killed an hour after their introduction. The lizards were found perfectly dead, and about to undergo the digestive process, at the end of fifteen minutes. ${ }^{3}$ It is safe to set down most of the cases referred to as cases of either self-

1 British and Foreign Review, Jannary, 1865, p. 236.

2 For an historical account of discoveries relating to trichine, and of experiments to infect different animals and to destroy the parasite by different medicines, vide article by John D. Jackson, M.D., in the Am. Jour. of Med. Scieuces, January, 1867.

3 American Journal of Medical Sciences, April, 1865. 
deception or imposition. Maggots, however, have been known to be discharged from the nasal cavity, the mouth, and the rectum. They may be developed in the nasal cavity from ova inhaled; but they may be developed in great numbers within the alimentary canal, the ova having been ingested with food or drink.

\section{H A P T E R X.}

Acute General Peritonitis-Anatomical Characters-Clinical History-Pathological Character -Causation-Diagnosis-Prognosis-Treatment. Partial Peritonitis. Chronic Peritonitis. Hydro-Peritoneum.

THE peritoneum in structure and functions does not differ materially 1 from other serous membranes. This, like other serous membranes, may be the seat of inflammation. Inflammation here, as elsewhere, may be acute, subacute or chronic. The whole or greater part of the membrane may be inflamed, and the inflammation is then said to be general; or the inflammation may be more or less circumscriber, and is then distinguished as partial. Inflammation of this membrane, agreeably to the nomenclature of inflammatory affections, is termed peritonitis, and this name is in common use. In treating of peritonitis, it will be convenient to consider separately acute general peritonitis, partial peritonitis, and chronic peritonitis. Some writers divide the disease into several varieties, according to its different causes or the circumstances under which it is developed. It will suffice to notice these diversities under the head of causation. Occurring as an epidemic in childbed, however, it claims separate consideration; but it does not fall within the scope of this work to consider puerperal peritonitis fully or separately. It is treated of at length in works on obstetrics and the diseases of women, and to these the reader is referred.

\section{Acute General Peritonitis.}

Anatomioal Charaoters.-The appearances after death, in different periods of the disease, are essentially the same as in other serous inflammations ; for example, in pleuritis or pericarditis. Redness, arborescent and punctiform, chiefly from hyperæmia in the subserous areolar tissue, exists, especially if death take place early. The degree of hyperæmia varies much in different cases, and is not to be taken as any criterion of the intensity, or otherwise, of the inflammation. Nor is redness from hyperæmia, be it never so marked, alone adequate proof that inflammation existed. More or less of the inflammatory product, lymph, is always present. The quantity varies much in different cases. It is sometimes abundant, forming a coating more or less thick, extending over the solid and hollow viscera and the parietal portion of the membrane; in other cases, the quantity is moderate or small, adhering to the membrane in patches. It is apt to be most abundant at, and may be limited to, the portions of the intestines which are in contact with each other, frequently agglutinating them together and to the abdominal walls. The 
quantity is sometimes slight and spread over the surface in a thin layer so as to escape observation without close attention and seraping the serous surfaces. It is soft and gelatinous if recently exuded, and dense according to its age. The peritoneal sac usually contains effused liquid, the quantity varying much in different cases. 'The liquid is turbid from lymph, and contains flakes of lymph in more or less abundance. Occasionally it is reddened by the arlmixture of blood. The serous membrane is more or less opaque and softened. The subserous areolar tissue is morbilly brittle, so that the membrane is more easily detached than in healtl. In certain cases, and especially if the duration of the disease have been considerable, the peritoneal sac contains pus in more or less quantity. If the peritonitis be connected with perforation of the stomach or intestines, fetid gas escapes on opening the sac, and the contents of these hollow viscera may be found within the serous cavity. Sphacelation of the membrane rarely, if ever, occurs, unless the peritonitis be connected with strangulation or invagination of the intestine. Ulceration from without is extremely rare. Lesions situated in other struetures than the peritoneum, and involved in the causation of the peritonitis, are found in a large majority of cases-such as intestinal perforation, invagination, rupture of the urinary or gall-blalder, abseess of the liver or in other situations, ete. etc. Associated lesions are always to be sought for, if not at once apparent. Finally, if life be sufficiently prolonged, morbid adhesions of the intestines to each other, to the solid viscera, and of the opposing parietal and visceral portions of the membrane, are observed. These adhesions are due to the formation of adventitious tissue, as in inflammation of other serous membranes. The adhesions may be more or less extensive. They may consist of bands or bridles of newly-formed membrane, leaving spaces, or loops, in which portions of intestine are liable to become strangulated, thus giving rise to fatal obstruction. The adventitious membranes resulting from inflammation here, as in other serous membranes, are firm and resisting in proportion to their age.

Clinical History.-A A cute peritonitis is sometimes developed gradually. Abdominal pain and soreness, progressively increasing, may be felt one, two, or three days before the local and general symptoms are sufficient to show the existence of the disease. But in most cases the attack is abrupt, and the disease quickly declared. Pain is usually a marked symptom. Commencing at a particular point, it extends over the whole abdomen. It is burning or lancinating in character. It is increased at times sufficiently to constitute marked exacerbations, and, not infrequently, in these exacerbations, the character of the pain is that of spasm or colic. Acute pain is produced by a deep inspiration. The respirations are usually shortened, and, by way of compensation, increased in frequency. The movements of the diaphragm are restrained, and those of the ribs proportionately increased; the breathing, in other words, is costal. Acts of coughing and sneezing occasion intense pain. Movements of the body are painful. The degree of suffering from pain varies in different cases. It is generally great, often extreme, occasionally moderate or slight, and this symptom may be wanting. Tenderness on pressure over the abdomen is usually marked. This symptom is very rarely wanting. The tenderness is often such that the slightest pressure is painful, but the degree of tenderness varies considerably in different cases. The pain and tenderness are frequently most marked in a particular circumscribed portion of the abdomen. These symptoms continue during the course of the disease. Owing to these symptoms the patient is apt to lie upon the back 
with the knees and thighs raised, in order to lessen the tension of the abdominal muscles and relieve the abdomen of the weight of the bedclothes. This decubitus, however, is by no means constant. The patient may lie on the side with the knees and thighs flexed, or even on the back with the lower limbs extended. More or less tympanites is generally present. The abdomen is often greatly distended and tense, and this condition persists during the course of the disease. If the tympanites be not great, the abdominal muscles, especially the rectus muscle on either side, is usually notably rigid and resisting. The bowels, as a rule, are constipated, but the exceptions to this rule are not very infrequent. Constipation may exist at first, and subsequently diarrhœa occurs, and occasionally diarrhoea exists at the outset. Vomiting is a frequent and in some cases a prominent symptom. The acts of vomiting occasion great pain. In the latter part of the course of the disease, the contents of the stomach are apt to be ejected by regurgitation rather than by vomiting. Thirst is usually a prominent symptom.

The pulse is more or less accelerated. It is apt to become quite frequent, numbering 120 or 130 per minute; but in some cases the acceleration is moderate. It is generally small and sometimes hard or wiry. The seuse of prostration is considerable or great. Perspiration is apt to occur. The countenance denotes gravity of disease; the expression is haggard and anxious. In some cases the upper lip is elevated and drawn tightly over the teeth. This appearance is characteristic, and, when present, points to the disease, but it is by no means uniformly present.

Difficulty in micturition is a frequent symptom, due, probably, in part to paralysis of the muscular tunic of the bladder, and partly to loss of power over the abdominal muscles in consequence of the abdominal distension. Moreover, the pain occasioned by the effort to micturate leads the patient to postpone it as much as possible. Retention of urine, requiring the use of the catheter, is not uncommon. Prof. Rogers, of the University of Louisville, has called attention to the occurrence of severe pain in the penis, in certain cases of peritonitis. ${ }^{1}$ This pain is sometimes extremely intense. I have observed this symptom in two cases, since the publication of Professor Rogers' paper, and I have met with several physicians who have observed it. Dr. H. Hunt, of Beloit, Wisconsin, met with an example in a boy six years of age. In some of the cases reported by Prof. Rogers, priapism and intense venereal desire existed. It remains to be ascertained how frequently these symptoms are present. I have ascertained their existence in two cases, since their occurrence was pointed out by Prof. Rogers.

The intellect, in cases of acute peritonitis, is generally not disordered. Slight delirium sometimes occurs, especially in fatal cases, toward the close of the disease.

Pathological Character.-Acute peritonitis, as regards pathological character, does not differ essentially from other acute, serous inflammations.

Causatron.-Acute inflammation of the peritoneum, exclusive of puerperal peritonitis, is rare. As a spontaneous or idiopathic affection, it is one of the rarest of diseases. The liability of the peritoneum to become inflamed is vastly less than of the pleura or the pericardium, a fact not easily explained, but which exemplifies a principle of conservatism, inas-

1 Western Tournal of Medicine and Surgery, Louisville, Aug., 18j5, vol. iv. No. 2. 
much as peritonitis is a much more serious disease than either pleuritis or pericarclitis. In the great majority of cases, peritonitis is incidental to some other affection of the abclominal viscera. The most frequent cause, exclusive of its occurrence in childhood, is perforation of the alimentary canal. Perforation of the ileum is incidental to the intestinal lesions of typhoid fever, and peritonitis thus produced is one of the occasional events pertaining to the clinical history of that form of fever. 'The intestinal ulcerations which occur in certain cases of tuberculosis, sometimes, although very rarely, lead to perforation, and, also, other ulcers situated in either the small or large intestine. Perforation from ulceration or sphacelation of the extremity of the appendix vermiformis of the cæcum is not of very rare occurrence; several examples have fallen under my observation. Perforation of the stomaeh is an occasional event in cases of gastric ulcer. Whatever be the seat of the perforation, the gaseous and other contents of the alimentary canal escaping into the peritoneal sac, peritonitis becomes at once developed. The escape of bile from rupture of the gall-bladder, or of the eystic, hepatic, or common duct, gives rise to peritonitis. Other causes are the discharge of pus into the peritoneal cavity from hepatic, ovarian, or other abscesses, rupture of the urinary bladder or ureter, and the evacuation of hydatid cysts. Inflammation, at first local, may become general, as in certain cases of invagination and strangulation of intestine. Injections into the cavity of the uterus have been known to give rise to the disease, the liquid injected passing into the peritoneal cavity through the Fallopian tubes. In certain cases of puerperal peritonitis, the inflammation conmences in the uterus and is propagated to the peritoneum. The disease may be produced traumatically by wounds of the abdominal walls, and violent contusions. It follows sometimes paracentesis, very rarely, however, except in cases in which the kidneys are diseased.

Excluding cases of acute peritonitis produced by these varions causes, together with all cases of puerperal peritonitis, the remaining cases which occur are exceedingly few. Of the few which remain, probably in most the disease is developed in connection with renal disease. Acute peritonitis is one of the local affections incidental to the morbid conditions of the kidneys collectively called Bright's disease. In a case under my observation at Bellevue Hospital, it was developed in connection with acute articular rheumatism, the patient recovering after a short duration of the disease. Exposure to cold may give rise to it. I have known its occurrence to be fairly attributable to this cause. The late Prof. Palmer, of Louisville, informed me that in the neighborhood of Woodstock, Vermont, where he formerly resided, cases of acute peritonitis were not very uncommon among persons employed in the winter and spring to repair water-wheels damaged by ice, this occupation requiring them to work standing in water.

Occurring as an epidemic affection, in childbed, it doubtless proceeds from a special cause. During the prevalence of puerperal peritonitis, epidemic erysipelas has been observed to prevail, showing some pathological relationship between the two affections.

In autopsical examinations, the various local causes of peritonitis are to be sought for. Without care they may be overlooked. A small intestinal perforation will be likely to escape detection unless careful search be made, and is probably not infrequently overlooked.'

1 For an analysis of 500 cases of peritonitis with reference to etiology and treatment, by Dr. Samuel O. Habershon, vide Medico-Chirurgical Transactions, pub- 
Diagnosis.-The diagnosis of acute peritonitis is not difficult in cases in which its diagnostic features are well marked, as they are in the majority of cases. 'These features are sometimes obscure or wanting, and it is then liable to be overlooked or confounded with other affeetions. From acute enteritis it is distinguished, generally, by a greater degree of pain, greater frequency of the pulse, more tenderness on pressure over the abdomen, more tympanites, rigidity of the abdominal nusscles, absence of diarrhœa, and the evidence, on all sides, of a graver malady. Rare as is acute peritonitis, acute enteritis, at least in the adult, is still more infrequent.

It is sometimes mistaken for functional colic. The pains, in some cases of peritonitis, are like those of colic. But colic laeks the continuous pain, the abdominal tenderness, the muscular rigidity, the tympanites, the frequency of the pulse, prostration, etc., of acute peritonitis. These symptoms, however, may not be immediately developed in peritonitis, and a little delay may, therefore, sometimes be necessary in arriving at a positive diagnosis.

Rheumatism, affecting the abdominal muscles, may give rise to certain of the local symptoms of acute peritonitis. Instances, however, of rheumatism limited to these muscles must be extremely rare. The diagnostic points, indicated by Genest, are as follows : ${ }^{1}$ In peritonitis, movements of the body occasion pain over the whole abdomen, whereas, in rheumatism, the pain is apt to be limited to certain muscles, and, perhaps, confined to one side of the abdomen. The same is true of tenderness on pressure. Moreover, in peritonitis the pain from pressure is proportionable to its amount, but in rheumatism, deep, firm pressure may be made without augmenting the pain.

Lumbo-abdominal neuralgia, with hyperesthesia of the abdominal walls or integument, sometimes simulates closely peritonitis as regards certain local symptoms. The pain may be severe, the tenderness great, the decubitus on the back with the thighs and knees flexed and, not infrequently, there is more or less tympanites. Cases presenting these symptoms are not uncommon. The patients are generally females, and manifest more or less of hysterical phenomena. The general symptoms of acute peritonitis, frequency of pulse, prostration, etc., are wanting in these cases; but attention to certain points connected with the local symptoms will suffice for the differential diagnosis. The tenderness in the neuropathic affeetion is more superficial; the patient shrinks from the slightest touch, but firm, prolonged pressure with the open palın is often well borne, and may even afford relief, whereas, in peritonitis, the pain is always proportionate to the amount of pressure. The museular rigidity of peritonitis is wanting. It is stated by Valleix that tenderness in these cases exists by the side of the vertebral column, and that the tenderness in front is especially marked at a point situated at or a little above the middle of the crest of the ilium.

I have known acute peritonitis to be mistaken for sporadic cholera in a case in which vomiting and purging occurred early, and were prominent

lished by the Royal Medical and Chirurgical Society of London, vol. xliii. 1860. From an analysis of these cases Dr. H. draws the following conclusions: Peritonitis is never idiopathic in its origin, but, when not traumatic nor due to perforation of viscera, it is dependent on either an extension of disease from adjoining viscera; or on certain blood changes, such as occur in albuminuria, pyæmia, etc. ; or on certain nutritive changes of the system connected with struma, cancer, etc. ; or, lastly, on local hyperæmia arising from cirrhosis, disease of the heart, etc.

i Valleix, op. cit. 
symptoms. In the exceptional cases in which these symptoms are sutficiently prominent to suggest that affection, the error of diagnosis is to be avoided by finding, associated with the vomiting and purging, the local symptoms of peritonitis, viz., pain, tenderness, tympanites, and muscular rigidity, together with general symptoms denoting an inflammatory affection, symptoms not belonging to the listory of sporadic cholera.

Acute peritonitis is sometimes remarkably latent, the local symptoms, usually so strongly marked, being obscure or wanting. Pain and tenderness may be slight or even absent; tympanites is not always present. Muscular rigidity in such cases may be a very valuable diagnostic symptom. As regards occasional latency, this disease resembles other serous inflammations, viz., pleuritis and pericarditis. Pain and tenderness may be obscure in consequence of the coexistence of other affections which occasion delirium or blunt the perecptions of the patient, and from the absence of these local symptoms, peritonitis may be overlooked. In some cases of peritonitis produced by perforation, in the course of typhoid fever, for example, the manifestations of pain and tenderness may not be sufficient to direct attention to the abdomen. In sucl cases, other local, in conjunetion with general, symptoms must be relied upon in the diagnosis.

It is desirable, in cases of peritonitis, to determine whether the discase be idliopathic or dependent on some one of the various local circumstances which stand in a causative relation to it in the majority of cases. In consequence of the relative infrequency of idiopathic peritonitis, the probabilities, in any case, are in favor of the cxistence of some one of these causative circumstances. Developed in the course of typhoid fever, it generally, but not invariably, procceds from perforation of the ileum. If developed in a case of pulmonary tuberculosis accompanied by diarrhoea, chronic peritonitis not preceding its development, perforation, connected with intestinal ulceration, is the probable cause. Perforation of the stomach is probable if the peritonitis occur suddenly in a case presenting the symptoms of gastric ulcer. Perforation of intestine, conneeted with latent ulceration, is to be very strongly suspected whenever the affection is developed suddenly, without any appreciable cause, in a person apparently in health; and if the pain and tenderness first occur in the neighborhood of the ereum, and continue most marked in this region, the seat of the perforation is probably the appendix vermiformis. The points just stated, however, do not positively clenote perforation of intestine. For example, they were present in a casc recently under my obscrvation, in which the peritonitis was due to perforation from ulceration of the gallbladder. Perforation of the ureter may be strongly suspected if the sulden development of the disease have been preceded by symptoms denoting obstruction from calculus or some other cause. In like manner, perforation of the ductus communis choledochus is the probable cause if symptoms denoting the impaction of a gall-stone in this duct have precerled. The disease is attributable to invagination or internal strangulation of the intestine when preceded and accompanied by symptoms denoting these causes of obstruction of the bowels. Its connection with renal disease is to be determined by an examination of the urine for albumen and casts from the uriniferous tubes. Finally, if evidence of the existence of any of the causative circumstances referred to be wanting, and the disease be fairly attributable to exposure to cold, it may be considered as idiopathic.

Prognosis.-Acute general peritonitis is always a grave disease, involving more or less danger to life. In the majority of cases, the danger is greacly cnhanced, either by the circumstances on which the development 
of the disease depends, for example, intestinal perforation, or by coexisting affections, for example, renal disease. We have no data for determining the amount of intrinsic tendency to death belonging to simple idiopathic peritonitis. There is reason to believe that the rate of fatality in all cases depends very much on the mode of management. Judiciously treated, in uncomplicated cases, the prospect of recovery is good. In complicated cases, including under this head cases in which the disease is connected with gastric or intestinal ulceration, Bright's disease, etc., the prognosis must allways be very unfavorable. Under certain circumstances, the disease runs rapidly to a fatal issue. It may destroy life within a few hours, developed as a result of pclforation. Recovery, however, is not impossible in cases of perforation, provided the opening be not large and closure be quickly effected by adhesions around it. The average duration in fatal cases is from five to six days.

The mode of dying is by either rapid or slow asthenia. An unfavorable progress of the discase is denoted by a progressive increase of the feebleness and frequency of the pulse, progressively increasing prostration, coldness of the extremities, hiccough, regurgitation, etc. On the other hand, a favorable progress may be predicated upon improvement as regards the general symptoms just referred to, together with diminution of the tympanites and muscular rigidity of the abdomen. Convalescence is apt to be slow, more or less tenderness and colic pains persisting during the progress toward recovery.

Treatuent.- Within a few years, an important change of practice, as regards the treatment of acute peritonitis, has taken place in this city (New York), and, to a considerable extent, throughout the country. There are grounds for the belief that, by means of the method of treatment which will be here recommended, this disease is managed much more successfully than heretofore. The change consists, first, in the disuse of bloodletting and cathartics; and, second, in relying mainly on the use of opium. Governed by the general principles which should regulate the employment of bloodletting in acute inflammations, the indications for this measure are very rarely, if ever, present in peritonitis, a disease which tends to destroy life, and sometimes very quickly, by asthenia. Having considered these general principles, I do not deem it necessary to enter into a consideration of the reasons for the opinion that, as a rule to which there are few, if any, exceptions, bloodletting, either general or local, is contra-indicated in the treatment of acute peritonitis. Bloodletting has been much employed in the treatment of this disease, and certainly, clinical observation has abundantly shown that it is not; to say the least, a curative measure. It is a measure which in this, as in other diseases, must do either good or harm; and in a disease, like this, involving always not a little danger to life, if it do harm instead of good, the amount of harm, even if not large, may bear heavily on the result. Cathartics, also, have been much employed in the treatment of this disease. Not only are they devoid of curative efficacy, but they are highly pernicious. An important point in the treatment of an acute inflammation of any part, is to secure for the part as much rest as possible. The friction incident to the peristaltic movements tends to perpetuate and intensify the inflammation in peritonitis. It is an object of treatment to arrest these movements, and thus secure rest for the inflamed membrane. Cathartics not only conflict with the means for effecting this object, but they aggravate the inflammation by increasing the peristaltic movements. Moreover, they are objectionable on the same score as bloodletting and other depletory and depressing measures. Clin- 
ical observation certainly furnishes no evidence of the usefulness of cathartics in the treatment of acute peritonitis. On the other hand, it has occurred to me, as to others, to sec the intensity of the inflammation reproduced by the too early administration of a cathartic, when the disease appeared to be progressing favorably, and the patient's life apparently lost in consequence. Cathartics, then, slould not enter into the treatment of this disease. The bowels should be allowed to remain constipated, if evacuations do not spontaneously oceur, during the career of the disease, even if this period be a week or more. In pursuing this course, the physician must expect, in some cases, to encounter the importunities of the patient or friends for medicine to open the bowels. If the patient suffer from an accumulation of feces within the rectum, its contents may be cautiously removed by repeated simple injections.

The use of opiates in the treatment of acute peritonitis is not of very recent date. Armstrong, more than a quarter of a century ago, adrocated full doses of opium in conjunction with bleeding and mercury, attaching the greater relative importance to the opium. Watson, in his admirable Lectures on the Principles and Practice of Physic, the first edition published more than 25 years ago, considers opium as of great value, and points out the evils of cathartics. This author refers to cases reported by a British physician, Bates, in which opium and rest were relied upon mainly in the treatment. More especially Graves, and afterward Stokes, of Dublin, called the attention of the profession to the subject, by reporting eases in which the disease was suecessfully treated with the free use of opium. They advocated the importance of this mode of treatment more particularly in cases of peritonitis due to perforation. But the credit of inangurating the treatment of acute peritonitis by opiates exclusively is, in a great measure, due to Prof. Alonzo Clark. Moreover, to Prof. Clark is due the credit of ascertaining that, in certain cases of peritonitis, there is a remarkable tolerance of opiates, and that, to secure their curative power, it is sometimes necessary to employ them in enormous doses. The attention of the profession of this country was first called to the efficacy of this plan of treatment, by the publication of cases of puerperal peritonitis treated by Prof. Clark, in the lying-in wards of Bellevue Hospital, in 1851-52. Prof C., however, was led to apply this method of treatment to these cases from his previous experience of its eflicacy in simple peritonitis.

An important object effected by opiates is the arrest or retardation of the peristaltic movements; this is a special object in this disease, but opiates are usefizl in this, as in other inflammations, by rendering the system more tolerant of the local affection. The latter is also an object to be effected. With reference to both these objects, opiates are to be given in doses sufficient to produce eertain effects. Relief of abdominal pain is generally evidence that the first object is effected. The doses, therefore, should be sufficient to produce this effect. The pain is to be relieved as quickly as possible, and the return of pain prevented by repeating the required doses at short intervals. Diminished frequency of the pulse is the best criterion of the desired effect upon the system. The success of the treatment will depend much on its being commenced early in the disease. Time should not be wasted by delay for any preliminary measures. A full dose should commence the treatment, the amount being regulated by the intensity of the pain. For an adult, from half a grain to a grain of a salt of morphia, or an equivalent close of some other form of opiate, may be given at oncc. In two or three hours the effects of the first close can be appreciated. If the patient be not then free from pain, 
snother close is to be given, the amount of this dose being determined by the degree of pain. And, during the course of the disease, the opiate is to be repeated, if required, at intervals of from two to three hours, in doses sufficient to prevent return of pain. Undue nareotism is, of course, to be avoided. In order to avoid this, the physician is to observe the effects as regard somnoleney, contraction of the pupil, and diminished number of respiration. It is never necessary to produce a degree of nareotism involving any danger. Judgment and careful watehing, however, are important, lest an unnecessary degree of nareotism be produced. The sommolency from the opiates should never become so great that the patient cannot be easily aroused. The respirations should not remain below ten or twelve per minute. If, as not infrequently happens, they are found below this number, or irregular, the intervals between the doses are to be lengthened or the doses diminished. In carrying out this method of treatment, it is desirable that the physieian should see the patient frequently. If this be impracticable, as it often will be in country practice, the administration of the doses must be intrusted, with minute instructions, to an intelligent and faithful assistant or nurse. The doses are to be gradually diminished in proportion as the local and general symptoms denote improvement, and, at length, discontinued.

The extent to which opiates are to be given in order to secure the objeets of the treatment will vary much in different cases. Other things being equal, the doses required will depend on the degree of pain. But the susceptibility of different persons to opium varies greatly, and in peritonitis, as in some other diseases, there is sometimes an extraordinary tolerance of this remedy. Thus, in one of the cases treated successfully by Prof. Clark, the patient took within the first 26 hours, of opium and the sulphate of morphia, a quantity equivalent to 106 grains of opium; in the second 24 hours, she took 472 grains; on the third lay, 236 grains; on the fourth day, 120 grains; on the fifth day, 54 grains; on the sixth day, 22 grains; on the seventh day, 8 grains, after which the treatment was sitspencled. 'This patient Prof. C. had reason to believe was not accustomed to the use of opium in health, and was not intemperate. As regards the amount of opium required, this ease is exceptional. Generally, according to Prof. C., in private practice, not more than half or two-thirds of a grain of a salt of morphia every two hours is needed. This accords with my experience. Often the requisite effects are produced with doses much less than those just named. The manner of conducting the opiate treatment may be illustrated by a tabular statement of the doses and symptoms at intervals of from two to three hours, in a ease in my service at Bellevue Hospital, the treatment being carried ont by the senior assistant.' In this ease, death took place on the tenth day, from the development of dysentery, the symptoms, as regards the peritonitis, denoting convalescence at the time the dysenteric affection became developed. The autopsy showed that the peritonitis was due to perforation of the gall-bladder from an abscess between its coats. The parts were agglutinated with recent lymph at the perforation, and the appearances of the peritoneum were consistent with convalescence from the peritonitis. Although fatal from an intercurrent disease, this ease exemplified the utility of the opiate treatment, and the autopsy leaves no room for distrust of the diagnosis.

The patient, a tailor, aged 30 , was suddenly attacked with sharp ab-

1 By Dr. George Engs. This case was reportcd by Dr. Engs in the American Medical Times, Tan. 9, 1864. 
dominal pain the day but one before his admission, and had had no treatment prior to entering the hospital. He was placed at once on the opiate treatment, and the following table, prepared by Dr. Engs, gives the doses, pulse, and respirations, with remarks, at intervals of from two to three hours :-

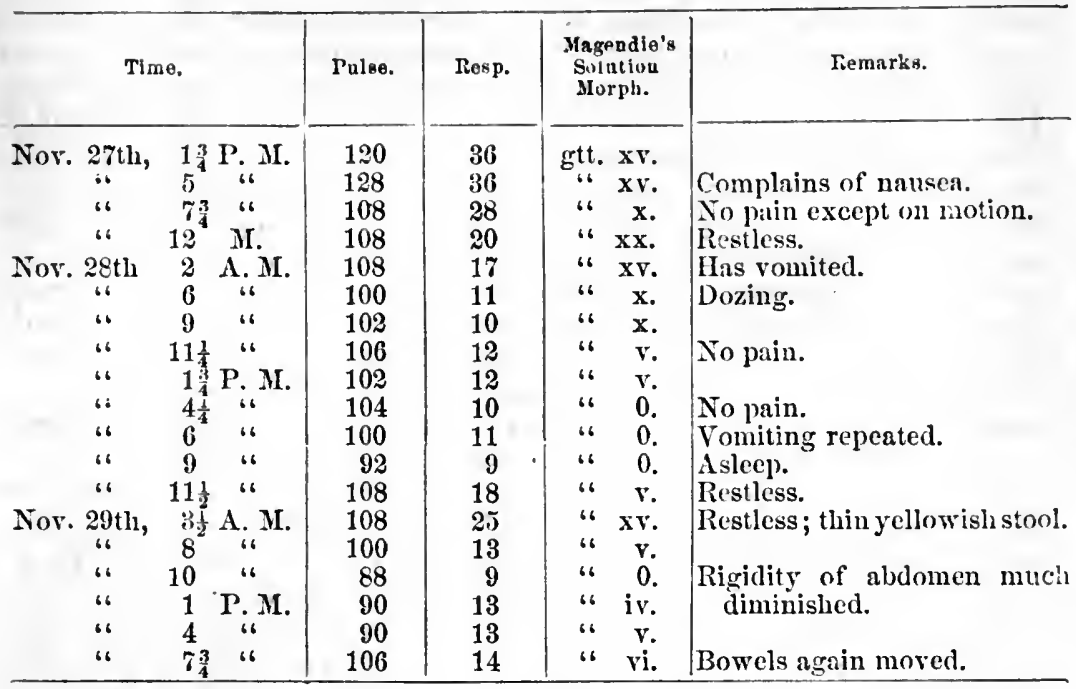

Dysenteric dejections occurred on the 30th instant, and death took place in six days afterward, the abdominal tenclerness, rigiclity, and tympanites having mostly disappeared.

In the treatment of this disease the main reliance is upon opiates. But other measures are important. Quietude of the body is to be enjoined; the bladder is to be relieved by the timely use of the catheter, if there be retention of urine. Rubefacients, sinapisms, or turpentine stupes may be applied over the abdomen; blisters are contra-indicated. All the good to be done in the way of revulsion will be effected by rubefacients, and counter-irritation beyond this does harm by increasing constitutional disturbance and contributing to depress the powers of life. Warm fomentations to the abdomen, if grateful to the patient, are useful; poultices are uncom. fortable from their weight; the water-dressing or the spongio-piline is to be preferred. Cold applications are recommended, after trial, by Grisolle and Allison. I cannot speak of their utility from observation, but I should be willing to trust to the feelings of the patient in deeiding between cold or warm applications. Cold applications, provided they are well borne by the patient, are recommended by Niemeyer, employed in the same way as in acute pleuritis, namely, by means of compresses dipped in cold water and renewed erery ten minutes. Sustaining measures are indicated in proportion as the symptoms denote failure of the vital powers. Tonies, alcoholic stimulants, and concentrated nourishment constitute the supporting measures, not less appropriate to this than to other diseases which tend to destroy life by asthenia. I have seen the life of a patient apparently saved by the free and persevering use of alcoholic stimulants, in conjunction with the use of opiates. The employment of eatharties in convalescence ealls for great circumspection; they may not be required, the bowels acting spontaneously. The free use of opiates continued throughout the 
disease does not appear to conduce to constipation, and does not prevent the occurrence of diarrhœa in a certain proportion of cases.

The employment of mercury claims a few remarks. Mercurialization has been deemed highly important in this disease by most practitioners heretofore, and it is still deemed important by many. It is supposed to be useful in this, as in other serous inflammations, by limiting exudation and promoting its absorption. The opinion has already been expressed that the so-called antiplastic and sorbefacient action of mercury has been much exaggerated, but that we are not authorized with our present knowlelge to deny to this remedy any curative power. And it is perhaps questionable whether, in so grave an affection as acute peritonitis, we are fully warranted in omitting this remedy. I must confess, however, that for some years past it has not entered into the treatment of the cases of which I have had charge. The employment of mercury should not diminish the reliance on opiates; and it should be so employed as not to interfere with the objects of the opiate plan of treatment. If given internally, it should be in fractional doses, so as to avoid a cathartic effect, but a preferable mode is its employment by inunction.

In endeavoring to determine clinically the relative value of different methods of treatment, cases should be gromped according to the different circumstances giving rise to the peritonitis. As regards the prospect of success from any treatment, cases of perforation, evacuation of pus into the peritoneal cavity, rupture of the gall-bladder, etc., are not to be classed with cases of simple peritonitis. Moreover, the disease, more especially in its simple form, is too rare for a clinical observer, however large his field of practice, to accumulate many cases. I have notes of thirty-five cases which have fallen under my own observation. Of these thirty-five cases, seventeen proved fatal, death being attributable to the peritonitis per se. In addition to these seventeen fatal cases, death was attributable in one case to dysentery which was developed during apparent convalescence; in one case the peritonitis supervened on acute pleurisy, the patient dying as if by "shock," and in one case death took place after the patient had recovered from the peritonitis sufficiently to be up and about, the immediate cause of death not having been ascertained. Adding these three cases and the whole number of those ending fatally is twenty. Of the fatal cases, exclusive of the three cases just referred to, the autopsy showed in four rupture of the vermiform appendix of the cæecum, in one case fracture of the liver from injury, in two cases suppuration of the ovaries, in both an abscess having opened into the peritoneal cavity; and in one case there was rupture of intestine from violent efforts made by the patient in reducing a hernia. In three cases there was no apparent local cause of this peritonitis. In six cases there was no autopsy. Of the three cases of simple peritonitis (as determined by the autopsy), and only those proving fatal, the opiate treatment was commenced in one case on the third day; in one case a cathartic was given on the third day and the patient was not kept quiet, and in the remaining case the opiate treatment was imperfectly carried ont. Of the six fatal cases in which no autopsy was made, three were treated with cathartics and bleeding. In two cases the opiate plan was moderately carried out, and in one of these cases the patient was considered as convalescent, and allowed to sit up, when a renewal of the symptoms took place, followed by speedy death. In the remaining case the opiate treatment was efficiently carried out until the patient appeared to be nearly convalescing, when, yielding to the importunities of friends, a cathartic was given, after which the intensity of the disease was reproduced, 
and the case speedily ended fatally. Of the fifteen cases ending in recovery the treatment was exclusively or chiefly by opium.

These analytical results are submitted as afforling eviclence of the efficacy of the method of treatment by opium after the plan pursued by Prof. Claik.

\section{Partial or Circumscribed Peritonitis.}

Inflammation limited to a circumscribed portion of the peritonenm is almost invariably a complication of a prior affection of the parts covered by the inflamed portion of the membrane. In this respect the same law holds good with reference to the pleura and peritoneum. Ulceration of the stomach, for example, leads to a peritonitis limited to the site of the ulcer, and the contiguous parts become adherent at that point-a conservative provision against perforation. Inflammation of the uterus extends to the portion of this membrane covering that organ. Affections of the liver sometimes lead to inflammation of the peritoneal investment of this viscus (peri-hepatitis). In consequence of this partial peritonitis, the liver sometimes becomes covered with lymph, and closely adherent to the adjacent parts; hence one source of atrophy with diminished volume of the liver, and consequent dropsy of the peritoneum. In like manner, partial peritonitis may be limited respectively to portions of membrane in relation with the spleen, the pancreas, the ovaries, ete. A small perforation of the intestinal canal may give rise to only partial peritonitis, the orifice becoming closed by exudation of lymph and adhesions.

The diagnosis of partial peritonitis is to be based upon cirenmscribed pain and tenderness, with perlaps more or less febrile movement. These symptoins are marked in proportion to the acuteness of the inflammation; but partial peritonitis is oftener subacute than acute. Physical exploration, in some cases, furnishes diagnostic evidence. The presence of lymph may oceasion a friction sound or a tactile sensation of rubbing due to the attrition of opposed surfaces with the diaphragmatic respiratory movements. It is most apt to be obtained when the peritonitis is situated over the liver and spleen. It is obtained in cases of partial oftener than of general peritonitis, and is of comparatively less importance in the latter, beeause the symptoms generally suftice for the diagnosis of gcneral peritonitis.

It must be difficult, if not impossible, to determine the existence of partial peritonitis not connected with an affection of the parts covered by the inflamed portion of peritoneum. Cases, however, are so rare that, the existence of partial peritonitis being ascertained, a complicating affection of the parts covered by the inflamed portion is to be inferred.

'The prognosis in cases of partial peritonitis will depend on the nature and extent of the affection of which the peritonitis is a complication. The allhesions resulting from the inflammation, as has been seen, may be either conservative or deleterious. for.

So far as the peritonitis is concerned, palliative treatment is alone ealled

\section{Chronic Peritonitis.}

Exclusive of partial peritonitis, inflammation of the peritoneum is oftener acute than chronic. Cases of idiopathic, chronic, general peritonitis certainly belong among the curiosities of medical experience. In cases of chronic peritonitis, the inflammation is generally either consecutive to a prior affection of the abdominal viscera, or it is sccondary to an 
affection situated elsewhere. Occasionally acute pertionitis eventuates in the chronic form of the disease. This is rare. In cases of acute peritonitis, if life be not destroyed by the acute disease, recovery, as a rule, takes place. The patient is liable to suffer for some time from weakness or disorder of the intestinal canal, and adventitious membranes resulting from the disease may occasion inconvenience; but the inflammation does not persist, save in exceptional cases.

Of cases of chronic, not succeeding acute, peritonitis, in the great majority the affection is developed in connection with the tuberculous cachexia. The affection is then commonly known as tuberculous peritonitis. Post-mortem examination, in these cases, generally reveals, in addition to appearances denoting peritoneal inflammation, either the yellow tubercle associated frequently with tuberculosis of the mesenteric and intestinal mucous glands, or the gray semi-transparent granulations. The latter, according to Robin, are produced consecutively to the peritonitis. 'Tuberculous products within the abdomen, however, are not invariably present in cases of peritonitis developed in connection with the tuberculous cachexia. The observations of Louis show that tuberculous patients are liable to chronic peritonitis irrespective of the presence of tubercle either beneath or upon the peritoneum, and irrespcctive of a tuberculous or other affection of any of the abdominal viscera, the peritonitis, in these cases, being attributable to the tuberculous cachexia. So generally is chronic peritonitis associated with the tuberculous cachexia, that, the existence of the former being ascertained, the latter may be inferred, provided the peritonitis be general, not traumatic, and acute peritonitis have not preceded. Moreover, since, after childhood, the local manifestations of the tuberculous cachexia almost invariably first take place in the lungs, pulmonary tuberculosis may be inferred with much positiveness from the existence of chronic peritonitis, with the qualifications just stated. For our knowledge of these relations of chronic peritonitis, we are mainly indebted to the clinical researches of Louis. ${ }^{2}$

In a certain proportion of cases, chronic peritonitis occurs in connection with carcinoma either seated in the peritoneum, or originating in other structures and extending to this membrane. The inflammation, in these cases, is excited by the carcinomatous disease. The affection, in these cases, may be called cancerous peritonitis.

After death, in cases of chronic peritonitis, more or less liquid effusion is frequently found in the peritoneal sac. 'The quantity, as a rule, is not large; not infrequently it is small, and in some cases there is no accumulation. An effusion frequently takes place in considerable quantity, which either diminishes or disappears before death. Exceptionally, the amount of effusion is so great that paracentesis is required. I have known this to occur in cases of cancerous peritonitis. The effused liquid contains lymph in more or less abundance. Merely serous effusion, here as elsewhere, is no evidence of inflammation. The peritoneal cavity may contain pus in greater or less quantity. A purulent collection here sometimes leads to ulceration and perforation of the intestine or of the abdominal walls, as in empyema the pus makes its way into the bronchial tubes or through the parietes of the chest. The intestines are agglutinated to each other, the solid viscera, and the abdominal walls by means of lymph, and permanent adhesions follow. The latter alone are evidence, not of existing but of past inflammation. If the disease have been of considerable or long duration, the intestinal convolutions are sometimes bound together,

1 Recherehes sur la Phthisie. 
or connected with the solid viscera, so as to form masses of considerable size, which are appreciable, by palpation, through the abdominal walls.

As regards the clinical history and diagnosis, chronic peritonitis is frequently obscure, belonging among the affections ealled insidious, and its existence is not always readily determined. If it follow acute peritonitis, it is less likely to escape detection than when it is subacute from the beginning. Under these circumstances, the persistence of pain and tenderness, the presence of liquid effusion in greater or less quantity, continued rigidity of the abdominal muscles, together with general debility, emaciation, in some cases febrile movement, etc., render the diagnosis sufficiently clear.

Tuberculous peritonitis is, from the first, subacute. The researehes of Louis show the symptoms to be as follows: Augmentation of the size of the abdomen from tympanites and liquid effusion, the latter denoted by fluctuation ; abdominal pains, often not marked, with tenderness on pressure; diminution of the enlargement from liquid effusion and perhaps disappearance of the sense of fluctuation after a time; the tympanites in some cases diminishing after a time, and followed by tension of the al)dominal walls ; the intestinal convolutions sometimes, after the tympanites has disappeared, apparent through the walls of the abdomen. Pains and tenderness are sometimes wanting, the patient making no complaint of the abdomen, and rough pressure being well borne. In some cases the abdomen has to the touch a doughy or boggy feel. Peritoneal friction is sometimes heard or felt. With these local symptoms are associated more or less acceleration of pulse, perspiration during sleep, hectic paroxysms, progressive emaciation, anæmia, general debility, etc. After childhood, pulmonary tuberculosis will be found to exist in a vast majority of cases. With the foregoing local symptoms, especially if tuberculous enteritis be excluded by the absence of diarrhœa, the existence of pulmonary tuberculosis tends to strengthen the diagnosis of chronic peritonitis. The tuberculous affection of the lungs in some cases is quite latent as regards pulmonary symptoms, and its existence is to be determined by means of physical signs.

In cancerous peritonitis, the local symptoms do not differ materially from those in tuberculous peritonitis. Febrile movement is less constant and marked in the former; perspiration is not so apt to occur. The age of patients is greater than in the majority of cases of the latter. The cancerous affection within the abdomen may be secondary to cancer in other situations open to examination. Pulmonary tubereulosis does not coexist.

The prognosis in all cases is unfavorable. A fatal termination, sooner or later, even if the peritonitis be not connected with either tubercle or cancer, is the rule. The duration of the disease in these cases may be long. I have met with a case in which the history appeared to show that the affection had existed for a great number of years before death. Tuberculcus and cancerous peritonitis are always fatal, the duration varying from one or two months to a year or more. The fatal termination is hastened by coexisting pulmonary disease, in cases of tuberculous peritonitis, and frequently by tuberculous enteritis and other local affections.

From what has just been stated with reference to the prognosis, it follows that chronic peritonitis is not to be treated with an expectation of recovery. If the peritonitis be simple, the indications are to palliate the local symptoms, and, by the use of tonic remedies, nutritious diet, and other hygienic measures, to strengthen the system and enable it to endure the local affection as long as possible. 
Considerable may be done in this way to prolong life, and this conservative plan of treatment will be most likely to etfect a cure if recovery be possible. Cathartics, mercurialization, local depletion, and all depressing remedies are contra-indicated. In cases of tuberculous peritonitis, the treatment embraces the measures, in addition to those just named, which have reference to the tuberculous cachexia, viz., cod-liver oil and alcoholic stimulants, provided these are well borne. Cancerous peritonitis claims essentially the same treatment. The local palliative treatment embraces opium, according to the degree of pain, soothing and moderately stimulating liniments applied to the abdomen, protection of the abdomen against cold by the application of plasters and flannel, and mechanical support with moderate compression of the abdomen.

Occasionally, in cases of chronic peritonitis, the accumulation of liquid in the peritoneal sac is sufficient to occasion inconvenience and even danger by compressing the abdominal viscera and interfering with the play of the diaphragm. Under these circumstances are inclicated measures to effect the diminution or removal of the liquid. 'These measures, including paracentesis, will be considered in treating of dropsy of the peritoneum.

Peritonitis is sometimes developed in newly-born children, and during intra-uterine life.

\section{Hydro-Peritoneum.}

The term hydro-peritoneum denotes peritoneal dropsy. The term ascites is commonly applied to cases of dropsy of the peritoneum, and also to cases in which liquid effusion is a result of peritonitis. In a pathological view, a dropsical differs essentially from an inflammatory effusion, and the distinction is of practical importance. The accumulation of liquid from inflammation has been noticed in treating of peritonitis. Under the present head, reference is had exclusively to cases of dropsical effusion, the effused liquid being purely a transudation, $i . e .$, not containing lymph, and the peritoneum free from inflammation. Like other dropsies, hydroperitoneum is a symptom or an effect of disease, not strictly a disease per se. But the accumulation of liquid may be the first and chief manifestation of disease; it occasions inconvenience and danger, and therapeutical measures are especially directed to it. Moreover, it is not practicable in all cases to trace the dropsy to its causative morbid condition. Hence the convenience and propricty of considering it as an individual disease. Peritoneal dropsy occurs in conjunction with dropsical effusion into the pleural sac, and the areolar tissue throughout the body, or anasarca. Hydro-peritoneum is, however, to be considered as an individual affection only when it occurs as a local dropsy, $i$. e., not as an element of general dropsy.

In the great majority of cases, hydro-peritoneum, occurring as a local dropsy, proceeds from morbid conditions seated in the liver. And, of these, the structural affection known as cirrhosis especially gives rise to peritoneal dropsy. This affection will be considered in connection with the diseases of the liver. Atrophy of the liver from compression by lymph upon its surface and morbid adhesions to surrounding parts leads to this form of dropsy. The dropsy in these cases is due to obstruction to the passage of blood through the liver, the transudation into the peritoneal sac being an effect of the hydraulic pressure upon the coats of the portal veins, caused by the hepatic obstruction. In so large a proportion of cases is hydro-peritoncum dependent on disease of the liver, that the 
latter is always presumable, assuming that general dropsy does not exist. It is generally considered that enlargement of the spleen may give rise to hydro-peritoneum. There is little or no foundation for this opinion. Enlargement of the spleen occurs in a small proportion of cases of dropsy dependent on eirrhosis of the liver, and occurring without the latter affection, as, for example, a sequel of intermittent fever, it is not accompanied by peritoneal dropsy. Obstruction of the vena portæ by the pressure of a tumor, by the presence of a thrombus, or as a result of adhesive phlebitis, gives rise to peritoneal dropsy in the same way as obstruetion due to hepatic lesions, viz., transudation taking place from hydraulic pressure. According to Frerichs, coagula in the portal vein take place, without inflammation, in consequence of weakened force of the circulation, from compression, exterior to the liver, by swellings or tumors, and in connection with various hepatic affections.' Cardiae and renal disease do not give rise to peritoneal, save as an element of general, dropsy. Clinical observation shows, in a small proportion only of cases of hydro-peritoneum occurring as a local affection, evidence of disease, either of the heart or kidneys. ${ }^{2}$ In the vast majority of cases, the dropsy is dependent on struetural lesions of some kind; that is, it occurs very rarely as a functional affection. Of forty-six cases coming under my observation, which I have analyzed, in one case only was the history consistent with the supposition that the affeetion was purely functional.

Varions causes may co-operate with the morbid conditions upon which peritoneal dropsy is dependent. Diseases of the heart, renal disease, anæmia resulting from intermittent fever, lactation, etc., may be mentioned as co-operating causes. It may be doubted, however, if these eauses alone ever give rise to the affection. Indirectly they favor its occurrence and determine the epoch when the dropsical effusion takes place.

The effusion of liquid and its accumulation take place usually without pain, tenderness, or any local subjective symptoms. The enlargement first direets attention to the abdomen. In the majority of eases, when this begins it goes on with rapidity, and the abdomen soon becomes considerably or greatly distended. The symptoms referable directly to the dropsy proceed from the mechanical pressure of the liquid, and, other things being equal, are in proportion to the quantity of effusion. The distension of the abdominal walls by the liquid, and its weight, oceasion more or less inconvenience. The functions of the abdominal organsstomach, liver, kidneys-are impaired by compression. Pressure on the veins within the abdomen gives rise to, or increases, cdema in the lower extremities. The movements of the diaphragm are restrained or arrested, breathing being carried on by the costal muscles, and, if the accumulation be very large, the eapacity of the chest is diminished by the upward pressure, and the embarrassment of respiration may be sufficient to destroy life. The appetite is usually more or less impaired. A sense of fulness is frequently felt after taking food in moderate quantity. Tomiting and diarrhœa are occasional symptoms. Hæmatemesis occurs in a certain proportion of cases.

Enlargement of the superficial veins on the anterior aspect of the trunk is a consequence of a portion of the blood from the portal vein passing into the systemic venous -system through anastomosing branehes. The

1 Clinical Treatise on Diseases of the Liver. Sydenham edition, vol. ii. p. 384.

2 For clinical facts on which these and other statements are based, vide paper by the author entitled Clinical Report on Hydro-Peritoneum, based on an Analysis of forty-six Cases, American Journal of Medical Sciences, April, 1863. 
superficial veins of the abdomen in some cases, of the chest in other cases, and sometimes in both situations, become more or less dilated, presenting a varicose appearance. Hernial protrusions at the umbilicus and in other situations are occasionally produced.

Edema of the lower extremities is a pretty constant symptom, the amount of cdema varying much in different cases. The limbs, scrotum, and penis sometimes become enormously swollen. Some œedema of the lower limbs preceded the enlargement of the abdomen in one-half of the cases (21), among those analyzed by me, the histories of which contained information on this point. An examination of these cases disproved a statement which has been made with respect to the significance of œdema of the lower extremities preceding the abdominal dropsy, viz., that it denotes disease of either the heart or kidneys. ${ }^{1}$ Edema of the face and upper extremities does not belong to the clinical history of hydro-peritoneum. If present, it denotes coexisting renal or cardiac disease. In the progress of the affection, emaciation and pallor become marked. The attenuation of the upper part of the body, the distended abdomen and the œdematous lower extremities, combine to render the appearance highly characteristic of the affection. Cholæmia or icterus is occasionally present; it oecurred in 7 of the 46 cases which I have analyzed.

Febrile movement does not attend the progress of this affection. The pulse may be more or less increased in frequency and proportionately feeble, or it is more or less enfeebled without acceleration. The urine is frequently scanty. Purpurine may be present in the urine, giving rise to an appearance of hæmaturia. The mind is usually intact, but, in a certain proportion of cases, toward the close of life, delirium, convulsions, and coma occur. These symptoms may be due to uræmia or to retention in the blood of the excrementitious principles of the bile (cholesteræmia).

The mode of dying, in the great majority of eases, is by slow asthenia. A rapid and large accumulation of liquid may destroy life by interference with respiration; and apnœa and asthenia are combined when coma precedes death.

The prognosis in most cases is extremely unfavorable. The cases in which a permanent recovery takes place are very few. The morbid conditions on which the affection is dependent are generally incurable, and lead to its return, sooner or later, in most of the cases in which the dropsy disappears or is removed. The duration of the affection is variable. In the fatal cases of those which I have analyzed, the duration varied from six weeks to seventeen months, the average duration being about five months.

The diagnosis of hydro-peritoneum is generally made without difficulty, yet there is a liability to errors which may lead to serious results. A distended bladder has been punctured, the case being supposed to be one of peritoneal dropsy. It is said that John Hunter committed this unfortunate mistake. On careful examination, the tumor formed by the distended bladder may generally be felt throngh the abdominal walls; but the introduction of the catheter is the diagnostic test. My colleague, Prof. Sayre, was called upon to puncture the abdomen in a case supposed to be one of dropsy. He was struck at once with the unusual appearance of the abdomen, which projected notably in front and was contracted at the sides. A little milky liquid was withdrawn by introducing the catheter and employing suction by means of an India-rubber bag. Death taking place, with the symptoms of uræmia, it was found, after death,

- Vide Report, op. cit. 
that the case was one of sacculated bladder, the saccular appendage being situated above and measuring twenty-eight inches in cireumference. Pregnancy, the liquor amnii being unusually abundant, has been mistaken for dropsy, and the uterus punctured. Careful examination through the abdominal walls and per vaginam, together with auscultation of the abdomen, should prevent this error. Great corpulency has led to error and the operation of paracentesis, as in the famous case of "dry tapping" related in the lectures of Sir Astley Cooper. Finally, tympanites, large abdominal tumors, and ovarian eysts are to be diseriminated from peritoneal dropsy.

The enlargement of the abdomen from dropsy commences at the lower part, and, if the abdomen be not largely distended, it is more marked below than above when the patient stands or sits. The enlargement on both sides is equal; the abdomen is symmetrical. If the patient lie upon either side, the weight of the liquid causes the depending side to sag. Percussion on one side, the open palm being placed on the opposite side, frequently causes a characteristic shock, called the sense of fluctuation. Sometimes this sensation is more appreciable when the palm is placed near the point at which the percussion is made. The former methor of pereussion gives rise to what is ealled diametrical and the latter to peripheral fluctuation. But a more satisfactory application of percussion is to eompare the results when the patient is placed in different positions. Pereussing first in the sitting or standing posture, tympanitic resonance, from intestinal gas contained in the intestines floating upon the liquid, is usually found at the upper part of the abdomen, extending below for a greater or less distance, and flatness from this point to the pubis. Placing now the patient on the back, the change in the relative situation of the liquid and intestines is shown by the greater extension of the tympanitic resonance toward the pubis. Similar proof of the presence of liquid is obtained by percussing upon one side of the abdomen suceessively when the body is inclined to that and the opposite side. The exeeptional eases in which this test afforded by percussion is not available are those in which the intestines are fixed by morbid adhesions. This is rare in eases of a purely dropsical affection. The several affections simulating dropsy which have been named may generally be excluded by the application of percussion as just described. Ovarian cysts of suffieient size to distend the whole abdomen are the most likely to be confounded with hydro-peritoneum. These are generally, however, distinguishable by the appreciation of the cyst through the abdominal walls, the existence of a tumor on one side before it extended to the whole of the abdomen, the absence of symmetry in the enlargement of the two sides, together with the want of evidence of the presence of liquid in the peritoneal sac afforded by percussion when the body is placed in different positions.

The treatment of hydro-peritoneum relates, first, to the dropsical effusion, and, second, to the morbid eonditions on which the effision depends. As regards the dropsical effusion, the object is to effect its removal or diminution. For this object, medicinal and surgical measures may be employed. The medicinal means consist of remedies to eliminate water from the blood, and thereby incluce absorption of the effused liquicl. The elimination of water from the blood is to be accomplished by diureties and hydragogue cathartics. Diuretics, in most cases, effect but little. It is diffieult generally to obtain much diuretic effect from any of the remedies of this class, the difficulty arising from the slowness with which the remedies enter the general eirculation, owing to the fact that the morbirl conditions giving rise to the dropsy usually involve obstruction to the 
portal circulation. Of 13 cases treated with diuretic remedies, in 8 no effect upon the dropsy was prodnced; in 5 more or less diminution of the dropsy followed, but in only 2 cases was the improvement marked and progressive. Little dependence, therefore, is to be placed on diuretics, but inasmuch as, if properly prescribed, they do not cause much disorder or prostration, they should be fairly tried. The saline and vegetable diuretics may be given in succession, and a diuretic effect is more apt to be produced if several are employed at the same time.

Hydragogue cathartics act more efficiently than diuretics ; but clinical observation does not furnish much evidence of success from their use. Of the different hydragogues, elaterium is the most reliable. I am accustomed to prescribe this remedy in doses of a quarter of a grain repeated at short intervals until abundant liquid evacuations are produced. Afterward the doses may be repeated according to circumstances. Of 17 cases in which this remedy was employed, in 9 no appreciable benefit followed; of the remaining 8 cases, in one case only was the dropsy removed, in the other cases more or less diminution taking place. In all the effect was only temporary. Owing to the disturbance and prostration caused by the prolonged use of hydragogues, they are liable to do harm, and should not enter largely into the treatment.

The apocynum cannabinum, or Indian hemp indigenous in this country, is considered by some an efficient hydragogue in this form of dropsy. From some trial of this remedy in Bellevue IIospital, I have been led to think it is less reliable than the elaterium. The decoction of the root may be employed, or the extract.

It is important to bear in mind that, in order to secure the desired effect of diurctics or hydragogue cathartics upon the dropsy, the ingestion of liquids is to be restricted, the patient drinking no more than the wants of the body require.

The surgical method of effecting the removal of the liquid is tapping. With respect to this operation, the views generally held are, that it is to be resorted to only when the abdominal distension occasions great distress or danger, and not until the indirect means have been thoroughly tried; that the dropsy generally increases more rapidly after the operation, and that the operation involves danger if the system be much prostrated. I have been led to think that these views are erroneous. Tapping effects promptly, without perturbation and without impairing the vital powers, the object for which diuretics and cathartics are employed, measures generally ineffectual, disturbing the digestive functions, and enfeebling the powers of life. The operation is a trivial one, involving little risk of accidents or of peritoneal inflammation. A patient came under my observation at the Long Island College Hospital, who had repeatedly tapped himself, using, as a trocar, a blade of a pair of scissors, and a piece of an ordinary clay pipe-stem as a canula. There is no danger from increased rapidity of effusion directly after the operation. The patient is spared, not only the inconvenience and distress, but the permanent injury caused by the prolonged pressure of the liquid upon the abdominal and thoracic viscera, and he is in a condition more favorable for other remedies than those which have special reference to the removal or diminution of the dropsy. Clinical experience shows that, in some cases, even when the dropsy is dependent on cirrhosis, the liquid does not accumulate for weeks or months after the operation. In a case which has come under my notice, after two tappings, there was no reaccumulation for six years. In another case which I saw in July, 1865, with my colleague Prof. J. C. 
IIutchison, and with Dr. Farnham, of New York, the patient, a female somewhat alvanced in life and feeble, was tapped, and, after the withdrawal of a large amount of liquid, a tumor was felt just below the epigastrium. The abdomen refilled in a short time, and tapping was again employed. Since the second tapping the dropsy has not returned, nearly a year having elapsed, although the tumor has increased in size. In this ease there was no ground for suspecting eirrhosis of the liver. It seems to me juclicious to resort to tapping as soon as the aecumulation of liquid is sufficient to occasion much inconvenience, adopting this direct method in lieu of the indireet means, provided the latter do not prove immediately eflicacious, and repeating the operation whenever the abdomen becomes again distended. 'These views are based on the results of tapping in 20 cases under my observation, the operation in these cases being performed but once in 11 cases, and in the remaining 9 cases being repeated from three to thirty times. 'The conclusions drawn from the facts contained in the histories of these cases are as follows: 1. 'Tapping may be resorted to as a palliative measure, when the eondition of the patient is such that only temporary relief is to be expected. 2. In a certain proportion of cases the dropsy returns more or less quickly, and it may be neessary to repeat the operation many times. The repetitions, howerer, are innocuous. This was illustrated in one case in which it was performed thirty times in 18 months. 3. In some cases the dropsy does not return for a considerable and even a long period. That it will prove a curative measure is not to be expected in the vast majority of cases.

The dropsical collection is sometimes discharged spontaneously, the walls of the abdomen becoming attenuater by distension, and rupture taking place. The perforation, in these cases, is most likely to occur at the umbilieus or at some point at the median line. In a case at Bellevue Hospital, a protrusion of a cyst-like tumor took place at the umbilieus, which, after attaining to a large size, burst, and the contents of the peritoneal sac were discharged through the opening. 'This patient is now under my observation, four months after the spontaneous discharge took place, and the dropsicul effusion has not yet returner.

The treatment relating to the morbid conditions on which the dropsy depends embraces, in the first place, measures to prevent an increase of the incurable lesion which exists in the great majority of cases, viz., eirrhosis of the liver. It will be seen, when we come to consider this lesion, that it is generally caused by spirit-drinking. Change of habits, as regards the use of spirits, is, therefore, the most important of the measures coming under this head. In the second place, certain accessory or co-operating causative conditions may be removed, causes which act by impoverishing the blood and impairing the forces which earry on the circulation. This part of the treatment embraces tonic medication with nutritious diet, and other measures to strengthen and invigorate the system. Well-directed treatment for this end, although rarely eurative, will often do much to prolong life, and to secure as much improvement of health as is compatible with existing structural lesions.

Scarification of the genital organs, when these become greatly œdematous, is apt to lead to gangrene and sloughing. This has oceurred in three cases under my observation.

' For details of these cases, vide Clinical Report, op. cit., from which the remarks on tapping are borrowed. During the perind from the publication of the first to the fourth edition of this work (six ycars), I have observed many cases which exemplify the correctness of the practical views presented in the text. 
Mechunical compression of the abdomen, after tapping, by means of a swathe or a laced supporter, is a measure of importance, as not only affording comfort, but tending to prevent a renewal of the dropsical effusion.

\section{CHA P TER XI.}

\section{DISEASES AFFECTING THE SOLID VISCERA OF THE ABDOMEN.}

Acute Diffuse Hepatitis-Anatomical Characters-Chnical History-Pathological CharacterCausation-Diagnosis-Prognosis-Treatment. Circumscribed Suppurative Hepatitis. Suppurative Portal Phlebitis. Cirrhosis of the Liver.

$\bigcap^{\mathrm{F}}$ the affections of the solid abdominal viscera, the greater number by far, and the more important, are seated in the liver. These will be considered, and afterward the affections of the spleen and pancreas. I shall first consider the hepatic diseases which are undoubtedly inflammatory; next structural lesions of the liver; and, lastly, functional affections of this organ. Acute inflammation of the liver, or hepatitis, is presented in two forms, viz., diffuse hepatitis, that is, the inflammation extending over the whole or greater part of the organ, and circumscribed, suppurative hepatilis or hepatic abscess. These two forms claim separate consideration. Suppurative inflammation of the portal vein, portal phlebitis, will be considered in this connection. Subacute or chronic hepatitis is generally supposed to be the essential pathological condition leading to the structural lesion known as cirrhosis of the liver. Aside from the latter, we have no precise clinical knowledge of subacute or chronic hepatitis. Congestion of the liver, which some authors recognize as a distinct affection, will be noticed among the functional affections of this organ (vide chapter xiii.) and, also, as an incidental element in treating of different affections. The first disease to be considered is acute diffuse hepatitis, called by some authors parenchymatous hepatitis. In view of the extreme infrequency of this disease, save in tropical climates, and the deficiency of exact information respecting it, in the present state of knowledge, a brief space only will be devoted to its consideration.

\section{Acute Diffuse Hepatitis.}

Of the rare affections, in the cold and temperate zones, this is one of the rarest. It is said to be not infrequent in tropical climates, but in the latter, inflammation of the liver is oftener circumscribed than diffuse. The inflammation in this affection is seated in the perenchyma of the liver. Inflammation of the portion of serous membrane which invests the liver, called peri-hepatitis, has been noticed under the head of partial peritonitis.

Anatomical Character.-Pathologists are not agreed respecting the morbid changes, apparent after death, which denote this disease. Mere hyperæmia or congestion is not adequate evidence that inflammation has existed. The same statement is to be made concerning simple softening 
of the hepatic parenchyma. Both, doubtless, are effects of inflammation. but both may be non-inflammatory. A fibrinous exudation is an effect of inflammation here as in other parenchymatous structures. This exudation takes place at the peripheral portions of the hepatic lobules. The organ is also infiltrated with a sero-albuminous liquid which escapes freely from cut surfaces. The presence of exudation and serous infiltration occasions, at first, more or less enlargement of the organ, but the atrophy induced by the pressure of the morbid deposit may lead to subsequent diminution of volume. The peritoneal covering participates in the inflammation, like the pleura in pneumonitis, and the surfice becomes opaque and roughened. The parenchyma is more friable than in its healthy condition. Microscopical examination shows destruction of the liver cells. Subsequently, piw may be formed, and the structure of the organ may be more or less broken down or destroyed. The pus may be collected in abscesses varying in number and size, and in some cases the appearance of the purulent collections shows that several abscesses, at first distinct, have coalesced. 'The abscesses are generally lined with a pyogenic membrane, but sometimes this is wanting. The pus is generally of the kind called laudable, but is sometimes. sanious and offensive. Gangrene has been observed, but is exceedingly rare. In favorable cases, the exuclation and effused serum are absorbed withont leading to the formation of pus and destruction of the parenchymatous structure. Death may take place, on the other hand, before the latter results take place. In a fatal case, which has fallen under my observation, the gross characters consisted of a considerable increase of the size of the organ from exudation and effusion, the enlargement having taken place, as shown by physical examinations, after the date of the attack. In this case there was no breaking down of the tissues nor production of pus, the life of the patient not being sufficiently prolonged for these effects. In another case the liver was much enlarged, weighing eight pounds, and in portions, which were estimated to be about three-fifths of the organ, the structure was broken down and mixed with bloody serum and purulent matter. The peritoneal covering was coated with lymph.

The changes belonging to the condition called acute or yellow atrophy of the liver are attributed by Bright, Frerichs, and others, to acute diffuse inflammation. The correctness of this view, however, is questionable. This condition will be noticed in connection with the structural affections of the liver.

Clinigal Mistory.--In describing this disease, writers have not been careful to distinguish diffuse from circumscribed inflammation ; moreover, the existence of the disease has frequently been based on morbid appearances after death which are of a doubtful character. Hence, it is difficult to determine its symptomatology with as much precision as is desirable; its clinical history is not, as yet, satisfactorily established.

The disease may be ushered in by a chill more or less marked, and a succession of chills sometimes oceurs. Febrile movement follows. Pain in the region of the liver is usually felt, but not invariably. The pain is sometimes dull or obtuse, and sometimes sharp or lancinating. If the latter character be marked, it is fair to infer that the peritoneal covering is implicated. Shooting of the pain into the right shoulder was formerly considered a pretty constant symptom, but later observations appear to show that this is the exception rather than the rule. More or less tenderness on pressure exists over the site of the liver. As regards the degree of pain and tenderness, there is much variation in different cases. 
$A$ sense of tension and weight is frequently felt in the right hypochondrium. Icterus, or jaundice, occurs in the majority of cases, but is not a constant symptom. The appetite is lost; thirst is usually marked; nausea and vomiting take place in a certain proportion of cases. Looseness of the bowels occurs in some cases, in other cases the bowels are constipated, and the two conditions may alternate. Respiration is not embarrasserl, as a rule, provided the hepatitis be not associated with some pulmonary complication. Considerable importance has been attached to a decubitus on the right side, with the head raised, together with a disinclination to change the position of the body, and especially to lie on the ieft side. These points relating to deeubitus, however, are not observed in all cases. The urine, if icterus be present, contains bile. Albuminuria is a frequent symptom. The pulse at first may be quite frequent, and afterward its frequeney may diminish, and irregularity be marked. Cephalalgia is generally a symptom having more or less prominence. Somnolency and delirium are apt to precede a fatal termination, but, in some cases, the mental faculties remain intact to the close of life.

Pathological Character.-The character of this disease is essentially the same as that of acute inflammation affecting other glandular organs, for example, the kidneys ; and, according to Frerichs, the disease is accompanied frequently with inflammation of the kidneys. 'To compare it with a disease less rare than acute nephritis, the local events are analogous to those in pneumonitis. Engorgement and exudation are the primary events, as in inflammation of the pulmonary parenchyma; if resolution take place, the exudation is absorbed, and the hepatic structure remains intact, but, if the clisease do not pursue this farorable course, either purulent infiltration or the formation of abscesses ensnes, involving more or less destruction of tissue, as in the third stage of pneuinonitis. It is probable that the point of departure for the inflammation in this variety of hepatitis is in the hepatic cells.

CaUsation.-Acute hepatitis is almost peculiar to tropical climates. Its causation, therefore, is in some way connected with climate; but the rationale of the climatic influence is not understood. Of the two cases noticed under the hearling Anatomical Characters, in the last the patient was attacked on shipboard in a vessel from a tropical climate. The disease rarely occurs under the age of 20 . Various eauses have been assigned by different writers, such as the abuse of alcoholic stimulants, the excessive use of purgatives, depressing emotions or great mental excitement. 'The supposed agency of these and other eauses is by no means established. Hepatitis is apt to accompany or follow dysentery in tropical climates, but the inflammation, under these ciremmstances, is generally circumscribed, not diffuse. In the ease just referred to, in which the patient was from a tropical climate, the disease was associated with dysentery. Acute diffuse hepatitis may be associated with other affections, such as pneumonitis, pleurititis, intermittent fever, etc. Contrary to the doctrine formerly held, viz., that the inflammation is generally propagated from the gastro-duodenal mucous membrane to the liver, this membrane is rarely inflamed in conjunction with acute hepatitis. The etiology of the disease requires further elucidation.

Diagnosis.-The diagnosis is sufficiently easy, provided all or most of the diagnostic symptoms be present. Pain in the right hypochondrium, and tenderness, with febrile movement, chills, anorexia, and the occurrence 
of jaundice, render the existence of the disease highly probable; and if, in addition to these symptoms, the liver be found to undergo an enlargement not attributable to congestion from disease of the lieart, the diagnosis is quite positive. Enlargement of the liver is an important point in the diagnosis, if it be certain that it has occurred after the date of the attack. But the enlargement maly not, in all cases, be suflicient to be appreciated by physical examination. and at a late period of the disease the organ may become diminished in volume. Pain, tenderness, and icterus are not always present. lcterus, in the great majority of cases, oceurs independently of hepatitis, and is, therefore, in itselt no evidence of the existence of the latter clisease. If the three symptoms just mentioned be wanting, the disease is latent and may readily be overlooked. Pain and tenderness in the region of the liver mily be due to other atfeetions, viz., pleuritis, pneumonitis, intercostal neuralgia, and muscular rhemuntism. The two former of these aflections were formerly, doubtless, not infrequently confounded with hepatitis; but, with our present knowledge of physical signs, they are readily excluded. It is, however, to be borne in mind that they may coexist with hepatitis. Intercostal neuralgia has its diagnostic eriterion, which has been considered, and this affection, therefore, is easily excluded. Muscular rheumstism is not likely to be limited to the site of the liver; careful examination will suflice to show that the pain and tenderness are in the walls of the abdomen, and this, with the absence of icterus and enlargement of the liver, renders the discrimination not difficult. A friction murmur, caused by the rubbing together of the peritoneal surfaces, when these are covered with lymph, is sometimes observed over the site of the liver. This physical sign was well marked in one of the cases under my observation, to which reference has been marle.

In our climate, the great infrequency of acute hepatitis is to be taken into account in the diagnosis. In view of its infrequency, evidence of the existence of the disease, which, in a tropical climate, should be deemed sufficient, may seem insufficient for a positive diagnosis. Hence, in a tropical climate, the liability to error in diagnosis is perhaps in deciding that the disease exists on insufficient grounds; whereas, in a cold or temperate climate, the error most likely to occur is failure to recognize it when present.

Prognosis.-Acute diffuse hepatitis is a disease attended with much danger. Data for determining the cleath-rate in this disease are wanting; but a fatal termination is uncloubtedly the rule if the disease proceed to the stage of suppuration. The fatality is increased by the frequent association of other diseases. The course of the disease is usually rapid. Its duration generally is not beyond three weeks. Life, however, in some cases, is prolonged for a much longer period; and if recovery take place after the formation of abscesses, the convalescence is apt to be tedions. If abscesses form, and life be sufficiently prolonged, the pus is liable to be discharged in different directions, viz., through the abdominal or chestwalls, into the colon, stomach, peritoneal cavity, ete. As regards the prospect of recovery, much will depend on the direction in which the pus is discharged. This topic will be considered under the head of eireumscribed hepatitis or hepatic abscess.

Treatment.-This disease is to be treated on the general principles applicable to the treatment of other inflammations. Bloodletting, local 
ol general, is to be employed, or not, according to the circumstances which either indicate or contra-indicate this remedy in inflammatory affections generally. Revulsive applications over the aflected organ, such as sinapisıns, dry cups, and stimulating liniments, are, to a certain extent, useful ; but counter-irritation by means of blisters, or other severe applications, is of doubtful propriety. Upium is indicated in proportion to the amount of pain and constitutional irritation. Mercury has been supposed to be specially adapted to the treatment of all hepatic diseases. 'There are no reasons, however, for its application to this more than to other acute inflammations. Whatever usefulness it may have, here as in other inflamniatory affections, consists in a so-called antiplastic and a sorbefacient influence. In so far as it may tend to increase the secretory action of the liver, its action is probably unfavorable; but the doctrine so long held, that mercury increases the secretion of bile, is, to say the least, open to much distrust. Of doubtful propriety in all cases if carried to the extent of inducing mercurialization, it cannot fail to be injurious in patients anæmic or with enfeebled, broken-down constitutions. Inasmuch as the danger lies especially in suppuration, tonic remedies and a nutritious diet are indicated early in the disease ; and, when suppuration has taken place, the reliance must be on the constitution of the patient, aided by efficient supporting measures.

\section{Circumscribed Suppurative Hepatitis, or Abscess of the Liver.}

In this variety of hepatitis the inflammation is limited either to a circumscribed portion of the parenchyma of the liver or to several distinct portions. Clinically, the existence of this variety of the disease is generally not known until suppuration has taken place, and an abscess is ascertained, either by manual exploration or by the escape of the pus from some outlet into which it is discharged. Practically, therefore, it suflices to consider circumseribed hepatitis under the head of hepatic abscess. The formation of pus within the liver, in the cases in which the veins of the liver have been supposed to be the seat of inflammation, differs, in certain respects, from suppuration in cases of ordinary circumscribed hepatitis, and will be considered under the head of portal phlebitis.

In the majority of cases a single abseess only is formed, but in some cases there are two or three, and rarely more than three. The size of the abscess varies much in different cases. It is usually of considerable, and not infrequently of large size. In a case under my observation, five and a half quarts were at once evacuated by an opening made through the thoracic walls. After a profuse purulent discharge for several months, this patient recovered, resumed the occupation of a hack-driver, and two years afterward was in robust health. A case came under the observation of Dr. J. R. Lothrop, at Rainsford Island Hospital, in which an abscess was found to contain, after death, eighteen pints of pus, the entire organ, except the left lobe, being transformed into a mere sac. The abscess may be situated at any part of the gland. It is much oftener seated in the right than in the left lobe, and oftenest at the posterior portion of the right lobe. It may be superficially or deeply seated. The purulent matter from an hepatic abscess may not differ in its characters from ordinary pus, but frequently it contains more or less of the detritus of the hepatic tissue, and it sometimes contains bile. The walls of the abscess at first consist of the exposed hepatic tissue, but, after a time, a cyst is formed which increases in thick- 
ness and firmness with age. The walls of the abseess oceasionally become gangrenous.

It is doubtful whether a considerable collection of pus within the liver be ever completely absorbed. It may remain in an indolent, latent condition for an indefinite period, but, sooner or later, if life eontinue, the pus makes its way, as in other abseesses, in the direction in which the pressure is least, and is at length discharged into some eavity, canal, or outlet.

In the larger proportion of eases, the evacuation is through the thoracic or abdominal parietes. When it takes this direction, a fluctuating tumor, after a time, makes its appearance upon the surface, eaused by the acenmulation of the pus beneath the integument. The tumor, if left to itself, increases, beeomes pointed, the skin becomes reddened, and finally the discharge is effected by ulceration. The most frequent situation of the tumor is below the ensiform cartilage, but it may appear at any point over the site of the liver. Sometimes the pus burrows beneath the integument, and the lumor is situated at a greater or less distance from the liver. It has been observed as high as the axilla, and below on the inner side of the thigh ; in the latter ease it is liable to be mistaken for lumbar abscess. Of course, partial peritonitis and adhesion over the cireumseribed space ocenpied by the abseess are essential in order that the pus may be discharged externally. This conservative provision does not always precede the opening of the abscess, and then the contents are discharged into the peritoneal cavity; peritonitis is at once developed, and, under these circumstances, death speedily follows. The direction, next in frequency, which the pus takes is throngh the diaphragm into the chest. Partial peritonitis preeedes the perforation of the diaphragm, and partial pleuritis ensues, frequently limited to a small space, preventing evaeuation into the pleural cavity. Occasionally, however, the latter conservative provision does not take place, and the pus, discharged into the pleural cavity, at once causes general pleuritis. If this be prevented by adhesion around the opening through the diaphragm, the lung becomes perforated, and the evacuation is into the bronchial tubes, the pus being diseharged by expectoration. 'The abscess is sometimes evacuated into the pleural eavity, and afterward perforation of the lung takes place, and the pus is expeetorated. Other less frequent directions in which the pus is discharged are the following: Into the colon, stomach, or duodenum, the pus being then discharged by vomiting or stool ; into the pericardium, followed, of course, by instant death; into the pelvis of the right kidney, the pus discharged with the urine; into a hepatic vein, or the vena eava, giving rise to purulent infection of the blood; into the gall-bladder or liliary duets, and thus, if there be no obstruction, the pus tinding its way into the duodenum. ${ }^{1}$ In a case which eame under my observation at Bellevue Hospital, an abscess pointed externally and was opened; subsequently perforation of the stomach took place, and liquids ingested eseaped through the external opening, the patient dying from inanition.

The clinical history of hepatic abscess is obscure. The symptoms rarely point distinctly to this affection. Pain exists if the hepatitis be near the surface of the liver, and whenever partial peritonitis ensues, but, even then, pain is not always a prominent symptom, and, if the inflammation be deep-seated, there may be no pain. Pain is felt in the right shoulder

1 For statistics showing the relative frequency of the evacuation of hepatic abscesses in these different directions, and other details, the reader is referred to $A$ Clinical Treatise on Diseases of the Liver, by Dr. Fried. Theod. Frerichs. Published by the New Sydenham Society, London, 1861. 
in a small number of cases. Rigidity of the abdominal walls over the liver, or of the right rectus muscle, is a sign to which much importance is attached by several observers. Febrile movement is rarely marked, and may be wanting. Chills usually occur during the suppurative process. 'These sometimes occur regularly, simulating intermittent fever, but generally they are irregular. Jaundice is rare. The appetite and digestion may be more or less disturbed, or they may be unaffected. The nutrition may be but little, or not at all, affected. Reference is now had to symptoms prior to the discharge of the pus or its appearance beneath the integument. The affection is not infrequently completely latent. It is not uncommon, in tropical climates, to find an abscess in the liver in bodies dead with various diseases, when hepatic disease had not been suspected during life; and the first intimation of the existence of this affection during life, in certain cases, may be the discharge of pus from the stomach, bowels, or air-passages.

The clinical history, after the discharge takes place, will, of course, depend upon the direction in which the abscess is evacuated. In certain cases the phenomena of the affection developed by the presence of pus are superadded, viz., of peritonitis, pleuritis, pyæmia, etc. If the evacuation take place either externally, into the alimentary canal, or through the bronchial tubes, symptoms are produced analogous to those connected with purulent discharges in other situations, viz., anæmia, debility, emaciation, and, in cases which pursue an unfavorable course, hectic fever, colliquative diarrhœa, exhaustion, the death taking place by asthenia.

Hepatic abscess is extremely rare in cold or temperate climates. It is an affection belonging par excellence to warm climates, although not very common in the latter. In warm climates it is apt to be associated with dysentery, and a causative relation has been supposed to exist between the two affections. Budd, and others, suppose that the abscess is consecutive to, and dependent upon, the dysenteric affection, and that suppuration takes place in the liver in consequence either of phlebitis induced by intestinal ulcerations or by the alsorption of septic matter which, carried to the liver in the portal blood, occasions inflammation there.' This view is conjectural. In the cases of hepatic abscess observed in cold or temperate climates, dysentery is very rarely associated, and of a large number of cases of intestinal ulceration from tuberculosis, typhoid fever, as well as dysentery, together with cases of gastric ulcer (324 cases) analyzed by Bristowe, the cases occurring in St. Thomas's Hospital, London, abscess of the liver existed in only 4. In each of these 4 cases it was associated with dysentery, but in 3 of the cases the dysentery was secondary. Of 31 cases of hepatic abscess analyzed by the same writer, in only 6 were intestinal ulcerations found; in 2 of these 6 cases the ulcerations followed the evacuation of the abscess into the bowel, leaving 4 cases in which dysenteric ulcerations preceded the hepatic abscess." When associated with dysentery in warm climates, the hepatic affection does not always follow, but, in a certain proportion of cases, precedes the intestinal affection. Annesley, and some other writers on diseases in the East Indies, are of the opinion that the hepatic affection generally precedes the dysentery, and that the latter is dependent on the former. The coexistence of dysentery and hepatic abscess in the East Indies does not occur as often as appears to be generally supposed. Of

1 On Diseases of the Liver. By George Budd, M. D., etc. Am. ed., 1853.

2 Vide Frerichs, op. cit. 
300 cases analyzed by Waring, dysentery existed in 82 . On the whole, it is perhaps questionable whether there be any special causative relation between the two affections. Dysentery is a very frequent disease in warm climates, and hepatic abscess vastly less infrequent than in cold or temperate climates; hence, in the former, mere coincidence is suflicient to explain their association in a certain number of cases. Dysentery, although not so frequent a disease in cold or temperate as in warm elimates, is yet not infrequent in the former, and were it capable of giving rise to hepatic abscess this affection should be less rare.

Ulceration of the gall-bladder or ducts, and the irritation caused by the presence of gall-stones, have been supposed to be adequate causes, but they have been found in only a very small number of cases, and they occur not infrequently without leading to hepatic abscess. Lumbricoid worms migrating into the hepatic ducts, is another supposed cause, but this is an occurrence of extreme infrequency. Contusions or other traumatic causes very rarely give rise to abscess. Of 318 cases analyzed by Morehead, only 4 were referable to mechanical violence. In the majority of cases, the causes of circumscribed hepatitis resulting in abscess are, at present, unknown, and the affection is, therefore, to be considered as spontaneous. This, however, is equally true of most other local inflammations.

The diagnosis of hepatic abscess can hardly be made with any degree of positiveness prior to its discovery by manual exploration, or the discharge of pus into the alimentary canal or bronchial tubes. The symptoms which have been named as belonging to its clinical history should suggest its existence as possible or perhaps probable, especially in a warm climate, but they are not sufficiently distingtive to render the diagnosis positive. Not unfrequently grounds of even a suspicion of its existence are wanting. When a fluctuating enlargement or tumor is perceived orer the site or in the neighborhood of the liver, it is to be discriminated from a subcutaneous, phlegmonous inflammation, carcinoma of the liver, a hydatid formation, and a distended gall-bladder. 'The first of these is distinguished by pain, hardness and redness of the integument, preceding the sense of fluctuation. Carcinoma offers a resemblance only when the medulary variety exists. The duration of the tumor and the nodulated condition of the liver, as felt through the abdominal walls, will serve for the recognition of this affection. A hydatid cyst is of slower development, and is not attended with the pain, tenderness, chills, and febrile movement which usually accompany an abscess. A hydatid cyst, however, may be the seat of suppuration, becoming converted into an abscess, and then the discrimination is difficult or impossible. A distended gallbladder, which is the most likely to be confounded with an abscess, is to be distinguished by its situation, the pear-shaped form, and by the fact that, in consequence of peritoneal adhesions rarely taking place, it has a mobility which an hepatic abscess has not. The antecedent history is, of course, always to be taken into account. Enlargement of the liver frequently, but not invariably, exists in cases of hepatic abscess, and the enlargement may be found to be limited to a particular direction, not affecting the whole organ, a point of some importance in the diagnosis.

If the abscess discharge through the bronchial tubes, the diagnosis is generally made without difficulty. The sudden expectoration of pus in large quantity, without evidence of antecedent and coexisting empyema or pulmonary abscess, and the expectoration coming from the bronchial tubes of the right lung, render the diagnosis altogether probalble; but the presence of bile or disintegrated hepatic tissue aflords demonstrative proof of the source of the purulent expectoration. If the abseess dis- 
charge into the pleural cavity, the diagnosis canno be positive, but is rendered probable by symptoms of hepatitis and suppuration having preceded the sudden development of general pleuritis with effusion.

The discharge of the abscess into the stomach, duodenum, or colon is followed by the vomiting of pus or its appearance in the stools. The diagnosis is to be based on these events, together with antecedent symptoms pointing to the existence of suppurative hepatitis. The discharge into the peritoneal cavity, pericardium, and vena cava gives rise to no phenomena distinctive of hepatic abscess. Its existence may have been suspected by the symptoms preceding the discharge, but the proof can only be afforded by an autopsical examination. The discharge into the pelvis of the kidney gives rise to pyuria, but it may be difficult or impossible to determine that the pus comes from the liver and not from the kidney or some other source.

The prognosis, even in the cases in which the abscess is evacuated in the most favorable directions, is unfavorable. When it discharges into the peritoneum, pericardium, and vena cava, death speedily follows. If it discharge into the pleural cavity, the termination is usually fatal. The cases which offer a fair prospect of recovery are those in which the discharge takes place through the integument, the bronchial tubes, or into the alimentary canal. Of 203 cuses collected by Rouis, 162 terminated fatally, in 39 there was a complete, and in 2 an imperfect, cure. This collection embraced cases in which the clischarge was into the peritoneal cavity and in other directions, as well as in those most favorable.' If complicated with dysentery, the prospect of recovery is diminished. The same writer gives the following statistics, showing the relative proportion of recoveries when the discharge took place in the most favorable directions, with and without dysenteric complication: Of 19 cases uncomplicated with dysentery, in 14 recovery took place, viz., 4 of 5 cases in which the evacuation was through the abdominal or thoracic walls, 6 of 8 cases in which the discharge was through the bronchial tubes, and 4 of 5 cases in which the abscess opened into the alimentary canal. Of 59 cases complicated with dysentery, in 25 recovery took place, viz., 13 of 29 cases in which the evacuation was through the abdominal or thoracic walls, 9 of 22 eases in which the discharge was through the bronchial tubes, and 3 of 8 cases in which the abscess opened into the alimentary canal. Thus, of the uncomplicated cases, 60 per cent. recovered, whereas, of the complicated cases, only 29 per cent. recovered.

The prospect of recovery is, of course, less when two or three abscesses exist than when there is but one.

Statistics show the duration, in fatal cases, to be longest when the discharge takes place through the bronchial tubes. The average duration, in fatal cases, is between three and four months. In cases which recover, statistics show the longest duration to be when the discharge is through the integument. The average duration in cases which recover is between four and five months.

From what has been said respecting the clinical history and diagnosis, it follows that there is seldom an opportunity to employ therapeutic measures with a view to prevent suppuration. Even if the existence of circumscribed hepatitis were early ascertained, it is doubtful if the formation of an abscess could be prevented. Cases in which the abscess is appreciable by manual exploration, and those in which the contents are discharged into the bronchial tubes and alimentary canal, alone admit of 
treatment with a positive knowledge of the existence of the affection. In the cases in which the abscess is ascertained to be making its way throngh the abdominal or thoracic parietes an early opening should be inade into it and the pus evacuated. Budd and others advise to allow the abscess to open spontaneously for fear of the entrance of air; but the air does little or no harm if the opening be free, and air is not excluded when the opening is spontaneous. If the opening be made before the pus has made it's way beneath the integument exterior to the abdominal or thoracic walls, there is danger of the pus escaping into the peritoneal cavity in consequence of the absence of peritoneal adhesions over the abscess. Under these cireumstances, to avoid this danger, an ineision may be made throngh the skin and muscles down to the peritoneum without opening the abseess, and the wound filled with lint. This procedure induces firm adhesions around the wound, and in three days the abseess may be opened with safety, if a spontaneous opening do not in the mean time occur. If, however, the walls of the abdomen or chest have been perforated by ulceration, a free opening may be made at once without hesitation. After the abscess is opened, the treatment consists of tonic and supporting measures. Good diet and ont-of-door life, in so far as the patient's strength will permit, are far more important than medication.

In cases in which the abseess evacuates through the bronchial tubes and alimentary canal, palliative remedies are called for, to relieve unclue irritation of the bowels and air-passages. In addition to these, measures, hygienic and medieinal, which tend to support, strengthen, and invigorate the system, are indieated, as the cases in which the discharge is through the integument.

\section{Portal Phlebitis.}

The name portal phlebitis denotes inflammation of the veins which compose the portal system. Inflammation here situated has been supposed to lead to the formation of coagula and consequent venous obstruction withont suppuration. The affection has been called suppurative portal phlebitis when the inflammation learls to the production of pus. In the opinion of Frerichs, coagula or thrombi take place oftener without than with phlebitis; and according to Virchow, the phenomena in cases of socalled portal phlebitis are due to thrombosis of the portal vein, occurring without as well as with inflammation of this vein. This affection has been supposed to give rise to the formation of pus within the liver, exterior to the hepatic vessels, in other words, to multiple hepatic abscesses, in the same way as phlebitis in a portion of the systemic venous system has been supposed to occasion purulent eollections in the lungs as well as in the liver, and in various other situations, called metastatic or pyæmic abscesses. These have been considered in treating of pyæmia, in the first part of this work.' The abscesses attributed to suppurative inflammation of the portal vein differ from ordinary hepatic abscesses in being numerous, whereas the latter very rareiy exceed two or three, and in the great majority of cases there is only a single abscess. They differ also in size, being generally small, varying in size from a pea to a hen's egg. They are found especially near the periphery of the liver. The veins within the liver and in other parts of the portal system have been found to contain purulent matter. Death sometimes takes place without the formation of pus within the hepatic parenchyma. 
The affection may proceed from injury of some of the branches of the portal vein, as in surgical operations within the rectum. It has followed violent efforts to replace a prolapsed rectum. It is referable, in some cases, to ulcerations in the stomach or intestines, to abscesses of the spleen, to suppuration in the mesentery and mesenteric glands, to ordinary abscesses of the liver, and to diseases of the bile ducts. ${ }^{1}$ But, in a certain proportion of cases, its source is not discoverable.

The clinical history is obscure. More or less pain is present, referable either to the epigastrium or right hypochondrium, but the character of the pain is not distinctive. Jaundice, more or less in degree, is usually a symptom. Diarrhœa occurs in the majority of cases. Chills with rigors occur, sometimes regularly, but oftener in an irregular manner, frequently recurring several times during the day. The pulse is more or less accelerated. The flesh and strength are rapidly lost. Hectic paroxysms and delirium take place during the latter part of the affection. The liver is generally found to be enlarged, and this is true also of the spleen.

The diagnosis cannot be made out with positiveness. The practitioner cannot do more than entertain a strong suspicion of the existence of the affection. From the symptoms the patient may for a time be supposed to have an irregular intermittent or remittent fever.

The prognosis is as unfavorable as possible. The affection is uniformly fatal if the inflammation extend over a considerable portion of the portal system, and give rise to hepatic abscesses. The fatal termination occurs after a duration varying from a week to six weeks. Happily the affection is extremely rare.

Palliative and supporting measures of treatment are indicated.

The following case of multiple abscess of the liver is of interest as showing their connection with thrombosis and suppurative inflammation caused by the presence of a fish-bone in the superior mesenteric and portal vein. The details of the case are from the autopsical records of Bellevue Hospital: Thomas Burn, aged 47, was admitted Feb. 11, 1867. Three weeks previous to his admission, after getting wet, he was seized with pain in the right side, accompanied with cough. On admission he had the symptoms and signs of pleurisy affecting the right side. Subsequently diarrhoa occurred, with tenderness over the abdomen. The urine contained albumen and granular cysts. Death took place Feb. 22d, 1867. On examination after death the brain was found to be healthy. The heart weighed $10 \mathrm{oz}$., and was normal. The right lung was adherent, and the pleural cavity contained a small quantity of purulent liquid. The abdomen was tympanitic. There was the evidence of recent peritonitis. The liver weighed $6 \frac{1}{2} \mathrm{lbs}$. Throughout the right lobe were scattered numerous spots of broken-down tissue, the spots ranging in size from that of a pea to a walnut. They contained a whitish purulent liquid. A great number were situated superficially, and from one of these the fluid escaped during the remoral of the liver. On cutting of the portal vein just before its division, a purulent-looking liquid escaped from both ends. In the left lobe several of the small branches of the portal vein were obstructed by clots. The portal vein on the left side contained a firm reddish clot over an inch in length, completely filling the vessel. On the right side there were the remains of a clot in the form of a cylinder of fibrin. The internal coat looked normal. The external coats were thickened, and the glands in the fissure of the liver were much enlarged. A fish-bone about two

I For examples of portal phlebitis from these several causes, vide Frerichs, on. cit. 
iuches long was found, one-half in the superior mesenteric and one-half in the portal vein, the central portion covered by a whitish clot which caused it to adhere to the wall of the vein. The end in the portal vein was pointed, the other end was blunt. The bone and clot together were half an inch in thickness. 'The place in the stomach or intestine where the bone had passed could not be found.

Multiple hepatic alscesses take place as a result, not only of portal phlebitis, but in cases of pyæmia. In the latter cases, abscesses always coexist in the lungs, and they are frequently found in other situations. In cases of portal phlebitis, abscesses, as a rule, are confined to the liver. There are, however, some exceptions to this rule, collections of pus taking place consecutively in the lungs and in other situations. Pyæmie collections existed in other organs than the liver in only four of twenty-five cases analyzed by Frerichs.

\section{Cirrhosis of the Iiver.}

Cirrhosis, a name introduced by Laennec, signifying a tawny or orange color, is in common use to clenote an affection of the liver generally supposed to involve chronic inflammation, and hence called chronic, diffuse, or adhesive hepatitis ; also called interstitial hepatitis. To corresponding affections of the kidneys and lungs the name cirrhosis is often applied. 'The name sclerosis of the liver is used by some writers. The latter term is applied to an aualogous affection of the nervous centres and some other organs. Fibroid aud indurated liver are also appropriate names distinctive of this affection.

A small or moderate amount of cirrhotic change gives to the organ an abnormal consistence; its size is unaffected, or it may be somewhat enlarged ; the capsule is apt to be more or less opaque and thickened; the surface is frequently studded with small prominences resembling granulations, varying from a pin's head to a pea in size, and a similar granulated appearance is observed in the interior. At a more advanced stage, the organ undergoes notable alterations as regards volume, form, and other appearances. Its size and weight are more or less diminished. Its size, in some cases, is notably reduced, and the weight may fall as low as, and even under, two pounds. It sometimes becomes much changed in shape, occasionally lohulated, and in extreme cases is so deformed as hardly to be recognized by its external aspect. The left lobe not infrequently is greatly reduced in size, and may entirely disappear. The surface of the organ, in a certain proportion of cases, presents numerous projections, sometimes pretty uniform, but, in other cases, varying in size and form. 'This nodulated aspect has given rise to the term hob-nailed, as applied to some examples of eirrhosed liver. The same nodulated appearance is apparent internally. This appearance does not belong to all cases of cirrhosis. In a certain proportion of cases, the surface of the organ is smooth. Morbid adhesions of the opposed peritoneal surfices are not uncommon, and in some cases the whole organ becomes firmly united to the surrounding parts. The coloration of the organ, especially of the nodules, gave rise to the name cirrhosis. Laennec supposed the orangeyellow nodules to be adventitious products, but more recent investigations have shown that they consist of the hepatic parenchyma, and that the yellowness depends on the accumulation of bile pigment from congestion of the minute biliary ducts. This yellowness, however, by no means characterizes all cases.

Microscopical examination shows an abnormal amount of fibrous tissue 
between the hepatic lobules, $i . e .$, in the interlobular spaces which contain the terminal branches of the portal veins, and the roots of the bile-ducts. There is also an abnormal accumulation of amorphous granular matter. The presence of this newly-formed tissue is manifest in the form of white lines to the naked eye. The pressure of this tissue upon the lobules induces atrophy; hence the contraction of the organ. Its shrinking causes the granular and nodulated appearance. Compressing the terminal branches of the vena portæ, it occasions obstruction to the passage of blood through the liver, and consequently portal congestion ensues, giving rise to dropsy and sometimes lemorrliage from the mucous membrane of the stomach and intestines. Pressure upon the bile-ducts causes biliary congestion and a jaundiced condition of the organ. The hepatic cells are diminished in size and number, and contain minute, greenish-colored oil. drops. These differ notably in appearance from the cells in cases of ordinary fatty liver. The fatty liver and cirrhosis, however, may be combined, and the organ may then be not diminished but increased in size. An enormous enlargement may also exist when cirrhosis is associated with the waxy or lardaceous liver.

The point of departure for the morbid changes just stated is supposed to be inflammation existing within the interlobular spaces; and cirrhosis is, therefore, regarded as a diffused, subacute inflammation of the areolar or connective tissue existing between the lobules, forming an extension of Glisson's capsule. This is the pathological view generally held at the present time. As a result of the inflammation, an abnormal production (hyperplasia) of the connective tissue takes place.

Enlargement of the spleen is found after death, and is determinable during life, in a certain proportion of cases. The enlargement is sometimes very great. Notable enlargement, howerer, in my experience, is the exception rather than the rule. More or less enlargement existed in 58 of 64 cases analyzed by Bamberger, and in 18 of 36 cases analyzed by Frerichs.

Coexisting cardiac disease has been supposed to be common. This has not been true in my experience. Of ten fatal cases, in seven the heart was free from disease; of the remaining three cases, in two, old pericardial adhesions existed, the heart being moderately enlarged in one and below the normal volume in the other case. In the third case, there was moderate enlargement (weight $11 \mathrm{oz}$.) with rigidity of the aortic valves. Of 22 cases in which autopsies were not made, in 15 there were no physical signs of disease of the heart, and of the remaining 7 cases, in 4 the only evidence was a systolic murmur at the base; in the other three cases, mitral lesions were denoted by the signs together with enlargement of the heart. Thus, of 32 cases in which the condition of the heart was noted, as determined by either autopsical examination or physical signs, in only 5 were there lesions involving enlargement of this organ. These facts go to show that cardiac disease and cirrhosis of the liver are not associated sufficiently often to denote the existence of any pathological connection between them. When they coexist, the association is probably merely a coincidence.'

Disease of the kidneys coexists oftener than cardiac disease. In 11 fatal cases under my observation, autopsical examination showed more or less renal disease in 6 . 'The concurrence would seem to be sufficient to denote either some pathòlogical connection between disease of the kidneys and cirrhosis, or that both are effects of the same causative conditions. As

' Clinical Report on Hydro-peritoneum, by author. 
will be presently seen, evidence of renal diseases is rarely afforded by albuminuria in cases of cirrhosis.

Cirrhosis as a rule gives rise to few or no symptoms which point to the liver as the seat of disease prior to the occurrence of hydro-peritoneum. Preceding this event, and afterward, pain in the region of the liver exists in only a small proportion of cases. Dropsical effusion is usually the first symptom to suggest the existence of the affection. And the dropsy may occur when the patient supposes himself to be in his habitual state of health, or it may be preceded and accompanied by indefinite ailments. The dropsical accumulation increases, and, usually, with considerable or great rapidity. It is rare for the quantity of liquid to remain at a moderate amount for a considerable period. Edema of the lower limbs, in a moderate degree, precedes the hydro-peritoneum in a large proportion of cases (11 of 21 cases). It has been stated that the coexistence of either cardiac or renal disease is to be inferred from the pre-existence of œdema of the lower limbs; but the facts developed by the analysis of cases which I have recorded are opposed to this statement. Of the 11 cases in which the odema preceded, 5 were fatal. Of these 5 cases, in three the heart and kidneys were found, on examination after death, to be free from disease; in one case there were old pericardial adhesions with moderate enlargement, and in the other case there was granular degeneration of the kidneys. In not one of the 6 cases which did not end fatally under my observation was the urine albuminous, or were there present physical. signs of cardiac disease. After the recurrence of hydro-peritoneum, œdema of the lower limbs is apt to occur, if not already existing, and it sometimes becomes great under these circumstances, being due, probably, to pressure upon the iliac veins. The genital organs may become more or less cedematous. Edema of the face and upper extremities does not occur, save in cases in which cardiac or renal disease coexists. Edema of the lower limbs does not occur in all cases.

Jaundice occurs in a small proportion of cases ( 7 of 46 cases); it is rarely great. The lesion does not necessarily occasion obstruction of the biliary ducts ; but it must diminish the secretion of bile, whether the secretion be effected, as generally supposed, by the hepatic cells, or, as considered by Robin, by the follicles of the membrane lining the biliary passages. Pallor of the prolabia and skin exists in the majority of cases. In general, the anæmic aspect is marked in proportion as other symptoms show the condition of the patient to be unfavorable.

Febrile movement does not accompany the disease in its progress. The pulse may be not increased in frequency, or it may be more or less accelerated; it is generally soft and feeble. When frequent, the other characters denote diminution of the vital forces or asthenia. The obstruction of the portal circulation is compensated for, in a measure, by new channels of communication between the portal and the systemic veins. The most important of these, as shown by Sappey, are anastomosing branches which pass to the liver between the folds of the falciform ligament and in the ligamentum teres, communicating with the veins of the abdominal parietes through the epigastric and the internal mammary vein. The course of the blood in these anastomosing branches, in cases of cirrhosis, is the reverse of that in health, being in the latter in a direction from, and in the former toward, the liver. Owing to this, the abdominal veins become dilated, and those superficially. situated in some cases are seen to be more or less enlarged. A collateral circulation, in a measure, is also established

' Bulletin de l' Académie de Médecine, tom. xxiv., Paris, 1859. 
through communications between the inferior mesenteric and the hypogastric vein in the hemorrhoidal plexus; by anastomosing branches between the vena portæ and the veins of the serous covering of the liver, and by newly formed vessels in the tissue by which the liver adlieres to the diaphragm. 'The size of the superficial abdominal veins, on either side of the median line, is sometimes enormous. The conservative effeet of this compensatory development of veins communieating with the two circulations is shown by the fact that the striking examples of great enlargement of the vessels apparent upon the abdomen are in those who are free from dropsy notwitbstanding a notable amount of cirrhosis.

Hemorrhage from the stomach and bowels is an oceasional event. Gastrorrhagia occurred in 6 of about 40 cases which I have analyzed, the histories in all the cases not being complete. It preceded the hydro-peritoneum in 3 cases. Enterorrhagia coexisted in 2 cases, and occurred alone in 2 cases. Vomiting is occasionally a prominent symptom, and diarrhœa is prominent in a small proportion of cases.

'The appetite is usually impaired. A sense of fulness after taking food is generally complained of, if the dropsy be sufficient to distend the abdomen. Progressive emaciation attends the progress of the disease, and, in an advanced stage, the attenuation of the upper portion of the body, the distended abdomen, and the lower limbs enlarged by odema, render the general aspect highly eharacteristic.

Albuminuria is rare in eases of eirrhosis. Of 28 cases in which the condition of the urine in this respect was noted, in only one case was albumen present. The urine is generally scanty, but in some cases abundant. The coloring matter of the urine is abnormally abundant, a fact attributable to the deficient secretion of bile ; the blood-pigment which serves for the production of the biliverdine, not being expended in the liver, is eliminated in the kidneys. The abundance of the coloring matter may be such as to give to the urine the appearance of being bloody. The urates are usually deposited in great quantity, and are notably red from the abundance of pigment.

In the great majority of cases there are no important symptoms pertaining to the nervous system. The mode of dying is usually by slow asthenia. If, however, the abdomen be greatly distended, or if the dropsical aecumulation take place very rapidly, death may be due to the extent to which the respiratory function is compromised. The mental faculties are generally preserved up to the last moments of life. To this rule there are exceptions. In 3 cases I have noted the occurrence of delirium several days before death. In one case the delirium was hilarious; in one case the patient appeared bewildered, and in one case the patient lapsed from childishness into imbecility. 'These cases ended in coma. In three other cases the patients died comatose. In one case convulsions occurred followed by coma. ${ }^{1}$

Cirrhosis, in the vast majority of cases, is due to spirit-drinking. Of 20 fatal cases in which the habits of the patients were ascertained, in 17 intemperance was admitted, and in one of the three remaining cases habits of drinking were admitted, but not to excess. Of all the cases, fatal and non-fatal, in which the form of alcoholic stimulant used is noted (24), in all save one the patients were accustomed to drink spirits. In the excepted case the patient stated that he drank only beer. Hence the signi-

1 This account of the symptomatology is based on an analysis of 46 cases of hydro-peritoneum, cirrhosis existing in nearly all the cases. Vide Clinical Report, already referred to. 
ficance of the term gin or whiskey liver, applied by British writers to this affection. In all the cases in which information as to the mode of drinking was noted (15), the custom was to take raw spirits at different periods of the day, beforre breakfast and at other times on an empty stomach, a little water being generally drank after the spirits. This accords with the observations of others as to the mode of spirit-drinking which gives rise to cirrhosis. As to the action of alcohol in producing this affection, the explanation now commonly received is, that, passing readily into the portal blood from the stomach, and carried at once to the liver, it excites, by contact, inflammation of a low grade in the interlobular spaces, and hence the production of adventitious tissue. So far as my observations go, they furnish no evidence of the agency of disease of the heart in the causation of this affection.

Cirrhosis very rarely occurs under thirty years of age, and, in the majority of cases the age exceeds fifty years. The affection is generally a result of the habitual abuse of alcohol continued for a long period. Males are affected much oftener than females, a fact readily explained by the dependence of the affection on the use of spirits in the manner stated.

The diagnosis in most cases of cirrhosis, after dropsy has occurred, is made without difficulty. Hydro-peritoneum, occurring as a local dropsy, that is, irrespective of general dropsy, in a person addicted to spirit-drinking, warrants an inference that this affection exists. Additional proof is afforded by the diminished size of the liver. 'This is ascertained by percussion. The upper margin of the liver is accurately determined by finding the line of hepatic flatness; the lower border is determined, not so accurately, but approximately, by the line of demarcation between hepatic flatness and the tympanitic resonance due to gas in the transverse colon. The latter is not exact, because tympanitic resonance is conducted for a certain distance above the lower margin of the liver. The vertical diameter of the liver in health, on the linea mammalis, is about four inches. Directly after the operation of tapping, while the abdominal walls are relaxed, the lower border of the liver may frequently be grasped by the fingers pressed upward beneath the false ribs, and its indurated, nodulated condition appreciated by palpation.

The physician is rarely called upon to make the diagnosis prior to the occurrence of dropsy. It may be practicable, in some cases, to determine, by palpation, induration, and a nodulated condition of the lower part of the organ before dropsy has taken place, and this information obtained, the diagnosis is highly probable if the patient be addicted to spirit-drinking. Hemorrhage from the stomach sometimes precedes the occurrence of dropsy, and, in a spirit-drinker, renders the existence of cirrhosis probable. In a case which came under my observation in Bellevue Hospital, the patient, a female, who presented anæmia with progressive debility, and died, at length, in the first stage of pneumonia, the liver was notably cirrhosed and hobnailed, the weight being two pounds and two ounces. Habits of intemperance had not been suspected in this case. She had had gastrorrhagia. In another case, the notes of which do not embrace the termination and autopsy, repeated attacks of gastric hemorrhage occurred, giving rise to notable anæmia, and the spleen was greatly enlarged, the vertical diameter of the liver was lessened, and the patient was a spirit drinker; but no dropsy had taken place. Other cases of gastrorrhagia from cirrhosis, without dropsy, have come under my observation. I have known most profuse hemorrhage, proceeding from cirrhosis, without dropsy, to be followed by the recovery of a fair 
condition of health. On the other hand, I have known it to cause sudden death.

Cirrhosis is to be regarded as an incurable lesion. After it has led to dropsy, in the majority of cases its course is progressively onward toward a fatal termination. The duration in sixteen fatal cases, dating from the occurrence of dropsy, varied from six weeks to seventeen months, the average duration being about five months. But doubtless in all cases the disease has existed for a considerable period before dropsy occurs. In a certain proportion of cases, the dropsy being removed, the patient may apparently regain comfortable or even good health, and remain free from any manifestations of the disease for months and years, but sooner or later, as a rule, to which there are very few exceptions, the dropsy returns, and, unless life be destroyed by some intercurrent affection, the disease ends fatally. Circumstances which preclude much expectation of improvement are, the coexistence of cardiac, renal, or any other important disease, considerable emaciation, sufficient debility to keep the paticnt in bed, greatly impaired appetite and digestion, speedy reaccumulation of liquid after tapping, and the occurrence of jaundice.

As regards treatment, in cases of cirrhosis and in the great majority of the cases of hydro-peritoneum, the indications are the same. The reader is, therefore, referred to the treatment of hydro-peritoneum considered in the preceding chapter.

\section{CHA P T E R XII.}

\section{DISEASES AFFECTING THE SOLID VISCERA OF THE ABDOMEN- (CONTINUED.)}

Fatty Liver. Waxy or Lardaceous Liver. Cancer of the Liver. Tuberculosis of the Liver. Acute Atrophy of the Liver. Hydatid Tumors of the Liver. Pigmentary Deposit within the Liver. Hypertropy of the Liver. .

$\mathrm{H}^{\prime}$ AVING considered the inflammatory affections of the liver, including cirrhosis, which is generally regarded as inflammatory, structural affections are next to be considered. The more important of these are incident to the abnormal deposit of fat, to the waxy or lardaceous alteration, to carcinoma and tuberculosis, to acute atrophy, and to the existence of hydatid tumors. They will be considered briefly in this chapter.

\section{Fatty Liver.}

The secreting cells of the liver, in their healthy condition, contain oildrops, and these are abundant, normally, in certain inferior animals, especially invertebrate animals and fishes. Experimental observations show that in logs and other animals, the quantity of fat which the liver contains varies considerably with diet, and it is fair to infer that this is true in man. It is a reasonable supposition that the fat which the cells contain in health, is, in some way, useful. An increase of the fat, beyond a certain amount, undoubtedly constitutes a morbid condition; but it is diff- 
cult to determine, with our present knowledge, the amount compatible with the state of health. A considerable quantity of fat has been repeatedly found after death in cases in which persons, apparently in perfect health, have been accidentally killed. It is probable that the quantity of fat in the same person, in health, varies considerably at different times, according to variations in diet and other circumstances. An extremely fatty liver is usually enlarged, but not in all cases; the hepatic cells are sometimes filled with oil, the size of the organ not exceeding, and even falling under, that of health.

The gross chabacters of a fatty liver are as follows: Usually, as just stated, the organ is more or less enlarged, the margins lose their natural sharpness and become blunted or more rounded than in health. The enlargement is pretty uniform, and the inferior border may extend more or less below the false ribs, so as to be appreciable by palpation in persons with thin and relaxed abdominal walls. The exterior is smooth. The capsule is tense, and when divided the edges of the wound separate. The consistence is less than in health, and pressure with the finger leaves an indentation as in œdematons parts. The specific gravity is light, so that the organ sometimes floats in water. Frerichs found the average weiglit in 34 cases to be 3 pounds, $8 \frac{1}{2}$ ounces avoirdupois. The weight, however, in some cases, is much greater thon in health; I have met with a case in which the organ weighed 17 pounds. The color is pale or whitish, compared by Rokitansky to that of autumnal foliage; the organ is anxmic.

The presence of an abnormal amount of fat is shown by burning a piece of the organ. When a piece is held in flame, after the water is driven off, it burns readily, throwing off blue sparks. $\Lambda$ thin slice placed on a piece of white paper and exposed to heat exudes oil in abundance, greasing the paper. 'The cut surfaces give to the finger passed over them an unctuous sensation. Fat adheres to the scalpel nsed in making an incision. The fat may be dissolved out of the parenchyma by means of ether, and the quantity thus ascertained. Frerichs hits found the proportion of fat to be four times greater than the remainder of the subst:nce after the water was driven off. But the readiest and most reliable test is the appearance of the hepatic cells under the microscope. Oil-drops are found, larger and more numerous than in health, and cells may be filled and eren distended by single globules of oil formed by coalescence of the drops. It is probable that, before the mieroscopic test was employed, other conditions were not infrequently confounded with fatty liver. The microscope shows a great accumulation of fat in the cells in some livers not increased, but even diminished iu size. 'The accumulation of fat is chiefly or exclusively within the hepatic cells. The situation of the fatty deposit was ascertained by Bowman in 1841 .

Fatty liver occurs in connection with different affections. Frerichs gives the results of the microscopical examinations in 466 bodies dead with various diseases. An extreme amount of fat existed in 28 , and a large amount in 164. The liver is oftener fatty in females than in males. It is abnormally fatty in a pretty large proportion of cases of pulmonary tuberculosis. Of 117 cases, analyzed by Frerichs, in 17 the organ was extremely fatty, and in 62 the amount was large. Lonis found the liver notably fatty in 40 of 120 bodies dead with phthisis. Persons addicted to intemperance are apt to have this affection. Of 13 fatal cases of delirium tremens analyzed by Frerichs, the liver was very fatty in 6 . It occurs, in a comparatively small proportion of cases, in connection with pneumonitis, pleuritis, emphysema, disease of heart, disease of kidneys, the continued and eruptive fevers, chronic dysentery, etc. Prof. Alonzo 
Clark, and others, have found the liver notably fatty in yellow fever. In newly-born infants and young children this has been observed; also in pregnant and puerperal females.

The pathological relations of futty liver to the divers affections with which it is liable to be associated, are not understood. In many cases, doubtless, the association is merely accidental. Undoubtedly the coexistence of fatty liver and pulmonary tuberculosis is not due to coincidence; a pathological relation exists between these two affections, but the conjectures which have been offered respecting the nature of the relation are not satisfactory. It is probable that in drunkards the liver becomes fatty as a direct result of the ingestion of alcohol, not as secondary to other affections to which intemperance gives rise. Fatty articles of food entering largely into the diet, conjoined with indolent habits, conduce to the accumulation of fat in the liver, the meehanism being the same as in the production of the foie gras of geese.

Our present knowledge is not sufficient to enable us to determine the pathological importance of fatty liver. The pressure of the fat appears to be sufficient to render the organ anæmic, but not to occasion obstruction enough to give rise to peritoneal dropsy. If the latter coexist with fatty liver, cirrhosis is also combined, or there exists some other cause for the hydro-peritoneum. The spleen does not become enlarged. Some obstruction to the passage of bile in the minute hepatic ducts is produced, and, in extreme cases, this is sufficient to occasion moderate jaundice. 'The function of the secreting cells must be more or less impaired, especially when these are filled with fat; and in certain cases atrophy of the organ is apparently a consequence of the accumulation of fat.

The clinical history of this affection remains to be ascertained. The affection has as yet not been studied, as regards its symptomatology, in cases in which it is not associated with other hepatic lesions, or with important affections situated elsewhere. With the imperfect knowledge which we possess, the affection, existing in a great degree, appears to give rise to impairment of digestion, defective nutrition, progressive loss of strength, and to prove fatal by slow asthenia. I have remarked in several cases in which the affection existed in a notable degree, slight or moderate, persisting jaundice, and mild, cheerful delirium preceding death.

The diagnosis of fatty liver can only be made when the organ is enlarged. There are no subjective local symptoms, other than those dependent on the increase of size: The enlargement may be determined by percussion, and, in certain cases, by manual exploration below the false ribs. The blunt or rounded lower margin may sometimes be ascertained. The smoothness of the surface is appreciable, and sometimes also the diminished consistence; or, at all events, the absence of induration may be ascertained. An enlarged liver presenting these characters, occurring in a patient affected with pulmonary tuberculosis, an intemperate person, or a person of luxurious, indolent habits, may be considered as in all probability fatty. The probability is greater in females than in males.

Fatty liver occurring in cases of pulmonary tuberculosis does not claim treatment. In fact, the treatment which is highly usefu in certain cases of pulmonary tuberculosis, viz., with cod-liver oil and alcoholic stimulants, may be supposed to favor the deposit of fat in the liver. Nor, as a general remark, with our present knowledge, does it claim treatment when associated with other important affections. Occurring in persons adclicted to the use of alcohol, and to over-feeding especially as regards fatty articles of diet, it is to be treated by a reformation of the habits of living, viz., by temperance, or total abstinence, in respect to the use of alcoholic beve- 
rages, by restricting the diet, in a great measure, to articles not abounding in fat, and, if the strength will permit, by more muscular exercise. 'The remedies indicated are those which will tend to strengthen and invigorate the system.

The nutmeg or myristicated liver, as it is called, in a clinical point of view hardly claims distinct consideration. The name is applied to a condition of the organ in which the cut surfaces resemble a nutmeg in appearance. Lines of white, red, and yellow are blended together. The appearance is due to congestion of the hepatic, as distinguished from the portal, venous system, rendering the intra-lobular veins prominent, together with congestion of the bile-rlucts, and a certain amount of fatty deposit at the periphery of the lobules. The venous congestion proecels from an obstruction at the centre of the systemic renous system, and the nutmeg liver is generally observer in association with disease of the heart, more especially with mitral obstructive lesions. The condition cannot be recognized during life, and is probably of no pathological importance beyond the hepatic coingestion which enters into it.

\section{Waxy or Lardaceous Liver.}

The different viers entertained by pathologists respecting the morbid change commonly called waxy or lardaceous, have been noticed in the first part of this work. 'The change is also called amyloid and albuminoil. Budd considers it under the head of scrofulous enlargement of the liver. The term colloid liver has also been used to designate it. The affection consists in the deposit of a morbid material, the nature of which is not yet satisfactorily ascertained. 'The primary seat of the infiliration is the muscular tunic of the arterioles; but it extends thence to the other anatomical constituents of the organ.

A liver which has undergone, to much extent, this morbid change is more or less enlarged. The enlargement is in some cases enormous. 'The weight is also much increased. Wilks refers to a case in which the weight was 14 pounds. The form of the organ is not altered. The margins are not as blunted or rounded as in fatty liver, unless the latter condition coexists. The organ is dense and resisting. The surface is smooth. The substance of the orgim is notably stiff, so that thin sliees are easily taken off, and do not readily eollapse. In making incisions, the knife meets with a certain resistance as in entting bacon. The interior of the organ is dry and anæmic. When thin sections are held to the light, they present a translucent appearance, compared by Wilks to that of a tissue which has been soaked in glycerine, and by Frerichs to thin slices of smoked salmon. The microscope shows the presence of a white, structureless, translueent deposit, supposed by some to be a form of albumen, by others a kind of fat, by others cholesterin, and by others a substance resembling regetable starch. This change is sometimes associated with ordinary fatty liver, and sometimes with cirrhosis. Usually, when the liver is the seat of this morbid change, other organs are also affected, more especially the kidneys and spleen, less often the intestines and lymphatic glands. The applicstion of a solution of iodine causes a deep-brown color, whereas, applied when the organ is in a healthy condition, it gives a yellow tinge. Iorline may thus be used as a test of the waxy or lardaceous change. 'The insufficiency of the iodine test with sulphuric acid, as evidence that the morbid material is analogous to starch or cellulose, has been already referred to."

1 Vide page 50.

2 Vide page 51. 
This affection occurs in young subjects oftener than in the middle period of life. It is rare in childhood and in old age. Of 68 cases analyzed by Frerichs, 19 occurred between 10 and 20 years; 19 between 20 and 30 years; 18 between 30 and 50 years; 9 after the age of 50, and 3 prior to 10 years. It is met with in eachectic persons who have suffered from caries or necrosis of bones arising from scrofula; hence, the name scrofulous enlargement applied to it by Budd. It appears to be ineidental to prolonged suppuration in different situations. It occurs in persons affected with syphilis, and is supposed, by Graves and Budd, to be produced by the injudicious use of mercury in conjunetion with the syphilitic virus. It has been observed to follow intermittent fever. It occasionally accompanies tuberenlosis of the lungs and intestines. In all these instances, it is supposed to be developed as a secondary affection, but, in a certain proportion of cases, it is not preceded by any other disease. It oceurs in males oftener than in females.

The pathological importance of waxy liver and its clinical history are to be based on the study of cases in which the affection is not associated with other serious affections. Such cases are not easily eollected, for, if the affection be not secondary, the kidneys, spleen, and other parts are apt to be similarly affected. It is undoubtedly an important affection. The functions of the liver must be compromised in proportion to the amount of the deposit. It may imperle the portal circulation through the liver sufficiently to give rise to peritoneal dropsy, but the dropsical effusion is not large, and is wanting in the majority of eases. Jaundice very. rarely occurs. ' Pain referable to the liver is wanting. If the kidneys be affected, general dropsy is likely to occur, and the patient is liable to uræmia.

'The diagnosis of this affection is to be based on the existence of enlargement and the exclusion of other affections of the liver involving increase of size. From fatty liver it is to be distinguished by the greater resistance on palpation, and by the margins being in a less degree blunt or rounded. 'The existence of peritoneal dropsy in some cases aids in the exclusion of fatty liver. From cancer of the liver it is distinguished by the absence of tumor and the smoothness of the surface. The absence of subjective local symptoms, especially pain, aids in the exclusion of cancer. Cirrhosis leads to contraction of the organ generally; but this affection is excluded by the smoothness of the surface, when it can be felt, and by either the absence of peritoneal dropsy or the existence of only a small amount of effusion. The coexistence of enlarged spleen, and the evidence of disease of the kidneys afforded by albuminuria, general dropsy, and perhaps uræmic symptoms, strengthen the diagnosis. The diagnosis is also strengthened by the coexistence of affections to which, as elinical observation shows, this affection is apt to be secondary, viz., prolonged suppuration, scrofulous affections of the bones and syphilis.

The prognosis is unfavorable. It is doubtful whether the deposit is ever removed by absorption. The lesion must be regarded in most cases as incurable. Frerichs, Graves, and Budd, however, have observed diminution of the size of the liver in eases in which this affection was supposed to exist. Data are insufficient for determining the duration when the affection is lisconnected from other diseases. Generally, the fatal termination is, to a greater or less extent, due to the diseases with which the affection is associated.

The treatment must have reference to the morbid conditions under which the affection is developed. The iodide of potassium and the iodide of iron are considered by Frerichs as sometimes suceessful in cases in which syphilis is involved. In scrofulous or tuberculous patients the therapeutic 
measures should be directed especially to the cachexia which is to be considered as standing in a causative relation to the affection. Budd recommends the muriate of ammonia, from five to ten grains three times daily. Frerichs attaches importance to mild alkaline remedies and the use of the sulphurous mineral waters. Dietetic and other hygienic measures are of the first importance. 'The body should be well nourished, the surface properly protected against vicissitudes, and as much out-of-cloor life as the strength will permit is advisable. I)isorders of any of the functions are to receive appropriate attention. Diarrhea occurs not infrequently, and this will elaim astringent remedies and perhaps opium in small doses. Albuminuria and general dropsy furnish inclications the same as when these symptoms occur in connection with any of the degenerative changes which, collectively, are called Bright's disease.

\section{Cancer of the Liver.}

The liver is not infrequently the seat of careinomatous disease. In point of relative liability to cancer, the liver lanks after the uterus, female breast, stomach, rectum, and lymphatic glands. Of 4000 autopsies, cancer in this situation was found by Oppolzer in 53 cases, the proportion being as one to eighty. In the great majority of eases, eancer of the liver is secondary to carcinoma in other situations. It is secondary to eancer of the stomach in more than one-third of the cases. Of 91 cases analyzed, with reference to this point, by Frerichs, in only 22 was the cancer of the liver primary. Of the three varieties of cancer, the medullary is the variety in the larger proportion of cases. Scirrhous, or hard cancer, is, however, not very infrequent, but the colloid or alveolar variety is exceedingly rare. In most cases the cancerous affection is circumscribed, but occasionally more or less of the organ is infiltrated; the latter is distinguished as diffuse hepatic cancer. When circumscribed, the affection is presented in the form of nodules or tumors varying in size from that of a pea to a child's head. 'There may be but a single nodule or tumor. This is rarely the case unless the cancer be primary. Usually, there are numerous nodules or tumors, differing in size, and some of more recent date than others. The whole organ.is sometimes studded with them. They are, as a rule, few in number in proportion as they are of large size. They are sometimes, but very rarely, melanotic. Here, as in cancerous affections elsewhere, blood is frequently extravasated. 'The cancerous affection generally give rise to more or less enlargement of the organ. The increase in size and weight is sometimes enormous. The weight has been known to exceed twenty pounds. There are exceptions to the rule as regards enlargement. Of 76 cases analyzed by Freriehs, in 10 the size was normal, and in 6 the organ was smaller than in health. The affection, in some cases, does not extend to the periphery, but in most cases the nodules or tumors give rise to prominence or projections on the surface of the organ. These vary much in size and number in different cases. They are flattened at their summits and depressed in the centre, so as to present an umbilicated appearance. If the cancer be of the hard variety, the bulging tumors are resisting to the touch, but, if medullary, they may communicate to the finger a sense of fluctuation, and the disease is liable to be mistaken for abscess of the liver. Partial peritonitis is generally developed when the tumors project beyond the surface, and, in some cases, the peritonitis becomes general.

Here, as in otber situations, cancer involves in its causation a special diathesis. Cases rarely occur in early life. Of 83 cases analyzed by Fre- 
richs, only seven were in persons under 30 years of age; the age in 14 cases was between 30 and 40 years; in 41 , between 40 and 60 years; in 19 , between 60 and 70 years; and in 2, the age exceeded 70 years. The two sexes are about equally liable to this affection.

Cancer of the liver being generally secondary, the symptoms, in most cases, are combined with those referable to other affected organs. It is much oftener secondary to an affection of other abdominal viscera than to a primary cancer of a part not connected with the portal circulation. When developed secondarily, under these circumstances, the hepatic affection is liable to be overlooked.

The general manifestations of the cancerous cachexia, viz., waxy pallor, progressive emaciation, and debility, are usually present, sooner or later, but in this, as in other situations, the affection sometimes makes considerable progress before cachectic phenomena are apparent. The affection in some cases remains for a greater or less period quite latent. The general symptoms may be manifest without local phenomena pointing to the seat of the affection ; but, in the majority of cases, the affection speedily gives rise to both general and local symptoms. Pain is usnally more or less prominent, referable to the liver, and shooting in different directions. Tenderness on pressure is a frequent symptom. Jaundice occurs in the minority of cases, viz., 39 of 91 cases analyzed by Frerichs. In the cases in which it occurs the jaundice persists until death. Peritoneal effusion occurs in the majority of cases, viz., 48 to 80 . This is generally due to peritonitis excited by the cancerous tumors, but sometimes by pressure upon the portal vein. In the latter case, the effused liquid is serum without lymph; in the former, more or less lymph is exuded. Occasionally blood, extravasated into the cancer, escapes into the peritoneal cavity. The accumulation of liquid may be so great as to require the operation of tapping. The appetite and ligestion are early impaired, and, at a later period, cliarrhca not infrequently occurs. Edema of the lower extremities is apt to occur in the latter part of life. Emaciation and loss in weight are progressive, and death takes place by slow asthenia, if the patient be not carried off by some intercurrent disease. A fatal termination may be hastened by peritonitis induced by the local affection, by abundant hemorrhage, by coexisting cancerous affections elsewhere, and by various accidental complications. Aside from these circumstances, the progress of the affection is sometimes rapid and sometimes slow. The duration in different cases varies from a few weeks to several years; sooner or later the termination is inevitably fatal.

In most cases, the diagnosis is made without difficulty. The enlargement of the organ, the presence of one or more nodules or tumors appreciable by the touch, with pain and tendcrness, and frequently the knowledge of the existence of cancer in some other situation, render the nature of the affection sufficiently clear. A large indolent tumor in the left lobe, however, may occasion considerable embarrassment, and I have met with cases in which different opinions were held by different persons of large experience in diagnosis. Such a tumor may be mistaken for an, aneurism, a fecal accumulation in the transverse colon, cancer of the stomach, or an enlarged pancreas. A medullary tumor, as already stated, may be supposed to be an hepatic abscess. The antecedent and coexisting symptoms are mainly to be relied upon in these cases.

The diagnosis is difficult when no evidence is obtained by palpation. If the cancerous growth be confined within the organ, and the latter be simply enlarged, still more, if the size be normal or below that of health, the existence of the affection can only be conjectured in view of more or 
less pain and tenderness over the liver, conjoined with a eachectic condition and the existence of cancer elsewhere. The diagnosis is impossible in the few eases in which there are no physical signs and the affection is latent as regards local symptoms.

The age of the patient, peritoneal effusion, persisting jaundice, and hereditary predisposition are to be taken into account in the diagnosis.

The treatment has reference only to the palliation of symptoms and the prolongation of life. Pain will often require the habitual use of opiun. An advantage of an early diagnosis consists in the avoidance of injurious medication. Every debilitating measure will tend to shorten life. The system $i$; to be supported, as far as practicable, by tonic remedies, nutritious diet, and other hygienic measures. The object in this, as in other chronic incurable affections, is to endearor to aid the system in resisting, as long as possible, the fatal termination.

\section{Tuberculosis of the Liver.}

Tuberculous collections are occasionally found in the liver. Embracing, under the name tubercles, the small, hard, semi-transparent granulations which have been described in the first part of this work, these are found, in some cases of so-called acute phthisis, here, as in numerous other organs, especially in children. In some cases of advanced ordinary tuberculosis, small masses of yellow, cheesy matter are found in the liver. These may undergo softening, but very rarely, if ever, are large collections found in this organ. The existence of tuberenlosis in this situation can only be ascertained by the scalpel after death; and as it is always secondary to the disease in other organs, tuberculosis of the liver does not claim consideration as an individual affection.

\section{Acute Atrophy of the Liver.}

Under the name acute yellow atrophy of the liver, Rokitansky has described a structural affection offering striking anatomical charaeters, and apparently involving a peculiar morbid process which is not fully understood. The affection has been studied clinically by Frerichs, and examples are to be found among cases reported by Bright, Alison, Graves, Budd, and Wilks in Great Britain. In this country eases have been reported by the late Prof. C. A. Lee, Prof. Horatio C. Wood, Dr. Isaac G. Porter, and Dr. John Homans.' 'The affection has heretofore been known as malignant or fatal jauredice, lyphoid icterus and hemorrhagic icterus.

The liver in this affection undergoes a rapid and notable reduction in size; hence the significance of the term acute atrophy. This diminution, appresiable by physicul signs during life, has been observed to take place within three or four days. The size is diminished in all directions, but more especially as regards thickuess, so that the organ is flattened. In extreme cases the size is reduced one-half and even two-thirds. The weight is proportionately lessened. Frerichs lias met with an example in which the weight was only 1 pound 13 ounces avoirdupois. This amount of reduction existed in a case under my observation to be noticed presently. The organ is notably flaccid, and folds up, or collapses from

1 Vide Buffalo Medical Journal, March, 1853. American Journal of Medical Sciences, April, 1867, and January, 1871. For an account of several additional cases observed in this country, and an analysis of 20 cases, see article by John Homans, M.D., in the American Journal of Medical Sciences, July, 1868. 
its own weight. The surface presents a puckered appearance. The consistence of the organ is much diminished, so that it is easily broken down with the finger, and it is sometimes almost pulpy. The cut surfaces present a yellow color which has been compared to ochre or rhubarb. 'The small vessels connected with the portal and hepatic vein appear to be destroyed, so that an injection thrown into either fails to reach the capillaries, and is extravasated into the glandular tissue. The hepatic cells are not discoverable on microscopical examination. In an advanced stage, the disorganization appears to be complete, the microscope showing granules, oil-drops, pigmentary matter, blood-corpuscles, together with crystals of lencine and globular masses of tyrocene, in addition to the vessels and the connective tissue. The gall-bladder and bile ducts are empty.

Enlargement of the spleen coexists in the majority of cases. The affection has, as yet, been insufficiently studied as regards its association with morbid conditions of other organs.

The clinical history, as determined by an analysis of thirty-one cases by Frerichs, is as follows: The affection, in about an equal proportion of cases, is either developed abruptly or preceded by a prodromic stage. The premonitions are symptoms denoting disorder of the digestive system, and are not significant of the affection. 'Without or' with premonitions which last from three to five days usually, but sometimes much longer, jaundice occurs. It is usually not intense, and does not differ, as regards the coloration of the skin, from ordinary jaundice. Intense cephalagia and delirium, either simultaneonsly with the jaundice or after a period varying from two to twenty-one days, become prominent symptoms. The delirium is usually active, patients crying out, and sometimes requiring restraint; but in some cases the delirium manifests itself only in quiet wandering. In a third of the cases, convulsions occur. These in some cases are epileptiform; in some cases they consist of general tremor or rigor, and in some cases they are limited to a few muscles. Trismus has occasionally been observed. To the delirium and convulsions, stnpor succeeds, eventuating rapidly in profound coma. The pupils are sometimes contracted, sometimes dilated, and in some cases unaffected, responding readily to light. When the jaundice first appears, the pulse is slow, but when delirium occurs, the pulse is more or less frequent. During the progress of the disease, the pulse fluctuates remarkably as regards frequency. At one time it may rise to 120 , at another time falling to 80 or 90 . The pulse varies also as regards other characters than frequency. In the latter part of the disease it becomes persistingly frequent, increasing progressively in frequency and becoming more and more attenuated and feeble. According to Frerichs, the temperature, as ascertained by the thermometer, is only raised during the early stage, and when the delirium and convulsions are excessive. In a case, however, reported by $\mathbf{A}$. Christy Wilson, resident physician of the Royal Infirmary, Edinburgh, the temperature flnctuated between $104^{\circ}$ and $105^{\circ} \mathrm{Fahr}$. up to shortly before death.' Hemorrhages take place in various situations, generally into the stomach and intestines, in females from the uterus, and occasionally hematuria occurs. Extravasations are not uncommon, giving rise to petechiæ and to ecchymoses fonnd after death in various situations. Pains in the epigastrium are common, extending to the right hypochondrium. Tenderness on pressure exists in these regions. The bowels are generally constipated. The breathing is apt to be early accompanied by sighing, and, after coma occurs, the respirations are irregular and sterto-

1 Vide Edinburgh Medical Journal, February, 1868. 
rous. The urine is not suppressed nor notably diminished. It contains bile elements, and there is a graduil disappearance of urea and the phosphate of lime. Leucine and tyrosine are found in large quantities. The urine is sometimes slightly albuminous. Coolness and dryness of the surface exist, except under the excitement of delirium and convulsions, when the heat of the skin is sometimes increased.

The duration of the affection is usually short. Of 28 cases, a fatal termination took place in 13 within a week, in 6 during the second week, in 5 during the third week, and in 4 during the fourth week.

The termination, in the vast majority of cases, is fatal.

The pathological character of the affection is not settled. Rokitansky and Henock attribute the morbid changes in the liver to an excessive production of bile, which, as they suppose, destroys the parenchyma by a process of liquefaction. Briglit, Frerichs, and others consider the morbid process as inflammatory, and, according to this view, the affection is neither more nor less than acute, diffuse, or parenchymatous hepatitis. Of these two explanations, neither is satisfactory. With our present knowledge, we must confess ignorance of the process or processes by which such remaikable anatomical changes are brought about. The symptomatic phenomena are doubtless in a measure due to the suspension of the hepatic functions. Cholesteræmia is probably a pathological element accounting for more or less of the nervous symptoms ; but the convulsions and coma may be due to uræmia.

The causation is not less obscure than the pathology. The affection is most apt to occur between 20 and 30 years of age. Of 31 cases, 20 were between these ages, 6 were between 10 and 20, and only 5 were over 30 . Various causes have been conjectured, but we are without any positive knowledge of its etiology. Females are more predisposed to it than males. Of 31 cases, 22 were females, and of these 22 cases, one-half were attacked during pregnancy. The latter is a striking fact, yet 33,000 cases of pregnancy, analyzed by Spaeth, afforded only two examples of acute atrophy of the liver.

The following is a condensed history of a typical case under my observation in Bellevue Hospital: A female, aged 21 years, was admitted February 18, 1865. When admitted, she was intensely jaundiced, and so lethargic as not to be able to communicate anything of the previous history. With much effort she could be aroused sufficiently to reply to some questions, but immediately relapsed into deep somnolency. She resisted attempts to administer remedies. The pulse was 65 , the surface cool, the pupils were natural, and there was sordes about the teeth. There was considerable tenderness over the liver, and, by percussion, the organ appeared to be diminished in size. At times she evinced a certain degree of consciousness by calling for food and facilitics for attending to other wants of nature. There were no convulsions. The somnolency erentuated in coma, and death took place February 25.

On examination after death, the liver was found to be much reduced in volume, more esperially in thickness; its weight was 1 pound 13 ounces. It had a mottled appearance, and was quite flaccid. On section, it presented a homogeneous, structureless appearance ; the color was deep ochre, and its consistence was almost pulpy. On microscopical examination, the liver cells had lost their characteristic appearance, and appearcd to be mere conglomerations of coarse brown granular matter, without nuclei, but with considerable oil. The field also contained free oil-drops and granular matter. The convoluted tubes of the kidneys had lost their epithelium, and were filled with granular matter. There was no disease 
of the duodenum, nor obstruction of the biliary ducts. The organs within the chest were healthy. The head was not opened.

The diagnosis, after the affection is developed, is to be made by attention to the striking features which belong to its clinical history, and by excluding other grave affections which are liable to be accompanied by jaundice. 'Typhus fever, remitting fever, pyæmia, and certain local inflammations, if jaundice occur, may present some of the symptoms of this affection, but, in general, they are, severally, to be recognized by diagnostic phenomeua which do not belong to the symptomatology of acute atrophy of the liver, or their exclusion is to be based on the absence of these phenomena. Progressive, rapid diminution of the size of the liver may generally be ascertained by physical exploration, and this is a capital point in the diagnosis. Frerichs attaches much importance to the presence of crystals of leucine and tyrocine in the sediment of the urine.

A few words will embrace all that is to be said with reference to treatment. With our present knowledge, little is to be expected, beyond palliation, from remedies. Drastie purgatives and emeties have been advised, but the propriety of these or other perturbatory measures is very doubtful. Symptoms which furnish indications for palliative remedies are vomiting and hemorrhages. For the vomiting and gastrorrhagia, ice in small pieces, bismuth, and anodynes may be prescribed. The mineral acids are appropriate. 'T'he symptoms referable to the circulation, viz., the frequency and feebleness of the pulse, with coolness of the surface, indicate tonics and stimulants. Inasmuch as the convulsions and coma may be due to uræmia, it would be desirable, if possible, to exeite the action of the kidneys; and, if this be not practicable, the hot-air bath and perhaps hydragogues, suggest themselves as means of effecting the vicarious elimination of urea. Hemorrhage from the bowels ealls for astringent remedies. Revnlsive applications over the site of the liver, by means of fomentations and stimulating embrocations or rubefacients, may be of some service.

\section{Hydatid Tumors of the Liver.}

A hydatid tumor consists of a sac, more or less thick and resisting according to its age, which contains a membranous bladder filled with a clear liquid resembling water. This bladder is not adherent to the surrounding sac; it may contain nothing but liquid, but generally it contains a greater or less number of floating globular or ovoid cysts, varying in size from that of a pea to a hen's egg. The number of these cysts contained within the membranous bladder, or the so-called mother cyst, varies greatly in different cases. They may be few, or the number may amount to hundreds and even thousands. The mother cyst and the cyst which it contains (which Frerichs calls the daughter vesieles), are now commonly known, as hydatids. They were formerly called, after Laennec, acephalocysts. Within the hydatid cysts are found, in more or less abundance, adherent to the inner surface, minute white particles, which Budd compares to diminutive fish spawn. Examined with the microscope, these minute bodies are found to be immature tæniæ, and they are called ech $i$ nococci hominis. The echinococcus, so long as it is contained within the hydatid cysts, remains undeveloped, but finding its way into the alimentary canal of certain animals, it is developed into a tapeworm. The hydatid cysts may contain only the remains of echinocoeci, viz., shreds of membrane, and the eharacteristic hooklets which encircle the head of the animal in its immature as well as its fully developed state. 'The only 
source of echinococci in any part of the human body is the introduction into the alimentary canal of the ova from trenia. The tania which furnish ova giving rise to the hydatid formations occurring in the liver and other orgaus in man, are found in the intestines of dogs and wolves (trenia echinococcus). 'The ova, ingested with food or drink, find their way from the alimentary canal to the liver or other organs where they are dereloped into echinococci or immature tapeworms.

For a fuller description of hydatids and echinococci, the reader is referred to treatises on morbid anatomy, or to works treating especially of entozoa-a subject which has acquired great interest and importance from recent researches. ${ }^{1}$

The liver is the most frequent habitat of hydatid tumors; they occur here oftener than in all other parts collectively. Usually there exists but a single tumor, but sometimes there are two, threc, or more. Similar tumors sometimes coexist in other organs, more especially in the lungs and spleen. They vary in size, in different eases, both in the liver and elsewhere, from a minnte cyst to a tumor as large as a child's head. They may exist in any part of the liver, being situated sometimes deep within the gland and sometimes near the surface. They are much oftener found in the right than in the left lobe of the liver.

The pathological history of hydatid tumors offers important differences. in different cases. The growth of the tumor in a certain proportion of cases is arrested by the thickness and density of the sac, which sometimes becomes calcareous. Under these circumstances, the contained hydatids shrivel up and are destroyed; the echinococei die, and an examination after death shows the debris of the former and the hooklets of the latter, with cholesterin, hematoidin, and inspissated bile. This is a mode of spontaneous cure occurring in a large proportion of cases. It is supposed that the destruction of the hydatids and echinococei is sometimes due to the passage of bile into the mother cyst.

In other cases the hyatid tumor continues to grow until it attains to a considerable or large size, projecting from the periphery of the liver and making pressure on the surrounding parts. The most frequent direction of the tumor is against the diaphragm, pushing it upward more or less within the thoracic space. The diaphragm has been observed to be pushed upward as high as the first rib, and even to the clavicle. Situated in the left lobe of the liver, it may push the parts before it into the left thoracic space, displacing the heart. Situated at the lower part of the liver, it presses against the colon and small intestines, and may extend nearly or quite to the brim of the pelvis. Situated near the anterior surface of the liver, it extends in an outward direction, and may appear as a fluctuating tumor at any point over the site of the organ.

Bursting of the tumor is an important event in its pathological history. Extending into the thoracic space, it may open and discharge its contents into the pleural sac, giving rise to acute pleuritis, which is apt to prove fatal. A far more favorable occurrence is an opening into the bronchial tubes, escape into the pleural sac being prevented by partial pleuritis followed by adhesions at the point of perforation of the diaphragm. The hydatids are then expectorated, and such cases offer a fair prospect of recovery. The opening may take place into the peritoneal cavity, and fatal peritonitis is then induced; or, the opening having been preceded by

1 The medical student or practitioner desirous of knowing all that is known respecting these and other entozoa should consult the work by Davainc, cntitled Traité des Entozoaires, etc., Paris, 1860. 
partial peritonitis and adluesions, it takes place into the stomach or colon. The hydatids are then discharged by vomiting or stool, and recovery may take place. It may burst into the pericardium, and a fatal result is immediate. Another fatal direction is into the vena cava. The hydatids then, getting into the venous 'circulation, are carried to the right side of the heart, and destroy life by plugging the branches of the pulmonary artery. It may open into the gall-bladder or biliary passages, and the hydatids may pass through the common duct, or, becoming impacted within it, give rise to obstruction to the flow of bile into the duodenum. And, lastly, the tumor may open externally through the abdominal walls, or in an intercostal space, under these circumstances recovery taking place in about one-half of the cases.

The bursting of the tumor inwardly may be the result of a blow or fall. This has repeatedly proved a cause of sudden death in cases in which the existence of an hydatid tumor had not been suspected. In some cases suppuration takes place within the tumor, converting it into an hepatic abscess.

The clinical history offers nothing which points to this or any affection of the liver until the tumor attains to a sufficient size to occasion inconvenience by pressing on the adjacent parts. So long as the tumor is contained within the gland, it is nsually completely latent. It gives rise to no pain or tenderness, and the hepatic functions are not appreciably disturbed. Tumors of considerable size are not infrequently discovered unexpectedly in autopsical examinations. If, however, a tumor be situated near the periphery, and project considerably bryond the organ, it is apt to give rise to local symptoms directing the attention to the part. Usually, under these circumstances, pain is slight or wanting, but a sense of fulness and uneasiness is felt in the neighborhood of the liver. When pain is present, it is dne mainly to inflammation developed by the pressure of the tumor. The pressure upon adjacent parts gires rise to symptoms which differ according to the direction in which the tumor extends. Extending into the thoracic space, it gives rise to dyspnoea on exertion, cough, and palpitation. Pressure on the stomach and intestines is liable to produce vomiting and constipation. If the vena cava be compressed, œdema of the lower extremities follows. The growth of the tumor is slow, and attended with little or no constitutional disturbance. 'There is no febrile movement, and the nutrition of the body may be unimpaired.

Important local and general symptoms, however, are incident to the bursting of the tumor, or the discharge of its contents by ulceration. These symptoms will depend on the direction in which the opening occurs. As already stated, pleuritis, pericarlitis, and peritonitis, are results of the opening, respectively, into the pleural, pericardial, and peritoneal cavities, and the two latter affections thus induced are uniformly fatal. The first of these three affections is very apt to prove fatal. Opening into the vena cava, it gives rise to the symptoms of obstruction of the pulmonary circulation from embolism. If the lung be perforated without general pleuritis, pneumonitis is apt to follow ; cough and expectoration are prominent symptoms, and in the latter are contained, from time to time, hydatids, together with bile in some cases. If perforation of the lung ensue after the evacuation into the pleural sac, the phenomena of pnenmo-hydrothorax are developed. .Perforation of the stomach or colon is usually attended with severe pain, and is followed by the discharge, either by vomiting or stool, of hydatids. The discharge of the hydatids into the biliary passages gives rise to jaundice. Jaundice, irrespective of this cause, is rare in cases of hydatid tumor. Dropsy of the peritoneum cloes not be- 
long to the clinical bistory of the affection. Both jaundice and hydroperitoneum, however, are occasionally effects of the pressure of the tumor on the biliary passages without the liver, and on the vena portre.

A diagnosis is impracticable so long as the growth does not extend beyond the gland. The affection can only be recognized when either the tumor is discoverable by physical examination, or hydatids are discharged through some outlet. $A$ tunor extending from the anterior surface, or from the inner or lower margin of the liver, after it has attained to a certain size, is apparent to the touch. The diagnosis now involves its discrimination from other tumors. When situated over the liver, it is to be discriminated from cancer and hepatic abscess. This discrimination may generally be made without much difficulty. From cancer it is distinguished by its smooth and globular form, its elasticity and the sense of fluctuation, freedom from pain, and absence of the cancerons cachexia. From hepatic abscess it is distinguished by the slowness of its growth, absence of pain and tenderness, and by its being unattended by the constitutional symptoms to which suppuration generally gives rise, viz., chills, febrile movement, etc. Extending from the inner border, it is to be discriminated from cancer of the stomach or pancreas, and from an aneurismal tumor. The comnection of the hydatid tumor with the liver, and the disconnection of other tumors, may generally be ascertained by palpation and percussion. Cancer of the stomach or pancreas is usually accompanied by pain, and, in the former situation, by notable gastric symptoms, and the cancerous cachexia may be apparent. The cancerous tumor is generally irregular or lobulated. An aneurismal tumor is distinguished by its anterior and lateral pulsation, the frequent occurrence of murmur, and generally by persisting gnawing pain referable to the back.

A physical sign, sometimes obtained by percussion, is highly distinctive of a hydatid tumor. If percussion be made upon the tumor, the fingers of the left hand or the whole hand being placed over the tumor, the collision of the floating hydatids with each other causes a characteristic tactile vibration known as the hydatid fremitus. The sensation is compared to that felt when percussion is made upon the hand resting on a mass of jelly. M. Briancon has shown, by filling an ordinary bladder with liquid, and introducing a greater or less number of hydatids, that this sign is due to the latter, and that, other things being equal, the fremitus is marked in proportion to the number of hydatids introduced. ${ }^{2}$. By placing the stethoscope over the tumor, and practising percussion, a peculiar sound is elicited, which in some cases has a musical intonation like the sonnd from a violoncello. These signs are obtained in only a certain number of cases, the proportion being about one-half, according to Frerichs.

An hydatid tumor, encrosching more or less upon the thoracic space, gives rise to flatness on percussion, and absence of respiratory murmur from the base of the chest upward in proportion to the height to which the tumor extends, with, perhaps, more or less displacement of the heart. The signs are those of pleuritis with effusion. Attention to the following point will suffice for the discrimination in many if not most cases. In pleuritis, the level of the liquid effusion, when the patient is sitting or standing, is denoted by flatness extending upward on each aspect of the affected side of the chest, to about the same distance; in other words, the upper limit of the flatness is indicated by a line passing horizontally, or 
nearly so, around the affected side. But the flatness due to a tumor extending into the thoracic space generally has an irregular limit-that is, the flatness extends higher at some than at other points. Moreover, the test of the presence of liquid effusion, afforded by the results of percussion when the position of the body is changed from the vertical to the recumbent, is not available in the case of a tumor.

The diagnosis in cases in which the tumor opens externally, or its contents are discharged by expectoration, vomiting, or stool, is established by the discovery of hydatids either entire, or the remains left after their destruction. If entire, they are easily recognized with the naked eye. If not entire, the microscope is to be employed to discover shreds of the characteristic membrane, and hooklets of echinococci.

There is still another means of arriving at a positive diagnosis, viz., by introducing into the tumor an exploring canula, and withdrawing a little of the liquid. If a clear liquid, like water, escapes, which does not coagulate by the addition of an acid, and on evaporation leaves crystals of the chloride of sodium, there can scarcely be room for doubt as to the character of the tumor. If the hydatids are clestroyed, the liquid may be turbid. In this case, microscopical examination may show hooklets of echinococci. An exploring puncture, made with a very small instrument, is attended with little or no danger.

A tumor caused by distension of the gall-bladder offers, in its general physical characters, a close resemblance to an hydatid tumor. But as a large accumulation of bile in the gall-bladder involves obstruction of the common duct, jaundice necessarily exists, and in an intense degree. This goes far in the differential diagnosis. Other points are, the form of the tumor resembling that of the gall-bladder, viz., egg-shaped, and its situation at the inferior border of the liver.

Age is of some account in the diagnosis. Hydatids occur mostly in middle life, rarely in childhood or old age. They occur most frequently among the lower classes of society. It may be stated, in this connection, that they occur more frequently in some countries than in others. They are certainly extremely rare in this country.

Hydatid tumors are not in themselves serious except from their size. They do harm by pressure upon adjacent parts. They prove serious, also, by opening into situations in which grave or fatal diseases are induced. The prognosis in these cases has been already stated. If the opening occur in a situation whence the contents are discharged from the body, viz., through the integument, into the bronchi, or the alimentary canal, recovery may be hoped for, or expected, according to the circumstances in individual cases. If recovery do not take place, the fatal termination is preceded by protracted irritation and progressive exhaustion due, generally, to suppuration within the sac.

The growth of the tumors is generally extremely slow, and hence, the duration of the affection is long. After the existence of a tumor is ascertained, many months, and in some cases many years, elapse before it attains to a size sufficient to cause serious results or great inconvenience. Of 24 cases analyzed by Barrier (cited by Frerichs), in 3 the affection lasted for 2 years; in 8 , for periods between 2 and 4 years; in 4 , from 4 to 6 years, and single cases exemplified its duration for $15,18,20$, and 30 years.

In the treatment of hydatid tumors of the liver, there are two objects to which measures may be directed. One olject is the evacuation of the tumor; the other object is to arrest its growth and promote absorption of its contents. Medicinal means are applicable to the last-named object 
only; the first object is, of course, to be effected exclusively by surgical interference.

Several internal remedies have been proposed as capable of destroying the hydatids. When this takes place, the tumor ceases to grow, and, gradually diminishing in size, may finally disappear. This is the mode in which a spontaneous cure not infrequently takes place. Calomel, common salt, and the iodide of potassium, have been thought to have the power of effecting the destruction of the hydatids, these remedies acting by being absorbed and exerting a poisonous effect upon the entozoa. Their efficacy, however, does not appear to have been established by clinical observation. Dr. Hjaltelin, of Iceland, reports that the tincture of kamela, an active tænifuge, given internally in doses of from thirty to forty drops three times a day in a tablespoonful of water, effected a cure in two cases under his observation, and in many other eases within his knowledge.'

The most effective surgical measure, in certain cases, is to make an incision into the tumor, sufliciently large to remove both the liquid and the hydatids which it may contain. This operation is warrantable, if not advisable, whenerer the tumor extends in an outward direction, provided adhesions have taken place around a space in which the opening is made, so that there is no danger of the escape of the contents into the peritoneal sac. A method of determining whether adhesions exist or not, is to ascertain whether the tumor, or the liver, be depressed by a forced inspiration, or remain fixed in the same position. This point is readily ascertained by marking on the chest or abdomen the situation of the tumor, or the lower margin of the liver, or its upper margin, successively at the end of an expiratory and an inspiratory act. After evacuating the tumor, cicatrization may be expected to follow, but this result may be preceded by suppuration within the sac, which may be continued so as to involve danger from constitutional irritation and exhaustion.

A better method of surgical treatment is to withdraw more or less of the liquid by means of a small exploring canula, employing a certain amount of suetion foree if neeessary. The effect of this procedure is to diminish the size of the tumor, and to destroy the hydatids, a cure taking place somewhat as it does spontaneously when the liquid is absorbed after the destruction of the hydatids. Dr. Murchison has collected the particulars of 46 reported cases in which this plan of treatment was pursued. In 35 of these cases the operation was perfectly successful. In 10 eases it was followed by suppuration requiring a free opening, and in eight of these cases recovery took place. In one case only did the patient die of peritonitis, and, in this case, the condition of the patient prior to the operation was unfavorable. The injection of medicated liquids with the view of destroying the echinococci is unnecessary, and increases the liability to suppuration. If suppuration occur a free opening is required.2

Evacuation by means of a small canula in cases in which the tumor extends within the thoracic space, or projects from the margin of the liver into the abdomen, may involve a certain amount of danger from the escape of the liquid into the pleural or peritoneal cavity. A precaution to be observed with reference to this accident is enforced by Boinet, viz., to make firm pressure over the tumor for some time after the canula is withdrawn; afterward a firm bandage with a compress should be applied. As a set-

1 Edinburgh Medical Journal, August, 1867.

- Clinical Lectures on Diseases of the Liver, etc. By Charles Murchison, M. D. Am. edition, 1868. 
off against some risk from the operation, the liability of rupture taking place, and the discharge of the contents of the tumor into these cavities, is to be borne in mind.

An Iceland surgeon, Dr. Thorarensen, has reported a case in which repeated shocks of electricity, conveyed within the tumor by means of steel needles, destroyed the entozoa and effected a eure. 'This method of treatment merits further trial. The surgeon just named has operated by perforating the sac with a trocar about 40 times, and of this number of cases not more than seven have terminated unfavorably. The large number of cases seen by this surgeon is explained by the prevalence of hydatid disease in Iceland; and the prevalenee in that country of the affection is explained by the fact that the sheep-dog in winter occupies often the dwelling of his master. Dr. J. Hjaltelin, inspecting medical officer of Ieeland, has operated sometimes with the trocar and sometimes with a bistoury in 36 cases, losing only six.'

Aside from surgical interference, the treatment of hydatid tumors of the liver embraces only such palliative measures as may be indicated by the circumstances pertaining to individual cases. The treatment of pleuritis, pneumonitis, and peritonitis indueed by the discharge of the contents of the tumor, does not claim distinct eonsideration.

The prophylaxis of hydatids, in the liver and elsewhere, is to be based on the source, viz., the ova of the trnia echinococcus developed in the intestine of the dog. The dog derives the tænia from meat infested with hydatids; hence, preventing this as far as praeticable, and burying or destroying the excreta of the animal, comprise the means for avoiding the introduction of ova into the human intestine.

\section{Pigmentary Deposit within the Liver.}

A deposit of dark-colored granules in the liver, giving to the organ a steel-gray, chocolate, or bronzed appearance, is observed not infrequently after death from the so-called malarious fevers. A similar deposit generally coexists in the spleen, and may be found in other organs. It is found in the blood, especially within the portal vessels. The source of the pigmentary matter, together with its pathological relations and effects, cannot be considered as settled. With our present knowledge, the pigment liver is hardly entitled to be reckoned in the list of hepatic aflections. It will be referred to hereafter as entering into the anatomical characters of the periodical fevers.

\section{H ypertrophy of the Liver.}

Hypertrophy of the liver was supposed to be not infrequent before the several degenerative affeetions to which this organ is liable were as well understood as now. The volume of the liver is often increased, but it may be doubted whether this ever proceeds from hypertrophy. 'The existence of true hypertrophy, as a morbid lesion of the liver, is questionable. At all events, the physician is never called upon to recognize and treat hypertrophy of this organ. The meaning of true hypertrophy is, of course, to be borne in mind, viz., abnormal growth of a part, with no abnormal change in composition or structure.

1 For an account of the echinococci endemic in Iceland, vide Edinburg Med. and Surg. Journal, Feb. 1870 . For a report of six cases in which the electrolytic treatment was successfully employed, by C. Hilton Fagge, M. D., and Arthur E. Durham, vide Trans. of Royal Medical and Chirurgical Society, Vol. LIV. London, 1871 . 


\section{CHA P TER XIII.}

\section{DISEASES AFFECTING THE SOLID VISCERA OF THE ABDOMEN- (CONCLUDED.)}

Diseases Affecting the Biliary Passages. Jaundice, or Icterus. Functional Affections of the Liver. Affections of the Spleen. Affections of the Pancreas.

\section{Diseases Affecting the Biliary Passages.}

THE diseases affecting the liver which have been considered relate, for the most part, to the parenchyma or substance of the organ. 'The biliary passages, within and without the liver, inclusive of the gall-bladder, are liable to disease. Inflammation of the mucons membrane lining the excretory ducts is probably an affection of not infrequent occurrence. Ordinary inflammation, that is, analogous to inflammation of other mucous membranes, constitutes, as there is reason to believe, the pathological condition giving rise to the affection called icterus or jaundice, in the majority of the cases in which this affection is not incidental to structural lesions of the liver or the surrounding parts. 'The opportunity of obtaining autopsical proof of this is rarely offered, because ordinary inflammation in this situation does not prove fatal. The swelling of the membrane and the accumulation of mucus within the ducts occasion more or less obstruction to the passage of the bile; hence its reabsorption, constituting jaundice. The clinical history of eases of jaundice referable to this pathological condition generally shows the inflammation to have had its point of departure in the duodenum, extending thence into the common duct, and perhaps to a greater or less extent into the hepatic duct and its branches within the liver.

In some cases of inflammation of the mucous membrane of the ducts an exuclation of lymph takes place, and occlusion of the ducts may follow. Complete closure of the common duct, in addition to jaundice, leads to an accumulation of bile within the gall-bladder. The gall-bladder, under these circumstances, may become enormously distended. In a case related by Van Swieten, it contained eight pounds of thick bilc. In a case recorded by Benson, the space occupied by the distended gall-bladder was so great that dropsy of the peritoneum was supposed to exist, and paracentesis was resorted to. Frerichs, who cites these cases, has obscrved a case in which there was an accumulation of eighteen ounces. The accumulation of bile within the gall-bladder may also occur from the impaction of a biliary calculus in the common duct, or from obstruction of this duct due to other mechanical causes. Obstruction of the cystic duct, preventing the passage of bile into the gall-bladder, but not into the common duct and intestine, does not give rise to jaundicc, but, under these circumstances, in some cases, the gall-bladder becomes greatly distended with a purulent liquid due to inflammation of the lining membrane of the gall-bladder. $\boldsymbol{A}$ case was reported by the late Prof. Pepper, in which the gall-bladder contained after death two quarts of purulent liquid mixed with bile, the cystic 
duct being completely closed by false membrane. ${ }^{1}$ In some cases of obstruction of the cystic duct the gall-bladder becomes distended with a serous liquid. In these cases the affection is considered as dropsical, and has been called hydrops cystidis felleæ. 'The liquid, however, is not purely a transudation, but contains more or less of inflammatory products.

The gall-bladder distended by liquid forms a tumor which is to be discriminated from a hydatid tumor and hepatic abscess. The situation of the tumor, its form before dilatation takes place sufficiently to distort it, and (exclusive of the cases in which the obstruction is in the eystic duct) coexisting jaundice are to be relied upon in making the discrimination. The antecedent history and the accompanying symptoms are also to be taken into account. 'l'he distending liquid may be presumed to be bile when coexisting jaundice and absence of bile in the stools show the common duct to be obstructed ; and the liquid is either purulent or sero-purulent if the absence of jaundice shows the obstruction to be in the cystic duct. An exploration of the tumor by means of a very small trocar or needle may be warrantable, in cases of doubt, as a means of diagnosis. The liability to mistake a distended gall-bladder for a moving or floating kidney is to be mentioned. A case in which several of my colleagues, as well as myself, fell into this error, came under my observation at Bellevue Hospital.

The treatment must have reference to the eircumstances in individual cases. Puncturing the gall-bladder, in order to discharge its contents, is to be resorted to when, from the degree of dilatation, there is danger of rupture taking place into the peritoneal cavity. The latter accident is followed by fatal peritonitis. If punctured, the same precautions are to be taken, with reference to peritoneal adhesions, as in opening an hepatic abscess.

Ulceration of the gall-bladder and of the excretory ducts, as an occasional effect of the presence of calculi, has been already referred to in treating of the passage of gall-stones or hepatic colic. Ulceration of the gall-bladder is sometimes the result of inflammation, irrespective of biliary calculi or distension from obstruction. The symptoms of cholecystitis, as it has been called, however, do not point to its existence, nor, perhaps, to the existence of any affection. I have met with a case of ulceration and perforation resulting, apparently, from an abscess between the coats of the gall-bladder, and in this case there were no symptoms of any disease prior to the sudden development of peritonitis due to the perforation. There were no calculi in this case.

The gall-bladder is sometimes the seat of cancer. In most cases, however, when seated here, it is secondary to cancer of the liver.

The ascaris lumbricoides, or round worm, occasionally migrates from the intestine into the excretory ducts of the liver, and may pass into the branches of the hepatic duct within the liver. Dilatation of these ducts is a lesion arising from persisting obstruction of the hepatic or common duct from any cause.

The entozoa known as the Distoma Hepaticum and Distoma Lanceolatum, or liver-flukes, which are common in the biliary passages of ruminant animals, have been occasionally found in man. They give rise to no symptoms by which their existence could be suspected. With our present knowledge, they have no interest or importance, in a pathological view, as occurring in the human species. 


\section{Jaundice, or Icterus.}

The presence of bile-pigment in sufficient quantity to give to the conjunctival membrane of the eye and to the skin a yellow color, greater or less in degree, constitutes the morbid condition known as lickruz, or Jaundice. The term cholæmia denotes the presence of bile in the blood, and, under this head, in treating of the morbid conditions of the blood in Purt First, has been considered the general pathology of the affection to be now bricfly noticed. Of the two terms, icterus and jaundice, the latter is more commonly used. Both terms, as regards their significance, relates to yellowness of the surfaee.

Jaundice is strictly never an inclividual disease. It is merely an effect or a symptom of disease. But as it is an obvious and striking morbid condition, dependent not on one affection, but on various affections, and occurring when its causation is not evident, for convenience it is regarded, in certain eases, as an individual disease. Not a few morbid conditions, nosologically classed as individual diseases, are, in like manner, in reality, only symptoms or effects of disease. In recognizing jaundice as an individual clisease, cases are to be excluded in which it is incidental to an affection the existence of which is clearly made out. Thus, jaundice occurs in certain eases of all the hepatic affeetions which have been considered, viz., acute hepatitis, cirrhosis, portal phlebitis, cancer, etc. etc. It occurs also in several general or constitutional diseases, namely, septicremia, puerperal, remittent, and relapsing fever. Under these cireumstanees, it is to be regarded as merely a symptom.

The presence of bile-pigment in the blood, or eholremia, is due to the reabsorption of bile, within the liver, after its secretion. The biliverdin and the biliary salts (the glyco-cholate and tauro-cholate of soda) are formed within the liver, that is, they do not pre-exist in the blood. 'The bile, however, does contain at least one excrementitious principle, viz., cholesterin. 'The latter constituent of bile will aceumulate in the blood, if the secretion of the liver be from any eause suspended. The other constituents just named are never present in the blood in consequence of suspension of the secretory function of the liver, but only from reabsorption after having been secreted. These, at least, are the views most consistent with our present knowledge. That the hematin of the blood may undergo a transformation into a substance analogous to, or identical witl, biliverdin, irrespective of the action of the liver, must be regarded as conjectural.

The reabsorption of bile within the liver, in the great majority of cases, is due to obstruction to its passage into the intestine. And, as already stated, in the majority of the eases in which jaundice is to be regarded as an individual disease, it probably depends on obstruction ineident to inflammation of the biliary ducts. Obstruction, however, may proeeed from other causes, such as the presence of a calculus in the common or hepatic duct, lumbricoid worms in these ducts, or the pressure of a tumor upon them. Is janndice, in all instances, attributable to obstruetion? Cases have occurred in which jaundice having been developed in connection with some other serious disease, and the opportunity of examination after death being afforcled, no appreciable obstruction was discoverable. Moreover, the occurrence of jaundice suddenly, after a violent mental emotion, has been considered as inconsistent with mechanical obstruction. Spasm of the ducts is hardly adequate to explain these cases, and it has hence been concluded that, under particular conditions of the circulation within the liver, the bile or certain of its elements may enter the hepatic 
veins when there is no mechanical obstruction. A more rational supposition is that the bile is absorbed from the alimentary canal, the usual metamorphosing therein not having taken place. ${ }^{1}$ Murchison accounts in this way for the jaundice following snake-bites occurring in relapsing fever, pyæmia, etc., as well as from fright and a hyper-secretion of bile.

Yellowness of the conjunctiva and skin takes place after a certain amount of bile has been reabsorbed. Prior to the appearance of janndice, the bile-pigment may be found in the urine, and in the liquid of serous cavities. The coloration of the surface depends mainly on the presence of the bile-pigment in the transuded liquid which infiltrates the tissues. Other things being equal, the intensity of the yellow color is proportionate to the degree of the cholæmia. The biliverdin is readily found in the blood-serum, but, according to Frerichs, not the acids which exist in bile in combination with soda. The presence of the bile-pigment in the serum of the blood is shown by the yellow color of the liquid beneath the epidermis, if a blister be applied.

The urine in cases of jaundice contains the coloring principle of bile in abundance. Its presence is apparent to the eye, and it gives to linen a yellow stain. The perspiration contains it, and the body-linen may also be stained yellow, especially from the abundant cutaneous secretion in the axilla. It is rarely, if ever, contained in the saliva, nor, as a rule, is it found in mucus, but it may be fonnd in the sputa of pneumonitis developed in a person affected with jaundice. A beautiful example of conservatism is afforded by the fact that the milk of nursing women very rarely contains it, although its presence in this seeretion has been observed. The humors of the eye do not contain it, except in some cases in which the jaundice is unusually intense. The "jaundiced eye" is so rare an event, that the phrase is to be regarded as a poetical license.

The tissues in the interior of the body are more or less colored. The coloration is seen especially in the areolar tissue, the serous membranes, and the muscles. The substance of the brain escapes, except in cases in which it is usually infiltrated with serum, that is œdematous.

Bile in the blood acts as a sedative on the cireulation and nervous system. The heart's action is usually diminished in frequency. The pulse frequently falls to 50,40 , or even much lower, in cases in which the jaundice is not associated with acnte inflammation or fever, and, if thus associated, the frequency of the pulse is less than if jaundice did not coexist. According to Köhrig this effect is due to the presence in the blood, not of the biliverdin, but of the bilious acid salts. The temperature of the body is unaffected. The respirations diminish in frequency in proportion to the effect on the circulation. The mental faculties are dull, and there is a disposition to somnolency. Pruritus of the surface, especially in the axilla and inguinal region, troublesome more particularly at night, is an occasional symptom. It is sometimes a source of great annoyance. An occasional complication is urticaria, denoted by the characteristic eleva. tions on the skin, or.wheals, which present a deeper yellowness than the surrounding integuments, and occasion intense pruritus.

1 The discussion of mooted pathological points relating to the transformation of the coloring matter of the blood, and of the biliary acids in the pile-pigment, within the vascular system, is inconsistent with the scope of this work. The treatise by Frerichs may be consulted on these as on other points relating to affections of the liver; also, the clinical lectures on diseases of the liver by Murchison. With reference to the question above, vide Prize Essay on the Patlology of Jaundice, by $\mathrm{S}$. Fleet Speir, M.D., in Transactions of the American Medical Association, vol. xv., 1864. 
The symptoms referable to the digestive system are frequently due to coexisting duodenitis or gastro-duodenitis. Anorexia, nausea, and sometimes vomiting, thirst, more or less pain referable to the neighborhood of the epigastrium, and tenderness in that situation, are symptoms denoting subacute inflammation of the gastro-duodenal membrane. In cases of jaundice disconnected from this affection, the appetite may be but little or not at all impaired, and certain articles of food are apparently digested without difficulty. Fatty food is imperfectly digested, and chemical examination of the evacuations from the bowels shows fat in more or less abundance. Patients have an antipathy to fatty articles of diet. The nutrition for a time may be but little affeeted, but sooner or later loss in weight takes place, and is progressive, if the jaundice continue.

The appearance of the dejections is important, as affording evidence of the completeness, or otherwise, of the obstruction to the passage of bile into the intestine. If complete occlusion exist, the stools are ash or clay colored. On the other hand, they have more or less of the yellowish or brownish color of health if the obstruction be only partial. The feces, when the intestines are devoid of bile, emit a fetid odor, due to putrefactive decomposition, and the formation of gas is frequently troublesome, one important purpose of the presence of bile in the intestiual canal being an antiseptic agency as regards the alimentary contents. Generally, the bowels are constipated, but this is by no means always the case even when complete occlusion exists.

Janndice may exist for a greater or less period without giving rise to notable disturbance. The system sometimes tolerates the accumulation of bile for a long time. Not infrequently patients are able to be up and about as in health, experiencing but little inconvenience. The symptoms and the gravity will depend, in a great measure, on the pathological conditions with which the jaundice is associated. If the jaundice depend on inflammation of the biliary ducts in comeetion with subacute gastro-duodenitis, the prognosis is always favorable. The affection usually continues from one to two or three weeks. If it depend on the impaction of a gall-stone in the common or hepatic duct, the recovery will depend on the passage of the stone into the intestine, or, backward, into the gallbladder. Permanent obstruetion from a gall-stone, a deposit of lymph in the dnets, or the pressure of a tumor, involves, of course, persistence of the jaundice. Under these circumstances serious results may not take place for a considerable or even a long period. Cases have been observed by Graves and Stokes, Budd and others, in which the nutrition of the body was not greatly impaired for one, two, and even four years. Life has continued under these cirenmstanees for a much longer period. Sooner or later, however, the body wastes, the patient becomes anmmic and cachectic, the vital powers gradually fail, and the termination is fatal, irrespective of either the morbid condition giving rise to the jaundice, or intercurrent affections of other organs than the liver. In a case now under my observation (1872) the jaundice has existed for more than two years, and the patient is in fair general health. The color of the skin becomes dark, or almost black, in some cases of long persisting jaundice. In the majority of the eases of jaundice from permanent obstruction, however, the duration of life does not extend beyond a few weeks or months.

When life is destroyed purely by the retention of bile, the liver is the seat of important lesions. The bile-ducts within the liver become dilated; the gall-bladder may, or may not, become distended with bile; 
the liver is at first increased in volume from the accumulation of bile, but subsequently its volume is diminished, and it become flabby and wrinkled. The persisting distension of the hepatic bile-ducts at length leads to an arrest of the secretion of bile. According to 1)r. Thomas Williams and Budk, ' the secreting cells of the liver undergo disintegration. The correctness of this statement is denied by Frerichs. In proportion as the secretion of bile is arrested, the jaundice will diminish, and the patient may suffer from the retention in the blood of the excrementitious portion of the bile, viz., cholesterin. In some cases death is preceded by convulsions and coma. In these cases cholesteræmia may be supposed to exist. But these phenomena, in a certain proportion of cases, are due to ura mia. Post-mortem examinations show an accumulation of granules of bilepigment in the uriniferous tubes, when jaundice has existed for a considerable or long period, and the uræmia proceeds from the morbid condition of kidneys. Hydro-peritoneum may occur in the latter period of the affection; and general dropsy is sometimes a result of the superinduced renal affection.

The diagnosis of jaundice is readily made if the examination of the patient be in the daytime. The yellowness may not be apparent by artificial light. The yellow tint of the surface seen in some cases of cancer, intermittent fever, and chlorosis, is readily discriminated from slight jaundice, by the absence of yellowness of the conjunctiva, the latter being always present in jaundice. But to determine the causative pathological conditions in individual cases, is not always easy, nor, in all cases, practicable. The dependence on inflammation of the biliary ducts connected with duodenitis or gastro-duodenitis, is to be inferred from the symptoms of the latter preceding and accompanying the jaundice, viz., anorexia, nausea, vomiting, and tenderness over, or in the neigh borhood of, the epigastrium. The presence of a gall-stone may be inferred from the pre-existence and coexistence of the symptoms of hepatic colic. A tumor which presses upon the common duct may be discoverable by manual exploration. From the character of the tumor, and the coexisting symptoms, its cancerous nature may sometimes be inferred. Occlusion as a result of inflammation of the ducts exclusive of duodenitis, from lumbricoid worms in the common duct, and other causes, cannot be determined during life ; the existence only of obstruction is determinable in such cases.

In the treatment of jaundice, it has been much the custom to prescribe active cathartics and emetics under an idea of removing the biliary obstruction by their operation. If, however, the jaundice be connected with duodenitis or gastro-duodenitis, not only are these measures ineffectual, but they can hardly fail to do harm. If the obstruction be caused by a gall-stone, its passage will not be hastened by these measures; and they will weaken the patient, without doing good, if the jaundice depend on a tumor pressing on the common or hepatic duct. As remedies, then, addressed to the obstruction giving rise to the jaundice, cathartics and emetics are contra-indicated.

The treatment of jaundice, in the majority of cases, is to be addressed to a subacute inflammation of the stomach and duodenum. Laxatives or cathartics are only required for the relief of constipation; and, when required, the mildest forms are to be preferred. There is no ground for the supposition that mercury exerts any special efficacy, but a few grains of calomel and blue mass frequently prove as mild and effective as any form of cathartic or laxative, followed, if necessary, by a saline draught. The 
latter alone will often suffice. The saline mineral waters, such as the Congress or Kissingen water, will frequently secure an adcquate laxative effect. Remedies to soothe the inflamed mucous membranes are useful, such as small doses of morphia or some of the succedanea of opium, viz., belladonna, hyoscyamus, conium, etc. Conium has been considered as a remerly having a special efficacy in jaundice. Its apparent efficacy may be accounted for, in part, by its usefulness as a soothing remedy, and, partly, by its supplanting active cathartics and emetics which are injurious. The diet should be bland and digestible. Fatiy articles of food should be excluded, and to these the patient generally has an antipathy. A blister over the epigastrium has seemed to me to be useful. Accidental symptoms will, of course, claim appropriate palliative measures. Gerhardt states that in cases of jaundice from gastro-duodenitis and inflammation of the ducts, as well as in the cases in which the affection is due to other causes, the gall-bladder, distended by the accumulation of bile, forms a tumor which is not infrequently perceptible to the touch; and that, under these circumstances, pressure upon the tumor, made with the fingers, will sometimes empty the gall-bladder by overcoming the obstruction in the ductus communis. He has also found the induced eurrent of electricity to be successful by exciting spasmodic contractions of the muscular coat of the gall-bladder. ${ }^{2}$ A bubbling sound, cansed by the passage of the bile into the duodenum, may sometimes be heard, occurring with the disappearance of the tumor. He compares the success of these measures, in some cases, to that of taxis in hernia.

In eases of jaundice connected with the passage of gall-stones, the palliation of pain is the chief object of treatment. The occurrence of jaundice does not add indications to those which have been already considered as pertaining to the passage of gall-stones, or hepatic eolic. (Vide page 459.)

Jaundice dependent on irremediable causes, sucl as permanent occlusion of the biliary ducts or the pressure of a tumor, does not admit of cure. And the existence of an irremediable cause is to be inferred when the jaundice persists for several months or years. Yet, I have repeatedly met with cases in which, after the persistence of jaundice for many months, recovery at length took place. A fecal tumor in the transverse colon has been known to give rise to jaundice by pressing on the common duct. Such a tumor may be removed, but, in general, tumors so situated as to produce obstruction are persisting. In cases not admitting of cure the objects of treatment, as regards the jaundice, are, the elimination of bile by the renal and cutaneous emunctories, and the support of the powers of life in order to postpone, as long as possible, the fatal termination. With a view to the elimination of bile, the action of the kidneys may be excited by the vegetable diuretic remedies, and the functions of the skin maintained by the use of the warm bath and guarding against vicissitudes of temperature. The powers of life are to be supported by a nutritious diet, together with all the hygienic means, moral and physical, of invigorating the system.

In cases of jaundice ending in recovery, it is to be borne in mind that the yellowness of the skin continues for some time after the reabsorption of bile has ceased. The coloring matter, being without the vessels, remains when the cholæmia no longer exists. Of the continuance of the cholæmia, or of the condition which occasioned the jaundice, the urine affords the most reliable evidence. The affection is to be considered as

1 Ueber Icterus gastro-duodenalis, von C. Gerhardt, Leipzig, 1871. 
having ended when the coloring matter ceases to be apparent in the urine. I have known yellowness of the skin to persist for several months after the disappearance of bile pigment from the urine. In the case in which this was observed the janndiee had existed for a year. The absorption is probably slow in proportion as the jaundice has been of long cluration. According to Frerichs, the bile acids are not to be found in the urine in cases of jaundice. Harley, however, states that they are present as long as bile is reabsorbed, and that when the secretory function becomes much impaired in cases of persisting jaundice, these acids are replaced by leucine and tyrosine. ${ }^{1}$

\section{Functional Affections of the Liver.}

The functional affections of the liver relate to the eirculation of blood within the organ, and to its sceretory functions. 'The consideration of these affections will be brief. They have heretofore held a prominent place in pathological speculations, and they are still often mentioned by physicians in conversing with each other and with their patients. But the amount of our actual knowledge of hepatic disorders, exclusive of the affections already considered, is extremely limited. In a clinical point of view, with our present knowledge, the importance of the functional affections of the liver is in striking contrast with the frequency.with which the organ is referred to as the source of morbid phenomena.

Congestion of the.liver is incident to lesions of the heart involving impediment to the venous circulation. This impediment arises especially from contraction of the tricuspid orifice, which is a rare lesion. It follows contraction at the pulmonic orifice, and this is not a frequent lesion. Mitral lesions lead to it by inducing dilatation of the right side of the heart. It also arises from over-distension and dilatation of the right sicle of the heart as a result of emphysema of the lungs. The accumulation of blood within the liver, in certain cases of cardiac disease, occasions enlargement of the organ as determined by physical examination, and the size of the organ is sometimes observed to vary considerably at different times, owing to differences in the degree of congestion. 'The organ is found to be congested after death, the intra-lobular veins being especially conspicuous, and contributing not infrequently to the appearance characteristic of the so-called nutmeg liver.

It is difficult to say how far the hepatic congestion in these cases is important as interfering with the secretory functions of the liver. The obstruetion to the flow of blood in the hepatic veins induces portal congestion, and the latter is supposed to give rise to indigestion, loss of appetite, and other symptoms referable to the digestive system. Saline laxatives are considered as useful by relieving temporarily the portal congestion. The congestion due to carcliac lesions is chronic, and admits of only palliative treatment.

Congestion, transient or less persisting, and not produced mechanically, as in diseases of the heart, is attributed to over-ingestion of food, especially of a stimulating quality, alcoholic drinks, and the use of condiments which excite the stomach, such as pepper and mustard. The heat in tropical climates, and the special cause of periodical fever are supposed to give rise to hepatic congestion. A sense of weight in the right hypochondrium, impaired appetite and digestion, sometimes nausea and vomiting, a bitter taste in the month, pain in the head, and a sallow com- 
plexion, are symptoms often attributed to hepatic congestion. It is by no means certain that this pathological conclition is represented by the foregoing symptoms. Hepatic congestion from other than mechanical causes doubtless occurs, but the agencies producing it and its morbid effects are not nnderstood. The diagnosis, with our present knowledge, is not practicable, and the physician, therefore, in treating it acts upon the suspicion of its existence.

Hemorrhage into the liver is an event extremely infrequent, which may be due to hepatic congestion. In most cases, however, this event occurs in comection with hemorrhages in other situations, as in purpura and yellow fever. It has been observed in some cases of pernicious intermittent fever. It may be a result of an injury over the liver. There are no means of determining its occurrence during life.

Functional disorder's of the biliary secretion arc a morbid excess, a morbid deficiency, ancl a vitiation of the bile. A morbid excess of bile is supposed to enter into the etiology of diarrhea, colic, and sporadic cholera in certain cases. Deficiency of bile is often treated as a functional alfection under the name torpor of the liver, remedies being given to increase the biliary secretion. A whitish or ashy color of the stools is probably evidence of deficient secretion, although this appearance may arise from undue absorption of bile from the intestinal canal. Every practitioner is familiar with this appearance of the stools in young children, and after the ardministration of opium. The production of the so-called bilious stools by the use of melcury is well known. Torpor of the liver is a phrase often applied to cases in which the morbid condition is obscure. It affords an acceptable explanation to patients, owing to the popular notion that the liver plays an important part in the ailments referable to digestion and the nervous system; and, whatever may be the rationale, a mercurial cathartic or laxative often affords relief of the symptoms which are vaguely called bilious. A deficiency of precise knowledge respecting excess and defieiency of the secretion of bile, as functional disorclers, must be confessed, but as regards vitiation of bile, the deficiency of knowledge is still greater. Acrid bile is supposed to be sometimes seereted, and the greenish color of the stools, sometimes observed, especially in children, has been considered to denote a morbid quality of bile. It is not improbable that a vitiated bile may be secreted, but it is impossible to determine whether morbid changes are clue to a faulty secretion or to causes acting within the alimentary canal; and nothing can be more vague than to regard a vitiated secretion of bile as a functional disorder furnishing therapeutical indications.

The great discovery by Bernard of the production of sugar within the liver suggests the inquiry whether important disorders may not relate to this function. A deficient production of sugar may be among the proximate morbid effects of disorganizing hepatic lesions, and of functional inactivity of the organ due to anrmia and defective innervation. But of this we have, as yet, no positive knowledge. The question whether an excessive production of sugar be not involved in the affection known as diabetes mellitus will come up in treating of the pathological character of that affection.

\section{Affections of the spleen.}

The spleen may be the seat of inflammation, acute and chronic, and of various structural affections; it is liable to vascular engorgement and to extravasation of blood. Irrespective of engorgement, we have no knowl: edge of purely functional affections referable to this organ. 
Acute inflammation of the spleen, or splenitis, must be an extremely rare affection in this eountry: I have no practical knowledge of it. Cases, however, have been repeatedly observed, in which the appearances after death have afforded ample proof of its existence. Simple enlargement, softening, and congestion of the organ are not sufficient to establish the existence of inflammation. 'The post-mortem proof requires the presence of an inflammatory product, viz., lymph or pus.

When suppuration takes place, the pus may be infiltrated or an abscess may be formed, or the pus may be in small disseminated collections; in the latter case, pyæmia is generally associated, and purulent collections are found in other situations. In addition to the presence of inflammatory products, the organ is more or less enlarged, softened, and congested. Inflammation of the peritoneal investment of the organ is generally involved. A splenic abscess may be evacuated in different dircctions, like an hepatic abscess, viz., into the chest, stomach, peritoneum, or externally through the integument. 'The quantity of pus in some cases of splenic abscess is enormous.

The symptoms which have been observed are, pain in the left hypochondrium and in the epigastrium, tenderness on pressure over the organ, more or less febrile movement, with anorexia, thirst, nausea, and vomiting.

The diagnosis is to be based upon these symptoms, together with the evidence of enlargement afforded by palpation and percussion, and, in some cases, the appearance of an abscess under the integument, or the discharge of pus by vomiting, stool, or expectoration, the source of the pus being traced to the spleen. Exclusive of the cases in which the existence of splenic abscess is ascertained, the diagnosis is confessedly difficult, and hence there is ground for distrusting the accuracy of the diagnosis in cases of supposed splenitis ending in recovery. The discrimination of splenic abscess from suppuration in the neighborhood of the spleen is not easy.

Owing to the uncertainty of the diagnosis, it would be difficult to collect trustworthy data for determining the proportion of cases in which the termination is fatal. If suppuration take place, the prognosis is unfavorable.

The general principles of treatment are the same as in acute inflammation affecting other parenchymatous structures, for example the liver. In the management of splenic abscess tending to open through the integument, the same precautions are to be observed as in cases of hepatic abscess.

Chronic splenitis is supposed to exist in certain cases of enlargement of the spleen. The existence, however, of inflammation in a chronic form is doubtful, if, after death, inflammatory products be wanting; and it is not easy to distinguish, during life, between inflammatory and non-inflammatory enlargement of this organ. More or less enlargement occurs as a concomitant or sequel of intermittent fever, and will be noticed in treating of the latter. This enlargement is commonly known in districts in which intermittent fever prevails as the ague cake. Enlargement has been already noticed as occurring in a small proportion of cases of cirrhosis of the liver. The increase of size in some of these cases is very great, and the spleen may become considerably larger than the contracted liver. Notable enlargement belongs to the history of leucocythemia. More or less enlargement and softening occur in typhoid fever and other affections. The organ sometimes is found greatly enlarged without any apparent connection with other affections. The enlargement may be of short duration, and it is then probably due mainly, or exclusively, to an accumulation of blood or engorgement. In general, when the enlargement is more or less persisting, an examination after death shows no 
material structural change, and, hence, the organ is to be considered as hypertrophied.

Chronic enlargement of the spleen is rarely attended with much pain or tenderness on pressure. If not greatly enlarged, there may be no local symptoms directing attention to an aflection of the organ. If much cnlarged, its weight occasions a sense of uneasiness and tension in the left hypochondrium. As alrealy stated, in treating of hydro-peritoneum, there is no ground for supposing that enlargement of the spleen alone ever gives rise to peritoneal dropsy. Disconnected from the pathological associations under which it is generally presented, it may exist without any notable general symptoms.

Splenic enlargement is gencrally ascertained without difficulty by palpation and percussion. In some cases the enlarged organ extends upward, pushing up the diaphragm, and causing an evident projection of the false ribs of the left side of the chest. Its upper border is accurately indicated by the line of demarcation between pulmonary resonance and flatness on percussion, and the lower borter is determined, approximately, by finding the upper limits of tympanitic resonance. In other cases the organ is dragged downward by its weight, and it may descencl as low as the brim of the pelvis. Under these cireumstances, if the abdominal walls be thin and relaxed, the organ may be grasped by the hand, and restored to its normal position. The form will serve to distingnish it from the other abdominal tumors. The hilus may sometimes befelt through the abdominal walls.

The treatment of enlargement due to either engorgement or hypertrophy, in the vast majority of cases, has reference to associated affections, viz., intermittent fever, lencocythemia, cirrhosis. In the cases met with in practice, the most frequent association by far is with intermittent fever, and the therapentical measures indicated will be considered in treating of the latter disease. The treatment in the rare cases in which the enlargement has no apparent pathological relations, must have reference to the symptoms and general condition in individual cases. There is no special course of medication to be pursued.

Extravasation sometimes occurs in connection with vascular engorgement of the spleen, and the hemorrhage may lead to rupture of the capsule and the escape of blood into the peritoneal cavity. 'This has been observed in cases of intermittent fever, of typhoid fever, and during an epileptic paroxysm. It occurs, also, in connection with pregnancy, both before and after labor. Death may take place immediately, from the loss of blood, and, in most of the cases which have been observed, the duration of life, after the rupture, has not cxceeded twenty-four hours. The symptoms rescmble those which follow intestinal perforation, and the differential diagnosis cannot be made with positiveness. Were it practicable to recognize the accident by the symptoms, the treatment would be the same as in cases of perforation, viz., opium in full doses, with perfect quietude of body. It would be proper to apply cold over the abdomen. Nothing is to be expected from treatment beyond palliation.

Most of the structural changes which have been considered in treating of the affections of the liver are occasionally found in the splecn. It is somctimes indurated, resembling, in resistance, the kidney, and microscopical examination shows a fibrinous deposit. This deposit, limited to. portions of the organ at its surtace, is not uncommon. The waxy or lardaceous deposit takes place in this organ, connected with a similar change in the liver, or with pulmonary tuberculosis. The whole of the spleen is rarely affected, the deposit being limited to portions of the organ, and 
hence the organ is not much enlarged. In cases of acute tuberculosis, miliary granulations are frequently tound in greater or less abundance in this organ, more especially in children. The yellow tubercle is sometimes found here. Cancer of the spleen is exceedingly rare, and is always seconclary. Hydatids have been observed in this organ, but their oceurrence here is very infrequent. The organ may be simply atrophied, and is sometimes extremely wasted. 'These structural changes do not claim consideration as individual affections. Anatomical changes due to obstruction of arterial branches (infarctus), are of frequent occurrenee in the spleen. These have been referred to in treating of embolism (vide Part I, Chap. V.). Emboli, taking their point of departure from the left side of the heart, are apt to become fixed in the splenic arteries. They occasion congestion or extravasation within the area which the obstructed vessel supplies with blood. 'This area is generally wedge-shaped and varies in size from that of a pea to an English walnut. Subsequent processes are, first, fatty degeneration and absorption, leaving a cicatricial contraction; second, in some cases, a cheesy mass which may either remain indefinitely, or undergo calcification; and, third, the result may be a puriform collection which contains more or less leucocytes.

Our present knowledge does not enable us to understand fully the consequences of the impairment or loss of the functions of the spleen. It is well known that this organ may be removed from inferior animals without the destruction of life or serious injury to health. This fact goes to show that whatever may be its functions they are adequately performed by other organs in its absence. There is reason to believe that this statement is applicable to man as well as to inferior animals. A case of much interest, as bearing on this point, has been communicated to me by a former pupil, Dr. John L. Alston, of Texas, who, during the late civil war, served in the army of the rebellion. 'The following account of the case is quoted from a letter written at Cairo, Illinois, where he was detained as a prisoner of war. "I will give you the history of" a very interesting case which came under my treatment after the battle of Perrysville. Wm. H. Worden, aged abont eighteen years, in fine health, was wounded on the 8th of Oct. 1861. The ball entered the integument one inch to the left of the spinous process of the fourth lumbar vertebra, and pursued a diagonal course upward, coming out between the ninth and tenth rib half-way between the sternum and spine. The ball must have entered the cavity of the abdomen, for at the orifice where it made its exit nearly the whole of the spleen protruded. The organ was not wounded, and it had a fresh and florid appearance as if it were not much strangulated. The protruding portion measured three and a half inches in length and two and a half inches in width. He came under my observation on the third day after the wound was received. My friend Surgeon Rice, of Gen. Cheatham's staff, agreed with me that the protruding substance was the spleen. I immediately threw a strong ligature around it, and each day tightened the ligature. It dropped off on the fourth day. The stump which remained plingged up the bullet-hole. No suppuration ensued, and he got well without a bad symptom. On the day after the ligature came off he was sitting up, and was walking about in five or six days afterward. He said that he had lost a great deal of blood when he received the wound. When he left the hospital he was entirely well, not seeming to suffer at all from the loss of his spleen."

I It would appear from Dr. Alston's account that about two-thirds of the organ was removed. 
It would be interesting to know whether the effects followed in this case which are sometimes observed after the removal of the spleen from the dog or cat, viz., a ravenous appetite and ferocity of disposition.

Since the first edition of this work was written, the spleen has been removed for great enlargement, by Spencer Wells, of London. 'The organ weighed, after nine ounees of blood had drained out of it, $5 \mathrm{lbs} .11$ ounces. 'The patient, a married woman, aged 34 years, survived the operation a little over six days. The annals of medical literature contain three additional cases in which this organ has been removed for enlargement. 'The first case was in Naples, in 1549 ; the operation was performed by a surgeon named Zaccarelli. The patient recovered. The authenticity of this case is perhaps open to distrust. The second case was in Germany, in 1826. The name of the operator was Quittenbaum. The organ weighed nine pounds. Death followed in six hours. The third case was also in Germany, in 1855; the name of the operator, Kückler; the weight of the organ was three pounds, and death followed in two hours. The result in all these cases certainly does not encourage a repetition of the operation, assuming it to be warrantable on pathological grounds, and the latter is by no means certain.'

Since the publication of the second edition of this work, four additional cases of spleenotomy have been reporter. In one of these cases the operation was entered upon with the expectation of finding an ovarian tumor. A large cyst was attached to the organ. A speedy recovery followed. The operator was M. Péan, and the case was reported for l'Union Medicale, Nov. 1867. The operation in the second ease was performed by M. E. Koeberle, and is reported in the Gazetle Hebdomadaire, October and November, 1867 . The patient was a woman aged 42 . The patient did not recover consciousness after the operation, and died evidently from hemorrhage. In the third case, the patient was a groom, aged 20 . The operation was performed by 'Thomas Bryant, and the case is reported in Guy's Hospital Reports, vol. xii., Third Series. 'The operation was begun at 2.30 P.x., and at 4.50 P.M. the patient died. The operation in the fourth case was performed by Thomas Bryant, and the report of the case is in Guy's Hospital Reports, vol. xiii., 'Third Series. The patient was a female aged 40. Death took place from hemorrhage, within a few minutes after the completion of the operation. ${ }^{2}$ M. Koeberle, in connection with the report of this case, cites two eases in addition to those which have been referred to, the operation being successful in each of the cases. In one case the spleen escaped through a wound from a sword. The patient six years afterward was in perfect health. Tliis was in 1678 . In the remaining case the spleen made a hernia through an opening of an abseess below the umbilicus. The patient lived five years after the removal. In the two last cases, it will be observed, there was no hypertrophy. It is to be hoped that there will be no further additions to the list of cases of removal of the spleen for enlargement.

\section{Affections of the Pancreas.}

Owing to their infrequency, and their obscurity as regards diagnosis, affections of the pancreas require, in this work, but a passing notice. This gland may be the seat of inflammation, acute or chronic. Of all the glan-

: Vide article by Samuel Wilks, in Guy's Iospital Reports, vol. xi., 1865 : also, the London Med. Times and Gazette, Jan. 1866, and the New York Med. Journal, May, 1866.

2 For a full account of these four cases, vide New York Med. Journal, June, 1868. 
dular organs-liver, ovaries, testes, kidneys, etc.-the pancreas is perhaps the least liable to become inflamed. Acute pancreatitis has been found, on examination after death, to occur in cases of continued fever, of puerperal fever, of pyæmia, and apparently as a result of the employment of mercury. The morbid appearances denoting acute inflammation are engorgement, softening, enlargement, and suppuration. Gangrene has been observed. 'The symptoms which have been observed are, pain referable to the epigastrium, vomiting, diarrhœa, chills, and more or less febrile movement. The data for determining the clinical history of the affection are insufficient: A discharge, by vomiting and stool, of a liquid resembling saliva, supposed to be the pancreatic secretion, has been thought to be a diagnostic symptom, but there is no reliable evidence of the correctness of this opinion. It remains to be ascertained whether the presence of fat in the stools is a diagnostic symptom. 'The diagnosis, with our present knowledge, is impracticable. Were it practicable to ascertain the existence of the affection before death, the indications for treatment would be the same as in other parenchymatous inflammations. An abscess of the pancreas has been known to open into the stomach. Chronic pancreatitis is, if possible, even more olsscure, as regards diagnostic symptoms, than the acute form of the disease. In a case observed by Wilks, death followed extreme emaciation without any symptoms pointing to the seat of the disease. ${ }^{1}$ From what has been stated with regard to the diagnosis, it follows that it is impossible to form an opinion as to the proportion of cases in which either acute or chronic pancreatitis ends in recovery.

The pancreas is sometimes the seat of cancer, which is generally secon. dary to cancerous deposits in adjacent parts. According to Wilks, in a certain proportion of the cases regarded as cases of scirrhus, the affection is chronic inflammation of the areolar tissue which enters into the composition of this organ, constituting an affection analogous to cirrhosis of the liver, and the two affections are apt to be associated. Enlargement of the head of the pancreas from chronic inflammation, cancerous dis. ease, or the formation of cysts, may constitute a tumor discoverable by manual exploration. The diagnosis involves its discrimination from other tumors in the same situation. It is most likely to be confounded with aneurism of the aorta and cancer of the pylorus. A pulsation may be communicated to the tumor from the aorta, and this will suggest the idea of aneurism. The diagnostic symptoms and signs of aneurism of the abdominal aorta are to be sought for, and this affection excluded by their absence. ${ }^{2}$ Cancer of the pylorus is to be excluded by the absence of the gastric symptoms which usually accompany this affection. ${ }^{3}$ The connection of structural disease of the pancreas and fatty diarrhøea has been considered in treating of the latter. The presence of free fat in the alvine dejections, taken in connection with a tumor supposed to be an enlarged pancreas, is undoubtedly a point of much weight in the diagnosis. The absence of fatty dejections, under these circumstances, is not proof against, but their existence is strong evidence for, the supposition that the tumor is pancreatic.

Enlargement of the head of the pancreas may give rise to serious results from pressure upon adjacent parts. The duct leading from the pancreas is sometimes compressed, and dilatation of its branches within the organ ensues. Pressure upon the ductus communis, involving obstruction to the flow of bile, is followed by the retention of this secretion, 
causing persistent jaundice, dilatation of the bile-ducts, and, at leugth, disorganization of the liver. Wilks states that in the cases of jaundice associated with enlargement of the head of the pancreas which have come under his observation, the obstruction and retention of bile have been due, not to the pressure of the pancreatic tumor on the common duct, but to associated disease of Glisson's capsule or cirrhosis of the liver. An enlarged pancreas may cause obstruction of the pylorus or duodenum, and this renders it difficult to differentiate the affection from cancer of the pylorus. Pressure upon the portal vein may exist to an extent to give rise to hydro-peritoneum.

Finally, calculi sometimes form within the pancreatic ducts, varying in size from that of a pea to an almond. These have been found in considerable number. In their journey along the exeretory passage to the duodenum, they may occasion severe pain, resembling that caused by biliary calculi. Paroxysms of pain due to the passage of pancreatic calculi, or attacks of pancreatic colic, are doubtless sometimes attributed to gallstones, nor is it practicable, with our present knowledge, to make the differential diagnosis. The differential diagnosis is not, practically, very important, since the indications for treatment are the same in either case. Clinical observation with reference to the presence of free fat in the dejections, may show this symptom to be present in cases in which, without special attention, it is overlooked, and it may be found that this symptom is of much valuc in the diagnosis of atfections of the pancreas. 


\title{
SECTION FOURTH.
}

\author{
DISEASES AFFECTING THE NERVOUS SYSTEM.
}

\section{CHA P T ER I.}

Preliminary Remarks. Congestion of the Brain, Active and Passive. Cerebral Anæmia.Apoplexy. Cerebral Hemorlliage and otliel Anatomical Conditions. Clinical History-Pathological Character-Causation-Diagnosis - Prognosis-Treatment. Insolation, or Sunstroke. Congestion and Anæmia of the Spinal Cord. Hemorrliage within the Spinal Canal.

THE parts composing the nervous system are, first, the brain and spinal cord, which, with the nerves therewith directly connected, form the cerebro-spinal system; and, second, a series of ganglia situated within the skull, and on each side of the spinal column, which, together with nerves distributed to the viscera and bloodvessels, constitute the division called the sympathetic or ganglionic system. Of the morbid conditions which relate specially to the latter, our positive knowledge is comparatively incomplete. The diseases affecting the nervous system, therefore, will be found to relate chiefly to the cerebro-spinal system. 'This system is divided into two parts, namely, 1st, the brain and spinal cord, distinguished as the central or centric portion, or the cerebro-spinal axis, and $2 d$, the nerves which are connected with the brain and spinal cord, the latter constituting the peripheral or eccentric portion of the cerebro-spinal system. The periphery of the nervous system, it will be observed, embraces, not merely the terminal branches of nerves, but the latter from their attachment to either the brain or spinal cord.

Physiological researches, within the last few years, have shown that the quantity of blood contained in different organs of the body, the functional activity of glands, the temperature of parts, spasm and paralysis of involuntary muscles, and the nutrition of different structures, are affected by influences communicated by means of the sympathetic system. 'These physiological relations involve corresponding pathological influences; and, at the present moment, many pathologists are disposed to attribute to the agency of the sympathetic and the reflex system a large share of morbicl conditions, and the phenomena of disease. Especially as regards reflex influences affecting the circulation of parts by means of the vasur-motor nerves, some pathologists consider that the sympathetic system plays an important part in the causation of many diseases. The discovery by Bernard, that section of the sympathetic nerve in the neck is followed by increase of blood and augmented temperature on the corresponding side of the head, and the fact subsequently observed by Brown-Séquard, that these effects are removed or diminished by transmitting the galvanic current through the divided nerve, seem to have been the point of departure for attributing many local processes of disease to either spasm or paralysis of the muscular coat of the smaller arteries, by means of reflex influences exerted through the vaso-motor nerves. Without entering into a discussion of this patho- 
logical doctrine, which would be here out of place, it is proper to state that it rests, as yet, mainly on conjecture. 'That spasm of the arteries contained in internal organs is an element of the diseases affecting them, has not been demonstrated. That this condition oecurs and persists sufficiently long to interfere materially with nutrition is not very probable. That, on the other hand, dilatation of these arteries leads to inflammation, hemorrlage, and degenerations of structure, is yet to be established; and the fact that the aceumulation of blood in parts open to inspection, consequent on division of the cervieal sympathetic nerve, is followed by neither transudation, exudation, nor notable impairment of nutrition, must eertainly be considered as rendering it highly probable that these morbid effects do not ensue from the same condition in internal parts. Moreover, it is not certain that the congestion of the vessels on the side of the head, consequent on division of the eervical sympathetic nerve, is due to paralysis affecting the vaso-motor nerves. Some physiologists consider the congestion as dependent on a prior effect in the strnctures involved. 'The doctrine of spasm and paralysis affecting the vaso-motor nerves thus resting on conjecture, it is needless to state that, to attribute to certain medicines or therapentical measures a power of inereasing or diminishing the quantity of blood in internal parts by exciting contraction or dilatation of the smaller arteries, is to indulge hypothetic views for which our existing knowledge affords no solid support.

Our knowledge of the susceptibility of the nervous system to morbid influences derived from an abnormal composition of the blood, and especially of the effects of toxical principles in the blood, has been much increased by late researches. Familiar illustrations are aflorded by the phenomena caused by the inbalation of anæsthetic vapors and gases, and by the introduction into the blood of minute quantities of certain poisons, such as strychnia, aconite, woorara, etc. The effects of the accumulation of urea in the bloor, now known to be frequent and vastly important, have been ascertained within late years; and it is probable that here is a field of pathologieal inquiry in which future researches will develop results of great value as regards the etiology of nervous diseases.

Of the diseases affecting the nervous system, the greater part relate to the nervous centres-the brain and spinal eord. Some important affections, however, are seated in the nervous trunks or their branches, that is, in the peripheral portion of the cerebro-spinal system. I shall consider, first, the diseases affecting the brain, or more properly, the encephalon; for the relations of the membranes within the skull to the brain are such, that their affections are to be treated of in this connection. Indeed, some of the most important encephalic diseases have their point of departure in the membranes. 'This statement applies equally to affections within the spinal canal. The brain and its membranes are subject to morbid conditions essentially the same as in diseases of other parts of the body, viz., hyperæmia or congestion, inflammation, hemorrhage, and various structural lesions. But there are affections of the nervous system which are peeuliar to this system, distinguished as the neuroses. These will be considered after having treated of the affections which, as regards pathological character, are common to this and other of the anatomical systems. Morbid eonditions pertaining to the circulation are of special importance in relation to the nervous centres. Cerebral congestion is to be reckoned among the affections of the brain, and its consideration will prepare the way for treating of other affections. After having considered congestion, a morbid defieiency of arterial blood within the skull will claim a brief notice. Hemorrhage occurring within the skull, eonstituting the most 
frequent morbid condition in apoplexy, will be considered in that connection, and will be also noticed as one of the morbid conditions giving rise to the form of paralysis known as hemiplegia.

\section{Congestion of the Brain.}

Certain peculiarities pertaining to the circulation within the craninm are of importance as favoring the occurrence of hyperæmia or congestion. A large proportion of blood goes to the head ; it has been estimated that onesixth of the whole mass is contained in this portion of the organism. The greater part of the intra-cranial blood is within the vessels of the membranes, including the reservoirs for venous blood, which are peculiar to this situation, called the sinuses. The brain is subject to intense functional activity in emotional and intellectual acts which increase the determination of blood to the head, in conformity with the general law that the demand for blood in parts is proportionate to the intensity of the exercise of their functions. 'The llood within the skull, after the fontanelles become ossified, is removed from direct atmospheric pressure. Hence, it has been inferred, a suction-force must keep the intra-cranial vessels full of blood. An inference to be drawn from the operation of this suction-force is, that, if blood be deficient in one class of vessels, it accumulates proportionately in another class. If the amomt of blood sent to the brain by the arteries be diminished, enough is detained in the veins and sinuses to make up the deficiency. Venous congestion, therefore, is necessarily incident to a defective supply of arterial blood.

Another physical point which has been assumed is, that the substance of the brain is incompressible by any amonnt of force which the heart can exert through the arteries, and, hence, that the blood within the skull can never exceed a certain amount. Taking this in connection with the absence of atmospheric pressure, the conclusion has been drawn that the amount of blood within the cranium can neither be increased nor diminished. In other words, assuming that the contents, exclusive of the blood, remain the same, that is, neither increased nor diminished, the blood which comes from, camnot exceed the quantity which enters within, the cranium; or, reversing the statement, the quantity which enters must be the same as the quantity which passes out. Limiting the attention, as some have done, to the blood and the solid structures within the skull, according to the doctrine just stated, the blood can only be diminished by a morbid increase of the solids, and can be increased only by a diminution of the space which the solids occupy in health. The cephalo-rachidian liquid is, however, to be taken into account with reference to the intra-cranial circulation. It is probable that an increase of the quantity of blood is immediately followed by a diminution of this liquid; and, on the other hand, this liquid is increased in proportion as the quantity of blood in the intra-cranial vessels is diminished.

That the blood within the cranium may be morbidly increased is abundantly shown by direct observation. In some cases in which death is attributable to cercbral congestion, the quantity of blood contained within the cranium is notably greater than is ever found under other circumstances. Assuming the brain-substance to be incompressible by any force which can be exerted by the heart, there is a force pertaining to the circulation which, in this connection, seems to have been overlooked, viz., a force acting directly upon the blood in the capillaries, which is increased in active congestion and inflammation. It is difficult to estimate the extent to which this force may be morbidly increased, but, in conjunction with the force 
of the heart's action, it is sufficient to overcome the resistance of the brain to compression. On the other hand, the experiments of Durham, Wm. A. Hammond, Donders, and others, in which portions of the skull in inferior animals were removed, have shown that the amount of blood within the skull is diminished during sleep. It has been supposed that the liquid contained within the cavity formed by the sheath surrounding the small vessels of the brain, pia mater, and spinal cord, may, by its variations according to the quantity of blood which the vessels contain, afford, to a certain extent, a protection against the morbid effects of their undue distension. Niemeyer reckons among the causes, obstruction to the flow of blood in branches of the aorta which are distributed to other parts than the head, or any interference with the distribution of the arterial blood in other organs. Under these circunstances, there is an undue determination of blood to the head, constituting what is termed by Virehow "collateral fluxion."

It may, perhaps, be rationally conjectured that paralysis affecting the vaso-motor nerves within the craninm belongs among the conditions which are causative of congestion. It may be suspected that in this way strong mental emotions occasion hyperamia; and, according to views held by some, when congestion is an effect of insolation, alcoholism, or other causes supposed to act by a local irritation, it is an effect of dilatation of the vessels from paralysis affecting the vaso-motor nerves. According to recent views which attribute much importance to variations in the size of the arteries, as affected through the vaso-motor nerves, an increase of the blood within the skull, as in other parts of the body, may be eaused by paralysis of the muscular fibres; and, on the other hand, diminution of the blood within the skull may be an effect of spasmodic contraction of these fibres.

Congestion of the brain, as of other parts, may be active or passive. In active congestion there is an undue determination of blood; that is, more blood enters the part through the distributing arteries than can be readily returned from the part by the veins. Physiological types of this pathological condition are blushing and the erection of the penis. In passive congestion, on the other hand, the blood accumulates in a part purely in consequence of some obstacle to its return by the veins, and the quantity of blood sent to the part may not be greater, but even less than in health.

Active congestion of the brain, in a degree to constitute an affection more or less grave, sometimes giving rise to sudden or apoplectic coma, may be connected with simple hypertrophy of the left ventricle of the beart. A striking case of sudden death, the only pathological conditions found being intense cerebral congestion and simple hypertrophy of the heart, has been given in another section.' Cases of this kind are extremely infrequent, for simple hypertrophy of the heart in a marked degree is a very rare affection. The occurrence of congestion sufficient to produce sudden death (congestive apoplexy), under these circumstances, may be determined by co-operating causes which increase the action of the heart, such as exercise or mental excitement, together with causes which, at the same time, imperle the return of blood to the heart, such as over-distension of the stomach. In the case just referred to, the sudden attack took place during exercise directly after a meal. In most cases of hypertrophy of the left ventricle, there exists either a mitral regurgitation or aortic insufficiency, which are compensatory as regards a liability to active ccrebral congestion. 
Insolation is another cause of active congestion. In certain cases of sunstroke, an excessive accumulation of blood within the head is found after death. Insolation, or sunstroke, will be hereafter considered as an individual affection.

Excessive mental or emotional activity, as in a fit of anger, is a cause of active congestion. Another cause is alcoholic stimulation. Active congestion is a pathological element in the maniacal delirium which is sometimes a direet effect of excessive indulgence in wine or spirits (delirium ebriosum), whereas the affection known as delirium tremens is an indirect effect, occurring usually when, from any cause, the accustomed stimulation is withdrawn or diminished.

Of the causes of active congestion of the brain, the foregoing are the more obvious. But congestion sufficient to produce speedy death may occur without any obvious cause. Of this fact I shall cite an example under the liead of congestive apoplexy.

The symptoms denoting active congestion of the brain of considerable intensity, but falling short of a degree sufficient to produce apoplexy or sudden coma, are as follows: Pain in the head, varying in degree, sometimes intense, with a sense of heat, fulness, bursting or weight. The pain is not limited to one side, nor to any particular part of the head. There is inability to sleep, or, if sleep be obtained, it is incomplete and nnrefreshing. Delirium sometimes occurs, and, if present, is active. This symptom occurs chiefly in cases in which the congestion is due to excessive alcoholic stimulation. Convulsions occasionally occur in the adult; they are less infrequent in infancy, but eongestion, as a primary affection, is not frequent in infantile life. The countenance is usually, but not invariably, flushed, the eyes are suffused or injected, with intolerance of light and sometimes scintillations. The head is hot; the carotids and temporal arteries pulsate strongly, and the patient is conscions of a throbbing sensation in the head. Inability to control mental operations, mental confusion, and momentary loss of consciousness are occasional symptoms ; also vertigo, tinnitus aurium, and some embarrassment in speeeh. Transient hemiplegia sometimes occurs in cases of active congestion, which may be explained by supposing the congestion, or odema dependent thereon, to be greater in one of the hemispheres. With the ophthalmoscope the vessels of the retina appear to be increased in number; they are enlarged and more tortuous than in health, and the optic disk may be more or less congested. ${ }^{1}$

The symptoms just enumerated point very distinctly to the existence of active congestion of the brain. The symptoms are those of the first stage of acute inflammation of the meninges of the brain, and the pathological condition is, in fact, the same. This discrimination cannot at once be made with positiveness, but the differential diaguosis is soon settled by the course of events. Practically, the discrimination is not of great importance. Reference is now had to congestion existing in considerable intensity. It may exist in a moderate or slight degree. The symptoms will be more or less marked in proportion as the congestion is greater or less in degree.

Simple congestion is merely a functional affection. It involves no lesion; if the congestion be removed, the structures are left intact. In a slight or moderate degree, it involves no immediate danger. As already stated, however, it may be developed rapidly in a sufficient degree to pro-

1 Vide Treatise on Discases of the Nervous System, by William A. Hammond, M.D., etc., 1871. 
duce apoplectic coma and sudden deatl. In a lesser degree of intensity, it may prove serious by eventuating in other pathological conditions, viz., extravasation of blood, serous effusion, and perhaps inflammation.

Intense active congestion calls for bloodletting. Relief is obtained most promptly by this measure, and promptness of relief may be of vast importance in preventing apoplectic coma or the pathological conditions just named. If the symptoms do not denote a degree of congestion sufficient to call for so prompt and efficient a measure as bloodletting, relief may be obtained by ice to the head, stimulating, hot pediluvia, and an active purgative. The bromide of potassium, of ammonium, of sodium, or of caleium, are remedies of power in cases of active congestion, the rationale of their operation probably being that they rliminish the amount of blood within the cranium. 'They may be employed, as substitutes for bloodletting, when it does not seem to be important to resort to the latter. The constant electrical current, employed so as to act upon the sympathetic nerve, appears to induce contraction of the bloodvessels within the skull.'

These measures are for the removal or diminution of the existing congestion. The treatment, in addition, should embrace, if possible, the removal of the causes; and, if the patient be of a full habit or plethoric, regulation of diet, and oceasional saline laxatives are advisable.

Passive congestion of the brain differs materially from the form just considered. Mechanical pressure from over-distended vessels exists alike in both; but there is superadded, in active congestion, the excitation of an overplus of arterial blood, and in passive congestion, the sedation of an excess of venous bloor. This difference accounts for a marked disparity in the symptoms. In passive congestion there is no increased determination of blood to the head, but the blood accumulates from detention. Of this form of congestion strangulation is the type.

In a degree to constitute a morbid condition, passive congestion may be produced by any meehanical impediment to the return of blood from the head. This may be due to pressure on the jugular veins in cases of goitre or enlargement of the lymphatic glands of the neck, and by the pressure of an aortic aneurism, or otler tumors, on the vena cava. Prolonged efforts of expiration, as in some forms of spasmodic congh, and in playing on wind instruments, have this effect. Diseases of the lungs occasioning interference with the pulmonary circulation, namely, pleuritis with large effusion and emphysema, give rise to passive congestion by leading to overloading of the right cavities of the heart. Over-distension of the stomach by the excessive ingestion of food or drink, and by the disengagement of gas, is another cause. It is incident to certain diseases of the heart, especially to obstruction at the right side of the heart from valvular lesions on that side, but more frequently from dilatation of the right ventricle and auricle, together with tricuspid insufficiency, as a result of mitral lesions. Obstruction or obliteration of the sinuses by coagula induces passive congestion in the communicating veins. Diminished arterial circulation, that is, weakness of the vis a tergo, is also a cause. The latter involves the suction-force peculiar to the circulation within the cranium. If this force tend to keep the encephalon filled with blood when the arterial supply is lessened, the venous blood is proportionally increased, and, hence, venous congestion. Passive congestion from this cause is supposed to exist in cases of various affections in which, 
in connection with feebleness of the circulation, head-symptoms become developed, more especially in diseases of children accompanied with exhaustion, as in certain cases of so-called cholera infantum. The occurrence of passive congestion, under these circumstances, in children especially, may be owing to the fact that the brain in early life has less functional activity, and the determination of arterial blood is consequently less than in after years.

The symptoms denoting passive congestion are, drowsiness, dulness of mind, blunted perceptions, and, in children, sometimes convulsions. Throbbing of the carotids and temporal arteries, active delirium, increased heat, and other symptoms denoting active congestion, are wanting. These symptoms are due, in part, to the pressure of the distended veins, and, in part, to the excess of venous, or the deficiency of arterial, blood. Passive as well as active congestion may lead to extravasation of blood or to serous effusion.

Passive congestion of the brain is to be considered as, not an individual affection, but a morbid element entering into various affections. When dependent on a mechanical impediment to the return of blood from the head, caused by cardiac lesions, tnmor of the neck, etc., the object of treatment is, if possible, to diminish the obstruction. It can do no good to diminish the mass of blood by bloodletting. It is doubtful if aught is to be effected by revulsive measures. Anything which weakens the force of the circulation tends to increase the congestion. If dependent on feebleness of the circulation, the therapentical indications are, to remove, if possible, the canses of exhaustion, and to increase the force of the circulation by tonics, stimulants, and nutritious diet.

In the foregoing account of cerebral congestion reference has been had to this condition as general, that is, affecting the entire encephalon, not limited to certain portions of the brain. Hyperæmia, more or less circrimscribed, occurs in connection with tumors which may produce obstruction of vessels by pressure, or cause a determination of blood within a limited area by irritation. Of these two modes, the former gives rise to passive, the latter to active, congestion. Passive congestion limited, more or less, in extent, is a result of thrombosis of veins, and, also, of both thrombosis and embolism of arteries.

The morbid appearances, after death, in case of cerebral congestion, are more or less distension of the vessels of the meninges, flattening of the convolutions in consequence of the turgescence of the brain, and red points disseminated in greater or less abundance over the cut surfaces of the cerebral substance. The latter appearance is caused by the open orifices of minute vessels abnormally distended. Microseopical measurement shows these vessels to be dilated sometimes to double their normal size. Serous transudation may take place into the pia mater and the substance of the brain, but not into either the ventricles or the subarachnoid space. It is to be borne in mind that, exclusive of congestion as a disease, or an element of disease, there is considerable variation in the fulness of the intra-cranial vessels, more especially those of the meninges. Differences, in this respect may be dne to physical conditions connected with the last hours of life, not having any direct pathological connection with the fatal disease. It is very common, in estimating morbid appearances within the skull, to err in considering as a morbid condition a degree of congestion not abnormal, or, if so, incident to the act of dying. To differentiate the latter is not always easy, without taking into account the phenomena during life. 


\section{A Morbid Deficiency of Arterial Blood within the Skull, or Cerebral Anmmia.}

The quantity of blood sent to the brain is diminished whenever the mass of blood in the body undergoes diminution, as after the loss of blood by hemorrhages, and in all diseases in which the constituents of the blood are expendel without a proportionate renewal by means of alimentation. An over-accumulation of blood in other organs than the brain has the same effect. Mitral obstruction or valvular lesions of the heart involving regurgitation also oecasion a defective supply of blood to the brain. An inerease of the subarachnoidean liquid, and œdema of the brain, which m:ly be effects of venous congestion, must diminish the amount of arterial bloorl by pressure on the cerebral arteries. It is conjectured that an obstacle to the passage of blood in the distributing arteries may arise from their spasmodic contraction exeited by influenees exerted through the vaso-motor nerves; ant that certain mental emotions which are accompanied by notable pallor of the face and a tendeney to syncope, may produce this effect.

A deficieney of arterial blood in either the whole or a part of the brain is an important pathological element in certain affections of the nervous system which are to be presently considered, the deficiency arising from plugging of a cerebral artery (embolism and thrombosis), the pressure of a clot, of the products of inflammation, namely, lymph, serum, and pus, and of tumors of different kinds. Inasmuch as the red globules are the constituents of the blood on which depends the normal eondition of the nervous centres, a paucity of these, as regards morbid-effects, is equivalent to a diminished supply of arterial blood to the brain. Cerebral anamia, therefore, is produced by the various causes which lead to impoverishment of the blood. Thus produeed, it is an important element in many affections. Ceplialalgia, mental depression, hysteria, and the phenomena to be presently notieed under the name nervous asthenia, are often attributable to a deficient supply to the brain of fully oxygenated blood, in consequence of its impoverishment, or, in other words, a morbid deficiency of the carriers of oxygen, namely, the red globules. Impoverishment of the blood, together with feebleness of the circulation, resulting from an abundant intestinal transudation, occasions, in children, that condition of semi-coma described by Marshall Hall under the name "hydrencephaloid affection," a condition involving, with deficiency of arterial blood, or of red globules, venous congestion.

The immediate effeet of a sudden and considerable diminution of arterial blood in the brain is syncope. Consciousness is lost, the face presents a death-like pallor, and slight convulsions may occur. This effeet is witnessed in cases of abundant hemorrhage, or after a copious venesection; and it may be produced by feebleness of the heart's action without any loss of blood. The phenomena of syncope are due to a sudden deficiency of arterial blood in the brain. It is especially apt to occur when the mass of blood in the body is deficient; and impoverishment of the blood also predisposes to it, inasmuch as the efliciency of blood in maintaining the functions of the nervous system depends on a normal quantity of the red globules. Coma and paralysis in cases of cerebral hemorrhage, embolism, ete., are attributable, in part at least, to an interruption of the free circulation of arterial blood in the substance of the brain. The interruption of the supply of blood to a portion of the brain in cases of embolism and thrombosis of the cerebral arteries leads to softening in consequence of a suspension of nutrition in the parts thus deprived of nutritive material. 
The treatment, in cases of syncopc, embraces measures to increase the quantity of oxygenated blood sent to the head. Recumbency, to secure the effect of gravitation, is important. Internal stimulants, to increase the force of the heart's action, are indicated. Dashing cold water upon the face is an efficient measure, which aets by causing deep inspiratory efforts. These measures generally are promptly suceessful, unless the syncope be caused by an excessive hemorrhage, or the loss of blood continue. If not suecessful, another efficient measure consists in compressing the abdominal aorta and the arteries of the upper limbs. In this way, advantage is taken of the principle of " collateral fluxiou."

Cerebral anæmia, as incident to impoverishment of the blood, giving rise to symptoms proceeding from defective stimulation and nutrition of the brain, calls for the remedies and hygienic measures which have been already considered in treating of anæmia as one of the morbid conditions of the blood. The importance of recognizing this condition, with reference, not only to a correct interpretation of pathological phenomena pertaining to the brain, but to disorders in other organs, and to its morbid agency when it is an element of venous affections, cannot be too strongly enjoined upon the practitioner.

It is to be noted that a deficiency of arterial or oxygenated blood may coexist with venous congestion within the skull. The former, indeed, as aiready stated, may lead to the latter in consequence of the contents of the sknll being free from atmospherical pressure; and, on the other hand, congestion of the cerebral veins dne to some imperliment to the return of blood from the head may be an impediment to freedom of eirculation in the distributing arteries, and thus occasion a defieiency of arterial blood.

\section{Apoplexy.}

The term apoplexy signifies a stroke or shock, and the latter terms are often tautologieally added; it is common to say, an apoplectic stroke or shock. It would be an improvement to discontinue the use of the term apoplexy as the name of an individual affection. As commonly used, it is applied to the sudden abolition of consciousuess, or sudden coma, dependent on different pathologieal conditions. The latter constitute different affections, and it wonld be better to consider the apoplectic phenomena as belonging to the clinical history of these affections. In conformity with long usage, however, apoplexy continues to hold its place in nosological classifications, and I shall therefore treat of it as a distinct disease, pointing ont the different pathological conditions with which it is connected, under the head of anatomical characters.

Anatomical Characters.-In fatal cases of apoplexy, the most frequent pathological condition is hemorrhage within the cranium. This pathological condition, which might with propriety be regarded as constituting an individual affection, is to be considered in connection with apoplexy, and hereafter with paralysis. Apoplexy, dependent on extravasation of blood, has been distinguished as apoplexia sanguinia vel gravis. The French writers especially employ the term apoplexy to denote extravasations in different organs, as apoplexy of the liver, kidneys, etc. With us the term is usually restricted to an encephalic affection, with the exception of pulmonary apoplexy. The hemorrhage may take place in different situations within the skull. It may take place into the substance of the brain, and between the meningeal membranes. In the latter situation it is comparatively infrequent. Hemorrhage may be distinguished as cerebral 
when it occurs in the substance of the brain, and as meningeal when it occurs in the meninges. The amount of extravasation differs much in different cases, varying from a few drops to several ounces and even pounds. Other things being equal, the gravity of the affection is proportionate to the quantity of blood extravasated. Occasionally blood is extravasated in isolated small points more or less numerous. This form of extravasation has been caller, after Cruveilhier, capillary apoplexy. Suclden coma, however, or a true apoplectic seizure, is not produced by the minute extravasations called capillary. The coma, if it follow, is developed gradually and may be preceded by paralysis.

All parts of the brain substance are not equally liable to be the seat of hemorrhage. It occurs oftenest by far in the motor traet, that is, the corpus striatum and thalamus opticus; next in frequency in the gray matter of the convolntions, especially in the middle lobe; rarely in the cerebellum; still more rarely in the pons Varolii; and other parts, viz., corpus callosum, fornix, hippocampi, etc., together with the medulla oblongata, are almost exempt from the liability to its occurrence. If the extravasation take place within or extend into the cortical portion, and it be abundant, blood will be likely to be found beneath the arachmoid, or, perhaps, this membrane giving way, blood may be found exterior to it. The hemorrhage occurs, in the vast majority of cases, in one of the hemispheres, and facts do not appear to slow a greater liability to its occurrence in either the right or left hemisphere. It may occur simultaneously in both hemispheres, but instances are exceedingly rare. Its occurrence successively, after an interval of greater or less duration, in the two sides, is not extremely infrequent. If the extravasation take place in the vicinity of the lateral ventricle, not infrequently the blood fincls its way into the latter, and, rupturing the septum lucidum in some cases it colleets in both ventrieles. In rare cases the blood penetrates the third, and thence to the fourth ventricle. From the latter it may be cliffused beneath the spinal pia mater, or, tearing the cerebral tissue, it may be diffused, more or less, over the base of the brain.

An examination, when death has followed within a few hours after the extravasation, shows a clot with bloody serum contained in a cavity produced by laceration of the substance of the brain. 'The clot is dark and friable, resembling a recent coagnlum in other situations, or without the body. If life be prolonged for several days, the clot is still darker and more clense. Generally it contains the debris of cerebral tissue in greater or less abundance. Sometimes, in proximity to the elot are minute points of extravasation more or less numerous. If the extravasation be large, the substance of the brain on the side in which the clot is situated is anæmic, sections presenting fewer' red points than the opposite hemisphere. After a still longer period, the clot is less deeply colored, and crystallized hematin or hematoidin is found on mieroscopical examination. 'l'he clot at length loses its color and resembles a mass of fibrin. In the mean time the serous portion of the extrarasated blood is absorbed. The fibrin becomes surrounded by a cyst, and is gradually absorbed. The cyst contracts as absorption of the clot goes on, and its surfaces adhere when the clot is completely absorbed, leaving a cicatrix, the substance of the brain having, of course, been permanently damaged by the laceration in proportion to the amount of the hemorrhage. 'The brain-substance surrounding the clot, if death take place within a short period, is usually more or less softened, and reddened by the imbibition of the serum colored with hematin. In some cases, this softening may have preceded the extravasation, but it is probably due, in most cases, chiefly to inflammation 
excited by the presence of the clot, which is, in effect, a foreign substance. The occurrence of circumscribed inflammation around the clot accounts for certain symptoms during life. The period required for the processes of recovery varies according to the quantity of extravasated blood, the degree and extent of softening from the inflammation which the clot ex. cites, and other circumstances. Many months must be required, under favorable circumstances, even when the extravasation is small. A very small extravasation of blood may produce, not laceration of the substance of the brain, but only separation of the cerebral fibres. Such an extravasation, however, would not be likely to give rise to an apoplectic seizure; and it is to be noted that, if the hemorrhage be sufficient to occasion laceration of the substance of the brain, a permanent lesion is left, althongh cicatrization takes place. Certain effects of the injury which the brain has received are inevitable. It sometimes happens that, after a clot has been encysted and while absorption is going on, a fresh extravasation takes place in the same situation. More frequently a subsequent hemoirhage takes place at a new point, and perhaps in the opposite hemisphere. Patients sometimes experience a series of extravasations at intervals more or less remote, and the appearances after death then exemplify the different stages in the processes of recovery. In some cases, after an apoplectic extravasation of ancient date, cysts are found which contain a bloody sermm. These may remain indefinitely. In other cases, the absorption of the clot ceases at a certain point, and the clot thereafter remains permanently, presenting the appearance, after death, of a cancerous or tuberculous deposit, with which, on a superficial examination, it is liable to be confounded. In some morbid specimens the brain-substance surrounding the apoplectic clot, cyst, or cicatrix, is indurated, or sclerosed, from hyperplasia of the connective tissue, as a result of inflammation. It is to be added that it is not always practicable to distinguish, from the morbid appearances alone, apoplectic cysts or cicatrices from those which may follow circumscribed softening, or necrobiosis, resulting from embolism and thrombosis.

The foregoing characters relate to hemorrhage taking place into the substance of the brain, or cerebral hemorrhage. In meningeal hemorrhage, the extravasation takes place generally beneath the arachnoid in the meshes of the pia mater. 'The blood may diffuse itself under the arachnoid; but, if the hemorrhage be abundant, rupture of this membrane takes place and the blood escapes into the arachnoid cavity. In this situation the hemorrhage does not occasion laceration of the brainsubstance, but produces compression in proportion to the amount of extravasation. The subsequent changes taking place in the effused blood are similar to those in cases in which the extravasation is into the substince of the brain. It is probable that absorption of the effused blood may take place in this situation if the quantity be small. Meningeal, or, as it is also called, peripheral apoplexy, occurs especially in infancy. After infantile life, it chiefly occurs in old persons, but it may occur at any period of life. Hemorrhage taking place between the dura mater and skull is always either traumatic or a result of caries of the bone, and therefore falls within the domain of surgery. An inflammatory affection of the dura mater, characterized by the formation of an adventitious membrane into which hemorrhage takes place from the newly formed vessels, is described by late writers under the names pachymeningitis and hematoma of the dura mater. An apoplectic seizure is sometimes incident to this affection. It will be briefly considered in the next chapter.

An apoplectic attack may be due to an intense and suddenly developed 
rongestion of the brain. Apoplexy dependent on this condition is distin guished as simple, congestive, or vascular. 'The anatomical characters in these cases consist of great fulness of the ressels of the surface of the brain, together with abundant red points when the brain-substance is divided with the scalpel. Blood escapes very freely from the divided integument in these cases. The sinuses are engorged. The smallest vessels of the pia mater are injected. 'The subarachnoid liquid is wanting. The gray portion of the brain is of a deep brownish-red color, and sometimes the white portion has a rosy hue. I have already referred to a case of fatal apoplexy from congestion occurring in connection with simple hypertrophy of the heart. (Vide page 597). The following case will exemplify the rapidity with which, in this form of apoplexy, life may be destroyed :-

Mr. B. aged 79, farmer, having always had robust health, retired to bed after spending the evening with his family, apparently as well as usual. He slept in a room adjoining to that in which his family were sitting, and, shortly after he had retired, his noisy respiration attracted attention. He was found to be unconscious, and breathing at lengthened intervals. Death occurred before any physician reached the house. On exanination after death, nothing was discovered save excessive fulness of the intracranial vessels. 'The heart was moderately enlarged; the aortic valves were somewhat rigid, with a small ealcareous deposit at the junction of the valvular segments. 'The heart-cavities contained but little blood and no decolorized fibrin. It will be hereafter seen that this variety of apoplexy is exemplitied in some of the cases grouped under the name insolation, or sun-stroke.

The sudden occurrence of serous effusion into the ventricles, arachnoid eavity, or subarachnoid space, has been supposed to be a pathological condition sometimes giving rise to apoplexy. Apoplexy attributed to this condition has been distinguished as serous apoplexy. It is doubtful whether serous effusion alone ever gives rise to coma taking place so suddenly as to constitute apoplexy, although, doubtless, coma induced more or less gradually, occurring in different affections, is attributable to this condition. Cases of apoplexy were reported by Andral, A bercrombie, Lobstein, Louis, and Alison, in which no appreciable pathological condition was discovered after death; and the affection in these cases has been distinguished as nervous apoplexy. These cases were observed when an occasional cause of sudden coma was comparatively but little known, viz., uræmia. It may be fairly presumed that certain of the cases of serous apoplexy, as well as of apoplexy without appreciable changes within the cranium after death, were cases of uræmic coma. Uræmic coma, suddenly developed, is to be reckoned among the pathological conditions giving rise to apoplexy. In the majority of the cases of uræmia the coma is developed more or less gradually, but cases occur in which the abolition of consciousness may be properly enough called apoplectic.

Another pathological condition which may give rise to an apoplectic seizure, is the sudden interruption of the circulation in a portion of the brain, by plugging of a cerebral artery with an embolus derived from the left ventricle of the heart, or possibly by arterial thrombosis. Owing to the absence of anastomosing vessels beyond the circle of Willis, the supply of blood within a certain area is cut off, for a time, by the plugging of one of the cerebral arteries. When we come to consider the pathological character of apoplexy, it will be seen that the production of apoplectic phenomena by this cause is not inconsistent with their occurrence from a superabundance of blood in the vessels. Three cases have been 
reported by M. Hayem, of Paris, in which an apoplectic seizure, proving fatal within a few hours, was apparently dependent on the suclden occlusion of the basilar artery by a coagulum formed in the vessel (thrombosis), in consequence, as supposed, of arteritis. No other lesion was found to account for the apoplexy and death. ${ }^{1}$ A similar case was reported by Prof. 'T'. G. Thomas, at a meeting of the New York Medical and Surgical Society, and an instance has fillen under my observation at Bellevue Hospital.

Cimical History.-The abruptness of the attack in apoplexy is implied in the name. In the great majority of cases the attack is without premonition. It is sometimes preceded by certain cerebral symptoms, such as a'sense of weight or fulness, vertigo, tinnitus aurium, flushing of the face-symptoms denoting eongestion of the brain. These symptoms, however, are not suffieiently significant to warrant the prediction of an impending attack, at least in cases in which one or more attacks have not already occurred. Of 63 cases analyzed with reference to prodromes by Rocheaux, they were wanting in all save 9. It is not uneommon for an attack to occur at a moment when the person attacked feels unusually well.

The symptom denoting the attack is the sudden loss of consciousness, usually aceompanied with slow stertorous breathing. The patient, if standing or walking, may fall to the ground, as if felled oy a blow; but, in general, the deveiopment of the coma is not absolutely instantaneous, and a sitting or reeumbent position is roluntarily assumed. The full development of the apopleetic state may occupy a period varying from a few seconds to half an hour or perhaps even longer. The attack is not infrequently during the night or when the person is alone, and the physician has the opportunity of observing it only by an accident which must happen very rarely. Such an opportunity has happened to me, and the following is the history of the case as noted at the time of its-occurrence:-

Mr. L., aged about 35, came in the evening to consult me, accompanied by my colleague, Prof. Childs. His health for some time had been impaired. The urine had intermittingly contained albumen and casts, and he had difficulty of vision, which Prof. Noyes had ascertained to be clue to fatty deposit in the retina. He had never had dropsy. When he entered my study, there was nothing in his appearance to denote disease. On sitting down, the conversation for a few moments was on general topics, and he remarked that he felt on that day unusually well. I had just begun my inquiries respecting the previous history of the case, when I noticed a change in his appearance and manner as if he were mentally agitated. He said, "I feel nervous, but it will pass off in a moment." Immediately I observed a change in his expression and that he winked with his left eye only. He attempted to bring his right hand before his face, as if to look at it; at the same time he reeled a little in his chair. Dr. Childs, who sat by his side, proposed to him to lie down on the sofa, and took his arm to assist him. He made an effort to rise, and fell from the chair. It was evident that the right side was paralyzed. We placed him on the sofa. He did not at once become unconscious, but was irrational, attempting to speak, but unable to articulate, and frequently making efforts to get up. The respiration soon became heavy, with slight stertor and puffing of the lips. The pulse was moderately full, and neither accelerated nor retarded. In about half an hour he was placed in a car-

1 Vide Archives de Physiologie Normale et Pathologique, Paris, May-April, 1848. 
riage and taken to a hotel in the immediate neighborhood. While being carried to the hotel, he vomited freely, and became profoundly comatose. He remained in the comatose state, and died on the following day.

An autopsy could not be obtained in this case, but it can hardly be doubted that the apoplexy was due to extravasation of blood, the order of events being mental confusion, paralysis of one side of the face, paralysis of an upper and lower extremity, stertor, delirium, vomiting, profound coma, the latter being developed in about half an hour, the hemor. rhage up to this point being probably progressive.

The coma, in difftrent cases of apoplexy, is more or less profound. When complete, the patient camnot be ronsed, in any degree, by efforts to awaken conscionsness; but in some cases properly falling under the head of apoplexy there is not this total loss of the mental faeulties. Volitional movements, in some cases, are entirely wanting; excepting the movements of respiration, the body is as motionless as a cadaver. But in the majority of cases, the patient exerts the will, although all manifestations of intelligence may be wanting. In this way the existenee of paralysis becomes apparent. The movements are limited to the upper and lower extremity of one side, and it is evident that hemiplegia is added to the apoplexy. Hemiplegia occurs in the majority of cases. The paralysis is at first complete; the paralyzed limbs are motionless as regards volition, but reflex movements ean generally be excited. 'The respirations are slow, the rhythm sometimes irregular, and the inspirations stertorous or snoring. If the coma be profound, the buceinator muscles and lips are flaceid, and are puffed out in expiration. The pulse is usually slow, full, and hard, the artery striking against the finger like a metal rod. The retardation of the heart's action is probably attributable to an influence exerterl through the par vagum analogous to that produced by the electrical eurrent. Exceptionally the pulse is small and feeble. The face is frequently flushed or injected, and, if respiration be much embarrassed, more or less livil ; but it is sometimes pallid. The surface in most eases is warm, but sometimes cool. For a certain period after an attack caused by cerebral hemorrhage, the temperature of the body, as determined by the thermometer, is lowered. The axillary temperature is, at first, somewhat greater on the paralyzed than on the opposite side, although subsequently, if the patient recover from the apoplexy, and the hemiplegia continue, the thermometer shows a decrease of temperature on the paralyzed side. The iris may be contracted or clilated, or, without either contraction or dilatation, it may be immovable, and a disparity between the two pupils is not uncommon. The muscles are generally flaceid, but in some cases rigidlity is observed. Convulsive movements are infrequent, but they sometimes occur. These are usually limited to one side, and to the side not paralyzed. To this rule there are exceptions. The paralyzed limbs are sometimes rigid, or the rigidity may be on the opposite side. Vomiting is usual at, or shortly after, the commencement of the attack.

These are the prominent symptoms which characterize the apoplectic statc. Its duration varies. It may last for a few moments only, the patient gradually emerging from it; or consciousness slowly returns after the lapse of a few hours; or the state may continue for days and then pass off. As a rule, if the coma persist withont any improvement for eight or ten hours, and, more especially, if, during this period, the coma beeome more and more profound, improvement is not to be expected, and the attack will prove fatal. On the other hand, the attack may prove fatal in a few moments, the mode of dying being by apnoa; or cleath takes place after several hours; 'or, again, the apoplectic state continues, and proves 
fatal, after the lapse of several days, in the latter case the dying being by asthenia and apnoua combined.

Pathological Character.-The symptoms in apoplexy dependent on cerebral hemorrhage are essentially the same as when coma from compression of the brain follows an injury to the head. An extravasation of blood has the same effect as compression by a depressed portion of the skull, owing to the peculiar situation of the brain, viz., its being inelosed in an unyielding bony case. The escape of blood without the vessels produces a shock or a stuming effect upon the brain, proportionate to the rapidity and the amount of the hemorrhage, and the pressure on any part affects the circulation in the whole mass of brain. 'The pressure interrupts the circulation in the substance of the brain, and the apoplectic phenomena are, measurably, due to the want of a proper supply of blood within the nervous mass. The latter is probably the rationale when the apoplexy depends on sudden and intense congestion. The pressure of the blood in the larger vessels interrupts the cireulation in the brain-substance; hence, the suspension of the cerebral functions. It is an apparent parodox that an over-accumulation of blood within the cranium should destroy life by depriving the mass of brain of a due supply of arterial blood, yet the faet is sufficiently intelligible. If this be the correct explanation, it is not inconsistent that apoplexy should be prodnced by the sudden withdrawal of blood in consequence of plugging of an artery, as well as by congestion. As regards the circulation in the brain-substance, the effect in either case is the same. The pathological condition standing in immediate relation to the apoplectic phenomena, is the same as in syncope. The production of apoplexy depends on the suddenness of extravasation, congestion, or the arrest of the circulation in a vessel of considerable size. If these events take place gradually, apoplexy is not induced, and, with regard to extravasation and eongestion, if they be not sufficient to destroy life within a brief period, the circulation may become restored sufficiently for the restoration of consciousness. This restoration generally takes place when the apoplectic seizure is due to an embolus or thrombus. An apoplectic seizure ineident to uræmia is probably due to the direct action of urea, or its elements, upon the brain. It is difficult to understand why, in uræmic apoplexy, the coma should occur suddenly, and, in some eases, without having been preceded by any symptoms denoting cerebral disturbance. As already stated, coma occurring so suddenly as to constitute an apoplectic seizure is exceptional in cases of uræmia ; in general, the patient more or less gradually falls into the comatose condition.

CaUsation.-The liability to apoplexy from hemorrhage into the substance of the brain, has a manifest relation to age. The liability progressively increases from the age of 20 years upward, and, in the majority of cases, the age is over 60 . It has been supposed that after 70 the liability diminishes, but the fewness of cases after this period of life is accounted for by the comparatively small number of persons living beyond the age of 70 years. Cases under 20 years are rare, yet they are occasionally observed. On the other hand, meningeal hemorrhage oftener occurs prior to, than after, middle life, exclusive of the cases in which it is traumatic. It occurs in children and in newly born infants. Males are more subject to cerebral hemorrhage than females.

Microseopical researches show that hemorrhage into the substance of the brain is often associated with either fatty or calcareous degeneration of the coats of the smaller cerebral arteries. Owing to their weakness or 
brittleness, rupture or fracture is liable to occur. The suddenness of the attack, its occurrence frequently without any obvious causes, and the absence of premonitions, are consistent with this explanation. In a certain proportion of cases, probably, softening of the cerebral substance precedes the hemorrhage, the softening being due to degeneration of the arterial coats, or, perhaps, to interruption of the circulation by an embolus. 'The existence of soltening prior to hemorrhage affords an explanation of the cerebral symptoms which sometimes precede the apoplexy. The dependence of extravasation on the conclition of the minute arteries of the brain explains the fact that apoplexy is most likely to occur in advanced life, the degenerative changes just named occurring especially in old age. 'The fact that hemorrhagic extravasation is especially apt to occur in the corpus striatum or in the opticus thalamus, is probably owing to the greater liability to fatty degencration of the vessels in these situations, and to the vascularity of these parts. Notable fatty degeneration is sometimes found here and not elsewhere in the brain. Another source of hemorrlage into the substance of the brain, to which attention has recently been directed by Charcot and Bouchard, and others, is the bursting of minute aneurismal dilatations of intra-cranial arteries, "miliary aneurisms" as they have been called. The formation of these is distinct from atheroma of the vessels although the latter may be associated. They may be so small as to require the microseope to discover them. They rarely occur before the age of 40 .

Disease of the heart has been supposed to stand frequently in a causative relatiou to apoplexy, exclusive of the cases in which an apoplectic seizure is caused by an embolus detached from the mitral or aortic valves; facts, however, do not sustain this opinion. Generally hypertrophy of the left ventricle is associated with mitral or aortic lesions which are conservative as regards the effects of the augmented power of this ventricle upon the cerebral circulation. Of over 70 cases of valvular lesions which I have analyzed, in only 7 did apoplexy occur, and in most of these other circumstances were involved in the causation.' Simple hypertrophy of the left ventricle, that is, disconnected from aortic or mitral lesions, there is reason to believe, may, in conjunction with other causes, give rise to apoplexy. But simple hypertrophy is rare. A striking example of the coexistence of congestive apoplexy with this form of cardiac disease has been referred to. ${ }^{2}$ Other lesions of the heart may act as auxiliary causes by inducing passive congestion of the brain, and the lesions which exert in this way a causative effect are those involving obstruction or weakness of the right side of the heart.

Various causes may co-operate with existing morbid conclitions within the cranium to determine the occurrence of the apoplectic seizure, causes which act by inclucing an undue accumulation of blood in the vessels of the brain. Mental excitement may, in this way, prove an exciting cause. Persous have been attacked while engaged in public speaking, or in a fit of anger. Straining at stool, violent muscular exercise, sexual intercourse, the throes of labor, drunkenness, have in some cases appeared to act as exciting causes. Fulness of the stomach is another auxiliary cause; not infrequently an attack occurs shortly after indulgence at the table. But, in the larger proportion of cases, the affection is not induced by any obvious exciting or auxiliary cause. Of 176 cases analyzed by Gendrin, in 97 the attack occurred during sleep.

Formerly much importance was attributed to a so-called apoplectic

Practical Treatise on Diseases of the Heart, by the Author.

2 Vide page 348. 
constitution, consisting of shortness of the neck, with considerable embonpoint, and what is known as a full habit. An analysis of a considerable number of cases shows that no reliance is to be placed on these or any other external characters, as denoting a predisposition to apoplexy. The larger number of persons attacked are either spare or of an ordinary habit of body.

The occurrence of apoplexy dependent on cerebral hemorrhage involves considerable liability to a recurrence. Of those who recover, a considerable proportion are again attacked at periods more or less removed from the first seizure. This fact is intelligible in view of the dependence of the affection, in the great majority of cases, on lesions of the cerebral vessels. As a rule, the hemorrhage in successive attacks is greater, rendering them more severe or dangerous.

Dragnosis.-The symptoms of apoplexy are striking and distinctive, but errors of diagnosis may be committed. It may be confonnded with several morbid conditions, other than those belonging to apoplexy, which involve coma more or less complete. Syncope is one of these. There is, however, little or no danger of confounding apoplexy and syncope except for a short time, for syncope is of brief duration. Syncope is characterized by death-like pallor, coldness, catching of the breath, and great feebleness or extinction of the pulse-symptoms which do not belong to the clinical history of apoplexy.

The coma which sometimes follows an epileptic paroxysm bears a resemblance to apoplexy. Knowledge of the fact that the comatose state has been preceded by violent convulsions, with difficult and noisy respiration from laryngeal spasm, will at once settle the character of the attack. Without this knowledge, the foam or blood on the lips and the wounded tongue show that epileptic convulsions have occurred. The doubt can only arise when the patient is a stranger found in an unconscious state. The pliysician generally is aware, or is able to ascertain, that the patient is subject to epileptic fits. A paroxysm of epilepsy may act as an exciting cause of apoplexy.

Hysterical coma is another condition which may suggest the existence of apoplexy. This is to be discriminated by characteristic phenomena of hysteria having precerled the comatose state; by convulsive movements in some cases, into which volition enters more or less; by the absence of stertor, the mobility of the iris, and the restoration of consciousness on resorting to the cold douche. The coexistence generally, in uræmic coma, of epileptiform convulsions, if dropsy do not coexist, suggests the probable existence of renal disease, and the urine is found to contain either albumen or casts, or both.

Profound alcoholic intoxication is the condition most likely to lead to error of cliagnosis. The following are the differential points: The odor of spirits or of wine in matters vomited and in the breath of the patient; the respiration not stertorous ; the pulse usually feeble or soft, and increased in frequency - not diminished in frequency and hard, as it is usually in apoplexy; the pupils dilated. The patient can generally be roused sufficiently to exhibit some of the manifestations of drunkenness in the manner of speaking. Pouring cold water upon the head from a considerable height is a ready mode of partially arousing the patient. It is important to make this discrimination. To call apoplexy drunkenness would be an unfortunate error, and the reverse would be sufficiently annoying to the practitioner.

In deep narcotism, the patient, by vigorous efforts, can generally be 
roused for a moment. The respirations are diminished in frequency, but there is no stertor. The pupils are contracted.

The existence of hemiplegia, which it is known did not exist prior to the occurrence of the comatose state, is a capital point in the diagnosis, to be referred to immediately in considering an object of diagnosis other than the recognition of the apoplexy.

Can the several pathological conditions giving rise to apoplexy, namely hemorrhage, active congestion, embolism, and sudden coma from uremia be differentiated by the symptoms? Hemorrhage into the brain-substance is accompanied by hemiplegial eaused by the laceration of nervous structure. The existence of hemiplegia may generally be ascertained during the apoplectic state. The patient is observed to move the extremities of one side, while those of the other side remain motionless. He may manifest sensibility only when the integument of one side is pinched or pricked. 'The face may be drawn to one side, especially if manifestations of pain can be produced. The paralyzed members, when raised and allowed to drop, fill inanimate. The eyes and the head are turned laterally in a direction opposite to the paralyzed side. On the other hand, meningeal lemorrhage and congestion do not, as a rule, give rise to hemiplegia. The existence, or otherwise, of hemiplegia is, of course, ascertained after the patient emerges from the apoplectic state. An embolus also gives rise to hemiplegia with the apoplectic seizure. This condition may be suspeeted if the apoplexy be transient, and still more if the hemiplegia disappear shortly after the patient emerges from the apoplexy; and it is rendered probable, under the circumstances just stated, by the coexistence of the signs of aortic or mitral lesions. In differentiating bemorrhage from embolism, the sicle on which the hemiplegia oecurs is to be taken into account. In the majority of the cases of embolism, the paralysis is on the right side (vide page 74 ). Vomiting rarely accompanies an attack of apoplexy from embolism. Age is another diagnostic point; embolism occurs in young subjects, whereas hemorrhage is rare under forty. Another diagnostic point relates to the occurrence in cases of hemorrhage of inflammation of the cerebral substance surrounding the clot. This occurs from the fifth to the serenth day after the hemorrhage and continues for about the same period. The axillary temperature is raised; the pulse becomes more or less frequent; there is cephalalgia ; vomiting may occur; pain is felt in the paralyzed limbs, and sometimes there is spasm or rigidity of the muscles on the paralyzed side.

In cases of apoplexy with extravasation of blood into the substance of the brain, can the situation and amount of the latter be ascertained hy the symptoms? The extravasation is situated in the hemisphere on the side opposite to that which is paralyzed; ancl, as a rule, it is situated in the motor tract. The situation of the clot, however, may be such as to oceasion paralysis of both sides. Bilateral paralysis has been observed in cases of extravasation into the cerebellum on or near the median line, and it may occur when the bloor breaks through the septum lucilum and is contained in both lateral rentricles. Paralysis of the face on one side, and of the members on the other side, has been observed. This eccentricity is attributed by Gubler to the elot being in the cerebellum, and so situated as to affect the facial nerve after its decussation and the motor nerves of the extremities before they decussate. Dr. J. Hughlings Jackson considers it as an evidence of the lesion being seated in the pons varolii. A small extravasation in the pons so situated as to press upon the medulla oblongata may produce great difficulty of deglutition and respiration, although the coma be not complete. A case illustrative of this fact has 
fallen under my observation. If the coma be profound, without a disproportionate disturbance of respiration or deglutition, and hemorrhage exist, it may be predicted with much confidence that the seat of the hemorrhage is the corpus striatum or the thalamus opticus of the hemisphere opposite to the side paralyzed, on the ground that it is far more likely to occur in these than in other situations. If death take place after the lapse of a few hours, profound coma having existed without notable disturbance of respiration or deglutition except toward the close of life, hemiplegia existing, it may be inferred that the amount of hemorrhage into the cerebrum is very large.

Meningeal apoplexy, in cases in which the early symptoms cannot be ascertained, may simulate subacute meningitis. 'Of this the following case is an illustration; the case being also of interest with reference to the cause of the hemorrhage:-

The patient, a printer, aged 26, was admitted into the Bellevue Hospital, February 3, 1866. He was in a semi-comatose condition, and the previous history was not ascertained. One of his friends stated that he was a very intemperate min, and had been lately on a debauch. He presented on admission the following symptoms: Countenance pale; respiration stertorous; surface cool, especially on the extremities; eyes half closed; pupils normal ; tongue tremulous and not coated ; pulse slow and feeble. With considerable difficulty, he could be aroused enough to answer some questions. There was no paralysis. Examination of the chest gave a negative result. The urine was free from albumen and casts. He remained without material change until the day following his arlmission, when convulsive movements of the limbs occurred; these increased until the next day, when there was a decided paroxysm of convilsions of an epileptiform character. He had two subsequent attacks within the space of three hours, and died in the last paroxysm.

On examination after death, a hemorrhagic effusion was found within the arachnoid cavity, situated over the anterior superior part of the left hemisphere, and depressing somewhat the brain beneath it. 'The blood was partly coagulated and partly liquid, the hemorrhage evidently having been of recent occurrence. The dura mater, in the situation of the hemorrhage, contained several bony or calcareous spiculæ, some shape- less, and others in the form of needles with sharp points. The points of some of the needle-shaped spiculæ had penetrated the arachnoid membrane covering the brain. There was no injury of the skull. The other viscera were healthy, except tliat the kidneys were congested.' It seemed rational in this case to attribute the hemorrhage to the wounding of vessels by the needle-shaped spiculæ. Uræmia being excluded by the absence of albumen and casts in the urine, and the early history of the case not being known, the symptoms seemed to point to the existence of subacute meningitis.

Cases of meningeal hemorrhage proving quickly fatal cannot be discriminated during life from cases of congestive apoplexy. The following case, which was observed in 1859 , is given in illustration. The patient, a merchant from the country, aged about 40 , had had repeated attacks of rleumatism, and for some time prior to the apoplectic seizure he was in ill health and under the care of a hydropathic practitioner of this city (New York). The seizure occurred directly after a luncheon of tea and

1 Case reported by Dr. S. Amabile, Senior Assistant, Bellevue Hospital, in New York Meclical Record, May 1, 1866. The thermometer might have aided in the differential diagnosis. 
toast. The servant who had brought his luncheon and left his room, on returning after a few moments, found him nearly unconscious, his licad resting on the table. He became at once completely comatose. When I saw him, an hour afterward, the breathing was stertorous and irregular, but the pulse had considerable volume and force. The patient was motionless; the respiration beeame more and more infrequent and irregular, the pulse retaining considerable force when the respirations were only two or three per minute, and death occurred two houls after the seizure.

On examination after death, a considerable amount of effused blood, within the arachnoid space, was situated on the anterior lobe of both sides. A layer of extravasated blood beneath the arachnoid extended over the greater part of the cerebrum, dipping down between the convolutions. 'The substance of the brain was remarkably pale, and presented no red points on section. 'The heart was moderately enlarged. The mitral eurtains were studded with small wart-like vegetations and fine granular deposits. 'Two of the tendinous cords were ruptured. Other organs presented no morbid appearances worthy of note. 'The vessels of the brain were not examined for emboli.

In cases of apoplectic coma from uramia, epileptiform convulsions in gener:ul speedily ensue. Death, however, in some cases takes place in an attack of uramic coma without convulsions. In uremic coma, hemiplegia is not likely to occur. 'The patient may have renal dropsy, or, if there be not dropsy, the cxistence of disease of the kidneys may be known. If not already known, an examination of the urine for albumen and renal casts will show either the existence of disease of the kidneys, or that these organs are not diseased. It is, however, to be borne in mind, that apoplexy dependent on extravasation of blood or other conditions, aside from uramia, may occur in persons affecter with disease of the kidneys.

The importance of a careful examination of the head for the evidence of injury, in cases of coma, when nothing is known of the history, may be here alluded to. A patient admitted into hospital, comatose, without. this precaution, may be supposed to have apoplexy when subsequently, perhaps at the autopsy, fracture of the skull, with meningeal hemorrhage, may be found.

Proanosis. - An attack of apoplexy may prove fatal within a very short period, sometimes within a few moments. When it proves suddenly fatal, the coma is at once profound and accompanied by great disturbance of respiration, with loss of deglutition, death taking place by apncea. A fatal termination frequently takes place after the lapse of from twelve to twentyfour hours. The coma, in these cases, continues and becomes more profound, the respiration is more and more embarrassed, tracheal rales occur, deglutition is gradually lost, the urine is retained or flows away involuntarily, involuntary dejections oceur, and the mode of dying is ehiefly by apnoea. If no improvement takes place within ten or twelve hours, the coma remaining or becoming complete, disturbance of the respiration and deglutition being more marked, there is little room for the expectation of amendment, and the ease will probably end fatally within a short period. In other cases, life is prolonged for several days, the patient not emerging from the apoplectic state, but more or less improvement as regards conscionsness in some cases taking place. The mode of dying, in these cases, is by apnoe and asthenia combined.

On the other hand, the apoplectic attack may pass off in a few moments or a few hours, and complete recovery at once take place. 'The pathological condition in these cases is congestion. Of the cases of apoplexy 
dependent on hemorrhage into the substance of the brain, when the apoplectic attack does not prove fatal, paralysis remains; the patient is left with hemiplegia. 'The paralysis at first is generally complete, and the sensibility on the affected side, as well as the power of motion, is often lost. The latter usually returns more or less speedily, and, more gradually, in a large proportion of cases, there is improvement as regards the power of motion. In most cases this improvement goes on progressively until a certain point is reached, and at this point, which varies much in different cases, the improvement stops. More or less of the paralysis remains permanently, as a rule. It is impossible to judge beforehand of the degree of improvement, as regards the paralysis, which may be expected. The completeness of the paralysis at first is probably due to the shock or contusion of the parts of the brain in proximity to the extravasated blood. After recovery from this, the amount of permanent paralysis will depend on the extent of the laceration which the brain-substance has received. An apoplectic attack dependent on embolism of the middle cerebral artery, gives rise to hemiplegia which remains after the apoplectic condition passes off. The paralysis may be temporary, complete recovery taking place after a variable perior, or it may be permanent. In the latter case, softening, more or less extensive, is a result of the arterial obstruction. In cases of meningeal hemorrhage, in which the amount and rapidity of the extravasation are sufficient to cause an apoplectic attack, the coma, as a rule, persists, becoming more and more complete, and, in a majority of cases, death takes place within twenty-four hours.

Patients emerging from the apoplectic state appear as if awakened from sleep, and it is remarkable that they rarely evince surprise or ask what has happened to them. Pain in the head, when consciousness first returns, is not usually a prominent symptom. After a few days, howerer, if there be cerebral hemorrhage, there is more or less pain associated with febrile movement, and delirium may occur. These symptoms denote inflammation of the cerebral substance surrounding the elot. This inflammation may lead to softening, or to induration, and thus the extent of injury to the brain may be increased.

Apoplexy with hemiplegia is followed by more or less impairment of mind. As regards this sequel, different eases differ much, but it is probably true that the strength of mind which existed prior to the attack is never fully regained. Nental weakness is shown by undue emotional manifestations. Weeping and mirthfulness are excited by trivial causes. Frequently the disposition undergoes a change. Irritability of temper and impatience are apt to be developed. The mind is more easily influenced by others. This fact is of importance in its medico-legal bearings.

A remarkable sequel in some cases is the loss of speech, dependent, not on paraly'sis of the muscles involved in speech, nor on mental imbecility, but an inability to use words. The term aphasia (aфarıs, speechlessness) denotes loss of speech, the vocal organs remaining intact, and intelligence preserved. This term is generally adopted in lieu of the terms alalia and aphemia, which have also been applied to this affection. The affection is to be distinguished from aphonia, a term denoting loss of voice as a consequence of either laryngeal disease or paralysis affecting the spinal accessory nerve, and, of course, also, from voluntary silence, or when loss of speech is either imagined or pretended. In aphasia the voice is not lost, but the patient is mute from an inability to use words expressive of his ideas. In some cases this loss of the power of using language is total, the patient being absolutely dumb; but the difficulty, in other eases, consists of more or less impairment of speech without complete mutism. 
Not infrequently the patient can speak but a single word, which is apt to be "yes," or" "no," and this word is uttered whenerer an effort to speak is made. Often the vocabulary is somewhat larger, but embracing only a few words.

It is certain that this inability to utter words exists when the intelligence is preservod. In some patients with aphasia the mental faculties seem to be not at all impaired; but in many cases there is more or less of the deterioration of mind which accompanies hemiplegia following an attack of apoplexy whether aphasia exist or not. Other differences than those relating to the degrec of the aphasia, and the general condition as regards intelligence, are to be noted. There are two varieties, at least, which present obvious and marked points of difference. In certain cases the aphasia consists in an inability to recollect words. The ideas may exist, but language is wanting. In these cases there is inability to write as well as to speak. This variety is distinguished as amnesic aphasia. Patients with this form of the affection are unable to read. Jaccoud calls this aphasia from "verbal ideation." On the other hand, in certain cases, the aphasia seems to depend, not on the loss of the meniory for words, but on the want of power to give them utterance, although they are in the mind. The difficulty, in these eases, is not due to want of muscular power, that is, to paralysis, but to a lack of the ability to communicate verbal ideas through the muscles of speech, or to co-ordinate these muscles for the purpose of speech. This variety is called ataxic aphasia. In cases of ataxic aphasia, patients, although mute, can write and read. As an example of the latter variety, a woman, aged 27 , the wife of a physician, could utter only the word no. As regards her mental faculties, she was evidently bright, and communicated intelligently by signs. She wrote without more difficulty than would be expeeted from using the left hand, there being incomplete hemiplegia of the right side. She had no difficulty in reading in silence, but she was unable to enunciate any of the letters of the alphabet.

In the great majority of cases, aphasia is accompanied by paralysis of the right side of the body. A French observer, Dax, has collected 140 cases in which the paralysis was on the right side.' Dr. J. Hughlings Jackson has reported 34 cases, in all of which, save three, the right side was paralyzed. ${ }^{2}$ Dr. E. C. Seguin, of New York, has collected 260 cases for analysis with reference to the relative proportion in which right and left hemiplegia coexist. Of these 260 cases, in 243 right, and in only 17 left, hemiplegia existed. A gain, of 545 autopsies, only 31 were exceptions to the rule that disease is seated in the left anterior lobe. ${ }^{3}$ of 80 additional cases collected by Hammond, in only 2 was there evidence of disease of the right hemisphere as determined either by post-mortem examination or the existence of right hemiplegia.

In a considerable proportion of the cases in which post-mortem examinations have been reported, lesions have been found to involve the third left frontal convolntion, situated, thus, near the island of Reil. The lesions generally found are either cireumscribed softening probably due in most, if not all, cases to embolism, extravasation of blood, or apoplectic cysts. Circumscribed cerebritis, suppuration or depression of bone, are lesions in traumatic cases. From the great preponderance of the number of cases in which the lesions are seated in the left hemispbere, it has been

1 Archives de Médecine, Jan. 1865, and Iondon Lancet, A ug. 1865.

2 Clinical Lectures and Reports of London Hospital, vol. i., 1864.

3 Quarterly Journal of Psychological Medicine, New York, Jan. 1868. 
inferred that the mental faculty of speech is to be localized in this side of the brain. Broca, of Paris, even considers the posterior part of the third left frontal couvolution "the convolution of articulate language." But aside from the improbability of this faculty being seated in either hemisphere to the exclusion of the other, such an inference is disproved by the fact that in a small minority of cases the coexisting hemiplegia affects the left side.

'The statistical researches of Dr. Seguin show that the third left frontal convolution is not involved in the majority of cases. Of 52 cases gatlered from different authors, this convolution was involved in 18 , and not in the remaining 34 cases. It is, however, a remarkable fact that lesions in this situation should have existed in 18 out of 52 cases. Our present knowledge does not offer any explanation of the fact.

The lesions giving rise to aphasia, it will be observed, are not exclusively those which belong to the history of apoplexy. It occurs, however, as a sequel of apoplexy in the larger proportion of cases, and, hence, the propriety of considering it in this connection. It is not always incidental to lesions which give rise to hemiplegia. It occur's sometimes in counection with paralysis limited to one or more of the cranial nerves, and, also, without paralysis anywhere of either motion or sensation. Does it occur without lesion, that is, may it depend on purely a functional disturbance, using this term functional as it has been defined, and as it is commonly used? 'The fact that it may occur without any other evidence of lesion, even without any other symptom pointing to cerebral disease, and the fact that it is sometimes of transient duration, seem to warrant an affirmative answer to this question.

As a rule, aphisia accompanying hemiplegia following apoplexy is permanent. If the hemiplegia be transient, the aphasia and the paralysis may disappear together. But patients sometimes recover from the hemiplegia without any improvement as regards the aphasia; of this several examples have come under my observation. I have met with a striking case in which the aphasia, at first complete, was unaccompanied by any other evidence of disease of the brain, and was of brief duration. The patient, having ridden all day in an open wagon, the weather being cold, after eating a hearty supper, found himself unable to utter a word. Excepting the loss of speech, he had no ailment whatever. He went to bed, hoping that his ability to speak would return in the morning; he slept perfectly well, but on awakening he was still nnable to utter a word. In the course of the day, however, he began to utter some words, often using wrong words, but, after a few days, he recovered his speceh, although, ever since, he has experienced some difficulty in seleeting right words. Nothing occurred afterward to denote any cerebral disease (more than a year after the aphasia having elapsed when I saw him) except that he was conscious of an uneasy sensation in the left side of the head. That this attack of aphasia was due to embolism, is rendered probable by the fact that there existed valvular lesions of the heart; he had an aortic regurgitant and a mitral direct murmur, with considerable cardiac enlargement.

In cases of persistent aphasia it is not always easy to determine to what extent, aside from the inability to use words, the mental faculties are preserved. The ability to comprehend fully what is said, and intelligence sufficient to perform routine duties, are certainly not incompatible with the loss of speech; but it is extremely difficult, in amnesic aphasia, to ascertain the mental condition as regards the reasoning powers, the judgment, and the sentiments. The affection in this aspect has an obvious and important medico-legal bearing. 
Treatment.-In the treatment of cases of apoplexy, an important question is, whether bloodletting shall be employed. If the apoplexy be dependent on active congestion, bloodletting is indicated, and the life of the patient may depend on the prompt abstraction of blood. Notable congestion of the face, a resisting pulse, and absence of hemiplegia, point to congestion as the pathological condition probably existing. If the patient be young, or below middle age, the probability of this condition, rather thin extravasation, is strengthened. On the other hand, if the apoplexy be dependent on cerelual hemomage, or on embolism. the propriety of bloodletting is always doubtful. It is contra-indieated by feebleness of constitution, advanced age, and if the completeness of the coma denote a large extravasation, or if its situation at the base of the brain be denoted by notable disturbance of respiration and deglutition. It will tend to destroy life if the tace be pallicl, the surface cold, and the pulse feeble. With the symptoms last named, stimulants are indicated. A large extravasation involves loss of blood, and the substance of the brain is anemic from the pressure of the cxtravasated blood. Bloodletting will neither remove nor diminish the clot. If the extravasation be not sufficiently large nor so situated as to occasion a fatal attack of apoplexy, the abstraction of blood may stand in the way of recovery from the slock, and the prolongation of life until the elot is absorbed. There is no reason to balieve that bloodletting tends to prevent or diminish the cerebral hemorrhage. Cruveilhier, Rostan, and Aussaguel have each reported a case in which hemiplegia ensued directly after bloodletting. I liave met with a case in which an attack of apoplexy with hemiplegia immediately followed a profuse hemorrhage from the rectum. Here, as in other affections, bloodletting is neither to be interdicted nor enjoined in all cases. It will be useful or pernicious accorling to the discrimination with which it is employed. In view, however, of the fact that apoplexy involves, in the majority of cases, cerebral hemorrhage, this measure is clearly inclicated in only a small proportion of cases.

Generally, it is advisable to administer an active cathartic. Croton oil, from the facility with which it is administered, its eflieiency and quickness of operation, is to be preferred to any other cathartic. 'Three or four drops may be given at once and repeated in three or four hours if free purgation be not produced. Its operation may be promoted by large stimulating enemas. An active cathartic should not be given if the pulse be notably small or feeble.

The patient should be placed in a cool, airy apartment; the head should be moderately raised; cold or evaporating lotions should be applied to the head, or, if the head be extremely hot and flushed, the ice cap) ; stimulating pediluvia may be employed if the extremities be cool, and everything constricting the neck and chest should be removed.

These are the measures to be pursued until it is decided whether the patient is to emerge from the apoplectic state. If, after the lapse of several hours, the symptoms denote a hopeless condition, the physician must content himself with measures which appear to contribute to the comfort of the patient, for he is, in fact, unconscious of suffering. Purgatives and other perturbating remedies, under these circumstances, will only tend to hasten the fatal termination. If, on the other hand, the attack pass off; and there be no paralysis, the after-treatment is that appropriate to prevent a recurrence of the attack. If the patient emerge and heiniplegia remain, either embolism or the presence of a clot is to be inferred. Embolism calls for no measures of treatment addressed to the head. Local inflammation exeited by a clot is to be treated by cooling lotions to the 
head, laxatives, mild revulsives, and an unstimulating diet. The vital powers, under these circumstances, should not be impaired by active measures of treatment. The recovery is to be effected by the absorption of the clot. The case is now one of paralysis which will be considered in a chapter devoted to that topic. There is an important consideration, however, which may be here mentioned, namely, attention should be speedily directed to the paralyzed limbs, and means employed to maintain their nutrition and functional capability. These means are, friction, or shampooing, and passive movements. Several weeks should elapse before electricity and remedies designed to excite the paralyzed muscles are resorted to.

The liability to recurrence of apoplexy dependent on cerebral hemorrhage, after recovery from an attack, renders it important to observe all possible precautions by way of prophylaxis. Placing the system in the best possible condition by means of a well-regulated diet and regimen, and avoiding exciting causes, will afford all the security which can be obtained. It is not probable that any protection is afforded by reducing the powers of the system, and other evils may thereby be induced. It is injudicious to adopt a diet which is insufficient for the wants of the system, or to resort to repeated bloodlettings, cathartics, or other lowering measures. In striving to avoid excesses and imprudences of all kinds, care must be taken not to err in the opposite extreme. Mental occupation within certain limits is advisable.

A liability to apoplexy, if an attack have never occurred, cannot be estimated with any degree of certainty. This is one of the affections which many persons are apt to apprehend, and, if certain cerebral symptoms be experienced, especially vertigo, the fear of apoplexy is often the source of much unhappiness. The suggestion by the physician that there is danger of this affection, is an indiscretion which I have known to prove most calamitous. When apprehension is felt, the physician is warranted in giving assurances that vertigo and other cerebral symptoms are sufficiently common without being followed by apoplexy, and that an apoplectic attack is rarely preceded by obvious premonitions. Needless uneasiness may oftentimes be removed by these assurances.

\section{Insolation-sunstroke.}

The terms insolation and sunstroke are applied to an affection, or, more properly, different affections, occasioned, not exclusively by exposure to the sun's rays, as the terms signify, but by the action of great heat combined generally with other causative eircumstances. The terms denote a sudden attack, in extremely hot weather, after exposure to the direct rays of the sun or to great heat, the attack characterized by loss of conseiousness, followed frequently by stertor and convulsions, death taking place, in a large proportion of cases, within the space of a few minutes or a few hours. Now, in a certain proportion of these cases the morbid condition is expressed by the term exhaustion; the vital powers are prostrated, the forces carrying on the circulation give way, and death takes place by syncope. In another class of cases the affection is analogous to congestive apoplexy. In still another class of cases the symptoms show a combination of apoplexy and exhaustion. The cases last referred to probably constitute the majority of the cases of so-called insolation, or sunstroke. Other names than insolation and sunstroke have been proposed, viz., solar asphyxia, heat-apoplexy, thermal or heat fever, etc.

$A \mathrm{~s}$ a rule, the vessels of the pia mater are found to be notably congested, 
the appearances being those of congestive apoplexy. Exceptionally, the vessels within the eranium are not unduly injected.' Notable congestion of the lungs is found generally, if not invariably. Pepper found the heart in four cases notably tlaccid, and its structure softened, but, generally, if the post-mortem examination be made shortly after death, the ventricles (more especially the left ventricle), are rigidly contracted, the ventricular cavities being empty. 'The blood coagulates imperfectly, if at all. Its alkalinity is diminished, and it gives often an acid reaction. It contains notably less oxygen than blood, in general, after death. Appearinees denoting inflammation within the cranium, or elsewhere, are wanting.

'The premonitions of the attack are slight or wanting. I). Swift, in a report based on the observation of 60 cases in the New York IIospital, gives the following account of the attack: "The patients are suddenly seized, while in the performance of their labors, with pain in the head, and a sense of fulness and oppression in the epigastrium, occasionally nausea and romiting, general feeling of weakness, especially of the lower extremities, vertigo, dimmess of vision, and insensibility. Surromnling objects appear of uniform color. In a great majority of cases, this was, so far as could be ascertained, blue or purple. In one instance every thing appeared red; in another green, and in another white." It is stated by Longmore that irritability of the bladder precedes the attack. ${ }^{3}$ Pain in the head, although generally present, does not always precede unconscionsness. It was wanting in the case of a medical friend of the author who had a severe attack, accompanied by violent convulsions.

The attack may consist of only a transient insensibility. But in severe cases the patient usually passes quickly into a comatose state. It is in this state that cases generally come under melical observation. The pupils are in some cases dllated, in some contracted, and sometimes the size is normal but the respondency to light is lessened or lost. There may be contraction and dilatation at different periods in the same case. The pulse may be at first infrequent and full, as in cases of apoplexy, but it often becomes frequent and feeble toward the fatal termination. I have observed death to take place purely by apncea, the pulse having considerable force until after the cessation of breathing. The respirations in some cases are stertorous, in other cases suspirious and accompanied by moaning; they are increased in frequency in the majority of cases. In most cases the temperature of the body is notably raised. The maximum rise, in cases at Bellevue Hospital, in July, 1868, was 10912 ${ }^{\circ}$ Fahrenheit, and the lowest observed was $99 \frac{1}{2}^{\circ}$. In a case observed in July, 1872, the temperature in the axilla was $110 \frac{1}{2}^{\circ}$. If the thermometer indicate a rise to $107^{\circ}$ or upward, a fatal termination is to be expected. In the case, however, just referred to, in which the temperature was $110 \frac{1}{2}^{\circ}$, the patient recovered. Convulsions and rigidity of the muscles are of frequent occurrence. The former occurred in 24 of 60 cases observed by Swift. Vomiting frequently oecurs, and, towarls the close of life, there are involumtary evicuations from the bowels. The prognosis is rendered extremely unfavorable by the occurrence of vomiting and purging. The duration varies from a few minutes to several hours. The average duration in the fatal cases observed by Swift was four hours. If the patient emerge from the comatose state, convalescence is usually speedy. Of 20 cases observed

1 Vide four cases reported by Dr. Pepper, Trans. Philadelphia College of Physicians, rol. iii.

\& Observations on Exhaustion from the Effects of Heat. By H. S. Swift, Resident Pliysician of the New York Hospital, N. Y. Journal of Jíedicine, July, 18.54.

3 London Lancet, March, $18 j 9$. 
by Pepper, in 3 insanity followed. This sequel did not occur in over 100 cases received at the New York Hospital. Temporary delirium succeeding the attack is occasionally observed. Paralysis is very rarely a concomitant or a sequel. I have, however, known of the oscurrence of incomplete transient hemiplegia in one case after recovery from the attack, and in one case before recovery. In the latter case it lasted but a few hours.

As already stated, the pathological character of the affection, in all cases of insolation, is not uniform. Cases of sudden death from exhaustion or syncope produced by heat and over-exertion are reckoned among cases in which the morbid condition is quite different. A certain proportion of cases seem to be truly apoplectic; the appearances after death, the character of the attack, and all the symptoms denote congestive apoplexy. In other cases, which, probably, constitute the majority, the pathological character is mixed; an apoplectic condition is combined with exhaustion. It is highly important to take cognizance of these differences as regards pathological character, in treating the different cases which are grouped together under the name insolation. The notably increased temperature of the body, perhaps, stands in a causative relation to certain of the phenomena. Regarded from this point of view, insolation is, in a marked degree, a febrile disease. That toxical changes in the blood play an important part in the pathology, is probable. The fluidity of the blood after death, its acid reaction, and the diminution of oxygen, are evidence of changes not yet understood. Vallin, of Paris, has reported a series of observations upon dogs and rabbits destroyed by insolation. These animals, placed upon the back, fastened in this position, and exposed to the direct rays of the sun, in the months of July and A ugust, in the climate of Paris, presented phenomena analogous to insolation in man, and died generally after an exposure of from half an hour to two hours. Examinations after deatlı showed hyperamia of the brain and lungs, the cavities of the heart, especially the left ventricular cavity, being empty. These experiments have been repeated by Prof. Horatio C. Wood, Jr. Vallin is led to attribute these conditions and death to coagulation of the liquid of the muscular tissue (suc musculaire. myosin) of the heart and diaphragm. He does not, however, apply this explanation to all the cases embraced under the name insolation. In some cases he attributes the death to toxical charges in the blood, the action of the heart being arrested by a morbid agency acting through the pneumogastric nerve. In the latter cases the heartcavities may be filled with blood. ${ }^{1}$ The observations of Prof. Horatio C. Wood, Jr., lead him to the conclusion that the contraction of the ventricles of the heart found after death, especially when autopsies are made within a short time after death, is, in most cases, of post-mortem, not of ante-mortem, occurrence. ${ }^{2}$

Excessive heat is the chief agency in causing insolation, but other circumstances co-operate in the causation. Muscular exertion, unduly great or prolonged, is a powerful auxiliary cause. Persons are generally attacked when engaged in labor, but there are exceptions to this rule. Dr. Swift states that, of the cases which he observed, a large proportion were attacked shortly after dinner. The concentration of innervation upon the processes of digestion, the distension of the stomach with food and liquids, the latter being frequently taken in large quantity, and perhaps, in some cases, the disengagement of gas from chemical changes arising from indigestion, will account for the fact stated by Swift. In most cases,

1 Vide Archives de Médecine, Fevrier, 1870.

On Thermic fever or Sunstroke, Boylston Prize essay, 1872. 
persons are attacked when exposed to the direct rays of the sun, but there are exceptions to this rule. Swift states that eleven patients were attacked on the same morning in the laundry of one of the large hotels in this eity, and several cases were brought to the hospital from a sugar refinery. Soldiers are not infrequently attacked after they have retired to their tents.

Cases are more frequent by far in tropical than in cold or temperate latitudes, and, in the latter, they occur during the heated term of summer when the weather is unusually and continuously hot. Atmospheric influenees other than heat have been supposed to be involved in the causation, viz., an unusual accumulation of electricity, dryness and rarefaction of the air ; but ficts showing the importance of these influences are wanting. 'The number of cases in the same place in different years varies greatly. Prof. Dickson states that in Charleston, S. C., more persons died from sunstroke in 1824 than in any twelve other years; and in the eity of New. York in the summer of 1853 there were 260 cases reported, whereas in no previous year had there been more than 36 cases.

The attack generally takes place at periods when the heat of the day is at its maximum. Of the 60 cases observed by Swift, 40 occurred between 11 A. M. and 4 P. M., 17 between 4 and 9 P. M., and 3 between 8 and 11 A. 1.

Feebleness from disease or other causes renders persons more liable to an attack.

The diagnosis is, in general, made without diffieulty. The circnmstances connected with the attack, and the symptomatic phenomena, are sufficiently distinctive. A poplexy with extravasation of blood is to be distinguished by the existence of hemiplegia. Persons deeply intoxicated are not infrequently brought into hospitals as cases of sumstroke, but the characters of alcoholic coma suffice for its discrimination.

Exclusive of mild eases in which there is transient stupor or insensibility, the danger in cases of insolation is always very great. The deathrate ranges from forty or fifty per cent. If, with deep coma, the breathing be stertorous, sighing, or moaning, the prognosis is extremely unfavorable. $G$ reat frequency and feebleness of the pulse, relaxation of the sphincters, tracheal rales, and complete immobility are forerunners of a fatal termination. Convulsions are extremely unfavorable; yet I have known recovery to take place after violent convulsions. In accordance with the difference in pathological character in different cases, the morle of dying is sometimes by rapid asthenia or syncope. This mode of dying is exemplified among the cases in which the death is notably sudden, taking place in the space of a few moments after the attack. 'The mode of dying in other cases is by apnœa, but, in the larger proportion of cases, by apnoea and asthenia combined.

The treatment is to be adapted to the pathological character of the affection, as represented by the symptoms, in individual cases. Pursuing this course, therapeutical measures, so far from being the same, will be diametrically opposite in different cases. In cases of nervous exhaustion, the danger being of death by rapid asthenia or syncope, complete rest is. of the first importance. The removal of patients to their homes or to hospitals, in this condition, may contribute in no small measure to a fatal result. Stimulants are to be administered by the mouth very cautionsly in order to avoid exciting vomiting. They may be given with less risk by the rectum. Alcoholic stimulants may be administered by enema.

I Elements of Medicine, 2d cdition, 1859. 
The spirits of turpentine given in this way are recommended. All restraints of dress are to be removed. The patient should be kept in as cool and pure an atmosphere as possible. If the surface be hot and dry, sponging the body with spirit and water should be employed. These should constitute measures employed during the attack. Cathartics, emeties, bloodletting, and all depressing ageneies are pernicious. The symptoms representing the condition which calls for the treatment as just stated are frequency and feebleness of the pulse, weakness of the heart-sounds, with absence of stertor and the embarrassment of breathing indicative of cerebral compression.

In well-marked apoplectic cases I believe bloodletting to be the measure especially indicated. The treatment called for is the same as in congestive apoplexy, which, in fact, in these cases, the affection is. The life of the patient may depend on the prompt employment of bloodletting. The bowels, should be frecly opened with croton-oil. Cold should be applied to the head, either by means of the ice-eap or the douche. The head should be elevated, and everything constricting the chest or neck removed. Revulsive applications should be made to the extremities. The symptoms representing the condition ealling for these measures are fulness of the pulse, slowness of the respiration, with perhaps stertor, heat of the surface, congestion of the face, and throbbing of the carotids and temporal arteries.

The question as to the propriety of bloodletting in cases of insolation has given rise to much diseussion and difference of opinion. The doctrine which I would inculcate is, that to abstract blood is vastly important in some and destruetive in other cases. Never to employ bloodletting, and to employ it in all cases, would be alike injudicious. It is, of course, for the judgment of the practitioner to discriminate between the cases in which bloodletting is called for, and the cases in which it will do harm. I have noted several cases which apparently exemplify the importance of bloodletting; and I am led to introduce a brief notice of two cases in view of the fact that, at the present time, some, and perhaps most, practitioners consider this measure improper in all cases of insolation.

On the 9th of August, 1862, eight cases of coma from sunstroke were admitted into Bellevue Hospital. Of these cases, 7 proved fatal. Bloodletting was employed in only one case, and in this case the patient recovered. This was the last case admitted on that day, and bloodletting was resorted to in that case by the house-physician, Dr. Martin, mainly in view of the fatal termination of the seven cases under other measures of treatment. In the case whieh reeovered, the coma was as profound, and the symptoms, in general, apparently as unfavorable as in the other cases. The breathing was stertorous, the pupils contracted, the skin hot and dry, and the pulse frequent, but full. Sixteen ounces of blood were taken from the arm, and the temples freely leeched. The patient came under my observation on the following day. He was then quite comfortable, and complained only of debility. He recovered his strength rapidly, and was discharged in a few days, well.

On the 13th of August, 1864, a patient was admitted into one of my wards at Bellevue Hospital, who had been found in a state of insensibility in the street. The pulse was 100 per minute, and had considerable force; the breathing was stertorous, the skin hot, the pupils neither contracted nor dilated, but not responding to light. Eighteen ounces of blood were taken from the arm, and the cold clouche applied to the head. These measures constituted the treatment. He recovered his consciousness in an hour and a half after the bleeding. On the following day he reported 
himself to be quite comfortable, and was discharged in the aftemoon of that day.'

In the majority of the cases of insolation, symptoms denoting congestive apoplexy are combined, in variable proportions, with those denoting exhaustion. The importance of bloodletting in these cases is to be measured by the predominance of the apoplectic phenomena, and it is contraindicated if predominance of exhaustion be denoted by great frequency or feebleness of the pulse. The success of the treatment will depend on the judgment of the practitioner in deciding whether bloodletting be called for, or otherwise, and, if indicated, in determining the amount of blood to be taken. Discrimination is also important in preseribing the croton oil. If the danger be from exhaustion, an active purgative is not indicated. Cold to the head is important in proportion as the symptoms of cerebral congestion predominate. On the other hand, in the cases in which the circulation is not:lbly feeble, stimulant remedies by the mouth or rectum are ealled for. In all cases quietude is important. In the cases in which convulsions occur, these may be excited by movements of the body. The most eflicient measure having reference to convulsions is the inhalation of chloroform.

The bromide of potassium has been employed freely in the treatment of cases at Bellevue Hospital, but without apparent benefit. For several years past considerable reliance, in that hospital, has been placed on sponging the body freely with cold water, in addition to ice to the heacl. During the present summer (1872), the application of cold water to the surface has been carried out more efficiently than hitherto, in the following manner: The patient is enveloped in a sheet wet with cold water, and placed on blankets covered with India-rubber cloth. With a sprinkling pot the cold water is applied over the whole body at intervals of a few moments. This is kept up for a period ranging from half an lour to several hours, the continuance being determined by the effect on the temperature, pulse, and other symptoms. The temperatute in the axilla is reduced more or less, and sometimes severnl degrees. The measure is repented as often as the temperature again rises. If the pulse be feeble, stimulants are given, more or less freely, during the applieation of the water. The good effect of this plan of treatment has been marked in some eases. A patient admitted at 2 P. M., unconseious, pupils contracted, pulse 150, breathing stertorous, the axillary temperature $110 \frac{1}{2}{ }^{\circ}$, and the borly cyanosed, was at once placed in the wet sheet and sprinkled. At half past 4 P. M., the tenperature in the axilla had fallen to $104 \frac{3}{4}^{\circ}$, the frequency of the pulse and the cyanosis persisting. He was taken out of the slieet, dry cups were applied to the ehest, and at 5 o'clock the wet sheet was again applied and continued until 15 minutes after 7 P. M. The temperature then was $1011^{\circ}$, and the pulse 120 . At $9.45 \mathrm{P}$. M. the temperature fell to $1003^{\circ}$. At miduight he became conscious. Progressive improvement followed, ending in recovery. The only arditional measures $\mathrm{em}$ ployed up to the restoration of consciousness, were an enema containing castor oil, with a few drops of croton oil, and the hypodermic injection of ten minims of the tincture of digitalis, the latter having reference to the frequency and feebleness of the action of the heart.

The employment of cold water in the manner just described was resorted to only in the cases in which there was a considerable increase of axillary temperature $-104^{\circ}$ and upward. When the temperature was less, spong-

1 The case of Dr. S. Weir Mitchell affords a striking example of the benefit of bloodletting. Vide Prize Essay by Prof. Ir. C. Wood, Jr. 
ing the body with cold water was employed. Further observations are required to determine the extent of the usefulness of abstracting heat from the body by the use of cold water in cases of insolation. That it is useful is certain, and, with dne care, the plan of treatment does not involve risk of doing harm.

Dry cups to the chest seem to relieve symptoms dependent on pulmonary congestion, namely, embarrassed breathing and cyanosis; applied over the spine, they are uséful in relieving convulsions and muscular rigidity.

In some of the cases treated in 1872, the Calabar bean was given in doses of one grain by hypodermic injection, and repeated two or three times after intervals of from half an hour to an hour. This remedy was tried with referenee to muscular rigidity and convulsive movements. It seemed to be useful, but the observations were too few to warrant any positive conclusions. Given in the dose just stated, it produced no marked effect upon the pupils. ${ }^{1}$

\section{Congestion and Anmmia of the Spinal Cord. Hemor- rhage within the Spinal Canal.}

Congestion and anæmia of the spinal cord will be noticed in connection with general spinal paralysis and paraplegia, affections to be considered after having treated of inflammation and struetural lesions situated within the skull and the spinal canal (vide Chap. V.). It is chiefly with referenee to the paralytic affeetions just named, that these pathological conditions are important; and, recognizing, as is done in this work, the different forms of paralysis as distinct diseases, although they are, in reality, only symptoms, it will suffice, in a practical point of view, to notice congestion and anæmia of the spinal cord, as entering into the etiology of the forms of paralysis referable to that part of the nervous system.

Hemorrhage within the spinal canal is an exceedingly rare event, especially when not dependent on injuries of the spine. Spinal hemorrhage may be meningeal (Hematorrhachis), or it may be in the substance of the cord (Hemomyelorrhagia). In either form, the lemorrhage constitutes what has been called spinal apoplexy. Its infrequency is in striking contrast to intra-eranial hemorrhage. In cases of meningeal hemorrhage, the extravasation may be between the bones and the dura mater, between the latter and the arachnoid, or between the arachnoid and the pia mater. The last-named situation is that in which it oftenest occurs. The amount of extravasation varies in different cases. The quantity is sometimes sufficient to fill the spinal canal. In contrast to this, there may be only a very small coagulum. Isolated, small clots have been observed, indicating, by their appearance, a series of extravasations occurring at different periods. In these cases, the appearances usually denote circumseribed spinal meningitis, the inflammation, in general, being secondary and exerted by the clot. It has been conjectured that sometimes the hemorrhage may follow spinal meningitis, giving rise to an affection corresponding to hematoma of the dura mater; a hemorrhage into the substance of the cord takes place in the gray portion, and gives rise to a clot, or, oftener, to two, three, or more clots, varying in size from that of a pin's head to a bean. If life be sufficiently prolonged, the same changes take

I For a report of 55 cases treated in Bellevue Hospital, July and August, 1872, prepared, at my request, by Dr. Katzenbach, house-physician, vide New York Journal of Medicine, Jan. 1873. 
place here as in cases of cerebral hemorrhage. These extravasations occur oftenest in the cervical portion of the cord, next in frequency in the dorsal portion, and very rarely below the dorsal.

In the larger proportion of cases of spinal hemorrhage, it is of traumatic origin. Meningeal extravasation has been observed in cases of tetanus and chorea. It is probable that apoplexy of the cord is attributable to the same conditions as extravasations within the substance of the brain, namely, atheroma of the arteries, miliary aneurisms, and softening. Aneurisms of the aorta sometimes, after having occasioned erosion of the vertebre, burst into the spinal canal. Extravasation into the cord sometimes coexists with cerebral hemorrhage. Spinal hemorrhage is one of the several morbid conditions standing in a eausative relation to general spinal paralysis and paraplegia.

\section{CHA PTER II.}

Pachymeningitis and Hematoma of the Dura Mater-Acute Cerebral Meningitis-Anatomical Characters-Clinicai History-Pathological Character-Causation-Diagnosis-PrognosisTreatment. Cluronic Meniugitls. Tuberculous Meningitis. Spinai Meningitis. CerebroSplnal Meningitis - Anatomical Characters-Olinical History - Pathological CharacterCausation-Dlagnosis-Prognosis-Treatment. Hydrocephalus. Hydroracilis.

TNLAMMATION within the cranium may be seated, primarily, either in the membranes investing the brain or in the cerebral substance. As regards the membranes, the dura mater is rarely the primary seat of an inflammation. When this membrane becomes inflamed, the inflammation, as a rule, follows either an injury of the skull, disease of the bones, or a morbid growth springing from the membrane, and the inflammation is circumscribed. Inflammation of the meninges is generally seated in the arachnoid and pia mater, and the term meningitis is applied to inflammation of these membranes. The relations of the arachnoid and pia mater are such, that one can hardly be inflamed to the exclusion of the other; and pathologists have differed as to which one of the two membranes is primarily or chiefly affected. It is not practically important to settle this question. The terms arachnitis and pieitis would be appropriate to denote an inflammation of the arachnoid and pia mater separately, but, in view of the difficulty of determining in which the inflammation has its point of departure, the term meningitis is to be preferred. Inflammation of the meninges of the brain should be called cerebral meningitis, in order to distinguish it from inflammation of the meninges of the spinal cord, or of the brain and cord. Inflammation of the meninges of the cord is spinal meningitis, and of the meninges of both the brain and cord, cerebro-spinal meningitis. Inflammation of the substance of the brain may be distinguished as cerebritis. Cerebral meningitis may be acute, or it may exist as a subacute and chronic affection. These two forms will claim separate consideration. A form of inflammation known as tuberculous meningitis will also be considered under a distinct head. In this chapter, after having devoted a little space to pachymeningitis and hema- 
toma of the dura mater, the three forms of cercbral meningitis just named will be considered; also, spinal meningitis and cerebro-spinal meningitis, with a brief notice of hydrocephalus and hydrorachis.

\section{Pachymeningitis-Hematoma of the Dura Mater.}

Under these names Virchow was the first to describe an affection which appears to be primarily and essentially an inflammation of the dura mater. The affection described under these names is, by some writers, considered as embracing inflammation situated on either the outer or the inner aspect of the dura mater; that is, on the surface in contact with the arachnoid membrime, and on the surface attached to the cranium. External pachymeningitis, or inflammation in the latter of these two situations, is probably never a primary affection; it is always secondary to either an injury, or disease of the cranial bones. Hence, if this be recognized as a variety of the affection, it belongs to surgical, rather than to medical, practice. The following account will, therefore, relate exclusively, to inflammation affecting the arachnoid aspect of the dura mater, or internal pachymeningitis.

Anatomically this affection is characterized, in the first place, by the formation of an adventitious membrane, or a collection of membranes, not "false," but organized and containing vessels. The membranes are so extremely thin and delicate, as to be compared to the spider's web; and, if hemorrhage has not taken place, they are liable to be overlooked if a careful examination be not made by the touch as well as by the eye. In some cases several membranes are suecessively found, and a series of layers may be detected; as many as twenty have been counted. 'They contain bloodvessels in greater or less abundance. In the second place, an anatomical characteristic, in a certain proportion of cases, is the presence of blood contained in the meshes of the adventitious membranes, and limited to the space, or spaces, which these occupy. The hemorrhage is attributed to rupture of the vessels contained in the adventitious membranes. From the appearances in some cases the occurrence of a single hemorrhage is inferred, and in other cases repeated hemorrhages have evidently taken place. The blood thus infiltrating the nembranes and retained in a kind of cyst, is the element of the affection denoted by the name hematoma. This is not an essential element, although its absence is exceptional. In some specimens, newly-formed, membranes are found without any evidence of hemorrhage. It may be supposed that in the cases which furnish these specimens, hemorrhage would have occurred had the life of the patient continued. The existence of pachymeningitis without hematoma, in some cases, goes to show that the latter is connective to, and dependent on, the former, and is in contradiction to another explanation, namely, that the hemorrhage occurs first, and the membranes are produced afterwarcls.

The inflammition in this affection is more or less circumscribed. It is usually seated at the cranial vault, by the side of the median line. The affection may be single or double; that is, it may be seated on one side of the medial line, or it may extend to both sides. In the latter case it often is equal in extent on the two sides, thus furnishing an example of the law of symmetry. The extent of surface affected varies in different cases. The quantity of blood which is encysted also varies, amounting sometimes to only an ounce, and sometimes exceeding a pound in weight. The eyst is generally oval in shape, its length being four or five inches, and the thickness, in some cases. heing half an inch. In proportion to 
its thickness, the corresponding surface of the cerebral hemisphere is clepressed, anemic, and not infrequently more or less softened. Small extravasations of blood also are often found beneath the arachnoid within the space corresponding to the hematoma.

The symptomatic phenomena embrace, first, those procecding from the inflammation prior to hemorrhage, and, second, those subsequently arising from the pressure of the hematoma on the surface of the brain. The affection may be divided into two periods, or stages, namely, before and after the hemorrhage. Cerebral symptoms belonging to the first of these periods are, cephalalgia, which is often notably intense, vertigo, tinnitus aurium, vigilanee, and disturbed sleep. There is muscular feebleness without paralysis. In general, contraction of the pupils exists in a marked degree. In young children there is febrile movement; after adult age this is wanting. Muscular contractions and convulsions are apt to oceur in children. 'This fever in children is short, lasting only a few days; in adults, on the contrary, it extends over weeks, and sometimes several montlis. The second period is distinguished by the supervention of symptoms denoting pressure. If the hemorrhage take place rapidly, it is likely to oecasion an apoplectic seizure from which the patient recovers. Repeated apoplectic attacks may occur, corresponding to suceessive extravasations of bloorl. If the hemorrhage take place slowly, it occasions somnolency, which continues for twenty-four or thirty hours, the mind, on awakening, being more or less confused. Suecessive periods of somnolence may mark repeated slow hemorrhages. Contraetion of the pupils persists during this period; and, if the hematoma be limited to one side, the contraction, although existing on both sides, is greater on the side corresponding to the seat of the affeetion. The pulse in this period becomes slow and irregular. Hemiplegia occurs in a certain proportion of cases, and several eases have been reported in which the hemiplegia was situated on the side of the lesion. In some cases paralysis is limited to the face, and it may be but temporary, sometimes, however, returning. Muscular contractions and convulsions do not occur unless some other cerebral affestion coincide with the hematoma.

The affection cannot be diagnosticated in some cases in which, during the first time, there are no marked cerebral symptoms, and a positive diagnosis is hardly ever warrantable. In young children it is likely to be confounded with tuberculons meningitis. Points which distinguish it from the latter are, a greater intensity and continuity of febrile movement, absence of constipation, and vomiting either slight or wanting. Convulsions are apt to be more prominent, and it is stated by Legendre that contraction of the muscles connected with the hands and feet is common. In adults, a probable diagnosis is to be reached by excluding other affections. The long duration of cephalalgia, without febrile movement, convulsions, or paralysis, points to this affection rather than to meningitis or cerebritis. Cerebral hemorrhage and embolism are excluded by the existence of cerebral symptoms prior to apoplexy or paralysis. The history, as regards paralysis affecting successively different parts, differs from that of tumors of the brain. 'The occurrence of successive attacks of apoplexy or prolonged somnolency is somewhat diagnostic.

Points relating to etiology are to be taken into account in the diagnosis. The affection is almost exclusively limited to infancy or early childhood, that is from one to five years of age, and to persons advanced in years; and in the majority of cases it occurs in males. In children the want of evidence of any tendency to tuberculous disease is a point to 
be considered in the differential diagnosis of this affection and tuberculous meningitis. In adults it has been observed to occur among insane persons, and among those addicted to the abuse of alcohol. It occurs, also, intercurrently in some cases of rheumatism and of the essential fevers. These facts are to be borne in mind in an endeavor to arrive at a diagnosis. The affection may be of traumatic origin.

The affection ends fatally in most cases. It proves fatal after a short duration in young children. In adults, more or less of the hematoma may be absorbed, and the brain become tolerant of the pressure, so that, even if complete recovery do not take place, life may continue indefinitely, with but few, if any, cerebral symptoms. As a rule, however, sooner or later the patient falls into a condition of persistent coma, ending in death.

Measures of treatment, in the first period, are those indicated by the existence of a circumscribed and, excepting in young children, subacute and chronic inflammation. Depletory measures, namcly, bloodletting and active cathartics, are rarely, if ever, advisable. Counter-irritation over the nucha may be useful, and should be tried, since, if judiciously employed, it will not do harm. The iodide of potassium and the bromides are to be tried. If not contra-indicated by the general condition of the patient, mercurialization may be resorted to. In the second period, an object of treatment is the absorption of the effused blood. Possibly the remedies first named may be useful with reference to this object. The prognosis, however, is so unfavorable that there is little encouragement to expect more than palliation of pain or other symptoms, and the prolongation of life by analeptic treatment. ${ }^{1}$

\section{Acute Cerebral Meningitis.}

The name acute cerebral meningitis denotes, conventionally, inflammation of the meninges of the brain exclusive of the dura mater, that is, inflammation affecting the arachnoid membrane and the pia mater. The name is applied to acute meningitis not associated with tuberculous disease. To distinguish it from the so-called tuberculous meningitis it may be designated simple meningitis. By some authors the name fibro-purulent meningitis is applied to it. As regards frequency, it is to be reckoned among the very rare affections.

Anatomical Characters. - The appreciable local results of acute inflammation in this situation are those of serous inflammation, viz., more or less redness from vascular injection, and the presence of serum, coagulated fibrin or Iymph, and pus. These inflammatory products, however, are beneath, not upon, the arachnoid membrane, that is, they are deposited in the meshes of the pia mater. 'The redness is from the injection of the vessels of the pia mater, not from red globules in the arachnoid. Lymph, in more or less abundance, is spread over the surface of the brain under the arachnoid. It is observer especially in the sulci between the convolutions, at the superior and lateral portions of the cerebrum, being less abundant or absent at the base of the brain. The presence of pus is denoted by a greenish color. A small quantity of exudation may be scraped from the outer surface of the visceral arachnoid, and, exceptionably, it is

1 This notice of pachymeningitis and hematoma of the dura mater is based mainly on Jaccoud's account (Pathologie Interne). Other authors, however, have been consulted. 
more or less abundant here, as well as beneath this membrane. The outer surface of this membrane is sometimes abnormally dry. There is more or less effusion of turbid serum in the subarachnoid space, rendering the arachnoid membrane opaque. The membrane is more easily detached than in a healthy condition. The ventrieles may contain a small or moderate quantity of turbid serum, or they may be empty. The cortical portion of the brain is sometimes softened, and it is sometimes infiltrated with either fibrinous exudation or pus. In most cases the two hemispheres are affected, and, often, the inflammation extends equally on the two sides ; it is, therefore, a bilateral afferstion.

It is to be borne in mind, in examinations after death, that the presence of serum in considerably larger quantity than is usually found beneath the arachnoid membrane is not adequate proof of meningitis. This occurs from atrophy, or wasting of the brain, incident to chronic disease in any part of the body, or from defieieney of blood in the vessels within the cranium. The criterion of inflammation is either lymph in quantity to be appreciable by the naked eye, or pus. Nor is mere opacity of the arachnoid meinbrane enough. As remarked by Rokitansky, "opacity and thickening of the arachnoid are very common post-mortem appearances; after midclle life, a moderate degree of them is almost constantly found, and their absence is the exception." In autopsical examinations, meningitis is apt to be considered as having existed on insuffieient grounds.

Clinical History.-Simple acute meningitis may be abrupt or gradual in its development. The premonitions are the symptoms of determination of blood or active congestion, and this, in fact, is the morbid condition. After the development of inflammation, there is a marked variation in symptoms at different periods of the disease, viz., prior to and after the sero-fibrinous exudation. The career of the disease is divisible into two stages, the clivision being based on the occurrence of the produets of inflammation. The first stage extends to the period when, owing to the pressure of these products, a marked change in the symptoms occurs.

The first stage has been distinguished as the " stage of excitement." It is characterized by pain, usually very intense, referred to the entire head, sometimes greatest in the anterior and sometimes in the posterior portion. The pain is the same as in cases of active cerebral congestion. Delirium frequently occurs; and the delirimm, in this stage, is generally active or maniacal, but in some cases hilarious. Owing to the prominence of the delirium, patients are sometimes carried to insane institutions, the affection being mistaken for functional mania. Convulsions may oceur, especially in young subjects. The special senses of sight and hearing are morbidly acute; light and sounds occasion distress, and increase the cerebral excitement. The eyebrows are corrugated to shield the eyes from light. 'The face is flushed. 'The carotids and temporal arteries pulsate strongly. The head is hot. Vomiting generally oecurs in this stage, and is often prominent as a symptom. The bowels are usually constipated. The abdominal walls are depressed. Febrile movement is more or less marked; the pulse is accelerated, strong, and full, and the axillary temperature may rise to or above, $104^{\circ} \mathrm{F}$. Exacerbations occur in which the delirium and cephalalgia are notably increased. The symptoms, in general, are those of active congestion, differing only in being more intense and persisting. The duration of this stage is short. It may continue but a few hours, and rarely beyond two or three days. 
The second stage has been called the "stage of oppression." The symptoms distinctive of this stage are those denoting pressure from the inflammatory products. 'The pain in the head is diminished. The morbid sensitiveness to light and sounds is lessened. The pupils may be dilated or contracted, and their respondency to light is diminished or lost. Strabismus and loss of vision may occur. Drowsiness succeeds the state of mental excitation. 'The pulse becomes slow and perhaps irregular. The respirations are disordered in rhythm and suspirious. Paralysis may occur, limited to the face, or extending over one side of the body. If the progress of the disease be unftrorable, coma is at length incluced and continnes until death. Convulsions may occur in the second, as well as in the first, stage.

Pathological Character.-The disease is essentially the same as acute inflammation affecting serous membranes in other situations. During the first stage, the distinctive symptomatic phenomena proceed fiom excessive and disordered activity of the cerebral functions. In the second stage the brain suffers from compression. This mechanical effect of the inflammatory products is not peculiar to inflammation in this situation, but, owing to the unyielding bony case which contains the brain, a small amount of effusion and exudation is vastly more serious than a much larger amount in other situations.

Causation.-A side from traumatic cases and those in which the disease is attributable to insolation, it is difficult to specify causes of this disease. Varions causes are named by different writers, but a difliculty arises from the fact that other affections liave been confounded with acute meningitis. It may arise from injuries of the head, and it appears to be sometimes produced by exposure to the direct rays of the sun. An analysis of a considerable number of cases, the diagnosis of which is not open to doubt, is a desideratum. It is probable that excessive indulgence in alcolıolic drinks may give rise to it. Of the cases which occur, very few are in early infancy. According to Barthez and Rilliet, examples in children are found between the ages of five and eleven. And according to Guersant, after the age of childhood, it occurs chiefly between adolescence and forty-five. 'The larger proportion of cases are among males.

Acute meningitis is sometimes developed in connection with acute articular rheumatism. Occurring in this commection, it is considered by some authors as a variety of the disease under the names rheumatic meningitis and cerebral rheumatism. The clinical history differs from that of acute meningitis not associated with rheumatism, in either the absence, or a much-less prominence, of cephalalgia and vomiting. In some cases the progress from the first to the second stage is notably rapid. Hallucinations are sometimes a marked feature, with tremulousness of the tongue and lips, as in cases of delirium tremens. Tremor of the muscles of the limbs is sometimes a marked feature. 'This, with pains in the back, points to inflammation of the spinal, as well as of the cerebral, meninges; and autopsical examinations have shown, in some cases, the existence of cerebro-spinal meningitis. Taking into account these distingnishing characters, it is perhaps fair to conclude that the causation involves the essential pathological condition in rheumatism; in other words, that the meningitis or cerebro-spinal meningitis is due to the same internal cause which determines the arthritic manifestations. The occurrence, however, of inflammation of the meninges of the brain, or of the brain and cord, in 
connection with acute articular rheumatism, is so rare that, judging from this point of view, the concurrence would be considered purely accidental.'

Dragnosis.-Acute meningitis may be confounded with active cerebral congestion. The pathological condition prior to exudation in meningitis differs from active congestion only in degree and persistency. If the inflammation go off by delitescence, that is, without passing into the second stage, it may be impossible to say, after recovery, whether the affection was meningitis or merely active congestion. In other words, more or less of the symptums belonging to the second stage are required for a positive diagnosis. Practically, it is not highly important to distinguish between active congestion and the first stage of meningitis, inasmuch as the same measures of treatment are indicated in either case.

Cases of typhoid fever characterized by early and active delirium, have, heretofore, not infrequently been considered as cases of acute meningitis. The points involved in this differential diagnosis are: The access or slow development of the fever; the distinctive events belonging to it, viz., diarrhoea, meteorism or tympanites, tenderness in the iliac regions, together with absence of morbid acuteness of the senses, and continuance of the delirium without the supervention of the phenomena of the second stage of meningitis. 'These points show the discase to be fever, and not meningitis.

Functional mania may be considered as denoting acute meningitis. The discrimination is to be based on the absence of morbid acuteness of the senses, together with the absence of febrile movement, and the continuance of the mania without the symptoms of compression.

Otlier affections are more likely to be considered acute meningitis than this to be mistaken for other affections. 'The ability of a practitioner to discriminate this from other afiections may fairly be suspected, if he believe that he has met with many cases of it.

Prognosis.-Acute meningitis involves much danger. It may destroy life quickly. Cases have terminated fatally in 36 hours. As a rule, the duration, in fatal cases, does not extend beyond eight or nine days. The symptoms denoting an unf.uvorable prognosis are, profound coma, notable disorder of respiration, difficulty of deglutition, general immobility and anæstlıesia, feebleness of the pulse, etc. Strabismus, hemiplegia, and convulsions may be present in cases which recover. In two cases received into Bellevue Hospital in 1862, reported by the late Dr. F. II. Lyman, one of the cases being in my service, and the other in the service of Prof. Thomas, a semi-comatose condition existed; in one case there was hemiplegia, in the other case strabismus and convulsions. Both cases.recovered. ${ }^{2}$ Marked strabismus existed in a case, ending in recovery, which I saw in 1862, with Dr. Purcell, of this city, the patient being a child 11 years of age. Data are wanting for determining the ratio of cases which end fatally, but it is certain that the fatal cases greatly preponderate. The association with acute articular rlıeumatism is considered as rendering the prognosis extremely unfavorable. I have, lowever, observed a wellmarked case, the symptoms denoting spinal as well as cerebral meningitis, which ended in recovery.

In cases which prove rapidly fatal, death may be due chiefly to apnoa,

1 For an analysis of 31 cases, vide Pathologie Interne, par E. Gintrac, tome huitième, 1869 .

\& American Miedical Times, vol. iv., 1862. 
but, if life be prolonged for several days, the mode of dying is by apnœa and asthenia combined.

Treatuent.-The treatment must have reference to the stage of the disease. 'The objects in the first stage are to lessen the determination of blood to the head, and to limit the amount of exudation. If the heart act with abnormal power, bloodletting, in this stage, is to be employed. As a measure of depletion, and perhaps revulsion, active purgation is indicated. Cold is to be applied to the head, either by means of the ice-cap, the cold douche, or constant ablution. Cutting the hair close to the head renders these applications more efficient. If, as appears to be shown, the bromide of potassium has the power of diminishing the amount of blood within the cranium, this remedy is rationally indicated. Hammond bears testimony to its value, as a remedy in this disease, and advises it to be given in doses of thirty grains three or four times daily.'

Bloodletting, in this disease, is indicated in view of the promptness of its operation and the short duration, frequently, of the first stage. Bearing in mind that the chief source of danger is from the products of inflammation, it is important that the measures employed with the hope of limiting these, should be resorted to more promptly and vigorously than in other acute inflammations, for example pneumonitis, in which the products of inflammation generally do not give rise to serious results. Moreover, there is not the same necessity for guarding the vital forces as in other acute inflammations; for in this disease there is danger that life may be quickly destroyed by apnœa caused by the pressure of effused serum and lymph, whereas in most other acute inflammations the danger is that they will destroy life by asthenia. It is not, however, to be forgotten that asthenia enters into the mode of dying in cases of meningitis which do not pursue a rapid career, and, hence, bloodletting and other measures of depletion may be pushed too far. These measures are only indicated in the first stage of the disease. Perfect quietness, exclusion of light, elevation of the head, and the avoidance of everything which may tend to increase the cerebral excitement by acting on the senses or mind, form an important part of the treatment of this stage. The existence of intense cerebral excitation suggests the inquiry whether it may not be advisable, in the first stage, to endeavor to allay the excitement by anodyne remedies. It remains to be ascertained by experience, whether opium and the inlalation of chloroform may not be nseful. Vomiting calls for palliative measures. The diet, in this stage, should be restricted to the blandest articles, taken cold.

In the second stage, the great object of treatment is to promote absorption of the morbid products, serum and lymph. Depletion is no longer admissible. Mercurialization is generally advised for this object, and, although the efficiency of mercury as a sorbefacient has doubtless been much exaggerated, we are not warranted in saying that it has no effect in that way. If it exert ever so small an effect, it is indicated in a disease like this, in which the object to be effected is of so much importance. Iodine is another remedy supposed to be useful, as a sorbefacient. I have witnessed, as I think, the good effect of the iodide of.potassium in several cases. In the case in my service at Bellevue Ifospital, reported by Dr. Lyman, and the case seen with Dr. Purcell, to which reference has been made, the patients recovered under the use of this remedy, mercury not being employed in either case. It may be given in doses of

1 Vide Treatise on Diseases of the Nervous System. 
from five to ten grains four or five times daily, increasing the doses, if improvament do not take place, up to the limit of tolerance. A blister may be applied over the nucha. The propriety of blistering the scalp is questionable.

Another object of treatment in the second stage, if death do not take place by apnati, is to support the powers of the system. Measures for this object are indicated in proportion as the tendency to death is by asthenia. Attention to the bladder is important in the second stage.

\section{Chronic Cerebral Meningitis.}

Simple chronic cerebral meningitis, that is, meningitis not incident to a tuberculous or any prior cerebral affection, is infrequent, although less rare than the acute form of the disease. This statement has reference to periods of life after infuncy, but it holds good also with respect to the latter period. In a great majority of cases, it is a subacute affection $a b$ initio.

The anatomical characters are essentially the same as in simple acute meningitis, consisting of the presenee of more or less serum and lymph, or pus, beneath the arachnoid, this membrane being thickened and opaque, together with more or less turbid liquid within the ventrieles. In some cases the attachment of the pia mater and arachnoid to the brain is sueh that, in detaching the membranes, more or less of the cortical substance is removerl with them. A large effusion into the ventricles may be accompanied by so small an amount of lymph that the latter without careful inspection is liable to be overlooked.

This affection may be ranked among the "insidious diseases." The symptoms may not point to any cerebral affection, or they are not distinetive of tlis form of disease. 'The diagnosis is diflieult. Pain in the head is usually more or less prominent as a symptom, but this is a symptom common to many affections. 'There may be little or no febrile movement. Vomiting may be a prominent symptom, but this, too, oceurs in numerous pathological connections. Mental apathy, amounting sometimes to stupidity, indisposition to either mental or physical exertion, and a notable cliange in clisposition, characterize certain cases. Paralysis of the face and hemiplegia sometimes occur, but they are effects of other forms of disease. This statement also applies to epileptiform convulsions.

I have known a case of chronic meningitis to be considered a case of chronic gastritis, vomiting being a prominent and persistent symptom. The patient fell into coma before death, but this was attributed to exhaustion. Meningitis was not suspected prior to the autopsy. In another case in which an ante-mortem diagnosis was not made, the patient dying suddenly with apoplexy from extravasation, the case had been considered one of latent or irregular intermittent fever, clills oceurring from time to time. Quite recently a patient was admitted into my division at Bellevue Hospital who had no symptoms referable to the head excepting mental inertia bordering on imbecility. He gradually fell into coma, and, after death, the case was ascertained to be one of chronic meningitis.

As regards diagnosis, close observation ought to lead to the conclusion that there exists some chronic cerebral affection, but the practitioner is hardly censurable for not being able to decide whether there be simply meningeal inflammation or some other chronic affection. Paralysis is less likely to occur than in cases of softening or of tumors within the skull. The absence of paralysis is, therefore, of some account in the diagnosis. This statement will measurably apply to convulsions. If cephalalgia, 
impairment of the mental faculties, and gradually developed coma follow an injury of the head, chronic meningitis is to be suspected rather than cerebral softening or tumors; a traumatic cause is more likely to give rise to inflammation than to the lesions just named. Chronic meningitis is developed in a small proportion of cases of the affections of the kidneys embraced under the name Bright's disease. The existence of renal disease is, therefore, of some account in the diagnosis. But, in cases of renal disease, cerebral symptoms referable to uræmia, viz., epileptiform convulsions and coma, are not to be considered as evidence of meningitis. Intermittency of cerebral functions may render the diagnosis diflicult. The following facts, in a case which came under my observation, will serve to illustrate this: A lad, 15 years of age, had vomiting and chills with pain in the head and face. He became somnolent to a degree almost amounting to coma. He emerged from this condition and appeared convalescent. A return of the cephelalgia, vomiting, and somnolency occurred. In a few days these symptoms again disappeared, and he again seemed to be convalescing. The head symptoms, howaver, returned a third time, he became comatose, respiration and deglutition were notably affected, and death took place 48 hours after the last attack, a month after the commencement of his illness. The autopsy showed much cerebral congestion, a little lymph at the base of the brain, without tubercles, and the ventricles greatly distended with liquid which was nearly transparent. The fourth ventricle was dilated. There were no tubercles in the lungs. Variations in the amount of effusion within the ventricles, probably, will account for the intermittency of the symptoms, and the pressure on the medulla oblongata by the liquid in the fourth ventricle explains the disturbance of respiration and deglutition, and the speedy death, in the last relapse.

The treatment consists of moderate counter-irritation, the iodide of potassium, and perhaps mercurialization, quietude of mind and body, and the hygienic regulations appropriate to chronic affections generally.

\section{Turberculous Meningitis.}

The researches of Guersant in 1823, and of Gerhard and Rufz in 1833 and 1834 , led to the recognition of a variety of cerebral meningitis differing from the simple acute and chronic forms which have just been considered. A distinctive feature of this variety is the presence of miliary granulations, in connection with meningeal inflammation. Hence the name tuberculous or tubercular meningitis. It has also been called granular meningitis. These names denote the connection of the meningeal inflammation with the semi-transparent, grayish bodies known as miliary granulations or gray tubercles. It is described by Troussean under the name "cerebral fever." This variety of meningitis affects chiefly children, and is treated of fully in works devoted to infantile cliseases. It is of rare occurrence during the first year of life, occurring especially after two, and under seven years of age. It is not, however, confined to childhood, and I shall therefore briefly consider it.

A distinctive feature of tuberculous meningitis is its situation in the majority of cases at the base of the brain. In this respect it differs from a simple acute or chronic meningitis, the latter affecting especially the superior and lateral portions of the cerebral hemispheres, and hence, it is called by some authors "basilar meningitis." The fibrinous exudation, very rarnly accompanied by pus, is found beneath the arachnoid membrane covering the pons Varolii, optic commissures, cerebellum, ctc., and 
notably in the fissure of Sylvius. The inflammation, however, may extend to the convex surface of the cerebrum. The amount of exuclation varies much in different cases. It is sometimes extremely small. The most characteristic anatomical feature is the presence, in greater or less number, of minute granulations which are generally considered to be gray tubercles, identical with the bodies, so-called, which are found in the lungs, the spleen, and other organs. In some cases of tuberculous meningitis, these granulations are so extremely minute that they are liable to be overlooked without close inspection. From their being usually of the size of millet seeds, they have been called miliary granulations. In some cases in which they cannot be discovered, it is possible that they are concealed by being imbedder in the inflammatory exudation. They are situated in the tract of the cerebral vessels, and, according to Bastian and others, within the perivascular sheaths peculiar to the vessels of the brain. There is a difference of opinion among pathological histologists as to whether these granulations are veritable gray tubercles. According to some observers, the microscopical characters show the two to be identical. But the constancy with which, associated with them, are gray tubercules in the lungs and other organs, goes to show the tuberculous character of the meningeal affection. In some cases, granulations in this situation are not accompanied by fibrinous exudation. In other words, the granulations may be developed here without other appearances denoting tuberculous meningitis. It would seem to be a fair inference from this fact that, when, as is usually the case, the granulations are combined with the ordinary products of a serous inflammation, namely, serum and lympl, the latter are secondary to, and dependent on, the former. Other morbid appearances are, congestion of the vessels of the pia mater and cerebral substances, more or less serous effusion beneath the arachnoid and into the ventrieles, the effused liquid generally turbid, flattening of the convolutions in proportion to the amount of ventricular effusion, and softening of the brain at different points due to pressure, to orlematous infiltration of the effused liquid, and also, in some cases, to an extension of inflammation to the cerebral substance. The intra-ventrienlar effusion is generally considerable, and sometimes quite large; and this distinguishes tuberculous from simple meningitis. It is owing to this that the affection has been heretofore included under the name "acute hydrocephalus." Minute extravasations of blood are, also, sometimes observed. In the majority of cases, granulations, or gray tubercles, with or without the product known as yellow tubercle, are found in the lungs, and frequently in other organs, viz., the spleen, liver, etc.

In the progress of this, as in simple meningitis, the symptoms undergo a marked change due to the pressure of the exudation and cffused liquicl; and this change will serve to divide the career of the affection into two stages. 'The development of the meningitis may be preceled by the symptoms and signs of pulmonary tuberculosis. Probably the latter always exists as an antecedent affection after infancy and childhood. The meningeal affection, when it does not distinctly supervene upon tuberculous disease of the lungs, is usually preceded by pallor, loss of weight, impaired appetite, irritability of temper, muscular debility, indisposition to exertion, and other general symptoms for a variable period. But, in a certain proportion of cases, the symptoms denoting the head-affection appear suddenly, the previous health having been apparently good. When the meningitis occurs after a prodromic period extending, as it does in some cases, over several weeks and even months, during which the general health becomes considerably impaired, an antecedent tuberculous aftection 
in other organs is to be inferred; or, it may be that the miliary granulations within the skull existed for some time prior to the development of meningeal inflammation. The latter is probable if the prodromic period have been marked by cerebral symptoms. On the other hand, the meningitis, when it is developed abruptly, that is, without a prodromic period, the previous health having been apparently good, is incident to a primary local expression of the tuberculous cachexia within the skull, or, at least, occurring simultaneously with manifestations in the lungs and other organs.

The development of meningitis, as a rule, is denoted by pain in the head, referred generally to the forehead. Convulsions are less likely to occur at the onset than in simple acute meningitis. Vomiting, in most cases, is an early, and sometimes a prominent symptom. More or less febrile movement occurs. The axillary temperature is raised, but the rise is less than in simple acute meningitis. Notable diurnal remissions are apt to occur, the exacerbations taking place at night. These symptoms continue for several days. The cephalalgia is frequently severe. Lancinating pains occur from time to time, causing infants to give a sudden, sharp, and short cry, which has been known as the cephalic cry. In children able to speak, a common exclamation is, "Oh! my head." The hands are often carried to the head. There is increased sensibility to light and sounds. The expression is frowning. In infants, raising the lids and exposing the eyes to the light occasion erying. The conjunctiva is often suffused. The pupils in this stage are frequently contracted. The face may be flushed, and, in some cases, paroxysmal flushing of the face is a notable symptom. I have observed this to precede convulsions. During this stage, vigilance is frequently a prominent symptom, and the sleep obtained is disturbed by dreams causing patients to awake suddenly in terror. It is important to note that vomiting and increased sensibility to light, although generally marked, may be wanting.

Drowsiness and somnolency mark the supervention of the second stage. There is now less suffering from cephalalgia, but occasional lancinating pains may still occur. The pulse may fall below the average frequency of health. It is not infrequently faltering; it fluctuates in frequency, and may be irregular in rhythm. The respirations which, in the first stage, may have been accompanied by sighing, are now often irregular in rhythm. In young children, masticatory movements are common, and after dentition, grinding of the teeth. Rolling the head, or boring the pillow, is a symptom in infants. The somnolency increases. The patients are reluctant to be aroused. If able to speak, they reply to questions slowly or in monosyllables, like a person half awakened from profound sleep. When aroused, they have an expression of stupidity. Incoherence is common, and occasionally drowsiness alternates with paroxysms of delirium. The pupils become dilated, and not infrequently present disparity as regards size. They respond sluggishly to light. As the somnolency deepens, the movements of the eyeballs are not in unison, strabis. mus frequently occurs, and oscillations of the balls are occasionally observed. The eyelids are but partially closed, the eyeballs being raised so that the cornea is partially covered by the upper lid, and in some cases the patients lie with the lids widely opened. Vision is gradually impaired, and frequently lost. Subsultus, carphologia, and fumbling with the bed-clothes are frequent symptoms. Convulsions are apt to occur, especially in children. They are either limited or general, and may be either severe or slight. Paralysis of one side of the face, and incomplete hemiplegia, are occasionally observed. Not infrequently the muscles of 
the posterior part of the neck become rigid, and the patient lies with the head drawn backward. Contraction of certain of the muscles of the limbs occurs in some cases. This is sometimes observed on one side, while the muscles of the opposite side are relaxed. Occasional vomiting is a symptom of the second stage. As a rule, there is complete anorexia, but, in some cases which I have observed, food has been taken with apparent relish. The teetl and lips often become covered with sordes. Constipation is the rule. In young children the dejections are sometimes greenish, and resemble spinach in appearance. The abdomen is retracted. Retention of urine is not uncommon.

Sooner or later the patient becomes comatose. But, in some cases, before the occurrence of coma, remissions occur, in which the improvement, as regards intelligence and in other respects, is such as to appear to the friends to afford ground for much encouragement in hoping for recovery. The occurrence of profound coma is followed by impairment and loss of deglutition, with more or less embarrassment of respiration. The pulse becomes frequent and feeble, and the mode of dying is by apnœa and asthenia combined.

The duration is variable. It rarely falls short of a week. In the majority of cases, it does not exceed two weeks. It rarely extends beyond three weeks, but, exceptionally, it may be prolonged considerably beyond this period. The termination is almost invariably, if not invariably, fatal. In the cases of supposed recovery which have been observed, it is probable that the affection was simple meningitis.

The diagnosis involves the discrimination from an essential fever. With the latter it is not infrequently confounded. In making this differential diagnosis, meningitis is to be excluded by the absence of sensibility to light, of the characteristic lancinating pains which belong to the first stage, and of the strabismus, affection of pupils, and other symptoms belonging to the second stage. In fever, drowsiness and delirium are not preceded and accompanied by the symptoms just referred to. Noreover, in typhoid fever, diarrhœe, abdominal tympanites, and iliac tenderness are likely to be present, symptoms not belonging to the clinical history of tuberculous' meningitis.

It is to be discriminated from the so-called hydrencephaloid affection incident, in children, to exhaustion from diarrhœa. With this affection it was formerly confounded. In this affection the symptoms denoting inflammation are wanting, and the drowsiness or stupor is not accompanied by strabismus, inequality of the pupils, embarrassment of respiration, irregularity of the pulse, and other symptoms, exclusive of the somnolence, which belong to the second stage of tuberculous meningitis.

To discriminate tuberculons from simple meningitis, is by no means always easy. The gradual development of the former, the prodromic symptoms, the longer duration of the first stage, and the coexistence of pulmonary tubereulosis, in a certain proportion of cases, must serve as a basis of this differential diagnosis. 'The meningitis is probably simple if it can be distinctly traced to a traumatic origin. It is, however, to be borne in mind that, in most cases, it is practicable to recall some injury of the head within a period of weeks or months prior to the occurrence of any disease in childhood; and it is customary to refer the affection to an injury often when there is not sufficient ground for such an opinion.

If the diagnosis be positive, the encouragement for successful treatment is exceedingly small. It is difficult, of course, to decide upon measures which will be likely to be useful in a disease tending intrinsically, like this, to a fatal issue, and when, in cases of apparent recovery, we are 
obliged to distrust the correctness of the diagnosis. The chief source of encouragement for successful treatment, in fact, in a case which the practitioner supposes to be one of this disease, is the possibility that the diagnosis may be incorrect, the affection being simple meningitis. With reference to this liability to error in diagnosis, the measures indicated in simple meningitis are admissible. If, however, the practitioner be thoroughly convinced of the correctness of the diagnosis, he is hardly justified in resorting to bloodletting, or measures which occasion suffering, such as blisters and frequent eatharties. Under these circumstances, measures to palliate distress and prolong life are alone indicated. In this country the iodide of potassium is generally given largely in the second stage of the disease. Cases have been reported in whieh this remedy was apparently successful ; but there is room for the supposition that in these cases either meningitis did not exist, or that the disease was simple meningitis. It is eertain than in the immense majority of cases the remedy is unsuccessful.

\section{Spinal Meningitis.}

In this affection the spinal membranes corresponding to those affected in cerebral meningitis, namely, the arachmoid and pia mater of the cord, are the seat of an inflammation which may be either acute, or subacute and chronic. Inflammation limited to the meninges of the cord, that is, the cerebral membranes not involved, exclnding cases of circumscribed spinal meningitis incident to injuries or diseases of the spine, and to structural affections within the spinal canal, is exceedingly rare. This statement is applicable both to aeute and chronic inflammation. Rare as is cerebral meningitis, spinal meningitis is still more infrequent. The anatomical characters of acute inflammation of the meninges of the cord, are essentially the same as in cerebral meningitis. The inflammatory products are within the inner sac, that is, between the spinal arachnoid membrane and the pia mater. The products are fibrinous exudation, pus, and more or less serous effusion. The latter is sometimes sanguinolent. Pus is present in only a certain proportion of cases. In chronic meningeal inflammation, the appearances are less marked, consisting of an increase of the subarachnoidean liquirl, which is sometimes opaque and sometimes bloody, with newly-formed membrane, which may occasion adherence of the membranes, or bridles of attachment. In acute spinal meningitis the inflammation, as a rule, extends over the whole or a greater part of the meninges of the cord. In chronic spinal meningitis the inflammation is not infrequently limited to a portion of the meninges.

The local symptoms of acute spinal meningitis, that is, symptoms standing in immediate relation to the affection, are chiefly referable to effects manifested throngh the spinal nerves; and they correspond to the functions of the posterior and anterior roots of these nerves. More or less pain is felt in the spine, and radiating therefrom to the extremities. The pain is increased, not so much by pressure over the spinal column, as by movements of the borly. The sensibility of the surface is morbidly increased (hyperæsthesia). These effects are manifested through the posterior roots of the spinal nerves. Other effects are manifested through the anterior roots, - these are tonic contraction of the muscles of the extremities, clonic in some cases, spasm of the muscles of the back so as to give rise to opisthotonos, and sometimes rigidity of the thoracic muscles causing dyspnœa. As regards the muscular contractions or spasms, there are exacerbations and remissions. These symptomatic phenomena are 
attributable to a morbid excitation of the spinal nerves, and they belong to the early part of the affection. Subsequently, paralysis may occur as the effect of pressure upon the cord by the products of the inflammation. 'The paralysis may be limited to the lower extremities (paraplegia), or it may be general, that is, affecting both the upper and lower extremities. The paralysis is rarely complete. It is usually limited to motion, and with it, indeed, exaggerated sensibility or hyperæesthesia may persist, as well as the tonic contraction of muscles. With these local symptoms there is more or less fever, as denoted by the axillary temperature, together with anorexia and prostration. The pulse is sometimes abnormally infrequent, and this may be found in striking contrast with the temperature of the body as determined by the thermometer. In the course of the disease, however, the pulse becomes frequent and feeble. Copious perspiratious are apt to oecur. If paralysis take place, reflex excitability and electric contractions are generally preserved.

The diagnostic features point to the spinal cord as the seat of the disease, and denote an inflammatory and grave affection. Hysterical opisthotonos lacks the evidenees both of inflammation and of gravity. Moreover, various antecedent and concomitant phenomena betoken the existence of hysteria. It is to be differentiated from tetanus, which is a very grave disease. The latter, however, is distinguished by the early occurrence of trisinus and tonic spasms; by the absence of local and general symptoms denoting inflammation, and by the tranmatic origin of the affection in the majority of cases. The diagnostic points involved in discriminating acute spinal meningitis from acute myelitis will be noticed in treating of the latter in the next chapter. It may, however, be remarked here that acute spinal meningitis and acute myelitis very rarely occur independently of each other. 'The two affections are generally associated; but the inflammation may be primarily seated in either situation, and it may predominate in one or the other.

Acute spinal meningitis runs a rapid course, its duration rarely extending beyond a week. It ends fatally as the rule. Life is destroyed by apncea in some cases in which the respiratory function is notably compromised by either spasm of the muscles concerned in respiration or an extension of the inflammation to the medulla oblongata. If the latter occur, dysphagia is likely to be a symptom. In either case death is preceded by intense dyspnnea, which, on a physical examination of the chest, is found not to proceed from any adequate pulmonary or eardiae complication. In other cases, asthenia predominates in the mode of dying.

The indications for treatment are essentially the same as in acute cerebral meningitis. Considering the rapid course of the disease, and the danger in the direction of apnca, bloodletting, general or local, or both, may be employed, if contra-indicating circumstances, relating to the pulse, the quality of the blood, and the constitutional strength, are not present. Mercurialization is warrantable, if not desirable. If employed, it should be rapidly induced; and, for this end, inunction may be added to the administration of calomel at short intervals. Ergot and belladonna are supposed by Brown-Séquard and others, to diminish hyperemia, by causing contraction of the small arteries, and, in this way, to be useful in inflammatory affections within the spinal canal. When the symptoms denote the presence of the products of inflammation, the vesieation, successively, of different sections of the spine, and the iodide of potassium in large doses are indicated. Sustaining treatment is, of course, indieated in proportion as the duration of the disease is extended, and the symptoms show a tendency to death by asthenia. 
Chronic spinal meningitis is a much less rare affection than the acute form of the disease. It follows from this fact, in connection with the fact that the acute affection generally ends futally after a rapid course, that in most cases of the chronic affection the inflammation from the beginning is subacute. In the chronic affection the inflammation rarely extends over the whole of the meninges of the cord, buc it is limited to a circumscribed space.

The symptoms will be noticed, in the next chapter, in treating of paraplegia and general spinal paralysis. The affection is chiefly important in that pathological connection. The treatment having reference to the meningeal inflammation, embraces counter-irritation, perhaps mercurialization, and the iodide of potassium.

\section{Cerebro-Spinal Meningitis.}

With acute inflammation of the meninges of the cord is almost always associated cerebral meningitis. The affection is then known as cerebrospinal meningitis. As a sporadic affection, it is extremely rare. A few cases only have fallen under my observation.' It occurs chiefly as an epidemic. Within the last quarter of a century, epidemic cerebro-spinal meningitis has prevailed, at different periods, in various parts of this country. Within and prior to the period just named, epidemics have occurred repeatedly in Great Britain and on the continent of Europe. Of late years this affection has excited attention anew in this country, in consequence of its reappearance in certain districts of Pennsylvania, New York, and other parts of the United States. It has been lately proposed to call the affection by another name, to wit, spolted fever. This name is applied by some writers at the present time to an epidemic affection which appears to be identical with that generally known as epidemic cerebro-spinal meningitis. For reasons which will be stated under the head of the pathological character of the affection, it seems to me preferable to continue to use the latter name. The name spotted fever derives its significance from the occurrence of petechial spots in a certain proportion of cases. It was applied, in this country, to an epidemic affection which prevailed in New England between the years 1807 and 1816 ; and it has been revived under the belief that the epidemic disease just referred to was the same as that prevailing at the present time.

Anatomical Characters.-As the name imports, the affection is characterized by meningeal inflammation within the skull and spinal canal. In the majority of cases, ample evidence of cerebro-spinal meningitis is afforded by the appearances after death. This evidence is the presence of the inflammatory products, lymph or pus. The exurlation in some cases is abundant and extensive; the greater part of the surface of the brain and cord has been found covered with a thick layer of lymph, situated chicfly beneath the arachnoid membrane, and extending into the sulci between the convolutions. In other cases, the exudation is more or less limited as regards amount and extent. When limited, it is oftener found at the base of the brain than upon its superior and lateral aspects. It is found especially in the tract of the vessels. The lower surface of the cerebellum is apt to be affected. Covering the medulla oblongata, the lymph or purulent matter may extend over the whole length

1 For an analysis of 48 cases of sporadic cerebro-spinal meningitis vide Pathologie Interne, par E. Gintrac, tome septième, Paris, 1868. 
of the corl; or a section only of the corl, more or less in extent, may present evidence of inflammation. When only a section of the cord is affected, it is oftener the lower than the upper part, but the reverse is true of a certain proportion of eases. The ressels of the pia mater and the substance of the brain and cord are congested. Ames noted in his autopsies a change of the vesicular neurine to a pink hue. ${ }^{1}$ Softening of the brain and cord at certain points is observed. A puruloid liquid is sometimes found in the ventricles and within the arachnoid cavity.

These appearances, essentially the same as in simple acute meningitis, differ much in different cases. The inflammatory products, lymph or pus, are sometimes wanting. This was true of one out of eleven eases examined after death by Ames. In a single autopsy witnessed by Gerhard, there were no products of inflammation within the skull or spinal canal. The absence of $1 \mathrm{ymph}$ and pus in a certain proportion of cases is attested, according to Valleix, by all European observers. In these cases, the morbid appearances relating to the nervous eentres consist of more or less congestion and serous effusion. 'The explanation of the absence of exudation, in at least a part of the eases in which it is wanting, is to be found in the short duration of the disease, death sometimes occurring within a few hours. In some cases, during the prevalence of an epidemie, the morlsid appearances denote cerebral meningitis only, the meninges of the cord presenting no evidence of inflammation.

Morbid ehanges frequently exist in other parts of the body. Serous effusion, and sometimes purulent liquid, are found in the pleural and pericardial eavities, in the joints, and in the tuniea vaginalis. Spots of ecchymosis are observed upon the pericardium, beneath the peritoneum, and in other situations. Peyer's glands and the follicles of Brunner are abnormally distinct, but not enlarged from a morbid deposit, or ulcerated, as in typhoid fever.

An analysis of the blood in four eases, by Ames, showed an increase of fibrin from 3.64 to 6.40 in a thousand parts. In three cases the red corpuscles were diminished. The coagulation of the blood drawn in renesection was rapicl, the color was unusually bright, and of 37 eases the buffy coat was observed in only four.

Clinical IIstory.-The characteristic features of epidemic cerebrospinal meningitis, in its visitations at different times and places, are remarkably distinetive. In some cases the disease commenees with a sudden attack, the patient being at once stricken down with grave symptoms, but in many cases the access is more or less graulual, the development occupying from one to eleven days. Dr. Githens, who has reported ninety-eight cases in the American Journal of Medical Sciences, July, 1867, states that the disease eame on gradually, with about a week of prodromata. Cephalalgia is the most frequent precursor. Chills without rigors preceded in 28 of 64 cases analyzed by Ames. In most of the cases reported by Upham there was chilliness, but not a pronounced chill at the onset. ${ }^{2}$ Other prodromes are nausea and vomiting, pain in the spine (rachialgia), pain in the limbs, vertigo, and diarrhoea. 'These are enumerated in the order of their relative frequency. There is nothing diagnostic of the disease in the precursory ailments. Cephalalgia is usually the most prominent symptom when the disease is developed. It was wanting in only one of

1 New Orleans Med. and Surg. Journal, 1848.

2 Epidemic Cerebro-Spinal Meningitis, in the Camps in and around Newbern, in 1862-6\%. By J. Baxter Upham, M. D. Boston, 1863. 
64 cases analyzed by Ames. The pain is intense, lancinating, and accompanied with a sense of tension; it is referred either to the frontal region, the occiput, or the whole head. The pain persists without intermissions. It is increased by light, sounds, and movements of the body. Pain referable to the spine, or rachialgia, is generally added to the cephalalgia. The pain may extend over the whole of the spine, or it is referred to either the lumbar, dorsal, or cervical portion. The extent and situation of the spinal pain probably correspond with the extent and situation of the spinal meningitis. The rachialgia is wanting, if the disease prove fatal without the inflammation extending to the spinal membranes. The spinal pain is not always increased by pressure over the vertebral column, but notably by movements of the body. In some of the cases observed by $A$ mes, pressure on the cervical vertebræ increased the pain in the head, and pressing on the dorsal vertebræ caused pain in the epigastrium, sternum, and umbilical region. Pain is frequently felt, irrespective of pressure over the spine, in different parts of the body, and especially in the lower extremities. The pain in other situations than the head and spine is often intense. Hyperæsthesia of the surface of the body is, in certain cases, a marked symptom, rendering the slightest contact a source of suffering. Not infrequently vomiting accompanies the cephalalgia at the outset of the disease.

Delirium in some cases quickly superrenes, but in other cases after a variable period. Sooner or later it occurs in a large proportion of the cases which are not quickly fatal. It was noted by Ames in 24 of 64 cases, and by Levy in 42 of 60 cases. It varies much in character andintensity. At first the patient simply manifests difficulty in collecting his ideas, but after a time he either remains taciturn, making no response to questions, or he replies incoherently, or he becomes actively delirious, shouting and struggling against restraint. The latter form of delirium occurs in paroxysms, the patient being calm at intervals. The paroxysms of delirium occur especially at night. They are accompanied by hallucinations, in some cases, and insane delusions. Occasionally the delirium is hilarious. The mind is usually desponding and apprehensive, if the intelligence be preserved. Loquacity and erotic desires with priapism were observed in several of Upham's cases. Repeated seminal emissions oceurred in a case which came under my observation. In a certain proportion of cases, the patient becomes quickly somnolent and falls into coma, which may be of temporary duration, or continue until the disease ends fatally. More or less stupor, frequently amounting to coma, occurred on the first day in 18 of the 64 cases analyzed by $A$ mes. The vision is rarely lost; double vision sometimes occurs; the pupils are frequently dilated, but sometimes contracted ; the conjunctiva is often injected; temporary deafness is an occasional symptom. In one of the cases of sporadic cerebro-spinal meningitis which I have observed, lateral and vertical oscillation of the eyeballs was a notable symptom. The coma in some of the cases which I have observed, has been without stertor, and resembled that which characterizes certain cases of hysteria. The patients seemed to be in a quiet sleep, crying out if any attempt were made to alter the position of the body. A patient in one case had remained in precisely the same position for 48 hours, and, in another case, for three days. Mobility of the iris may remain, althongh the patient is in this comatose or deeply somnolent state.

Important symptoms relate to the muscles. Tonic contraction of the muscles of the neck and back is of frequent occurrence, giving rise to retraction of the head and opisthotonos in a greater or less degree. This 
was observed by Levy in 18 of 60 cases. Trismus occurred in 17 of these cases, and pleurosthotonos in 2 cases. Contraction of more or less of the museles of the extremities was noted in 16 cases. Of the 98 cases observed by Githens, marked opisthotonos occurred in 17, and slight contraction of the muscles of the back was observed in 38. Muscular pains, usually seated in the posterior muscles of the legs and thighs, the spinal museles and those of the neck, existed in nearly all of Githen's cases. Rigidity of the recti muscles was observed by Ames in several eases. Tremor of the muscles is an occasional symptom. Strabismus occurred in 9 of the 64 cases analyzed by Ames. In the cases in which the disease is protracted, subsultus and carphologia are frequent. Convulsions are not of frequent occurrence; of the cases analyzed by Ames, they were observed toward the close of the cliscase in 3 cases, and at the beginning in one case. Paralysis is also infrequent; it occurred in only three of 99 cases observed by 'lourdes. Incomplete ptosis on one side occurred in one of the cases analyzed by Ames, and incomplete hemiplegia in another case.

Nausea and vomiting are more or less prominent throughout the disease in a minority of eases; vomiting was noted by Ames in 15 cases, and nausea without vomiting in 3 cases. Constipation is the rule. Diarrhoa was observed by Ames in only one case; and in this case it was probably due to the employment of tartar-emetic given for a pneumonic complication. The appetite is generally lost; however, a desire for food was expressed in 8 cases observed by Ames, convalescence not being near at hand. Of Githen's cases, nausea alone existed in 9, and vomiting, to a greater or less extent, in 40 . In 40 cases the stomach was perfectly quiet. Constipation existed in 70 of these cases; diarrhoa in 13; in 10 cases diarrhoea and constipation alternated, and in 13 eases the bowels were regular. The tongue presents a variety of morbid appearances. It is frequently large and flabby, showing indentations made by the pressure of the teeth. It becomes coated, and, in the progress of the disease, dry and dark. The teeth and lips frequently present sordes.

The respirations are sometimes suspirious. They are usually increased in frequency even when no pulmonary complication exists. Irregularity of the respirations was noted in eases reported by Upham. Stertor is rare. The pulse at the commencement of the discase is generally not more frequent, and often slower, than in health. Intermittency of the pulse was observed in some of Uphan's eases. Tumultuous action of the heart was observed in some cases. In the progress of the disease the pulse becomes moderately accelerated, rarely exceeding 100 per minute, until toward a fatal termination, when it is frequent and small. Softness of the pulse is stated by Ames to be a constant feature. Fluctuations in frequency at different periods of the day are stated to be a striking feature by Tourdes and Ames. Palpitations are occasionally observed. The quantity of urine is greater than in health, and the urates are deposited in abundance. In a fatal case among those which I have observed there was hematuria, the hemorrhage being abundant, and not occurring in any other situation.

The temperature of the skin varies in different cases and at different periods in the progress of the disease. At the beginning the temperature rarely exceeds, and often falls below, that of health. Notable heat of the surface is very rarely observed: the thermometer in the axilla, however, shows the heat of the body to be more or less raised. Thermometric examinations were made by Githens in 44 cases at $6 \mathrm{~A}$. M., at $12 \mathrm{M}$., and at 6 P. M., daily, with the following results: The maximum of increase was $105^{\circ}$ Fahr.; in only two cases was this height reached. The maxi- 
mum in 15 cases was between $104^{\circ}$ and $105^{\circ}$; in 7 cases between $102^{\circ}$ and $103^{\circ}$; in 6 cases between $101^{\circ}$ and $102^{\circ}$; and in two cases it was below $100^{\circ}$. The difference between the morning and evening temperature was less than in most of the essential fevers. "A rapid fall was the sure precursor of collapse." As regards dryness and moisture, cases vary on different days and at different periods of the same clay. Sweating appears to be a rare event, except in some cases shortly before death. The skin was moist and hot in most of Upham's cases. 'The face was suffused and often of a dusky hue.

Petechial spots, which have of late especially excited attention, are frequently observed, but they are by no means constant. They appear to be of more frequent occurrence in some epidemics than in others. 'They vary in size from that of a pin's head to spaces a quarter of an inch in breadth or even larger. They are evidently due to an extravasation of hematin, in other worls, they are ecchymoses such as occur in scorbutus, purpura, in some cases of continued fever, and occasionally in various affections. They are hardly entitled to be called an eruption. According to Tourdes, a rose-colored papular eruption, resembling that of typhoid ferer, is occasionally observed. The same observer has noticed herpes labialis in onethird of his cases. Ames observed this eruption in only 3 cases. Urticaria, erythema, and bullæ have been observed, but their ocemrence is evidently accidental ; that is, they do not belong to the clinical history of the disease. In one of the cases which I have observed, sudamina were abundant.

A survey of the clinical history of this affection, exclusive of the cases in which deatlı quickly ensues in apoplectic coma, shows a correspondence with the symptoms of acute cerebral meningitis, and the addition of the phenomena due to an extension of the inflammation to the meninges of the spinal cord. The duration of the disease is very variable. Of fatal cases, the minimum duration is stated by Tourdes to be twenty hours. Ames states the minimum to be fifteen hours. The maximum duration in fatal cases is stated by Tourdes to be 100 days, and by Ames over forty days. Of the cases analyzed by Ames, 16 terminated fatally on the second or third, and 19 on the fourth day. Of 160 cases analyzed by Dr. S. B. Hunt, 12, or one in 13, died within the first twenty-four hours; 92, or more than half, died before the close of the fifth day; 14, or one in 11 , died before the close of the tenth day; 4 , or one in 40 , before the close of the fifteenth day, and 18 survived for various periods thereafter.' In Upham's cases the duration varied from thirty-six hours to six weeks. Thus, in the majority of fatal cases, the disease runs a rapid course, ending prior to the fifth day. In cases which end in recovery, the convalescence is generally tedious, and the patient is apt to remain for a long period in feeble health. Relapses have not been observed.

It is not easy to divide the career of the disease into well-marked stages. And the arrangement of cases into different classes, such as inflammatory, congestive, malignant, etc., does not seem to me to be attended with any practical advantage. Remissions are stated to occur by different observers, especially by Ames. They vary as regards the time of their occurrence, their duration, and the degree of amelioration of the symptoms. Not infrequently the improvement for a few hours, and sometimes for a day or so, is so marked as to give encouragement to hope that convalescence is about to take place. These remissions are most apt to occur on

\section{War.}

Article by Dr. Hunt in the medical volume of the Sanitary Memoirs of the 
the second or third day. In a case which I saw with Dr. O. White, of this eity, the temperature and pulse beeame normal, the intelligence returned, and for twenty hours the patient seemed convaleseent; the symptoms, however, returned and the case ended fatally.

Pathological Charafter.-The name of this disease implies the occurrence of inflammation of the cerebral and spinal meninges; but it is not to be inferred that the disease is purely local. Inflammation of the parts just named is doubtless a local manifestation, or an effect, of an underlying, general morbid condition. This statement will apply to all epidemic diseases characterized by local affections, and, also, to not a few sporadic diseases which are nosologically considered as local. Moreover, the existence of an underlying, general morbid condition is declared by the occurrence, in a certain proportion of cases, of local imflammation in other situations than within the head and spinal canal. It is in vain to inquire, in what consists this general morbid condition; but we are not more ignorant of the essential pathological character of this than of most other cliseases which belong in the same category. A morbid condition of the blood is probable, and, in view of the disease being an epidemic, it is reasonable to conclude that the morbid condition of the blood is of a toxical character.

Some writers are of opinion that the disease belongs more properly in the class of fevers than among the affections of the nervous system. Bondin, in 1849, proposed to call the disease cerebro-spinal typhus. Dr. W. H. Draper has ingeniously advocated its identity with typhus. ${ }^{1}$ 'This view of its pathologieal character does not seem to me to be tenable. The anatomical characters of cerebro-spinal meningitis are exceedingly rare in cases of typhus; as regards clinical history, the points of contrast with typhus are more numerous and striking than those of resemblance; and facts are opposed to the supposition of community as regards causation. The distinetive features of the disease relate to the nervons system, and in a large proportion of cases the symptomatic phenomena are due to inflammation of the meninges of the brain and spinal cord; henee, in the existing state of our knowledge, it has seemed to me most convenient and appropriate to continue to use the name cerebro-spinal meningitis, and to consider it in the section of this work devoted to diseases affecting the nervous system.

Causation.-The prevalence of the disease as an epidemic implies the existence of a special catuse. With respect to the source of the special cause, and the cireumstances concerned in its production, we have no positive knowledge. Nearly all who have observed the disease concur in the belief that it is not propagated by contagion or infection. This suffices to establish its non-identity with typhus, for the latter is undoubtedly communicable from one person to another. Moreover, epidemic cerebral-spinal meningitis is developed in rual districts where typhus is never generated. Nor is the cause identical with that which gives rise to periodieal fevers, for the disease has prevailed where intermittent and remittent fevers are never produced. It may be, however, that, in so-called miasmatic districts, the special cause may be combined with that giving rise to periodical fevers, and, hence, the phenomena of the latter may be associated with those belonging to cerebro-spinal meningitis.

In France, the disease has been observed to prevail especially among

- Bulletin of the New York Academy of Medicine, April, 1864. 
solcliers, and more particularly among new recruits. Recently, in this country, it has prevailed among troops stationed at several different points. It attacks persons in all conditions, and at all periods of life. Infunts are not exempt, but the liability is increased after seven years of age. In a series of cases the larger number are between the ages of 20 and 30 years. Epidemics have been observed in all climates, and in all seasons of the year. Of the persons attacked the larger proportion are males. 'The difference, however, is not great. The statisties of Sanderson, cited by Hunt, show 164 eases among males, and 154 among females.

Epillemics occur much oftener in the winter and spring than during the summer months. Of the epidemics which have oceurred in this councry nearly all were in the spring and winter months. Of 52 epidemics in Europe, analyzed with reference to this point by Dr. John Simon, 10 were in August and September ; 24 were in October and November; 46 were in December and January; 48 were in February and March; 30 were in $A$ pril and May, and 24 were in June and July. The disease, as stated by Hunt, who cites the foregoing statistics, "has its favorite habitat in prisons and barracks." "The four or five years following 1937 it invaded nearly all the erowded barracks in France. It has appeared frequently among the galley slaves of Toulon."

Auxiliary causes which may give rise to the disease by co-operating with the special cause remain to be ascertained.

Diagnosis.-Diffieulty in making the diagnosis of cerebro-spinal meningitis relates chiefly to sporadic eases, to eases of an unusually mild character, or to the cases which occur at the commencement of an epidemic. Exclusive of mild cases, the diagnostic features are striking and distinctive.

The disease should never be confounded with typhoil ferer. The suddenuess of the attack in many cases, the absence of febrile movement at the commeneement, the pulse being often at first less frequent than in health, the increase, instead of diminution, of the cephalalgia as the disease progresses, and the occurrence of symptoms denoting inflammation of the meninges of the brain and corl, distinguish it alike from typhoid and typhus fever. On the other hand, the characteristic abdominal symptoms of typhoid tever are wanting, viz., diarrhoa, meteorism, and iliac tenderness. Absence of the latter symptoms does not exclude typhus; but other diagnostic points belonging to the latter disease are wanting. The characteristic eruption of typhus, often eopious, appearing on the third or fourth day after taking to the bed, does not occur in cerebro-spinal meningitis. The spots of eechymosis on which the name spotted fever is based cannot be confounded with the eruption of typhus. The dusky hue of the surface, and the characteristic physiognomy of typhus, are wanting. 'Tonie contraction of the muscles of the neck' and back, rachialgia, convulsions, and furious delirium, which occur not infrequently in cases of cerebro-spinal meningitis, are very rare in cases of either typhoid or typhus fever.

In the cases in which the patient falls quickly into coma and dies after the lapse of a few hours, the affection resembles, in this fact, a paroxysm of pernicious intermittent fever. In general, however, the latter does not destroy life in the first paroxysm, and the occurrence of a paroxysm from which the patient has emerged, suffices to establish the diagnosis. It is of course only in districts in which periodical fevers exist, that the practitioner is called upon to make this differential diagnosis.

The occurrence of trismus and opisthotonos may suggest tetanus. But 
in tetanus the cerebral symptoms which belong to cerebro-spinal meningitis are wanting. Moreover, tetanus, in other than tropical climates, is exceedingly rare save as a traumatic affection, and never prevails anywhere as an epidemic.

Prognosis.- There are few epidemic diseases so destructive to life as cerebro-spinal meningitis. In the epidemic observed by Tourdes, the proportion of deaths was 60 per cent. $\Lambda$ mes gives the same proportion of fatal cases in the epidemic which prevailed in Alabama in 1848. In an epidemic observed by Leferve, four-fiftlis died at the commencement, and two-thirds toward the end of the epidemic. Of 366 cases analyzed by Dr. S. B. Hunt, the deaths were 243 , and the recoveries 123 , making the percentage of fatal cases 70. Stillé states that ten epidemics between 1838 and 1848 gave an average mortality of 70 per cent., and a similar number of epidemies between 1855 and 1865 gave an average of about 36 per cent. He cites these statistics as showing a gradual decline of power in the epidemic cause. All observers agree as respects a large denth-rate in cases of this disease. The prognosis in all cases in which severe symptoms occur-such as notable delirium, coma, convulsions, and muscular contraction-is exceedingly grave. 'The disease is most apt to prove fatal in infants, and in persons in middle and advancer life. It is stated that the proportion of fatal cases is greater among females than males.

The mode of dying, in the cases in which coma and death take place speedily, may be by apnœa, but in the majority of eases it is chiefly by asthenia. Deafness and blindness are not very rare sequels of this disease. . In the large majority of eases, the deafness is bilateral. The blintness may be in both eyes, but oftener it is unilateral. The blindness is due to purulent choroiditis, and the deafness to purulent inflammation of the labyrinth of the ear..

Treamuent. - In a disease of śo severe and fatal a character, it might be anticipated that various potent therapentical measures have been abundantly employed. Bloodletting has been largely practised without favorable results. Indications for bloodletting, derived from the cireulation, as a rule, are not present; the pulse is usually deficient in force. Still, there may be cases in which this measure is of great utility. At an early period, if there be considerable febrile movement, and a pulse denoting power of the heart's action, it is admissible, if not demanded.

Quinia, in large doses, has been frequently employed. Experience has not furnished satisfactory evidence that this remedy possesses curative power over the disease. In some cases of reported suecess from its use, there may be reason to suspect that pernicious intermittent fever has been mistaken for the disease. In miasmatic districts, the possibility of this error in diagnosis, or of the association of the malarious poison with the special canse of the disease, may render the employment of quinia advisable, without placing reliance on it as a curative remedy for the cerebrospinal meningitis.

With respect to opium in large doses, testimony is discrepant. Chanffard, Boudin, and others affirm that this remedy exerts a favorable influence upon the disease, and reduces the rate of mortality. Its safety and usefulness by way of palliation is stated by Ames and others who do not attach to it a curative power. Prof. Stille, who studied abont one hundred and twenty cases in the Philadelphia Hospital, during the winter of

' Vide Article by Dr. H. Knapp in the N. Y. Medical Record, Aug. 15, 1872. 
1867 , states that he was in the habit of giving one grain of opium every hour in severe, and every two hours in cases of moderate gravity, neither narcotism, nor any approach to that condition, being produced in any instance; and under the influence of the medicine, the pain and spasm subsided, the skin grew warmer, the pulse became fuller, and the entire condition was generally more hopeful.

Mercury, tartar-emetic, active purgatives, extensive vesication over the nucha and back, ice applied to the head and spine, are among the measures which have been more or less largely employed; but it is difficult to determine, from the literature of the subject, their agency, respectively, for good or harm. In the recent epidemic visitations in this country, practitioners have resorted to the use of alcoholic stimulants freely, but sufficient facts have not, as yet, been communicated to warrant a judgment concerning their value.

In the present unsettled state of opinions and practices respecting the treatment of this disease, the most judicious course for the practitioner is not to adopt any plan to be followed indiscriminately, but to employ different therapentical measures with reference to the indications in individual cases. Bloodletting may be employed under the circumstances which should govern the use of this measure in the first stage of simple acute meningitis, and in congestive apoplexy. Opium may be tried, and continued or not, according to its apparent effects. Mercury is advisable on the same grounds as in simple acute meningitis, but it is not to be relied upon sufficiently to take the place of other measures. Cold is to be applied to the head and back when grateful to the patient, and not followed by an aggravation of symptoms. Vesication of the neck or spine by means of a blister, or the strong aqua ammonia (the latter, from its quickness of operation, is to be preferred), may be employed, except in young children, and it may be repeated if it appears to afford relief. Revulsive applications to the extremities, by means of sinapisms or stimulating pediluvia, are always admissible. Active purgation is of questionable propriety after the bowels have been freely evacuated. Finally, stimulants are indicated whenever the symptoms denote failure of the vital forces; and they are urgently indicated in proportion as the tendency is to death by asthenia.

Without any definite knowledge of the special cause of epidemic cerebro-spinal meningitis, or of the accessory causes, the prophylaxis will embrace attention to all sanitary measures relating to individuals and to public health. Prudence dictates, whenever practicable, removal beyond the sphere of the epidemic influence.

Prior to the revision of this work for the present (fourth) edition, I had had no opportunity of observing epidemic cerebro-spinal meningitis. The prevalence, to some extent, of the disease in New York and its vicinity, in the spring and summer of 1872 , enabled me to see a considerable number of cases. As regards the treatment, the remedies which were generally relied upon, and which were considered as useful by the physicians with whose practice I became acquainted through professional intercourse, are opium, the iodide of potassium, and the bromides. Some, in whose judgment and accurate observation I have confidence, relied chiefly on opium. They who relied, measurably or chiefly, on the iodide of potassium or the bromides, generally prescribed these remedies in large doses. My personal observations are not, as yet, sufficiently extensive to warrant any positive inferences therefrom in respect of the efficiency of therapeutical measures. 


\section{Hydrocephalas. Hydrorachis.}

The term hydroceplalus has been loosely applied to all accumulations of liquid, in different situations, within the cranium. Meningitis, with abundant effusion, has been called acute hydroeephalus. 'The term should be applied exclusively to a non-inflammatory or dropsical effusion. 'This may take place within the arachnoid cavity, in the subarachnoid space, and in the ventricles. It occurs most frequently in the two latter situations. The morbid conditions giving rise to hydrocephalus are local. There is but little tendency to dropsical effusion within the cranium in cases of general dropsy. It oceurs as a congenital affection, or in early infancy, leading, in some cases, to an enormous enlargement of the cranium, and giving rise to a characteristic deformity of the head. The rearter is referred to works on morbid anatomy, or on the diseases of children, for an account of this affection.

Exclusive of the affection thus referred to, an effusion of serum takes place as a result of wasting or atrophy of the brain-substance, called by Rokitansky effusion "ex vacuo." Under these circumstances, as the effused serum rimply fills an unoccupied spaee, the brain does not suffer from compression. 'The supposition that serous effusion sometimes occurs suddenly in sufficient quantity to occasion apoplexy (serous apoplexy) has been already referred to, with an expression of cloubt as to its correctness. An abundant serous effusion is an important element in certain cases of meningitis in children, and sometimes in adults. The exudation of lymph in some cases is very small, and the amount of effused serum large; but these are not, properly, cases of hydrocephalus. Dropsical effusion may occur as an element of various structural lesions within the skull, and in these cases it is probably due to obstruction of some of the intra-cranial vessels.

A dropsical accumulation within the spinal canal is called Hydrorachis. In cases of hydrocephalus in which the effused liquid is contained within the arachnoid cavity, the dropsical accumulation is also, of course, in the spinal canal. Exclusive of these cases, under the head of hydrorachis, are embraced cases of congenital deficiency of portions of the vertebral column, constituting the affection eommonly known as spina bifida. This affection belongs among the diseases of children. It is also treated of in works on surgery.' Much importance is attached by some writers to pathological conditions relating to the circulation within the spinal canal. It is probable that an undue accmunulation of blood, or congestion, and an abnormal deficiency of blood, or ancemia, are pathological conditions which are more or less important. It is. however, difficult, with our present knowledge, to estimate, with anything like precision, the degree of importance belonging to these conditions, or to indicate the symptomatic phenomena connected with them. These conditions will be referred to in connection with paralysis.

1 Vide System of Surgery, by Gross. 


\section{CH A P TER III.}

Cerebritis, and Abscess of the Brain. Structural Lesions within the Cranium. Indnration or Sclerosis. Non-Inflammatory Softening of the Brain. Morbid Growths or Tumors within the Cranium. Psammona of the Brain. Hypertrophy and Atroply of the Brain. Myelitis. Structural Lesions within the Spinal Canal.

TNFLAMMATION seated in the substance of the brain, or cerebritis, and 1 various structural affeetions situated within the eranium, are the topics which will occupy the first part of this ehapter. Afterward, inflammation of the substance of the spinal cord, or myelitis, and structural affections within the spinal eanal, will be noticed. For full details respecting these topics, works on morbid anatomy must be consulted. In the present state of our knowledge, it is hardly practicable to treat of the different cerebral and spinal lesions as individual affections. In a clinieal point of view, we are situated with respect to these as formerly, before the introduction of physical exploration, physicians were situated with respect to pulmonary and cardiac lesions. The diagnostic characters belonging to them separately are imperfeetly ascertained. Their differential diagnosis often cannot be made out with positiveness. In medical praetice, the different lesions affecting the brain and spinal cord are of importance as leading to impairment and disorder of the mental faculties, and to paralysis. The latter are effects, or symptomatic phenomena, but, with our existing knowledge, it is convenient to consider them as individual affections. My plan, therefore, will be to devote a limited space to cerebritis and myelitis, and to notice very briefly the structural affections of the brain and spinal cord, referring to them under the head of paralysis, and devoting to the latter a more extended consideration.

\section{Cerebritis. Abscess of Brain.}

The term cerebritis denotes inflammation of the substance of the brain In cerebral meningitis, the inflammation frequently, if not generally, extends more or less from the pia mater to the brain-substance. In this respect meningitis differs from other serous inflammations, for example pleuritis, in which the inflammation involves only to a very limited extent, if at all, the parenchyrna. The cerebritis thus induced secondarily to meningitis is more or less diffused. Cerebritis is also incidental to tumors which encroach upon the brain, and to extravasations of blood. In these cases, the inflammation is limited to the portion of cerebral substance surrounding the tumor or clot. In a large proportion of the cases in which the inflammation is circumscribed and gives rise to abscess of the brain, it is secondary to caries of the cranial bones, generally connected with disease of the internal ear, the orbit, or nose. It is oftener a result of injury of the head than of any other eause. As a primary affection, it is much rarer than has heretofore been supposed. In a large proportion of the cases which, until lately, were considered to exemplify this affection, the morbid condition was not inflammatory, but softening from suspended nutrition (necrobiosis), attributable to either thrombosis or embolism. The knovledge of late acquired respecting the accidents just named leads 
to the conclusion that primary cerebritis, that is, exclusive of the cases in which it is due to the presence of a clot or a tumor, or when it follows caries of the bones, and of traumatic cases, is exceedingly infrequent. The inflimmatory affection of the brain now referred to is that leading to either softening or suppuration. Induration or sclerosis of the brain, which is generally considered as resulting from chronic inflammation, will be embraced among the structural lesions to which a portion of this chapter will be devoted. Circumscribed cerebritis is either limited to a single space greater or less in extent, or it may exist in more than one situation. The most frequent seat is in the white portion of the middle cerebral lobes, but there is no portion of the brain in which it may not be seated. Next to the part just stated, it is most apt to occur in the corpora striata, the thalami optici, the pons Varolii, and the cerebellum.

The fir'st physical change in the inflamed parts after hyperæmia is a certain amount of induration, from the presence of exudation-matter. The amount is small, owing to the fact tlat the substance of the brain contains very little areolar or connective tissne. An ulterior effect is softening, and from this effect cerebritis has been considered under the name inflammatory softening of the brain. The softening varies in degree, from a slight diminution of consistency to pulpiness or complete difluence. The best test of a slight or moderate degree of softening is the effect of allowing a small stream of water to fall upon the part. The softened portion is reddened by blood globules, or infiltrated hematin. Hence, inflammatory softening has been called red softening. The red coloration, however, is not distinetive of the softening from intlammation ; softening from necrobiosis may, also, have a red color dne to the presence of blood or hematin. 'The softened portion becomes yellowish or yellowish brown from a change of color in the hematin, such as occurs after a bruise attended with an extravasation of blood. Examined microscopically, when the softening amounts to diflluence, there are found red globules, leucocytes, the debris of the elements of the nervous tissue, granular matter and cells, free nuclei and fatty granules. Microscopical observers, however, admit that it is not always easy to differentiate inflammatory from non-inflammatory softening, without taking into account the ante-mortem history, and the absence of appearances in the vessels pointing to embolism or thrombosis. If the cerebritis be seated near the surface of the brain, eircumscribed meningitis may occur, as, in other situations, circumseribed inflammation of the serous investment takes place over an inflammation originating in the parenchyma of organs. Finally, the inflammation may be suppurative, and a collection of pus mixed with liquefied cerebral tissue constituting abscess of the brain. Surrounding the abscess in certain cases is a pseudo-membranous cyst, but in other cases the walls are formed by the cerebral tissue, or the latter is infiltrated with the pus. In the majority of cases, abscess of the blain is single, but sometimes there are two or three distinct abseesses. The pus is sometimes fetid. This is more apt to be the case when the abscess is connected with caries of the bones of the ear or nose, or after fracture of the skull.

The development of cerebritis is generally preceded by local symptoms, namely, pain and a sense of fulness in the head, vertigo, tinnitus, disturbances of vision, unilateral contractions and convulsive movements of the muscles of the limbs, hesitancy or uncertainty of gait, and embarrassment in speech. Febrile movement exists when the inflammation is developed. The pulse is more or less accelerated, and the axillary temperature rises, but the thermometer rarely shows an increase of heat 
above $103^{\circ}$. Vomiting occurs at intervals; the bowels are constipated ; cephalalgia is usually more or less marked, although it may be wanting. Epileptiform convulsions are apt to occur. These symptoms represent a stage of the disease corresponding to the "period of excitement," or the first stage, in meningeal inflammation. This stage continues rarely beyond a week. Softening, or the formation of pus, gives rise to paralysis, mental dulness, somnolency, coma. This stage may be of brief duration, or life may be prolonged for many weeks and even months, death being perhaps caused by some intercurrent affection. I shall content myself with this enumeration of the more prominent events entering into the clnical history. The symptomatology of cerebritis calls for renewed study, inasmuch as, until within a very late period, observers have not differentiated cases of this affection from those of non-inflammatory softening following embolism and thrombosis.'

Coma, in connection with cerebritis, is sometimes sudden, and, hence, authors have reckoned this disease among the several morbid conditions giving rise to apoplexy. Doubtless, in some of the cases of so-called apoplectic cerebritis, the apoplectic seizure was due to embolism. Softening from inflammation, however, may occasion cerebral hemorrhage taking place with sufficient rapidity and in sufficient amount to cause apoplexy. Another event giving rise to sudden coma and death is the rupture of a cerebral abscess, the pus being discharged into one of the ventricles, or upon the surface of the brain. In some cases of cerebral abscess connected with disease of the internal ear, orbit, or nose, the pus from the abseess is discharged externally in these situations.

Aphasia may be caused by inflammatory softening or abscess of the brain, as well as by other cerebral lesions, especially when the seat of the morbid changes is in the left anterior lobe of the cerebrum, near the island of Reil. (Vide page 615).

The causation of circumscribed cerebritis, and abscess of the brain, exclusive of the cases in which it is connected with tumors or the presence of a clot, and of the cases in which it proceeds from injuries of the head or affections of the bones of the cranium, is obscure. It occurs oftener in persons of middle or advanced age than in young subjects, in this respect affording a contrast to meningitis. It is observed oftener when the constitution is impaired by antecedent affections than in healthy persons, but clinical observation has not established relations with any particular affections. Suppurative inflammation here, as in other organs, may be incident to pyæmia. In cases of pyæmic cerebral abscess, numerous small collections, the size varying from that of a pin's head to a hazelnut, may be found in different parts of the brain.

The diagnesis involves the exclusion of embolism and thrombosis, acute cerebral meningitis, cerebral hemorrhage, and tumor of the brain. The existence of prodromes pointing to some affection within the skull will seem to exclude embolism and thrombosis, these accidents occurring without prodromic symptoms. They are also excluded by the presence of symptoms denoting an inflammatory affection, namely, acceleration of the pulse and rise of temperature, symptoms not belonging to embolism and thrombosis. Cerebral meningitis is attended by more intense local symptoms-cephalalgia, delirium, etc.-and it gives rise to greater intensity of fever, together with other evidence of general disturbance. Moreover, muscular contractions and convulsive movements in cerebritis

1 For a tabulated report of 73 cases of the alsscess of the brain, vide article by Drs. Gull and Sutton, in Reynolds' System of Medicine, Vol. II., 1868. 
are generally unilateral, whereas, in meningitis they are bilateral. Hemorrhage into the substance of the brain, when it is not a sequel of cerebritis, occurs without prodromes, and the symptoms denoting inflammation around the clot oceur several days after the apoplectic seizure. The differentiation from tumors is the most difficult. If the cerebritis pursue a regular course, the first stage well marked, continuing for a week or somewhat longer, and the symptoms denoting softening or suppuration following, tumor may be excluded. The course of cerebritis, however, is not always regular, but it is subject to great variations. Abscess of the brain is sometimes notably latent, being found after death when it had not been suspected during life. As regards the differential diagnosis of abscess and tumor, much weight belongs to the following points: If the cerebral affection be connected with disease of the internal ear, orbit, or nose, or if it have a traumatic eause, it is abscess ratber than tumor. If there be room for doubt, the probabilities are in favor of tumor, inasmuch as abseess is much more infrequent than tumor.

Cerebritis leading to softening or suppuration, ends fatally, sooner or later. Recovery, under these cireumstances, is perhaps possible. Cases have been reported in which the symptoms and progress seemed to denote inflammatory softening, and the patients recovered, save that more or less paralysis remained. But in these cases there is always room for doubt as to the correctness of the diagnosis. It is by no means improbable that circumscribed cerebritis may occur and end in recovery without having eventuated in notable softening or suppuration; but, in such cases, the symptoms cannot be relied upon for the diagnosis.

The duration in fatal cases varies greatly. Of 109 cases of softening, 3 died within twenty-four hours from the time when the symptoms denoted an attack, and, in the majority of cases, death occurred before the twelfth day. In only 16 cases the duration was longer than a month. This list, however, may have included eases of softening without inflammation. ${ }^{1}$ These facts suffice to show that, as a rule, inflammatory softening runs a rapid course.

A few words will embrace all that is to be said with reference to treatment. At a period when a probable diagnosis may be made, that is, after inflammation has eventuated in softening or suppuration, the possibility of recovery involves sufficient prolongation of life for the absorption of the destroyed eerebral tissue with the inflammatory products and for cicatrization. The greai object, then, of the management, is the prolongation of life. Keeping in view this object, depletory and debilitating measures are likely to do harm. Mild revulsives, tonic remedies, a nutritious diet, repose of the mental faculties, and stimulants, if the patient be in clanger from depression of the vital powers, should constitute the treatment.

\section{STRUCTURAL LESIONS WITHIN THE CRANIUM.}

Under this heading I shall consider, briefly, the different lesions which are liable to oceur within the cranium, in addition to those incident to the cerebral affections which have been considered.

\section{Induration or Sclerosis of the Brain.}

This structural affection corresponds to cirrhosis of the liver and of the kidneys. It corresponds to affections in other parts, called by different 
names, such as scleroderma of the skin, pseudo-hypertrophy of the muscles, etc. Wherever situated, the structure affected is the comnective tissue. The essential anatomical condition is a morbid production (hyperplasia) of this tissue. 'The underlying pathological condition is supposed to be a chronic inflammation. Sclerosis of the brain is, therefore, characterized by a hyperplasia of the interstitial nerve cement, the analogue of the connective tissue in other situations, called by Virchow the neuroglia; and the affection is supposed to be a form of chronic cerebritis.

In rare cases, the lesion cxtends over an entire lobe, or even over an entire hemisphere. The affection is then called diffuse cerebral sclerosis.' As a rule, however, it occurs in isolated, disseminated portions which are more or less numerons, and it is then distinguished as multiple sclerosis. The indurated or sclerosed nodules rary in size from that of a small pea to an almond. They may be few, so that, if small, they are liable to be overlooked, or the number may be great. As regards their number and size, different cases offer great variations. 'They are found in the white or medullary substances of the brain, very rarely existing in the cortical substance. The color of the modules is grayish-white, and in density they vary from that of the albumen of an $\mathrm{egg}$ boiled hard, to a leathery consistence. Sections under the microscope lave a fibrillated appearance, and show fragments of nerve fibres, granular masses, nucleated cells, free nuclei, and frequently amyloid corpuscles. These appearances denote hyperplasia of the neuroglia and atrophy of the nerve structure, the latter, doubtless, dependent on compression by the former. From the color, the lesion has been called gray degeneration. The notules may be found in any and in all parts of the medullary substance of the brain. They may be limited to the brain, but they often exist in both the brain and the spinal cord, occurring primarily and predominating sometimes in one and sometimes in the other of these situations. The name cercbro-spinal sclerosis expresses this concurrence of the affection in the brain and cord.

Symptomatic effects of this lesion are pretty constantly manifested in the mental condition. The faculties of the mind, as a whole, may be more or less enfeebled. The impairment may be especially apparent in failure of the memory. Melancholy and a morlid emotional impressibility are the prominent mental manifestations in some cases. Cephalalgia and vertigo enter into the clinical history. Like other lesions of the substance of the brain, this gives rise to paralysis. As to the muscles which are affected with paralysis, this will depend on the situation, the size, and the number of the sclerosed or indurated nodules. A diagnostic feature is, the paralysis affects different muscles successively, not simultaneonsly, and in an irregular manner. This is explained by the dissemination of the lesion which causes the paralysis, and by the successive occurrence of sclerosed nodules. Generally, at first, the paralysis is confined to certain of the muscles of one of the lower limbs; then, certain muscles of an upper limb, or of the corresponding member on the opposite side, may become affected. The paralysis may extend to the muscles of the trunk. Strabismus may occur. There may be facial paralysis on one or both sides: The muscles involved in speech are often affected, and sometimes those of deglutition and respiration become implicated. 'This irregularity in the successive paralytic manifestations, corresponding to the multiple character of the lesions, is highly significant of the affection. The cutaneous sensibility may be but little, or not at all, affected. The sense of

1 Vide Hammond on Diseases of the Nerrous System. 
tact may be diminished, while the susceptibility to pain is preserved. Defective vision, sometimes even amaurosis, and deafuess are occasional effects. Other of the cranial nerves, exclusive of the facial already referred to, may be involved.' Muscular tremor is a very constant symptom. It precedes and accompanies the paralytic effects. It is manifested with voluntary movements, and, also, irrespective of these, especially under the influence of emotions or mental excitement. During the progress of the affection, convalsive movements and spasms sometimes occur, but they are rare. Defective co-ordinating power over certain muscles (ataxia) is sometimes observed. The paralyzed parts are susceptible to reflex excitation, and to the electrical current; they also do not show defect of nutrition. The nutrition of the body may be well maintained, and patients may even grow fat. The attitule of the patient when standing, and the manner of progression, are characteristic. In standing, the body is bent forward, and, instead of walking, the patient has a jog-trot gait.' This symptom is called festination. Generally, the trotting is forward, but sometimes the patient moves backward for several steps before going in a forward direction. This is a marked symptom in a case under my observation.

The duration of the affection is long, extending, in the majority of cases, from five to ten years. 'The duration may execed considerably the latter period. In a case now under my observation, the disease has exister already sixteen years. Sooner or later it ends fatally, if the patient be not carried off by some intereurrent aflection.

As regards treatment, it must, in the first place, have reference to symptomatic indications, together with attention to the general health. In the second place, although medication will not effect a cure nor prevent a fatal ending, certain remedies are useful. The phosphide of zinc, in a case under my observation for two years, the patient still living, has been unmistakably and decidedly beneficial. Hammond recommends the chloride of barium. S. Weir Mitchell has given the bichloride of mercury with advantage.

\section{Non-Inflammatory Softening of the Brain.}

Circumscribed softening of the brain, exclusive of traumatic cases, and excluding also the cases in which it is secondary to an antecedent local affection such as tuinor, hemorrhage, and caries of the cranial bones, is, in most instances, non-inflammatory. Heretofore it was mooted whether softening of the brain substance ever occurs independently of inflammation. This was before our present knowledge of thrombosis and embolism had been acquired. It seems now certain that circumscribed softening is a result of suspended nutrition from an arrest of the circulation in the part affected, this being an effect of arterial obstruction caused by either coagulation in the ressel at the site of the obstruction (thrombosis), or infarction from a plug (embolus) derived from the heart or larger arteries. The softening is sometimes called atrophic; another name is yellow softening, and it is also called softening from mortification, or nccrobiosis. The softening involves a fatty degeneration of the anatomical elements of the part affected. It is oftener seated in the white than in the gray cerebral substance. It differs, in degree, in different cases, as well as in extent; in an extreme degree, the softened brain-substance has the consistence of a thick, yellowish, creamy liquid. Absence of a red 
coloration has been considered as distinguishing non-inflammatory softening, and, hence, in addition to the several names already cited, it has been called white softening. As already stated in connection with cerebritis, this is an error; hematin may be contained in softened cerebral tissue when inflammation has not been involved, and, therefore, the same coloration may exist as in inflammatory softening. In differentiating, after deatl, the two kinds of softening, namely, the inflammatory and noninflammatory, the evidence afforded by the microscope is essential as showing, in the former, inflammatory products-granular fibrin, and leucocytes.

Softening gives rise to more or less impairment of the mental faculties, and to paralysis. This is the lesion in certain cases of hemiplegia, and it will be referred to in treating of that form of paralysis. Occurring as a result of thrombosis, which is generally an effect of calcareous degeneration of the cerebral arteries, the patients are generally aged, and the paralysis is preceded by impairment of the mental faculties. Following embolism, the paralysis takes place suddenly either with, or without, an apoplectic seizure. Softening as a result of embolism may occur at any age, and this is a point of importance in differentiating apoplexy and hemiplegia from extravasation of blood into the cerebral substance; the latter rarely occurs prior to the middle period of life. An embolus is most apt to become lodged in the left middle cerebral artery, and, hence, the hemiplegia affects the right side. The existence of right hemiplegia, therefore, is of some valne in determining the lesion under present notice. The points involved in the differentiation, during life, of non-inflammatory softening from that incident to cerebritis, are the occurrence of hemiplegia, with or without an apoplectic seizure, or impairment of the mental faculties, not having been precerled by prodromes referable to the brain; the absence of fever as denoted by the pulse and temperature. Cerebral symptoms are likely to precede the paralysis which is an effect of inflammatory softening, and the inflammation is accompanied by more or less acceleration of the pulse, and of increase of heat as determined by the thermometer.

Non-inflammatory softening, as well as other lesions, may give rise to aphasia (vide page 614 ). This effect renders it probable that the situation of the lesion is in the left anterior cerebral lobe, near the Island of Reil.

\section{Tumors within the Cranium.}

For a full account of intra-cranial tumors, as regards their classification, anatomical appearances and relations, histological characters, etc., the reader is referred to works which treat more comprehensively of the diseases of the venous system, or to treatises on morbid anatomy and histology. This section will embrace only a brief notice of the different kinds of tumor occurring in this situation, together with the symptomatology and diagnosis. The nodules which are found in cases of sclerosis may, with propriety, be reckoned as tumors of the brain; these, however, have been considered in another section of this chapter.

'Tubercles aggregated and fused, forming either a single mass, or masses more or less numerous, but rarely exceeding 20 in number, very infrequent in children, are extremely rare in adults. If in a single mass, they may vary in size from that of a filbert to a hen's egg. As a rule, the size of tuberculous nodules is small in proportion as they are numerous. They may be situated in either the cerebrum or the cerebellum, oftener in the latter. They may be cheesy as regards consistence, or they may have become diffluent. A collection of liquefied tubercle may be confounded 
with abscess of the brain. Carcinomatous and other morbid products which have undergone the cheesy metamorphosis, have been often cousidered to be tuberculous.

Carcinomatous tumors are less infrequent than the tubereulous. The tumors are situated in the cerebrum oftener than in the cerebellum. The three varieties of cancer, namely, scirrhous, medullary, and colloid, are represented in different cases. In general, a single cancerous tumor only exists, but in some cases there are several; and in these cases the tumors are usually in corresponding situations in the two hemispheres. If multiple, eaneer is generally found in other parts of the body. The medullary variety is the most frequent. 'The tumors, in some cases, are developed within the substance of the brain, and in other cases their point of departure is in the meninges, more especially in the dura mater. They may originate without the cranial eavity in the neighboring cavities, more especially in the orbit, and make their way within the eranium; and, on the other hand, intra-cranial cancerous tumors sometimes extend into the surrounding cavities.

Fibroid or fibro-plastic tumors are among those developed within the cranium. They may be single or multiple, and they sometimes attain to a considerable size. Of these tumors, called, also, glioma and sarcomatous tumors, a certain proportion, according to Robin, consist of a morbid abundance of eells which this observer describes as found in health in the gray substance, and which he calls myeolocytes. These cells exist normally in the gray substance of the cerebellum, constituting an acessory anatomical element. The tumors are produced by a hypergenesis of these cells. According to the same authority, a certain proportion of the socalled fibro-plastic tumors are epithelial, and each of these two kinds of tumor is not infrequently mistaken for cancer. According to Robin, these tumors form the majority of those found within the eranium, and also within the spinal canal.

A species of tumor consists of an accumulation of cholesterin. A tumor composed of this substance may attain to the size of a hen's egg. It is called cholesteatoma, or, sometimes, pearly tumor. Hydatid cysts and eysticerci form other rare species of tumor. These are either connected with the meninges or situated within the ventricles. Osseous growths, or exostosis, lipomatous, enchodromatous, and those called gummatata, or syphiloma, are to be included among the intra-cranial tumors. The cerebral arteries are occasionally the seat of ancurism, aside from those distinguished as miliary. Aneurismal tumors are generally connected with arteries at the base of the brain, the basilar, the internal carotid, the trunks which form the circle of Willis, and the middle cerebral artery. Generally the tumor's are small, but they soinetimes are of the size of a pullet's egg.'

The different tumors produce morbid effects by pressure on the brain, and their effects, thus produced, other things being equal, are proportionate to the size of the tumor and the rapidity of its growth. Special morbid effects depend on the situation of the tumor. But they eause additional injury to the brain either by exciting circumscribed eerebritis and thereby inducing softening of the brain-substance surrounding them, or by interfering with nutrition and thus leading to non-inflammatory softening; and as a result of the softening, hemorrhagic extravasation is not uncommon. These morbid etfects are produced alike by different tumors; that is to say, the morbid effects have no relation to the particular kind of tumor.

1 For an analysis of 33 cases of intra-cranial aneurism, vide article by Dr. James II. Hutchinson, in the Pennsylvania Hospital Reports, vol. ii., 1869. 
Hence, the kind of tumor in any case cannot be determined by the symptoms which it occasions. For this reason the several tumors are considered collectively, instead of receiving each a separate consideration.

Of the symptoms which accompany tumors within the skull, cephalalgia is usually a prominent symptom. The pain is frequently limited to a circumscribed space, but the locality may not correspond to the site of the tumor. The pain, however, is generally on the side of the head corresponding to the situation of the tumor; and is always referred to the occiput if the tumor be situated in or upon the cerebellum. Other cerebral symptoms which are likely to be present, and more or less prominent, are, vertigo with more or less difficulty in preserving an equilibrium in wailking, momentary loss of consciousness, tinnitus aurium, the fecling of formication, numbness in different parts of the body, and scintillations, liyperæsthesia of the surface followed by a blunted general sensibility, mental irritability, to which succeed dulness and progessive impairment of the faculties of the mind; sometimes active delirium, and, frequently, epileptiform convulsions. Vomiting may be a prominent symptom, occurring irrespective of the ingestion of food and drink, and not accompanied with symptoms denoting gastritis. This symptom is apt to be prominent in the cases in which cephalalgia and vertigo are unusually marked.

Paralysis, more or less extensive, affecting the special senses and voluntary muscles, occurs sooner or later. Hemiplegia, generally incomplete, is the most frequent form of. the paralysis. In some cases the two sides are successively affected, constituting double hemiplegia. The hemiplegia may have been preceded by facial paralysis, and, if the latter do not precede, it may either accompany or follow the hemiplegia. The tumor may be so situated as to press upon certain of the cranial nerves exterior to the brain; the paralysis of the parts supplied by these nerves will then be on the same side as the tumor, and opposite to the hemiplegia, whereas, if the paralysis be due to pressure of the tumor within the substance of the brain, the cranial nerves affected are those on the same side as the hemiplegia, and the side opposite to the hemisphere in which the tumor is situated. The paralysis may be limited to the eighth or portio dura nerve, or other of the cranial nerves may be involved. Loss or impairment of vision in one or both eyes occurs when the tumor either involves destruction of the optic nerve or interrupts its function by pressure. Paralysis of sensation on one side of the face results from similar effects upon the fifth nerve. The third and the fourth nerves may be involved; and sometimes, although rarely, the paralysis affects, separately or collectively, the sense of hearing, of smell, and of taste.

The differences in different cases, as regards the parts affected with paralysis, of course depend on the seat of tumors, their size, and the direction in which they extend. If the tumors be situated within one of the cerebral hemispheres, the paralysis will be likely to be limited to hemiplegia, with perhaps paralysis of the facial muscles on one side. If situated at the anterior, middle, or posterior portion of the base of the brain, tumors are likely to give rise to paralysis of the several cranial nerves. With reference to the differential diagnosis of tumors and inflammatory softening or other lesions, the extension of the paralysis to different parts in succession, after variable intervals, is a highly significant fact. The paralysis affecting successively parts supplied by different cranial nerves ; the members, in some cases, becoming affected first on one side and then on the other side, and the continued increase of the degree of the paralysis of different parts, point with significance to tumors, because such a history is in eorrespondence with their continued growth, and the progres- 
sively increasing amount of pressure and of destruction of cerebral substance. A phasia, in some cases, is an effect of tumors, and this effect renclèrs it probable that they are situated in the left anterior cerebral lobe. The ophthalmoscope is useful in determining the existence and the situation of intra-cranial tumors. Morbid changes in the optic clisks, either congestive, inflammatory or atrophic, are found in a large proportion of cases, even although the vision be not appreciably affected. For details relating to this source of diagnostic information the reader is referred to works on ophthalmoscopy.

With respect to determining the character of the tumor in individual cases, carcinoma may be very strongly suspected if the patient be of middle or advanced age, if cancer be herelitary, or if it exist elsewhere. The evidence afforded by the symptoms of a rapid growth of the tumor is in favor of the conclusion that it is carcinomatous. The tuberculous character of the tumor is to be inferred from the existence of tuberculous disease in the lungs and other organs, and from the youth of the patient. 'Tumors of this kind rarely occur after puberty or under three years of' age. The syphilitic character of the tumor is rendered probable by the fact that the patient has had syphilis, and by the absence of evidence of carcinoma and tubercle. Ancurismal tumor's rarely occur except in persons between fifty and sixty years of age. There are no circumstances to be cited as pointing to the pathological character of the tumor in the cases in which it is fibro-plastic or sarcomatous, and in cases of cholesteotoma, cysticerci, or hydatids.

As regards digestion, nutrition, and the functions of the body in general, different cases differ. Patients may retain their weight and an aspect of health for a considerable period after the occurrence of paralysis and other symptoms referable to the brain. In other cases, more or less impairment of the functions of assimilation, leading to debility and emaciation, occurs early. The general condition will be likely to deteriorate speedily in the cases in which the tumor is either carcinomatous or tuberculous, other parts than the brain being the seat of the disease. It is to be added, that the tumors within the skull may remain latent, as regards both local and general symptoms, until they have attained to a considerable size. The slowness of their growth favors their latency. On the other hand, local morbid conditions incident to the growth of the tumors, namely, inflammation and softening of the surrounding brain-substance, congestions, circumscribed meningitis, and extravasation of blood, give rise, not only to paralysis affecting different parts in succession and progressively increasing in degree, but to exacerbations of pain and other cerebral symptoms, varying much in frequency and intensity in different cases; to somnolency or coma, in some cases, either of transient duration, or lasting for several days, and occasionally to active delirium.

The termination, in most cases of tumor within the skull, is, sooner or later, fatal, the duration of life varying much in different cases, according to the nature of the tumor, the rapidity of its growth, and the inciclental local morbid conclitions; in some cases, death takes place after a few months, and in other cases not until after many years. An exception to this statement is to be made in behalf of syphilitic tumors. These are amenable to curative treatment, if they have not attained to much size, and have not led to damage of the cerebral substance in proximity to them.

The indications for treatment are derived from the symptoms, and relate chiefly to palliative measures. In the progress of the disease, analeptic and supporting measures are indicated. It is important to make trial of 
anti-syphilitic remedies, especially the iodide of potassium, if there be ground for suspecting a syphilitic cachexia. That these remedies are useful and in some cases effect a cure when the history and symptoms point to intra-cranial syphilitic disease, is a fact which the practitioner should bear in mind.

Psammoma of Brain.-The name psammoma denotes a tumor characterized by the presence of lime-concretions, together with other morbid anatomic elements. I use the name, for want of a better heading, in order to introduce an account of a case which, from its being extremely rare, if not unique, and in view of the striking features of the clinical history, is of much interest. The case occurred in the practice of Prof. William $\mathbf{H}$. Thomson. I saw the patient in consultation. The brain was exhibited at a meeting of the New York Pathological Society, June 12th, 1872.

The white substance of the entire brain, more especially of the cerebellum, was infiltrated with bodies like grains of sand, generally of the size of a pin's head, but a few as large as a pea. They were composed of the carbonate and phosphate of lime. There were no appearances denoting meningitis, except that the arachnoid membrane of the superior part of the cerebrum was slightly opaque. There was no morbicl effusion into the ventricles. The arteries were not atheromatous. The brain was notably anæmic. Other organs than the brain were not examined.

The patient was a merchant aged abont 40 years. Excepting that he was subject to attacks of gout, he had had good health up to Dec. 1871 . He then became melancholic, and had delusions, fancying that he was financially ruined and that he had committed frauds. 'These passed away in about a month; he was afterward taciturn, and the mind acted slowly, but he gave no evidence of mental derangement. A remarkable feature of the case was a tendency to extraordinary movements, and positions of the body. He often sat in bed with his head between the knees, and sometimes with his knees on the bed and his head on the floor. When not in these positions, much of the time he performed saltatory movements, not infrequently throwing himself out of bed, so that constant watching to prevent this became necessary. Sometimes he performed a somersanlt on the bed. These movements were voluntary, not convulsive or automatic, and there was no apparent want of co-ordinating power. He never complained of pain either in the head or elsewhere. He ate well, and the digestion was good, until near the end of life. There was no paralysis except of the bladder. The urine was withdrawn by the catheter twice daily for five months; but, toward the close of life, he recovered the ability to expel it by his own efforts. Examinations of the urine for the evidence of renal disease were negative. A few days before his death he became comatose, and died in that condition. The duration of his illness was about six months.

\section{Hypertrophy and Atrophy of the Brain.}

It may be doubtful if true hypertrophy of the brain occurs; that is, an abnormal increase of the cells and fibres which compose the proper cerebral substance. In cases of so-called hypertrophy, the increase of volume is probably caused by an undue development of the interstitial or connective tissue.

Atrophy is a more definite lesion. It affects the cerebrum, the cerebel-

1 For a fuhler report of this case, vide Now York Medical Record, No. for Aug. 15,1872 . 
lum being very rarely affected. It is incident to old age, and occurs in persons bedridden with various chronic diseases. It may be due to a deficient supply of blood from disease of the vessels, and, thus produced, may be limited to one hemisphere. It may proceed from an arrest of development in fotal life, and is then called cerebral agenesis. Serous effusion suflicient to make up for the diminished volume of the brain is found in cases of atrophy. The effusion is usually within the subarachnoid space, but sometimes in the ventricles. The effused liquid, under these circunstances, does not do harm by pressure. Congenital hemiplegia may depend on atrophy of the hemispliere opposite to the paralyzed side; when unilateral, the atrophy is oftenest on the left side. Atrophy, however, is connected especially with deficiency of the mental powers, or imbecility. (Edema of the cerebral substance is a pathological condition incident to effusion into the cavities, the effusion penetrating the substance by imbibition.

\section{Myelitis.}

Inflammation of the substance of the spinal cord is called myelitis. This affection stands in the same relation to spinal meningitis as cerebritis to cerebral meningitis. $A s$ in the latter affection the inflammation frequently, if not generally, extends more or less from the meninges to the substance of the brain, so spinal meningitis involves, to a greater or less extent, myelitis. The point of departure of the inflammation in myelitis is the gray substance of the cord. As spinal meningitis may involve, as a secondary affection, myelitis, so in myelitis the inflammation may extend to the membranes in immediate relation to the inflamed portion of the cord; that is, meningitis occurs as a complication of myelitis. If cases ever occur in which an inflammation, having its point of departure in either the substance of the cord or the investing membrane, is limited exclusively to the one or the other, they are certainly very rare exceptions to the rule. Therefore, when spinal meningitis and myelitis are described as different affections, it is to be understood that, generally, in the former the inflammation begins and is predominant in the meninges, whereas, in the latter, the inflammation begins and is predominant in the cord.

The morbid appearances denoting inflammation of the cord are analogous to those in cases of cerebritis. With hyperæmia, and increased density from exudation-matter, is associated softening. The softening, in some cases, apparently has its point of departure on the superficies, and extends more or less toward the centre; in other eases, the central gray portion is the primary seat, and it is sometimes limited to this portion. In either situation the softening very rarely, if ever, extends continuously over the whole cord, but is limited either to a single segment, or to segments which, in different eases, vary in number, size, and situation. The degree of softening varies. The coloration differs according to the presence and the amount of either extravasated blood or infiltrated hematin, and the changes in color which the latter undergoes. For a time softened portions are reddened by hematin; but afterward the color is brown or yellow, and, finally, they present a white color. 'The microseope shows disintegration, to a greater or less extent, of the nervous structure, granular matter, fatty granules, hyperplasia of the connective tissue, the so-called "granular bodies," and sometimes lencocytes, or pus globules. Here, as in cerebritis, softening, coloration from blood, or infiltrated hematin, the presence of fatty granules, and the "granular bodies," do not constitute adequate evidence of inflammation. To constitute such 
evidence there must be present inflammatory products, namely, either exudation matter or leucocytes. Corroborative evidence of myelitis is afforded by coexisting hyperæmia and the appearances which denote spinal meningitis in portions of the meninges corresponding to the softened segments. If no inflammatory products be found on microscopical examination, softening of the cord, as well as of circumscribed portions of the brain, may be non-inflammatory, that is, due to defective nutrition or necrobiosis.

Excluding cases of myelitis when it is secondary to spinal meningitis, and, also, when it is of traumatic origin, the disease, in an acute form, is extremely rare. Prolonged exposure to cold, as in sleeping upon the damp ground, has produced it. The adequateness of other agencies which have been supposed to be causative, such as muscular efforts, excessive coition, and exposure to a high temperature, is problematical.

The clinical history of acute myelitis embraces symptoms which belong alike to spinal meningitis. This is true of pain referred to the spine and to the limbs, to paralysis which may be general or confined to the lower limbs, and to fever as denoted especially by the temperature of the body. The differential points which distinguish myclitis are the following: The pain in the back is not diffused, but localized in correspondence with the situation and number of the segments of the cord in which the inflammation is seated. The pain in the back is less intense than in spinal meningitis, and it is excited by percussion of the spine, the application of hot vater or ice, rather than by movements of the body, hyperæsthesia of the surface is more rare, and, on the other hand, diminution of the sense of touch and of pain is far more frequent. Muscular spasms and convulsions are less frequent and less marked. When these symptoms do occur, they proceed from the coexisting meningitis. Paralysis occurs earlier, in some cases within a few hours, and it is greater in degree. Its extent depends on the situation of the inflamed segments. If these be not situated above the lumbar and the lower dorsal region, the paralysis is limited to the inferior limbs and the abdomen. If the upper dorsal and the cervical region be affected, the paralysis embraces the upper limbs, and it may embrace the muscles concerned in respiration and deglutition. The sphincter muscles of the bladder and anus are oftener paralyzed. Portions of the body exposed to pressure are more prone to become gangrenous. Reflex movements, after a short time, cannot be excited, and the electrocontractility of the paralyzed muscles is quickly impaired or lost. The urine is often alkaline.

Acute inflammation of the cord is in most cases speedily fatal. The duration of the disease, in fatal cases, depends on the number, the situation, and the size of the segments inflamed. If situated in the cervicooccipital sections, life may be quickiy destroyed by an arrest of the respiratory movements, that is, by apnœa. In other cases, the mode of dying is by asthenia. After considerable softening has taken place, it is not probable that the disease ever ends in complete recovery. If, however, the inflammation be confined to a segment or to segments in the lumbar portion of the cord, the acute may end in a chronic affection of indefinite duration.

The therapeutical indications do not differ materially from those in cases of acute spinal meningitis.

Chronic myclitis, in some rare cases, as just stated, is a sequel of acute inflummation of the cord. In these cases it is limited to a segment, or to circumscribed portions of the cord. In the affected segment or segments 
there is softening. 'This inflammatory softening is distinguisled from that which is non-inflammatory by the presenec of inflammatory products-granular fibrin and lencocytes-by hyperplasia of the conneetive tissue or the neuroglia, and by thickening or adhesions of the spinal meninges at the situation of the softening. The softening, when it proceeds from inflammation which at the outset was subacute, is distinguished by the same appearances.

Chronic myelitis gives rise to paralysis which may be general, that is, affecting both the upper and lower limbs (general spinal paralysis); but, in the great majority of cases, the paralysis aflects the two lower limbs (paraplegia). These forms of paralysis are considered in Chapters $V$. and VI.

Another morbid condition attributed to chronic myelitis is induration, or sclerosis. This will be presently noticed among the structural lesions within the spinal canal.

The therapentical indications in chronic myelitis are essentially the same as in chronic spinal meningitis.

\section{Structural Lesions within the Spinal Cord.}

Lesions take place within the spinal cord corresponding to those noticed in this chapter as taking place within the cranimn, namely, induration or sclerosis, non-inflammatory softening, various morbid growths or tumors, and atrophy.

Induration or Sclerosis of Spinal Cord.-This lesion is supposed to proceed from chronic inflammation, and to consist in an abnormal production of the connective tissue or neuroglia, the latter involving more or less atrophy and other degenerative changes in the nervous strueture. The obvious morbid alterations are notable hardness of the portion or portions affected and a grayish coloration. An infiltrated viseid liquid eseapes on section. The meninges are often adherent at the affected portions of the cord.

The sclerosis may be limited to a single segment of the eord. It is then distinguished as circumseribed. Existing in isolated patches more or less numerons and differing in size, in different cases, it is distinguished as irregular, diffused, disseminated, or multiple selerosis. On the other hand, the sclerosis may he seated exelusively in the posterior columms, each column being generally, although not invariably, affected, the lesion extending over a greater or less space. When limited in extent tle lumbar section is that generally affected. Again, the lesion may be seated cxclusively in the antero-lateral portion on both sides. Sometimes, however, the posterior and the antero-lateral portion are both affected. Sclerosis of the spinal cord and of the brain are not infrequently associated. The lesion of the cord may extend from the superficies a limited depth which varies in different cases, or it may embrace the whole of the affected. segment or segments. At an advanced stage, the obvicus appearanecs are marked; but at an early period, when the lesion is comparatively. slight, it may escape observation without the aid of the mieroseope.

Sclerosis affecting the antero-lateral portions of the cord is one of the morbid conditions giving rise to general paralysis and to paraplegia. This is intelligible in view of the fact that these portions contain the fibres which transmit volitions to the museles of the extremities. Affeeting the posterior columns, it constitutes the anatomical characteristic of the disease known as locomotor ataxia which will be treated of in chapter V. Portions of the cord adjacent to softened segments ave sometimes 
sclerosed, these two conditions attributable to chronic inflammation, namely softening and induration, being thus combined.

Non-inflammatory Softening of the Cord.-Softening of circumscribed portions of the cord, as of the brain, occurs without the evidence, afforded by either morbid appearances or symptoms, of myelitis having existed. From the anatomical characters, independently of those obtained by means of the microseope, it is not always easy to determine whether the softening be, or be not, inflammatory. 'Thickening or adhesions of the membranes corresponding to the portion or portions softened, renders the existence of myelitis probable. This is also true of induration or selerosis of the adjacent structure. The proof eonsists of the presence of inflammatory products within the softened tissue, namely, granular fibrin and leucocytes. It is a rational supposition that softening of portions of the cord, as of the brain, proceeds from embolism and thrombosis. Aside from this supposition nothing positively is known of the causation. This, like the other lesions within the spinal cord, is among the conditions giving rise to general spinal paralysis and to paraplegia.

Tumors within the Spinal Canal.-The different tumors which occur within the cranium, namely, tubereulous, carcinomatous, fibroid, hydatid, aneurismal, etc., are also sometimes found within the spinal canal. All are, however, in the latter situation much more infrequent. Osseous or calcareous plates formed on the visceral portion of the arachnoid are not very uncommon. All are embraced amoug the morbid conditions giving rise to either general spinal paralysis or to paraplegia. The obstruction caused by pressure on the cord may produce paralysis ; but, in addition, they are apt to give rise to circumscribed meningitis, myelitis, or meningomyelitis. It is important to take cognizance of the fact that morbid growths in this situation may be of syphilitic origin. These alone are notably amenable to remedies. There is but little ground to expect that tumors, other than those due to syphilis, will be removed or even diminished by any known remedies.

Atrophy of the Spinal Cord.-The foregoing lesions within the spinal canal lead to atrophy of the cord as a consequence of functional disuse of certain of its fibres. The disused fibres become attenuated and undergo fatty degeneration, as a result of suspension of their functions. This is in accordance with a pathological law applicable to all the organs and structures of the body. An illustration is afforded by the wasting of the muscles and the degeneration of nerves in cases of paralysis. A lesion of a section of the cord leads to atrophy of the antero-lateral columns, or the motor fibres, below, and of the posterior columms, or sensory fibres, above the seat of the lesion. A lesion confined to the antero-lateral columns leads to atrophy limited to the fibres composing these columns below and not above; and so a lesion confined to the posterior columns leads to atrophy limited to the latter alone. In other words, the consecutive or secondary atrophy is in a retrograde direction as regards the posterior, and in an onward direetion as regards the antero-lateral columns; and it affects the antero-lateral and posterior columns, singly or combined, according as the lesion is either limited to the one or the other, or invades both.

Atrophy of the cord also follows cerebral lesions which lead to functional disuse of the fibres entering into the antero-lateral or posterior columns, in accordance with the same pathological law as regards the limitation to either or the affection of both. Inasmuch as cerebral lesions occasion paralysis of motion vastly oftener than of sensation, the atrophy is much oftener seated in the antero-lateral than the posterior columns. 
These facts respecting atrophy of the fibres of the cord also apply to the sensory and motor fibres within the cranium.

In consequence of the atrophy being greater in some fibres than in others, the paralysis resulting from lesions within the cranium and spinal canal is apt to be greater in certain muscles than in others; hence, one reason for the distortions, especially of the wrists and fingers in certain cases of hemiplegia and general spinal paralysis.

It is an interesting fact that morbid changes in the optic nerves, occur in a number of cases of the different forms of disease within the spinal canal sufficient to show a pathological connection. The changes are atrophy, and the appearances denoting chronic neuritis. They occur after a considerable duration of the intra-spinal affections. For a discussion of different opinions as to the nature of the pathological connection, the reader is referred to works on ophthalmology.

\section{CHA P T E R I V.}

Paralysis, General Remarks on. Paralysis of the Third Cranial Nerve. Paralysis of the Fourth Cranial Nerve. Paralysis of the Fiftl Cranlal Nerve. Paralysis of the Sixth Cranial Nerve. Paralysis of the Seventh Cranial Nerve. Paralysls of the Eighth Cranial Nerve. Palalysis of the Ninth Cranial Nerve. Local Paralysls of other than Cranial Nerves. Paralysis of the Cervical Sympathetic Nerve. Paralysls from Lead.

THE term paralysis, in general, denotes loss or impairment of the power of muscular action. The term, however, embraces absence or diminution of either general or special sensibility. In the former application it is distinguished as paralysis of motion, or motor paralysis ; in the latter, as paralysis of sensation, or sensory paralysis. 'The latter, however, is expressed by other names, viz., anæsthesia and analgesia. The term akinesia denotes motor paralysis.

Motor paralysis relates to either the voluntary or involuntary museles. The stomach, intestines, bladder, and heart may be paralyzed. $\mathbf{A}$ paralyzed condition of these organs respectively has been considered incidentally in treating of their diseases. In the present connection the forms of motor paralysis to be considered will relate chiefly to voluntary power over muscles. Motion and sensation are affected conjointly in certain cases of paralysis. Such cases are far less frequent than cases in which motion is alone affected, but they are more frequent than the cases in which the paralysis affects sensation without motion. Cases in which sensation is alone paralyzed, exclusive of the special senses, are extremely rare.

Parylysis may be complete or incomplete. It is complete when there is total loss, and incomplete when there is more or less diminution, of either motion or sensation. The paralysis may be general, partial, or local. It is general, as applied to motion, when the power of volition over the greater part of the voluntary muscles is lost or impaired. Loss of power over both the upper and lower extremities constitutes general 
paralysis. The paralysis, however, may be still more general. A medical friend, Prof. Coventry, related to me a case which came under his observation, in which power was lost over all the voluntary muscles, with the exception of the orbicularis palpebrarum. Such a case exemplifies, in a striking manner, the excito-motory or reflex action of the nervous system, disconnected from volition ; respiration, deglutition, digestion, and all the functions necessary to existence going on, but, so far as voluntary manifestations are concerned, the patient being in a state of living death.

In partial paralysis, a limited division of the voluntary muscles is affected. The majority of the cases of paralysis belong to this category. The most frequent forms of partial paralysis are hemiplegia and paraplegia. In hemiplegia the paralysis affects the power over the muscles of a lateral half of the body. In paraplegia the paralysis affects the power over the two upper or lower extremities, almost invariably the latter.

A paralysis is local when it is still more limited as regards the number of muscles affected. Examples are, loss or impairment of power over the upper eyelid, or ptosis ; of more or less of the muscles of the face on one side, or facial paralysis ; of the muscles of the forearm, as in lead paralysis. A paralysis of any of the organs supplied with muscular structure not under the control of volition is an example of local paralysis, and also a paralysis limited to any one of the special senses.

Paralysis of sensation may embrace the two kinds of sensibility, viz., tactile sensation and the sense of pain, or it may be limited to the latter. The skin and the mueous membrane of the mouth and other outlets are endowed in health with both kinds of sensibility. Now, the capacity in these structures to receive painful impressions may be more or less impaired or lost, the sensibility as regards tact remaining. Paralysis of the sense of tact is called anæesthesia, and paralysis of the sense of pain analgesia. Analgesia may exist without anæsthesia, but, whenever the sense of tact is lost, the sense of pain is wanting. 'The loss of diminution of the sense of pain is readily determined by pinching and pricking the part or parts affected. Loss of the sense of tact is ascertained by finding that touching or bringing any substance into contact with the affected part or parts causes no sensation. To determine diminution of the sense of tact, the method introduced by Weber is to be employed. 'This consists in measuring the shortest distance at which two points of contact are felt. An instrument devised for this purpose by Sieveking, called the æesthesiometer, may be used; but the common compasses used by the draughtsman answer perfectly well. The further removed the two points of contact in order that each may be separately felt, the more the sensibility to tact is diminished; or, per contra, the sense of tact is shown to be unaffected if the patient be able to appreciate two points of contact approximated as closely as they are appreciable in health. The degree of tact, as thus measured, differs in different parts of the cutaneous surface in healthy persons. The deviation from health, therefore, is to be cletermined, whenever practicable, by a comparison of corresponding situations on the two sides of the body. ${ }^{1}$

Our knowledge of the pathology of paralysis cannot be complete until the normal functions of the brain and nerves are more fully understood. But the rationale of the production of paralysis is, in most cases, intelligible. Generally it depends on an obstruction in the course of either the nerves or the nervous filaments entering into the composition of the brain

1 A very convenient resthesiometer, devised by my colleague, Prof. Wm. A. Hanmond, may be obtained of Tiemann \& Co., of New York. 
or spinal cord, the transmission of volitions or the passage of sensitive impressions being, separately or conjointly, thereby restricted or prevented. In motor paralysis either motor nerves or motor nervous filaments arc obstructed; and obstruction of sensory nerves or filaments occasions paralysis of sensation. To render the pathological explanation still more comprehensive, in paralysis of either motion or sensation, or of both, the nervons ganglia standing in immediate relation to the mental perceptions and to volition fail to receive, as in a healthy state, sensitive impressions or to transmit the dietates of the will. This statement will include morbid conditions seated in the ganglia themselves, as well as obstruction in the nerves and nervous filaments. The statement, however, does not embrace cases in which involuntary movements are paralyzed; the conditions giving rise to the latter relate to the ganglionic or sympathetic division of the nervous system.

Limiting attention to the cerebro-spinal system, if a ligature be applied to a particular nerve, e.g. the seiatic, paralysis is produced, affecting both the voluntary power over the muscles through the motor filaments which this nerve contains, and the sensibility communicated by its sensitive filaments. The same effeets follow division of the nerve, olstruction from the pressure of a tumor, or disorganization from the disease of the nerve. So, complete obstruction at any part of the spinal cord cuts off sensation and voluntary motion in parts supplied by the nerves comnected with the cord below the point at which the obstruction is seated. So, likewise, an obstruction from any cause within the skull will affeet parts in proportion to the number and situation of the nervous filaments obstrueted. The existence of paralysis does not involve any inability to exert aets of volition or to perceive sensations. These mental powers are not dependent on the entire integrity of the nerves or even of the cerebral organs. Whenerer there are any manifestations of mind, there exists the power of willing and the capability of feeling; in other words, the morbid conditions giving rise to paralysis of either voluntary motion or of sensation pertain to the channels of communication between the mind and muscular organs or sensitive parts. A paralysis is said to be central or centric when it depends on a lesion or a morbid condition seated in the central or centric portions of the nervous system, that is, in either the brain or the spinal cord ; on the other hand, a paralysis is said to be peripheral or eccentric when it depends on an affection of a nervous trunk or its branches.

The morbid conditions giving rise to obstruction in eases of paralysis are generally apparent after death; but, in some cases, they are not appreciable, and this is the ground of a division into, first, paralysis dependent on obvious lesions, and, second, functional paralysis. In cases of socalled functional paralysis, something more than a purely dynamic change is doubtless involved. Molecular changes or histological lesions probably exist, but they are not determinable with our present means of examination or, at all events, they are not yet fully cletermined. The application of the microscope enables the observer now to discover lesions which were formerly inappreciable. The term functional, here as in other applications, means simply that adequate sensible changes are not as yet determined.

The term reflex paralysis has been applied to cases in which a paralyzer condition of eertain parts is attributed either to a wound or shock received upon other and more or less remote parts, or to a local disease situated elsewhere than in the paralyzed parts. It is diflieult to account for the paralysis in certain cases, save by supposing a reflex influence. It has been conjectured that this influcnce is exerted through the vaso-motor 
nerves, and that the paralysis is due to contraction of the bloodvessels of the nervous centres, in consequence of which an anæmic state of the latter is induced and their nutrition impaired. This explanation has been suggested by Brown-Séquard. Others have suggested as a more probable conjecture, paralysis of the bloodvessels of the nervous centres as an effect of the reflex influence on the vaso-motor nerves. ${ }^{1}$ Certain cases of paraplegia are supposed to exemplify the occurrence of reflex paralysis from disease, and I shall notice the grounds of this supposition together with the pathological explanations in treating of paraplegia. In cases of so-called reflex paralysis, the morbid condition, whatever it may be, is to be considered, in the present state of our knowledge, as functional. Handfield Jones and others consider that in certain of the cases of functional paralysis the morbid condition is expressed by the term exhaustion. 'The term paresis has come into use, as expressing this condition. Cases of so-called paretic paralysis are among the cases distinguished as functional.

As so far considered, paralysis proceeds exclusively from morbid conditions pertaining to the nervous system. This is true of the majority of cases of paralysis. The voluntary muscles, over which the power of the will is in abeyance, may not have lost their capability of motion. They may contract quickly and vigorously under the stimulus of electricity. They would contract equally under the stimulus of volition, were this stimulus to reach them. But, in some cases, the morbid condition giving rise to the paralysis is seated in the muscles. This appears to be, in part at least, the explanation of paralysis from lead. In cases of lead paralysis, the affected muscles are found not to be excited to contraction by the electrical current as in their healthy condition. As a means of measuring the capability of contraction in the muscles, and testing the conducting power of nerves, electricity affords important information in cases of paralysis. The ability to excite reflex movements, that is, movements taking place without volition, is also important in certain cases, as showing that muscles have not lost the capability of contraction.

A highly important fact relates to an effect of long-continued paralysis upon the nutrition of the affected muscles and nerves. Diminished bulk or wasting is an obvious result of long disuse, with which clinical observers have always been familiar. But recent microscopical researches have shown that fatty degeneration of the muscular and nervous fibres takes place when paralysis has been of long continuance. This degenerative change, when considerable, is a permanent structural lesion, rendering recovery impossible. The practical bearing of our knowledge of the remote local effects is obvious. It enforces the importance of effecting improvement, if practicable, before this change takes place, and of endeavoring to keep up the functional activity of the parts in order to prevent atrophy: and disorganization. Parts completely paralyzed in consequence of division, injury, or disease of a nervous trunk (peripheral paralysis) undergo degeneration more rapidly than when the paralysis is central. S. Weir Mitchell has pointed out the occurrence of chronic arthritic inflammation in paralyzed limbs, the affection being apparently the same as in articular

1 Reflex Paralysis, Circular No. 6, Surgeon-General's Office, March, 1864. Gunshot Wounds and other Injuries of Nerves, by S. Weir Mitchell, M.D., George R. Morehouse, M.D., and Wm. W. Keen, M.D., in charge of United States Army Wards for Diseases of the Nervous System, Turner's Lane Hospital, Philadelphia, 1864. See, also, article by Dr. S. Weir Mitchell, on the Diseases of Nerves resulting from Injuries, in the "Sanitary Memoirs of the War," medical volume published by the United States Sanitary Commission. 
rheumatism.' Certain speedy local effects of paralysis are apparent. The temperature of a portion of the body affected by paralysis is soon lowered, and the temperature is more easily affected by that of the surrounding medium than in bealth. The quantity of blood which the paralyzed portion receives is lessened, and the cireulation within it is sluggish. The activity of the cutaneous glands is diminished.

In the great majority of cases, paralysis has no intrinsic claims to be considered as an individual disease per se. It is a symptom or an effect of various morbid conditions, and the latter are, in reality, the diseases. But, in the existing state of our knowledge, it is convenient to consider paralysis as giving rise to a number of affections which I shall now proceed to notice. I shall first notice paralysis affecting the different cranial nerves, local paralysis of other than cranial nerves, and paralysis from lead; next, the two forms of partial paralysis known as hemiplegia and paraplegia, and general spinal paralysis; and, lastly, glosso-labial paralysis and general cerebral paralysis.

Paralysis of either of the nerves of special sense, viz., the olfactory, the optic, or the auditory nerve, is frequently dependent on abnormal conditions relating to the apparatus provided for the exercise of smell, sight, and hearing. This remark applies more especially to the eye and ear. Affections of the eye and ear, involving loss or impairment of sight and hearing, belong in the province of surgery, and treatises are devoted specially to them. It is important, however, for the physician to take cognizance of paralysis affecting these senses, as denoting intra-cranial disease. Paralysis of the senses has been already noticed in connection with some of the affections of the brain which have been considered. It may proceed from the varions intra-cranial lesions enumerated in the preceding chapter, these lesions affecting either the brain at the points at which the optic and auditory nerves emanate, or the nerves in their course from their points of origin to the organs of sight and hearing. In most cases, paralysis of these nerres of special sense, if due to intra-cranial disease, is associated with paralysis of other parts. Paralysis of the other cranial nerves severally elaim brief consideration. It is more correct to say paralysis of the parts to which the branches of a nerve are distributed than to apply the term to the nerve, but the latter is a convenient mode of expression, and is sanctioned by usage.

\section{Paralysis of the Third Cranial Nerve.}

Paralysis of this nerve, the motor oculi communis, gives rise to dropping of the upper eyclicl or ptosis, diverging strabismus, protrusion of the eyeball, and dilatation of the pupil. These effects correspond with the distribution of the several branches of the third pair of nerves. The internus rectus muscle being paralyzed, the eyeball is turned outward by the externus rectus, and the dilatation of the pupil is a result of paralysis of the circular fibres of the iris. The effects are so obvious and characteristic as at once to be referable to paralysis of this nerve. Paralysis of this nerve, existing alone, may be produced by an injury in the neighborhood of the eye, by the action of cold, and by over-exertion in occupations which require especially the use of the eyes. If produced by cold or over-use, it is probably functional, and will not be likely to be permanent. Double vision frequently accompanies paralysis of this nerve. It very rarely, if ever, exists alone, if it proceed from intra-cranial lesions;

1 Injuries of Nerves and their Consequences, 1872. 
it is associated with paralysis affecting other parts, or with other marked effects of cerebral disease. It is a rare event in cases of hemiplegia. Occasionally it is the first event pointing to grave cerebral lesions. This fact is to be borne in mind. It may be a forerunner of hemiplegia, convulsions, or coma. If ptosis exist without strabismus and dilatation of the pupil, it may be inferred that the paralysis is not central but peripheral.

\section{Paralysis of the Fourth Cranial Nerve.}

Paralysis of this nerve, the patheticus, produces effects less marked than those of the third nerve. The power of rotating the eye in the orbit is lost, and there is donble vision. Paralysis of this nerve is rarely an isolated affection, at least when symptomatic of intra-cranial disease.

\section{Paralysis of the Fifth Cranial Nerve.}

The effects of complete paralysis of this nerve are shown by its division within the skull in inferior animals. Loss of sensibility on one side of the face, on the mucous membrane within the mouth, the conjunctival membrane on that sicle, and the anterior portion of the tongue, with paralysis of the masticatory muscles on the same side, immediately follows. If the division be made anterior to the Casserian ganglion, remote effects are inflammation of the conjunctiva leading to ulceration of the cornea with discharge of the humors of the eye, and loss of the sense of smell on the paralyzed side. These remote effects do not follow if the division be made posterior to the Casserian ganglion, a fact which may serve to explain their non-occurrence when paralysis is due to lesions affecting the nerve at its point of origin, or between this point and the ganglion just named. The immediate and remote effects of paralysis of this nerve are now made familiar to medical students by the experiment of dividing the nerve in connection with physiological teaching.

Complete paralysis of this nerve from disease is extremely rare. The loss of power over the buccinator muscle, which frequently occurs in cases of hemiplegia, was considered by 'Todld as due to paralysis of the motor portion of the fifth nerve. Facts, however, disproving this statement will be presented in connection with hemiplegia. The motor portion may be affected alone, and, per contra, the sensitive may be affected without the motor portion. The different divisions of the sensitive nerve may be affected separately, viz., the supra-orbital, the superior, and the inferior. maxillary. The loss or impairment of sensibility will then be limited to the portions of skin and mucous membrane supplied by the branches of the paralyzed division.

Considered in itself, paralysis of this nerve is important in proportion to its completeness and its extent; that is, the paralysis may extend to the entire nerve or to only a single portion, and one or two of the divisions of the sensitive portion may be alone affected. It wonld seem also that its importance, as regards remote effects, depends on whether lesions giving rise to the paralysis are so situated as to obstruct the nervous filaments derived from the Casserian ganglion, that is, whether they are situated behind or before this ganglion. As significant of cerebral disease of some kind, paralysis of this, as of other cranial nerves, is of much importance.

Double paralysis of this nerve, that is, paralysis of the nerve on both sides, is sometimes observed. In a case reported by Althaus, the com- 
mon sensation of the face and scalp were lost, together with the sense of temperature and of tact; the mucous membrane of the eyes, nose, and mouth was completely anresthetic; the sense of taste was preserved, although the exercise of it seemed less prompt than in health; the muscles of mastication were completely paralyzed. The tongue presented a frightful spectacle, having been bitten and lacerated in every direction during the act of taking food. The patient experienced no pain when a tooth was drawn. The lips were covered with froth. The sense of smell and of sight were not affected, except that the latter was obstructed by thick letcoma of both corner. He suffered much from photophobia. There was an abundant secretion of mueus from the Schneiderian membrane. Hearing was intact, but the patient was greatly annoyed by tinnitus aurium. The paralysis was produced by long exposure of the face to a keen wind, the morbid condition being supposed to be inflammation of the neurilemma where the nerves emerge from between the transverse fibres of the pons Varolii at the base of the brain. The brain was unaffected, and the paralysis was limited strictly to the distributions of the fifth pair. At the end of three months, under a treatment consisting of the systematic application of the continuous galvanic eurrent to the several branches of the affected nerve, the recovery was nearly complete. ${ }^{1}$

\section{Paralysis of the Sixth Cranial Nerve.}

Paralysis of this nerve, the motor externus, leads to converging strabismus, the eyeball being turned inward by the action of the internal rectus or adductor muscle, from the loss of the antagonism of the external rectus or abductor muscle. The patient is unable to turn the eyeball outward, whereas, in ordinary cases of strabismus, this can be done if the other eye be closed. Paralysis of this nerve sometimes occurs in cases of cerebral meningitis, and in connection with lesions giving rise to hemiplegial. This nerve, however, escapes in the majority of the cases in which paralysis of other parts occurs from intra-crinial affections. When the nerve is paralyzed, paralysis of the third cranial nerve is frequently associated. 'The effect of paralysis of both these nerves is immobility of the eyeball, as regards lateral movements; it can neither be turned inward nor outward. Double vision is likely to exist if the paralysis be limited to the sixth nerve. Dr. J. Hughlings Jackson has reported a case in which paralysis of the sixth nerve on both sides accompanied paralysis of the facial nerve on the two sides. This is explained by the fact that the sixth nerve arises from the same nucleus as the facial, in the floor of the fourth ventricle.

\section{Paralysis of the Motor Portion of the Seventh Cranial Nerve.}

Paralysis of this nerve, the portia dura, or the motor facial nerve par excellence, is much less infrequent than paralysis of the sensitive portion of the fifth nerve. Complete paralysis of this nerve produces eharacteristic appearanees, by means of which the affection is at once recognizerl. The expression of the physiognomy peculiar to the person is abolished on the affected side. In speaking or smiling, the mouth and sometimes the extremity of the nose are drawn to the opposite side. This appearance 1869.

I Transactions of the Royal MIcdical and Chirurgical Society of London, vol. iii, 
is so striking and distinctive, that the existence of the paralysis is immediately apparent. The power over the orbicularis oculi being lost, the patient is unable to wink or to close the eye; when the attempt to close the eye is made, the eyeball is turned upward so that the cornea is concealed by the upper lid; the upper lid is abnormally elevated and the lower lid depressed, so that the eye appears to project more than in health. Owing to the loss of power over the orbicular muscle, the tears are not diffused over the conjunctiva as in health, and hence results epiphora. From the constant exposure of the conjunctiva in sleep as well as in waking hours, it becomes inflamed, and opacity of the cornea may be a result of the conjunctivitis. The eyebrow is lowered, and the patient is unable to frown or to wrinkle the forehead on the affected side, from loss of power over the corrugator supercilii and the anterior portion of the occipito-frontalis muscle. The alæ nasi cease to dilate, but may contract in inspiration, and dilatation, if it occur, is in expiration. Loss of power over the orbicularis oris on the affected side, renders the patient unable to whistle ; expuition is imperfect, the saliva dribbles from the angle of the mouth, and the pronunciation of certain letters, viz., o, b, p, is diffcult. Loss of power over the buccinator renders the eheek flaccid; it is liable to be wounded in mastication, and the patient is annoyed by the accumulation between the cheek and gums of food which he is obliged to remove with the finger. The cheek and lips on the affected side are sometimes puffed outward by the expired breath, as in the act of expelling tobacco smoke from the month. These obvious appearances are readily understood by reference to the anatomical distribution of the branches of this nerve. They are, of course, less marked in proportion as the paralysis is incomplete. The velum pendulum palati on the paralyzed side is sometimes abnormally flaccid, and the uvula deflected to the opposite side. The anditory sense on the paralyzed side is more acute than in health.

Paralysis of this nerve is not very infrequently an isolated affection; that is, it exists independently of intra-cranial disease. It may be due to the pressure of a tumor on the nerve after it emerges from the stylomastoid foramen. Before the discorery of the motor character of this nerve, surgeons sometimes divided it for the cure of tic douloureux, thas producing facial paralysis. The nerve may be affected within the petrous portion of the temporal bone, in connection with caries of this bone, and internal otitis. In such cases the paralysis is peripheral, not central. Facial paralysis occurs, as a functional affection, from the action of cold upon the face. I have known two instances of its occurrence in connection with anæmia, in one case at the end of gestation, and in the other case during lactation. In both cases the recovery after a few weeks was complete. It is sometimes produced in newly-born children by the employment of the forceps in labor. Facial paralysis may proceed from the varions intra-cranial affections which have been considered. In these cases, it is frequently associated with paralysis affecting other parts. It is sometimes a forerunnepo of arave cerebral affection.

It is desirable to determine, in individual cases, whether the paralysis be symptomatic of an intra-cranial affection, or due to an affection of the nerve after it leaves the cranial cavity, in other words, whether the paralysis be central or peripheral. If central, the paralysis is generally preceded or accompanied by symptoms denoting a cerebral affection. A point bearing on this question relates to the sense of taste. If the nerve be affected at its origin, or behind the situation at which the corda tympani is given off, the sense of taste on the lateral half of the anterior portion of the tongue corresponding to the paralyzed side, is diminished, 
as shown by comparing the sensations produced by placing successively on the two sides of the anterior extremity of the tongue some bitter substance in powder, for example aloes. . This diminution of taste is due to the corda tympani being involved in the paralysis. The taste is not affected if the cause producing the paralysis act upon the nerve anterior to the situation at which the corda tympani is given off. Flaccidity of the palatine arch on the affected side, and deflection of the uvula to the sound side, denote that the cause of the paralysis acts upon the nerve, either within the aqueductus Fallopii, or before it enters this canal, the branches supplying the levator palati and the azygos uvulæ museles being here given off. According to Duchenne and others, if the facial museles respond readily to electricity, the paralysis is dependent on an intra-cranial affection, whereas, the paralysis is due to an affection of the nerve when the muscles do not readily contraet under the electrical stimulus. This difference may be explained by the fact that in peripheral paralysis, as after the division of a nerve, the nerve and paralyzed parts speedily undergo degenerative change, whereas this change occurs more slowly when the paralysis is central. Paralysis of the sixth nerve accompanying paralysis of the facial nerve, denotes a central lesion, these nerves arising from the same point in the floor of the fourth ventricle. The absence of reflex morements is evidence that the paralysis is peripheral. If winking be not produced by touching the eyeball, the sensibility of the latter being preserved, the nerve is affected after leaving its central connection; on the other hand, if the eye be closed involuntarily by an impression made on the conjunctiva, when the voluntary power is lost, the paralysis is central.

According to Todd, the portio dura generally escapes in eases of hemiplegia. He attributes the facial paralysis whieh occurs in the latter affeetion to the motor portion of the fifth nerve. The power over the orbicular muscle, as a rnle, is either intact or but slightly impaired in eases of hemiplegia. As already stated, this opinion held by Todd is not tenable, as will be seen in treating of hemiplegia. Paralysis of the portio dura sometimes precedes the occurrence of hemiplegia.

In eases of functional facial paralysis, recovery may be expected after the lapse of several weeks. If the paralysis continue without improvement for two or three months, it is probably not functional, but, if not dependent on inter-cranial lesions, it proceeds from either disease of the nerve or something pressing upon it and occasioning obstruction. Under these circumstances, it will remain a permanent affection.

Paralysis of the seventh facial nerre on both sides has been repcatedly observed, but examples are extremely rare. ${ }^{1}$ This may result from injury or disease of the two nerres, or from intra-cranial lesions. The expression is then abolished on both sides of the face. The face remains inmovable, the only changes which are observed relating to the circulation. It is as if the face were covered by a mask. Relaxation of the soft palate may coexist, rendering the voice unnatural. Moreover, articulation is clifficult from the immobility of the lips. Mastication is difficult from the sane cause. The saliva dribbles from the mouth. The eyes are constantly open and staring. Aside from the great inconvenicnce which bilateral or double facial paralysis occasions, the prognosis is serious, or otherwise, according to the situation and nature of the morbid conditions on which the paralysis is dependent. In a case which came

1 For a report of 28 cases of double-faced paralysis, see article by Dr. Pierreson, in Arclives Générales de Médecine, Aout et Septembre, 1867. 
under my observation, with the double facial paralysis was associated incomplete general paralysis, and the patient died by apnoa from inability to expel, by coughing, matter in the air-passages. Paralysis affecting the alæ nasi may lead to an amount of depression with inspiration sufficient to occasion notable obstruction. This is exemplified in a case under my observation at the present moment. The cartilaginous portions of the nostrils on both sides are sucked in with the inspired breath to such an extent as to constitute a serious obstacle to respiration. A German writer, Wachsmuth, applies to paralysis of the serenth facial nerve on both sides the name facial diplegia.

\section{Paralysis of the Eighth Cranial Nerve.}

The paralysis affecting the pharynx, which occurs as a sequel of diphtheria, in certain cases, and which is sometimes limited to this situation, is to be considered as a paralysis of that portion of the eighth nerve called the glosso-pharyngeal. This local paralysis will be referred to in treating of diphtheria. Occurring in that connection, it is functional, and ends in recovery.

Paralysis of this nerve, together with the par vagum, occurs in various cerebral affections which produce deep coma, giving rise to impairment and loss of power of deglutition and diminished frequency of the respirations. This paralysis precedes a fatal termination. It may occur from a small cxtravasation of blood into or near the medulla oblongata. I have met with an example, the apoplectic attack being characterized by notable disturbance of respiration and inability to swallow, without complete loss of consciousness.

Paralysis of the spinal accessory nerve, giving rise to aphonia, has been already referred to in treating of the affections of the larynx. Incomplete aphonia may be a result of paralysis of this nerve.from the pressure of an aneurismal or other tumor on the recurrent laryngeal nerve, and it is sometimes produced by the surgeon in operations on the neck.

\section{Paralysis of the Ninth Cranial Nerve.}

Paralysis of this nerve, the hypoglossus, on one side, involves loss of power over the genio-glossus muscle on that side. The effect is a deviation of the tongue from a right line when protruded, the apex pointing to the side of the paralysis. The direction in which the apex points is readily understood when the action of the muscles which protrude the tongue is considered. These muscles extend from the chin to the base of the tongue, and when, from paralysis, the protrusion is effected by the muscle on one side, the base is drawn toward that side, and, as a result of the base moving in one direction, the apex turns to the opposite direction, the latter being the paralyzed side. Paralysis of this nerve rarely, if ever, exists as an isolated affection, and is generally symptomatic of intra-cranial disease. It exists frequently in cases of hemiplegia.

In determining the existence of this paralysis, it is to be borne in mind that the tongue may deviate from a right line in consequence of absence of teeth on the side to which the apex is turned, or of irregular inward projection of teeth on the other side. Dropping of the upper lip on one side produces an appearance as if the tongue deviated to the opposite side; and to obviate liability to error on this score, the upper lip should be raised with the finger while the tongue is protruded.

Paralysis of the hypoglossus nerve on both sides is extremely rare, but 
cases are sometimes observed.' In complete paralysis of this nerve on both sides, there is inability to protrude the tongue, and the power of speech is lost from in:lbility to articulate. Ineomplete paralysis renders protrusion of the tongue and artieulation more or less imperfect. Paralysis of this nerve on both sicles enters into a paralytic affection to be presently considered under the name glosso-labial paralysis.

\section{Local Paralysis of other than Cranial Nerves.}

Paralysis may be produced by injuries or diseases affecting nervous trunks in any part of the body. The loss or impairment of motion or sensation, or both, extends over the parts supplied with the nervous filaments given off by the nerve below the situation of the injury or clisease. Traumatic local paralysis follows division or wounding of nerves and contusions. If a contusion give rise to paralysis by simple shock or stunning the nerve, recovery may be expected. But paralysis due to palpable injury of the structure of a nerve is apt to be permanent, although reunion, with a restoration of function, may take place after the division of a nerve of considerable size. Diseases which give rise to local paralysis by affecting nerves are tumors or morbid growths situated within and without the neurilemma, and inflammation of the neurilemma, or of the nerve-substance. The latter occur's by extension of inflammation seated primarily in the structures adjacent to the nerve affected. Idiopathic neuritis is one of the rarest of rare affections. Paralysis due to the pressure of a tumor may be recovered from if the pressure be removed before disorganization or atrophy has resulted.

Pressure on a nervous trunk, continued steadily for several hours, may lead to temporarý or even permanent paralysis. Paralysis of an upper extremity has repeatedly occurred from remaining in profound sleep, or in a state of intoxication, with the arm hanging over the back of a chair, the paralysis being due to pressure on the brachial plexus. I have met with many examples of paralysis of the forearm attributable to lying, intoxicated, with the weight of the body upon the arm. Paralysis of a single muscle, or a small group of muscles, when not traumatic, is significant of syphilis as the cause.

The following interesting case of local sensory paralysis from injury has fallen under my observation. The injury was received by falling from a considerable height upon the deck of a ressel, and striking upon the nates, in a sitting posture. Permanent paralysis of the sensibility of the rectum and anus followed the contusion. Excrescences, which at one time existed around the anus, were removed without any sensation of pain. The patient has no consciousness of the accumulation of feees in the rectum, and never experiences the desire for defecation. Hence, the bowels are habitually constipated. 'The contraction of the sphincter, due to reflex influence, is intact. Involuntary evacuations occur only when diarrhoea exists, and when voluntary power over the muscles is requisite to prevent their occurrence. There is no paraplegia, no difficulty in evaenating the bladder, and no sexual deficiener. The accident occurred more than twenty-five years ago, and in the mean time the person has had good health.

A statement which has already been made with reference to paralysis of the facial nerve has a general application, namely, in cases of complete motor paralysis. When peripheral, the affected muscles become more

1 Vide Art. by Dr. Keyes, in the New York Med. Journal, Nov. $18 \pi 0$. 
speedily insensible to the electrical current than when the paralysis is central. In case of the latter, contraction of the paralyzed muscles may be excited during several months, whereas, in cases of the former, contractions cease to be produced after a few weeks. Hence, electricity may be made useful in determining whether the paralysis be central or peripheral. The explanation of this difference, as already stated, is, that, in cases of peripheral paralysis, atrophy and fatty degeneration of the affected muscles take place sooner than when the paralysis is central.

\section{Paralysis of the Cervical Sympathetic Nerve.}

The experiment of dividing the sympathetic in the neck, repeated by Bernard in 1851, and its effeets studied more fully than by previous observers, is now made familiar to medical students by those teachers in physiology who employ vivisections. The reader is, therefore, presumed to know that the immediate results of this experiment are, congestion of the head on the side corresponding to the section, increase of temperature clue to an exaggeration of the processes of nutrition, and contraction of the pupil on the same side. The effeets on the cirenlation and nutrition are attributable to paralysis of vaso-motor nervous filaments. Paralysis of this nerve oceurs from pressure upon it of a tumor, and it has followed surgical operations and other wounds. Contraction of the pupil is one of the symptoms of aortic aneurism when the tumor is so situated as to press upon this nerve. Dr. S. Weir Mitchell has reported a case in which paralysis of the right sympathetic was eaused by a wound from a musket ball. The right pupil was contracted, and this eye became myopic. On exercise, the right sicle of the face became flushed, while the left side was pale.' Dr. William Ogle has reported a case in which, from the local appearance and the phenomena, it was inferred that the right sympathetic had been destroyed by an abscess in the neck. In this ease, the right pupil was contracted, and the eyeball somewhat retracted. The right side of the head was redder and warmer than the left, especially after exercise. A singular feature in this ease was, the patient sweated on the left side of the face only. This was observed repeatedly after active exereise, and during exposure to great heat in taking a Turkish bath. Although this condition had existed for two years, no inflammatory process had followed. ${ }^{2}$ Functional paralysis of the sympathetic system, in different situations, has been supposed to be involved in certain eases of paralysis affecting the upper and lower limbs, and to enter largely into the pathology of nervous affections accompanied by disturbances of circulation, secretion, and nutrition. In the present state of our knowledge, however, such views have a physiological rather than a pathological basis, being founded chiefly on the relations of the sympathetic system to the vaso-motor nerves, as shown by experimental observations on inferior animals. ${ }^{3}$

\section{Paralysis from Lead.}

The accumulation of lead within the system may give rise to partial and even general paralysis, but, as a rule, the paralysis due to this cause is local. In the great majority of cases, the paralysis is limited to the ex-

Vide Injuries of Nerves, by S. Weir Mitchell, M.D., 1872.

2 Vide Trans. Royal Med. and Chirurg. Society of London, vol. lii., 1869.

3 For a report of several cases of unilateral sweating of the head attributahle to paralysis of the cervical sympathetic nerve, vide Art. by Roberts Bartholow, M.D., in Quarterly Journal of Physiological Medicine, Jan. 1869. 
tensor muscles of the lower or upper extremities, and the extensors of the upper are much more frequently affected than those of the lower extremities. The usual form of lead paralysis is that commonly known as wrist-drop. 'This name expresses the peculiar appearance of the upper extremities. The patient is unable to raise the hand on a linc with the forearm. Frequently the first series of the phalangeal bones of the hand cannot be brought on a line with tle back of the hand, but the second and third series can generally be extended. The dropping of the hands is characteristic of this affection. Whenever the peculiar appearance referred to is presented, the physician is justified in assuming that the paralysis is from lead. Paralysis of the extensors of the lower limbs is shown by inability to raise the feet with the heels resting on the floor, or raising the toes; the feet drop when the patient walks, and he therefore steps high to avoid tripping. If the extensors of the legs be affecter, the patient throws them forward with feebleness as compared with the force with which they may be flexed. Paralysis of the extensors of the lower limbs is not coinmon. In a case now under my observation, the extensors of both the feet and hands are paralyzed; the patient is affected with dropping at the ankle as well as at the wrist.

The appearance is diagnostic, but other evidence of lead-poisoning may almost always be obtained. As a rule, paralysis succeeds other characteristic effects of lead, more especially the affection known as leaclcolic. The blue line on the gums, referred to in treating of lead-colic, is always to be sought for. Generally the occupation of the paticut suggests the source of the paralysis, but in some cases the mode in which the lead has been introduced into the system is ascertained only after eareful investigation.

Lead-paralysis belongs among the symmetrical or bilateral affections. It is very rarely limited to one side, and corresponding muscles on the two sides are usually affected, but, in general, differently as regards the degree of paralysis. The paralysis may be complete or incomplete, and different cases differ much in respect of its degree. The power over the flexor muscles is frequently more or less impaired, but in some cases, with almost complete loss of power over the extensors, the flexors are but little, if at all, affected. This fact was illustrated in a striking manner in a case under my observation at Bellevue Hospital. Wishing to group several cases of paralysis for a clinical lecture in the hospital amphitheatre, which is situated at the top of the building, I desired to have a paticnt affected with complete paraplegia carried up from a ward on the ground-floor. Four flights of stairs were to be mounted. One of the patients, who was to make part of the group, was affected with lead-paralysis, the extensors of the hand being nearly powerless. He was a man of great strength, and he voluntecred to carry his fellow-patient to the amphitheatre on his back. It was necessary to assist him in obtaining a firm grasp of the patient, and when this was obtained, he performed the feat with ease, and, after the lecture, brought the patient down to the ward.

The paralysis is supposed to be caused by the presence of lead in the affected museles. It is difficult to understand its limitation to the extensor museles, and why the museles of the upper should be affected much more frequently than those of the lower extremities. The fact of its being a bilateral affection is consistent with the fact of the cause being brought to the affected muscles in the blood. The affected muscles are found to be feebly or not at all excited to contraction by the electric current. There is deficiency or loss of, not only electro-muscular contrac- 
tility, but electro-muscular sensibility ; that is, the sense of pain produced by passing a strong current through the affected muscles is slight compared with that felt in the unaffected muscles. If the paralysis be complete or considerable in degree, the affected muscles waste, and at length, if the paralysis continue, they become atrophicd and degenerated, the affection, under these circumstances, being permanent. The supposition that the paralysis is due to the presence of the lead in the affected muscles is not accepted by all pathologists. Lead has been found after death in both the muscles affeeted, and in the nervous centres. Hammond is of the opinion that the difficulty is in the motor nerve cells of the spinal cord.'

It is to be borne in mind that other muscles than those involved in the common form of lead-paralysis, or the wrist-drop, may be paralyzed by this cause. The evidence of lead-poisoning is therefore to be sought after in cases of paralysis in which other adequate causes are not evident, that is, in cases in which the paralysis is not traumatic nor connected with evident lesions. Amaurosis is one of the occasional effects of lead-poisoning. Anresthesia and analgesia of the surface have also been observed, affecting limited portions of the integument of the body and extremities. The muscles of the larynx concerned in phonation have been known to become affected, leading to aphonia; also, the lips and lingual muscles, rendering articulation difficult.

The duration of lead-paralysis is very variable. It is sometimes of short duration, but much oftener continues for a considerable period, and improvement, if it take place, is very gradual. If no improvement take place for several weeks, the prognosis is unfavorable as regards recovery; the paralysis will be likely to be permanent. If the paralysis continue long enough to lead to notable atrophy and degeneration of the muscular structure, the condition, as regards recovery or improvement, is hopeless. The proportion of cases in which this result takes place is not small.

The treatment of paralysis from lead involves, in addition to the measures applieable in general to paralysis, remedies having reference to the elimination of lead from the system, and especially the iodide of potassium. These measures have been considered in connection with lead-colic.

\section{CH A P T E R V.}

Hemiplegia. Paraplegia.

THE forms of partial paralysis of most frequent occurrence are hemiplegia and paraplegia. This chapter will be devoted to the consideration of these affections exclusive of their treatment, deferring the latter until general paralysis has been considered.

\section{Hemiplegia.}

The term hemiplegia denotes paralysis of a lateral half of the body, or the upper and lower extremity on one side. Muscles of the face may,

1 Diseases of the Nervous System, 1871. 
or may not, be involved. The paralysis may embrace both motion and sensation, or it may be limited to motion. In the great majority of eases the paralysis is limited to motion, or, if sensation be at first lost, it is shortly regained, although the motor paralysis eontinue. The paralysis may be complete or incomplete, and, if incomplete, it may be of every gradation, as regards degree, between the slightest appreciable diminution of motor power and the closest approximation to eompleteness. The paralysis may be eomplete in one and incomplete in the other extremity. If ineomplete in both extremities, the degree of paralysis is generally greater in one than in the other extremity, and in the great majority of cases the greater degree is in the upper extremity.

In cases of complete motor paralysis of the members, movements may frequently be produced by pricking the integument, tickling the sole of the foot, or applying a heated iron to the surface. These movements take place without volition, and much to the surprise of the patient, if his mental faculties be intact. They are purely reflex. They are produced in the lower, but rarely in the upper extremity. There is a notable difference in different eases as regards the condition of the muscles of the paralyzed limbs. They are, in some cases, soft and flaceid, offering no resistance to the movement of the limbs in different direetions; but, in other eases, they are hard and rigid, resembling, sometimes, museles in tetanic contraction, and offering much resistance to movements of the limbs.

If the paralysis involve the muscles of the face, the side affected, as a rule, corresponds to the paralyzed limbs. Exceptionally, the limbs on one side and the face on the opposite side are affected. When this is the case, disease of the pons Varolii is indicated. The following explanation is quoted from Dr. J. Hughlings Jackson, in the report of a case of paralysis of the facial nerve on the right, and of the limbs on the left side: "Sinee the nerve fibres for the limbs cross below the pyramids, those of the left arm and leg will pass in the right side of the pons, on their way to the higher parts of the motor-tract. But the facial nerve of the right side runs through the lower part of the right side of the pons to its nucleus on the floor of the fourth ventricle. So that a clot which damages the right facial nerve damages also the motor fibres which have come over from the left arm and leg."

On the paralyzed side of the face, the expression is abolished, and when the facial muscles are excited to action, as in speaking or smiling, the mouth is drawn to the opposite side. Voluntary power over the orbienlar muscle of the eye is rarely lost, although the paralysis of the limbs may be complete; the patient is generally able to close the eye on the affected side, but he eannot, as a rule, close the eye on the affected side while the other eye remains open. The power over the orbicular muscle, in a certain proportion of eases, is very obviously impaired. The patient usually winks on the affeeted side. It was the opinion of Todd that in the paralysis of the face, in eases of hemiplegia, the buecinator muscle is alone affected, and he attributed the paralysis to the motor division of the fifth, and not to the facial or portio dura nerve. This opinion was held by John Shaw, the relative and co-laborer of Charles Bell. The opinion is based on the wide differenee, as regards the extent and degree of the paralysis, between cases in which the facial nerve is alone affected, and the cases of hemiplegia in which the facial mnscles are involved. In cases of paralysis affecting exclusively the portio dara or facial nerve, the power of closing the eye is lost, winking does not take

1 Clinical Lectures and Reports of the London Hospital, vol. i. 1864. 
place, and the face is often much distorted by the loss of the antagonism of the muscles on the affected side. It is not easy to explain fully this difference, but the opinion of Todd is abundantly disproved by clinical and physiological facts. The limitation of the paralysis of the face in cases of hemiplegia to the buccinator muscle will not account for the appearances in a certain proportion of cases in which there is considerable distortion when the patient talks or smiles, and in which the orbicular muscle of the eye is to some extent affected. Moreover, if the motor branch of the 5th pair were involved, the masticatory muscles, exclusive of the buccinator, should be notably affected, and clinical observation does not show this to be an effect of the paralysis in cases of hemiplegia. Finally, physiological experiments have shown that the buccinator muscle receives no motor filaments from the fifth, but is supplied entirely by the facial nerve.' The fact that the involuntary act of winking frequently continues on the side affected with hemiplegia, is thus explained by Sanders: This act is reflex and is dependent on fibres arising from the medulla oblongata, which are more or less independent of the cerebral bemispheres. The same writer considers that the inmunity of the orbicular muscles of the eye, in certain cases, is to be explained by supposing that the nervous fibres of the 7th pair which are distributed to this muscle arise from parts of the brain not involved in the lesions giving rise to hemiplegia. The fact that the orbicular muscle of the eye is affected, as a rule, much less than other muscles of the face, is not more strange than that the upper extremity, as a rule, is notably more paralyzed than the lower, if the paralysis in both be not complete. In view of the considerations just presented, the conclusion is, that in hemiplegia the paralysis of the face is due to an affection of the portio dura or facial nerve, the motor branch of the 5th pair remaining unaffected." In some cases of hemiplegia the loss of power over the orbicular muscle is complete, and the face is distorted when the muscles are at rest.

If the patient protrude the tongue, the extremity frequently points to one side, the side on which the limbs are paralyzed. The reason of this has been already stated in connection with paralysis of the ninth nerve. The velum pendulum palati, on the side of the paralyzed limbs, is sometimes involved in the paralysis. This is shown by an abnormal flaccidity of the velum on one side, which remains motionless when the muscles of the other side are seen to contract on depressing the tongue with a spatula. The uvula in these cases is deflected to the opposite side; but this alone is not evidence of paralysis, as deflection of the urula to one side is frequently observed in healthy persons. Occasionally the third cranial nerve is involved, giving rise to ptosis, diverging strabismus, and dilatation of the pupil. When this nerve is involved, it is sometimes on the same side, but in the great majority of cases, on the side opposite to that of the paralyzed limbs. The coexistence of paralysis of the third nerve on the side opposite to the hemiplegia denotes that the centric cause is seated in the crus cerebri. The other nerves distributed to the eye are very rarely involved. The fifth nerve usually escapes. The eighth nerve is unaffected, save in certain cases of hemiplegia with coma about to terminate fatally. The nerves of special sense almost always escape. The muscles of respiration are not usually involved in the paralysis; the respiratory movements on the two sides are equal. The muscles of the neck and trunk in general remain unaffected.

1 Vide Physiology of Man, by Austin Flint, Jr., vol. iv.

2 For an able discussion of this question the reader is referred to a paper by $\mathrm{Dr}$. Wm. R. Sanders, in the London Lancet, January, 1866. 
Hemiplegia may be dereloped suddenly or more or less gradually. In the great majority of cases, it is suddenly developed in connection with an apoplectic attack. The patient is stricken down with apoplexy; the paralysis is apparent during the continuance of the apoplectic state, and remains after the patient has emerged from the apoplexy. But the paralysis may be equally sudden, withont the occurrence of apoplexy. 'The patient is then said to have a paralytic shock or a stroke of palsy, which the term hemiplegia derivatively signifies. 'The patient falls to the ground, but without losing consciousness. In some cases there is loss of consciousness for an instant, or for a few moments only. Frequently, if not generally, when sudden hemiplegia occurs without apoplexy, there is more or less disturbance of mind, denoted by confusion of ideas, or bewilderment.

The clinical history of hemiplegia, during its continuance, presents much diversity in different cases. It is impossible to judge at first of the prospect as regards the duration of the paralysis, or the amount of improvement which will take place. If life be not speedily destroyed by the lesions giving rise to the paralysis, more or less improvement takes place in the majority of cases. The improvement is usually gradual. The sensibility, if this have been lost, is first restored, and it may become abnormally increased on the affected side. 'The paralyzed condition of the face may diminish or disappear in a short time. Improvement, as a rule, begins sooner and progresses more rapidly in the lower, than in the upper extremity. Frequently the patient is alle to walk without much difficulty while the upper extremity remains almost completely paralyzed. The manner of walking varies in different cases. If the rotating muscles of the thigh be relatively but little affected, the limb is thrown forward so as to describe the arc of a circle; but frequently the body is inclined to the opposite side and the limb is dragged after its fellow. Sometimes it is brought forward with a jerking movement. Tremnlousness of the limb in walking is sometimes observed. The patient often experiences difficulty in walking from a depression of the toes, which renders him liable to trip.

In some cases of hemiplegia, complete recovery takes place after a few days or weeks. These cases form a small minority. In the great majority of cases complete recovery never takes place. The limit of improvement varies much in different cases. Persons with permanent hemiplegia not infrequently live many years. I have at the time I am writing two cases under observation of ten years' standing. I have records of several cases in which persons of middle and advanced age had been attacked with hemiplegia in infancy or childhood, a certain amount of paralysis remaining.

Remote effects upon the paralyzed parts are deficiency of volume or wasting and degeneration of structure, these effects being proportionate to the degree of permanent paralysis. Other remote effects relate to the contraction of certain muscles. Permanent contraction of the flexor muscles, especially of the forearm, is frequently observed. This effect, occurring at a late period in cases of hemiplegia, was considered by Todd as resulting from cicatrization with the loss of a certain amount of cerebral substance. It is in some cases due to a force of contraction sufficient to overcome antagonizing muscles, and, in other cases, to a greater degree of paralysis of the extensor muscles, the flexor contracting simply because they are not antagonized. In the latter case, the flexed parts are restored with a little force; in the former, the contracted muscles offer resistance to efforts for extension. Dr. S. Weir Mitchell has been led to 
observe the interesting fact that in cases of hemiplegia from cerebral lesions, the growth of the nails on the limbs of the affected side is arrested, and that a resumption of their growth is a forerunner of a return of power over the paralyzed muscles.

The mental condition in different cases of persistent hemiplegia presents great variation. The faculties of the mind are generally, if not invariably, more or less impaired. Patients are moved to tears or laughter by trivial circumstances. A marked change in character is frequently observed. They are apt to become impatient and irritable. They lose self-reliance, and are easily influenced by those around them. This fact sometimes has important medico-legal bearings. Exclusive of the cases in which hemiplegia has existed from early life, the tendency is to progressive impairment of the mental faculties, ending at last in imbecility. Cases differ much as regards the rapidity of progress toward this result. The mental deterioration doubtless often depends, in no small measure, on the deficiency of the exereise of the mental faculties after paraly'sis has occurred.

Loss of speech (aphasia) has been already considered as an occasional sequel of apoplexy. It characterizes certain cases of hemiplegia not developed in connection with apoplexy. Difficult articulation may result from the persistence of a paralyzed condition of the tongue and lips, and I have observed imperfect phonation attributable to paralysis of the spinal accessory nerve; but the loss of speech now referred to depends on inability either to recollect words, although their meaning is understood when used by others, or to co-ordinate the movements involved in the utterance of language. In thesc cases, often, the patient uses the same word or words whenever he attempts to speak.

If life be prolonged in cases of hemiplegia, subsequent attacks may be experienced. In these repeated attacks, the same side of the body may be affected, or the two sicles may be successively affected. Several cases of alternate hemiplegia have fallen under my observation. Simultaneous paralysis of both sides, or double hemiplegia, is occasionally observed. The lesion in these cases is seated in the pons Varolii, the explanation of the double hemiplegia being that the fibres from each side of the body are not widely separated in the pons as they are in the crus cerebri, thalamus opticus and corpus striatum. An anomalous form is the occurrence of motor paralysis on one side, and paralysis of sensation on the other side. This must be exceedingly rare. Cases, however, have been reportecl. The seat of the lesion is spinal in those cases which exemplify the decussation of the sensory fibres in the cord, according to the views of Brown-Séquard. I have met with an example of motor paralysis affecting the lower extremity of one side, and the upper extremity of the other side. The patient was a child two years of age. Preceding the paralysis, an abscess formed behind the ear on one side. The paralysis was at first nearly complete. Six months afterward, the patient had nearly regained full power over the lower extremity, and the paralysis of the upper extremity was much diminished. At this time there were no symptoms pointing to cerebral clisease, exclusive of the paralysis, and the general health was good.

The diagnosis of hemiplegia offers no difficulty. A very slight degree of paralysis may escape observation if the attention be not directed to it. The patient may not be aware of its existence. It is ascertained in such cases by causing the patient to grasp, with all his force, with each hand in succession, the hand of the physician, and to stand successively on each leg. The comparative sensibility to tact on the two sides is to be measured by compasses as already described, and the sensibility to pain contrasted by pinching or pricking the corresponding extremities. Paralysis 
is liable especially to be overlooked in young children, even when it is not very slight, the more because hemiplegia in the young child is extremely rare.

Malingerers sometimes feign hemiplegia. They are apt to pretend that sensation, as well as motion, is lost; this should excite suspicion. 'Hughlings Jackson has pointed out a mode by which this deception may generally be discovered. The malingerer keeps the arm close to the body when asked to stoop forward. In doing this, he exerts a voluntary power over the limb. In true hemiplegia, when the patient stoops, the palsied arin falls forward.

Having determined the existence of hemiplegia, the next point is to ascertain the morbid condition on which it is dependent. This is an important point in its bearing on prognosis and treatment. It is not always easy to determine whether hemiplegia be functional or not, and, if dependent on lesion, to arrive at a positive conclusion respecting its character. In a certain proportion of cases, its functional character may be isferred from its brief duration, the completeness of recovery, and the absence of symptoms, exclusive of paralysis, denoting lesion.

The lesions which give rise to hemiplegia are various. With rare exceptions, they are intra-cranial. They must be situated either within the cranium or at the upper part of the spinal canal. A lesion of the spinal cord, in order to give rise to hemiplegic paralysis, must be limited to an antero-lateral quarter of the cord. It is obvious that a lesion so limited is not likely to occur. Yet cases have been reported. An example is given by Todd.' In spinal hemiplegia, the lesion of the cord is on the same side as the paralysis. Of course, in this form of hemiplegia the cranial nerves are not involved ; the face is unaffected. A point of distinction relates to the intercostal and abdominal muscles. These are apt to be affected, whereas they are rarely affected in hemiplegia from intra-cranial lesions. The diaphragmatic movements also are apt to be affected in spinal hemiplegia. Another differential point is, that in cerebral paralysis the electro-contractility and electro-sensibility of the affected muscles is preserved, whereas, they are more or less impaired if"the paralysis depend on a lesion of the cord. This point is only available within a period of four months from the occurrence of the paralysis.

Almost invariably the lesions are intra-cranial and situated above the point of decussation of the pyramidal columus of the medulla oblongata. The paralysis is, therefore, on the side opposite to that of the lesions. Lesions found after death are laceration of brain-substance from extravasated blood, inflammatory exudation and softening from circumscribed cerebritis, non-inflammatory softening, pressure of exuded lymph in meningitis, and morbid growths, or tumors either within the brain or connected with the meninges and pressing on the brain. To these are to be added compression of the brain from depression of bone, and wounds of the brain, the consideration of which belongs to surgery. The different intra-cranial lesions give rise to hemiplegic paralysis when they are either seated in, or are so situated as to interfere by pressure with the functions of, those parts which contain the motor and sensory fibres in the hemisphere opposite to the paralyzed sicle, viz., the corpus striatum, the thalamus opticus, and the crus cerebri. In the great majority of the cases of hemiplegia, the centric cause affects either the corpus striatum or the thalamus opticus of the opposite side. A limited number of observations appear to show that lesions seated exclusively within the corpus striatum give rise to mo- 
tor paralysis only, and that lesions confined to the thalamus opticus give rise to paralysis of motion and sensation, that of the latter predominating. ${ }^{1}$

of the foregoing lesions, the most frequent is extravasation of blood into the substance of the brain. This is the lesion in a large proportion of the cases in which hemiplegia aceompanies and remains after apoplexy, and, in the majority of cases, hemiplegia and apoplexy are thus associated. Extravasation, however, is sometimes so small, or occurs so slowly; that hemiplegia is produced without apoplexy. With or without apoplexy, if the paralysis occur suddenly, especially in a person of middle or advanced age, and without having been preceded by symptoms denoting cerebral disease, the chances are that it is dependent on extravasation. This is almost certainly the case, if fever, together with the symptoms of cerebritis, be developed shortly after the attack and continue for several days, and if the patient be over forty years of age.

Hemiplegia produced by a clot in the brain is generally at first complete. The completeness is due to a stunning or contusion of the brain, and to the anamia caused by the pressure of the extravasated blood on the vessels in addition to the laceration of brain-tissue. The improvement which takes place is explained partly by the recovery from the stunning or contusion, and, in part, by the absorption of the serous portion of the extravasated blood. The recovery in such cases is never complete. The approximation to completeness of recovery will be close, other things being equal, in proportion as the permanent damage which the brain sustains is small. Assuming that the clot is absorbed and cicatrization takes place, the amount of permanent damage to the brain differs much in different cases, according to the size of the clot together with the extent of inflammation and softening in the substance of the brain surrounding it. In a case under my observation at Bellevue Hospital, in which the recovery was nearly complete, an apoplectic cyst was found after death, as large as an Ameriean walnut, situated in the corpus striatum.

The dependence of hemiplegia on inflammatory softening is to be inferred from the paralysis having been preceded by symptoms of circumscribed cerebritis. It is doubtful if complete recovery ever take place in these cases. The hemiplegia is not likely to be produced so suddenly as in the cases of extravasation. Extravasation, however, may occur in connection with inflammatory softening.

Hemiplegia is an occasional effect of the pressure of lymph in cases of meningitis. If it occur without extravasation or softening, the local conditions do not preclude complete recovery. It also occurs in cases of induration or partial sclerosis of the brain.

Tumors of the brain give rise to hemiplegia by pressure and by exciting circumscribed inflammatory softening. The hemiplegia in these cases is developed gradually, different parts being suceessively affected. The face is apt to be affected out of proportion to the paralysis of the limbs. Amaurosis, loss of hearing, and paralysis of the nerves distributed to the muscles of the eye, occur oftener than in cases of hemiplegia dependent on other lesions. Epileptiform attacks are not infrequent. The paralysis is progressive, and there is no ground for an expectation of recovery.

In a certain proportion of the cases in which hemiplegia is to be considered as functional, the paralysis is due to an interruption of the circulation in a portion of the brain by either an embolus or a thrombus in one of the cerebral arteries. The interruption of the circulation in a portion of the brain from embolism may, however, lead to sbftening; the paraly-

1 Vide Interne Pathologie par Jaccoud, Tome I. 
sis then persists and is, of course, no longer funetional. An embolus from the left side of the heart is likely to enter one of the carotid arteries, and, carried onward with the current of bloor, it is apt to pass into the middle cerebral artery, which is almost a direct continuation of the carotid. Plugging of one of the middle cerebral arteries at once deprives a portion of the brain of blood, because between the arteries beyond the cirele of Willis there are no anastomoses, and, therefore, the supply of blood by the collateral circulation is slow and difficult; hence, the oceurrence of softening from defective nutrition. An embolus is much oftener carried into the left than into the right middle cerebral artery, the reason being that the left carotid artery takes its origin from the arch of the aorta, and is in a direct line with the current of blood in the latter vessel, whereas the arteria innominata, from which the right carotid springs, forms an angle with the aorta. An embolus may also be derived from an aortic aneurism. Plugging of the cerebral artery may also arise from coagulation of fibrin within the artery, the clot, or thrombus, being caused hy the roughened interior surface of the vessel from calcareous degeneration. The paralysis from a thrombus is, in general, not sudden, but is developed more or less slowly. Non-inflammatory softening is to be inferred when the paralysis persists in cases of either embolism or thrombosis. Points which are diagnostic of embolic hemiplegia are the following: The paralysis occurs suddenly, either with, or without, an apoplectic seizure. The hemiplegia is oftener on the right, than the left, side, for the reason just stated. The hemiplegic attack is not followerl by fever and the local symptoms denoting cerebritis. The latter point is important, becanse the diagnosis involves especially differentiation from hemiplegia caused by a clot. Other points which are significant, as tending to exclude the latter, are the age of the patient, and the coexistence of either valvular lesions of the heart or aortic aneurism. If the patient be under middle age, the chances are in favor of embolism ; and cerebral embolism is generally assoeiated with the signs of cardiac valvular lesions, or with aortic aneurism. The short duration of the hemiplegia, and the completeness of the recovery, corroborate the diagnosis of embolism; yet it is to be borne in mind that non-inflammatory softening resulting from embolism involves persistence of the hemiplegia. With due attention to all the foregoing points, it must be confessed, the differentiation of cerebral hemorrhage from embolism is not always easy. In thrombosis, the hemiplegia, as a rule, is not sudden, but developed more or less gradually. This is the chief point of difference as contrasted with embolism. The latter causes a sudden and more or less complete paralysis, which is likely to diminish and entirely disappear if it be purely funetional, that is, if softening do not ensue. On the other hand, thrombosis causes, at first, a slight paralysis which increases more or less rapidly. To each of these rules, however, there are exceptions. Thrombosis may occur so quickly that the hemiplegia is sudden, and more or less complete, afterward diminishing. An embolism may occasion, at first, a partial occlusion of the vessel, and the obstruction be afterward made more complete by coagulation of blood at the point of the obstruction. In the latter case, embolism and thrombosis are continued; and the liability to this combination is to be borne in mind.

Embolism and thrombosis are among the causes of hemiplegia when it is to be considered as functional. Other examples of functional paralysis are to be mentioned. Todd distinguished certain cases as cases of emotional hemiplegia, the paralysis taking place during strong mental excitement, as in a violent fit of anger, recovery taking place, but sometimes 
slowly. Hemiplegia sometimes follows epilepsy, usually, in this connection being of transient duration. In a case which came under my observation in which epileptic paroxysms recurred at short intervals for several successive days, hemiplegia occurred before the paroxysms ceased, the face, as well as the limbs, on one side, being paralyzed. The paralysis completely disappeared after a few days. Functional paralysis sometimes occurs in connection with hysteria. The form of hysterical paralysis is generally paraplegia, but hemiplegia is sometimes observed. The face and tongue are not involved in this form of paralysis, and, as a rule, the left side is the side paralyzed. Hemiplegia has also been observed to follow chorea, the limbs paralyzed being those on the side most affected with the choreic movements. The paralysis in these cases does not extend to the face or tongue. Finally, functional hemiplegia is sometimes a sequel of diphtheria. I have already referred to a case in which hemiplegia, supposed to be functional, followed insolation (vide page 620.)

The prognosis in functional hemiplegia is always favorable. The recovery is a question of time; it may take place quickly, or the paralysis may be more or less protracted. In view of the contrast, as regards prognosis, between functional cases and those in which lesions are involved, it is desirable to discriminate between them in practice. The comnection with diphtheria, chorea, hysteria, or epilepsy, is a ground for the supposition that it is functional. The points distinctive of embolism and thrombosis are to be considered; and, finally, the cireumstances which go to exclude the varions lesions-clot, cerebritis, meningitis, sclerosis, tumor-are to be taken into account. In view of the importance of this discrimination, I shall subjoin brief abstracts of a few illustrative cases of functional hemiplegia:-

CASE 1. Mr. S., aged 75, merchant, formerly subject to attacks of rheumatism, a man of active habits, attending daily to business, left his home in the morning, to go to his place of business, in liis usual health. He was observed to act strangely; he walked with difficulty, and had difficulty in moving his hands. He insisted that nothing was the matter, but was prevailed upon to return home in a carriage. He got up stairs with considerable difículty, and immediately became somnolent, but was easily roused, giving incoherent replies to questions. I saw him an hour after he had been bronght home. He was somnolent, but without stertor. The face when he spoke was drawn to the right side; enmuciation was diffieult; the left upper and lower limbs were incompletely paralyzed, and the tongue turned to the left side. Yawning was freqnent. Pulse 70 and compressible. He swallowed with some difficulty. There had been no vomiting. When roused, he persisted in saying that nothing was the matter. He complained of no pain.

On the following day there was marked improvement. He had slept through the night and awoke perfectly rational. The distortion of the face and deviation of the tongue were much less, and the paralysis of the limbs was less. With assistance he walked to the water-closet. He ate a light breakfast. The heart-sounds were extremely feeble, and no cardiac murmur was discoverable.

The improvement continned, and, on the sixth day after the date of the attack, he insisted upon going out and attending to his business as usual. The paralysis had entirely disappeared. A little laurel-water and aconite were the only remedies employed. He was allowed at once a nutritious diet.

This case occurred more than six years ago. The patient has had no 
return of the paralysis, and is still living and well. The pathological condition in this case was, probably, thrombosis.

CASE 2. Mr. S., aged about 70, his general health good, and his mental faculties well preserved, took a long walk in the afternoon, returned at his usual hour, feeling as well as usual, and slept well during the night. On awakening in the morning, he complained of numbness in the right side of the body, and of deficient muscular power on that side. He was able to walk without diffienlty, but the grasp with his right hand was considerably impaired. There was no distortion of the face, and the tongue was protinded in a right line. The most marked difficulty related to the speech. There was no trouble in enunciation, but the difficulty was in the recollection of worls. On undertaking to speak, after a few words, he hesitated, and finally was unable to express what he desired to say, leaving a sentence so ineomplete that it was not easy to comprehend him. He was greatly distressed by his inability to converse. A side from the foregoing symptoms, he appeared to have no ailment. He had no pain, and had his usual appetite.

He remained in the condition just described for several days, and gradually recovered. This was in March, 1862. He has had no return of the paralysis, and is now (Nov. 1865) well. No remedies were given. The probable diagnosis, in this case, lies between embolism and thrombosis. My notes do not embrace the condition of the heart.

CASE 3. The following case, which came under my observation at Bellevue Hospital, is a good example of functional hemiplegia conmected with hysteria.

Susan C. aged 18, dressmaker, was admitted June 20, 1866. Three weeks before her admission, she retired at night perfectly well; but on awakening in the morning she found sensation and the power of voluntary motion completely lost in the left leg. Toward evening of the same day she suddenly lost sensation and the power of motion in the loft upper extremity. The complete paralysis of the upper extremity continued for a week, and then she gradually recovered the use of it. On her admission she could move this extremity freely, but there was complete loss of sensation and the power of motion in the left lower extremity. In walking with a crutch she dragged the paralyzed limb. There was not, nor had there been, any facial paralysis. The tongue was protruded in a right line. There was no rigiclity of the paralyzed muscles; no disparity between the two limbs as regards temperature, and the pupils were of equal size. Her intellect was intact. Soon after her admission she had retention of urine, and the catheter was resorted to for several days; but she succeeded in passing her water, on the physician in cliarge refusing to continue the use of the catheter. She was notably hysterical, the globus hystericus being well marked. She complained much of wandering neuralgic pains.

July 23d. During a thunder-storm the patient was much frightened, and had a hysterical convulsion. The cold douche was resortel to, which promptly arrested the convulsions, and, on recovering her conscionsness, she regained at once, almost entirely, power over the paralyzed limb. The next day she walked without diffieulity, and on the following day went out of the hospital on a pass. She remained well for about a week, and then suddenly lost the power of moving the left upper and lower extremity.

Aug. 6. Under the use of the shower-bath, she has recovered nearly complete power over the upper extremity, and partially over the lower extremity. 
This patient subsequently became affected with paraplegia, loss of the sense of pain and of tact coexisting with motor paralysis. She recovered completely from the latter, and was discharged from the hospital well.

CAse 4. A case at my hospital clinic, Nov. 24, 1871, illustrated the occurrence of two successive attacks of hemiplegia, the first attack being attributable to embolism, and the second to thrombosis. The first attack was sudden, the patient at the time of its occurrence being apparently well. The right side was paralyzed. There was no apoplectic seizure. The hemiplegia was, for a few moments, complete. In an hour it had entirely disappeared. Several months afterward, another attack occurred. In this attack, the paralysis was developed gradually, becoming nearly complete in the course of a week. He began shortly to improve, and, in a few weeks, he had entirely recovered. A slight diminution of motor power remained.

CASE 5. The probable interpretation of the following case is that the first attack was due to embolism from which resulted softening, and, as a consequence of softening, hemorrhage took place giving rise to the second attack.

A young woman, in December, $18 \uparrow 1$, was affected suddenly with left hemiplegia. In two weeks she had nearly recovered; but there remained slight diminution of motor power after a second attack which occurred in the July following. 'The second attack was sudden, the paralysis being on the same side. During the succeeding four months, gradual improvement took place. The patient could then walk with difficulty, but the recovery of voluntary power over the upper limb was small.

Case 6. A case referred to me by Dr. John L. Campbell, October, 1871, presented the following facts: 'The patient, a clerk, was 40 years of age. He had had rheumatism several years before. He was, as he supposed, in perfect health, when, at breakfast, he had sudden right hemiplegia, the paralysis being complete. It disappeared entirely within an hour. There was no pain in the head, nor loss of consciousness, nor confusion of ideas. After this for two weeks he had recurrences of the hemiplegia in like manner without any cerebral symptoms, the paralysis incomplete and lasting for a few moments only. During the period just stated, there were ten or twelve attacks, the health otherwise being perfectly good. The attacks then ceased, and I learned several months afterward that he was well. There were no signs of cardiac disease in this case.

In cases of hemiplegia dependent on intra-cranial lesions, the prognosis as regards the duration of life, will depend on the nature and extent of the lesions, and other circumstances. The persistence of the hemiplegia does not preclude the continuance of life for an indefinite period. Hemiplegia which has lasted from early life to middle age may not interfere at all with the duration of life. But, occurring in middle or advanced life, the lesions which exist in the majority of cases, viz., extravasation of blood, or softening, or both, generally involve more or less immediate danger to life; and, if the attack do not prove fatal, other attacks are apt to recur, from the persistence of the morbid conditions to which the primary attack was attributable. The connection of hemiplegia with syphilis, in certain cases, is a highly important fact as regards treatment and prognosis. Syphilitic hemiplegia occurs at variable periods after the date of the primary disease, the average time being about five years. The paralysis is preceded, for several weeks, by pain in the head. Dilatation of the pupil on either the paralyzed or the opposite side is of frequent occurrence, not associated with ptosis or strabismus. In most cases the hemiplegia comes on gradually; but it is sometimes sudden, and then 
usually without an apoplectic seizure. Improvement or recovery under anti-syphilitic treatment goes to confirm the diagnosis. $A$ highly important practical injunction is to suspect a syphilitic connection whenever a patient has had syphilis. Of the different lesions giving rise to hemiplegia, those which are of syphilitic origin are especially amenable to medicinal treatment. ${ }^{2}$

\section{Paraplegia.}

Bilateral paralysis, that is, paralysis affecting members on the two sides of the body, almost always affects either both the upper and lower extremities, or the two lower extremities. Paralysis of the two upper extremities, the lower limbs remaining unaffected, is extremely rare, but examples have oceurred. In such cases the paralysis must depend on a lesion or a norbid condition limited to those fibres of the cervical portion of the spinal cord which go to form the brachial plexus on the two sicles. To such cases the term paraplegia is applicable; but this term, in general, is used to express paralysis affecting the two lower extremities, and, in some cases, involving the bladder and reetum. The paralysis inay be motor, or sensory, or both motor and sensory. The paralysis is limited to motion in the larger proportion of cases, but both motion and sensation are lost or impaired much oftener than in cases of hemiplegia. There are cases in which the paralysis is limited to sensation, but, exclusive of cases of locomotor ataxia, they are exceedingly infrequent. Of 35 eases which I had recorded, and which I analyzed, in preparing the first edition of this work (1866), with respect to points pertaining to the clinical history of the affection, in none was the paralysis limited to sensation; in 11 cases, both sensation and motion were either impaired or lost, and in the remainder of the cases the paralysis was limited to motion. Sensibility as regards pain may be lost, and tactile sensibility preserved. 'This was noted in the histories of two cases. In several of the cases the sensibility was morbidly increased, that is, hyperasthesia existed. In the cases in which both motion and sensation were not lost, but more or less impaired, the paralysis of motion, in general, predominated. In one case, sensation was more impaired than motion.

Paraplegia may occur suddenly, but, in the great majority of cases, it is developed more or less gradually. Of the 35 cases, in six it occurred suddenly; in all but one of these six cases the patients fell to the ground with a paralytic stroke, complete paralysis, in the excepted case, being found to exist on awakening from slecp in the morning. In the cases in which the development was gradual, the period during which the paralysis attained to its maximum varied from a few days to several months. In several cases a sense of weakness on any unusual exertion, as in ascending stairs or in running, was noticed for some time before paralysis was suspected. Laborers continued to work for weeks, and even months, when it was evident paralysis existed. A liability to trip and fall in walking was sometimes observed before paralysis was recognized. In one case, sudden and complete paralysis occurred in one limb, and paralysis was gradually developed in the other limb, the paralysis becoming complete in the latter in three months.

In the majority of cases the paralysis was incomplete. In only 6 cases did it become complete, that is, so long as the cases were under observa1870

1 Vide art. by Prof. E. L. Keyes, in the N. Y. Medical Journal, Narember, 
tion. The cases varied greatly as regards the degree of the paralysis. If the paralysis be slight, or moderate, it occasions only a certain amount of diffieulty and insecurity in walking. In walking over uneven ground, or whenever a misstep is made, the patient is unable to excrt quickly enough a sufficient degree of muscular effort to recover himself, and he is therefore liable to fall. This gives him a sense of insecurity. It is difficult for him to walk in the dark. With a greater amount of paralysis, locomotion is impracticable without the aid of a cane, and, with a still greater amount, crutches are necessary. If the paralysis approximate to completeness, the patient is confined to the bed and to the chair. If the paralysis affect sensation as well as motion, a difficulty in walking, in addition to the impairment of muscular power, arises from the inability to feel the contact of the feet with the ground. The patient is guided in his movements by the sight, and is unable to walk without the aid of the eyes. Not infrequently more or less impairment of the power of coordinating voluntary movements is associated with the paralysis in cases of paraplegia.

A marked point of difference in different cases relates to reflex movements. These may be sometimes exeited to a great extent in cases of complete paralysis. I have seen the limbs flexed quickly so as to bring the thighs to a right angle with the trunk, in a case of complete paralysis, by irritating the inguinal region of the abdomen. In some cases reflex morements are slight, and in some altogether wanting. Tremulousness of the paralyzed muscles in cases of incomplete paralysis falls under the head of reflex movements. This is a marked feature in some cases. Whenever the patient undertakes voluntary movements, tremor of the muscles is excited. Involuntary twitching of the affected muscles is a symptom in some cases. Cramps not infrequently occur, especially at night. In some cases there is rigidity of the muscles, and in other cases the muscles are flaccid. Spontaneous movements, i.e., without excitation by tiekling the soles of the feet or other parts, are sometimes frequent and extensive. In a case at Bellevue Hospital the legs were drawn up and the thighs flexed at a right angle with the body, and these movements were repeated at short intervals, so that, at the patient's request, the limbs were mechanically confined in an extended position. These spontaneous movements were much more marked on some days than on others. At length the flexion of the thighs and legs became permanent, and continued until the death of the patient. The paralysis in this case was complete, and followed a strain from lifting a heavy weight. An examination after death, in this case, revealed softening to diffluence of the cord below the sixth dorsal vertebra, connected with caries of the spine, the seventh dorsal vertebra being separated into two pieces, and these projecting into the spinal canal.

A marked difference in different cases relates to pain in the back and limbs. Pain in either the back or limbs, or in both situations, is a prominent symptom in some cases, and in other cases it is wanting. Pain sometimes precedes and accompanies the development of the paralysis, and afterwards disappears. Patients sometimes complain of a sense of coldness, and sometimes of a burning sensation in the affected limbs. A sense of numbness, a feeling such as exists when the limbs are said to be asleep, and the sensation commonly known as of pins and needles, are other symptoms more or less marked in certain cases. The temperature of the paralyzed limbs is sometimes higher, but often lower, than that of health.

An important difference relates to the pelvic organs. In a minority of cases these organs remain unaffected. They are more or less implicated 
in a majority of cases. Constipation usually exists. The sense of the presence of the feces in the rectum is not infrequently lost, and after a purgative, or if diarrhœa occur, evacuations take place involuntarily. The mildest affection of the urinary system consists in the need of strong voluntary abdominal efforts to expel the urine. This sometimes precedes the paralysis of the limbs. Retention is not uncommon, requiring the use of the catheter. Incontinence of urine is common, the urine dribbling away, especially at night. In some cases of slight or moderate paraplegia, the patient, whenever the desire is felt, is obliged to urinate in haste to avoid involuntary micturition. The accumulation of urine in the bladder, in some cases, leads to the decomposition of urea, and the urine is ammoniacal when passed. In these cases the urine, rendered irritating by changes within the bladder, sometimes gives rise to cystitis. The urine is then found to contain mucus and pus in more or less abundance. Irrespective of cystitis, or retention, the urine is in some cases alkaline, and in some cases acid. If allialine, it may deposit the phosphates more or less abundantly.

Priapism, that is, erection without venereal desire, is an occasional symptom. As regards venereal desire, different cases differ. Complete paraplegia, affecting both sensation and motion, is not incompatible with sexual passion and procreative power. Not infrequently there is loss of the desire and capacity for sexual intercourse. This I have noted in several of the cases which I have recorded. In one of my cases, in which recovery took place after five years, there was absence of venereal excitement for that period, and reappearance of sexual appetite shortly before complete recovery from the paralysis. A sensation of stricture across the abdomen, as if it were encircled with a tight girdle, is an occasional symptom.

Different cases present great variation as regards the course of the affection and its duration. Exclusive of the eases in which complete paralysis occurs suddenly, the affection increases sometimes rapidly, but oftener slowly, until it has attained to a certain degree, which may be slight or moderate or considerable. The paralysis remains at this point stationary for a variable period-weeks, months, or even years-and then, in a certain proportion of cases, improvement begins, which may progress to a greater or less extent, but without ending in recovery ; or recovery may take place. In another class of cases, after the paralysis reaches its maximum, either becoming complete, or, if incomplete, greater or less in degree, it remains permanently at that point. And in this class of cases life may continue for an indefinite period, the functions of digestion and nutrition being well performed. The muscles waste, other things being equal, in proportion to the degree of paralysis and its duration. In cases in which complete paraplegia has existed for many years, the general health being good, the attenuation of the lower limbs is in striking contrast with the body and upper extremities. The continuance of paraplegia, although complete, does not lead to imbecility or notable mental deterioration. Persons, under these circumstances, preserve their mental powers unimpaired. In this respect, paraplegia is in striking contrast with hemiplegia.

Paralysis of the lower limbs, limited to sensation,. may be due to a morbid condition of the nerves at their periphery. I have met with a case in which, apparently from the action of cold, there was loss of sensibility below the knees, motion being unimpaired. The patient was obliged to be guided by the visual sense in walking. The paralysis may be due to the condition of the muscles, as when it depends on the presence of lead. But, as a rule, paraplegic paralysis proceeds from an affection of the spinal 
cord. To attribute it to an intra-cranial affection, is to suppose a morbid condition to exist in both hemispheres sufficiently limited in each to obstruct the motor nervous filaments going to the lower extremity without interfering with the filaments going to the upper extremities. Such a coincidence is possible, but vastly improbable. Even when paralysis is limited to one lower extremity, the morbid condition is far more likely to be seated within the spinal canal than within the skull. That paraplegia may be a direct effect of cerebral lesions has been maintained by distinguished writers-Serres, Esquirol, Durand-Fardel, Watson, Romberg, and others-the opinion being based on cases presenting amaurosis or other evidence of cerebral disease; and on the fact that eerebral lesions are sometimes found after death, and no morbid appearances in the spinal cord are discoverable. But inasmuch as paraplegia may exist without any lesions of the cord which are diseoverable after death, it is rational to consider the paraplegia as functional in these cases rather than as a direct effect of cerebral lesions. Inflammation of the meninges of the cord (spinal meningitis), or of the substance of the cord (myelitis), and the intra-spinal lesions noticed in a preceding chapter, viz., softening, sclerosis, hemorrhage, tumors or morlid growtls, may respectively stand in a causative relation to paraplegia. But paraplegia sometimes exists and proves fatal, and no appreciable changes are found after death. Careful microseopical examination has shown, in some cases, changes not apparent to the naked eye. Doubtless there are molecular changes even when the microseope, with our existing knowledge, fails to reveal them; but we must admit, for the present at least, the existence of functional paraplegia. Practically, it is highly desirable to determine, in individual cases, whether the paralysis be functional or not, and if not functional, whether it depend on meningitis, myelitis, or on some one of the lesions just enumerated. However, to determine these points with positiveness will often be found difficult, and sometimes impossible, with our present means of differential diagnosis.

The sudden occurrence, without premonitions, of complete paralysis, involving sensation and motion, accompanied with more or less pain, traumatic causes being excluded, suggests, as a probable morbid condition, hemorrhage within the canal or cord. Brown-Séquard has eited two cases in which a hemorrhage limited to one lateral half of the gray matter of the cord produced loss of movement on the side of the hemorrhage, and loss of sensibility on the opposite side. ${ }^{1}$

The symptoms which denote acute inflammation of the substance of the cord (myelitis), or circumscribed inflammation of the meninges (spinal meningitis), as the causative condition are, of course, those belonging to the clinical history of these affections. (Vide Chapter II. and Chapter III.)

The development of paralysis dependent on inflammation of the meninges of the cord, or of the cord itself, is more or less gradual. The inflammation may be caused by a blow or a fall upon the back. Caries of the spine gives rise to paralysis, by causing meningitis or myelitis, or, if angular curvature follow, by pressure on the cord.

Induration or sclerosis of the cord gives rise to paralysis differing, as regards degree, according to the extent to which the antero-lateral columns are affected. If the lesion do not extend beyond the superfices, reflex movements may be excited, and the electro-muscular contractility of the paralyzed muscles is preserved. It is otherwise if the lesion extend to the

' Lectures on the Central Nervous System, Philadelphia, 1860. 
centre of the cord. Sensation is not diminished in the affected limbs, if the lesion do not extend to the posterior columns. If, however, the posterior columns be affected, the paraplegia is associated with more or less impairment of the ability to co-ordinate the movements of the paralyzed museles, the latter constituting locomotor ataxia. ${ }^{1}$

In cases of paraplegia due to non-inflammatory softening of the cord, the development of the paralysis is usually slow and nnaccompanied by the pain in the back and limbs, spinal tenderness, cramps, convulsive movements of the paralyzed muscles, and rigidity of the muscles of the back, which characterize cases of local myelitis or meningitis.

Tumors, intra-spinal in their origin or extending into the canal from without, may give rise to paralysis by exciting inflammation of the meninges, or of the cord, or of both, and the symptoms then will be those of local meningitis or myelitis ; or tumors may give rise to paralysis by pressure on the cord, and the symptoms will then be analogous to those of non-inflammatory softening. To determine the existence of a tumor of which there is no external appearanee, is hardly practicable. A tumor injuring a lateral half of the cord may give rise to loss of motion in one limb and loss of sensation in the other limb. The existence of a syphilitic tumor may be suspeeted if the patient have had syphilis, and nodes have appeared in other situations. This suspicion should suggest remedies having reference to syphilis. Notable improvement under the use of mercury, or the iodide of potassium, corroborate the diagnosis.

Paraplegia may be considered as functional when the absenee of inflammation and structural lesions is to be inferred from the symptoms and course of the affection. Pathological relations are to be taken into account in arriving at this opinion. Paraplegia occurring in connection with hysteria, called hysterical paraplegia, is devoid of the evidence of inflammation, and the completeness of recovery is proof that the lesions which have been named did not exist. I have met with a case in which repeated attacks of functional paralysis, the paralysis involving sensation as well as motion, had occurred between the ages of seven and of ten years, the patient being subject to hysterical manifestations. In like manner, when paraplegia occurs as a sequel of diphtheria, it is to be considered as functional. Cases have been reported by Graves, Romberg, Rayer, Brown-Séquard, and others, within late years, with paraplegia apparently referable to various affections of different organs, viz., the kidneys, bladder, utei'us, ovaries, intestines, ete. A causative connection between the paralysis and diseases seated in these organs is inferred from the fact that recovery from the former takes place after the latter are cured. It is supposed that the local cliseases induce paralysis by a morbid influence transmitted through the reflex system of nerves, and hence the term reflex paraplegia has been used to distinguish the affection in these cases. In order to establish a pathological connection between different local diseases and paralysis, it is necessary to show that the former precede the development of the latter in a proportion of cases too large to be explained by merely aceidental coineidence. It is questionable whether facts sufficient to show this have been, as yet, aceumulated. And when, on the other hand, it is considered that the various local diseases, supposed to be adequate to the causation by a reflex infuence are not accompanied by paralysis in the vast majority of cases, the existence of a calusative

I For an account of sclerosis, non-inflammatory softening, and tumors, sec Structural Lesions within the Spinal Cord, Chap. III. 
relation may reasonably be doubted in the cases in which the association exists.

It has been conjectured that diseases of organs more or less remote from the spine may give rise to paraplegia by inducing spasm of the arteries of the cord, in this way diminishing the supply of blood and impairing nutrition. This is the explanation of the so-called reflex paraplegia given by Brown-Séquard. That spasm may be produced by a reflex influence emanating from cliseased organs is inferred from the facts observed in experiments on inferior animals. This inference may fairly be questioned; but, admitting its correctness, the explanation of BrownSequard assumes that a degree of anæmia sufficient to occasion notable impairment of the functions and nutrition of the cord may be due to spasm of the arteries, that the spasm may be a persisting morbid condition, and that it may be limited to a segment of the cord. These are conjectures which certainly admit of much doubt. On the other hand, it has been conjectured that the supposed reflex influence induces congestion of the cord, by paralyzing the vaso-motor nerves. ${ }^{1}$

Congestion of the cord is generally enumerated among the morbid conditions giving rise to paraplegia. It may be doubted whether simple congestion of the cord is ever sufficient to produce paralysis, and, still more, whether simple congestion is ever so limited to a portion of the cord as to produce paraplegia. The tortuous course of the veins within the spinal canal, the absence of valves in these veins, and their relation to the venæ cavæ, would seem to render it probable that passive congestion is peculiarly liable to take place in this situation, and it might be supposed, $\grave{a}$ priori, that active congestion would be here of frequent occurrence, taking into view the excitations to which the spinal cord is subject. But general spinal paralysis, exclusive of traumatic cases, is extremely infrequent, and paraplegia is not observed under the circumstances which must give rise to more or less congestion, sueh as the cold stage of a paroxysm of intermittent fever, prolonged exposure to cold, cirrhosis of the liver, abdominal tumors of large size, pregnancy, disease of the heart, etc. In the present state of our knowledge it is, to say the least, conjectural to reckon congestion of the cord among the causative conditions standing in a causative relation to paralysis.

The production of paraplegia by the use of flour made from the bean of the lathyrus sativus in some parts of India, suggests the reflection that it may sometimes be produced by poisons not yet ascertained. As already stated, Dr. Handfield Jones considers that functional paralysis may depend on simple exhaustion of the nervous centres, and he employs the term cerebral or spinal paresis to denote this condition, distinguishing cases in whieh this is supposed to be the morbid condition as cases of paretic paralysis. ${ }^{2}$

As further means of distinguishing in practice cases of functional paraplegia, in addition to the exclusion of inflammation and lesions, the following points are to be mentioned: The paralysis is generally, but, by no

1 For an able discussion of the theory of reflex paralysis, by Dr. S. Weir, Mitchell, see New York Monthly Journal of Medicine, numbers for February and March, 1866. Dr. Mitchell shows that in several of the cases cited by Graves and others the evidence that the local affections preceded the paralysis is insufficient; and in some of the cases it is not clear that paralysis existed. See, also, paper by Dr. William Gull, in Guy's Hospital Reports, London, vol. vii., 1861 ; also Prize Essay of the New York Academy of Medicine, by Robert T. Edes, 1869.

2 Clinical Ohservations on Functional Nerrous Disorders. London, 1864. Republished by H. C. Lea. 
means invariably, incomplete, the bladder and rectum are rarely implicated, motion is alone impaired in the majority of cases, but not invariably, and the nutrition of the muscles is but little affected. The favorable progress, under judicious treatment, goes far toward contirming the correctness of the diagnosis in cases considered as functional. To the foregoing diagnostic points in differentiating functional paraplegia, however, there are exceptions. A young woman who came under my observation at Bellevue Hospital, found herself completely paraplegic on awakening in the morning. She was subject to epilepsy at night, and a paroxysm occurred during the night when the paralysis took place. She was a prostitute, and had had sexual intercourse with several persons before falling asleep. The paraplegia was complete as respects motion and sensation. Very slight reflex movements could be excited. There was retention of urine, and partial want of control over the rectum. On the eighth day after the paralytic attack, sensation began to return, and on the ninth day the recovery of both sensation and motion was complete. On the thirteenth day she was well enough to be discharged, having had no treatment directed specially to the paralysis.

Functional paraplegia is attributable, in some cases, to excessive venereal indulgence or to masturbation. Among the cases which I have recorded, is one in which the patient, a male, aged 50 years, admitted an almost daily indulgence in the venereal act for twenty-five years. Males are more subject to paraplegia than females. Of 150 cases observed by Brown-Séquard, in 110 the patients were of the male sex.

The prognosis in cases of paraplegia must have reference to the different morbid conditions of the cord on which the paralysis depends. Cases in which the paralysis is due to hemorrlage or spinal apoplexy generally end fatally within a short period. Recovery from myelitis, without injury to the cord suffieient to render permanent a greater or less degree of paralysis, is rare. The prospect in cases of local meningitis of the cord is better. If the inflammation be limited to the meninges, and the paralysis be caused wholly by the pressure of lymph, with the cessation of the inflammation and absorption of the lymph the causative condition is removed, and complete recovery may take place. In cases of sclerosis notable improvement may take place, but recovery is never to be expected. In cases of non-inflammatory softening, neither recovery from the paralysis nor improvement is to be looked for. The best that can be expected, in such cases, is that the paralysis will either remain stationary at a certain point, or increase very slowly. In the cases in which the paralysis depends on the pressure of a tumor, or of the bone in displacement from caries, there may be improvement in so far as the paralysis may have been increased by local meningitis, but in proportion as the pressure of the tumor or bone produces obstruction, the paralysis will be permanent, because most tumors are persistent and are more likely to increase than to decrease. Syphilitic tumors form an exception to the rule just stated. These may diminish or disappear ; and if they have not led to destruction of any portion of the cord, recovery may take place. In cases of functional paraplegia, that is, paraplegia not dependent on either inflammation or manifest lesions, the prognosis is always favorable.

In cases of complete paraplegia, extensive sloughing of the integument on the nates is liable to occur if great pains be not taken to prevent it. In this way the paralysis may prove fatal. Exclusive of this incidental effect, the paraplegia does not tend to destroy life, irrespective of the morbid conditions which stand in a causative relation to it, or with which it may be associated. So far as concerns the paralysis, the prognosis has 
reference mainly to recovery or improvement, on the one hand, and, on the other hand, increase or a stationary condition. In general, if the paraplegia have existed for a series of months or years without improvement, and still more, if it have progressively increased, the probability is that it will be permanent. But there are exceptions to this rule. Among the cases which I have recorded is one in which paraplegia became gradually developed six months after an injury from a fall whieh led to angular curvature of the spine. The paralysis continued for three and a half years. The patient then recovered perfectly, and was able to work as a laborer for five years. At the end of this period, almost complete paraplegia again occurred, involving sensation and motion. He again recovered after a year and a half, and worked without difficulty for four years. At the end of this period he had a second recurrence of the paralysis. After two months there was improvement, and the case was then lost sight of.

Another of the cases exemplifies recovery after a still longer duration of the paralysis. This patient, a German emigrant of considerable intelligence, became paraplegic shortly after his arrival in this country, and was sent to Ward's Island. He remained there five years. During three of these years he was confined mostly to the bed. During the last two years there had been gradual improvement. He came under my observation at Bellevue Hospital at the end of the five years, and after a few months was discharged, having perfectly recovered.

Such cases as these afford ground for encouragement, notwithstanding the paralysis may have existed for a long time.

Almost every practitioner is acquainted with cases of paraplegia, either complete or incomplete, of many years' standing, in which digestion, nutrition, and all the bodily functions, together with the mental faculties, remain unaffected. The following case, reported by the late Prof. Childs, is one of the most remarkable which have fallen under my notice:-

"M. G., on the 6th of November, 1804, being then a stout and healthy man 22 years of age, while painting the tower of a church, was precipitated, by the fall of a scaffolding, a distance of forty feet, striking the frozen ground upon his back and hips. Paralysis of the lower part of the body was found to be complete and loss of sensation entire. A careful examination showed depression corresponding to the twelfth dorsal vertebra. In short, the patient exhibited the usual symptoms of fracture and dislocation of the spine with compression of the medulla. Vigorous but unsuccessful attempts at reduction were made. The treatment consisted of local depletion, careful attention to the bladder and bowels, and afterwards baths, liniments, friction, electricity, etc. etc. The patient soon became comfortable, and lived from November, 1804, to August, 1857 ; when he died of double pneumonia. During this period, although completely paraplegic, he married, had six children, and by his energy and industry amassed a little fortune for his family. At the post-mortem examination, the entire spine was removed and carefully examined. The specimen is preserved in the museum of the Berkshire Medical College. The injury was found to consist of fracture with displacement of the twelfth dorsal vertebra, and below this point the cord was completely atrophied.

"I have now to describe the phenomena during the long period extending from the injury to the death of the patient-fifty-three years. These may be conveniently grouped under four heads: ist. The effect of the injury upon sensation and voluntary motion. 2d. Upon nutrition and secretion. $3 \mathrm{~d}$. Upon the evacuations. $4 \mathrm{th}$. Upon the generative function. 
"I have said that the loss of sensation and motion was at first complete below the site of the injury. Within a year sensation gradually returned to the front of the abdomen, the front of the scrotum, and the front of the thighs down to within two and a half inches of the knee-joint, and extending about half-way around the thighs. At the same time the power of the extensor muscles of the leg (quadriceps extensor cruris) was recovered. This corresponds exactly with the distribution of the anterior crural nerve. No further restoration of sensation or voluntary motion ever took place. In a narrative by the patient he says: 'I have frequently cut, burned, bruised, and injured my legs in various ways without knowing it till I discovered the wounds by sight, and some ten or fifteen years ago I fell and broke both bones of the right leg, and knew nothing of it until I attempted to get up and found it swinging about like a leather strap. I immediately took my bed, sent for a surgeon, had it bandaged, splintered, and dressed in the usual manner, and after lying on the bed the usual time, I again got upon my crutches and walked about with it as usual, without having experienced, during the whole time, the least sensation in it more than in the crutch that I walked upon.'

"With respect to the effect upon nutrition, the lower extremities showed a moderate atrophy, while the head, trunk, and upper extremities were largely developed. In regard to the secretions and the excretions, the only peculiarity observed was that the alvine evacuations were uniformly in the form of very dry, small scybalæ. The catlieter was used for four weeks after the accident, but afterwards he was able, by a powerful contraction of the abdominal muscles, aided by a loop of rope tightly twisted with a stick over the hypogastric region, to nearly empty the bladder.

" $\mathrm{As}$ to the phenomena exhibited in the reproductive functions, the patient shall speak for himself; he says : 'At the age of 36 I married, and my wife in due time presented me with a son, and in 1823 with a daughter; in 1826 with another son; and in 1828 with twins (a son and daughter); and in 1831 (my age being 49 and the age of my wife 43) she presented me another daughter, which was the last. You will probably ask whence came all these children. I reply that for many reasons, perfectly satisfactory to myself, I have not the least doubt of their legitimacy.' The patient, in his narrative, goes on to detail irrefragable proofs of his virility. I have no doubt of the correctness of his conviction of the legitimaey of his children. They all resemble him closely. The mother, throughout her married life, sustained an excellent character; besides, there is no physiological reason why a paraplegic should not procreate, the productive, like the other organic functions, being independent of, though greatly influenced by, the cerebro-spinal system. This was well illustrated in this case. Thongh capable of coition and fruitful connection, he never experieneed the venereal orgasm.

" He frequently experienced vague distress in his lower extremities, and it was only by experience he came to connect this with the action of cold. So when the rectum or bladder required evacuation, he did not experience the usual sensation pointing to these needs, and by accident only came to associate these feelings of discomfort with the conditions which they represented."

A not less remarkable case of complete motor and sensory paralysis below the fifth dorsal vertebra, following an injury, was reported by Dr. William D. Purple, in the New York Journal of Medicine, 1853. The patient, in this ease, finding his lower limbs burdensome, persuaded a surgeon to amputate them near the hip-joint. The operation was wholly without pain, and the stumps healed readily. 
In the rare cases in which one lower limb is the seat of paralysis from a morbid condition limited to a lateral half of the cord, the affection is distinguished as hemiparaplegia. It seems to be settled that when a lateral half of the cord is alone affected, the motor paralysis is on the same side as the lesion, and the sensory paralysis is on the opposite side.

\section{CHAPTER VI.}

General Spinal Paralysis. Treatment of Paralysis. Glosso-labial Paralysis. General Cerebral Paralysls. Progressive Locomotor Ataxia. Progressive Muscular Atroplyy. Pseudo-hypertrophic, or myo-sclerotic paralysis. Scleroderma. Neuritis.

$\mathrm{O}^{\mathrm{F}}$ the different forms of paralysis, it remains to consider the forms dis(1) tinguished as general. After having briefly considered that variety of general paraly sis which, from its dependence on morbid conditions of the spinal cord, is called spinal, the treatment of paralysis will claim attention. •

\section{General Spinal Paralysis.}

Paralysis is called general when the four extremities are affected. Not only the four extremities, but the face on one side or on both sides may be paralyzed in cases of double hemiplegia, to which reference has already been made. In these cases the lateral halves of the body become paralyzed by successive attacks of hemiplegia. Such eases are rare. An extravasation of blood or circumscribed softening, occurring simultaneonsly in each of the hemispheres of the brain, may give rise at once to double hemiplegia or general paralysis, which wonld be likely to involve, to a greater or less extent, the museles of the face. General paralysis, involving the cranial nerves, either proceeds from, or is connected with, intra-cranial morbid conditions. It is possible that such a paralysis may be functional. But the term general paralysis is applied to a paralysis limited to the four extremities, and dependent on intra-spinal morbid conditions. This form may, therefore, be distinguished as general spinal paralysis. The affection known as progressive general paralysis, or the paralysis of the insane, will be considered separately, in this chapter, under the name general cerebral paralysis.

General spinal paralysis is by no means of frequent occurrence, yet it is not extremely rare. My clinical records, when the first edition of this work was prepared (1866), furnished thirteen cases. In all these cases the brain was apparently unaffected, that is, the cranial nerves were not implicated, and the intelligence was unimpaired. In this form of paralysis the intra-spinal morbid condition must either be seated within the upper portion of the canal, as high as the connection of the nerves which form the brachial plexus, or the whole of the cord below this point must be affected. Local spinal meningitis, circumscribed myelitis, disseminated or multiple sclerosis, extravasation of blood, either meningeal or into the cord ; also, non-inflammatory softening, and tumors pressing on the cord, if situated in the cervical portion, may give rise to general paralysis. But 
in the majority of cases these various lesions are situated in the dorsal or lumbar portion, and hence give rise to paraplegia. Judging from the cases which I have observed, general paralysis is apt to be functional, and the prognosis is, therefore, as a rule, far more favorable than in most cases of either paraplegia or hemiplegia. Of my 13 cases two ended fatally; in five cases the recovery is known to have been complete; in five the recovery was nearly complete at the last date of obscrvation, and in one case the patient, at the time of writing, had nearly recovered.

of the two fatal cases, in one no autopsy was made. In the other case, an examination of the brain and spinal cord showed no morbid appearances; but a microscopical examination was not made. In this case, reduplication of the two heart-sounds was observed for several successive days, but this disappeared before death. The heart presented no morbid appearances. The patient died suddenly, probably from syncope. The autopsy revealed no explanation of the sudden death. In a fatal case which came under my observation in Bellevue Hospital in 1868, the spinal cord presented no morbid appearances appreciable by the eye or touch; and in this case the antopsy showed no disease elsewhere to account for death.

The derelopment of the paralysis was gradual in 5 cases, and sudden in 4 cases, the histories not containing information on this point in 4 cases. In most of the cases the paralysis was developed without premonitions. Headache preceded for a single day in one case, and in another case pain in the head and back for a day. In one case the patient had suffered much from rheumatism. In two cases the paralysis was preceded by diarrhœa. In three cases the paralysis was attributable to exposure to cold and wet, in one of these cases the patient having been in the water for three days. In one case intermittent fever and pleuritis preceded the paralysis. In one case the patient, a female, attributed the cliscase to mental inquietude. In one case it followed an injury, and was due apparently to shock.

In some of the cases in which the development of the paralysis is gradual, the different limbs become affected not simultaneously, but suecessively. In one of my cases the paralysis was first apparent in the thumb of one hand. In another case, the right upper limb was first paralyzed, next the left upper limb, and afterward both lower limbs became affected. In another case the paralysis commenced in one lower limb, next extended to the other lower limb, and afterward to the upper extremities. In several cases the different members were not equally affected. In most cases it is noted that the paralysis was greater in the lower than in the upper limbs. In one case the reverse is noted. In some cases an upper and a lower limb on the same side were more paralyzed than the limbs on the other side, but in two cases an upper and a lower limb on opposite sides presented a greater degree of paralysis. The sensibility was in not one of the cases diminished, and hyperæesthesia of the surface was noted in one case. In but one of the cases was there difficulty in urination, and in this case the difficulty was of very brief duration. Pain in the back and spinal tenderness are noted in only two of the cases. In most of the cases the appetite and digestion were but little or not at all affected, and the body was well nourished.

The thoracic respiratory movements were not in any case diminished. In one case they were increased, and the diaphragmatic morements were wanting. The breathing in this case, for several weeks, was costal, physical exploration showing the absence of any pulmonary or cardiac disease. The paralysis evidently involved the diaphragm. The patient was unable 
to sneeze. When snuff was put into the nostrils, he felt intensely the desire to sneeze, but the act did not take place. Afterward, when notable improvement had taken place as regards the paralysis of the limbs, the power of sneezing returned, and the diaphragmatic movements in respiration were apparent. In three cases phonation was notably affected. In two of these cases there was complete aphonia, and in the other case the voice was impaired but not lost.

As regards the duration of the paralysis, of the two fatal cases, in one it existed for 17 months, and in the other case for only a week. Of three cases in which the recovery was complete, it is a curious coincidence that in each case the duration was six months. The duration in five cases, respectively, at the date of the last record, the improvement in all having been so great as to render it probable that complete recovery would take place, is as follows: five months, sixteen months, and in three cases two years.

One of the cases embraced in the foregoing analysis is interesting as illustrative of functional paraly sis produced by exposure to cold and muscular exertion. The following is a synopsis of the case: Mr. M., aged 45 , a broker, during a violent snow-storm, Dec. 12 th, 1867 , rode from the city to Morrisania, a distance of about $8 \mathrm{miles,} \mathrm{in} \mathrm{a} \mathrm{horse-car.} \mathrm{'The} \mathrm{jour-}$ ney was much protracted on account of the snow, and he was often obliged to get out in consequence of the car getting off the track. The car was crowded and he stood upon the platform of the car, his back exposed to the storm. He had not been well for two or three weeks, having symptoms denoting disturbance of the stomach, and he had been on restricted diet. He managed to reach his house, feeling; as he expressed it, as if he had been stoned almost to death. He shortly became insensible, but after a short time consciousness returned, and there was now general paralysis which was complete, involving both motion and sensation, extending over all the body below the head. He said it was difficult to breathe, and that he felt at times as if he would lose his breath. There was a disposition to sommolency, but he was easily aroused and his mental faculties were intact. I saw him in consultation with the attending physician, Dr. Horton, of Morrisania, on the fifth day after the attack. Under a treatment consisting of stimulants, frictions, and nutritious food, he had in a measure recovered from the paralysis. These measures of treatment were continued, and the recovery was in a short time complete.

A case of general spinal paralysis which came under my observation in Bellevue Hospital, in 1860, illustrated the danger which may arise from diminished power of the respiratory movements, and, also, recovery under circumstances which seemed to render the prognosis extremely unfarorable. The patient, an unmarried woman, aged 27 , began to lose power over the limbs shortly after an attack of acute articular rheumatism which had confined her to her bed for about four weeks. The paralysis progressively increased for two months, when she was admitted into the hospital. The paralysis of the limbs was incomplete, but the muscular power was so much diminished that she was quite helpless. 'There were no cerebral symptoms, and the intelligence was unaffected. The breathing was exclusively costal, and, at times, the suffering from dyspnœa was extreme. Scotch snuff introduced into the nostrils caused an intense desire to sneeze, and grimaces, but there was no sneezing act. There were no signs of pulmonary or cardiac disease. She was able to speak only in a feeble voice, and with jerking efforts. At one time the want of power to carry on respiration was so great that, apparently, she was almost moribund. At this time the inhalation of nitrous oxide gas secmed to be useful. Dry cup- 
ping over the spine appeared to be of service. In addition, she was treated with tonies, stimulants, and good alimentation. After a fortnight there was marked improvement. She was then attacked with pneumonia affecting the lower lobe of the right lung. At this time she had not recovered diaphragmatic movements. She passed safely through the pneumonia. The ability to sneeze soon returned, and, after remaining in hospital several months, she was discharged well.

\section{Treatment of Paralysis.}

The treatment of the several forms of paralysis which liave been considered will, in the first place, have reference to the morbid conditions on which the paralysis is dependent, to coexisting affections in any part of the body, and to the state of the system. The treatment, in these points of view, is addressed, not directly to the paralysis, but to causative and concomitant affections. Measures to be addressed to morbid conditions on which the paralysis depends, must, of course, vary according to the nature of these conditions ; hence, it is important, if possible, to determine their nature in individual cases. The questions which the practitioner is to endeavor to settle, as preparatory to this part of the treatment, are : Does the paralysis depend on an inflammation, or a lesion of some kind, and, if so, where is the inflammation or lesion situated-in the tract of a nerve, within the cranium, or within the spinal canal? If the paralysis depend on a lesion, what is its character and its extent? Is there ground for the suspicion, if not belief, that the paralysis is the effect of lesions caused by syphilis? Does the paralysis depend on poisoning by lead? Is there ground for the suspicion of some other toxical agent, such as arsenic, copper, or mercury? Do the history ard symptoms render it probable that the paralysis is functional? Are there concomitant affections which may possibly stand in a causative relation, either directly or indirectly, to the paralysis? What is the state of the system as regards nutrition, debility, and, more especially, anæmia?

Measures of treatment, differing and quite opposite in character, will be indicated according to the conclusions at which the practitioner arrives, after propounding to himself the foregoing questions. It is not necessary to consider here the various measures which may be indicated in different eases. The success of the treatment will depend, in some cases, on the indications being correctly interpreted and judiciously fulfilled. But, in a certain proportion of cases, the paralysis depends on lesions which are irremediable, and complete success of treatment is not to be expected. It is to be added, that if there be ground to suspect the existence of syphilitic lesion, the remedies which experience has shown to be specially eurative, in many cases, namely, mercury, and the iodide of potassium, are indicated. The former may be carried to mild jtyalism, and the latter to the point of toleration. The curative effect of these remedies, in cases of doubt, confirms the supposition of the dependence on syphilis.

The treatment, in the second place, has reference directly to the paralysis, and consists of measures addressed to the paralyzed parts. The objects are to rouse or restore the function of the paralyzed parts, promote circulation in the parts, and secure, as far as practicable, healthy nutrition. The more important of the measures are, friction with stimulating embrocations, or shampooing, electricity, passive movements, strychnia, and, above all, whenever practicable, active exercise under the influence of the will. 
These measures cannot be effectual, and some of them may be hurtful, so loug as the paralysis is sustained by the morbid conditions which have produced it. But it is to be borne in mind, a portion of the immediate paralyzing effect of certain of these conditions is of transient duration. The paralysis, for example, which follows extravasation of blood, is measurably due to the shock or contusion, and to a temporary local anæmia. These incidental causative conditions are temporary. Even functional paralysis is usually stationary for a certain period, before improvement begins. On the other hand, paralyzed parts will not, of themselves, resume at once their normal condition, even after all the causative conditions are removed. This is a highly important fact. Nerves and muscles may become permanently paralyzed from disuse. Having been for a considerable period paralyzed, they may remain so indefinitely if appropriate efforts be not made for their restoration.

In the treatment of paralysis the praetitioner is liable to err, first by resorting immediately, or too soon, to measures to rouse the paralyzed parts, while the paralysis is still sustained by the causative conditions, and restoration at that time is out of the question. A second error is, after the paralysis has existed for some time, to overlook the fact that the restoration will not take place spontaneously, but that persevering efforts may be required for recovery. A third error is, to expect success when the paralysis has existed so long that the muscles and nerves involved have undergone degenerative changes.

The researches of Duchemne and others, within late years, on the application of the induced electrical current (Faradization) in cases of muscular paralysis, have shown that, by means of this agency, important information may be obtained respecting the condition of the affected muscles. When the current from an electro-magnetic or electro-galvanic battery of sufficient power is passed through healthy muscles, contractions are excited. This susceptibility to the electrical current is called the electro-muscular contractility. The passage of the current through the muscle causes more or less pain, not due to the contraction excited by the current, and this muscular pain denotes what is called the electro-muscular sensibility. Now, the state of paralyzed muscles as regards electro-muscular contractility and electro-muscular sensibility is to be taken into account in the application of not only electricity, but other therapeutical measures addressed to the paralysis. In muscular paralysis from intra-cranial affections, as a rule, the paralyzed muscles respond to the electrical current not less, and in some cases even more, than in health, until they begin to suffer from prolonged inaction or defective nutrition. The electrical current in such cases is, to some extent, a test of the state of the muscles as regards their capacity for contracting under the influence of volition. The statement just made appears to be the rule, but there are exceptions. In some instances volitional contractility is regained while the electro-contractility is defective or lost. Examples have been reported by Dr. S. Weir Mitchell. Dr. Mitchell has observed variations on different days as regards the electro-muscular contractility. He states: "It is not uncommon to find a muscle very exeitable to-day, and to-morrow far less so. It is also quite common to notice that a muscle temporarily inactive under currents may be aroused by the electrization of a neighboring muscle or . group of muscles." Again, it seems to be a rule that, when museular paralysis is due to intra-spinal affections, to injury of nerves, or traumatic causes affecting the muscles themselves, the electro-functions are impaired

d Sanitary Memoirs of the War. 
or lost irrespective of changes arising from defective or disordered nutrition. Loss of electro-contractility and electro-sensibility has already been stated to characterize paralysis from lead. Finally, in proportion as muscles and nerves undergo degeneration of structure as an effect of longcontinued paralysis, they respond but feebly, or not at all, to the electrical current. In certain cases, therefore, electricity gives information of the hopelessness of paralysis. The electro-contractility of paralyzed muscles is sometimes lost, and the electro-sensibility remains intact; under these circumstances, the prognosis is less unfavorable than when both are wanting.

The judicious employment of electricity, as a theraputical agent in cases of paralysis, is undoubtedly of great importance. To consider, however, this part of the treatment fully would require details which do not properly fall within the scope of this work. For these the reader must consult treatises devoted specially to electricity considered in its applications to pathology and therapeutics. A few practical points only can be here introduced. ${ }^{2}$

The ends to be attained by electricity, it is to be kept in mind, are the restoration of the ability of the paralyzed muscles to respond to the will, and the maintenance of the circulation, together with healthy nutrition, within the affected organs. Electricity is therefore not required when the paralyzed muscles respond to the current as actively as the corresponding muscles which are not paralyzed, and still less is it required when the electro-muscular contractility and sensibility are greater than normal. Under these circumstances, electricity may do harm. In cases of paralysis from intra-cranial morbid conditions, the employment of this measure is to be deferred until the muscles begin to suffer from prolonged inaction, as denoted by diminished electro-contractility, electro-sensibility, and nutrition. Electricity is not to be employed when the paralyzed muscles are rigid from irritation or inflammation at the nervous centre.

In cases in which the paralyzed muscles fail to respond to the electrical current from defective innervation, as an immediate or speedy result of morbid conditions affecting the spinal cord or nerves, recovery is not to be expected so long as the paralysis is sustained by the persistence of these morbid conditions. Yet, under these circumstances, the judicious employment of electricity may promote circulation and nutrition, and thus tend to keep the muscles in a state of preparation for resuming their capacity to act in obedience to the will, when the conditions sustaining the paralysis are diminished or removed. A response to the electrical current, as regards contractility or sensibility, or both, is evidence of returning innervation; and then the persevering employment of electricity may prove of great service.

In cases in which the causative morbid conditions, such as non-inflammatory softening or the pressure of a tumor, preclude any expectation of improvement, or when, from the long duration of the paralysis, clegeneration of structure in the paralyzed parts has ensued, it is useless to persist in the employment of electricity.

1 For the convenience of readers desirous of entering into a full consideration of medical electricity the following references are given: The writings of Duchenne and Althaus; Meyer's treatise translated by Hammond; Electro-phrsiology and Electro-therapeutics by Alfred C. Garratt; Practical Treatise on the Medical and Surgical Uses of Electricity, by George M. Beard, M.D., and A. D. Rockwell, M. D. ; On Injuries of Nerves, etc., by S. Weir Mitchell, M.D.; Hammond's treatise on Diseases of the Nervous System; and Lectures by J. Russell Reynolds, M.D., London Lancet, 1870. 
Much benefit, in cases of paralysis from lead, may be hoped for from electricity, and its employment may do much toward hastening recovery in cases of functional paralysis.

Ths foregoing remarks have had reference chiefly to paralysis of motion. The judicious application of electricity in cases of paralysis of sensation is often useful. The success of electrical treatment in paralytic affections will depend on the exercise of judgment in determining the indications for it, and of tact in its employment. The advantage of experience in the practical application, together with the time which it requires, renders it desirable that some members of the profession should give to it special attention. Unfortunately the employment of electrotherapentics, in this country, has been too much in the hands of uneducated practitioners, who know but little of the agent which they employ, and still less of the human organism.

Friction of the surface, and, in muscular paralysis, kneading with deep pressure, or shampooing, are important measures of treatment. Stimulating liniments facilitate the friction, or rubbing, and are, to a certain extent, in themselves useful. These measures are serviceable by promoting the circulation and maintaining nutrition. They should be faithfully and perseveringly employed by persons properly instructed by the physician.

Passive movements in cases of complete paralysis are not less important; that is, movements of paralyzed limbs by the hands of others, and, when practicable, by the hand or hands of the patient. The effects of inaction on the circulation and nutrition are in this way diminished, and paralyzed muscles are kept in a state of preparation for volitional contraction. The importance of this measure will not, of course, be appreciated by the patient, and it is to be explained and enforced by the physician. Drs. Mitchell, Morehouse, and Keen attribute much value to passive movements in the treatment of paralysis from gunshot wounds and other injuries of nerves. They state that in cases in which motion of the paralyzed parts was very painful, they were accustomed to give ether. These authors attach importance to the douche, using alternately hot and cold water.

In cases in which the paralysis is not complete, the most effective measure for effecting either recovery or the utmost degree of improvement compatible with permanent lesions is voluntary exercise. A patient who has been for some time a paralytic is in a condition not unlike that of the infant who has not yet learned to use the muscles. Persistent exercise in the one case, as in the other, is the means by which development and power are to be acquired. 'There is reason to believe that, in not a few cases, paralysis continues until it becomes incurably fixed at a certain point, when persevering exercise, in conjunction with other measures, might have led to recovery, or to more or less improvement. Gymnastic or calisthenic exercises are to be recommended. It is often difficult to enforce this measure. It fails to secure confidence on account of its simplicity. Drs. Mitchell, Morehouse, and Keen state that, in treating soldiers affected with paralysis, they found it necessary to place patients in charge of intelligent orderlies trained for the purpose, and by this means an efficient system of exercise was carried out.'

Remedies addressed to the paralysis are of far less importance than

In an interesting paper on the treatment of paralysis by systematic exercise, published some years since by the late J. P. Batchelder, M.D.. of New York, the successful application of this method of treatment is illustrated by several cases. 
the foregoing measures. From its power of exciting contractions in paralyzed muscles, strychnia has been much employed in cases of paralysis. 'The expectations which led to its employment have not been realized, and, at the present time, there appears to be a tendency to consider it as a remerly of little or no value. Clinical experience furnishes but little evidence of its possessing any special curative power, but, as a tonic remedy, it appears to be useful in certain cases. In a case of paraplegia, among those which I have collected, in which the patient was confined, for the most part, to the bed for three years, he took no remedy during this period. He began to improve after commencing the use of strychnia and quinia in small doses; and he continued to use these remedies steadily for two years, the improvement progressing and complete recovery taking place. Strychnia should not be given when the conditions sustaining the paralysis involve inflammation or irritation of the brain or spinal cord. As regarls closes, it should be given within the limit of producing involuntary twitchings of the palsied muscles. In farorable cases, its use, in moderate or small doses, should be continued for a long period.

Phosphorus is useful in some cases of paralysis. In a case, under my observation, of general paralysis dependent on supposed multiple selerosis of the cord, the affection having existed for sixteen years, the patient being seventy years of age, marked benefit has followed the employment of the phosphide of zinc given in doses gradually increased from onetenth of a grain to half a grain three times daily. This remedy has been continued most of the time for the space of two years. Its usefulness has been proved by repeatedly suspending it for a short period, and contrasting the condition during this period with the condition before the suspension and after the resumption of the remedy. The syrup of the lacto-phosphate of lime is an eligible preparation for the introlluction of phosphorus into the system.

Other remedies are ergot and belladonna. These remedies are arlvocated by Brown-Séquard in cases of paraplegia dependent on inflammation of the cord or its meninges. They are supposed to act by inducing, through vaso-motor nerves, contraction of the arteries of the corl. This supposition is hypothetical, and clinical experience furnishes but little evidence of their possessing curative efficacy.

In all cases of paralysis whenever the morbid conditions are supposed to be due to syphilis, anti-syphilitic medication is indicated, especially the iodide of potassium. If syphilis have existed, a possibie connection of the paralysis with syphilitic conditions should be suspected, and a trial of the remedy made on this ground. Small doses of mercury, continued for a considerable period, are sometimes useful in these cases.

\section{Glosso-Labial Paralysis.}

A form of paralysis described by Duchenne in 1861 is entitled to be considered as a separate paralytic affection. This form of paralysis is characterized by diminished power of voluntary motion over the tongue, the muscles of the palate, and the lips. The loss of power, at first slight, progressively increases. After a time much difficulty is experieneed in articulation; the patient is unable to pronounce distinctly words and the letters of the alphabet, more especially the consonants requiring for their enunciation movements of the tongue and lips. Speech may be at length impossible, and the only means of communication is by signs or writing. The paralysis of the orbicularis oris renders the lips immovahle during emotions of grief or mirthfulness; the mouth no longer expresses these . 
emotions. The saliva dribbles over the lips. Food collects between the teeth and cheeks, and the fingers are required to dislodge it. The ability to protrude the tongue or to eurl upward the lip of this muscle is lost. When the paralysis of the tongue and orbicular muscles of the mouth has increased to a certain extent, or even from the outset, and before the paralysis has extended to the lips, the muscles of the palate are affected. Deglutition then is more or less difficult, food and drink being often returned through the nostrils in the progress of the affection. The laryngeal muscles are liable to become affected, leading to aphonia, and to diminution or cessation of the respiratory movements of the glottis. Paralysis of the muscles of respiration may follow; the acts of respiration are feeble, and the power of coughing is impaired.

Portions of food and drink, and the salivary fluids, are apt to be drawn into the larynx, and are with difficulty expelled owing to the inability to cough effectively. For this reason, if the patient be attacked with bronchitis, there is danger from the accumulation of mucus in the bronchial tubes. Paralysis may occur in other parts of the botly, and may extend, more or less, over the voluntary muscles. The affection is sometimes associated with that to be presently considered under the name progressive muscular atrophy. The progress of the affection is unattended by febrile movement. General debility, after a time, exists in a marked degree. The affection advances steadily, but more or less gradually, toward a fatal termination. Death may be caused by apnoea from impaired ability to carry on respiration, and accumulations in the bronchial tubes; or the mode of dying is by asthenia, of which innutrition may be a causative element, arising from inability to ingest a sufficient amount of aliment to sustain life. Sudden death by syncope may take place.

The nerves involved in this affection are the pneumogastric, hypoglossal, and the lingual. From the bilateral character of the affection it is a rational inference that its seat is at the nervous centres, in the medulla oblongata, where these nerves are associated. Autopsical examinations sustain this inference. The nerves going to the paralyzed muscles have been found, after death, atrophied ; but this is to be regarded as secondary to the central lesion. The muscles themselves have been found free from lesion, and preserving their electro-contractility. The central lesions are incluration (sclerosis) with atrophy of the nerve cells, and fatty degeneration.

A case under my observation at Bellevue Hospital exemplified the characteristic features of this affection at an early period of its progress. The patient, a widow, aged 57 , domestic, entered the hospital January 14th, 1868. About two months before her admission she began to have diffieulty in speaking, which gradually inereased, and, on this account, she was compelled to leave her place of service. Her speech, on her admission, was very slow and labored, the enunciation being quite indistinct. The vowels she could pronounce pretty well; the labial consonants were uttered very slowly, but with tolerable distinctness; the linguals were enunciated with the most difficulty and incompleteness. She was tronbled with the accumulation of saliva in the mouth, but she was able to prevent it from dribbling. She could protrude the tongue only to the inner edge of the teeth. She could not raise the tip of the tongue, but she erected the organ by raising the posterior portion. There was no impairment $\mathrm{cf}$ deglutition, but there was a difficulty in conveying food and drink from the mouth to the pharynx; the food collected between the teeth and lips, so that she used the finger to dislodge it, and liquids sometimes escaped from the mouth. For these reasons she ate very slowly, and apart from 
the other patients in the ward. There was no defect of phonation, and no paralysis manifested elsewhere than in the tongue and lips. The intellect was unaffected, and the general health good. After remaining in the hospital for four and a half months, she was discharged at her own request, the affection having distinctly but not greatly increased during her stay in the hospital, and the paralysis limited to the muscles of the tongue and month. About a year afterward she again came under my observation at Charity Hospital, Blackwell's Island. The paralysis of the tongue and lips was then nearly complete. The mouth was elongated, and the lips somewhat separated. She was unable to articulate, but uttered guttural sounds when questions were addressed to her. Her intelligence appeared to be intact. She had great difficulty in swallowing, especially liquids, much of the latter running out of the mouth. Death took place apparently from inanition resulting from insufficient alimentation. The cluration of the disease was about twenty-two months.

The name glosso-laryngeal paralysis, given to this affection by Trousseau, is open to the objection that laryngeal paralysis is among the late events of its clinical history, and does not always occur. The glossal and labial paralysis constitute the primary and most distinguishing feature. The affection may readily be distinguished from double paralysis of the facial nerve, because the orbicular muscles of the eye and the muscles of the face other than the orbicularis oris are not affected. In its progressive character and the occurrence of paralysis in other situations, it bears a resemblance to the affection to be next considered, namely, general cerebral paralysis, in which the muscles coneerned in speech are apt to be first affected. It differs from the latter affection, howerer, in the fact that the intellect remains unaffected; and the occurrence of paralysis of other muscles than those of the tongue, mouth, pharynx, and larynx, appears to be incidental to the disease, rather than a constitnent of it. From progressive muscular atrophy it is distinguished by the absence of degeneration of the affected muscles. In a case reported by Duchenne, however, the affection just named coexisted with the affection under consideration.

With reference to cansation, facts are wanting. Judging from the cases which have been reported, there is no ground to expect either recovery or improvement. Further clinical study of the affection may perhaps show that it is less hopeless than it appears to be with our present knowledge of it.

Rational indications for treatment would learl to the employment of electricity with a view to prevent or retain the progress of the paralysis, and to measures having reference to the general health and constitutional rigor. In several cases treated by Duchenne with the induced electrical current, there was a temporary improvement, but the cases afforded no ground for encouragement in expecting a cure from this remedy.

The affection is described by Duchenne under the name "Progressive muscular paralysis of the tongue, the palate, and the lips." The name glosso-labial paralysis is convenient from its brevity, and it expresses the obvious primary features of the affection.

'See De l'Electrisation Localisée, etc. Deuxième Edition. Paris, 1861. 


\section{General Cerebral Paralysis, or the Paralysis of the Insane.}

A form of general paralysis, distinguished by its association in most cases with mental derangement, has been known heretofore as the general paralysis of the insane. It has been proposed to eall this form of paralysis general paresis. ${ }^{1}$ The term paresis, however, has been applied by some writers to paralysis dependent on a functional condition of the brain or spinal cord. In this sense the term is not appropriate as applied to the general paralysis of the insane, inasmuch as the latter is probably never a purely functional affection. The name paralysis of the insane is exceptionable, inasmuch as cases may present the same phenomena as regards the paralysis, without being associated with mental derangement. Sueh cases, however, are extremely rare. In distinction from general spinal paralysis, the affection may be called general cerebral paralysis. This form of paralysis is usually developed very gradually. It commences imperceptibly, and has slowly made a certain amount of progress before becoming sufficiently developed to be distinctly recognized as a paralytic affection. The same is true of the mental aberration associated with the paralysis. The evidence of mental aberration in the incipiency of the affection is generally extravagance in ideass and actions, which, for a time, may pass simply for ecentricities of eharacter. The extravagances usually relate to the supposed acquisition of wealth, extraordinary mental powers, or superior personal advantages of some kind. Such ideas, however, are in some cases preceded by mental depression. Writers consider the first stage of the disease to extend to the period when the affection is so far developed that its symptomatic characters are well marked.

The mental characteristics, in the second stage, are those of the first stage increased so as to constitute unmistakable insanity. The patient is apt to imagine that he is immensely rich, or that he is some clistinguished personage, or that he is the recipient of some extraordinary good fortune. The kind of insanity is that styled by French writers delire des grandeurs. It does not consist in any fixed delusion, but the insane ideas are constantly shifting. Paroxysms of maniacal fury sometimes occur, and may lead to homicide. At times the insanity may have the form of melancholia, and in some cases its prevailing character is of the latter form.

The paralysis, as the name of the affection implies, affects the whole or the greater part of the system of voluntary muscles on both sides. It is not limited to the extremities, but embraces muscles animated by the cranial nerves. The paralyzed muscles are affected, not sucressirely, but simultaneously. The paralysis in the second stage, and, indeed, throughout the disease, is incomplete, and varies much in degree in different cases. The tongue is protruded with effort, and is tremulous. Twitchings of the facial muscles accompany the acts of speaking. The articulation is thick or mumbling, resembling the speech of atrukenness. 'The muscles of the face are relaxed, causing elongation of the mouth, and more or less impairment of the facial expression belonging in health to the person affected. Paralysis of the lower limbs is manifested by an increase of exertion in walking, by a straddling, shambling, tottering gait, and by the body in standing being poised on both legs widely separated. 'The muscles of the upper extremities are involved, as shown by defective power of grasp, and by clumsy movements of the fingers. The special nerves, as a rule, are not notably affected. General sensibility, however, appears to be some-

I From rapası, exhaustion or relaxation. 
what blunted. In this stage of the affection, the pupils frequently vary in size, and are sometimes irregular in form. They respond sluggishly to light. 'The ophthalmoscopic observations of Albutt and others show in almost all cases sclerosis and atrophy of the optic nerves. In the early stage the optic dise is intensely hyperæmic. ${ }^{1}$

The functions of the body, irrespective of mental aberration and paralysis, may be but little, or not at all, disturbed in the second stage. 'The appetite and digestion are frequently unaffected. The pulse is regular. Patients often become fat.

The third stage is characterized by an increase of the paralysis to such a degree that there is inability to walk or stand, and generally by mental imbecility. The patient at length passes his evacuations in bed, and is insensible to ideas of cleanliness and clecency. The limbs, in some cases, become contracted. Difficulty of deglutition now becomes a feature more or less prominent, and suclden death has repeatedly been caused by the impaction of food in the pharynx. Death, if not caused by this accident or some intercurrent affection, is a result of either extreme exhaustion gradually induced, or of inability to carry on respiration from paralysis of the respiratory muscles.

It is stated by Brierre and Duchenne that the paralyzed muscles in this affection respond to the eleetric current, whereas they do not in general paralysis dependent on spinal lesions. Bucknill and Sankey, however, have found that excito-motory movements are not readily produced by tickling the soles of the feet.

During the progress of this affection, a sudden attack of coma is liable to occur, from which the patient may emerge and nearly regain his condition prior to the attack, but, in the majority of cases, the coma is the forerumner of deatl. Sudden hemiplegia is another event which is apt to occur. From this not infrequently the patient recovers after free purgation, and in some cases frequent hemiplegic attacks take place, which may affect alternately the two sides. Another form of sudden attack consists of a notable increase of the general paralysis, with obstruction of the mental faculties, without unconsciousness. Remissions, in the course of the affection, as regards the mental derangement and the paralysis, either or both, have been observed. The improvement manifested in these remissions is sometimes such as to seem to afford much hope of recovery.

The prognosis is extremely unfavorable. A few cases of recovery only are on record, and in these cases the affection had not progressed far." There is scarcely any ground for holding out encouragement as regards recovery. In a large proportion of cases death is due to some intercurrent affection. If the inuscles of the chest have become notably affected, any pulmonary affection is likely to prove fatal. Even ordinary bronchitis, under these circumstances, occasions much embarrassment of respiration from the accumulation of mucous secretion within the bronchial tubes, and it may prove fatal.

The duration of this affection is extremely variable. Patients sometimes live for many years in a condition of either incomplete or complete dementia. In the great majority of cases, however, death takes place within the space of three years, and the affection sometimes proves fatal within a few months or even weeks. Deatl may occur, of course, at any time from intercurrent affections.

1 Vide lecture by Prof. Henry D. Noyes, on the relations of diseases of the eye with other diseases, N. Y. Medical Record, No. for $\Lambda$ ugust, 1872.

2 A well-marked case, ending in recovery, was reported several years ago by Dr. Pliny Earle. Bee Amer. Journ. of Med. Sciences, July, 1857. 
Examinations after death in cases of this affection show invariably more or less marked morbid appearances of the brain. Histological observations show degenerative, granulo-fatty changes of the nerve cells of the cerebrum, more especially of the cortical substance. 'There is hyperplasia of the connective tissue, or neuroglia. The smaller vessels are thickened and rigid. Wilks describes them, in a case which he observed, as "standing out from the cut surface of the brain like so many bristles from a brusl.." The membranes are more or less adherent. Wilks considers the disease as taking its point of departure from the pia mater; Meschede, and others, place its primary seat in the cortical substance. The essential pathological process is a subacute, chronic inflammation; hence, the names chronic cerebritis or meningo-encephalitis, which have been applied to it, are not inappropriate.'

General paralysis of the insane occurs much oftener in males than in females. In the great majority of cases, the age is between thirty and fifty; but the affection occurs at all periods of life. There seems to be ground for imputing the affection, in a considerable proportion of cases, to painful moral shocks arising from loss of friends, disappointment, bankruptcy, remorse, etc. In a certain proportion of cases it is attributable to intemperance. In the histories of cases the mental derangement is found to be sometimes the first manifestation of disease, the paralysis occurring at a variable period afterward; on the other hand, the paralysis sometimes has precedence in point of time, and, again, the mental derangement and the paralysis sometimes oceur simultaneonsly.

This affection is to be discriminated from general paralysis dependent on morbid conditions seated in the spinal cord. 'The paralysis of the insane is dependent on cerebral lesions ; hence, differential points are mental disorder in connection with the latter, and paralysis of muscles supplied by cranial nerves, whereas in spinal paralysis insanity does not occur except by aceidental association, and the facial muscles are not affected. The affection known as progressive locomotor ataxia presents some analogous features. As will presently be seen, however, the latter is not a paralysis, but its distinctive feature is deficieney of the power of co-ordinating voluntary movements. Moreover, insanity does not belong to the natural history of this affection, and groups of the voluntary muscles are affected, not simultaneously but successively. The affection which will presently be noticed under the name progressive muscular atrophy differs essentially in the fact that the loss of muscular power is due to an atrophic lesion of the affected muscles. It is only, however, within late years, that the form of paralysis under consideration has been clearly separited from the three affections just named, and the latter distinguished from each other.

The great majority of patients affected with paralysis of the insane, sooner or later are received into lunatic asylums. Not only is there scarcely any ground for curative treatment, but permanent improvement is hardly to be hoped for. The treatment consists of palliative measures, together with the regulation of diet, regimen, etc., with a view to retarding the progress of the affection and contributing, as far as practicable, to the comfort of patients during its progress.

1 For further details respecting the morbid anatomy, vide Isectures by W. $\mathbf{H}$. O. Sankey, M. D., London, 1866 ; $\Omega$ work entitled "Practical account of General Paralysis," etc., by Thos. I. A ustin, London, 1859 ; Art. by M. Magnan in Archives de Pliysiologie, normale et pathologique, Mar-Aine, 1868 ; Art. by Dr. Franz Meschede in Journal of Mental Science (England), Oct. 1866 ; Art. by Dr. Wilks, in Guy's Hospital Reports, 1866, and Art. by Dr. S. G. Webber in the New York Quarterly Jourual of Psychological Medicine, Oct. 1865. 


\section{Infantile Paralysis.}

In leaving the consideration of paralysis, I shall notice the affection called Infantile Paralysis only with reference to its distinctive features. This belongs among the diseases of infancy and childhood, and the reader is referred to treatises devoted to these diseases for a fuller account of it.' The affection has been known as the essential paralysis of infaney. It occurs between the age of six months and of three years. The usual history is of a sudden attack, the maximum of the degree of the paralysis being attained at once or quickly. Generally the paralysis affects the two lower limbs (paraplegia); sometimes an upper limb is also paralyzed. The paralysis is limited to voluntary motion. The bladder and rectum are not involved. The paralysis is sometimes limited to a single group of muscles, as either the flexors or extensors. The symptoms do not denote any cerebral affection. In most cases the paralysis is preceded by a fever of short duration, but in some cases it occurs when the child is apparently well, and it may occur during sleep. The temperature of the paralyzed limbs falls from three to eight degrees. In most cases spontaneous improvement soon begins, and ends in recovery in from three to four weeks. But in some cases the restoration is confined to only certain of the affected museles, others remaining paralyzed. The muscles which remain paralyzed soon undergo atrophic or degenerative changes; they lose electrocontractility, and recovery is then impossible. The pathological condition which gives rise to the paralysis is seated in the spinal cord. The nature of the affection of the cord has not been ascertained, but, of course, as recovery generally takes place, and, with efficient treatment, would probably be the result in nearly all cases, it is evident that a structural lesion of the cord is not involved.

The indications for treatment relate, first, to associated morbil disturbances, and to circumstances which may be suspected to have a cansative influence, such as dentition and intestinal worms; second, to the timely employment of passive movements, kneading the affeeted muscles, and, above all, electricity to promote the return of the muscles to the control of the will, and prevent the degenerative changes incident to the paralysis. Systematic voluntary exercise of the paralyzed muscles is to be enjoined, as soon as the influence of the will is restored, in order that the recovery may be complete.

\section{Locomotor Ataxia.2}

The affection now known as locomotor ataxia is so called after the name proposed by Duchenne. Duchenne entitled it progressive locomotor ataxia ; but as the affection is by no means always progressive, in respect of either its degree or diffusion, the term progressive is hardly appropriate. It was described by Duchenne, in 1858 and 1859 , more fully than by any previous writer; hence, Troussean designated it Duchenne's disease. The affection, however, was clearly defined, as regards its distinctive character and its seat, by the late Dr. Todd, prior to the researches of Duchenne. ${ }^{3}$ Romberg has described the affection under the name tabes dorsalis-it has also been called myelo-phthisis.

1 Vide Treatise by Prof. J. Lewis Smith, second edition, 1872 ; also Hammond on Diseases of the Nervous System.

\& From $a$ and $r a \xi_{c}$, " order."

3 Vide Cyclopædia of Anatomy and Physiology, London, 1830-1847, vol. iii., p. 721 . 
This affection is not a form of paralysis, but it is generally associated with diminished sensibility and not infrequently with more or less diminution of motor power. The distinctive characteristic of the affection is impairment or loss of the ability to combine and direct muscular movements. The patient may be able to exert as much muscular strength as in health, but he finds it difficult or he is unable to co-ordinate the action of the muscles for the execution of the acts of volition.

The affection has certain premonitions, viz., fugitive shooting pains in different parts of the body, which are regarded as neuralgic or rheumatic, nocturnal incontinence of urine, spermatorrhœa, anaphrodisia, or, on the other hand, a morkid excitability of the sexual organs, transient paralysis of certain nerves, such as the sixth or the third pair, and sometimes impairment or loss of vision. These paralytic affections are in some cases permanent.

The ataxia is generally first manifested in the lower extremities. It may appear first in the upper extremities. This was true of a case which I have observed. One lower limb is sometimes affected before the other, and the two lateral limbs may be first affected. When one limb is first affected, whether it be a lower or an upper extremity, it is on the left much oftener than on the right side. The defect of co-ordination is apparent when any combined movements are undertaken. In proportion as the affection is marked, the patient's gait in walking is uncertain, irregular, and grotesque. The lower limbs are thrown forward by forcible jerks, without any definite direction. The body is swayed from side to side in the attempt to maintain an equilibrium, and the arms are thrown out like those of a person balancing on the tight rope. In an extremely marked case which was under observation for several years at Bellevne Hospital, whenever the patient attempted any volitional acts, the violent contortions of the limbs and body were such that he appeared to be performing for the surprise and amusement of the spectators, and it was difficult at first for the medical class to repress manifestations of mirth. In cases less marked, the greatest difficulty is experienced in beginning to walk, and after getting under way, the patient is unable to advance slowly, but walks with precipitation; the legs are thrown forward with a quick, jerking movement, the feet brought to the ground with force, and liable to strike against each other. Notwithstanding the violence of the exertions, the muscular strength being retained, patients are sometimes able to travel long distances. In an extreme degree of the affection, voluntary progression, even with the aid of a cane or assistants, is impossible, and the patient is confined to the chair or bed. The shooting pains into the affected limbs continue, patients often comparing them to electric shocks.

The defect of co-ordinating power over the upper extremities is shown by an inability to execute acts which require the combined movements of the fingers. The patient is unable to button or unbutton his clothes; yet he may grasp with not less force than in health. A patient whom I exhibited at a clinic at Bellevue Hospital tried in vain to unbutton his vest, but his grip was too strong to be borne. In extreme cases the patient is unable to bring his hand to his mouth, and it is necessary for others to feed him.

Sooner or later the speech is liable to become affected. The articulation is difficult and imperfect; the tongue, when protrnded, is tremulous. The speech may become so impaired as to be unintelligible; mutism is thus a feature of some cases.

In a certain proportion of cases, the affection progressively extends over the voluntary muscular system, and increases more or less slowly. The 
defective co-ordinating power may be much more marked at some times than at others. A patient who was for some time under my observation, and who had had this affection for five years, habitually walked with a certain amount of uncertainty and irregularity, and enunciated with a sense of labor and slowly, but distinctly. This patient at times, for the space of an hour or so, walked with great difficulty, presenting in a marked degree the characteristic gait, and the embarrassment in speech was such that he was with difficulty understood. These paroxysms recurred on some days repeatedly, and he was sometimes exempt from them for several successive days. This patient had occasionally double vision and nightblindness.

A distinctive feature of this affection, even when it exists in a slight degree, is inability to walk or stand with the eyes closed. If the patient, standing with the feet in apposition, close the eyes, he shortly reels, and would fall if not supported. This has been attributed to the coexistence of more or less anæsthesia of the soles of the feet. This, however, is not the correct explanation, as is shown by the fact that patients with notable loss of sensibility of the soles, but who have not ataxia, are able to stand without difficulty when the eyes are closed. ${ }^{1}$ A moderate amount of ataxia oecasions want of eonfidence in the locomotory powers. A patient of mine has the disease only in a sufficient degree to walk usually with what seems an eccentric gait; but were he to cross a street when it beeaure necessary to hasten in order to get out of the way of carriages, he would scarcely be able to make any headway, and he has to avoid carefully such a contingency. Dilatation of the pupils immediately following pinching, pricking, or irritation by galvanism of the trunk or limbs has been observed hy Voisin, Eeheverria, and Clymer. ${ }^{2}$ In a case under my observation at Belleve Hospital, the ataxia being limited to the two lower extremities and the right upper extremity, the pupil of the left eye was contracted nearly to a pin's point, and there was slight converging strabismus, with defective vision of the left eye. Impairment of sight exists in a considerable proportion of cases, and deafness sometimes occurs. The ophthalmoseope shows in most eases atrophy of the optic nerves. Strabismus and ptosis frequently occur, sometimes being permanent and sometimes of temporary cluration. These symptoms are explained by reference to the morbid anatomy in cases of this affection.

In most, if not all cases, the cutaneous sensibility is more or less impaired. The amount of sensory paralysis varies in different cases; generally both anesthesia and analgesia are combined. This occasions an additional difficulty in walking, from inability to feel the contact of the feet with the ground. Hence, the eyes are directed to the feet or ground in walking. The upper as well as the lower limbs may be deficient in tactile sensibility. 'The affection is essentially distinct from motor paralysis, but the latter may be associated with it. In certain cases of reritable paraplegia, the difficulty in walking depends measurably on defective power of co-ordination.

Incontinence of urine, and sometimes retention, occur in connection with the ataxic affection. Impotence in most cases oceurs sooner or later, if it have not preceded the affection. There is inability to retain the contents of the rectum at an advanced period of the affection. The muscles, for a considerable period, show increase of reflex excitability and of electro-contractility.

1 Vide report of cnse hy Dr. Pepper in Am. Jour. of Med. Sciences, July, 1871.

2 Echeverria on Reflex Paralysis. 
The diagnosis involves a discrimination from paraplegia, and, also, from general paralysis. The differential diagnosis may be easily made if the ataxic characters be marked. The ataxia is not a paralytic affection; the difficulty in walking, or in using any of the affected muscles, depends, not on deficiency of muscular power, but on the lack of ability to combine and clirect the movements of the affected muscles. The difference in this respect is strikingly shown by causing two patients, one affected with paralysis and the other with locomotor ataxia, to walk side by side. The paralytic walks with a feeble tottering or shuffling gait; the patient affected with ataxia throws out his limbs with force, but in an irregular, uncertain manner. The preservation of voluntary motor power, in cases of ataxia, is shown by the resistance which the patient is able to make when the physician tries to bend the limbs, and, also, by the freedom and strength of movements when the patient is recumbent. The coexistence of mental aberration with general cerebral paralysis, in the great majority of cases, is a diagnostic feature of the latter. In that disease the voluntary muscles of the limbs, face, etc., are simultaneously affected; in locomotor ataxia different parts of the voluntary muscular system are successively affected, the affection being generally manifested first in the lower extremities.

The affection is one of great gravity. The prognosis as respects recovery is as unfavorable as possible. The most to be hoped for in the majority of cases is that it will remain stationary or advance very slowly. Its progress is usually very gradual. The duration varies much in different cases. Exceptionally, it rapidly increases, but, as a rule, it continues mally ycars before leading to a fatal result. In the majority of cases, patients are cut off by some intercurrent or superadded disease. A certain amount of improvement sometimes takes place. In a case which was under my observation for several years at Bellevue Hospital, the ataxia being confined to the lower limbs, the patient for several months was obliged to keep the bed; but the affection did not extend to other parts, and the improvement was such that he acquired the ability to walk without much difficulty. In a case which has been under my observation in private practice for over ten years, the affection has not extended beyond the lower limbs, and the increase in degree has been slight. Ataxia, affecting all the extremities, and in a degree sufficient to render them useless, is not incompatible with good general bealth. A striking illustration of this fact was afforded by a case for several years under my observation at Bellevue Hospital, the patient being at length taken off by typhus. When death is attributable to the spinal affection, paralysis becomes superadded to the ataxia, portions of the cord connected with the motor nerves being implicated.

The anatomical characters of locomotor ataxia consist of induration or sclerosis of the posterior columns of the spinal cord, involving the gray substance and the posterior roots of the nerves. The sclerotic lesion may extend to the optic and the anditory nerve, the tubercula quadrigemina and the motor nerves of the eye. The hyperplasia of the connective tissue, constituting the sclerosis, involves atrophy and degeneration of the nerve fibres. In the degenerated structures, oil globules and amylaceous bodies are found in more or less abundance. The parts affected present to the naked eye a grayish or a translucent appearance. With sclerosis of the posterior columns, is associated, in some cases, more or less of the same changes in the anterior columns, a fact which accounts for the coexistence, with ataxia, of more or less motor paralysis in these cases. The sclerosis is sometimes limited to one of the posterior columns, and this 
fact will account for the ataxia being unilateral, althongh, in the vast majority of cases, it becomes bilateral. In cases of pure ataxia, that is, ataxia without paralysis, the lesion being limited to the posterior columns, the voluntary muscular power is preserved because the function of the anterior columns, as conductors of the will, is not affected. 'The defective power of co-ordination in these cases, shows this faculty to be dependent on the posterior columns of the cord.

The calsation of locomotor ataxia remains to be ascertained. The disease has been observed to follow various affections, viz., rheumatism, pellagra, epilepsy, hysteria, syphilis, ete. ; but the diversity of the antecedent affections renders it doubtful if the sequence denote any etiological connection. Exposure to cold and excessive muscular exereise have been supposed to produce it. It has been attributed to venereal excesses, but conjecturally rather than as a conclusion drawn from clinieal facts. Males are affected oftener than females. Of 60 cases analyzed by Carre, 42 were males and 18 females. It occurs very rarely prior to adult life. It may, however, occur in infancy. It is rare between the age of 10 and 30 years. In the great majority of cases the age is between 30 and 50 years. Trousseau has cited a case in which the patient was 80 years of age. Duchenne and Troussean cite cases which appear to show that in females it is most apt to occur among those who inherit a predisposition to nervous diseases. The disease has been observed to follow a fall producing an injury of the loins, and a strain from carrying a heavy burden. Baillarger, in France, and several German observers, have reported eases in which the locomotor ataxia was associated with general cerebral paralysis. In some of these cases the ataxia, and in other cases the paralysis, had precelence in point of time. I have met with a case in which it followed spinal paralysis.

The treatment will require but a few words. There is no special plan of medication as yet established. Benefit may be derived from the judicious employment of electricity. Duchenne and others in France bear testimony to the utility of the induced or interrupted eurrent of electrieity (Faradisation); on the other hand, Remak claims to have sueceeded in several cases by employing the continuous current administered by means of a powerful battery. Hammond considers galvanism a main feature of the treatment. According to Tronssean, the persevering use of sulphur baths has proved useful. This author states that hydropathy has been faithfully tried without any advantage; Carre, lowever, states that he has known this treatment to prove highly useful in two cases. Methodic gymnastic exercises continued perseveringly for several months are stated by Eisenmann to have been successful in two of six eases. Prof. Wunderlich adrocates the use of the nitrate of silver as a curative remedy, and this remedy has been used by others to some extent. With reference to the cases on which the supposed curative efficacy of this remedy rests, a distinguished anthor in this country states that he has examined them with great care without being able to accept their conclusions as demonstrated.' The ioride of potassium appeared to be useful in a case reported by Dr. Hughlings Jackson, of London, and in a case treated by Prof. Bartholow, of Cincinnati. In several cases the use of phosphorus, continued for a long period, has seemed to me highly beneficial. Hammond has prescriber with benefit the chloride of barium.

1 Prof. Alfred Stille ; Review in the Am. Journ. of Mled. Sciences, January, 1864. The reader is referred to this review for a digest of the recent literature of the discase. 
Ergot, belladonna, the bromide of potassium, cod-liver oil, oil of turpentine, and strychnia are also recommended. Experience has shown that counter-irritation by blisters, issues and the hot iron, is of no utility.

In the absence of any special medication, measures to maintain the nutrition and tone of the muscular system, to invigorate the general health, to remove coexisting disorders, and to palliate incidental symptoms, may doubtless do much toward retarding the progress of the affection and prolonging the life of the patient.

Duchenne has indicated a differential feature by which, as he obserres, ataxia dependent on disease of the spinal cord, may be distinguished from disease of the cerebellum. He thinks that the difficulty pertaining to movements in certain cases of disease of the cerebellum, is like that which exists in alcoholic intoxication, being, in the former, as in the latter, dependent on vertigo; whereas, in cases of ataxia there is no vertigo, but simply a deficiency of the co-ordinating power. ${ }^{1}$ The validity of this distinction admits of doubt.

\section{Progressive Muscular Atrophy.}

The distinctive characters of the affection now generally known by the above name, were first pointed out in 1848 by Cruveilhier, and hence it is sometimes called Cruveilhier's disease. 'The name progressive muscular atrophy was given to it by A ran in 1850. Cruveilhier called it gradual paralysis of motion with muscular atrophy. It is the affection called by English writers wasting or creeping palsy. Like the affection last considered, it is not strictly a form of paralysis. The impairment or loss of motion is due to atrophy or degeneration of the affected muscles. In cases of paralysis, the paralyzed muscles become atrophied, and their structure degenerates; but the defective and perverted nutrition, in these cases, results from the paralysis, whereas, in the affection now under consideration, the structural changes in the muscles precede their functional incapacity.

The atrophied muscles are more or less diminished in volume. The muscular fibres become pale, like those of cold-blooded animals. Examined microscopically, the fibrillæ of the affected muscles are reduced to a third or half of their normal size; the transverse striæ are diminished or lost; molecular granules are substituted for the proper muscular substance, and these finally undergo the fatty metamorphosis. Duchenne applied to it the name, muscular atrophy with fatty degeneration. The changes do not exist uniformly in all the fibres of the affected muscles. Intermingled with fibres which are atrophied and degenerated are those which have undergone little or no change. The loss of functional capacity in the affected muscles is, of course, proportionate to the number of fibres involved and the amount of structural change.

Progressive muscular atrophy generally commences in one of the upper extremities, oftener the right than the left. The affection is at first usually limited to a certain number of muscles. In the larger proportion of cases, some of the muscles of the hand are primarily affected. The muscles forming the ball of the thumb and the interosseous muscles are atrophied. In other cases the muscles of the shoulder, or the arm, or the forearm, are the first to become atrophied. Corresponding muscles of the other

1 Vide Diagnostic differentiel des affections cérebelleuses et l'ataxie locomotrice progressive, par le docteur Duchenne (de Boulogne). Extrait de la Gazette Hebdomadaire de Médecine et de Chirurgie, 1866. Vide Physiology of Man, by A. Flint, Jr., vol. iv., on functions of cerebellum. 
extremity are apt to become subsequently affected, the affection thus exemplifying the law of parallelism. More or less gradually the affection extends over the muscular system. Not only the muscles of the extremities, but those of the trunk are liable to become atrophied, and in some cases the latter are first affected. The intercostal muscles and the diaphragm may be involved, and death caused by apnoa. The muscles of deglutition may become involved, and the affection prove fatal by causing inanition. The affection is limited to the voluntary muscles.

A marked degree of atrophy of external muscles renders the appearance striking and characteristic. In a case which I saw with my colleague, Prof. Taylor, the atrophy was limited to the museles of the left shoulder, arm, and forearm. The right scapula was well covered by its muscles, but on the left side this bone was conspicuous, its boundaries distinct, and the spinous ridge projecting. The whole of the upper limb was attenuated, presenting a remarkable contrast with its fellow. In a case reported by Dr. Reade, the patient, a young man, when stripped to the waist, "exhibited the neck, chest, and arms to the elbow-joints, reduced to the most abject degree of emaciation. 'The emaciation was perfectly symmetrical; the greater and lesser pectoral muscles were little more dense than the strongest brown wrapping-paper; the muscles of the neck, anterior and posterior, proportionately attenuated ; the muscles on the scapulæ, particularly the supra- and infra-spinatus, were so much diminished as to show the spine of the bone with distinctness only less than the dry bone; all prominences from the deltoids were gone, and the muscles of the humeri were reduced to the cellular membrane, the mere elementary outline of the muscles, the biceps and triceps especially. From the elbows, the muscles of the forearms and hands displayed the full development of a robust and vigorous man of his stature, with all the concomitant power, sensibility, and aptitude for use. All the museles outside the pelvis, and those of the inferior extremities, were full, strong, and well formed." A patient in Bellevue Hospital presents great attenuation of the arms and thighs, the forearms and legs retaining a large muscular development. In this case the muscles of the back are affected, so that in walking the spine is curved backward, and on raising the upper extremities the scapule are thrown outward in a remarkable manner. In another case the atrophy is limited to the feet and about two-thirds of the muscles of the calves, the remaining third of the latter being unaffected. The feet have the appearances of bones covered only with integument. Cases have been reported in which the affection extended over the greater part of the voluntary muscular system. The tongue and muscles of mastication, the facial muscles, and the muscles of the larynx, may be included in the general atrophy. In a case cited by Thourenel, the power of moving the limbs and body was lost, and the patient was only able to move the head feebly. The tongue was involved in this case, and deglutition difficult. Trosseau refers to a case treated by Bretonneau, in which the power of speech and all voluntary movements, exeept of the head and of the forefinger of the right hand, were lost. This patient, a female, held communications with her family by indicating letters, in this way forming words and sentences. She dictated in this manner her last will and testament. When the atrophy is limited to a few muscles, the loss of substance is rendered striking by the normal size of the adjacent muscles. $A$ trophy of one class of muscles, for example the flexors or extensors of the limbs, causes distortion by the contraction of the muscles which are not affected, 
the contraction arising from the loss of antagonism. Distortions of the head, trunk, and extremities may be produced by the limitation of the atrophy to certain muscles, or by the progress of the atrophy being much greater in some muscles than in others. The characteristie appearances belonging to the affection give to it a physiognomy by which it is readily recognized in well-marked cases. In the early part of its progress it may escape recognition, if a close examination be not made.

In general, the first evidence of trouble is weakness of the affected muscles, arising from the atrophy. Pain or soreness precedes and accompanies the affection in a certain proportion of cases. Generally the pain is not severe. It is referred to the affected muscles, and is of a neuralgic character. The affected parts are notably sensitive to cold, and their temperature is below that of other parts. Defective capillary circulation is sometimes shown by congestion of the surface. Quivering movements of the fibres of the affected muscles are often observed, and sometimes subsultus of the tendons and slight twitehings of the parts to which the tendons are attached. These movements occur irrespective of the will, occasion no pain, and the patient may not be conscious of them. The sensibility of the surface is unaffected. The mental faculties remain intact, and there are no symptoms pointing to intra-cranial disease. The appetite, digestion, and nutrition may continue unaffected. There is no loss of power over the bladder or rectum, even when the affection is far advanced, nor does the heart become affected. Constipation may be produced by atroplyy of the abdominal muscles. The affected muscles lose their electro-contractility only in proportion as they become atrophied and degenerated; the fibres which are unaffected respond to the electrical current. This serves to distinguish the affection from the wasting of muscles due to paralysis firom lead and some other forms of paralysis.

The progress of the affeetion is slow. More or less slowly increasing, it is, thus, as a rule, progressive, as the name implies. This rule, however, is not without exceptions. Sometimes it remains stationary after having progressed to a certain extent. In a few cases recovery may be said to take place; that is, the affection ceases without leaving any permanent deformity or notable debility. It may continue, slowly progressing, a great number of years before leading to a fatal result. The prognosis is extremely unfavorable. The most to be hoped for is that it will cease to progress, or that its progress will be very gradual. Retrogression is not to be expected after much change in the muscles has taken place. Life may be destroyed by apnœa, if the respiratory muscles become affected, or by inanition from atrophy of the muscles concerned in deglutition. In other cases, death takes place after a long and tedious confinement to the bed, if the patient be not carried off by some intercurrent disease.

As regards duration, the following are the results of the analysis of one hundred and five cases by Roberts: Mean duration of cases ending in recovery, one year and two months; of cases ending in permanent arrest, two years and three months; of cases ending fatally, five years and two months. Of the cases which recovered, the longest duration was two and a half years, and the shortest eight months. Of the cases proving fatal, the longest duration was twenty-three years, and the shortest twelve months.' The longest case ending in arrest continued active for seven years, and the shortest for four months.

' An Essay on Wasting Palsy, Cruveilhier's Atrophy. By William Roberts, B. A., M. D., etc., London, 1858. Dr. Roberts' work embodies our existing knowledge of this disease. 
This affection is connected with, and, probably, dependent on, certain lesions of the nerrous system. Cruveilhier's researches showed atrophy of the anterior nerves of the spinal cord, and he was led to consider this lesion as preceding, and giving rise to, the muscular atrophy. Later observers have found the anterior spinal nerres reduced in volume, owing to the disappearance of a greater or less number of their component fibres. Changes have also been observed in the spinal cord, namely, atrophy and softening of the gray portion, with the presenee of granular matter and amyloid bodies. These changes may be seated in either the anterior or posterior cornea, in the posterior commissure, and in the central portions of the cord. They may be found in general on all of these situations, and they may either extend over the cord, or be limited to isolated portions. 'They are most apt to be marked in the cervical portion of the cord. Portions of the sympathetic system have also been found in a state of fatty or granulo-fatty degeneration. It has been observed that when the muscular atrophy is limited to, or most marked on, one side of the body, the changes in the nervous system are either exclusively situated, or greater, on the corresponding side.

It is conjectured that the lesions of the nervous system, in this affection, are confined, ehiefly or exclusively, to cells and filaments distinguished as trophic, their function being to preside over the nutrition of the muscles. Some writers (Jaccoud, Hammond) entitle the affection a disease of the trophic nervous system. This view of the pathological character is rational; but it is to be borne in mind that the existence of trophic cells and nervous filaments has not, as yet, been demonstrated.

The causation is obseure. The affection rarely occurs under adult age, and it is of rare occurrence after middle life. Males are much oftener affected than females. There is no connection with social condition or particular occupations. Several members of the same family, in repeated instances, have been affected. It has been supposed, in some cases, to proceed from undue muscular exertion. Like most affections, it is sometimes attributed to the action of cold.

The great object of treatment is the arrest of the progress of the affection. The measures for this object have reference to the affected muscles and the general condition. Undue exertion of the affected muscles is to be avoided, but a certain amount of exercise is important. The circulation and nutrition within the affeeted muscles are to be promoted by friction and shampooing, with the use of stimulating embrocations. The induced electrical cuirent, according to Ducheme, applied to the muscles aftected, is of much value. Remak states that the application of the constant galranic current upon the inferior cervical ganglion of the great sympathetic has produced under his observation a hilppy effect. Measures having reference to the general condition are those which invigorate the powers of the system. Here, as in cases of progressive locomotor ataxia, judicious management, although it may fail to effect reeovery, may do much toward retarding the progress of the affection and prolonging the life of the patient. 'The point in the progress of the affection at which the arrest is effected is of vast importance to the patient; hence the desirableness of an early diagnosis.

In one of the cases at Bellevue Hospital, which has been referred to, in which the muscles of the arm, thigh, and back are greatly atrophied, not only has there been no progress of the disease during three years, but the patient is greatly improved, and now discharges the duties of a ward orderly man or nurse. 


\section{Pseudo-Hypertrophic, or Myo-sclerotic, Paralysis.}

The characteristics of this affection were first pointed out by Meryon, in 1851. It was, however, more fully described, and its nosological individuality established subsequently by Duchenne. It is characterized anatomically by hyperplasia of the connective tissue of muscles, together with atrophy of the muscular fibres.' Jaccoud proposes, as a better name, progressive muscular sclerosis. As observed by Duchenne, this affection is peculiar to infancy and childhood. Dr. Dyce Brown, of A berdeen, Scotland, has, however, reported a case occurring at the age of twenty-six years. ${ }^{2}$ Like the affection last considered, this is not primarily a paralysis, but the loss of motor power is an effect of atrophy of the muscular fibres. This atrophy is a result of the increased production of areolar tissue between the fibres of the affected muscles. Witl this hyperplasia and consequent atrophy, there may, or may not, be an abundance of fat. 'The affected muscles are apparently enlarged, but the apparent increase of size is due to the increased quantity of connective tissue, that is, the selerosis. As regards the muscles, therefore, the affection is a false hypertrophy. The affected muscles are abnormally hard and firm. According to Duchenne, the enlargement is preceded by muscular weakness.

The structural ehanges involve diminution of motor power in the muscles which are affected, proportionate to the amount of compression and consequent atrophy. Seated in the museles of the lower limbs, the affection causes a peculiar waddling gait, the limbs in walking being widely separated. If the extensor spinal muscles are affected; a lumbo-sacral anterior curvature takes place when the posture is upright. Frequently, the affection predominating in the flexor museles of the foot, equinism, or talipes equinus, is a result of the greater action of the extensors, and with this is a claw-like deformity of the toes. After having reached a certain degree and extent, the affection may remain stationary for sereral years; but, at successive epochs, it is apt to increase and to become more and more diffused, until patients are reduced to a helpless condition. Death is generally caused by some intercurrent clisease. - The progress of the affection is unattended by pain. There is neither hyperæsthesia nor anasthesia. The functions of the bladder and rectum are unaffected. In an advanced stage the electro-contractility of the affected muscles is notably diminished or lost. The affection is, in most cases, incurable; the chief object of hope is that it may not make further progress. Duchenne advises faridization of the museles, shampooing, and hydropathic treatment, together with the iodide of potassium and phosphorus. Jaccond cites a case, reported by Benedikt, in which a cure was effected by galvanism. ${ }^{3}$

\section{Scleroderma, or Sclerema.}

The affection called scleroderma or sclerema, also sclerodermia and sclerenia, may be here noticed, as it is analogous, in its pathologieal character and certain of its effects, to that just considered. Other terms than those already given have been applied to it, to wit, scleremus, scleria, sclerosis,

- De la Paralysie Pseudo-Hypertrophique ou Paralysie Myo-sclérosique. Par le Dr. Duchenne (de Boulogne). Paris, 1868.

2 Edinburgh Med. Journal, June, 1870.

3 For a report of a case, and the facts in 41 reported cases tabulated by Dr. William Ingalls and Dr. S. G. Webber, vide Boston Medical and Surgical Journal, Nov. $17,1870$. 
scleroma, and sclerymus, all derived from the Greek word ox ripos (hard). Its anatomical characteristic is the production, in the lower portion of, and beneath, the skin, of dense fibrous tissue in great abundance. This renders the integument notably rigid and resisting to pressure. It is closely adherent to the muscles. It camnot be noved over these, nor pinched up in folds. The muscles are restrained in their movements by the density of the structures above them, as if a firm bandage were closely applied. More or less wasting of the muscles is caused by the pressure. Pigmentary matter is, in most cases, deposited in the cells of the rete mucosum, produeing a discoloration whieh is yellowish, yellowisl-gray, or dark-brown, more marked in some situations than in others; but, in some cases, the skin becomes decolorized. These changes may be either limited to certain parts or diffused over the whole surface but varying in amount in different places.

The affection is extremely rare. A case which was under my observation was reported by Dr. Walter De F. Day in the American Journal of Medical Sciences, April, 1870. Dr. Day was able to collect for analysis reports of only thirty-three eases; and the case reported by him was the seventh on reeorl in which there was an examination after death. The affection is not to be confounded with that called by some writers acute sclerema which is peculiar to infints. The latter is a variety of cedema. The affection under consideration occurs generally in middle life. It occurs much oftener in women than in men. It is a chronic affection, usually beginning insidiously, and progressing slowly, but exceptionally developed rapidly with a sudden invasion. The upper part of the body is oftenest first affecterl, but it may appear primarily in any part.

The affection is not necessarily associnted with disease of any of the internal organs; and, if uncomplicated, it is not incompatible with good general health. Existing in a notable degree, it obstructs musenlar movements by mechanieal pressure and restraint, leading, at length, to more or less impairment of motor power by inducing atrophy. The surface is usually eool or eold, but the heat at times may be greater than normal. Sensibility is not impaired, and transpiration continues. The duration is variable. It may continue for months and years. A fatal termination is due to some complication or intereurrent affection. It continues, in some cases, indefinitely without improvement, and, in other cases, more or less improvement takes place without recovery ; but complete recovery may be hoped for. The number of cases reported is, as yet, too few to determine the ratio of recoveries.

Various measures of treatment were employed in the cases which have been reported. It remains to be ascertained what are the remedies, if there be any, which exert a curative effect. In the case under my observation, arsenic was given for some time, and it seemed to be of service; but, in this case, there occurred grave complications, namely, pleuritis, pericarditis, and peritonitis. An analeptie course and tonic medication are rationally indicated, together with alkaline baths, and emollient applications to the skin.

\section{Neuritis.}

Before passing to consider certain functional affections of the nervous system, in the next chapter, inflammation of the nerves claims a brief notice. Neuritis, exclusive of the cases in which the inflammation is either of traumatic origin, or propagated from surrounding parts which are inflamed, is extremely rare. There is, however, room for the conjec- 
ture that it may exist in a certain proportion of the cases in which the diagnosis is neuralgia - this term denoting a functional affection. If the inflammation be seated in a sensory nerve, the only symptom, at first, may be pain extending along the affected nerve, perhaps to its terminal branches. Subsequently, il some cases, as a result of inflammatory lesions, namely, suppuration, hyperplasia of connective tissue, and secondary atrophy, there is anæsthesia, or diminished tactile sensibility, with which more or less pain may continue, that is, analgesia may be wanting.

- Neuritis seated in motor nerves, or those containing motor fibres, may give rise to muscular contractions, and paralysis of motion may be a secondary effect. Other effects are loss of reflex and of electrical conduction. The paralyzed muscles, in these cases, rapidly become atrophied. Erythema of the skin, vesicular eruptions, and dark-colored patches are supposed to be sometimes dependent on a neuritis.

The diagnosis involves a discrimination from neuralgia. Points which distinguish a neuritis are as follows: The pain being more continuous, that is, not occurring in paroxysms, or the exacerbations less marked; the occurrence of paralysis when motor or mixed nerves are affected, and the loss of reflex and electric conduction. If the affected nerve be superficial, or accessible to pressure, there is tenderness over it and from the inflamed part along the whole tract, the tenderness not confined to certain isolated parts as in neuralgia. The inflamed nerve may be felt to be like a hard cord. The skin is reddened over the site of the inflammation. According to Benedikt, general electric tenderness of the nerve is distinctive of neuritis. In most cases, neuritis is due to the presence of a foreign substance, a wound, the pressure of a tumor, or the existence of inflammation in an adjacent situation. Of course, in these cases, the treatment has reference to the local causative circumstances. When the affection is spontaneous, revulsive and soothing local applications are indicated. Subcutaneous injections may be required to relieve the intensity of pain. The anæsthesia and motor paralysis which may follow, call for the employment of electricity.'

\section{CHAPTER VII.}

\section{THE NEUROSES. NEURALGIA.}

Trifacial Neuralgia. Cervico-Occipital Neuralgia. Cervico-Brachial Neuralgia. Lumbo-Abdo. minal Neuralgia. Crural Neuralgia. Sciatic Neuralgia. Dermalgia. Myalgia. Cephalalgia. Vertigo.

CERTAIN affections of the nervous system are distinguished as the Uneuroses. The affections so called are peculiar to the nervous system, and occur without either inflammation or any morbid changes in the nervous structure as yet definitely ascertained; in other words, the neuroses are, in the existing state of our knowledge, to be considered func-

I For a fuller account of neuritis, vide Injuries of Nerves, etc., by S. Weir Mitchell, M.D., 1872 
tional affections, using the term functional as it has been defined (page 20 ), and as it has been hitherto used in this work. Some of the disorders of function, however, which nosologically rank as individual affections, occur also as symptoms of diseases involving either inflammation or lesions of structure. For example, pain referable to nervous trunks, convulsions, and paralysis, are, severally, symptoms of diseases which have been considered. Inflammation and lesions within the skull and spinal canal may give rise to these disorders of nervous functions. Now, pain in a nervous trunk, not occurring as a symptom of inflammation or any appreciable change, signalizes a functional affection or one of the neuroses, viz., neuralgia. Convulsions, of a certain character, taking place, in like manner, irrespective of any inflammatory or structural disease, denote a functional affection, another of the neuroses, viz., epilepsy. So, paralysis is sometimes functional, and then it belongs among the neuroses. Several of the neuroses have been already considered in treating of the affections manifested in connection with the respiratory, the circulatory, and the digestive system, as well as the affections of the nervous system to which the preceding chapters of this section have been devoted. This has been done for the sake of convenience. It remains to consider the functional affections which it is most convenient to arrange in a separate class.

The different functional affections of the nervous system differ according to the functions disordered. 'The latter may be classified as follows: 1. The mental faculties. 2. Special and general sensibility. 3. Voluntary motion. 4. Involuntary or reflex movements; and 5. Innervation.

Mental disorders do not usually enter into courses of instruction in the Principles and Practice of Medicine. They are, however, to be included within the range of the studies of the physician. Treatises are devoted to these disorders, and their management constitutes one of the specialties of medical practice; but all physicians are called upon, more or less, to treat affections of the mind, filling short of well-marked, confirmed insanity. Melancholia and hypochondriasis in their lighter grades, i. e., not amounting to insanity, come constantly under the notice of the practitioner. Want of energy, lack of buoyancy - mental apathy and inertiaoften exist without any obvious disease. The physician should consider these as generally denoting corporeal ailment. And this should be impressed on the minds of patients, since they are thereby led to reformations and measures of management which will be likely to afford relief, and they arc enabled to bear better and strive against mental disorder. $\boldsymbol{A}$ vast amount of unhappiness is due to canses which, under intelligent medical direction, may be removed, or to functional morbid conditions which judicious treatment may relieve. It is a grave error to attribute mental disorders exclusively to mental causes. These few remarks open up a subject of great importance. Insanity is to be prevented by the general practitioner. They who devote themselves to the treatment of insane patients have not the opportunity of preventing the development of insanity.

The study of mental disorders is important to the physician as involving questions of medical jurisprudence. The existence, or otherwise, of insanity sufficient to require or warrant confinement in institutions for the insane, the exemption of moral responsibility for criminal acts, incompetency to perform legal obligations, to take carc or dispose of property, etc., are questions concerning which physicians are called upon to give testimony; but for information on these questions the student and practi- 
tioner are referred to works treating of disorders of the mind, and of medical jurisprudence.

Disorders manifested in the organs of voluntary motion are, paralysis, spasm, convulsions, and defective co-ordination. These have been already considered as symptoms of certain diseases, and they will also enter into some of the functional affections of the nervous system which remain to be considered, viz., epilepsy, chorea, and hysteria. Functional paralysis of motion has been already considered. Convulsions occurring in the puerperal state, or puerperal eclampsia, and in children, do not come within the scope of this work.

Disorders relating to the involuntary movements of the heart, stomach, intestines, etc., and to the tonic contractility of the voluntary muscles, involve the reflex, true spinal, or diastaltic portion of the nervous system, and the sympathetic or ganglionic system. These are cousidered in connection with the affections treated of in other sections, together with the nervous affections exclusive of the neuroses. This statement will apply to disorders of the imperceptible influence communicated to the different organs of the body, known as innervation, which also involves the portions of the nervous system just named.

Disorders of the special senses do not come within the scope of this work except as symptoms of discases. An important functional disorder of general sensibility, viz., neuralgia, gives rise to several individual affections, some of which have been considered. Under this head I shall proceed to present certain general considerations, and notice briefly neuralgic afiections not embraced in other sections.

\section{Neuralgia.}

The name neuralgia, a term of modern date, embraces a group of local affections characterized by pain which may occur without inflammation or any appreciable changes in the parts affected. All that can be said of the pathological character of the affection, in these cases, is, they involve a morbid condition of sensibility. The neuralgic affections are of frequent occurrence. Apparently they are much more frequent at the present time than heretofore; but many of the affections now called neuralgic were formerly considered as inflammatory, spasmodic, or rheumatic. Any organ or part of the body endowed with seusitive nerves may be the scat of neuralgia. Other things being equal, the organs most abundantly supplied with this class of nerves are most liable to be affected. Visceral neuralgias, as they are called, that is, neuralgic affections seated in organs belonging respectively to the respiratory, circulatory, and digestive systems, have been already considered. The affections which are here to be consiclered are scated in nervous trunks not involved in the organs composing the foregoing anatomical systems. As regards clinical history, diagnosis, causation, prognosis, and the general principles of treatment, whatever is true of one of these affections is mainly true of all. To aroid repetitions, therefore, I shall consider, first, these neuralgic affections collectively, or neuralgia in general, under the several aspects just named, and afterward notice briefly the more important of these affections individually.

Neuralgia may be abruptly developed, but in the great majority of cases there are premonitions. The development was sudden in one-seventh, and more or less gradual in the remaining six-sevenths of the cases analyzed by Valleix. 'The premonitions consisted of a feeling of weight, a dull

1 Op. cit.; also Traité des Névralgies. 
pain, a sense of heat or some uncomfortable sensation in the seat of the affection. The ehief characteristic symptom of neuralgia is pain. The pain is either ehiefly or exclusively in paroxysms, or there may be more or less continued pain with exacerbations in which the pain is much increased. The eontinned pain may be clull or contusive, but during the paroxysms or exacerbations the pain is described as darting, tearing, lancinating, and it is often exerueiating. The paroxysms or exacerbations are of variable duration, lasting in some cases for a few seeonds only, and in other cases for several hours or even days. The intermissions or remissions are not less variable in different eases; their duration may only be a few seconds or moments, and they may extend to days. The lancinating pain shoots in the direction of a nervous trunk and its branches; patients sometimes delineate the tract and distribution of the nerve affeeted, in defining the directions in which the pain extends. In the great majority of cases neuralgia is unilateral.

Tenderness on pressure, generally limited to eireumscribed points, is an important diagnostic feature of neuralgia. Valleix was the first to show that, as regards their relations to the nerves, the tender points are situated as follows: 1st. Where nervous trunks or branches emerge from the skull or spinil camal ; 2 l. Over branches which penetrate muscles on their way to the integument; $3 \mathrm{~d}$. At the termination of branches which are lost on the surface; and, 4 th. In places over trunks near the surface. 'The situation of the tender points in intercostal nemalgia has been stated in treating of that affection. The diagnostic evidenee to be derived from this souree in other neuralgic affections will be stated when these are severally noticed. Pain may not be produced by pressure made with the open palm ; indeed, firm pressure thus made often relieves the pain. The tenderness is shown by pressure made with the ends of the fingers or by percussion. The pain thus produeed is often aente, cansing the patient to cry out or to try to eseape from the exploration. If, however, the pressure be continued, the tenderness is often found to diminish or temporarily to disappear. The tender points are frequently quite limited, the end of the finger being often suffieient to cover them. As a rule, the pain produced by pressure is acute in proportion to the acuteness of the spontaneous pain. The tenderness is most marked during paroxysms or exacerbations of pain. In the intervals between paroxysms, the patient being entirely free from pain, the tenderness on pressure may disappear.

Paroxysms or exacerbations of pain are excited or inereased by sudden or violent movements of parts in which the affected nerves are situated. A concussion of the whole body, as in coughing or sneezing, excites or increases the pain. The application of cold or lot substances to the surface over the seat of pain, in some cases, is insupportable.

Febrile movement or any notable constitutional disturbanee does not belong to the elinical history of neuralgia. The finetions of parts to which the affected nerves are distributed may not be notably disturbed. A neuralgic attack, however, sometimes induces congestion in the site of the pain, as shown by temporary redness of the conjunetiva in cases of neuralgia affeeting the ophthalmie division of the fifth pair. Involuntary contractions of museles and eramps sometimes oceur. An inereaserl secretion of tears, saliva, and mueus may accompany paroxysms of neuralgia affecting the several divisions of the fifth pair; and glandular organs within the body are probably in like manner excited in the visceral neuralgias.

Neuralgic affections have no fixed duration. 'They may end spontaneously or under the influence of treatment in a few hours or days, and they may continue for years or during life in spite of all methods of 
treatment. The prognosis is almays favorablc as regards a fatal termination. Persons who suffer attacks at variable intervals for a lifetime are at length carried off by other maladies not developed in consequence of the continuance of the neuralgia. Of the cases analyzed, with reference to termination, by Valleix, in seven-ninths a permanent cure took place; in one-ninth there was improvement without recovery, and in the same proportion there was neither cure nor improvement.

Neuralgic pain may proceed from a palpable lesion, such as a tumor either involving or pressing on the affected nerve, the presence of a foreign body, the traction of a cicatrix from neuritis, etc. In such cases, the neuralgia does not properly belong among the neuroses. As a functional malady its causation differs in different cases. It proceeds, in certain cases, from the action of the special cause of periodical fever, commonly known as malaria. This is always to be suspected in cases occurring in so-called malarious districts. When due to this cause, the paroxysms sometimes recur with the same regularity as the paroxysms of intermitting fever. But it may proceed from this cause when the paroxysms do not exemplify a law of periodicity. It is one of the effects of lead-poisoning. Next to lead colic (and this is, in fact, a neuralgic affection), nemralgia is the most frequent form in which the poisonous effect of lead is manifested. Anamia, by whatever cause induced, favors the occurrence of nenralgia. A large proportion of those who suffer from neuralgic affections are anremic." Persons are most apt to suffer from neuralgia between the age of twenty and of fifty. Prior to ten years of age it is extremely rare. Women are more subject to it than men, and, of the different species of neuralgic affections, some occur most frequently in the male and some in the fomale sex. Females, for example, are more subject to intercostal neuralgia, and males to sciatic neuralgia. A neuralgic attack is sometimes referable to exposure to cold, particularly to a current of cold, moist air continued upon the part which becomes affected. Cases occur more frequently in cold than in warm seasons.

The situation of the pain is not evidence of the seat of the morbid condition giving rise to neuralgia. The mind may refer the pain to the terminal branches, or the parts to which they are distributed, when the morbid condition is seated at any point in the course of a nervous trunk, or at the point of connection of a nerve with the spinal cord. This is illustrated by the fact that, after an amputation, the condition of the extremities of the divided nerves gives rise to pain which the patient refers to the amputated limb. Anstie believes that, as a rule, in the neuralgic affections denominated functional, there is some morbid change at the central connection of the nerve or nerves to which the pain is referred. $\mathrm{Hc}$ is also led to think that, in a large proportion of cases, a hereditary tendency is involved in the causation; and, further, that the development of these affections is favored by exhaustion and debility, aside from anæmia. ${ }^{2}$

The diagnosis of neuralgia is to be based on the character of the pain, its situation in the course of a nervous trunk or its branches, and the limitation of tenderness to circumscribed isolated spaces on the surface. Other diagnostic points are the recurrence of pain in paroxysms or marked exacerbations, shifting of the situation of the pain and tenderness, and the difference, as regards pain, between pressure with the ends of the

1 With regard to the dependence of neuralgia on anæmia, Romberg says : "It seems as if pain were the prayer of the nerve for healthy blood."

2 Neuralgia and the Diseases that Resemble it, 1872. 
fingers and with the open palm. In the shifting of pain from one nerve to another, which is a highly diagnostic feature, the nervous trunks successively affected do not always have a common origin; thus, the neuralgia may be transferred from a cranial to a spinal nerve. These are the positive diagnostic points. Negative points are those by which inflammation and structural lesions are excluded, such as the absence of febrile movements, etc. In determining the affection to be functional neuritis is to be excluded, and it is to be ascertained, if practicable, that the pain is not clependent ou a local mechanical cause, such as a tumor pressing on the nerve at some point in its coursc. Syphilitic periostitis is also to be excluded.

The treatment of neuralgia is rational and empirical. 'The rational treatment consists, in the first place, in fulfilling indications which relate to morbid conditions suspected of standing in a causative relation to the neuralgia. The physician, in individual cases, is to seek for evidence of malaria or lead-poisoning, conditions having diagnostic criteria irrespective of the neuralgic affection. Appropriate measures of treatment are to be addressed to these or any other associated morbid conditions under the supposition that they may have more or less to do with the existence of the neuralgia. Diathetic conditions, especially the rheumatic and gouty, are to be considered as probably contributing to the neuralgic affection, and, with reference to the cure of the latter, these conditions claim appropriate measures of treatment. With reference to these conditions, $\mathrm{my}$ experience coincides with that of Anstic; they are concerned in the production of neuralgic affections far less frequently than many practitioner's and writers seem to suppose. Attention is always to be directed to the coexistence of ansemia, which the venous hum in the neck may denote when the anremic condition is not manifested by pallor of the face and prolabia.' If aniemia coexist, preparations of iron should be continued for a long period. An eligible plan is to give the pulvis ferri in the form of dragées, for several months, enjoining upon the patient not to expect restoration to health until after a long continuance of the remedy. 'This treatment addressed to the anæmia is indicated, provided the anæmic condition continues, notwithstanding the neuralgia may cease. The disappearance of the venous hum is evidence that the anæmic condition is removed.

The relief of pain enters into the rational treatment. In severe paroxysms or exacerbaticns, opiates are called for. Large doses are sometimes indicated by the intensity of pain. The most prompt and effective methol of affording relief is the hyportermic injection of a solution of morphia. In the use of opiates for the relief of neuralgic pain, the risk of the formation of the habit of taking opium is always to be borne in mind, yet in serere cases, which resist therapentical measures, this risk must sometimes. be incurred. If the pain be not intense, relief may be obtained by belladonna, hyoscyamus, stramonium, conium, the preparation known as chlorodyne, and the tincture of aconite. Local applications will often do much toward the relief of pain. Of these, the most efficient are liniments. of the tincture of aconite, chloroform and opium. Belladonna, stramonium, veratria and aconitia, applied in the form of ointment, are sometimes efficacious.

An arrest of the paroxysms of pain by opiates is sometimes enratire. that is, the paroxysms or exacerbations do not recur. If the derelopment of the affection have been recent, it is not easy to decide whether a cure be effected by the remedy or whether the affection has no intrinsic ten-

- For the method of obtaining this physical sign, see page 63 . 
dency to continue; but the paroxysms sometimes cease to recur, after having been once or repeatedly arrested, when the affection has existed for a greater or less period, and in these cases it is fair to attribute a curative efficacy to the arrest. In order to arrest a paroxysm, a full opiate should be given as soon as the paroxysm begins, or, if practicable, the occurrence of the paroxysm may be anticipated by a full opiate. Before abandoning this plan of treatment, it should be employed in several recurring paroxysms. The continued employment of some form of opiate, or of some one of the narcotic extracts, especially belladonna, during the intermissions or remissions, in some cases effects a cure.

The administration of opiates by means of hypodermic injections, to which reference has been made repeatedly in the foregoing pages, is especially applicable to the treatment of certain cases of neuralgia. As simply securing temporary freedom from pain, that is, as a palliative measure only, its advantages over administration by the mouth are, the greater promptness with which relief is obtained, the smaller quantity of medicine required, a less amount of interference with the digestive fumctions, and avoidance of the unpleasant after-effects of opiates in some of the cases in which these effects follow the administration by the mouth or rectum. These advantages are not peculiar to the employment of the hypodermic method in the treatment of neuralgia, but belong equally to the employment of this method in other affections. As regards the curative effect of opiates administered hypodermically, they appear to be oftener eflicacious than when administered by the mouth or rectum. In view of the greater effects of opiates administered hypodermically, as compared with the administration by the mouth, an important caution is, not to inject at first too large a quantity. It is a safe rule not to use in the first injections more than half the stomachic dose for males, and not more than a third for females. Sufficient time should be allowed between a first and second injection, to judge of the narcotic effect of the first. If the salts of morphia be used, the quantity in the first injection may vary, according to the intensity of pain and other circumstances, from one-sixth to one-fourth of a grain. The amount of morphia which may be tolerated without narcotism in cases in which the quantity injected has been gradually increased, is truly marvellous. Dr. T. B. Townsend, of New Haven, Conn., has reported a case of obstinate neuralgia, with intense pain, in which, after continuing daily injections, the doses being progressively increased, at the end of four months 50 grains per diem were required to subdue the pain, and this enormous amount produced no symptoms of narcotism. ${ }^{1}$

The empirical treatment of neuralgia consists in the use of remedies which experience has shown to be sometimes curative, and which are to be tried without following any particular rational indications. These remedies are in some cases efficacious and in other cases devoid of any curative effect; and often there are no means of determining beforehand which one of the remedies will be most likely to prove successful. In rebellious cases, the different remedies are to be tried in succession. Methodic counter-irritation is undoubtedly efficacious in a large proportion of cases. Of the different modes of counter-irritation, Valleix prefers the actual cautery, the hot iron being passed along the course of the affected nerve, taking care that the cauterization shall be superficial so as not to lead to deep eschars and suppuration. To avoid the pain occasioned by the cautery, he advises etherization. He states that he has resorted to

I American Med. Times, Dec. 27, 1862. 
the actual cantery in more than 150 cases, and always with success, if the neuralgia be functional. This method may be resorted to in cases which resist milder means of counter-irritation, together with other empirical remedies. Milder means should first be tried. Of the latter, a succession of small blisters, applied over the painful and tender points, will frequently prove successful. Prof. Alfred Stillé advises blisters of only balf an inch in diameter, the period of application to be from one to two hours only, the vesicated surfaces to be healed as rapilly as possible, and the blisters to be repeated in a few days if the neuralgia continue. This writer states, as a result of the employment of this method of treatment in almost every case which has come under his observation in public and private practice for many years, that " in no single instance has it failed to mitigate the symptoms, and in very many it alone has achieved a cure."s

During the process of vesication the neuralgic pain is frequently increased. When quickness of resication is desirable, it may be effected in a few moments by the strong aqua ammonix, and still more quickly by means of a hammer heated by hot water. By the latter means, or by an instrument devised for the operation known as firing, a rubefacient effect may be quickly and eonveniently produced, and in mild cases this may suffice without vesication. Dry eupping is another effective method of mild comnter-irritation. Sinapisms are proverbially useful, and, in certain cases, will suffice, in conjunction with internal remedies.

Electrieity has been often found efficacious by those who have given special attention to electro-therapeutics. A pplied in conjunction with aeupuncture it has been successfully resorted to in some rebellious cases. The palliative effect of electricity is probably due to a temporary blunting of the excitability of the nerve or nerves to which the pain is referred; their capacity either to originate or to conduct painful sensations is diminished or annulled by passing through them the electrical current. This measure, however, in many cases, has not only a palliative, but a curative effiracy. The testimony of those who have had great experience in its employment in neuralgias is stronger than that which can be cited in behalf of any other remedy. Hammond says, " $A$ bove all local means, not only for relieving the pain of any particular paroxysm, but also for effecting a permanent cure, electricity stands first."' This author considers the primary galvanic eurrent as vastly preferable to the induced current. Anstie holds the same opinion as regards the superior effieacy of the primary current, and he is hardly less strong in his estimate of its value. 'To quote his language, "The constant current, as I now estimate it, is a remedy for neuralgia unapproached in power by any other, save only blistering and liypodermic morphia, and even the latter is often surpassed by it in permanence of effect." Drs. Beard and Rockwell, ${ }^{3}$ who have found both forms of electricity efficacious, affirm that, by its use, "The majority of cases will be cured or permanently improved." " Strong pressure upon an affected nervous trunk sometimes has a palliative effect; the pressure, at first, may occasion great pain, but, continned or repeated at short intervals, it is horne without suffering and diminishes for a time the excitability of the nerve.

Of the internal remedies which experience has shown to be efficacious, the more important are quinia, arsenic, the precipitated carbonate of iron,

1 Therapeutics and Materia Medica.

Op. cit.

Op. cit. Vide Treatise by Anstic and that by Beard and Rockwell for practical detrils concerning the employment of electricity.

- op. cit. 
the preparations of zinc, strychnia, the essential oil of turpentine, and the iodicle of potassium.

Quinia, as already stated, is especially indicated when the neuralgia is attributable to malaria, and it will be most likely to prove successful when paroxysms occur periodically. But it is efficacious in eases in which there is no ground for suspecting a malarious causation, and in which there is no observance of the law of periodicity. 'To be fairly tried, this remedy should be given in full doses-from 15 to 30 grains daily to an adultand continued for a week or ten days. Of the foregoing list of remedies, this is entitled to the first rank. Arsenic may be tried in cases in which there is an intolerance of quinia, or when the latter fails to effect a cure. It is a remedy of great value in neuralgic affections. To be fairly tried, it should be carried to the extent of producing some of its characteristic toxical effects.

The precipitated carbonate of iron, advocated some years ago by Hutchinson, is sometimes efficacions. It is to be given in large doses, viz., from one to two drachms twice or thrice daily. Ferruginous remedies are rationally indicated by coexisting anæmia, and this condition is present in a very large proportion of the cases of neuralgia. The efficacy of the preparation named is doubtless, in a measure at least, attributable to the coexistence of anæmia. The hydrochlorate of ammonia has been found in some cases notably efficacious. I have known the nitrate of ammonia to be signally successful. The valerianate of ammonia is a useful preparation.

Of the preparations of zinc, the valerianate is best suited to cases of neuralgia. Strychnia may be given internally, or applied locally upon a blistered surface. In the latter mode of administration, the benefit may in some cases be measurably, and perhaps chiefly, due to the counter-irritation. The oil of turpentine is but little used in neuralgic affections, but I have known it to prove promptly successful, in doses of a drachm, combined with castor oil, given on alternate days, after a variety of remedies had been employed without success. The iodide of potassium has been advocated by Graves, Watson, and especially by Nencort. ${ }^{1}$ The writer last named advised its use in large doses. The coexistence of the rheumatic diathesis is regarded as indicating specially this remedy. The bromide of potassium has been found efficacious in certain cases. According to Anstie, this remedy is especially useful in young persons who may be supposed to suffer from the nervous consequences of unsatisfied sexual desire. He thinks its efflcacy is rarely secured under a less quantity per diem than ninety grains.

In conclusion, a permanent cure in cases of neuralgia will often depend on the correct appreciation and judicious management of associated morbid conditions, together with hygienic measures to invigorate the general health. Good alimentation is always indicated, and it is desirable that fatty articles of food should enter pretty largely into the diet. Cod-liver oil, if well tolerated, is useful.

In noticing the different neuralgic affections to which, collectively, the foregoing remarks have had reference, I shall adopt the classification of Valleix. This author divides these affections into species, after the different nerves of the body in which the pain is seated; and the localization of pain chiefly or exclusively in the different branches of these nerves he considers as giving rise to varieties of the several species. Thus arranged,

'De la Névralgic lombaire, etc., Archives Générales de Medecine, 1858. 
the more important species are as follows: 1 . Neuralgia affecting the fifth nerve, or trifacial neuralgia. 2. Cervico-occipital neuralgia. 3. Brachial neuralgia. 4. Dorso-inter'costal neuralgia. 5. Lumbo-abdominal neuralgia. 6. Crural neuralgia. 7. Femoro-popliteal or sciatic neuralgia.

The neuralgic affection called angina pectoris has been considered in the section devoted to diseases of the heart. ${ }^{1}$

\section{Trifacial Neuralgia.}

In this species of neuralgia the pain is seated in the fifth or trifacial nerve. Pain and tenderness on pressure are either limited to, or most marked in, certain circumscribed spaces, situated as follows: The point of exit of the frontal branch above the orbit, the upper eyelid, the upper and lateral portion of the nose, the point of exit of the infra-orbitar branch, the lower margin of the malar bone, over the temporal bone, the point of exit of the inferior maxillary branch, and over the parietal bone. It is rare that pain and tenderness are seated in all these spaces, but generally in at least one of the spaces within the range of the three branches of this nerve whence it receives its name, trifacial. In the different varieties of this species, the pain is either limited to, or especially marked in, the different branches respectively of the nerve, viz., the supra-orbital, infraorbital, infra-maxillary, etc. It is rare for the affection to be limited to a single branch, with the exception of that portion of the inferior maxillary which is contained within the inferior maxillary bone.

When the first of the three divisions of the nerve is the seat of the nenralgia, during the paroxysms of pain there is intolerance of light, with redness of the eye on the affected side, and more or less abundant secretion of tears. In this situation the neuralgic affection has been called migraine, hemicrana and brow-ague. Increased heat of the nostril and an abunclant secretion of mucus are effects of the neuralgic affection when seated in the second of the three divisions, and salivation sometimes attends an affection of the third division. The branches which accompany the facial nerve (portio dura) may be the seat of pain, a fact which formerly led to the supposition that the latter nerve was affected. Spasmodic contraction of the muscles of the face on the affected side is an occasional effect ; hence the affection was formerly called tic douloureux, or painful spasm. The affection in the vast majority of cases is limited to the nerve on one side, that is, it is unilateral. The right and the left side are affected in about an equal proportion of cases.

Trifacial ncuralgia in point of frequency ranks with intercostal and sciatic neuralgia. An occasional canse peenliar to this species is caries of the teeth. It is by no means frequently referable to this cause, but that it is so occasionally cannot be doubted. Teeth, however, are often needlessly sacrificed from a supposed causative connection. It is not sufficient to show such a connection that there is tenderness on striking one or more of the teeth. If the neuralgia proceed from this cause, touching the offending tooth or teeth will excite a paroxysm of pain extending into more or less of the branches of the nerve.

Trifacial neuralgia is to be discriminated from inflammation of the membrane lining the supra-orbital and the maxillary antrum, and from syplilitic periostitis. 'The two former of these affections are distinguished by the different character of the pain, its fixed situation, and the occurrence of a discharge into the anterior nares. The latter affection is recognized

- Vide page 358. 
by the fixed tenderness on pressure, the existence of swellings or nodes, the aggravation of pain in the night-time, and other signs distinctive of the syphilitic cachexia. Neuralgia in this situation is often dependent on malaria, and the paroxysms of pain sometimes exemplify the same laws of periodicity which govern the recurrence of the paroxysms of intermittent fever. 'Trifacial neuralgia rarely occurs in infancy. In the majority of cases the age is between 30 and 50 years, and it oftener occurs in women than in men.

This neuralgic affection is curable in the majority of cases. Exceptionally it resists all the therapentical measures which have been considered as applicable to neuralgic affections in general. The branches which are generally affected, in these rebellious cases, are the frontal and the inferior maxillary. As a last alternative, division or excision of a portion of the affected branch is sometimes resorted to. The long-continued and extreme suffering from frequently recurring paroxysms, in some cases, warrants this procedure after other measures have been faithfully tried. Mere section of a nervous branch usually affords only temporary relief. 'The affection returns after the divided extremities have united. A portion of the nervous branch must be removed to prevent or delay reunion. The removal of a portion of the inferior maxillary nerve within the inferior maxillary bone has sometimes proved suceessful after the failure of all other measures; but in a considerable proportion of cases the affection returns sooner or later. In a case under my observation, in which the patient had suffered from paroxysms of intense pain reeurring at intervals of a few moments for the greater part of ten years, the removal of a portion of the nerve by trephining the inferior maxilla, the operation being performed by my colleague, Prof. Hamilton, procured relief for several months, but the pain subsequently returned. Prof. Carnoehan, of this city, has reported several cases in which, by a bold and difficult operation, he removed a portion of the second branch of the fifth nerve beyond the ganglion of Meckel.'

Facial neuralgia, persisting indefinitely, resisting medieation, and characterized by spasmodic movements of the muscles of the face during the paroxysms of pain, is distinguished by Trousseau as epileptiform neuralgia. There are few forms of disease more distressing than this, or more hopeless as regards the prospect of effecting a cure. A case which recently came under my observation exemplified the persistency of this form of neuralgia, and also exemption from suffering for an unusually long period procured by simple division of the nerve. The patient, a man aged about 60 , had suffered intensely from neuralgia affecting the supraorbital branch of the fifth pair on the left side for more than two years. The paroxy'sms of pain recurred at intervals of a few minutes, and were often excruciating beyond description. After trying a great variety of remedies and different systems of practice, the nerve was divided by the late Valentine Mott, and the patient was comparatively free from suffering for the space of three years. At the end of this period, while under treatment for enlargement of the heart and general dropsy, the neuralgia suddenly returned with the same violence as before the operation. Paroxysms were produced by mastication, by movements of the lips in pronouncing words containing labial letters, by touching the face, and they occurred without any exeiting canse. The nerve was divided a second time by my colleague, Prof. J. R. Wood, with immediate and almost com-

Vide American Journal of the Medical Sciences, January, 1858. 
plete relief, which continued until the patient's death, several wecks after the operation.

Facial nenralgia, after having persisted for a long period, sometimes occasions disturbance of nutrition on the affected side of the face, denoted by tumefaction, an increase of adlipose tissue, thickness and firmness of the beard; and it sometimes appears to give rise to pustular or erythematous inflammation. An analysis of one hundred and twenty-eight cases by Notta, in 1854, showed as occasional complications, in addition to those already mentioned, paralysis, either complete or incomplete, of the retina, iritis, glancoma, inflammation and ulceration of the cornea, tumefaction of the tongue on one side, paralysis of the motor oculi, deafness, and anesthesia of the face on the affected side. ${ }^{1}$ Certain of these complications are attributable to paralysis of the vaso-motor nerves. Anstie, who has verified the correetness of Notta's observations, has observed, in addition, grayness of the hair on the affected side, and discoloration of the skin from the presence of dark pigment."

\section{Cervico-0ccipital Neuralgia.}

In this species, the neuralgia affects the posterior branches of the first four of the eervical nerves, and especially the branch of the second cervical nerve, called, from its size and distribution, the occipitalis major.

Painful and tender points denoting this species of neuralgia are situated as follows: Between the mastoid process and the cervical vertebre, over the posterior cervical plexus, on the parietal portion of the head, on the mastoid proeess, and in the concha auris. Shooting pains start from the cervical vertebra just below the oceiput, and extend thence over the posterior and snperior portion of the cranium. Darting pains are sometimes referred to the external meatus auditorius. In cases of neuralgia affecting primarily the cervico-occipital nerves, the pain is apt to extend to the branches of the trifacial distributed to the frontal portion of the head, and in like manner, in cases of neuralgia affecting primarily the superior division of the trifacial, the pain is apt to extend to the branches of the cervico-occipital nerves.

Cervico-occipital ncuralgia is to be discriminated from rheumatism affecting the muscles of the neck, or torticollis. In the latter affection, the pain is limited to the affected muscles, the tenderness on pressure is more diffuse, and not confined to cireumscribed disseminated points; the pain is excited exclnsively by movements of the affected muscles, and, so long as the head remains immovable, there are no painful paroxysms.

\section{Cervico-Brachial Neuralgia.}

In cervico-brachial neuralgia sensitive fibres of the four lower cervical nerves and of the first dorsal nerve are affected. The pain is frequently referred to the shoulder, and it may extend to the neck. It shoots downward along the arm, to the forearm and hand, following the course of one or more of the nervous trunks of the upper extremity. The affection may be limited to either the ulnar, radial, median or musculocutaneous nerve, giving rise to different varieties of the species. Of these varieties the most frequent is neuralgia affecting the ulnar nerve. Pain-

'Archives Générales de Medécine, 1854. Fide, also, Treatise by Anstie.

2 For a report of cases observed and collected by Prof. Henry D. Noyes, with reference particularly to the effect upon the eye of paralysis of this nerve, vide New York Journal of Medicine, Vol. XIV. p. 163. 
ful and tender points in this species of neuralgia are situated as follows: Near the last cervical vertebra, near the acromial end of the clavicle, over the upper part of the deltoid muscle, in the axilla, at the inner condyle of the humerus, near the lower end of the ulna and of the radius.

Pain referable to the cervico-brachial nerves is sometimes an effect of injury of the upper extremity by a wound, a burn, or a contusion. Func. tional neuralgia is to be discriminated from the pain incident to neuroma or other morbid growths affecting the periphery of these nerves, by the failure to discover a tumor in the course of the nerves. Pain referred to these nerves may be symptomatic of vertebral lesions, and, also, of cervical arthritis. In cases of the latter, movements of the head, especially those of rotation, even in the intervals between the paroxysms, are painful. Another feature is, the patient when lying down, is obliged to raise the head with a hand on the occiput, to aroid pain. Rheumatism affecting the muscles of the upper extremity is to be discriminated by the larger extent of surface which is tender on pressure, by the absence of paroxysms of pain except when provoked ky movements of the affected muscles, and the limitation of the pain to the affected muscles.

Herpetic vesicles in patches, constituting Herpes Zoster, sometimes occur in the course of the nerves involved in this affection.

\section{Dorso-Intercostal Neuralgia.}

This species of neuralgia has been considered in the section devoted to diseases affecting the respiratory system, to which the reader is referred. ${ }^{2}$

\section{Iumbo-Abdominal Neuralgia.}

This species of neuralgia, which has its seat in the branches of the five lumbar nerves, is of not infrequent occurrence. It occurs much oftener in females than in males. Pains in this species are apt to be referred to the hypogastrium, and may resemble those of colic. Uterine pains are incident to this species. In males the affection is sometimes characterized by pains extending into the scrotum, accompanied by notable tenderness of the testicle.

Painful and tender points are situated near the upper lumbar vertebræ, a little above the middle of the crest of the ilium, in the neighborhood of the inguinal ring, in the scrotum, and, in the female, on the labia majora. Pains are excited by muscular efforts, the acts of coughing or sneezing, straining at stool, and sometimes by micturition.

Rheumatism affecting the lumbar muscles or lumbago is to be discriminated by the existence of pain and tenderness on both sides, whereas in neuralgia they are generally confined to one side; by the tenderness being diffused over the affected muscles and not limited to certain isolated points, and by the absence of paroxysmal pain except when produced by movements of the affected muscles.

In the case in which pain extends to the uterus, inflammation of this organ, metritis, and carcinoma are to be excluded. In uterine neuralgia the os uteri is tender on pressure, and the tenderness is either limited to, or most marked on, one side. The pains in this species of neuralgia may simulate those caused by the passage of a calculus along the ureter or nephritic colic. The pains in the latter are more intense and persisting; they are suddenly developed, whereas the development of a neuralgic 
affection is generally gradual, and the signs of gravel afforded by an examination of the urine are wanting in cases of neuralgia.

\section{Craral Neuralgia.}

This species of neuralgia is rare. It is characterized by shooting pains in the direction of the trunk of the crural nerve and its branches, and by painful and tender points in the groin, the inner aspect of the thigh, knee, and ankle.

\section{Sciatic Neuralgia.}

Sciatic neuralgia, or, as it is commonly called, sciatica, in frequency ranks with intercostal and faeial neuralgia. It occurs in males oftener than in females, the relative proportion of cases being as three to two. It very rarely oecurs before the age of twenty and rarely after sixty. It is sometimes symptomatic of pregnaney, of enlargement of the rectoperitoneal lymphatic glands, of aceumulation of feces or foreign substances in the sigmoid flexure of the colon, and intra-pelvic tumors, but, in the great majority of eases, it is a functional affection.

As in the foregoing neuralgie affections, shooting pains, in paroxysms or exacerbations, extend along the trunk and branches of the affected nerve. In indicating the course of the pain, the patient oft in delineates with accuracy the anatomieal distribution of the sciatic nerve. The character of the pain is sometimes described as burning, and sometimes as a sensation of coldness; the patient feels as if a eurrent of hot or cold liquid were flowing in the course of the affected nerve. A continued, contusive pain, more or less intense, exists in the larger proportion of cases. The latter, as in the other species of neuralgia, is referred expressly to the circumseribed spaces which are tender on pressure.

Painful and tender points in this neuralgic affection are found in more or less of the following situations: On the sacrum, over the sacro-iliac junction, about the middle of the crest of the ilium, at the sciatic notch, behind the trochanter major, on the thigh along the track of the nerve, in the popliteal space, on the external border of the patella, over the articulation of the fibula with the tibia, where the nerve passes around the fibula, over the lower and posterior part of the external malleolus, on the dorsum and outer portion of the sole of the feet. The points situated at the sacro-iliac junction of the troclianter major, the head of the fibula, and the external malleolus, are those most frequently painful and tender. Pressure with the finger upon some of the foregoing points may not only be more or less painful, but excite a paroxysm or exacerbation of pain extending to other parts in the course of the affected nerve.

The intensity of the pain in this, as in other species of neuralgia, varies much in different eases! The movements of the affected limb, in some cases, occasion such an amount of suffering that the patient remains in bed keeping the limb as nearly immovable as possible. In other cases, the patient is able to walk, but with more or less difficulty; pain is felt especially when the weight of the body falls upon the affected limb, and relief is found in the use of a cane. Sometimes difficulty in walking is only felt in beginning to walk, and after a short time the patient walks with ease; the suffering is occasionally relieved by exercise. In severe cases muscular twitchings and cramps accompany the paroxysms or exacerbations of pain.

Sciatic neuralgia rarely, as compared with the other species, more espe- 
cially facial neuralgia, exemplifies, in the recurrence of paroxysms, the law of periodicity. Like other neuralgic affections, its duration is very variable. As a rule, in favorable cases, it persists for several weeks, disappearing, as it is nsually developed, gradually. In the vast majority of cases, sooner or later it ends in recovery, but, in some cases, it continues indefinitely in spite of all remedial measures. If it continue for a considerable period so severely as to lead the patient to avoid, as much as possible, movements of the affected limb, the repose of the muscles may render them for a time incompletely paralyzed. The muscles, under these circumstances, become flaceid and diminished in volume.

The diagnosis of sciatic nemralgia involves its discrimination from muscular rheumatism and from the affection of the hip-joint commonly known as morbus coxarius. Muscular rheumatism here, as in other situations, differs from neuralgia in the limitation of pain to the affected muscles, in the diffusion of tenderness over a considerable space, viz., a space corresponding to the number of muscles affected, and in the fact that parox$y$ sms of pain are excited exclusively by movements of the affected muscles.

Morbus coxarius is distinguished by the pain in the hip-joint produced hy pressure upon the great trochanter and by flexion and extension of the thigh. Moreover, febrile movement, general debility, emaciation, etc., in conjunction with the local symptoms, point to the existence of chronic inflammation within the joint.

The name sciatica, as commonly used, is applied to cases in which pain is referred to sacral nerves, although the sciatic nerves may be unaffected. Pain, of a neuralgic character, in the nerves composing the sacral plexus, is often symptomatic of lesions within the pelvis and abdomen, such as aneurismal, carcinomatous or other tumors, enlargement of glands, etc.; also, of lesions of the sacrum and vertebral colnmn, and those occurring within the spinal canal. Here, too, as in other situations, it may be dependent on neuritis. This is to be inferred from the speedy oceurrence of paralysis and atrophy of the muscles to which the nerves are distributed.

The coexistence of two or more of the foregoing different species of neuralgia, or their successive occurrence, is frequently observed. Proximate or inosculating nerves are apt to be simultaneously or successively affected. Thus, trifacial and cervico-occipital neuralgia are not infrequently associated, and this is true of sciatic and crural neuralgia. A cure or spontaneous cessation of neuralgia in one situation is apt to be followed by the development of the affection in another situation. These facts go to sliow the dependence of neuralgia on constitutional or general morbid conditions.

\section{Dermalgia.}

Neuralgia sometimes appears to be limited to the skin. Cases are occasionally observed in which pain is felt within a circumscribed space, or within circumscribed sjaces, on the surface of the head, trunk, or extremities, together with notable tenderness on pressure, the nervous trunks being free from pain or tenderness. This form of neuralgia has been called dermalgia. The hyperæsthesia of the abdomen occurring in females and simulating peritonitis appears in some cases to be a neuralgic affection of the periphery of the sensory nerves in that situation. Beau, of Paris, has described the affection distinguished as dermalgia.'

1 Archives Générales de Médecine, 1841. 


\section{Myalgia.}

Neuralgia may affect especially or exclusively sensory nerves in museular organs. The affection thus situated is distinguished as myalgia. 'The muscles of the back are especially apt to be the seat of the affection. The local symptoms in cases of myalgia are analogous to, if not identical with, those of museular rheumatism ; in faet, myalgia and muscular rheumatism are often eonfounded, and there is reason to believe that in certain of the cases of so-called muscular rheumatism, the affection is essentially neuralgic. I shall consider muscular rheumatism in the section devoted to general or eonstitutional affections, and in that connection shall again refer to myalgia.

\section{Cephalalgia-Headache.}

Pain in the head is a symptom oceurring in many affections, such as, active cerebral congestion, eerebral meningitis, cerebritis, all the essential fevers, acute dyspepsia, syphilitic periostitis of the head, intra-cranial tumors, etc. ete. When not symptomatic of some affection, that is, oeeurring idiopathieally, it is frequently due to neuralgia affecting either the cervico-oecipital nerves or the supra-orbital division of the fifth pair. Pain limited to one side of the head, or hemierania, is due to neuralgia affecting the nerves just named. Aceording to Valleix, idiopathic eephalalgia or headache is in all eases resolvable into hemicrania. This opinion does not seem to me to be correct. In eertain cases the pain does not follow the direetion of these nerves, it is not limited to one side of the head, disseminated tender points may not be found, and in its behavior the affection differs from neuralgia affeeting these or other of the nervous trunks and branches. Henee, eephalalgia or headache may denote a functional neuropathic affection distinet from hemicrania. The seat of the affection when confined to one side, in eertain eases, has been supposed to be the branches of the trifacial nerve which are distributed to the dura mater. It has also been supposed that the sensory filaments entering into the composition of the brain may be the seat of neuralgia.

Cephalalgia is extremely common. It occurs in paroxysms, lasting usually from 12 to 24 hours, in some cases being of shorter duration, and occasionally continuing for two or three days. There are few persons who do not oceasionslly experience an attack after childhood or adult age, and there are very many who are subject to attacks recurring sometimes at regular, but usually at irregular, intervals for many years, or during lifetime. A host of persons suffer from it who either do not consider it of sufficient importance to resort to medical treatment, or, having found medical treatment ineffectual, resign themselves to it as an irremediable malady.

The pain in different cases, or in different attaeks in the same case, differs much in degree. An attack is often not severe enough to prevent habitual oceupations, but in some instanees the intensity of the pain is such that the patient is unable to be about, and perhaps is obliged to take to the bed. The pain is sometimes referred to the forehead, sometimes to the occiput, and sometimes to the whole head. The character of the pain, as described by patients, differs. It is sometimes sharp or lancinating, like the pain in neuralgic paroxysms; in other cases it is a dull, heavy pain. Patients sometimes say that they feel as if the head would burst. Firm pressure upon the head, especially over the temples, often affords momentary relief. Frequently rubbing or shampooing the sealp with considerable force diminishes the pain. There is frequently an abnormal 
sensitiveness to light and sounds, and in severe attacks the patient seeks perfect quietude in a dark room away from all noise. The appetite during the attack is either impaired or lost. The odor of food is often disagreeable. Nausea and vomiting occur in a certain proportion of cases, and then it is common to call the affection sick headache. Without nausea or vomiting, the affection is commonly known as nervous headache. The face is in some cases flushed, the eyes suffused, and the temperature of the head raised; in other cases, especially if nausea and vomiting occur, the face is pallid and the head may be cool. Free vomiting; although the stomach may not contain any aliment, is sometimes followed by relief.

Accompanying the foregoing local symptoms, is more or less general debility or prostration. There is rarely any febrile movement; the pulse is generally small and feeble, and the surface of the body cool. Occasional shiverings are not uncommon. Disturbed action of the heart, or palpitation, is not uncommon during the attack.

The attack gradually passes off, sometimes with the occurrence of free evacuations from the bowels, or an abundant secretion of urine, or a slight perspiration. The attack is apt to be developed in the morning, pain being experienced on awakening from sleep, and it is apt to continue until after sleep is obtained on the following night. Persons subject to the affection are able, sometimes, to predict its advent by a feeling of lassitude, depression of spirits, or a sense of indefinite discomfort. A fter the attack has passed off, there is frequently, for some time, a sense of soreness in the head, with more or less general debility. Often, however, the mental faculties are unusually clear and spirits buoyant after recovery from an attack.

The affection involves a constitutional predisposition. Persons are subject to periodical attacks of headache, as to asthma or epilepsy. The predisposition existing, various exciting causes may determine an attack, such as mental excitement, deprivation of sleep, bodily fatigue, exposure to the sun's rays, and dietetic imprudences. Not infrequently attacks occur withont any obvious exciting causes. Females are more subject to the affection than males, and it is apt to occur at the menstrual period. The predisposition varies in degree in different cases, as shown by the frequency or rareness of the attacks. The predisposition frequently ceases after the age of 50 or 60 ; persons who have experienced attacks, more or less frequently, from adult age up to the periods of life.just named, thereafter are exempt from their recurrence. This may be stated as a rule, but, unhappily, there are not a few exceptions.

The affection is neuralgic in its character, but it is difficult to determine its precise seat. It is a reasonable supposition that it involves a toxical agent in the blood. Cephalalgia, it is well known, is produced by different toxical agents, viz., opium, alcohol, strychnia, quinia, and the accumulation of urea. Assuming the correctness of this supposition, the nature and source of the toxical agent, or agencies, are unknown.

Periodical headache, as regards successful treatment, belongs among the opprobria of medical art; yet, in not a few cases, much benefit may be derived from treatment. The treatment embraces measures to be employed at the time of an attack, and during the intervals.

If patients be not affected unpleasantly by opiates, an attack may be sometimes warded off, or abridged, or its severity lessened, by a full dose of opium in some of its forms, or of its alkaloids, morphia or codeia. The carbonate of ammonia is sometimes successful in affording relief. A saline purgative, taken at the commencement of the attack, is in some cases an effectual remedy. The bromides given in full doses are some- 
times efficacious. This statement will also apply to the hydrate of chloral, in doses of fifteen grains, repeated once or twice after an hour's interval, if relief be not obtained.

Various palliative measures may be resorted to with advantage. The inhalation of a little of the vapor of chloroform is sometimes an efficient palliative. Evaporating lotions to the head - alcohol, spirits, vinegar, ether-in some cases aftord marked relief. In some eases a towel or napkin wrung out in water as hot as can be borne, and wound around the head, is more efficient than cold applications. Warm stimulating pediluvia are useful. Strong coffee or tea affords marked relief in some cases. It is stated that the application of the galvanic or the electro-galvanic current will often succeed in breaking up an attack of headache.

During the intervals, remedies which are sometimes useful, by way of prophylaxis, are, nux vomica or strychnia in small doses, arsenic, small doses of quinia, belladonna, and the preparations of zinc, more especially the valerianate. These remedies may be tried in succession. Ifygienic measures are important. In general terms, the liability to attacks is less, the more the laws of health are observed. A voidance, as far as possible, of everything which experience shows, in individual cases, to act as exciting causes, is an important part of the management.

\section{Vertigo.}

Tertigo, occurring under certain cireumstances, may be appropriately considered as a neuropathic affection. A person apparently in good health, or, at all events, without any definite malady, suddenly becomes giddy, surrounding objects appear to be turning round, or the person seems himself to be performing movements of gyration. The sensation is like that produced by a long-continued series of rapid rotatory movements of the body. If the vertigo be moderate or slight, it simply renders the person unable to walk steadily; be recls like a drunken man. In a greater degree, walking is impossible, and there is inability to stand without support. It is accompanied by a sense of prostration which is frequently due, in part, to a feeling of apprehension oceasioned by the attack. Generally nausea and sometimes vomiting occur. An attack varies much in duration. It may last for a few moments, or it may continue for hours and days. After the attack passes off, there may follow more or less pain in the head, or there may be freedom from any ailment.

Successive attacks oeeur at variable intervals. In some cases, days, weeks, or months may intervene. In other eases, for a certain period, attacks are liable to occur frequently from slight exciting causes, such as any intellectual effort, sudden muscular exertion, looking upward, a bright light, etc. The patient may be comparatively free from vertigo while in the recumbent posture, but experience it whenever he attempts to sit up or stand.

These attacks generally give rise to great apprehension. The patient fears that apoplexy, paralysis, or a fit of some kind is impending. If his fears be not removed by positive assurances from the physician, he is apt to become despondent; he is afraid to walk or to be left alone; he avoids all public assemblages lest something may happen to him to excite disturbance, and he may pass months or years in a state of anxiety, the more to be regretted because it is quite gratuitons.

It has occurred to me, as doubtless to most physicians, to meet with numerous examples of the affection thus briefly sketched. I have made notes of a number of cases which came under my observation many years 
ago. I have never known it to prove a premonition of apoplexy, paralysis, epilepsy, or any grave affection. The affections just named are not, as a rule, preceded by attacks of vertigo. The physician is fully warranted in giving positive assurances that the attacks are of no importance beyond the inconvenience which they occasion. These assuranees will do much good, not only by removing needless apprehensions, but in the way of effecting a cure, for the mental anxiety occasioned by the attacks increases the liability to their recurrence.

It is important, with reference to proper management, for the physician to appreciate the pathological character of this affection. It is not an effect or a symptom of cerebral congestion. The measures of treatment based on such a supposition will prove, not only ineffectual, but injurious. It is not an effect or a symptom of disease of the heart. Coexisting disorder of the heart's action, which is not uncommon, might lead to the suspicion of such a pathological connection. It proceeds from a functional morbid condition of the brain, and, like the other affections included among the nemroses, this, with our present knowledge, is all that is to be said respecting its pathological character.

In a certain proportion of cases, the attacks appear to be incident to dyspepsia. This causation, however, is certainly not as constant as it is regarded by 'Trousseau, who calls the affection stomachal vertigo (vertigo a stomacho laeso). Long-continued overtasking of the mental faculties appears to give rise to it in some cases. Among the cases which I have noted are two in which it was referable to the cause just stated. In these two eases, the persons affected were distinguished members of the medical profession. One of the persons referred to was the late lamented John Ware. Some ten years before his death, for sereral months, any intellectual effort, even reading a book, occasioned an attack of vertigo. Absence from professional duties, a voyage to Europe, and life in the country, effected a cure. Of the cases which I have noted, in this one only did either apoplexy or paralysis occur afterward; but, in the case of Dr. Ware, the apoplectic seizure occurred ten years after recovery from the affection under consideration. In most of the cases the patients are still living and well. The other person referred to was the late Amariah Brigham. Many years before his death he was, in like mamner, for several months, subject to attacks of vertigo, which were especially excited by any intellectual effort. He died of dysentery.

I have been led to attribute the affection, in some cases, to excessive venery. The immoderate use of tobacco appears to be the cause in some cases. It is sometimes incident to the debility which remains after convalescence from a severe disease, for example, typhoid fever. Cases are observed in hospital and dispensary practice, and in some of these cases the affection is attributable to deprivations, hardships of various kinds, and mental anxiety.

The treatment involves, as the first and most important measure, the removal of the cause or causes. As already stated, measures addressed to cerebral congestion, viz., bloodletting, counter-irritation, purgation, and reduced diet are hurtful. On the contrary, tonic remedies, a nutritious diet, and other hygienic means of invigorating the general health, are indicated. The practitioner should bear in mind the importance of assuring the patient positively of the absence of all danger. This assurance will sometimes do much toward effecting a cure. Generally a cure is soon effected, but in some cases the affection continues for a long period. 


\section{CHAPTER VIII.}

\section{Chorea. Anomalous Muscular Movements. Epilepsy.}

CERTAIN of the neuroses are charaeterized by disordered muscular $U$ movements. Of the affections embraced in this group, the more important are, chorea, epilepsy, hysteria, tetanus, and rabies or hydrophobia. The first of these (chorea) may be reckoned among the affections occurring especially in early life, and is treated of fully in works devoted to the diseases of children. Hysteria very rarely affects males, and belongs, therefore, among the diseases peculiar to females. Tetanus, in the great majority of cases, is a traumatic affection, and, as such, falls within the provinee of surgery. A brief space only will be accorded to these three affections in this work. As regards the other affections named, directing attention chiefly to points which are of practical importance, they will not claim very extended consideration.

\section{Chorea.}

The affection called chorea, chorea sancti Viti, or St. Vitus's dance, is characterized by irregular contractions of more or less of the roluntary muscles, giving rise to movements which are either involuntary or not under the dircetion of the will. The manifestations of the affection are usually at first limited to a portion of the body, to one of the upper extremities oftener than elsewhere, and sometimes they are confined, for a time, to the fingers of one hand. The movements increase and progressively extend to both upper extremities, the muscles of the face, and frequently to the lower extremities and to the trunk. The appearances consist of frequently recurring or unceasing movements of the parts affected, producing in the face varied and ludicrous grimaees, and in the extremities and trunk grotesque contortions and fantastic gesticulations.

The spectaele afforded by the manifestations of this affection is comical, and it is difficult to realize that it is not a performance for the amusement of observers. The condition, however, if the affection be severe, is truly distressing. The incessant aetivity of the muscles induees fatigue. The patient may be unable to perform the voluntary aets which the wants of the body require. $\mathrm{He}$ is completely dependent upon others. Speech may be impaired or lost. Locomotion may be impossible. The isolation is sometimes more complete than in cases in which a considerable portion of the body is paralyzed or several senses abolished. Want of ability to direct and harmonize voluntary movements is a feature of this affee. tion. The condition, in this respect, is analogous to that in progressive. locomotor ataxia. Acts of volition give rise to the irregular movements ; but movements, to a greater or less extent, take place without the stimulus of volition, that is, they are involuntary. In the latter respect ehorea differs from locomotor ataxia, the movements in ataxia being never wholly involuntary. The movements in chorea are usually suspended during sleep, but they sometimes continue, althongh always more or less diminished. They are increased generally when the patient is conscious of being observed, and under any emotional excitement. The movements 
are frequently more marked on one side of the body, and this is generally the left side. They are sometimes confined to this side, and the affection is then called hemi-chorea. The choreic manifestations are confined to the voluntary muscles. The sphincters are rarely affeeted; and this is true of the muscles concerned in respiration, inclusive of the laryngeal muscles, as, also, those of the pharynx.

The affection is not accompanied by febrile movement. If this exist, it is due to an intercurrent or coexisting affection. The appetite is usually more or less impaired and the bowels are constipated. Anæmia often coexists. Irritability of temper is a notable feature. The mental faculties are weakened. The grimaces give to the physiognomy an appearance of idioey, and, if the affection become ehronie, patients fall into a condition of apparent imbecility. The mind fails, in these cases, from the deficiency of exercise of the mental faculties. If the speech be lost, it is difficult to estimate the amount of intelligenee which is preserved. The power of the affected muscles is more or less diminished, and this impairment of power, or ineomplete paralysis, is most marked on the side most affected with the chorea. Anæsthesia of the surface, on one or both sides of the body, is sometimes observed; oftener, however, there is hyperæsthesia which is especially marked in the tract of nerves. Tenderness along the spinal column exists in a large proportion of cases. There is an exaggerated suseeptibility to reflex excitations, and the electrical excitability, of both the muscles and skin, is abnormally increased. Pressure upon tender points over nervous trunks excites the ehoreic movements.

Endocardial murmurs are not infrequently found in cases of chorea. If these be aortic or pulmonie, accompanying the first sound of the heart, they are to be regarded as inorganic or anæmic murmurs. They do not constitute evidenee of endocarditis. Nor is a mitral murmur, in this affection, evidence of endocarditis. I have observed a mitral systolic murmur during the continuance of chorea, and the disappearance of the murmur after recovery from the chorea. This murmur may perhaps, as has been conjectured, depend on temporary insufficiency at the mitral orifice caused by irregular contractions of the papillary muscles.

As one of the neuroses, this affection, of course, has no anatomical characters. In cases which have proved fatal, morbid changes, not uniform in character or seat, have been found in the venous centres. Minute embolic obstructions in the corpus striatum have been observed by J. Hughlings Jaekson and Bastian in hemi-chorea. It seems to me fair to conclude that the different morbid appearances found after death are either coincidences, or developed secondarily as effects of the disease.

The causation of this affection is obscure. It is common to suspect the presence of worms in the alimentary canal, but there is very little evidence showing that the affection ever depends on worms. In some cases the exciting cause appears to be a fright. It has been observed to follow - a violent fit of anger. Numerous observers have noticed an apparent connection with rheumatism, and a French writer, M. Sée, has been led, by extended researches, to the conclusion that the affection is to be considered as essentially rheumatic.' Facts, however, do not warrant this conclusion. The most to be said of the connection with rheumatism is that children who have had this disease are apt to become affected with chorea. ${ }^{2}$ There is abundant reason to believe that the development of the

1 De la Chorée, etc., Mém. de l'Acad. de Méd., 18:0, and Medico-Chir. Review, Jan. 18\%2.

2 For a series of articles by Dr. Henri Roger, Physician of the Children's Hospital of Paris, in which the writer deduces from a collection of cases the conclusion 
affection is favored by anæmia. Oecurring sometimes in pregnancy, the latter is to be considered a cause. When thus occurring it has been called chorea gravidorum.

Chorea occurs especially between 10 and 15 years of age. It rarely occurs under 6 years of age. It may oceur at any period of life. Henri Roger has reported a case oceurring in a female aged 84 years. Graves observed a well-marked case in a male aged 72 years. Girls are more liable to it than boys, the ratio being as three to one.

The duration of the affection varies from a few weeks to several months. The average duration is from two to three months. In the vast majority of cases the termination is in recovery. After a variable duration, the affection tends intrinsically to this termination. In a very small proportion of cases it becomes chronie, and is incurable. Life may be destroyed by intercurrent affections, which, in general, do not suspend the choreic phenomena. A fatal result may take place without any superadded disease, the patient being worn out by prolonged irritation, loss of sleep, and inanition. The incessant movements sometimes give rise to contusions and excoriations which may lead to erysipclas and abseesses, and these may contribute to a fatal result. Occurring in pregnancy it is apt to lead to abortion or premature delivery, and, hence, is not without danger. Of thirty-one cases collected by Jaccond four ended fatally.

Relapses are apt to occur. Of 158 cases analyzed by Sée, they oceurred in 37 . In 13 of these cases the affection recurred twice, and in 6 cases there were four attacks. The relapses occur after intervals varying from a few months to two or three years. As a rule, the relapsing are shorter than the primary attacks.

A diversity of remedies have been advocated by different authors as effecting a cure of this disease. In general, when different methods of treatment of any disease are found to be successful, it is a fair inference that the disease ends favorably from an intrinsic tendency. This is undoubtedly true of chorea. Of the diverse remedies indicated, none can be relied upon for promptly arresting the course of the disease, and it is doubtful if any exert a special curative agency. It by no means follows, however, that much benefit may not be derived from judicious treatment. The greater part of the remedies are doubtless more or less useful, and the physician is to exercise judgment in adapting them, severally, to individual cases, and in employing them successively in the same ease.

Rational indications, in the first place, are to be sought for in coexisting disorders or morbid conditions. If there be symptoms, aside from the chorea, pointing to rheumatism, remedies addressed to this condition are called for. Anæmia, in a large proportion of cases, calls for appropriate treatment. Constipation, if present, claims attention. Purgative remedies are among those advocated as efficacions in curing the disease.

Of remedies, other than purgatives, which experience lias shown to be useful, the more important are, arsenic, the carbonate and prussiate of iron, zine, strychnia, opium, eannabis Incliea, and various antispasmodics. Of the efficacy of arsenic there is abundant and strong testimony. Fowler's solution is the most eligible preparation. Commencing with three

that there exists a pathological connection between choren, rheumatism, and endocarditis, vide Archives Générales de Médecine, Janvier, Février, Mars, et Avril, 1868. Dr. John W. Ogle of London, has reported 16 fatal cases of chorea will the morbid appearances found after death, in the British and Foreign Medico-Chirurgical Review, Jan. 1868. In ten of these cases fibrinous deposit or granulations existed upon the valves or some portion of the lining membrane of the heart. 
or four drops of this preparation, three times daily, the doses should be gradually increased until the characteristic effects are observed. Of the preparations of iron, the carbonate and prussiate have been supposed to exert a curative effect aside from their usefulness with reference to coexisting anæmia. Of the preparations of zinc, the oxide has been especially advocated as curative in this affection. Strychnia is advocated especially by 'Trousseau, who prefers the sulphate dissolved in syrup. He commences the treatment with a very small dose, viz., one twenty-fifth of a grain once daily, and increases the dose very gradually. Hammond gives to this remedy a decided preferenee over all others. Opium is advocated by Troussean as a curative remedy. He gives it in a full dose, producing slight narcotism, which is to be maintained for several days. He affirms that this method of treatment is often extraordinarily successful. ${ }^{t}$ To the efficacy of cannabis Indica, testimony is borne by Corrigan, Walshe, and Prof. D. Humphreys Storer, of Boston. Cimicifuga, in some parts of this country, has been a popular remedy for ehorea, and its value is attested by numerous eminent physicians. Of antispasmodic remedies, those whieh have been found especially useful are valerian, assafoctida, and eamphor. Keeping the patient daily under the influence of chloroform for half an hour or more has been found serviceable. This measure is hardly to be advised save in cases in which the movements are unusually violent and constant. The application of ether spray to the spine, for four or five minutes at a time, daily, or on every alternate day, as recommended by Dr. Benj. Richardson, has been found efficacions by Hammond, Jaccoud, and others. The testimony respecting the usefulness of electricity is conflieting. Benedikt and Jaccoud claim signal success by galvanization of the spine. Beard and Rockwell have found general faradization uniformly successful. The result of galvanization of the spine in a few cases was unsatisfactory in their hands, and also in the experience of Meyer. Hammond states that he has used both the primary galvanie and induced current in sixty cases, and that, in his opinion, they are inefficacious, except when paralysis is associated with the chorea. This discrepancy may be, perhaps, in a measure explained by differences as regards the mode of employing both the direct and indueed eurrent; and also by differences as regards the period of the disease when the treatment was entered on, bearing in mind that this disease may be said to have a self-limited duration. The bromides are advised by Jaecoud, Hammond, and others. In my experience they have failed to meet the expectations which I had been led to form of their usefulness in this affection. Prof. A. Clark has reported a case of unusual severity, the affection having existed for four weeks, in which whiskey administered in intoxicating doses was followed by immediate relief, and under the subsequent use of tonics, by a rapid recovery. ${ }^{2}$

Hygienic measures are certainly not less important than remedies. Alimentation as nutritious as possible is desirable. Daily exposure, and, if practicable, exercise in the open air, are highly important. The cold sponge bath is useful, provided it be not dreaded by the patient, and it be followed by brisk reaction; the shower bath, with the same provisions, is admissible. On the other hand, the tepid bath, as a palliative measure, is sometimes highly useful. In the Children's Hospital in Paris for the last 20 years much reliance has been placed on gymnastic exercises for

i Vide Traité de Thérapeutique, etc., par Trousseau et Pidoux, Paris, 1854.

2 American Medical Times, August 2, 1862. 
the cure of chorea. Of course, these are practicable only in the cases in which the ability to co-ordinate movements by the will, although more or less impaired, is not lost. The exercises eonsist of systematic movements performed under the direction of assistants, and enlivened by music. ${ }^{\prime}$ After the choreic movements have ceased, more or less inability to co-ordinate movements, together with museular weakness, remains, and these effects of the disease are to be removed by exereise. The power of again clireeting the muscles is to be acquired as in infancy, but it is acquired rapidly.

\section{Anomalous Muscular Movements. Tremor and Local s pasms.}

Certain ahnormal muscular movements are considered, incorrectly, as denoting varieties of chorea. Persons, sometimes, without motive or purpose, impelled apparently by an irresistible impulse, perform extraordinary acts. One of these is leaping suddenly and violently, and sometimes jumping upon chairs or tables. Such movements have been considered as denoting a variety called chorea saltatoria. A patient subject to epilepsy was brought to me for examination, a young man aged about 20, who, at variable intervals, had paroxysms of jumping vigorously for a few seconds. He deelared he was unable to avoid these movements. In other cases there is a propensity to run, and the patient, unexpectedly, when walking, rushes forward with a rapid pace for several minutes. These movements have been considered as denoting another varicty, called chorea festinans vel procursiva. Rotating and vibrating movements are performed in other cases. Moving the head and body alternately backward and forward, constituting what has been called Salaam convulsions, belongs in the same category.

These movements, although abnormal, are systematic and regular, and proceed altogether from the action of the will. They denote a species of mental aberration. They are manifestations of a delirious volition, resembling in this respect certain of the convulsive movements in hysteria. They are sometimes connected with cerebral disease, but in most cases they are purely funetional. In some cases it is undoubtedly true that patients are unable to resist the impulse which impels to the abnormal acts, but a morbid propensity to excite wonder and interest doubtless enters into the rationale in some cases.

The treatment embraces remedies and hygienic measures to invigorate the nervous system, together with efforts to overcome by the will the propensity which gives rise to the abnormal movements.

Sudden, brief movements, directed by a delirious volition, belong among the multiform epileptoid attacks, and will presently be referred to in that connection.

Allied to the cases just referred to are those in which twitching of certain parts becomes habitual. Thus some persons acquire the habit of jerking the head, raising the shoulders, or making other motions, especially under mental excitement. In many, if not most, of these eases, the habit may be overcome by persevering efforts before it beeomes confirmed. Convulsive movements of certain of the muscles of the faee, producing sometimes notable grimaces, may be involuntary, and are closely allied to chorea. These are apt to become confirmed and irrcmediable. Spasm of

' For details concerning gymnastic exercises, vide papers by M. Sée, and by M. Blache in Mémoirs de l'Acad. de Médecine, Paris, 1855. For a summary of these details, vide Meigs on Diseases of Children. 
the eyelids is called blepharospasmus. When the angles of the mouth are raised, the peculiar expression is significantly styled risus caninum, or the sardonic grin. The convulsive movements or spasms of the inuscles of the face, called by Romberg mimic or histrionic, may be either clonic or tonic. 'They are oftenest the former. The involuntary grimaces which are sometimes produced, occasion for the patient much mortification. The clonic spasms come on in paroxysms which generally last but an instant. They are apt to occur especially under mental excitement. Sometimes they interfere with mastication and articulation. They are almost alway's unilateral, and they may be arrested by pressure on the facial nerve. Involuntary or spasmodic movements of the head, with or without grimaces, characterize some cases. 'In a case at Bellevue Hospital, there was violent jerking of the head from side to side at intervals of a few seconds, and with these there were rapid lateral oseillations of the eyeballs. Aside from these symptoms, there was no evidence of cerebral disease. They followed a debauch. The patient soon left the hospital, the movement continuing. In another case, the patient moved the head spasmodically to the left side at short intervals, with some contraction of the facial muscles on that side. Notable improvement, in this case, took place under the use of the bromide of potassium.

A spasmodic affection of the muscles engaged in the act of writing has been called chorea scriptorum, or writer's cramp. Whenever the act of writing is attempted by one laboring under this affection, the flexor muscles especially, but sometimes the extensors of the thumb and fingers, either become rigidly contracted or contract irregularly, and guiclance of the pen is impossible. The affection proceeds from undue exercise of the affected muscles in writing. Timely and entire rest of the affected muscles may effect a cure. When the affection becomes confirmed, it continues permanently and is irremediable. Mechanical appliances sometimes succeed in measurably overcoming this difficulty. It is a curious fact that all other acts may be performed by the fingers and hand without any abnormal movements being excited.

Persons who employ certain muscles constantly in other acts than writing are sometimes similarly affected. Thus, pianists are no longer able to play, printers cannot compose, tailors and seamstresses lose the power of guiding the needle, and turners are unable to move the lathe with the foot, other movements, calling into exercise the same muscles, being performed without difficulty.

Painful contraction of muscles, irrespective of affections of which this is a symptom, such as tetanus, epiclemic cholera, etc., and not produced by any obvious cause, is known as cramp. Some persons are liable to suffer much from cramp of the muscles of the legs or feet, occurring especially at night. A method of treatment proposed by Dr. Bardsley, of Manchester, England, is said to be generally successful. This consists of sleeping on an inclined plane-the bed being twelve inches higher at the head than at the feet.

Under the name essential or idopathic spasm of the muscles of the extremities (contracture essentielle ou idiopathique des extrémités), French writers describe an affection characterized by tonic contraction occurring with either intermissions or marked exacerbations. It occurs especially in young subjects between the age of 17 and 25 , and in children from one to three years of age. Females are subject to it more than males. The puerperal state and lactation predispose to it. It is observed as a sequel of certain diseases, such as the continued or eruptive fevers, articular rheumatism, and cholera. In children it appears to be connected with 
dentition, intestinal worms, and gastric disorders. The spasm or cramp affects first the muscles of the upper extremities, and especially the flexors of the forearm. It extends generally to the lower extremities, and here the extensors are the muscles most apt to be affected. The hand is often firmly closed, the fingers concealing the thumb which is in close apposition to the palm, and the flexion may embrace both the phalanges and the metacarpal bones; or, more rarely, the fingers are separated and partially flexed so as to give rise to the claw-like appearance. Sometimes the position of the fingers is like that in the act of writing. Exceptionally, the extensors of the wrist are affected. The contractions are powerful. Generally they are bilateral, but they are sometimes confined to one side. In some cases the spasm extends to the muscles of the arm, shoulder, thigh, trunk, and neck, together with those of the jaws. In these cases the affection simulates tetanus. It has been called intermittent tetanus. In tetanus the trismus is primary as regards the muscles affected, whereas, in this affection, it is secondary. Moreover, in this affection, the associated symptoms denoting gravity of disease are wanting. The intermissions sometimes observe the laws of periodicity governing the different types of intermittent ferer. The affection belongs among the neuroses, that is, it is a functional affection, the duration varying generally from one to three weeks. Relapses are apt to occur. The spasm sometimes leads to paralysis of the muscles which are affected. The affection tends to a farorable termination. Cases, however, have been observed in which a fatal result has been due to paralysis, following spasm, of the muscles concerned in respiration, and to spasm of the glottis. The indications for treatment relate to the pathological conditions with which it is associated. Quinia in full doses is indicated, if the affection be intermittent. Belladonna, opium, or the hypodermic injection of morphia may be resorted to for palliation of the pain and spasm.

Tremor of certain parts, caused by alternate contraction and relaxation of muscles in rapid succession, is a symptom of certain lesions of the nervous centres, especially of multiple or disseminated sclerosis of the brain and spinal cord. It is not infrequently incident to advanced age; and it is then observed especially in the upper extremities, but, in some cases, constant lateral motions of the head take place during waking hours, and they do not always cease during sleep. The senile trembling, as it is called, is irremediable. It involves degenerative changes at the nervous centres. Tremulousness, especially of the hands, occurs in early and middle life, as a result of the abuse of alcohol, the habitual use of opium, sexual excesses, and other causes which tend to depress or disorder the nervous system. Under these circumstances it may be purely functional. The trembling sometimes extends to the lower extremities and to the trunk. Paralysis agitans, or shaking pasly, is a name used to denote this affection. The affection, however, does not necessarily involve more diminution of muscular power than is incident to the general debility which may exist. I have met with several cases of unilateral tremulousness, that is, confined to the upper and lower extremity of one side. In the cases which I have seen, the muscular power of the limbs affected was not much diminished, and the general health was good. In one of these cases the tremulousness was diminished by bandaging the limbs, and by applying to them the tincture of aconite, small doses of strychnine being also given.

The treatment of muscular tremulousness consists in removing, if practicable, the cause or causes, and employing tonic remedies in conjunction with invigorating hygienic measures. Hammond advises strychnia and 
phosphorus, together with galvanization of the spine. The latter is advised by Remak, Reynolds, and Benedikt.

Mercurial tremor denotes trembling movements of the limbs, the muscles of the face, the tongue, and sometimes the trunk, due to poisoning by mercury. The tremor occurs especially when the patient makes voluntary movements ; in this respect it is analogous to chorea. This affection, formerly not infrequent, of late years is rarely seen, in consequence of improvements in mechanical processes intolving the use of mercury, and the better observance of precautionary measures. 'The inhalation of vaporized mercury is most apt to give rise to it, but it has been produced by the use of mercury as a medicine and by mercurial inunctions. In the only case which has fallen under my observation, the gums were swollen, reddened, and spongy, and the teeth had been loosened. These appearances, together with other symptoms of the mercurial cachexia, and, generally, knowledge of the fact of exposure to mercurial poisoning as incident to the occupation of the patient, will lead to the diagnosis.

Recorery may be expected, provided the continued introduction of mercury into the system be prevented, under the use of tonic remedies with an invigorating regimen. The iodide of potassium is supposed to effect the elimination of mercury from the body.

My colleague, Prof. Hammond, has described a curious affection, which properly comes under the head of anomalous movements, characterized by an inability to retain the fingers and toes in any position in which they may be placed, and by their continual motion. He proposes to call this affection athetosis (A $\theta \varepsilon \tau$ os, without fixed position). He has observed two cases. In both the patients had at first epileptic paroxysms; and in both there were head symptoms, with tremulousness of the tongue, numbness on the affected side, and pains in the spasmodically affected muscles, paralysis not existing in either case. It remains to determine the nature and seat of lesions in this affection, or its functional character.

\section{Epilepsy.}

The affection now to be considered, which has heretofore received various names, is universally known, at the present time, as epilepsy, a term signifying a sudden seizure. It is a paroxysmal affection, the paroxysms being characterized by complete loss of consciousness, with convulsive movements, the latter being both tonic and clonic, and affecting more or less of the voluntary muscular system. Convulsions analogous to those of epilepsy are symptomatic of certain affections, especially those involving uræmia, and they are sometimes incident to injuries of the head. These are called epileptiform convulsions. Epilepsy, as one of the neuroses, is a functional affection, that is, not necessarily connected with either inflammation or any appreciable lesions. Related to the affection, sometimes preceding its development, and sometimes occurring in persons affected with the ordinary form of epilepsy, are attacks in which the convulsive movements are either slight and limited, or altogether wanting. This mild form of seizure, important especially from its relationship to fully developed epilepsy, is called, by French writers, epileptic vertigo, a name which hardly expresses its character. I know of no better designation than to call these attacks epileptoid. The symptoms which belong to ordinary epileptic paroxysms first claim consideration.

In the majority of cases, a paroxysm occurs without any premonition. The loss of consciousness is as sudden as if produced by a stunning blow upon the head. The person falls, and, hence, the affection has been popu- 
larly known as the "falling sickness." In a minority of eases, there is a brief warning of the approaching fit. Generally the premonition consists in a sensation which the patient is unable to describe. The sense of a "cold vapor" emanating from some part of the body and mounting to the head, has been ealled the aura epileptica. This sensation was first described by Galen, and, as remarked by Herpin, "the commentators of Galen and those who copied from them handed down the phrase, until the existence of an aura seemed to be aceepted as an ascertained fact." It is a traditional error to consider such a sensation as a frequent warning of an epileptic paroxysm, and it is certainly extremely rare for the patient to experience any sensation emanating from a particular part of the body. The onset of the paroxysm is generally marked by a loud, short, and piercing shriek or cry, which, from its intensity and unnatural character, is sometimes truly terrific. The face, at the time of the seizure, becomes notably pale. The direction in which the fall takes place is almost always forward on the face. The face is not infrequently wounded or bruised by the force of the fall, and severe burns are sometimes caused by falling upon heated stoves or into the fire. The convulsive morements at once begin. These are at first tonic, that is, the muscles are forcibly contracted, and the contraction persists for several seconds. The muscles of the face, neck, arms, forearms, the lower extremities, the abdomen and chest, are frequently involved, and present a tetanic rigidity. In most cases the museles of one side of the body are more affected than those of the other side, and sometimes, although very rarely, the convulsions are limited to one side. The tonic convulsions last for a period varying from a few seconds to half a minute or somewhat more. The convulsions then become clonic, that is, the muscles present forcible contractions and relaxations in rapid succession. The face is hideously distorted, the head is mored upon the trunk, the upper and lower limbs and the body jerk with violenee. The tongue is sometimes caught between the teeth and wounded by the tonic contraction of the muscles of the lower jaw. This more frequently happens during the clonic convulsions. Respiration is nearly suspended by the tonic, and is irregular and difficult during the clonic convulsions. A quackling noise accompanies the respiratory acts. The face during the convulsions becomes deeply congested and livid. Convulsive masticatory movements are accompanied by the ejection of foamy saliva, frequently commingled with blood from the wounded tongue, or from a portion of the cheek being eaught between the teeth. The violent clonic convulsions continue for a period varying from half a minute to three minutes. The period to the observer seems to be much longer, but, if observed by the wateh, it is rarely found to exceed three minutes. The convulsive movements now become less rapid and violent, the embarrassment of respiration diminishes, the patient fetches a deep sigh, and the paroxysm, so far as the convulsions are concerned, is ended. The paroxysms, in different cases and different paroxysms in the same case, differ much as regards the violence of the convulsive movements, and the extent of the muscular system involved. The convulsions are sometimes especially marked in the face and upper extremities, and they may be limited to these parts. Paroxysms differ also as regards duration. The violence of the convulsive movements of the muscles of the shoulder is sometimes sufficient to produce luxation of the humerus. A case in which this repeaterly oceurred has fallen under my observation. During the convulsions the urine and feces are sometimes expelled, and an emission of semen may take place. The pupils, during the paroxysm, are dilated and do not contract on ex- 
posure to a bright light. The eyelids, however, close when the conjunctiva is touched, showing the preservation of the reflex junction.

After the convulsions have ceased, consciousness may be speedily regained, and the patient only experiences a sense of fatigue, with, usually, a disposition to sleep. But in most cases the state of coma continues for a variable period. Frequently the respiration for some time is stertorous, and the lips are puffed out with expiration. The patient remains in this state for a few minutes, or for half an hour; the pupils are still dilated and do not contract on exposure to light, and he cannot be roused from the comatose state. Gradually he emerges from this state, opens his eyes, appears confused, and some time elapses before he is able to appreciate his situation and reply to questions. If he attempt at once to walk, he reels like a person intoxicated. Occasionally the patient passes from the apoplectiform state into one of delirium. . He talks incoherently, manifests hallucinations, and sometimes the delirium assumes the form of mania during which he may be dangerous to himself or others. After recovery of the mental faculties, there is complete inability to recall anything which has transpired during the paroxysm.

The paroxysms recur after intervals which are extremely variable in different cases. The intervals may extend over many months or years. On the other hand, they recur, in some cases, daily and even many times daily. When a paroxysm has once occurred, other paroxysms are almost sure to follow sooner or later. In most cases the paroxysms at first are more widely separated than after they have repeatedly recurred; they become, as a rule, more and more frequent until the affection is confirmed. In some cases the recurrence is periodical. In females sometimes paroxysms occur regularly at the menstrual period. Periodicity, however, is not the rule, the intervals generally varying considerably in the same case; but in certain cases of confirmed epilepsy, about the same number of paroxysms take place in successive periods of a quarter, a half, or the whole of a year. Exceptionably, the recurrence of the paroxysms becomes less frequent after a time, and a patient who, for several years, has been subject to paroxysms after short intervals, may afterward have long intermissions. Occasionally, having recurred more or less frequently for months or years, they cease altogether for a considerable period, and then return with not less, and perhaps greater, frequency than before.

In some cases paroxysms occur in rapid succession during a day or during several successive days. As many as one hundred and even a larger number of paroxysms have been known to occur during twenty-four hours. In a case which came under my observation at Blackwell's Island Hospital, paroxysms took place at intervals of a few moments for fourteen consecutive days. The patient recovered, and the paroxysms did not again recur for several months. When a series of paroxysms thus occur, the mind is notably impaired for a time ; the patient is childish and perhaps idiotic for several days, but gradually recovers the habitual state of the mental faculties. Hemiplegia occasionally follows a series of paroxsyms. This occurred in the case just referred to. The paralysis, in such cases, is usually of brief duration.

Epileptic paroxysms occcur at all hours of the day or night. They are apt to occur during the night. In some cases, for a greater or less period, they occur only at night. In these cases the affection may have existed long before its existence is ascertained. I have known a case in which, the paroxysms never occurring in the daytime, the existence of the affection was for a long period concealed from the patient. It is a curious fact that the paroxysms are usually suspended during the course of an 
acute disease. A patient, for example, sulject to daily paroxysms, became affected with typhoid fever. During the career of the fever no paroxysms occurred, and among the events denoting convalescence was the return of the epilepsy.

Of the essential pathological condition in epilepsy we have no positive knowledge. This affection is not to be excepted in the statement that our present knowledge of the functions of the nervous system warrants only conjectures with respect to the nature of the morbid perversions which constitute the neuroses. Paroxysms, in all respects like those of epilepsy, are, in some cases, connected with, and doubtless in some way dependent upon, certain intra-cranial lesions, such as tumors of various kinds, syphilitic and other lesions, meningitis, and they sometimes follow fracture with depression of the skull. It is fair to infer that, in these cases, the various lesions simply stand in a causative relation to the unknown pathological condition giving rise to the paroxysms. The researches of Schroeder Van der Kolk show the existence, after death, of induration of the medulla oblongata from exudation in some cases, and softening from fatty degeneration in other cases, with enlargement of the capillary vessels. These changes, however, are to be considered as effects of the disturbance of the circulation incident to the paroxysms, and it is difficult to say how much pathological importance belongs to them. An interesting fact with respect to the enlargement of the vessels is pointed out by the author just named. In epileptics who bite their tongues in the paroxysms, the capillary vessels are enlarged in the course of the hypoglossus nerve, and in those who never or very rarely bite their tongues, the enlargement of the vessels is in the course of the vagus. ${ }^{1}$

The functional condition which constitutes epilepsy can only be said to exist at the time when the paroxysms occur. It may be presumed that this condition is essentially the same when similar paroxysms are symptomatic of cerebral lesions, or when they occur in the course of other affections, for example, uræmia, as when epilepsy is an irliopathic affection. The agencies producing this condition are supposed to be, in some cases, seated at the centre of the cerebro-spinal nervous system, and the affection has then been distinguished as "centric;" in other eases the condition is supposed to be due to morbid influences transmitted to the centre of this system from some part of the body, and the affection has then been called "excentric," or " centripetal." With regard to these points, however, we have no positive knowledge. Without entering into any diseussion of the pathology of the affection, the author will simply state that the humoral doctrine, as enunciated by Todd and some others, seems to him to be rendered probable by analogical reasoning. Todd attributed the affection to an abnormal development of nervous force which manifests itself in the epileptic paroxysm, as a Leyden jar, when charged with electricity to a certain state of tension, gets rid of the disturbance by the disruptive disclarge. This undue nervous force he attributes to the accumulation of some material in the blood which, acting on the brain as a poison, excites the "disruptive discharge," leaving the nervous system free from disturbance until a fresh accumulation excites a new paroxysm. The following facts are cited by Todd as supporting this humoral doctrine of epilepsy: 1. The influence of certain toxic agents in producing artificial epilepsy. 2. The frequent connection between epileptic convulsions and imperfect eliminatory action of the kidneys. 3. The correspondence be-

I On the Minute Structure and Functions of the Spinal Cord and Medulla Oblongata. Publications of the. New Sydenham Snciety, London, vol. iv., 1859. 
tween the paroxysmal character of epilepsy and that of other diseases confessedly humoral, such as ague and gout. 4. The fact that the introduction of certain animal poisons (as those of the exanthemata or of typhus) into the system may produce epileptic convulsions. $A$ side from the humoral doctrine just stated, an explanation with which many are satisfied refers the paroxysms to irritation of the medulla oblongata, extending to the brain, the irritation being either developed within the medulla or communicating through afferent nerves. Some have supposed that the paroxysms are attributable to an active congestion of the medulla; on the other hand, the paroxysms have been considered as arising from anæmia of this part, caused by spasm of the muscular fibres of the vesscls. The marked pallor of the face which is a striking feature of the onset of the paroxysm, and the brief duration of the paroxysm, favor the hypothesis of a morbid inflience exerted through the vaso-motor nerves and producing spasm of the vessels. Assuming this to be a correct interpretation of the phenomena, there must be, of course, a morbid agency underlying the influence transmitted to the vaso-motor nerves. This prior ageney, as it seems to me most reasonable to suppose, is toxical in character. Judging from the anatomical relations of the medulla oblongata, and from what is known of its pathological relations, the morbid condition giving rise to the bilateral convulsions is seated in that part of the central nervous system. The coma, however, which accompanies the paroxysms, denotes a morbid condition of the cerebrum.

The causes of epilepsy are not equally operative at all periods of life. Of 995 eases analyzed with referenee to the age when the affection was developed, by Moreau, in 87 it existed at birth, it was developed in infancy in 25 eases, from 2 to 10 years in 281 cases, from 10 to 20 years in 364 cases, from 20 to 30 years in 111 cases, from 30 to 40 years in 59 cases, from 40 to 50 years in 51 cases, from 50 to 60 years in 13 cases, and from 60 to 70 years in 4 cases. ${ }^{1}$ The period, according to these statistics, most favorable for the development of the affection is from 10 to 20 years, and next to this period it occurs most frequently from 2 to 14 years, and next from 20 to 30 years. As regards sex, statistics show that, prior to 10 years of age, each sex is about equally represented, but after 14 years the number of cases in females predominates. Hereditary predisposition, if the question be limited to epilepsy, is not established by statistical data, but facts go to show that the progenitors of epileptics in a large number of cases have been subject to affections of the nervous system.

Of particular canses, when the affection is idiopathic, little is positively known. It is certain that intemperance, as regards the use of alcohol, may give rise to it. Epileptic paroxysms occur not infrequently in drunkards, and cease to reeur in cases in which reformation of intemperate habits is effected. It is one of the occasional effects of lead-poisoning. Venereal excesses, and especially habits of masturbation, have been supposed to be not infrequent causes. The supposition is highly probable, but an accumulation of facts establishing this causative connection is desirable. A female patient, 23 years of age, unmarried, who had been subject to paroxysms occurring almost nightly for two years, stated to me, voluntarily, that she was taught to masturbate at six years of age, and continued the practice almost daily until she was fourteen. At this

1 Mém. de l'Acad. de Méd., Paris, 1853, vide Valleix, op. cit. For an analysis of 104 cases by Edward H. Sieveking, M.D., vide Med.-Chir. Trans. Published by the Royal Med. and Chirurg. Soc. of London, vol. xxvi., 1861. 
age she became religious, and realizing the sinfulness of the habit, discontinued it. She was afterward tormented with sexual desires, and ex. perienced the venereal orgasm almost nightly during sleep. She was led to disclose this private history by a conviction, at which she had arriver of her own accord, that it was important with reference to the source of the epilepsy. To what extent the antecedent history in cases of epilepsy would furnish corresponding facts can only be conjectured. Epilepsy has becn known to occur in persons affected with tania, and to cease after the expulsion of the worm.

In persons subject to epilepsy, the paroxysms, in the great majority of cases, occur without any obvious, adequate, exciting causes. The causes determining the attack, as a rule, are intrinsic. Persons not infrequently are able to judge, by experience, from certain indefinite sensations, that they will ere long have a paroxysm. These sensations disappear after the paloxysm has occurred; and it is not uncommon for persons to feel better after recovery from the immediate effects of a paroxysm than for some time prior to its occurrence. It has been supposed that the aura which sometimes precedes the paroxysm denotes the existence of an exciting cause emanating from the part to which the aura is referred. This supposition rests only on conjecture. A case has fallen under my observation in which the patient, a female, experienced the first paroxysm during the first act of sexual congress after marriage. Subsequently, a paroxysm occurred at each marital connection, but, after a time, paroxysms took place without this exciting cause, and the patient become a confirmed epileptic. It is a remarkable fact that an attack may occur from imitation. An instance has fallen under my observation: A female was employed as an attendant on a lady who was subject to epilepsy. The lady, on one occasion, had a scries of paroxysms in quick succession. Her attendant, while these paroxysms were in progress, first manifested hysterical phenomena, and finally experienced a distinet epileptic paroxysm. She had never before had an attack of epilepsy, nor did anotlier paroxysm take place, the person remaining within my observation for several years afterward. It is well known that $\operatorname{logs}$, cits, and other domestic animals are liable to epilepsy. Prof. Dalton has related to me the following interesting illustration of the effect of imitation in the canine race. A dog, not previously affected with epilepsy, was in company with another dog, who was subject to this affection: the latter being seized with an epileptic paroxysm, the former immediately afterward had a similar attack. This occurred under Prof. Dalton's observation.

The diagnosis of epilepsy, in its ordinary form, rarely offers much difficulty. The disease is, in general, readily ascertained, when it has become confirmed. In cases in which the practitioner has not an opportunity of witnessing any of the paroxysms, he may generally obtain sufficient information respecting the distinctive features to feel sure of the diagnosis. The prominent points of inquiry are, the sudden seizure, the cry at the beginning of the paroxysm, the loss of consciousness, the tonic and clonis convulsions, embarrassed respiration, ejection from the mouth of frothy saliva often mixed with blood, and the short duration of the fits. An examination of the tongue, if repeated paroxysms have occurred, in some cases shows cicatrices, resulting from the wounds of this organ, which are quite diagnostic.

The affection is to be discriminated from hysterical convulsions; the latter present points of difference which are usually sufficiently marked. The convulsive movements are not purely automatic, as in epilepsy, but proceed from a delirious volition. They are of longer duration than the 
convulsive movements in a paroxysm of epilepsy; they are developed gradually. Foaming at the mouth does not accompany hysterical convulsions; the pupils are not dilated. Epilepsy may be developed in persons subjeet to hysteria, and the two affections may be associated.

If ealled to a patient, not known to be an epileptic, in the comatose state which suceeeds the convulsions, apoplexy might at first be suspeeted; but if the convulsions which precede this state have been observed by any one, information respecting their occurrence suffices for the diagnosis. If the convulsions have not been observed, the appearance of foam and blood upon the lips is diagnostic. The age of the patient is to be considered, and the absence of hemiplegia which so often oceurs in cases of apoplexy and very rarely in cases of epilepsy.

Epileptiform convulsions, or eclampsia, occurring in children, in pregnant women, or in connection with the affections grouped under the name Bright's disease, are to be discriminated by means of the antecedent and coexisting symptoms. Judging from the convulsions and associated venous phenomena, uræmia and epilepsy might easily be confounderl. Uræmic convulsions, however, are rarely of as brief duration as an attack of epilepsy; but examinations of the urine will suffice for this differential diagnosis. A paroxysm of convulsions in an infant or child often, with good reason, oceasions anxiety lest it may prove the beginning of epilepsy. It is not always practicable to determine this point at once. There is more reason to apprehend epilepsy in proportion as the convulsions are not otherwise to be aceounted for. Their non-recurrenee affords the only positive proof that they are not epileptic.

It is desirable to determine whether epileptic paroxysms are connected with lesions affecting the cerebro-spinal system, or whether they are idiopathic. With referenee to this point, symptoms denoting lesions are to be sought for in the intervals between the paroxysms. Persisting pain in the head, paralysis, and disturbance of the intellect greater than the epileptic paroxysms will account for, are symptoms pointing to cerebral lesions. Lesions are to be inferred if the epileptic paroxysms succeed an injury of the head.

Epilepsy is one of the affeetions which are sometimes simulated by malingerers. More or less of the appearances which are presented in epileptie paroxysms may be voluntarily produced, but a malingerer must be very well acquainted with the disease to represent accurately the phenomena in the order in which they suceed each other, and certain of the phenomena are beyond the power of the will. To determine a case of deception, let it be observed if the face beeome at first pale and afterward deeply congested and livid, if a cry be uttered, if the fall be forward on the face, if the convulsions be at first tonic and afterward clonic, if foamy saliva be ejeeted from the mouth, and if the tongue or cheek be wounded by the teeth. Let the pupils be examined; these cannot be voluntarily dilated, nor their mobility on the approach of light prevented. Let the duration of the fit be noted; the convulsions will be likely to be unduly prolonged. Let it be ascertained if, accompanying and sueceeding the convulsions, there be coma with insensibility to pricking, burning, or pinching the skin. Let the attention be directed to the time and place in which the paroxysms take place. Malingerers will not seleet the night, nor a situation in which they will not be observed. They are not likely to fall in a way or in a position to reeeive injury. The aspect of confusion and shame which is manifested on recovering consciousness after an attack of epilepsy is not easily feigned.

Epilepsy may exist for a long time before its existence is aseertained 
If the paroxysms occur only at night. The disease is to be suspected when patients complain of arakening from time to time with headache, lassitude, and a wounded tongue. Under these circumstances, the diagnosis is to be settled by causing the patient to be watehed during the night.

Owing to the terrible nature of the malady, ancl the apprehensions felt by those in company with an epileptic lest a paroxysin may occur, persons are naturally seusitive with respect to themselves or their relatives being said to have epilepsy. For this reason there is sometimes a disposition to conceal the nature of the paroxysms even from the physician. To avoid the depressing effect upon the mind of the patient which a knowledge of the disease is likely to produce, it is sometimes judicious to refrain from calling it epilepsy. In the case of a lady who was under my observation for many years, the nature of the disease was never communicated to her. The paroxysms, which were often frequent and severe, were always called nervous attacks, and she finally died of an intercurrent affection, without knowing that she had been an epileptic. In this case, ignorance of her disease enabled her to participate in many of the enjoyments of life from which, owing to her sensitive nature, she would have shrunk had she been aware that she was aflicted with epilepsy.

The prognosis, in cases of epilepsy, as regards immediate danger from the paroxysms, is always favorable. It is exceedingly rare for a paroxysm to prove fatal. When it does prove fatal, death is eaused by apnoa arising from prolonged tonic spasm of the muscles of respiration and the laryngeal muscles. The liability to any serious result is small, but intercranial hemorrhage sometimes occurs. Nor does epilepsy tend to give rise to any particular disease or diseases of a dangerous character. Epileptics, as a rule, are not short-lived, and, in the majority of cases, death arises from other diseases, to the development of which the epileptic paroxysms have not contributed. In another point of view, however, the prognosis is most unfavorable, viz., as regards the persistence of the malady. In the vast majority of cases, the disease teuds to become confirmed, and, as a rule, the intrinsic tendency is to increasing frequency of the paroxysms.

In another point of view, the prognosis is unfavorable, viz., as regards the mental condition. To a certain extent, the disease teuds intrinsically to impairment or disorder of the faculties of the mind. Epileptics, especially if the paroxysms recur at short intervals, sooner or later fall into imbecility. To a greater or less extent this is due to causes other than the intrinsic tendency of the disease. If epilepsy occur in early life, the mental faculties are not developed by education and those occupations which involve their exercise; and, in the eases in which the disease occurs at a later period, the mental faculties progressively fail from disuse. The unhappy sufferer from this terrible disease is apt to lose interest in those objects or pursuits in life which incite to the exercise of the mental faculties. This explanation, in part, of the apparent effect of epilepsy on the mind has an important practical bearing.

Reference has been already made to mild attacks having an evident relationship to ordinary epilepsy, which may be called epileploid attacks. It is important for the practitioner to recognize and appreciate the significance of these attacks. They are considered by French writers as belonging to a variety of epilepsy which they call petit mal, in contradistinction from the ordinary form which they call the grand or haut mal. These attacks sometimes precelle the development of ordinary epilepsy, and they sometimes occur, intercurrently, in persons who are confirmed epileptics.

The epileptoid attacks are multiform. A sudden loss of consciousness for a few seconds occurs in some cases, during which the body may remain 
fixed, the person apparently being lost in deep abstraction, the mental faculties resuming their operation, as if nothing had taken place, directly the attack passes off. In a case of confirmed epilepsy under my observation, the patient, at one time, in the intervals between the paroxysms, was subject to frequent attacks of the kind just described. During the attacks she remained fixed in the position in which she happened to be when they occurred. For example, if standing and arranging her hair, the hands were fixed precisely as they were at the instant of the seizure. On some days these attacks took place a great number of times, and the patient did not appear to be conscious of their occurrence. In the fixation of the body and limbs these attacks resemble catalepsy. In the case just referred to, the only convulsive movements were of the muscles of mastication. Similar attacks are sometimes accompanied by convulsive movements of other of the facial muscles, of the muscles of the neck, and slight laryugeal spasm. In some cases the patient falls, but recovers and rises in a few seconds. Loss of consciousness and falling, in some cases, are unattended by any convulsive movements. Trousseau relates examples of this kind. Of course, in such cases, epileptoid attacks are to be discriminated from syncope.

In other epileptoid attacks, sudden delirinm occurs, continues for a few seconds, and passes off, leaving the mind in the condition in which it was at the instant of the seizure. The delirium is manifested in different ways. Trousseau eites a case in which the patient uttered a burst of laughter, and when asked why he laughed, he looked surprised, having no knowledge of what he had done. The same author eites examples of persons engaged in eonversation, debate, or other occupations, abruptly, as if struck by a sudden thought, going into the street bareheaded, and walking until they recovered themselves, when they were able to return and proceed with the matter in which they were engaged, as if nothing had happened. ${ }^{2}$ The following example came under my observation in one of the wards of Bellevue Hospital : A female patient quietly standing in the ward, suddenly uttered a cry, and ran from one end of the ward to the other. The urine was at the same time expelled, leaving on the floor traees of her course. Having reached the end of the ward, she stopped, looked confused, and quietly returned. The following is another example: Some years ago a young man, who had been subjeet to ordinary epilepsy for.twelve years, came to see me accompanied by his attending physician. On the previous evening, from time to time, he had paroxysms of jumping up and down with violence for a few seconds. In walking to my residenee, now and then he stopped and performed his jumping movements in the street. After sitting down and replying quietly to my questions, he began to jump violently in the chair for a few seeonds, and continued to do so at short intervals. He was desired to go into an adjoining room during the consultation, and while there we could hear him jumping from time to time with much noise. This case has been already referred to in connection with "anomalous muscular movements."

The following example of an epileptoid attack is interesting from its presenting very distinctly the aura epileptica. A young man, aged 30, had been for ten months in disordered health, suffering from palpitation and mental depression. He described paroxysms of frequent occurrence during the time just stated, in which he felt a flash, as he termed it, originating sometimes in the chest, sometimes in the abdomen, and sometimes in one of the lower extremities, rising upward, giving rise to a sense of

1 Vide Clinique Médicale, tome ii. 
suffocation, and, on reaching the head, followed by momentary confusion of mind. He had never fallen. In some of these attacks he had felt stiffness of the muscles of the jaws and of the forehead. The attacks were irregular, but had recurred more or less frequently every week for the preceding ten months. On being questioned, he said that the "flash" gave the sensation of a stream of air or gas. He had never heard or resl aught of the epileptic aura. Nine months afterward, this patient had recovered his former health, and no longer had the paroxysms above described.

Other attacks consist of convulsive movements of certain muscles without loss of consciousness or delirium. In a case under my observation, the patient, a young man apparently in perfect health, first consulted me for the occasional occurrence of convulsive movements of the upper extremities. From time to time, the hands werc violently jerked, and whatever he might happen to hold at the time was thrown with great force. It was unsafe for him to hold fragile articles. A careful examination in this case revealed nothing abnormal save these convulsive movements. They became more and more frequent, and, shortly, they extended to the lower limbs, so that he was repeatedly thrown down for an instant, still without loss of consciousness. At length, after the lapse of several weeks, he had a severe paroxysm of epilepsy, and the malady is now established. In a case under observation, when this chapter was written, 1864, paroxysms occurred daily, more or less frequently, in which the head was forcibly drawn toward the right shoulder, and retained there for a few seconds. This affection had existed for five years, without eventuating in epilepsy. The paroxysms, however, had increased in frequency and severity, and he appeared to have loss of consciousness for a few scconds, although he did not fall. In other respects the patient was apparently well. Being a young physician, he suffered from the anxiety incident to a knowledge of the fact that these attacks may be premonitory of the truly epileptic paroxysms. Thiee years afterward he wrote to me that the paroxysms were not more frequent, but more severe, causing him to fall. He stated that they were controlled by large doses of the bromide of potassium.

In the case of a girl, aged 12 years, in which I was consulted, the patient afterward having been seen by Dr. J. Hughlings Jackson, the attacks presented the following features: Almost daily, often at the breakfinst table, she suddlenly manifested a changed expression of countenance, appearing as if frightened, sometimes uttered some exclamation as if surprised, and ber consciousness was lost for a few seconds. Sometimes the face was distorted. When consciousness returned, she almost invariably asked "what time is it?" There had never been any convulsive movements of the limbs, but they were sometimes rigid. There was never any spasm of the glottis.

Epileptoid attacks, occurring in persons who have never had epileptic paroxysms, are to be considered as denoting a pathological condition which, fully developed, constitutes epilepsy. Hence, the obvious importance of not overlooking their significance, in order to prevent, if possible, the development of the malady which is foreshadowed. Facts showing how frequently epileptoid attacks occur without being followed by epilepsy, are wanting, but it is certain that the latter by no means invariably follows. The physician, therefore, should not excite unduly the fears of the patient or friends, by predicting the development of epilepsy.

The treatment of epilepsy relates to the management of the paroxysms and to measures for effecting a cure. The latter are to be employed in 
the intervals. Curative treatment, as in other of the neuroses, may be conveniently considered as rational and empirical.

The rational treatment consists in deriving, as far as possible, indications from an investigation of each case with regard to the state of the system, coexisting disorders, and circumstances which may be suspected of standing in a causative relation to the malady. If the patient be of a full habit or plethoric, dietetie and other measures addressed to this state, are rationally indicated. If, on the other hand, the patient be anæmic, measures of an opposite character are indieated. Disorders of the digestive system, uterine affections, and, in short, morbid disturbanees anywhere in the economy, should reeeive appropriate treatment; they may, possibly, be in some way concerned in the development or perpetuation of the malady. The presenee or otherwise of trenia is to be ascertained. Inquiries with respect to syphilis are not to be forgotten. Habits of life, with respect to the probable or possible causation, are to be scrutinized. Overtasking of mind or body, undue exposure, on the one hand, and sedentary habits on the other hand, the facts with regard to mental and moral influences, the abuse of alcoholic stimulants, the immoderate use of tobacco, and especially over-indulgence in venery or the practice of masturbation, are to be inquired into. In brief, an important part of the rational treatment consists in enforcing observance, in all respects, of the laws of health. It is unnecessary to consider the numerous and varied details which this part of the treatment involves.

The empirical treatment consists in the employment of remedies or therapeutical measures which experience has shown to be sometimes curative. These are exceedingly numerous. It would require many pages simply to enumerate the medieines, the curative effieacy of whieh, in certain cases, has been attested by honest and competent observers. The list is so extensive, the testimony with respeet to particular remedies is so conflicting, and the instances of ineurable epilepsy are so numerous, that practitioners are apt to enter upon the treatment of a case without much expectation of suceess. For the reasons just stated, the disease is not infrequently allowed to continue without persevering efforts to effect a cure, and this fact may in a measure account for the number of cases in whieh patients remain hopelessly epileptics. Of the host of remedies which have been employed, many have doubtless proved successful in a certain proportion of cases. But without any disposition to deceive either themselves or others, practitioners have often been led to overestimate the success of different remedies by not making allowance for the following facts : Any active remedy will frequently prevent recurrence of the paroxysms for a considerable period. Under the use of a certain remedy, a patient passes weeks or months without a paroxysm; he is ready to believe himself cured, and the physician is happy to participate in the belief. Now, sooner or later, the paroxysms, in most cases, return; the patient despairs of benefit from treatment, or consults another physician. The return of the malady may not be known to the physician who had supposed that a cure was effected, and, in the mean time, he may have reported the case as one cured by the remedy employed. Again, the paroxysms cease, in some cases to recur for weeks or months or years, without any treatment. I have notes of a case in which paroxysms more or less in number every month, and sometimes fifteen or twenty in twentyfour hours, had occurred for sixteen years, a great rariety of remedies having been tried without avail; at length, after all treatment had beeu discontinued, the paroxysms ceased to recur for ten or twelve years, when they again returned, but at long intervals. Making, however, due allow- 
ance for the temporary interruption of the malady either by the treatment or spontaneously, there remain a certain number of undoubted cures effected by different remedies. In view of this fact, cases should not be abandoned without the faithful trial of many remedies. Of the various remedies which have been considered as curative, those, within late years and at the present time, in most repute, are the following: The nitrate and other preparations of silver, preparations of zinc, digitalis, opium, the narcotic extracts, more especially belladonna, and the bromide of potassium.

The nitrate of silver has long been a remedy for epilepsy, and its occasional efficacy rests on abundant testimony. That it will not effect a cure in the majority of cases, is not a sufficient reason for failing to make trial of it in cases which resist other remedies. Commencing with a fraction of a grain three times daily, the dose may be gradually increased to three or four grains. An effect of the prolonged use of this remedy, in some cases, is to be borne in mind, viz., permanent blueness of the skin. 'To avoid this effect, the remedy should be suspended for a time, after having been continued for two or three months. The oxide of silver is less likely to produce discoloration of the skin, but is less powerful as a remedy. The ehloride of silver is preferred by Dr. Perry, of Philadelphia.

Of the preparations of zinc, the oxide has been found curative by different observers, and is advocated especially by Herpin,' who claims that, in his hands, a cure was effected by this remedy in 26 of 42 eases. The mode of administration advised by Herpin, is to give at first from six to eight grains daily in divided doses an hour after each meal. The quantity given daily is to be increased by two grains each week, until it reaches forty-five grains, this quantity to be continued for three months. The remedy is not to be discontinued after the cessation of the fits.

The prospect of effecting a cure according to Herpin, by this or any remedy, other things being equal, is proportionate to the small number of fits which have occurred. If there have been more than 500 fits, the probability of a cure is extremely small. In a few cases, under my observation, in which the treatment as laid down by Herpin was faithfully carried out, the remedy proved unsuccessful. The cases reported by this author, however, afford indubitable evidence of a certain amount of curative power; and with reference to any remedy for this disease, there is no known means of determining beforehand whether it will be likely to prove successful in any particular case. In the cases in which I have tried this remerly, it has been difficult to continue it long after the maximum of quantity is reached, on account of the strong antipathy to it which the patient acquires. In a paper published after the prize essay by Herpin he advises the lactate, in preference to the oxide of zine. Babington prefers the sulphate of zinc, and has carried the quantity given per diem for several weeks in succession to $36^{\prime}$ grains without producing nausea. ${ }^{2}$ The phosphate of zinc is recommended by Dr. Barnes." ${ }^{3}$ The valerianate of zinc is an eligible preparation.

The ammoniated copper has been found curative. Of 12 cases treated exclusively with this remedy by Herpin, four were eured. The dose is half a grain, increased gradually to four or five grains. The sulphate of copper has also been employed successfully.

' Op. cit. Vide Analytical Review in British and Foreign Medico-Chir. Review, London, 1853.

S Braithwaite's Retrospect, Part iii. p. 21.

- London Lancet, January, $18 \overline{8} 8$. 
Digitalis is said to have been long a remedy of much repute in Ireland for epilepsy, and its efficacy is attested by Sharkey, Crampton, Cormack, and Corrigan. The infusion is the preparation to be preferred. The quantity given daily is to be increased to the amount which is tolerated, and continued steadily for several months.

Opium is among the remedies recommended as sometimes curative, and, perhaps, it deserves a trial more extended than appears as yet to have been given to it. The evils of the habitual use of opium are to be considered, but, with a fair probability of effecting a cure of the epilepsy, the physician would be warranted in exposing his patient to this danger.

Of the different narcotic extracts, belladonna has the strongest claims, based on the testimony of different observers. Trousseau is an ardent advocate of this remedy, as capable of effecting a cure in a certain proportion of cases, and frequently ameliorating the condition of epileptics when it fails to prove curative. 'Trousseau insists that a fair test of this remedy requires its continuance for a period not limited to months, but embracing several years. His mode of administration is to begin with a small dose of the extract (gr. 1-5) once daily, which is to be continued for a month withont increase. At the end of each month the dose is to be rloubled, until as large a dose as can be conveniently borne is reached. The tolerance of the remedy differs in different cases. After the disease is perceptibly modified, the doses are diminished in the same manner as they were increased. Atropine may be employed in lieu of belladonna, the mode of administration being similar. With the use of belladonna or atropine, 'Trousseau frequently combines the employment of nitrate of silver, copper, and the lactate of zinc. ${ }^{1}$

When the first edition of this work was issued (1866), the bromide of potassium had but recently come into vogue as a remedy in epilepsy. A few clinical facts, showing the value of the remedy in this disease, were cited in that edition. Additional facts had occurred between the publication of the first and the second edition ; and in revising the work for the third edition (issued in 1868), I was able to state, that " many cases coming under my observation have been added to those previously observed, in which the effect of this remedy has been more or less marked, and in some cases truly marvellous." At the present time (1872), taking into view the abundant testimony of practitioners in different countries, who have had large experience in the treatment of the disease, this remedy takes precedence of all of the various remedies known to have any curative power in epilepsy. It is certain that, in a proportion of cases not very small, a permanent cure may be effected by this remedy; and that in a large majority of cases it is more or less useful, as shown by the increase of the intervals between the paroxysms. In a small proportion of cases the remedy is neither curative nor useful. To secure all its curative or useful effect, it must be given in full, and often in large, doses. As a rule, at least fifteen grains, twice or thrice daily, for an adult, should be given at first. The doses should be increased, if the paroxysms continue, and the remedy be well borne. The tolerance of the remedy varies in different persons. The doses should be increased to the limit of tolerance, if the desired effect be not obtained by smaller doses. Patients will often tolerate from one to two drachms daily; and Hammond has carried the doses, in one case, to the extent of giving three drachms daily, the paroxysms being arrested only when this amount was reached. The remedy is to be continued for a year or longer. If, however, the

1 Clinique Médicale. 
paroxysms fail to recur for several months, the doses may be diminished; and, at length, it may be suspended, to be again resumed should the paroxysms return. If, after continuing the remedy for several weeks, increasing the doses to the limit of tolerance, the paroxysms contimue as before, it may be considered as having failed, and its further continuance is useless. It is desirable to produce the phenomena of bromism, if the paroxysms do not otherwise cease. These phenomena are physical inertia, mental depression, and a constant tendency to somnolency. Carried beyond this period, the remedy causes difficulty of walking, patients staggering as if drunk, and insane delusions which give rise to apprehensions and terror. ${ }^{2}$ These effects continue but a short time if the remedy be discontinued. The other bromine salts, namely, the bromide of sodium, ammonium and lithium, have, as yet, been used in this disease much less than the bromide of potassium. They have been used sufficiently, however, to show that they possess a similar power, although their relative efficacy has not been determined. They may be given as substitutes for the bromide of potassium, if the latter be not well borne by the stomach, or if it give rise to a pustular eruption sufficiently to occasion much annoyance.

The fact of syphilis having existed at a period even remote, should lead to the employment of the antisyphilitic medication, namely, the iodide of potassium and the bichloride of mercury.

It will suffice to mention some other remedies which are worthy of trial, in the cases in which the bromides fail, as follows: Strychnia, or nux vomica, recommended in small doses, especially by Marshall Hall; cotyledon umbilicus, an ounce of the fresh juice or half a drachm of the inspissated juice to be given twice daily ; indigo, commencing with moderate doses and increasing to the amount which the stomach will bear; valerian, in large doses, continued for a long period; musk, camphor, cimicifuga, and the oil of turpentine.

Van der Kolk asserts that counter-irritation over the nucha by means of wet cupping, setons, and issues, has proved in his hands successful. Others have claimed for these measures success.

Under the supposition that the paroxysm depends upon a morbid agency springing from some part more or less remote from the ncrvous centres, and that the aura indicates the point of departure of this agency, it has been proposed to interrupt the nervous communications with the part by means of ligatures, section of nerves, the actual cautery, and even amputation of an extremity. But in the great majority of cases paroxysms are not preceded by an aura, and when this exists there is no ground for the conjecture that it denotes a causative agency. Moreover, it does not appear that treatment based upon this supposition has proved successful.

Surgical measures for the cure of epilepsy, in addition to those just alluded to, are, ligation of the carotids, tracheotomy, and after injuries of the head, trephining. Ligation of one or both of the carotids has been repeatedly tried, but the results do not warrant its being recommended. The same is true of tracheotomy, which was warmly advocated by Marshal Hall. Trephining, when there is reason to believe that the epilepsy may depend on depression, fraeture of the internal table of the skull, spicula of bone, or a collection of blood or pus, is a warrantable operation. In 35 cases collected and analyzed by my colleague, Prof. Stephen Smith, the

1 Vide cases reported by Prof. Hammond, in the Quarterly Journal of Psycholo. gical Medicine, New York, January, 1869. 
results of this operation were as follows: "Relieved, but not cured, 3. Immediate relief after operation and no further note of result, 2 . Relieved for one month or under, when last-seen, 3 ; between one and six months, 3 ; between six months and one year, 6 ; between one and five years, 3. Set down cured, but lapse of time from date of operation to time last seen not given, 7." "

It remains to consider the treatment called for during the paroxysms. These are rarely witnessed by the physician, save when a series of paroxysms occur in quick succession. The duration of a single paroxysm is so short that, if the physician do not happen to be present, it ends before he reaches the patient. The instructions to friends or attendants should be, to place the patient on a bed, if practicable, and not to attempt to restrain by force the convulsive movements. All articles of dress which cons'ringe the neck or chest should be removed or loosened. To prevent wounding of the tongue, a roll of linen or a piece of soft wood may be introduced between the teeth. A congregation of spectators around the patient should be prevented, and free ventilation provided for. The patient should be kept quiet after the paroxysm has ceased, and soothed by cooling lotions to the head.

Epilieptics should sleep in beds with raised sides to prevent injury from being thrown upon the floor by the convulsive movements, should a paroxysm occur during the night. They should be visited, as far as practicable, during the night, for death from suffocation has resulted from the face being pressed into the pillow during the paroxysm.

When a series of paroxysms occur, measures may be employed to prevent their recurrence. Ether, valerian, belladonna, and opium may be given for this object. The inhalation of chloroform may be tried. Compression of the carotids may prove successful, if other measures fail. The method of employing this measure is as follows: Compression is to be made upon one of the primitive carotids at a time, and if the convulsive movements are most marked on one side of the body, the carotid on the opposite side should be first compressed. The compression with the finger, pressing the artery against the vertebral column, may be continued steadily for fifteen or twenty minutes, then the artery on the opposite side may be compressed for the same period, and thus, alternately, the compression made on either side. If the convulsions be severe, both arteries may be compressed simultaneously. Dr. S. Weir Mitchell has reported a case in which the inhalation of the nitrite of amyl appeared to arrest the paroxysms.

It is a comfort to patients to be provided with a remedy to ward off an impending paroxysm. Chloric ether, Hoffmann's anodyne, the compound spirits of lavender, or the extract of valerian, may be prescribed for this purpose. A paroxysm may perhaps be postponed, but it is doubtful if it be preventable by any measures resorted to when premonitions are experienced. In a case under my observation, a friend sat by the side of a patient the whole of an afternoon, and, when the premonition was felt, dashed a little water into the face. The paroxysm was apparently prevented for the time, but it occurred directly the effort to prevent it was discontinued.

A highly important point connected with the management of epileptics

I Vide Article on the Surgical Treatment of Epilepsy, etc., New York Journal of Medicine, March, 1852. Prof. Smith, in this article, gives a summary of facts with respect to setons, issues, cauterizations, and moxas, operations on the scalp, tracheotomy, amputation, operations on the nerves, operations on the arteries, and trephining. 
relates to measures to prevent deterioration of the mental faculties. In so far as impairment of mind proceeds from disuse of the mental faculties, it may be obviated or retarded by taking pains to sccure as much exercise of these faculties as practicable. It conduces greatly to an alleviation of the unhappy condition of epileptics for them to participate, as far as practicable, in the interests and social enjoyments of life; and, if practicable, it is sometimes desirable to conceal from them the nature or the extent of the malady with which they are afflicted. I lave known marriage to be advised by the pliysician in the case of a young lady. Such advice cannot be too strongly censured. There is no ground to expect that the malady will be favorably affected by marriage. With reference to this step, it is the duty of the physician to explain fully to the family of the patient, if not to the patient, the nature of the malady.

The existence of epilepsy is an important fact in certain medico-legal cases. In the transient delirium which sometimes succeeds epileptic paroxysms, and which may be incident to epileptoid attacks, violence to others, and even homicide, may be committed. The occasional occurrence of delirium, lasting for hours or days, in connection with the disease, is to be borne in mind in giving medical testimony. The treatment in cases of epileptoid disease is essentially the same as in cases of epilepsy.

\section{CHA P T E IX.}

Hysteria. Catalepsy. Ecstasy. Somnambulism. Tetanus.

\section{Hysteria.}

TYSTERIA is reckoned among the diseases peculiar to females. The A phenomena embraced under this name are however observed in males, but examples are comparatively rare. Referring the reader to treatises on the diseases of females, or to monographs, for a full cousideration of the affection, I shall consider it briefly.

The name hysteria, as commonly used, embraces a multiplicity of morbid phenomena. It is used to denote an abnormal condition of the nervous system and of the mind, entering largely, as a morbid element, into a variety of affections. The hysterical condition, as regards the mind, involves a morbid susceptibility to emotions and a defective power of the will to restrain their manifestations. A person, whether male or female, is said to be hysterical who weeps or laughs irrepressibly on inadequate occasions, and when these emotional acts are incongruous. Frequently these manifestations of opposite emotions occur in alternation, and with an abrupt transition. Weeping and laughter are physiological acts when they represent emotions which are natural, that is, excited by adequate causes, and not exceeding the bounds which observation shows to be consistent with health. These acts are pathological when they do not represent corresponding emotions, or when they proceed from emotions which are unnatural in view of the circumstances under which they occur. The emotions may be real, but morbidly excited or in morbid excess. On the other hand, the hysterical manifestations, in some cases, simulate emotions 
which do not exist. Patients sometimes weep violently without experiencing mental anguish, and laugh immoderately without enjoyment. An intelligent and estimable lady, in advanced life, who was under my observation for many years, was subject to spells of uttering loud explosive sounds resembling those of laughter, from which she declared she was unable to refrain. They were called, in the family, her laughing or screaming fits. These were the only hysterical phenomena in the case, and it was frequently necessary to administer a full opiate to arrest them.

A tendency to exaggerate subjective symptoms of disease often enters into the hysterical condition, proceeding sometimes from an exaggerated sense of existing symptoms, and sometimes from a morbid desire to excite interest or sympathy. It is an important part of the knowledge and tact of the practitioner to make due allowance for this tendency in his investigation of symptoms. In some cases a morbid perversion of the mind leads patients to undertake to practice gross frauds as regards their ailments. They pretend to have extraordinary disorders, and resort to ingenious and persevering efforts of deception. Here, too, the knowledge and tact of the physician are called into requisition. He meets not infrequently with hysterical malingering as well as hysterical self-deception. Owing to the facts just stated, hysteria is associated in the minds of many with cither imaginary complaints or imposition ; hence, many are loth to consider themselves as affected with hysteria, and the physician learns reserve in the use of this term in his intercourse with his patients.

Other symptoms of the hysterical condition are, a painful sense of constriction in the epigastrium, and the sensation of a foreign body in the throat, known as the globus hystericus. To these a great number and variety of morbicl sensations might be added, such as cephalalgia, tinnitus aurium, eructations, abdominal tympanites, irregular chills with yawning and stretching, diuresis, twitching of muscles, cramps, etc. etc. Now, the nervous and mental disorder which constitutes what is commonly called the hysterical condition, may be associated with a host of affections. This condition may enter more or less as an element into almost any disease. There are certain affections with which it is frequently associated, viz., neuralgia, especially supra-orbital, cervico-occipital, and dorso-intercostal, functional disturbance of the heart, hyperæsthesia of the abdominal walls and of the surface of the body elsewhere, functional paraplegia, diminished sensibility to pain in portions of the cutaneous surface (analgesia), loss of voice from paralysis, or nervous aphonia, a peculiar nervous cough which has been considered in a previous part of this work, etc. Associated with the bysterical condition, these affections are often considered as varied forms of hysteria; the name hysterical is applied to them. Properly considered, they simply coexist with the hysterical condition. The circumstances under which these affections are developed are often favorable for hysterical manifestations; but the occurrence of the latter is not sufficient ground for calling the affections hysterical. Practically, however, it is important, always, to take into account the coexistence of the hysterical condition.

What I have called the hysterical condition embraces the milder manifestations of hysteria. Several forms of the affection are hysterical paroxysms characterized by convulsive movements, by coma more or less complete, and by delirium. In some cases the convulsions constitute the most prominent feature of the paroxysms, coma, in these cases, usually coexisting ; in other cases coma occurs without convulsions, but the coma 
may precede or follow convulsions, and in some cases delirium is the prominent feature. The severer forms of hysteria, therefore, may be considered as threefold, viz., hysterical convulsions, hysterieal coma, and hysterical delirium.

Hysterical convulsions occur in persons subject to the milder manifestations of hysteria, and the latter are frequently premonitions of the former. The development of the paroxysm is generally gradual ; the convulsions are preceded by notable distress referred to the epigastrium, by a sense of choking or suffocation, and sometimes by a feeling as if a solid body, or ball, ascended from the abdomen to the throat (globus hystericus). The convulsive movements are often first manifested in the muscles of the eyelids and eyeballs. Other of the muscles of the face are rarely affected, and, hence, movements more or less rapid of the eyes and constant nictation are highly distinctive of an hysterical paroxysm, if the movements be limited to these facial museles. These muscles may be alone affected, but firequently the convulsive morements extend to the extremities and trunk.

A distinctive feature of the convulsive movements of the body and limbs is, they are not purely automatic. Patients throw their limbs in various dircetions, they tumble about the berl with violence, rolling from the bed to the floor, and striking with force against solid substances, if not prevented; they struggle with those who attempt to restrain them, and sometimes show a prodigious degree of strength. After continuing active movements for several minutes, they remain comparatively quiet for a time, and the convulsions are then renewed. At times they grasp the throat as if to remove an obstruction, strike the breast, or tear the hair. These movements are in great measure roluntary; they are directed by a delirious volition, in this respect differing essentially from the convulsions of epilepsy, or eclampsia, which are purely automatic or involuntary. Automatic movements, however, in certain cases, enter, more or less, into hysterical convulsions. Tonic spasm of the posterior museles of the trunk, bending the body backward, so that the trunk, head, and lower limbs form the arc of a circle, constituting what is known as opisthotonos, occurs in some cases of hysteria. The jaws are sometimes firmly closed, as in trismus. In the intervals between the active movements the muscles are often rigid.

Consciousness during the active motions, and in the intervals, appears to be lost, but generally the patient has more or less cognizance of what is said and done, a fact which the practitioner may often turn to good account in the management. The face is sometimes flushed and sometimes pale. Respiration is often notably disturbed, in some cases being extremely rapid and irregular, in other cases slow and suspirious. It is extremely rare for foamy saliva to be ejected from the mouth, or for the tongue to be bitten. 'The spasm of the larynx and muscles of respiration, which characterizes epilepsy, is wanting, the nostrils are usually dilated nictation and movements of the eyes generally continue. The pulse is frequently but little, or not at all, affected. The heart, however, sometimes acts with violence and irregularity. The tranquillity of the eirculation, in some cases, is a striking feature. The duration of the paroxysm, varies much in different cases. It very rarely, if ever, is limited to a few moments ; it may continue for a period varying from half an hour to several hours. The convulsive movements gradually go off, and the disappearance of the paroxysm is frequently accompanied by cries, groans, sighs, loud weeping, or spasmodic laughter. Copious diuresis is apt to occur at the termination of the paroxysm. 
It is important to discriminate hysterical convulsions from epileptic paroxysms and epileptiform convulsions or eclampsia. The foregoing sketch presents certain points which, in general, suffice for the differential diagnosis, viz., the evidence of volition in the character of the movements, the gradual development of the attack, absence of convulsive movements of the muscles of the face, excepting those of the eyes, absence of foamy saliva and blood upon the lips, the respiration disordered, but not suspended by spasm of the larynx and respiratory muscles, the long duration of the paroxysm, the incomplete loss of consciousness, and the occurrence of symptoms denoting the hysterical condition prior to and following the paroxysm. It is a fact, however, not to be lost sight of, that epilepsy or eclampsia may be conjoined with hysterical convulsions.

Hysterical coma enters more or less into the paroxysms just described. It may precede and follow them. It is sometimes accompanied with convulsive movements limited to the eyelids or eyes, but it occurs without any convulsions. In a case of simple, ordinary hysterical coma, the patient lies apparently in a state of profound, tranquil sleep. The countenance presents a natural appearance; the respiration is quiet, with perhaps occasional sighs. The pulse is regular, and is neither accelerated nor retarled; there is frequently more or less rigidity of the muscles. Attempts to ronse the patient from this state are either ineffectual, or answers to questions are obtained with much difficulty in fecble whispers. The muscles offer resistance to efforts to separate the jaws. This quasi comatose state continues for a variable period, often for hours and sometimes for days, if not successfully treated. Patients in this state are not completely unconscious; they are cognizant of what is said and done. Sensibility to pain is blunted, but not abolished; pinching or pricking the skin may be borne without flinching, but stronger impressions, such as are produced by the application of a heated hammer or the prolonged cold douche, occassion suffering.

Attacks of this kind occasion great alarm, and the physician. will naturally participate in the apprehensions of friends if he fail to determine the nature of the affection. It is to be discriminated from apoplexy, and the diagnostic points are as follows: The attacks do not present the stertor of apoplexy, and hemiplegia is wanting; the pupils respond readily to light; the patient is perhaps known to be subject to hysteria. The symptoms of the hysterical condition precede the coma. The age of the patient will be likely to be under the period when apoplexy is most apt to occur; and in ordinary hysterical coma the nature of the affection is generally speedily declared by the efficacy of certain measures of treatment to be presently mentioned.

Uræmic coma is to be excluded. Antecedent and coexisting symptoms relating to the urine, general dropsy, in some cases, and the absence of hysterical premonitions, will suffice for this differential diagnosis. Abercrombie relates cases of fatal coma, occurring in females, which are liable to be mistaken for hysteria, nothing being found after death to account for the coma except cerebral congestion. Williams describes these cases in the following terms: "A young female becomes anæmic, and after exhibiting various symptoms of feeble general circulation, with headache, drowsiness, and impaired sensorial functions, suddenly becomes worse, passes into a state of stupor, with dilated pupils, sometimes varied by slight manifestations of delirium, throbbing of the carotids and partial heat of the head, and dies comatose. On opening the head, a small quantity of serum is found under the arachnoid and in the ventricles, sometimes with a little lymph (in one case there was none). The vascularity 
of the membranes is remarkable, but the vessels most distended are the veins, and in the larger of these and in the longitudinal sinus there is a firm coagulum." "I had met with two cases answering to this description many years ago, before uramia was as much understood as now. The existence of uræmia in sueh cases would seem now to afford a rational interpretation of the phenomena; yet $I$ have recently met with a case in which repeated careful examinations of the urine gave no evidence of renal disease.

There is a possibility of confounding the coma dependent on meningitis for hysterical coma. Meningitis is to be excluded by the fact of the coma not having been preceded by the diagnostic symptoms of that disease, viz., pain in the head, intolerance of light, suffusion of the eyes, throbbing of the carotids, etc. The thermometer will aid in the exclusion of meningeal or any other acute inflammation. If an acute inflammation exist, the heat of the body is more or less raised; whereas, hysteria, existing alone, does not give rise to any increase of heat. By this, also, meningitis may be excluded in cases of hysterical delirium.

Under the head of hysterieal coma may be embraced certain extraordinary cases, in which the mental faculties, the senses, and sensibility are suspended more completely and for a longer period than in orclinary cases. In view of the infrequency of such cases, and the embarrassment to the young practitioner which they are likely to occasion, I shall subjoin an account of two cases which have fallen under my observation, the patient in one of the cases being of the male sex :-

CAsE I.-Jacob Humble, German, aged 25, meehanic, was admitted into hospital in a state of apparent unconsciousness, and the previous history was not ascertained. On the morning after his admission he seemed to be in quiet sleep; he could not be roused to any manifestation of consciousness. On raising the eyelids, they remained open for some time, but he appeared to take no notice. Flies creeping on the face and even over the conjunctiva did not disturb him. The pupils were not dilated nor contracted, and responded fully to light. The respiration was perfectly normal, pulse 76 and regular, skin natural, no distension nor tenderness of abdomen. Drinks introduced into the mouth were retained there for some time, and mostly escaped, a small portion being swallowed. He lay motionless, not changing his position, and giving no manifestation of suffering. There were no physical signs of disease of the heart. An enema of spirits of turpentine and assafotida was directed. The cold douche was applied for some time to the head with no effect.

On the second day he was in the same state, and had so remained. Deglutition was not impaired; he swallowed when liquid was introduced into the mouth, but did not call the act into exercise by any voluntary effort. The vapor of ammonia applied to the nostrils occasioned contraction of the orbicular muscle of the mouth and flushing of the face, but no manifestation of consciousness nor effort to escape from the inhalation. No remedy was presented, but efforts to introduce nourishment directed.

On the third day there was no material alteration. He remained apparently unconscious and nearly motionless. The eyes were now, for the most part, open, but he seemed to take no notice. The eyes were fixed in one direction. He seldom winked. Drink and nourishment, introduced into the mouth, were mostly lost. There had been no evacuation from the bowels or bladder since his admission. The catheter was introduced without difficulty and with no voluntary resistance, and a quart of

Principles of Medicine. 
urine withdrawn. The iris was mobile. The finger could be brought quite into contact with the conjunctiva without causing him to wink. During my visit, while engaged in examining other cases, he changed his position in bed, which he had not been observed to do before. No treatment directed, except the administration of a little brandy and nourishment.

On the fourth day there was marked improvement. The eyes were open and denoted more intelligence, but he did not speak, nor appear to give heed to questions. He made slight and ineffectual efforts to protrude the tongue when requested. He took nourishment freely and with apparent relish. On the previous evening he asked for tea after some had been forcibly administered. He had on this evening a paroxysm of violent weeping.

On the fifth day the aspect and symptoms were not so good. He resisted the introduction of nourishment. He lay with the eyes open, but appeared to take no notice. Urinated in bed. No dejection since his admission. Croton oil, gtt. j, every two hours until operation, was directed.

On the sixth day there was no material change. The croton oil, together with brandy and nourishment, were foreibly administered, the patient resisting, holding the articles in the mouth, but finally swallowing them. Four drops of croton oil had bcen given without effect. On making preparations to introduce the catheter, he urinated, discharging a pint of urine.

On the seventl day there was no material change. He had had a free evacuation from the bowels, which he did not pass in bed, but disposed his body so as to pass it on the floor. "Firing" was resorted to on this day by means of a hammer heated by dipping it in boiling water. He bore the heated iron for some time without manifesting pain, but, on continuing the application, and stating that it was intended to carry the firing over the whole body, he began to make vigorous resistance, and at length protruded the tongue when requested, and took brandy and water freely. The firing was then discontinued, with the threat of repeating it, and resorting to the red-hot iron, if marked improvement did not ensue.

On the eighth day the improvement was marked. He took notice, drank without difficulty, had a free evacuation from the bowels, and manifested by signs his wish for that purpose. On the ninth and tenth days the improvement continued. He took, however, but little notice, and replied to questions slowly in a feeble whisper.

On the eleventh day he was made to sit up. Without any effort on his part, he was dressed and placed in a chair. He remained sitting in the same position, the eyes fixed in one direction, the lips somewhat separated. He protruded the tongue partially and slowly when requested, but did not reply to questions. Nourishment was administered with difficulty. The expression of countenance was that of the deepest dejection. On the twelfth day he remained the same, and was carried out of the ward to the balcony.

On the thirteenth day there was a marked change. He took notice, greeted me with a smile, and offered his hand. He walked of his own accord. But on the fourteenth day he again became indifferent, the eyes fixed in the same direction, and he resisted nourishment. He was in this state on the fifteenth day. He took no notice. Flies creeping over the fice, lips, and conjunctiva seemed to occasion no annoyance. At times there was constant nictation. He continued to resist the introduction of nourishment. Firing was again employed. This operation caused wri- 
things and exclamations of pain, and on discontinuing it he took nourishment as directed. There was no material change on the sixteenth day. The patient was made to sit up, and presented an air of intense abstraction. Firing was resorted to from time to time as a means of making him take nourishment.

On the seventeenth day the patient took food without difficulty, but remained in the same state as regards silence, motionless position, and an expression of abstraction. He was led about the ward, walking slowly with his eyes fixed upon the groind. Firing was continued, and it was apparently by the moral effect of this remedy that he was made to eat and walk about. The experiment was tried of leaving him unsupported in the centre of the ward. After remaining perfectly motionless for several minutes, he dropped down, but in a way not to receive any injury.

On the eighteenth day there was improvement. He talked and walked about on his own accord. On the nineteenth day he again relapsed into silence, with an expression of abstraction, and reluctance to take food. Firing was resumed, and, by means of this remedy, he was made to walk in the open air. On the twentieth day he walked about, ate, and talked freely. After this date he progressively improved, and was discharged well at the end of three weeks.

Nothing could be ascertained respecting the cause of this attack. The patient stated, after his recovery, that he recollected what had occurred during his illuess. I did not discover that the mind was exclusively occupied by any dominant iclea, as in ecstacy. Consciousness was not lost, but the exercise of the faculties dependent on the cerebro-spinal system were, in a great measure, suspended. A morbid moral perverseness appeared to be an element in the case, as in most cases of hysteria, but it was certain that the patient was not a malingerer.

CASE 2.-This case came under observation in the practice of the late Prof. Peniston, of New Orleans. The patient was a married lady, aged 27. She had had seven children, and, within the preceding eighteen months, two miscarriages. Three years before she had had an attack of hysteria, followed by mental aberration for a few weeks. I saw the patient but once. She passed immediately from the care of Prof. P. into other hands, and I did not learn the subsequent history. She was in a state of apparent mental abstraction. During my visit she sat in the same position, remaining motionless, with the eves closed. She made no reply to questions, and gave no indication of taking the least notice of persous or things around her. She could not be made to open the eyes or month. When the eyelids were raised, the eyeballs were rolled upward so as to conceal the greater part of the cornea. She resisted efforts to depress the lower jaw. The pulse was regular and not accelerated. At times she opened her eyes, and replied briefly to questions. She signified her desire for food and drink, and for conveniences to minate and evacuate the bowels. She took nearly as much food as in health.

My notes do not contain information respecting the condition as regards sensibility of the surface in this case. There appeared to be a suspension of the exercise of the faculties of the mind, save as connected with the instinctive wants. The condition had existed, with temporary periols of improvement, for several weeks. I have met with a case presenting, essentially, the same features as case No. 1. The patient was a young man whom I saw in consultation with Dr. J. S. Crane, of Elizabeth, New Jersey, and another similar case, except that catalepsy was added, has come under my observation at Bellevue Hospital. Both these cases ended in recovery. 
Hysterical delirium occurs generally subsequent to, or in alternation with, the paroxysms of convulsions or coma, which have been described; but it also occurs alone. It is preceded and followed by symptoms characteristic of the hysterical condition. The delirium is active, and is manifested, in some cases, by wild, excited talking, the mind passing rapidly from one topic to another. The mind may run on either gay or grave topics, or there may be an incongruous union of both. Patients are sometimes violent. Occasionally they use vulgar and obscene language. In some cases the mind acts under the influence of insane delusions, and these sometimes involve spectral illusions. A girl ten years of age, during convalescence from dysentery, after complaining for some hours of pain in the abdomen, presented symptoms which greatly alarmed her friends. She declared that she was dying; desired to kiss the members of the family for the last time; said she was going to her heavenly home, and, exclaiming, "I see my mother, I am coming," she fell into a state of unconsciousness. Being summoned in urgent haste, I found her in hysterical coma, from which she emerged in a short time, and recovered without any relapse. The mother of the patient had died two years before.

Of the pathology of hysteria in the severer forms just described, all that can be said, with our present knowledge, is, it involves a functional morbid condition affecting the nervons system, especially in its relations with the mental faculties. An important element of the affection is disorder and weakness of the will ; the affection, however, is by no means purely mental, but proceeds from a pathological state of the cerebral organs with which the perceptive and emotional faculties, together with the will, are connected. The three forms of hysteria which have been described differ considerably as regards the kind of morbid manifestations, but that they are different forms of one affection is shown by their coexistence or occurrence in alternation, by certain symptoms, characteristic of the hysterical condition, which occur alike in connection with each form, and by the fact that what we know of the causation applies equally to the three forms.

Hysteria may occur at any period of life, but in the great majority of cases, patients are between fifteen and twenty years of age. I have referred to a case in which it was well marked in a girl ten years old. It is certainly of rare occurrence either before the evolution of the sexual instinet, or after the period when it may be supposed that venereal desires have ceased; but it sometimes continues into advanced life, and an instance has been referred to in the second paragraph of this chapter. It is an affection peculiar to females, but it occur's in males. I have notes of several cases, in addition to the case of hysterical coma which has been detailed, in which men were affected with well-marked hysteria in its severer forms. The symptoms which characterize the hysterical condition are not very uncommon in males. The name hysteria, therefore, which, in its etymology, signifies the uterus, is a misnomer.

The causation appears to require a peculiarity of constitution. There are some persons who are constitutionally prone to hysteria, and there are many who are incapable of having it. The hysterical diathesis is apt to exist in mernbers of the same family. A great variety of causes may contribute to the development of the affection, among the more frequent being anæmia, overtasking of mind and body, mental anxiety or grief, and the prostration incident to various diseases. The influence of causes pertaining to the sexual system has doubtless been much exaggerated; but the agency of excessive venereal indulgence or masturbation, on the one hand, and of continence on the other hand, in certain cases, is not to be doubted. Sudden disappointment, aflliction from loss of friends, violent 
anger, jealousy, and other kinds of strong mental excitement, often act as exciting causes of an hysterical paroxysm. A desire to excite in the minds of friends or others anxiety or alarm, and to furnish occasions for interest or sympathy, contributes to the abandonment or perversion of the power of the will, which enters into the paroxysms. The hysterical constitution may be developed, and is often fostered, by the absence of wholesome restraint and discipline in the management of youth, and by an unfortunate habit to which females, more than males, are apt to become addicted, namely, of concentrating unduly the attention on personal sensations and sentiments.

As regards prognosis, hysterical attacks are proverbially devoid of danger. 'The praetitioner, however, is not to lose sight of the fact that hysteria may be associated with affections which are serious. He should not be too rearly to set down morbid phenomena to the account of hysteria. It behooves him to be careful not to confound bysteria with other affections.

The treatment of hysteria is to be considered with reference, first, to the hysterical condition, and, second, to the paroxysms which have been described.

The hysterical condition calls for remedies to soothe the nervous system, but opiates are to be avoided. Valerian, assafotida, lactucarium, ether, belladonna, hyoseyamus, and the valerianate of ammonia are appropriate remedies. Of these, the first two are the most efficient. The bromide of potassium, in large doses, has, in some cases, a remarkably soothing effeet. Alcoholic stimulants should be recommended, if at all, with great reserve, lest the patient take advantage of the sanction of the physician, and resort to them more frequently and freely than prudenec warrants. The most important part of the treatment is hygienic. The object is to invigorate both body and mind. With regard to bodily vigor, good diet, abundant exercise in the open air, and regular habits as regards sleep, are important. With regard to mental vigor, healthful occupation of mind, and avoidance, as far as possible, of everything calculated to produce undue development of the sentiments and passions, or to excite the imagination, are to be enjoined. The causes which may be suspected, in individual cases, are to be removed as far as practicable. The patient should be encouraged to endeavor to increase the power of the will to resist a tendency to give way to emotional disturbance. For this purpose, the discreditalle character of the affection may sometimes be referred to with advantage. The moral management will involve details suited to inclividual cases, which must be left to the discretion, delicacy, and tact of the practitioner. Cases in which ailments are exaggerated or simulated with a view to excite attention, curiosity, or commiseration, are unhappily not infrequent. As the determination of the patients, in these cases, is not to get well, the varied measures of treatment which are adopted prove, of course, ineffectual. These cases sometimes tax most severely the patience and temper of the physician.

In treating cases of hysterical convulsions, it is to be borne in mind that, if left to pursue their course, simply preventing the patient from the selfinfliction of wounds or bruises, they would end of their own accord, and it would perhaps be as well for the patient if the paroxysm were allowed to exhaust itself. But the anxiety and alarm of friends generally require a resort to measures to arrest the convulsive movements. The prolongel application of the cold douche to the head is generally successful. The head of the patient is to be held over a tub, and cold water poured upon it continuously until the convulsions cesse, and the patient admits being 
relieved and begs that the measure may be discontinued. Recollecting that consciousness is not usually abolished, and that the convulsions depend upon a delirious volition, the physician should take care to state repeatedly that the douche is to be persisted in until the patient is able to express relief, and is to be repeated if the relief be not permanent. It is unquestionable that the efficiency of this measure depends, considerably if not chiefly, on its moral effect. The physician should also make to the friends, within the hearing of the patient, positive assurances that there is no danger, and that the paroxysm will certainly be arrested. After the convulsions cease, if the patient be much distressed, an opiate may be given, either by the mouth or by the hypodermic method, but if valerian, assafoetida, or ether will suffice, they are to be preferred.

In an ordinary paroxysm of hysterical coma, the douche, as just described, will almost invariably succeed in restoring consciousness. The same course is to be pursued, with respect to moral management, as in paroxysms of hysterical convulsions. The treatment, after consciousness is restored, is the same as in cases of convulsions after these have ceased. In cases of prolonged hysterical coma, "firing," repeated from time to time, is the most effective measure. Nourishment should be given forcibly, if necessary, and as sensibility and consciousness return, the exercise of the will in taking food, sitting up, and performing other voluntary acts, should be enforced.

In hysterical delirium, if mild, the remedies which have been mentioned, valerian, assafœtida, etc., with soothing managcment, may suffice. If the delirium be violent, the tartrate of antimony carried to the point of nausea, will be likely to tranquillize the patient.

After the paroxysms have ceased, in each of the three forms, the treatment resolves itself into that called for by the hysterical condition.

\section{Catalepsy.}

The affection called catalepsy, as this term is now commonly used, is evidently allied to one of the forms of hysteria, viz., hysterical coma. In the cataleptic state, the sansory functions, volition and consciousness, are mostly or entirely suspended. So far, the state is essentially the same as in some cases of hysterical coma ; but superadded is a peculiar rigidity of the voluntary muscles, retaining the limbs and trunk in a fixed position, the different parts of the body preserving the positions in which they may be placed by the hands of another. This superaded feature is characteristic of catalepsy.

The cataleptic state is generally preceded by symptoms of the hysterical condition, but it is sometimes developed suddenly, that is, without premonitions. The patient, while in this state, remains immovable, preserving the position in which the body happened to be at the time of the attack. In some cases the rigidity of the muscles is such that they offer considerable resistance when efforts are made to alter the position of any part, but in other cases the different parts are easily moved. The trunk or limbs are retained in positions which, in health, would require a strong exertion of the will, and for a longer period than would be possible in health. Thus, the limbs are sometimes kept for a long time extended, or, the patient lying on the back, the lower limbs may be raised and the trunk elevated, so that the body rests only on the sacrum, and this position, which, voluntarily' assumed, would soon become insupportable, is preserved for a consiclerable period. 'This remarkable feature is more or less 
marked in different cases-in other words, the catalepsy may be complete or incomplete.

As in cases of hysterical coma, the vital functions may be but little or not at all disturbed. The circulation may be regular, respiration natural, the temperature maintained, and, if food be ingested, the processes involved in nutrition may be well performed.

The affection is paroxysmal, but different cases differ widely as regards the frequency and duration of the paroxysms. They are sometimes short, lasting but a few moments; not infrequently they continue for several days, and they may extend to weeks and even months. In the majority of cases the duration is three or four hours. A single paroxysm only may occur, and, on the other hand, paroxysms have been known to be repeated daily for a long period. $\Lambda$ case is cited by P'uel, in which 1200 paroxysms occurred within twenty-eight months.

Catalepsy, in this country, exclusive of cases of so-called Mesmerism or animal magnetism, must be extremely rare. Irith pretty large opportunities for clinical observation for more than thirty years, I have met with but two well-marked cases. 'l'he statistical researches of Puel, embracing results of an analysis of 148 cases, show that it is not peculiar to females. Of these 148 cases, 80 were in females and 68 in males. ${ }^{*}$ In one of the cases which I have seen the patient was of the male sex. A large majority of cases are between 10 and 30 years of age. The affection, however, may oecur under 10 years, and at an advanced period of life. It appears to be induced especially by mental causes. Melancholy, over-taxing of the intellect, and violent excitement of the passions favor its development, and eauses frequently determining an attack are, hatred, jealousy, fright, domestic affliction, reverses of fortune, etc.

The prognosis is always favorable, as regards danger to life. It has, however, been observed to precede the occurrence of insanity, paralysis, and epilepsy.

The principles of treatment are essentially the same as in cases of hysterical coma. The condition of the muscles calls for the use of friction with stimulating liniments. Forcible alimentation may be required if the cataleptic paroxysm be prolonged.

An abnormal state, analogous to that of catalepsy, is among the curious phenomena which, within late years, have excited much attention, and are commonly known as the phenomena of Mesmerism or animal magnetism. The consideration of these phenomena does not come within the scope of this work.

I shall content myself with a bare allusion to certain abnormal conditions which properly belong to the domain of psychology. One of these is the condition known as ecstasy. In this condition, the mind, absorbed in a dominant idea, becomes insensible to surrounding objects. In some cases, during the ecstatic state, the body remains immovably fixed, as in catalepsy. Extraordinary visual hallucinations occur in some cases. The mental condition differs from that of catalepsy in this, the mind is active and thoughts or visions which occur are recollected afterward, whereas in catalepsy the action of the mind is suspended and the period passed in the paroxysm is a blank in the patient's memory. Another name for the ecstatic state is trance. As in some cases of catalepsy, the respiration and circulation may beeome so feeble that, without close examination, life may be supposed to be extinct. If the breathing be not realily ascertained, and

1 Mém. de l'Acad. do Mid., Paris, 1856, vide Valleix op. cit. 
the pulse be inappreciable, auscultation with the binaural stethoscope can hardly fail to reveal the heart-sounds in such cases.

Another condition is that called somnambulism. This condition is incident to sleep. It embraces the mental and physical performances, sometimes very extraordinary, which are observed in sleep-walkers. Similar phenomena are observed in the condition known as the Mesmeric or magnetic sleep.

\section{Tetanus.}

The term tetanus is applied to a functional affection characterized by persisting rigidity of the muscles of the jaw, and frequently of the greater part of the voluntary muscular system, with paroxysms of tonic spasms, the intelligence being preserved. In the great majority of cases, this affection follows a wound or local injury of some kind. It is then distinguished as traumatic tetanus, and when not traumatic, it is distinguished as idiopathic tetanus. Its symptomatic characters are essentially the same whether traumatic or idiopathic. From the large preponderance of traumatic cases, it belongs among the surgical affecions, and is treated of at length in comprehensive works on surgery. ${ }^{1}$ I shall briefly consider it as an idiopathic affection.

Idiopathic tetanus is everywhere rare, and in cold or temperate climates is one of the rarest of affections. It is less rare in tropical climates. It is one of the affections to which newly-born children are subject, especially in tropical climates, but occasionally everywhere. In adults it seems sometimes attributable to exposure to cold, and, with this apparent causation, it is ealled by some writers Tetanos a frigore. It has been conjectured that the disease is sometimes a manifestation of rheumatism.

In most cases idiopathic tetanus is developed abruptly, withont premonitions ; but in a certain proportion of cases it is preceded by indefinite ailments, such as chilly sensations, sense of fatigue, etc. The muscles first affected are those of the neck and lower jaw. The affection is sometimes limited to these muscles, and it is then distinguished as trismus. The jaws are firmly shut by the rigid contraction of the muscles, and hence the affection is known as lock-jaw. The month in some cases cannot be opened by any force which it would be prudent to employ; and drink, nourishment, or remedies can only be taken through the spaces between the teeth. The muscles of the face are frequently involved, giving rise to distortions of the features which are sometimes terrific. The angles of the mouth are drawn backward and upward giving rise to the expression called risus sardonicus. The tongue is sometimes severely bitten by being eaught between the teeth. The pharyngeal muscles are not infrequently involved, preventing deglutition.

If the affection extend to the muscles of the trunk, the abdomen bebecomes retracted and rigid, and the respiratory movements of the chest are restrained. The limbs become rigidly extended. The entire body is sometimes immovalle and stiff, so that it may be raised by the head or feet as if it were a statue.

Rigidity of the museles is persistent. But, at intervals, spasm is added to the persisting contraction. In the paroxysms of spasm or convulsions, the body is frequently bent backward by the predominant force of the posterior muscles of the trunk; and, in extreme cases, the body forms the arc of a circle, resting on the head and sacrum, and sometimes on the

1 Vide Gross's System of Surgery; also Hamilton's Surgery. 
head and heels. This is called opisthotonos. A much less frequent form of curvature is produced by the predominant contraction of the muscles on the anterior portion of the trunk. This is called emprosthotonos. A lateral curvature, still more rare, is called pleurosthotonos. The paroxysms vary in duration, and occur at intervals which vary much in different cases. They are attended with severe pain. The paroxysms are apt to be excited by any mental emotion, and by impressions transmitted from the surface of the body. In this respect, as also in the character of the convulsions, they resemble those which take place in cases of poisoning by strychinia.

As already stated in the enumeration of the characters distinctive of tetanus, the intelligence is preserved; delirium and coma are wanting. In general, the paroxysms are more frequent during the day than during the night. If deglutition be prevented by pharyngeal spasm, the patient suffers from thirst and hunger. Foamy saliva, under these circumstances, collects and escapes from the mouth. The bowels are usually constipated, but, in some cases, involuntary dejections occur. Retention of urine may occur, or, on the other hand, it may be passed involuntarily. The circulation may be but little, or not at all, disturbed, save as an effect of disturbance of the respiration. Respiration is embarrassed in proportion as the respiratory muscles are affected. The embarrassment is usually great during the paroxysms, and fatal apncea may be induced. 'The temperature of the body is notably raised, the thermometer in the axilla showing an inerease from $105^{\circ}$ to $110^{\circ} \mathrm{F}$. This high temperature is measurably, if not chiefly, an effect of the tonic contraction of the muscles. As regaris temperature, this disease differs from essential fevers in the absence of any regular thermometric fluctuations. The temperature is always raised during exacerbations in which the muscular contractions are increased. Profuse sweating occurs in the exacerbations, or fits of spasm, and more or less perspiration exists in the intervals. Contraction of the pupil to the size of a pin's point during the exacerbations is often observed, the contraction going off in the intervals. $A$ frequent symptom is pain at the pit of the stomach, piercing through to the back. Radcliffe, who thinks this symptom is rarely wanting, attributes it to a tetanic condition of the diaphragm.

The disease sometimes destroys life within twenty-four hours. On the other hand, in some very rare cases, it continues for several weeks; when it is called chronic tetanus.

In a large proportion of cases the affection ends fatally, after a duration varying from twenty-four hours to twenty days, the average duration being not far fiom ten days. The fatality is somewhat less from idiopathic than from traumatic tetanus. The inode of dying may be by apnœa or asthenia. The danger of apncea is in proportion to the amount of embarrassment of respiration. Death by asthenia is cansed by the exhaustion incident to long continuance of the rigidity and spasms, together with the difficulty, in some eases, of alimentation. 'It has been noticerl that, in some cases, rigor mortis succeeds the spasms during life, without any appreciable interval of muscular relaxation.

The distinctive characters of the affection render the diagnosis sufficiently easy. It is to be distinguished from spinal meningitis and cerebro-spinal meningitis, by the absence of local symptoms denoting inflammation, and from the latter of these atfections by the absence of cephalalgia, delirium, and coma. Absence of coma distinguishes it from epilepsy. Moreover, the latter is purcly paroxsymal, the muscles not heing contracted in the intervals. Absence of characters distinctive of hysteria 
sufficiently distinguish it from that affection. It is, however, to be recollected that trismus, with or without convulsions, is an occisional manifestation of hysteria, and, under these circumstances, is unattended with danger. 'The phenomena produced by strychnia in poisonous doses bear the closest resemblance to those of tetanus, and to discriminate between them is of great importance in a medico-legal point of view. Within late years strychnia has been repeatedly employed for homicidal and suicidal purposes. The following differential symptoms are quoted from 'lodd:-1

"As regards the tetanoid state from strychnine.-a. The rapid supervention of tetanoid convulsions affecting chiefly, and with most intensity, the muscles of the trunk and spine, causing an active and violent opisthotonos rarely met with in tetanus. $b$. A rigid and tetanoid state of the muscles of the lower extremities, with somewhat less intensity than those of the trunk; the limbs extended and the feet drawn powerfully inward by the action of the tibiales postici muscles. The upper extremities aflected also, but in a less degree, and the hands generally semiflexed. c. 'The trismus existing only imperfectly, and the facias tetanita very slightly or not at all; swallowing perfect, but the mode of deglutition peculiur, the patient snapping at the liquid offered, and gulping it down with an effort, in a manner very similar to that in which hydrophobic patients swallow. $d$. The attacks of opisthotones very frequent, seemingly exquisitely painful, and ushered in by a cry more or less loud.

"As reyards tetan $u s .-a$. The symptoms coming on gradually, and the trismus the earliest, the most promiment, and most important one. $b$. The facies tetanica a very characteristic symptom. c. The attacks of opisthotonos less frequent, less extensive, and less severe than in poisoning by strychnine. $d$. The extremities the last parts affected and suffering much less from the tonic spasms than other parts. e. Deglutition slow and difficult, and sometimes impossible, owing to the spasmodic closure of the mouth."

To these differential points it is to be added, that a fatal dose of strychnia kills quickly, usually within an hour, whereas, in fatal cases of tetanus, life is prolonged generally for two or three days.

Examinations after death, in cases of tetanus, have not established, as yet, definite, uniform lesions. Lockhart Clark is of the opinion that degeneration of the cells of the cord is constant. It is, however, a question, when lesions have been found, whether they are primary, or secondary, or, again, whether they are not associated with the disease merely by coincidence. With respect to the pathological condition, the analogy of the phenomena of the disease to those produced by strychnia is a strong point in favor of the doctrine which refers most of the neuroses to a morbid material in the blood. 'The symptomatic phenomena point to the medulla oblongata and the spinal cord, as the seat of the affection.

The treatment of tetanus, by different methods, has proved unsuccessful in a large proportion of cases. Among the measures which have seemerl to be sometimes successful are, opium given in large doses, aconite. alcoholic stimulants carried to the extent of producing intoxication, and the inhalation of chloroform. Data are at present insufficient for determining the relative value of these or other ineasures. Other remedies, which have been advocated as sometimes successful, are belladonna, cannabis Indica, and quina in large doses. Bloodletting and counter-irritation over the spine have been largely tried, and are not to be recommended. Ice applied to the spine has been found useful by Todd and others. Cold

1 Clinical Lectures on the Nervous System. 
- affusion has been employed with success. The high temperature of the body suggests the inquiry whether eold baths or the wet sheet, repeated at short intervals, with a view to maintain a reduction of the lieat of the body, as determined by the thermometer, may not be an effective plan of treatment. One advantage of this measure is, it does not conflict, and may therefore be employed eonjointly, with the various medicinal remedies which are advocated.

In addition to the remedies already named, others have been more recently employed, namely, the hydrate of chloral, the Calabar bean, woorara, and the nitrite of anyl, the latter by inhalation. Cases whieh have ended favorably under treatment by these remedies have been reported; but clinical facts do not, as yet, warrant positive conclusions respeeting the remedial power which they respeetively exert over the disease. The great number of remedies which have been advocated as possessing eurative efficacy in tetanus, suggests a reflection which has been already suggested in other connections. It is, when cases of a disease have ended fiavorably under a host of remedies, it may be reasonably conjeetured that the ending, in these eases, was due more to an intrinsic tendeney of the disease than to the remedies used. It remains to be determined in how large a proportion of eases tetanus would end in recovery without any actual medicinal interference.

A highly important part of the treatment relates to the quietude of the patient. The body should be moved as little as possible, and all excitement of the mind should, as far as practicable, be avoided. Light, eurrents of air, and noise should be excluded. A former eolleague, Prof. Brickell, of New Orleans, assured me that he treated a ease of icliopathic tutanus successfully in a negro man, simply by support and the utmóst care to exelude all sources of excitation. Nutritious alimentation is an important part of the treatment. If deglutition be impossible, food should be introduced by means of a stomach-tube which may be passed through the nostril.

A case of idiopathic tetanus of which I have notes is interesting and instructive in regard to the recovery after all treatment had been abandoned. I shall subjoin an account of this ease.

The patient was a boy, aged 7 years, the son of a washerwoman. The affeetion had existed for nearly a fortnight at the time of my visit. I saw the cuse with $\mathrm{my}$ friend and colleague, Prof. White, of Buffilo. At short intervals he was seized with opisthotonotic spasms, during which the limbs were rigid with the thumbs pronated. Rigidity of the muscles of the abdomen was marked, the penis was erected, and the jaws firmly closed. The embarrassment of respiration during the paroxysms was great. The paroxysms passed off gradually after lasting two or three minutes. They recurred spontaneously every few minutes, and oftener during the night than during the day. They were always brought on whenever the body was moved. During the paroxysm the body could be lifted, like a statue, by raising one leg. The angles of the month were depressed, and the features presented an expression of great clistress. A certain amount of trismus was constant; the mouth could not be opened sufficiently for the patient to take food freely or protrude the tongue. The pain was severe during the paroxysms. The mind was clear. He obtained but little sleep. The pulse was not accelerated. The disease

1 As bearing on this point, vide analysis of cases of traumatic tetanns treated by different measures, by $\mathrm{Dr}$. $\dot{\mathrm{D}}$. W. Yandell, in American Practitioner, Sept. 1870 ; also Treatise on Diseases of the Nervous System, by Hammond. 
could not be referred to any cause; he had not received any wound or injury.

Prof. White had treated the case with morphia, quinia, and the valerianate of zinc. It was resolved to try the effect of chloroform. The patient was brought under the influence of chloroform, and kept under it for two or three hours. 'The effect was to relax the muscles of the jaw, and to render the paroxysms less firequent. After this trial of the chloroform, the patient was left with directions for a report of the condition to be sent the following day. Nothing was heard of the case, and, from a misapprehension as to who was to take charge of it, the patient was not seen again for about ten clays. At the end of that time he was found to be greatly improved. The improvement continued, and recovery took place. After the trial of the chloroform, no treatment was employed; the mother did nothing but give nutritious food. The improvement was very gradual, slight spasms occurring from time to time after he had convalesced sufficiently to be up and about.

\section{H A P T E R X.}

Rabies. Delirium Tremens. Alcoliolism. Nervous Asthenia. Hypochondriasis. Pathophobia.

THE affections known as rabies canina, or hydrophobia, and delirium tremens, are to be included among the neuroses. The consideration of these, and of a morbid condition which I shall designate nervous asthenia, will conclude the section of this work devoted to diseases affecting the nervous system.

\section{Rabies.}

The affection called rabies canina, and more commonly known as hydrophobia, is due to the action of a special poison, a virus generally derived from the mouth of some animal of the canine or feline race, viz., the dog, cat, fox, or wolf. The virus is communicated by inoculation, that is, by the bite of an animal affected with the disease. An animal affected with the disease in this way communicates it to other animals, herbivorous as well as carnivorous. The former rarely communicate it, becanse they rarely bite other animals or man. That it may be communicated by herbivorous to carnivorous animals appears to be proven. The virus is contained exclusively in the fluids of the mouth; there is no proof that other fluids of the body are capable of communicating the disease. It would appear, also, that the virus enters the body only by inoculation; it is not absorbed from the healthy cutaneous or mucous surface. It has been repeatedly communicated from man to inferior animals by inoculation. The disease in man is much oftener derived from the dog than from any other animal; hence the name, rabies canina. Owing to the pains taken to confine or destroy animals suspected of having the disease, it is rarely seen in man. Only two cases have come under my observation. Of these cases I made copions notes at the time of their occurrence.

The disease does not make its appearance for some time after the bite. 
The usual period of incubation is stated to be from thirty to forty days. There is considerable variation in this period in different eases. In one of the eases which I have observed, the patient was bitten seven weeks, and in the other case eight wecks, before the disease appeared. There is ground for distrusting the accuracy of statements in eases in which the disease is said to follow very quickly after the bite, and, also, when several years have been supposed to elapse. The error in the first of these cases probably consists in eonfounding the disease with tetanus. The utmost possible duration of the period of incubation cannot be stated, and this is a point in the natural history of the disease of no little consequence with reference to the anxiety of persons after having been bitten by an animal known to be, or suspected of being, rabicl. It is probable that the period is very rarely, if ever, under eight or ten days; and that it very rarely exceeds a year.

The development of the disease is rather gradual. It is stated that certain morbid sensations emanating from the eicatrized wound preeede other symptoms; but, if this be true of some, it is eertainly not of all eases. There were no such premonitions in the eases which I have observed. The premonitions are, notable depression of spirits, so that this premonitory period has been called the stadium melancholicum, change of disposition, restlessness, mental agitation, vigilance or disturbed sleep, eephalalgia, chilly sensations, loss of appetite, a sense of distress referred to the epigastrium, and sometimes nausea and vomiting. Two or three days elapse before the distinctive characters of the disease are fully declared.

When the disease is developed, the most prominent feature is laryngeal spasm excited by the effort to swallow water. The patient has a dread of water because efforts to swallow it oceasion violent paroxysms of suffoeation and are generally unsuceessful. It is the association with these paroxysms which gives rise to the fear of water, or hydrophobia. After they have repeatedly been thus produced, the mere sight of water may be sutficient, to provoke them; but this is not always the case. In neither of the eases which I observed did the patients suffer any inconvenience from seeing, or hearing the sound of, water. Patients are sometimes able to swallow other liquids than water; in one of the cases whieh I have noted, spirit was taken without much inconvenienee, and in the other case, at times, water was taken from a teaspoon without exciting spasm. Pieces of iee were taken without any ineonvenienee. The paroxysms excited by attempting to swallow water are extremely violent. The patient, with a nervous determination, drinks precipitantly, and instantly respiration is arrested, the whole frame is agitated, terror and distress are depieted on the countenance, and the water is forcibly ejeeted from the mouth and nostrils. In some eases, paroxysms of laryngismus oeeur spontaneously; in one of the cases which I have noted, they were repeated 40 or 50 times in an hour, the patient suddenly and quickly raising himself in bed, the spasm lasting a few seconds, and in the intervals the respiration was hurried and panting. In other cases, the patient is free from paroxysms if he do not attempt to swallow liquids. A current of air is apt to excite laryngeal spasm; the patient complains that it takes away his breath. A bright light, the reflection from a mirror or any polished surface, and any mental exeitement, have the same effect. The countenanee expresses excitement, anxiety, and terror.

An abundant secretion of tenacious mucus from the fanees, together with an increased flow of saliva, leading to frequent and sometimes almost constant expuition, is a distinctive feature. An examination of the throat shows the fauces to be more or less reddened. At first, the mind is simply 
excited, but, in the course of the disease, delirium becomes developed. The patient talks wildiy and incoherently. Sometimes the delirium assumes the form of mania; the mind acts under the influence of hallucinations, and forcible restraint in some cases becomes necessary. There is no foundation for the vulgar notion that patients assume the eharacter of the animal from which the virus was received, and attempt to bite persons around them. In some cases delirium is manifested by excessive tenderness and affection. Salyriasis has been observed in males, and nymphomania in females.

Convulsions occur in a large proportion of cases. These are sometimes clonie, extending more or less over the voluntary muscular system, and they are sometimes tonic, as in tetanus. The pulse becomes frequent and small. Febrile movement does not belong to the history of the disease; the temperature of the body is inereased but little, or not at all. The surface presents capillary congestion and is sometimes covered with clammy perspiration. The vital forces progressively fail; the patient is worn out with continued vigilanee, the paroxysms of laryngismus, the convulsions and innutrition, the mode of dying being by asthenia.

Of all the diseases in the nosology, there is no one in which the intrinsic tendency to death is greater than in this. It may perhaps be doubted if any well-authenticated case has ended in recovery. The disease runs a brief career, death usually taking place on the second or third day, and always within five days after it has become fully developed. The disease has no anatomical characters. It does not sppear to exert a special tendeney to the production of any secondary local affection.

The diagnostic features of rabies are highly distinctive. It is distinguished from tetanus by the absence of trismus, and the occurrence of delirium. In some cases of tetanus, the deglutition of liquids is impossible; but the difficulty arises from spasm of the pharyngeal, not involving the laryngeal, muscles, the latter being affected in rabies. Moreover, convulsions occur at a late period in rabies, and the convulsions are tetanoid only in a certain proportion of cases. The only difficulty of diagnosis relates to the discrimination of cases of true rabies from those in which, to a greater or less extent, hydrophobic phenomena are simulated. Notable repugnance to the deglutition of liquids, susceptibility to currents of air and to light reflected from a mirror or some polished surface, frequent sputation, together with delirium and convulsions, are occasionally incident to various acute diseases. But the hydrophobic phenomena are especially simulated under intense apprehension of rabies after a bite has been inflicted by an animal suspected of being rabid. Excessive fear leads to the belief, in the mind of the patient, that the disease exists, and this belief leads to the production of certain of the phenomena, especially horror of liquids, delirium, and sometimes a fancied disposition to bite others. Among the points to be considered in this diserimination is the time which has elapsed after the bite. If the morbid phenomena occur immediately or quickly afterward, or, on the other hand, after a very long period, the reality of the disease is very doubtful. If it be evident that the horror of liquids is purely mental, or dependent entirely on spasm of the pharynx, as in some cases of hysteria; in other words, if laryngeal spasm be excluded, the affection is not true rabies. The continuance of the hydrophobic phenomena is another point. If they continue for many days or weeks, the affection is not true rabies, for the latter affection ends fatally within five days. The opinion, which some have entertained, that rabies may be developed in man spontaneously, probably has no foundation other than the cases in which hydrophobic phenomena have been inci- 
dental to other diseases: The disease, however, is spontaneously developed in the dog and other animals.

Of the various measures which have been resorted to in the treatment of rabies, none have proved successful. This disease has, as yet, baflled all the resourees of therapeutics. In one of the cases which I have observed, the trial of chloroform was commenced, but the inhalation appeared to increase the laryngeal spasm, and it was therefore abaudoned. In the other case, tracheotomy was performed early in the disease, as suggested by Marshall Hall, but without suecess. With our present knowledge, we must be content with fulfilling rational indications, viz., palliating the symptoms as far as possible, excluding all controllable causes of mental or physical disturbance, and supporting the powers of the system by alcoholie stimulants and alimentation.

There is no prophylactic treatment to be relied upon, save excision or effectual caterization of the wound as quickly as possible after it has been inflicted. Amputation of fingers or toes, and even of larger members, is advisable, if the extent or character of the wound be such that the exeision and eauterization cannot be effectually employed.

It is desirable, of course, to know whether an animal which has inflicted a wound be really rabid. Dogs and cats are often supposed to be rabid when they are affected with epilepsy or some disease other than rabies. It is a popular error that dogs are especially apt to beeome rabid during the so-called dog-days, that is, the hot montlis of summer. Cases are quite as likely to oceur at other periods of the year. When rabies is suspected, the animal should be confined and not killed until the character of the disease is fully declared. Rabies in the $\log$ is to be suspected when the animal manifests a notable change of habits, becoming shy and irritable, eating straw, bits of paper, etc., and refusing food. When the disease becomes developed, the appearance is much changed; the look is depressed and haggard, the ears and tail droop, the quality of the bark is altered, the eyes are watery, the fauces are reddened, saliva flows from the mouth, and there is febrile morement. Dread of water is no test of the malady in the dog and other animals. They frequently lap water without difficulty. Delirium supervenes, and then the dog snaps at his master as well as others, and at any animal which comes in his way. He seldom becomes ferocious, but bites and runs away. He appears sometimes to have hallucinations, and snaps at invisible objects. Convulsions may or may not oceur. Death takes place usually within five days.

Persons bitten by an animal known to be rabid are by no means invariably affected with rabies, even if no preventive measures are resorted to. Of 145 persons who were bitten by rabid animals in Wurtemberg, only 28 were affected with the disease. ${ }^{1}$ The chances of escape, thus, are as about five to one. The exemption from the disease may proceed from an insusceptibility to the poisonons action of the virus, but probably often, in those who escape, the inoculation does not take place. Of 100 inoenlations made by Herting, only 23 were suecessful. If the bite be inflicted through elothing, the virus will be likely to be wiped away from the teeth before they penetrate the skin. The inoculation is more likely to take place from the bite of the wolf than from that of the dog. Of 250 cases, collected by Renault, of persons bitten by the wolf, 164, about two-thirds, became affected by rabies. ${ }^{2}$ 


\section{Delirium Tremens. Alcoholism.}

This affection, like that last considered, has a special causation. It proceeds from the abuse of alcohol. The various pathological effects of alcohol are considered as incident to a toxical condition, called alcoholismus or alcoholism. These effects enter directly into the causation of many affections, such as cirrhosis of the liver with hydro-peritoneum, fatty liver, epilepsy, muscular tremor, gastritis, pyrosis, various dyspeptic disorders, and the lesions of the kidneys embraced under the name Bright's disease. Indirectly, alcoholism favors the production of nearly all diseases, by lessening the power of resisting their causes; and it contributes to their fatality by impairing the ability to tolerate and overcome them. Taken in sufficiently large quantity, alcohol produces certain immediate effects which belong to the state commonly known as intoxication or drumkenness. The opportunities of observing these effects are, unhappily, but too common. The coma which exists when a person is profoundly intoxicated, or, in common parlance, dead drunk, is liable to be mistaken for apoplexy. The differential points have been presented in treating of the latter. Intoxication falling short of coma sometimes comes under the cognizance of the physician under circumstances in which the nature of the difficulty is not suspected by friends, as in females and children. Knowledge of the characteristic phenomena is then brought into exercise. And the physician, in these cases, is sometimes bound by delicacy and prudential considerations to withhold an enunciation of the diagnosis.

The habitual use of alcohol, beyond certain limits, produces a deleterious influcnce on the whole economy. In cases of chronic alcoholism, the digestive powers are weakened, the appetite is impaired, the muscular system is enfeebled, the gencrative function decays. The blood is impoverished, nutrition is imperfect and disordered, as shown by flabbiness of the skin and muscles, emaciation or an abnormal accumulation of fat. l'ain in the limbs and back is a symptom of frequent occurrence. The breath and emanations from the skin have a characteristic odor. The deleterious influence on the mental is not less marked than on the physical powers. The perceptions are blunted, the intellectual and moral faculties progressively deteriorate, until, at length, the confirmed inebriate, miserably cachectic in body and imbruted in mind, has but one object in life, viz., to gratify the morbid craving for alcohol. He exemplifies a variety of the form of mental derangement called dipsomania, from which recovery is extremely rare.

Delirium tremens, called, also, mania à potu, is an affection incidental to alcoholism. A mooted question is, whether the affection, ordinarily known by these names, be due to the sudden withdrawal of an habitual amount of alcoholic stimulation, or whether it be a direct consequence of the long-continued action of alcohol on the brain. Both explanations are correct. In a large proportion of cases, its development is evidently owing to the use of alcohol being suspended or much diminished. Thus, it occurs in persons who voluntarily undertake to abandon intemperate habits, or who are unable to obtain liquor, or who are prevented from drinking by the occurrence of some disease or accident. It is notoriously common among inebriates who are thrown into prison, and among those admitted into hospitals. It is apt to follow paroxysms of intemperance, in periodical drinkers, when the stomach refuses further alcoholic libations. On the other hand, it is sometimes developed notwithstanding the continued use of the habitual amount of alcohol. 
The symptoms attending the access are anorexia, insomnia, muscular tremor, more especially tremulousness of the tongue and notable dejection. This state is known among drunkards as " the horrors." 'The mental depression is so great as not infrequently to lead to suicide. There is no pain in the head nor febrile movement; the pulse is generally feeble, and the surface cool. This stage continues for one, two, or three days.

The development of the disease is characterized by mental aberration of a peculiar character. The derangement is characteristic, although presenting considerable diversity in different cases. The expression becomes wild, the eyes either vacant or staring. The movements are quick. The patient is constantly in motion. He talks incoherently. His mind wanders from one subject to another. He fancies that he has important business to attend to, wishes to go out, and requires to be constantly watched. To these manifestations of mental disorder are added illusions of the senses, phantasms and hallucinations of various kinds. He sees imaginary objects, such as mice, dogs, cats, lice, snakes, and ferocious animals. He hears noises of animals or men, answers imaginary questions, and is apt to fancy the proximity of persons who are bent on insulting or ridiculing him, or from whom he apprehends personal violence. Uneouth and unnatural representations appear to him to be going on. At first, he may be alle to appreciate the unreality of what he sees, hears, and imagines ; but, after a time, his morbid perceptions become delusions, and, however extraordinary and absurd, they are real to the sufferer. They are sometimes of a character to inspire the greatest possible terror. Thus, patients not infrequently leap from windows and are dashed in pieces, believing that they are pursued by wild beasts or by men who seek their lives. A patient who escaped in his night-clothes, under a sense of personal danger, ran barefooted over frozen ground for fifteen miles before he was overtaken by men on horses who followed in pursuit. 'The degree of terror must be fearful to lead to such an amount of physical effort and endurance. In some cases the delusions are ludicrous. Thus, a patient declared that he should be able to sleep, but whenever he fell into a dose there were persons under his bed who tickled his fundament with straws. Under the apprehensions which the delusions excite, patients are sometimes violent and dangerous, but they are usually controlled without much difficulty.

The insomnia continues. The patient gets no sleep for two, three, or four days, and sometimes for a still longer period, after the development of the affection. The delirium is always much worse during the night. Tremulousness of the muscles continues, but this is not always a marked symptom, although it enters into the name. Vigilance being a more constant symptom, it has been proposed to call the affection delirium vigilans, instead of delirium tremens. Anorexia continues, the bowels are usually constipated, the skin is cool, the pulse is feeble and, except under mental excitement, may be but little, or not at all, accelerated; the patient makes no complaint of pain in the head or elsewhere, and generally declares that he is well.

If the affection end favorably, sleep at length occurs. If the patient once become soundly asleep, he will be likely to remain so for many hours, or, if he awake, the speedy return of sleep may be counted upon. After a protracted sleep, generally the delirium is found to have disappeared; occasionally it continues, but is diminished, and disappears after renewal of sleep. Sleep appears to be, not merely a criterion, but a means of convalescence. After sleeping much of the time for one or two days, convalescence is established, and the patient recovers more or less slowly or rapidly according to the duration of the affection and his physical condi- 
tion at the time of the attack. If the affection pursue an unfavorable course, the insomnia persists and notable prostration ensues. The condition is not unlike that in an advanced stage of typhus; the delirium continues, with efforts to get out of hed, subsultus tendinum occurs, the pulse becomes more and more frequent and feeble, the skin is sometimes bathed in perspiration, and death takes plaee by asthenia, although the fatal termination is usually preceded by coma.

The prognosis is generally favorable. When not associated with other affections, a fatal termination is rare. The affeetion destroys life per se, chiefly in cases in which repeated attacks have occurred, and the constitution is broken with a long existing alcoholic cachexia. Occurring after wounds, surgical operations, or accidents, and assoeiated with other affections, as, for example, with pneumonitis, it adds much to the gravity of the prognosis.

The symptomatic phenomena show the affection to be cerebral, but the nature of the morbid condition, as in the other neuroses, is unknown. It is important to bear in mind that inflammation is not involved, nor is there ground to believe that congestion is an essential element. All that can be said of the pathological charaeter with our present knowledge is, that the prolonged toxical action of alcohol on the brain induces a peculiar morbid condition, giving rise to those manifestations of disorder which are characteristic of this affection, especially when the use of alcohol is from any cause suspended. According to Dr. Bence Jones, deficiency of the phosphatic constitneuts of the urine is distinctive of this affection, as compared with meningitis, in which the phosphates are increased. There is reason to believe that the morbid phenomena relating to the mind, as well as the progressive impairment of the vital forces, are to a considerable extent due to the insomnia. This is to be inferred from the fact that the delirium generally ceases after sleep lias continued for several hours. Inability to sleep is an essential feature of the affection, and the chief obstacle in the way of a favorable termination.

In general, the diagnosis is not attended with difficulty. The characteristics of the delirium render the affection easily recognizable when fully developed; moreover, the known habits of patients generally lead the praetitioner to expect it. Tremulousness of the tongue and limbs, with vigilance, if the patient be a drunkard, denote an impending attack. If now the patient complain of having remarkable visions or manifest illusions of the senses, the development is progressing. The cases in which the practitioner hesitates in coming to a diagnosis are those in which the habits are unknown. The affection may be developed in secret drinkers, and in persons who are supposed to be perfectly temperate. I have known a person to be attacked who was supposed by his friends never to taste any alcoholic beverage. As the diagnosis implies intemperance, caution in forming an opinion in some cases is advisable, and prudence in expressing the opinion when formed. Of course, it is a breach of medical ethics to reveal the character of the affection save to the patient, and, under certain circumstances, to his friends.

Excesses in drinking sometimes occasion a form of delirium, differing, pathologically, from delirium tremens, but apt to be confounded with it. The delirium now referred to is an active delirium, characterized sometimes by violence or fury, and sometimes by hilarity. It is a form of acute mania due to active cerebral congestion, conjoined with the immediate effect of alcohol on the brain. It is occasioned by a protracted paroxysm of drinking rather than by the habitual use of alcohol. This form of delirium has been distinguished as delirium ebriosum. It is 
accompanied by pain in the head, increased heat, throbbing of the carotids, febrile movements ; in short, by the symptoms of active cerebral congestion. It is of brief duration, ceasing when alcoholic stimulants are discontinued; it thus ccases under the circumstances which are apt to occasion delirium tremens. It may be followed by delirium tremens. Aside from the discontinuance of stimulants, it claims the treatment indicated by active congestion of the brain, viz., bloodletting, purgatives, etc., measures not suited to the treatinent of delirium tremens.

Finally, with respect to the diagnosis of delirium tremens, meningitis is excluded by the absence of cephalalgia, intolerance of light and sounds, throbbing of the carotids, febrile movement, etc. Acute gastritis, which may present some of the phenomena of impending delirium tremens, is to be excluded by the absence of vomiting, gastric pain, tenderness over the stomach, etc. Typhoid and typhus fever are excluded by the absence of the diagnostic symptoms, other than delirium, of these diseases.

Delirium tremens was formerly treated by bloodletting and other of the so-caller antiphlogistic measures, under the belief that it involved either inflammation or cerebral congestion. These measures are now abandoned as not only uncalled for, but injurious. Within late years, various curative methods of treatment have been advocated on the grounds of success; and, in endeavoring to julge of their respective merits, it is to be borne in mind that, under favorable circumstances, the intrinsic tendency of this affection is to recovery. It may be said of this affection, as of others tending in like manner to recovery, that a large proportion of recoveries cannot be cited in evidence of the curative efficacy of any particular method of treatment, but a large proportion of fatal cases favor the presumption that the treatment was injurious. Excluding cases in which the affection occurs in connection with wounds, surgical operations, and accidents, or with other affections, and the cases in which it occurs in persons whose constitutions are shattered by intemperance, judicious management without therapeutical measures would, in the great majority of cases, prove successful. It does not follow, from this statement, that remedial measures are not often nseful.

The great object of treatment is to procure sleep. Opium has been employed largely for this object. The statistical researches of the late lamented John Ware showed that the fatality was greater under the opiate plan of treatment than when an expectant or eclectic plan was adopted, and that the fatality is proportionate to the excessive use of opium. Opium used indiscriminately and largely is undoubtedly injurious, but that it is useful in certain cases, moderately used, is not to be doubted. The use of opium as advised by Prof. Stille may be safely arlopted. This author says: "The best mode of exhibiting the medicine (opium) is undoubtedly to begin with a quarter of a grain, or its equivalent, and progressively to augment the dose by small and hourly additions until sleep is produced, or a sufficient degree of tranquillity obtained." I have not infrequently found a full dose, repeated, if required, after intervals of several hours, to act very favorably. The remedy should never be pushed to the extent of inducing narcotism, and if, on a fair trial, it be not found to tranquillize, it should be discontinued.

Cases have been treated by Gerhard and others satisfactorily by simply continuing the use of alcoholic stimulants in moderate quantity. In general, it is injudicious to discontinue entirely the use of stimulants so long as the affection continues. The time for breaking off the habitual use is after sleep has taken place and the patient is convalescent. Stimu- 
lants are to be given freely in cases in which the symptoms denote failure of the vital powers.

Tartar emetic, in nauseating doses, is useful in certain cases. This remedy, if not pushed too far, exerts a sedative influence, diminishes delirium, and disposes to sleep. It is suited to cases in which there is considerable constitutional vigor, and it should be very cautiously given to patients much enfeebled, or in cases in which there is a tendency to prostration. Emetics have been arlvocated as curative, but the evidence of this is unsatisfactory, and they are now very rarely employed. They are objectionable on the score of perturbation. Cathartics are objectionable on the same score. These evacuant remedies have been employed under the idea that they elininate the aleohol from the system. But the affection is due to the toxical effeets of alcohol, not to its actual presenee in the system; indeed, the attack often appears to proceed from a diminution of the amount of alcohol habitually present.

Cold affusions, the cold douche to the head, and the shower-bath are useful in some cases. They are of doultful expediency if employed against the will of the patient; he should be induced to concur in the propriety of making trial of them. They may do harm to feeble subjects, or if the powers of life be much depressed. My colleague, Prof. Sayre, has found immersion in cold water until the sedative effect of cold is apparent, an effective method of treatment in eertain cases. This measure should be resorted to only in eases in which there is considerable vigor of constitution ; and its employment requires much care and judgment.

Digitalis has been advocated as a curative remerly. It was introduced by Ir. G. M. Jones, of Jersey, Englanr, who was led to ascertain its efficacy by a patient affected with delirium tremens having taken an ounce of the tineture by mistake. Subsequently he employed it in seventy cases with the loss of only one case. No practitioner would have ventured to give this remedy in the doses which experience has abundantly shown may be given without any umpleasant consequences in this disease. 'The dose advised by Dr. Jones is half an ounce, which may be repeated, if required, after an interval of four hours. Numerous case in which this plan of treatment has apparently proved efficacious have been reported. 1 have tried it in a few cases, in one of which it appeared to act like a charm, but in the others no curative effect was apparent.

The inhalation of chloroform, in some cases, tranquillizes the patient and induces sleep. This is a remedy to be employed with great circumspection. Its effect has been happy in several instanees under my observation. In one case the effort to employ it excited such a degree of mania that I was led to desist. The hydrate of chloral may be employed, as a substitute for the inhalation of chloroform. The use of this remedy calls for circumspection. The bromide of potassium, given in large doses, appears to be a valuable remedy in the treatment of this disease. This remedy, for the past two or three years, has been relied upon in a large majority of the cases treated at. Bellevne Hospital.

As regards the measures which have been referred to, and others, they a re to be employed according to circumstances proper to individual cases. Wach of the measures is suited to some cases and not to other cases. 'They are to be employed tentatively, the practitioner being guided by the effect in deciding whether to continue or discontinue them. Not infrequently they may be tried successively in the same case. In short, the appropriate treatment, as regards these measures, is expectant and eclectic, and they will be employed judiciously or otherwise according to the judgment of the practitioner. 
The management in other respects is important. Quietude is to be enforced. Visitors, who are often actuated by curiosity, are to be excluded. The circumstances surrounding the patient should, as far as possible, be regulated with a view of promoting sleep. Much may be done by the exercise of tact in soothing the patient, combing the hair, bathing the face, resorting to warm pediluvia, ete. I have known museular exeleise, carried to the point of fatigue, useful. Alimentation is highly important. The patient should be encouraged, as much as possible, to take nutritions and easily digested food.

It is the duty of the physician, after recovery from this disease, to inform the patient of its character, and to point out the inevitable consequences of the habits which have induced it. And the patient will be aided in an effort to emancipate himself from the slavery of intemperance, by remedies and hygienic measures which tend to invigorate the body, thereby strengthening the mental powers.

\section{Nervous Asthenia.}

The term nervous asthenia may be used to denote a morbid condition sufficiently common in this conntry, especially in the larger eities. The propriety of considering the condition as a functional affection, and the name, have been suggested by my collengue, l'rof. Forlyce Barker. As the name signifies, debility or prostration affecting especially the nervous system, constitutes the affection. It oceurs without anemia or any notable disorder of the vital functions. It proceerls from undue functional activity of the nervous system as connected with the exercise of the intellectual and emotional faculties.

Physicians are often consulted by patients who, althongh far from being well, have no well-defined malady. 'They complain of languor, lassitude, want of buoyancy, aching of the limbs, and mental depression. They are wakeful during the night, and enter upon their daily pursuits with a sense of fatigue. Under the pressure of mental excitement, they may be able to exert themselves with their usual energy, but when the excitement subsides, they are jaded and worn out. 'They become apprehensive that their powers are giving way, and are apt to fancy the existence of some serious malady. An investigation of the different organs of the body reveals no evidence of disease; the lungs, heart, kidneys, etc., are sound. None of the affections embraced in the nosological catalogne may be discovered; yet, the morbid condition is real. 'This brief sketch represents a class of cases with which every physician is familiar.

An inquiry into the habits and cireumstances connected with the daily Iife in the cases now referred to, will slow that the mind has heen for a long period unduly taxed by the eares and responsibilities of business, over-exertion in the pursuit of wealth or other olyects of ambition, longcontinned anxiety or inquietude-in short, the patient is suffering from wear and tear of the nervous system. The action of mental caluses is increased by other causes which tend to diminish the vigor of the body, such as irregular habits with respeet to diet, the hours divoted to sleep and rest, ete. The causes which act directly through the mind are especially operative in this country, owing to the early age at which persons engage in the active pursuits of life, the great inducements to excessive exertions, and the emulation excited by the activity of others. It is not uncommon to meet with persons who have overtasked the nervous system, without any rest or recreation, for many consecutive years.

The condition of nervous asthenia is, in itself, not a small evil, causing 
much discomfort and unhappiness to the patient, and rendering him uncomfortable to others by inducing depression, irritability, or moroseness. That this condition favors the development of diseases to which there may be a predisposition, or co-operates with the various causes of diseases, is highly probable. It is apt to engender a desire for stimulants, and thus lead to intemperance. It may eventuate in confirmed melancholia, hypochondriasis, or other forms of mental derangement.

An essential part of the treatment relates to the causation. Frequently it is desirable to endeavor to procure, temporarily, complete relief from exertion and care. It is, however, extremely injudicious to advise permanent relinquishment of active occupations. After rest and recreation for a season sufficient for recuperation of the nervous system, a return to accustomed pursuits is advisable, with injunctions to observe proper moderation, and to comply with the laws of health as regards a fair apportionment of time to recreation, and a proportionate exercise of all the faculties of the mind. It is, of course, desirable that causes of mental uneasiness and anxiety should be removed, but this part of the treatment often is not within the range of medical control. Halits tending to impair bodily vigor are to be corrected, such as dietetic imprudences, late hours, sexual errors or vices, the immoderate use of tobacco, etc. Hygienic measures to invigorate the body are important, such as nutritious alimentation, out-of-door life, sea-bathing or the sponge-bath, etc. Tonic remedies, varied from time to time, and long-continued, are serviceable both morally and medicinally. As a calmative remedy in cases in which there is want of sleep, the bromide of potassium, and of ammonium, are reliable remedies, the former given in doses of from 20 to 30 grains shortly before bedtime.

A full consideration of nervous asthenia would require much more space than can be accorded to it in this work. The topic belongs properly to mental hygiene, a subject of vast importance in its pathological, social, and moral bearings. I must content myself with having offered a few hints for the reflections of the medical reader.

\section{Hypochondriasis. Pathophobia.}

Although hypochondriasis belongs in the list of disorders of the mind, the mental aberration is not regarded as amounting to insanity, and patients with this affection are not proper subjects for treatment at lunatic asylums. Cases are not infrequent, and they claim the services of the general practitioner. In view of these facts, it is appropriate to consider the affection as one of the neuroses. The name hypochondriasis, which from its derivation is supposed to have reference to morbid sensations sometimes felt in the hypochondriac regions, has very little significance as indicating the character and seat of the affection. The name pathophobia is much more expressive of the malady.

The characteristic feature of the affection is a morbid apprehension of either the existence of, or a liability to, some serious disease. In its mildest form it consists of a feeling of extreme delicacy of constitution, or of a peculiar susceptibility to the causes of disease. Existing in this form, it leads to an anxiety concelning health, and over-precautions for its preservation. In the severest form of the affection, patients suffer from the conviction that they have an incurable malady, and that life is in imminent danger. Different cases represent gradations between these extremes. The mental repression or despair which may be produced by a knowledge of the fact that a serious or fatal disease actually exists, does 
not constitute hypochondriasis. The hypochondriac is the victim of a delusion with respect to his pathological condition ; and the mental suffering arising from this error of belief often exceeds that which would be likely to be experienced if the fancied diseases really existed. Generally the hypochondriacal delusion is accompanied with more or less mental depression, and often with marked melancholia; but in some cases, paticnts are resigned and eheerful. Fancied diseases are sometimes the occasion of deep and pure religious sentiment, calling forth the manifestations of pious resignation.

The alfection is presented to the practitioner in different phases. In some eases the imagination becomes fixed upon a particular disease, and the patient's belief in the existence of the disense defies all efforts to remove it. Disease of the heart, pulmonary consumption, cancer, syphilis, tapeworm, softening of the brain, and diabetes are among the diseases which, in different cases, are supposed to exist. 'The physician may strive in vain by assurances and arguments to dispossess the mind of his patient of the delusion. In support of his conviction the patient sometimes not only exaggerates ailments which are present, but insists on the presence of symptoms which are wanting. The pertinacity and ingenuity with which, in some cases, patients hold to their faith and strive to convince the physician of its truth, is remarkable. Not infrequently the conflict between the physician and patient continues until it becomes intolerable, and each is well pleased to break off the professional relation. Hypochondriacs are apt to consult many physicians in succession, finding, for a time, greater attention and apparent interest in a new medical adviser; naturally enough, in their mental condition, they become dissatisfied when the patience of the physician becomes exhausted, and they have given up all expectation of converting him to their belief. 'The absurdity of the delusion in some instances is truly surprising. I have known two cases in which the patients, females of intelligenee and good sense, for a long time could not be persuaded that they were not affected with cancer of the breast, although nothing was to be seen or felt to give color to the delusion. It is a noteworthy faet that hypochondriasis is not confined to the weak-minded. l'ersons of education and strong mental powers are as liable to the affection as those who are ignorant and of feeble intellectual capacity.

In another phase of the affection there is not a fixed belief in the existence of a particular discise, but a shifting of the delusion from one disease to another. The physician may succeed in convincing the patient of the non-existence of an imagined disease, but no sooner is this done than the patient's imagination runs upon another disease, and so divers diseases are in turn imagined, until, at length, the physician is discouraged and is apt to give up the contest. Again, patients sometimes keep the house and take to the bed, complaining of ill health, prostration, and various morbid sensations, without selecting any particular disease. With eases of bedridden patients who have no definite malady, but a host of subjective symptoms, all practitioners of much experience are familiar. Such cases occur much oftener among females than males, and the phenomena embraced under the name hysteria are generally more or less involved.

The causes of hypochondriasis are physical and mental. Some persons are constitutionally prone to morbid apprehensions in this direction. Whenever they are ill with any affection, their mental constitution leads to a sense of danger and to despondency as regards recovery. Not infrequently this state of mind exerts a depressing influence which may interfere seriously with the favorable progress of discase. Persons thus 
unhappily constituted are apt to exaggerate the permanent consequences of dissipated habits in early life, and more especially of abuses of the sexual function. Delusions with respect to the latter are fostered by publications and lectures designed by unprincipled persons to produce this effect. Apprehensions on this score, often with little or no foundation, are a source of nefarious profit to a host of charlatans. If a person with a hypochondriacal predisposition either have had or imagines that lie may have had syphilis, he is liable to fall into the belief of supposing that his system is irretrievably ruined. This delusion also is encouraged by empiries whose business is to prey upon the fears which they foster and excite. 'There is a class of wretched liypochondriacs whose minds are absorbed with the idea that they are the hopeless victims of the tlooughtless error's of youth, and patients of this class, much as they are to be pitied, often tax severely the temper of the physician. It is to be adiled that the practice of masturbation, excessive sexual indulgence, and the intemperate use of alcoholic stimulants, are undoubtedly sometimes involved in the cansation, not merely by an effect upon the imagination, but by aiding to produce a morbid condition of the nerrous system.

'The reading of legitimate medical works is to be mentioned among the causes of hypochondriasis. Want of mental occupation contributes indirectly to the affection. Persons who have relinquished active pursuits are apt to become hypochondriacs. Success in the acquisition of wealth, when it leads to a determination to forego labor and to enjoy leisure, may thus endanger a far greater evil than poverty. On the other hand, the loss of fortune, or want of success in the pursuits of life, and severe affliction of any kind, are sometimes concelned in the eausation of this affection. 'These various causes may prodnce the affection in those who are not, as well as in those who are, constitutionally predisposed to it; but, of course, their efficiency will be marked in proportion as the predisposition is strong.

The morbid physical conditions often existing in cases of hypochondriasis, and more or less concerned in its causation, are varions. Among these may be mentioned anæmia, nervous asthenia, and dyspeptic disorders. The latter, there is reason to believe, are frequently to a considerable extent, effects of the morbid conclition of the mind.

The diagnosis of hypochondriasis must rest on an exclusion of the maladies which are apprehended or imagined, and of any serious disease. The different organs of the body are to be interrogated by means of symptoms and signs; and it is obvious that the accuracy of the diagnosis will depend on the ability of the physician to exclude the affections to which the different organs are subject. Subjective symptoms are to be admitted with cireumspection, and a certain amount of distrust. The incongruity of the previous history and the present condition, as described by the patient, with the laws of any definite disease, exclusive of mental disorder, is often at once significant of the affection under consideration; or the subjective symptoms are incongrnous with the physical disorders which may be associated with the hypochondriasis. From want of sufficient knowledge or skill to reach the diagnosis by exclusion, practitioners are sometimes misled by the statements of patients, and thus contribute unwittingly to establish more firmly existing delusions.

In a certain proportion of the cases in which some serious disease is either apprehended or imagined, the positive assurances of the physician suffice to dispel the delusion. In this way the pluysician who is confident in his ability to interrogate accurately the different organs of the body has the power of doing much good. 'The fear of the existence of somc 
grave disease oppresses especially those who are dependent for the means of living upon a certain measure of health; and a decided opinion, in which they have confirlence, is a great blessing to patients of this class.

In cases of confirmed hypochondriasis, the first object with reference to management is the renioval of any supposed causes. Over-exertion, mental or physical, if possible, is to be avoided; sexual abuses, intemperance, and any violation of the laws of health, are to be inquired into and reformed. Aside from the causal inclications, the measures of treatment relate to both body and mind. Any coexisting physical disorders are to be remedied as far as practicable. Anæmia and dyspeptic ailments often claim remedial measures. In addition to the physical operation of remedies, they undoubtedly have a useful moral effect within certain limits. Patients are apt to crave remedies, and it is important not to encourage this disposition; but it is often not judicious to withhold remedies altogether, even when the medicinal part of the treatment is comparatively unimportant. Alcoholic stimulants and opiates should not be prescribed, or, if indicated for a temporary object, they should be given with great circumspection.

The most important part of the treatment is that which relates to the mind. A rguments, assurances, and combined medical opinions may be brought to bear upon the delusions with more or less effect. It is, however, not always judicious to act upon a determination that the patient shall be at once reasoned out of the affection. It is not wise to attempt to dispel the delusions by ridicule or indifference. A fundamental part of the moral management is to direct the patient's attention from himself. This is to be effected by inducing occupation, if the patient has been living a life of indolence, and by well selected recreations, if the patient be of industrious habits. It is more difficult to effect this in cases of hypochondriasis among females than among males. Change of scene and new associations are often effectual, and, wherever practicable, should be advised. With respect to the particular plans to be pursued in different cases, the habits, intelligence, education, and social position are to be considered in each case, an'l the success of the treatment will depcud much on the judgment and tact of the physician. 


\section{SECTION FIFTH.}

\section{DISEASES AFFECTING THE GENITO-URINARY SYSTEM.}

\section{CH A P T E R I.}

Suppurative Nephritis and Pyelitis-Anatomical Characters-Clinical History-Pathological Character-Causation-Diagnosis-Prognosis-Treatment. Paroxysms of Pain caused by the Passage of Calculi along the Ureter, or Nephritic Colic, and Lithiasis or Gravel. Acute Desquamative Nephritis, Acute Albuminuria, Acute Bright's Dlsease, or Acute Inflammatory Dropsy-Anatomical Characters-Cliuical IIistory-Pathological Character-CausationDiagnosis-Prognosis-Treatment.

A LARGE proportion of the affections of the genito-urinary system do A not fall within the scope of this work. Excluding affections peculiar to females, the diseases of the male generative organs, and of the bladder in either sex, are to be excluded as belonging to surgical, rather than medical, practice. The diseases which remain are chiefly those affecting the kidneys. Abnormal changes in the urine are highly important, as denoting morbid conditions relating to the kidneys and the blood. These changes constitute important symptoms in various diseases; they are not to be considered as individual affections, and in general it suffices to notice them in connection with other symptomatic events which make up the clinical history of different diseases. 'There is, however, one disease which, in the present state of our knowledge respecting its pathological character and seat, it is convenient to consider as a urinary affection, although it is certain that the abnormal change in the urine expressed by the name of the disease is merely a symptom. The disease referred to is glycosuria or diabetes mellitus. It is common also to apply the term albuminuria to certain affections of the kidneys, characterized by the frequent presence of albumen in the urine.

Proceeding to treat of diseases affecting the kidneys, I shall consider, first, acute inflammation of the pelvis and calices, and of the interstitial tissue of these organs. The latter I shall consider under the name suppurative nephritis; inflammation of the pelvis and calices is called pyelitis. These are distinct affections, although the former rarely exists without the latter. It will suffice to consider both affections under the one heading.

\section{Suppurative Nephritis. Pyelitis. Pyelo-Nephritis.}

Suppurative inflammation of the kidneys is one of the rarest of diseases, if we exclude cases of pyæmia, those in which it is produced by extcrnal wounds or injuries, and, also, the cases in which the inflammation has extended from the pelvis and calices. The primary seat of the inflammation is the areolar or connective tissue which unites the convoluted tubes and the malpighian bodies; hence the affection is also called acute interstitial nephritis. Pyelitis, although a rare disease, is less infrequent. The 
latter occurs without the former, but suppurative nephritis is generally consecutive to pyelitis. Both affections, in the majority of cases, arise from the presence of renal calculi, and the terms calculous nephritis and calculous pyelitis have been used to denote the affections as thus produced; in these cases the inflammation is, in fact, traumatic. Next to the local action of calculi, distension of the pelvis and ealices from olsstruction of the ureter is the most frequent cause of pyelitis and suppurative nephritis.

Anatomical Characters.-The organ is more or less enlarged, the capsule is easily detached, and, on section, the ent surfaces present abnormal redness, softening, and ecchymoses together with suppuration. The pus is sometimes in numerous minute disseminated points, sometimes in distinct abscesses of greater or less size, and sometimes in the form of purulent infiltration. It is to be borne in mind that purulent formations in the kidneys occur, as in other organs, in eomnection with the morbid condition known as pyemia. It is rare for a single large abscess to be found in the substance of the kidney, and the inflammation rarely extends to the peritonemm over the kidney. Inflammation of the peritoneal covering of the kidney is called perinephritis.

Inflammation of the pelvis and calices causes redness, thickening, and softening of the lining membrane. If obstruction exist, the cavities contain more or less liquid which consists of muco-purulent matter and urine. According to Robin, urine in the pelvis and caliees, holding in suspension desquamated epithelium from the uriniferous tubes, presents the gross characters of pus, and is often mistaken for the latter in examinations after death. In cases of considerable and long-continued obstruction, the pelvis and ealiees beeome greatly dilated, the parenchyma is progressively atrophied by pressure, and at length the whole of the organ may be converted into a sac. Not infrequently the pelvis contains one or more calculi. Perforation may take place in different directions, viz., behind the peritoneum, leading to an abscess which may make its way externally through the integument; into the peritoneal cavity, giving rise to futal penitonitis; into the colon, stomaeh, or through the diaphragm, either into the pleural cavity or bronchial tubes. The retention of urine, without pyelitis, within the pelvis and calices, from obstruction of the ureter, is called, after Rayer, hydro-nephrosis. The accumulation of pus within the pelvis and calices is called pyo-nephrosis. The name pyelonephritis denotes the combination of pyelitis and interstitial nephritis.

Clinicat History.-In acute suppurative nephritis there is severe lumbar pain shooting toward the bladder and thigh, and accompanied sometimes by retraction of the testicle, together with notable tenderness on pressure over the affected kidney or kidneys. With these local symptoms are associated chills, or chilly sensations, fever more or less marked, and, at the commencement, often, vomiting. The secretion of urine is diminished especially if the kidney's are affected. The patient has a desire to urinate frequently, although the bladder may contain little or no urine. If the nephritis have followed a penetrating wound or other injury, the urine may be bloody. Exelusive of the cases in which it contaius blood, assuming that pyelitis does not coexist, the urine is not albuminous. The urine contains pus (pyelitis being absent) only when there is eoexisting inflammation of the bladder, or when a renal abscess has discharged into the pelvis of the kidney.

In pyelitis there is more or less pain in the lumbar region, but the pain 
is not usually severe, and it may be but slight. The pain produced by the presence of calculi, and especially by their passage along the ureter, is to be distinguished from the pain incident to inflammation. Distension of the pelvis and calices, if the ureter be obstructed, occasions an obscure, dull pain in addition to the pain due to the inflammation. The urine is often greatly increased in quantity, and contains muco-pus or pus in more or less abundance, provided there he not complete occlusion of the ureter. In determining that the muco-purulent or purulent matter comes from the kidneys, inflammation of the bladder, or cystitis, is to be excluded. With these local symptoms are associated more or less febrile movement and other symptoms denoting constitutional disturbance. The pyelitis may succed an attack of pain cansed by the passage of a calculus along the ureter, called nephritic colic, or the latter may occur during the continuance of the pyelitis.

Pyelitis may be acute, and, running its course, end in recovery, or the inflammation may continue and become chronic. In chronic pyelitis, the local symptoms persist, leading to progressive emaciation and debility with hectic fever.

In cases of suppurative nephritis, the primary affection is generally pyelitis; pus and urine accumulate within the pelvis and calices, leading, in some cases, to destruction of the kidney from extension of the inflammation and from pressure. This accumulation involves either occlusion or great obstruction of the ureter. If the obstruction be removed, a sudden and copious discharge of purulent matter with the urine takes place. If the obstruction continue, the contents of the abscess may be discharged in various directions, as already stated. The symptoms show the pyclitis to be either single or double.

- Pathological Character.-The inflammation, in suppurative nephritis and in pyelitis, is seated in different structures. In the former, the areolar or connective tissue, especially of the cortical portion, is the seat of the affection. In pyelitis, the mucous membrane lining the pelvis and calices is the structure affected. As already stated they are different affections, and either may exist without the other; but almost invariably the nephritis is secondary to, and dependent upon, pyelitis, excluding cases in which the nephritis follows an external wound or injury, and exclusive of cases of pyæmia.

Causation.-The causation of suppurative nephritis, in the great majority of eases, has just been stated; it is dependent on pyelitis. The affection, therefore, may be produced, secondarily, by the causes which give rise to pyelitis. As a primary affection, it may be caused by wounds and contusions. Possibly it may arise from excessive doses of certain remedies which act directly upon the kidneys, such as the nitrate of potassa, turpentine, and cantharides. It has been known to follow exposure to cold.

Pyelitis may be produced by wounds penetrating the pelvis of the kidney, but rarely, if ever, by contusions. The most frequent cause of this affection is the local action of calculi. It may arise from the presence of the entozoon of the kidneys, the strongylus gigas, and from a hydatid prodnction. Next to renal calculi, the most frequent cause is obstruction leading to distension of the pelvis and calices from an accumulation of urine. The obstruction may be due to the impaction of a calculus in the ureter, a morbid growth within the canal, a tumor pressing upon it; or to retention of urine within the bladder from stricture of the urethra, 
enlarged prostate, or paralysis of the bladder in cases of paraplegia. In some eases of cystitis the inflammation extends along the ureters to the pelvis of the kidneys. This has been observed to follow suppression of gonorrhœal urethritis. Among the causes of suppuration is to be mentioned the existence of the hrmic affection known as pyamia, multiple abscesses oceurring in the kidneys, in some cases of this affection, as well as in other organs:

Diagnosis.-Lumbar pain, more or less constitutional disturbance and bloody urine, after a wound or injury has been received in the region of one of the kidneys, render the diagnosis of inflammation of the substance of the kiclney quite positive. Except in traumatic cases, the disease, if primary, affeets usually both kilneys; and the diagnosis is to be based on the existence of lumbar pain and general symptoms, together with notable diminution or suppression of urine. If coma and convulsions supervene, they are to be attributed to uramia.

The diagnosis of pyelitis is to be based on the presenee of pus or mucopus in the urine, in conjunction with more or less pain and constitutional disturbance, cystitis being excluded. The occurrence of paroxysms of pain eharacteristic of the passage of a calculus along the ureter, and the expulsion of calculi or gravel from the bladder, are important diagnostic events. If the pyelitis be due to caleuli, the urine maly be bloody, but casts of the uriniferous tubes will not be found in the sediment of the urine. The sudden discharge of pus, in more or less abundance with the urine, shows that an accumulation in the pelvis of the kichey has eseaped through the ureter into the bladder.

Pyelitis caused by caleuli will be likely to be limited to one kidney, but if it follow eystitis, or retention of urine from an obstruction beyond the ureter, both kidneys will be likely to be affected. Complete or very great obstruction, causing an accumulation of urine within the pelvis of both kidneys, would lead speedily to uremia. Complete obstruction, however, limited to one kidney, may exist for an indefinite period, without leading to uramia, the other kidney being sufficient for the elimination of urea. The accumulation in the pelvis of one kidney may be sufficient to occasion a fluctuating tumor which may be felt through the abdominal walls. This tumor may attain to a large size, and in the female, it may simulate ovarian disease. It is to be differentiated from perinephritic abcess.

An accumulation of purulent matter within the pelvis and ealices, if the ureter remains permanently obstructed, eventuates in perforation and the discharge of the pus in some direction, provided the life of the patient be sufficiently prolonged. The source of the pus is to be determined by the antecedent and coexisting symptoms which point to the seat of the affection. I shall subjoin a condensed account of a case of suppurative pyelitis, in which the pus made its way outwardly, forming a subcutaneous abscess which was opened, perforation subsequently taking place into the peritoneal cavity. The history of the case was noted at the time it came under observation in 1857.

CAsE.-The patient was a mechanic aged 23 years. A bout a year before his admission into the hospital, he began to pass bloody urine. There was little or no pain in the region of the kidneys, but a sense of weakness and general debility. The blood in the urine disappeared after a few days, but recurred from time to time. About six months after the hamaturia first occurred, the urine became cloudy, and a white ereamy deposit was observed. The latter condition of the urine existed on his admission, and the creamy deposit was found to be pus. Shortly after his admission, 
a fulness and sense of fluctuation were found in the left lumbar region. In a few days, the fulness and fluctuation becoming more marked, the abscess was opencd by my colleague, Prof. Hamilton, and a quart of pus was at once discharged. The left lower limb was consiclerably œelematous. This nearly disappeared after the abscess was opened. His condition, in all respects, was improved, and he appeared to be progressing favorably, the discharge of pus externally and also with the urine continuing, until, nineteen days after the opening was made, he was seized suddenly with all the symptoms of acute peritonitis, and death took place in forty-eight hours afterward. An examination after death showed a membranous sac in place of the left kidney, the substance of the organ being completely destroyed. The right kidney was not enlarged, and was healthy in appearance. A perforation of the sac into the peritoneal cavity existed, and the appearances denoted recent peritonitis.

Prognosis.-It is probable that acute interstitial inflammation of the kidneys may be developed and end favorably by resolution withont suppuration. This may be conjectured from the clinical history, in certain cases, but there must always be room for distrusting the correctness of the diagnosis. A purulent collection, if small, may undergo the granulofatty degeneration, and be absorbed, recovery in this way taking place. Death sometimes occurs speedily, more speedily when the suppurative inflammation has a traumatic origin. But, in most cases which end fatally, the affection becomes chronic, the pus discharging in some direction, if life be sufficiently prolonged, and death follows a gradual decline with hectic fever and emaciation. If both kidneys are the seat of acute interstitial inflammation, fatal uræmia may occur even before suppuration takes place. Uræmia may be the immediate'canse of death after suppuration, if this involve great damage to both kidneys. The discharge of the pus into the pelvis of the kidney is the most favorable direction, and, next to this, the evacuation of a renal abscess externally. If the pus be discharged into the peritoneal or the pleural cavity, a fatal result is almost inevitable. In some cases the abscess opens into the alimentary canal, and pus is discharged by stool. Recovery has taken place under these circumstances.

The gravity of pyelitis depends on the causes which give rise to the affection. If produced by an extension of inflammation in cystitis, and there be no permanent obstruction to the flow of urine and pus into the bladder, a favorable termination may be looked for. If due to the presence of calculi, and these make their way into the bladder before destruction of the kidney ensues, recovery will be likely to take place. If from the size of the calculi their escape be impossible, the inflammation will be apt to continue, and the prognosis is unfavorable. Cases have been observed of pyelitis caused by a large calculus, in which, after having led to complete destruction of the kiclneys, the inflammation has ceased, and the calculus has remained encysted by the renal capsule, giving rise to little or no inconvenience, the remaining kidney doing all the work of renal elimination. Permanent obstruction to the flow of arine from the pelvis and calices of both kidneys, dependent on cystitis or retention of urine in the bladder from whatever cause, leads to destruction of the renal parenchyma and death from uramia. An accumulation of urine and pus in the pelvis and calices of one kidney may prove fatal by the perforation into the peritoneal cavity, as in the case of which I have given a condensed account. If the discharge take place either into the intestinal 
canal or externally, the patient is likely to be worn out by the prolonged drain and constitutional disturbance.

Treatuent.-Acute inflammation of the substance of the kidneys claims the measures of treatment appropriate in other parenchymatous inflammations, viz., local depletion in some cases, counter-irritation, fomentations, rest, restricted diet, etc. The danger of uræmia is to be considered. This will be proportionate to the diminution of the secretion of urine. If the urine be suppressed or greatly diminished, measures to effect the vicarious elimination of urea are indieated. The most efficient measures for this end are hydragogue cathartics and the hot air bath. Diuretics cannot be relied upon, and the propriety of making trial of them is questionable.

The treatment in cases of pyelitis must have reference to the causation. If dependent on retention of urine from stricture of the urethra, enlarged prostate, or paralysis, measures to relieve the bladder are of prime importance. If consecutive to cystitis, the latter claims appropriate treatment. Quietude is to be enjoined especially in cases in which the affection depends on calculi. Soothing applications to the loins, and anodyne remedies are indieated in proportion to the amount of pain. If the accumulation of pus and urine form a tumor which presents itself in an accessible situation, it should be opened. Tonic and supporting measures are required after the opening of the abscess either externally or into the alimentary canal.

\section{Hydronephrosis.}

This name should be limited to cases in which the nrine aceumulates in the pelvis of the kidney withont pyelitis. It is, however, one of the cansative conditions giving rise to the latter affection; the detention of the urine, and the changes which it undergoes, occasion inflammation, and this may extend to the interstitial renal tissue, giving rise to suppurative nephritis. Cases are, however, met with, in which the chief effeet of the hydronephrosis is atrophy of the organ from the pressure of the liquid. The pelvis and calices are more or less dilated, and the renal parenehyma is proportionately diminished. The latter, in some eases, is in fact destroyed, the whole organ being converted into a membranous sac.

Hydronephrosis is the result of an ohstruction to the passage of urine through the ureter. The obstruction may be due to various causes, such as the impaction of a calculus in the ureter and the pressure upon the latter of a tumor. From these causes the affection is unilateral. Recovery may take place with the loss of the kilney, the other organ being sufficient for the renal functions. In a specimen taken in the dissecting room from the body of an aged woman, the left kidney was destroyed by atrophy, the pelvis and infundibula were enormously clilated, and the leit ureter was greatly enlarged. The right kiclney was healthy. With these appearances, the specimen showed no obstruction. Obstruction had doubtless' existed, leading to the changes just stated, but the obstructing cause had disappeared. The most rational supposition is that the obstruetion was due to a calculus which at length found its way into the bladder and was discharged.

A specimen was exhibited at a meeting of the New York Pathological Society, by Prof. J. C. Hutchison, which illustrated the fact that one of the kidney's may be completely destroyed by hrdronephrosis without any manifestations of disease. The specimen was from a case in which death 
was caused by injury of the chest from a street car. The parenchyma of one kidney was destroyed by atrophy. Moreover, the other kidney (which was not exhibited) was considered as diseased; and the urine from the bladder after death was found to contain albumen and casts. The patient up to the time of receiving the fatal injury was apparently in perfect health, daily working as a laborer. I have met with a specimen of congenital hydronephrosis with atrophic destruction of the kidney.

Bilateral hydronephrosis, with more or less wasting of both kidneys, may occur from obstruction incident to persistent over-distension of the bladder and hypertrophy of this organ. In a case which came under my observation at Bellevue Hospital, the patient passed a very large quantity of limpid urine of a low specific gravity, and the case was supposed to be one of polyuria or diabetes insipidus. Uræmic coma and convulsions occurred, and the case ended fatally. On examination after death, the bladder was contracted, and the muscular coat enormonsly hypertrophied; both ureters were greatly dilated; the pelvis and calices of both kidneys were much enlarged, and the renal parenchyma nearly destroyed by atrophy.

Another case, occurring in private practice, presented the following facts: the patient was a gentleman 54 years of age, who had experienced more or less difficulty in micturition from boyhood. For some time previons to his coming under my observation, his general health had failed. He had become feeble ; his appetite was gone, and he suffered from diarrhœa. An examination of the uriue showed nothing except a very low specific gravity. A large, globular tumor was felt, extending upward from the pubes; this completely disappeared after two gallons of urine had been withdrawn by the use of the catheter. After this, vomiting became a prominent symptom, and he died by asthenia. The bladder, in this case, was found, after death, to be enormously thickened; both ureter's were dilated, together with the pelvis and calices of each of the kidneys; the pyramidal bodies were mostly absent, and the cortical portion much wasted. These changes were more marked in the left than in the right kidney. At the neck of the bladder there was notable thickening from hypertrophy of the submucous structure, evidently of long standing.

Unilateral hydronephrosis, with atrophy of the kidney, can hardly be diagnosticated. In making this statement I exclude, of course, cases of pyelitis and pyelonephritis, in which the pelvis of the kidney becomes distended sufficiently to form a fluctuating tumor which can be appreciated by manual exploration. These are not cases of hydronephrosis, although the latter may have preceded and given rise to the inflammatory affections. Bilateral hydronephrosis, with atrophy of the kidneys, may be inferred, when, after long-continued distension of the bladier, uræmic symptoms occur, and exuminations of the urine show notable diminution of urea, without the presence of either albumen or casts.

The treatment in the cases last referred to consists in meeting symptomatic indications, and averting impending danger from uræmia by measures to favor the vicarions elimination of urea through the skin and the alimentary canal. The liability to hydronephrosis and wasting of the kidneys, as a remote consequence of urethral impediments to the evacuation of the bladder, enforces the importance of removing these impediments, if practicable, by appropriate surgical treatment. 


\section{Perinephritic Abscess.}

Inflammation is sometimes developed in the areolar tissue surrounding the kidney, and eventuates in abscess. Owing to its infrequency, suppurative inflammation in this situation has received but little attention from medical writers. Dr. Henry J. Bowditch has reported three cases, the only cases which he has seen, and, singularly enough, they came under his observation within a period of nineteen months.

In each of Bowditch's cases the disease commenced in the neighborhood of the right kidney. Pain was a prominent symptom in all, the pain being referred to the situation of the disease, and extending down the limb. The patients were confined to the bed, and lay with the knees flexed. A tumor was found in the renal region. The pus, in each case, extended upward toward the chest, behind the liver; and, in one of the cases, pleurisy was developed on the right side.

In all these cases an external opening was made, and the three cases ended in recovery. $\Lambda$ considerable hemorrhage followed the opening in one of the cases. In two of the cases the kidney was felt by the finger at the bottom of the abscess. In two of the cases recovery took place in about six weeks after the abscess was opened. In the third case (the hemorrhage occurring in this case) convalescence was much more protracted.

In Bowditch's cases the patients were debilitated when the disease commenced ; in two of the cases it could be referred to a strain of the muscles, and in one case it followed exposure to cold and wet.

The diagnosis is to be based on the presence and situation of a tumor, in conjunction with the local and general symptoms denoting inflamm:tion. 'The symptoms resemble closely those of suppurative nephritis and pyelitis. These affections are to be excluded by examinations of the urine. In the former, the quantity of urine is notably diminished, whereas, in perinephritic abscess there is no interference with the renal secretion. In pyelitis, with which suppurative nephritis is usually associated, the urine contains pus.

Bowditch's cases show the importance of opening the abscess. If this measure be unduly delayed, the pus may be discharged into the peritoneal or the pleural cavity. Next to opening externally, the direction most favorable would be into the intestinal canal.

\section{Paroxysms of Pain caused by the Passage of Calculi along the Ureter, or Nephritic Colic, and Lithiasis or Gravel.}

Urinary concretions, or calculi, are generally formed within the renal cavities. Calculi of small size may pass from the kidneys to the bladder, giving rise to little or no inconvenience, and, having reached the bladder, they are either discharged with the urine, or, remaining in the bladder, they increase in size and require for their removal surgical interference. In some cases, calculi within the renal cavities, increasing in size, become too large to pass along the ureter, and are consequently retained. As has been seen, retention of urine within the pelvis and calices of the kidiney, pyelitis, renal abscess, and destruction of the renal parenchyma, are sometimes attributable to the presence of calculi or a calculus. A calculus retained in the pelvis of the kidney may continue to increase in size, and, leading to atrophy of the parenchyma, it may acquire a bulk nearly as great, or perhaps even greater than that of the healthy organ. Calculi not too 
large to pass through the ureter, and not small enough to pass with facility, occasion during their passage more or less pain and constitutional disturbance. The amount and duration of the pain caused by the passage of a calculus is in proportion to its size and the roughness of its surfaces. The pain begins when the calculus enters the duct and ends when it reaches the bladder. The paroxysm is analogous to that occasioned by the passage of a gall-stone from the gall-bladder to the duodenum. The latter is commonly known as hepatic colic, and the paroxysm of pain occasioned by the passage of a renal calculus is called nephritic colic.

A paroxysm of nephritic colic is usually developed suddenly. The attack, however, may be preceded by mole or less pain or a sense of uneasiness, due to irritation or perhaps inflammation caused by the presence of calculi in the pelvis of the kidney. The paroxysm quickly attains to its maximum of intensity. The pain in some cases becomes exceedingly severe. It is referred to the situation of the kidney, usually on one side, that is, by the side of the last dorsal and the first lumbar vertebra. The pain radiates from this point in different directions, but especially along the course of the ureter, extending frequently to the groin and thigh. In the male, pain is generally felt in the testicle, which is drawn upward by the contraction of the cremaster muscle. Frequently the pain is described as lancinating or tearing. It is sometimes so excessive as to force the patient to groan and cry aloud; he tries a variety of positions to obtain relief, walks about the clamber, and compresses the abdomen with his hands as in intestinal colic. The pain continues without intermission, but there occur exacerbations and remissions. The urine in most cases is notably diminished. 'The patient experiences a frequent or constant desire to micturate, passing only a few drops at a time. The sensation is well expressed by the term tenesmus of the bladder. Not infrequently the urine is bloody. If it contain pus or muco-pus, either pyelitis or cystitis coexists.

With these local symptoms are associated those denoting more or less constitutional disturbance, viz., anorexia, thirst, nausea and vomiting, coldness of the surface with sweating, and feebleness of the circulation. The countenance is pallid and expressive of anguish. The bowels are usually constipated. Suddenly, after a duration varying considerably in different cases, the paroxysm ends. The pain abruptly ceases. An abundant discharge of nine takes place, which may contain more or less purulent or mnco-purulent matter. The calculus has reached the bladder, and if care be taken to examine the urine, it may be found at the bottom of the vessel. The general symptoms rapidly disappear, and in one or two days the recovery may be complete. In some cases, however, one or more additional paroxysms follow, other calculi contained within the pelvis of the kidneys getting into the ureter in consequence of the dilatation caused by the ealculus which has just passed. A person who has experienced one paroxysm is liable to recurrences at periods more or less remote. The paroxysm is commonly known as a "fit of the gravel."

The duration of an attack of nephritic colic varies in different cases, but it is usnally short. In the majority of cases it lasts but a few hours, but in some cases it continues for one, two, or three days. Although the suffering is often extreme, it very rarely, if ever, proves fatal. The recovery is complete, unless calculi remain, which may occasion pyelitis, obstruction of the ureter, and destruction of the kidney.

The diagnostic characters of nephritic colic are the suddenness of the attack and termination, the situation of the pain and its extension to the groin, thigh, and testicle, the retraction of the testicle, the diminution of 
urine, the tenesmus of the bladder, the hæmaturia, and the discovery of the calculus. 'The fuct of antecedent attacks having occurred will assist in the diagnosis. The affections to be excluded are the form of muscular rheumatism called lumbago, lumbo-abdominal neuralgia, enteralgia, and hepatic colic. In lumbago the pain is less severe, is provoked especially by movements of the body, and is felt on both sides. Lumbo-abdominal neuralgia is characterized by tendemess at certain points. ${ }^{2}$ In enteralgia, the seat of the pain is within the abdomen. This statement also applies to hepatic colic. In all these affections the characteristic features of nephritic colic are wanting.

The treatment of an attack of nephritic colic consists of palliative measures. Complete relief depends on the escape of the ealeulus into the bladder, and there are no means of aiding direetly in effecting its passage. The propelling foree is derived from the accumulation of urine behind the calculus. The eopious ingestion of liquids may perhaps, by inereasing the urinary secretion, hasten its progress. Fomentations to the loins, opium given in full doses, and, if the suffering be excessive, the inhalation of chloroform, constitute the measures for palliation.

The discharge from the body of urinary concretions, either with or without the symptoms of nephritic colic, constitutes lithiasis or gravel. The term gravel is properly applied only to concretions formed within the body, not to those which form in the urine after its emission. Not infrequently coneretions, varying in size from that of a pin's head to a small pea, pass without giving rise to sufficient pain to constitute an attack of nephritic colic. The composition of the concretions varies in different eases. The most frequent form of gravel is that in which the concretions consist of urie acid. The concretions being usually of a red color, this variety is sometimes distinguished as red gravel. The urine in these cases always gives an acid reaction. The concretions may consist of the earthy salts, viz., the ammonio-phosphate of magnesia, phosphate of lime, and the carbonate of lime. 'The color of these is usually either grayish or white. The urine in these cases is frequently alkaline. In another variety the concretions consist of the oxalate of lime. These are of a yellow, brownish, or dark color. In cases of nephritic colie, the composition of the calculi which occasion the paroxysms may be inferred from the knowledge of concretions whieh have been already passed, and from an examination of the sediment which the urine deposits. If the urine contain uric acicl crystals or abound in the urates, the calculus is probably composed of uric acid. So, the urine will be likely to contain the phosphates in more or less abundance when the calculi consist of the earthy salts; and oxalate of lime caleuli will be likely to be preceded or accompanied by oxaluria. It may happen, however, that tho caleuli giving rise to nephritic colic are not of recent formation, and the condition of the urine giving rise to their formation may not exist at the time when the attack of colic oecurs.

The treatment of gravel, irrespective of attacks of nephritic eolic, will depend on the nature of the eoncretions or the character of the urine. In cases of uric acid gravel, alkaline remedies are indicated. The liquor potassa, in half drachm doses thrice daily, may be given, but the biearbonate of potassa, from a seruple to half a drachm thrice daily, is preferable. The latter may be given during effervescence with a vegetable acid. The Vichy water is well suited to this variety of gravel, and its effieacy is due to the presence of the carbonate of soda. The artificial is 
probably as efficacious as the natural water. The phosphate of soda and ammonia, introduced by Dr. Buckler, of Baltimore, is considered as having the power of keeping uric acid in solution in the urine, given in doses of ten grains thrice daily. The benzoic acid is another remedy, given in doses of eight or ten grains thrice daily. The carbonate of lithia was introduced by Garrod as a remedy which promised to be especially valuable from its forming with uric acid a highly soluble compound. Experience has shown it to have much value. It may be given in doses of five grains three times daily. All these remedies act as solvents, and preventing uric acid concretions. In addition to these remedies, attention to the digestive organs, the skin, and the functions generally, constitutes an important part of the treatment.

In cases in which the concretions are composed of the earthy salts, the mineral acids are indicated, either the sulphuric or hydrochloric. The nitro-liydrocloric acid is especially indicated in cases of oxalate of lime gravel. Diuretic remedies and the ingestion of water abundantly form an important part of the treatment in all cases of gravel. The phosphatic and oxalate of lime concretions are apt to occur in persons suffering from nervous asthenia, and the latter condition claims appropriate management. A bstinence from articles of diet containing oxalic acid is to be erijoined if the concretions consist of the oxalate of lime.

As already stated, the sedimentary deposits which occur in urine after its emission are not properly called gravel. It is important, however, to take cognizance of these as constituting evidence of disorder, and as involving a liability to the formation of concretions within the kidney or bladder. The conditions of the urine known as lithiuria, oxaluria, and phosphuria, standing respectively in relation to the different varieties of gravel just noticed, claim appropriate treatment, although concretions within the body have not taken placc. For further details respecting these and other disordered conditions of the urine, the reader is referred to works devoted to urinary affections. ${ }^{1}$

\section{Acute Desquamative Nephritis. Acute Tubal Nephritis. Acute Albuminuria. Acute Bright's Disease. Acnte Inflammatory Dropsy.}

These several names denote an affection characterized by the presence of albumen in the urine in more or less abundance, and general dropsy. These characteristic features belong alike to the clinical history of eertain chronic affections of the kidneys, our knowledge of which dates from the published researches of Richard Bright in 1827. The latter affections will be considered in the next chapter. From these the affection now to be considered differs in being acute, and not involving degenerative structural ${ }^{\circ}$ changes. The acute affection, although not devoid of a certain amount of danger, in a large proportion of cases ends in complete recovery. The chronic affections, involving irremediable structural lesions, sooner or later end fatally. The former has long been familiar to practitioners as the dropsical affection which is apt to occur as a sequel of scarlatina. The character of the affection, however, was not understood prior to the writings of Bright. Much additional light upon its character has been shed by the researches of George Johnson.

'Thudicum on the Urine, and Roberts on Renal and Urinary Diseases, may be especially mentioned. 
Anatomcal Ciraracters.-The kidneys are increased in volume and weight. The weight of one of the kidneys sometimes amounts to or exceeds eight ounces, the normal weight being from three to five ounces. The renal substance is in some cases everywhere engorged with blood, but in some cases the cortical portion presents cverywhere an anrmic appearance; generally the cortical portion presents engorged and exsanguine patches, in varying proportions in different cases. Red points, more or less in number, are observed upon the surface, and within the cortical portion. These are due to minute ecchymoses. The cortical portion is more friable than in healthy organs. 'The cones are deeply congested, and the tubes, separated at their bases by the swollen cortical substance, present an appearance which has been compared to a sheaf of wheat. The surface is smooth, and the capsule is readily removed.

Microseopical examination shows an aceumulation of epithelial cells within the convoluted tubes, especially marked in the parts which present an anxmic appearance. Some of the tubes contain what has been supposed to be exuded fibrin. The contents of the tubes, when squeezed out, resemble the epithelial casts found in the sediment of the urine. The microscope shows the red points, apparent to the naked eye, to depend on extravasated blood, and not merely on congestion of the Malpighian bodies. The extravasation takes place into the tubes and also in the intertubular spaces. The epithelium in some of the tubes contains drops of oil. As regards the gross and microscopical appearances, both kidneys are affected in a similar manner and in about an equal degree. It will be noted that the morbid appearances in this form of disease do not denote any structural change in the kidneys. The anatomical changes are due chiefly to the accumulation of epithelium together with an exuded material within the tubes, and to more or less congestion.

Clinical History.-_Subcutaneous oedema is a pretty constant symptom, and is usually the first symptom pointing to the existence of this -affection. The odema is generally first observed on the face, particularly below the eyes, but it is speedily observed in the lower extremities, and sometimes occurs first in the latter situation. Coincident with the appearanec of the dropsy is more or less febrile movement, frequently preceded by chills or shiverings, together with thirst, anorexia, and pain in the loins. Vomiting is a frequent symptom at the commencement of the affection. The skin is dry, and the countenance becomes pallid. The dropsy increases and becomes general, varying considerably in amount in different cases. In some cases the anasarca is great, the limbs, scrotum, and penis, or extermal labia, becoming enormously swollen. In other cases it does not exceed a moderate amount. More or less dropsical effusion usually takes place into the peritoneal and the pleural cavity. Hydrothorax sometimes occurs to such an extent as to occasion great suffering from dyspnca, and endanger life.

The urine furnishes important symptoms. The quantity is usually scanty. It may be quite small, and suppression sometimes oceurs. Tho specific gravity generally exceels that of health. Tested with heat or nitric acid, the urine is found to contain albumen usually in considerable, and frequently in great, abundancc. Not infrequently the urine contains blood. A small quantity of hematin gives to it a smoky or sooty appearance, which is quite characteristic of this affection. Rayer compares this appearance to that of bouillon de bœuf.

Microscopical examination of the sediment of the urine shows frequently blood-globules, together with renal epithelium and crystals of uric acid. 
But the most characteristic objects are cylindrical bodies which are evidently moulds of the convoluted tubes, and are commonly known as casts. These casts are of different kinds. Those regarded as especially characteristic of this affection are composed chiefly of desquamated epithelium from the convoluted tubes. Johnson distinguishes them as epithelial casts. Their average diameter is about $\frac{1}{0} \sigma$ inch. Other casts consist of an exuded material resembling fibrin. These are diaphonous or wax-like, and are known as hyaline or waxy casts. To these casts, epithelial cells, in more or less abundance, are frequently adherent. The waxy casts are of large or small size. If large, the diameter is about $\frac{1}{50} \overline{0}$ inch. If small, the diameter is about $\frac{1}{100}$ inch. Large waxy casts are comparatively few in number in this affection. Occasionally the casts appear to consist of coagulated blood, that is, of exudation with adherent blood-globules. These are called blood-casts. Robin affirms that the hyaline or waxy casts do not consist of fibrin. He states that they are not striated or fibrilated like recently coagulated fibrin, nor are they uniformly granular like fibrin which has been for some time coagulated. Moreover, according to this observer, the granular, the hyaline, and the epithelial casts are found in eertain of the straight and convoluted tubes, in the contents of pelves of the kidneys, and sometimes in the urine contained in the bladder in bodies dead with different affections, when there is no ground to suspect the existence of renal disease. He states, also, that they are found in the kidneys of animals killed for food. ${ }^{1} \quad$ The renal epithelial cells in the sediment of the urine sometimes contain a few oil-globules.

Coma and convulsions occur in a certain proportion of cases. These are symptoms of uræmia. Owing to an insufficient climination of urea by the kidneys, it may accumulate in the blood to an extent sufficient for the manifestations of its toxical effects upon the brain. Vomiting and purging may precede, or occur without the cerebral symptoms of uræmia, being due to an effort to eliminate urea through the gastro-intestinal $\mathrm{mu}$ cous membrane. Impaired vision and amaurosis are among the effects of uræmia occasionally observed. Blindness, which may be complete, when it occurs in acute albuminuria, is generally sudden; it is often of temporary duration, and recovery is complete. The ophthalmoscope, under these circumstances, shows no morbid appearances within the eye. The morbid condition is central. It is otherwise, as will be seen, in amanrosis occurring sometimes in the acute affection, but oftener in cases of chronic albuminuria. The amaurosis which is not associated with changes appreciable with the ophthalmoscope, is called uræmic amaurosis. It occurs especially in cases of acute albuminuria developed in connection with scarlatina and pregnancy.

Complications considered as occurring sufficiently often to show, not merely coincidence, but a pathological connection with the affection, are bronchitis, and serons inflammations, especially pleuritis and pericarditis. Pulmonary œdema is an occasional complication.

In the course of the affection, the symptoms are apt to present considerable variation on different days. The quantity of urine, the amount of albumen, etc., vary, and so with respect to the œdema, the effusion into the cavities, and the febrile movement. If uræmic effects and important complications do not occur, the affection continues for a period varying between a few days and a couple of months, the average duration being about four weeks. The approach of convalescence is denoted by a notable diminution or disappearance of the dropsy, cessation of febrile movement, 
return of appetite, and an abundant secretion of urine. Albuminuria usually continues, but in a lessened degree, after the dropsy has disappeared. At length the urine becomes normal and casts disappear from the sediment. The latter sometimes continue to be found for some time after the albumen has disappeared. The affection may end in complete recovery, or it may eventuate in a chronic affection. The latter is rare. As a rule, if the patient be not cut off by uramic poisoning or the complications which are liable to occur, the recovery is complete and permanent.

Pathological Character.-This affection is an acute inflammation of the membrane lining the convoluted tubes; and, as a prominent feature is the desquamation of the renal epithelium, Jolinson suggested, as an appropriate name, acute desquamative nephritis. In this alfection, as in the clironic affections which involve structural changes, he regards the secreting cells of the kidneys as the primary seat of the loeal morbid manifestations, these depending on "an effort made by the cells to eliminate from the blood some abnormal product." According to this pathological view, morbid blood-changes underlie the local affection. The obstruction of the tubes and the loss of secreting cells lead to the transudation of blood-serum, causing the albuminuria, and to the defieient elimination of urea, causing in some cases uramic poisoning. Exudation takes place in more or less of these tubes; hence, the hyaline, or waxy casts. The diminished density of the blood-serum from the loss of albumen, totogether with the embarrassment of the capillary circulation flom the retention of urinary principles and the aceumulation of water in the blood in consequence of the scanty secretion by the kidneys, occasion the dropsy. An examination of the blood in cases of acute albuminuria shows a lessened proportion of albumen, a low specific gravity, paucity of red corpuscles, and an augmented amount of urea. According to Picard, assuming the normal proportion of urea in the blood to be 0.0177 in 1000 parts, the morbid increase varies between 0.0846 and $0.0215 .^{1}$ The bronchitis and serous inflammations which are apt to occur as complications, are supposed to be due to the action of urea accumulating in the blood. Vomiting and purging are attributable to an effort on the part of the gastro-intestinal mucous membrane to eliminate the urea vicariously.

Both kidncys being alike affected, the affection is bilateral, a fact which goes to sustain the rloctrine of dependence on a morbid condition of the blood. As exemplifying the pathological law of parallelism, and in other respects, the affection is analogous to bronchitis, and the name renal catarrh has been applied to it. The epithelial and other easts contained in the seliment of the urine may be compared to the matter of expectoration in cases of bronchitis. Niemeyer ealls the affection croupal nephritis. The name implies that a fibrinous exuclation takes place upon the surfice of the membrane lining tubes. $A \mathrm{~s}$ it is doubtful whether sueh an exudation oceurs, the propriety of the term is questionable. M. Cornil suggests, as the name of the affection, albuminous nephritis. In the able treatise on albuminuria by Dr. William H. Dickinson, the affection is called acute tubal nephrilis.

Causation.-This affection may occur at any period of life; young infants are not exempt from it. It oecurs oftener in males than in females. As already stated, in the majority of cases it is a sequel of scarlatina. It may occur during the progress of scarlatina, or follow

1 Traité des Mraladies à Urine Albumineuscs, etc., par J. Abcille, Paris, 1863. 
directly the stage of desquamation, but it is most apt to occur in the second or third week after the date of convalescence. It is an important question whether this sequel proceed exclusively or chiefly from an agency pertaining intrinsically to scarlatina, or whether it depends on extrinsic causes, such as the action of cold. It occurs in cases in which the utmost care is taken to place the patient beyond the ageney of extrinsic causes, but it is probable that the latter are frequently involved. In may be developed in a person much exposed to the scarlatinous infection, without any affection of the throat or an eruption, the person having already experienced an attack of scarlatina many years previously. I have notes of a case exemplifying the facts in this statement.

Exclusive of the cases in which it is a sequel of scarlatina, it occurs in various pathological connections; it is an occasional sequel of diphtheria, and of epidemic cholera. It sometimes occurs during the development of pulmonary tuberculosis, in the course of articular rheumatism, in cases of typhoid and typhus fever, erysipelas, and measles. Albuminuria and general dropsy occurring in pregnancy depend, in a certain proportion of cases, on this affection. As a primary affection it occurs especially in persons addicted to intemperance. In these cases it is sometimes difficult to say how much causative agency is to be attributed directly to the action of alcohol, and how much to the exposure incidental to intemperance. It is observed not infrequently to become developed after lying on the ground in a state of intoxication. It appears in some cases to be caused by exposure to cold in persons who are temperate, and, finally, it sometimes has no obvious causation.

Diagnosis.-The existence of albuminuria is not, in itself, adequate evidence of either the affection under consideration or of the chronic affections of the kidneys, which remain to be cousidered. Clinical observation shows that albumen may be present in the urine, in a small or moderate quantity, and for a brief period, in the conrse of a great number of diseases. In general, the cliagnosis is easily made, being based on the occurrence of anasarea, developed rapidly, accompanied with febrile symptoms, and an examination of the urine showing albumen in abundance. Dropsy is an early symptom in the great majority of cases, but it is sometimes wanting. The diagnosis is then to be based on symptoms pertaining to the urine, in conjunction with general symptoms. In addition to the presence of albumen in the urine, the quantity is usually notably lessened; it frequently has a smoky or sooty appearance from the presence of hæmatin, and it is sometimes distinctly bloody. The sediment of the urine, examined microscopically, is found to contain, in greater or less abundance, the casts which have been described. The presence of these, in conjunction with other characters pertaining to the urine, and the general symptoms, render the diagnosis positive.

Prognosis.-The danger in cases of this affection is from uræmia and the complications which are liable to occur. Coma and convulsions always denote imminent and great danger to life, but recovery sometimes takes place notwithstanding these effects of uræmia. Pleuritis, pericarditis, peritonitis, meningitis, and pneumonitis, developed in the course of the affection, are apt to prove fatal. Edema of the lungs may prove a cause of death. Dropsical effusion into both pleural cavities may take place to such an extent as to destroy life. An instance of death from this cause has not fallen under my observation, but I have repeatedly met with cases in which the dyspnca seemed to involve imminent danger to life. 
Exelusive of uræmia and serious complications, the prognosis is favorable. 'The affection does not tend to disorganize the kidneys. It is a self-limited affection, seldom continuing more than two months, and in some cases ending in from one to two weeks. It very rarely assumes a chronic form. Cases of the chronic affections which remain to be considered, as a rule, do not originate in an acute attack.

Uramic poisoning is to be apprehended when the urine is scanty; in other words, when the quantity of urea eliminated by the kidneys is small. Suppression of urine lasting twenty-four or forty-eight liours places the patient in imminent danger, and, if it continue, is a fatal symptom. Danger from uramia is not passed, although the albumen may have disappeared from the urine, if the sediment still contain easts. I have known fatal uræmic convulsions to occur under these circumstances.

Treatment.-The treatment embraces the following leading objects: 1. Diminution of the intensity of the renal imflammation, promotion of resolution, and restoration of the secretory function of the kidncys. 2 . Diminution or removal of dropsical effusion. 3. Elimination of urea through the skin and gastro-intestinal mucous membrane, if uræemia exist or be threatened.

With reference to the first of these objeets, rest and warmth of the surface are important. In general, the patient should keep the bed. The diet should be unstimulating. Water and other bland liquids should be allowed as freely as the thirst dictates. Opium should be given very circumspeetly, and, as a rule, is inadmissible on account of its tendency to diminish the secretion of urine. Dry-cupping over the loins is the best method of revulsion or counter-irritation, and, if the patient be not feeble or anæmic, the searificator may be employed. General bloorletting is advisable only in cases in which the patients are plethoric and attacked when in good health. As a rule, after scarlatina, in intemperate persons, and whenever the system is enfeebled from any cause, this measure is inadmissible. Fomentations over the region of the kidneys by means of moistened cloths covered with oiled muslin and several thicknesses of flannel, or the spongio-piline, are useful. Saline laxatives are useful by way of depletion.

With reference to the dropsy, either saline catharties or more active hydragogues are indieated in proportion to the amount and situation of the dropsical effusion. In cases in which suffering and danger are incident to hydrothorax, the elaterium is the most prompt and reliable hydragogue. I have repeatedly obtaineil, by means of this remedy, effectual relief in a few hours when the pleuritic effusion was sufficient to occasion alarming dyspnœea. Gamboge is highly recommended by Abeille, given at first in small doses, progressively increased to fifteen or twenty grains. Jalap and the bitartrate of potassa form an efficient hydragogue. Diuretics are not to be relied upon for the relief of the dropsy; the kidneys will not be likely to respond to them. Moreover, they are considered by some as inadmissible, beeause their aetion on the kidneys may increase the inflammation. They are, however, recommended as safe and frequently useful by Christison, Bennett, Gairdner, Dickinson, and others. A rational indication for their use is afforded by the faet that the renal tubes are obstructed; a free secretion of urine will be likely to remove the obstruction by washing away the contents of the tubes. So far as my experience goes, digitalis and the saline diuretics do no harm and are often highly useful.

Measures for the vicarious elimination of urea are indicated whenever 
the diminished quantity of urine and its low specific gravity show the elimination by the kidneys to be greatly deficient. By timcly meeting this indication, uræmic poisoning may perhaps be forestalled. Of course, measures for this object are urgently indicated when uræmic poisoning has taken place. Hydragogue cathartics are the most efficient, and next to these the hot-air bath. Both are to be employed in alternation if uræmic coma or convulsions have occurred. If the indication be not urgent, salines and sudorific remedies may suffice. An eligible sudorific remedy is the liquor ammoniæ acetatis in half-ounce doses thrice daily. The statement concerning the use of diuretics, with reference to dropsy, of course, is equally applicable to their use for the elimination of urea. 'They are not to be relied upon for the elimination of urea when uræmia exists or is threatened. Under other circumstances diuretics may be employed, selecting those which do not act as irritants of the kidneys. The bitartrate of potassa, digitalis, and the decoction of broom are eligible diuretics; and with reference to a diuretic effect, pure or distilled water should be taken as a drink as freely as can be done without inconvenience.

During convalescence, carefulness as regards diet, exercise, and exposure to cold, is important. Tonic remedies, and especially preparations of iron, are generally indicated.

My clinical records furnish illustrations of complete recovery from this affection when the dropsy was excessive, when life was threatened by hydrothorax and œdema of the lungs, and also after the occurrence of uræmic coma and convulsions.

\section{CH A P TER II.}

Degenerative Changes or Lesions of the Kidneys generally characterized by persistent Albuminuria, collectively termed Chronic Bright's Disease or Chronic Albuminuria-Anatomlcal Characters-Clinical History-Pathological Character-Causation-Diagnosis-PrognosisTreatment.

$7 \mathrm{HE}$ researches of Bright form a memorable epoch in the history of pathology and practical medicine. Prior observers had been led to the discovery of albuminuria and its connection with general dropsy, and it had long been known that the suppression of urine was followed by coma, convulsions, and death. But Bright was the first to point out the existence of certain morbid conditions of the kidneys, generally characterized by the presence of albumen in the urine, and, leading to secondary affections, in addition to general dropsy, and to fatal toxical effects dependent immediately on the accumulation of urinary principles in the blood. The morbid conditions of the kidneys described by Bright have bcen considered as constituting an affection commonly known as Bright's disease. Subsequent observers have added much to our knowledge of these conditions, especially as regards the information obtained by means of the microscope. Our knowledge of the blood-changes induced by these conditions, and of ulterior morbid phenomena, has also been much increased by continued clinical observation. The vast importance of the researches of Bright is manifest when it is considered how largely, at the 
present time, the morbid conditions embraced under the name Bright's disease enter into medical practice.

Under the name Bright's disease, are commonly embraced the acute affection considered in the last chapter and the chronic renal lesions which are now to be considered. With respect to the morbid conditions in the acute and chronic form of Bright's disease, there are differenees of opinion among pathologists, some regarding them as different stages or modifications of one disease, and others considering them as essentially different affections. The grounds for considering the acute disease as distinct from the chronic have already been referred to. It seems to me sufficiently clear that the term chronic Bright's disease embraces different affections; but with our present knowledge, it is not always practicable to diseriminate them at the bedside. I shall therefore consider them under one head, noticing ineidentally ascertained points of difference, relating to anatomical characters, clinical history, etc.

Chronic Bright's disease, considered as embraeing degenerative ehanges or lesions of the kidneys, and characterized generally by albuminuria, is of rather frequent occurrence; it is not, at all events, rare. It is important as standing in a causative relation to dropsy in a certain proportion of the cases met with in practice, as giving rise to uræmia, and as liable to be associated with various affections.

Anatomical Characters.-The morbid appearances of the kidneys in different eases of ehronic Bright's disease are far from uniform. The differences, as regards gross appearances, have led pathological observers to divide the disease into several forms. Bright deseribed three forms which he considered as " different modifications of diseased action." This arrangement is followed by Christison and Frerichs, who regard the three forms as representing different stages of the disease. In these divisions the acute affection is inclucled. Including the latter, Rokitansky described eight, Martin Solon five, and Rayer six different forms. Johnson, basing his divisions in a great measure on microscopical appearances, considers that there exist five forms. Waiving, for the present, the pathologieal relations of different forms to each other, it answers all practical purposes to reckon them as four in number, to wit: 1. The form called, after Bright, the large white kilney; 2. The cirrhotic, fibroid, or contracting kidney; 3 . 'The fatty kidney; and, 4. The lardaceous, amyloid, or waxy kidney. These forms are not infrequently mixed, that is, two or more forms may be represented in the same kilney. I shall notice, briefly, the appearances, gross and microscopical, which belong to these forms severally, referring the reader for a fuller deseription to works on morbid anatomy or monograms treating of the disease under consideration.

1. Large white kidney.-The organs are more or less enlarged and their weight is increased. The increased weight of each kidney may reach or exceed twelve ounces, the normal weight being from three to five ounces. The surface of the kidney is smooth, and it has been called the large smooth kidney. The color, as the name imports, is white or whitish. The color in some cases is uniform, but in other eases the surface presents a mottled or marbled aspect. The enlargement is in the cortical portion. The cones sometimes preserve their compact appearance and well-defined outline, but in some cases they are more or less compressed, obliterated, and the tubes separated from each other. The cortical portion, as a rule, and sometimes the cones, are devoid of blood, or anæmic. The surface is frequently dotted with numerous white or grayish points, ancl, when the organ is bisected longitudinally, these arc also observed-on the incised 
surfaces; occasionally the exterior surface presents small granulations. The veins upon tle exterior surface present an arboreseent or stellate arrangement; on the incised surfaces vessels are scaircely visible. The organs are rather flaccid, less resisting tlan the healthy strueture, but not notably softened. A variable amount of turbid serum exudes on section.

Microscopical examination shows an accumulation within the uriniferous tubes of disintegrated epithelium, granules, and exuded matter, with a small or moderate proportion of fatty granules or oil-drops. There appears to be no morbid product nor abnormal change in the intertubular structure in this variety of clisease.

The large white kidney is the form of disease when the chronic has been preceded by the acute affection. It is held by many pathologists that the large white kidney precedes the fatty kidney, the products within the tubes unclergoing the granulo-fatty metamorphosis, and, also, that, after the latter change has taken place, the contents of the tubes may be absorbed, leaving the organs more or less atrophied. According to these views, the atrophy thus produced is to be distinguished from that incident to the second form of lesion, namely, the fibroid, cirrhotic or contracting kidney. These pathological views are to be considered as having only an inferential basis; however rational they may be, they do not rest upon demonstration. Under the title "the inflammatory form of Bright's disease," are reckoned, in addition to the appearances belonging to "acute tubal nephritis," those of the large white kidney, the fatty kidney, and atrophy, these three last named forms being regarded as representing successive stages of one disease, namely, inflammation within the tubules. ${ }^{1}$

2. Cirrhotic, fibroid, or contracting kidney.-The kidneys, in this form, offer a striking contrast to the appearances just stated. The organs are . sometimes notably reduced in volume and weight. Both kidneys, in a case of extreme atrophy cited by Wilks, weighed only an ornce and a half. The cortical portion esrecially is diminished, and, in extremely marked cases, the cones extend to the exterior surface, and their sides may be nearly in contact. The pyramids, or cones, are but little, or not at all, affected. The organs are abnormally dense and resisting. The capsule is with difficulty detached, and, when forcibly removed, brings away portions of the torn parenchyma; the exterior surface is lobulated, or roughened by irregular elevations which may present a hob-nailed appearance. The microscope shows an abnormal production of fibroid or the connective tissue. Hyperplasia of this tissue is the primary lesion, the contraction of volume being due to atrophy from pressure of the newly produced interstitial tissue. 'The arteries are thickened.' The secretory tubes are shrunken, and more or less of them are obliterated. Frequently numerous cysts are seen with the mieroscope, and, not infrequently, they are visible with the naked eye. Crystals of the urate of soda are not infrequently seen in the pyramidal, and, more rarely, in the cortical portion, being contained both within, and exterior to, the tubes. They are more apt to be present when the disease of the kidney is associated with gout. This form of disease is called by some English authors, the "gouty kidney." It is also called the "granular kidney," and it was described by Dr. George Johnson under the name "chronic desquamative nephritis." As an antithesis to the "large white kidney," it has been called the "red small kidney." As regards appearances within the secreting or convo-

1 Vide Treatise by T. Grainger Stewart, M.D. 2d edition, 1871. Also, Pathologie Interne, par Jaccoud. 
luted tubes, different observers differ, some having found the epithelium wanting, the tubes containing an abundance of granules, others finding the epithelium neither absent nor essentially changed. It is intelligible that different specimens may differ in this respect, the difference being explained by the coexistence of tubal nephritis in some, and not in other, cases. They who consider as successive stages of inflammation of the tubes, atrophy, the appearances belonging to the large white and to the fatty kidney, differentiate this atrophic condition from that which belongs to the cirrhotic kidney, in part, by the evidence, afforded by the microscope, of disease within the tubes, but, chiefly, by the existence in the former of only a relative excess of fibroid or connective tissue, not an actual increase of it as in the cirrhotic form. The cirrhotic kidney, as this name implies, is analogous to cirrhosis of the liver, and to other affections to which the terms cirrhosis and sclerosis are applied. The pathological process giving rise to hyperplasia of the intertubular comnective tissue is, by most pathologists, considered to be inflammatory. Some, however, are of the opinion that hyperplasia of this tissue here, as elsewhere, may take place withont inflammation.

At an early period of the disease the size of the kidneys is not diminished; the contraction is proportionate to the duration and the adranced progress of the disease.

3. Fally kidney.-Oil-drops or granules of fat are contained in the epithelium of the convoluted tubes of the large white kidney and in the kidneys after death from the acute affection. They are in notable excess in the so-called fatty kidney. The organs are more or less cnlarged, rather soft and yielding to pressure, and of a yellowish or fawn color. The incised surfaces have a greasy feel. When the organs are bisected, if serum escape, oil-globules are sometimes seen in abundance, floating upon the liquid. The weight of a single kidney may reach fourteen ounces, or even more.

The microscope shows epithelial cells distended with oil, together with free oil and granules of fat within and between the tubes.

The propriety of considering this as a distinct form is denied by some, on the ground that the fat is produced by a degeneration of other morbid products. It is, however, quite certain that the fat is not always exclusively thus produced. The organs are, in some cases, enlarged nuch beyond their volume in cases of the large white kidney. This fact shows a production beyond a mere degenerative change. But in cases in which the volume is but little, or not at all, increased, it is a theoretic conclusion that the fat is the result of the metamorphosis of inflammatory products, and that a fatty kidney is the second stage of a disease, the first stage of which is represented by the large white kidney, and the third stage by atrophy. Moreover, analogy favors the recognition of the fatty kidney as a separate lesion. The condition is identical with that of the fatty liver; in fact, the fatty liver and the fatty kidney are often clinically associated. For these reasons, it secms to me an elror to consider the fatty kidney as always incidental to other forms of disease, and that it has, therefore, no claim to be reckoned among the different lesions which give rise to the phenomena of chronic Bright's disease.

4. Lardaceous, amyloid, or waxy kidney.-The appearances have been described in a former part of this work.' This form of disease is generally associated with a similar condition of the liver and spleen. The organs may or may not be increased in sizc. They are dense and firm, 
the cut surfaces are smooth and present a translucent appearance like wax. "In extreme forms, the organs look as if they had been soaked in a substance like glycerine or balsam, which had subsequently hardened and enveloped all the tissues."

On microscopical examination, the Malpighian tufts, and the small arterial branches elsewhere, together with more or less of the other structures entering into the composition of the kidney, are found to be infiltrated with a peculiar product which has been already considered.

In examining bodies dead with various diseases, especially in hospitals, the kidneys are frequently found to present lesions which had not been suspected during life. It remains to be ascertained how far morbid changes in these organs may proceed without giving rise to serious morbid effects.

Of the four forms of disease just noticed, the point of departure for the local morbid process or processes, in the large white kidney and the fatty kidney, is within the secreting tubes. In the cirrhotic, or fibroid form, the primary seat of the disease is the connective tissue which binds together the convoluted tubes, Malpighian bodies, and vessels. In the waxy form of disease, the morbid material is first deposited in and around the walls of the vessels, and afterward in the surrounding tissues. It is to be noted that both kidneys, in all the forms, are, as a rule, affected in the same mamer, and in about an equal degree. These morbid conditions, therefore, are among those distingtished as symmetrical or bilateral. To this rule, however, there are exceptions.

The different forms of disease or morbid conditions of the kidneys agree as regards certain immediate pathological effects which are liable to ensue. A brief consideration of these immediate effects will prepare the reader to understand the symptomatic phenomena which make up the clinical history of chronic Bright's disease. The same effects are also involved in the clinical history of the acute affection. They are resolvable into two classes, viz., first, interference with the secretory function of the kidneys, especially as regards the elimination of urea; second, interference with the circulation in the kidneys.

The secretory function is interfered with in two modes. One mode is mechanical, viz., the obstruction or plugging of the convoluted tubes by disintegrated epithelium, granules, exudation-matter, and fat. The other mode is the destruction, to a greater or less extent, of the secreting structure; in certain cases the epithelium of the convoluted tubes is injured or lost, the tubes being sometimes completely denuded of their epithelial lining, and, in cases of atrophy from cirrhosis, a greater or less number of the secreting tubes are obliterated, or their tubularity no longer exists. The circulation is also interfered with in two modes. One mode is the pressure on the inter-tubular veins of detached epithelium, granules, exudation-matter, fibroid tissue and fat, and a consequence of this pressure is congestion of the veins, and especially of the Malpighian tufts. The other mode involves the dependence of the circulation on the functional activity of the kidneys. If the secretory function be impaired, congestion follows in accordance with a general law, viz., that whenever in any organ the function of that organ is interfered with, the interference extends to the circulation. Much importance has been attached by Johnson and others to congestion produced in this way by pathological conditions of the kidneys interfering with their secretory function.

Tracing the consequences of the morbid conditions of the kidneys a step further onward, two classes of ulterior effects are liable to follow. First, uræmia. This ulterior effect is attributable to the non-elimination 
of urea. Second, albuminuria. This ulterior effect is attributable to the interferenee with the circulation in the kidneys. Instead of the transudation of water holding in solution the solid constituents of the urine, the serum of the blood transudes, containing albumen.

The symptoms now to be considered are rendered, in a great measure, intelligible by connecting them with the immediate and ulterior effects just noticed.

Ceinical History.-The morbid conditions embraced under the name chronic Bright's disease sometimes follow the acute affection. 'This statement applies chiefly, if not exclusively, to the large white kidney. But, as a rule, they are dereloped imperceptibly, constituting, from the first, a subacute affection. In a large proportion of cases, the event which first directs attention to the existence of renal disease is dropsy. Prior to this event the patient for some time may have been sensible of impaired strength, and the appearance may have denoted anæmia; but not infrequently, up to the time when dropsy is noticed, the patient was not aware of any deterioration of health. The first appearance of dropsy is ædema either around the eyes, in the lower extremities, or in both situations simultaneously. As regards the amount of dropsy and the rapillity with which it increases and is diffused, different cases differ. It may exist and remain in so slight a degree that its existence is only ascertained by close examination, and it may escape the attention of the patient. On the other hand, in certain cases, anasarca occurs either rapidly or slowly, the limbs, body, and especially the genitals, beconing enormously swollen, together with more or less effusion into the serous cavities. A convenient place for determining, by pressure with the finger, a small amount of anasarca is over the sternum.

The different forms of disease do not equally give rise to general dropsy. It occurs most constantly, and is most marked, in connection with the large white kidney. It is wanting in a certain proportion of cases, and in these cases the most frequent form of disease is the cirrhotic kidney. It is frequently, also, wanting for a considerable period during the progress of the waxy form of the disease. Of 89 , out of 102 recorled cases under my observation, in which facts respecting dropsy were noticed, it existed in 74 , and was absent in 15 cases. The dropsy was slight or moderate in 21 , and considerable in 36 cases. In 17 cases the degree and the extent of the dropsy were not noted. ${ }^{1}$ Of 430 cases collected from different authors and analyzed by Frerichs, general dropsy existed in 376 and was wanting in 34. The rationale of renal dropsy has been considered in another part of this work." Suflice it to repeat here that this event has relation especially to the excretion of albumen with the urine. The loss of albumen diminishes the density of the blood-serum which has been observed to fall from 1.030, the normal standard, to 1.010. Moreover, the loss of albumen impedes the capillary circulation, and this leads to congestion, the viscosity of the blood, due measurably to the presence of albumen, being necessary to its free passage through the capillary vessels. And, again, not infrequently, the amount of water eliminated through the kidneys is much diminished. It is intelligible that the low density of the blood-serum, the venous congestion arising from capillary

1 Vide "A Clinical Report on an Analysis of One Hundred and Two Cases of Bright's Disease," by Austin Flint, M.D., one of the Attending Physicians of Bellevue Hospital, etc., published in the Bellevue and Charity Hospital Reports, 1870, p. 226.

Vide page 47. 
obstruction, and the accumulation of water in the blood, give rise to dropsical effusion. The almost invariable occurrence of dropsy and its great amount in connection with the large white kidney, as well as in cases of the acute affection, are explained by the interference with the circulation in the renal organs. On the other hand, the lesser amount of dropsy and its occasional absence in connection with the cirrhotic and the waxy kidney are explained by the fact that the interference is not so much with the circulation as with the secretory function of the kidneys.

General dropsy and albuminuria are symptomatic of the morbid conditions in Bright's disease in the great majority of cases. The presence of albumen in the urine, however, is by no means distinctive of these conditions. Albuminuria occurs in the course of many diseases. But, occurring independently of Bright's disease, it is transient, and the quantity of albumen in the urine is small. As a symptom of Bright's disease, the albuminuria is persistent. Frequently the quantity of albumen is large, but this by no means holds true in all cases; the quantity of albumen is sometimes quite small in cases of Bright's disease, and albuminuria does not invariably exist.

The appearance of dropsy, in the majority of cases, leads the practitioner to examine the urine for albumen. But the albuminuria, in these cases, doubtless had existed for a considerable and perhaps a long period prior to the appearance of dropsy. In testing the urine for albumen by the usual metloods, viz., by nitric acid and heat, certain sources of fallacy are to be kept in mind. Heating the urine, if it be alkaline, sometimes causes the phosphates to be thrown down, which present an appearance resembling that of coagulated albumen. This fallacy is detected by adling a few drops of nitric or any acid which causes the re-solution of the phosphates. Again, if the urine be alkaline, heat carried to the boilingpoint will not always coagulate the albumen, unless the alkalinity be removed by a few drops of acid. Adding nitric acid to a fresh specimen of urine sometimes leads to the disengagement of uric acid in abundance, which may render the urine opaque as if albumen were present. To avoid error, specimens should be tested with both heat and nitric acid.

The albumen in the urine is especially abundant in connection with the large white kidney. It may be small in quantity in cases of the cirrhotic and the waxy kidney. Not very infrequently, in the latter forms, the urine is not albuminous; and the evidence of renal disease, obtained by an examination of the urine, must rest, in these cases, on other points, particularly on the presence of casts in the sediment.

As a rule, general dropsy is marked in the cases in which the albumen in the urine is very abundant, and, per contra, general dropsy is slight or wanting in the cases in which the urine contains but little albumen or none whatever. ${ }^{1}$

The quantity of urine varies much in different cases, and at different periods of the same case. The acts of micturition are fiequently increased, but in many cases the quantity is below the average of health, which is from 30 to 60 ounces. Not infrequently the quantity is much diminished. In a certain proportion of cases it is increased. The latter occurs especially in connection with the cirrhotic and the waxy kidney; in cases of the latter a considerable increase of urine is the rule. The color of the urine also varies, but, as a rule, it is limpid or devoid of color. "The specific gravity is an important point as denoting deficiency, or otherwise, of the solids, more especially urea. In most cases, the specific gravity is

' For facts sustaining this statement, vide my Clinical Report of 102 cases, op. cit. 
more or less diminished. An increase of the specific gravity, which is sometimes found, is due to diminution of the water of the urine. Diminution of the specific gravity, the quantity of urine not being increased, denotes deficiency of urea. Of course, the deficiency of urea is great in proportion as the quantity of urine is diminished and the specific gravity reduced. By ascertaining the quantity of urine passed in the 24 hours, and determining the specific gravity with the urinometer, the condition, as regards the amount of urea, is determined ronghly, but with sufficient precision for ordinary practice. A table drawn up by Prof. Haugliton indicates at a glance the quantity of urea excreted per day, provided the number of ounces voided in the twenty-four hours and the specific gravity be ascertained.' The amount of urea may be determined with much greater accuracy, and without much inconvenience, by volnmetrie analysis. The method proposed by Dr. Edmund W. Dary ${ }^{2}$ will be found readily available. The amount of urea excreted in 24 hours varies between four and six hundred grains. The range of variation within healthy limits is widle, and the fluctuations in different diseases are yet to be ascertained. In affections of the kidneys, to determine the amount of urea in the urine is important as showing to what extent the affections interfere with the excretory function of these organs. The liability to the phenomena and dangers of uræmia may be measurably determined by aseertaining the deficiency of urea in the urine. As evidence of defective renal elimination, it is stated by a French author, Dr. Beauvais, that the characteristic odor of violets after copaiba has been taken, and the peculiar offensive smell after eating asparagus, are wanting in certain eases of Bright's disease. Uline deficient in urea decomposes slowly, and has not the strong ammoniacal odor of putrescent, healthy urine.

Deficiency of urea bears no uniform relation to the albuminuria in cases of Bright's disease. The urea may be greatly defieient, when the urine contains but little albumen or none whatever. This oecurs especially in connection with the cirrhotic, and not infrequently the waxy kidney.

A microseopical examination of the sediment of the urine may reveal important morbid appearances. In certain cases, in which albuminuria is wanting, morbid products derived from the secretory tubes, in the shape of casts, eonjoined with a deficiency of urea, may be the chief evidence, afforded by the urine, of the existence of serious renal disease. And the casts are important to be considered in comnection with the presence of albumen in the urine. Epithelial casts have been stated to be characteristic of the acute affection. Casts composed in part, or chiefly, of granular matter denote a chronic affection. They are distinguished as granular casts. Casts in which epithelial cells are filled with oil, or to which oil-globules are adherent, called fatty casts, are significant of the presence of an abnormal amount of fit within the secretory tubes. $H y$ aline or waxy casts are likely to be present. These have an important significance according to their size. If small in size, that is, not over roo of an inch in diameter, they are formed in tubes in which the epithelial structure is intact; but if large, they are formed in tubes in which the epithelium is lost. When only $\frac{1}{5} \sigma \sigma$ of an inch in diameter, they are nearly as large as the tubes themselves, and, hence, the latter must be denuded of their epithelial lining. Granular and large waxy casts in

${ }^{1}$ For this table, vide Treatise on Urinary and Renal Diseases, by $\mathrm{Wm}$. Roberts, M. D. Republished by Henry C. Lea, 1866.

Vide Manual of Chemical Examination of the Urine in Disease, by Austin Flint, Jr., MI.D. 
abundance are symptomatic of destruction of the secretory tissue, and they may be present when the urine is not albuminous. Waxy casts may be dissolved by urine which has become alkaline from the decomposition of urea.

In addition to casts, the sediment of the urine may be fcund to contain renal epithelial cells more or less deformed, or containing oil-drops and granular matter. Other microscopical appearances, such as uric-acid crystals, crystals of the oxalate of lime, spores of penieillium glaucum, etc., have no special pathological significance.

Passing to other symptoms, pallor of the countenance, due to anæmia, is generally more or less marked. The anæmia is, doubtless, measurably, if not chiefly, owing to the loss of albumen; it is most marked in cases in which albuminuria and general dropsy are marked. On the other hand, in certain of the cases of cirrhotic and of waxy kidney in which albuminuria and dropsy are slight or wanting, pallor is not a marked symptom. Edema and pallor combined give to the face an appearance highly characteristic of Bright's disease.

Symptoms referable to the digestive system are more or less prominent. Indigestion or dyspeptie disorder is common. Flatulency of the stomach is a frequent symptom. Vomiting and purging are prominent and persistent symptoms in certain cases. The probable explanation in these cases is, the urea, being insufficiently excreted by the kidneys, is ricariously eliminated by the gastro-intestinal mucous membrane. The experiments of Bernard and Barreswil which have been often repeated-viz., removing the kidneys in inferior animals-show romiting and purging to occur before death takes place from uræmia, and either urea or the carbonate of ammonia is found in the dejections and the contents of the alimentary canal. The romiting is often sudden and violent, called by Goodfellow "explosive vomiting." Occurring sometimes without dropsy, it may lead the practitioner to suppose that the stomach is the seat of the disense. Prof. Clark cites a case which came under his observation, in which, from the persistency of the vomiting and notable pallor, the patient was supposed to have cancer of the stomach. ${ }^{1}$ In view of the explanation just given, the romiting and purging are to be regarded as conservative ; yet, in the cases in which the vomiting is so constant that everything is rejected from the stomach, it may prove an immediate cause of death by interfering with alimentation; and the vomiting and purging are probably occasioned by the presence of the carbonate of ammonia, produced by the decomposition of the urea. Vomiting is a more frequent symptom than purging. A case under my observation at the Long Island College Hospital appeared to exemplify this cause of death. In the case cited by Clark, it became neeessary to attempt to nourish the patient by injections. Vomiting and purging are likely to be prominent in the cases in which the secretory function of the kidneys is interfered with ; in other words, when the quantity of urea in the urine is notably diminished. These symptoms, however, do not always precede or accompany uræmic poisoning. Urea is also eliminated vicariously by the skin, and the odor of urine is in some cases distinctly appreciable in the perspiration.

Symptoms referable to the respiratory system may be produced by pleural dropsical effusion, or hydrothorax, by pulmonary œdema, and by oedema of the glottis. These might be embraced under the head of the complications of Bright's disease. Pleuritic effusion and œdema of the lungs sufficient to occasion notable embarrassment of respiration are apt

1 Vide American Medical Times, 1862. 
to occur more especially when disease of the heart, involving mitral contraction or regurgitation, or both, coexists with the renal disease. Under these circumstanees, the pleural cavities may become nearly filled with serum; adema of the lungs, under these circumstances, is sometimes developed to such an extent as to prove the immediate cause of death. The occurrence of the odema is sometimes very sudden, and it may prove quickly fatal. Not infrequently, howerer, the odema disappears, even when life has been placed in imminent danger. EEdema of the glottis is an accident liable to oceur and prove rapidly fatal. It sometimes oceurs in cases in which the general dropsy is moderate, or slight, or even wanting.

Dyspnoea may exist in a marked degree when it is not referable to any of the conditions just named, but proceeding, apparently, from a toxical effect upon the ganglionary nervous centre presiding over involuntary respiration. I have met with several eases in which this nervous dyspnoa was the most prominent symptom; and I have known it to exist when no other symptom was present to direct attention to the kidneys as the seat of disease. The patients suffer from a feeling of apprehension of "losing the breath," and are afraid to sleep on this account, the exertion of the will being required for respiration. Disturbance cf the rhythm of respiration characterized by the frequent oceurrence of a long interval between the respiratory acts, followed by series of rapid respirations, is not infrequent, occurring in connection with uranic stupor and coma.

Symptoms referable to the nervous system are among the most important of those belonging to the clinical history of the disease. Pain in the back is rarely prominent, and it is generally wanting in chronic Bright's disease. Cephalalgia is a frequent symptom. It may precele as well as accompany, the symptoms which point to the existence of renal disease. Neuralgic pains in other parts of the body are common; attacks of vertigo are not infrequent. Troubles relating to vision constitute, in certain cases, important symptoms. Diplopia or double vision, hemeralopia or night blindness, myopia, and presbyopia are attributed, and perhaps fairly attributable, to the disease. Incluching these forms of disorder, it would appear that vision is more or less affected in a pretty large proportion of eases; but, exclusive of these, either obscmity or loss of sightthat is, amaurosis, complete or incomplete-is not of extremely rare occurrence. The amaurosis affects both eyes. When not complete, and it rarely is so, the degree varies in different eases, and in different periods of the same case. In chronic, as in acnte, albuminuria, the impairment of vision may depend on an intra-eranial morbid condition, the ophthalmoscope showing no morbid appearances within the eye-called, then, uramic amaurosis. Under these circumstances, it may be temporary, and it is liable to recur from time to time. I have known it to occur repeatedly, in the same case, as a precursor of uræmic coma and convulsions. More frequently, however, it is comnected with changes within the eye, which, by means of the oplithalmoscope, have been brought under elinieal observation. The appearances denote a neuro-retinitis, characterized by congestion, ecchymosis, spots of exulation, fatty degeneration, and sclerosis." 'The appearances are, in a measure, distinctive of renal disease, so that a practised ophthalmoscopist is led by the former to suspect the latter. It has happened, in several cases which have come under my observation, that disease of the kidney was discovered by means of the ophthalmoscopic appearanees, the patients having consulted oculists for 
defect of rision. Amaurosis dependent on neuro-retinitis may diminish, and recovery is possible; but; so far as my observation goes, it is almost always persistent, either progressively increasing or remaining stationary after it has reached a certain degree. 'The development is usually gradual, whereas, the so-called uræmic amaurosis occurs suddenly. The patient describes the defect of visions as like that of a mist before the eyes. Deafness, hemiplegia, paraplegia, and paralysis limited to a single member, have been observed to follow or accompany uræmic coma and convulsions. Muscular cramps are sometimes a source of suffering, and I have known them to constitute the chief ailment for some time before other symptoms led to direct attention to the kidneys.

The symptoms just mentioned, viz., coma and convulsions, are the most important of those referable to the nervous system. They occur in a pretty large proportion of cases, and are generally forerunners of a fatal termination. In chronic Bright's disease, as in the acute affection, they proceed from uræmic poisoning; they are, therefore, among the ulterior effects of interference with the secretory function of the kidneys. They are consequently most apt to occur when the secretory structure of the organs is extensively destroyed, and, hence, especially in connection with the cirrhotic kidney. In acute Bright's disease they alway's denote great danger, but in chronic Bright's disease the danger is vastly greater.

Coma may precede or follow convulsions, and it occurs in some cases, and death takes place, without the occurrence of convulsions. Occasionally coma occurs suddenly, and it is probable that, before uræmia was understood, in certain of the cases of so-called nervous apoplexy the morbid condition was uræmic coma. Generally it is developed gradually, the patient being more or less sommolent, dull, or lethargic for several days before becoming profoundly comatose. The coma is sometimes quiet and in some cases accompanied by stertor. Marked dilatation of the pupils is sometimes observed. The face is congested, sometimes becoming livid, and death is preceded by tracheal rales.

Convulsions, in some cases, are preceded by coma, and, in other cases, they precede coma. They are epileptiform in character, varying much in degree and extent in different cases. They occur in paroxysms, which may be repeated at short intervals, the patient, in the intervals, being completely comatose, the convulsions at length ceasing, and the coma continuing until death. This is the rule, but exceptionally, after a paroxysm or paroxysms of convulsions, the patient recovers intelligence, and hours, days, weeks, or months elapse before a recurrence takes place. Convulsions not following coma are generally preceded by symptoms referable to the head, such as cephalalgia, vertigo, and mental excitation sometimes amounting to delirium. Contraction or rigidity of certain muscles has been observed to precede convulsions; but they sometimes occur suddenly without any premonitions. Of 70 cases, among those which I have analyzed, selecting those only the histories of which cmbrace either death or recovery, coma or convulsions were noted in 26.

The uræmic phenomena, if neither an acute inflammation nor an essential fever exists, are not, as a rule, accompanied by notably increased frequency of the pulse, or an increase of the temperature of the body; the action of the heart is sometimes retarded; I have known the pulse to fall to 32 per minute.

Delirium, which has just been mentioned as sometimes preceding convulsions, is occasionally a prominent feature in cases of uræmic poisoning, occurring in connection with both acute and chronic Bright's disease. It is characterizerl, in some cases, by remarkable hallucinations. Abeille 
cites a case in which the patient for several successive nights fancied he heard numerous voiees singing a particular song. The delirium may be quiet, or gay, or sad, and it is sometimes furious or maniacal.

Uramic poisoning, as manifested by delirium, coma, and convulsions, occurs in eases of Bright's disease with abundant general dropsy; but it is more apt to occur in eases in which dropsy is slight or wanting. Of the 26 eases, above referred to, in which coma or convulsions occurred, in $1 \mathrm{l}$ there was no dropsy. It may follow eopious vomiting and purging, but it is observed to occur oftener in eases in which these symptoms are sliglit or wanting. The symptoms of uræmic poisoning are sometimes the first to direct attention to the kidneys as the seat of disease. In illustration of this fact the following case is cited by Prof. Clark: "A merchant in rather delicate health, but still sufliciently strong to pursue his business, was suddenly and unexpectedly seized one evening with convulsions. IIe had not previously been sick enough to consult a physician. He had several of these attacks in the course of the night, and died in the course of the next day. 'The post-mortem examination revealed the existenee of contracted kilney and no other disease which could have caused his death. One of the kidneys weighed only an ounce and a half."

The oceurrence of violent delirium (which is rare) and a remarkable intermittency of the uramic phenomena, were strikingly illustrated in a case which I saw in consultation with Dr. Charles D. Smith, of New York. The patient, a vigorous man, aged about 55, suffered for sereral weeks from intense eephalalgia, with entire loss of appetite. He harl at this time moderate adema of the legs. He complained, of his own aceord, of a taste of ammonia in his mouth. The urine eontained albumen in abundance, and waxy easts. The heart was enlarged without evidenee of valvular lesions. This was in Mareh, 1865. Under treatment with purgatives and tonies, the headache disappeared, together with the odema, the appetite improved, and he seemed nearly well; the urine, however, was still albuminous and contained easts. In May the headache returned, and on getting up at night to go to the water-closet, he fell upon the floor in a state of unconsciousness, and had a slight convulsion. In a short time he beeame furiously delirious, shouting violently, and struggling so as to require foreible restraint. He was at length brought under the influence of chloroform, and kept under its influence for several hours. In the course of the following day he became rational. Ile progressively improved, and after two or three weeks he seemed again almost well. The urine, however, was still loaded with allumen. In June he went to his country-seat, and there lie had several attacks of convulsions, followed by active delirium, the latter continuing for several days. In the intervals the improvement was such that he seemed to be on the road to recovery. At length romiting became a prominent and persisting symptom, and he died by asthenia early in Oetober. The delirium was not preeeded nor accompanied by symptoms denoting eerebral meningitis.

The following ease, which I saw with my colleague, Prof. Barker, illustrates fatal uræmic coma and convulsions suddenly developed without having been preeeded by any symptoms pointing to disease of the kidneys, save vomiting and suppression of urine for twenty-four hours prior to the coma. The patient, a banker, aged 52, apparently in good health, was seized with symptoms which appeared to denote acute dyspepsia, or, as it is commonly called, a bilious attack. The chief symptom was romiting. On the fourth day the vomiting had ceased, but he had frequent and forcible belehing of wind from the stomach. With the cessation of romiting the urine was suppressed. An hour before my visit his condition in every 
respect seemed favorable, aside from the suppression of urine; but at the time of my visit he had become nearly comatose, lying with the eyes open, and with great difficulty aroused sufficiently to protrude the tongue. Convulsions soon followed, and deatl took place in about four hours from the occurrence of coma. The intermittency of uræmic phenomena, and of the evidence of renal diseases afforded by an examination of the urine, is strikingly illustrated by the following case: Mr. D., aged 56, while transacting business in a bank, had an apoplectic seizure, followed by epileptiforn convulsions. Leeches were applied to the head, and free lyydragogue evacuations were obtained promptly by podophyllin and elaterium. On the following day he had emerged from the coma. The urine was found to be albuminous, and to contain hyaline, fatty, and granular casts. On the fifth day he had recovered sufficiently to be carried to his country house, distant seven miles. Both the albumen and casts had now disappeared from the urine. Examinations of the urine, made repeatedly during several weeks, showed always absence of albumen and easts; and, in the mean time, he had regained his acenstomed apparent health. Five months afterward, at the card-table with his family, he was seized with coma and convulsions. He emerged but partially from the comatose state, and died on the fourth day after the attack. Examinations of the urine prior to the last uræmic attack showed the presence of albumen and casts. This ease also illustrates the sudden occnirence of coma and convulsions, not preceded by the elimination of urea by the alimentary canal, or any symptoms of uremia. A case under my observation at Bellevue Hospital afforded a striking illustration of the latency of the disease, and, also, of rapid recovery from uræmic coma and convulsions. A stonecutter, of midclle age, while at his work, was seized with coma and convulsions, and bronght, in this state, to the hospital. The coma and convulsions continued for several hours, and the disturbance of respiration was such that the patient seemed at one time moribund. The urine, withdrawn by the eatheter, was found to contain albumen. He was purged with elaterium; and, on my visit the day following, he was up and dressed, had eaten a fair breakfast, and wished a discharge in order to return to his work. 'The day after this he was discharged.'

As regards uræmic phenomena, different cases present a striking difference in the amount of degenerative lesions with which their occurrence is connected. Coma, convulsions, and death may take place when the kiclneys are not greatly diseased, and, per contra, the amount of disease sometimes becomes very great before any uræmic phenomena are manifested. So, also, there is a great difference in different cases with respect to the relation between the diminution of the secretion of urea, and the effects of its accumulation in the blood. A rational explanation of this difference is given by Dr. George Johnson. ${ }^{2}$ It is, that the occurrence of uræmic phenomena depends much on the rapidity with which the accumulation of urea in the blood takes place. Coteris paribus, uræmia is quickly induced if the urea accumulate rapidly. On the other hand, if the accumulation take place slowly, the system acquires a tolerance of this, as of other toxic substances, and hence, fatal uræmic effects may be for a long period postponed.

The complications of the disease form an important part of the clinical history. Several affections, included among the symptomatic events, might

' For other cases illustrating the latency of renal disease, vide Bellevue Hospital Clinical Reports, op. cit.

2 Vide London Lancet, Am. Ed., Jan., 1857, p. 13. 
be considered in the light of complications, viz., œedema of the lungs, odema of the glottis, muscular paralysis, and amaurosis. Other secondary affections are liable to be developed. Among the more important of these are pericarditis, peritonitis, pleuritis, and meningitis, all serous inflammations. With respect to the relative frequency of the occurrence of these affections as complications, statistics from different sources are not available, because cases of the acute affection are, in general, included. Moreover, statistics based on the coexistence of other affections, as ascertained after death, do not show their development to be secondary; they may have existed prior to the existence of the renal disease. Cirrhosis of the liver and fatty liver are not infrequently associated with Bright's disease, but they are to be considered as concomitant, not secondary, affections. This statement is applicable to waxy liver and waxy splecn, which, in a very large proportion of eases, accompany the waxy form of disease of the kidneys. Waxy disease of the alimentary canal coexists in a certain proportion of cases. Cerebral hemorrhage, although not of frequent occurrence, occurs sufficiently often to show a pathological connection. It is a complication of the cirrhotic form oftener than of the other forms of renal disease. Pulmonary phthisis is a complication of the waxy kidney sufficiently often to show a pathological comnection; this does not hold true of the other forms of renal disease. Fenwick and Wilson Fox's researches show that the appearances after death denote often gastritis. This may be attributed to the presence of decomposed urea in the stomach. Gastritis occurs especially as a complication in cases of the cirrhotic kidney. Epistaxis and hemorrhages in other situations may be here mentioned as of not infrequent occurrence.

The coexistence of eardiac lesions, in a certain proportion of cases, has given rise to discussion as to whether they sustain a relation of eanse or effect to the discase of the kidneys. Lesions of the valves, either without or with an affection of the walls of the heart, and more especially enlargement without valvular lesions, are found to be associated with Bright's discase in a pretty large proportion of eases. An analysis of the records of the fatal cases at St. George's Hospital, London, during ten years, by Dr. 'Thos. K. Chambers, showed disease of the heart in 274 out of 454 cases of Bright's disease, that is, 60 per cent. On the other hand, an analysis of 1707 fatal cases in which Bright's disease dicl not exist showed 512 cases of diseased hearts, that is, 30 per cent. It would seem to be an inference from these facts that the liability to disease of heart in a person affected with Bright's disease is much greater than the liability to bright's disease in a person affected with disease of the heart. Again, Dr. Chambers analyzed 1371 cases in which the heart was healthy, with reference to the existence of Bright's disease, and found the latter in 175 cases, or a fraction over 12 per cent.; whereas, in 785 cases in which the heart was diseased, Bright's disease existed in 273 cases, or 34 per cent. From these data, Dr. Chambers deduces, as a probable conclusion, that "Bright's disease is a frequent cause of cardiac affections, but cardiac affeetions are not a frequent cause of Bright's disense." The cases analyzed were of the acute as well as chronic.form of the disease.

In these statisties, doubtless, the heart was considered as diseased whenever lesions of any kind were notel. Now, valvular lesions, together with lesions of the walls of the heart to a certain extent are often either imocnous or give rise to no serious results, and only the lesions which do occasion a certain amount of disturbance of the circulation can be supposed to stand in a eausative relation to disease of the kidneys. Fliminating cases in which lesions are not of a character to occasion notable 
disturbance of the circulation, the number of cases exemplifying the coexistence of disease of heart and disease of the kidneys would probably furnish but little, if any, ground for the conclusion that the former stands in a causative relation to the latter. Of the 100, fatal cases studied by. Bright, cardiac disease coexisted in 49 . But of these 49 cases, only 8 had valvular lesions with enlargement. In the remainder either the heart was considered as simply hypertrophied, or valvular lesions existed without enlargement. With reference to the suspicion of causation derived from pre-existing cardiac disease, all the cases save 8 should be excluded. In 30 fatal cases reported to the New York Academy of Medicine by Prof. Clark, cardiac disease existed in only 3. In one of these three cases the lesions were those of old periearditis with enlargement, and in another of these cases there was simply hypertrophy. As a rule in the cases in which a relation of eause and effect exists between disease of the heart and Bright's disease, that is, when the association be not merely a coincidence, or both are not effects of a common causation, the cardiac disease is to be considered as secondary. And if the renal disease tend to produce disease of the heart, in view of the fact that, in a pretty large proportion of the cases in which the two diseases are associated, the cardiac lesion is simple hypertrophy, the explanation given by Bright is still the best that can be offered. Bright, remarking the number of eases in which simple hypertrophy (a rare cardiac lesion) coexists with the disease which has received his name, and regarding the former as an effect of the latter, explains the causation by supposing that the "altered quantity of the blood so affects the minute and capillary circulation as to render greater action necessary to force the blood through the distant subdivisions of the vascular sy'stem." It may be added that Bright's disease has probably been supposed to become developed consecutively to disease of the heart in not a few cases incorrectly, from the oceurrence of slight albuminuria which is not an infrequent symptom of cardiac disease, without denoting renal lesions. Hypertropliy of the heart without valvular lesions occurs especially in cases of the contracted kilney. Considering the infrequency of cardiac hypertrophy without valvular lesions, except in conmection with renal disease, the former constitutes always presumptive evidence of the existence of the latter. In my analysis, out of 26 fatal eases in which the condition of the heart was noted, in 11 there was enlargement without valvular lesions.

Chronic rheumatism is sometimes associated with chronid Bright's disease. This association was observed by Christison. It may, however, be doubted whether it occur often enough to establish any pathological connection. Todd, and other British writers, have observed the frequent association of gout and the contracted kidney. This form of Bright's disease was called by Todd the gouty kidney. Gout in this country is, comparatively speaking, a rare disease, but the contracted kidney is by no means extremely rare. The union of these diseases is not of frequent occurrence with us. ${ }^{1}$

Pathologtoal Character.-In respect of the pathological character of chronic Bright's disease, a point of inquiry, already adverted to, at once arises, viz., does the name denote one disease, or does it embrace sereral distinct affections? Bright regarded the different conditions of the

1 For statistical researches on the complications of the different forms of Bright's disease. hy T. Grainger Stewart, M. D., see British and Foreign Med. Chir. Rev., Jan., 1867. See, also, treatise by Dickinson. 
kidneys, in the acute and chronic affection, as representing different modifications or phases of disease. Christison regards the condition as representing successive periols of one disease, viz., an incipient, middle, and advanced stage. Frerichs, adopting the doctrine previously advanced by Reinhardt, of Berlin, advocates the unity of the acute and chronic affection, and also recognizes three stages. 'The first stage, according to this author, is characterized by congestion or hyperamia, and exudation ; in the second stage the exuded matter undergoes fatty degeneration, and in the third stage the fatty matter is absorbed. The different appearanees, after this theory, are explained by various cireumstances pertaining to the deposit, its transformation and removal by absorption. 'This doctrine is adopted by most of the German pathologists. In contrast with these views, Johnson, Wilks, Dickinson, and others, consider that different affections are embraced under the name chronic Bright's disease. The propriety of considering the acute and chronic affection as distinct has already been considered. Without entering into discussions which would be inconsistent with the plan of this work, I shall simply offer some considerations in behalf of the opinion that the different morbid eonditions enumerated under the head of the anatomical characters of chronie Bright's disease represent different affections.

The forms of disease just referred to are, the large white kidney, the cirrhotic or fibroid kidney, the fatty kidney, and the lardaceons, amyloid, or waxy. Directing attention to the two first of these four forms, evidence that they are not different stages of one affection is afforded by the fact that the previous clinical history in cases of the cirrhotic kidney does not present the symptoms which pertain to the large white kidney. As has been seen under the head of the clinieal history, the symptoms of these two forms are to a considerable extent distinctive; so much so that the differential diagnosis, as will presently be seen, may be made with much confidence. The immediate pathological effects in the kidney differ, in the cirrhotic kilney the secretory function, and in the large white kidney the circulation, being especially interfered with. Cases of the following description are met with in practice: A patient is suddenly attacked with uræmia, there having been no manifestations of any affection of the kidneys prior to the attack, and the patient, perhaps, having been up to that time apparently in good health; death speedily follows, and a post-mortem examination reveals cirrhotic kidneys. In such cases, it seems altogether improbable that the large white kidney has existed without any of the symptoms belonging to that form of disease. A gain, cases are not infrequent in which albuminuria exists for a long period, with general dropsy, and the large white kidney is found after death. Now, when the disease is thus prolonged, if the views held by Christison, Frerichs, and other's be correct, the post-mortem examination should have revealed the cirrhotic kidney. The opinion that the fatty kidney is intermediate between the large white and the contracted kidney is conjectural. There is as much ground for the opinion that cirrhosis of the liver is always preceded by a fatty liver. The condition, as regards fibrous tissue and the secretory structure, which exists in the cirrhotic kidney, may be combined with the fatty condition, notwithstanding the two conditions are pathologically distinct, just as fatty liver and eirrhosis may be combined. Finally, the anatomical characters of the lardaceous, waxy, or amyloid kidney are suflicient to show this to be a distinct pathological condition.

The pathological character of the morbid conditions, severally, of the kidneys into which Bright's disease is resolvable, is another point of in- 
quiry. In the large white kidney the affection is probably inflammatory, the chronic inflammation being seated within the uriniferous tubes. This is a fair inference from the fact that this form of disease is found in cases in which the acute affection has preceded. In the cirrhotic kidney, the newly:formed tissue implies intertubular or interstitial chronic inflammation as in cirrhosis of the liver. In the fatty kidney, fat is deposited, as in other organs, probably in consequence of its undue accumulation in the blood. The lardaceous deposit is a result of a prior blood-change. In all the morbid conditions the affection of the kidneys is to be regarded as the localization or local manifestation of the constitutional affection which probably involves blood-changes, the latter being dependent on perverted assimilation. That the pathology embraces blood-changes may be inferred from the law of parallelism which these affections exemplify. This law holds good certainly in the vast majority of cases. If one kidney be enlarged, the other is likewise cnlarged, and so with respect to contraction we rarely find one kidney increased in size, and the other atrophied. If one kidney be fatty, so is the other, etc. Of the nature of the antecedent blood-changes we are ignorant; but we are not more in the dark here than with respect to other affections which, in like manner, are considered as constitutional.

Causation.-In a small proportion of cases, chronic Bright's disease follows the acute affection. The causes, in these eases, are those which give rise to the latter. Exclusive of these cases, the causation is often obscure. In a considerable proportion of cases, there are no symptoms denoting impaired health prior to the manifestations of the renal discase, that is, prior to either dropsy or uremic symptoms. This was true in 30 of 53 cases, among those which I have analyzed, information on this point being contained in the histories of these 53 cases. In 6 of these 30 cases, uræmic coma or convulsions occurred either when the patients were supposed to be in good health, or when these events were preceded by other ailments only for a period not longer than three or four days. In the 23 cases in which ailments preceded the manifestations of renal disease, excluding the cases in which other diseases existed, the patients had complained of general debility more or less marked for a period ranging from a few weeks to several years. It is very probable that in many of the cases in which the manifestations of renal disease occurred in persons apparently in good health, the urine would have been found for weeks, or months, possibly even for years, to furnish proof that the kidneys were not healthy. The disease occurs very rarely in infancy and childhood, a fact showing that the acute affection in early life seldom becomes chronic. The disease is also infrequent in old age. In the vast majority of cases the patients are either young or in middle life. My analysis of 102 recorded cases showed not a single case under fourteen or over seventy years of age. One patient only was 70 years of age. The mean age was 34 years. Of 491 cases collected from different authors and analyzed by Frerichs (inclusive of the acnte affection), the ages were under ten in 10 cases, under twenty and over ten in 44 cases, under forty and over twenty in 263 cases, under sixty and over forty in 146 cases, and over sixty in 28 cases. A difference relating to age exists between the different forms of renal disease. The large white kidney, which is to be considered as a chronic tubal nephritis, occurs especially in early life, although extremely rare under two years of age. On the other hand, the cirrhotic kidney is seldom developed in early life. Dickinson states that the earliest age at which he has met with a fatal case of the latter is twenty-four. It is most 
apt to occur in the neighborhood of fifty. Men are much oftener affected than women. Of my 102 cases, 67 were males and 35 females. As regards habits of intcmperance, of the 102 cases which I have analyzed, in 38 the histories contained information on this point. In nearly all these cases the patients were males. In 18 cases the liabits, as regards the use of alcoholic stimulants, were good; in 13 cases the patients were spiritdrinkers and intemperate; 3 patients were moderate drinkers; 4 drank malt liquors freely, and in one ease it was simply noted that the patient was a "free liver." So far as these facts go, they certainly do not show any special causative influence pertaining to the use of alcoholic stimulants. It is probable that the abuse of alcoholic beverages contributes to the production of the disease, but it is also probable that their direct agency has been much over-estimated. Clinical observation in this country certainly does not warrant the name spirit-kidney, as applied to the cirrhotic kidney by Goodfellow.' The disease is much oftener observed in hospitals than in private practice among the better classes of society, a fact going to show that the exposures, hardships, and deprivations incident to the lives of those who seek for hospital relief in illness are involved in the causation. Persons whose occupations involve exposure to cold and wet are more liable to be affected than those who are protected against the vicissitudes of weather. Cases are more numerous in humid, marshy climates, and on the sea-coast, than in dry, elevated, and inland situations. Malaria, however, does not seem to be involver in the causation. Of 78 cases observed in situations in which intermittent fever was of frequent occurrence, this disease had existed antecedently to the renal affection in only ten. Dickinson shows by statistical clata that chronic albuminuria is of more frequent occurrence in temperate than in either cold or tropical countries, and that variableness, as regards temperature, favors its production.

It has been common to consider chronic albuminuria as not infrequently dependent on cardiac disease, but, as has been seen, on insufficient grounds. It may be developed in the course of various other diseases. Prof. Clark has slown that it occurs not infrequently as a complication of chronic dysentery. It sometimes is developed during the progress of pulmonary tuberculosis, but, exclusive of the waxy kidney, the proportion of the cases in which after death tubercle in the lungs or elsewhere is found to be associated, is not greater than in fatal cases of other diseases. Of the cases of the waxy kidney, pulmonary tuberculosis exists in a considerable proportion, and it precedes the renal disease sufficiently often to denote a causative influence. ${ }^{2}$. The waxy kidney is developed secondarily to syphilis in such a proportion of cases as to show that the latter has a causative agency. Caries and necrosis of bones have a relation of causation to this form of renal disease; and a similar relation has been attributed to chronic suppuration. ${ }^{3}$ Todd, Garrod, and other British authors, have observed the frequent occurrence of the eirrhotic kidney in persons affected with gout. That the latter affection is often involved in the causation is doubtless true; but the connection is by no means sufficiently constant to warrant, as a distinctive title of this form of renal disease, the name "gouty kidney." Facts appear to show that the absorption of lead into the system may lead to the development of the cirrhotic kidney.

Chronic albuminuria not infrequently supervenes in protracted cases of

1 For facts relating to alcohol as a cause of renal disease, see Dickinson, op. cit.

2 Vide Treatise by Stewart, op. cit. $\quad$ Vide Treatise by Dickinson, op. cit. 
diabetes mellitus. In these cases the latter is probably causative. In two of the cases which I have analyzed diabetes preceded the renal affection. In both these cases the histories showed a remarkable tolerance of the disease of the kidneys.

Dingnosis.-The diagnosis of chronic, as of acute, Bright's disease, is to be based ehiefly on the evidence afforded by an examination of the urine. But it is always to be borme in mind that transient albuminuria is a symptom occurring in the conrse of many diseases. Albumen in the urine is found in eases of all the essential fevers, of pneumonia, diphtheria, erysipelas, etc. ete. Generally the amount of albumen is small, but it is sometimes considerable. On the other hand, it is to be borne in mind that albuminuria is not an invariable symptom in cases of chronic Bright's disease. In these cases renal easts constitute the direct evidence of the disease. It is yet to be ascertained to what extent epithelial and waxy easts are liable to be found when Bright's disease does not exist. In general, the diagnosis is rendered suffieiently easy and positive by persistent albuminuria, and, frequently, easts of the different kinds which have been deseribed, together with dropsy and other diagnostic symptoms. The occasional latency of the disease, exelusive of symptoms referable to the urine, is not to be lost sight of. Not very infrequently albuminuria and easts show the disease to exist when dropsy and other diagnostic symptoms are wanting. The disease is always to be suspeeted in cases of coma or convulsions, of cephalalgia, or neuralgia in other situations, of vertigo and other forms of cerebral disorder, of amamrosis and other forms of paralysis, of serous inflammations, of persistent romiting or purging, of anxmia and impaired muscular strength, whenever the etiology is not otherwise intelligible. In short, it is a good rule in practice to examine the urine for albumen in all eases, whether symptoms point to disease of the kidneys or not, and, if praeticable, whenever there are any grounds for suspecting the kidneys to be at fault, the mieroscope should be employed. To determine the coexistence of Bright's disease, or to exclude this disease, in all affections, is of much importance. Of course the urine is to be examined repeatedly in order to ascertain the persistency of albumen or casts.

To determine the particular form of chronic Bright's disease which exists is not always practicable with our existing knowledge, and hence the propricty, for the present, of treating of the different forms collectively, although they are, in fact, different affections. The form ealled the cirrhotic kidney, however, has several distinguishing symptomatic features which have been already referred to in connection with the clinical history, and these features sometimes warrant at least a highly probable diagnosis. 'This form of disease is most likely to exist in the cases in which the disease is latent up to the development of the effects of uræmic poiscning. Persistent vomiting or purging, serous inflammations, coma and convulsions, with but little or no dropsy, point to this form of Bright's disease. Notable defieiency of urea in the urine, with but little or no albumen, granular and waxy casts, and especially the large waxy easts in abundance, are other diagnosties. The age of the patient has a bearing on this differential diagnosis. The cirrhotic kidney occurs very rarely before middle life, whereas the large white kidney occurs especially in early life. The waxy kidney does not oceur in infaney, but after this period it may occur at any age, being most frequently between 20 and 30 . The fact of the coexistence of hypertrophy of the heart without valvular lesions, points to the cirrhotic kidney. Impaired. 
vision, or amaurosis, is especially apt to oceur in this form. The waxy kidney is to be diagnosticated by the coexistence of waxy liver and spleen, and by the prior existenee of either syphilis, disease of the bones, tuberculous disease of the lungs, or ehronic suppuration. Other diagnostic points are an excessive secretion of urine with a low specific gravity, and with little or 110 albumen, and the small amount or absence of general dropsy if the heart be not affeeted. 'Tliese points, however, are also involved in the diagnosis of the cirrhotic kidney. The existence of gout or the evidence of lead poisoning render it probable that the clironic renal affection is cirrhotic. Oily casts or renal epithelial cells containing oil in abundance, are significant of fatty kidney.

Uræmic coma, with or without convulsions, sometimes occurs suddenly in persons not known to be affected with renal disease. To determine that the attack is clue to uramia, in these cases, the urine is to be examined with reference to albumen, the presence of casts, the specifie gravity, and the quantity, as soon as practicable; and the eatheter should be resorted to, if necessary, in order to obtain a specimen with as little delay as possible. Apoplexy, congestive or from extravasation of blood, meningitis, epilepsy, and alcoholism are the affections to be excluded in cases of uramie coma oceurring suddenly, or when the previous history cannot be ascertained; the latter is liable to happen in hospital cases.

Prognosis.-In a large proportion of cases, the different forms of chronic Bright's disease destroy life sooner or later, if the patient be not cut off by some intereurrent or superadded affection. The duration in fatal cases varies greatly in different cases. It is diffienlt to determine the actual duration in many cases, because the disease is usually developed imperceptibly, and has existed for a greater or less period before its manifestations are observed. Dating from the time when its existence is declared, the fatal termination may take place in a few hours on the one hand, and, on the other hand, the disease may continue many years. In the majority of cases, the duration is between six and eigliteen months. Although recovery may not take place, not infrequently the progress of the disease appears to be stayed, or it progresses impereeptibly, the prominent symptoms disappear, and the patient may have comfortable health for an indefinite period, the albumen and easts sometimes disappearing from the urine. 'The kidneys, like other important organs, such as the heart, liver, lungs, etc., may be disorganized to a certain extent, and still be competent to fulfil their functions sufficiently for the continuance of life and tolerable health.

A fatal termination is frequently due to aceidents or secondary affections, such as pneumonia, pericarditis, œclema of the glottis, ete. Coexisting clisease of the heart, of the liver, or other important organs, often hastens the fatal termination. The prospects of improvement will also depend much on the general eondition of the system. As regards the mode of dying, it is by apnoa in some cases, and in some cases purely by asthenia. In the former cases, uremic coma, hydrothorax, pulmonary œdema or œdema of the glottis, are the immediate causes of death. In the latter cases, long-continued and excessive general dropsy, persistent. romiting or complete loss of appetite and digestion, precede death, symptoms of uræmie poisoning being absent. Of 45 fatal cases among those which I have analyzed, in 18 the death was attributable to uræmic coma or convulsions; in 6 cases the death was due to exhaustion irrespective of complieations, and in 21 eases the causes of death related, measurably or entirely, to complications or intercurrent affections. 
Examinations of the urine furnish important prognostics. If the urine continue to be loaded with albumen, this, in eonjunetion with persisting dropsy and marked anæmia, denotes that the disease will probably not be of very long duration. Danger from uræmia is to be apprehended in proportion to the deficiency of urea in the urine. A scanty seeretion of urine with a low speeifie gravity points to imminent danger in that direction: Another mode of ascertaining the liability to uræmic poisoning, or the existenee of uræmia, is to analyze the serum of the blood with reference to the quantity of urea which it contains. The perspiration sometimes has a distinet urinous odor. The prognosis is not the same in the different renal affections embraced under the name ehronic Bright's disease. It is most unfavorable in eases of cirrhotie kidney; yet, it is eertain that, with the kidneys considerably damaged from this form, life may continue with eomfortable health for an indefinite period. Chronic tubal nephritis, represented by the large white kidney, may exist to a greater or less extent, and recovery takes pluce with little or no permanent injury of the organs. It is probable that reeovery may take place in cases of fatty kidney; nor is it settled that, existing within certain limits, the waxy kidney is neeessarily permanent. Whatever may be the form of disease, it is to be borne in mind that the kidneys may be damaged to an extent equivalent to the loss of the whole of one of the organs, and the remaining portion be quite suffieient for life and health.

Of my 102 reeorded cases, in 77 the histories extended to either death or a condition whieh was considered as denoting recovery, and of these 77 eases, in 22 the latter termination was noted. In a few of these cases, the affection was aeute, and in some, instead of a permanent reeovery, there may have been only a temporary suspension of the symptomatic phenomena. Making due allowance for these eases, the result of the analysis affords encouragement to hope for recovery in not an insignificant proportion of cases of ehronie Bright's disease ; and in many cases may be entertained the hope of an arrest of further progress of the disease.

Treatuent.-In cases of ehronic, as well as of acute, Bright's disease, therapeutieal indications relate to general dropsy and uræmia. General dropsy, when great or considerable, calls for the same measures as in cases of the acnte disease. In general, hydragogue catharties constitute the most effieient and reliable means of affording relief. If the dropsy be slight or moderate, the saline cathartics may suffiee-viz., the sulphate of magnesia or soda, or the citrate of magnesia ; but if the dropsical effusion be large, more active hydragogues are required, viz., elaterium, gamboge, or the bitartrate of potassa with jalap. The acrid or drastic eathartics are to be avoided. The hydragogue may be given in doses sufficient to produce free purging, and repeated after intervals of two or three days, or it may be continued in small doses daily, so as to keep up a moderate draining away of liquid. Elaterium is sometimes well borne in doses sufficient to produce a few watery evacuations daily for a considerable period. When it is important to obtain an effieient hydragogue effeet as promptly as possible, the elaterium is invaluable. It may be preseribed in doses of from ${ }_{16}^{1}$ to $\frac{1}{4}$ of a grain hourly until the cathartic operation begins. It should then be at once suspended, in order to avoid vomiting. There is an unneeessary apprehension, in the minds of many physicians, of dangerous prostration from the use of this drug. I have been led by a pretty large experience to consider it quite safe, employed in the manner just stated. I have repeatedly seen a very marked result from eroton oil when a prompt and efficient effect is important. This drug acts as a hydra- 
gogue, but it is also drastic in its operation, and the cathartics which are more purely hydragogues are to be preferred.

Diuretics are less reliable than hydragogue cathartics. Frequently the kidneys will not respond to them, but in some cases these remedies act efficiently. Experience shows that they may be tried without risk of doing harm. 'Their effect upon the kidneys is not injurious; indeed, there is reason to believe that when they produce diuresis they may do good by washing away accumulated epithelium and morbid products which obstruct the uriniferous tubes. The bitartrate of potassa in doses not large enough to purge, or the acetate of potassa, may be given in conjumction with digitalis, the infusion of broom, or other vegetable diureties. I have known the infusion of parsley root (Petroselinum) to be remarkably effective as a diuretic remedy. If the state of the stomach and bowels preclude the internal use of diuretics, their external application may be tried after the plan proposed by Christison, viz., an infusion of digitalis $\tilde{z} \mathrm{j}$ in water $\tilde{3} \mathrm{xx}$, applied to the abdomen by cloths soaked in it, or by the spongio-piline. I have known this application to be followed by copious diuresis and complete disappearance of general dropsy. A liniment of digitalis, iodine, and squill rubbed over the abdomen and limbs will, in some instances, increase notably the quantity of urine. If continued, however, it is apt soon to excite soreness of the skin. As regards digitalis, it may be useful, in certain cases, not only as a dinretic, but by limiting the production of urea. Experimental observations by Mégevaud and Rabuteau, uncler the direction of Robin, of Paris, show a marked diminution of urea in the urine as an effect of this drug, this effect being greater in proportion to the retardation of the circulation which the drug occasions. The inhalation of the vapor of juniper has proved useful as a diuretic, in some cases under my observation, when remedies were not tolerated by the stomach. The vapor may be produced by dropping 30 minims of the oil on a sponge dipped in hot water and squeezed. The inhalation may be repeated several times daily.

Sudorifics must be relied upon if hydragogne cathartics be not borne and the kidneys do not respond to diuretics. The hot-air bath-that is, heated air introduced under the bedclothes-is the most eflicient mode of producing diaphoresis. This may be employed in alternation with cathartics or diureties. Patients often express a sense of marked relief after perspiring freely under the use of the hot-air bath, but in some cases it occasions a disagreeable heating of the surface without free perspiration, and, if carried too far or repeated too often, it produces prostration. Experience in individual cases must be the guide as to its utility and the extent to which it should be carried. The warm bath, perspiration being kept up for some time afterward by corering the patient with an abumdance of clothing, may be employed as a substitute for the hot-air bath when the latter caunot be conveniently employed, or when its operation is unsatisfactory.

In cases of excessive distension of the integument of the lower limbs and genitals, temporary relief may be obtained by making numerous minute punctures of the skin with a pin, the punctures being so superficial as not to draw blood. The amount of water which drains a way through these imperceptible punctures is surprising. This method is to be preferred to deep punctures or incisions, which are apt to lead to erysipelas and gangrene.

Hydragogue cathartics, diuretics, and sudorifics are the means, also, for the elimination of urea in the cases in which symptoms denoting uremia are present. If uræmic poisoning be manifested by coma or convul- 
sions, diuretics are too uncertain to be depended upon. Active hydragogues and the hot-air bath are indicated. Impending death is to be warded off only by the prompt and efficient action of these measures. We have no knowledge of remedies which will neutralize the toxical properties of urea accumulating in the blood. Elimination, so as to reduce the quantity below the amount requisite to produce poisoning effects, is the only resource. It is a therapeutical object to forestall the development of uræmia sufficient to give rise to poisoning effects in the cases in which an examination of the urine shows a notable deficiency of urea, or an examination of the blood-serum shows an excess of this excrementitious principle. A moderate hydragogue action may be maintained for this object, or the hot-air bath may be employed once or twice daily. Under these circumstances diuretics may be adequate, if the kidneys respond to them. It might be supposed that diuretics are objectionable on the score of their increasing the excretion of albumen in the urine. Experience shows that they do not produce this effect; although the quantity of urine is angmented, the amount of albumen is generally not greater than before, and it may even be diminished.

So far the treatment relates, not to the disease itself, but to its effects. There are no special therapeutical measures to be employed for the removal of the morbid conditions of the kidneys. Certain remedies have been supposed to have a curative effect, viz., the tincture of cantharides, nitric acid, and preparations of mercury. Experience has failed to establish the efficacy of these or other remedies addressed directly to the kidneys. Much, however, may often be done by judicious management toward arresting the progress of the disease, prolonging life, and, if recovery do not take place, securing comfortable health for an indefinite period. Tonic remedies are indicated. The preparations of quinia and iron are the most efficient. Heve, as in other affections in the treatment of which the longcontinued use of tonics is important, a change of remedies from time to time is advisable.

Hygienic measures are of the first importance. Alimentation as abundant and nutritious as the powers of digestion will admit of, clothing to secure miform warmth and activity of the functions of the skin, and such an amount of out-door life as the strength of the patient will bear, constitute the lyygienic management. Abeille states that hydropathic measures have been found useful in France. Travelling, especially a long sea-voyage, has been found highly useful. Some striking illustrations of the eflicacy of the latter are cited in Johnson's treatise. The fact that renal disease appears to be much less frequent in tropical, and, also, in cold climates, suggests the inquiry, whether it may not be advantageous for patients to exchange for these climates one which is unfarorable from variability and humidity. It is hardly necessary to add that all causes of debility or disorder are, as far as possible, to bel removed. Overtasking of body or mind, exposure to vicissitudes of weather, the use of alcoholic stimulants, save as a means of aiding appetite and digestion, and taken for these objects, if at all, very sparingly, venereal indulgences, and dietetic imprudences, are to be interdicted.

Symptoms, other than those already referred to, in certain cases, call for treatment. Cephalalgia and neuralgic pains in different situations call for palliative measures. With regard to the use of opium to relieve pain, or procure rest, some writers have inculcated the importance of great circumspection, under the belief that, eren in moderate doses, it is liable to produce undue, and even fatal, narcotism, if the blood be surcharged with urea. Apprehensions on this score have been greatly exaggerated, if, 
indeed, there be any foundation for them. I have known opium to be taken, habitually, in large doses, for several months, by a patient who suffered much from vomiting and diarrhœa, the urine being loaded with albumen, and the dropsy very great, and, so far from its being injurious, life was apparently prolonged by its use. At Bellevue Hospital opium has been given largely, and chiefly relied upon, in the treatment of uræmic convulsions, by my associate, Prof. Alfred L. Loomis, who has been led by his experience to consider this plan of treatment eminently successful. There is reason to believe that, so far from opium having a poisonous action, it renders the nervous system more tolerant of the uræmic poison.

Vouniting and purging, if excessive, claim interference, but it is to be recollected that generally these symptoms denote the vicarious elimination of urea through the alimentary canal. It would be injudicious to attempt to arrest the eliminatory process in this direction, but, as excessive vomiting and purging preclude adequate alimentation, palliative measures are to be employed. Relief may be procured indirectly by favoring elimination through the cutaneous surface by means of the hotair bath. Direct palliation may be obtained by pieces of ice taken into the stomach, by rubefacients or dry cups applied to the abdomen, and by the internal use of hydrocyanic acid, creasote, the oxalate of cerium, and the preparations of bismuth. The latter are often highly useful. The dilute sulphuric or the hydrochloric acid, given with a view to neutralize the ammonia, is recommended by Dr. George Johnson. Dr. Johnson also advises copious libations of warm water, in order to facilitate romiting, and thus remove the ammonia from the stomach.

Astringent remedies have been advised with a view to restrain the exeretion of albumen in eases in which the urine is highly alluminous. Gallic acid, the acetate of lead, and the astringent preparations of iron, have been used for this purpose. I have repeatedly made trial of gallic acid for the end just stated, but with little or no effect. Dr. Horatio C. Wood has found the albumen to diminish in a marked degree under the use of this remedy given to the extent of forty-five grains daily. Tannic acid produces no effect in that way, and, as shown by Dr. Wood, it is objectionable on account of its coagulating albumen.'

Dr. Arthur Scott Donkin elaims that the skim-milk treatment effects a cure in eases of the large white kidney, and that marked benefit is derived from it in the other forms. He has found it to occasion abundant diuresis, carrying off the dropsy within three weeks, and the albumen in the urine subsequently disappearing. The eases, however, which he adduces are too few to justify his encomiums on this treatment, but it should be tried until its merits are determined by a suffieiently large number of clinical facts. The loss of the albumen in the blood, from its elimination by the kidney, has suggested, as an important indication, the endeavor to make up this loss by a highly albuminous diet; and the evidence of experience has been cited in behalf of the usefulness of this measure of treatment. ${ }^{2}$

The treatment of complications, such as serous inflammations, or of affections which may be accidentally associated with chronic Bright's disease, is to be modified by the coexistence of the latter. Active measures, which, under other circumstances, might be admissible, are contra-indicated. General or local bloodletting, mercury, and other depressing mea-

1 Philadelphia Med. Times, Fehruary 1, 1871.

"Vide article "On Albuminuria and its Ferro-Albuminous Treatment," by Dr. David Nelson, New Sydenham Society Year-Book of Medicine and Surgery for 1860. 
sures, as a rule, are injudicious. If there be exceptions to this rule, they are of rare occurrence.

In conclusion, the management, as has been seen, does not embrace special medication, but, in addition to remedies for particular symptoms or effects, and the treatment of consecutive affections, it consists, in a great measure, in the removal of all morbific causes and in hygienic regulations. The object is not to effect recovery from damage that has been done, but to prevent further injury of the diseased organs, reeollecting that the existence of a certain amount of disease is not incompatible with the continuance of life and comfortable health for many years. The advantage of an early diagnosis is very great in this disease. The same consideration is applicable here as in cases of pulmonary tuberculosis, viz., inasmuch as the object to be hoped for is an arrest of the progress of the disease, the earlier its existence is ascertained the less will be the amount of damage before the disease is stayed.

\section{CHAPTER III.}

Simple Renal Cysts. Hydatids in the Kidney. Renal Tuberculosis. Carcinoma of the Kidneys. Movable Kidney. Hæmaturia. Diabetes Mellitus-Auatomical Characters-Clinical History-Pathological Character-Causation-Diagnosis-Prognosis-Treatment.

THE kidneys are sometimes the seat of affections involving structural changes other than those already considered. Carcinoma and the tuberculous affection occasionally occur in these organs. Hydatids and simple cysts are addlitional affections in this category. Their interest for the pathologieal anatomist is greater than their practical importance to the physician, inasmuch as it is often difficult or impossible to determine the fact of their existence clinically. On this aceount they claim, in this work, only a passing notice. Having noticed these affections, the remainder of this chapter will be devoted to the consideration of diabetes mellitus.

\section{Renal Cysts.}

Cysts discoverable with the aid of the microscope, or microscopical cysts, are frequently found in chronic Bright's disease, occurring chiefly in the contracted kidney. Cysts apparent to the naked eye are not infrequent, varying in size from that of a pin's head to an almond or even larger. The manner in which they are produced has excited considerable discussion. The most rational theory is that which attributes them to dilatation of portions of the renal tubes. Either with or without the morbid conditions which belong to Bright's disease, cysts are sometimes produced in sufficient number and size to involve considerable, and even total, destruction of the renal parenchyma. The volume of the kidney is, in some cases, greatly increased. A cyst may project from the kidney and form a tumor considerably larger than the kidney. There may be a single cyst of greater or less size, or numerous cysts. One kidney or both kidneys may be affected, oftener the latter. 'The cysts contain a 
liquid which may be limpid and colorless, of a citron color, or sometimes quite dark. Occasionally the contents are thick, and sometimes nearly or quite solid. They sometimes contain crystals of cholesterin, and I have seen a tumor of the size of an English walnut projecting from the kidney, which contained a solid mass of pure cholesterin. The liquid contents of renal cysts rarely contain the constituents of the urine.

The symptoms may point to the existence of renal discase if the cysts be sufficiently numerous or large to occasion considerable interference with the circulation in the kidneys, or with their secretory function. The urine may be seanty or abundant; it will be likely to contain albumen, and will be more or less deficient in urea and the other solid constituents. General dropsy may occur and the effects of uræmia. Under these circunstances, the disease will be considered as Bright's disease prior to the autopsy. I have met with a case in which death from sudden uræmic coma was attributable to extensive cystic destruction of the kidneys.

Renal cysts are often discovered in the deal-house, when there had been no symptoms during life pointing to disease of the kidneys. If the cysts be not large nor numerons enongh to involve considerable destruction of the renal parenchyma, and Bright's disease be not associated, they may not have occasioned any appreciable inconrenience.

Were their existence discoverable during life, the indications for treatment would relate to the symptoms and effects.

\section{Hydatids in the Kidney.}

Hydatids in the kidney are, in all respects, similar to those in other organs, for example the liver, and a description of them need not be here repeated. Their occurrence in the kidney is extremely rare. They occur in only one kidney, as a rule to which there are very few, if any, exceptions. They do not, therefore, diminish the excretion of urine sufficiently to occasion uræmia. If they interfere with the secretory functions of the kidney, the augmented functional activity of its fellow makes up for the deficiency. So long as the liydatids remain intact within the renal parenchyma they give rise to no well-marked symptoms, and they may remain for an indefinite period completely latent. They are found after death when no renal trouble has been manifested during life. They sometimes attain to a sufficient size to form a tumor perceptible by palpation. The symptoms to which they give rise depend on inflammation, ulceration, and the discharge of the hydatids in different direetions.

If the hydatid cysts open into the pelvis of the kidney, the hydatids pass through the ureter into the bladder, and are discharged with the urine. In their passage along the ureter they may give rise to the same train of symptoms as the passage of a renal calculus, in other words, to nephritic colic. Owing to their elasticity, hydatids of considerable size may pass into the bladder and through the ureter withont great difficulty, and without much pain. Cases have been reported in which they were discharged in great numbers. Their passage may be accompanied with hæmaturia and pyuria. The latter is due to suppuration of the hydatid cyst.

The hydatid eyst sometimes develops in a direction toward the surface of the kidneys, and opens into the post-renal areolar tissue. Suppuration takes place and an abscess forms in the lumbar region, which, when opened or allowed to open spontaneously, gives exit to hydatids together with pus in greater or less quantity. Hydatid cysts in the kidneys have been known to open into the peritoneal cavity, the stomach, intestinal 
canal, and even into the lungs, the hydatids, in the latter case, being expectorated. And, again, hydatids here, as in other situations, sometimes die, the cyst contracts and becomes obliterated, or contains a certain amount of calcareous matter, a spontaneous cure in this way taking place.

The diagnosis of this affeetion cannot be made prior to the discharge of the hydatids in some one of the directions just stated. The prognosis, in cases in which the hydatids are discharged through the renal passages, or in the lumbar region, is not very unfavorable. The treatment does not embrace any special therapeutical measures, but is to be governed by the symptoms and circumstances proper to individual cases.

\section{Renal Tuberculosis.}

A tuberculous affection of the kidneys is always secondary to pulmonary tuberculosis. It is rare for secondary tuberculosis to occur in this situation, but specimens of extensive tuberculous disease are occasionally met with. I have seen both kidneys crammed with yellow tubercle which was present in abunclance within the pelvic cavity as well as within the renal parenchyma. In this case the vesiculæ seminales were also filled with tuberculous matter. In another specimen the kidneys contained numerous tuberculous eavities, the patient dying of perforation of lung incident to pulmonary tuberculosis.

The diagnosis can hardly be made with any degree of positiveness, and the therapentical measures, were the existence of tubercle in these organs determinable, would be those indicated by the tuberculous affection of the lungs.

\section{Carcinoma of the Kidneys.}

Cancer is rarely seated in the kidneys, save when it extends to these organs from an adjoining situation. In the cases in which the affection occurs in these organs, the form of cancer is usually the encephaloid. The cancerous growth may be presented in one or more masses of variable size, situated in the cortical portion, without much enlargement of the organ; or the kidney may be greatly enlarged so as to form a tumor discoverable by the tonch. Encephaloid cancer in the kidney is comparatively frequent in children. Dr. C. Hilton Fagge has reported a case, the patient being four and a half years old, in which the affected kidney weighed $4 \frac{1}{2}$ lbs. A kidney, in the case of a child eleven months old, weighing $5 \frac{1}{2}$ lbs., was presented to me by my colleague Prof. Mott.

Hrmaturia, the source of the blood being evidently the kidney, with or without preceding and accompanying pains in the renal region, calculous pyelitis being excluded, and a tumor in the abdomen near the site of the kidney being felt, which is not movable, not lowered by the descent of the diaphragm, and not fluctuating, render the diagnosis quite certain. The diagnosis, under these circumstances, is positive if cancer exist in some other situation.

Palliative measures of treatment only are indicated.

\section{Movable Kidney.}

One of the kidneys is sometimes loosened so as to form a movable tumor which may be felt through the abdominal walls. This is to be recollected in determining the nature of obscure tumors within the abdomen. The right kidney oftener than the left is movable; it occurs in females oftener than in males. Of 35 cases analyzed by Fritz and Becquet, in 30 the pa- 
tients were females. ${ }^{1}$ In the vast majority of cases the age is between 18 and 45 years. The movableness is attributable to violence, to increased weight of the organ from disease, to relaxation of the abdominal walls from pregnancies, and to the use of corsets ; these causes may act singly or in combination.

A movable kidney occasions pain in the lumbar region, especially after muscular exertion, and a sense of uneasiness in the abdomen. The latter is probably due to twisting of the meter and retention of urine in the pelvis of the organ from the temporary obstruction thereby occasioned.

'The diagnosis must rest on the situation of the tumor, a correspondence with the kidney in its size and form, together with a tympanitic resonance on perenssion, showing the presence of intestine posteriorly in the site of the kidncy.

Mechanical compression of the abdomen by means of a bandage, belt, or abdominal supporter, obviates, in a measure, the inconvenience attending this anomaly.

\section{Hæmaturia.}

Hæmaturia, or bloorly urine, occurs in various diseases, as well as after wounds and other injuries of the different parts composing the urinary apparatus. The hemorrhage may take place from the kidneys, the uterus, the bladder, or the urethra. When it takes place from the bladder or urethra, whether it be traumatic or symptomatic of disease, it comes within the province of surgery; but the means of determining that the hemorrhage is either cystic or urethral are of importance to the physician as aiding him, by exclusion, to decide either that the hrematuria is renal, or that the blood comes from the ureter. Urethral hemorrhage is easily discriminated. The blood escapes without any effort of micturition, and hæmaturia exists only in so far as blood remaining in the urethra is washed away with the flow of urine. Moreover, the seat of the disease or injury may be ascertained by surgical exploration. Cystic hemorrhage is discriminated by attention to the following points: The passage of pure blood, with acts of micturition, after the discharge of urine, obstruction to the free escape of urine from the presence of congula within the bladder, the existence of calculus as determined by surgical exploration, the symptoms of either cystitis, ulceration, or malignant disease of the bladder, and finally, the fact of the bladder having been the seat of a wound or injury.

Cystic hemorrhage is of frequent occurrence in certain towns at the Cape of Good Hope and on the borders of the Nilc in Egypt. The name endemic hrmaturia lias been applied to it in these situations. The researches of Dr. John Harley render probable the dependence of the hemorrhage in the cases of so-called endemic hrematuria or an entozootic disease of the bladder. Examining the urine in a number of cases occurring at the Cape of Good Hope, Dr. Harley found invariably the eggs of an entozoon, and in some samples examined ciliated animalculæ were discovered, belonging to the family Distomum, of the class Frematodia. ${ }^{2}$ The larvæ of this parasite are contained in the river-water drank by the inhabitants of the places in which the disease prevails. This parasite has been found in other organs than the urinary bladcier, namely, in the portal vein by

I Archives de Médecine, Janvier, 1865.

2 Vide London Medical Times and Gazette, February 6, 1864 ; also, the American Journal of Medical Sciences, April, 1864. Vide second article by Harley in Trans. Royal Med. and Chirurg. Society of London, yol. lii., 1869 ; do. third communication in do. 1871, and in London Lancet, Dec. 3, 1870 . 
Bilhar, of Cairo, and in the veins of the mesentery and pelvis of the kidney by Griesinger, Reinhart, and Lantner. ${ }^{1}$

Excluding the urethra and the bladder as the source of the hemorrhage in cases of hæmaturia, it remains to be determined whether the blood comes from the ureter or from the kidney. Hemorrhage from the ureter generally involves the presence of a calculus in this situation. If, therefore, symptoms denoting this causation be wanting, and the hemorrhage be not cystic or urethral, in all probability the source of the hemorrhage is renal. In most of the cases of hæmaturia in which the bloody urine denotes a medical, as distinguished from a surgical, affection, the source of the hemorrhage is the kidney. In certain cases, however, the hemorrhage is both renal and eystic. In traeing blood in the urine to the kidney, aside from the exclusion of the urethra, bladder, and ureter as sources of the hemorrhage, changes in the urine, in addition to those dependent on the presence of blood, and also other symptoms of renal disease, are, of course, to be taken into aceount. The mieroseope is sometimes essential in determining the presence of blood in the urine. Pu'purine. may give to the urine a color resembling that eaused by blood. Various articles ingested give rise to an appearance of bloody urine, namely, the prickly pear, beet-root, madder, logwood, ete. On the other hand, blood may be present in so small an amount as not to produce a bloody appearance to the naked eye, when the microscope shows blood-corpuscles in the sediment. The presence of blood-easts in the sediment of the urine is proof that the hemorrhage is renal; so, also, the presence of fibrinous casts of the ureter, the latter being large enough to be seen with the naked eye. The action of the urine upon the blood oceasions a smoky or sooty appearance of the urine in certain cases.

Renal hemorrhage is generally to be regarded merely in the light of a symptom. As such, it enters into the clinical history of acute albuminuria or tubular nephritis. Hæmaturia from either renal or cystie hemorrhage, or both, is a symptom of diseases which are characterized hy hemorrhage from different mucous surfaces. Thus it is symptomatic of scorbutus, purpura hemorrhagica, and yellow fever. By several writers, however, it is considered as oceurring under eircumstances which warrant its being recognized as an individual affection, which has been named intermittent or paroxysmal hæmaturia. An account of the affection so called, based on an analysis of seven cases observed by himself, has been published by Dr. Edward Headlam Greenhow. ${ }^{2}$ The data for the description which follows are derived from Dr. Greenhow's paper.

The affection in all cases was uniform as regards the symptoms and course; and in all, the existing canse appeared to be the same, namely, exposure to cold or wet. As the names of the affection imply, the affection is characterized by paroxysms. These commence with coldness of the extremities, followed by a chill, which is sometimes accompanied by rigors. During the chill the patient compliains of a feeling of weight and pain in the loins, with weakness and stiffness in the lower limbs. Generally, but not invariably, the chill is followed by imperfectly marked febrile movement. "From half an hour to two hours after the chilliness or rigors, the patient has never failed to pass the first dark-colored urine, which has always been highly albuminous, and has eontained numerous crystals of oxalate of lime, with more or lees of brownish or yellowishred amorphous granular matter, and a few hyaline casts, but only occa-

1 Tide Aitken's Science and Practice of Medicine.

2 Vide Edinburgh Medical Journal, May, 1868. 
sionally some stray bloorl-corpuscles. At each succeeding micturition after the chilliness, the urine has invariably shown unore or less diminution of eolor, of albumen, of oxalate of lime, and of its other abuormal constituents, resuming its natural character and appearance by the second or third micturition, after slight attacks, and usually by the fourth or fifth after severer paroxysms. By the second day after an attack, the patients, as a rule, have regained their ordinary degree of health and strength, and have continued well until some fresh exposure has brought on a new attack of their complaint. To this summary I may add, that, so far as my observation extends, all the patients suffering from this disease have had the same pale, sallow, cachectic aspect, two of them having been distinctly jaundiced, and the others having had at times an icteroid tint of skin."

The evidence of hrematuria in this affection is ehiefly the presence in the urine of the brownish or yellowish-red amorphous granular matter, which is supposed to consist of disintegrated blood-corpuscles; the latter are not uniformly found, and when present they are not numerous. The short duration of the affection shows the absence of inflammation or any important lesion of the kidneys. It is evidently a constitutional affection of which the hamaturia is a local expression.

From its paroxysmal character, and its occurrence sometimes in malarions districts, it has been supposed to be a variety of ague. Of the cases observed by Greenhow, not one had ever had intermittent fever; and it occurs among those who have never been exposed to malarious inflnence. Occurring, however, in malarious distriets, it may be associated with intermittent fever.

The treatment consists of measures to restore warmth during the elill, and, afterward, of quinia and chalybeate tonics. Remedies with a view to the arrest of the renal hemorrhage do not seem to be required. After an attack, exposure to cold and wet should be avoided, in order to prevent a recurrence of the affection.

Intermittent or paroxysmal hematuria is exceedingly rare. 'The seven cases reported by Greenhow eame under his observation during a period of ten years; and he states that in English medical records he was able to fincl only ten or eleven cases. Cases have been of not infrequent occurrence during certain seasons in different situations in the Southern States, as I have learned in personal intercourse with medical practitioners at the Sonth. From its apparent connection with intermittent fever, it has been called "miasmatic hamaturia." 'The medical literature of our country is deficient as regards clinical facts relating to this affection, or, perhaps, more properly, this symptom of disease.

\section{Diabetes Insipidus. Polyuria.}

The term diabetes signifies simply increased flow of urine, or diuresis. The term is applied to two affections which differ essentially each from the other. In one of these affcetions notable diuresis exists, a given quantity of the urine containing a small proportion of solid eonstituents; that is, the specific gravity is low, being sometimes but little above that of spring-water. The morbid abundance of urine in this affection is due to an excessive elimimination of water by the kidneys. In this affection thirst is excessive, the quantity of fluids drank being in proportion to the abundance of the urine; so that the affection is also called polydipsia, and has becn already noticed under that head.' Considered as a urinary 
affection, it is called diabetes insipidus, or polyuria, the latter being the better name. 'The other affection to which the term diabetes is applied is characterized by the presence of sugar in the urine. This affection will be presently considered.

Diabetes insipidus, or polyuria, occurs in various pathological connections. Of 72 cases analyzed by Roberts, in 6 the affection followed an injury of the head by a blow or fall, and in 5 cases it was connected with cerebral diseases. ${ }^{1}$ In the remaining cases the affection was attributed to various causes, viz., intemperance, exposure to cold, and to the sun's rays, etc. All ages are represented in the cases analyzed by Roberts, in seven cases the patients being infants, and in four cases the ages being between 50 and 70 years. In six of these cases only was the condition of the kiudeys ascertained post-mortem. Of these six cases, in three the kidneys were atrophied and degenerated; in one case the granular tissue of the organs was entirely wanting; in one case multiple abscesses were found in the kidneys, and in the remaining case the organs were simply hyperæmic and somewhat enlarged.

In a case which came under my observation in Bellevue Hospital, November, 1864, the kidneys, as in one of the fatal eases collected by Roberts, presented almost entire destruction of the secreting substance, having the appearance of empty sacs. Both ureters were much dilated, and the walls of the bladder were greatly hypertrophied. The patient, a tinsmith, aged 42 years, entered the hospital seventeen days before his death, and stated that cliuresis and excessive thirst had existed for two years, but that he had kept at his work until about three weeks prior to his admission. The urine was considerably, but not exceedingly, abundant; the sp. gr. was 1008 , and it was free from albumen and sugar. He was pale and moderately emaciated; the appetite was good; the bowels were constipated, and he complained only of debility. On the fourteenth day after his admission he took to the bed, and was sommolent. On the next day he became nearly comatose, and he had epileptiform convulsions. He became completely comatose and died on the following day. The brain, lungs, and heart, on autopsical examination, presented no evidence of disease. This case has been aiready referred to in connection with hydronephrosis.

In the cases in which polyuria is associated with lesions of the kidneys, it is a question whether the diuresis be the cause or an effect of the lesions. Roberts is of the opinion that the lesions are produced by the long-continued diuresis. That the diuresis is an effect of the lesions in these cases, is perhaps equally, if not more, probable. The affection, however, is associated with serious lesions of the kidneys in only a certain proportion of cases, and it is probable that in many, if not in most, cases the kidneys are not diseased. In a certain proportion of cases the affection is to be considered as polydipsia rather than polyuria; that is, the diuresis occurs in consequence of the excessive ingestion of fluids. That this is not true of all cases is shown by the continuance of the diuresis notwithstanding abstinence from fluids is enforced.

Of the 72 cases analyzed by Roberts, sixteen ended in complete recovery; thirteen ended fatally, and the remaining forty-three cases were in progress when reported, considerable amelioration having taken place in several of them. Inasmuch as in all the fatal cases in which post-mortem examinations were made renal lesions were found, it is fair to infer, from the fatal result, disease of the kidneys. The symptoms preceding

1 Practical Treatise on Urinary and Renal Diseases. 
death in several of the cases denoted uromia, as in the case occurring under my observation; the duration of the affection in the fatal cases collected by Roberts varied from seven weeks to twenty years. In the eases in which the affection appeared to be congenital, it had existed in fiftynine, and in the other fifty, years at the date of record.

A point of practical importance relating to polyuria is the discrimination of this affection from cliabetes mellitus, or saccharine diabetes. The differential characters which belong to the former are the low speeific gravity and the absence of sugar in the urine, as determined by resorting to the proper tests. Cases, however, have been observed which seem to present an intermediate affection between insipid and saccharine diabetes. In these cases there exists polyuria, and the urine is of a low specific gravity, but containing, either transiently or persistently, a small quantity of sugar.

\section{Diabetes Mellitus, or Saccharine Diabetes. Glycosuria. Melituria. Glycohæmia.}

An increased flow of urine, or diuresis, is generally a notable feature of the affection now to be consirlered. The ristinctive feature, however, is the presence of grape sugar or glucose in the urine; hence the significance of the term mellitus. Glycosuria and melituria are names applied to the affection characterized by diuresis and saccharine urine, and they are to be preferred to diabetes mellitus. 'The term glycohæmia, denoting a saccharine condition of the blood, is, however, to be preferred to either. 'The affection is far from being of frequent oecurrence, yet it is not so rare but that cases oceasionally come uncler the observation of every physician.

Sugar in the urine, like albuminuria, oceurs not infrequently as a symptom in various pathological connections. 'The sugar, under these circumstances, is usually not abundant, and the urine contains it for a brief period only. As the distinctive feature of an individual affection, the sugar which the urine contains is in greater or less abundanee, and it continues persistingly in the urine. Even when the affection exists, the saccharine urine is, in fact, merely a symptom. It does not constitute the affection. It is incidental to, or an effect of, the real disease. The sugar exists in the urine because it pre-exists in the blood. Existing everywhere in the vascular system, it is excreted by the kidneys. But, with our present knowledge, the true seat and nature of the disease are not clearly established, and, therefore, provisionally, it is named as if it were a disease of the urinary system, and is included, for the sake of convenience, among the discases affecting this system.

Anatomical Characters.-A side from lesions belonging to concomitant or consecutive affections, this disease has no constant anatomical characters. The kidneys are often enlarged, without structural changes, that is, they are hypertrophied. 'This is doubtless a result of their longcontinued increased functional activity. But this condition of the kidneys is not invariable. Glycosuria and Bright's disease are sometimes associated. In these cases, the latter is generally, if not invariably, developed after the former. Since the discovery by Bernard of the glycogenic or sugar-producing function of the liver, and the effect upon this function produced by irritating the floor of the fourth ventricle, attention has been directed to the part of the nervous centre just named to see if changes there are not discoverable after death; and cases have been reported in which the microscope has appeared to reveal morbid appearances in that 
situation.' An examination of the blood shows the presence of sugar. It has been found, also, in the saliva, in the liquid contained in serous cavities, in the humors of the eye, and even in the spermatic secretion. During life, sugar is found in the serum of blisters, and sometimes in the sweat.

Cuinical History.-Notable increase of the urine, or diuresis, is usually the symptom which first awakens suspicion of the existence of the disease. Not only are the acts of micturition more frequent, but the quantity of urine passed in a given time is much greater than in health. The urine is deficient in color in porportion to the amount of sugar which it contains. The appearance is generally elear, as well as pale. It has frequently a sweetish odor like that of whey. The presence of sugar is perceptible to the taste. Flies and bees are attracted to it, if emitted on the ground. The density exceeds that of healthy urine, in proportion to the amount of sugar. The specific gravity varies between 1.030 and 1.074 , the latter being au extreme of increased density. The reaction is in most cases feebly acid; it is sometimes neutral, and in rare cases alkaline. The water of the urine is more or less inereased; hence, the increased quantity of urine. The increase in the quantity of urine is generally very great, and sometimes enormous. It may amount to thirty pints, or even more, in the 24 hours. The frequent calls to micturate are a source of great discomfort, and especially during night they constitute a serious evil by interfering with sleep. The action of the sugar on the urethra is apt to oceasion a sense of heat and stinging pains ; excoriation of the prepuce and glans is sometimes produced, and may lead to phimosis. In the female, eczema of the vulva is a frequent effect, giving rise to distressing pruritus. It has been stated that more liquid is contained in the urine, in some cases, than is ingested. This may be true for a limited period, but examinations extending over several days show that, large as is the quantity of urine, it falls short of the amount of the fluid contained in food and drink. As regards the normal solid constituents of the urine, they are not, as a rule, diminished. The sugar in the urine does not displace these constituents, viz., urea, uric acid, etc.; the urea, in fact, has been found considerably more abundant than in health." If albuminuria exist, it shows either the existence of coincident renal disease, or those conditions w'ich, under other circumstances, give rise to the presence of albumen in the urine.

Different cases differ in respect of the quantity of urine and the amount of sugar. Generally, at the commencement of the disease, the quantity of urine and the amount of sugar are not large, but the increase of both is progressive as the disease advances. Eceptionally the quantity of the urine is not increased, although abounding in sugar. The quantity of both urine and sugar also varies at different periods of the day, being generally largest within a certain period after meals. According to Bonchardat, the average proportion of sugar to the quantity of urine is 32 to 35 to 1000 . In two cases under my observation, a quantitative analysis of the urine, with reference to the amount of sugar, made by Prof. Austin Flint, Jr., gave the following results: In one case, there were

I Vide cases reported by MM. Luys and Martineau, in Abeille, op. cit., and Cliniqué Médicale par Trousseau. Vide, also, account of morbid appearances in 5 cases by Dr. Wm. Dickinson, in Trans. of Royal Med. and Chirurg. Society, Lon.: don, yol. Jiii., $18 \% 0$.

1860 . 
39.82 grs. to an ounce of urine, and the amount of sugar contained in the urine passed in twenty-four hours was 12 ounces, 413.07 grains. The quantity of urine in twenty-four hours was 155 ounces and 6 drachms; the sp. gr. 1035 ; the amount of urea was 343.627 grs. In the other case, the quantity of urine passed in twenty-four hours was 89 onnces; the sp. gr. 1037. The amount of sugar in an ounce was 36.363 grains; the amount in the whole quantity, 6 ounces, 356.16 glains.

The urine, when preserved for several days, unclergoes the acetic fermentation ; it remains acid, and is devoid of the ammoniacal odor which is emitted by decomposed healthy urine. Vegetable sporules and filaments are developed after a few hours.

Emaciation is usually marked after the disease has existed for some time. Different cases differ as regards the rapidity of the emaciation. In general, it is in proportion to the abundance of sugar in the urine, and the duration of the disease; but it is affected in a greater measure by the condition of the digestive organs. It is evident that alimentary principles which in health contribute to the growth and repair of the body, are, in some way, lost to nutrition in this disease. The development of other affections, especially pulmonary tubereulosis, increases the emaciation. The occurrence of diabetes is not infrequently preceled by embonpoint, more or less excessive. Prof: Seegan observed this in 30 of 100 cases coming under his observation.

Increase of the appetite is usually a prominent symptom. The patient ingests much more food than in health. The abnormal appetite may amount to bulimia or polyplagia. This, coupled with progressive decrease of weight, should alone excite suspicion of the disease. Exceptionally the appetite is not increased, but, on the contrary, more or less impaired. Not infrequently, in the conrse of the disease, the appetite fluctuates, being sometimes greater and sometimes less than in health. It fails as the disease approaches a fatal termination.

Thirst is one of the first symptoms, occurring as soon as the quantity of urine is increased. It is urgent in proportion to the degree of cliuresis. The quantity of drink taken is sometimes enormous. The necessity of drink during the night contributes, with the calls to urinate, to prevent sleep. Dryness of the mouth is a source of considerable inconvenience. The tongue is sometimes moist and coated, and sometimes dry and reddened. The gums are generally altered; they are softened, and either pale or reddened, bleeding readily on pressure. The teeth are apt to become loosened. The saliva is usually acid.

Digestion, until the disease is far advanced, or until serious complications occur, generally appears to be well performed. Dyspeptic ailments, however, are not uncommon. If vomiting oceur, the vomited matter is found to contain sugar. Sugar is also contained in the feces. Constipation is the rule, but, in some cases, diarrhœa alternates with constipation. The dejections show a deficiency of bile. Caries of the teeth is apt to occur early in the progress of the disense.

The respiratory function is not disordered, sáve by the pulmonary affections with which the disease is liable to become complicated, especially pulmonary tuberculosis. Phthisis is liable to occur especially in patients under the age of 30 years. The respirations are somewhat under the normal frequency if there be no pulmonary complication. It is stated that the amount of carbonic acid in the expired air exceeds that of health. The breath has a mawkish, sweetish odor, which is quite characteristic. I have recognized a patient whom I had examined at a clinic months before, and whose features I had entirely forgotten, as soon as he came 
sufficiently near for the odor of his breath to be perceptible. The condition of the gums and teeth renders the breath in some cases fetid.

The circulation offers no symptom of importance. The pulse, if at all affected, is oftener retarded than accelerated, exclusive, of course, of the effect of complications. I have observed capillary congestion of the surface in a notable degree. The temperature of the body is lowered; the thermometer in the axilla shows a temperature of $96^{\circ}$ or $95^{\circ} \mathrm{F}$. in some cases in which the disease is uncomplicated. The skin is usually dry and rough. It is extremely rare for perspiration to occur except when pulmonary tuberculosis becomes developed. Furfuraceous desquamation of the epidermis is not uncommon. Diabetics are subject to carbuncles, boils and various eruptions, viz., psoriasis, lichen, eczema, and impetigo. Spontaneous gangrene of the lower limbs is an occasional event. Ulcerations of the lower limbs, without gangrene, have been observed. In protracted cases œedema of the legs frequently occurs.

Muscular feebleness is more or less marked, and progressive. Aside from the effect of complications, the patient is enfeebled in proportion to the excessive secretion of the urine, the amount of sugar which it contains, and the duration of the disease. Troubles of vision are not very infrequent. Amblyopia, or feebleness of vision, sometimes occurs early, and may eventuate, as the disease advances, in confirmed amaurosis, either complete or incomplete. The vision was more or less affected in 20 out of 162 cases of cliabetes observed by M. Fauconneau Dufresne. In the majority of cases in which the vision is affected, the defect is slight or moderate. Complete amaurosis is extremely rare. In the cases in which amaurosis, complete or incomplete, exists, the ophthalmoscope shows atrophy of the optic disks. Retinitis is a ground for suspecting that albuminuria coexists. ${ }^{1}$ The diabetic condition also appears to favor the development of cataract in some cases. Dr. S. Weir Mitchell has shown, by injecting syrup beneath the skin of the frog, that sugar has a direct action on the erystalline lens. ${ }^{2}$ Prof. Seegan has observed three cases in which recovery from defect of vision occurring in diabetes took place after the sugar had disappeared from the urine. The mental condition frequently undergoes a marked change; the patient becomes irritable, sad and hypochondriacal, the intellectual faculties are enfeebled, and sometimes the mental weakness amounts nearly to imbecility. Diminution and, at length, extinction of venereal desire belong to the clinical history of the disease. This effect frequently occurs early in the history of the disease, before the general powers of the system are much reduced. It may be the first symptom noticed by the patient. It is not, however, a constant symptom. In the female, the menstrual discharge gradually diminishes and is at length suppressed.

The march of the disease is generally slow. Its development is imper. ceptible, and, when discovered, it has probably, in most cases, existed for a considerable period. It is, therefore, difficult to fix the date of its commencement. But, dating from the time when its existence is known, in most cases it continues for many months and not infrequently for many years before ending fatally. Its duration is indefinite and variable. Exceptionally, it runs rapidly to a fatal issue. Generally in these cases the patient is cut off by some intercurrent affection. Any important disease supervening on this is very apt to destroy life; for example, pneu-

1 Vide Allbutt, op. cit.

2 Vide Am. Jour. of Med. Sciences, Jan. 1860. 
monitis in a diabetic patient is usually fatal. Griesinger found the mean duratiou in 225 cases to be from 2 to 3 years. Of 700 cases noted by Prout, only 2 were living at the end of ten years from the time when the disease had been ascertained.' During the progress of intercurrent diseases, the sugar is frequently observed to disappear from the urine. Under these circumstances, this is not to be considered as farorable.

Pathologicar Character. - The pathological character of this disease has already been briefly considered in connection with the morbid conditions of the blood in Part I. ${ }^{2}$ Notwithstanding the facts relating to digestion which seience has acquired within late years, and the important discoveries by Bernard relating to the glycogenic funetion of the liver, the pathology of diabetes is still obscure. It is not certain that the disease does not consist, in a measure at least, in an abmormal production of sugar from the ingesta within the alimentary canal, as maintained by Prout, Bouchardat, and others. The notable effect of excluding fiom the ingesta substances readily converted into sugar goes to support this pathological view. Bermard considers that the disease is due to hyperproduction of sugar by the liver, dependent on morbid excitation received through the nervous system. The known essential pathological condition is the existence of sugar in more or less abundance everywherc in the blood. But it is not certain that this condition is due to the entrance into the circulation of an exeessive quantity of sugar ; it may be rl:e to interruption of the processes by which the sugar existing in the blood in the right side of the heart is decomposed during the passage of the blood through the pulmonary organs. This is the view hold by Mialhe and Raynoso, the former attributing the deficient destruction of the sugar to a want of sufticient alkalinity of the blood. The good effect of alkalies given as remedies, in some cases of diabetes, affords some support of this doctrine. Other theories, which attribute the disease to a deficiency of oxygen in the blood, due to a morbid conclition of the red globules, and to disease of the pancreas, rest only on conjectures. Jaccoud holds that the disease is one of nutrition; the production of sugar being the result of a vice in the process of destruetive assimilation. ${ }^{3}$ It seems clear that the essential pathological condition inyolves either, 1. An undue introduction of sugar into the blood, whether from hyper-produetion of sugar in the liver, in the alimentary canal, in the tissnes of the boly, or from all these sources; or, 2. Deficient destruction of the sugar, the quantity not being increased; or, 3. An increased introduction and deficient destruction combined. Our present knowledge does not enable us to say in which of these explanations lies the true pathology of the disease. The great amount of sugar excreted in the urine in some cases renders it probable that the quantity which enters the blood exceeds that in health.

Causatron.-Our knowledge of the cause of this disease is completely barren of well-ascertained facts beyond the influence of age. In the vast majority of cases the age is between 30 and 50 years. The disease occurs in infuncy, youth, and advanced life, but instances are extremely rare. $A \mathrm{~s}$ regards pathogenetic influences pertaining to climate, season, diet, temperament, habits of life, etc., we have only conjectures, without any precise

1 Archiv. für Physiologische Heilkunde, 1859-60-62.

2 Vide page 83.

3 Vide Pathologie Interne and Nouveau Dictinnaire de Medicine et de Chirurgie Pratique, art. Diabète Sucré. 
information. Men are mueh oftener affected than women. Of the 225 cases analyzed by Griesinger, in 172 the patients were men, and in $\mathbf{5 3}$ women.

Cases have been reported in which injuries reeeived on the posterior part of the head have been followed by saccharine urine. But it is doubtful if in these cases diabetes, considered as an individual affection, existed. The saccharine urine oecurring under these circumstances denotes a transient condition which occurs in various other pathological connections.

The disease in some cases has been attributed to violent mental emotions.

Diagnosis.-It is to be borne in mind that the fact of sugar existing in the urine is not alone sufficient evidence of the existence of the disease under consideration. Sugar, generally in small quantity, and for a transient period, is not infrequently found in the urine in the course of various maladies. In aged persons the urine sometimes temporarily contains a considerable amount of sugar. A distinguished member of the medical profession in this country, of advanced life, three years ago, was led to observe that the quantity of his urine was notably increased. On examination, it was found to contain a large amount of sugar. Without any medicinal treatment or change of dict, the diuresis ceased; there is now no evidence of diabetes, and the health is in all respects good. I lave known much needless uneasiness to be occasioned by announcing to a patient the discovery of a trace of sugar after an analysis of the urine.

If diabetes exist, saecharine urine is persistent, the amount of sugar is unusually large, and generally the quantity of urine is notably increased. These points relating to the urine render the diagnosis sufficiently easy and positive. The diagnosis is confirmed by other symptoms belonging to the clinical history of the disease. If the quantity of urime be considerably increased, and, on testing with the urinometer, the specific gravity be found high, sugar is probably present. In eases of diuresis, in which only the water of the urine is inereased, that is, in cases of socalled diabetes insipidus, the specific gravity is extremely low, sometimes but little above that of spring-water. Moreover, the fragrant odor of diabetic urine is characteristic, and it froths readily on agitation, the airbubbles remaining for some time. But the practitioner should not be satisfied without demonstrative proof of the presence of sugar. Of the numerous methods of testing urine for sugar, one readily available, and as reliable as any, is the fermentation test. A common test-tube or phial containing a little ordinary yeast is to be filled with uriue, inverted and placed in a saucer containing the urine, care being taken to prevent the entrance of air. Kept at a temperature of $70^{\circ}$ Fahr., fermentation ensues, and the gas formed rises in the tube and displaces the urine. The gas is shown to be carbonic acid from its failure to support combustion. For other tests, the reader is referred to works on chemistry, or treatises devoted to urinary diseases. ${ }^{1}$ Christison states that a cubie inch of the carbonic acid formed in the fermentation test represents a grain of sugar. It may be desirable to ascertain more accurately the amount of sugar excreted in a given period. For this purpose, Fehling's method of analysis may be employed. Examinations of urine are to be repeated, in the first place, to settle the persistence of the saccharine condition, and, in the second place, with reference to improvement or otherwise. In

1 Vide Bird on Urinary Deposits, etc. Edited by Birkett. Am. Ed. 18.59. Do. Roberts on Urinary and Renal Diseases. Do. Manual of Chemical Examination of the Urine in Disease, by Austin Flint, Jr. 
general, it suffices for these oljects to ascertain the quantity of urine passed in the twenty-four hours, and its specific gravity, the existence of sugar having been already ascertained.

Prognosis.-Diabetes mellitus is a disease which, in a large majority of cases, offers very little encouragement as regards the prospect of permanent recovery. Of course, in this statement reference is had to cases in which the diagnosis is based on persistency of the diabetic condition. The prospect, in most cases, is more hopeless than in cases of pulmonary tubercuiosis. Undoubtedly recoveries have taken place. Data are wanting for letermining the proportion of radical cures, but they form a very small minority. In the great majority of eases, however, the cliabetic condition may be notably ameliorated. Not infrequently the quantity of urine may be reduced nearly or quite within the normal limits, the sugar being proportionately lessened or completely disappearing, and the patient regaining, to a greater or less extent, weight and strength. But in the larger proportion of these cases relapse occurs sooner or later, and the disease ultimately proves fatal.

Patients are generally cut off by intercurrent affections. The existence of this disease impairs the ability to cope with affections which, under other circumstances, might not be serious. If the disease continue for a long time without complications or supervening affections, it induces an extreme degree of debility and marasmus. In a considerable proportion of cases in which the disease is of long duration, pulmonary tuberculosis becomes developel, and this hastens the fatal issue. Phthisis was the immediate cause of death in 43 per cent. of the cases analyzed by Griesinger.

An early diagnosis diminishes somewhat the gravity of the prognosis. The chances of arresting the disease are greater if it be recognized before the general condition of the system has undergone much deterioration. 'This fict enforces the importance of examining the urine for sugar in all cases in which there are any grounds for suspecting its presence; and it is to be recollected that sugar is sometimes persistingly present when the quantity of urine is not much, nor even at all, increased. Examination of the urine for sugar should not be omitted whenever failure of strength or emaciation occurs without apparent cause, and especially if the appetite be increased rather than climinished. Unusual thirst, dryness of the mouth or fauces, acidity of the saliva, dryness of the skin, impairment of vision, diminution or extinction of venereal desire, in men irritation or inflammation of the prepuce or glans penis, and in women pruritus vulva should suggest the possibility of the existence of this clisease. Notable increase in the quantity of urine, of course, should excite strong suspicion, but a certain amount of increase may take place without the attention of the patient being excited, or the patient may not deem it a symptom of importance, and it is therefore necessary for the physician to direet inquiries to this point.

Some cases have come under my observation exemplifying, apparently, recovery from the disease. Two illustrative cases were introduced in the last edition of this work. These patients have since died, but without a return of the diabetes. Roberts, in his work on urinary and renal diseases, has cited several such cases. As stated by this author, age has an important bearing on the prognosis: "The younger the patient, the less hope of ultimate recovery. The disease is less formidable in corpulent than in spare persons. It is less serious in proportion as the diuresis is small." Roberts adds that "cases which can be traced to mental anxiety 
and traumatic lesions, appear to be somewhat more hopeful than those for which no tangible cause can be assigned."

Roberts and others attach to the supervention of albuminuria much additional gravity. Some cases which $I$ have met with illustrate a remarkable tolerance of diabetes and Bright's disease in combination. The diuresis ineident to the former seems to afford a certain amount of protection against uræmia. Undoubtedly the development of structural disease of the kidneys increases the hopefulness of recovery. In a case, however, of which an abstract was given in the last edition of this work, the sugar disappeared from the urine, and the patient many months afterward died of renal disease accompanied with dilatation of the heart and general dropsy.

Treatment.-Of the great number and variety of remedies which have been employed in the treatment of this disease, several are more or less useful. 'The disease maty he favorably modified by medication, but experience has thus far failed to establish the curative efficacy of any known remedial agent. Certain therapeutical measures have been employed, which are not only devoid of usefulness, but hurtful. Among these are general and local bloodletting, mercurial preparations, emetics, and active purgatives. Other measures are of doubtful utility, and some exert no appreciable effect, either for good or harm.

Among the useful remedies are alkalies. Whether the theoretical views which led to their introduction as remedies in this disease by Mialhe be correct or not, to wit, that sugar fails to undergo adequate destruction on account of a deficient alkalinity of the blood, experience has abundantly shown that they sometimes producc in a notable degree a favorable modification of the disease. The bicarbonate of soda may be given, commencing with a drachm, and increasing to two, three, or even four drachms daily. It may be continued for a long period, if, under its use, the quantity of urine and amount of sugar decrease, and improvement take place as regards thirst and other symptoms. But, to avoid risk of harm from the too prolonged use of the alkili, it should be suspencled for several days at short intervals. Trousseau advises that it should he given for ten consecutive days only in each month. I have known this plan of treatment signally beneficial. If the sorla occasion diarrhœa, the quantity should be diminished or lime-water substituted. The Vichy water has long been considered useful in this disease. The natural or artificial water, if circumstances permit, may enter with advantage into the alkalize treatment. Other alkaline waters are also useful.

The carbonate of ammonia has proved a useful remerly in the hands of Barlow, Birch, Bouchardat, and others. This remedy has been observed to produce a farorable effect upon the functions of the skin. The citrate of ammonia in the effervescent form is highly recommended by Camplin.

Opium is a useful remedy in certain cases. Under its use the saccharine condition of the urine is sometimes lessened in a marked degree. Opium has been given in large quantities, even to the extent of narcotism, in the hope that it might prove curative. All the advantage to be derived from it, however, is obtained from moderate doses. Of course, patients who tolerate this remedy well will be likely to receive most benefit from its use. Belladonna has also been found useful, and may be employed in cases in which opiates are not well borne. Pavy has reported 13 cases treated by either opium or some of its constituents. He found codeia preferable to other preparations. Beginning with half a grain of codeia, he increased the doses gradually, and he has given as much as ten grains three times daily. He has not found this remedy objectionable on the 
score of either constipation or impairment of the appetite. The details of his cases show a marked effect as regards the reduction of the quantity of sugar in the urine, the diet not being restricted. In none of the 13 cases, however, was it ascertained that a permanent cure was effected, although, in one case, the urine was free from sugar after the lapse of a year from the date of the discharge of the patient from hospital.'

Arsenic has been extolled as a useful remedy by Devergie, Foville fils, Jaceond, Titon, and Bronardel in France; also, by Leube in Germany. I have prescribed this remedy in several cases apparently with some benefit, but without results showing any curative eflicacy. It should be given in small doses, and continned without increase of dose. Given thus, it is tolerated for a long period.

From the effect of the bromide of potassium in some cases, I was led to propose it as entitled to be ineluded among the different remedies which are nsefin in this disense." I have found it useful in allaying the nervous irritability which the disease is apt to oceasion, and in procuring sleep. I do not, however, claim for it curative power.

Prof. Alnaldo Cantani, of Naples, has introduced, as a new remedy, lactic acic. In explanation of the remedial effieacy which he attributes to this drug, he offers the following theory. In diabetes there is not an excess in the production of sugar, but it is produced in a morbid form to which he gives the name paraglucose. In this form it is not transformed in the lungs into lactic acid and fat; hence, it passes off by the kidneys, and the patient consumes his own fat as well as the albuminates and fat of his food, in order to keep up, by combustion, the production of animal heat. The diminished temperature of the body, in cases of diabetes, is a ground for this theory; also, the diminished frequency of respirations which is observed in some, but not in all eases. In the lactic acid, a combustible element is supplied, and, thereby, the fats and albuminates of the body are saved. The use of the drug is to be conjoined with a rigid antidiabetic diet. The quantity to be given per diem is from 150 to 250 grains. ${ }^{3}$ Dr. Balfour, of Edinburgh, has reported seven cases which appeared to show notable benefit from this remedy.* I)r. Forster, of Birmingham, on the other hand, has found no decided effect from a trial of it on either the quantity of urine, the amount of sugar, the temperature of the body, or the weight of the patient. Forster has observed, as an effect of this remedy, pains of a rheumatic character in the joints and limbs. ${ }^{5}$

The peroxide of hydrogen has been used under the belief that an essential part of the pathology of the disease is the diminution of oxygen in the blood. Clinical experience, however, has failed to furnish satisfactory proof of any therapentical value in this remedy. A more effectual mode of introducing oxygen is the inhalation of the gas. Proof of the efficacy of this measure is wanting.

The cod-liver oil, if tolerated by the stomach, and digested, is useful by preventing the consumption of the fat within the body (autophageny).

Strychnia has been observed to exert a marked effect upon the amount of sugar contained in the urine. In a case under the observation of the late Ir. Frick, of Baltimore, for nine months, the relative effects of several remedies successively tried were tested by a careful analysis of the urine

1 Guy's IIospital Reports, 1870.

2 Vide American Med. Practitioner, vol. i., No. 1.

3 Vide Year-hook of Therapeutics, etc., edited by Horatio C. Wood, Jr., M. D., 1872 ; also. Dobell's Reports on Progress of Med., vol. ii.

1 Vide Edinburgh Med. Jnurnal, Dec. 1871.

- Year-book by Wood, 1872. 
at the end of each week. The remedies tried, in addition to strychnia, were the tincture of the chloride of iron, ammonia, iodicie of potassium, creasote and naphtha, cod-liver oil, ergot, and calomel and opium. of these remedies the strychnia effected by far the greatest diminution of the amount of sugar excreted.'

A simple enumeration of some other remedies will suffice. Rennet, introduced by Dr. Gray, of Glasgow, Scotland, given in doses of a teaspoonful after each meal, the supposed utility of this remedy being explained by its power of converting sugar into lactic acid. The article known as pepsin has also been used. Yeast has been suggested by Prof. George B. Wood. Cod-liver oil, recommended by Babington and others. Creasote, rhatania, and other vegetable astringents ; camphor, assafœtida, valerian, and other of the so-called antispasmodics; iodine, quinia, the mineral acids, and the various preparations of iron, are to be added to the list. Each and all these remedies are doubtless more or less useful in certain cases. The vapor and the hot-air bath have been found useful. Abeille states that the use of water after the hydropathic method has been found, in France, to be useful in this disease, as well as in affections attended with albuminuria.

The remedies which experience has shown to be useful in diabetes are to be selected, combined, and varied in adaptation to circumstances in individual cascs. In addition, dietetic and regimenal measures are indicated, and these, indeed, constitute by far the most important part of the treatment. Saccharine and amylaceous substances are, as far as practicable, to be excluded from the diet. Under judicious dietetic restrictions in this respect, the quantity and density of the urine are generally notably diminished. Adequate alimentation is, of course, important, and the object is to nourish the body as well as possible with as much exclusion of starch and sugar from the aliment as is compatible with nutrition. Meat of all kinds, including fish, oysters, crabs, etc., may enter into the diet, with butter, cream, curd and cheese, eggs, and all vegetables deficient in starch and sugar, such as lettuce, cabbage, chicory, the tops of beets, spinach, celery, water-cresses, onions, etc. Mushrooms, cauliflower, and turnips in moderate quantity are admissible. Vegetables which abound in starch or sugar, such as rice, potatoes, beets, and beans, are to be taken very sparingly or not at all. Bread or cakes made of wheat or rye flour, corn or oatmeal, belong in the same category. The force of the phrase "staff of life," as applied to bread, is appreciated when the attempt is made to exclude it persistingly from the diet. The want of bread, in cases of this disease, becomes almost imperative; hence, various methods of preparing a substitute from flour or meal with the amylaceous part removed. Of the different kinds of diabetic bread, that recommended by Camplin is to be preferred. This kind of bread, or cake, was devised by Camplin, a medical practitioner of I ondon, for himself, being affected with diabetes, and to its use, in conjunction with other measures, chiefly hygienic, he attributes his apparent recovery and exemption from a return of the disease for ten or twelve years. ${ }^{2}$ Pavy has employed rusks or bis-

1 American Journal of the Med. Sciences, July, 1852.

2 Mr. Camplin's little book may be consulted with much interest and profit. For the convenience of the practitioner, his formula for bran cakes to be used as a substitute for bread is subjoined : "Take a sufficient quantity (say a quart) of wheat bran, boil it in two successive waters for a quarter of an hour, each time straining it through a sieve, then wash it well with cold water (on the sieve) until the water runs off perfectly clear; squecze the bran in a cloth as dry as you can, then spread it thinly on a dish and place it in a slow oven; if put in at night let it remain until 
cuits made of the sweet almond finely ground. $\Lambda \mathrm{s}$ a change this is found advantageous. In regulating the diet of cliabetics, the articles not on the proscribed list should be varied from day to clay in order to avoid the disgust occasioned by using too continuously the same kinds of food. Different modes of cooking should be resorted to with a view to variety. From time to time the restrictions should be cautiously relaxed in order to see how a change of diet is borne. 'Tea and coffec, without sugar, but with milk or cream, are allowable. Claret and Burgundy wine, and dry, sound sherry wine, are not only allowable, but generally useful; or spirit, in small quantity, may be tried instead of these kinds of wine. The acid or subacid fruits may sometimes be moderately indulged in. In carrying out the dietetic treatment, success will depend in a great measure on the pains taken to combine and prepare the admissible articles, so that the appetite and taste will be satisfied. The physician should enforce the importance of this, and enter into details with patients sufficiently to secure it. With a diet earefully arranged, I have known it to be continued for several years without being felt as a hardship; and, under dietetic treatment alone, I have known the urine to be reduced to the normal quantity, and the general health so fully restored that, without the knowledge of the fact that the urine contained sugar, the patient would consider himself perfectly well. Some patients, however, eonsicler the diet insupportable, and, if persisted in, they decline in spirits and strength. Under these circumstanees, it is not judicions to insist upon a rigid adherence to it. Patients are apt to feel the want of bread more than any other article of food, and it is sometimes advisable to allow it in moderate quantity.

The free use of sugar in cases of diabetes was tried many years ago in Guy's Hospital by Richard Bright, and more recently in Franee and other countries. Experience has shown, that, while sugar is tolerated in some cases much better than might have been anticipated, in many, if not most, cases it is evidently produetive of harm.

Attention to the skin is important in eases of cliabetes. The surface should be efficiently protected, in cold or variable climates, by flannel or silk, and during the winter season, by the chamois leather or buckskin waisteoat. Sponging the boly, followed by brisk friction, is useful. The occasional use of the warm bath is to be advised. A change from a cold and variable climate to one uniform and warm is often highly advantageous. A long sea voyage in a warm latitude has been found signally beneficial. Exercise in the open air is a highly important part of the regimenal treatment. Gymnastic exercises may sometimes be resorted to with profit. Last, but by no means least in importance, mental relaxation and recreation are to be enumerated in the list of measures of regimen. Professor

the morning, when, if perfectly dry and crisp, it will be fit for grinding. The bran thus prepared must be ground in a fine mill and sifted through a wire sieve of such fineness as to require the use of a brush to pass it through. That which remains in the sieve must be ground again until it becomes quite soft and fine. Take of this bran powder three ounces (some patients use four ounces), three eggs, one and a half or two ounces of butter, and about half a pint of milk; mix the eggs with a little of the milk, and warm the butter with the other portion; then stir the whole well together, adding a little nutmeg and ginger, or any other agreeable spice. Bake in small tins, which must be well buttered, in a rather quick oven for about half an hour. The cakes, when baked, should be a little thicker than a captain's biscuit. 'I'hey may be eaten with meat or cheese for breakfast, dinner, and silpper. At tea they require a free allowance of butter, or they may be eaten with curd or any of the soft cheeses.", 
Seegan, who has studied over one hundred cases at Carlsbad, Germany, states that the waters of this place are generally useful, as shown by a diminution of the sugar in the urine, and general improvement, but that they do not exert a curative effect.'

The treatment of this disease, as regards diet and regimen, unfortunately, in many cases, is practicable only to a limited extent. The prospect of improvement and cure is vastly better when the eircumstances of patients permit their having recourse to the measures which have been mentioned, to the fullest extent. These measures are not fully available in hospital or dispensary practice and in cases among the poorer classes of society. If, happily, the affection be removed, as shown by the disappearance of sugar from the urine, and a corresponding improvement in all the symptoms, the liability to a return is to be borne in mind. The usual diet of health and the habits of life in other respects are to be cautiously resumed, and great care to aroid all the causes of disease, for months, years, and indeed during lifetime, is to be enjoined.

As an addendum to the foregoing account of the treatment of diabetes, what is known as the "milk-treatment" claims notice. An exclusively milk diet has been found by Arthur Scott Donkin, George W. Balfour, Dr. Karrel, of St. Petersburg, and others, notably effective in this disease. I can testify to its utility, having made trial of it in several hospital cases. In my cases the quantity of urine diminished greatly under its use, and there was general improvement, but without, in any cases, an approach to recovery. The milk used was that known as " condensed milk," brought to the ordinary standard of pure milk by the addition of water. 'The writers just named, however, eonsider it important to remove the cream from the milk; hence, the term "skim-milk treatment" is used by them. Donkin claims, in behalf of this treatment, that if the disease be not far advanced, the sugar will disappear from the urine in from 12 days to 5 or 6 weeks; and that, if the disease have been of long standing, its progress will be arrested, although a eure is not to be expected. The patient is to be restricted to skim-milk for 3 weeks or a month, then, in addition, from two to four pints may be taken in the form of curd made by rennet. A fter a time, varying in different eases, lean meat and green vegetables are added. Gradually, the patient is allowed eggs, fish, game, poultry, potterl bread, and a variety of dishes made of lean meats. After recovery, vegetable substances containing starch and sugar are to be excluded for a long time, and, in some eases, this must be permanent. There is always loss of weight for the first 2 or 3 weeks after commencing the skim-milk treatment. ${ }^{2}$

It is to be added, that the elinical evidence, addnced by Donkin, of the success of this treatment, is comprised in a report of seven cases. In one of these cases sugar was absent from the urine, and the general condition was good three months after the date of recovery. In another case, freedom from the disease was known to have continued for several months. In one case, the disease returned nine months after apparent recovery. In another case, the health was good, and there was no sugar in the urine, after the lapse of seven months. In the remaining three cases, there was improvement but not recovery.

These cases, while they certainly furnish encouragement for the trial of

1 Vide Archives Géenerales de Médecine, Mars, 1867.

2 The Skim-milk Treatment of Diabetes and Bright's disease, by Arthur Scott Donkin, M.D.; etc. London, 1871. 
the treatment, are by no means sufficient for determining the extent of its curative power; nor is it eertain that a permanent cure was effected in a single case. Further clinieal facts are requisite as a basis for a fair estimate of the value of the treatment.

\section{CH A P T ER I V.}

Disease of the Supia-Renal Capsules-Addison's Disease. Involuntary Seminal Emisslons, Spermatorrhoea. Impotence.

THE supra-renal capsules have no known connection with the kidneys other than the proximity expressed by the name. As, however, the functions of these organs are not known, the diseases to which they are subject may with propriety be noticed in this section. A portion, therefore, of this chapter will be devoted to disease of the supra-renal capsules, as entering into the affection described by Addison. Diseases affecting the generative system are, for the most part, embraced either among the diseases peculiar to women or the diseases which are properly surgical. But there are certain affections of the male generative system which may be considered as belonging to medical practice rather than to surgery, viz., involuntary seminal emissions, spermatorrhoea, and impotence. A brief notice of the latter affeetions will conclude the section embraeing the diseases of the genito-urinary system.

\section{Disease of the Supra-Renal Capsules-Addison's Disease.}

The late Dr. Thomas Addison, of Guy's Hospital, London, in 1855, described a form of anæmia characterized by a dingy or dark discoloration of the skin. This form of aniemia, which has been already referred to in connection with the morbid conditions of the blood, is described by Dr. Addison as follows: "For a long period I had, from time to time, met with a very remarkable form of general anæmia, occurring without any discoverable cause whatever-cases in which there had been no previous loss of blood, no exhausting diarrhœa, no chlorosis, no purpura, no renal, splenic, miasmatic, glandular, strumous, or malignant disease. Accordingly, in speaking of this form of anæmia in clinieal leetures, I-perhaps with little propriety - applied to it the term 'idiopathic,' to distinguish it from cases in which there existed more or less evidence of some of the usual causes or concomitants of the anæmic state. The disease presented in every instance the same general character, pursued a similar course, and, with scarcely a single exception, was followed, after a variable periorl, by the same fatal result. It occurs in both sexes, generally, but not exclusively, beyond the middle period of life, and, so far as I at present know, chiefly in persons of a somewhat large and bulky frame, and with a stronglymarked tendency to the formation of fat. It makes its approach in so slow and insidious a manner, that the patient can hardly fix a date to his earliest feeling of that languor which is shortly to become so extreme. The countenance gets pale, the whites of the eyes become pearly, the general frame flabby rather than wasted; the pulse perhaps large, but 
remarkably soft and compressible, and occasionally with a slight jerk, especially under the slightest excitement; there is an increasing indisposition to exertion, with an uncomfortable feeling of faintness or breathlessness in attempting it; the heart is readily made to palpitate; the whole surface of the body presents a blanched, smooth, and waxy appearance; the lips, gums, and tongue seem bloodless; the flabbiness of the solids increases; the appetite fails; extreme languor and faintness supervene, breathlessness and palpitations being produced by the most trifling exertion or emotion; some slight œelema is probably perceived about the ankles; the debility beeomes extremc, the patient can no longer rise from the bed, the mind occasionally wanders, he falls into a prostrate and halftorpid state, and at length expires; nevertheless, to the very last, and after a sickness of perhaps scveral months' duration, the bulkiness of the general frame and the amount of obesity often present a most striking contrast to the failure and exhaustion observable in every other respect."

In connection with anæmia and symptoms denoting an extremely grave cachetic condition, Dr. Addison was led to observe a discoloration of the surface, which he describes as follows: "With more or less manifestation of the symptoms already enumerated, we discover a most remarkable, and, so far as I know, characteristic discoloration taking place in the skin - sufficiently marked, indeed, as generally to have attracted the attention of the patient himself or his friends. 'This discoloration pervades the whole surface of the body, but is commonly most strongly manifested on the face, neck, superior extremities, penis, and scrotum, and in the flexures of the axillæe and around the navel. It may be said to present a dingy or smoky appearance, or various tints or shades of deep amber or chestnut-brown; and in one instance the skin was so universally and so deeply darkened, that, but for the features, the patient might have been mistaken for a mulatto."'

An examination of the bodies after death failed to reveal lesions of imporant organs adequate to explain the symptoms and the fatal termination in the cases described. At length Dr. Addison was led to observe the coexistence of disease of the supra-renal capsules. In the monograph from which the foregoing extracts are taken, he gives an account of eleven cases, in all of which the supra-renal capsules were more or less diseased. In some of the cases the disease was limited to one of the capsules, and in other cases both organs were affected.

Since the publication of Addison's researches, the attention of clinical observers in different countries has been directed to the coexistence of disease of the supra-renal capsules, with the peculiar cachectic condition, accompanied by discoloration of the skin. And, regarding the condition as constituting a newly-discovered affection, it is commonly known as the bronzed-skin disease; it has also been called, in honor of the discoverer, Addison's disease; supra-renal melasma is another name. Numerous cases have been reported. The affection, although not of frequent occurrence, is not extremely rare. My colleague, Prof. Isaac E. Taylor, has reported seven cases, six of which were observed in Bellevue Hospital, in the course of a service of two months. A post-mortem examination was made in only two of these cases, in both of which the supra-renal capsulcs. were diseased.

Of the reported cases in which the coloration of the skin, or melasma,

1 On the Constitutional and Jocal Effects of Diseases of the Supra-renal Capsules, by Thomas Addison, M.D., Senior Physician to Guy's Hospital, London, 1855.

2 Ibid. 
together with the anæmia and other symptoms, have corresponded with the cases observed by Addison, the supra-renal eapsules have not been uniformly found diseased. On the other hand, disease of the supra-renal capsules is by no meaus invariably accompanied by the bronzed-skin and cachectic condition deseribed by Addison. 'The proportion of cases in which the bronzed-skin disease and disease of the capsules are associated, are, however, sufficient in number to show that the association is due, not merely to coincidence, but to a pathological connection. Of the nature of the pathological connection existing between the bronzed-skin disease and disease of the supra-renal capsules, facts at present ascertained do not warrant a conclusion. 'The pathological importance of the disease of the capsules can only be conjectured. The lack of positive knowledge on this point corresponds with our ignorance of the physiological importance of the capsules. Brown-Séquard, finding that speedy death followed the removal of these organs in inferior animals, was led to conclude that they performed some function indispensable to life. 'The experiments of Harley and others, however, render this conclusion untenable. Animals have recovered and remained perfectly well after these organs had been removed. We are hardly warranted in ealling the disease of the capsules, when associated with bronzed-skin disease, the analomical characteristic of the latter, in the same sense as, for example, disease of the Peyerian glands is the anatomical eharacteristie of typhoid fever. The association is not snfficiently constant to establish the relation expressed by that term. The pathological eonnection would seem rather to resemble that existing between enlargement of the spleen and intermittent fever.

The disease of the supra-renal capsules in certain cases of the so-called Addison's or bronzed-skin disease consists in the presence of a softish, gray, translucent matter, which sometimes becomes liquefied into a creamlike matter, and sometimes becomes solidified into a cretaceous mass. The deposit replaces the tissue of the organs, and, if extensive, involves their disorganization. ${ }^{1}$ These organs are also occasionally the seat of the tuberculous deposit, of cancer, and fatty degeneration. Aside from the connection with the bronzed-skin disease, there are no symptoms which point to disease seated in these organs.

Bronzing of the skin, melasma, corresponding to the description by Addison, may exist without the anæmia and cachectic condition which are essential to the affection which he describes. It is, therefore, to be borne in mind that melasma, alone, is not evidence of Addison's disease, and, in itself, does not involve a grave condition. The melasma which is a symptom of Addison's disease is to be discriminated from the eruptive disease known as pityriasis niga. 'The latter is accompanied by furfuraceous desquamation of the cuticle, and is attended by a troublesome pruritus. Discoloration of the skin caused by the prolenged ariministration of silver is, also, not to be confounded with the bronzed skin of Addison's disease. Microscopical examination of specimens of skin affected with the bronzed discoloration, made by Wilkes, Kobin, and Dalton, has shown that the morbid change in color is due to pigmentary granules in the rete mucosum, identical with those in the skin of the negro.

As regards the treatment of the bronze-skin disease, the indications are derived from the aniemia and the cachetic condition. But, thus far, thera-

'Vide Wilkes' Pathological Anatomy. 
peutical measures have proved unavailing. Were the pathology of the affection understood, it might perhaps lead the way to successful treatment, but, in the existing state of our knowledge, it is to be reckoned among the incurable diseases. In a case which I saw with Dr. A. D. Rockwell, the treatment by general faradization and galvanization of the sympathetic was accompanied by marked improvement as regards the muscular strength and endurance; the sexual desire and capacity, which had been lost, returned, and the bronzing became sensibly lighter. This inprovement continued for two years, when the patient failed rapidly and died within a few weeks from the time he began to decline. The autopsy in this case showed characteristic lesion of the capsules.

Dr. Edward Headlam Greenhow, in a monograph published in $\mathbf{1 8 6 6}$, gives the results of an analysis of 196 cases, these being all the cases which he was able to collect, of disease of the supra-renal capsules of whatever kind, and all the cases of bronzed skin without any supra-renal disease. In this collection of cases were embraced ten in which discoloration of the skin existed without disease of the supra-renal capsules; twenty-four in which the disease of the capsules was considered as cancerous; ten in which the disease of the capsules was not cancerous nor yet true Addison's disease, and twenty-four in which the disease was imperfectly described as of a doubtful character. Excluding these sixty-eight cases, the remainder (128 cases) were cases in which the supra-renal capsules had undergone the peculiar morbid change characteristic of Addison's disease. Of these 128 cases, in 46 the disease of the capsules was uncomplicated; in 59 the disease was complicated with vertebral and tubercular disease, and in 23 phthisis, or some other scrious disease, was associated. In 18 of the 128 cases there was no discoloration of the skin; in 85 the characteristic discoloration was marked, and in 25 it was slight. In 28 cases there were no constitutional symptoms ; in 17 cases the coustitutional symptoms were slight, and in 83 cases the constitutional symptoms were marked. These facts are considered by Greenhow as showing a pathological "relation between a certain train of constitutional symptoms, attended by discoloration of the skin, and one particular organic lesion of the supra-renal capsules."

According to Greenhow, the lesion in true Addison's disease is peculiar to that disease. Cancerous and other affections which are not characteristic are unattended by discoloration and the constitutional symptoms proper to Addison's disease. He describes the morbid appearances as follows : "They (the capsules) are generally hard and nodulated. On section, they scarcely ever present any trace of the distinction between cortex and medulla, or any remains of the natural tissue. It is evident that there has been an exudation which has obliterated the original structure. In a large proportion of the accurately recorded cases, the appearance of the capsules on section is said not to have been uniform, but marked by the admixture of two different looking deposits. One of these is semi-transparent, firm, and, when first cut into, of a grayish color, rapidly assuming a pinkish hue on exposure to the air; the other is generally seen in the form of irregular, roundish, opaque, yellow, or creamcolored masses, emberded in the translucent portion. Often, on careful examination, various gradations will be met with, intermediate between the translucent tissue and the opaque friable nodules, and sometimes there are harder portions of a cretaceous character, either in the form of granules or of larger masses. Sometimes, also, collections of thick, creany fluid, called abscesses by some observers, are found occupying larger or smaller 
portions of the diseased organs, which, in exceptional, probably older, cases, are shrivelled instead of being enlarged, and contain only this fluid with a little cheesy matter, or else are hardened throughout into cretaceous masses. Under the microscope the translucent portion is generally found to consist of a more or less fibrous basis, mixed with much granular matter, containing shrunken cells and nuclei, some of the nuclei being occasionally elongated into spindle-shaped bodies. The yellow opaque nodules consist, in differeut proportions, of amorphous matter mixed with irregular shaped, shrunken cells, nuclei, and oil. When the creamy liquid from cavities, or so-called abscesses, has been examined, it has been found not. to be true pus, but to consist mainly of oily debris."

Both capsules are generally diseased. The exceptions in Greenhow's cases were only 11 in 128 . The disease, however, is by no means in the same stage in both organs. Greenhow attributes the disease to an extension of inflammation from disease or injured adjacent parts to the capsules. In a large proportion of cases, evidences of inflammation in the cellular envelopes of the capsules are found after death, namely thickening and firm adhesions to neighboring organs-diaphragm, liver, pancreas, vena cava, kidneys, stomach, ete. It is, perhaps, more probable that these effects of extrinsie inflammation are secondary to disease of the eapsules.

Greenhow describes the constitutional symptoms as follows: "Gradual progressive asthenia, often originating without any apparent causc, and seldom dating from any definite period ; great languor and indisposition for exertion, with, in advanced cases, breathlessness and palpitation, frequent sighing or yawning, and generally faintness on unaking any muscular effort, sometimes even on being raised up in bed. There is almost invariably great weakness of the heart's action and remarkable feebleness of pulse ; loss of appetite ; irritability of stomach, with nausea ; and, toward the close of the illness, at least occasional, and often persistent, vomiting. The mind is generally clear to the last, but so great is the prostration in the latest stages of the disease that the patient often lies in a drowsy, apparently semi-comatose state, from which, however, he can be roused by questions, and to these he generally gives pertinent, though slow and reluctant, answers. There are in many cases pains in the loins, hypochondria or epigastrium; and, more rarely, dimness of sight, vertigo, and, near death, a tendeney to ineoherence or delirium. Death takes place from asthenia, and, often, rather suddenly." "There is, in uneomplicated cases, comparatively little or no emaciation. The skin is soft and cool. The tongue is usually clean and moist until the last day of life; the bowels are seldom disordered, and the urine is normal."

Other conclusions from Greenhow's investigations are as follows: "In many cases the hair becomes visibly darker with the darkening of the skin."

"As yet no satisfactory conclusions ean be drawn from the hitherto insuffieient investigations into the condition of the blood."

Males are oftener affeeted than females. The disease seems not to occur in childhood; the earliest age of death was 11 years. It is eqnally rare in advaneed life. Only seven males and four females died above the age of 50 years; and of these only two males and one female were over 60 years of age. The disease is in a great measure confined to classes engaged in active manual labor.'

- For analysis of 202 cases, and facts bearing on the theory that the semilunar ganglion and solar plexus are involverl in the pathology of the affection, vide article by Jaccond in Nouveau Dict. de Méd et de Chirurg. Pratique, tome v. (Bronzée maladie), 1866. 


\section{Involuntary Seminal Emissions. Spermatorrhoa. Impotence.}

Involuntary seminal emissions, spermatorrhœa, and impotence frequently depend on abuormal conditions which belong to surgery, but not infiequently they fall properly within the sphere of the practice of medicine. By involuntary seminal emissions is meant the ejaculation of semen and the venereal orgasm, without any voluntary effort, either natural or umatural. Involuntary emissions occurring during sleep are common, taking place in connection with erotie dreams. Oecurring after intervals of several weeks or days, in persons of continent habits, they are pliysiological rather than pathological. They denote simply a certain amount of functional activity of the generative organs. 'They do not imply a morbid condition, nor do they lead to any morbid effects. 'They are apt especially to oeeur when habitual sexual intercourse is, from any cause, interlupted. Under these circumstances, they are manifestations of health rather than disease. The physician is warranted in giving such assurances to patients, who often are rendered needlessly apprehensive and unhappy by popular works or lectures, the object of which is to excite fears for a mercenary purpose.

Occurring mole frequently than within the limits just stated, the emissions denote a morbid erethism and weakness of the organs of generation. They occur sometimes nightly, and sometimes even repeatedly during the same night. They then eall for remedial measures, although, under these circumstances, their morbid effects have been much exaggerated. Occurring frequently in persons debilitated from any cause, they may take place without erection, and with little or no venereal excitement, the patient sometimes becoming aware of their oceurrence only by the seminal stains. They occur sometimes during the day when the venereal desire is excited. These are called diurnal, in distinction from nocturnal, emissions. $\Lambda$ patient stated to me that they had occurred, in his case, from excitement caused by shampooing the head in a barber's shop. Persons who have been addicted to venereal excesses or to the ummatural abuse of the sexual function, are especially subject to this morbid frequency of involuntary emissions. The mind, in such cases, particularly if the affection be attributable to unnatural abuse, is apt to become greatly depressed; the patient is apprehensive of impotence, or fancies that he is already impotent; thus attention is absorbed with the affection; he suffers from a sense of pollution and degradation; he believes that his constitution is ruined, and there is danger of insanity. Patients in the condition just sketched form an unfortunate class with which every practitioner is more or less familiar. They are apt to go from one physician to another, and upon patients of this class quacks prey largely. 'The affection sometimes exists in a still greater degree. 'The emissions are diurnal, as well as nocturnal. They are produced by anything which provokes the sexual passion, and may take place with little or no exeitement of the external organs.

Spermatorrhœa means, strictly, the discharge of semen without the occurrence of the orgasm. It is an affection superadded to morbid seminal emissions, the seminal secretion passing into the urethra, and sometimes into the bladder, without the consciousness of the patient. In cases of spermatorrhœa, the semen is expelled in the acts of micturition and defecation. Cases of true spermatorrhoea are far less frequent than has hitherto been supposed. Persons have been regarded as having this affection whenever a mucilaginous fluid bearing some resemblance to semen is discharged from the urethra. The microscope affords the only reliable 
mode of determining that the liquid is seminal. Were this mode of examination generally employed, cases of spermatorrhoa would be found to be extremely rare. In examining the sediment of the morning urine, it is to be borne in mind that spermatozoids will be likely to be found if sexual intercourse or a seminal emission have taken place during the night. The presence of the spermatozoids, under these circunistances, is not evidence of spermatorrhoa. Veritable spermatorrhoea doubtless occurs, but probably always in persons who are affected with a morbid frequency of seminal emissions. Muco-purulent matter escaping from the urethra, or observed in the urine, in cases of cystitis, may give rise, in the mind of the patient, to the apprebension of spermatorihoa. A transparent viscid liquid escaping, in a small quantity, from the urethra, especially after prolonged erection, not infrequently occasions much uneasiness in the minds of persons whose attention is directed to the sexual org:ms with reference to spernatorrhoa, and of those who have had gonorrhoea. 'This is treated as a gleety discharge by empiries. The discharge is a secretion from the bulbo-urethral glands, or the glinds of Cowper, and is of no practical importance. It is sometimes of importance in a medieo-legal view to determine whether stains on linen are caused by dried semen. The physical characters of suspected stains are not to be relied upon; nor is chemical analysis reliable. If, however, water be slowly adder to the stains, and portions be examined by the microscope, spermatozoids will be recognizable when the stains are seminal. ${ }^{1}$

Involuntary seminal emissions and spermatorrhœa are fequently associated with symptoms denoting various kinds of functional disorder, and more especially disturbance of the nervons system. These symptoms have been considered as clirect morbid effeets of seminal losses. Nore or less general debility is undoubtedly a result of an abnormal discharge of semen, but the latter is often incidental to disorder of the system otherwise produced; and the disturbance of the nervous system, so frequently associated with seminal losses, is measurably due to the mental depression and apprehensions which they are apt to oceasion.

In the treatment of seminal emissions, the distinction which has been made between their physiological and pathologieal character is to be kept in view. Oceurring infrequently in a person of full health, in addition to assurances of their innocuousness, it will generally suffice to advise moderation in the use of wine or spirits and stimulating articles of food, saline laxatives occasionally, cold ablution of the genital organs at night, avoidance of undue warmth from the bed or bedclothes, and the mind to be diverted as much as possible from provocatives of sexual clesire. If these measures do not suffice, certain remedies which appear to exert an anaphrodisiac influence may be prescribed. Remedies which may be given for this purpose are camphor, lupulin or the tincture of hops, conium, belladonna, and the bromide of potassium. The remedy last named has seemed to me to be distinetly efficacious. Marriage is always remedial in these cases.

Cases in which the emissions depend upon a morbid erethism and weakness of the generative organs, and cases of spermatorthoea, are of more importance, and the treatment is more difficult. The anaphrodisiac remedies are indicated in these cases; but in these cases much benetit is derived from tonic remedies and an invigorating regimen. Quinia, small doses of strychnia or nux vomica, and the preparations of iron are useful ; seabathing or the sponge-bath and out-of-door life or gymnastic exercises are 
useful regimenal measures. The diet should be nutritious, but not stimulating, and alcoholic stimulants are to be taken very moderately or interdicted. Everything calculated to provoke sexual desire should, as far as practicable, be avoided. Healthful mental occupation and chaste associations do very much toward effecting a cure. It is obvious that, to carry out the moral part of the treatment, requires on the part of the patient a determination and perseverance which are often wanting. Taking into view all the circumstances in individual cases, it is sometimes judicions to encourage or recommend marriage.

Attention is to be directed to the condition of the urethra in cases of seminal emissions and spermatorrhœa; they are apt to follow gonorrhœa, and are sometimes dependent more or less on urethral stricture. According to Lallemand, seminal losses often depend on an abnormal sensibility at or near the prostatic portion of the urethra, and the treatment in these cases requires the use of the bougie and cauterizing applications. The opinion of Lallemand is held by distinguished surgeons at the present time. For information on this point the reader is referred to surgical works. Trousseau advises in some cases a mechanical method of treatment, consisting in the introduction within the rectum of a kind of pessary of an elongated oval form, varying in size from that of a pigeon's to a hen's egg, connected by a stem with a metal plate applied to the perineum and coceyx, the object being to compress the urethra at the orifices of the ejaculatory ducts. ${ }^{1}$

Impotencè means inability to perform the act of coition. The term does not mean want of the power of proereation; the latter may be wanting when there is no incapacity for sexual intercourse. Exclusive of malformations and other abnormal conditions of a surgical nature, impotence, in the first place, may involve absence of sexual desire. Different persons in health differ widely as regards the venereal propensity; in some persons the sexual desire is intense and importunate, in others it is feeble, and sometimes it is altogether wanting. The propensity may, on the one hand, be increased by indulgence, and by fostering in various ways ideas connected with it; and on the other hand, it may be diminished and extinguished by an opposite course. In the marital relation, frigidity, as regards this propensity, may depend on personal antipathy, and hence, a not infrequent source of domestic unhappiness. A bsence of sexual desire may arise from general debility, and is incident to various diseases. If the diseases with which it is connected be not serious nor permanent, a return of sexual desire may be expected on recovery of the accustomed health. Intense mental preoccupation, the depressing emotions, and exaltation of the higher sentiments, tend to repress sexual desire.

As the loss of virility is generally dreaded, physicians are often consulted when a notable change as regards the venereal propensity is experienced. The circumstances which have led to the change, in individual cases, are to be investigated, and the conclusion with respect to the probability of impotence, together with the treatment, is to be based on these circumstances. In a large proportion of cases, the deficiency or absence of sexual desire is due to temporary causes. Premature decline or extinction may result, on the one hand, from early and excessive indulgence or abuse of the generative function, and, on the other hand, from prolonged continence. Under certain circumstances aphrodisiac remedies are desired. The remedies so called are of doubtful efficacy. Phosphorus, cantharides, and nux vomica have been considered as special stimulants acting on the 
organs of generation. They should be given circumspectly. It is sometimes useful to secure the moral effect of remedies supposed to have this effect.

Impotence may exist without loss of sexual desire. Temporary incapacity sometimes proceeds from timidity or deficient self-confidence. The physician is not infrequently consulted by persons about to enter upon marriage, who are apprehensive of incompetency to accomplish coition. Assurances of capability, if it be evident that the apprehensions are imaginary, may be all that these cases require. Persons who have practised masturbation in boyhood, and those who are subject to involuntary emissions during sleep, are not infrequently led to believe that they are impotent by pernicious publications or lectures, designed to alarm sensitive minds; and apprehension on the score of ability to accomplish the act of coition creates a temporary incapacity. A failure to accomplish the act is apt to be followed by the conviction that impotency exists, and, practically, it cloes exist as long as this convietion continues. In such cases all that is requisite is that the act of coition should be undertaken without any anxiety as to success. It is useful, in such cases, to interdiet sexual intercourse for a certain time, in order to prevent tentative efforts which, if unsuccessful, fasten the conviction of impotency more and more upon the mind. If a certain period of abstinence be enforeed, it will be likely to happen that the patient is impelled by his desires to demonstrate his capacity before the period expires. A remedy prescribed with an assurance of a cure, cloubtless sometimes proves curative by a moral effect. In certain cases, impotence, with sexual desire, is a result of the erethism and weakness of the generative organs, associated with seminal emissions and spermatorrhoa. The ejaculation and orgasm take place at the beginning of, or prior to, the act of coition. This kind of impotence is sometimes, temporarily, a result of prolonged continence. The measures of treatment in such cases are those indicated by seminal emissions and spermatorrhoa. Persistence in the employment of these measures, together with a proper degree of reserve, as regards the frequeney of the attempts to consummate sexual intercourse, will generally prove successful.

Excessive indulgence in venery, when it does not lead to involuntary seminal emissions, spermatorrhoa, or impotence, is a not infrequent eause of ill health. Dyspeptic ailments, attacks of vertigo, functional clisorder of the heart, melancholia, and hypochondriasis, are among the morbid effects of this cause. It is the cluty of the physician to inquire into the habits of patients in this regard. The evils of excessive indulgence, aside from moral consequences, are greater when it is illicit than when it is marital ; moreover, the former is far more apt to be excessive. 'The limits of healthful indulgence doubtless vary according to constitutional vigor, but it may be considered as excessive if the acts of coition are repeated several times a week.

The physician is sometimes appealed to by unmarried persons and married persons debarred, for various reasons, from marital intercourse, to sanction illicit indulgence, on the score of health. It would be out of place to consider here this topic in its moral aspects, but they are by no means to be lost sight of by the physician. Irrespective of these, in view of the fact that inactivity of the generative organs can never be considered as a cause of disease, and also of the fact that, as a rule, the functional power of these organs is not lost by being held in abeyance, he is not justified in giving his professional approval of fornication as a hygienic measure. 
The unnatural abuse of the sexual function, or masturbation, as a cause of involuntary seminal emissions, spermatorrhœa, and sometimes impotence, has been already referred to. 'The effects on mind, as well as body, are pernicious. It is plainly the duty of the physician not only to endeavor to discover and arrest this practice in individual cases, but to enjoin upon parents, teachers, and associates the importance of watchfulness, in this respect, over those for whose welfare they'are, to a greater or less extent, responsible. There is also another duty of the physician connected with this important topic. Many sensitive persons who have been addicted to self-abuse in early life suffer greatly from the belief that their mental and physical powers have been irreparably injured. The evils, great as they sometimes are, are greatly exaggerated by popular works and lectures designed to excite fears for a mercenary end. The physician may often do much good by removing undue apprehensions connected with the errors of youth. 


\section{SECTION SIXTH.}

\section{FEVERS AND OTHER GENERAL DISEASES.}

\section{CH A P T E R I.}

Classification of Fevers. The General Pathology of Fever. Fabricula. Typhold Fever.Anatomical Cluaracters and Clinical History.

THIS concluding section will be devoted to fevers and other general diseases. The discases to be considered in this section differ from those which have been considered in the preceding sections in this, viz.: They do not, so far as at present known, proceed from an affection of any particular part; hence they are distinguished as general diseases. The diseases known as fevers are plainly thus distinguished from diseases of which the primary manifestations are always scated in some organ or structure; hence the latter are ealled local diseases. Diseases other than fevers, which it is convenient to distinguish, nosologically, as general diseases, are those which have no fixed or uniform seat, as regards their local manifestations. Diphtheria, acute and chronic rheumatism, gout, scorbutus, and purpura belong in this category. These diseases will be considered in this section.

Of the general diseases, fevers will be first considered. At the present time, it is unnecessary to discuss the grounds for recognizing the claims of fevers to be regarded as idiopathic affections. Few, if any, pathologists now hold to the doctrine of Broussais and others, advocated within the last half century, that fevers are always symptomatic of some local inflammation. It is sufficient to state the distinction between an idiopathic fever and the morbid condition, also commonly called fever, which is dependent on a local affection. The latter is distinguished as symptomatic fever or febrile movement. It embraces elevation of temperature and increased frequency of the heart's action, together with other morbid phenomena therewith connected which have been seen to enter into the clinical history of acute inflammations wherever seated. The fever, under these circumstances, is sccondary to the local affection; it is symptomatic of the latter; whereas fever, when idiopathic, is primary, local affections, if they coexist, being sccondary. The term essential is also used to distinguish a fever which is not secondary or symptomatic, but primary or idiopathic. A primary, idiopathic, or cssential fever is one of the elementary forms of disease. ${ }^{1}$

The number of fevers is sufficient to render a classification of them desirable. The arrangement generally adopted, althongh open to criticism, is sufficiently natural and convenient.

First. A form of fever characterized by its short duration and mildness, is called febricula. 
Second. Certain fevers are distinguished by the unbroken continuity of the febrile phenomena, and, hence, they are called continued fevers. This class will embrace the fevers known as typhus and typhoid. Relapsing fever and erysipelatous fever may be included in this class.

Third. In contrast with those of the preceding class, certain fevers are distinguished by the occurrenee of clistinct paroxysms or exacerbations as regards the febrile phenomena, and, hence, they are known as periodical fevers. This class embraces intermittent fever and remittent fever. Yellow fever is usually included in this class. The fever called dengue may be here placed. In this connection will be noticed the form of fever recently deseribed in this country under the name typho-malarial fever.

Fourth. An eruption on the surfaee of the body is a prominent feature of eertain fevers. These are distinguished as eruptive fevers or the exanthemata. This class embraces variola or smallpox, scarlatina or scarlet fever, rubeola or measiles, and roseola or roserash.

The foregoing list of fevers does not eomprehend all that exist. It comprehends, however, those which, from their prevalence and established individuality, it is desirable to consider in this work. Nistinet species of fever, not now recognized as such, may be hereafter ascertained; and it is not improbable that new species may be developed.

It is somewhat neeessary to refer to certain distinetions and names heretofore in vogue, in order that the meaning of terms, still more or less used, may be understood. Fevers have been distinguished and named aecording to the organ or system prominently affected. Hence the terms gastric fever, mucous fever, nervous fever, etc. These names were used when the distinction was not elearly made between essential, or idiopathic, and symptomatic fever. The names gastric fever and mueous fever are still used by some authors to denote cases of either transient gastritis, or of febricula attended with notable disorder of the stomach. The general feature or complexion, so to speak, arising from the predominance of certain symptoms, has served as the basis of certain distinctions and names. Thus, fevers characterized by great prostration have been called adynamic. Fevers have been said to be ataxic, when symptoms referable to the nervous system, such as delirium, etc., are prominent. These names are still employed to denote the general character of different fevers. Fevers tending intrinsically to a fatal result have been, and are still, called malignant. Fevers supposed to furnish evidence of septic changes in the fluids have been called putrid. Fevers of unusually long duration have been called slow; if attended with unusual depression, they have been called low, this term having the same sense as adynamic; when the febrile phenomena are intense, the fever is said to be high. The term congestive fever has been much used to denote an aceumulation of blood within the vessels of internal organs. This term is still often applied, in this country, to certain cases of periodical fever. Again, names have been applied to fevers, expressive of their source, or the places in which they prevail. Thus, typhus has been known as jail, ship, or camp fever; and periodical fevers, in different localities, have been called Boa Vista fever, Walcheren fever, jungle or coast fever, swamp fever, Panama fever, etc.

Before treating of the different species of fever separately, it will be advisable to present some general eonsiderations relating to fevers collectively, in other words, to consider, briefly, the general pathology of fever regarded as one of the elementary forms of disease. As regards our present knowledge of the essential nature of the several fevers, what has been ascertained respecting one is alike applicable to the others; or, 
to vary the expression, we know no more of the nature of any one than of all. The few preliminary considerations relating to the general patho$\log y$ of fever which will now be presented, will render it unnecessary to consider the pathologieal eharacter of the individual fevers.

The following questions at once arise: What morbid phenomena are necessary to establish the fact of the existence of an essential fever? What are the characteristic symptoms of this elementary form of clisease? Practically, the problem generally is to determine whether a fever be symptomatic or essential. An enumeration of the obvious phenomena belonging to an essential fever, that is, of features common to all essential fevers, embraces the following points: In most cases the invasion is not abrupt or sudden, but there is a period of access, or a forming stage. Ditferent fevers cliffer as regards the time oceupied in the development of the disease, and certain prodromic symptoms are peculiar to particular fevers. Generally, the development is attended by a chill or by chilliness, and frequently by rigor's. The pulse becomes more or less aceelerated, and usually there is a very pereeptible inerease of the heat of the surface. The thermometer shows, as a rule, to which there are no exceptions, more or less inerease of temperature; henee, the source and significance of the name fever. This increase of temperature is measurably due to a diminished loss from evaporation on the surface of the body; but it is chiefly owing to an abnormal production of animal heat. Anorexia and thirst are usually present; uausea is rarely wanting, and often vomiting oecurs. Other symptoms are, frontal headache, pain in the loins and limbs, lassitude, restlessness, vigilance, or imperfect sleep. The urine and most other secretions are diminished. The scantiness of urine, however, is due to deficiency of water, the specific gravity being from 1020 to 1025 , or more. The coloring matter of the urine is generally increased. The abnormal amount of excrementitious products which represent the waste of the tissues corresponds to the degree of animal heat above that of health. The urea and urie acid are increased, the amount of the latter being double that of health. 'This shows auginented waste of the tissues. The chlorides are diminished, at least, one-lialf. There is more or less rapid and notable loss of weiglit, the loss being greater than the reduetion of alimentation. The amount of carbonic acid eliminated in expiration is notably increased. In most of the fevers the morbid phenomena continue for a limited period and then spontaneously end. The museular debility is usually sufficient to eause the patient to take to the bed.

The combination of all these phenomena would not denote an essential, as distinguished from symptomatic, fever, without this negative condition, viz., the absence of any local discase adequate to account for them. We judge, then, that a patient has an essential fever of some kind, when he presents an ensemble of certain symptoms with the proviso just stated. But it will be seen hereafter that the different speeies of fever present, severally, certain symptoms which are characteristic of eacl species, that is, listinctive, on the one hand, of fever as contrasted with other diseases, and, on the other hand, of a particular species of fever as contrasted with other species.

The foregoing sketch of the phenomena of fever shows it to be a general disease; that is, it affects, in its manifestations, the entire organism. The language of Fordyce, with reference to this point, is not less pertinent now than when penned more than half a century ago: " $A$ fever is a disease which affects the whole system. It affects the head, the trunk, and the extremities. It affects the circulation, the absorption, and nervous 
system. It affects the skin, muscular fibres, and the membranes. It affects the body, and it affects likewise the mind. It is, therefore, a disease of the whole system in every kind of sense."

The causes of fever will be considered in treating of individual ferers. There is reason to believe that each species of fever has its own special cause, that is, a cause which gives rise only to that particular form of disease; hence there are as many special causes as there are different species of fever. Concerning the essential nature of these special causes, their generation, the manner of their entrance into the systcm, and their primary action within the body, our present knowledge is very limited. Some of the causes are evidently of terrestrial origin, and some consist of either a virus or miasm produced within the body of another. The special causes appear to be aided in their morbific action by various auxiliary eauses. The latter often seem to be required to give efficiency to the special causes.

Of the nature of the pathological condition which constitutes an essential fever we have no positive knowledge. Can we not, however, go further than merely to say it is one of the elementary forms of disease? Here, as in respect of another elementary form of disease, viz., inflammation, we approximate, in some measure, toward an explanation of the pathological condition if we are able to trace its primary seat in the organism; in other words, to ascertain the portion of the body first acted upon by the special cause. The phenomena which constitute the manifestations of fever are evidently, to a great extent, independent of each other; they do not follow in a chain of successive sequences, but are obviously alike effects of a pathological condition which must be seated in a portion of the body having extensive relations within the organism. There would seem to be, in fact, in the boly only two anatomical systems having relations so extensive as to be able to give rise to the train of morbid phenomena in fever, viz., the nervous system and the blood. Now, the phenomena of fever point to the existence of a morbid conclition of both of these anatomical systems. The question then arises, which is primarily affected, or in which is the essential pathological condition in fever seated? The answer to this question which would be given by most pathologists at the present time is, the blood is the portion of the body first affected, and the essential pathological condition in fever is here seated. The correctness of this doctrine is not demonstrable. It has not been proven by direct observation that the special causes giving rise to the different species of fever act primarily upon the blood. But the doctrine is most consistent with our existing knowledge, if, indeed, it be not logically certain. The following are the grounds for considering fever as primarily and essentially a blood-disease :-

1. The existence of a period of incubation renders the introduction of a materies morbi into the blood probable. According to the theory of late years held by most pathologists, the special causes of fevers, as well as of certain other diseases, act in the blood as ferments, or on the principle of eatalysis; hence the signifieance of the term zymotic, as applied to these diseases by Farr. This theory is consistent with the fact that an inappreciable amount of the matter which constitutes the special causes or poisons giving rise to fevers, as well as other diseases, is adequate for their production; and the existence of a period of incubation is consistent with the theory that the special causes are organic germs, either vegetable or animal. A difficulty, however, pertaining to the theory of zymosis, or the doctrine which attributes diseases to organic entities, arises from 
the movements of the blood in its circulation, and the constant mutations of which it is the seat in the changes incident to assimilation.'

2. One of the essential fevers, viz., variola, or smallpox, may be produced at will by the introduction into the circulation of a palpable material, or virus, and an immense quantity of this material, or virus, is reproduced. Now, reasoning analogically, it is to be inferred that, in like manner, a morbific matter is introduced whenever other species of fever are produced.

3. Fevers generally are self-limited. A duration, within certain limits, is among the laws by which they are governed. This fact is consistent with the supposition of either certain chemico-vital processes or the multiplication and extinction of organic entities in the blood. On the other hand, affections of the nervous system are irregular in their development and duration. They do not have a definite, self-limited career.

4. Fevers, as already stated, are, in general, gradually developed, having a forming stage, or prodromic period. They do not, as a rule, occur abruptly, as if from an impression on the nervous system. Their gradual development suggests, as probable, chemico-vital processes or organisms in the blood.

5. Impressions made on the nervons system, however violent, are not adequate to occasion any of the essential fevers.

6. Of the different species of fever, each presents in its clinical history a definite and pretty uniform series of symptomatic events. Nervous affections, on the other hand, are characterized by the diversity and irregularity of the phenomena which accompany them.

7. It is difficult to understand how external causes giving rise to fevers can possibly come into contact with the nervous system so as to produce upon this system morbid impressions, except through the intervention of blood-changes.

8. There is reason to believe that most of the affections of the nerrous system involve prior blood-changes.

Many of the phenomena of fever are referable to the nerrous system; but it is most rational to consider the morbid condition of the nervous system giving rise to these phenomena as consecutive to a morbid condition of the blood, the latter inducing, secondarily, certain morbid effects on the nervous system.

The phenomena of a chill, or ague, seem to involve contraction, or spasm, of the ressels of the periphery of the body from a morbid agency excited through the vaso-motor nerves ; and, on the other hand, paralysis of these vessels may rationally be inferred from the existence of capillary congestion which is more or less marked in fever when chill, or ague, is - not present. Assuming the correctness of these explanations of phenomena referable to the circulation in fever, it is reasonable to refer the agencies exerted through the vaso-motor nerves to the etfect of morbid conditions of the blood on the nervous system.

\section{Febricula. Ephemeral Fever. Simple Fever.}

The term febricula, a diminutive of febris, is perhaps the best name to denote a mild, short fever which is everywhere of not infrequent occurrence. If very short, lasting only one or two days, it is appropriately called an ephemeral fever. When not ephemeral, the fever may continue

' For some account of the germ-theory of the causes of disease, vide Part I., Chap. VIII. 
for a period varying from three to eight, or even ten, days. It has been known by a variety of names; it is frequently called simple continued or simple fever. Prof. Wood describes it, in his work on Practice, under the name irritative fever. It is the synocha or inflammatory fever of Cullen and other nosologists.

This fever has no known anatomical characters; the opportunity of post-morten examination is very seldom offered, for the disease very rarely, if ever, of itself ends fatally, at least in cold or temperate climates. No lesions belonging to the fever have been found in cases in which, owing to complications, death has taken place.

The invasion in cases of febricula is generally abrupt; but, in a certain proportion of eases, lassitude, loss of appetite, and general malaise exist for two or three days before the fever is dereloped. Irregular chilly sensations may accompany the attack, but there is seldom a well-pronounced chill. The febrile movement is more or less intense ; not infrequently the acceleration of the pulse and heat of the surface are greater than at the commencement of typhus and typhoid fever. Pain in the head is a prominent symptom, the pain being generally frontal; there is more or less pain in the loins and limbs. Anorexia is usually complete. Constipation usually exists, and the abdominal symptoms which belong to typhoid fever are wanting. The urine is scanty and deep-colored; the intelligence remains unaffected ; the hebetude which eharacterizes typhus and typhoid fever is not observed. There is no eharacteristie eruption ; herpetic vesicles about the mouth sometimes occur, and bluish spots (tâches bleuatres), from three to eight lines in diameter, not elevated and not disappearing on pressure, have been observed. These occur oceasionally in typhoid fever.

The febrile career ends suddenly after one, two, three, or more days, the limit of the duration being ten days. Frequently with the eessation of fever there is a copious perspiration; epistaxis and occasionally hemorrhage from the uterus and rectum have been observed at the end of the fever. The convalescence is usually rapid, and there are no sequels. The thermometer indicates a sudden rise of temperature four or five degrees, and as rapid a decline, the heat passing, in one or two days, to the normal standard.

This species of fever is supposed to occur irrespective of any special cause; that is, ordinary causes are supposed to be adequate to produce it. It is observed to follow over-exertion, dietetic excesses, and exposure to the sun's rays. This view of the causation is, howerer, conjectural. If a special cause be required, the process by which the morbific material and its effects are gotten rid of are speedily completed. It is probable that certain cases which, from their short duration, are considered as cases of febricula, are in fact cases of typhoid or typhus fever, the disease ending without going through its usual career; in other words, cases of ephemeral fever may be cases of abortive continued fever.

The diagnosis of febricula is of importance with reference to the success of measures employed to arrest or abridge the duration of typhoid and typhus fever. In cases in which measures employed for these ends appear to prove successful, the question is, was the disease typhoid or typhus fever, or was it a febricula? The following are considered as diagnostic points: 1. The abrupt development of the fever. 2. The attack succeeding obvious and ordinary causes. To these is to be added absence of diagnostic traits belonging to the clinical history of either typhoid or typhus fever. Cases however occur in which it is difficult or impossible to decide, during the progress of the ferer, whetler it be typhoid, or 
typhus, or a febricula; and, after the fever ends, whether it have been a febricula or an abortive or arrested typhoid or typhus fever.

Febricula in cold and temperate climates is rarely, if ever, fatal, except firom an accidental complication of a serious nature. It is stated to prove fatal not infrequently in tropical countries.

As regarls the treatment of febricula in cold and temperate climates, lyygienic and palliative measures only are called for. A saline laxative, refrigerants, and anodynes are the remedies indicated.

\section{Typhoid Fever.}

The name typhoid signifies typhus-like. The pertinency of this name, as applied to the fever to be now considered, consists in the resemblance of the disease to typhus fever. It might therefore seem most appropriate to consider, first, typhus fever; but typhoid fever prevails very extensively in this country, and has been known with us as common continued fever, whereas the prevalence of typhus is comparatively limited, and the latter is very rarely, if ever, with us an indigenous disease. For this reason, reversing the order which the name suggests, I shall consider, first, typhoid fever, with referenee to its anatomical characters, clinical history, causation, diagnosis, and prognosis ; afterward typhus, under the several aspects just named, consirlering in this connection the nonidentity of these two fevers; and, lastly, the treatment of typhoid and typhus fever.

The name typhoid is open to objection. It is customary to apply this name to a condition or state which is incidental to many diseases, and hence has arisen not a little confusion. Of other names which have been proposed within late years, no one has been generally adopted, and probably the disease will continue to be ealled typhoid fever. 'This name was introduced by Louis, whose clinical researches were of great value, not merely with reference to our knowledge of this clisease, but as inaugurating the true method of the study of the clinical history of all diseases, viz., by means of the analysis of carefully recorded cases. 'The correspondence of the results of the researches of Louis, as regards typhoid fever, with the results of similar researehes by others in different countries, furnishes the best possible proof of the value of the method of study, and, at the same time, shows the remarkable uniformity of the disease at different times and places. By the German writers, the disease is called abdominal typhus. Prof. Geo. B. Wood has suggested, as an appropriate name, enteric fever. Both these names relate to the intestinal lesions which are characteristic of the disease. As remarked by Murchison, these names are objectionable because they are apt to convey the impression that the fever is the result of these lesions. Murchison has proposed, as a substitute, pythogenic fever, a name which implies the supposed source of the disease to be putrescent matter. It is an objection to this name that the source of the special cause, which the name implies, has not been conclusively established.

Anatomical Characters.-Typhoid fever has certain special lesions which are highly interesting and important. They are seated in the Peyerian or agminated, together with the solitary, glands of the small intestine, and in the mesenterie glands. The first ascertained step in a series of changes occurring in the patches of Peyer and the solitary glands, is enlargement from a morbid produet within the glandular saes. It is supposed that increased vascularity precedes the deposit, but this is not fully 
ascertained. The enlargement is considerable, causing an elevation one, two, three, and even fonr lines above the plane of the aljacent mucous surface. 'The enlargement is chicfly due to hyperplasia of the cellular elements within the closed sacs which form the Peyerian and solitary glands. The mucous membrame over the affected patches is of a pinkish or purplish hue, and the corresponding portions of the peritoneum are much injected. Usually the patches are inclurated to the touch; they are sometimes smooth, and sometimes present an irregular, granulated aspect. These appearances are presented early in the disease. They have been observed as early even as the second dity. The glands nearest the cacum are first affected, and, successively, those situated above. Sloughing away of the glands and mucous membrane is the next step. 'This probably occurs in the great majority of cases. When death takes place during the second week, the slonghing is in progress. Generally, at this stage of the disease, portions of the patches have separated, other portions are partially detached, and portions are still adlerent. The sloughs are sometimes dark from the presence of blood, and sometimes yellow from the inbiljition of bile. The rapidity of the sloughing varies in different cases. It is very rarely completed before the end of the first week. It takes place earliest in the glinds nearest the cacum, and, usually, different degrees of progress are presented in the series above the cacum. It is sometimes nearly or quite completed in the lowest, while it has hardly commenced in those which are uppermost. The slonghing is evidently due to decomposition (necrosis) resulting from an arrest of the cireulation (ischamia). Uleeration is a consequence of the slonghing. After the slonghing is completed uleers take the place of the Peyerian and solitary glands. The ulceration first occurs in the glands nearest the caeum, and sucessively in the ascending series. Ulceration in the lowest glands rarely takes place before the end of the second week of the disease, but, exceptionally, it may occur during the first week. Gencrally the uppermost glands do not become the seat of ulcers until the third week or eren later. 'The occurrence, successively, of ulceration in the glands from below upward is one of the distinctive features of the disease. The edges of the nlcers are not elevated and hardened as in tuberculous ulcers in the same situation. Another distinctive feature is an overlapping of the mucous membrane at the margins of the ulcers, presenting an appearance as if an undermining material had been dissolved from beneath the shelving border. Frequently at the bottom of the ulcers the muscular fibres are exposed; the muscular layers and the peritoneum then form the only support of the intestine at the ulcerated patches. Cicatrization is the last step in the series of processes. A thin, serous-like membrane is formed at the bottom of the ulcers. This membrane gradually becomes thicker and firmer, and at length the excavations produced by the sloughing away of the glands are filled up. The cicatrization never learls to stricture of the intestine. It is not probable that the glands are reproduced. The process of cicatrization usually begins in the third week of the disease, and is going on during convalescence. The time occupied in the completion of this process varies in different cases. It is sometimes unusually slow, a fact which accounts for the persistence of the abdominal symptoms, in certain cases, for some time after the career of the fever is ended.

Perforation of the intestine is liable to occur in one or more of the ulcers. It may be due to an extension of the ulceration to the muscular and peritoneal tunics, to sloughing of these tunies, or to rupture. The opening is sometimes extremely small, if due to ulceration ; it may be no larger than a pin's point. If caused by sloughing or rupture, the opening 
may be of considerable size. Tith intestinal perforation are associated appearances denoting acute peritonitis, the latter being occasioned by the escape of the gaseons and other contents of the intestine into the perito. neal sac.

The series of changes which have been described occur generally both in the Peyerian and the solitary glands. 'The latter are the seat of these changes in a large proportion of cases, but not invariably. Some cases have been observed in which the lesions were seated exclusively in the solitary glands. The number of both the Peyerian and solitary glands which are affected in different eases, varies. It may be stated here that there is no constant relation between the extent of the intestinal lesions and the severity of the disease, as denoted by the abdominal and other symptoms. Not infrequently the solitary glands of the crecum, and in other portions of the large intestine, are the seat of similar morbid changes; and in some very rare cases the characteristic lesions are confined to the large intestine, the small intestine remaining maffected. Exceptionally corresponding lesions occur within the jejunum and luodenum, and they may be limited to these sections of the intestinal tube. The lesions have also been observed within the pyloric portion of the stomach.

More or less enlargement of the mesenteric glands is always associated with the intestinal lesions. The mesenteric glands which are in immediate relation to the affected Peycrian and solitary glands, are especially enlarged. 'Their volume is often much increased; not infrequently they attain to the size of a pigeon's egg. They diminish in size as the sloughing process is going on within the intestine, and gradually the morbid product or growth which produced the enlargement is entirely absorbed. Exceptionally, sloughing takes place within the mesenteric glands, the slough separating and being discharged into the peritoneal sac. This is an occasional source of peritonitis developed in the course of the disease.

The foregoing lesions are peculiar to typhoid fever. In a greater or less degree they are always present, and they occur in no other disease. l'hey are characteristic of typhoid fever, as the eruption in suallpox is characteristic of that disease. 'They do not constitute the disease, more than the eruption constitutes smallpox; but they sustain to the disease a definite, fixed pathological relation, the nature of which is unknown. It has been conjectured that slougling and uleeration within the intestine do not always take place, but that the deposit is sometimes absorbed here as it is within the mesenteric glands. With regard to this point we lack positive information.

It has been supposed that certain of the symptoms and complications of typhoid fever are attributable to septicxmia from resorption of the typhus material. This supposition is without any solid foundation. The symptoms and complications attributed to septicamia thus induced oceur in typhus as well as in typhoid fever, and in the former of these affections there is no morbid product analogous to that which is characteristic of the latter.

The spleen is more or less enlarged and softenerl. It is sometimes the seat of hemorrhagic extravasation; and examples have occurred of rupture of the capsule, followed by the escape of blgod into the peritoneal cavity. The other abdominal viscera present only contingent or aecidental lesions. Minute ulcerations in the pharynx and osophagus are observed in a small proportion of cases, and inflammation of the gall-bladder sometimes occurs.

Important lesions situated elsewhere than in the ligestive system are not of a special character, but cither depend on complications or are acci- 
dentally connected with the disease. Uleerations in the larynx and trachea are found in a very small proportion of eases. The bronchial mucous unembrane is more or less congested. Hypostatic congestion of the depending portions of the lungs is sometimes observed. Pulmonary œedema occasionally occurs. Pneumonitis is a not infrequent complication. The bronchial glands are apt to be enlargerl when pneumonitis occurs. The enlargement of these glands, in some eases, is considered as corresponding in pathological character to the enlarged mesenterie glands.

As regarls the nervous system, more or less cereloral eongestion is not uncommon, together with serous effusion in the arachnoid cavity, lateral ventricles, and subarachnoid space. The effusion, however, is not turbid from lymple, and those appearances are not sufficient to show the existence of meningitis. Meningeal inflammation is a very rare complication. The morbid appearances within the cranium are not greater than are found after death frorn varions other diseases, and they have no relation with the symptoms referable to the nervous system. Parts of the nervous system other than the brain present nothing abnormal.

In some cases the walls of the heart are notably flaceid, and the muscular strueture is softened. The blood contained in the heart-eavities, when death has occurred purely from the fever, is often unusually dark and liquicl. Bacteria in the blood have been observed by Coze, Feltz, and Tigri; and Hillier has described large and small cells which he regards as organisms, and includes under the name micrococens. The endocardial membrane and the lining membrane of the aorta and pulmonary artery are often stained by the imbibition of the blood pigment. Fibrinous clots are found in cases in which life is destroyed ly a eomplieating inflammation such as peritonitis or pnemmonitis. The fibrin of the bloorl is diminished in this, as in other essential fevers, when not complicated with an acute influnmation. The albumen is diminished ; the white corpuscles are increased, and the red globules are deficient.

The kidneys are in some eases notably eongested and in other cases unusually pale. They are sometimes enlarged, and the uriniferous tubes filled with exfoliated epithelium. The latter may occur post-mortem.

Zenker and Hoffinan have described notable changes in more or less of the fibres of certain voluntary museles as occurring not infrequently. From the transparent or lightly opalescent appearance under the microscope the name waxy degeneration has been applied to one of two morbid conditions. The other condition is one of granular degeneration. These changes have been observed in the museles of the thigh, the abdominal walls, the chest, tongue, diaphragm, etc. It is attributed to congnlation of the contents of the myolemma. As an effect of this change rupture of vessels and hemorrhage sometimes occur. ${ }^{1}$

Clinical History.-Typhoid fever, as a rule, is developed gradually. The time occupied in its development is eonsidered by some as a preeursory or prodromic period, and by others as a stage of the disease. The latter seems to me more correct. After the development of the disease, the most convenient division of stages is into septenary periods. The career of the disease will embrace the first, second, third, and sometimes a fourth, fifth, sixth, or even a seventh week.

In a large proportion of cases, patients are unalle to fix the precise date of their ailments; so imperceptible is the commencement of the discase. It is, therefore, often not easy to determine accurately the duration

1 Vide Jaccoud, Pathologie Interne. 
of the forming stage. This stage ends when the disease is fully developed. How is the latter to be determined? My rule has been to eonsider the fever as established when the patient takes to the becl. 'This is an arbitrary criterion, and not always accurate, but the instances in which patients take to the bed before and after the full development of the disease will about compensate for each other. The first chill, or chilly sensations, and an increase of the axillary temperature, are more correct criteria; but, in a large majority of cases, patients do not come under observation until the fever is developed, and they often cannot recall the first chill or chilly sensation. Judged by the rule just stated, the forming stage varies from one to ten days, and the mean duration is about five days. Exceptionally, the attack is abrupt, the patient taking at once to the bed.

The following are the symptoms belonging to the forming stage: Chills, more or less pronounced, or chilly sensations, recurring irregularly, sometimes followed by perspiration and sometimes not. Cephalalgia, the pain generally referred to the frontal region. Mental irritability, with difficulty of concentrating the attention or exerting the faculties of the mind. Loss of appetite, occasional nausea, and sometimes slight vomiting. Epistaxis, in a pretty large proportion of cases. Pain in the loins and limbs. Looseness of the bowels, or hypercatharsis if a mild purgative be given. Lassitude and progressive debility until, at length, the patient, who, up to this time, has kept up with more or less elfort, feels compelled to take to the bed.

Of the symptoms in the foregoing list, those especially of diagnostic importance are looseness of the bowels and epistaxis. 'The duration of this stage is of importance in diagnosis.

Proceeding to notice the symitoms which make up the clinical history, after the forming stage, it will be convenient to arrange them accorling to the different anatomical systems to which they are referable. They are distributed, after this plan, into those referable to the comntenance or general aspect, the nervous system, the digestive system, the skin, the respiratory system, the circulation, and the urinary system.

Countenance and General Aspect.-There is no very marked alteration of the countenanee for the first few days. The face in the early period of the disease is usually more or less flushed. Afterward, the countenance presents a dull or listless expression, and in severe cases there is lack of any expression, or an appearance of stupidity. The latter corresponds with the gravity of the disease, and is more and more marked as the disease continues. These alterations of the physiognomy are dependent on the mental condition.

The surface presents slight or moderate capillary congestion, such as is produced by the action of cold. This is most marked on the face, and especially on the cheeks. Distinctly circumseribed redness of the cheeks is rare save in the cases in which the disease is complicated with pnenmonitis. 'The redness due to the congestion disappears on pressure, and returns more or less quickly. In some cases the hue of the surface is slightly dusky. The capillary congestion observable on the face is diffused over the body, but, next to the face, it is most marked on the hands and arms.

The symptom is much less marked than in typhus, but, in view of its constaney, in a lesser clegree, in this disease, it must be considered as having a pathological significanee. It is rational to consider it as denoting paralysis affecting, through the vaso-motor nerves, the peripheral arteries. 
Slight or moderate congestion of the conjunctiva is not infrequently observed.

Nervous System.-Pain in the head is more or less complained of during the first week. It is not always a prominent symptom, and never has the intensity which belongs to the pain in the first stage of acute meningitis. It gradually becomes less and less a sulject of complaint, and usually ceases during the second week. The cephalalgia is not accompanied with notable intolerance of light and sounds. Pain in the back is also eomplained of in the early part of the disease, but is rarely a very prominent symptom.

Delirium is manifested in the majority of cases which I have observed. Of sixty-four cases analyzed with respect to the presence or absence of this symptom, it existed in $47 .^{2}$ As a rule, it is not manifested until the second week, and sometimes not until the third or fonrth week. Exceptionally, it is manifested in the first week, and even when the patient first takes to the bed. Different cases differ much as regards the degree of delirium ; it may be slight, or, on the other hand, a very prominent symptom. Generally, the first evidence of mental aberration is temporary confusion on awakening from sleep, the patient being unable to recall where he is, and asking incoherent questions. Inereasing, the patient talks incoherently, and mutters like a person talking in sleep. Efforts to get out of bed are common, and when asked where he wonld go, a frequent answer is. he wishes to go home. In most cases he is easily persuaded to lie down, but after a few moments the effort to get up is repeated. If not watehed, patients sometimes get up and succeed in getting on their clothes. The delirium is always greater, and may be manifested only, during the night. In the great majority of cases, the delirimm is of a quiet, passire. kind. It apparently proceeds from weakness of mind ; there is inability to carry on connected trains of thought. The incoherent talking or muttering usually relates to habitual pursuits. The patient is in a dreamy state, a succession of disjointed ideas passing through the mind.

'There are occasional exceptions to the rule regarding the kind of delirium. It is sometimes noisy and active; the patient shouts, and makes strong and persistent efforts to get up. Constant forcible restraint is sometimes necessary. Fixed delusions, either of the senses or ideas, during the progress of the disease, are certainly rare; they may occur at the time of convalescence. In one of my cases the patient fancied that he had become immensely rich, and this delusion continued for several days after convalescence. The delirium is in general marked in proportion to the intensity of the disease. So far as my observations go, persisting, active, or violent delirium, requiring restraint, is an extremely unfavorable symptom.

Patients are sometimes perfectly coherent, hut not rational. They reply to questions at hap-hazard, and no reliance is to be placed on their statements with respect to the events of their illness. In some cases there are no manifestations of delirium during the whole course of the disease, but, after recovery, there is very little recollection of anything that transpired. The delirium sometimes has a hysterical character, consisting in emotional manifestations-weeping and sobbing-the emotions. being very rarely mirthful.

Delirium, however prominent as a symptom, is no evidence of encephalic inflammation. The appearances of meningitis are not found after death in

1 Clinical Reports on Continued Fever, based on an analysis of 164 cases, etc., by Austin Flint, M.D., 1852. 
cases characterized by very active delirium, nor is there an abnormal amount of congestion. The statistical researches of Louis have shown that delirium is not constantly associated with any complications of the fever, and hence, it is to be eonsidered as dependent on the morbid condition of the blood in which consists the essential pathology of the disease.

Irrespective of delirim, the mental condition in the second, third, fourth or subsequent weeks is characterized by hebetude, indifference, inanimation. Blunted perception is shown by various circumstantes; the patient asks for nothing-not for drink, although the mouth may be dry and clesiccated, nor for the position of the borly to be changed; flies creeping over the face may occasion little or no amnoyance, etc. In grave cases the urine and feces may be passed in bed, not from paralysis of the sphineters, but through indifference and a reluctance to make any effort. 'Jhe special senses are inpaired, especially the sense of hearing; deafness in one or both ears is firequently marked. General and special sensibility are diminished, as a rulc, in proportion to the intensity of the disease. Jemner and Gairluer have noticed the fiequent oceurrence of dilatation of the pupil in this clisease.

Wakefulness is complained of cluring the first few days; the patient experiences discomfort from the want of refreshing sleep. Afterward, the want of sleep unay not be appreciated, owing to the mental condition. 'The patient, incleed, may seem to cloze most of the time, but without obtaining any true sleep. IIe is easily aroused, but chirectly relapses into a pseudosommolent state. 'This state, being one in which the patient may be said to be both sleeping and wakeful, is called coma-vigil, the significance of the name depending on the incongruity of the words which it combines. 'This name is also applied to a state occasionally observed, in which the patient is unconscions with the eyes open. In coma-vigil, or the pseudosomnolent state, from which the patient is readily aroused, the mind is frequently occupied with a series of discomneeted ideas or dreamy delusions which give rise to the muttering deliriun. This combination of stupor and delirium is expressed by the tern typho-mania. The want of true sleep doubtless contributes not only to the exhanstion, but to delirium and other disordered nervous phenomena, as in delirium tremens.

Coma-vigil and typho-mania do not involve a tendency to true coma ; but in a certain proportion of cases, coma is gradually developed in the latter part of the career of the fever, and under these circumstances a fatal result is to be expected. In a certain proportion of cases coma is surldenly induced; it occurs unexpecterlly, and in mild as well as in severe cases. These cases generally end fatally, the molle of dying being by apnoa caused by the loss of the besoin de respirer. This apoplectic coma is often preceded by disturbance of the rhythm of respiration, the inspiratory act being shortened and quickened.

Other symptoms referable to the nervous system, or ataxic symptoms, denoting gravity of disease, are, grasping at invisible objects or carphologia, pulling-up of the bedclothes or fumbling with the body linen, visible twitchings of the museles of the face and of the extremities, and movements of the tendons of the wrist perceptible to the touch, the latter called subsultus tendinum, rigidity of the muscles of the neck or extremities, and convulsions. Muscular rigidity and convulsions are very rare, and betoken a fatal termination. There is reason to believe that the pathologieal condition giving rise to convulsions with coma is generally, if not always, uræmia. In most of these cases the urine will be found to be albuminous, but uræmia may' occur without albuminuria. Paraplegia, 
either temporary or persisting, dependent on spinal congestion or veritable inflammation, is an extremely rare complication.

Digestive System.-Anorexia is the rule, but to this rule there are exceptions; the appetite is sometimes preserved throughout the disease. Foor is sometimes acceptable when, owing to the mental condition, it is not asked for. Thirst is usually a prominent symptom until perception becomes blunted. Drink, although not asked for, is often taken with avidity when given. 'The condition of the mouth would involve a desire for drink were the ability to perceive morbid sensations not impaired.

The tongue generally presents morbid appearances. It may be simply furred or frosted, but is oftener covered with a coating more or less thick, which, in different cases, is whitish, yellowish, brownish, or even black. Not infrequently the coatings are thrown off once or repeatedly, and the surface is then usually reddened. Exfoliation of the coating or its gradual thinning, the surface being moist and of a natural color, betokens convalescence. The varied appearances of the coatings have no special significance. The surface of the tongue is sometimes reddened and smooth or glazed. It sometimes becomes dry and hard. This occurs in coma-vigil; the patient breathing with the mouth open, the surface is desiccated. It sometimes becomes cracked and deeply fissured. Tremulousness of the tougue, as in cases of delirium tremens, is sometimes observed, usually preceding or accompanying grave ataxic symptoms. The tongue, in some cases, at an advanced period, is protruded with-apparent hesitation and difficulty, and when protruded, may not be withdrawn, apparently from forgetfulness. The delay in protruding and withdrawing the tongue represents the weakness and slowness of the mental acts.

Dark or black matter, called sordes, after the first week, is apt to collect upon the teeth and lips, especially in grave cases. Hemorrhage from the gums is an occasional symptom, occurring in mild, as well as severe, cases. Redness of the gums and bleeding on slight pressure are common.

A rare but somewhat characteristic complication is inflammation of one or both of the parotid glands. It leads to notable enlargement, and the appearance is like that of ordinary parotiditis or mumps. But, unlike the affection just named, in the great majority of cases suppuration takes place, and not infrequently more or less sloughing of the areolar tissue. 'This complication adds to the danger and retards convalescence. It may occur at any period of the febrile career or during convalescence. It is not to be regarded as a critical event. The discharge of pus is sometimes into the meatus auditorius. . This complication occurs in typhus as well as in typhoid fever. It occurred in 5 of 30 cases of typhus and typhoid fever which I recorded in the winter of 1849-50. Prior to that year I had never met with an example, and since that year I have met with a very few examples only, in a much larger field of observation.

Vomiting is an occasional symptom: Generally it is a result of overingestion. As.regards the occurrence of this symptom, typhoid fever is in striking contrast with remitting fever.

Diarrhoea or looseness of the bowels exists in a large majority of cases, and belongs among the diagnostic symptoms of the disease. It was more or less marked in 48 of 74 cases of those which I have analyzed. In cases analyzed by Louis and others, it existed in a still larger proportion. It is a prominent symptom in proportion as the intestinal lesions are extensive. The dejections have a yellow, ochre color. They give an alkaline reaction. Exceptionally, constipation exists, and I have known the dejections to have a natural appearance throughont the disease. The borrels are sometimes evacuated in bed from indifference, and sometimes from 
paralysis of the sphincter ani. The involuntary evacuations denote great gravity of disease.

Hemorrhage from the bowels occurs in a certain proportion of cases. It oecurred in 3 of 73 cases which I have analyzed. Aclding to these the cases analyzed by Louis, Jenner, and Murchison, the number is 312 . Of these cases, hemorrhage occurred in 32. As regarls the rate of fatality among the cases in which hemorrhage occurs, facts collected by different observers differ. Of the three cases in the collection of cases which I have analyzed, two ended in recovery. Since that analysis I have recorded three cases in private practice, in two of which the hemorrliage was so profuse as to induce syucope and loss of pulse at the wrist for several hours. All these cases ended in recovery. So far as ny experience goes, therefore, it is in aceordance with that of Graves, Tronsseau, and Kennedy, going to show that this event is not of unfayorabie omen. The facts, however, upon which this conclusion is based are negative; and facts observed by Louis, Chomel, Jenner and others show that the loss of blood sometimes proves fatal. Of 303 cases analyzed by James Jackson, hemorrhage from the bowels occurred in 31, and of these cases 20 terminated favorably. 'The hemorrhage oceurs late in the disease, and, in most instances, is from the intestinal ulcers. The occurrence of dejections of bloor may be foreshadowed by a sulden and notable decline of the temperature of the boly. I have met with a case in which the decline of the axillary temperature to $98^{\circ}$, was, for a time, considered as evidence that the fever had ended; but within a few hours profuse hemorrhage from the bowels occurred, and the patient died from the loss of blood.

Other abdominal symptoms are, meteorism or tympanites, tenderness or pain on pressure, and gurgling. 'The abdomen is always resonant from the presence of intestinal gas; frequently there is more or less distension, and sometimes the tympanitic enlargement is great. 'Tenderness on pressure in the iliac region, especially on the right sirle, is very rarely entirely abseat. Sudden and foreible pressure may be required for the manifestation of pain, if perception be much blunted. The tenclemess is often marked, and sometimes extends over the greater part of the abdomen. Gurgling on pressure in the iliac region, as a sounc, and as a tactile sensation, is a frequent symptom. These three symptons, riz., meteorism or tympanites, iliac tenclerness and gurgling, especially the two former, conjoined with diarrhoea or looseness, and ochre discharges, form a group of abdominal symptoms highly diagnostic of this form of fever. The tympanites, tenderness, and gurgling, as regards their prominence, are not proportionate to the amount of intestinal lesions.

Perforation of the small or, in rare instances, the large, intestine occurs in a certain proportion of cases. Of my 73 cases it took place in only two. Of fatal eases collected by Louis, Murehison, Bristowe, together with the records of the London Fever Hospital, making in all 185, perforation occurred in 38 , the proportion being a fraction under one-fiftl. Of 231 cases (20 cases fatal) analyzed by Prof. Pfeufer, of Munich, perforation occurred in 5 . It oceurs late in the disease or during convalescence, and sometimes even after apparent recovery from the fever. It occurs in cases in which the fever appears to be mild as often as when the fever is serere, a fact which is consistent with a statement already marle, viz., that the severity of the fever does not correspond with the amount of intestinal lesions. It occurs oftener in men than women, and almost never in children.

Perforation gives rise to peritonitis which is generally developed abruptly. The sudden occurrence of notable tenderness diffused over the abdomen, and abdominal pain, increased tympanitic distension, rigidity 
of abdominal walls, with marked frequeney of pulse, prostration, haggard expression of countenance, ete., point to peritonitis from perforation. In some cases, the perforation being quite small, the development of the peritonitis is gradual ; it may be circumscribed under these circumstances. The existence of peritonitis and its sudden development do not invariably denote perforation. Peritonitis in typhoid fever may be caused by the evacuation into the peritoneal sac of liquefied matter within a mesenteric gland. It may oceur irrespective of either this cause or perforation. In a case at the fever hospital on Blackwell's Island, in which death took place from typhus fever, the disease occurring during convalescence from typhoid fever, the post-mortem examination showed recovery from recent peritonitis, adhesions over the Peyerian patehes indicating the point of departnre of the peritoneal inflammation to have been the intestinal ulcerations. Probably in most of the reputed cases of recovery after the occurrence of peritonitis, perforation was not involved. Peritonitis from perforation is almost certainly fatal.

Skin.-_Of the symptoms referable to the skin, the most important is a characteristic eruption. The eruption consists of isolated papules, generally limited to the trunk, of a rose or pink color, called by Louis lenticular rose-colored spots. They are slightly elevaterl, and therefore are papulæx, not maculæx. The elevation is distinctly felt if the finger be carried lightly over them. They are slightly oval in form, and are from one to two lines in diameter. The redness momentarily disappears on light pressure. The number of papules is generally small, in the majority of cases not exceeding fifteen or twenty, and sometimes only four or five can be discovered. Exceptionally they are abundant over the trunk, and the eruption sometimes extends to the extremities. Fresh papules appear from time to time during the course of the fever, taking the place of those of anterior date. 'They are to be sought for over the chest and abdomen in front, and over the back; they are sometimes found exclusively in the latter situation.

Reckoning from the beginning of the forming stage, the eruption appears between the seventh and fourteenth day. Dating from the time of taking to the bed, it is observed between the second and twelfth day, the average time of the appearance being about the seventh day. Papules continue to disappear and appear during the career of the clisease, and sometimes the eruption persists into the stage of convalescence. The papules are not apparent after death.

The eruption is not invariably present. In the 73 cases which $I$ have analyzed, it existed in 49 . The proportion of cases in which it occurs varies in different years, as my statistics show. It appears to be less frequent in the comntry than in the eity. According to Jenner, it is less frequent in children than in adults. The copiousness of the eruption is no evidence of severity of the fever, and, on the other hand, it is as likely to be absent in mild as in severe cases. A scarlet rash occasionally precedes the characteristic rose eruption.

Miliary vesicles or sudamina are not infrequently observed in the middle and latter part of the disease, on the neck, chest, and abdomen. Lonis attributed to this eruption considerable diagnostic value, but on insufficient ground. It is observed in various aflections in which perspiration occurs. Petechiæ or minute ecchymoses are occasionally observed. These do not denote unusual gravity of the disease; they occur in mild as well as severe cases.

Bluish patehes of irregular form, from three to eight lines in diameter, are occasionally observed on the abdomen, back, and thighs. They are 
not peculiar to this disease, and are more likely to occur in mild than in severe cases.

Perspiration more or less abundant, and recurring more or less frequently, is observed in about one-half of the cases, exclusively of its occurrence at the time of convalescence or just before death. It occurs in about an equal proportion of fatal cases and of those ending in recovery. Observation does not show a connection between this symptom and any particular antecedent events; but taking the frequency of the pulse as a criterion of the gravity of the disease, and comparing the pulse before and after the occurrence of perspiration, in the majority of cases improvement follows. While this symptom, therefore, has no bearing on the prognosis, it often betokens some amelioration. It is not certain whether the perspiration produce the amelioration or proceed from it. It is most apt to occur at night. As a rule, during the greater part of the career of the fever, the skin is dry. The temperature of the body will be noticed in connection with the circulation.

Gangrene is apt to occur in situations exposed to pressurc. Spontaneous gangrene of the extremities has been observed, the probable explanation being arterial thrombosis. Gangrene, and troublesome ulcers, are liable to follow vesication. 'The liability to bed-sores on the nates, hips, and shoulders is to be borne in mind. 'These may be prevented by changing from time to time the position of the boly, the use of air-pillows, etc. Erysipelas is an occasional complication of typhoid ferer.

Respiratory System.-Cough is not a plominent symptom, mless some unusual pulmonary complication exists ; but slight or moderate cougl is almost invariably present, proceeding from subacute bronchitis, or, perhaps, from congestion of the mucous membrane similar to that of the skin. A sibilant rale is frequently heard over the chest on auscultation.

Pneumonitis is a not infrequent complication. It occurred in 12 of 73 cases which I have analyzed. This complication is rendered probable by prominence of cough and accelerated breathing; but the proof of its existence is afforded by the physical signs. This complication is sometimes quite latent as regards diagnostic symptoms, and is determinable only by physical exploration. The existence of pneumonitis adds to the danger, but by no means precludes recovery. Irrespective of any pulmonary complication, except subacute bronchitis, the frequency of the respirations is increased, the average being about 21 per minute.

Frequent sighing in the early part of the disease is apt to be a forerumer of ataxic symptons of grave omen. A spasmodic or jerking inspiration, when pneumonitis does not exist, is an important symptom as a precursor of coma. 'This statement holds good in other forms of fever than typhoid. Of the cases which I have analyzed, this symptom was noted in 16, and in one-half of these cases, death by coma followed within a period varying from twelve hours to four days. The importance of this symptom is enlaneed by the fact that it may sometimes be observer when no other symptoms denote impending coma. 'This was true of several of the cases which I have observed.

Epistaxis has been mentioned as a symptom of diagnostic value in the forming state. It occurs not infrequently afterwarl, especially in the early part of the febrile career; and in some cases it occurs repeatedly. Of the 73 cases which I have analyzed, it occurred once or repeatedly in 21. Generally the hemorrhage is small, but occasionally it is profuse, and it may even require to be arrested by mechanical means. When slight or moderate, it does not appear to exert any influence on the progress' of the disease. 
Laryngitis and œedema of the glottis are oceasional complications of typhoid fever.

Circulation and Temperature--More or less acceleration of the pulse belongs to the history of the disease. The acceleration varies considerably in different cases and at different periods of the disease in the same case. An analysis of my cases shows a greater average frequency in fatal cases than in those ending in recovery. $\Lambda$ s a rule, the gravity of the disease is in proportion to the acceleration of the pulse. The danger is usually considerable if the pulse exceed 120 per minute, and the danger is augmented in a geometrical ratio as the frequency rises ligher than this. The mean frequency in the eases not proving fatal, of those which I have analyzed, was 93 ; in the fatal cases 110 . In some mild cases the average frequency during the whole of the clisease is less than the average frequency of health. In one of my cases the average frequency was 69 . But in these eases the pulse at times exceeds the healthy standard ; hence, the pulse may at times fall below the staudard of health. This is not infrequently observed at or near the time of convalescence. I have noted it as low as 64,60 , and 40 . Others have observed it to fall still lower. Oftener the pulse continues more or less accelernted after convalescence is declared. A sudden and considerable increase of the frequency of the pulse denotes the ocenrrence of some important event, such as pueumonitis or peritonitis. 'The frequency of the pulse may vary at different periods of the dlay. The increase is sometimes in the morning and sometimes in the evening. So far as my observations go, regular exacerbations of fever, as indicated by the pulse, or remissions, are rarely observed. The thermometer, however, shows throughout the disease a variation in temperature at evening and morning, the maximum being in the evening, the minimum in the morning.

As regards eharacters of the pulse other than frequency, it is often quick and vibratory, but rarely hard or resisting. It denotes increased action, but not increased power of the ventricular systole. It becomes feeble or compressible in proportion as it is frequent. The foree is always notably diminished if the frequency much exced 120. An unfavorable progress of the disease toward asthenia is shown by progressive increase in frequency and diminution of force. Under these circumstances, the first sound of the heart, on auscultation over the apex, is notably lessened, and may be inappreciable. The observations of Marey show that the form of the pulse, as traced by the splyggmograph, presents the following characters : a short and nearly vertical line of aseent, indicative of weakness and quickness of the ventricular systole; a line of descent denoting the free passage of blood from the arteries in the capillaries, and notable dicrotism. The dicrotism of the radial artery is sometimes appreciable by the touch. These characters belong to the graphic pulse also in cases of typhns, and in all affections with which is associated the typhoid condition.'

More or less increase of the lheat of the body is invariable. The rule that dnring the greater part or the whole of the career of the disease the temperature is raised, is without any exceptions; but, under certain circumstances, the heat may not exceed, and it may even fall below, the standard of health. In general, the thermometer in the axilla shows a daily increase of temperature for the first five or six days. At the end of this period, the temperature at least rises to $103^{\circ}$ Fahr. If, in the progress of the disease, the thermometer show much increase above this,

I Fra a general account of the spluy gmograpli, sce page 114. 
the prognosis is unfavorable, whereas, a decline of the temperature in the morning to near the normal standard is evidence of convalescence. During the progress of the disease there is, as a rule, an oseillation of temperature between morning and evening, the difference being, however, less than a degree up to the time of convalescence, when the oscillations bacome much greater, the difference being four to five degrees, the temperature in the morning falling to the normal stanclard $98^{\circ}$ or lower. 'The difference between the morning and evening temperature at the time of convalescence has been observed to be over nine degrees. A high elevation of temperature in the morning, viz., $106^{\circ}$ to $108^{\circ}$, indicates the approach of death. The laws of the disease, as regards temperature, are sufficiently characteristic, to render the thermometer useful in diagnosis as well as prognosis. A sudden and considerable rise of temperature, during the progress of the disease, points to the occurrence of some inflammatory complication. A sudden and considerable fall of temperature, other symptoms not denoting convalescence or improvement, is unfavorable. This has been observed to precede hemorrhage from the bowels. It is important, however, to note that, irrespective of complications, the temperature, in some cases, becomes increased and the rise may continue for several days; and, on the other hand, a decline of from one to two and a half degrees sometimes takes place, lasting for several hours, without any obvious cause and having no special significance.

Urine - The urine is usually scanty and of high specific gravity until the approach of convalescenec, when it becomes abundant and of a low specific gravity. During the progress of the disease, as a rule, the urea and uric acid are absolutely increased. The coloring matter is also more abundant than in health. The presence of albumen during a portion of the febrile career is not very uncommon. Abeille found it in 12 of 95 cases. Of 282 cases observed by Murchison, Parkes, Solon, and others, albuminuria occurred in 93 , being about 32 per cent. Abeille, who has studied with care the occurrence of transient albuminuria in this and other affections, states that it is a symptom denoting always gravity of the disease. Of the 12 cases noted by this observer, in 6 the disease was fatal. The abundance of albumen denotes a proportionate gravity. This symptom occurs in the midlle or latter part of the disease, and may continue for a period varying from 24 hours to 12 days. In the fatal cases in which it occurs, it contimues up to the time of death. In the cases studied by Abeille, the kilneys after death were simply congested, no deposit nor structural change existing. He supposes that the albuminuria proceeds, partly from congestion of the kidney, and in part from a morbid cliange in the blood.

Renal casts in the urine are sometimes observed. These denote diseases of the kidneys, either existing prior to the fever, or developed as a complication. If, with or withont renal casts or albuminuria, the urine be notably deficient in urea, uræmia is to be apprehended. It is probable that coma and convulsions occurring in the progress of typhoid fiever are due to urremic poisoning.

The urine is sometimes retained in cases of typhoid fever, and, owing to the blunted perception, the bladder may become greatly distended. The practitioner should not omit to ascertain the condition of the bladder, by manual examination of the abdomen, during the progress of the disease, and resort seasonably to the use of the catheter when required. On the other hand, the urine is often passed in bed, sometimes from indifference and sometimes from incontinence.

In order to determine the duration of the disease, it is necessary to fix 
upon certain points which shall mark the beginning and the end of the febrile eareer. The time of taking to the bed is a convenient, and, in a collection of cases, a tolerably correct eriterion of the full development of the fever. It is difficult to find any circumstances which will answer equally well to denote the date of convalescence. The career of the fever rarely ends abruptly; the termination in convalescence, like the development, is gradual. The decision that a patient is to be considered convalescent is a matter of judgment, and is to be based on the ensemble of symptoms. Ditferent practitioners would differ in individual cases as to the precise day of convaleseence. This fact disposes at once of the doctrine of critical days. A believer in this doctrine would be likely to fix upon a critical day as the date on which the fever ends, whereas a non-believer would be as likely to fix upon some other day; hence, it is elear that observations colleeted by believers in critical days would go to support the doctrine. Of 42 eases, ending in recovery, which I have analyzed with reference to the duration of the fever, dating from the time of taking to the bed to the time when the improvement in all the symptoms was sufficient for the patients to be considered convalescent, the average duration was 16 days. The maximum duration was 28 days; the minimum 5 days. The longest duration in any case under my observation was in a female patient at Bellevue Hospital, the disease continuing, in this ease, without any inportant complication, about 58 days. The mean duration in 75 cases observed by Murchison, was a fraction over 24 days. This greater duration, as compared with my eases, is probably owing to the commencement being fixed at an earlier period than the date of taking to the bed. Of 45 fatal cases in my collection, the mean duration was a fraction over 14 days, the maximun being 26 , and the mininum 9 days. The mean duration in 12 fatal cases observed by Murchison was a fraction over 22 days. The average duration of convalescence is from one to two weeks. The return of the axillary temperature to the normal standard, that is, complete defervescence, denotes, of course, that the febrile career is ended. Sometimes the defervescence is rapid, and the temperature quickly falls to that of health. But generally it is gradual, and paroxysms or exacerbations of fever, as indicated by the thermometer, are liable to occur for some days after the appetite, mental condition, and other symptoins denote convalescence. The temperature may be kept up by complications which persist after the career of the fever is ended. A temporary fever is apt to accompany the change of diet from liquid to solid animal food.

Relapses of typhoid fever are sometimes observed. Several examples have fallen under my observation. A return of the fever may take place after ten days or a fortnight from the date of convalescence, and the patient pass through a second carcer, the eruption and other claracteristic symptoms being reproduced. The duration of the second career is usually shorter, and the severity greater than the first, but a fatal termination is rare. In 231 cases oecurring in six months, in Munich, in 1856-57, analyzed by Prof. Pfeufer, there were seven examples of relapse. Of these 7 cases 4 were fatal, the large rate of fatality being a deviation from the rule in relapsing cases. "A fter death two sets of ulcers were found in the intestines, corresponding to the two attacks of fever." I

The complications which are apt to arise in the course of typhoid fever have been noticed in connection with the symptoms referable to different anatomical symptoms. Typhoid fever may be associated with scarlatina,

1 Am. Journ. Med. Sciences, July, 1861. 
rubeola, diphtheria, and perhaps with typhus. Occasional sequels are, pulmonary tuberculosis, and subcutancous abscesses. Progressive emaciation and death from inanition have been known to follow ; but in many instances, nutrition becomes extremely active after recovery, and the patient attains to a greater weight than ever before. 'The mental powers in some cases are enfecbled for a considerable period.

\section{CHAPTER II.}

Typhoid Fever Continued-Causation-Diagnosis-Prognosis. Typhus Fever-Anatomical Characters-Clinicai History-Causation-Diagnosis-Prognosis. Non-identity of Typhus and Typhoid Fever.

THE anatomical characters and clinical history of typhoid fever have $I$ been consiclered in the preceding chapter, and it remains to consider the causation, diagnosis, and prognosis of this disease before entering on the consideration of typhus fever.

Causation.-Typhoid fever is not restricted within any geographical limits ; it is endemic in every quarter of the globe. In certain of the socalled malarious districts it is not observed, as distinct from remitting fever ; but there is reason to believe that, in these districts, it is obscured by being blended with periodical fever. It is certain that in some situations in this country, well-marked cases of typhoid fever were hardly known so long as malarious fevers were rife, but the former became the common form of fever after intermitting and remitting fevers ceased to prevail. Of this fact, I have been personally cognizant in two situations, viz., Buffalo and Louisville. As regards the seasons of the year, it shows a decided predilection for the autumn. Of 45 cases which $I$ have analyzed with reference to this point, 16 were in October, 9 in November, and 12 in December. It is stated that a warm dry summer favors the occurrence of the disease in the following autumn.

A decided predisposing influence pertains to age. Young persons are chiefly liable to it. Murchison states the mean age, in 1,772 cases admitted into the London Fever Hospital, to have been a fraction over 21 years. 58.08 per cent. of cases admitted into this hospital in ten years, occurred in persons between the ages of 15 and of 25 , and only 27.76 per cent. above the latter age. It occurs but rarely in infancy. It is not uncommon in childhood. A considerable proportion of the cases of so-called infantile remitting fever are cases of typhoid fever. It is extremely rare after 50 years of age; but well authenticated cases of its occurrence after that age, and even in old persons, have been reported by Lombard, Trousseau, Wilks, and others. I have met with a case in which all the diagnostic characters were well marked, the patient being a woman 53 years of age. It is stated that the glands of Peyer begin to disappear after adult life, and traces of their existence only are apparent after forty-five years. This will account for absence of the lesions of these glands after fifty years of age. 
Both sexes appear to be about equally liable to the disease. It was observed by Lovis to occur especially among persons who had resided for a short period only in Paris. It has been observed to prevail among recent residents in other eities. A rational explanation of this fact is, that, having been exposed to the action of the cause of the disease in a small quantity, the older residents have thereby aequired an insusceptibility to the action of it in a larger quantity, as persons by use are able to tolerate certain remedies in enormous doses, for example, the narcotic remedies. Generally patients are in good health when attacked; other diseases do not seem to predispose to it. No cansative influences relating to social position, occupation, or habits of life, have been ascertained. There does not appear to be any solid ground for the notion which has been held by Carnot and some other French writers, that the diminished prevalenee of smallpox, in consequence of vaccination, has rendered typhoid fever more prevalent.

With respect to the contagiousness of the disease, there has not been unanimity of opinion. That it may be communicated under certain cireumstances is certain, and that it frequently or generally originates spontaneously, that is, irrespective of contagion or infection, is perhaps equally certain. Assuming these statements to be correct, this is one of the diseases, the special cause of which may be generated without the body and reprodueed within the body.

The eontagionsness of typhoid fever is proven by instances in which persons, having contracted the disease in one locality, go to another in which the disease was not prevailing, and, of the residents in the latter locality with whom they are brought into contact, a greater or less number become affected. Many instances of this kind have been reported, ${ }^{\prime}$ but the most remarkable of any on record eame under my observation, in 1843, in a little settlement called North Boston, situated eighteen miles from the city of Buffalo, consisting of nine families, all being within an area of a hundred rods in diameter; but the few houses in which the disease oceurred were elosely grouped together around a tavern, the house farthest removed from the tavern being only ten rods distant. A stranger from New England, travelling in a stage-coach which passed through this settlement, had been ill for several days, and on arriving at this stoppingplace was unable to proceed further. He remained at the tavern, and, after a few days, died. He was seen by several pliysicians of the vicinity, and there can be no doubt that his disease was the same as that with which others were subsequently affected. Up to this time typhoid fever had never been known in that neighborhood. The sick stranger was seen by the members of all the families in immediate proximity to the tavern, with a single exception. One family named Stearns, having quarrelled with the tavern-keeper, had no intercourse with the family of the latter and very little with the other families, all of whom were tenants of the tavern-keeper. No member of the family of Stearns saw either the sick stranger or any of those who were taken ill after the stranger's death. Members of the family of the tavern-keeper were the first to become affected; the first case occurring 23 days after the arrival of the stranger. Other cases speedily occurred in the surrounding families. In a month, more than one-half the population, numbering 43 , had been affected, and ten had died. Of the families immediately surrounding the tavern, that of Stearns alone escaped; no case occurred in this family.

The occurrence of the disease produced great excitement in the neigh-

1 Vide Murchison, on the Continued Fevers of Great Britain. 
borhood; poisoning was suspected, and Stearns was charged with having poisoned a well used in conmon by all the families except his own. $\boldsymbol{A}$ fact which encouraged this suspicion was, the common well being owned by the tavern-keeper, he had refused permission to use it to Stearns, who had in consequence been obliged to dig a well for his own use. An examination of the water from the common well showed it to be perfectly pure. The disease was undoubtedly typhoid fever. Visiting this settlement during the prevalence of the clisease, and recording the symptoms of several cases then in progress, the clinical history furnished abundant evidence of the nature of the disease. Moreover, I made an examination of the body of one of those who had died with the disease, and found the Peyeri:m patches uleerated and the mesenteric glands greatly cnlirged.'

Now, were it proposed to devise an experiment to test the communicability of a discase, no better plan could be adopted than to introduce a case into a district where it was not at the time prevailing, and to procure free exposure on the part of some of those residing in the district. The experiment would be more satisfactory if the disease introduced had not been hitherto endemic in that locality. And to render the experiment as perfeet as possible, by divesting it of any moral influence, it should be made without the knowledge of those who were to be the subjects. If, under all these cireumstances, a large proportion of those of the inhabitants of the district who had been bronght into contact with the disease were attacked simultaneously or in quick succession, and thus a new and hitherto unknown affection be suddenly developed, which spreads rapidly over a limited circuit, affecting those only who have been in habits of intercourse with the imported case or with the persons who were subsequently attacked, others residing in the same district, but not brought into contact with the disease, uniformly escaping - what is the logical deduetion from the facts? In the absence of all evidence of epidemic or endemic agencies, contagion offers the only adequate explanation. The laws of probabilities would not anthorize the supposition that the events depended on mere coincidence. To undertake such an experiment would be neither practicable nor justifiable: but it will be seen, on reviewing the facts connected with the development and diffusion of typhoid fever at North Boston, that they embrace all the conditions for a fair experiment to test the contagiousness of the clisease. If every circumstance had been deliberately selected and arranged, they could hardly have been more complete.

Although this disease may be undoubtedly communicated in some way from the sick to the well, under ordinary circumstances it is not diffused by contagion. As a rule it is not communicated to those who come into contact with private patients, nor is it propagated among hospital patients, thus differing widely from typhus fever. There is ground for the conjecture that, when communicated, the special poison is derived from the intestinal discharges. Dr. William Budd and others entertain the bolief that the disease invariably proceeds from a special virus contained in the excreta, and they account for the prevalence of the disease by the dissemination of the virus in emanations from water-closets, privies, and drains. In the instance just given of the diffusion of the clisease at North Boston, the fact that all the families in which the disease prevailed were supplied with water from a common well, and the fact that the family in which no case occurred did not obtain water from this well, afford ground for sup-

' For fuller details, vide Clinical Reports on Contiuued Fever, etc., 1852, and American Journal of the Medical Sciences, July, 1845. 
posing that a virus nerived from the excreta was conveyed in the water drank. That the alvine excreta are the media of communication in the rare instances in which the disease is evidently communicated is highly probable, but the doctrine that this is invariably the causation is inconsistent with facts which appear to show conclusively the spontaneous generation of the causative agent in the great majority of cases.

Murchison cites several striking outbreaks of typhoid fever in localities in which it appeared to be fairly attributable to emanations from obstructed drains and cesspools, or from drinking-water tainted with sewage. The following is a striking instance, apparently, of this kind: Mr. C., a wealthy citizen, spent the summer in 1867 at his country-house, leaving his house in the city in charge of two persons. He returned, with his family, to town in the middle of September. After his return, five of his children within a few days fell ill, and the disease proved to be typhoid fever. A nephew who left town shortly after the return of the family was taken ill after leaving, and had typhoid fever. A child was ill for a few days with vomiting and fever, but the disease in this case did not prove to be typhoid fever. With exception of the person last referred to, every member of the family, except the parents, had typhoid fever. All were attacked within a few days. All recovered except one, who had profuse hemorrhage from the bowels. The ages of the patients were between 7 and 22 years. On examination of the premises, the waste-pipe in the cellar was found to be defective, and at times a bad odor from it had been pereeived. The house had been shut up during the summer. The disease was not prevailing in the neighborhood. The cases were treated by my colleague, Prof. MeCreedy. Regarding the poison as frequently thus generated, Murchison proposes, as a name for the disease, pythogenic fever. That putrescent substances, animal or vegetable, and excreta, exert a causative agency, is, to say the least, highly probable, but whether by giving rise to the special poison or acting as auxiliary causes, cannot be considered as settled; hence, the name is objectionable for the reason that it implies a doctrine which is hypothetical. The probable agency of noxious gases from drains, cesspools, and privies, or of water polluted from these sources, and of emanations from the intestinal discharges of fever patients, enforces the importance of measures of prophylaxis, consisting of effective sewerage, providing against obstructions of housedrains, preventing leakages from these and cesspools, taking care that the trapping of sinks and water-closets is complete, and the speedy removal of the excreta of patients affected with the disease.

The interval from the introduction of the poison into the system and the first manifestation of disease, or the period of incubation, is most commonly about two weeks. The duration is longer than this in some instances, but, probably, it is never several months, as has been supposed. On the other hand, the duration sometimes dnes not exceed one or two days. ${ }^{1}$ This disease is one of those which are very rarely experienced twice; a person who has had the disease, as a rule, is thereafter unaffected by the special cause. As already stated, however, a relapse sometimes occurs shortly after the date of convalescence.

Diagnosis.-Typhoid fever is to be discriminated from other essential fevers, more especially typhus and remittent fever. The differential diagnosis cannot be fully considered without anticipating the diagnostic events which belong to the clinical history of other fevers which remain

1 Vide Murchison in St. Thomas's Hospital Reports, vol. ii. 
to be considered. The discrimination is to be made by ascertaining the presence of more or less of the events which are diagnostic of typhoid fever, and the absence of events diagnostic ct other fevers. The points in the clinical history which are clistinctive of typhoid ferer are as follows: the gradual development, the absence of marked remissions, the abdominal symptoms, viz., diarrhoa with ochre-colored dejections, tympanites, tenderness in the iliac regions and gurgling; the occurrence of epistaxis and the characteristic eruption. As regards the eruption, a fact important to be borne in mind is, that rose-colored papules, like. those belonging to the history of typhoid fever, are sometimes found in other affections. Other points to be taken into account are, the antumnal season, and the youth of the patient. The foregoing points, taken in connection with the absence of features distinctive of other fevers, generally render the discrimination easy, after a certain duration of the disease. In the early part of the disease, the discrimination is not always easy, and some delay may be necessary in order to arrive at a positive diagnosis.

The diagnosis involves discrimination from several local affections. Certain local affections may be supposed to constitute the disease when they are actually present, not as idiopathic affections, but as complications, or when certain organs are functionally disturbed in a marked degree without any local affection more than is incident to the fever. In determining whether an existing disease be an essential fever or a local affection, the following general considerations are to be kept in view. They are applicable alike to typhoid ferer and other essential fevers.

1. 'The presence of an access, or forming stage, of greater or less duration, is somewhat distinctive of an essential fever. Local affections, as a rule, are comparatively abrupt in their development. This consideration is especially applicable to typhoid fever.

2. 'The events which denote fever of some kind are to be sought after in the details of the early history prior to the occurrence of symptoms pointing to any local affection.

3. It is to be observed, if the general symptoms, such as prostration, febrile movement, delirium, etc., be not greater than would be expected as symptomatic of the particular, local affection which is suspected.

4. 'The events which are distinctive of particular fevers, and which do not belong to the history of local affections, are to be sought after; for example, the events proper to typhoid fever, such as the eruption, epistaxis, and abdominal symptoms.

5. It is to be observed whether symptoms are not absent which would probably be present if the suspeeted local affection existed, or, assuming its existence, if it were a primary or illiopathic affection.

6. 'The laws of the disease with respect to animal heat, as determined by the thermometer, are to be taken into account. This disease is characterized by a progressive daily increase of heat for the first three or four days, and an increase to $103^{\circ}$ or more Fahr. during the second week. If during a period too short for the career of the disease the temperature fall to the normal standard, the disease is not typhoid fever.

Local affections with which typhoid fever is liable to be confounded are, meningitis, bronehitis, pneumonitis, acute tuberculosis, and enteritis.

Acute meningitis, as distinguished from typhoid fever, is characterized by more intense cephalalgia, by intolerance of light and sounds, early and active delirium, frequently by vomiting as a prominent symptom, and sommolency and coma succeeding the delirium. There is generally constipation; the abdomen is not tympanitic, but frequently depressed, and 
iliac tenderness is wanting. This differential diagnosis is required chiefly in children.

Subacute bronchitis is an element of typhoid fever. If the bronehitis be unusually prominent and the fever unusually mild, the latter may be overlooked and the disease considered a primary bronchitis. This error can only happen in the early part of the fever; the duration and progressive inerease of the disease, together with the characteristic events of the fever, will lead to a correction of the diagnosis.

Typhoid fever may be confounded with pneumonitis, when the latter exists as a complieation. 'The existence of pneumonitis is shown by its physical signs. The differential diagnosis is to be based on facts which show the existence of fever prior to the oecurrence of the pneumonitis, and on the events characteristic of typhoid fever, viz., the abdominal symptoms and eruption. Cases of primary pneumonitis, in which the existence of typhoid fever may be suspeeted, are cases of so-called typhoid pneumonitis, that is, pneumonitis with phenomena denoting the typhoid state, viz., low delirium, prostration, subsultus tendinum, etc.

Acute tuberculosis has not infrequently been mistaken for typhoid fever. The former is to be discriminated by the notable frequency of respirations, the prominence of the cough, the occurrence of hxmoptysis in some cases, the abundance of subcrepitant rales, by marked lividity in some cases, and by the absence of the abdominal and other characteristie events of typhoid fever.

Typhoid fever may be considered as primary enteritis when the abdominal symptoms are unusually prominent from the beginning. The liability to this error is chiefly in children. Events, other than the abdominal symptoms, belonging to the history of typhoid fever, are to be sought after. Some of these are not as readily appreeiated in children as in adults, especially those relating to the mind, and the eruption is oftener wanting. Hence, typhoid fever in children is not infrequently called enteritis. With due care in tracing the development and progress of the disease, this error of diagnosis should generally be avoided.

It is evident that familiarity with the phenomena and laws of typhoid fever and of the diseases with which it is liable to be confounderl, viz., other fevers and the local affeetions just referred to, is an essential qualification for accuracy in diagnosis. This remark is of general application to diagnosis.

The use of the thermometer may be brought to bear upon the diagnosis of this disease at an early period, before the eruption of other distinetive features is fully declared. In a case in which typhoid fever is suspected, if the thermometer in the laxilla show a notable increase of heat, the diagnosis may be made with much confidence, provided acute inflammations are excluded. On the other hand, in such a case, if the thermometer do not show increase of heal, it is certain that the disease is not typhoid fever.

There is a very small proportion of cases in which the disease is likely to be overlooked in consequence of the febrile phenomena being imperfectly developed. The cases now referred to were called by Louis cases of "latent typhoid fever." They are called by some German writers cases of "typhus ambulatorius;" and the term "walking cases" is in use in this country. The patients do not take to the bed, and perhaps they continue their usual avocations. They complain only of debility, loss of appetite, and mild diarrhœa. Under these circumstances, death sometimes takes place from intestinal perforation, profuse hemorrhage, or some grave complication, and, on post-mortem examination, the characteristie typhoid lesions of the intestine are found. The only explanation of these 
cases to be given is, that the effects of the special cause are concentrated on the glandular elements of the small intestine.

Proarosis.-Of 18,612 cases aggregated by Murchison, occurring at the London Fever Hospital in 14 years, Guy's Hospital, St. 'Ihomas's Hospital, and King's Hospital, of 'London, at Strasbourg, Paris, and the provinces of France, and the Infirmary at Glasgow, the mortality was 18.62 per cent., or 1 in 5.4. The separate collections of cases which make up the above aggregate show a variation in mortality between 16 and 32 per cent. Excluding 190 cases at Strasbourg, in which the mortality was 23 per cent., and 147 cases at Paris, giving a mortality of 32 per cent., the variation in the remaining collections was between 16 and a fraction over 20 per cent. Of 303 cases received in the Massachusetts General Hospital, and analyzed by James Jackson, 42 were fatal, being 1 in a fracction over 7 , or a fraction under 13 per cent. Of the 73 cases which $I$ have analyzed, 18 were fatal, nearly 1 in 4 , or about 24 per cent.

These facts show considerable variation in the death-rate in different collections of cases occurring at clifferent places and periods. The variation is often considerable at different seasons in the same place, and under similar circumstances as regards surroundings and treatment. 'This fact is shown by the ratio of deaths in suecessive years in the Massachusetts General Hospital.' In 1828, of 22 eases, 2 died ; in 1829, of 25 cases, 1 died ; in 1830, of 14 cases, 4 died; in 1831, of 29 cases, 2 died; in 1832 , of 23 cases, 4 died ; in 1833 , of 37 cases, 6 died ; and in 1834, of 34 cases, 6 died; and in 1835, of 35 cases, 6 died. To a certain extent, therefore, differences in the intrinsic tendency of the disease to a fatal issue at different periods and places must be admitted-a fact to be considered in estimating the influence of therapentical measures.

As regards age, Murchison's statistics show the lowest rate of mortality to be from 10 to 15 years, and the next lowest from 5 to 10 years; the number of cases analyzed uncler 5 years being insignificant. The highest rate under 55 years is from 30 to 35 years. Jackson's statisties show the death-rate to be greater above than below 30 years of age; and they show a greater mortality in the cold than in the warm months. It is a matter of common observation, that vigorous persons are more likely to succumb than those of feeble constitution.

A futal result is rarely due to the intensity of the disease. Neath, generally, is attributable to complications or accidents, such as pneumonitis, peritonitis, and occasionally hemorrhage. Pre-existing disease may lead to a fatal result-for example, chronic disease of the kidneys. Unusual severity of the abdominal lesions is sometimes the cause of death. In the great majority of fatal cases the morle of dying is by asthenia. Apnoa is combined with asthenia in the cases in which sudden coma precedes death.

Coma, probably uræmic in most cases, and convulsions render the prognosis extremely unfavorable. Other marked ataxic symptoms, such as carphologia, subsultus, etc., are ominous. Cases characterized by active persistent delirium usually end fatally. The prognosis is unfavorable whenever the pulse becomes extremely frequent and feeble, and the first sound of the heart is notably weakened. Great prostration is evidence of great danger. In eases of pregnancy, abortion generally takes place, and death follows. The chances of recovery may be good, notwithstanding the occurrence of pneumonitis limited to one lobe. Intestinal hemorrhage is less serious than has been supposed by some; recovery takes

1 Report on Typhoid Fever, by James Jackson, M.D., Boston, 1838. 
place in the majority of the cases in which this event occurs. Death may be caused by gangrene or bed-sores in parts exposed to continued pressure from the weight of the body. An increase of the axillaly temperature above $105^{\circ}$ denotes great danger; a temperature of $106^{\circ}$, or $107^{\circ}$, or upward, is a fatal prognostic. On the other hand, a considerable decrease of temperature below the minimum of health is extremely ominous. In general, after the third day of the disease, the temperature having daily increased up to this time, the heat as shown by the thermometer varies in the morning from $102^{\circ}$ to $103^{\circ}$, and in the evening from $103^{\circ}$ to $105^{\circ}$.

A favorable prognosis may be entertained so long as ataxic symptoms are not marked, the adynamia not great, the pulse not very frequent or feeble, and there are no serious complications; but, under these eircumstances, the prognosis should alway's be guarded, in view of the liability at any period of the disease to serious complications or accidents. And it is to be borne in mind that sudden coma and perforation of intestine are not less liable to occur in mild than in severe cases. The liability to perforation cluring convalescence is to be recollected. On the other hand, recovery may be hoped for in cases in which the symptoms denote the utmost gravity.

\section{Typhus Fever.}

The fever called typhus, known from the earliest antiquity, has received a great variety of names. The name typhus, introduced by sauvages in 1759 , and now generally adopted by writer's of all countries, has the negative merit of not involving any hypothesis concerning the nature and seat

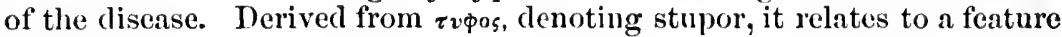
of the disease which is usually more or less prominent. In this country, from the fact that the disease is imported in vessels bringing emigrants from Ireland, it has been commonly known as ship fever.

'This fever has many features in common with that just considered, viz., typhoid fever. The identity or non-identity of the two affections has been, within late years, a mooted question. They present points of contrast sufficient to show that they are distinct diseases. The grounds for concluding that they are not identical will be stated, after having considered the anatomical characters, clinical history, causation, diagnosis, and prognosis of typhus. In considering this species of fever under the aspects just enumerated, it will suffice to present the traits by which it is distinguished from typhoid fever.

Anatomicat، Characters.-In typhus, the abdominal lesions which are characteristic of typhoid fever are wanting. The Peyerian and solitary glands of the small intestine are either unaffected or they are simply more conspicuous than usual, sometimes presenting numerous black points, giving rise to what has been called the shaven-beard appearance. The mesenteric glands do not contain the typhoid material, and are generally healthy.

The disease has no known special anatomical characters; that is, there are no lesions peculiar to the disease and constantly present. Morbid appearances in different parts are frequently found after deatl, but they are due to pre-existing disease or to complications, or they occur in a proportion of cases sufficient only to show an accidental connection.

The spleen is frequently enlarged and softened; the heart is often flabby and softened. The blood in the heart and large vessels is unnaturally 
dark and fluid; coagula, if they exist at all, are dark and soft. Hypostatic congestion of the lungs and pulmonary odema are more frequent than in typhoid fever. Slight extravasation of blood into the arachnoid eavity occurs in a small proportion of cases. Cerebral congestion and effusion of serum into the ventricles of the brain, the subarachuoicl space, and the arachnoid cavity are not uncommon, but unattended with the exudation of lymph. The kidneys are apt to be congested; they are sometimes enlarged, and the convoluted tubes may be filled with desquamated epithelium. 'The latter, probably, not infrequently occurs post-mortem. The granular and waxy changes in fibres of voluntary museles, noticed as occurring in typhoid fever, have been observed by Dr. George Buchanan in typhus.

Clinicat History.-The duration of the aceess or forming stage is shorter than in typhoid fever, and eases of an abrupt invasion are not as rare. Patients in the great majority of cases take to the bed on the second or third day after the first manifestation of illıess. 'l'he symptoms during the development of the disease are essentially the same as in typhoid fever, with this important differenee, viz., the abdominal symptoms of typhoid fever-diarhoea, meteorism, iliac tenderness, and gurgling-are wanting.

Countenance, and General Aspect.-Capillary congestion of the face, extremities, and trumk is more marked in typhus than in typhoid fever. It gives to the surface, especially on the face, a dusky or ilingy liue so distinctive of typluus as compared with typhoid fever, that even nurses and attendants, after becoming conversant with the two diseases, learn to discriminate them by the physiognomy. The conjunctiva is more frequently and deeply congested. A besotted or expressionless comtenance is earlier and more strongly marked.

Nervous System.-The symptoms referable to the nervous system which belong to the clinical history of typhoid fever occur in typlius. Comavigil and typho-mania are observed in a large proportion of eases of typhus, they are oftener present in a marked degree, and occur earlier in the eareer of the discase. Other attaxic symptoms, such as subsultus and carphologia, are of more frequent occurrence. I'rostration is apt to be morc marked and earlier manifested. Persistent active delirium, in my experience, has occurred oftener in typhoid than typhus fever. Contraction of the pupil often accompanies delirium or stupor, and is sometimes extremely marked, called by Graves the pin-hole pupil. I have observed in two cases oscillations of the eyeballs, occurring in a marked degree, the movements being lateral and notably rapid. The oscillations oceurred whenever the patient was raised. Both eascs ended fatally. Coma and convulsions occurring in typhus, as in typhoid, fever, are generally attributable to uræmia. Hyperesthesia of the surface, even when the mental pereeption is notably blunted, is sometimes manifested.

Digestive System.-The tongue, more frequently than in typhoirl fever, becomes covered with a thick, brown, or black coating. It is less frequently reddened, glazed, and fissured. It is oftener with diflieulty protruded. Sordes occurs more frequently, earlier, and in greater abundance. As regards the abdominal symptoms which are highly diagnostic of typhoid fever, the two diseases present striking points of contrast. Diarrhoea is often absent in typhus, and, when present, it is almost invariably slight. The stools have not the ochre color of those in typhoid fever. Tympanites is oftener wanting, and generally when present, slight in typhus. This statement applies also to iliac tenderness. Intestinal per- 
foration does not occur in typhus. Hemorrhage from the bowels is one of the rarest of events, exclusive of cases in which dysentery is a complication.

Parotiditis is liable to occur, and the remarks made with reference to this complication in typhoid fever are equally applicable to its occurrence in typhus.

Skin.-A characteristic eruption occurs in a larger proportion of cases of typhus than of typhoid fever. Murchison states that of 3506 cases received at the London Fever Hospital, it was not observed in 403, or a fraction over 11 per cent. Of 65 cases which I have analyzed, it existed in 57. It is oftener wanting in children than in adults. The eruption appears earlier in typhus. The avernge period from the time of taking to the bed to the first appearance of the eruption is somewhat under three days. The eruption in typhus is much oftener abundant, frequently being copious over the extremities as well as on the trunk, and sometimes appearing on the face.

Other distinctive points relating to the eruption are as follows : It is a maculated, not a papular, eruption. The spots become of a dull, dingy or dark red color, and, after the first two or three days, the redness cannot readily be made to disappear by pressure. In some cases, in the latter part of the disease, the spots become truly petechial. They are smaller than the papules of typhoid fever. They do not come and go like the rose papules of typhoid; they all appear in the course of three or four days, and remain during the greater part or whole of the disease, sometimes even continuing into convalescence. They are often apparent after death.

The copiousness of the typhus eruption represents generally a corresponding gravity of disease. This statement does not hold good with respect to the eruption in typhoid fever.

In a certain proportion of cases of typhus, intermingled with the characteristic macula are more or less of the rose papules which are characteristic of typhoid fever.

Petechiæe are observed in some cases of typhus, as in cases of typhoid fever, as well as in various other diseases. As already stated, the characteristic typhus-spots sometimes become petechial. The eruption of typhus has been called by some a petechial eruption. This is incorrect. Petechix are spots caused by minute extravasations of blood, that is, they are ecchymoses, and these are not distinctive of typhus. Vibices are occasionally observed in cases of typhus. Sudamina or miliary vesicles occur in cases of typhus as well as typhoid fever.

The statements with respect to sweating and moisture of the skin in typhoid are also applicable to typhus fever.

The odor from typhus patients is stated to be characteristic. I have met with persons who declared that they were able to discriminate cases of typhus from typhoid fever by the sense of smcll. I can bear testimony to an offensive odor emanating from patients affected with either disease, but $I$ have not been able to appreciate its value as a diagnostic symptom.

Erysipelas sometimes occurs in typhus as in typhoid fever. Bed-sores are less liable to occur in consequence of the shorter duration of typhus. Spontaneous gangrene, not produced by pressure, is an occasional event. I have seen a case in which gangrene of both feet took place. The nose, penis, and scrotum have been known to slough away. Ulceration or slonghing of the cornea has been observed: The affection known as noma, or cancrum oris, is another serious complication occurring sometimes, 
not only in ehildren, but adults. These complications occur in hospitals among patients who, prior to the disease, were suflering from innutrition.

Respiratory System.-The account of symptoms referable to the respiratory system in typhoid fever is mainly applicable to typhus. The signilicance of a spasmodic inspiration, oceurring without any pulmonary complication, is the same in the latter as in the former disease. Cough from bronchitis is apt to be more prominent than in typhoid fever. Pseudo-pneumonitis, that is, hypostatic congestion and odema, are more frequent, whereas, true pneumonitis appear's to be less frequent than in typhoid ferer. Epistaxis oceurs less frequently. It appears to be estalblished that the expired breath contains more ammonia than in health.

Circulation and Temperature.-The arerage frequency of the pulse in cases of typhus is greater than in typhoid fever. The significance of frequeney and other charaeters of the pulse, and of weakening or absence of the first sound of the heart over the apex, as representing the state of the vital forces, is not less in typhus than in typhoirl fever.

The temperature of the body, as determined by the thermometer, is more or less raised, varying from $102^{\circ}$ to $107^{\circ} \mathrm{Fahr}$. The increase of temperature corresponds to the intensity of the disease. A rapid deeline of temperature is coincident with the occurrence of convalescence. The defervescence in some cases is notably rapirl, but in other cases it is gradual. At the time of convalescence the temperature frequently falls below the standard of health. As in typhoid fever, and in other essential fevers, a sudden and considerable rise of temperature renders probable some inflimmatory complication; and, on the other hand, a sudden fall of temperature below the average range of health denotes a serious change for the worse. As a rule, the inerease of temperature daily is progressive from the commencement of the disease to about the fifth day, and the daily oscillations of the morning and evening temperature are not observed prior to this period. An analysis of 58 eases ending in recovery, by Dr. 'T. J. Maclagan, gave as the average temperature in the morning a fraction over $102^{\circ}$, and in the evening a fraction over $103^{\circ} \mathrm{Fahr}$. 'The highest point reached in any of these cases was a fraction over $105^{\circ}$. In typhus, as in other fevers, a fall of temperature not infrequently denotes approaching convaleseence, prior to any appreciable improvement in the pulse and other symptoms.

Urine.-The urea and uric acid are generally increased as in cases of typhoid fever. According to Murchison, the chlorides are greatly diminished or disappear entirely.

Albuminuria is of more frequent occurrence in typhus than in typhoid fever. Murchison found it in 20 of 28 cases, or a fraction over 71 per cent. In about one-half of the cases the amount of albumen was considerable. In most of the latter cases there was no evidence of pre-existing renal disease. In typhus, as in typhoid, fever, the early appearance of albumen in the urine, its abundance and duration, denote gravity of disease. Epithelial and blood-casts are sometimes observerl.

The duration of typhus is less than that of typhoid fever. Of $53 \mathrm{nn}$ complicated cases ending in recovery, analyzed by Murchison, the duration varied between 8 and 20 days, the mean duration being a fraction over 14 days. The mean duration in 10 fatal cases was 15 clays. Of 45 cases ending in recovery which $I$ have analyzed, the maximum duration was 26 and the minimum 9 days, the mean being 14 days. The mean duration in 9 fatal cases was 10 days. In my cases the commencenent of the disease was reckoned from the time of taking to the bed.

Relapses of typhus are extremely rare. 'They were, however, observed 
repeatedly at Bellevue in 1847-48 by Prof. Alonzo Clark. Examples of this fever having been experienced more than once are also very infrequent. There are no speeial sequels, and, in the great majority of cases, the health is excellent after recovery. Temporary loss of the hair is a common consequence of both typhoid and typhus fever. Mental imbecility sometimes remains for a certain period after the general condition of the body denotes recovery. Dr. Upham, of Boston, observed the frequent occurrenee of a dysenteric affection, during convulescenee, in cases occurring among Irish immigrants at the Boston Quarantine Hospital in $1847-48 .^{1}$

Typhus fever is sometimes associated with dysentery. Typhus and variola have been known to be combined. Probably typhus and typhoid fever may coexist.

Causation.-A striking point of contrast between typhus and typhoid fever relates to communicability. Diffusion by infection appears to be an exception to the rule in typhoid fever, whereas it is the rule in typhus fever. The contagiousness of typhus is shown by the successive oceurrence of cases in particular houses and neighborhoods, by the number who are attacked of persons brought into contact with typhus patients, espeeially in hospitals, either as physicians, attendants, or fellow-patients, and by the importation of the disease in localities where it did not previously exist. Referring the reader to works on fever for the evidence establishing conelusively the contagiousness of typhus, I shall simply subjoin certain illustrative facts falling within my own knowledge.

In 1850, '51, and '52 I was engaged in the elinical study of typhus and typhoid fever in the Buffalo Hospital of the Sisters of Charity. I recorded during these years sixty-five cases of typhus. Twelve Sisters of Charity were assigned to this hospital during the period just named. Of these twelve Sisters, five contracted typhus fever. These five Sisters alone had charge of the fever patients; the remaining seven Sisters, performing other duties, were not brought into contact with the fever patients. Thus, every Sister who nursed fever cases had an attack of the disease. During the last of these years, eleven patients, admitted for other affections, contracted the disease, the number of cases of typhus received during this year being considerably larger than during the two preceding years.

Typhus fever has prevailed in eertain parts of the city of New York, as an epidemic, from the summer of 1861 to the present date (1865). Prof. A. L. Loomis, one of the visiting physicians of Bellevue Hospital, in a paper on "The History of Typhus Fever, as it occurred at Bellevue Hospital, etc.," states that, for the two years prior to 1861 , not a single case of typhus in the hospital had fallen under his observation. In June and July, 1861, there were 21 cases, and from this time forward the number of cases increased, so that from Jan. 1, 1861, to Nov. 1, 1864, 1428 eases had been admitted, over 500 eases having been received during the six months preceding Nov. 1, 1864. As ehairman of a committee appointed by the Hospital Medical Board to investigate the origin of the disease, Prof. Loomis ascertained that the first case known to have occurred was of a child who had come to this country from Ireland two weeks before being taken ill. From this ease the disease extended over the tenement house in which the patient lived and an adjoining house, sixteen cases occurring in both houses within three months. During the follow-

1 Records of Maculated Typhus, or Ship Fever, etc., with plates, by J. B. Upham, M.D., New York, 1852. 
ing two years the cases received at Bellevue were mostly from the immediate neighborhood of the locality where the discase first appeared; and during the whole period this neighborhood furnished the highest number of cases.

Of 22 members of the house staff during the time above stated, 15 contracted the fever; of these 15,10 contracted the disease while on duty in the fever wards, 2 while taking care of a fellow-member of the staff' sick with fever, one while on cluty in the quarantine fever ward, one from a single case occurring in a general medical ward of which he had charge, and one was accustomed to visit daily the fever wards. Of jersons employed in the hospital as orderlies, watehers, etc., 16 were attacked with fever, and "in every case the dnties of those attacked brought them in direct contact with fever patients, or within the concentrated poison of the fever wards." From June, 1863, to June, 1864, 26 patients, admitted into the hospital for various affections, contracted the fever. Of these cases, "so far as the exact cireumstances attending the development of the disease in each could be reached, none sickened unless brought into direct contact either with the personal exhalations of a typhus patient or within the limits of the concentrated poison of the fever ward." "During this period, 175 cases were developed in the general medical wards within ten days after admission, and were transferred to the fever wards as soon as the nature of the disease was apparent." At length, " on the 17th of May, 1864, owing to the great increase in the number of typhus patients and the increasing mortality of those brouglit into contact with the disease, the Commissioners of Public Charities and Correction, upon the recommendation of the medical board through a special committee composed of Drs. Clark, Wood, Hamilton, and Flint, established tents on Blackwell's Island in which all the fever cases able to be removed from Bellevue were placed, and to which all fever cases thereafter admitted were sent."

The infectious miasm emanating from typhus patients rarely communicates the disease except to those who are in immediate proximity to patients. Concentration of the miasm is generally necessary. Hence, the disease is apt to be contracted by those who render personal attentions to patients, and especially by those who are exposed to the atmosphere of ill-ventilated fever-wards containing a large number of cases. A single patient in a spacious, well-ventilated apartment seldom communicates the disease. All persons at all times, moreover, are not equally susceptible, and, hence, some contract the disease after comparatively slight exposure, while others who are much exposed escape. There is no proof that the disease is diffused from one house to another, or from hospitals to arljoining houses, except by intercommunication. Facts show that the disease may be communicated by fomites; but a considerable impregnation of articles of clothing, etc., is necessary. Prof. Loomis states that of those in Bellevue Hospital who had charge of the clothing of the hundreds of typhus cases admitted into that institution, not one has contracted the disease. Exposure of infected clothing to a dry heat of $200^{\circ}$ Fahr., probably destroys the miasm. Some facts appear to show that the disease may be contracted by dissecting bodies dead with typhus; but this is not certain. The period of the disease in which it is most apt to be communicated is after the first week. Facts appear to show that it is liable to be communicated turing convalescence.

There is considerable variation in the estimation by different observers,

' Vide Bulletin of the New York Academy of Medicine, vol. ii., Nos. 21-24,1865. 
of the period of incubation. Probably this period varies considerably in different cases. Murehison has determined the average period to be about twelve days. Exeeptionally it is longer than this, but it rarely, if ever, exceeds three weeks. On the other hand, in many cases, it is less than twelve days, and occasionally the symptoms commence almost at the instant of exposure. Persons have sometimes had an impression that they reeeived the poison at a particular moment when they were conscious of a peculiar sickening odor inhaled from the body of a typhus patient. Little or no reliance is to be plaeed on this impression as an evidenee of the reception of the poison. Probably physicians have very many times experienced this sickening odor without having had typhus. Such has been repeatedly my experience.

As a rule, in this comtry, typhus ferer is an imported disease and diffused by contagion, using the latter term in its broad, popular sense, as applied to a miasm as well as a virus. It is, however, difficult to trace all outbreaks of the disease to eontagion, and, hence, the opinion is generally held that the special cause is sometimes of spontaneous origin. Facts appear to render it highly probable that the disease is sometimes developed as a eonsequence of overcrowding and deficient ventilation; in other words, that the concentrated emanations from the bodies of healthy persons may suffice for the generation of typhus miasm. ${ }^{2}$ The development of the disease in jails, hospitals, workhouses, ships, and unventilated tenement louses erammed with occupants, is thus accounted for, irrespeetive of contagion.

In 1841 I reported four cases of typhus developed in the Erie County almshonse. They occurred during the winter months. The wards in this institution were quite small and crowded, and, for the sake of warnth, fireplaces, which had previously been used, had been bricked up and close stoves substituted. There was an abundant eruption in all these cases, and an examination after death, in the only case which proved fatal, showed the absenee of the typhoid lesions. These cases seemed fairly attributable to overerowding and want of ventilation. ${ }^{3}$ No eases of typhus occurred ont of the almshouse, and the disease was not imported.

Overerowding, defieient ventilation, especially if conjoined with innutrition, are powerful anxiliary causes acting in conjunetion with eontagion. The causative influence of destitution and starvation is strikingly shown by the prevalence of typhus in Ireland in periods of famine, and by the large proportion of eases, among those received into hospitals, in which the deprivations and hardships of poverty have been experienced prior to the attack of ferer.

Typhus attacks the two sexes in about an equal proportion. Persons are more liable to be attacked after adult age, but children are not exempt, and this fever does not, like typhoid, spare those who are beyond the middle period of life. Of the eases admitted into the London Fever Hospital during ten years, 53.58 per cent. occurred at ages above 25 years." It is more likely to prevail in the winter and spring months than in autumn, in this respect differing from typhoid fever.

Diagnosis. - The remarks on the discrimination of typhoid fever from remittent fever and several local affections will apply to typhus fever.

I Vide St. Thomas's Hospital Reports, Vol. II.

2 For facts bearing on this point, vide Murchison, op. cit.

Vide Boston Med. and Surg. Journal, June, 1841.

- Anstie on Epidemics. 
The discrimination of typhoid and typhus fever is to be here noticed. The following are the more important points in this differential diagnosis: The relatively long duration of the forming stage in typhoid, and its short duration in typhus. Absence of the characteristic abdominal symptoms of typhoid, in typhus, viz., diarrhcea, meteorism, iliac tenderness and gurgling, or their presence in comparatively a slight degree. The dusky or dingy hue of the surface in typhus. The appearance of the eruption earlier in typhus. The different characters of the eruption, viz., in typhoid, papular, rose-colored, the reduess disappearing on pressure; in typhus, after two or three days, if not at first, maculated, the color dark red, and the redness not disappearing on pressure. Other distinctive points relate to the eruption, viz., the sparseness in typhoid, and the copiousness in typhus; the frequent extension, in the latter, of the eruption over the extremities; the persistence of the spots in typhus, and the disappearance of papules with the production of fresh papules in typhoid. It is to be borne in mind that in some cases of typhus, rose papules are intermingled with the characteristic maculæ.

Corroborative diagnostic points are, the occurrence of peritonitis and hemorrhage from the bowels in some cases of typhoid, and the earlier occurrence of delirium, coma-vigil, and other ataxic symptoms in typhus.

If the eruption be present, typhus is generally recognized without difficulty. The eruption is not always present, and the diagnosis may then involve delay and difficulty. The prevalenec of typhus and exposure to contagion are to be taken into account in doubtful cases.

Prognosis.-Of 18,592 cases aggregated by Murchison, occurring at the London Fever Hospital in 14 years, at King's College Hospital, the Edinburgh Infirmary, and at Glasgow, Scotland, the mortality was 18.78 per cent., or 1 in 5.27. This rate of mortality differs only by a small fraction from the rate in about the same number of cases of typhoid fever. ${ }^{2}$ The death-rate is found to vary, at different times and places, between 9 and 25 per cent., the variations being due to differences as regards an intrinsic tendency of the disease to a fatal result. In epidemies of typhus, as of other diseases, it has been often observed that the fatality is greatest at first, and decreases as the number of cases diminish. The ratio of fatality is greater among males than females. The fatality is less in early life than subsequently. It is greater under 10 years of age than between 10 and 20 years. After 30 years the fatality progressively inereases, and after 50 years the proportion of deaths is nearly one-half.

Of the 63 cases which $I$ have analyzed, 12 were fatal, that is, 1 in a fraction over 5 , or a fraction over 18 per cent.

A fatal result is oftener due to the intensity of the discase, that is, occurring irrespective of complications, in typhus than in typhoid fever. I'eritonitis, which destroys a certain proportion of patients with typhoid fever, does not occur in typhus. This is true, also, with respect to intestinal hemorrhage. Pneumonitis is less frequent in typhus, and there are no complications peculiar to the latter. Uræmic coma, and sometimes convulsions, both generally followed by death, are as liable to oceur in typhus as in typhoid fever. A fatal result appears to be oftener attributable to feebleness of constitution, or to causes which impair the power of resisting the disease, in typhus than in typhoid ferer. Of 38 cases which, within the past three years, have occuired among the members of the resident medical staff and persons employed in Bcllevue Hospital, 17 
have ended fatally, whereas, of 1428 patients admitted with fever during the same period, the number of deaths was 243 , or 1 in 5.87. This difference in the mortality in the two classes of cases is the more remarkable because the resident physicians and persons employed in the hospital were treated for the disease under more favorable circumstances than the hospital patients. It is difficult to account for the greater fatality among the former elass, except by supposing that living in the hospital involved exposure to nosocomial depressing influences which rendered the system less able to resist the disease.

In general, the symptoms pointing, on the one hand, to a favorable, and, on the other hand, to an unfavorable prognosis, are the same in both typhoid and typhus fever.

\section{Non-identity of Typhus and Typhoid Fever.}

Discussion of the reasons for considering typhus and typhoid fever as distinct diseases is less called for now than heretofore, because, at the present time, very few hold to the opinion that they are identical. It will suffice to state the more important of the facts on which the doctrine of their non-identity is based.

1. Typhoid fever is characterized by peculiar and remarkable abdominal lesions which are not found in cases of typhus. The difference between a fever with and a fever without these lesions is hardly less striking than the difference between a fever with and a fever without the cutaneous lesions, that is, the eruption, characteristic of smallpox. 'The abdominal lesions of typhoid fever are of so special a character as, in themselves, to constitute a valid claim for the individuality of the disease.

2. The events of the clinical history in the two diseases show points of contrast which denote the distinct individuality of each disease. The more striking of these points of contrast relate to the abdominal symptoms and the eruption. The characters of the eruption, alone, suffice to show that the diseases are not identical. The eruption in each disease belongs to a different class, viz., in typhoid to the papula, and in typhus to the maculæ. The difference is nearly as great as between the eruption of rubeola and that of scarlatina; and it is worthy of note that the two diseases just named have been considered as identical within the past century.

3. There is reason to believe that typhus and typhoid fever have each its own special cause or causes, that is, a cause or causes which will not produce the other disease. Jenner traced the origin of cases received into the London Fever Hospital during two suecessive years (1848 and 1849 ), in order to determine whether two or more cases coming from the same habitation afforded examples of the same kind of fever or of different fevers. Forty-four localities in 1848 furnished 101 eases of typhus, and one only of these houses furnished a case of typhoid fever. Eighteen localities in 1849 furnished 51 cases of typhus, and none of these houses furnished a case of typhoid fever. During these years nine localities furnished 19 cases of typhoid fever, and these houses furnished only one case of typhus. Similar investigations pursued by Murchison, Gairdner, Peacock, Wilks, and others, have led to similar results, showing that the two fevers have no community of origin.

4. Neither typhus nor typhoid fever, as a rule, is experienced twice, but neither exempts from the other. Patients admitted with typhoid fever into hospital fever-wards containing cases of typhus are liable to 
contract the latter, and pass successively through both diseases. Several examples of this kind liave come under my observation.

5. Certain laws with respect to cansation go to show their non-identity. Typhus is chiefly diffused by contagion, typhoid is rarely communicated. Typhoid fever is indigenous in many regions where typhns is very rarely, if ever, generated. After 50 years of age the susceptibility to the typhoid poison is almost nil, whereas typhus is often contracted after this age. Typhus prevails as an epidemic, but typhoid is usually an endemic disease.

\section{CHAPTER III.}

Treatment of Typhns and Typhoid Fever. Relapsing Fever-Anatomical Characters-Clinical History - Causation - Diagnosis-Prognosis - Treatment. Erysipeiatous Fever. Epidemic Fever characterized by mild Erythematic Pharyngitis.

TIIE general principles of treatment in typhoid and typhus fever are essentially similar, and, indeed, are applicable to all the essential fevers. It will suffice to notice, incidentally, indications pertaining exclusively either to typhus or typhoid fever, without considering the treatment of each fever separately.

It must be admitted that the known resources of therapentics do not afford reliable means for the arrest of these fevers, nor even for shortening the duration of the febrile career. Measures proposed for these ends within late years are, quinia in large doses, full doses of opium, and the use of the wet sheet after the hydropathic method. The first of these, viz., large doses of quinia, has been abundantly tried, and found to be unsuccessful as an abortive plan of treatment. The opiate plan I have tried in a few cases. These cases have not furnished evidence of success in arresting the fever, but in some of the cases the disease appeared to be favorably modified. This plan of treatment claims further trial. The wet sheet-after the mode practised by the hydropathists, commonly called packing-I have tried in a small number of cases. A distirict amelioration of the symptoms followed immediately in every case, and in two cases immerliate cessation of the fever followed. As an effective measure for diminishing heat, reducing the frequency of the pulse, tranquillizing the ' nervous system, and, perhaps, promoting elimination by means of copious perspiration, water applied in this mode claims a fair trial at the hands of physicians in typhoid and typhus fever as well as in other affections. There is room for the supposition that in my two cases just referred to the disease was either febricula, or spontaneously aborting typhoid fever. Emetics were thought to be sometimes suecessful in either arresting or affecting favorably the progress of typhoid fever by the late James Jackson. Ir. T. K. Chambers advocates them both as abortive and curative. It is claimed by Wunderlich, Pfeuffer and Niemeyer, that a full dose of calomel, repeated several times during the first wcek, will, in a small proportion of eases, render the disease abortive. There is ground for the belief that in typhoid and typhus fever, as well as in the other essential fevers, and, indeed, in all diseases characterized by a continuous and considerable increase of the temperature of the body, the gravity of symp- 
toms, certain of the lesions, and the danger are due directly, in a greater or less degree, to the calorification. In so far as this is true, antipyretic measures of treatment are rationally indicated; moreover, clinical experience furnishes abundant evidence of their value. Foremost among these measures is the use of cold water, either in the form of affusion, as advocated by Currie nearly a century ago, the cold bath, the wet sheet, or sponging the surface of the body. Refrigeration by one of these methods is inclicated whenever the thermometer shows a temperature of $103^{\circ}$ or upwards. The mildest mode of refrigeration is that last mentioned, namely, sponging the body with cool or tej,id water, to which may be added with advantage either citric acid or alcohol. Repeated once, twice, or oftener, daily, the temperature is somewhat lessened, and a corresponding improvement in the symptoms is manifested. The other modes of refrigeration are more effective; and they are indicated in proportion as the temperature is higher than that just stated. Affusion and the cold bath involve often inconvenience, as well as, on the part of the patient, fatigue, and generally a suflicient effect may be procured by the wet sheet, which recommends itself by its freelom from the objections to the two modes just named. A wet sheet, spread over India-rubber cloth, with woolen blankets beneath, may be placed either upon a eot or on the flcor; upon this the patient, with the clothing removed, is laid, and the body enveloped in the sheet. As the latter acquires heat from the body, it may be sprinkled with cold water. After remaining in the sheet for a period varying from a few moments to half an hour, having wiped the body with dry towels, the patient is removed to the bed, and covered with the bed-elothes. In a slort time, the patient may be dressed as before the bath. If the circulation be unduly depressed, as shown by feebleness of the pulse, a stimulant may be given-wine or spirit-before, or direetly after, the patient is removed to the bed: 'The temperature of the body is invariably diminished either a degree, or two degrees, or even more, by this measure, and it may be repeated as often as the temperature again rises. It is a measure devoid of risk of harm; and the immediate good effect is shown by general improvement as regards symptoms other than the temperature. Statisties cited by Liebermerster appear to show a consiclerable reduction of the death-rate by the cold water treatment in conjunction with antipyretic remedies.'

As accessory to the use of cold water, there are certain remedies which are useful by diminishing the temperature of the boly, namely, quinia, digitalis, and the veratrum viride. The two latter diminish, at the same time, the frequency of the heart's action, and, of the two, the veratrum viride has especially this effeet. Given for an antipyretic effect, the dose of quinia must be large-from 15 to 25 grains for an adult. Aside from some risk of producing disturbance of the stomach, no harm can arise in making trial of this remedy with a view to its effect on the temperature. Digitalis may be given for this effect without any risk of doing harm. The veratrum viride, if used for this purpose, should be given very circumspectly in order to avoid risk of causing niusea and general prostration.

The mineral acids are considered as highly useful by Huss, Chambers, Richardson, Murchison, and others. The dilute phosphoric acid is preferred by Huss, ten or fifteen minims given hourly, or every second hour. Chambers and Richardson prefer the dilute hydrochloric acid, a fluidounce to be taken daily. Murchison combines the hydrochloric and the

1 Vide über die Behanflung des Fiebers, von C. Liebermerster, Sammlung Klinischer Vorträge herausgegeben von Richard Volkman, No. 31, Leipzlg, $18 \% 1$. 
nitric acid, giving 20 minims of the former witl ten of the latter every three hours. The supposed curative efficacy of these mineral acids has been accounter for on the supposition that the blood is in a state of superalkalinity. I have employed the dilute sulphuric acid largely in the treatment of typhus at the Blackwell's Island and Bellevue Hospitals, and to some extent in cases in private practice, and $I$ can bear testimony to its apparent utility. With a view to determine whether this acid exerted a curative influence, Dr. Irving W. Lyon, then a member of the resident medical staff, instituted, under my direetion, a comparison of the rate of mortality in the male fever-ward at Bellevue Hospital during six months without, and nearly six months with, the use of the acid. Eliminating all the cases in which death took place within 48 hours after admission, from January 1 to July 1, 1863, 70 cases of fever were treated in this ward withont acil, and with 14 deaths, the rate being 20 per cent., or 1 in a fraction over 5 , which is about the average fatality in both typhus and typhoid fever. From July 1, 1863, to January 1, 1864, 78 cases of fever were treated in this ward with acid and with 8 deaths, the rate being 10.25 per cent., or 1 in a fraction over 9.' 'The treatment in these two collections of cases was essentially the same, aside from the acid, consisting chicfly in the use of alcoholic stimulants according to the indications in individual cases. 'There was no reason to believe that the disease was more severe during the first than during the second six months. The reduction of the fatality nearly one-half scemed fairly attributable to the use of the acid. The mineral acids sufficiently diluted, with the addition of simple syrup or the syrup of orange-peel, are generally reished by fever patients.

Ir. Chambers, in his "Lectures Chiefly Clinieal," gives statisties illustrative of the results of the acid treatment in typhoirl fever as follows: 'Two hundred and thirty eases treated at St. Mary's Iospital were an:lyzed. Of these the first 109 cases were treated with nentral salines, chalk, and mereury, in the early part of the disease; at a later period, with bark, ammonia, ether, and wine; leeching and cupping being sonetimes employed, and food given foul times daily. The remaining 121 cases were treated with strong beef-tea and milk given freely, and twenty drops of diluted nitro-hydrochloric acid every two hours. The average mortality in the first groul) of cases was 21 in 109 , or 1 in 5 , or $19 \frac{1}{2}$ per eent. In the second gronp the fatality was only 3 in 121 , or 1 in 40 , or $2 \frac{1}{2}$ per cent. Dr. Henderson, of Shanghai, gives a reduetion of the mortality from 28 to 7 per cent., by the acid treatment. Dr. Cotting, of Boston, out of 307 cases treated without drugs of any kind, lost 31 , or 10 per cent. ${ }^{2}$

Prof. George B. Wood attributes a curative influence to the oil of turpentine in typhoid fever. He supposes that this remedy exerts its curative influence upon the intestinal uleerations. He recommends it in the strongest terms in the latter part of typhoid fever, and especially when the tongue is dry and the abdomina symptoms marked. It is to be given in doses varying from 5 to 20 drops, in mueilage, repeated every lour or two. It is to be gradually diminished during convalescenee.

'The sulphites introduced by Prof. Polli as exerting a curative influence in all the diseases considered as zymotic, have been employed sufficiently to show that they do not arrest the course of these fevers. Whether they mitigate the intensity of the disease, or possess any prophylactic power,

I Vide American Medical Times, February, 1864.

2 London Lancet, May, $18 . i 6$. 
remains to be determined by further clinical observation ; at the present time (1873) the opinions of practitioners differ, the majority, perhaps, finding little or no evidence of their utility. The carbolic acid has been used, as an antiseptic remedy, to some extent, but without any effect going to show its utility.'

Asirle from abortive and direct curative measures, indications are derived from particular symptoms or events and complications in individual cases. The more important of these indications are to be noticed.

In the early part of the disease cephalalgia claims palliative measures. This symptom is relieved by ice-water, spirit and water, distilled vinegar, or cologne water freely applied to the head, and, if more efficient measures be required, the cold donche or ice-cap may be employed. As soon as the existence of either typhus or typhoid fever is declared, the hair should be closely cut. This renders the patient more comfortable, and facilitates local applications.

Vigilance is a symptom calling for treatment. To procure sleep is desirable, not only for immediate comfort, but as a means of averting ataxic symptoms. A full opiate will often prove beneficial. An opiate sometimes procures refreshing sleep in the place of pseudo-somnolence, or coma-vigil. The latter condition, not denoting a tendency to true coma, does not contra-indicate the trial of opium.

Delirium, if slight or moderate, does not call for remedies; but if prominent as a symptom, as shown by constant talking and attempts to get out of bed, it leads to other ataxic symptoms and to exhaustion. Opium, in some form, and other anodynes, are indicated. If these fail, the combination of antimony, in small doses, with opium, as recommended by Graves, will often prove efficacious. An eighth or a sixteenth of a grain of the-tartrate of antimony and potassa may be given hourly or half-hourly until the patient becomes quiet, unless nausea or vomiting occur, when it is to be at once suspended. 'The bromides, in some cases, have a tranquillizing effect. The treatment for delirium is generally called for during the night-time.

Dr. Mowry has reported a case of typhoid fever, characterized by active delirium, in which digitalis given in large-doses appeared to have an extremely salutary effect. ${ }^{2}$ Half-drachm doses of the tincture were given hourly for several successive hours, the patient taking about two ounces in thirty hours. The remedy produced no bad effects. The inquiry suggests itself whether even larger doses of the remedy may not be safe and useful in the delirium of fevers, as they have been found to be in cases of clelirium tremens. ${ }^{3}$ The hydrate of chloral may be tried in cases characterized by persistent, violent delirium.

Nausea and vomiting seldom occur spontaneously in typhoid or typhus ferer to an extent to call for more than regulation of the ingesta. Diarrhœa in typhoid fever may require to be restrained, and, for this end, opiates and astringents, given by the mouth or rectum, may be employed. Mere looseness of the evacuations, without undue frequency, does not call for treatment. On the other hand, in typhus, and sometimes in typhoid fever, constipation may furnish an indication for treatment. As a rule, cathartics, or even laxative remedies, are not required for constipation; simple enemata suffice, and are to be preferred. Evacnations may be delayed for two or three days, or even longer, without injury, if there

1 Vide Samson on the Artiseptic System.

2 American Journal of Medical Sciences, April, 1865.

3 Vide Treatment of Delirium Tremens. 
be no evidence of discomfort, and the abdomen be not distended. Tympanites, if great or consirlerible, occasions discomfort, and embarrasses respiration by interference with the descent of the diaphragm. Large enemata may suffice to relieve this symptom, depending, as it does, chiefly on acenmulation of gas in the lirge intestine. Turpentine should enter into the enemata. If these fail, a saline laxative may be given. A stomach-tube introduced into the rectum, and carried as fur as practicable, sometimes occasions the discharge of gas in considerable quantity. Turpentine, given by the mouth, is supposed to relieve this symptom. 'l'urpentine stupes to the abdomen are useful. Mechanical compression, by means of a band applied over the abdomen, sometimes aflords rilief. Compresses applied over the abdomen and kept wet with cold water, in cases of typhoid fever, are highly recommended by Iluss.

'Two important events connected with the abdominal lesions are liable to occur in cases of typhoid fever, viz., perforation of intestine followed by peritonitis, and intestinal hemorrhage. The probability of the successful treatment of the former is almost nil. The small chance of success depends on the free use of opium and measures to support the powers of life. Local depletion and blisters are not admissible. Peritonitis not dependent on perforation claims the same treatment, and is not so hopeless. Intestinal hemorrhage, if profuse, involves a certain amount of danger. This event calls for astringent remedies, given in conjunction with opiates, by the mouth or rectum, such as tamnic acid, the acetate of lead, and the persulphate or pernitrate of iron. Cold applications may be made to the abdomen. IRigid quictude is to be enforced.

Cough, although generally existing, is very rarely sufficiently troublesome to require palliation. Pneumonitis should not be treated with local or general depletion, blisters, or mercury, nor with nauseant sedatives given to the extent of producing vomiting or nausea. This, as well as other serious complications, contra-indicates depressing measures. Epistaxis is sometimes so profuse or persisting as to indlicate astringent remedies, or even plugging of the anterior and posterior nares.

The pulse, in the early part of the disease, is sometimes moderately accelerated and strong. These characters of the pulse, however, should not lead to the employment of bloodletting. Notable frequency and feebleness of the pulse will be presently referred to as denoting the propriety of stimulants. The veratrum viride is of doubtful propriety under these circumstances. Diaphoreties are of doubtful utility; clinical observation shows their operation to be uncertain, and, if diaphoresis be produced, it does not essentially modify the course of the disease. The importance of producing diaphoresis has relation chiefly to the elimination of urea.

Important indications may be derived from examinations of the urine, especially with reference to urea. If urea be notably deficient, uramia is to be apprehended and, if possible, forestalled. If, with deficient elimination of urea, somnolency, coma, and other ataxic symptoms be present, they are to be attributed to uræmia. The existence of uræmia is confirmed by an abundanee of ammonia in the expired breath. Deficiency of urea in the urine indicates the importance of diuretic remedies, and, under these circumstances, if the kidneys do not respond, it may be important to produce diaphoresis. If uræmic phenomena become developed, the hot-air bath is to be employed until free diaphoresis is obtained. Somnolency and coma, in addition to measures for the elimination of urea, call for sinapisms on various parts and vesication of the nucha. 
Strong coffee and tea, under these circumstances, are useful. Retention of urime is an event liable to occur, and is to be provided against.

Hygienic and supporting measures belong among the most important part of the treatment of typhoid and typhus fever. Hygienic measures indicated are, complete ventilation, cleanliness, regulation of temperature, which should not be allowed to rise above $60^{\circ}$ Fahr., changing frequently the position of the body, and the varied attentions embraced under the head of good nursing.

Foremost among the hygienic conditions for passing safely through the disease is an abundance of pure air. In private practice, the sick room should be large, and ventilated as completely as possible. In hospitals, the fever-wards, in addition to ample provisions for ventilation, should not be crowded; 1500 culvic fect should be allowed to each bed. The evacuations should always be instantly removed. When practicable, it is advisable that the bed and body linen should be changed daily.

The importance of air is shown by the reduced rate of mortality when patients are treated in sheds and tents, as compared with the death-rate in even well-ventilated hospital wards. Striking evidence of this was afforded by the transfer of fever-cases (mostly typhus) from Bellevue Hospital to tents on Blackwell's Island. This course was adopted, in accordance with the recommendation of the Hospital Medical Board, in May, 1864, and a large number of fever-cases were treated in tents during the following winter, as well as the summer and autumnal months. A comparison of the mortality in 520 cases treated in tents from May 17 to November 1, 1864, with the average mortality in the Bellevue Hospital wards, shows a marked difference. The average death-rate in the hospital wards was 1 in 5.97 ; in the tents, 1 in 16.77 , excluding the cases in which death took place within 48 hours after admission. Nearly all the eases in the tents were treated without alcoholic stimulants, and mostly with little or no medication.' Making the fullest allowance for the conjecture that the cases in the tents were milder than those in the hospital wards, and for difference of treatment as regards the use of stimulants, it can hardly be doubted that the superior ventilation in the tents was the means of saving many lives.

In a paper communicated to the New York Academy of Medicine, in June, 1853, by Dr. John H. Griscom, an account is given of 82 cases of typhus transferred from an emigrant ship at Perth $\Lambda$ mboy, N. J., to wooden shanties with sail " roofs." The facts were communicated to Dr. Griscom by Dr. Charles M. Smith. They occurred in 1837. The emigrant ship brought over between three and four hundred passengers, and a number had clied on the passage. There being no hospital nor other accomodations in the town, two shanties were erected, "thirty feet long and twenty feet wide, boarded on three sides about four feet up, and over them old sails were stretched." Eighty-two fever patients were transferred to these shanties, of whom twelve were in a state of insensibility when removed. On the night after their removal there was a violent thundergust accompanied by torrents of rain, and the following moruing the clothes of all were saturated with water. The medical treatment consisted " of an occasional laxative or enema, vegetable acids, and bitters were liberally administered, together with the free use of cold water, buttermilk, and animal broths." Four sailors who sickened after the arrival of the vessel were removed to an ordinary dwelling-house, and, of these

1 Vide papers by Prof. A. L. Loomis, already referred to, page 892. 
cases, two proved fatal. Of the 82 cases treated in the shanties, not one proved fatal.'

Cold water, or iced water, or barley water acidulated with lemon or orange juice or some other vegetable acid, Gr carbonated water should be freely given, care being taken that the quantity taken at a time be not suflicient to incommode the stomach.

'The importance of support is based on the plain fact that, typhus and typhoid fever being self-limited diseases, if the patient can be kept alive, after three, four, or more weeks recovery must take place provided there be no serious complication. In a case of severe uncomplicated fever, the patient is in a situation not unlike that of a person in danger of drowning not far from, or perhaps very near, the shore; if he drown, it is because his strength gives way before the shore is reached. As a person in this situation requires only to be broyed up by some support, so the fever patient, in a similar emergency, may only need supporting measures to live until the disease ends. Death may take place at a juncture when, could it have been averted a few days, or, perchance, even a single day, the period of danger might have passed.

Supporting measures embrace tonic remedies, alcoholics, and alimentation, more especially the two latter. Of tonic remedies, the most eflicient and convenient of administration is quinia, which may be given in doses of from one to three grains twice or thrice daily.

Alimentation ranks first in importance. It is desirable for the patient to take as much nutritious food as will be digested and assimilated. Inmutrition in cases of fever, as of other affections, enhances and adds to the morbid phenomena belonging to the disease. In the early part of the disease there is generully a repugnance of food, and afterward, owing to the mental state and the condition of the month, taste and appetite are wanting. Under the latter circumstances food is to be given although not desired by the patient, and even when he is disinclined to take it. 'The indifference to food may proceed, measurably, from an unwillingness to be disturbed and a reluctance to make any excrtion.

The supporting diet should be in a liquid form, and embracing the necessary variety of alimentary principles. 'The animal broths or essences, eggs and milk, with the addition of some farinaceous form of food, fulfil these requirements. Different articles should be given in alternation, and at regular intervals. The intervals should be from two to four hours. It is injudicious to give food every half hour or hour, or at even shorter intervals, as is not infrequently done. The amount of food given at a time must vary according to circumstances, the object being to give as much as can be given without risk of either vomiting, discomfort from over-accumulation in the stomach, or indigestion. If the patient be in a condition to feel and express a desire or choice for particular kinds of food, the preference should, as far as practicable, be consulted. 'The physician should have an oversight of the preparation of the articles of food, lest, through ignorance or carelessness, they be unpalatable or innutritious. Precise directions and attention to the details of dietetics form an important part of the physician's duty, not only in fevers, but in all cases of disease in which supporting treatment is indicated. Of the different articles of food just named, milk is by far the most valuable as entering into the fever diet. It is taken, if not with relish, at least with less reluctance than the other articles; and it has the great advantage of embracing, in proper combination, all the alimentary principles required for nutrition.

1 Transactions of the New York Academy of NIedicine, vol. i. Part. ii., 1853. 
The "beef tea," as it is often prepared, is little more than warm water flavored with the meat. 'The different so-called meat extracts contain but little nutriment. It is clained, indeed, that they are injurious from the amount of excrementitious principles entering into them. Prepared in any mode, and as carefully us possible, the essence of meat or broths are vastly inferior, in nutritive value, to inilk. They should, therefore, never be relied upon to the exclusion of the latter. If the same article of animal diet, for example, beef essence, be given day after day, the patient acquires for it, after a time, an intolerable disgust. The form should, therefore, be varied, beef essence, mutton broth, and chicken soup being given in alternation. It is desirable that from one to two quarts of milk per diem should be ingested.

'The importance of " feeding fevers" was enforced especially by Graves, whose writings had no small agency in leading practitioners of all countries to avoid the danger of innutrition. It is, however, but justice to say that there were practitioners in this country who appreciated, and exemplified in their practice, the dictetic management as advocated by Graves, prior to his most valuable clinical teachings.

Alcoholic stimulants have entered largely into the treatment of fevers in this country during the last thirty years. 'That they have been used too freely and indiscriminately can hardly be cloubted. As a natural consequence, there is perhaps at the present moment a tendency to undervalue their importance. Observation shows that under the free ventilation afforded by tents, the rate of mortality from typlus in cases treated without alcoholic stimulants is small. In the valuable report of Prof. Loomis, already referred to, it is stated that, of 520 cases treated in tents on Blackwell's Island, alcoholic stimulants were given in only a few instances, and the mortality, deducting the deaths within forty-eight hours, was, as already stated, 1 in 16.77. It does not, however, follow that alcoholic stimulants are never useful because, under most favorable hygienic conditions, the rate of mortality is small in a series of cases in which alcoholic stimulants were not used. Observation of the immediate effects of alcoholic stimulants in certain cases shows their utility often in a very striking manner. Indiscriminately and excessively used, they are not useful, but used with proper application and moderation, they form an important part of the supporting treatment of fevers, as well as in cases of all other diseases which destroy life by asthenia.

The supporting effect of alcoholic stimulants is directed chiefly to the circulation. Hence the indications respecting their use are derived especially from symptoms relating to the heart. Feebleness of the circulation, as denoted by the pulse and the heart-sounds, calls for the use of stimulants, and they are to be given, as regards quantity, in proportion to the degree of feebleness of the circulation, and according to their effect. The characters of the pulse denoting feebleness of the heart's action are smallness and compressibility, frequently conjoined with considerable or great frequency. A pulse in the adult exceeding 120 per minute never denotes strength, and, above this number, it is feeble in proportion to frequency. Above 130, the pulse always denotes a degree of feebleness of the circulation rendering supporting measures highly important. The heart-sounds also constitute a good criterion for estimating the degree of feebleness of the circulation. In proportion as the action of the heart is weakened, the intensity of the first sound over the apex is diminished; it is shortened and in quality resembles the second sound. If the heart be notably weakened, the first sound may be inappreciable over the apex. Stokes was the first 
to direct attention to these changes as criteria for the administration of alcoholie stimulants.

Aleoholic stimulants are rarely indicated in the early part of either typhus or typhoid fever. They are oftener and earlier indlicated in typhus than in typhoid fever. Brandy, or some form of spirit, or wine, sherry or Madeira, may be employed. Commeneing with a moderate quantity, half an ounce of spirits or an ounce of wine, the effect on the eirculation and other symptoms should be watehed. A good effect is shown by inereased force, with perhaps diminished frequency, of the pulse, perhaps some reduction of the temperature of the body, diminution of delirium, etc. 'The intervals should vary, according to the urgeney with which this supporting measure is indicated, between six or eight hours and each successive hour. The quantity given at a time should rarely exceed that just statel. Guided by the true indications and by the effect, alcoholic stimulants will not do harm, and will often do mueh good in the treatment of typhus and typhoid fever. I have repeatedly tried their suspension for a few hours in enses in which they appeared to be urgently indicated, in order to become fully satisfied of their ntility, and luave found the pulse become more frequent, with increase of delirium and other ataxic symptoms, until their use was resumed. While a eertain proportion of cases do well without them, and they are, therefore, not always inclicated, I eannot doubt that $I$ have seen cases in which life was saved by their employment. Wine or spirit may often be combined with nourishment with advantage, for example, in the form of wine-whey, milk-punch, eggnog, etc.

In these fevers, as in some other dliseases, the tolerance of alcohol is notably greater than in health. A patient may take from one to two pints of spirit per day who, in health, could not have taken one-fourth of this quantity without ineloriation. The ordinary effects of alcohol in health should never be produced by their use in eases of fever. If these effeets be produced, the indications for alcohol are not present or it is too largely given. In extreme cases an ounce, or even more, of spirit, given hourly, may be neeessary, but, in the majority of the eases in which alcoholie stimulants are indieated, not more than six or eight oumees in the twenty-four hours are needed. The observations of Ringer and Richards show a slight diminution of temperature in febrile diseases under the free use of alcohol.'

During convaleseenee, alcoholic stimulants should always be given moderately. If they have been used freely during the career of the fever, when convalescence begins their exeitant effeet may be for the first time apparent. Frequently malt liquors or light wine are best suited to convalescence. Solid food should be allowed as soon as convalescence is declared. The processes of digestion and assimilation are usually very active, and the appetite is apt to be voracious. The cantions to be observed relate to the kinds of food and the quantity taken at one time. The patient should be restrieted to plain, wholesome, well-eooked articles of diet, and excess may be avoided by taking food at shorter intervals than in health. Convalescence from typhoid, more than from typhus fever, requires care as regards diet, in view of the probable existence of intestinal uleerations. Gestation in the open air may be allowed early in the stage of convalescence, and, as soon as their strength will permit, patients may walk out of doors. Care with respect to much muscular

I Braithwaite's Retrospect, Part 1, IV., page 31. 
exertion is important in convalescence from typhoid fever, in consequence of a liability to perforation from rupture of the intestine.

The prevention of typhus fever, except among those who are necessarily exposed to infection in rendering medical and other aid to patients in fever wards or hospitals, is attainable by proper hygienic reforms. If overcrowding be prevented, and free ventilation secured, the fever miasm is not generated, nor is the disease likely to be communicated. One of the great objects to be accomplished by improved sanitary regulations, especially in large towns, is the prevention of typhus. There is no evidence that the special cause of typhoid fever is generated by overcrowding or defective ventilation, but these may act as auxiliary causes rendering the system more susceptible to the typhoid miasm, and they may contribute to the diffusion of the disease by infection. In view of the risk of the diffision of the disease, patients with typhus should not be treated in hospital wards with other cases. This statement does not apply to patients affected with typhoid fever; the risk of the diffusion of the latter among other patients, provided wards are well ventilated, is extremely small. The custom of transferring to ferer wards with cases of typhus, patients affected with typhoid fever, is objectionable in view of the risk of the latter contracting typhus during convalescence. Repeated instances in which this has occurred have fallen under my observation.

\section{Relapsing Fever.}

A form of continued fever, heretofore known by a variety of names, but now called relapsing fever, received very little attention in this country prior to 1870 . It has never been indigenous in this country. In June, 1844, fifteen cases were admitted into the Philadelphia Hospital presenting the characters of relapsing fever, this name, however, at that time not being in use. These were, probably, the first cases reported in this country. The patients were Irish immigrants, all coming over in the same vessel. The cases were observed by Dr. Meredith Clymer, and were reported in the work on fever by this author. ${ }^{1}$ In addition to these so far as I know, the only cases observed are a few reported by Dr. A. Dubois, in $1848,{ }^{2}$ and fifteen cases which came under my observation in 1850-51, an account of which is contained in my "Clinical Reports on Continued Fever. ${ }^{3}$ The cases reported by Dr. Dubois and by me were among recent Irish immigrants. It is highly probable that other causes have occurred among immigrants, which have not been reported, and which may have been confounded with cases of typhus and typhoid fever. In the winter of 1869-70 the disease was imported by forcign immigrants, and it prevailed, to a considerable extent, in New York, during this winter and the following summer. It prevailed, also, to some extent, in other of the large cities of this country. At this time, the wards of Bellevue Hospital afforded an ample opportunity for the clinical study of it; and my observations were the basis of a lecture published in the spring of 1870 , to which was appended a statistical report of 103 cases, prepared, at my request, by Dr. Thomas J. Moore, then one of the hquse physicians of the hospital. ${ }^{4}$

I Fevers; their Diagnosis, Pathology, and Treatment. By Meredith Clymer, M.D., Prof. of the Principles and Practice of Medicine in the Franklin Medical College of Philadelphia, etc. etc. Philadelphia : Lea \& Blanchard, 1846. Page 99.

2 Vide Transactions of American Medical Association, Vol. II.

3 Vide Clinical Reports on Continued Fever based on analyses of 164 cases, by Austin Flint, M. D., 18\%?.

- Vide New York Medical Journal, March, 18 ro. 
It is not long since the distinctive features of relapsing fever have been pointed out, and its claims to be considered as a distinct species of fever established. It is by no means, however, a new disease, and there is evidence of its having existed from a very early period in the history of medicine. In modern times it has prevailed, at diflerent epochs, more especially in Ireland, Scotland, and England. On the contincnt of Europe it is rare. It prevailed as an epidemic in Upper Silesia, a province of Prussia, in 1847, and among the troops in the C'rimea during the late war of France and England with Russia. Its claims to be considered as a distinct species of fever rest on the following facts: It does not present either the anatomical characters or the diagnostic events of typhoid fever, and, as regards its non-identity with typhus, the two diseases are separately communicable, but neither communicates the other, nor cloes the occurrence of either secure an immunity from the other; each disease appears to have its own special cause. Kelapsing and typhus fever, however, are apt to prevail together. Among the cliflerent names heretofore applied to the disease are the following: "Five day fever," "seven day fever," "short fever," "mild yellow fever," "famine fever," "liungerpest." The name relapsing fever is based on one of the most striking of the peculiarities of the disease, and involves no hypothesis; it is, therefore, to be preferred to any of the other appellations.

Anatomical Characters. - There are no distinctive morbid appearances found in the rare cases in which the disease proves fatal. Lesions which may exist are due either to pre-existing disease or to complications. The spleen is enlarged and softened, even to a greater extent than in cases of typhoid fever, but this is not a distinctive lesion. Ecchymoses within the stomach have been observed in cases characterized by hamatemesis or black vomit.

Crinical History.-Under this head, I shall state suceinctly the symptomatic features which are characteristic of this fever. 'These relate to two or more successive paroxysms of fever, separated by an apyrexial period or intermission. The recurrence of the paroxysm of fever, or the relapse, is the distinetive feature whence the name of the disease derives its significance.

Abruptness of invasion characterizes the disease. The attack is sudden. There is no prodromic period. The seizure is almost always marked by a well-pronounced chill, which is immediately followed by febrile movement. Usually, the patient at once takes to the bed; but, in some cases, one, two, or three days pass before there is this evidence of yielding to the disease. Moderate perspiration occurs shortly after the fever begins, in a considerable proportion of cases. The perspiration in some cases is abundant, and it may recur repeatedly during the continuance of the febrile paroxysm.

The fever attains quickly to either considerable or great intensity, as denoted by the pulse and axillary temperature. Thus, of two cases in which the disease was developed in Bellevue Hospital, in one, the pulse on the first day was 120 , and the temperature $103^{\circ}$; in the other case, the pulse on the first day was 130 , and the temperature was $103^{\circ}$. During the continuance of the first paroxysm, the pulse and temperature generally denote a persistent intensity of fever, the pulse ranging, in different cases, from 100 to 140 , and the temperatmre from $100^{\circ}$ to $105^{\circ}$. The oscillations are rarely great, and those which occur are irregular in their occurrence. 
The cessation of the fever is as abrupt as the accession. The pulse and temperature quickly fall to nearly or quite the normal standard. The transition from high fever to complete apyrexia takes place, often, in a few hours; usually this is accompanied by profuse perspiration, which continues for several hours, and even an entire day. Not infrequently, the pulse and temperature fall below the standard of health. I have observed the pulse to fall as low as 54, and the temperature as low as $95^{\circ}$. In a day or two both the pulse and temperature rise again to the normal standard.

The duration of the primary paroxysm, in the majority of cases, is from five to seven days. Exceptionally, it is sometimes only two days, and it may be twelve days.

During the apyrexial period or intermission, the absence of fever is complete. It is ineorrect to call this period a remission; the fever does not remit, but it intermits. The average duration of this period is about seven days; but it may not exceed two or three days, and it may extend to twelve days, or even more.

The relapse, like the primary attack, is sudden. It is generally ushered in by chilly sensations, but not so constantly by a well-pronounced chill as the first paroxysm. The ferer in the relapse quickly becomes more or less intense. The intensity may exeeed that of the first paroxysm. But, in the majority of cases, the intensity is less. The relapse also ends suddenly, and in most cases with profuse perspiration.

The duration of the relapse varies between three and five days. It is sometimes, however, only twenty-four hours, and it may extend to ten days.

It is to be borne in mind that the relapse does not always take place. On the other hand, a second, a third, and even a fourth and a fifth, relapse have been observed. In none of the cases which $I$ have seen has there been more than a single relapse.

The foregoing points are those most highly distinctive of relapsing fever. It remains to notice other points, belonging to the clinical history, which are more or less characteristic.

Of symptoms referable to the digestive system, nausea and romiting oceur sufficiently often to by somewhat distinctive, especially when this disease is contrasted with typhus and typhoid fever. Not infrequently, these symptoms are prominent and persistent during the febrile paroxysms. The matter vomited is green or yellow from the presence of bile. The tongue presents nothing distinctive; it is generally coated, and in some cases becomes dry and fissured. The vomiting of blood presenting the character of " black vomit" has been observed; but it is a very rare symptom. It is generally associated with hemorrhage in other situations, and it is to be considered as an accidental event, not as an element of the disease. It probably denotes a scorbutic complication. Diarrhœa occurs very infrequently; constipation is the rule. 'The diarrhœa, when it occurs, is evidently accidental. Meteorism, in a moderate degree, is not uncommon. Tenderness on pressure over the epigastric region is frequent. Slight tenderness in the iliac region is not uncommon. Notable tenderness exists in some cases over the liver and spleen; and enlargement of these organs is sometimes determinable by palpation and percussion. I have not observed a craving for food during the paroxysms, which, according to some writers, is distinctive of this form of fever. The appetite, however, returns during the intermission, and the digestion may be active in this stage.

The occurrence of jaundice may be mentioned in this conneetion. This event occurs in a small proportion of cases, but its infrequency in the 
other continued fevers renders it somewhat distinctive of relapsing fever. It is doubtful if the statement that this event is an element of gravity be correct. The event is much more frequent in some epidemics than in others. The name "mild yellow fever" derives whatever pertinency it has from the occurrence of jaundice sometimes in a considerable proportion of cases, and also from the occasionil occurrence of black vomit.

A symptom referable to the nervous and the muscular system is highly distinctive of the disease under consideration. I refer to arthritic and muscular pains, more especially the latter. During the first paroxysm, pains in the loins, the calves of the legs, and the muscles in other situations, are generally much complained of. They are never wanting, although, as regards intensity, they differ considerably in different cases. The muscular pains do not cease with the ending of the paroxysm, but they continue cluring the intermission; they are more or less prominent during the relapse, and they are apt to persist into convalescence.

The inental condition, perhaps, in a measure, accounts for the suffering from these pains. The perceptions are not blunted in this disease as they are in typhus and typhoid fever. This is a negative point of distinction in contrast with the fevers just named. Another negative point is the absence of the delirium which characterizes typhus and typhoid fever. Delirium is by no means absent in all cases of relapsing fever; but the delirum is such as is apt to occur whenever there is high febrile movement, whether the fever be essential or symptomatic, and it is generally manifested only at night. In the daytime the mental faculties are generally intact. The condition known as coma-vigil does not belong to the clinical history of relapsing fever. This statement is also true of subsultus, earphologia, and other ataxic symptoms which oceur in grave cases of typhus and typhoid fever. Deafness is also a rare symptom in relap. sing fever.

A distinctive point, in comparison with typhus and typhoid fever, is the absence of a characteristic eruption. In most cases there is no eruption. Sudamina or miliary vesicles are sometimes observed at the time when profuse perspiration occurs; but this eruption is incidental to various affections. The same is true of petechial spots which oceur in some cases of relapsing fever. Other kinds of eruption are sometimes accidentally associated. Fven the rose papules of typhoid fever sometimes oceur in this as they do in various other diseases.

The physiognomy presents nothing distinctive. The face is more or less flushed, as in cases of symptomatic fever. There is not that degree of capillary congestion, marked especially on the cheeks, which exists in typhoid fever, nor the dingy complexion which characterizes typhus. The expression of indifference, vacuity, or stnpidity, which is a notable characteristic of the fevers just named, is rarely observed in relapsing fever.

The urine, as a rule, is increased, and the urea is in larger proportion than in health. Great diminution, and even suppression of the urine, however, are sometimes observed, uræmic coma and convulsions taking place, but these cases are exceedingly rare.

It may be stated, as a point somewhat distinctive of relapsing fever, that there is very little liability to serious complications. In this fact we have an explanation, in part at least, of the very small rate of fatality from the disease.

What has just been stated with respect to complications holds true as regards sequels. As a rule, important sequels do not occur. A pecnliar form of ophthalmia, which was described as following the cases reported by Dr.'A. Dubois, of this city, in 1848, is an exception to the rule. It 
has been repeatedly observed as a sequel of relapsing fever. The peculiarities of the ophthalmia are described in works which treat of the diseases of the eye. 'This sequel, however, has not occurred in any of the cases which I have observed. Relapsing fever, when it attacks pregnant women, almost always leads to miscarriage or abortion. The mother almost invariably recovers, but the child, no matter how near may be the end of gestation, as a rule, is either stillborn, or dies shortly after birth.

Diagnosis.-The distinctive features of relapsing fever are such as to leave hardly any room for doubt concerning the diagnosis after the disease has ended. Whatever difficulty in the diagnosis exists, relates to the discrimination during the primary paroxysm.

The diagnosis involves certain distinctive characters which belong to the primary paroxysm; but it is to be based, also, on negative points; in other words, on reasoning by way of exclusion. The distinetive characters are the abruptness of the invasion, the rapid increnent of fever, the frequent occurrenee of moisture on the skin, or perspiration more or less abundant, withont any marked abatement of the fever, the prominence of muscular and arthritic pains, and, in certain cases, the occurrence of jaundice. These are the positive points which are diagnostic. The fevers to be excluded are the eruptive fevers, febricula, remittent fever, typhus and typhoid fever. Scarlet fever and measles are readily excluder by the absence of the eruption and of the characters which are distinctive of these fevers during the period of invasion. Smallpox is excluded after the third day by the continuance of fever and the absence of the eruption. It is not always practicable to decide at once that the disease is not a febricula; but the intensity of the fever and the notable musenlar pains do not belong to the latter. Donbt, however, is soon removed by the persistence of the fever. Typhoid fever is excluded by the absence of a prolonged access, and the absence of the abdominal symptoms, before sufficient time has elapsed for the appearance of the eruption, and of the characteristic mental condition. Typhus is excluded by the absence of the eruption, which appears earlier than in typhoid fever, by the absence of the dusky complexion, and by the absence of the mental eonditionthe latter also being apparent earlier than in typhoid fever. Remittent fever is excluded by the absence of remissions, these being determinable by a notable diminution of the axillary temperature in some cases in which they are not distinctly denoted by the pulse. Of course, the prevalence of relapsing fever is taken into account in arriving at the diagnosis.

Causation.-Relapsing fever is undoubtedly communicable from the sick to the well. This statement rests on facts derived from the different sources of evidence, exclusive of inocnlation, which establish the contagionsness of other diseases ; for example, typhus fever. Of those who are attacked during the prevalence of the disease, a large proportion are known to have been brought into contact with, or close proximity to, patients affected with it. The disease is diffused in hospitals among fellow-patients and those who have charge of the sick. During the period in which cases were received in Bellevue Hospital, after the disease began to prevail in this city, namely, between November 14, 1869, and February 6,1870 , twelve persons contracted the fever in the hospital. These twelve persons were especially brought into contact with patients affected with the disease, and in no instance did it attack one who had not been thus exposed. One of the senior assistant physicians residing in the hospital had it. The orderly in one of $m y^{r}$ wards contracted it; and his wife, who 
came to nurse him, was attacked by it. The disease has often been diffused in localities in which it did not previously exist, after the importation of a case.

Facts, however, go to show that it is not a higlily contagious disease. Considerable exposure is, in general, necessary. 'I'he area of the infecting disease appears to be limited, and it remains to be ascertained whether it may be transported by fomites. Some facts, cited by Murchison, render it probable that it may be cliffused in the latter mode. It is especially communicable when the miasm is derived from a number of patients in the wards of a hospital, or in elose apartments. The disease is not likely to be contracted from single patients in well-ventilated rooms. 'The investigations of the Metropolitan Board of Health in this city, during the prevalence of the disease, showed that it is diffused chiefly among those living in overcrowded, illy-ventilated tenement-houses. Of the propriety and importance of removing these patients to hospitals or wards devoted specially to cases of this disease, there can be no doubt.

Destitution, deprivations, and especially deficient alimentation, are powerful predisposing canses. Of this fact, the past history of the disease, at different times and places, furnishes abundant evidence. 'The significance of the names "famine fever" and "lhunger pest" relates to this fact. Statisties dọ not show any notable etiological influence pertaining to age, sex, or season.

Although its diffusion involves generally, and perhaps always, a contagious principle, the history of the disease in other comutries shows that it occurs as an epidemic, and, after continuing for a certain time, it clisappears completely. Thus, in the eity of London, for the fourteen years preceding the winter of $1868-69$, there had been no eases of the disease. 'The complete disappearance of the disease for so long a period goes to show the necessity of predisposing and eo-operating eauses in conjunction with the special poison on which the production of the disease depends. The effective means for arresting the spread of the disease are, thinning the population of overcrowded tenement-houses, dispersing the oecupants of insalubrious dwellings, relieving destitution, especially as regards food, and promptly removing patients to hospitals devoted to cases of relapsing fever. An attack of this disease does not exempt from subsequent attacks. The period of incubation in more than one-half of the cases in which it is determinable does not exceed five days. It rarely exceeds nine days. Occasionally, as in typhoid and typhus fever, the symptoms commence almost immediately after the exposure. ${ }^{1}$

Prognosis.-It is, at first view, remarkable that a fever of such intensity, and prevailing especially among a elass of persons whose powers of tolerance are impaired by previous hardships and want, should have such a small rate of mortality. The mortality, in different collections of cases, varies from two to four per eent. Dr. Moore, in his statistical report, states that, of one hundred and three cases admitted into Bellevue Hospital between November 14, 1869, and February 6, 1870, only two proved fatal. What is the explanation of this low rate of mortality? An explanation, which perhaps is sufficient to account for the fact, has been already given, namely, the disease is very rarely accompanied by any serious complieations. The comparatively much greater fatality of typhus and typhoid fever is due mainly to complications. These fevers rarely

' Vide Murchison, St. Thomas's Hospital Reports, vol. ii. 
kill per se, that is, death is not purely an effect of the intensity of the disease.

Of the fatal cases of relapsing fever, the death, in a certain proportion, is attributable either to complications, such as pneumonia and dysentery, or to antecedent affections, for example, disease of the kirneys. But this fever may destroy life, irrespective of any important complications or antecedent affection. Several observers have reported cases in which sudden death occurred apparently from syncope. Dr. Moore's report exemplified this fict. 'The death was sudden and unexpected during the niglit, on the seventh day of the disease. At the time of death, the primary paroxysm seemed about to end, free perspiration having taken place. Neither coma nor convulsions occurred, and, so far as information conld be obtained, the dying was by syncope. The autopsy revealed no important lesions except that the kidneys appeared to be fatty.

Suppression of urine followed by uræmic coma and convulsions is sometimes a cause of death. This fact was exemplified by one of the two fatal cases occurring in Bellevue Hospital. It remains to be determined whether the suppression of urine may result from simply functional inactivity of the kidneys, or whether in all cases disease of these organs exists, as either an intereurrent or an antecedent affection.

Treatment.-There are no known means which can be relied upon for cutting short a relapsing fever. With our present knowledge, there are no remedies which, employed in the intermission, will prevent the relapse. Quinia has been used freely, but with no success. Its inutility, as a prophylactic, has been abundantly shown by different observers. Reasoning by analogy, I am led to think that the mineral acids may exert somewhat of that modifying influence, in this disease, which is so manifest in typhus and typhoid fever. They were, to some extent, used in the cases treated at Bellevue Hospital, but not sufficiently to warrant any conclusions based on experience. The treatment, with our present knowledge, must be expectant, meaning, by this term, that it is to consist of palliative measures, and those addressed to the particular indications in individual cases.

The intensity of the fever during the paroxysms may be lessened by sponging the body with water, and by the wet sheet. Cold water may be taken into the stomach freely. Ice-cold carbonic-acid water is an acceptable and useful drink to allay thirst. Cephalalgia may be relieved by cold applications to the head. The bowels should be kept open by saline laxatives. The muscular and arthritic pains call for the use of opium, especially if it produce no unpleasant after-effects. Irrespective of the pains, opiates are indicated to relieve sleeplessness.

The dietetic treatment is important, more especially in the cases in which deficient alimentation has been a predisposing cause of the disease. In this fever, as in other fevers, when alimentary support is indicated, milk is the form of diet to be preferred. And it is to be borne in mind that in this, as in most diseases, there is never any danger of the overappropriation of nutriment; the only risk is in the ingestion of more food than can be digested. It is desirable that, during the paroxysms, from one to two quarts of milk should be taken daily. In the intermission, when the appetite returns, as much substantial food as can be digested should be allowed; and the more food is appropriated in this stage, the better is the patient enabled to tolerate the relapse. Tonic remedies are indicated throughout the disease, and especially in the intermission. 
Quinia in small doses, and some preparation of iron, are the tonics to be preferred.

If the symptoms denote asthenia, alcoholic stimulants are indicated. The occasional occurrence of death from syncope renders it important to watch carefully for this indication. When there is any room for supposing that alcoholic stimulants may be useful, they should be given tentatively and continued, or not, according to the effect, especially on the pulse and temperature. 'They are, of course, urgently indicated if the symptoms denote danger in the direction of asthenia.

Attention to the urine is important. It is to be recollected that a cause of death is suppression of urine. If the urinary excretion be deficient in quantity, or if, the quantity being sufficient, there be a deficiency of urea, diuretics are indicated. Should the kidneys not respond to cliuretics, hydragogue laxatives or cathartics are indicated, with a view to eliminate urea through the alimentary canal. Active hydragogues are, of course, indicated, if uræmic coma or convulsions should supervene.

It is stated that convalescence from relapsing fever is notably slow, patients remaining for a long time much enfeebled, and tolerating with difficulty affections which may oceur before the recovery is complete. 'This has not been a marked peeuliarity in the cases which have come under my observation. It is doubtless more likely to be marked in the cases in which there had been impairment of the vital powers from innutrition and hardships before the fever was contracted; and, also, it is more likely to be marked where abundant alimentation and the judicious use of stimulants bave not entered into the treatment of the disease.

\section{Erysipelatous Fever.}

Erysipelas, considered as a local affection, belongs to surgery rather than medieine; for an account of this form of inflammation the reader is referred to surgical works. It oceurs as an occasional complication of the continued fevers which have been considered. A form of continued fever, distinct from the fevers which have been considered, is charaeterized by the frequent occurrence of erysipelas, and hence it is called erysipelalous fever. As a distinct form or species of fever, this is to be distinguished from erysipelas occurring as a local affection and accompanied with more or less febrile movement. The fever in the latter is symptomatic, whereas, in erysipelatous fever it is primary or essential. On the other hand, erysipelatous fever is to be distinguished from typhus, typhoid, and relapsing fever, with erysipelas as a complication.

Erysipelatous fever occurs as a sporadic and an epidemic disease. As a sporadic disease it is rare, and has not been as yet sufficiently studicel by means of the analysis of recorded cases. The invasion is either abrupt or there is a forming stage of variable duration. The erysijelas may be developed within a few hours from the attack, or after the lapse of one, two, or three days. The head is oftenest the seat of the erysipelas, but it may be developed in any part of the body. Different parts may be suecessively invaded; I have known it to travel over the whole surface of the body. The febrile movement is more or less intense. Passive delirium and other ataxic symptoms are not infrequently developed; in other words, the typhoid state exists. The abdominal symptoms of typhoid fever are wanting; the career of the disease is shorter than that of typhoid fever. The symptoms are modified, and the disease, as regards duration and danger, is affected materially by the degree of intensity, the extent, and the local results of the erysipelas. The general principles of treatment 
are the same as in other of the continued fevers. In the existing state of our knowledge of this fever, I shall pass it by with this brief notice. It is sometimes classed among the eruptive fevers, but the erysipelas is hardly to be considered as a cutaneous eruption.

Epidemic erysipelatous fever, I shall also notice very briefly. It prevailed extensively in this country from 1841 to 1846 . It was not confined to a particular section, but prevailed in certain districts in the New England, Middle, Western, and Southern States. In some localities great numbers were affected, and the mortality was large. It was commonly known in certain parts of the country as the "black tongue," from an appearance of the tongue occasionally observed. The medical journals of this country, during the years just named and afterward, contained many articles relating to the prevalence of this disease in different localities. Of these, an article by Drs. Hall and Dexter, giving an account of the disease as it prevailed in Vermont, ${ }^{1}$ and a paper by the late Dr. Bennett, of Bridgeport, Conn., are especially deserving of notice. The latter gives an analysis of various articles contained in different medical journals. ${ }^{2}$

The epidemic prevailed in isolated sections. It did not appear to migrate or to be transported from place to place. Its course was "irregular and erratic." Dr. Bennett, however, states that it sometimes appeared to follow the direction of rivers, small streams, and lakes. The attack was sometimes sulden and sometimes preceded by premonitions; the latter rarely existing longer than 24 hours, and being the same as those belonging to the access of other fevers. A promounced and frequently prolonged chill, with or without rigor, ushered in the disease, accompanied often by pains in the extremities, and in some cases by great prostration. Pharyngitis was at once or speedily developed; this local affection was constant. It varied much in different cases in intensity. Not infrequently it was attended with great swelling of the tonsils, and sometimes sloughing occurred. The inflammation sometimes involved the larynx, and in a certain proportion of cases death was due to either laryngitis or œdema of the glottis. The lymphatic glands of the neck became more or less swollen, the swelling in some cases being very great, and occasionally suppuration taking place.

Erysipelas was far from being coustant. The frequency of its occurrence varied at different times and places. Dr. Bennett observed it in only one-sixth of 150 cases. In view of the fact that erysipelas occurs in only a certain proportion of cases, the propriety of calling the disease erysipelatous fever rests upon the absence of any other and better name. The erysipelas was seated in different parts. It was apt to lead to suppuration, gangrene, and slonghing. The clanger and protractedness of the disease depended on the degree, extent, and local results of the erysipelas.

The febrile movement was more or less intense. Erratic pains, like those of neuralgia and muscular rhenmatism, were common during the course of the disease. Typhoid delirium and other ataxic symptoms occurred in severe cases. Petechiæ were sometimes observed. Abscesses, withont erysipelas, occurred in some cases. The disease was not infrequently complicated with inflammation of serous membranes-the pleura, peritoneum, and the cerebral meninges. Pneumonitis was an occasional complication.

Different cases differed much as regards the gravity of the disease. In

I Vide American Journal of Medical Sciences, January, 1844.

2 Vide New York Journal of Medicine, July, $18 j 3$. 
many cases the disease was mild and ended in five or six days. The severity and danger were generally due to the complications. Of the cases complicated with laryngitis, or adema of the glottis, extensive erysipelas, serous inflammations, and pneumonitis, a large proportion ended fatally, the duration being indefinitely prolonged.

Puerperal peritonitis prevailed in conjunetion with this epidemic so uniformly as to show a pathological relationship between these two affections. 'I'he opinion was generally held that the former was eaused by a virus or miasm earried by the obstetrician from patients affected with the latter. Assuming this to be true in certain cases, puerperal peritonitis was by no means uniformly to be accounted for in this way. A rational explanation in many, if not in all cases, is that labor acterl as an exciting eause and determined the situation of the local affection in those predisposed to the disease from the action of the epidemic influence. Many, if not most, practitioners held that the disease was communicable. Its contagiousness, however, cannot be considered as established.

$\Lambda \mathrm{s}$ regards treatment, there was a diversity of opinion. Some practitioners advocated bleeding and other of the measures ealled antiphlogistic. But the majority, before the epidemic ceased to prevail, were convineed of the impropriety of these measures. In view of the symptoms, the nature of the disease, the tendency to suppuration and gangrene in the cases in which erysipelas occurred, and the other complications which were liable to occur, there can harclly be a question as to the propriety of measures of an opposite character. Tonic remedies, alimentation, and alcoholic stimulants, in other words, supporting measures of treatment, were indieated. Mild cases required little or no treatment; but in grave eases, the principles which shonld govern the management of other essential fevers with serous local complications were applicable.

\section{Epidemic Fever Charaoterized by Mild Erythematic Pharyngitis.}

In the winter and spring of 1857 an epidemic fever prevailed in the western part of the State of New York, in the adjacent parts of Pennsylvania, and in Canala, characterized by inflammation of the pharynx, of a mild grade of intensity, and nnaceompanied by either diphtheritic exulation or much submucous infiltration. I reported the results of an analysis of 23 cases which $I$ observed and recorded. A report of this epidemic, based upon notes of 37 cases, was at the same time made by Prof. Rochester to the Buffalo Medical Association.' 'The different cases presented great uniformity as regards the general and local symptoms. 'The fever commenced with a chill without rigors. The febrile movement, in all the cases, was considerable or marked. The severity of the disease was generally sufficient to keep the patients in bed for several days. The lymphatic glands of the neck were in most"cases moderately enlarged, but without suppuration. Soreness of the throat was not a prominent symptom, even when the pharyngitis was marked. There was in no case an eruption. Fomiting did not oceur, and generally the bowels were constipated. There were no symptoms of importance referable to the pulmonary and nervous system. The duration of the fever was from three to six days. It affected persons of either sex, and of all ages. The epidemic continued about two months, reaching its acme gradually, and gradually declining. In no case under my observation did it prove fatal.

I For both reports, vide Buffulo Med. Journal, 1857. 
The discase was not scarlatina, as shown by its affecting persons who had had that disease, by the fact that scarlatina did not prevail at the time, by the absence of an eruption in all the cases, by the non-occurrence of dropsy as a sequel in any case, and the fact that middle-aged and aged persons were attacked as well as children and infants. There were no cases of epidemic pharyngitis with the exudation of lymph, or diphtlieria in that part of the country. The fever resembled the mild cases of erysipelatous fever which occurred during the epidemic prevalence of that disease, but erysipelas was not developed in any case. The cases bore a close analogy to those of influenza, except that the local affection was seated in the pharynx, and not in the Schneiderian and bronchial mucous membrane.

Palliative measures of treatment only were indicated.

Dr. Harvey E. Brown, Asst. Surgeon U. S. A rmy, kindly communicated to me an account of an epidemic similar to that just described, which prevailed among the United States troops, stationed at Hart's Island, Long Island Sound, in Jamuary and February, 1866. The garrison of the island numbered about 1000 men, the greater part being new recruits. During the months named there were 181 cases of the fever. The attack commenced with a slight chill, not infrequently accompanied by nausea and vomiting. Considerable febrile movement followed, the pulse being quick and full, and its average frequeney being about 100. Generally the bowels were constipated, but diarrhoa occurred in a few cases. Of the 181 cases, in 81 there existed marked inflammation of the tonsils and pharynx. In the remainder of the cases pharyngeal inflammation existed, but it was comparatively slight. Swelling of the lymphatic glands of the neck was a marked feature of many cases, accompanied by much pain, but in no case did suffocation occur. In several cases external otitis occurred with discharge of purulent matter. In seven cases erysipelas of the face supervened. Hoarseness existed in most cases, and in two or three cases there was complete aphonia. Physical exploration showed absence of any pulmonary complication in all the cases. In several cases there was more or less difficulty in passing urine, and in one case there was complete retention. Examinations of the urine showed no abnormal condition. The disease varied much in severity in different cases, but in no case was it fatal. The duration varied from three to seven days. The epidemic commenced about the 10th of January, reached its maximum about the 15 th of February, and at the date of Dr. Brown's communication, March 2, was gradually declining.

The treatment was, at the outset, a saline cathartic; this was followed by the liquor ammoniæ acetatis, and pulvis ipecacuanhæ et opii was given at bedtime. Gargles of tannin and capsicum were employed, and in some cases the throat was brushed with a weak solution of the nitrate of silver. The enlarged lymphatic glands were sometimes painted with the tincture of iodine, with good effect. 


\section{CH A P T E R IV.}

Periodical Fevers. Intermittent Fever-Anatomical Characters-Clinica] History-Causation -Diagnosis-Prognosis-Treatment. Pernicious Intermittent Fever.

T'HE periodical are distinguished from the continued fevers by the occurrence of febrile paroxysms or marked exacerbations in a regular o:der of sueession, thereby exemplifying a law of periodicity. 'This clistinction applies more especially to the fevers called intermittent and remittent. Yellow fever is generally included among the periodieal fevers, and belongs perhaps more appropriately here than in any other nosological division. The intermittent and remittent fevers are often distinguished as malarial fevers. These fevers will be found to present many striking points of difference, as contrasted with the fevers which have been considered in the preeeding chapters. They differ not only in the events of the elinical history, but as regards the laws governing their causation, duration, eomplication, sequels, and the extent to which they are controllable by known remedies.

\section{Intermittent Fever.}

An intermittent fever is characterized by the occurrence of febrile paroxysms in regular succession, and by the absence of febrile movement between the paroxysms. The intermission is the distinctive feature of this form of fever, as its name implies. Popularly, the disease is known as "fever and agne," "chill fever," "the shakes," ancl, by names expressive of the locality in which it is produced, as, in Louisiana, "swamp fever," "Panama fever," ete. Although essentially the same disease, intermittent fever, as ordinarily presented in practice, is unattended with danger to life, but occasionally it is one of the most dangerous of maladies. Hence I shall consider first simple or ordinary intermittent fever, and afterward, the grave forms of the discase under the head of pernicious intermittent fever.

Anatomical Charactens.-Bronzing of the liver, spleen, and brain which is found after death from remittent and from pernicions intermittent fever, together with the presence of dark pigment in the blood (melanomia), may be supposed to exist in more or less of the cases of simple intermittent fever in which the disease ends in recovery. Aside from these there are no known lesions which are characteristic of intermittent fever.

Chinicar History.-The clinical history will embrace an account, first, of the paroxysm, and, second, of the intermission.

In the majority of cases the attack is sudden. In a certain proportion of eases, however, there are premonitions for a variable period. The premonitions are not very distinetive of this disease, consisting of pain in the head, yawnings, indisposition to exertion, loss of appetite, and general malaise. Although not very definite, they sometimes suflice to lead pa- 
tients who have been repeatedly affected with intermittent fever to anticipate an impencling attack.

A paroxysm, when complete, consists of three distinet periods or stages, viz., the cold, the hot, and the sweating stage.

Cold Stage.-This stage commences with a feeling of chilliness, beginning in the loins and extending thence over the back and limbs. The chill is more or less intense, in some cases consisting of a slight creeping sensation of coldness with shiverings, and in other cases extremely severe. Museular tremor commonly known as rigor may or may not aecompany the ehill. I have met with a single example of distinet rigor without a sensation of ehilliness. I) uring the chill, bristling of the hairs over the borly, or horripilation, and the appearance familiar as "goose-skin" are often observed. Notwithstanding the sensation of coldness which the patient experiences, and the feeling of eoldness when the hand is placed on certain parts, the thermometer placed in the axilla shows an increase of the temperature of the body. 'The thermometer, however, applied to the extremities, shows a decrease of temperature below that of health: The inerease of the temperature of the body begins prior to the paroxysm. 'The sensation of coldness is sometimes limited to a part of the boly, as the back, or to the extremities. During the rigor frequently the teeth chatter, and the movements of the body and limbs are sometimes sufficient to shake with considerable violence the bed on which the patient lies.

During this stage the patient frequently sighs, the pulse, which is usually aceelerater, is small and feeble, the countenance has an expression of anxiety, the prolabia and face are pale, or frequently livid, and lividity is often marked at the roots of the nails. The general appearance is as if the person had been exposed to intense cold. The circulation is sometimes completely suspended in the fingers, which are waxy in appearance, devoid of sensibility, and do not blecd if wounderl. Other synptoms are, mental irritability, a sense of oppression referred to the præcordia, pain in the head and limbs, and palpitation.

The duration of this stage varies from a few moments to two hours or even longer. 'The average duration may be estimated at from a half to three-fourths of an hour. The transition to the next stage is sometimes abrupt, but generally gradual ; flushings of heat are felt, the rigor ceases, the coldness, as it were, melts away, febrile movement is developed, and the eold stage is then enderl.

The eold stage is not infrequently wanting, the paroxysm beginning with the hot stage. I have known a state of intense nervousness take the place of the cold stage. Gastralgia and gastrie irritability, as denoted by incessant vomiting, are other morbid conditions which sometimes occur instead of this stage; also drowsiness or stupor and a condition resembling hysterieal coma. Veritable coma is liable to occur, in this stage, in a form of the disease which will be noticed separately under the head of pernicious intermitlent fever. In young children convulsions are apt to occur in the cold stage.

Congestion of internal organs must necessarily be involved in the cold stage, inasmuch as there is less blood in the vessels of the surface, and the whole mass of blood is not diminished. But the primary and essential morbid condition underlies the congestion; in fact it is probable that the latter is an effect of an aetive contraction or spasm of the peripheral ves: sels, the blood accumulating in the internal organs in consequence of its being driven from the surface of the body. That the congestion incident to this stage does not stand in a causative relation to the other stages is 
shown by the fact that the cold stage is not infrequently wanting, and the other stages in these cases are not less marked than when the cold stage occurs.

Hot Slage.-This stage is characterized by more or less intense febrile movement. The skin becomes hot, the pulse is accelerated and full or bounding, the face is flushed, the cephalalgia continues, but the pain in the limbs and the præcordial oppression disappear. The thermometer in the axilla indicates a rapid increase of heat usually to $105^{\circ}$ or $106^{\circ} \mathrm{Fahr}$. Thirst is usually a prominent symptom. The intensity of the febrile movement varies considerably in different cases. The duration of this stage is from three to eight hours. It is evident, in this stage, the spasm of the vessels of the periphery, which existed in the cold stage, has ceased, and, in place of that morbid state, the distributing vessels of the surface of the body are abundantly dilated. Whether the dilatation be due to a paralyzed condition of the vessels or to an increased attraction of the tissues for blood, our present actual knowledge does not enable us to decide.

Sweating Stage.-Perspiration appears first on the face and afterward on the trunk and extremities. The febrile movement gradually abates, and at length disappears. The heat of the surface, cephalalgia, thirst, restlessness, etc., cease. The thermometer indicates a rapid defervescence, the heat falling to the normal standard. The patient obtains refreshing sleep. With this stage the paroxysm ends. As regards the amount of sweating, different cases differ; it is sometimes profuse, and sometimes slight. The durating of the sweating stage is variable, the average duration being three or four hours. The sweating is evidence that the febrile movement is about to cease. That it is the means of bringing the paroxysm to a close is by no means certain; it is a sign of the approaching intermission, and it may be an effect, rather than a cause of the decrease of the febrile movement. During and succeeding the paroxysm, urea, uric acid, and the chlorides in the urine are increased. 'The urine is not infrequently albuminons, and occasionally hæmaturia exists.

The intermission, called also the apyrexial period, is the space of time between two successive paroxysms. The period from the beginning of one to the beginuing of the next paroxysm is called the interval, in distinetion from the intermission. The duration of the interval is the basis of a division into varieties commonly known as the different types of the diseasc. Each variety of type observes a law of periodicity in the succession of paroxysms-a wouderful fact belonging among the pathological mysteries of which our present knowledge affords no explanation. There are three simple types of intermittent fever, viz., the quotidian, lertian, and quartan type. In the quolidian type the interval is about twentyfour hours, or the paroxysm reeurs daily, as the name imports. In the tertian type the interval is abont forty-eight hours, or the paroxysm recurs on the third day, reckoning in the days on which two successive paroxysms take place. In the quartan type the interval is about seventytwo hours, or the paroxysm recurs on the fourtl day. As a rule, the paroxysms are uniform in each individual case as regards the occurrence of the cold stage, the duration of the several stages respectively, the severity of each etc.; but they present considerable diversity in different cases. Of the three simple types, the quotidian and tertian type are by far the most frequent. Examples of the quartan type are comparatively rare. Of 98,237 cases of intermittent fever in the United States army, only 1757 were of the quartan type.'

1 Vide Woodward, Camp Diseascs of the United States Army. 
As regards the relative frequency of quotidian and tertian cases, either may predominate at particular seasons. In the same locality the majority of cases are, at one season, of the quotidian, and, at another season, of the tertian type. In an aggregate of cases occurring in a series of years the quotidian type predominates. Of 98,237 cases in the United States army, 51,623 cases were of quotidian and 44,857 cases of tertian type.' Cases have been observed in which the 1 aroxysm has recurred on the fiftl, sixth, seventh, and eighth day, and hence there may be a quintan, a sextan, a heptan, and an octan type of intermittent fever. Such cases, however, are among the rarest of the rare curiosities of clinical experience. In some cases, during the continuance of the disease, the type changes, the quotidian succeeding the tertian, or vice versa; such cases, however, are extremely rare.

Cases occasionally are presented in which one of the simple types is duplicated; that is, two sets of paroxysms of the same type coexist. Thus, one compound type is called a double quotidian, two paroxysms occurring daily. Another compound type is a double tertian. In a double tertian a paroxysm occur's daily, but the paroxysms on successive days take place at different hours, and may differ in various ways, whereas on alternate days they oceur at the same hour, and correspond in other respects. Again, a tertian is doubled or duplicated when two paroxysms occur on alternate days. Another compound type is a double quartan. In this type a paroxysm occurs on two successive days, and on the third day there is no paroxysm. Finally, a triple quartan is a compound type in which a paroxysm occurs on three successive days, the paroxysm on each day presenting notable points of diflerence, but each paroxysm corresponding with that which recurs on the fourth day. These compound types, except the double tertian, are extremely rare, and merely interesting as curiosities of clinical experience. The double tertian is not very infrequently met with.

Paroxysms may occur at any hour of the day. They very rarely occur during the night. In a large majority of the cases of the quotidian and tertian type the paroxysms occur before noon; quartans are as likely to occur after as before the middle of the day. In infants the type is almost miformly quotidian. As a rule, the successive paroxysms recur precisely or nearly at the same hour; but sometimes there is a variation from this rule, and the variation is in accordance with a law when the paroxysms are said to be either anticipating or retarding. They are anticipating when each successive paroxysm recurs earlier, by the same period, than the last. 'Thus, they may regularly anticipate half an hour, an hour and a half, etc. The paroxysms are retarding when each successive paroxysm is, in like manner, delayed. This variation in the time of recurrence is generally evidence that the disease is about to end.

During the intermission there is much difference in different cases as regards freedom from ailments. In some cases the patient complains only of a certain amount of debility; the appetite and digestion are good, and there is no apparent disorder of any of the functions. In other cases morked prostration follows; the appetite and digestion are more or less impaired, and various disturbances exist.

Enlargement of the spleen is an occasional complication. The enlargement is often sufficient for the organ to be readily felt through the abdominal walls, constituting what is commonly known as the "ague cake" in districts where the discase prevails. The organ in some cases becomes

1 Vide Woodward, Camp Diseases of the United States Army. 
enormously enlarged. This complication is not very frequent. It oceurs in some cases when the disease has been of short duration, and it is by no means developed in the majority of the cases in which the discase has existed for a long period. Its pathological connection with the disease is not understood. It may continue for a consiclerable period after the paroxysms cease.

Aniemia is incident to the disease, especially if it have been of considerable duration. 'The pallor of the face in eases in which the rlisease has been protracted, or in which frequent relapses have taken plaee, is often associated with a yellowish or sallow tint, and with more or less oedema, giving rise to a characteristic physiognomy. These characters denote what is called the malarious cachexia. General dropsy is an occasional coneomitant or sequel. Notable anasarca, with effusion into the serous cavities, is sometimes incident to the disease, and does not, therefore, denote either a cardiac or renal affection. An herpetic eruption about the mouth is a frequent coneomitant.

The duration of the disease is indefinite. It not infrequently cnds spontaneously after a few paroxysms, but in many cases it continues for weeks and even months, if not arrested by curative means. Its inclefiniteness of duration is a striking point of difference as contrasted with the contimued fevers; its self-limitation is not, as in the latter, restricted within a certain period. Another not less striking point of difference relates to the liability to relapses. Subsequent attacks oceur in the great majority of cases. The liability to relapse remains for many years. In some cases suceessive attacks oceur regularly after a certain period, showing that in these eases the relapses take place in accordance with an intrinsic tendeney, that is, irrespective of external causes. 'The notion was formerly entertained that the tendency to relapse was lessened if the disease be left to end of its own aceorl, or "wear itself ont.". So far from this being true, I believe that clinical experience shows a diminished tendeney to relapse in proportion as the paroxysms are speedily interrupted by therapeutical interferenee. After the paroxysms have ceased to recur, either spontaneously or from the use of remedies, thermometric observations may show more or less increase of the heat of the boly at the time when the paroxysm was to be expected. So long as these fluctuations of temperature occur, the disease has not completely disappeared, and relapses are liable to occur.

Plysicians in malarious districts become familiar with eases which may be called cases of latent intermiltent fever. In these cases paroxysms are not fully developed, but the patient complains of indefinite ailments, to which the term bilious is frequently applied. 'The appetite is impaired or lost; there may be nausea and occasional vomiting, with pain in the head, indisposition to exertion, etc. The tongne is frequently covered with a thin and very white coating. A close examination will sometimes disclose evidence of periodicity in an increase of ailments at regular periods on successive days or on alternate days, and slight manifestations of the several stages of a paroxysin may be observed. The thermometer in the axilla is useful, as a means of diagnosis, in these cases, showing a periodical rise of temperature when the surface of the body may not show increase of heat, and the pulse is but little accelerated. In a ease under observation at Bellevue Hospital, at the time of writing, the morning temperature in the axilla was $105^{\circ}$ Fahr., the pulse being only 80 , and at evening the temperature was $100^{\circ}$. Under the free use of quinia, the daily increase of temperature rapidly climinisherl, and in a short time ceased. The prompt relief afforded by treatinent addressed to a sus- 
pected malarious condition shows the correctness of the suspicion, and this treatment may be resorted to tentatively in cases of doubt. The affection, in these cases, is sometimes called dumb ague. This term is also applied to paroxysms in which rigors are wanting.

Intermittent fever is said to be masked in certain cases in which it is associated with other affections. It may exist in combination with various affections-bronchitis, pneumonitis, dysentery, etc. The association may render the periodical disease irregular, and interfere with the full development of the paroxysnis. In these cases, it is not correct to apply to the associated affections the term malarial, meaning thereby that these affections are due to malaria. They are simply accompanied by phenomena proceeding from malarious disease existing in combination. In malarious districts the influence of malaria is apt to pervade all affections, and claims special measures of treatment. Of this fact practitioners in these districts become fully aware. Paroxysms of neuralgia sometimes appear to take the place of the paroxysms of intermittent fever, recurring with the sane regularity after intervals corresponding to those in the different types of intermittent fever, and a cure is effected by the remedies which are efficacious in the latter disease.

Causation.-The causation involves a special morbific agent, commonly known as malaria. The existence of a special cause is logically inferred from the peculiar character of the disease, its limitation to certain localities called malarious, and the fact that it is controlled by remedies having a specific operation. The production of the special cause was attributed to vegetable decomposition in marshy localities, and called marsh miasm, in 1717, by Lancisci, an Italian writer. This doctrine, and the name, have since been very generally adopted. But that something more than ordinary vegetable decomposition is requisite for its production is sufliciently proved by the disease being indigenous in certain localities, whereas, in certain districts and countries in which vegetable decomposition must take place abundantly, the disease never occurs. If produced in connection with vegetable decomposition, it depends on incidental circumstances peculiar to certain localities. Observation shows that it is generated more especially in marshy situations, but its production is not confined to such situations, and, hence, the name marsh miasm is open to criticism. The following statements embody points relating to our existing knowledge of malaria, which are of practical importance :-

" 1 . It affects, by preference, low and moist localities."

"2. It is almost never developed at a lower temperature than $60^{\circ}$ Fahr."

" 3 . Its evolution or active agency is checked by a temperature of $32^{\circ} . "$

"4. It is most abundant and most virulent as we approach the equator and the sea-coast."

"5. It has an affinity for dense folinge, which has the power of accumulating it when lying in the course of winds blowing from malarious localities."

" 6 . Forests or even woods have the power of obstructing and preventing its transmission under these circumstances."

"7. By atmospheric currents it is capable of being transported to considerable distances, probably as far as five miles."

" 8 . It may be developed in previously healthy places by turning up of the soil, as in making excavations for the foundations of houses, tracks for railroars, and beds for canals."

" 9 . In certain cases it seems to be attracted and absorbed by bodies 
of water lying in the course of such winds as waft it from the miasmatic soul'ce."

"10. Experience alone can enable us to decide as to the presence or absence of malaria in any given locality."

"11. In proportion as countries, previously malarions, are cleared up and thickly settled, periodical fevers disappear, in many instances to be replaced by typhoid fever."

The telluric source of malaria is proven by facts contained in the foregoing statements; the fact of its being endemic in eertain localities is alone sufficient to establish this source. It is generated only during the summer season. Facts appear to show that its specific gravity keeps it near the surface of the earth. P'ersons sleeping in an lipper story may escape, when those sleeping on a level with the ground become affected. It is more abundant in the night-air than during the day, and hence the disease may often be avoided by avoidling exposure in the evening, night, or early in the morning. ${ }^{2}$ The special cause of this disease is never reprodnced in the bodies of those affected ; that is, the causation never involves the existence of a virus or an infectious miasm. Facts abundantly prove that the disease is in no sense communicable from one person to another.

A remarkable paper on the cause of intermittent and remittent fevers appeared, in 1866, from the pen of Dr. J. II. Salisbury, Professor of Physiology, Histology, and Pathology in the Charity Hospital Niedieal College of Cleveland, Ohio. ${ }^{3}$ In this paper P'rof. Salisbury elaims to have ascertained that these fevers are caused by the introduction into the system of cells or spores emanating from eertain species of algoid plants, ealled Palmellx, which belong to the lowest known vegetable organisms. To these species of plants he applies the generic name Gemiasma, signifying earth miasm, and he also calls them ague-plants. Prof. Salisbury claims that this cliscovery is based on the following facts : $\mathbf{A}$ mieroscopical examination of the salivary secretions and mucous expectoration, in the morning, of persons living in a malarious region, showed cells of an algoid type, resembling strongly those of the palmella, to be the only constant bodies present; and these bodies were invariably absent whenever the mucous secretions were examined from persons resilling above the summit plane of ague. These palmelloid cells were obtained by suspending plates of glass, near broken ground, over night, in places whence malarious emanations were know to arise. The so-called ague-plants were invariably found in numerous localities in which intermittent fever prevailed, and in no instance were they found where this disease did not occur. Cakes of surface soil from a malarions locality, which were covered with the palmellæ, were carried to a high, hilly district, situated five miles from any malarious locality, where a case of ague had never been known to occur. These cakes were exposed on the sill of an open second story window opening into the sleeping apartment of two young men. A plate of glass suspended over them during the night was found to be covered with palmelloid spores and cells. Both the young men had intermittent fever, one on the twelfth and the other on the fourteenth day, no other

1 Report on the Nature and Treatment of Miasmatic Fevers, by John T. Metcalfe, M.D., published by the United States Sanitary Commission, 1862.

${ }^{2}$ For an elaborate exposition of facts pertaining to malaria, the realer is referred to the alle and learned work by La Roche, entitled, "Pneumonia; its Supposed Connection with Antumnal Fevers : including an Inquiry into the Existence and Morbid $\Lambda$ gency of Malaria." Philadelphia, 1854.

American Journal of Medical Sciences, Jan. 1866. 
members of the family becoming affected. The ague-plants were found in the urine of persons affected with intermittent fever.

The eryptogamic theory is consistent with well known facts relating to the causation, namely, the production of the clisease in the summer season; its prevalence in marshy situations; the absence of the,cause when parts known to be malarious are submerged, and, on the other hand, the abundanee of the cause in these parts in a dry season; the fact that certain countries and sections are never malarious, etc. During the six years which have elapsed, however, since the appearance of Prof. Salisbury's paper, his data have not been confirmed by the observations of others, and the validity of the diseovery is not, as yet (1872), generally recognized.

The period of incubation is indefinite; it may be a few days only, or a few weeks, but it is not infrequently many months, and probably years may elapse after the reception of the malaria before its morbid effects are manifested. The latter is one of the most wonderful of the striking facts pertaining to this disease. Auxiliary causes appear to be often necessary to give efficiency to the speeial cause. An attaek is apt to occur after exposure to cold, over-exertion, exeesses in eating or drinking, etc. The activity of the special cause is also often awakened by the occurrence of some other affection. The disease oecurs espeeially in the spring and autumn, probably in eonsequence of the action of anxiliary causes in these seasons. The vernal intermittents are in general milder than the antumnal.

The disease shows no preference for either sex, and it affects all ages.

Diagnosis.-In weil-marked eases the diagnosis offers no difficulty. The type is to be determined only by the duration of the intervals and a comparison of the paroxysms. Latent and masked intermittents are not so easily recognized, and in arriving at a positive diagnosis the effect of treatment is sometimes to be considered. Febrile paroxysms resembling those of intermittent fever are sometines observed in connection with pulmonary tuberculosis; they may present the three stages well marked, and recur at regular intervals. They oftener oecur in the afternoon than in the forenoon, whereas the reverse obtains in intermittent fever. The coexistence of tuberculous disease is to be taken into aceount in making this differential diagnosis, also the known exposure to malaria or otherwise. The treatment appropriate to intermittent fever will sometimes arrest the paroxysms connected with tuberculosis. Chills connected with suppuration in some of the internal organs may suggest, as probable, the action of malaria in malarious districts. The irregularity of their occurrenee, the inefficacy of treatment specially directed to a malarious affection, and the discovery of the local affection will in time clear up the diagnosis. Happily the remedies indicated in malarious diseases are rarely hurtful in the cases in which intermittent fever is incorreetly supposed to exist. Operations on the urinary passages, such as catheterism and lithotrity, are apt to produce paroxysms resembling those of intermittent fever. If the observations of Prof. Salisbury be accepted as accurate, the presence of the so-called ague plant in the urine is a diagnostic eridence of the disease.

Prognosis.-Ordinary or simple intermittent fever, as regards immediate danger, is not a grave affection. Whenever the disease involves immediate danger, it is to be considered as pernicious, and not therefore belonging under the head of ordinary or simple intermittent fever. An important fact is, an intermittent fever at first devoid of immediate danger may become pernicious. An ordinary or simple intermittent may 
prove remotely serious, if it continue long or recur frequently, by indueing anamia, general dropsy, or the malarious eachexia. 'The remote efleets of the disease, however, are very rarely in themselves fatal; deatl may result from the association of intermittent fever with other affections. It has been supposed that this disease exerts a proteetive influence against the developinent of pulmonary tubereulosis; so far from this being true, there is reason to believe that it promotes the development of the affeetion just named in those predisposed to it. It is not a small ealamity to coptract intermittent fever on aceount of the liability to relapse, and to recurrenee of the disease in connection with other affections.

T'Reatuent.-For the cure of intermittent fever, medicine possesses specifics, if any remedies are entitled to this appellation. 'This statement applies especially to the salts of quinia, of which the sulphate is the one almost universally used. The sulphate of quinia will promptly interrupt the recurrenee of the paroxysms of intermittent fever in the vast majority of cases. It is always clesirable to arrest the disease as speedily as possible. Its morbid effects are less in proportion as it is quickly arrested, and the liability to relapses is diminished. There is no need of preparatory treatment. 'This position was taken by the author in an article published more than 30 years ago." An experience embracing many hundred eases in clifferent climates, since the date of that publication, has abundantly confirmed the correctness of this position. Aside from the clelay in arresting the disease, the measures heretofore employed to prepare the system for the sulphate of quinia or other special remedies were injurious. These measures were, mercurial cathartics, emetics, and sometimes bleeding. 'They are not indicated in the treatment of intermittent fever. A consideration of no small importance, as enforeing an immediate employment of the abortive treatment, is the possibility of an intermittent fever, at first simple or ordinary, becoming, after several paroxysms, pernicious.

With respect to the time of giving the anti-periodic remedy, my experience has led me to the conclusion at which Bretonneau, Graves, Briquet, 'Trousseau, Murehison, and others have arrived, viz., that it proves most effective when given as near as possible to the paroxysm which has passed. It is probable thit if it be given in the sweating stage, the chances of preventing the next paroxysm are greater than if the administration be delayed until after this stage. As regards doses, the most effective plan is to give the remedy so as to produce evidence of cinchonism as speedily as possible. 'l'his object may be accomplished by giving at a single dose the quantity supposed to be required. 'This quantity for an adult is from ten to twenty grains. A dose of from ten to twenty grains is generally tolerated by the stomach as well as smaller doses. An objection, however, to this method is, the quantity estimated as sufficient may not be large enough, or it may be larger than is required. A preferable method, therefore, is to give the remedy in smaller, but not in small doses, which are to be repeated at intervals of two or three hours until cinchonism is prodncel. The latter is indicated by tinnitus aurium, and this effect is, in general, evidence that a sufficient quantity has been administered. For an adult, five grains may be given at a dose, and repeated once, twice, or thrice, if necessary for the effect just stated. By this method of treatment, in a case of quotidian type, the chances that another paroxysm will or will not occur are about even. In a case of tertian type

I Vide Amcrican Journal of the Medical Sciences, October, 1841. 
the chances that another will not occur preponderate. If another paroxysm occur, the same method of treatment is to be repeated.

As regards the form in which the sulphate of quinia may be administered, it is most effective when given in solution, its solvency being secured by the addition of a minim of the aromatic sulphuric acid for each grain of the salt. It may be given, however, in powder or in pills ; if the latter be preferred, they should be recently prepared. If, owing to the irritability of the stomach, the romedy be not retained when given by the mouth, it may be given per enema. It is readily absorbed from the intestine; and the opinion has been lield by some that it acts more efficiently when thus given. According to most observers, however, the quantity when given by the rectum should be larger than when given by the mouth.

The plan of treatment just described is almost invariably successful. If it fail, there is always reason to suspect that the preparation of quinia employed is spurious or adulterated. A diflicnlty, in some cases, relates to an intolerance of the remedy. Some persons are affected very unpleasantly by even small doses of the sulphate of quinia. This intolerance is not infrequently imaginary, but it is sometimes real. In such cases small doses must be given, or some one of the remedies to be presently mentioned substituted. As a rule, small doses are curative in the cases in which the remedy is tolerated only in small doses. On the other hand, some persons are affected by the remedy only when full or even large doses are given; and, as a rule, such persons require larger doses than others. Cinchonism is the test of the requisite quantity having been given, but this need not be produced except in a slight degree. The coexistence of any affection does not contra-indicate the plan of treatment which has been described. In all cases in which intermittent fever is complicated with other affections, the primary object of treatment is to interrupt the paroxysm as speedily as possible. In children and infants the doses of the sulphate of quinia are to be less than in adults in proportion to the age. In young children, owing to the difficulty of administration by the mouth, the remedy may be given per enema. It may also be employed effectively by inunetion, an ointment containing it being rubbed into the axilla, groins, and over the abdomen.

The hypodermic injection of a solution of quinia may be resorted to in cases of ordinary intermittents, when the remedy is not retained either by the stomach or rectum. This mode of administration, moreover, has the advantage of economy as regards the quantity of the remedy required, late experimental observation appearing to show that the effect is three or four times greater when thus administered than when taken into the stomach. The effect is also more quickly induced. It is, however, in cases of pernicious intermittent and remittent fever that the hypodermic injection of a solution of quinia promises to prove of special value, by reason of the ccrtainty and promptness with which cinchonism may be induced by this mode of administration.

After the paroxysms are interrupted, the remedy should be continued in small doses, from two to four grains daily, for a considerable period. It is desirable to continue it for several weeks. If anæmia exist, a chalybeate should be conjoined. The citrate of iron and quinia is an eligible preparation. Relapses are prevented by this after-treatment. The diet should be nutritions, and a little wine with meals is advisable. Cathartics are to be avoided; given before the interruption of the paroxysms, they conflict with that object. If there be constipation, it should be remedied by mild laxatives or enemas. 
Preparations of cinchona other than the sulphate of quinia are more or less effective in arresting intermittent fever. 'The sulphate of cinchona appears to possess an anti-periodic power scarcely less than that of the sulphate of quinia. Of 100 cases treated with this salt by Dr. A. Paul Turner, about a scruple being generally given in the intermission, the paroxysms were at once interrupted in 79 per cent., and in only two cases did Inore than two paroxysms occur after the treatment was commenced. Dr. Turner is of the opinion that gastric disturbance and unpleasant cephalic symptoms are less likely to be produced by cinchona than by quinia. Amorphous quinia, called quinioidia, chinioidine, or the preeipitated extract of bark, is also reliable in doses of double the size of the sulphate of quinia. Crude or impure quinia may be used effectively in the same doses as the sulphate of quinia, and having, comparatively, but little bitterness, is sometimes to be preferred for administration to children.

There are several succedanea of the preparations of cinchona, which are capable of arresting the disease. Salicin appears to be successful in a eertain proportion of cases. From 30 to 40 grains are to be given in an intermission. The sulphate of bebeerine, an alkaloid of the bark of a tree in Guiana called bebeeru, is said to be frequently successful, and it has been claimed in behalf of this remedy that it does not produce the unpleasant effects which are sometimes caused by the preparations of cinchona. From 15 to 30 grains are to be given in the apyrexial period. The ferrocyanide of iron or Prussian blue I have found an efficient antiperiodic in doses of from one to two drachms. Patients take it with reluctarce, its appearance suggesting the idea of poison. Stryehnia and nux vomica often sueceed. Chloride of sodium, given to the extent of from eight to ten drachms in the apyrexia, effects a cure in a certain proportion of cases. ${ }^{2}$ Piperine sometimes proves curative. Many praetitioners give this remedy in combination with the preparations of cinchona. Arsenic is, probably, next to the preparations of einchona, the most reliable. Fowler's arsenical solution is the form generally employed, given in doses of ten or twelve drops three times daily to an adult. The hydrochlorate of ammonia, in doses of a drachm in the intermission, appears to exert an anti-periodic power. Nitric acid in doses of from 5 to 8 drops continued during the intermission and paroxysm, once in six hours, has been found to be efficacious by Dr. E. S. Bailey, of Indiana. Dr. W. A. Hammond has reported the result of this remedy in 32 cases, the dose being 10 drops three times daily. In all these cases the disease was arrested within three days. ${ }^{3}$ The iodide of potassium has proved successful, in some cases, when the disease has resisted quinia." This list might be exten led. It is to be borne in mind, in judging of the effect of remedies in this disease, that it tends intrinsically to end after a few paroxysms in a certain proportion of cases. All the known remedies which may be employed as suceedanea of the preparations of cinchona are inferior to the latter. None are to be preferred except in some cases in which, from an idiosyncracy, the preparations of cinchona are not tolerated. 'They may sometimes come in play where the preparations of cinchona cannot be procured, and they have the advantage of being less costly than the latter.

I American Jnurn. of Med. Sciences, April, 1864.

2 Vide cases reported by Prof. Joseph C. Hutchison, M.D., New York Journal of Medicine. March, 18.54.

3 Vide Maryland and Virginia Med. Jonrnal, February. 1861. For the experience of Dr. George Mendenhall, vide Western Isancet, August, 1854.

4 Vide Archives de Médecine, Aout, 1869. 
Prof. Polli, of Milan, has reported cases in which the sulphites have proved curative. It is not claimed that they arrest the disease like quinia, but that the severity of the paroxysms is progressively diminished and the disease ends in a short tirne. He recommends the sulphite of magnesia as the most efficacious, which may be taken to the extent of an ounce or an omnce and a half in the twenty-four hours, in divided doses. Several American physicians of large experience in malarious situations have reported highly successful results from the employment of the sulphite and hyposulphite of soda.' In order to test fairly the efficacy of these remedies, I.employed them faithfully, in 1868 , in twenty suecessive cases in Bellevue Hospital, an ounce of the sulphite of magnesia being given daily. A report of these cases was made, at my request, by Dr. D. C. Cliandler, then one of the house physicians of the hospital. ${ }^{2}$ of the 20 cases, in only 2 did the paroxysm cease under the use of the remedy. In 4 cases there was improvement while the remedy was continued. In 14 cases there was neither an arrest of the paroxysm nor any improvement.

So far, only the curative measures of treatment have been considered. Palliative measures are indicated cluring the paroxysms. A full opiate at the beginning of the cold stage often appears to shorten and modify the severity of the paroxysm. It sometimes, indeed, acts as an abortive measure, and may even effect a cure of the disease. I have known a sinapism applied over the whole length of the spine, at the commencement of the cold stage, arrest the paroxysm and effect a cure. This result has been obtained by dry cups applied to the spine, and by friction with the spirits of turpentine. The methor of MeIntosh, viz., bleeding in the cold stage, was sometimes effectual, but, for obvious reisons, it is not to be recommencled. A paroxysm appears to be sometimes warded off by taking to the bed before the hour when it is expected, and keeping up warmth of the body by bottles of lot water or other means. Fxternal warmth is useful in the cold stage, and stimulants may be given internally, if tolerated by the stomach.

The internal use of chloroform has been highly recommended by Dr. A. P. Merrill, formerly Professor of Medicine in the Medical College of Memphis, Tenm., now a practitioner in New York. Given in drachm doses (either alone, followed immediately with cold water, or suspended in mucilage), at the commencement of the paroxysm, it has been found to arrest the chill and. induce a refreshing sleep, from which the patient awakens without prostration. The duration and severity of the paroxysm appear to be lessened by this treatment. Dr. McClellan, Asst. Surg. U. S. Army, has reported several cases illustrative of the happy effect of this remedy. Dr. Henry Hartshorne, and Dr. D. Scott, of Iowa, have borme testimony to the value of this remedy as a prompt and sate hypnotic given in the doses recommended by Drs. Merrill and McClellan. "These doses may be repeated at short intervals if necessary.

During the hot stage, relief is procured by sponging the body with cold or tepid water. Pieces of ice, ice-water, or carbonated water may be taken freely. During the sweating stage, comfort is promoted by wiping the body with warm flannel, and changing the linen and bedclothes, taking proper care to avoid exposure to cold.

Anæmia and general dropsy, occurring as sequels, claim tonic remedies,

1 Vide Am. Jour. of Med. Sciences, April, 1867, and January, 1868.

2 Vide N. Y. Med. Record, 1869.

3 American Jourual of Medical Sciences, Jnly, 1866.

- Ibid., January, 1854, and April, 1868. 
into which the preparations of einchona and iron should enter, with nutritious rliet and other hygienic measures cilculated to restore the strength and invigorate the body. Enlargement of the spleen is to be treated witl the sulphate of quinia or other preparations of cinchona, and a belladonna plaster or anodyne embrocations applied over the enlarged organ. If these measures fail, the iodide of potassium may be tried. Dr. Maclean states that the ointment of the biniodide of mereury has, of late, in India, been found to be highly efficacious in the treatment of enlargement of the spleen following intermittent fever. The ointment is to be applied over the enlarged organ, by means of a smooth spatula, and the surface is then to be exposed to the heat of the fire as long as the smarting thereby oceasioned ean be borne. Dr. Maclean states that in soine cases where the spleen has extended down into the pelvis, it has been redueed almost to its normal size by several applications of the ointment; and he has not observed any unpleasant constitutional action in any case. ${ }^{2}$

The preparations of cinchona, and other anti-periodic remedies, are efficacious as prophylacties. Taken in small doses daily by persons exposed to inalaria, they ward off attacks of intermittent fever, and they prevent relapses." There is no foundation for the popular notion that the prolonged use of the sulphate of quinia, in small or moderate doses, is prejudicial to health. I have known an instance in which two or three grains had been taken daily for 20 years, the person liaving found by experience that by this means he was exempt from relapses of intermittent fever. Certain means of prophylaxis have been alrealy stated in treating of the causation.

\section{Pernicious Intermittent Fever.}

Simple or ordinary intermittent fever, as regards immediate danger, is not a grave disease. But the disease is sometimes presented in forms attended with more or less immediate danger, and it may prove fatal within a few days or hours. These forms of the disease are to be considered apart from simple or ordinary intermittent fever, and, collectively, they constitute a variety of the disease called pernicious, malignant, and congestive. Of these names I adopt the first, because it is the one most commonly adopted by writers. The term congestive, although commonly used in the southern and western portions of this country, is objectionable, as implying that the primary or essential pathological state is congestion, a doctrine which is probably incorrect.

Pernicious intermittent fever oceurs in isolated cases wherever the simple or ordinary variety of the disease prevails; happily, such cases are extremely rare. But it prevails at certain epochs in malarious regions. Of the cases of intermittent ferer which occur at these epochs, the proportion in which the disease is pernicious is more or less large. In some seasons the fatality from the disease is very great, and it constitutes one of the most formidable of the maladies which the physician has to encounter. The pernicious variety of the disease prevails at particular seasons in all malarious regions. In this country it lias chiefly prevailed in the Southern and Western States. Drake states that, of the interior valley of North America, the regions in which it has most frequently prevailed are, "the

I System of Medicine. Edited by Reynolds.

2 For facts establishing the correctness of this statement, see paper on "Quinine as a Prophylactic against Malarious Diseases," by Prof. W. II. Van Buren, M.D.; in "Military Medical and Surgical Essays," prepared for the United States Sanitary Commission. Philadelphia, J. B. Lippincott \& Co., 1864. 
level portions of Alabama, Mississippi, and Louisiana, the southern shore of Lake Michigan, from Chieago around to St. Joseph River, and of Lake St. Clair and Lake Erie, from Lake Huron to Lake Ontario, near the estuaries of the creeks and rivers." In view of the great danger attendant on pernicious intermittent fever, and of the fact that the suecess of prompt and efficient treatment is perhaps more conspicuous in this than in any other malady involving an equal amount of clanger, the disease is one of great importance. It will, however, suffice to notice the symptomatic features by which it is distinguished from simple or ordinary intermittent fever, together with the therapentical indications.

Different observers agree that in many, if not in most cases, a pernicious paroxysm is preceded by one or more paroxysms not characterized by any symptoms denoting gravity of disease ; hence the vast importance, especially whenever cases of the pernicious variety are known to have occurred, of resorting in every case at once to prompt and efficient measures to interrupt the paroxysms. But, in a certain proportion of cases, the pernicious character of the disease is manifested in the first paroxysm, and death may take place in that paroxysm. Drake states that the pernicious character is sometimes foreshadowed by some slight anomaly in the first paroxysms, such as, "a partial numbness or coldness of the great toes, instead of a regular chill, or a disposition to sleep at the access of the paroxysm." According to this author, in the cases in which the pernicious character is gradually developed, the cold stage in the early paroxysms is not strongly marked, the hot stage is imperfectly developed, and in the intermissions the patient may be pursuing his usual arocations. Both patient and physician are apt to be thrown off their guard by the mildness of the paroxysms preceding the one which is pernicions.

The symptomatic features of pernicious paroxysms vary much in different cases, and from these diversities writers have considered this variety of the disease as embracing several forms. More or less complete coma characterizes some cases ; these cases are distinguished as comatose, soporose, or apoplectic intermittents. In a fatal case at Bellevue Hospital, of the comatose variety, the cortical portion of the brain was found to be deeply bronzed, together with the liver and otlier organs. Frerichs and Niemeyer, however, have observed cases in which cerebral symptoms were prominent, and, after death, the capillaries of the brain were not found to denote melanæmia. Other cases are characterized by delirium preceding coma. The delirium is sometimes active, the patients requiring to be restrained. Two examples have fallen under my observation. Epileptiform convulsions occur in some cases. A form called algid is characterized by notable reduction of temperature, the extremities becoming cold as marble, or the coldness being like that of a cadaver. Profuse sweating characterizes some cases. Vomiting and purging are, not infrequently, prominent symptoms, leading to a state of collapse like that in cases of epidemic cholera. The secretion of urine is sometimes suppressed, and albuminuria is not infrequent. Of 51 cases observed by Frerichs, albuminuria existed in 20, and suppression in 5. Hemorrhage from the stomach, bowels, and kidneys is a feature of some cases. Notable embarrassment of respiration and great restlessness are sometimes marked features. The regular succession of the different stages is often wanting in pernicious paroxysms. The pulse is small, fechle, irregular. The development of well-marked febrile movement, with a full, regular pulse

\footnotetext{
1 On the Principal Diseases of the Interior Valley of North America, second series, 1854 .
} 
and heat of skin, is evidence that the patient will struggle through the paroxysm. The danger to be apprehended from another paroxysm is proportionate to the severity of that from which the patient emerges with safety; if the latter have involved very great risk, the next will be likely to prove fatal. The prostration in the intermission is in proportion to the severity of the paroxysm.

of the pathology of pernicious as compared witl simple or ordinary intermittent fever, all that can be said with our present knowledge is, the essential morbid condition existing in the latter varicty in a degree not involving inmediate danger exists in the former variety in such intensity as to prove highly dangerous. Intermittent fever is not the only affection which, withont any essential change in the nature of the disease, is in some cases extremely mild and in other cases extremely formidable. Scarlet fever may be cited as another striking example. The difference, as regards symptomatic phenomena and gravity, between pernicious and simple or ordinary intermittent fever is not explained by lesions found after death.

Pernicious intermittent fever is often preventalble, and many lives are saved by timely, eflicient treatment. It is cloubtless prevented by promptly interrupting paroxysms before they assume a pernicious eharacter. In scasons when pernicious eases prevail, there is mueh risk of lives being sacrifieed by the delay in arresting the disease, incident to the employment of the so-ealled preparatory measures of treatment. In these seasons, especially, the curative treatment should be resorted to at once in all eases of iutermittent fever, and the disease arrested as speedily as possible. When the disease has become pernicious, there are two great objects of treatment. One of these oljects is to carry the patient safely through an existing paroxysm, the other is to prevent another paroxysin.

With reference to the first of these objects, the indications differ according to the differences of form which pernicious paroxysuns assume, and according to the symptoms, whatever may be the form. Enfeebled action of the heart always calls for stimulants, alcoholic, ethereal, and aromatic, severally or collectively. In many cases the danger of the disease is manifested chiefly by the symptoms referable to the eirculation. In these cases the all-important indication is to strengthen the heart's action. Coma, unaccompanied by augmented force of the circulation and increased heat, does not call for bloodletting. Bloodletting has been employed in the cold stage of pernicious, as well as ordinary, paroxysms of intermittent fever. That patients have recovered under this treatment, by no means proves that it is devoid of danger. It is admissible only when the symptoms denote active cerebral congestion. Coldness of the surface is an indication for the external application of heat by means of warm blankets, bottles of hot water, etc., and for interual stimulants. Vomiting and purging are to be restrained by opiates given by the mouth or rectum. Opiates are also indicated by restlessness, deliriuin, and convulsions. In connection with the latter, the kidneys are to be interrogated with reference to the existence of uræmia. Emeties given at the commencement of the paroxysm, although they have been much used, are of doubtful utility, and cathartics should never be given. Chloroform, in drachm doses, as recommended by Merrill, McClellan, and others, deserves further trial as a means of abridging the cold stage. Merrill considers it a remedy of great potency in diminishing the intensity and duration of the paroxysm. The doses should be repeated until the liypnotic effect of the remedy is obtained.

The second object, viz., to prevent another paroxysm, should enter 
into the treatment before the paroxysm, attended with more or less immediate danger, is ended. With reference to this object, nothing can take the place of the preparations of cinchona. The succedanea of these preparations are not to be relied upon, if the latter are to be obtained. If the sulphate of quinia be used, it should be given during the paroxysm. It should be given in larger doses than in cases of ordinary intermittent fever, in view of the vastly greater importance of arresting the disease, and because the tolerance of the remedy is often much greater. It is to be given during the paroxysm, not for its immediate effect, but to render more sure the prevention of another paroxysm. A scruple of the sulphate of quinia may be given at once, by the mouth, to an adult, and a larger quantity if given by the rectum. After three or four hours, if there be no evidence of einchonism, the dose may be repeated. It is to be continued after the patient has emerged from the paroxysm, in doses of from ten to twenty grains, the intervals between the doses being sufficient to judge of the effect of each dose. Cinchonism should be produced and maintained during the intermission without referenee to the quantity required; but to produce this effect the enormous doses which have sometimes been given are never neeessary. Promptness and boldness are important for the suceessful management of this formidable disease, but excessive einchonism is not advisable, and it is to be borne in mind that permanent blindness and deafness have resulted from the use of quinia in excessive doses. By pursuing the plan just stated, the remedy may be employed as freely as may be neeessary to obtain, to the fullest extent, its remedial effect, without incurring much risk of injury from its toxical effect. In a case which I saw with Prof. T. G. Thomas, in 1867, in which it was eonsidered that the life of the patient depended on the prevention of another paroxysm, one hundred and twenty grains of quinia were given, in divided doses, in about twenty-four hours. 'The paroxysm was prevented, but suddenly, without having previously had symptoms of much cinchonism, he became completely blind and almost completely cleaf. As may be imagined, great anxiety was felt; but happily, at the end of eight hours both the blindness and deafness had disappeared, and the patient recovered without any further untoward symptoms.

The administration of quinia by means of hypodermic injeetions has been found effective in eases of pernicious intermittent characterized by coma and inability to swallow. It may be resorted to when, from irritability of the stomach, the remedy will not be retained if given by the mouth. Facts appear to show that for the requisite effect a much smaller quantity by the hypodermic method suffices than when the remedy is given by the mouth or rectum. This advantage, in view of the cost of the drug, is often of sufficient importance to be considered in treating ordinary intermittent fever. The hypodermic method, also, will be acceptable to some patients who have a strong antipathy to the bitter taste of quinia. The employment of this method on a large seale may lead to the knowledge of other advantages. For injection a solution of thirty grains of quinia in an ounce of water, with ten or twelve drops of sulphuric acid, may be used, the amount injected being from half a drachm to a drachm. An objection to this solution is the bulk. Lorent, of Bremen, advises a solution of thirty grains in two and a half drachms of water, the solution being effected by means of heat.: In some cases

1 For some facts and remarks on this topic, see American Journal of Medical Sciences, No. for April, 1866, p. 436 . Alsn, Archives de M d., Janvier, 1866. Also, Article on the Treatment of Malarial Fever by the Subcutancous use of the Sulphate of Quinia. By E. C. Seguin, M.D., New York Medical Journal, Dec. 1867. 
abseesses and phlegmonoid swellings have followed the hypodermic use of quinia, occurring at the situations in which the injections were made. Dr. Seguin, in his account of the experience at the New York Hospital, attributes these consequences to the presence of undissolved erystals of quinia, and particles of dust in the solutions employed. My colleague, Prof. McCready, has used in a number of cases a concentrated solution of quinia in sulphuric ether, prepared as follows: Pure quinia is dissolved in an excess of ether, and the latter is evaporated until the solution contains a drachm of quinia in two fluidrachms of ether. Two minims of this solution eontain a grain of quinia. Prof. McCrearly has not observed any unpleasant local effects from the use of this solution. If the hypodermic syringe become clogged by the use of the solution, dipping the instrument in ether speedily removes this difficulty.

A pernicious paroxysm having been experienced, at the time when another paroxysm is to be expected, provided the type be known, and on the second and third day, if the type be not ascertained, the patient should be in bed at the hour when the recurrence is likely to take place, the borly kept warm by artificial heat, and hot, stimulating drinks. An opiate will render more sure the prevention of the dreaded paroxysm.

After the interruption of the paroxysms, some one of the preparations of cinchona should be continued in tonic doses, with a nutritions diet and other restorative measures. After both the pernicious and ordinary variety of intermittent fever, if the malarious cachexia remain in spite of appropriate measures of treatment, remoral without the sphere of the malarious influence, during the season when this influence is rife, is to be advised.

\section{CH A P T ER V.}

Periodieal Fevers Coneluded. Simple Remittent and Typho-Malarial Fever-Anatomical Characters-Clinlcal History-Causation-Dlagnosis-Prognosis-Treatment. Pernleious Remittent Fever. Yellow Fever-Anatomical Characters-Clinical History-Causatlon-DlagnosisPrognosis-Treatment.

A FORM of periodical fever is distinguished from that considered in A the last chapter, viz., intermittent fever, by the occurrence of remissions instead of intermissions, and, hence, called remittent fever. It is alsa known as bilious remittent or bilious fever. The term bilions is superfluous, and, so far as it has any significance, tends to perpetuate a pathological idea, the correctness of which is, to say the least, problematical. It is desirable, therefore, that the term be dropped. Between simple remittent and intermittent fever there is a close relationship. These two forms of fever are mutually convertible into each other, they undoubtedly involve the same special cause, and they are controlled by the same speeific remedies. The propriety of recognizing remittent as distinct from intermittent fever is denied by sqme writers. Bonillaud calls it a " real nosological superfœetation!" "There is, however, a practical convenience in considering the two forms as separate affections, admitting that they are essentially identical. 
Remittent fever and typhoid fever may be associated. Not only may the former present typhoid phenomena or the typhoid state, but the two affections occur not infrequently in combination. This statement embodies an opinion held for a long time by practitioners in malarious sections of this country and inculcated especially by Prof. Dickson. The late Dr. Drake maintained this opinion, calling the combined affection remitto-typhus fever.' I have for twenty-five years advocated this opinion in medical teaching. More recently it has been proposed to employ a name denoting the mion of the two affections, and the term typhomalarial fever has been introduced by Dr. Joseph J. Woodward.2 I shall adopt this name, and consider remittent and typho-malarial fever conjointly. The facts which show the blending of remittent and typhoid fever will be noticed under the head of pathological character and causation.

\section{Simple Remittent and Typho-Malarial Fever.}

$\mathrm{By}$ the term simple remittenit fever is meant remittent, not associated with typhoid fever, and not accompanied by symptoms of gravity entitling it to be called pernicious. Pernicious remittent fever will claim a brief, separate notice. Typho-malarial ferer will be considered in conjunction with simple remittent fever.

Anatomical Ciraracters. - The lesions in simple remittent fever, which, from their constancy, may be considered as essential, are found especially in the liver and spleen and in the blood. The liver is more or less softened, and presents a bronze, chocolate, or slate color. This change in color was pointed ont as an anatomical characteristic of the disease by Dr. Thomas Stewardson. ${ }^{3}$ Facts confirmatory of the correctness of the observation by Stewardson, have been communicated by Prof. Alfred Stillé, and the late Dr. Swett. ${ }^{4}$ The condition is described by Frerichs under the name pigment liver, and it has been already referred to in treating of hepatic disenses. Frerichs thinks that the pigmentary matter is contained, not in the hepatic cells, but in the capillary network of the portal and hepatic reins, and, also, sometimes in the branches of the hepatic artery. According to Virchow, the liver cells contain pigment. In a specimen from a case at Bellerue Hospital, examined by Dr. Francis Delafield, one of the curators of the Hospital Museum, who kindly furnished me with drawings of the microscopical appearances, the pigment was contained in the liver eells, and also in the hepatic and portal vessels. A discoloration of the surface of the liver resembling that of the bronzed liver is sometimes caused by the action of the intestinal gases in cases of peritonitis from perforation. In these cases the coloration does not extend below the surface. In cases of remittent fever, the blood contains black granules and dark-colored cells, to which reference has been made in Part I. of this work under the head Melanæmia. The spleen is more or less enlarged, softened, and is rendered dark, or black, by pigmentary deposit. Bronzing of the brain and spinal cord is found, sometimes in a marked degree, in cases of malarial fever, the coloration being due to the presence of pigment both within and without the vessels of the gray sub-

1 Vide The Principal Diseases of the Interior Valley of North America, vol. ii. p. 557.

2 Vide Outlines of the Chief Camp Diseases of the United States Armies, etc. By Joseph Janvier Woodward, M.D., Assistant Surgeon U. S. Army, etc., 1863.

3 Vide Am. Journ. of Med. Sciences, 1841 and 1842.

Ibid., 1846. 
stance. The question arises whether the pigmentary deposit in the viscera named occurs in other than malarious diseases. Dr. J. Forsyth Meigs failed to find it in cases of cholera, typhus and typhoid fever, Bright's disease, jaundice, cirrhosis, cancer, fatty degeneration of the liver, alcoholism, white cerebral softening, and disease of the heart." It remains to be determined whether it is not sometimes observed in affections other than those just enumerated. In two cases in which more than a year before death remittent fever had existed, the liver, inspected by Prof. A lonzo Clark, presented the characteristic bronzing, although less intense than in recent cases. From these cases it may be inferred that the pigmentary deposit is very slowly removed. The appearanees of the mucous membrane of the stomach in some cases denote subacute inflammation. Enlargement of the glands of Brumner, or the mucous follicles of the duodenum, were observed by Stewardson, and in twelve cases noted by Anderson and Frick, which were analyzed by Stillé. Softening of the muscular walls of the heart has been frequently observed. Lesions denoting meningitis and puemmonitis are occasionally observed, but these and other affections are to be regarded as complications.

In typho-malarial ferer, in addition to appearances characteristic of malarial disease, the abdominal lesions which are essentially those characteristic of typhoid fever are found after death.

Ceinicar History.-Under this head I shall present a brief sketch of simple remittent fever, as regards especially its distinctive features, and, afterward, of typho-malarial fever.

Simple remittent fever often commences abruptly, but in a certain proportion of cases, has a brief forming stage, the prodromes being the same as those attending the development of other ferers. 'The febrile career commences with a chill, more or less pronounced, either with or without rigors. During the chill, as in the cold stage of an intermittent paroxysm, the temperature of the body is actually raised, as shown by the thermometer in the axilla. The attack is oftener before than after mid-elay. Febrile movement follows, varying in intensity in different cases, accompanied with eephalalgia, pain in the loins, etc. The thermometer in the axilla shows an increase of temperature from $2^{\circ}$ to $10^{\circ}$ above the range of liealth. The febrile movement continues unabated for $6,12,18,24$, and even 48 hours, and then notably subsides; the pulse falls in frequency, the skin becomes moist, the patient is comparatively confortable, and may obtain refreshing sleep. The febrile movement, however, does not disappear, there is not apyrexia; there is a remission, not an intermission. 'The temperature in the remission, as compared with the exacerbation, undergoes a notable decrease, sometimes falling nearly to the normal standard. The remission is apt to occur during the night. The degree of abatement of febrile movement varies in different cases, sometimes approximating to apyrexia, and sometimes the remission being slight. The duration of the remission varies from two or three hours to one or two days. At the end of the remission, another chill may occur, but it is often wanting, and, if present, is generally slight; the febrile movement is renewed with as much or more intensity than before the remission. A series of remissions may ensue, recurring in regular succession, and, as regards the intervals, corresponding to the quotidian, tertian, or, frequently, the double tertian type of an intermittent ferer. I have observed

I Penn. Hospital Reports, rol. i. 1868. 
a case in which the double quotidian type was represented by the remissions, that is, two distinct exacerbations occurring daily. The remissions sometimes recur irregularly. Different cases differ as regards the number of remissions. There may be but a single remission, or, on the other hand, remissions may occur regularly through the whole course of the disease. The latter is rare; in general, the remissions cease to be distinct after a few days, and, sooner or later, the fever becomes continuous. The febrile career ends during the second or third week. It eventuates in intermittent fever in a certain proportion of cases. In some cases, the disease is preceded by intermittent fever, remissions taking the place of the intermissions. During the remissions there is a marked abatement of the temperature of the body, the temperature during the exacerbations being notably increased. In this respect remittent ferer differs, in a striking manner, from typhoid ferer exclusive of the period of defervescence in the latter disease. The fall in temperature is sometimes evidence of remissions when they are not rendered very apparent by the general symptoms.

Early in the fever, nausea and vomiting generally occur, and are frequently prominent symptoms, continuing more or less througlout the febrile career. The matter vomited is greenish or yellowish in color. Pain or uneasiness is usually referred to the region of the stomach, and there is tenderness on pressure over the epigastrium. Diarrhoa, tympanites, and iliac tenderness are wanting in the majority of cases. Sordes is rarely observed. Delirium is rarely a prominent feature, and, when present, is due to the intensity of febrile movement. The urine is scanty, its coloring matter is increased, and its specific gravity is high. It is rarely albuminous. Jaundice occurs in a certain proportion of cases.

If simple remittent fever be protracted, certain symptoms denoting the typhoid state may appear, viz.; low delirium, sordes, subsultus tendinum, etc. These symptoms are not sufficient to show the union of typhoid fever and remittent fever. They are liable to occur in all fevers and in various other affections.

In typho-malarial fever, the symptoms distinctive of typhoid fever are intermingled with those of periodical fever. The symptoms referied to are those connected with the abdominal lesions of typhoid fever, viz., diarrhca, tympanites, and iliac tenderness. These symptoms become more or less marked during the second week of the career of the disease. The characteristic eruption of typhoid fever is sometimes observed. The ataxic symptoms belonging to the typhoid state, viz., low delirium, deafness, subsultus tendinum, etc., occur more frequently, earlier, and are more marked than in connection with simple remittent fever. Enlargement and suppuration of one or both parotids are sometimes observed in cases of typho-malarial fever. This form of fever is preceded by a forming stage longer than in cases of simple remittent fever. The duration of the febrile career is apt to be longer, and the gravity of the disease is greater. Perforation of the intestine is liable to occur. Bronchitis is a common complication, and pneumonitis is developed oftener than in simple remittent fever. Convalescence is apt to be protracted in cases of typho-malarial fever. In different cases of the latter, the phenomena of typhoid fever and of periodical fever are intermingled in varying proportions ; in some cases the periodical, and in other cases the typhoid, phenomena preponderate. According as the phenomena of the one or the other disease predominate, cases will approximate, on the one hand, to simple remittent, and, on the other hand, to typhoid fever. 
Pathologidal Character and Causation.-Simple remittent fever, as regards its essential pathological character, does not differ from intermittent fever. Both are clue to the special poison known as malaria. True remittent fever is never contracted elsewhere than in malarious situations. The distinction between the two diseases is nosological rather than pathological.

Typho-malarial fever is caused by the combined action of malaria and the special cause of typhoid fever. Practitioners in malarious situations have been accustomed to say that remittent becomes eonverted into typhoid fever. This mode of expression is not accurate; there is not a metamorphosis of the one disease in the other, but a combination of both diseases, the phenomena of the one or of the other disease predominating in different cases.

Cases of typho-malarial fever occur in most, if not all, malarious regions. This fact shows that the special cause of typhoid fever is not held in abeyance by the prevalence of malaria, and generated only after the latter disappears. The extinetion of malaria is followed by cases of unmixed typhoid fever, and hence it has appearod as if the latter follows in the wake of the periodical fevers. The typho-malarial fever prevailed largely among the United States troops in certain situations, during the recent civil war, especially in the Army of the Potomae, in the Peninsular campaign of 1862 , when it was known as the Chickahominy fever. It was called elsewhere, during the war, camp fever.

Simple remittent and typho-malarial fever prevail especially in the antumnal season. Persons of all ages are liable to be affected with simple remittent fever. There is no evidence that this fever is ever contagious, nor that the malarious poison producing it is portable. Having once experienced it affords no protection against its recurrence.

Diagnosis.-Simple remittent fever is readily discriminated from simple intermittent fever by the occurrenee of remissions, instead of intermissions, and by the fever generally becoming continuous. It differs from continued fever (typhus and typhoid) in the occurrence of remissions, but these are liable to be overlooked, and cases sometimes come under observation after the remissions have ceased to recur. The thermometer in the axilla may show the occurrence of remissions when these are not distinctly manifested by symptoms aside from the temperature of the body. A notable variation in temperature at different periods of the day in the early part of the disease, the mercury rising to a high point at one period and falling nearly or quite to a point within the range of health at another period, warrants the exclusion of typhoid fever. Exclusive of remissions, the differential diagnosis involves the following points :-

The abdominal symptoms of typhoid fever are wanting in simple remittent fever, and in place of these are the gastric symptoms distinctive of the latter, viz., nausea and vomiting, with tenderness over the epigastrium. The ataxic symptoms of the typhoid state are oftener wanting in simple remittent fever, and, if present, occur at a later period. The typhoid eruption is wanting. The access is much oftener abrupt, and aceompanied by a more pronounced chill. Paroxysms of intermittent fever are liable to occur at the close of a remittent fever. Finally, remittent fever is developed only in malarious distriets, or in persons who have been exposed to malaria. There can be no question as to this differential diagnosis in cases of disease occurring in a situation where malaria is known not to exist, and when the patient is known not to have been within a malarious influence. 
Typho-malarial fever is discriminiated, on the one hand, from simple remittent fever by the characteristic events of typhoid ferer, viz., the abdominal symptoms, the eruption in some cases, the earlier occurrence of ataxic phenomena, epistaxis, and occasionally intestinal perforation. It is discriminated, on the other hand, from unmixed typhoid fever by the characteristic.events of periodical fever, viz., remissions, gastric symptoms, jaundice, and the eventuation in some cases in intermittent fever.

Prognosis.-Simple remittent fever, as a rule, is attended with very little danger to life. In fatal cases, the termination is generally due to coexisting affections. Of course, it is to be understood that cases of pernicious remittent fever are not included under the head of simple remittent fever. An attack of remittent fever leares behind it a liability to subsequent attacks of intermittent fever, and is liable to be followed by the sequels of the latter disease, viz., enlarged spleen, anæmia, and general dropsy. This disease differs much in severity at different times and places, and in different eases at the same time and place. As a rule, it is apt to be more severe in tropical than in temperate climates.

Typho-malarial fever is a much graver affection than simple remittent ferer; it is certainly not less grave than typhoid disconnected from malarial fever. Like the latter, the rate of mortality differs at different times and places, owing to differences as regards the intrinsic tendency of the disease and a variety of circumstances. Data for determining the average death-rate are not available, inasmuch as it has not been customary to separate cases of this disease from cases, on the one hand, of simple remittent fever in which the typhoid state occurs, and, on the other hand, of typhoid fever without the combined action of malaria.

Treatment.-The first and leading object in the treatment of simple remittent fever is the arrest of the disease by anti-pericdic remedies, of which the preparations of cinchona are by far the most reliable, the sulphate of quinia being the preparation to be preferred. As soon as the character of the disease is determined by the occurrence of a remission, the sulphate of quinia should be given in a full dose, viz., from ten to twenty grains to an adult. The remedy should be continued in doses of from five to ten grains after intervals of from two to four hours, until it produces slight deafness or ringing in the ears, suspending the remedy when these manifestations of cinchonism appear. When other preparations of cinchona are used, they are to be given, in equivalent doses, in the same manner. If the remedy be not tolerated by the stomach, it should be given per enema. If einchonism be not produced during the remission, the remedy may be continued during the exacerbation of fever. This is preferable to waiting for another remission. In a case in which the practitioner is satisfied that a remission has already occurred, in other words, whenever the diagnosis is clear, the remedy should be given at once, notwithstanding the intensity of febrile movement, without waiting for a remission. Time need not in any case be lost in order to resort to cathartics or other measures preparatory to the exhibition of an anti-periodic remedy. In short, the treatment is essentially the same as in cases of intermittent fever. And this plan of treatment will succeed, in a large proportion of cases, in promptly arresting the disease.

Aside from the abortive or curative treatment just stated, palliative measures are to be employed according to the indications in individual cases. The indications will relate to pain in the head, nausea, and vomiting, vigilance, and restlessness, heat and dryness of the skin, etc. The 
same measures are to be addressed to these symptoms as when they are present in other fevers. It is unnecessary to consider these measures in detail in this connection.

If the disease be not arrested and the febrile movement become continuous, the principles of treatment are essentially the same as in the continued fevers. Anti-periodic remedies should be continued in moderate doses. Supporting measures are indicated by symptoms denoting failure of the vital powers, precisely as in other fevers. If the disease eventuate in intermittent fever, the latter is to be treated as when it occurs under other circumstances. The treatment during convalescence offers nothing peculiar, except that a preparation of cinchona should be employed for some time, in conjunction with a chalybeate, as after an attack of intermittent fever.

The plan of treatment which has been sketched does not embrace measures heretofore considered important in this clisease, viz., emetics, cathartics, bleeding, and the use of mercury. Emetics are contra-indicated by the condition of the stomach. Cathartics are not indicated by the disease per se; they are required only to overcome constipation, and generally simple enemata or mild laxatives will suffice for this object. Bleeding is called for only by active eerebral congestion or intense febrile movement with augmented power of the heart's action, the patient being robust and plethoric. Under the latter circumstances, sedative remedies, saline laxatives, and cold to the surface will generally be sufficient without the abstraction of blood. The external use of cold water is highly useful when the skin is notably hot and dry. The wet sheet may be employed as in eases of typhoid fever. Opium, given in pretty full doses early in the disease, appears sometimes to exert an effect beyond that of a palliative, by inducing a more marked remission. The pathological views which formerly led practitioners to employ mercury freely in this disease are not tenable, and it may fairly be doubted if elinical observation afford any ground for regarding this remedy as specially indicated.

In typho-malarial fever the periodic element claims the treatment indicated in simple remittent fever. The object is to eliminate this element by means of anti-periodical remedies. A side fiom this object, the hygienie and medicinal measures inclicated are the same as in cases of unmixed typhoid fever. 'To consirler these measures here would be to repeat what has been presented in the chapter in which the treatment of typhoid fever is considered. The reader is, therefore, referred to that chapter.

\section{Pernicious Remittent Fever.}

A brief notice of pernicious remittent ferer will suffice. This ferer is distinguished as pernicious under cireumstances similar to those which constitute grounds for the application of the same name to intermittent fever, viz., circumstances gi ving to the disease unusual gravity and danger. This variety of the disease is also called malignant and congestive. Pernicious remittent fever presents the same diversities, as regards symptomatic phenomena, as pernicions intermittent fever. In eases in which death takes place quickly, it is impossible to determine whether the disease be intermittent or remittent; a fatal result may take place before either a remission or an intermission occurs. The occurrence of an intermission or a remission is the chief differential point. Pernicious remittent fever, however, is attended with more danger than pernicious intermittent fever. The two forms of disease, in a pathological view, are essentially; the same, and they claim the same treatment. The importance of 
pernicious remittent ferer is by no means to be measured by this brief notice of it; but inasmuch as all that is of practical importance in relation to this disease is embraced in the consideration of pernicious intermittent fever, the reader is referred to that portion of the preceding chapter which treats of the latter.

Remittent fever, especially the pernicious variety, has received, in different parts of the world, a variety of names denoting its geographical relations, such as Walcheren fever, African fever, Hungarian fever, jungle fever, lake fever, etc.

\section{Yellow Fever.}

The fever to be now considered has received a great variety of names, but it is at this time everywhere known as yellow fever, a name, the sig. nificance of which is derived from the frequent occurrence of yellowness of the surface of the body. Although the name is open to criticism on the score that it relates to a symptom not constantly present, and occurring occasionally in other fevers, it has the merit of not involving any hypothesis concerning the nature or causation of the disease.

'The question whether this be a distinct species of fever has been much mooted. It has becn considered as identical, on the one hand, with typhus or typhoid, and, on the other hand, with remittent fever. Cullen applied to it the name typhus icterode. Grisolle calls it typhus d'Amérique. Now that typhus and typhoid fever have been so thoroughly studied, it is evident that differenees, as regards the symptoms and laws, show these fevers to be entirely distinet from yellow fever. Few, if any, at the present time, contend for the identity of yellow fever with either of these fevers. The doctrine that yellow fever and remittent fever are identical, in other words, that both originate from the special cause commonly known as malaria, has had more tenacious adherents, and this doctrine is not, at the present time, utterly abandoned. That these two diseases may exist in combination is undoubtedly true. It is also true that remittent fever, at certain times and places, assumes more or less of the characters of yellow fever, so that the two affections are not always easily discriminated, and that much confusion has arisen from their having been not infrequently confounded. But that they are essentially different diseases, each being a distinct species of fever, is conclusively shown by the following facts :-

1. Yellow fever, as a rule, prevails in towns; whereas, remittent fever prevails especially in rural districts.

2. Yellow fever has been known to prevail in places where neither remittent nor intermittent fever was ever known to occur; and, per contra, remittent fever prevails to a great extent and in numerous places where yellow fever has never been known to occur.

3. There is abundant ground for the belief that the special cause producing yellow fever is portable, and that the disease is thus liable to be imported. There is no evidence of the importation in this way of remittent fever.

4. Remittent fever is characterized by the occurrence of remissions, whence is derived the name of this disease, and it is apt to eventuate in intermittent fever. Yellow fever is devoid of remissions, and does not end in intermittent paroxysms.

5. Yellow fever attacks chiefly unacclimated persons. Acclimation does not protect against remittent fever. 
6. An attack of yellow fever exempts from a subsequent attack in the immense majority of cases. This is not true of remittent fever.

7. The remedy or remedies, exerting a controlling influence over remittent fever, which may be called specific, viz., the preparations of cinchona and other anti-periodies, do not control yellow fever. 'This statement is not inconsistent with the belief that these remedies are more or less useful in yellow fever, as they doubtless are in many diseases which they do not control.

8. They who contend that yellow fever is a form of remittenit fever, regard it as a severe or malignant form of the latter. But during the prevalence of yellow fever a liost of cases occur in which the discase is milder than even mild cases of remittent fever.

Other facts relating to differences as regards the symptomatology and laws of the two diseases might be addueed. The foregoing, however, it is believed, are quite sufficient to establish their non-identity. Assuming, therefore, yellow fever to be essentially distinct from all otler diseases, I shall proceed to consider, succinctly, its anatomical characters, clinical history, causation, diagnosis, prognosis, and treatment.

Anatomiont Cinaracters.-The morbid appearances after death which are most frequent are situated in the stomach, alimentary canal, and liver. Antopsical examinations made by Louis and Troussean, at Gibraltar, Nott, of Mobile, Thomas Hewson Bache, of Philadelphia, and many others, show that morbid appearances in the stomach are frequent, consisting of redness, softening of the membrane, thickening, and the conclition known as mamellonated. The morbid appearances are not uniform, and in a certain proportion of cases there are no morbid appearances in this organ. The appearances canıot be considered as always denoting gastritis. Some of the appearanees are doubtless cadaveric. The stomach often contains more or less of the matter vomited, which is known as the black romit. This will be noticed in connection with the gastric symptoms, under the head of the clinical history. The intestinal canal in some cases presents no morbid appearances. Redness in patches, ecchymoses, and softening are occasional morbid appearances. Similar appearances are sometimes found in the osophagus. The Peyerean and solitary glands of the small intestine, as a rule, present no morbid alteration. 'i'he intestines contain black matter similar to that found in the stomach.

The liver was found by Louis and Trousseau in the epidemic at Gibraltar, which they studied in 1828 , to present a morbid appearanee somewhat peculiar. 'The color' was unnaturally yellow, compared to the color of butter, coffee and milk, or mustard. This being the only constant morbid appearance found by these observers, they were disposed to attach to it considerable importance, regarding it as the anatomical characteristic of the discase. Subsequent observation has shown that this coloration, althougl existing in a large proportion of cases, is not constant. Prof. Alonzo Clark, in 1853, was led to observe "a fatty state of all the secretory epithelial cells, and an abundance of free fat globules" in the liver of a subject dead with yellow fever, and he raised the inquiry, "Is not the change so constantly observed in the livers of those dying of yellow fever an acute fatty degeneration?"' Since this observation by Prof.

1 Vide La Roche on Yellow Fever, 18īis. The author would here acknowledge his constant indebtedness to the great work of La Roehe, in treating of Yellow Fever. ' $l$ 'he reader is referred to this work as a mastcrly digest of the whole vast literature of the subject. To save frequent references, the autlior would also here 
Clark, an abundant fatty deposit in the liver has been found by Dis. T. H. Bache and Leidy in numerous cases in the Pennsylvania Hospital. Riddell, of New Orleans, found this condition existing in a certain proportion of cases. The pathological significance and importance of the change in color and the abnormal amount of fat, cannot be explained with our existing knowledge. 'T'he spleen is sometimes enlarged and softened, and in some cases unaltered.

'The brain and its membranes present nothing distinctive; the same variations, as regards the quantity of blood and serous effusions, are found in this as in other affections. 'This statement applies also to the spinal cord and its membranes. In the pulmonary organs black patches, and, in some instances, solid dark masses, have been found by different observers; these appearances, by some of the older writers, were described as gangrenous spots ; they denote extravasation of blood, or pulmonary apoplexy, due to the hemorrhagic tendency manifested in the clinical history of this disease. The kidneys in a certain proportion of cases present nothing abnormal; they are sometimes congested, extravasations of blood have occasionally been observed, and sometimes matter resembling pus.

This disease has no known special anatomical characters; the lesions which occur are eontingent or inciclental. We cannot, therefore, with our present knowledge, look to morbid anatomy for a satisfactory explanation of the phenomena observed during life.

Clinical History.--An attack of yellow fever is usually abrupt. In a minority of cases it is preceded, for one, two, or three days, by languor, lassitude, loss of appetite, pain in the head, and chilly sensations. The attack is generally denoted by a chill, with or without rigors; the chill, in most cases, is of moderate intensity. Febrile movement succeeds. The febrile movement varies in intensity in different cases; it is sometimes quite intense, oftener inoderate, the heat of the surface rarely being much raised, and the pulse seldom exceeding 100. The tongue is moist, and more or less coated. Vomiting occurs in a certain proportion of cases early, but it rarely is a prominent symptom until a later period. It is less prominent at an early period than in most cases of remittent fever. Tenderness on pressure over the epigastrium is more or less marked. The bowels, as a rule, are constipated. Cephalalgia, the pain especially referred to the supra-orbital region, is usually present and is sometimes intense. Frequently pain in the loins, and pain in the lower limbs, especially in the calves of the legs, are prominent symptoms. This fever bears an analogy to smallpox in the frequent prominence of lumbar pain. The mind is usually clear, but occasionally delirium is manifested, rarely violent or active, and sometimes mirthful. The eyes are reddened, irritable, watery or tearful; this is quite constant, and so marked as to constitute a diagnostic feature. Otherwise, the organs of sense are not disturbed. In general, there are no pulmonary symptoms.

The febrile movement continues for a period varying from a few hours to three days. Then follows either a marked abatement or, generally, entire cessation of fever. A remission is said to take place; but this term is not accurate, inasmuch as there is generally complete apyrexia, and, in a certain proportion of cases, there is no return of febrile movement. The condition following the febrile paroxysm has been called "the state

express acknowledgment especially to the following authors : Bartlett and Clark, Dickson, Fenner, Dowler, Simons, Reviewer in Brit. and For. Med.-Chir. Review, 1847, Mitclel, Barton, and Wragg. 
of calm." This is to be reckoned as the second stage of the disease, the first stage embracing the period of the febrile movement, the latter called "the stage of reaction," the "febrile period," the "stage of excitement," or "the paroxysm." "The duration of the first stage, in different cases, is very variable, and the average cluration is found to vary in different epidemies. Dickson considers this stage as constituting the disease proper, and whatever may follow, as sequels of the disease; he calls the disease a fever with a single paroxysm. In mild cases, convalescence takes place at the end of this stage.

In grave cases the cessation of febrile morement is deceptive; the pains, etc., may cease, and the patient may feel as if convalescence had begun. But after a period, varying from a few hours to 24 hours, or longer, new symptoms become developed, more distinetive of the affection than those occurring cluring the febrile paroxysm. The pulse in this stage sometimes falls below the normal frequeney; it has been observed to fall as low as 40, and even 30 per minute. It is sometimes small and weak, and sometimes vibratory. It is always notably compressible, and has been called a "gaseous pulse." The characters show, in a marked degree, deficient power of the circulation. The surface is usually cool, especially of the parts of the body which are exposed. In the few cases which I have noted, capillary congestion of the skin existed in a marked degree. Lividity of the back, attributable to hypostatic congestion, has been observed by Simons.

of the symptoms referable to the digestive system, those most characteristic pertain to the stomach. Vomiting occurs, if not already present, and, if present, becomes more prominent. In a large proportion of fatal cases, and in a few of the cases ending in recovery, the black romit occurs. This, taken in connection with other symptoms, is pathognomonic of the disease. Heretofore there has been much discussion respecting the nature of the vomited matter, but it has been abundantly settled, by chemical and microscopical examinations made by different observers, that the characteristic appearance is due to blood changed by the action of the gastric fluids. As regards the gross appearances, the matter vomited is a thin liquid of a reddish, brown, claret, or blackish color, with sediment resembling coffee-grounds. Occasionally the liquid is bright red, the blood having undergone but little change. It rarely, if ever, has a greenish or yellowish tinge from the presence of bile. The secretion is acid, and it lias an acid taste without any bitterness. It is sometimes acrid, excoriating the throat, tongue, and lips. The microscope shows the sediment to contain mucus, epithelim, hematoidin, and deformed blood-disks. The black vomit may be produced artificially by adding an acid to blood out of the body. The bilious matter vomited in some cases of remittent fever presents very similar gross characters, but chemical and microscopical examination in these cases show the presence of bile and the absence of blood constituents. The black vomit in different eases of yellow fever is more or less abunclant. It is sometimes ejected with force, and sometimes by an act of regurgitation rather than of vomiting. It generally ceases for a period of from 12 to 24 hours before death. Vegetable formations are sometimes found in the matter vomited. The black vomit rarely oceurs until the first stage is passed; it oceurs at variable periods during the second stage, but generally not until toward the close of the disease. Blair describes a matter romited prior to the black vomit, limpid or slightly opalescent, which he terms the "white vomit." Tendelness over the epigastrium is more or less marked in the second stage, ind the tenderness is sometimes extreme. The tongue is frequently 
reddened, dry, and cracked; in some cases it is moist and covered with a creamy coating; and sometimes it preserves its natural appearance.

During the second stage, the evacuations from the bowels are frequently of a brownish, approaching to a black, appearance; they resemble sometimes tar or molasses. This appearance is due to the presence of blood, so altered, however, in its passage through the intestinal canal that its morphological characters are not demonstrable. The dark color is due to hematin or hematoidin, discoverable on microscopical examination. Certain constituents of the urine, viz., uric acid and the triple phosphates, are found in the evacuations from the bowels; sometimes blood but little altered is passed from the bowels. Diarrhoa in this, as in the first stage, is rare. The abdomen is soft and seldom meteorized. The melænic discharges generally precede the occurrence of black vomit; they have been called the black romit stools.

Important symptoms referable to the urine have only of late enlisted much interest, for the reason that full knowledge of the significance of urinary changes is of recent date. The frequent occurrence of albuminuria in grave or fatal cases has been pointed out, particularly by Blair.: Blair's statement that almost invariably albuminuria exists when the disease ends fatally is based on examinations in more than five hundred fatal cases. Porcher found that this statement did not hold good in an epidemic at Charleston, South Carolina; but that albuminuria is of frequent occurrence in grave cases, and is to be regarded as evidence of the gravity of the discase, appears to be sufficiently established. Casts of the renal tubuli and disintegrated renal epithelium are sometimes found in the sediment of the urine. A scanty secretion of urine or suppression not infrequently precedes a fatal ending. Uræmia doubtless occurs in a certain proportion of cases. The urine in certain cases becomes of a yellow or orange color, and it is sometimes bloody. Further clinical study of the urine in this cliscase, by means of chemical and microscopical examination, is a desideratum.

Yellowness of the surface of the body, whence the disease derives its name, oceurs in the second stage. The conjunctiva becomes yellow, and this, with the redness, gives to the eye a striking and peculiar appearance. The yellowness of the skin is especially marked on the chest and upper extremities. The yellowness is doubtless due to biliverdin. It exists in only a certain proportion of cases; it occurs in a much larger proportion of fatal than of non-fatal cases -in fact, it occurs in a small proportion of the latter. This symptom, although by no means constant, is quite characteristic of the disease, occurring very rarely in the continued and malarial fevers. The yellowness continues after death and into convalescence.

Hemorrhage in various situations, other than the stomach, intestines, and the kidneys or bladder, to which reference has been already made, is often a striking feature of the disease. It occurs from the nostrils, gums, uterus, wounds or abrasions of the skin, and sometimes from the eyes, meatus auditorius, finger nails, holes bored in the ears for ear-rings, etc. Petechi:e and vibices are sometimes observed.

As regards symptoms referable to the nervous and muscular system, coma and convulsions are of occasional occurrence. They are probably dependent on uræmia. Delirium is observed, but frequently the mind, in the second stage, remains clear. Delirium may exist in the first, and dis-

1 Report on the Yellow-Fever Epidemic of British Guiana, by Daniel Blair, M.D., British and Foreign Medico-Chirurgical Review, 1850 ; vide Bartlett's work, edited by Clark. 
appear in the second stage. As a rule, when it occurs after the first stage, it is toward the elose of life. Generally there is notable muscular prostration, but to this rule there are remarkable exceptions. Patients sometimes do not take to the bed, but keep about their usual avocations, not thinking themselves much ill, often a few hours before death. These have been called "walking cases." Rush relates a case in which the patient stood up before a glass and shaved himself on the day of his death. In a case cited by Bartlett, the patient, a soldier, continued to do duty until black vomit took place. In another case, the patient dictated and signed a letter a quarter of an lour before deatl. Cartwright gives an instance of a shoemaker who nearly finished a shoe the day before his death. Fenner has seen a patient in the New Orleans Charity Hospital quietly reading a book after the black vomit had oceurred. 'These eases exemplify a kind of cheerful delirium, as it is called by La Roche, in which the patient fancies himself well or but little ill.

The physiognomy of patients affected with this disease is described by different writers with a good deal of metaphor, but nearly all state that it is characteristic. The face is flushed, or presents a red coloration. The appearance of the eyes is described by different witers as injected, brilliant, transparent, fiery, and glassy. To the facies is applied the following terms : suffering, dejection, anxiety, anguish, despair, terror, stupidity, vacancy, astonishment, sullenness, etc. The diversity of these terms raises a suspicion that the peculiarity of the physiognomy is less distinetive than observers have generally supposed; at all events, it appears to be difficult to convey a clear idea of the characteristic appearance by language.

The duration of the second stage is very variable; it varies from twelve hours to three or four clays. In cases of great gravity, the cluration is brief, the disease ruming rapidly to a fatal termination.

The third stage, in fatal cases, is sometimes called the stage of collapse, or the stage of exhaustion. The collapsed condition is denoted by prostration, feebleness and irregularity of the pulse, coldness of the extremities, low delirium in some cases, and the intellect remaining elear in other cases, mental indifference or apathy generally existing as in cases of epidemic cholera-these symptoms showing adynamia and tendency to death by asthenia. Coma and convulsions sometimes occur in this stage, attributable, probably, to uræmia. In some cases this stage is characterized by great restlessness, jactitation, and general distress; but in other cases the patient remains quiet and seems free from suffering.

If death do not take place, the third stage is the stage of convalescence. When the discase passes to the second stage, and presents the grave symptoms belonging to this stage, viz., black vomit, hemorrhage, ete., the recovery is always slow, and is frequently preceded by fever having more or less of the phenomena of the typhoid state. Relapses sometimes occur after convalescence appears to be declared.

The disease is divided by different writers into varieties accorcling to differences as regards gravity and other circumstances. Some writers make numerous varieties. A division, arbitrary, but convenient from its simplicity, is into simple or mild, inflammatory, and maliguant. In the simple or mild variety, the disease ends in convalescence directly after the paroxysm of fever. Whenever an epidemic prevails, there is a certain proportion of cases which are devoid of any gravity. In these cases a slight chill occurs, followed by mild febrile movement continuing for one or two days, and recovery quickly takes place. These eases would not be reeognized as cases of yellow fever, except from the fact of their occurrence during the prevalence of an epidemic. When this mild form of the 
disease occurs sporadically, it is not infrequently a matter of doubt whether patients have had the disease or not.

The inflammatory variety is distinguished by the intensity of the febrile movement. 'The term inflammatory, however, as applied to these cases, is not correct, inasmuch as the intensity of the febrile movement is not due to inflammation, but to the fever per se. Malignant cases are those characterized by black vomit, or hemorrhage in other situations, followed by collapse or exhaustion, and also cases in which coma or convulsions occur.

The duration of the disease is variable. Shortness of its career is a distinguishing feature. In the shortest cases, death or convalescence takes place in from two to three days. According to La Roche, the duration varies from three to nine days. The average duration is less than a week.

Causation.-The peculiar features of this disease, its limitation within certain geographical boundaries, and its occurrence as an epidemic, are grounds for the inference that it requires for its production a special cause. And the question at once arises, does the special cause emanate from the bodies of those affected with the disease; in other words, is the disease contagious? This question has given rise to much discussion. Volumes have been written by contagionists and non-contagionists in defence of the two opposing doctrines. It is evident that the question is one of great importance in its bearing on commerce and quarantine laws, as well as on the precautionary measures respecting exposure in visiting or attending upon those affected with the disease. For a full consideration of this topic the reader is referred to works treating in extenso of yellow fever, and especially to the elaborate and admirable treatise by La Roche. I shall simply state the grounds which seem to me to substantiate the non-contagiousness of the disease.

1. The disease is confined within certain territorial limits. In this respect it differs from most, if not all diseases, the contagiousness of which is established. Even in localities in which it is prevailing as an epidemic, it is sometimes restricted to a circumscribed area.

2. The rise and progress of epidemics are not consistent with its diffusion by contagion. For example, my former colleague, the late Prof. Fenner, with great zeal and fidelity traced the first 30 or 40 cases in the epidemic at New Orleans, in 1853, and ascertained that the disease broke out in different places among persons who could have had no communication with each other. Prof. F. investigated the origin and spread of the disease in New Orleans for twelve years, and states that he never found the least proof of personal communicability. 'This is alike true of epidemics in other places. ${ }^{2}$

3. In certain places, within the yellow fever zone, sporadic cases occur almost every year. Prof. Fenner states that a summer never passes in which there are not a greater or less number of sporadic cases in New Orleans, but the disease prevails as an epidemic only in certain years. Were the disease contagious, it should be diffused more or less whenever there are any cases of it.

4. When it prevails as an epidemic, it spreads too rapidly to be diffused by contagion. If diffused wholly or chiefly in that way, it must be highly contagious, and this is opposed by well-known facts.

5. Persons going from a district where it prevails into a district where

'Vide article on "Epidemiological Conclusions and Suggestions," in Brit. and For. Medico-Chirurg. Review, Oct. 1870, page 484. 
it does not exist, and becoming attacked in the latter, do not communicate the disease. There is abundant evidence that this is the rule, and the apparent exceptions are so few, and of such a character, that it is most logical to explain them otherwise than by the supposition of contagion. It is true that real exceptions to the rule would have the force of positive facts, but, in view of the overwhelming mass of negative facts, it is fair to conclude that the apparent exceptions are not real.

6. Of persons brought into close contact with yellow fever patientsphysicians, nurses, other hospital patients, etc., the proportion attacked is less than would be the case were the disease communicable.

7. Epidemics run a certain course, as respects duration, and abruptly end, in this respect resembling epidemics of eholera. 'The disease should prevail longer and disappear more slowly, if propagated by contagion. Like other epidemies, the disease, as a rule, becomes milder by continuance; it appears to absorb other diseases while it continues, in this respect resembling epidemic cholera, and its prevalence is arrested by cold. These facts, especially the two latter, are not consistent with the doctrine of contagion.

8. 'The great majority of those who have had an extensive practical acquaintance with the disease believe the disease to be non-contagious. In councction with this fact, it is, however, to be stated that the doctrine of contagion is advocated by some distinguished teachers and writers who have had abundant opportunities for observing the disease.

9. Numerous experiments to test the question of the communicability of the disease, by inoculation, by swallowing the black romit, and by the utmost possible exposure, have led to negative results. To cite one among many experimental observations, Dowler gives the following account: In 1805, Don Cabanellos, a Spaniari, slept at night with his children in beds, in the Lazaretto, in which yellow fever victims lad died. For submitting the question of contagion to this personal test he was made physician to the Royal Household, with an annuity of 1200 dollars. A number of galley slares who accompanied him had one year's imprisonment remitted. The whole party amounted to fifty, and no one suffered any harm.

10. Complete seclusion has proved ineffectual to prevent the disease.

In view of the foregoing considerations, to which others might be added, yellow fever is not communicable by means of a virus or miasm generated in the bodies of those affected with the disease. But another question arises in connection with that of contagion, viz., may not the special cause be carried from one place to others in clothing, merchandise, etc., and the disease be in this way imported? Facts appear to establish the affirmative to this question. The special cause is especially apt to be transported in connection with the cargoes of ships, and facts appear to show conclusively that the special cause is sometimes generated in ships at sea. In either case, clothing and articles of merchandise in infected vessels are to be regarded in the light of fomites. Among many instances may be cited the occurrence, in 1856, of over 500 cases in the neighborhood of the quarantine grounds of New York, the disease in the great majority of the cases being distinctly traceable to infected vessels.'

The fact of the disease being portable renders judicious quarantine restrictions of vital importance, but in view of the non-contagiousness of the disease, the restrictions need not include the detention of persons arriving at ports in infected ships. With respect to the points just stated,

1 Annual Report of Elisha Harris, M.D., Physician-in-Chief of the Marine Hospital at Quarantine, presented to the Legislature, February 4, 1857. 
the following resolution adopted by the National Quarantine and Sanitary Convention held in the eity of New York, in 1859, may be considered as embodying the views held by the great majority of the medical profession of this country: "Resolved, That in the absence of any evidence establishing the conclusion that yellow fever has ever been conveyed by one person to another, it is the opinion of this Convention that the personal quarantine of cases of yellow fever may be safely abolished, provided that fomites of every kind be rigidly restricted." Of the members of this Convention, 85 voted for this resolution, twenty-three of this number not being members of the medical profession, and 6 voted in the negative, four of the latter not being medical men.

Of the nature and source of the special cause of yellow fever we have no positive knowledge, beyond the fact that it originates without the body. Something, however, is known of the conditions under which it is produced. It is indigenous chiefly, if not exelusively, in warm climates. Yellow fever is rarely developed south of $20^{\circ}$ (south) or north of $40^{\circ}$ (north) latitude. It prevails more in the Eastern than in the Western Hemisphere, and in certain parts of Europe and $\Lambda$ merien than in Africa. In the Western world it prevails especially in the commereial towns on the Atlantic coast sonth of Charleston, S. C., on the Gulf of Mexico, and in the West India Islands. It is indigenous, and very rarely prevails as an exotic disease in rural situations. It prevails only in the summer season in other than tropical climates, and in the latter chiefly during the hottest months. $\Lambda$ high temperature is essential to its cansation, but in yellow ferer loealities epidemies do not always occur in the years in which the temperature is highest. Humidity has been supposed to faror the development of the disease, but this is not conclusively established. There does not seem to be ground for connecting the eausation with any known meteorological conditions other than a high temperature. As stated by Dr. Nott, the special cause is most active near the ground; persons on a ground floor are more likely to be attacked than those in stories above. The cause is more active at night than in the daytime. ${ }^{2}$

In localities where the disease prevails often, the conditions for its development do not uniformly exist during the hot seasons. Epidemies oceur only in certain years. It would seem that the efficiency of this, as of other special causes of disease, depends on the co-operation of other causes. The latter may be controlled, and in this way the disease may be preventable. Acting in this way, sanitary measures-drainage, sewerage, removal of filth, avoidance of overerowding, etc.-will be likely to prevent the oceurrenee of yellow fever epidemics.

Unacclimation is a condition pertaining to individual susceptibility. Natives of yellow fever localities are rarely attacked. Particular epidemics, horrever, have been characterized by a considerable number of cases among the native population. Not having experieneed the disease is another personal condition. The diseasc laving been once experienced, the susceptibility to the special cause, as a rule, is extinguished. The number of persons who have the disease more than once is probably not greater than the number of exceptions to the rule that smallpox and typhus render the system insusceptible ever afterward to the special cause of these diseases. Acclimation, as regards this disease, is complete only when the disease has been experienced.

1 The word fomites in this resolution, of course, is considered as embracing the presence in merchandise, clothing, etc., of miasms other than those generated in the body. The term has sometimes been restricted to the latter.

2 Vide New York Med. Record, Dec. 1, 1871. 
It would appear that the negro race is singularly exempt from a liability to this disease. Statistics show the mortality among negroes to be insignificant. Fenner states that the insusceptibility is not nearly as great as mortuary statistics would seem to show, in consequence of the fact that the disease in the negro is almost invariably mild, and not likely to prove fatal. The susceptibility is not affected by age; persons of different ages, inclusive of infancy, are liable to the disease. Statistics show a larger proportion of cases among males than females.

The special cause is destroyed by cold. It is a matter of common observation that an epidemic is arrested by one or two hard frosts. "It is killel like insects and plants by a temperature of $32^{\circ} \mathrm{F} . "$ Epidemies, however, have a self-limited duration. Fenner states that, at New Orleans repeatedly, the disease has disappeared before the occurrence of frost. An epidemic rarely continues longer than from 60 to 70 days. Barlow has calculated the average duration of yellow fever epidemics, and finds it to be 58.33 days, being somewhat longer than the mean cluration of epidemies of cholera.

To sum up the most important points relating to the causation, an unknown special cause-a poisonons miasm-is involved; the doctrine that this special cause is reproduced within the body, does not rest upon adequate proof; the special cause requires for its development or efficieney conditions peculiar to certain localities, and a high temperature is an essential condition; auxiliary causes which exist especially in cities or large towns exert a powerful agency in the production and perpetuation of the disease, and by the removal of auxiliary causes epidemics may be prevented or divested of much of their fatality ; finally the special cause may be transported by means of infected vessels or fomites, and, in conjunction with a high temperature and auxiliary causes, the disease may prevail in places where it is not indigenous.

The period of incubation is variable. It varies between two and fifteen days, and in the majority of cases it is nearer the first than the second of these numbers.

Dragnosis.-The access of the disease, and the symptoms during the febrile or first stage, present nothing highly distinctive. All observers agree that it is often difficult to arrive at a positive diagnosis during this stage. In a considerable proportion of cases, the disease, at the end of this stage, terminates in convalescence. These cases would not be considered as eases of yellow fever except during an epidemic. The disease in these cases presents the characters of febricula. 'The symptoms which have diagnostic significance in these cases are, the abruptness of the attack, pains in the back and limbs, and suffusion or rediness of the eyes.

In cases progressing beyond the first stage, and presenting the grave characters of the disease, the diagnostie features are sufficiently distinctive. They are, yellowness of the conjunctiva and skin, black vomit, hemorrhages elsewhere than within the stomach, epigastrie tenderness, slowness of the pulse in certain cases, coldness of the extremities, suppression or a scinty secretion of urine, and the phenomena denoting collapse. All these events are not present in every case, but generally enough of them to render the diagnosis positive. The march of the disease to a fatal issue, from the end of the first stage, is, in well-marked cases, peculiar, and it can hardly be confounded witl any other affection.

Yellow fever, although essentially distinct from intermittent and remit- 
tent fever, may be associated with the latter. The late Dr. Lewis, of Mobile, and other's, have described cases in which the disease presented modifications due to this combination, differing from ordinary yellow fever chiefly in the occurrence of intermissions and remissions. The existence of this combination, in certain cases, has an obvious bearing on the treatment.

Prognosis.-The mortality from yellow fever varies much in different epidemics. The range of variation is from 10 to 75 per centum. ${ }^{1}$ The average mortality, according to calculations by La Roche, is 1 in 2.32 . The rate of mortality differ's in different periods of the same epidemic, the rule being a decrease in the rate as the epidemic approaches its termination.

Unfavorable prognostics are, yellowness of the surface, black vomit, great diminution or suppression of urine, abundant hemorrhage in any situation, coldness of the extremities, jactitation, hiccough, delirium, convulsions, and coma. Of these symptoms, black vomit and suppression of urine are almost invariably forerunners of death. Coma and convulsions are fatal symptoms dependent on uræmia.

A favorable prognosis cannot be confidently entertained in any case of this disease. Unfavorable erents are liable to occur when, up to the time of their oceurrence, the symptoms appeared to be favorable. Black vomit, hemorrhage elsewhere, uræmic convulsions or coma, are liable to occur unexpectedly in cases which appear to be progressing favorably.

Exclusive of cases in which the immediate cause of death is uræmic coma, the mode of dying is by asthenia.

Treatment.- With reference to the treatment of yellow fever, it is to be premised that no specific remedy has been, as yet, discovered; there is no known plan of treatment on which reliance can be placed to cut short the disease. Within late years it has been claimed by some that quinia and opium are effectual as abortive remedies. Clinical experience has failed to substantiate this claim. The remedies may be in a greater or less degree useful. They may appear to render abortive cases in which the discase ends after a brief career by self-limitation. They may arrest remittent fever existing in combination with yellow fever. They will be likely to prove effectual in cases of remittent ferer incorrectly considered to be cases of yellow fever. It is difficult to decide as to whether they have any remedial power over yellow fever from the facts which have been publisherl.

Another consideration to be premised is, the disease, exclusive of malignant cases, tends to recovery. Mild yellow fever is a very mild disease, not tending to destructive lesions of either the solids or fluids; nor does it tend to continue indefinitely, if not arrested, like intermittent fever. Judicious observers agree that no active interference is required in mild cases.

A third preliminary consideration is that, in a considerable proportion of malignant cases, the disease is necessarily fatal. The blood changes and their consequences are too great to admit of recovery. Hence, a large proportion of fatal cases is by no means proof of injudicious treatment. And a fourth consideration is, that different epidemics differ as regards the relative proportion of mild and malignant cases. In some epidemics the majority of cases are mild, in others malignant. It follows

1 Da Costa, Medical Diagnosis. 
that the ratio of recoveries under a certain plan of treatment may be no test of the superiority of that plan. Measures which appear to be eminently successful in one, may appear equally to fail in another epidemie, the difference being due to variations as respeets the tendeney of the disease to a fatal issue.

The inclieations for treatment may be considered as relating respeetively to the three varietics of the disease, viz., mild, inflammatory, and malignant yellow fever.

1. Mild Yellow Fever.-As already stated, in this variety of the disease no active interference is required. Quietude, restricted diet, ventilation, and other hygienie regulations, together with such palliative measures as particular symptoms in individual cases may indicate, suffice for the treatment. The palliative measures will embrace anodyne and refrigerant remedies, eold applieations to the head, sponging of the body, laxatives in some eases, ete. Dr. Stone, of New Orleans, says, the only treatment which in his hands has appeared to be useful is that designed "to faror the efforts of nature in prolonged sweating, ealm, and rest of the system." He recommends "foot baths under the bedelothes and sponging the body with tepid water."

Many eases of mild yellow fever in New Orleans are managed by creole nurses without medical aid. It is, however, to be borne in mind that the disense may become malignant in eases which are at first mild in appearance. It is impossible always to distinguish at the outset the cases which will prove to be mild; hence, it is of vast importance to take every precaution to prevent the development of grave symptoms. For this encl, hygienic measures are espeeially or eliefly important. Patients should at once give up to the disease and take to the bed. $A$ s complete rest of body and mind as practicable is to be enforced. The services of a faithful and experienced nurse are of more importanee than any medication. Free ventilation, eleanliness, and other sanitary measures are not less important in this than in other forms of fever.

2. Inflammatory Yellow Fever.-The propriety of bloodletting in yellow fever relates to this variety of the disease, which is eharacterized, not as the name might imply by the existenee of inflammation, but by an unusual intensity of the febrile movement in the febrile stage of paroxysm. Bloodlctting has been advoeated and largely enployed as a curative measure in yellow fever. This view is now abandoned, and all that can be claimed for it is promptness and effieiency in abating the intensity of the febrile movement. 'The short eareer of the fever is a reason why it is less objectionable, on the seore of ulterior injurious effects, than in some other forms of fever, but, in general, as in other fevers and aeute inflanmations, the salutary ends of bloodletting may be secured by other measures which are to be preferred. The measures which may take the place of bloodletting are those noticed in treating of other fevers, viz., saline laxatives, sedatives, cold ablutions, or the wet sheet. As regarls other measures, they do not differ from those indicated in eases more or less severe, which are not cases of the so-called inflammatory variety of the disease.

3. Malignant Yellow Fever.-Under the head of malignant cases, are to be embraced all which are charaeterized by symptoms denoting great severity and danger. In the absence of known remedies or any therapeutical measures which exert a specific influence over the disease, the treatment in these cases must be expectant, in the sense in which this term has been litherto used in this work.

Purgatives have been much employed in this as in most other diseases. 
Drastic or active purgatives will be likely to do harm by increasing the gastro-intestinal irritation and produeing exhaustion. Even if well borne, it is difficult to see what indieation they fulfil which may not be equally fulfilled by mild laxatives or simple enemas. The latter are indicated by constipation, and sometimes during the febrile stage saline laxatives are useful as depletants. Purgation, therefore, except in the eases in which depletion is indicated, or, in some cases, for the purposes of eliminating urea, is to be avoided. Emetics, which have also been much used, are contra-indicated by the gastric phenomena which belong to the history of yellow fever.

Mercury has been considered by many as highly important in this disease. Calomel in large doses has been much used, and it has been thought to be desirable to produce ptyalism. This practice has now but few advoeates. Facts do not warrant the opinion that mercurialization is in any sense curative, and the testimony of most physicians who have had a practical acquaintance with this disease is adverse to the use of mereurial preparations, except as laxative remedies.

Measures to relieve gastric irritability are often indicated. For this end, counter-irritation over the epigastrium may be employed by means of small blisters, dry-cupping, sinapisms, and stimulating liniments. Anodynes, given either per orem or by the endemic or hypodermic method, are indieated for this end. Other remedies are, chloroform in small doses, prussic acid, and creasote. Ice swallowed in small pieees has been found useful in allaying irritability of the stomach. To prevent black vomit, and with a view to arrest gastric hemorrhage when it has occurred, the acetate of lead has been much extolled. The persulphate and pernitrate of iron may be useful as hæmostatics when hemorrhage takes place in the stomach and elsewhere.

Great restlessness and vigilance call for opium and other anodyne remedies. Opiates, however, are to be given with much reserre, if the secretion of urine be seanty and danger from uræmia be apprehended. Deficieney of the urinary secretion is an indication for diuretic remedies if they be tolerated by the stomach, and for measures to produce diaphoresis if diuretics be not borne or if they prove inoperative. If symptoms be present denoting uræmia, the hot air-bath should be employed. Under these circumstances elaterium or some other hydragogue may be advisable, if relief be not procured by diaphoresis.

In view of the tendency of blood to internal organs, and of the hemorrhages which in part perhaps result therefrom, measures to determine to the surface are indicated, such as stimulating and warm pediluvia, sinapisms, the hot air-bath, and liniments. These measures are indicated especially when internal congestion is denoted by coldness of the extremities and surface of the body.

Sustaining measures are indicated in proportion as a tendency to collapse or death by asthenia is denoted by the symptoms. In this, as in other fevers, it is desirable to forestall a notable depression of the vital forces by the timely use of stimulants and by alimentation. The irritability of the stomach may interfere with the sustaining treatment. The forms of stimulus and nourishment are to be selected which, on trial, are found to be best borne. Alcoholic stimulants may be givell per enema, if not retained by the stomach. Two of my colleagues in the New Orleans School of Medicine, who had experienced this disease in its severe or malignant form, attributed their recovery to the free use of alcoholic stimulants. In one of these cases, when a fatal termination was regarded as 
imminent, a favorable change occurred immediately after champagne wine had been given freely.

It is proper to state that my opportunities for observing and treating cases of yellow fever have been quite limited. During the three years, however, of my connection with the New Orleans School of Medicine, I became acquainted with the practical views of my colleagues and others who had treated a large number of cases of the disease. These views were adverse to bleeding, purgatives, or other active remedies. Perfect rest, careful and efficient nursing, and sanitary conditions, were considered as of prime importance. Measures to promote gentle diaphoresis were deemed highly useful. For this end, the body is kept well covered, and a mild stimulant diaphoretic given, the infusion of the orange-leaf being the remedy commonly used. These, together with sustaining measures and snch palliatives as the particular circumstances in individual cases may indicate, constitute the treatment generally pursued at New Orleans. Prof. Fenner was led to attach considerable value to the use of the chlorine mixture, as recommended by Watson in scarlatina. ${ }^{1}$ He also regarded the veratrum viride as useful when the pulse is frequent, given in doses sufficient to act as a cardiac sedative.

Yellow fever associated with remittent fever claims the employment of anti-periodie remedies, especially the preparations of cinchona ; these may be useful as tonic remedies in cases of unmixed yellow fever.

The measures for the prevention of yellow fever epidemics have been incidentally referred to in treating of the causation of the clisease. The indigenous development of the disease is to be prevented by removing, as far as practicable, all auxiliary morbific eauses relating to individuals and to the community at large; in other words, by measures of public and private hygiene. There is reason to believe that complete sanitary regulations render the disease preventable. ${ }^{2}$ The importation of the disease is to be prevented by judicious quarantine regulations which embrace efficient measures for the disinfection of vessels and merchandise. Free ventilation is important as a means of disinfection. Disinfecting agents may be employed. ${ }^{3}$ Clothing, bedding, ete., are disinfected by being subjected to a temperature of $210^{\circ}$ to $250^{\circ} \mathrm{Fahr}$. In view of the promptness and completeness with which cold destroys the special canse of the disease, it is probable that the disinfection of vessels would be effectually accomplished by reducing the temperature below the freezing point.

1 This mixture is prepared as follows: "Put eiglit grains of the chlorate of potassa into a pint bottle, and pour upon them one drachm of strong hydrochloric acid. Keep the mouth of the bottle closed until the violent action has ceased, then add an ounce of water and shake the bottle well; then add another ounce of water, and so on until the bottle is full. The chlorate should be pulverizd, and in cold weather the bottle should first be warmed. A tablespoonful or two of this mixture, according to the age of the patient, may be given for a dose, frequently. An adult may take the whole pint in a day." - Vide Watson's Practice.

2 Vide paper by Dr. Elisha Harris, entitled "Hygienic Experience in New Orleans during the War," Bulletin of the New York Academy of Medicine, No. 30, Sept. 1865. See, also, a paper by the late Prof. E. D. Fenner, of New Orleans, written just before his lamented death, entitled "Health of New Orleans during Military Rule," in the Southern Journal of Medical Sciences, vol. i. No. 1, May, 1866.

3 Vide page 504. 


\title{
CHAPTER VI.
}

\author{
ERUPTIVE FEVERS.
}

Variola, or Smalipox-Anatomical Characters-Clinical History-Causation-Diagnosis-Prognosis-Treatment. Varioloid, or Modified Smallpox. Vaccinia, or Cowpox. Varicelia, or Chicken-pox.

THE fevers which remain to be considered are characterized by an eruption or exanthem, and hence they are called eruptive or exanthematous fevers. The continued fevers, typhus and typhoid, have also an eruption, but the eruption is less constant and less prominent than in the eruptive ferers. The eruptive fevers are variola, or smallpox, including the modified form known as varioloid ; varicella, commonly called chicken-pox; rubeola, or measles; scarlatina, or scarlet fever, and roseola, or rose-rash. 'The eruption in two of these fevers is moist, that is, resicular or pustular; this is true of variola and varicella. In the remainder the eruption is dry, and is properly an efflorescence or rash. To the latter kind of eruption the term exanthem is, strictly speaking, restricted.

All the eruptive fevers were formerly considered as varieties of one disease, and the individuality of all of them was not fully settled much before the present century. Each is a distinctive species of fever, having phenomena and laws which are distinctive, and its own special cause. All, with the single exception of roseola, are propagated by special causes, reproduced within the body ; that is, they are communicable diseases.

The division of the career of the disease into stages is the same in all the eruptive fevers. The first stage is the stage of invasion or the access; this stage begins with the first manifestations of clisease, and ends with the first appcarance of the eruption. The second stage is called the stage of eruption, and extends from the time when the eruption first appears to its disappearance; this stage is subdivided in variola. The stage of desquamation, or desiccation, follows the eruptive stage, and either constitutes or is followed by the stage of convalescence. The consideration of these fevers will also embrace a period of incubation, and events liable to occur after the disease, or sequels.

\section{Variola. Smallpox.}

The discovery of vaccination, by the immortal Jenner, toward the close of the eighteenth century, has divested this disease of much of the importance which it had in the two previous centuries, when it ranked first among the acute affections destructive of human life. It is not, however, so rare, even in its unmodified form, at the present day, but that cases are liable to come under the observation of every practitioner. The gravity and loathsomeness of the disease, together with its contagiousness, render highly important an early diagnosis and judicious management. In treat. ing of the disease, I shall confine myself mainly to matters which have direct relation to the practical duties of the physician, devoting very little space to questions of merely historical, controversial, or speculative interest. I shall consider first unmodified variola, and afterward, under a 
separate head, the modified form of the disease known as varioloid. The latter head will embrace the consideration of variola produced by inoculation.

Anatomical Characters.-This disease has no known special anatomical characters other than those comected with the eruption on the skin and mucous membrane. These character's will enter into the clinical history. Aside from these, morbid appearances found after death are due to complications which are not peculiar to this disease.

Cuinical Histony.-Differences pertaining to the ermption, and other erents embraced in the clinieal history, in different eases, are so great that writers have instituted several varieties of the disease. The division into confluent and discrete or distinct variola is generally adopted. In the latter variety the vesicles and pustules are separate or distinct from each other. In the former variety coalescence of the vesicles or pustules takes place. In eases in which coaleseenee exists to a greater or less extent, but not over the whole or the greater part of the surface of the body, the disease is said to be semi-confluent. Without considering these varieties under separate heads, I shall notice their distinctive features in proceeding to consider the symptomatology of the several stages of the disease.

Stage of Invasion.-The disease is ushered in by a chill in the great majority of eases, and the chill is usually marked, more so than in the other eruptive fevers. In some eases a series of chills oecurs, alternating with flushes of heat. Febrile movement follows, accompanied generally by more or less perspiration. The latter is apt to continue or recur more or less frequently up to the maturity of the eruption, a feature distinetive of this, as compared with the other eruptive fevers. The febrile movement is known as the fever of the eruption, or the primary fever. In this disease, as in other febrile affeetions, the temperature of the body is the criterion of the intensity of the fever. The fever, as juclged by this eriterion, is often intense, the thermometer sometimes showing a rise to $104^{\circ}$ or $105^{\circ}$. Nausea and romiting are apt to be prominent symptoms in this stage. The tongue becomes coatel. Pain is referred to the epigastrium, accompanied with tenderness on pressure, either with or withont notable nausea and vomiting. Generally the bowels are constipated, but diarrhœa is sometimes present, especially in children. Cephalalgia, pain in the limbs, and general debility are more or less marked, as in the early part of other essential ferers ; but in this fever pain in the loins is usually a marked symptom and possesses diagnostic importance. Ineomplete paraplegia is occasionally observed, generally disappearing with the development of the eruption. Paralysis of the bladder, giving rise to retention of the urine, sometimes exists without paraplegia. Convulsions often attend the development of this disease in children, and sometimes oceur in adults. Delirium is an oceasional symptom.

If the symptoms in this stage be mild, the eruption will probably be discrete, but if the chill be notably marked, the febrile movement intense, the lumbar pain very severe, etc., it may be expected that the clisease will prove to be of the confluent variety.

The duration of this stage, as a rule, is two days. The eruption begins to appear on the third day after the attack. The exceptions to this rule, however, are numerous. In a considerable number of cases the eruption appears on the second or the fourth day. In a small proportion of cases it appears on the fifth day. The duration is sometimes extended to the 
sixth day, and occasionally even longer. The eruption, if it appear on the second day, may be expected to be confluent, and, on the other hand, in proportion as the appearance of the eruption is protracted beyond the third day, mildness of the disease is to be expected. Two events, relating to the skin, are of occasional occurrence in this stage. One of these is a cutaneous efflorescence or rash. The whole or the greater part of the surface of the body may be covered with this erythematic redness, closely resembling the appearance in scarlatina. The disease is liable to be mistaken for scarlatina when this oceurs. In other cases the rash is in isolated patches, and it may then bear some resemblance to measles. The patches, however, are not elevated, that is, they are not papular. Small vesicles, likc sudamina, are sometimes observed. The efflorescence appears on the second day and continues only eighteen or twenty-four hours, giving place to the eruption proper of the disease.

The other event is the appearance of petechiæ, that is, ecchymoses. These may be more or less abundant. They are sometimes very numerous, eovering both the trunk and the extremities. In some cases these foreshadow subsequent hemorrhages; but they by no means have always this significance. They do not always denote severity of the disease; they are sometimes observed in mild cases. They continue during the greater part of the stages of the eruption proper of the disease.

Stage of Eruption.-The cruption, as a rule, appears first on the face, especially about the lips and chin, and nearly at the same time on the neck aud wrists; next on the chest and arms; then over the body, and last on the lower extremities. Its diffusion over the whole cutaneous surface occupies from one to three days. Exceptionally it is first observed on the genital organs, chiefly in young children, and on the loins or nates. It may appear first in the neighborhood of a blister or sore existing in any part of the body. Several successive changes take place in the physical characters of the eruption, which, in different stages of its progress, represent nearly all of the cutanei. At first it appears in the form of small red spots or specks, sometimes having a purplish or livid color. It is now a maculated eruption. The central part of the maculre becomes hard, slightly elevated, and pointed. A change has taken place from a maculated to a papular eruption. In this stage of its progress, the eruption is not unlike that of measles, and the disease is liable to be mistaken for the latter. The papulæe are smaller, rounder, and harder, and lack the crescentic or curvilinear arrangement which characterizes the eruption of measles. They feel like small shot under the skin. Next, the cuticle becomes elevated at the apices of the papulæ by a drop of liquid. The papula now become vesiculæ. This change is observed 24 hours after the first appearance of the eruption. After 24 hours more, i.e., 48 hours from the first appearance of the eruption, the vesicles have acquired considerable size. On the fifth day of the stage of the eruption, the resicles have attained to nearly or quite their full development, measuring from $\frac{1}{4}$ to $\frac{1}{3}$ inch in diameter, and are raised from $\frac{2}{10}$ to $\frac{4}{10}$ inch above the level of the skin. More or less of the vesicles present a depression in the centre. They are said to be umbilicated. This appearance is highly characteristic, indeed, almost pathognomonic. It is observed in no eruption other than that of variola, excepting the vaccine vesicle, and sometimes ecthyma. This umbilicated appearance may be discovered in some of the vesicles, often as soon as the latter are discoverable. The vesicles are multilocular, consisting of five or six cells. They contain an opalescent serum. The interior is lined by a layer of lymph, forming a pouch or pock. The form of the vesicles is that of a truncated cone. 
This is the history of the eruption, up to the period when the vesicles reach the maximum of their development, if the eruption be discrete. In the confluent variety, a diffused redness of the surface preceles the appearance of papules and vesicles. This diffused redness is apt to lead to the error of supposing the disease to be measles. The vesicles when they first appear have not the determinate form of those in discrete smallpox, but run together or coalesce to a greater or less extent. At the end of the period for the full development of the vesicles, the face and other parts are covered with patehes of greater or less size, in which the euticle is uniformly raised by opalescent serum, resembling a blister. The whole of the face and the greater part of the cutaneous surface may be covered with coaleseent vesicles.

After the full development of the vesicles, there is another important change in the eruption. The vesicles become pustules. In other words, suppuration takes place. With this change the external characters are altered. The pocks are more distended. The cells are broken up. The central depression is lost, and the pustules become pointed. This change from vesicles to pustules is accompanied by general symptoms which show it to be an important epoch in the elinieal history of the disease. Hence, with this change begins another stage, viz., the suppurative stage, or the stage of maturation. This will elaim consideration under a distinct head. It will be observed that of the different forms of cutaneous eruption, all except the squamæ and tuberculæ are represented in the suecessive changes in the ertution of smallpox, viz., enumerating them in the order of their sequence, maculæ, papulæ, vesiculæ, pustulæ, and, in the contluent eruption, bullæe.

The eruption is not limited to the skin. Simnltaneously with its appearance on the cutaneous surface, it may be observed, to a greater or less extent, on the mueous membrane of the mouth and throat. It appears here in the form of round opaque spots caused by a deposit of lymph, the epithelium giving way from the aceumulation of serum beneath it, so that vesicles are rarely seen on a mueous surface. The membrane surrounding the spots is inflamed, and ulceration sometimes follows. The spots are observed especially on the tongue and soft palate. They are liable to occur in the larynx, trachea, and bronchi; on the conjunetiva, leading sometimes to nleers and destruetion of the eye; within the nostrils and on the prepuce or vulva. Aceording to Beraud and Trousseau, the eruption sometimes takes place, in the male, within the tunica vaginalis, giving rise to a complication which has been termed variolous orchitis, and in the female, upon the peritoneum surrounding the ovaries, giving rise to variolous ovaritis. In the larynx, the eruption may cause laryngitis which may prove fatal. An instance of this kind has fallen under my observation.

A striking feature of this eruptive fever is the cessation of febrile movement, or a notable remission, on the appearance of the eruption. The pulse falls to nearly or quite the normal frequency; the heat of the skin is but little or not at all above that of health, and the thermometer in the axilla shows a reduction to $100^{\circ} \mathrm{Fahr}$. This is a highly diagnostic feature of sinallpox, provided the eruption be not confluent. In the confluent variety this feature is either wanting or it is much less marked. As the eruption progresses the febrile movement is gradually developed or increased.

Stage of Suppuration.-With the change of the eruption to pustules, usually on the sixth day after the first appearance of the eruption, there is a recurrence or notable increase of febrile movement, constituting what is kuown as the suppurative fever, or the secondary fever. The pulse 
becomes more or less frequent, the heat of the skin is sensibly raised, and the thermometer denotes increase of temperature, especially in the evening as compared with the morning. The perspirations, which up to this epoch are apt to occur, now cease. In mild cases of discrete smallpox, the secondary fever continues only for three or four days; if it continue longer, it is kept up by some complication. But it continues longer in the confluent variety irrespective of any complication. The febrile movement is symptomatic of the affeetion of the skin, and its intensity, other things being equal, is proportionate to the degree of cutaneous inflammation incident to the suppurative process. More or less redness of the skin is apparent in the spaees between the pustules. Tumefaction of the face oceurs, and is often considerable. The swelling of the eyelids is often sufficient to close the eyes, as in cases of erysipelas. Swelling of the hands and feet oecurs in severe cases, especially if the eruption be confluent. The swelling of the face and extremities, and the appearance of inflammation around the pustules, belong to the natural history of the disease, and, if wanting, the disease will be likely to prove fatal. Trousseau states that reeovery from confluent smallpox almost never takes place if swelling of the extremities do not occur. The redness and swelling are accompanied by a burning pain, as in crysipelas. Salivation is a frequent symptom, and in severe cases of the confluent variety it is often very profuse, and accompanied with more or less swelling of the salivary glands. It is measurably or chiefly dependent on the eruption in the mouth and fauces. It is rarely observed in children. In severe cases delirium is apt to occur in this stage. The delirium is generally passive like that in most cases of typhoid fever, but it is sometimes active or maniacal. Other ataxic symptoms, viz., subsultus, earphologia, and comavigil, may also occur, denoting always a condition of greater gravity. Coma occurs in a certain proportion of fatal cases. Other symptoms are due either to complications or to anomalous occnrrences which will presently be noticed. Diarrhœa is an occasional symptom, and is always an unfavorable omen. The duration of the stage of eruption is four or five days.

Stage of Desiccation.- This stage commences on about the twelfth day of the disease. The exceptional cases in which it commences much earlier or later are very few. The tumefaction and redness of the skin diminish. The pustules, for the most part, break, and the pus concretes into a thick scab. Patches in which the eruption is confluent become covered by a continuons scabby crust. The whole face, in severe cases, is covered, as if by a mask, presenting a hideous appearance, and the greater portion of the surface of the body may present a similar aspect. The diminution of the inflammation and the formation of scabs are first observed on the face, afterward on the trunk and upper extremities, and last on the lower extremitics. During this stage the skin exhales a sickening characteristic odor. In cases in which the eruption is confluent the stench is extremely offensive, and may be perceived at a considerable distance. The spectacle in this and the preceding stage and the fetor render smallpox the most repulsive and loathsome of diseases. In mild cases the stage of desiccation is the stage of convalescence. The scabs fall off, leaving the skin beneath unbroken, but presenting a discoloration which continues for a long time, and is especially apparent when the surface is exposed to cold. But in severe cases the skin beneath the scabs is excoriated or ulcerated, the seabs are liable to be renewed, and troublesome ulcerations sometimes follow. More or less febrile movement, in severe cases, is continued into the stage of desiccation. In mild cases there may remain no permanent traces of the eruption, but in the great majority of cases, there are left 
cicatrices, either cireular or linear, which are known as "pitting" or "pock marks." The amount of pitting will depend on the abundance of the eruption, the size of the pocks, and the depth of the ulcerations. Some of the pocks do not break, but harden, and their contents are absorbed. It is probable that in these suppuration does not take place, that is, the vesicles are not converted into pustules.

To recapitulate the relative duration of the several stages in the majority of eases: The duration of the stage of invasion is from 2 to 4 days, of the stage of eruption about 5 days, of the stage of suppuration 4 or 5 days, and of the stage of dessieation 6 to 10 days, making the duration of the disease, in cases ending in recovery from 17 to 24 days. In mild, uneomplicated cases, the recovery is usually rapid, but the convalescence may be indefinitely prolonged by ulcerations of the skin and complications.

Various complications and anomalous events are liable to occur in the several stages of the disease. Pharyngitis, dependent on the eruption within the pharynx, is sometimes suffieient to occasion pain and difliculty of deglutition. Laryngitis, dependent on the eruption within the larynx, is of not infrequent occurrence. It is denoted by huskiness of voice, or aphonia, with, in some cases, more or less difficulty of respiration. The laryngeal inflammation is sometimes diphtheritic. Edema of the glottis is an occasional accident. Bronchitis, also from the eruption, occurs in a certain proportion of cases. Pneumonitis, pleuritis, and pericarditis, are rare complications. Furunculi, or boils, and subcutancous abscesses are not uncommon, both as concomitants and sequels. Erysipelas on the face, neck, limbs, or borly was noted in 47 of 287 cases recorded and analyzed by Sargeant. $\quad$ Gangrene may occur on parts exposed to pressure, and sometimes in other parts. Ophthalmitis is an important complication, sometimes leading to permanent blindness from opacity or perforation of the cornea. The inflammation in the throat sometimes extends through the Eustaehian tube and gives rise to internal otitis which is liable to eventuate in deafness. Hemorrhage in various situations occurs in a certain proportion of cases. Epistaxis is an occasional early symptom, and does not betoken unusual gravity of the disease. Hrematuria is sometimes an early and always a grave symptom. Hemorrhage ocenrring, at any period of the discase, from the mouth, throat, air-tubes, or bowels, aceompanied by peteehiæ or vibices, and sometimes by an extravasation of blood into the pocks, characterizes certain malignant cases. Hemorrhagic, scorbutic, and black variola are names applied to the disease as exhibited in these eases. In females menstruation is apt to occur, and if not irregular or profuse it is not an untoward event. Metrorrhagia is apt to occur in hemorrhagic cases. Albuminuria is a symptom of not infrequent occurrence in severe cases. It is probable that the coma which occasionally occurs is sometimes due to uremic poisoning. The oceasional occurrence of symptoms denoting orchitis and ovaritis has already been referred to. These complications are supposed to be due to the occurrence of the eruption in the parts affected. It appears not to be clearly settled whether or not the eruption ever takes place within the stomach and intestinal canal.

Causation.-Smallpox is a highly contagious disease. It may be communicated by means of a virus, that is, by inoculation, and by means of a miasm emanating from the body. It is, therefore, propagated by both

1 Report of the cases of Smallpox received into the Philadelphia City IIospital in 1845-46. Vide American Journal of Medical Sciences, 1849. 
contagion and infection, as these terms have been defined in the first part of this work. The disease is readily transported by means of fomites which may retain the virus or miasm in an effective condition for months and even years. A very transient and slight exposure often suffices for the production of the disease. Thus, it is not infrequently taken by passing in the street or meeting in yublic conveyances persons who either are or have recently been affected with it. In this city it is not infrequently contracted in liackney-eoaches which have been used for carrying patients to hospitals. The disease is generally supposed to be communieable in all its stages, but undoubtedly it is most highly so during the stages of suppuration and desiccation. Dr. S. B. Hunt, Surgeon of Volunteers and Medieal Director in the Army of the Southwest, during the late civil war, says: "Smallpox is not contagious in its early stages. If the patient be secluded at the earliest period when the disease can be recognized by the scientific eye, another and an unprotected person can sleep in the same bedding with very little danger. We have seen this tested on a large seale." The contents of the vesicles and pustules and the crusts and scabs formed by desiecation contain the virus; and the miasm, it is probable, not only emanates from the cutaneous surface, but is contained in the expired breath, and perhaps in the intestinal exereta. The disease may be communicated from the dead body by means not only of the virus, but the miasm. Thus it has been contracted in the dissecting-room from subjeets dead with the disease. It is stated that a cadaver may retaĭn the virus in a condition to communicate the disease for an indefinite period-even for several years. ${ }^{2}$ The following instance, related by Diekson, is interesting as showing the communication of the disease from the dead body, death having taken place before the derelopment of the eruption: "During the winter of 1848-49, a young man, a member of the medical class of New York University, died suddenly and unexpectedly in the night under the care of a physician who had not thought him seriously ill. I was invited to the autopsy, and observed, when the corpse was uncovered, a few dark-red spots on the surface, which were supposed to be petechial; the principal symptoms of this attack having been gastric with great clebility, as we were informed. The coffin was taken home to a New England village for burial, where, at the funeral, some of the relatives approached and opened it to see the face of the deceased before it was inhumed. Of this number, eight were attacked with smallpox, no other persons in the neighborhood being assailed." 3 The facts in this instance militate against the statement by Dr. Hunt just quoted. Whether the disease be ever produced otherwise than by means of contagion or infection is, and must always remain, an open question; for, with respect to the many reported cases in which the disease has appeared to originate spontaneously, it can alwaýs be said that there may have been some unsuspected and untraceable exposure. And this explanation is perhaps more rational than the supposition that the special cause is generated de novo.

The susceptibility to the disease exists in all ages, but it is greatest in children. It is a curious fact that some persons are wholly insusceptible to it, exposing themselves as fully as possible, with impunity. Cases have been observed in which persons have become susceptible after having been insusceptible for many years. Unknown causes peculiar to certain times and places co-operate with infection in aiding the diffusion of the

1 Sanitary Memoirs of the War, p. 149.

2 Vide Nouveau Dict. Art. Contagion.

3 Vide American Journal of Medical Sciences, July, 1862. 
disease, so that it prevails as an epidemic. Reference, in these statements, is had to the production of the disease in the natural way, that is, exclusive of inoculation. 'The disease produced by the infectious miasm is distinguished as natural smallpox. The negro and Indian races appear to be particularly susceptible to the cause of the clisease. As a rule, the occurrence of the disease once cxtinguishes the suseeptibility ever afterward. Instances, however, are not extremely infrequent of the disease having been experienced twice, and it has been known to occur thrice.

Certain interesting facts relate to the susceptibility of the child in utero. A pregnant woman affected with the disease may, or may not, communicate it to the fotus. The development of the disease before birth generally causes the death of the child, and cases have been reported of still-born children presenting the different stages of the eruption. 'The death of the child, however, does not always occur.

IIealthy children have been born presenting the evidence of having passed safely through the disease in the uterus. In some instances the disease has been contracted before birth, but not developed until several days after birth. Recovery has taken place under these circumstances. The disease may be conmunicated to the unborn child by the mother after the fourtl month of pregnancy and perhaps before. Finally, the special eause may be received into the system of the mother, and the foetus become affected without the mother experiencing the disease. This fact has been observel in cases in which the susecptibility of the mother had been extinguished, either by the disease having been experienced, or by vaccination.'

The special cause of this disease remains for a certain period latent, that is, there is a period of incubation. The duration of this period varies from one to nearly three weeks, the average duration probably being from twelve to fourteen days. Facts appear to show that the special cause of rubeola or of scarlatina may be in operation simultaneously with the operation of the special eause of variola; in other worls, smallpox may be combined with either measles or scarlet fever. Cases, however, exemplifying these combinations are extremely rare."

During the epidemic prevalence of smallpox, cases are sometimes observed in which all the symptoms of the stage of invasion occur without being followed by an eruption. The disease appears to abort spontaneously at the end of the stage of invasion. These attacks resemble febricula, but it has been observed that persons who have had these attacks during the prevalence of smallpox are thereafter insusceptible to the disease, although not protected by vaccination. Hence, it has been considered that the disease sometimes occurs without an eruption; and writers have bcen accustomed to recognize as a variety of the disease, variola sine variolis.

The question in what consists the essential principle, the contagium, in the virus and the infectious miasm, is one of great pathological interest. It is claimed by the advocates of the germ-theory of disease that the eflicient agents arc living organisms, either animal or vegetable; and, according to the late researches of Coze and Feltz, the blood contains a partieular species of vibrios. The discussion of the question, however, in the present state of our knowledge, would be here out of place.

I For a collection of cases exemplifying these facts, vide appendix to Gregory on the Eruptive Fevers, by the American Editor, Dr. Bulkley.

\& Vide nppendix to Gregory's work, by Bulkley. 
Diagnosis.-The diagnosis of smallpox presents no difficulty after the characters of the eruption are fully manifested. It is not so easy at an early period of the stage of the eruption before the vesicles are distinct. In seeking to determine the character of the eruption, vesicles should be looked for, and, next, their umbilicated appearance. This appearance, it is to be borne in mind, is almost pathognomonic. The distinctive characters of the papules, as determined by the touch, are also to be borne in mind, viz., they are granular, hard, and deep-seated. The duration of the stage of invasion is to be considered, viz., from two to three days. The prominence of lumbar pain and vomiting, and especially the remissions or cessation of febrile novement at the time of the appearance of the eruption, are important diagnostic features. An examination of the throat and mouth should not be omitted. The round, whitish, or ashy spots, which are characteristic of the eruption on a mucous surface, are generally apparent here as soon as, or even before, the eruption appears on the skin. The appearance of the cutaneous eruption successively, on the face or neck, the trunk and upper extremities, and the lower extremities, is to be recollected.

The diagnosis cannot be made with positiveness prior to the stage of the eruption. Pain in the loins with febrile movement, taken in connection with the absence of the symptoms which attend the access of the other eruptive fevers, and of typhoid fever, should excite a strong suspicion of smallpox, especially if the patient be not protected either by vaccination or by having once had the disease. The exclusion of rubeola and scarlatina by the absence of the symptoms characteristic of the stage of invasion in these diseases is an important step in the diagnosis prior to, and in the early part of, the stage of eruption. When the eruption first appears, the differential diagnosis lies chiefly between these fevers. In rubeola the Schneiderian membrane and air passages, and in scarlatina the fauces are prominently and early affected, as will be seen in treating of these fevers. Moreover, in rubeola the stage of invasion is longer, and in scarlatina it is shorter, than in variola. In neither rubeola nor scarlatina does the febrile movement remit or cease when the eruption appears. Other affections liable to be confounded with smallpox, are lichen, accompanied with febrile movement, and a secondary syphilitic eruption. The occasional occurrence of an efflorescence and of petechire, to which reference has been made, preceding the eruption proper, is to be borne in mind.

The diagnostic points of smallpox should be fixed in the memory, as, in view of the danger of the diffusion of the disease by exposure before its character is ascertained, an early diagnosis is immensely important. $\Lambda$ s another inducement for being prepared to recognize the disease promptly, it may be added that the ability of the physician is thereby strikingly shown, while, on the other hand, delay or failure to recognize it brings upon him popular censure. Of the liability to error of diagnosis in considering cases of other affections as cases of smallpox, the following statistics, kindly obtained for me by Dr. Henry G. Piffiard, formerly of the resident medical staff at Bellevue Hospital, may be cited: Of 1494 patients admitted into the smallpox hospital on Blackwell's Island during the years 1860 , ' 61 , and ' 62 , in 48 the disease proved not to be smallpox. Of these 48 cases, the disease in 10 was scarlatina, in 9 rubeola, and in 29 various other affections.

Prognosis.-Unmodified or natural smallpox is attended with much danger to life, the ratio of fatal cases being as 1 to 3 or 4 . The mortality 
is rery great in cases of the confluent, and considerably less in the discrete variety, the ratio in the latter being as 1 to 10 . The death-rate varies between wide limits in different epidemics. The disease is much more fatal to children than to adults, and is especially fatal in early infancy. It is also extremely fatal to aged persons. With respect to the period of the disease when death is most likely to take place, Gregory's analysis of 168 fatal cases gives results which are probably of general application. Of the 168 cases, death took place in 99 during the second week, in 32 during the first week, in 21 during the third week, in 9 during the fourth week, and in 7 cluring the fifth week.

Death is sometimes attributable to the intensity of the disease per se. Life is then destroyed before the eruption is matured, and witlout any important complication having taken place. Instances of this kind are rare. If death take place before the stage of suppuration, it is generally due to some important complication. Complications which are likely to prove fatal are, acute laryngitis, odema glottidis, pneumonitis, and an affection of the kidneys involving uræmia. 'The latter is to be suspected in cases in which coma or convulsions occur.

Death not attributable tc complications is generally due to inability of the powers of the system to support the eutaneous affection; hence, the danger, other things being equal, is proportionate to the amount of the eruption. Confluent smallpox involves a very extensive suppurative inflammation which is apt to lead to a fatal result by exhaustion. Unfavorable prognostics are, great prostration, frequency and feebleness of the pulse, typhoid delirium and other ataxic symptoms, absence or subsidence of redness and tumefaction of the face and extremities, cessation of salivation, diarrhoa, hemorrhage from the mueous membrane and extravasations on the cutaneous surface.

Death taking place during or after the stage of desiceation is attributable to ulcerations of the skin, boils, abscesses, erysipelatous inflammation, gangrene, continued cliarrhœe, etc.

In pregnant women the disease in its severe form is apt to induce abortion or miscarriage, and, under these circunstances, the result is generally fatal.

Treatment.-The treatment of smallpox embraces measures having direct reference to the eruption, and those indicated by the phenomena of the disease, irrespective of the eruption; in other words, the treatment is local and general.

The general treatment involves the same principles as the continued fevers. There is no special treatment. The disease will run its course, and, hence, the expectant plan is to be pursued. During the stage of invasion, the intensity of the febrile movement may be lessened by the use of cold drinks, ice-water, lemonade, carbonated water, allowed as freely as the condition of the stomach will permit, by refrigerant remedies, and sponging the surface of the body with cold or tepid water. The latter is not objectionable on the score of interfering with the appearance of the eruption. Bloodletting is very rarely, if ever, called for. Cathartics are not indicated, but constipation is to be removed by mild laxatives or simple enemata. Remedies to relieve nausea or vomitiug and cephalalgia are fiequently indicated in this stage. Prof. Polli claims in behalf of the sulphites in this, as in other essential fevers, that they exert a modifying effect upon the disease, lessening the severity and danger. Sansom thinks that the sulphite of sodium in doses from 20 to $30 \mathrm{grs}$. given, every third or fourth hour is useful. He also regards as an useful 
remedy the sulpho-carbolate of sodium given in the same doses and with the same intervals.

During the stage of the eruption, prior to suppuration, palliative measures are indicated by continued nausea or vomiting, vigilance, restlessness, diarrhœa in some eases, etc. The various complications which are liable to oceur will also furnish therapeutical indications. It is probable that the mineral acids are useful in this fever as well as in the continued ferer's. Alimentation and supporting measures in some cases are important in this stage. These measures are important in the suppurative stage and the stage of desiccation in proportion as the eruption is copious and eonfluent, and the general symptoms are indicative of failure of the vital forees. The prineiples which should govern the use of aleoholic stimulants and tonic remedies in conjunction with concentrated nutriment are the same as in all the essential fever's and other discase in which danger in the direction of asthenia is more or less imminent.

All the liygienic conditions which are so important in the treatment of typhus and typhoid fever are not less important in the treatment of smallpox; and, of these conditions, the freest possible ventilation is of especial importance.

The local treatment embraces measures to render the eruption abortive on the face, or to prevent the disfiguration cansed by pitting. The treatment for this end is called ectrotic, this term signifying to miscarry. 'The eetrotic treatment extending more or less over the body has been supposed to be useful by limiting the amount of cutaneous inflammation, and thereby the degree of secondary fever and danger from exhanstion. of the varions eetrotic measures which have been tried, the following have been found measurably successful :-

1. The careful evacuation of the resicles by means of a fine needle. This plan is advocated by Rayer. It calls for much patience on the part of the operator.

2. Evacuation of the vesicles and cauterization by means of a finepointed stick of the nitrate of silver. 'This, also, is a very tedions operation. It is highly recommended by Velpeau, Bretonnean, and others.

3. The application of the tincture of iodine, once or twice daily by means of a brush, while the eruption is papular. This plan was introduced by Dr. Crawford, of Montreal, and Dr. Samuel Jackson, of Northumberland, Pa. The effect was observed by Sargeant in thirty cases, the treatment being limited to one side of the face. There was but little swelling on the side on which the iodine was applied; the resicles were flattcned, and the pitting, although not prevented, was eridently dininished.

4. The exclusion of light and air was recommended as an ectrotic measure by the late Dr. Picton, of New Orleans. This is effected by covering the face with a plaster of some kind. Covering the face with gold-leaf, as practised by the Egyptians, according to Larrey, must bc useful chiefly by excluding light and air.

5. The application of a mild mercurial ointment, spread on cloth, has been highly recommended by Stewardson.' It is advocated strongly by several French observers. Sargeant found this plan useful, but less effective than the application of iodine. The risk of producing the constitutional effects of mercury is an objection of considerable moment to this plan. Mercurialization is certainly not desirable in smallpox. In view of this risk, Bennett was led to substitute, for the mercurial ointment, calamine saturated with olive oil, and this application he found to be

I Vide American Journal of the Medical Sciences, January, 1843. 
equally effective. Skoda and Hebra recommend the application of compresses dipped in a solution of corrosive sublimate (grs. ii-iv, to water $\tilde{\jmath}$ vi). Niemeyer prefers a weaker solution in gr. $i$ to $\xi$ vi.

6. The application of the subnitrate of bismuth and prepared chalk, in equal parts, twice daily, often smearing the surface with sweet oil, is stated to be an effective ectrotic measure by Dr. Hamilton, of Illinois.'

7. Dr. Stokes advises the application of poultices over the face, as the surest method of preventing disfigurement. They should be applied at the earliest period and continued to an advanced stage of the disease. $\mathrm{He}$ thinks that linseed meal is the best article for the poultice. It should be spread on a soft material, such as French wadding, and covered with gutta-percha paper or oiled silk.

8. 'The application of collodion, as an ectrotic application, was first suggested in a letter to me by Dr. S. B. Brinkerhoff, of Sinesville, '’a. ${ }^{2}$ It was subsequently employed by Aran, of Paris. The collodion is to be applied once or twice daily by means of a brush. To be successful, the application must be made while the eruption is papular, or while the vesicles are quite small. Applied at a later period, it seemed to me in one case to increase the pitting. In the few cases in which I have seen this plan tried, the application was agrecable to the patient.

A solution of gutta-percha in chloroform has been used by Stokes, Graves, and Wallace. 'The modus operandi of the gutta-perela is similar to that of collodion, the effect being produced by means of the exclusion of air and, mechanically, by the compression caused by the applications.

9. Carbolic acid has been used with success by Lemaire and Sansom. The acid is to be liquefied by alcohol, and applied by means of a camel's hair pencil brush, as soon as the contents of the resicles begin to appear puriform.

A side from ectrotic measures, the local treatment embraces eraporating lotions to alate undue heat, a weak solution of the chloride of lime as an antiseptic and to allay pruritus, soothing unguents in the suppurative stage, and absorbent powders. If ulcerations follow the falling off of the scabs, they require the same applications as ulcers occurring under other circumstances.

It should be an object of treatment to prevent the eruption from appearing on the conjunctival membrane. The continued application of compresses wet with cold water is advisable for this object. Niemeyer advises a weak solution of corrosive sublimate.

With a view to prevent diffusion of the disease, patients should not be discharged from hospital, or, if treated at home, allowed to go abroad, until the surface is completely frec from scabs, and repeated, thorongh ablutions have been employed. All articles of clothing worn during the disease should be left at the hospital, or, in cases in private practice, destroyed. Fumigations, as a means of destroying the virus or miasm, should not be relied upon. Physicians, after visiting patients affected with this disease, should not see other patients until after having been sufficiently in the open air for the miasm to be dissipated by free rentilation. The hands should be washed before leaving the hospital or house, and change of dress before visiting other patients, especially young children, is to be recommended, if not enjoined.

1 American Journal of the Medical Sciences, Oct., 1865.

2 Vide Buffalo Medical Journal, vol. vi., 1851. 


\section{Modified Smallpox, or Varioloid.}

Thus far, smallpox has been considered in its ordinary form as produced by an infectious miasm, that is, natural smallpox, in persons unprotected by vacciuation. Materially modified, as respects the events of its clinical history, and its severity notably diminished, the disease is commonly known as varioloid. This term is not to be understood as applied to a disease essentially distinct from, although resembling, smallpox. 'The name would imply this. But in all cases to which the term varioloid is correctly applied, the disease is neither more nor less than variola.

Smallpox is modified, as a rule, in a remarkable degree when produced by inoculation, or, as it is also called, variolation. As a means of rendering the disease so mild as to be measurably divested of danger, and of preventing pitting, inoculation had been practised from time immemorial in China and Persia. Imported into Turkey, the practice was introduced thence into Great Britain by the Lady Mary Wortley Montague, in 1721. It was not adopted in France until 1764. In the same year of its introduction into Great Britain, a large number of persons in Boston and the vicinity were inoculated by Ward Nicholas Boylston. Boylston was the first to note this important fact relating to variola produced by inoculation, viz., the average period of incubation is shorter than in cases of natural smallpox. Hence, the production of natural smallpox may be anticipated and prevented by inoculation after known exposure to the miasm of the disease.

The eruption in cases of inoculated smallpox, or after variolation, is usually slight. The pocks rarely exceed 100. Many of them abort, that is, they do not proceed to suppuration. The secondary fever is slight or wanting. There is very little constitutional disturbance, and the disease leaves little or no pitting. These statements are true in the main. Exceptionally, when inoculation was largely practised, the disease was severe. The rate of mortality was exceedingly small, varying, according to Shattuck, in 23,000 cases, in Boston, from 5 to 30 deaths in a thousand. Transferring the virus from persons successively inoculated, the disease becomes progressively more and more modified, until at length, as a rule, to which there are exceptions, the eruption consists only of the pustule formed at the point of inoculation, with a few pustules developed around this mother-pustule.'

As a rule, variolation affords complete protection, ever thereafter, against smallpox. For those who availed themselves of it, therefore, it was a great blessing prior to the discovery of vaccination. But it contributed to the diffusion of the disease by multiplying the foci of contagion and infection, and, since the discovery of vaccination, it has ceased to be resorted to as a means of avoiding the danger and disfigurement of natural smallpox. In England the practice of inoculation is interdicted by law, with severe penalties. Under certain circumstances, however, the physician is not only justified, but it is his duty to practise inoculation. These circumstances are: the known exposure to the miasm of smallpox of persons who have never had the disease, and who are unprotected by vaccination, and an inability to obtain the vaccine virus. Inoculation should then be resorted to in order to secure the great advantages of modified smallpox. The late Prof. Dickson advocated a restoration of the practice of inoculation, in addition to vaccination, with a view to test

1 Vide Trousseau, Clinique Médicale, tome premier. 
the protective power of the latter, and destroy, as completely as possible, all susceptibility to the disease. ${ }^{1}$

Inoculation has been sueeessfully practised in eows, as a means of rendering less severe and dangerous the epizoötic disease affecting these animals, which is modoubtedly the same disease as variola in man, viz., vaccinia, or cowpox. And this practice, with similar success, has been resorted to in a disease affecting sheep, which is probably the same disease. ${ }^{2}$

$A t$ the present day, in most eases of varioloid, the modification is due to vaceination. Vaccination, as will presently be seen, does not always afford complete protection against smallpox, but, in general, the disease is materially modified.

In varioloid the primary or eruptive fever, in the stage of invasion, is often as marked as in cases of natural smallpox. The duration of this stage is longer than the averagn duration in ordinary variola. The eruption is usually far less eopious; a few poeks only may appear, and the eruption may even be limited to a single poek. The eruption passes more rapidly through the suecessive changes, the maturation being completed in five or six days. Frequently the eruption aborts to a greater or less extent. It may stop at the vesicular, and even at the papular, period. More or less of the vesicles dry up without breaking. The general symptoms, as a rule, are much milder than in most benign cases of natural smallpox. The seeondary fever is either slight or wanting, and the convaleseence is speedy. Exceptionally eases are severe, and the disease proves fatal in a ratio varying from seven to ten per cent.

Smallpox is modified in a certain proportion of eases spontaneously, that is, irrespective of inoculation or vaceination. Cases presenting the characters of varioloid oceurred prior to the practice of inoeulation and the diseovery of vaceination. They were, however, rare. Finally, modified smallpox or varioloid is apt to be the form of the disease when it oceur's in those who have before experieneed it. In all eases of varioloid, it is to be borne in mind that the disease is essentially variola or smallpox, and that the virus or miasm, however mild may be the cases, is capable of producing, in persons unprotected, a severe and fatal form of the disense.

The diagnosis, is cases of varioloid, offers diffieulties which do not obtain in cases of nmmodified smallpox. The disease most likely to be confounded with varioloid is rubeola. The different points will be stated in treating presently of the latter disease.

In eases of varioloid oftener than in ordinary variola, the eruption is liable to be preceded and aceompanied by an efflorescence bearing considerable resemblance to scarlatina. Until the variolous eruption is developerl, the disease may be supposed to be scarlatina. The oceurrence of papules in the midst of the efflorescence, and the remission or cessation of febrile movement, are the differential points. Moreover, an examination of the mouth and throat may show the characteristic eruption here before its distinetive characters on the skin are apparent. The oceurrence of a scarlatiniform eruption explains, in some cases, the supposed coexistence of variola or varioloid and searlatina.

The treatment of varioloid does not claim separate consideration. The same principles are involved as in the treatment of ordinary smallpox,

1 On Smallpox and the means of protection against it. By S. Henry Dickson, M.D., Professor of the Practice of Medicine in Jefferson Mcdical College; Am. Journ. of Medical Sciences, July, 1862.

2 Vide Aitken, op. cit., and Trousseau, Clin. Médicale. 
but the comparative mildness, in the great majority of cases, renders active treatment unnecessary. Usually little is required except hygienic measures.

\section{Vaccinia, or Cowpox.}

Vaccinia and cowpox are names of a disease of the cow, which, communicated to man, destroys, in the great majority of cases, for a certain period, the susceptibility to smallpox, and, in the cases in which it does not offer complete protection against the latter clisease, renders it, as a rule, mild and devoid of danger. Cowpox is transferred to man and from one person to another by the introduction of a virus, and never, at a distance, by infection. The operation for its communication is called vaccination. For the employment of vaccination as a means of preventing smallpox, the world is indebted to the immortal Jenner. The discovery was promulgated by Jenner in 1798, after a series of observations and experiments which had occupied his attention for twenty years. The fruits of this transcendently important discovery have been the saving of an incalculable number of lives which would otherwise have been destroyed by one of the most loatlisome of discases, and the prevention, to an extent which cannot be computed, of the disfigurement and other distressing effects which smallpox is apt to produce when it does not prove fatal. of all the benefactors who have ever lived, no one has conferred on mankind such immense, palpable, and time-lasting benefits. Yet, out of the ranks of the medical profession, how many are ignorant of the name of the discoverer of vaccination! The practice of vaccination met at the outset with much hostility, but Jenner lived to see it triumph over all opposition, and to witness the beginning of beneficent results which will accumulate from age to age until the end of the world. The practice was introduced into this country in 1799 , notwithstanding a bitter popular prejudice, by Benjamin Waterhouse, of Boston, the first professor of medicine in Harvard University. Connected with vaccinia and vaccination are questions of great interest and importance, which have given rise to extended experimental and statistical researches, together with much discussion. 'They have occupied a considerable share of medical literature since the time of Jenner. Certain points are still unsettled and claim further investigation. The scope and character of this work allow of only a brief statement of facts and the opinions most consistent with existing knowledge, considered with direct reference to the practice of medicine.

Directing attention first to the descriptive history of vaccinia in man, on the third day after vaccination (the operation being usually performed on the arm near the inseriion of the deltoid musele), red points, slightly elevated, that is, small papules, are apparent at the spots where the vaccine virus was inserted. On the fourth day the papules are more developed and reddened. On the fifth day vesicles are discoverable. The vesicles increase, presenting an umbilicated appearance, and on the eighth day they attain to their full development, being elevated from two to three lines, and measuring one-third of an inch in diameter. The vesicles, like those of smallpox, are multilocular, flattened at the summits, and contain a transparent, viscid liquid called the vaccine lymph. On the seventh or eighth day a red areola is apparent, extending from one to three inches around the pocks, increasing in redness until the ninth or tenth day, and the contents of the vesicles become more or less purulent. At this time there is usually slight febrile movement, with some local pain and pruritus. The lymphatics of the arm become swollen, and the glands in the axilla 
may become enlarged and tender. On the tenth or eleventh day the febrile movement subsides and the redness around the pocks diminishes. $A$ dark spot soon appears on the centre and gradually extends over the whole of the pocks. The pustules desiceate, and by the fifteenth day they are converted into black hard scabs which fall off usually by the twenty-fifth day from the date of the vaccination. During the progress of the vaccine affection, vesicles having the distinctive characters of vaceinia have sometimes been observed in other parts of the body. It is probable that these are cansed by the patient scratching the vesicles on the arm and carrying therefrom lymph containing the virus, on the finger nails, to parts where, owing to the abrasions of the skin, self-raccination is the result. Experiments show that between the fourth and the ninth or tenth day, the characteristic vesicles may be multiplied at will by revaccinating with lymph from the vesicles produced by the primary raccination. ${ }^{1}$ Lichenous or vesicular cruptions, the latter lacking the distinctive features of vaccinia, not infrequently accompany or follow vaccination. Finally, permanent cicatrices denote the situation of the vaccine pocks. The eicatrix, provided the vesicles have pursued a regular course, and subsequent ulceration have not oecurred, is characteristic, presenting a series of depressions or pits, each of which represents the site of one of the multilocular cells composing a vaccine vesicle. 'The period of incubation in vaceinia is sometimes considerably protracted in consequence of the existence of some other affection, or from causes which are not apparent.

A most important practical point connected with raccination is the discrimination of veritable and complete vaccination from an incomplete or a spurious affection. The development of resicles of full size, the successive stages, and the duration of the affection, together with the characteristic appearance of the resicles and the eicatrices, are the eriteria for determining this point. Simple sores having no specific character may be the result of vaccination. Ineomplete vaccinia, vaccinoid, as it is termed by Trousseau, is evidenced by the vesieles being developed more quickly and progressing to desiccation more rapidly, by their swollen size and conoidal form, and by the absenee of cicatrices, or of the appearance distinctive of the true vaccine cieatrix. In riew of the immense importance of a true and perfect vaccination to those who suppose themselves to be protected against smallpox, the responsibility of performing vaccination should never be undertaken by those not practically familiar with vaceinia. It is obviously far better that a person should not have been vaccinated, with a knowledge of the fact, than that he should incorrectly believe himself to have had veritable and complete cowpox, since this belief involves a false sccurity leading to exposures which wonld otherwise be avoided.

Certain interesting questions relate to the nature and source of cowpox. Did it originate in the eow, or come from the human species? Does it affect other animals? What are its essential relations to smallpox? With respect to these questions, I clesire to qualify certain statements, made in the previous editions of this work, namely, that vaccinia in the cow may be produced by inoculation with the smallpox virus taken from man, and that the disease thus produced in the cow, transferred to man, gives rise to vaccinia in the latter; and that vaccine virus obtained in this way has been found effectual in preventing smallpox. These statements were made as the basis for the conclusion, that the opinion held by Jenner, that vaccinia and variola are essentially identical, is proved to be true. 
There are, perhaps, facts, supporting these statements, sufficient to warrant the foregoing conclusion; but the practical inferences, as I am now satisfied, are incorrect and dangerous. 'The virus obtained from the cow by inoculating with the smallpox virus (retro-vaccination as it is called), is not only unreliable, as affording protection against smallpox in man, but it has been found repeatedly to communicate the latter disease. Vaccine virus, thus obtained, is, therefore, never to be employed in vaccination.' Experiments have confirmed the correctness of the opinion held by Jenner that an affection sometimes seen in horses, known as grease, called by the French des eaux jambes, is identical with cowpox, and that vaccinia in man may be derived from this source. It is not, however, settled that, as supposed by Jenner, corpox in the cow was derived from the horse. It has also been ascertained that sheep are affected with an epizootic disease, called by the French clavelée, which is analogous to, if not identical with, cowpox. As to the origin of the disease in these and perhaps other domestic animals, it is not certain that it was primarily a human disease, but this view is perhaps the most rational with our existing knowledge.

Other questions, the importance of which is obvious, are, what extent of protection against smallpox is afforded by cowpox, and what are the causes of the protection not being universal and complete? In a large miajority of cases vaccination affords, for a time at least, absolute protection against smallpox. In these cases the situation of persons vaccinated, as regards susceptibility to the latter disease, is precisely as if natural or inoculated smallpox had been experienced. But in a minority of cases the protection is only partial; the susceptibility to smallpox is not destroyed, but the disease is materially modified, constitnting what is called varioloid. At certain times and places, the causes which give rise to the epidemic prevalence of smallpox being unusually efficient, a considerable number of vaccinated persons become affected with varioloid. Owing, howerer to the comparative mildness of varioloid, the rate of mortality is very small, and, therefore, fails to represent the relative number of vaccinated persons who become affected with modified smallpox. It is to be remarked that smallpox, in a certain proportion of cases, is experienced a second time. - From the following statistics it would appear that not much more protection is offered by the occurrence of variola than by vaccination: Of 5774 boys admitted into the Royal Military Asylum at Chelsea (England) in 48 years, 1950 had, on admission, marks of smallpox, and 3824 either had marks of vaccination or they were at once vaccinated. Of the former, 6.15 per 1000 , and of the latter, 7.06 per 1000 , contracted smallpox subsequently during their residence in the Asylum.'

'The opinion is largely held, and it appears to be well founded, that the extent of protection afforded by cowpox is less in the latter than in the former part of the period which has elapsed since the discovery of vaccination; that is, a larger proportion of vaccinated persons have had varioloid of late years than heretofore; hence many have been led to entertain the belief that the vaccine virns has deteriorated in consequence of its transmission from person to person. This belief has suggested, as important, obtaining, from time to time, virus direct from the cow.

Another reason for the increase of varioloid among those who have been vaccinated is, the protective influence of vaccination diminishes after

I Vide Journal of the Gynæcological Society of Boston, April and June, 1872. Report of discourse by Dr. Henry A. Martin.

$\&$ Vide Reynold's System of Medicine. 
the lapse of a certain number of years. Varioloid occurs much oftener among those more or less advanced in life than among the young. The length of time which has elapsed after a vaccination may fairly be considered as entering into the etiology of varioloid. The importance of revaccination rests upon this fact. Revaccination, after the lapse of a greater or less number of years, is completely successful, as regards the production of the vaccine disease, in a considerable proportion of cases, and partial success, as shown by imperfect or vaceinoid vesicles, is still more common. The epidemic prevalence of smallpox, embracing numerous cases of varioloid among vaccinated persons, has been promptly and completely arrested by universal vaccination and revaccination.

The importance of revaccination being admitted, the inquiry at once arises, how often should it be resorted to? There is no known law governing the cluration of the protective influence of a single vaccination. Probably the duration varies widely in different persons; and some persons are protected by one vaccination for life. Some have thought that revaceinations should be practised as often as every three years, and others that an interval of twenty years or more is allowable. It is evidently better that the period should be needlessly short than too long. With our present knowledge, the propriety, if not importance, of revaccinating every five year's is to be advocated. In case of known exposure, or when smallpox prevails as an epidemic, it is proper to revaecinate without regard to previous vaccinations. Revaccination, in fact, is always proper as the readiest and safest test of insusceptibility to smallpox.

There is ground for the belief that the frequent occurrence of varioloid, and also unmodified smallpox, among those who suppose themselves to be protected by having had cowpox, is due, in no small measure, to imperfect or spurious vaccinations. In this country vaccinators are often unqualified persons. The operation is performed, not infrequently, by those who make no pretensions to medical knowledge, and to a great extent by irregular practitioners. In many cases, persons vaccinated are never seen after the operation, they or their friends undertaking to judge for themselves of its success. Hence, a host of persons are either wholly unprotected, or but partially protected.

$A$ cause of inadequate protection is an insufficient number of vaccine vesieles. Many have supposed that a single vesicle is all that is required, and that the only advantage of inserting the virus in more than one spot is to diminish the risk of failure to procure a single vesicle. In many parts of this country it is not customary to procure more than one or two vesicles. Facts appear to show that the number of true vaccine vesicles has a decided influence on the amount of protective influence of cowpox. The following results of the analysis, by Simon, of nearly 6000 cases of smallpox contracted after vaccination, with reference to the proportion of deaths to the number of cicatrices, is interesting as connected with the present topic: The ratio of deaths among those who stated that they had been vaceinated, but who presented no vaccine cicatrix, was $21 \frac{3}{4}$ per cent. ; among those who had one cicatrix, $7 \frac{1}{2}$ per cent.; among those who had two cicatrices, $4 \frac{1}{8}$ per cent. ; among those who had three cicatrices, $1 \frac{3}{4}$ per cent., and among those who had four or more cicatrices, $\frac{3}{4}$ per cent. ${ }^{1}$ In a primary vaccination at least four or five vesicles should be obtained. A still larger number is advisable both in primary vaccinations, in persons who have been exposed to smallpox, and when the disease prevails as 
an epidemic, provided it be extremely important to secure the utmost possible protection, as in cases of pregnant females.

Since the publication of the first edition of this work (1866), a highly important practical question, relating to vaccination, has arisen in this country. In 1866 a case of spontaneous cowpox was discovered at Beatgency, in France. The virus obtained from this animal was perpetuated, throngh successive inoculations in heifers, conducted by Dr. Depaul, under the sanction of the French Academy of Medicine, and with the aid of the French government. The virus from this stock having been imported into this country by Dr. Henry A. Martin, of Boston, in 1870, he has since kept up the supply through a continued series of heifer vaecinations; and more recently the supply has also been kept up from virus derived from this stock, by Dr. Frank P. Forster, under the auspices of the New York Dispensary. The terms animal and bovine virus are used to distingnish that derived immediately from the heifer; that derived from the human subject being called humanized virus. The important practical question is, whether the animal virus affords greater protection against smallpox than the humanized virus. 'That the latter has deteriorated in consequence of removes from the cow, seems very certain. Vaceination with the long humanized virus is less constantly successful in producing vaccinia than formerly; the revaccination of persons who have been vaceinated with this virus is oftener successful, and a diminished protection is slown by the greater number of vaccinated persons who become affected with varioloid. The opinion that vaccination with the animal virus will secure a more complete and a longer exemption from the clanger of contracting smallpox, is shared by many, and this virus is now (1872) extensively used in this country, instead of the long humanized virus. The positive proof of the correctness of this opinion, of course, can only be obtained at a future period. It remains to be determined by the results of a long experience whether, assuming that, at the present time, the animal virus has a greater protective power, this power may not become deteriorated by distant removes from Beaugency stock, in heifers, as there is reason to believe has been the case in the human race. This, however, is a consideration which concerns the future; and a deterioration, if it occur, would only render it important to take advantage, at different periods, of other instances of vaceinia, in the cow, of spontaneous origin, ${ }^{2}$ aud in this way to renew the stock.

Vaccinia in man produced by the animal virus, differs, in certain respects, from the disease as produced by the long humanized virus. The full development of the vaccine pock requires a longer period; the pock continues to inerease for several days after the eighth day, this being the period required for the full development of the pock after vaccination with the long humanized virus. The pock is of larger size. The crusts are not formed until the eighteenth or nineteenth day, and they remain adherent from the trenty-fifth to the thirty-fifth day. The vesicle does not spontaneously rupture, and the crust preserves the form of the vesicle, with the umbilicated centre. The constitutional symptoms are more marked. In a small proportion of cases (one in fifty) a vesicular eruption occurs, and the vesicles, on close inspection, are found to be umbilicated. With respect to these points of difference, the disease, as now produced by the animal virus, corresponds with the disease as described

1 The term spontaneous is here used to distinguish from the disease produced by retrovaccination. Whether cowpox is ever in a broader sense spontaneous, is an open question. 
and delineated by the earliest vaceinographers-a fact which, in itself, renders probable the deterioration of the humanized, and the superiority of the animal, virus. ${ }^{1}$

Assuming that the animal or bovine virus is equal to the liumanized in its protective power (and at least this must be conceded), there are certain advantages which the former has over the latter. There is fur less liability to local accidents, namely, ulcerations and erysipelas. Dr. Martin states that, in April, 1872, there had been, at least, 400,000 revaccinations made, in this country, with the Beaugency virus, and no case of death, of erysipelas, or of post-raceine rariola had been reported among them. Another advantage is a more uniform success in both primary vaccinations and revaccinations. Another is, the supply of virus can always be made to meet the demand. Again, an advantage which has only to be named for its importance to be apparent, is the fact that the virus from the heifer is incapalble of communicating syphilis. Granting that the risk of syphilis is small when humanized virus is used, there is, nevertheless, a risk; syphilis has been repeatedly communicated. It is an immense advantage to eliminate all rlanger in this direction.

Deterioration of the long humanized virus is to be added to the causes, previously enumerated, of the apparent inadequateness of vaccination to afford complete protection against smallpox. Assuming that this obstacle may be overcome by the use of animal virus, the other causes are removable; and, were they to be entirely removed, it is not improbable that the expectation which Jenner cherished of exterminating smallpox might be realized. Certain it is, that, were all the requisites for the most perfect vaccination to be invariably observed, and if all persons living were vaccinated and revaccinated, smallpox, if not exterminated, would be insignificant as regards its prevalence and severity. The difliculty of securing for all mankind a protection against the disease almost complete, is not so much in the insufficiency of the means, as in enforeing their adoption to the fullest possible extent. Vaccination and revaccination, in order to be universal, must be compulsory, and authoritative measures to secure the proper and successful performance of the operation are essential. To consider the most efficient legal provisions to accomplish these desirable ends would be to open up a wide field for discussion, which would be inconsistent with the oljects of this work.

It remains to notice some practical points relating to the operation for vaccination. The situation for the introduction of the vaccine virus should always be that usually selected, viz., on the arm near the insertion of the deltoid muscle. Uniformity of practice in this respect enables physicians and others to ascertain without delay or doubt the fact of a previous vaccination, and to julge of its success by an examination of the cicatrices. Either the vaccine lympl or crust may be used. The lymph is the most active, and, when available, is to be preferred. The lymph is usually taken on ivory points or pointed quills, but the virus is preserved in this way only for a short period. A better mode, with a view to preservation, is to withdraw the lymph from the vesicles in glass capillary tubes, which are afterward sealed by means of a spirit-lamp. A crust inclosed in wax and covered with tin-foil retains its activity for a considerable period.

I Vide Discourse by Dr. Martin in Journal of the Gynæcological Society of Boston. Do. Practical Remarks on Vaccination by Dr. Frank P. Forster, in Neumann's Inandbook of Skin Diseases, translated by Bulkley. Also, Sur le Cowpox découvert a Passy, par Bousquet, Paris, 1S36. 
As regards the mode of vaccinating, if dried lymph be used, or lymph contained in capillary tubes, it may be inserted by means of slight punctures, or by scraping away the epidermis, or by minute scarifications; the latter method is preferable. Having made, with a common lancet, numerous linear and transverse scratehes, not deep enough to canse the escape of more than a drop or two of blood, the lymph is to be applied over the scarified spots, which should be five or six in number, and not larger than half a dime. If the incisions be sufficiently delicate, the opcration gives no pain, and allowing the spots to dry before being covered by the clothing is all that is required. If the crust be used, a small portion should be dissolved, always at the time of the operation, in a drop or two of water, and applied by means of the lancet over the scarified spots. This is preferable to making punctures and introducing small pieces of the undissolved crust. The latter mode is apt to cause sores, which, aside from the annoyance they occasion, may interfere with the specific action of the virus.

The age for the performance of a primary vaceination is a point of importance. Between the second and the third month of infancy is the period to be preferred. A liability to exposure to smallpox, however, renders the operation advisable at an earlier period. It is desirable that the child, when vaceinated, should be in good health, but the operation should not be delayed on this account if there be al liability to exposure to smallpox. If the operation prove unsuccessful, another trial should be made, and, if this fail, the virus may be introduced a third time. Some persons are insusceptible to cowpox; an insusceptibility is to be inferred from two or three successive failures. The attempt to vaccinate, however, should be repeated from time to time, for susceptibility may afterward exist. So long as there is insusceptibilty to cowpox, there is little danger of smallpox being contracted. Aside from want of susceptibility, and exclusive of inertmess of the lymph or crust used, the common cause of failure is undue bleeding from the scarifications, the flow of blood preventing the absorption of the virus.

Troublesome ulcerations occasionally follow vaccination, arising from irritation by the friction of clothing, or from an unhealthy condition of the system. These are to be treated like ordinary sores. Frysipelatous inflammation and abscesses may occur. These concomitants or sequels are not always evidence of any fault in the operation or impurity of the matter used. Dr. Henry G. Piffard, however, has reported cases in which the disease of the skin known as impetigo contagiosa lias occurred as a sequel of vaccination with the virus in crusts both animal and humanized; and on the examination of a considerable number of crusts he has found, not infrequently, in them, vegetable organisms which, as he has demon. strated, constitute the contaginm of the cutaneous affection just named. The liability to the communication of this affection is a reason for preferring lymph to crusts for vaccination. ${ }^{1}$ Ignorant persons are sometimes prone to attribute to vaccination diseases of any kind which may occur within a few weeks or months afterwards, and to charge the physician with having used impure vaccine matter. This impression may be so rooted as not to be removed, and, hence, a vaccination may very unjustly prove the occasion for a withdrawal of the confidence which the physician had previously possessed.

After a known exposure to smallpox, if the person exposed have not

1 Vide articles by Dr. Piffard in the New York Med. Journal, Nos. for June and July, 1872. 
been recently vaccinated, or if there be any reason to distrust a previous vaccination, revaccination should, of course, be promptly performed. It is proper to perform it without reference to previous vaccinations, with a view to test the susceptibility and render the security as complete as possible. It has occurred to me repeatedly to vaccinate, under cireumstances involving the utmost possible exposure, persons who had never been vaceinated, and thereby to prevent the oceurrence of smallpox. In one instance an unvaccinated child lived in the same room and slept with a person who had contracted smallpox. The first operation proving unsuccessful, it was repeated three days afterwarls with suceess, and the child escaped. In another instance a patient with measles was supposed to have smallpox and sent to a smallpox hospital. He had never been vaccinated, and vaccination was at once performed; the vaccine disease was deferred until the measles had run its course, it then became developed, and smallpox did not occur. Owing to the relatively short duration of the period of ineubation in cowpox, it anticipates the development of smallpox. I have not observed the coexistence of the two affections under these circumstances.

\section{Varicella. Chicken-Pox.}

Varicella, or, as it is commonly called, chicken-pox, is an affection quite insignificant as regards danger or distressing symptoms. It is important with reference, first, to the question as to its having pathological relations with smallpox, and, sccond, to its discrimination from modified variola or varioloid.

It is an eruptive disease affecting for the most part, children, but oceasionally adults. The eruption is preceded generally by slight constitutional symptoms for about twenty-four hours. The eruption appears first on the body, and afterwards on the head. It is usually more abumdant on the neck, body, and scalp, than on the face. The eruption is frequently quite small, and very rarely abundant. It is almost invariably discrete. It is a vesicular eruption from the first. The vesicles contain, at first, a transparent liquid which afterward becomes opaline, but not purulent except when they are irritated by scratching. Desiccation occurs from the fifth to the seventh day. The erusts are granular, and are rarely followed by cicatrices or pitting. Sometimes bullæ of greater or less size form instead of vesicles. New vesicles frequently continue to appear during the first three days of the stage of the eruption, and even after the desiccation of those which first appeared has commenced. During the eruptive stage, constitutional symptoms are slight or wanting. 'The disease is contagious as regards children, but very rarely as regards adults. Experiments made to determine its communicability by inoculation have frequently, if not invariably, failed. The period of incubation varies from twelve to seventeen days. Writers have instituted several varieties based upon clifferences as regards the size and shape of the vesicles. The ordinary variety, in which the vesicles are about the size of split peas and flattened at the top, has been distinguished as varicella lcntiformes, vel lenticularis, or lenticular chicken-pox. The variety called varicella coniformis, vel conoides, or swine-pox, is characterized by the conoidal form of the vesicles. Varicella globularis, vel globata, is the name of a variety in which the vesicles are unusually large and globular in form. The latter varicty has been called "the hives." The different vesicles in the same case not infrequently present the characters 
of these three varieties. The division into varieties is of not the least practical consequence.

The name varicella, which is a climinutive of variola, meaning little smallpox, implies that the disease is essentially variolous. It has been considered as a modified form of variola, and is so considered by some eminent teachers and writers at the present time. The non-identity of the two affections is established by the following facts: The miasm of varicella is never known to give rise to ordinary variola, whereas the severest form of the latter disease may be derived from cases of varioloid. Varioloid prevails only during the prevalence of ordinary smallpox, but varicella not infrequently prevails when there are no cases of smallpox. Taricella is very rarely communicated to adults. This is not true of varioloid. Varicella affords no protection against variola, and, conversely, persons who have had smallpox contract varicella. Vaccination does not protect against varicella, and the latter does not interfere with the success of vaccination. It does not seem to be indubitably settled that varicella is ever communicable by inoculation. Other points of difference, showing the non-indentity of varicella and varioloid, relate to the clinical history, and upon these points the differential diagnosis is to be based.

Although pathologically distinct, it must be confessed that the discrimination of varicclla from varioloid is not always easy. The two affections are liable to be confounded. Cases of varioloid are not infrequently, at first, considered as cases of varicella, and vice versa. In view of the great importance of making this differential diagnosis promptly and positively, the following diagnostic points claim the careful attention of the student and practitioner:-

The stage of invasion in varioloid is as long, or longer, than in cases of ordinary smallpox, viz., from two to three days. The short duration of this stage in varicella is a distinctive feature. The constitutional symptoms, in this stage, are often as marked in varioloid as in cases of ordinary smallpox. The mildness of these symptoms in varicella is a diagnostic point. The vesicular character of the eruption from the start is characteristic of varicella. In varioloid the vesicles are preceded by papules. In varioloid, as in ordinary smallpox, the eruption appears at first and especially on the face; in varicella, it appears first on the body, and is apt to be more abundant elsewhere than on the face. A capital diagnostic point relates to the central depression of the umbilieated appearance; this is generally discoverable in more or less of the vesicles in varioloid, and it is generally, if not invariably, wanting in varicella. The duration of the eruptive stage is less in varicella than in the great majority of cases of varioloid. Finally, traces of vesicles in varicella are very rarely found on the mucous membrane of the mouth and fauces.

During the prevalence of smallpox, cases of varioloid in which the eruption is small, are ofter considered as cases of varicella. The cases of so-called varicella in adults, when smallpox prevails, are generally cases of varioloid-hence, one source of the diffusion of smallpox.

Varicella claims no treatment. It is important to recognize it only in order to relieve apprehensions by the assurance that the disease is not varioloid. 


\section{CHAPTER VII.}

\section{ERUPTIVE FEVERS-(CONTINUED.)}

Scarlatina, or Scarlet Fever-Anatomical Characters-Ciinical History-Causation-Diagnosis -Prognosis-Treatment.

THE exanthematous fever called, from the color of the eruption, scarlatina, or scarlet fever, is remarkable for the wide diversity which it presents, in different cases, as regards symptoms and fatality. In its mildest form it is a trivial affection; in its severest form there are few diseases more appropriately styled malignant. The disease has been divided into several varieties based on difrerences in symptomatic characters and intensity. 'Three varieties are generally recognized by writers, viz., scarlatina simplex, scarlatina anginosa, and scarlatina maligna. Widely different as are these varieties in respect of gravity and distinctive characters, they are not distinct diseases; essentially they are identical. A simpler division is into mild and severe scarlatina, but it will suffice to consider incidentally the events which belong to the latter, without treating of it separately.

Anatomical Cinaracters.-The eruption on the surface and morbid appearances in the throat constitute the anatomical characters in this disease. Lesions in other situations, which may be found after death, are due to concomitant affections or complications. The researches of Drs. Samuel Fenwick and Murchison show the frequent occurrence of a condition of the mucous membrane of the stomach and intestines analogous to that of the cutaneous surface. The former observer found, on microscopical examinations in sixteen cases, accumulation of granular and fatty matter in the stomach tubes and the follicles of Lieberkïhn; fibrinous casts of these tubes in the contents of the stomach and intestine, and desquamation of epithelium. Appearances similar to these were wanting in all save one of forty-fivc cases in which examinations were made of subjects dead with other diseases; and the excepted case was a case of gastritis. ${ }^{1}$ Some writers consider hyperæmia of the kidneys, frequently associated with inflammation of the convoluted tubes, as bclonging essentially to the disease, insead of being embraced among the complications.

Clinical History.-The symptomatic events of the several stages separately will be first noticed, and, afterward, anomalous events and complications liable to occur in either of the three stages.

Slage of Invasion.-The attack may commence with a chill, more or less pronounced, but not infrequently it is wanting. Vomiting is generally an early symptom, especially in children, with, in a certain proportion of cases, diarrhoa. The febrile movement, as a rule, is notably greater than in the other eruptive fevers. Epistaxis is not uncommon. Redness of the fauces, more or less vivid, may gencrally be observed in

1 Proceedings of the Isondon Royal Medical and Chirurgical Society, London Lancet, July $23,1864$. 
this stage, either with or without a sense of soreness, and pain in the act of swallowing. The affection of the throat is due to the efflorescence, which appears in this situation before making its appearance on the skin. As regards the intensity of febrile movement, the increased heat of skin, pain in the head, prostration, and general malaise, different cases differ widely, not including eases of unusual severity. In very mild cases, the symptoms are so slight that the patient does not take to the bed.

The average duration of this stage is about twenty-four hours; the eruption usually appears on the second day. Exceptionally it appears, on the one hand, a few hours after the attack, and, on the other band, it may be delayed one, two, three, or more days after the time when it usually appears. A considerable delay in the appearance of the eruption is generally attributable to some complication.

Slage of Eruption.-This stage, in children, is sometimes ushered in by a transient convulsion which, at this period, is not an uræmic symptom. In its development and diffusion over the body, the eruption does not observe the same regularity as smallpox and measles. It oftener appears on the body and limbs before making its appearance on the face and neck, but in a certain proportion of cases it appears first on the latter situations. It is rapidly diffused, extending more or less over the whole cutaneons surface in twenty-four hours. As regards the degree and extent of the efflorescence, different cases differ much. The first appearance is in the form of minute dots or specks. These coalesce, forming irregularly distributed patches, which vary much in size and shape, having irregular or serrated margins. Exceptionally, the eruption remains punctiform, that is, in the form of distinct macula or spots. The redness is most vivid and remains longest in situations where the skin is delicate, as the inner surfaces of the arms and thighs and the flexures of the joints. The redness is vermilion or scarlet; it is not uniform, like erysipelatous redness, but, on close inspection, the patches are seen to be studded with points, which are distinguished by a more deeply-red color. The redness momentarily disappears on pressure, and white lines are produced by drawing a pointed hard substance-for example, a pencil-over the reddened surface. In some cases the whole cutaneous surface is covered with the efflorescense, presenting an appearance which has been compared to that of a boiled lobster. Generally, on passing the fingers over the reddened surface, it is felt to be smooth, but in some cases there is a sensation of minute elevations or papules; these are the papilla abnormally developed, as in the condition commonly known as goose-skin. Occasionally miliary vesicles are seattered, more or less abundantly, over the surface. The integument is slightly swollen; this is evident on the face, and is apparent to the patient when the hands are closed. The feet are also evidently somewhat swollen. The eruption is sometimes accompanied with more or less burning and pruritus. The eruption attains to its maximum of intensity and diffusion on the third day after its first appearance.

There is much variation in different cases in the amount of cutaneous eruption: It is sometimes slight. This is apt to be the case when the throat affection is unusually severe; the eruption then seems to be concentrated within the throat. Occasionally the efflorescence in the skin clisappears shortly after its appearance, and afterward returns. It fluctuates as regards vividness, being especially marked in febrile exacerbations, and it is generally more vivid toward evening than in the early part of the day. In anomalous cases, which will presently be referred to, the eruption is wanting. 
The throat is more or less affected in the vast majority of cases; but in some very mild cases there is no throat affection. If the throat be but slightly affected, there is simply redness over the tonsils, the posterior part of the pharyux, and extending more or less over the soft palate, with little or no swelling. The affection is then only an efllorescence. In most eases, lowever, there is more or less swelling of the tonsils, together with a white or ash-colored exudation in a greater or less abundance. This exudation is generally not like that which characterizes diphtheritic inflammation. It adheres less closely; it is pultaceous, and camnot be detaehed in strips. It may receive a dark color from matters vomited and from the oozing of blood. In proportion to the amount of throat affection there is pain in deglutition, and the voice becomes nasal. The submaxillary and lymphatic glands at the angle of the jaw are frequently swollen and tender. A still greater degree of throat affection belongs among the anomalous events of the disease, and characterizes the variety distinguished as scarlatina anginosa.

The tongue early in the clisease is generally coated. While the coating remains, frequently the papilla projecting through it have the appearance of numerous red points; the surface of the tongue looks as if cayenne pepper or red sand had been sprinkled over it. This is seen in other affections. Another appearance is quite clistinctive of scarlet fever. In the progress of the clisense, the coating exfoliates, leaving the surface of the tongue clean and reddened; and, the papilla being enlarged, the appearance is strikingly like that of a ripe strawberry. 'The strawberry-like tongue is a pathognomonic symptom; it is peculiar to this disease. It is often but not uniformly present. It is due to a condition of the mucous membrane analogous to that of the skin in the cases of so-called rough, as distinctive from smooth, scarlatina.

The febrile movement is not diminished, but, as a rule, increased after the appearance of the eruption. The frequency of the pulse and increased heat of the skin are more marked in this than in any other of the essential fevers. The pulse varies in different cases which, although more or less severe, pursue a favorable course, from 100 to 130 or 140 per minute. In extremely severe cases, especially in children, it rises still higher. A side from frequency, the pulse is quick, often vibratory or thrilling, but always compressible, these characters showing exeited action without augmented power of the ventrieular contractions. Marey's observations with the sphygmoglaph show that in scarlatina, and in all the eruptive ferers, the form of the pulse denotes strong arterial tension, the absence of dierotism being the character which especially has this significance. The skin is usually dry, and the heat, as felt by the hand, is often intense. The thermometer in the axilla shows an increase of temperature to $105^{\circ} \mathrm{Fahr}$, and in severe eases a still greater increase; it may even reach $112^{\circ}$ Fahr.

During the stage of the eruption, anorexia continues, vomiting is not uncommon, constipation exists in some cases, and in some cases diarrhoa. Thirst is usually urgent. Delirium, manifested by incoherency, generally exists, save in very mild cases. I have known a patient, not consiclered ill enough to require a wateher during the night, get $u$ ) and wander out of doors in a state of delirium. A melancholy instance, illustrative of the importance of watchfulness in the care of patients in the delirium of fever, occurred, many years ago, at the Louisville Marine Hospital during my term of service. A female adult patient affected with scarlatina, but not considered as seriously ill, had been removed to a room, by herself, in the uppermost story of the hospital building, with a view to isolation. During the absence of her attendant for a few moments, she precipitated 
herself from the window, and, falling upon the pavement, was instantly killed. Restlessness, jactitation, and insomnia are apt to be more or less marked. Albuminuria occurs in a certain proportion of cases during the eruptive stage. Of 21 eases in which the urine was found to be albuminous by $\Lambda$ beille (the whole number of cases analyzed being 53 ), in 8 cases the albuminuria was noted in the stage of the eruption. 'Transient, slight albuminuria occurs in scarlatina, as in various other affections, without denoting renal disease; but disease of the kidneys forms an important complieation in certain cases of scarlatina, and will presently be noticed.

The duration of the stage of eruption, in the majority of cases, is from 4 to 6 days. It is sometimes shorter; oftener it is prolonged to 8 or 10 days, and occasionally it continues for a longer period. Of 54 cases analyzed by Jenner, the ernption disappeared on the 5th day of the disease in 1 case, on the 6th day in 3 cases, on the 7th day in 5 cases, on the 8th day in 13 cases, on the 9th day in 12 cases, on the 10th day in 8 cases, on the 11th day in 4 cases, and on the 13th, 14th, and 16th days respectively in 2 cases.'

Stage of Desquamation.--The commencement of desquamation marks the beginning of this stage. Desquamation frequently commences with the decline of the eruption, and prior to its disappearance. The degree and extent of the desquamation is, in general, proportionate to the intensity and diffusion of the efflorescence. Desquamation is rarely wanting, save in the cases in which the efflorescence fails to appear. The cuticle is exfoliated either in the form of minute branny scales, called furfuraceous desquamation, or in pieces of epidermis of greater or less size. Occasionally the epidermis of the hands is detached entire, and may be stripped off like a glove. This is true also of the epidermis of the feet. Sometimes several successive exfoliations occur.

In cases which pursue a favorable course, the febrile movement diminishes with the commencement of exfoliation and gradually ceases, corresponding improvement taking place in other symptoms. The decrease of animal heat, or defervescence, is sometimes rapid, but in most cases gradual. ${ }^{2}$ If the disease be uneomplicated, and there be no untoward events, convalescence is declared during the stage of desquamation. The desquamative process is frequently accompanied with pruritus, which, in some cases, is excessive. Albuminuria occurs in this stage more frequently than in the stage of eruption; $A$ beille found it in 13 of the 21 cases (the whole number of cases being 53) in which this symptom was present at some period of the disease. As in the stage of eruption, so in the desquamative stage the presence of albumen in the urine may or may not be indicative of an important renal complication. A recurrence of the eruption may take place during the stage of desquamation. I observed this in the case of a son of a colleague (Prof. Mott), the secondary eruption being marked and diffused over the entire cutaneous surface, but without febrile movement, or any return of the efflorescence in the throat. There was no albuminuria in this case.

The duration of this stage is indefinite. It may end in five or six days. The desquamation is generally completed in from ten to twelve days, but, exceptionally, it continues for several weeks.

The foregoing sketch of the clinical history of scarlet fever applies to cases which are either mild or not extremely severe, and in which the

1 Gulstonian Iuectures, 1855.

2 Vide On the Temperature in Scarlet Fever, by Dr. Sydney Ringer, in Trans. of Med. and Chir. Society of London, vol. xlv., 1862. 
course of the disease is regular, that is, without amomalous events or complications. Certain eases are characterized by extreme gravity of the symptoms at the outset of the disease. The pulse becomes very frequent, numbering $140,150,160$, and more per minute. 'The thermometer shows great elevation of temperature. Great restlessness, delirium, coma, and occasionally convulsions, are other symptoms denoting severity of the disease. The vital forees seem to be overwhelmed, and death may take place in one or two days, or sometimes even within a few hours from the attack. A fatal result may take place before the eruption appears on the skin or any pharyngeal reduess is apparent. The disease, in such cases, is truly malignant. Cases of this description are liable to oceur in eertain epidemies. The gravity in these cases is not due to any complieation, but to an unusual intensity of the essential morbid conditions which constitute the disease. $\Lambda$ taxic or nervous symptoms, in like mamner dependent on the intensity of the disease, are liable to be developed at a later period, viz., active delirium, typhomania, carphologia, and subsultus tendinum. These symptoms characterize certain cases of severe scarlet fever.

Convulsions, coma, and delirium may be developed as effects of uræmia. Disease of the kidneys is an important complication, or a local manifestation, in a certain proportion of eases. Albuminuria occurs, either during the stage of eruption or desquamation, in nearly a proportion of 1 to 2. It may continue for two or three days only, or during the whole career of the disease, and afterward. A beille found its average duration, in 21 eases, seven or eight days. This symptom may not indicate any important affection of the kidneys, and, on the other hand, grave disease of the kidneys may exist withont albuminuria. Renal epithelium and cylindrieal, waxy, epithelial or granular easts are more or less abundant in the sediment of the urine, with or without albuminuria, in a pretty large proportion of eases. 'These are of more significance as denoting renal disease than the presence of albumen in the urine. The liability to uræmia, and the evidence of its existence, here, as under other circumstances, are denoted by the deficient elimination of urea. Examinations of the urine with reference to this point are of more consequenee than with regard to albumen and easts. Here, as under other eireumstances, the deficiency of urea is to be inferred from a scanty sceretion and a low specific gravity; or, if the quantity passed in a given time can be ascertained, the amount of urea may be determined by volumetric analysis after Davy's method.' Clinical facts show that scarlatina involves a special tendency to renal clisease, not only as a sequel but as a coneomitant. And it is a rational supposition that the lining membrane of the secretory tubes of the kidneys takes on a morbid condition analogous to that of the mucous membrane of the pharynx, constituting a locil affection serious in proportion as the excretion of urea is interfered with. General dropsy sometimes occurs cluring the progress of the disease, as well as afterward. Symptoms pointing to uræmia, in addition to the events already stated, viz., convulsions, coma, and delirium, are cephalalgia, disturbance of respiration occurring without either bronchitis, pneumonia, œdema of lungs or any appreciable pulmonary affection, defective vision, vomiting, and oceasionally diarrhœe: These symptoms should direct attention to the urine as regards albuminuria, the presence of easts, and, more especially, the deficiency of urea. Uremia, taking place either as a concomitant or sequel of scarlet fever, may occur with-

' Vide Manual of Chemical Examination of the Urine, by A ustin Flint, Jr. 
out albuminuria, and without dropsy. Hence the importance of examinations of the urine in all cases, with reference, not alone to the presence of albumen, but of casts and the quantity of urea.

Certain cases of severe scarlatina are characterized by a great amount of throat affection. The name scarlatina anginosa is applierl to these cases. The tonsils are greatly swollen. The exudation is unusually abundant, and in some cases the pharynx offers all the characters of diphtheretic inflammation. Deglutition is difficult, and liquids taken into the mouth are returned through the nostrils. The inflammation may extend to the posterior and anterior nares, and acrid pus flows from the nose, excoriating the face. The breath emits in intolerable fetor in some cases. Sometimes, but rarely, the inflammation extends into the larynx and bronchial tubes. Edema of the glottis is an occasional accident in adults. The structures of the eye may become involved, and destruction of both eyes has been known to follow. An extension of the inflammation through the Eustachian tube into the internal ear is liable to occur. I have met with a case in which the tympanum in each ear was perforated, and the ossicles were discharged through the external mentus. Permanent deafness, facial paralysis, and, in some cases, caries of the temporal bone followed by circumseribed meningitis and abseess of the brain, are effects of an extension of the local affection to the internal ear. The submaxillary and lymphatic glands of the neck become more or less enlarged, and sometimes suppuration takes place, constituting what have been called scarlatinus buboes. I have met with a case, in the practice of $\mathrm{my}$ friend, Dr. Dudley, of Brooklyn, of extensive sphacelation of the lips and cheeks, the patient being a child well nourished and healthy up to the date of the attack. Gangrene and ulceration within the throat are very rare events. The local events belonging to scarlatina anginosa, inclusive of inflammation and suppuration of the glands of the neck, may occur early in the disease, or, on the other hand, they may be developed during the stage of desquamation, the symptoms having previously denoted a mild form of the disease. A fibrinons exudation in the fauces characterizes certain cases, and denotes that constitutional state which exists in diphtheria, or the supervention of the latter disease, the danger from which is enhanced by its occurring as a concomitant or sequel of the scarlet fever.

In cases of so-called scarlatina anginosa, the cutaneous eruption is apt to be slight and sometimes wanting. Albuminuria is more apt to exist in these cases. Uræmic phenomena are liable to occur, but, exclusive of these, and of laryngitis or œedema of the glottis, the severity of the throat affection may be sufficient to lead to a fatal result.

Hemorrhage from various situations, together with petechiæe and vibices on the skin, cliaracterize certain cases. The name hemorrhagic scarlatina lias been applied to these cases. These cases almost invariably prove fatal. Hæmaturia occurs in connection with renal disease, and, if not accompanied with hemorrhage elsewhere, is not necessarily of grave omen.

Complications other than those which have been noticed, and of rare occurrence, are articular rheumatism, usually confined to a few joints, vaginitis, pleuritis or pyo-thorax, pericarditis, and endocarditis, the two latter affections being associated with either rheumatism or renal disease.

In irregular cases of scarlatina the disease is often, but by no means always, severe. The only local manifestation of the disease may be a slight soreness of the throat, no cutaneous eruption taking place. In these cases the disease has becn distinguished as scurlatina faucium and scarlatina sine eruptione. Cases of the throat affections, more or less 
severe, without any eruption on the skin, not infrequently occur during the prevalence of epidemies of scarlatina. An attack of this kind appears, in some cases at least, to render the paticnt thereafter insusceptible to the disease, but it is not uncommon for persons who have once had searlatina in its regular form to suffer from the throat affection when exposed to the infectious miasm. In cases of scarlatina sine eruplione the nature of the disease is not infrequently overlooked. Another irregularity in very mild cases consists in the absence of any affection of the throat, the cutaneous affection bcing more or less marked. In these cases, doubtless, the disease sometimes exists without being recognized, or it is confoumded with roseola. It has been supposed that the disease may exist without either an affection of the throat, a cutaneous efflorescence, or febrile movement. The name scarlatina latens has becn applied to such cases. The evidence of the existence of the disease in this latent form is the occurrence of characteristic sequels, viz., albuminuria and anasarca, in persons who had been exposed to the scarlatinous infection, without presenting auy of the usual phenomena of scarlatina. The local manifestation of the discase, in these cases, is supposed to be exclusively in the kidneys. Assuming that such cases occur, and that this explanation be true, it is more correct to say that the searlatinous infection sometimes gives rise to acute albuminuria without causing scarlet fever. I have referred to cases cxemplifying this fact in treating of acute albuminuria.

Scarlatina has various sequels. The one which oceurs most frequently has just been alluded to, viz., albuminuria with general dropsy. This follows the disease in a considerable proportion of cases, the proportion being found to vary at different times and places. The time of its oceurrence is from ten to twenty days after the date of desquamation. The symptoms are those of acute albuminuria, or, after Johnson, acute desquamative nephritis, an affection which has been considered in connection with.the diseases of the urinary system. ${ }^{1}$ (Edema of the face and lower extremities is first observed, and anasarca frequently follows. Effusion takes place into the serous cavitics if the anasarca be considerable. Hyclrothorax sometimes exists to sueh an extent as to embarrass seriously respiration. The urine is scanty, and not infrequently contains blood. It is usually loaded with albumen, but cases have been reported by Rayer and others of dropsy after scarlatina without albuminuria. Epithelial, granular, or waxy casts are generally found in the urinary sediment. There is usually notable anxmia, the appetite is deficient, the temper is irritable, and muscular debility is more or less marked. Febrile movement, varying in degree in different cases, accompanies these symptoms. Urremic coma and convulsions, pulmonary celema and celema of the glottis are grave accidents ineident to the renal affection. They occur happily in but a small proportion of cases, and, under judicious management, this affection is rarely fatal. Temporary blindness is a rare event occurring in connection with uramic convulsions following this clisease. ${ }^{2}$ A eute albuminuria after scarlet fever rarely ends in chronic renal clisease. It is more rational to consider the renal affection which follows scarlatina as a special effect of the latter, than to attribute its production to cold or other causes acting after the searlatinous poison has ceased to act.

The opinion has been held by Graves and others that the kidneys are oftener affected after mild than severe cases of scarlatina. 'This sequel certainly occurs after mild cases, and sometimes when, from the absence of either the throat affection or of the cutaneous eruption, scarlet fever 
had not been supposed to exist. A probable explanation, however, of its occurrence in a larger proportion of mild cases is afforded by the fact that, of severe cases, a considerable number end fatally during the progress of the fever. It is to be borne in mind that coma and convulsions may occur as sequels of scarlatina, due to uræmia taking place without either albuminuria or dropsy. That the renal affection has no necessary connection with the eruption on the skin, is shown by its occurrence in eases in which the scarlatinous rash was wanting. Dr. R. Hubbard, of Bridgeport, Conn., has related to me a case of this kind which eame under his observation.

Pleuritis, pericarditis, and acute articular rheumatism are oceasional sequels as well as concomitants of scarlet fever. Chorea is also to be reckoned among the occasional sequels. External otitis is not uncommon. A purulent discharge takes place from the ears, and sometimes there is considerable deafness. If the inflammation be wholly withont the tympanum, the deafness will be temporary, and, under judicions management, the affection may generally be removed. It is apt to return, from time to time, for a considerable period after recovery.

Causation.-The communicability of scarlet fever is established by irrefragable proof, and, exclusive of inocnlation, it is probable that its eausation always involves infection. Of those who are known to be brought into contact with cases of the disease, a much larger proportion eontract it than of those not exposed. The following facts, given by Rilliet and Barthez, afford a striking example of this kind of proof of communicability. "Out of 53 children who contracted scarlet fever in the Children's Hospital at Paris, 34 were in wards into which scarlet fever cases were admitted, 14 were in wards in elose communication with the preceding, and only 5 were in wards having but slight communication with those first mentioned." Equally strong proof is afforded by the importation of the disease into localities previously free from it. For the following example I am indebted to a former colleague, the late Prof. $B$. R. Palmer: A farmer living several miles from Woodstock, Vermont, on a by-road, which was not a thoroughfare, had but a single neighbor. He visited, with his family, some friends at a town about twelve miles distant. Scarlatina existed in the families with whom he visited. After his return home, all his children (the precise number is not noted) had, in succession, scarlatina. The children of his only neighbor contracted it. Five children in these two families died with it. The cases were under the care of a physician living at Woodstock, distant some six or seven miles. Scarlatina did not previously exist in Woodstock. Shortly two children in the physician's family were attacked and one died. A family in Woodstock was related to one of the families in which the disease first appeared, and the husband and wife watched one night with the ehildren of their relative. Shortly afterward their children were attacked. Communication with this family and the family of the plyysician was suspended throughout the village, and the only additional person affected was a boy who was exposed by going to the physician's house during the time his children were ill. Proof of this kind might be multiplied. Finally, it appears to be certain that the disease has been communicated by inoculating with the serum contained in the vesicles which sometimes appear in conjunction with the cutaneous efflorescence.

That the disease may be communicated by means of fomites is unquestionable. The infections miasm may be carried in the clothes of physi-

1 Cited by Murchison, Braithwaite's Retrospect, London, 1865. 
cians, nurses, and others. The disease is probably in this way often diffused. Facts show that the miasm adheres to material substances and preserves its morbific power for a long period. Persons have been attacked on returning to houses in which cases have occurred, for weeks or months afterward, and even when elaborate efforts had been made to remove the miasm. Benjamin W. Richardson gives some striking examples. ${ }^{1}$ The following instance came under uny own observation. $A$ child in a family living in a private boarding house in this city had scarlatina. The mother and child of another family, boarders in the same house, were on a visit in the country. They remained away for a month in order that the child might not be exposed. There was but a single case of the disease in the house. At length, deeming it safe, they returned, and three days afterward the child was attacked with scarlatina.

The period of incubation is undoubtedly, as a rule, shorter in scarlatina than in the other eruptive fevers. Its duration, however, varies in different cases. In the instances just cited, it was three days. 'Trousseau cites a case in which the attack took place within twenty-four hours after exposure. In a case under my observation, the patient, an infant of nineteen months, was attacked twenty-four hours after the first symptom of illness in a young girl who assisted in taking care of the infant. It is probable that the latent period rarely, if ever, exceeds six days.

Statisties show that the clisease occurs most frequently in the third and fourth year of life; that the liability to it diminishes rapidly after the fifth year, and becomes very small after forty. Out of 2042 cases admitted into the London Fever Hospital during twenty years, only four were above fifty, and one above sixty. Children under two years of age and infants at the breast are not, as has been incorrectly stated, insusceptible to it. Of 431 cases collected by Richardson, 15 were under two years of age. Within a few months from the time of writing, two instances have come under my observation. In one of these, the clild was a year old; in the other instance, a child only three weeks old contracted the disease from her mother, who was attacked a few days after confinement. Both children recovered. As bearing on this point, the following statistics, cited by the author just named, are interesting: Of 12,962 deaths from scarlet fever in children under fire years of age, according to the mortality tables of the Registrar-General of Great Britail, the number of cases under one year amounted to 1289 , and the number between one and two years 2874, making the total under two years 4163 . Case have been reported in which the disease has been contracted in utero.

As a lule, this clisease is experienced but once; but, making due allowance for cases in which roseola may have been confounded with scarlatina, the exceptions to this rule are not extremely rare. Richardson states that he has himself had the disease three times. My colleague, the late Prof. Elliott, witnessed the occurrence of the disease three times in the same subject. It is stated that secondary or tertiary attacks are never fatal.

Dragnosis.-Attention to the following diagnostic points renders the discrimination of the disease, in the great majority of cases, sufliciently easy: The short duration of the stage of invasion, the intensity of the febrile movement, the appearance of the efflorescence in the throat prior to the cutaneous eruption, the appearance of the efllorescence on the body and upper extremities often before or simultancously with its appearance 
on the neck and face, the rapid extension of the efflorescence over the cutaneous surface, the scarlet color of the efflorescence, the irregular and serrated margins of patches of efflorescence, the persistence or increase of the febrile movement after the appearance of the efflorescence on the skin, and the exalted heat of the body. 'These are positive diagnostic features belonging to the clinical history of scarlet fever as contrasted with smallpox and measles. On the other hand, the two latter eruptive ferers are excluded by the longer period of invasion in both, pain in the loins not marked in this period, absence of the characteristic eruption of variola in the month and fances, the non-occurrence of variolous papules and resicles with depression in the centre, and of the remission of fever when the eruption appears; absence of coryza and bronchitis which belong to the history of rubeola, and of certain distinctive characters pertaining to the eruption of rubeola, which will be stated in treating of the latter.

The difficulties in the way of diagnosis relate, in the first place, to extremely mild cases, with a slight eruption, and without any affection of the throat. In these cases the disense is liable to be confounded with roseola. The differential points will be noticed in treating of the latter in the next chapter; and, in the second place, the diagnosis may be difficult in cases in which the local manifestation is confined to the throat; that is, cases in which the cutaneous efflorescence is wanting. It must be confessed that the diagnosis in some of these cases must be, in a measure, based on their occurrence during the prevalence of scarlatina, and sometimes on the occurrence of well-marked cases in members of the same family. In some cases of doubt or error, the sequels of scarlatina, especially those pertaining to renal disease, serve either to correct or establish the diagnosis.

A highly probable diagnosis, before the efflorescence appears on the skin, may be based on a sudden attack of vomiting, with high febrile movement, and redness of the fauces, in a subject between three and five years of age, especially during the prevalence of scarlatina.

Prognosis.-There is, perhaps, no disease in the nosology which presents, in different cases, wider extremes, as regards gravity, than scarlatina. In malignant cases the disease is rapidly fatal. The proportion of these cases varies much at different times and places. Richardson has advancel the opinion that the relative proportion of severe and mild cases is always about the same, viz., as 1 to 5 , there being a sufficiently large number of mild cases to equalize the ratio whenever the number of fatal cases is large. This opinion is opposed to common observation. The prevailing type of the disease, in some seasons, is mild, and in other seasons, severe. Whether this variation be due to a difference in the virulence of the miasm, or to adjunctive influences, we are unable to say. A side from the intensity of the disease, the danger depends on complications liable to occur in cases which, as regards the general symptoms, may appear to be mild. The symptoms which denote imminent danger from the intensity of the disease, are, excessive frequency of the pulse, jactitation, active delirium and prostration, the mode of dying, in these cases, being by asthenia.

The prognosis is unfavorable in cases in which the throat affection is unusually severe. It is extremely unfarorable if diphtheria become developed, either during the progress of the disease, or as a sequel. If laryngitis ensue, a fatal result is to be expected. Great enlargement and suppuration of the glands of the neck, irrespective of diphtheritic inflam- 
mation of the fauces, denote great danger. If gangrene of the throat or month occur, recovery is the exception rather than the rule. Cases characterized by hemorrhage from nueous membrane and petechiæe or vibices are generally fatal.

Uræmic coma and convulsions involve imminent danger. Uræmia may become developed in cases which, as regards the general symptoms, appear to be mild. In the following case, which I noted twenty-five year's ago, sudden death occurred when, from the apparent mildness of the disease, there were no apprchensions of immediate danger. I was requested to see, in consultation with my friend, Dr. Pride, a girl aged eighteen, whom $I$ found in deep coma, the respirations irregular, the pulse having considerable foree. Death took place two hours afterwards. Dr. Pride stated the history as follows: She had been in delicate health for some time. Ou the day but one preceding her leath, she was attacked with scarlatina. The eruption was copions and the throat affection slight. Dr. Pride saw her at ten o'clock on the evening of her death. She was then sitting up, scemed very comfortable, and shortly after retired to bed. At eleven o'clock her mother, who had not retired, while conversing with her daughter, observed a slight spasm, the eyes rolled upward, and found that she was unconscious. Dr. Pride was immediately sent for, and arrived in a few moments. On his arrival, the patient was fully conscions, declared she was quite comfortable, and wondered at the anxiety of those around her. She had aroused a few moments after her mother had become alarmed, and was not conscious that anything unusual had occurred. Dr. Pride remained to observe the patient for a time. After talking jocosely for a few moments, and laughing at her mother's fears, she appeared to drop asleep, and Dr. Pride observed that she breathed heavily and groaned. He was told that this was not unusual. She awoke repeatedly, and remarked upon her audible respiration. At length Dr. Pride observed a slight tremor and a rolling upward of the eyeballs. He immediately attempted to arouse her, with partial snccess, but she specdily fell into complete coma, and cleath took place within three hours. The coma and convulsions, in this case, it is presumed, were due to uræmia, but the symptoms relating to the urine had not been ascertained at the time the case occurred.

Daily examinations of the urine, in cases of scarlatina, are highly important. The presence of albumen or casts should always excite apprehension, but the immediate danger is proportionate to the deficiency of urea. 'The patient is by no means safe after albumen has disappeared from the urine, if casts continue to be found in the sediment, more especially if the quantity of urine be small and the specific gravity low. Occurring after confinement, the prognosis is extremely unfavorable. A single instance only has fallen under my own observation. This occurred in the practice of Dr. J. O. Stone, of this city. The patient passed through the career of the disease, and clied of uræmia. On inquiry of sereral physicians of large experience in this city, none had met with an instance in which recovery took place.

Richardson and others have reported cases of scarlet fever in which a fatal result was due to the formation of a heart-clot in the right cardiac carities.

With regard to the fatality from scarlet fever, the author just named states that the mortuary tables of the Registrar-General of Great Britain show the disease to be more fatal in towns than in the rural districts, and that amongst the communicable diseases it ranks second ou the list, the fatality being exceeded only by the returns of typhus and typhoid fever. 
Treatuent.-Cases of mild scarlatina do not call for active measures of treatment. The often-quoted saying of the English Hippocrates, that scarlatina is only dangerous through the officiousness of the physician, is, however, harlly true even of these eases, for, be the discase never so mild, there is a liability to grave complications and sequels. On the other hand, in certain cases of severe scarlatina the malignancy of the disease renders any treatment unavailing. With respect to this as well as the other eruptive fevers, it is to be premised that there are no known specific remedies by means of which it may be arrested or controlled; but the inference by no means follows that judicious measures of treatment may not do much toward affording relief and diminishing the fatality from the disease. It is to be added that a comparison of different collections of cases with reference to the rate of mortality under diffcrent methods of treatment is of little value, on account of the wide diversity as regards severity of the disease.

Certain measures which have heretofore been employed in the treatment of scarlatina are now regarded as, in general, opposed by sound reasoning and experience. 'This statement applies to bloodletting, active purgatives, mercurialization, blisters, and emetics. 'These are very rarely, if ever, indicated, and, if not indicated, must of necessity prove hurtful. From the fact that measures are not addressed to the disease per se, it follows that the expectant plan is to be pursued, and the indications are to be drawn from particular symptoms, events, and complications in individual cases.

In the majority of mild cases, hygienic treatment is alone required. In addition to free ventilation, cooling drinks, and light nourishment, the hygienic treatment should embrace measures to maintain the functions of the skin. In view of the fact that renal disease, as a complication or sequel, is a source of danger in these cases, attention to the skin is important, however mild may be the disease. The tepid bath once or twice daily is to be recommended. If the bath be not practicable, the application of a sheet wet with tepid water or repeated sponging of the body may be employed in its stead. There is reason to believe that the more the functions of the skin are maintained by these measures, the less is the liability to an affection of the kidneys either during or after the disease. Exposure to cold is to be avoided both while the disease is in progress and in convalescence, but care in this respect should not lead to error in the way of free ventilation, or of oppressing the patient with an overplus of elothing. Vigilance or restlessness, if not relieved by the nse of the bath, the wet sheet, or sponging, may indicate anodyne remedies. Constipation is to be relieved by simple enemas; other symptoms may indicate appropriate palliative remedies. For the affection of the throat the chlorate of potassa may be prescribed, internally and as a gargle.

In some cases, the leading indication is derived from the intensity of the febrile movement as denoted by the increased heat of the body and the frequency of the pulse. Insomnia, restlessness, and delirium are metsurably dependent on the febrile movement, and in general the latter is a criterion of the severity and danger of the disease. To diminish the heat and the frequency of the heart's action is an important object of treatment. This object may be most effectually accomplished by the use of water. It was in this disease that Currie was led, more than half a century ago, to employ cold affusions with a happy effect. This mode of applying water is strongly advocated by Trousseau, who states that he has employed it with highly satisfactory results in a large number of cases in private and hospital practice. It is doubtless the most efficient measure 
to be employed for the relief of the active delirium which sometimes occurs in this disease. The apparent boldness of the practice renders it frequently objectionable on the score of popular prejudice. Frequent sponging of the body secures, to a considerable extent, the same results; but the use of the wet sheet is not less efficacious than the cold affusion as practised by Currie. Employed as an efficient antipyretic measure, the wet sheet is applied over the whole body, without any other covering, and the surface is sprinkled with cool or cold water at short intervals, in the. manner described in connection with the treatment of typhoid fever and insolation.' 'The temperature is always reduced, one, two, or more degrees, by this measure, and it may be repeated as often as the thermometer shows a return to great intensity of heat. There is no occasion for fear on the ground of danger of repelling the eruption. In employing the wet sheet as a sedative and diaphoretic measure, water is to be used at abont the temperature of $70^{\circ} \mathrm{Fahr}$. ; the patierit, stripped of all clothing, is enveloped in the sheet and closely covered with several blankets. The procedure is that practised by the hydropathists and called by them the wet pack. Usually, after remaining in the pack for about an hour, free perspiration is induced. The pack is to be removed when this effect occurs, the body wiped dry, and the patient placed in bed. The frequency of the pulse is often notably lessened by this measure; the patient is tranquillized, and obtains refreshing sleep. The measure may be repeated once or twice daily. Its effect is so agreeable that patients often desire it to be repeated.

In malignant cases in which the vital powers are overwhelmed, and whenever the intensity of the disease involves a tendency to death by asthenia, the chief reliance must be on sustaining measures. Alcoholic stimulants are indicated in proportion to the frequency and feebleness of the pulse, together with general prostration. I have witnessed the same striking benefit from the free use of wine or spirits in certain cases of scarlatina as of typhus or typhoid fever. Alcoholic stimulants, however, are not to be given without discrimination, and the physician is to be guided in their use by watching their immediate apparent effects, precisely as in other essential fevers. In children, wine-whey, milk-punch, and egg-nog are eligible forms for the administration of stimulants and nourishment.

Cases of so-called scarlatina anginosa, in addition to sustaining treatment, call for measures addressed to the affection of the throat. The chlorate of potassa is now generally considered, and probably with justice, an important remedy in these cases. From one to two drachms may be given daily. The chlorine mixture is highly recommended by Watson and others. The formula for this mixture has already been given. ${ }^{2}$ Stimulating or caustic applications to the pharynx are of doubtful utility. The difficulty of making them in children, and the perturbation occasioned by making them forcibly, in spite of the resistance of patients, are not small objections; but, aside from these objections, it may be cloubted if they produce a beneficial effect. They have been deemed important with a view of preventing the resorption of decomposed matter and the production of septicxmia; but it is by no means certain that a putrid infection of the blood thus induced is ever an element of the clisease. It is proper, however, to state that local applications within the pharynx are deemed useful by many, if not most, physicians. Of cauterizing applications, the hydrochloric acid is generally preferred. Alumen, capsi-

- Vide pages 623, 898.

2 Vide page 953. 
eum, tannin, and the borate of soda, applied in solution or in powder, have been much used. Antiseptic applications, by gargling, if the patient be not too young, or made by means of a sponge if the resistance be not too violent, contribute at least to comfort. The solution of ehlorinated soda may be used for this purpose, diluted with eight or ten parts of water. 'The permanganate of' potash may also be used in the proportion of half a grain of the salt to five ounces of water. The free use of iced drinks is useful as regards the condition of the throat. The patient may be allowed to take into the mouth small pieces of ice almost ad libitum; and for young children a convenient plan is to confine pieces of iee in a gauze bag which may be held in the month. Externally compresses kept wet with cold water may be applied. It has been reeommended to apply ice to the sides of the neek. I have been aceustomed for many years to advise the pretty free use of the tineture of iodine to the neck, especially if there be much glandular swelling. If suppurative inflammation ensue, poultices or the water dressing should be applied. Prof. W. T. Gairdner attaches more importance to the inhalation of the steam of hot water, continued from the begimning to the end of the fever, than to any other measure addressed to the throat affection. He states that in his practice this supersedes all other local applications. ${ }^{2}$

Extreme pruritus of the skin is, in some cases, a souree of mueh annoyance, preventing sleep and inereasing the eonstitutional disturbance. With reference especially to this symptom, some years ago a plan of treatment proposed by a German physician, Sehneemann, eonsisted in the applieation of lard to the surface of the body, the rind of bacon being used for this purpose. This plan was adopted to some extent in this eountry. It relieves, in some cases at least, the pruritus, and diminishes the febrile excitement. Other applications, however, less disagreeable, are equally effieacious. Glycerine and the rose-rvater ointment, or glyeerine and cologne-water, in the proportion of one part of the former to four or five parts of the latter, may be substituted.

Convulsions are generally due to uræmia, but, occurring early in young children, they may be incident to the development of the disease without denoting a renal complication. They are less frequent in children in the early part of this disease than in variola. Whether urremic or otherwise, if they continue, the inhalation of chloroform is indicated. I have notes of two eases in which this measure was employed with immediate relief, and recovery followed. Troussean extols compression of the carotids, in the manner already described, as highly efficacious in arresting convulsions in this disease. ${ }^{2}$

Uræmic phenomena, in addition to convulsions, viz., cephalalgia, disturbance of vision, or coma, render prompt measures of treatment vastly important. Saline purgatives or elaterium are to be employed at onee to eliminate urea, and diaphoresis should, if possible, be procured by the use of either the warm or hot air bath or the wet sheet. Vomiting and purging should always suggest the inquiry whether they be not due to uræmia, and, if so, they are not to be arrested. Albuminuria, and especially a cleficiency of urea in the urine, should lead to measures with a view of forestalling the effects of uræmia. Fomentations are to be applied over the loins, the bowels are to be kept loose with saline laxatives, and, with reference to this object, it is important to produce diaphoresis by means of the warn bath or the wet sheet.

Restlessness and vigilanee, if not relieved by measures of treatment

1 Clinical Medicine, Edinburgh, 1862.

2 Vide page 762. 
already noticel, may require anodyne remedies. The succedanea of opium are to be preferred, viz., belladonna, hyoscyamus, etc. If, however, they are not eflicacious, opiates are to be given, but cautiously in young children. With reference to the liability to heart-clot, Richardson advises the carbonate of ammonia, given in small doses frequently repeated. Several writers have strongly advocated this remedy as exerting a special curative influence in scarlatina.

The treatment of the sequels of scarlatina need not be here considered. The most frequent of the important sequels, viz., acute albuminuria, has been considered in a former part of this work, to which the reader is referred. In the treatment of pruritus, pericarditis, rheumatisin, and chorea, are involved essentially the same principles as when these affections occur in other pathological comnections. External otitis is often neglected, and is apt, therefore, to continue, and, in the encl, lead to impairment of hearing. Daily cleansing the ear by the injection of tepicl water and soap will nsually prove sufficient to effect a cure; but if the affection continue in spite of this simple measure which from its very simplicity it is often diflicult to enforee, mild astringent injections shonld be employed.

I will add a remark with reference to the prevention of renal disease as a sequel of scallatina. The occurrence of this sequel being generally attributed to cold, it is common to confine patients within doors, and sometimes even to the bed, for two or three weeks after convalescence. Regarding, however, the sequel as a remnant of scarlatina, this extreme care is unnecessary. It is sufficient to observe tle same precautions with regard to exposure to cold or other morbid agencies as during convalescence from any acute affection. Gestation, out of doors, has seemed to me to be useful even when patients are suffering from albuminuria and general dropsy following scarlet fever.

It remains to notice the protective influence against scarlatina, which has been imputed to belladouna. For the conflicting statistical data bearing on the question whether minute doses of this remedy tend to render the system, for the time, insusceptible to the disease, the reader is referred to works treating of materia medica and therapeutics. In estimating the force of evidence for and against the assumed prophylatic nature of the romedy, it is to be considered that the failures to afford protection are the positive facts. A certain proportion of persons do not contract scarlet fever, however great the exposure. We have no data for determining the proportion of persons among those exposed at any particular time or place, who are thus natimally insusceptible. Of those who, under the use of belladomna, fail to contract the disease, it may be said that they might have been naturally insusceptible, whereas, all the instances in which the disease is contracted notwithstanding the use of belladonna militate against the protective power of this remedy. Giving dne weight to this consideration, satisfactory proof of the proplrylactic power of belladonua requires that the number of failures shall not be large; and, judged by this rule of evidence, it is extremely doubtful if there be any ground for imputing to this remedy a prophylactic power. It is, however, to be considered that to prescribe belladonna as a proplyylactic is always a harmless experiment, and is, therefore, only objectionable on the score of supererogation. Moreover, as the popular mind has been directed to this question, the wishes of friends are often better satisfied if the remedy be prescribed. These considerations may properly influence the physician. 'The following are the directions for the use of the remedy: "I)issolve from one to thee grains of fresh and well-prepared extract of bellaclonna in an ounce of cinnamon water, adding a few drops of alcohol to prevent 
fermentation. Of this solution may be given, two or three times a day, one drop for each year of the child's age, to be so administered for two weeks, or longer, if the danger should continue." ' The only reliable way of protection against the disease is to avoid the infectious miasm. 'To do this effectually, it is necessary, not only that persons do not come within an infecting range of patients, but that they avoid those who have been within this range, the houses in which there have been cases, and everything that may prove to be fomites. Removal without the limits of the prevalence of the disease is advisable when it is found that cases are apt to prove malignant or severe. On the other hand, if eases be pretty uniformly mild, it is to be considered that the period is favorable for having the disease.

\section{CHA P TER VIII.}

\section{ERUPTIVE FEVERS-(CONTINUED.)}

Rubeola, or Measies-Cinical History-Causation - Diagnosis-Prognosis-Treatment. Roseola. Summary of the more important of the Differential Characters of Variola, Scarlatina, Rubeola, and Roscola. Dengue.

R UBEOLA, so called from the red color of the eruption, called also 1 morbilli, and commonly known as the measles, is a disease of less importance than the eruptive fevers which have been considered. It is generally a mild and often a trivial disease; but it is desirable as early as possible to discriminate it from other eruptive fevers ; it is of frequent occurrence ; it is sometimes accompanied by grave complications ; it is occasionally followed by serious sequels, and, in a certain proportion of cases, the disease is intrinsically severe, or even malignant. Hence, rubeola is by no means an unimportant disease.

Rubeola, like scarlatina, has no special anatomical characters exclusive of the internal and external efflorescence which is most conveniently considered as belonging among the symptoms of the disease. I shall pass, therefore, at once to the clinical history.

Cuinical History.-The career of this disease is subdivided, like the other eruptive fevers, into the stages of invasion, eruption, and desquamation, each stage claiming separate consideration.

Stage of Invasion.-The distinctive local symptoms in this stage resemble those of a common cold, or rather an attack of influenza. There is coryza, with frequent sneezing, and an acrid muco-serous discharge from the nostrils. The eyes are irritable, reddened, and watery. Epiphora is sometimes marked, the tears excoriating the face, and there is more or less intolerance of light. Subacute laryngitis, denoted by hoarseness, frequently occurs, and the symptoms of bronchitis are generally present, the cough being dry, sonorous, and painful. Occasionally the pharynx is the seat of subacute inflammation. The bronchitis, laryngitis, and coryza are due to the efflorescence which takes place on the mu-

I Stillé, op. cit. 
cous membrane of the air-passages prior to its appearance on the skin. With these local affections, which vary much in intensity in different cases, there is associated more or less febrile movement, accompanied by irregular chilly sensations and shivering, but rarely ushered in with a distinct clill ; the appetite is impaired or lost, and in some cases nausea and vomiting occur; pain in the head and limbs, with debility and lassitude, are, in general, proportionate to the amount of febrile movement. The general are often out of proportion to the local symptoms, as in cases of influenza; they are less intense than in the stage of invasion in variola and scarlatina, and in certain cases they are slight. Constipation exists in some cases, and in other cases there is diarrhœa. Convulsions sometimes occur in this stage, chiefly in children. In general they are not indicative of danger. Epistaxis is, in some cases, a prominent symptom. Spasm of the glottis, or false croup, is an occasional symptom in young children.

The average duration of this stage is four days. The duration is, therefore, twenty-four hours longer than in variola, and forty-eight or more hours longer than in scarlatina. Cases are not very infrequent in which the duration is five, six, seven days, and even longer, and, on the other hand, it may be but three or two days, and even one day only.

Stage of Eruption.-A transient convulsion may usher in this stage in children, but it occurs more rarely than in scarlet fever. The efllorescence first appears on the temples and forehead. In a few hours it extends over the head and neek. It is gradually diffused over the body and extremities, its full development occupying from thirty-six to forty-eight hours. In the slowness of the extension of the rash over the cutaneous surface, this fever differs from variola and scarlatina.

The eruption at first presents an appearance of minute red dots or specks which soon enlarge, become slightly elevated, and tend to arrange themselves in circular or crescentic forms. The papules resemble those in smallpox prior to the appearance of vesieles; but they are larger and softer. They bear a resemblance to flea-bites. The redness momentarily disappears on pressure. Increasing in number and size, they coalesce to a greater or less extent, and form blotches of variable dimensions, with curvilinear or semilunar borders, contrasting in the latter respect with the irregular or serrated patches of efflorescence in scarlatina. Aside from cases in which a petechial eruption occurs, the eruption proper to the disease sometimes shows, not only hyperæmia, but infiltrated hematin, the redness being with difficulty removed by pressure, and a brownish color remaining after the eruption has disappeared. The eruption in some cases is everywhere confluent, being uniformly diffused over the whole or the greater part of the cutaneous surface. The color of the eruption is dull or decp red, offering a contrast to the crimson or scarlet redness of the scarlatinous efflorescence. The portions of skin not occupied by the eruption retain the normal appearance. In proportion to the abundance of the eruption there is swelling, which is most marked on the face. The eruption is sometimes attended with considerable pruritus. Occasionally vesicles are intermingled with the rubeolous papules. On the fourth day of the eruption it begins to fade on the face, and successively on the trunk and extremities, and the stage of desquamation commences.

During the stage of the eruption, the symptoms denoting coryza, laryngitis, and bronchitis continue. The latter, in this stage, gives rise to a more or less abundant expectoration, consisting of greenish or yellowish sputa which are frequently nummular. Dry and moist bronchial rales are frequently heard on auscultating the chest. The irritability of the 
eyes continues, and not infrequently there is conjunctivitis. Pharyngitis, in some cases, continues, and sometimes the inflammation extends into the Eustachian tube, occasioning partial deafness. The febrile movement does not disappear nor diminish with the development of the eruption. On the first and second day of the stage of the eruption, the temperature in the axilla may rise to $106^{\circ} \mathrm{Fahr}$. and eren higher. From this date, in the natural eourse of the disease, the temperature rapidly diminishes. Other symptoms denoting constitutional disturbance are proportionate to the amount of febrile movement. The latter, save in exceptional cases, is much less in degree than in scarlatina. The tongue is generally coated, and not infrequently the elongated papillæ, projecting through the coating, present the appearance of numerous red points.

Stage of Desquamation.-This stage may be considered as having commenced when the eruption begins to fade. The duration of the stage is from four to eight days. In uncomplicated eases pursuing a favorable course, this stage is the stage of convaleseence. Desquamation, in fact, oceurs in only a certain proportion of cases. It may be more or less marked. It is always furfuraceous, the epidermis being exfoliated in bramny scales, not in patches or flakes. Coincident with the decline and disappearance of the eruption, the febrile movement diminishes and ceases. More or less cough and expectoration are apt to continue after the cutaneous eruption lias disappeared, and persisting conjunctivitis is not uncommon. Occasionally the eruption on the skin reappears after having existed for the usual period and disappeared. Diarrhœa, usually mild, is apt to occur in this stage.

The career of the disease thus extends from twelve to sixteen days.

The foregoing sketch of the clinical history embraces cases in which the disease pursues its ordinary regular conrse. Anomalous cases occur, the disease in some being very severe, or malignant, and in others unusually mild. The affections of the Schneiderian membrane, larynx, and bronchial tubes are sometimes wanting. Writers have considered these cases as constituting a variety of the disease called rubeola sine catarrho. To a certain extent, doubtless, cases which have been considered as of this variety have been eases of roseola. On the other hand, it is supposed that eases occur in which the local manifestations are limited to the mucous membrane; hence, another variety called rubeola sine eruptione. It must be difficult to decide positively that rubeola exists when the cutaneous efflorescence is wanting. In the cases belonging to the two varieties just named, the disease is unusually mild. A severe form of the disease is cliaracterized by the occurrence of petechiæ and hemorrhage in various situations. The phenomena of purpura are associated with measles in these cases. Of this rare form of the disease the following; case is an example: The patient was an adult aged about 25 years. I saw him, in consultation, during the stage of the eruption. The rubeolous eruption was abundant, presenting the characteristic appearances, but was somewhat darker than usual. Seattered among the patches of efflorescence, over the whole surface of the body, were petechiæ varying from the size of a pin's head to a split pea. There was hemorrhage from the nose and mouth; blood also was discharged from the bowels and was contained in the urine. The tongue and gums were pallid except at certain points which were stained with exuding blood. Blood was effused beneath the conjunctiva, infiltrating the whole surface of the eyeball except the cornea. I saw the patient on two successive days. On the first day there was preternatural heat of the skin, and the pulse had considerable force, although frequent. On the second day the surface of the body was 
cool, the pulse was very small and feeble, and there was great prostration. The case terminated fatally. The form of the disease is distinguished as hemorrhagic rubeola. It has been called also rubeola nigra.

Gangrene attacking the mouth and occasionally the anus, vulva, nose, lungs, and larynx, sometimes occurs in connection with rubeola. Other morlbid conditions than those belonging to the latter, it is to be presumed, must concur in giving rise to this event. It has been observed chiefly in young children in eleemosynary institutions. Aside from any such loeal events, or important complications, this disease sometimes assumes a severe form, presenting the ataxic and adynamic symptoms of typhus or typhoid fever, and ending fatally from an intrinsic tendency to death. Such cases, however, are extremely rare.

In the great majority of the cases in which the disease is grave, the gravity is dependent on complications. The important complications most likely to occur are seated in the respiratory system. Diphtheritic laryngitis or true croup is sometimes developed. Valleix observed a considerable number of cases in which death was due to this complication, the cases occurring at a particular season among foundling children. Capillary bronchitis is another fatal complication liable to occur in ehildren; and occurring in children under three year's of age, it generally clestroys life. Trousseau states that during an epidemic which prevailed in Neckar Hospital in 1845 and 1846, of twenty-four ehildren attacked with rubeola, twenty-two were carried off by this complication. It is more liable to oceur in hospital than in private practice. Pnemmonitis, however, is the most frequent of serious complications. This, in young children, is likely to prove fatal, and it is by no means devoid of danger to adults. Pleuritis is an occasional complieation, but is far more infrequent than pneumonitis. Croup, eapillary bronchitis, and more especially pneumonitis, are not less likely to occur as sequels than as concomitants of rubeola.

A delay in the appearance of the eruption is generally an effect of some important complication. And the occurrence of an important complication during the stage of the eruption is apt to lead to a notable diminution, and sometimes retrocession of the eruption. Irregularity as regards the situation in which the eruption first appears is sometimes observed. A former colleague, the late Prof. B. R. Palmer, related to me a case coming under his observation, in which the efflorescence first made its appearance on the lower extremities, and extended thence gradually over the body, upper extremities, and head.

After recovery from rubeola, pulmonary tuberculosis and the affection known as tuberculous meningitis are observed in a proportion of cases, not large, but suffieient to show that the system is left in a condition favorable for the development of these affections, provided the tuberculous diathesis exist. Affections distinguished as scrofulous are apt to follow as sequels, namely, scrofulous ophthalmia, coryza, and otorrhoea, enlargement of lymphatic glands, etc. After an extensive prevalence of measles in any place, it is observed for some years that the mortality among children exceeds the average mortality at other times. The practical importance of these facts is at once obvious.

Causation.-Rubeola, like scarlatina and variola, is a communicable discase. The infectious miasm is not only recieved by those brought into close proximity to persons affected with the disease, but it may be transported to a distance by means of fomites. Persons contract the disease 
from the miasm adherent to the clothes of those who may have recently visited rubeolous patients. Physicians may in this way diffuse the disease.

It appears to be settled that the disease may be communicated by inoculation. Prof. John E. McGirr, many years ago, reported fourteen cases in which the discase appeared to be produced by inoculation; the cases were observed in the Catholic Male and Female Orphan Asylums at Chicago, Illinois. The inoculation was made with blood drawn from an exanthematous patch at the height of the eruption. The disease was developed after a period, from the date of the inoculation, varying from four to nine days. 'The disease was mild and in no case proved fatal, whereas of fifteen cases in the same institution in which the disease occurred without inoeulation five proved fatal. ${ }^{1}$

The duration of the period of incubation varies, according to different authors, within widely separated limits, viz., between one day and thirty days. Probably in the majority of cases the attaek occurs from six to. ten days after exposure. The duration was within these limits in a dozen cases under my observation, in which the time of exposure could be definitely fixed, with a single exception, in which the duration was fourteen days. When produced by inoculation, the period of incubation is somewhat shorter than when the disease oceurs naturally.

The disease may occur at any period of life, but it is by far most frequent in young subjects after the period of dentition. It is rare before the epoch just named. After fifteen years of age the liability to it diminishes. It may prevail, however, very extensively among adults of different ages, who are closely thrown together, as among soldiers in military camps. Thus, during the two first years of the late civil war in this country, 38,021 cases were contained in the official returns to the SurgeonGeneral. ${ }^{2}$ Prevailing as an epidemic among bodies of troops in different sections of the country, it attaeked nearly all who had never experienced the disease, as well as, in not a few instances, those who had previously had it. $A$ s a rule, this disease, like scarlatina and variola, renders the system ever afterward insusceptible to it, but exceptions to this rule are not very rare. Dr. Minot, of Boston, has reported the case of a child who, having had the disease well marked and communicated it to three persons, was attacked six weeks afterward, the eruption reappearing, and the disease again passing through its usual course. Well-authenticated cases in which the disease has occurred three and even four times have been reported. Prevailing in camps, schools, hospitals, and communities as an epidemic, this disease presents at different times a great diversity as regards mildness or severity, and the tendency to particular complications ; in other words, the character of different epidemics of rubeola, as of other epidemics, is by no means uniform, but varies much in different seasons and localities.

Dr. J. H. Salisbury, of Ohio, has communicated certain observations as showing that the inhalation of the fungi of wheat straw is capable of producing measles; that by inoculating with the spores and cells of the fungi of wheat and rye straw an affection may be produced resembling measles, and that persons in whom this affection is in this way prodnced are rendered insusceptible to measles. ${ }^{3}$ Referring the reader to the communication of Dr. Salisbury, it will be found that the facts, although interesting

1 North western Medical Journal, 1851. Vide, also, Jaccoud, Pathologie Interne, 1871.

2 Woorward, Circular No. 6, Surgeon-General's Office, Nor. 1865.

3 American Journal of Medical Sciences, July, 1862. 
and worthy of consideration, are hardly sufficient to warrant the conclusions just stated. Out of 100 cases occurring among soldiers, analyzed by Dr. Roberts Bartholow, 85 had not, and only 15 had, slept or lain on straw or hay sinee enlistment. ${ }^{\prime}$ At the present time we have no positive knowledge of any cause capable of producing this disease, exclusive of contagion and infection.

Dragnosis.-The diagnosis of rubeola, after the appearance of the efflorescence on the skin, is generally unattended with any difficulty. The diagnostic characters which have been embraced in the clinical history are sufficiently distinctive. The more important of these are : the long duration of the stage of invasion, the affection of the air'passages, the appearance of the eruption almost invariably first on the face, and its gradual diffusion over the body; the color of the eruption, its papular charaeter, the softness of the papules, and the tendency to assume a crescentic arrangement. So far as the eruption is concerned, early in the eruptive stage there is some resemblanee to the papules of smallpox ; but the differential points which have been presented in treating of the latter disease should enable the practitioner to make the discrimination. In a case of rubeola in which the cutaneous efflorescence is not abundant, and the air-passages are unaffected, the disease is liable to be confounded with roseola; but the eruption in these two affections differs, and, moreover, such cases are extremely rare. Cases in which the air-passages are alone affected, that is, in which there is no eutaneous efflorescence, hardly arlmit of a positive diagnosis, but such cases are also extremely rare. Their occurrence during the prevalence of rubeola in persons known to have been exposed is chiefly the ground on which the diagnosis is to be based.

The diagnosis camnot be made with positiveness prior to the appearance of the efflorescence on the skin. The occulrenee, however, of coryza, irritability of the eyes, and bronchitis, with more or less febrile movement, in jersons who have never had measles, at a time when the disease is prevailing, renders a diagnosis during the period of invasion higlily probable. The diagnosis is sometimes delayed by the early oceurrence of complications which interfere with the regular course of the disease, especially as regards the cruption. The appearance of the latter, however, sooner or later, certainly, in the great majority of cases, removes the difficulty which may previously have existed.

Of this, as of the other eruptive fevers, the diagnosis in the negro must, of course, be based on other points than those relating to the color of the eruption. "In the pure negro, the eruption appears as yellowish spots slightly elevated, and giving a sensation of roughness; in the mulatto, as a dusky-brown, ill-defined ; and in the lighter shades, more distinct, redlishbrown spots, approaching the characteristics of the eruption in the white."

Prognosis.-In the majority of cases, as this disease is ordinarily presented in civil practice, it is either mild or unattended with danger. But in a certain proportion of cases the disease is severe and dangerous. The severity and danger are due to the anomalous events and complications which have been noticed in connection with the clinical history. The number of cases fatal from the coexistence of capillary bronchitis, diphtheritic laryngitis, pneumonitis, etc., is not inconsiderable. The rate of mortality differs greatly at different times and places, so that the statis-

I Synopsis of a Report upon Camp-Measles, based upon an analysis of 100 cases. American Medical Times, May, 1864. 
tics of any particular epidemic would not furnish results applicable generally to the disease. It is apt to prove a serious disease in camps. Woodward states that of the 38,021 cases contained in the official returns from the armies of the United States in the two first years of the late civil war, there were 1864 deaths. Prof. Bartholow is of the opinion that these figures do not express the actual fatality due directly or indirectly, that is, by complications and sequels, to this disease during the war; he fixes the ratio of deaths to cases as 1 to $5 .^{1}$

During the war the prevalence of the disease was great among recruits, especially those from the country; and the sequels, or the effect upon the general health, incapacitated a considerable proportion for remaining in the service. Taking into view the mortality and the enfeebled condition remaining for a long time in many of the cases which did not end fatally, measles proved to be one of the most formidable of camp-diseases. With reference to the efficiency of troops in active service, it would seem to be desirable either to expose new reeruits to the disease when they can pass through it under the most favorable hygienic conditions, or to isolate cases at once wherever they occur in camp. Doubtless the rate of mortality and the disqualifying effects of the disease were often due to the coexistence either of seorbutus or malarial poisoning.

Treatment.-In ordinary regular cases, the disease calls for nothing but palliative measures and attention to hygiene. The cough may be palliated by anodynes, and these may also be indicated by restlessness or vigilance ; and for the febrile movement, if considerable, gentle diaphoretic and refrigerant remedies may be prescribed. The object of treatment in these cases is simply to render the patient as comfortable as possible; the disease pursues a favorable course withont any therapentical interference. Many cases are treated with domestic remedies without coming under the cognizance of the physician. It is a widely diffused popular notion that saffron (crocus sativus) is a valuable remedy in this, as well as the other eruptive fevers, given for the purpose of promoting the eruption. There is little ground for this notion, but the remedy is harmless. Cathartics in this, as in the other eruptive ferers, are not indicated, and are likely to do harm. Emetics are rarely, if ever, indicated. The patient should be confined to a room in which the temperature is agreeable, but free ventilation should be sccured. The air of the room, in the treatment of cases of this, as of the other essential fevers, and, indeed, of all acute diseases, should be effectually changed scveral times daily by opening the windows, protecting the patient against drafts by covering the face as well as the body. The body may be sponged, in portions at a time, with tepid or cool water. This may be done without any fear of causing the eruption to disappear prematurely. As a protection for the eyes, the room should be moderately darkened.

In cases in which the disease is rendered severe by complications, the treatment will have reference to the latter. The general principles of treatment, in these cases, are the same as when the complicating affections occur in other pathological connections. It would, therefore, be a repetition to consider the indications derived from the different complications. A practical question relates to the retardation, diminution, or retrocession of the eruption in some complicated cases. Is it important to resort to active measures designed to promote the appearance of the eruption? The importance of this object of treatment has doubtless been much exagge- 
rated. Certain measures employed for this object, such as emetics, active stimulants, the hot or vapor bath, and overloading the body with clothing, are not to be employed. 'This general rule may be adopted, viz., measures should not be employed for this object if, aside from the object, the measures are likely to be hurtful. The tepid bath, stimulating pediluvia, and sinapisms, or other rubefacient applications to the skin, are not excluded by this rule. It is to be considered that the complications do not occur in consequence of the eruption being delayed or insufficient, or striking in, but the latter are rather effects of the former.

Cases presenting ataxic or adynamic symptoms, either with or without complications, claim the tranquillizing and supporting measures indicated in other diseases with the same symptoms.

It is important to bear in mind the liability to serofulous and tuberculous affections after recovery from this disease. Patients should be placed under hygienic influences which will tend to invigorate the system, with a view to obviate a tendency to the development of these affections.

\section{Roseola.}

The eruptive fever called roseola, or rose rash, sometimes called false measles, is an affection of very little importance, exclusive of the liability of confounding it with rubeola and scarlatina.

The eruption is preceded by symptoms of constitutional disturbance for one or two days. These symptoms are, cephalalgia, loss of appetite, with sometimes nausea and vomiting, and occasionally diarrhœa, chilly sensations, febrile movement, and general malaise. In young children, convulsions may occur. The constitutional disturbance is more or less marked, but it is frequently slight. The affection of the throat and the affection of the air-passages, which belong to the history of scarlatina and rubeola in the stage of invasion, are wanting in roseola.

The eruption appears in the form of rose-colored spots or patehes which are not elevated, the redness disappearing momentarily on pressure. It appears on different parts of the body, not commeneing on the head and extending over the body as in rubeola. Frequently it appears on the body and extremities and not on the fice. It continnes for twenty-four or forty-eight hours only, in a majority of cases. It disappears without desquamation, and is liable to return. It is sometimes accompanied with considerable pruritus. The affection has neither sequels nor complications. It may, however, occur in the course of other affections, especially in the stage of invasion in varioloid, in articular rheumatism, and in epidemic cholera after reaction. It has no gravity. One attack affords no protection against recurrences. It is ofiener presented in females than in males. It prevails not infrequently as an epidemic, and chiefly during the summer season. Some anthors consider it to be contagions.

Attention to the foregoing points will suffice for the discrimination of the affection from scarlatina and rubeola. It does not call for treatment.

The use of the balsam of copaiba by some persons incluees an efflorescence analogous to that of roseola, and more rarely the nse of eubebs, the iodide of potassium, turpentine, and the sulphate of quinia. A variety of the affection belongs to the history of syphilis. 


\section{Summary of the more Important Differential Characters of Variola, Scarlatina, Rubeola, and Roseola.}

Period of Incubation.- The average period in variola from 10 to 14 days; in scarlatina from 1 to 6 days; in rubeola from 6 to 10 days.

Stage of Invasion.-Duration in variola from 2 to 3 days; in scarlatina from 1 to 2 days; in rubeola from 4 to 5 days; in roseola from 1 to 2 days. In variola, whitish or ash-colored spots in the mouth and fauces, and sometimes a cutaneous efflorescence; lumbar pain in a marked degree. In scarlatina, efflorescence and sometimes exudation in throat; febrile movement often notably intense. In rubeola, coryza, irritability of eyes, hoarseness, and lond barking cough.

Stage of Eruption.-In variola, eruption appearing first on face and wrists and gradually extending over cutaneous surface. The eruption at first maculated, next papular, next vesicular, more or less of the vesicles umbilicated, and finally the eruption pustular. The eruption aborting at different stages in cases of varioloid. The eruption in varicella differing in being vesicular at first, the vesicles not umbilicated, and not becoming pustular; also in the short duration of the stage of erniption. The febrile movement in variola remitting or ceasing with the appearance of the eruption. In scarlatina the eruption frequently appearing on the chest and upper extremities before, or simultaneously with, its appearance on the face and neck. The eruption rapidly diffused over the cutaneous surface. The eruption of a scarlet color, not elevated, assuming the form of patches with irregular or serrated borders, or existing continuously over the whole surface. The tongue resembling a ripe strawberry. In rubeola the eruption appearing first on the head and gradually extending over the cutaneous surface. The color a dull or deep red. The eruption elevated, and the coalescing papules groupet in crescentic forms. Febrile movement not diminished at the time the eruption appears. In roseola the eruption appearing irregularly on different parts of the cutaneous surface. The color rose-red. The eruption not elevated. The febrile movement slight. No affection of the throat or air-passages.

Stage of Desquamation.-In variola, concretion of pus and the formation of crusts or scabs. In scarlatina the epidermis exfoliated sometimes in branny scales and sometimes in large flakes or patches. In rubeola, desquamation frequently wanting, and, when it occurs, always furfuraceous. In roseola, no desquamation.

Complications and Sequels.-In variola, no special tendency to any particular complication or sequels, but erysipelas, furunculi, and subcutaneous abscesses not uncommon. In scarlatina, a special tendency to renal disease, leading frequently to general dropsy and involving danger from uræmia. In rubeola, tendency to pulmonary affections, viz., capillary bronchitis, diphtheritic laryngitis, and pneumonitis, more especially the latter. Liability to tuberculous affections after recovery. In roseola, no complications nor sequels.

\section{Dengue.}

The following succinct account of the epidemic affection commonly called dengue, an unclassical name of uncertain derivation, is based chiefly on communications by Prof. Dickson, Dr. Wm. T. Wragg, Dr. Henry F. Campbell, and Prof. Fenner, describing the affection as it occurred in Charleston, S. C., Augusta, Ga., and New Orleans, in the year 1850. Prof. Dickson's name is especially identified with the affec- 
tion from his having been the historian of its visitation in this country in 1828, and he contributed more largely to what is known respeeting it than any other writer. Collated abstracts from the communications just referred to are contained in a report of the Committee on Practieal Medicine to the American Medical Association in 1851, of which committee the author of this work was chairman. For a fuller account of this affection than is consistent with the scope of this work, the reader is referred to that report, and to the writings of Dickson. ${ }^{1}$

The affection prevailed extensively in the West India Islands in 1827 and 1828, and about the same time in many parts of the Southern States of this country. An affection supposed to be the same prevailed in Philadelphia in 1780, and was described by Rush. It was then, as since, frequently known as the breakbone fever. Other epidemics supposed to be identical have occurred at various periods in other parts of the world. Cocke and Copland apply to an epidemic affection supposed to be the same the name scarlatina rheumatica.

The development of the affection is either abrupt or slow. The symptoms attending its development are anorexia, chilly sensations, but rarely a pronounced chill, languor, lassitude, and general malaise. These symptoms exist in some cases for only twenty-four hours, but in other cases for several days before the affection is fully developed.

After the access or forming period follows a febrile stage or a paroxysm of fever. The duration of the febrile movement varies from nine hours to three or four days, the average duration being about thirty-six hours. During this stage, and sometimes during the access, acute, often excruciating, pains in the head, eyes, museles of the neck, loins, and extremities are prominent traits of the affection; hence the name breakbone fever. The pains diminish or disappear with the cessation of the fever, and the patient, who had taken to the bed with the onset of fever, is now able to sit up, and complains only of debility; perhaps he returns to his accustomed avocation. But in four or five days the pain often returns, with frequently a recurrence of the febrile movement, debility, and malaise, compelling a return to the bed.

In the great majority of cases, an eruption occurs at a variable period after the febrile paroxysm. The eruption presents, in different cases, a diversity of characters. It resembles in some cases very closely the efllorescence of scarlatina. In other cases it is not unlike the eruption of rubeola. It is sometimes papular, like either lichen or urticaria, and it is sometimes vesicular, like either sudamina or varicella. Erysipelas and purpura are occasionally observed. Hemorrhage from the nose, mouth, bowels, and utcrus occurs in some cases.

The convalescence is often tedious; the recovery of strength, appctite, etc., is apt to be slow. The average duration of the disease is about eight days. Relapses are not infrequent. As regards incidental events and sequels, convulsions, in children, occasionally usher in the attack; delirium, like that of delirium tremens, has been observed succeeding protracted vigilance, and in pregnant women miscarriage is apt to take place. A rheumatic condition of the joints, abscesses, boils, and carbuncles are not infrequent scquels.

The extent of prevalence of this epidemic in Charleston and other places is remarkable. Wragg computes the number of cases at one time in Charleston at 10,000, and during the epidemic seven or eight-tenths of the population were affected. All classes are attacked, persons of

1 Transactions Am. Med. Association, vol. iv. 1851. 
either sex, children and octogenarians. As an illustration of its universal prevalence, the editor of the Southern Medical and Surgical Journal, in his issue for December, 1849, apologized for typographical errors by saying that "the editor, publisher, and printers were all suffering from breakbone fever." In the village of New Iberia, Louisiana, the population in 1851 did not exceed 250. Dr. Duperier states that in six weeks 210 of the inhabitants of this village, and about 40 from the neighborhood, had gone through the disease.

The duration of epidemics is brief, ceasing usually in six or eight weeks. of the circumstances giving rise to the special cause, nothing definite is known. The late Prof. Dickson regarded the affection as contagious. This opinion is opposed by the rapid and almost simultaneous diffusion of the affection, by the limitation of its prevalence to towns or within a circumscribed area, and the short duration of epidemics. This distinguished author also was of the opinion that, as a rule, immunity from subsequent attacks is secured when the disease has been once experienced. In proof of this opinion, he stated that, at Charleston, where the disease prevailed in 1850, those only escaped who had experienced it during the epidemic of 1828. The occurrence of a few cases of the disease among those who had it in 1828, he accounts for by supposing that after an interval of 22 year's, the susceptibility was renewed.' It is an affection chiefly occurring in warm climates, and prevails especially in cities and large towns. To the latter rule there are, howerer, striking exceptions.

This affection, although extremely distressing, and presenting not infrequently serere symptoms, is very rarely, if ever, fatal. Its apparent intensity is in striking contrast to the absence of danger.

The affection is self-limited, and there are no known means of arresting or abridging it. The treatment, therefore, consists of palliative measures. Opiates to relieve the pains, restlessness and vigilance, are indicated. 'The degree of suffering, in some cases, warrants the use of chloroform by inhalation. Rubefacients to the spine and in other situations are useful. Alcoholic stimulants, given pretty freely, have been found beneficial. During convalescence, tonic remedies and hygienic measures to promote appetite and the recovery of strength are indicated.

\section{CHA PTER IX.}

Diphtheria-Anatomical Characters-Clinical History-Pathological Character-CausationDiagnosis-Prognosis-Treatment.

$\mathrm{O}^{\mathrm{F}}$ the constitutional affections, exclusive of the essential ferers, which U remain to be considered, one of the most important is the affection now commonly known as diphtheria. This affection has prevailed at times as an epidemic, in various parts of the world, from a remote period. It has been described by writers in different countries and epochs, under a variety of names, such as, ulcus Egyptiacum vel Syriacum, cynanche maligna, angina maligna, angina gangrænosa, morbus suffocans vel strangulatorius,

1 Elements of Medicine, 2d edition, page 748. 
Garrotillo, malignant sore-throat, epidemic croup, etc. In this country, until within the last few years, it has occurred rarely, and to a limited extent, during the present century. It was described by Bard, in 1789, under the name angina suffocaliva. 'The distinctive characters of the affection were very clearly elucidated by Bretonneau, in 1821, 1825, and 1826, who applied to it the term diphtherite, whence originated the name diphtheria. This name, the significance of which relates to the most characteristic local event, viz., the formation of a false membrane, has, to say the least, the negative merit of not involving any hypothesis concerning the pathology of the affection.

Anatomical Characters.-The characteristic feature, as just stated, is the presence of a false membrane. The fauces in the vast majority of cases is primarily affected, and, in a certain proportion of cases, the local affection is limited to this situation. The first appearance is redness of the fances accompanied by more or less swelling of one or both of the tonsils. The pseudo-membrane, at first thin and semi-transparent, soon becomes opaque and more or less thick. It is white or ash-colored, but it may become dark and even black from decomposition and imbibition of blood. It may also be colored by remedies and by matters vomited. The patches of false membrane present well-defined abrupt margins. When usually thick it resembles wet parchment or chamois leather.

Different cases differ in the extent of the affection within the fauces. One or both of the tonsils may be alone affected. These bodies are sometimes greatly swollen so as almost to meet, and in other cases they are only moderately enlarged. The false membrane may extend over the posterior part of the pharynx, the palatine arches, the uvula, and, forward, over the greater part, or the whole, of the soft palate. It may be uniformly diffused in these situations, but is oftener in distinct irregular islets or patches. Redness extends beyond the margins of the exudation, and the parts affected, other than the tonsils, are somewhat swelled. With the forceps the false membrane may be torn away, and frequently bloody points are apparent on the surface from which the membrane has been detached. The false membrane, when thick, loose, and dark or black, presents an ulcerated or gangrenous appearance, which is implied in some of the names formerly given to the affection. Sooner or later, if life be sufficiently prolonged, the false membrane is thrown off. The exfoliation sometimes takes place in three or four days, and it is sometimes delayed even to twenty days. Not infrequently it is followed by a second, and sometimes a third, and even a fourth formation of false membrane. of late, histological pathologists distinguish the diphtheritic false membrane from the exudation which occurs within the larynx and trachea in fibrinous laryngitis, or true croup. The former is made up chiefly of cellular clements, and does not consist of fibrin or lymph. These cellular elements are regarded as modified epithelial cells. The affection is not only on the surface of the membrane, but it is interstitial, extending more or less beneath the superficies, and with the exfoliation of the false membrane there is a gangrenons separation of a layer of the mucous structure, leaving an ulcerated surface. On the otlier hand, in fibrinous laryngitis, the false membrane, in part at least, consists of exnded lymph; the microscope shows fibres as well as cells, and there is no loss of substance after the membrane separates.

'Vide Pathological Histology by Rindfleisch, and Manuel d'Histologie Patho. logique, par Cornil et Ranvier. Paris, 1869. 
The lymphatic glands of the neck, and especially those behind the angle of the lower jaw, are more or less enlarged, painful, and tender, in the great majority of cases. As a rule, the amount of enlargement corresponds with the affection within the throat. If the latter be limited to one side, the glands on the same side may be alone affected. The glandular affection rarely proceeds to suppuration.

The diphtheritic formation within the throat is to be discriminated from an accumulation of follicular secretion which is sufficiently common, especially over the tonsils. The latter is a pultaceous deposit, not membraniform, nor removable in strips or patches, and may be seen to dip into the follicles. Pharyngitis with follieular secretion is often called diphtheria. The term, however, is correctly applied only to cases in which a true diphtheritic membrane exists. During the epidemic prevalence of diphtheria, cases of simple pharyngitis, or of pharyngitis with follicular secretion, are often rife. The affeetion in these cases may be due to the epidemic influence, but the disease is comparatively trivial, and, hence, the rate of fatality in different collections of eases of so-called diphtheria will vary according to the accuracy of diagnosis, or the strict observance of the proper application of the name.

In mild cases of diphtheria the disease manifests itself locally in the fauces alone, but in cases of more or less severity and danger the diphtheritic affection occurs in other situations. The posterior and anterior nares, on one or both sides, may be affected. The Eustachian tube is sometimes involved. The mucons membrane of the cheeks and gums may present patehes of, or be completely coated with, false membrane. The eye is sometimes invaded, and the diphtheritic conjunctivitis involves considerable danger of destruction or impairment of vision chiefly from opacity of the cornea. The diphtheritic inflammation sometimes invades the œsophagus, and may extend quite to the stomach. A more serious extension is into the larynx. The disease then involves the morbid conditions which exist in true or diphtheritic croup. As in croup, the inflammation and false membrane extend to the trachea, and may extend into the bronchial tubes, reaching, in some cases, the tubes of small size. As a rule, however, it does not extend beyond the trachea. In the cases in which the parts adjacent to the fauces become affected, the affection is not to be considered as spreading to these parts, but they are consecutively invaded, that is, the affection of these parts, as of the fauces, is a local manifestation of the constitutional disease. This consideration has an important practical bearing.

The parts communicating with the fauces are not the only parts liable to become involved in cases of diphtheria. The meatus auditorius externus, the anus, the vulva, and prepuce, are sometimes affected. The affection also appears in some cases on the skin in parts excoriated, as behind the ears, on vesicated surfaces, ulcers, leech-bites, in short, wherever the skin is deprived of the epidermis. Exceptionally it may be seated in some one or more of the parts which have been named, exclusive of the fauces, the latter remaining unaffected. Different cases differ as regards the number of parts affected. Parts other than those primarily affected are liable to be invaded at any time during the career of the disease.

There are no ascertained anatomical characters of this disease in addition to those belonging to the diphtheritic inflammation of certain portions of the mucous membrane and skin. Morbid appearances of internal organs, which may be found after death, are due to coincident affections or complications. 
Clinical History.-Diphtheria presents in different cases such differences as regards general and local symptoms, the latter incident espeeially to the localizations of the diphtheritic affection, that some writers have instituted several varieties of the disease. Without adopting formally any of these, it will suffice to indicate the important diversities in connection with the symptomatology.

$A$ marked point of difference relates to the development of the disease. The attack is sometimes acute, commencing with a chill more or less pronounced, which is followed by high febrile movement. But not infrequently the development is gradual and insidious, the patient complaining of indefinite ailments, and the characteristic affection of the throat being discovered on inspection, when there were few or no local symptoms pointing to the existence of the disease. After the development of the discase, the symptoms are naturally divided into general and local, the latter being referable especially to the parts affected with diphtheritic inflammation.

The affection of the fauces is rarely accompanied with notable pain or soreness, hence a liability to overlook its existence. The sensibility of the parts appears, in some cases, to be climinished. Deglutition is sometimes painful, but, in other eases, unattended with pain. Incomplete paralysis of the muscles concerned in deglutition, which will be noticed as a sequel, is sometimes a concomitant of the disease, giving rise to more or less difficulty of swallowing, especialiy liquids. These may regurgitate through the nose. If the exudation be abundant and loose, pharyngeal rales with respiration occur, especially during sleep. The breath in some cases is notably fetid, suggesting the idea of gangrene of the throat or lungs, events not frequent in the history of the disease. The extension of the false membrane over more or less of the buccal mueous surface occasions more or less pain in connection with the introduction into the mouth of food or drinks, together with ptyalism, stiffness of the parts, involving difficulty of articulation and absence of taste. The affection of the anterior nares is preceded and accompanied by a discharge which produces irritation and swelling of the upper lip, limited to one side of the lip if one nostril only be affected. In other situations accessible to view, on a mucous surface or on the skin, the diphtheritic inflammation gives rise to more or less pain or soreness, and is attended by a sero-purulent discharge, variable in quantity, frequently ichorous and sometimes fetid. As a rule, the gravity of the disease is commensurate with the extent of the local affection and the abundance of the exudation. It does not follow from this fact that the gravity depends on the local affection-the latter is a criterion, not the cause, of the former.

The symptoms denoting an invasion of the larynx are essentially those which belong to the history of diphtheritic laryngitis or true croup. These have been already considered. ${ }^{1}$ Cough, having more or less of the characters known as croupous, feebleness of the voice with elevation of pitch, or aphonia, and labored, stridulous respiration, point to this most serious localization of the affection. The embarrassment of respiration may be increased paroxysmally, as in simple laryngitis or in true croup, from the addition of spasm of the muscles of the glottis. Swelling of the tonsils and pharynx may be sufficient to occasion some obstruction to respiration without invasion of the larynx. It is to be borne in mind that laryngitis may be excluded so long as the voice remains unaffected. I have all along referred to diphtheritic laryngitis or true croup as an affection distinct from the disease under present consideration. The 
reasons for this will be stated in comnection with the pathological character of diphtheria. Diminished respiratory murmur and embarrassment of the respiratory function out of proportion to the amount of laryngeal obstruction, with the presersee of moist bronchial rales, denote an extension of the affection to the bronchial tubes.

Passing to symptoms not directly referable to the parts affected with diphtheritie inflummation, different cases differ widely as respects the amount of constitutional disturbance. Irrespective of an affection of the laryux, which of necessity involves great danger, cases are either mild or more or less severe. In some mild cases the disease is almost trivial, patients not taking to the bed and even scarcely heeding the disease. But, however mild cases may be at the outset or during more or less of the eareer of the disease, there is always a liability to the occurrence of diphtheritic inflammation in new situations, to the development of grave symptoms, and to important sequels. In the cases in which the disease is mild throughout its career, the diphtheritic inflammation is confined to the fauces, or, exceptionally, to a limited space in some other situation.

In cases of greater or less sererity, the clinical history embraces symptoms referable to the different anatomical systems, viz., the circulatory, cutaneous, respiratory, digestive, nerrous, and urinary.

The pulse in some cases becomes very frequent; in other cases the acceleration is moderate, and it is sometimes slight. In the course of the disease the pulse is sometimes at first more or less rapid, afterward suddenly falling below the arerage of health. Aside from frequency, the pulse is soft or compressible, denoting diminished power of the helart's action. It is sometimes irregular-a symptom of unfarorable omen, if it be independent of pre-existing organic disease of the heart. Hemorrhage from the nostrils is not an infrequent symptom; it occurs occasion. ally from the throat and mouth. Epistaxis is sometimes so profuse as to require mechanical means for its arrest. Under these circumstances it denotes great gravity and danger, and hemorrhage in other situations is to be regarded as ominous.

The skin rarely presents much increase of heat. In some cases there is no increase, and not infrequently in the course of the disease the surface becomes cool or cold. The range of temperature, as determined by the thermometer ${ }^{1}$ in the axilla, is less elevated than in most other acute diseases. Petechial spots or ecchymoses are sometimes observed, occurring generally in connection with hemorrhage from mucous surfaces. In grave cases the skin presents an anæmic aspect, even when hemorrhage has not occurred. In the cases in which the air-passages become affected, there is more or. less lividity of the surface, especially marked on the face and prolabia. The disease has no characteristic eruption; rose-spots are sometimes observed and other forms of eruption, viz., erythema nodosum, urticaria, and sudamina, but these are accidental.

The symptoms referable to the respiratory system are due to diphtheritic inflammation of the larynx, trachea, and the bronchial tubes. These have been already referred to among the symptoms incident to the local affections characteristic of the disease. If life continue sufficiently, false membrane, in these cases, is expectorated, sometimes in the form of hollow tubes like maccaroni, but generally in patches of irregular size and shape.

The desire for food is generally either notably diminished or lost. Aside from pain and difficulty in deglutition, or the suffering caused by food

1 Vide Clinical Thermometry in Diphtheria. By Joseph G. Richardson, M.D. New York Med. Record, July 15, 1867. 
and drinks when the mouth is, affected, there is often an invincible repugnance to nourishment, so that, in children, aliment, if given at all, must be forcibly administered. Vomiting is a frequent, and in some cases a prominent, symptom. This, as well as anorexia, is serious from the interference with alimentation. Diarrhœa is not uncommon, and is a symptom of bad omen. In the cases in which the diphtheritic affection extends into the osophagus, false membrane is expelled by vomiting in the form of ribbous, provided death do not take place before the exfoliation is completed.

In the majority of cases the mind is unaffected. Slight passive delirium occasionally occurs, and sometimes the delirium is active. The latter denotes great gravity of the disease. Convulsions and coma are rare, but both are of occasional occurrence. Convulsions have been observed at all periods of the disease; conia is the forerumer of a fatal termination. It is probable that coma and convulsions, occurring in this disease, are generally due to uramia.

Albuminuria is a frequent symptom. The proportion of cases in which it occurs is variable. A beille found the proportion two-thirds in one year and one-third in another year. Of 48 cases studied by this author in different years, it existed in 19. Generally, the albumen contained in the urine is small, but it is sometimes abundant. In the cases in which it is likely to be abundant, the diphtheritic exudation is large and the swelling of the glands of the neck unusually great. General dropsy in eonnection with albuminuria is rare; it has, however, been observed in a small number of eases by $\Lambda$ beille and Sée. Fibrinous casts arc rarely found in the sediment of the urine. Hrematuria has been observed in cases presenting the symptoms of purpura. As in the other febrile diseases the urine contains a larger amount of urea than in health, a fact showing an increased waste of the tissues.

The duration of the disease is from one to two weeks. In fatal cases the duration may fall short of a week, death sometimes taking place within forty-eight hours. On the other liand, the illness may continue for an indefinite period beyond the maximum duration of the eareer of the disease, owing to consecutire affections or sequels. A side from the characteristic diphtheritic affections, which are properly components of the disease, complications are rare and accidental. I'neumonitis is occasionally developed; and the affection of the kidneys giving rise to an abundance of albumen in the urine and sometimes to general dropsy is perhaps to be regarrled as a complieation. The disease, however, may be associated with other diseases. Its concurrence with scarlatina, measles, and smallpox has been repeatedly oloserved.

The sequels of this discase form an important part of the clinical history. Anæmia and general debility are apt to persist for a considerable period. Feebleness of the action of the heart sometimes exists to such a degree as to lead to sudden death from syncope. 'The feebleness appears, in some cases, to be due to a paralyzed condition of the heart.

Paralysis affecting more or less of the voluntary and sometimes the involuntary muscles is a characteristic sequel. The muscles of the soft palate and pharynx are oftenest affected, and paralysis here generally precedes its occurrence elsewhere. The palatine muscles are sometimes affected during the course of the disease, but generally not until after a period varying from a week to four weeks from the date of convaleseence. In a slight or moderate degree, the paralysis in this situation is denoted by a certain amount of difliculty in rleglutition and the regurgitation of liquids through the nostrils. In a greater degree, which is happily rare, 
there is very great difficulty in swallowing, and it may be even necessary to introduce aliment by means of the stomach-tube. Under these circumstances, sudden death has been caused by the passage of aliment into the larynx. On inspection, the soft palate is seen to be relaxed and immovable. The paralysis affects the sensibility of the parts, so that the palate remains motionless when irritated with a pointed instrument. The existence of paralysis in this situation is indicated by a nasal intonation of the voice, and sometimes by a snoring sound in respiration. A patient who was affected with moderate paralysis in this situation complained of the fatigue which deglutition oceasioned, the labor of swallowing being so great as to render it difficult to eat sufficiently to satisfy the appetite. The paralysis in some eases affects the tongue and muscles of the face, so that articulation is difficult, and the patient may be unable to perform the acts of suction and whistling. The act of gargling is also difficult or impossible.

The paralysis is sometimes limited to the museles of the palate, pharynx, and mouth, but not infrequently it extends to other museles. Exceptionally, the paralysis is manifested primarily, or solely, elsewhere than in the throat and mouth. The lower limbs are most apt to be affected, and paralysis here generally takes precedence of its occurrence in the upper extremities. The paralysis of the limbs is developed gradually, and is preceded by tingling, numbness, and a sensation of coldness. The loss of power over the muscles may be complete, or the paralysis may have different degrees of incompleteness. The paralysis sometimes assumes the form of hemiplegia. One of the npper extremities may be paralyzed in connection with paraplegia. The upper limb on one side and the lower limb on the opposite side may be alone affected. Trousseau has observed the paralysis to affect successively and recurrently the different extremities. Finally, the paralysis is sometimes general, that is, all the extremities are paralyzed, and, in addition, the paralysis may involve the face, the muscles of the throat and mouth, of the neck and trunk, and certain of the involuntary muscles. Cases in which the paralysis is so extensive are extremely rare.

In connection with the paralysis of motion, the sensibility in the affected parts is often notably diminished. The power of feeling pain is either lost or more or less impaired (analgesia), and also the tactile sense (anæsthesia).

Exeeptionally, the sensibility of the integument is morbidly inereased (hyperasthesia). The special senses are liable to be affected, more especially the sense of sight. Vision may be affected by paralysis of the external rectus muscles causing converging strabismus. Aside from this cause of disturbanee, the most frequent form of disorder is presbyopia. The defect of sight is then obviated by the use of convex glasses. Oceasionally the form of disorder is myopia. In these cases the disorder consists in defective power of adjustment in the eye for distant or near objects. But in some cases the impairment of vision is amaurotic. Complete amaurosis oceasionally occurs. There is reason to believe that the amaurosis is sometimes attributable, in part at least, to uræmia. Disordered vision is in some cases the first of the paralytic sequels, and the paralysis is sometimes limited to the organs of sight. The other special senses, viz., taste, smell, and hearing, are sometimes affected, but rarely as compared with the sense of sight.

The paralysis sometimes affeets the intercostal muscles and diaphragm, giving rise to dyspnoa, and involving danger from inability to carry on the respiratory acts. 'The muscles concerned in defecation are sometimes 
paralyzed, causing retention of, or inability to retain, the feces. In like manner the paralysis may affect the bladder, causing retention of urine, or, on the other hand, the sphincter muscles may be affected, giving rise to ineontinence. Notable impairment or loss of virile power may be here mentioned as one of the sequels of diphtheria.

The paralysis in the different situations in which it occurs after diphtheria, is functional, that is, it is not dependent on appreciable lesions. In fatal cases, lesions are not discoverable, and, if death do not take place, the paralysis is temporary; recovery may be confidently expected after the lapse of a few weeks or months. The pathological explanation of the paralysis cannot be given. It is in some way connected with the special condition which exists in diphtheria, for functional paralysis is extremely rare as a sequel of other diseases. It is not associated constantly with urinary changes which point to uræmia ; on the other hand, uræmia occasions convulsions and coma oftener than paralysis, and these are not among the sequels of diphtheria. As regards the frequency of the paralytic sequels, more or less impairment of deglutition is common, but paralysis elsewhere occurs in a minority of cases. These sequels take place in cases of mild as well as severe diphtheria.

Pathological Character.-Diphtheria is manifestly a constitutional disease. 'The diphtheritic affection is the local expression of a special morbid condition of the system. And in this, as in other constitutional diseases, the essential morbid condition is supposed to involve bloodchanges, the nature of which remains to be aseertained. Symptoms denoting constitutional disturbance precede the occurrence of diphtheritic inflammation. The latter, therefore, is not to be regarded as giving rise to the former. Moreover, the constitutional disturbance is often out of proportion to the local manifestations. It is true the diphtheritic local affections, as regards their extent and amount, bear a certain relation to the gravity of the disease, but this is consistent with the fact that they constitute the local expression of the disease.

By some French writers, under the name diphtheria is embraced the affection of the larynx and trachea known as diphtheritic laryngitis, or true croup. The latter affection is identical with diphtheria in so far as the inflammation is characterized by the formation of a false membrane. As already stated, the false membranes in the two affections present certain points of difference. Other circumstances, however, show the nonidentity of the two diseases. Diphtheria occurs and may prove fatal without any affection of the larynx. If the term true croup be applied to laryngitis whenever the inflammation is psendo-membranous, it is proper to say that croup is liable to occur as an intercurrent affection in certain cases of diphtheria. These cases differ materially from cases of croup occurring irrespective of diphtheria. In cases of diphtheria with croup, the exudation is more abundant and extensive in the fances than in cases of croup without diphtheria. In the latter, the exudation does not extend to the nares, into the œsophagus, etc., nor does it occur in other situations. The enlargement of the cervical glands is greater in cases of diphtheria. Albuminuria is not of frequent occurrence in primary croup; the febrile movement and other general symptoms are more symptomatic of the local affection, and the affection is not followed by paralysis. Moreover, primary croup is a spasmodic affection, whereas diphtheria prevails as an epidemic, and the latter does not, like the former, affect almost exclusively children.

Diphtheria has been considered by some as identical with scarlatina. 64 
The non-identity of these two affections is shown by the absence, in the former, of the efflorescence which is present in the great majority of the cases of the latter; by the recurrence of diphtheria in the same person once or frequently in numerous instances, and by the occurrence of dipltheria in those who have had scarlatina. Moreover, the following important points of clifference relate to the elinical history: Searlatina is developed rapidly, and diphtheria often gradually. In diphtheria there is a tendency to an extension of inflammation within the larynx; this very rarely occurs in scarlatina. The characteristic sequels of scarlatina, viz., albuminuria and general dropsy, very rarely follow diphtheria, and, on the other hand, the characteristic sequel of diphtheria, viz., paralysis, very rarely follows searlatina.

$\dot{A}$ diphtheritic affection of the throat, however, occurs as an element of scarlatina, and, as already stated, the two affections may exist in combination. The following facts, which came under my observation in the practice of Dr. John A. Brady, of Brooklyn, seem, at first view, to point to the identity of these affections, but probably exemplify their combination. I was requested by Dr. Brady to see in consultation a boy aged 4 years, affected with complete hemiplegia and general dropsy. The urine abounded in albumen. This boy had recently had diphtheria, the false membrane in the fauces having been well marked. There had been no eruption. After his convalescence a sister was attacked with scarlatina, the rash being abundant. The boy liad the eharacteristic sequels of both diphtheria and scarlatina, and there was ground for the supposition that he communicated the latter affection to his sister. He recovered from the dropsy and the paralysis.

An important pathological question relates to the agency of the local affections in inducing more or less of the constitutional disturbance in cases of diphtheria. The view appears to be held by many that septicamia is produced by resorption of the exudation. The practical bearing of this view is obvious. If the view be correct, it is immensely inportant to limit, if possible, the local diphtheritic affections, and to effect as speedily as practicable the separation of the false membrane. The view is, however, conjectural; and it is more rational to attribute the constitutional symptoms chiefly to the primary and essential morbid condition which constitutes the disease, rather than to suppose a reinfection of the system by the morbid products on the inflamed parts.

Causation.-Diphtheria rarely occurs sporadically. It is eminently an epidemic disease. Like other epidemic diseases, it has its special cause. Is the special cause a contagious virus or an infectious miasm; in other words, is the disease communicable? The affirmative is beld by many distinguished clinical observers. Examples of persons becoming affected after having been brought into close contact with cases of the clisease are numerous; but such cases, during the prevalence of an epiclemic, do not prove communicability of the disease. Striking instances are reported, in which the disease appeared to be produced by the accidental introduction of some of the diphtheritic exudation into the mouth or nostrils ; yet repeated experiments made by Trousseau and Peter, to produce the disease by inoculation, proved invariably ineffectual. Peter even went so far as to apply freely to the throat lint charged with diphtheritic matter. The strongest proof of contagiousness is afforded by instances which appear to show importation of the disease. Such instances are cited by Greenhow, Trousseau, and others. It is questionable whether the number of these instances precludes the supposition that they only show coinci- 
dences, or whether the special cause may not have been transported without having been produced within the bodies of those affected. Regarding the question of communicability as sub judice, it is certainly proper that precautions should be taken to aroid needless and excessive exposure.

Epidemies of diphtheria are irregular in their occurrence. It is a remarkable fact that they were scarcely known in America from the epidemic in 1771 , described by Bard, up to about 1856, and since the latter date they have occurred in all parts of the continent. The disease is often limited, in its prevalence in particular sections, to a circumscribed area. For example, it has been observed to be restricted to a narrow strip of land on the banks of a stream. The special cause seems to require auxiliary causes, at present unknown, which are peculiar to certain localities.

The susceptibility to the disease is not equal in all periods of life. In the great majority of cases, patients are between three and twelve years of age. Persons of any age are, however, liable to be attacked. The following analysis of 461 cases, by Daviot, will serve to show the relative liability to the disease at different periods of life: 'The number of cases under 5 years age was 148 ; between 5 and 10 years, 112 cases; between 10 and 15 years, 86 cases; between 15 and 20 years, 46 cases; between 20 and 30 years, 32 cases; between 30 and 40 years, 28 cases; between 40 and 50 years, 8 eases; and between 50 and 60 years, 1 case. ${ }^{1}$ The number of females in these cases exceeded somewhat the number of males. Circumstances pertaining to social position, occupation, etc., do not appear to be connected with the causation.

Different epidemics differ as regards certain features of the disease and the rate of fatality. In some epiclemies there is an unusual tendency to an invasion of the larynx, and the amount of exudation in other situations is nnusually large in the majority of cases; in other epidemics the local affection is confined to the fauces, as the rule, and the disease is generally mild. In respect of variations at different times and places, diphtheria resembles other epidemic diseases.

Diagnosis.-Prior to the occurrence of inflammation and exudation, the disease presents no distinctive traits, and the characteristic local manifestations are preceded by general symptoms for a variable period. These symptoms are sometimes slight, but in some cases violent. For example, in a case which I attended with the late Prof. C. R. Gilman, the affection of the throat was preceded by high febrile movement and delirium, and, as the attack commencel shortly after confinement, the diseasc was at first supposed to be puerperal fever. In a case which came under the observation of Dr. Bogert, of this eity, the patient, for twenty-four hours after the attack, presented such an alarming degree of prostration that death appeared to be averted only by the persistent and free use of stimulants. Pain and tenderness existed over the abdomen, and peritonitis was suspected. After twenty-four liours, however, the nature of the disease was declared by the occurrence of pseudo-membranous pharyngitis. In both these cases recovery took place. It is not improbable that death is sometimes due to the overwhelming violence of the disease, at its onset, before the diagnostic features are developed.

The diagnosis is sufficiently easy when the local manifestations have taken place. The only liability to error has been already referred to, viz., confounding with the true diphtheritic affection cases of pharyngitis

1 Cited by Prof. A. Clark; vide American Med. Times, vol. ii. 1861. 
with the deposit of abundant follicular secretion. The distinctive points have been stated under the head of anatomical characters. It may be here repeater, that cases of simple pharyngitis occurring during the prevalence of diphtheria are not to be considered as cases of the latter disease; in other words, the false membrane is an essential diagnostic criterion. Ordinary sore throat, with more or less constitutional clisturbance, which is of frequent occurrenee when diphtheria prevails, is doubtless due to the epidemic influenee, just as ordinary diarrhœa during the prevalence of epidemic cholera arises from the choleraic influence, and the former should not be called diphtheria more than in cases of cholerine the disease should be called ebolera.

Prognosis.-Statistics, communicated by different observers, showing the rate of mortality from diphtheria, present a very wide range of variation. 'This is measurably due to the fact that, irrespective of treatment, the fatality in different epidemies actually varies within widely separated limits. The variations in statistics are also due in a measure to the error of confounding with diphtheria pharyngitis with follicular secretion, and the error of applying the name diphtheria to all cases of sore throat occurring during an epidemic. Without attempting, therefore, to express the average death-rate in figures, it will suffice to say that it is sometimes very large and sometimes comparatively small.

The danger is, first, from an invasion of the larynx. Of the cases in which the air-passages become involved, the vast majority end fatally. The prognosis in these cases is always exceedingly unfarorable. Apnoa either canses or contributes to the fatal result whenever the larynx becomes involved. The patient cannot be considered as secure against diphtheritic laryngitis so long as the career of the disease continues, but the danger from this source progressively diminishes after the first week.

The danger is, second, from asthenia or exhaustion. The violence of the disease may destroy life within forty-eight hours from the date of the attack, or perhaps at an earlier period. In these cases, the disease is truly called malignant. Using the language of metaphor, for the want of precise knowledge of the general conditions on which the fatality in these cases depends, the vital powers are said to be overwhelmed with the force of the disease. But in the larger proportion of the cases in which the disease proves fatal by asthenia, the powers of life are gradually exhausted, and death takes place in the second week. In the cases in which the larynx is unaffected, the prognosis is always unfarorable if the diphtheritic membrane extend from the fauces into the posterior and anterior nares, if it pervade extensively the buccal membrane, or if it exist abundantly in other situations. Other unfavorable prognostics are, frequent vomiting, diarrhœa, copious epistaxis or hemorrhage in other situations, great frequency, irregularity, or feebleness of the pulse, coldness of the surface, abundance of albumen in the urine, convulsions, delirium, and coma. It would appear from the observations of Dr. Joseph G. Richardson that the axillary temperature when not above $102^{\circ}$ or $1022^{\circ}{ }^{\circ}$ Fahr. is not to be relied upon as evidence that the disease will not be likely to prove fatal:

A fact important to be borne in mind is the liability to sudden, fatal syncope in this disease. This has occurred unexpectedly in two cases under my observation. It may occur in cases which, as regards the general symptoms, do not present an appearance of great gravity. It generally occurs on some unusnal exertion, as in getting out of bed ; it has been known to occur even during convalescence. It is probable that, 
in some of the cases in which a fatal result occurs suddenly or unexpectedly, the death is occasioned by the formation of a thrombus in the right cavities of the heart. Dr. J. Forsyth Meigs has reported three cases in which death was attributed to this accident. ${ }^{1}$

The paralytic affections which are liable to supervene do not, in general, involve danger. The danger incident to these affections relates to innutrition in the cases in which deglutition is extremely difficult or impossible, and to apnoen when the respiratory muscles are involved in the paralysis.

Treatuent.- With reference to the therapeutical indications, it is convenient to divide cases into those with and those without a diphtheritic affection of the larynx. And, first, of the treatment of cases in which the larynx does not become affected. The indications in these cases relate to the general condition and to the local affection.

Bretouneau, 'Trousseau, with many others, attach chief importance to the local treatment. 'Theoretically, one reason for this is the belief that the system becomes infected by absorption of the diphtheritic matter. Regarding this belief as unfounded, local treatment with a view to prevent reinfection of the system has no rational ground for support. Another object of local treatinent is to arrest the progress of the diphtheritic inflammation and prevent its extension. But with reference to this object, it is to be considered that the local affection does not, properly speaking, spread, but the progressive extension and successive invasion of different parts are due to the agency of an interual determining influence. The restriction of the (liphtheritic affection in one case to a small space, and its wide diffusion in another case, depend on the essential morbid condition which constitutes the disease, and, if so, topical treatment will be likely to excrt little or no effect in limiting the amount or extent of the local manifestations. Turning from theoretical views to clinical experience, a large proportion, if not the great majority, of the practitioners of this country have been led to discontinue the cauterizing and irritating topical applications which hare been heretofore in vogue, and to content themselves with soothing and antiseptic local measures. So far as my own opportunities for observation enable me to julge, this practice is to be preferred. In the place, therefore, of a strong solution or the solid stick of the nitrate of silver, hydrochloric acid, the sulphate of copper, alum, and the astringent preparations of iron, applied repeatedly in the twenty-four hours, a gargle of a solution of the chlorate or nitrate of potassa may be employed if the patient be not too young or the act of gargling do not occasion much annoyance; ice may be taken into the mouth in small pieces frequently if the effeet be agreeable, and if there be fetor, a weak solution of the chloride of soda may be applied, or a solution of the iodide of bromine, two drops to a fluidonnce of mucilage, the application being made by means of a camel's hair brush, as recommended by Prof. Metcalfe. A weak solution of cither creasote or carbolic acid is doubtless a useful antiseptic application. A mild astringent application may sometimes be employed with advantage, and probably the best preparation is tannin combined with glycerine. A solution of the hypo-sulphite of soda, applied to the diphtheritic patches, has been found useful. The utility of this application is supposed to depend in part on its power of destroying vagetable parasitic productions. 'The microscope shows the presence of these in the psendo-membranous formation. Three drachms of the salt may be dissolved in two drachms of glycerine and six drachms

I American Journal of Medical Sciences, April, 1864. 
of water. A gargle of half a drachm of the salt dissolved in half a pint of water and half an ounce of glycerine may be used at short intervals. It may be added, a German author, L. Letzerich, maintains that the distinctive character of the diplitheritic inflammation depends on the local effect of cryptogamic productions, and that clinical experience shows the false membrane may be prevented if the vegetable spores and spornles are either timely removed by emetics, or destroyed by topical applications.'

With reference to general treatment, it is to be considered that the disease is not controllable by any known specific remedy. In view of this fact, and also of the danger being from asthenia, it follows that the great object of treatment is to support the system, endeavoring to keep the patient alive until the career of the disease is ended. The therapeutical measures indicated, therefore, are tonics, stimulants, and alimentation. Of remedies, the sulphate of quinia, the tincture of the chloride of iron, and the chlorate of potassa are especially to be named as having been fonnd useful. Their usefulness is not to be denied, although they do not exert a specific influence over the disease, as some have supposed. The permanganate of potassa has been advocated as a useful internal remedy in this disease by Prof. Samuel Jackson, Dr. Mackall, and others. Of a solution consisting of a drachm of the salt in a pint and a half of water, a teaspoonful may be given every three hours. A weak solution of this salt is also useful as a local antiseptic application. There is reason to attach much importance to alcoholic stimulants employed with proper discrimination. 'The principles which should govern their employment are the same as in the essential fevers and other cliseases tending to destroy life by asthenia. These principles need not here be repeated. In this disease, as in the essential fevers, there is sometimes a notably increased tolerance of alcoholics. Jenner cites a case in which two drachms of brandy were given hourly, with advantage, to a child three years of age. In a case of great severity which I saw with my colleague, the late Prof. Enos, half an ounce of brandy was given hourly, the patient being thirteen years of age, without any excitant effect, the case ending in recovery. In the use of alcoholic stimulants the physician is to be guided by the indications and by carefully watching the immediate effects. Alimentation is an essential part of the treatment. The diet should be concentrated, highly nntritions, and embracing the necessary variety of alimentary principles. Milk meets pre-eminently these requirements. A serious difficulty in the treatment often arises from the invincible repugnance to nutriment, and sometimes from the persistence of vomiting. Owing to the difficulty of alimentation in some cases, and sometimes to a want of appreciation of its importance, death takes place from innutrition.

All the measures heretofore known as antiphlogistics, viz., bloodletting, general or local, purgation, mercurialization, emetics, and nauseants, are rationally contra-indicated by the simple fact that the danger is not from the inflammatory manifestations of the disease, but from the general condition which tends to destroy life by asthenia. Clinical experience has abundantly shown these measures to be injurious. Blisters and other counter-irritant measures are not to be employed, occasioning, as they are apt to do, new foci of diphtheritic inflammation.

Symptoms incidental to the disease claim palliative treatment. Vomiting is to be relieved by bismuth, creasote, or hydrocyanic acid. Diarrhœa calls for opium and astringents. Anodynes, that is, opium or its succe-

1 Vide Virchow's Archives, 1868 ; also article by Dr. H. Beaunis, in Gazette Médicale de Paris, 4 Juin, 1870. 
danea, the bromicies, or the chloral hydrate, are often required by restlessness and vigilance. Hemorrlage indicates the use of hemostaties topically and internally.

The treatment of eases in which diphtheritic laryngitis becomes developed involves the same general principles as cases without the laryngeal affection. But, in addition, are inclicated measures to hasten the separation of the false membrane within the larynx, precisely as in cases of laryngitis with false membrane disconnected from diphtheria, that is, in eases of true sporadic or primary eroup. The most efficient of the measures for this end is the inhalation of warm vapor or steam. With reference to this measure, and other points relating to the treatment directed to the laryngeal affection, the reader is referred to the chapter of this work deroted to pseudo-membranous laryngitis. ${ }^{1}$

The rules with respeet to tracheotomy or laryngotomy, when the danger from laryngeal obstruetion is imminent, are the same as in cases of primary eronp. There is undoubtedly less hope of success from surgical interferenee than in the latter affection. With reference to the propriety or importance of the operation, however, the simple question is, are lives ever saved by it? This question is undoubtedly to be answered in the aflirmative. The question, how many lives are saved, is of less importance in its practical bearing. If lives are ever saved by tracheotomy or laryngotomy in diphtheria, a practitioner is reprehensible if he allows a patient to die from laryngeal obstruetion without opening the larynx or trachea. The patient is entitled to the chanee of being saved by an operation, however small that chance may be.

The propriety of precautions against contagion has been already referred to. When practicable, removal, especially of persons between three and twelve years of age, beyond the epidemic prevalence of the clisease is to be enjoined.

\section{CHA P T ER X.}

Acute Articular Rheumatism-Clinical History-Pathological Character-Causation-Diagnosis-Prognosis-Treatment. Subacute and Chronic Articular Rheumatisin. Muscular liheumatism.

THE term rheumatism is used to denote a constitutional disease characterized by ecrtain local manifestations seated in the articulations and fibrous tissues in other parts ; the term has also been applied to neuralgic and other affections supposed to arise from the constitutional morbid conditions existing in the ordinary form of the disease. I shall consider, first, the affection to which the term rheumatism is par excellence applied, riz., acule articular rheumatism; second, the affection, or affections, called subacute or chronic rheumatism; and, third, the aflection known as muscular rheumatism. The disease called gout is in certain respects analogous to rheumatism, although presenting essential points of difference. After having considered the several forms of rheumatism just 
named, I shall consider gout, and, finally, an affection to which is frequently applied the name rheumatic gout.

\section{Acute Articular Rheumatism.}

Aeute articular rheumatism, as the name imports, involves an affection of the articulations or joints. The affected joints present the phenomena of inflammation, and the affection is also called acute rheimatic arthritis. The lining membrane of the joints is, in fact, inflamed, but rheumatic inflammation in this situation has certain peculiarities, viz., it rarely leads to the exudation of lymph, suppuration, production of new tissue, or structural lesions of any kind; and the inflammatory condition not infrequently completely disappears in the course of a few hours. The disease per se very seldom, if ever, proves fatal ; but in cases in which life is destroyed by concomitant affections of internal organs, the affected joints are found to present, as a rule, only more or less effusion, which is sometimes opalescent, within the articular cavity, with thickening of the synovial membrane, and infiltration of the surrounding parts. Aside from these appearances the discase has no ascertained, constant, anatomical characters, except that the quantity of fibrin in the blood is notably increased, with, perhaps, an abnormal tendency to congulation (inopexia). In the course of the disease certain internal organs are liable to become inflamed. The inflammation is generally seated in the fibro-serons tissues, and especially in the endocardium and pericardium. The inflammation in these situations differs from that of the joints in this, viz., it is apt to give rise to the exudation of lymph, and sometimes to suppuration. In a pathological view, the endocarditis, pericarditis, and other internal inflammations which occur in cases of acute articular rheumatism are local manifestations of the disease, like the arthritic affections, but it is most convenient to consider them in the light of complications.

Cuinical History.-Acute articular rheumatism, in the majority of cases, commences with a sudden attack, and in nearly one-half of the cases in which the attack is sudden it takes place at night. In a small proportion of cases, febrile movement, more or less marked, precedes the local manifestations for a period varying from a few hours to one or two days; hence one reason for the name rheumatic fever, instead of acute rheumatism. In most cases, however, the fever and the local manifestations are simultaneous in their occurrence. Sometimes pain and soreness of the joints precede the development of the disease for a variable period.

The development of the disease is denoted by an affection of one or more of the articulations. The local phenomena are pain, tenderness, increased heat, swelling, and redness of the skin. The pain differs in intensity in different cases, but, as a rule, it is not intense so long as the parts are perfectly quiet. Pain is especially excited by morements of the affected joints. In severe cases the slightest motion is insupportable; even jarring the bed or room occasions suffering. Pressure over the joints is painful. Owing to the pain occasioned by movements, the patient feels obliged to maintain, as long as possible, a fixed position; changes of the decubitus cause often extreme suffering. Swelling is most apparent in joints not covered with muscle, viz., the knee, wrist, elbow, ankle, and the smaller joints of the hands and feet. It is less apparent in the hip and shoulder. The swelling is due, in part, to a morbid increase of liquid within the synovial cavity, and in part to effusion into the surrounding 
structures. The redness is due to an erythema in the form of patches or zones which do not present well-defined borders. The redness, like the swelling, is not apparent over the hip and shoulder, but only over joints comparatively superficial. 'The swelling and redness are, in general, proportionate to the acuteness of the rheumatism.

In some cases several joints are affected either simultaneously or in quick suicession (Polyarthritis); in other eases the affection is limited to a single joint for a greater or less period (Monoarthritis). In the course of the disease, in inost cases, various joints are successively affected, and frequently a greater or less number of joints are affected at the same time. It is a peculiarity of the rheumatic inflammation to leave one or more joints abruptly, the local phenomena sometimes disappearing within a few hours, and to attack as abruptly other joints. Different eases differ as regards the number of joints affected in the course of the disease; in some cases few or no joints escape, and in other eases the rheumatic inflammation is limited to a few joints or to a single joint. The fibrous tissues elsewhere than within and around the joints, and exclusive of the visceral organs, are liable to be attacked. Thus, those of the clorsum of the hand and of the instep, the ligaments of the neek and back, and the sclerotic tunic of the eye are sometimes involved.

The disease is extremely irregular as regards the number of joints affected, either simultancously, or suceessively, the order in which they are attacked, etc.; yet the local manifestations are governed by an inlportant pathological law, viz., the law of parallelism. Corresponding joints are often affected together, and when this is not the case, the different affected joints are either on one side of the body, or joints on the two sides which are analogous, viz., the knee and elbow, wrist and ankle, hip and shoulder, are affeeted in eombination. In an analysis of 21 cases with reference to this point, ont of 88 instances in which either a joint was affected singly or more or less joints affected in eombination, there was but a single violation of the law of parallelism. ${ }^{1}$ This disease, therefore, is eminently one of the bilateral or symmetrical diseases. As respects the relative liability of the different joints to become affected, the analysis just referrel to showed the order to be as follows: The knee, ankle, wrist, shoulder, hip, elbow, phalangeal joints, first of the hands, and second of the feet, and the maxillary joint.

Acute articular rheumatism is always accompanied with more or less febrile movement. As already stated, the disease is sometimes called rheumatic fever. In addition to the fact that the fever sometimes precedes the local manifestation, a reason for the propriety of this name is to be found in the fact that the intensity of the febrile movement is not always in proportion to the number of joints affected or the intensity of the rheumatic inflammation, as denoted by pain, soreness, heat, ete. The researehes of Louis show the fever to be less intense, judging from the pulse, than in typhoid fever, pneumonitis, the eruptive fevers, and erysipelas of the face. The pulse rarely exceeds 90 per minute. The temperature, as shown by the thermometer in the axilla, according to the observations of Dr. Sidney Ringer, vacillates irregularly between $102^{\circ}$ and $110^{\circ}$ Fahr. The intensity of this, as of other acutc or febrile diseases, is in proportion to the increase of temperature as indicated by the thermometer in the axilla. Sweating is generally a symptom more or less prominent, occur-

1 Analysis of twenty-one cases of articular rheumatism, by the author. Buffalo Medical Journal, Mareh, 1854. 
ring especially at night. The sweat emits a notably sour odor. In conneetion with more or less profuse sweating, sudamina or miliary vesicles not infrequently appear on the neck and trunk. 'The appetite is either lost or greatly impaired. 'Thirst is more or less urgent. The tongue is frosted or coated. 'The saliva becomes acid. Generally the bowels are constipated, but exceptionally there is diarrhœa. 'The urine is diminished in quantity; its specific gravity is high, owing to increase of urea and decrease of water; the coloring matter is increased, and, on cooling, the urates are deposited in abundance. Owing to the increase of the piginent of the urine, the sedimentary deposit is usually of a reddish color. The deposit of this lateritious sediment does not show an increase of the amount of uric acid in the urine, but only the diminution of water. Quantitative analyses of the urine have shown the quantity of urie acid not to be greater than in health. The chlorides are sometimes deficient, and oceasionally the urine is slightly albuminous. Pain in the head is not common. The intellect, as a rule, is not disturberl unless a cerebral complication occur, which is rare; but mild delirium is sometimes observed. In general, the strength is well preserved. Vigilance is usually a source of discomfort and more or less prostration, sleep being prevented by pain on change of position, and copious perspirations.

An important feature of acute articular rheumatism is its tendency to invarle certain of the structures of the heart. Endocarditis occurs in a considerable proportion of cases. It is, however, less frequent than has been supposed by some, as already explained in treating of that affection. The inflammation, as a rule, is limited to the membrane lining the left carities of the heart, and it affects especially the portion of this membrane which enters into the composition of the mitral valve. In a large proportion of the cases of valvular lesions of the heart, more especially mitral lesions, their point of departure is rhenmatic endocarditis. Pericarditis occurs less frequently, the proportion being about one-sixth. As a rule, when pericarditis becomes developed, endocarditis cocxists. For a consideration of these affections, as developed in rheumatism, the reader is referred to the section devoted to diseases affecting the circulatory system.' It may be repeated here that pericarditis and probably endocarditis sometimes precede the local manifestations elsewhere, for, although it is convenient to speak of these affections as complications, they are, properly speaking, to be reckoned among the local manifestations of the disease; that is, they are dependent on the same internal, determining cause to which the affection of the joints is to be referred. The structures of the heart and other internal organs are not affected in consequence of a change of place from the joints, or a metastasis, as formerly supposed, but in consequence of an elective affinity in the disease for certain tissues, among which the endocardium and pericardium are included. But it is to be noted that rheumatic endocarditis and pericarditis differ from rhemmatic arthritis in this, they present, as a rule, the ordinary results of inflammation, viz., exudation of lymph, formation of false membrane, and sometimes suppuration, results extremely rare in the articular manifestations. The structures of the heart are apt to become involved in proportion to the acuteness of the rheumatism. They may become involved at any period in the course of the disease, but the liability is greatest in the carly and middle period.

Bronchitis, pleuritis, and pneumonitis are oceasionally developed. They are, however, so rare that it may be a question whether they be not acci-

1 Vide pages 316,326 . 
dental complications. Of 21 cases which I analyzed several years ago, and 13 cases more recently analyzed, the disease in the latter cases being left to pursue its natural course, making in all 34 cases, pneumonitis occurred in only one case; and these eases furnished not a single example of pleuritis, irrespective of pneumonitis, nor a single example of bronchitis.

The meninges of the brain are sometimes affected. Examples, however, are infrequent. Active delirium, convulsions, and coma are events observed in a small number of cases. In some of these cases the appearances denoting meningitis have been found after death, but in other cases the cerebral struetures are devoid of the evidence of inflammation. The eveuts just named are sometimes associated with pericarditis, and are probably connected pathologically with the latter affection. It may be conjectured that rheumatic inflammation may attack the meninges of the brain, leaving no traces after death, as has been observed with respect to the joints. There is also room for the supposition that, in some cases, delirium, convulsions, and coma may be due to uræmia, disease of the kidneys being accidentally associated with rheumatism. That the morbid condition of the blood proper to rheumatism may determine these events toxically, as conjectured by Fuller and others, is hardly probable in view of the infrequency of their occurrence. Meningitis, distinguished as rheumatic, has some distinctive features which have been mentioned in a previous section (vide page 630). Cerebral embolism from masses of fibrin detached from within the left cavities of the heart is an event of rare occurrence. In the ease of a male patient under my observation, peritonitis was developed in the course of the affection, the peritoneal inflammation encling in recovery.

The duration of acute articular rheumatism varies greatly. The disease ends from self-limitation, but the minimum and maximum of duration are widely apart. These statements have reference to the course of the disease when left to itself, that is, no active measures of treatment being employed. In 1862, I observed, at Bellevue Hospital, 13 eases which were allowed to pursue their course uninfluenced by therapentical interference, some palliative measures being alone employed. I am not aware that, prior to this date, any one had undertaken to study, in this way, the natural history of the disease. I reported the 13 cases in the American Journal of Medical Sciences, No. for July, 1863, entitling my article "A Contribution toward the Natural History of Articular Rheumatism." In making an analysis, in $\mathbf{1 8 5 4}$, of the cases which I had, at that time, recorded (24) and to which reference has been already made, I was impressed with the importance of obtaining knowledge of the natural history of the disease, as the point of departure for the study of therapentical measures. With reference to this point, in my report of the analysis of 24 cases, in 1854, I used the following words: "At the present moment we cannot answer the question, "what are the intrinsic tendencies of articular rhenmatism as respects its continuance, its complications, and its remote consequences in the organism?' Were we able to answer this question by an appeal to facts, we should then have a criterion by which to estimate the f:worable or unfavorable influences of different methods of treatment pursued in a series of cases; as it is, in bringing statistical information to bear on the therapeuties of the disease, we can only study the immediate effects of different remedies, and institute comparisons, in this point of view, and also with reference to the duration of the disease, etc., in different series of cases treated by different methods." In my "Contribution

1 Vide Buffalo Med. Journal, March, 1854, vol. ix. p. 557. 
to the Natural History of Articular Rheumatism," published in 1863, I premised a report, in full, of all the cases (13) by the following account of my plan of study: "On entering on duty at Bellevue Hospital in August, 1862, I resolved to treat with palliative measures only, the cases of articular rheumatism which should be reeeived into my hands, so long as circumstances might lead me to conclude that, by continuing this plan, no injustice was clone to the patients whose relief was, of course, paramount to any other object. The cases thus treated progressed so satisfactorily, that I found no ground for a discontinuance of the plan. The last of the cases, in fact, was the only one in which any important complication occurred. Some of the cases were recorded by myself, and the remainder by my zealous clinical assistant, Lr. Shiverick." . . "The reported cases were treated, throughout the whole course of the disease, with only palliative measures. 'These measures consisted of opium in some form given in small or moderate closes, the application, generally, of dry flammel to the affected joints, and the external use of either the soap and opium liniment, or the tincture of aconite. But to secure the moral effect of a remedy given specially for the disease, a 'placebo' was given consisting, in nearly all the cases, of the tincture of quassia very largely diluted. This was given regularly, and became well known in my wards as the 'placeboic remedy' for rheumatism. The farorable progress of the cases was such as to secure for the remedy generally the entire confidence of the patients. I may add, that all the cases were brought before the medical class in attendance during the winter."

of these 13 cases, an endocardial murmur existed in 11 ; but the murmur was limited to the base of the heart in all but 3 cases. Moreover, all the patients but two were women; and in women affected with articular rheumatism, a systolic basic murmur is the rule, with very few exceptions, the murmur being generally of hæmic origin. In only one case was pericarditis developed. In this case, the pericarditis occurred directly after the patient's admission. Pneurnonia occurred subsequently. The patient, however, recovered. ${ }^{\mathbf{2}}$

The duration in these cases, respectively, from the date of attack to convalescence, excluding the case in which pericarditis and pneumonitis occurred, was as follows: In three cases under 15 days, in one case between 15 and 20 days, in thrce cases between 20 and 25 days, in three cases between 25 and 30 clays, and in the remaining two cases 45 and 56 days. The mean duration was a fraction under 26 days.

'These facts show that acute articular rheumatism belongs among the self-limited diseases. Facts subsequently published, by Dr. Henry G. Sutton, in Guy's Hospital Reports, 1865, confirmed this conclusion. Dr. Sutton's communication embraced a tabulated report of 41 cases treated, chiefly, with mint water. 'Twenty-two were males, and ninetcen females. The average duration from admission to the cessation of pain, the skin being cool, and no relapse taking place, was, in the male cases, 13.5 days, and in the female cases, 15.5 days. The average number of days in hospital was, for males, 27.6 days, and for females, 26.3 days. The duration prior to admission into hospital, in many of the cases, was considerable, the longest being six weeks. Pericarditis became developed in four cases, and pleurisy in five cases. In a second report by Dr. Sutton of cases treated, in the same manner, in a subsequent volume of Guy's Hospital Report, the average duration to convalescence was 9 days; and, in a third

1 Fide A Contribution toward the Natural History of Articular Rheumatism, by the Author. American Journal of Medical Sciences, July, 1863. 
report of 25 cases in the Trans. Royal Med. Chirurg. Society, 1869, the average duration was 10 days.

The illness of patients affected with rheumatism may be indefinitely prolonged by pericarditis and pleuritis. But of the cases which 1 analyzed in 1854, embracing five cases of endocarditis and one case of endo-pericarditis, different methods of treatment being employed, the minimum duration was 7 days, the maximum 26 days, and the average duration was a little less than of all the cases, viz., a fraction over 16 days.

A peculiarity of rheumatism is, it does not, like the essential fevers, for example, pursue a steady continuous march from the beginning to the end, but its course is often marked by notable fluctuations as regards the general and local symptoms, more especially the latter. Not infrequently, after a few days, the affected joints are nearly free from pain, soreness, etc., the febrile movement subsides, and convaleseence appears to be near at hand, when suddenly, the local manifestations and the ferer are renewed with perhaps as much or even more intensity than before. This may occur repeatedly during the career of the disease.

Patiological Character.-In view of the number of parts in which the local manifestations are often seated, the apparent migration of the inflammation from part to part, and more especially the observance of the law of parallelism in the arthritic affections, it is a reasonable supposition that the pathology of the disease involves a morbid principle in the blood. The view now held by most pathologists is, that a materies morbi does exist in the blood. With respect to the nature of the morbid material, all pathologists are not agreed. It has been supposed to be uric acid; but an analysis of the blood in forty typical cases of rheunatism, by Garrod, failed to show the presence of this principle in larger quantity than exists in health, whereas in gout the presence of this principle in a morbid quantity is demonstrable. ${ }^{1}$ Others have adopted the supposition of Prout, that the morbid principle is lactic acid. The majority of pathologists, at the present moment, appear to regard this supposition as probably correct, and it is generally accepted as a rational basis of treatment. 'The supposition is, however, in the existing state of our knowledge, hypothetical ; that is, its correctness is yet to be established by demonstrative proof. Assuming the hypothesis to be true, it is evident that the presence of lactic aeid in the blood is an effect of morbid conditions of which nothing positive is at present known. Lactic acid is supposed to be formed during the destruetion of sugar in the lungs in health; it may be conjectured, therefore, that its presence in the general cireulation depends on circumstances which either occasion its undue formation or which interfere with its entering into combinations leading to its disappearance. It has also been conjectured that the lactic acid is formed in the decomposition of the gelatinous and albuminoid tissues. Diseussion of these or other conjectures would be here ont of place; suffice it to say that the morbid principle in the blood, whatever it may be, is doubtless in intrinsic production; that is, it is not received from withont the boty. The pathologieal essence of the disease underlies the produetion of this principle. So long as its production goes on, the career of the disease continues, and the disease ends when the prineiple is no longer produced. 'This principle, eirculating with the blood, acts upon certain parts and gives rise to the local manifestations of the disease; and in its action upon parts it shows an election for the fibrous tissues and espe-

1 On Gout and Rheumatic Gout. London, Second Fdition, 1863. 
cially those entering into the composition of joints. The lactic acil theory seems to have received support from the oecurrence of articular rheumatism in cases of diabetes treated with this acid. ${ }^{1}$

Causation.-Rheumatism is one of the diseases supposed to be frequently, if not generally, eaused by suppression of the functions of the skin, and attributed especially to the action of cold upon the surface of the body. This view of the eausation has but little foundation. It is true that patients are apt to refer the disease to cold, but they are often led to fix upon some particular exposure under the belief that this agency is of course involved, for it is a popular notion that rheumatism generally proceeds from cold. A careful interrogation will show in many, if not most, eases, that the statement has little to support it. And how often is exposure to eold not followed by rheumatism! That the causation may involve the agency of eold is not to be denied in toto, but the considerations which are to follow render it probable that cold acts only as an exciting cause.

A special predisposition seems to be requisite for the causation; in other words, the disease involves a rheumatic diathesis. This predisposition or diathesis may be congenital and inherited, or it may probably be acquired. There are persons eontinually prone to the disease. This is shown by the occurrence of the disease in early life, and by its recurrenee more or less frequently in the majority of the persons who are once attacked by it. Statistics establish conclusively the hereditary transmission of the disease. Of 208 eases analyzed by Fuller (excluding cases in which information was uncertain or not noted), in 71 there was evidence of hereditary predisposition, this evidenee existing in a much larger pro. portion of the cases under, than over, thirty-five years of age. It is a matter of common observation that the disease prevails among the different members of certain families. Age has a decided influence in the eausation. It occurs very rarely under the age of five years. It may, however, occur in infaney. I have met with a case in which it oceurred at one year of age, aortic lesions and hypertrophy of the heart existing at the age of two years. Dr. Henri Roger, Physician to the Children's Hospital, in Paris, states that he has met with but one case at the age of two, and one at the age of three years. ${ }^{2}$ In the majority of cases, patients are between fifteen and thirty years of age. If a person have not experienced one or more attacks before the age last named, the liability to the disease diminishes afterward, and beeomes quite small after fifty. The number of males attacked by this disease exceeds the number of females, but the difference is not great. Of 425 cases analyzed by Fuller, 226 were males and 199 were females. External influenees pertaining to season play a small part in the causation. Fuller analyzed 289 cases with reference to the months in which they respectively occurred, and the variation in the different months was between 14 and 28 cases. The disease prevails most in humid, changeable climates. It is a disease of frequent occurrence in all climates within the temperate zone, but comparatively rare in tropical and extremely cold climates.

These statements express briefly the sum of what is actually known with respect to the causation. In the existing state of knowledge we are alike ignorant of the speeial perversion in which consists the essential pathology of the disease, and of the special agencies upon which these perversions depend.

2 Vide Archives Généralcs de Médicine, Janvier, 1867. 
Disanosis. - The diagnosis requires but a brief consideration. The disease is to be diseriminated from gont and rheumatic gout. For the differential points involved in this diserimination, the reader is referred to the ehapter treating of the latter disease. In general, the diagnostie features of acute artieular rheumatism are so obvious that the disease is at onee recognized. The chief difficulty in diagnosis, exclusive of the discrimination from gout and rheumatic gont, relates to cases in which the rheumatic affection is confined to a single joint. The problem, in these eases, is to distinguish rheumatism from a purely local affection of the joint, or what is commonly known as synovitis, or simple acute arthritis. The following are the points involved in this differential diagnosis: The fixedness of the inflammation in a single joint, from the first, in the latter, whereas in artieular rheumatism it is rare that the mobility of the disease is not to some extent manifested. The existence of fever in some cases of articular rheumatism prior to the affection of the joint. The want of correspondence between the febrile movement and the local phenomena in rhenmatic cases. The longer durntion of the local affection in cases not rhenmatic, and, in eertain eases, the swelling and redness being greater than in rheumatic cases. The rapid disappearance of the local affection, often, in eases of rheumatism, and the speedy recovery of the use of the affeeted joint, the disappearanee and recovery being more slow in eases not rheumatic. The oeeurrence of endocarditis or pericarditis in a certain proportion of rheumatic cases. The age of the patient and the previous oecurrence of rheumatism are points to be taken into aecount.

Cases of pyæmia in which a purulent effusion takes place into certain joints, are sometimes mistaken for cases of rheumatism. Attention to the antecedent and eoneomitant cireumstances should prevent this error. Purulent collections within the joints in eases of puerperal fever are embraced in this eategory.

Prognosis.-This disease is very rarely fatal; the immediate danger to life is exceedingly small. When death takes place during the eareer of the disease, it is due to complications, including under this head pericarditis. But the majority of cases in which periearditis oceurs do not end fatally. Active delirium, convulsions, and coma denote imminent danger, but it has been seen that these are extremely infrequent events. Pulmonary eomplieations may prove serious.

The disease, aside from the complications just referred to, and the suffering attending it, is serious in view of the. liability to endocarditis which may lay the foundation for incurable and grave lesions of the heart. The immediate danger from rheumatic endocarditis is slight; the lesions resulting from this affeetion rarely oceasion inconvenience until after the lapse of several years. Grave cardiac lesions do not invariably follow rheumatic enclocarditis, and in some cases there remains no evidence of any permanent injury from that disease.

The liability to embolism is to be mentioned as incidental to endocarditis. The emboli may be derived from the left side of the heart, being due to the formation of fibrinous masses in connection with enclocarclitis; or a clot may form in the right side of the heart, and cause plugging of the pulmonary artery. Sudden death, during the eourse of acute rheumatism is sometimes caused by pulmonary embolism. An example has fallen under my observation. The patient, a robust man, aged about 50, was apparently convalescing from a mild attack of rheumatism. He had been treated with alkalies and opium. There was no evidence of endocarditis. Suddenly, in the evening, he was seized with acute pain 
in one side of the chest, which was followed by febrile movement. The pain was relieved by an opiate. On the following morning, being quite coinfortable, he was giving some directions with regard to his business, when he suddenly complained of a sense of suffocation and said he was dying. Death took place in a few minutes after this sudden change. On examination after deatl, the pulmonary artery was found to be plugged with a firm black clot. The heart was free from disease. The lower lobe of one of the lungs was in the first stage of pneumonitis. Nothing was found to account for the sudden death except the pulmonary embolism. A similar case of embolism occurring in the course of acute rheumatism, is reported in the London Lancet, May, $1865 .^{1}$

Acute articular rheumatism seldom ends in the chronic form of the disease.

Treatment.-There is scarcely any other disease in the nosology, the treatment of which, during the last quarter of a century, has undergone such mutations as that of acute articular rheumatism. Among the measures which have been more or less in vogue during the period just named are the following: Bleeding, general and local, mercurialization, colchicum carried to the extent of producing romiting and purging, nitrate of potassa, an ounce or more given daily, opium in large doses, large doses of quinia, and the use of the veratrum viride. Cases in which reliance has been chiefly placed on each of these measures have pursued a favorable course, and the treatment has seemed to be successful. Want of knowledge of the natural history of the disease, that is, the history based on the observation of cases in which the disease has been allowed to pursue its course under favorable hygienic circumstances, uninfluenced by therapeutical interference, has rendered it difficult to judge of the effect of different methods of treatment. $\Lambda \mathrm{s}$ a contribution toward the knowledge just referred to, my report of thirteen cases at Bellevue Hospital (to which reference has been already made) possesses value. In these cases palliative measures solely were employed. In one case only was the heart affected, pericarditis occurring in this case directly the patient was admitted ; and exclusive of this case, as already stated, the mean duration of the disease was 26 days, the minimum being 12 and the maximum 56 days. These facts go to show an intrinsic tendency in the disease to end within a shorter period, and a less degree of liability to complications than have been hitherto generally supposed. And, in view of these facts, it may be reasonably concluded that the influence of different methods of treatment over the duration of the disease and the occurrence of complications has been much over-estimated. Each of the measures above enumerated probably are, to a certain extent, useful in certain cases, but a special controlling influence over the disease is exerted by none, and clinieal observation has shown the development of endocarditis, pericarditis, and other complications, under all of them. It is not worth while, therefore, to discuss their merits and demerits severally, considered as constituting exclusively or chiefly the treatment of the disease. Moreover, at the present time, few, if any, practitioners rely upon any one of these measures, exclusively or chiefly.

Of late years the practitioners of this country have generally adopted the alkaliue treatment, first systematically employed by Dr. Wright in 1847 , and afterward, on a large scale, by Garrod and Fuller. The bicar-

1 Vide leport of annther case in the Nouveau Dictionairé, art. Endocarde, page 274, Paris, 187. Jaccoud (Pathologie Interne) states that he has met with two cases. 
bonate of potassa, the bicarbonate of soda, and the tartrate of potassa and soda, or the Rochelle salt, are the remedies usually employed. Garrod's report of fifty cases treated with alkalies largely given showed an average duration of six or seven days; and in no case, after the patient had been under the influence of this treatment for forty-eight hours, was any affection of the heart developer.' 'The alkaline treatment, provided it be not pushed too far, nor continued too long, has the negative merit of doing no harm if it fail to do good. It would, however, be an erroneous inference, from the foregoing statement of Fuller, that cardiae complications never occur after the patient is fairly under the influence of this treatment. I have notes of several eases seen in consultation in which pericarditis was developed when alkalies had been freely given, the urine having been rendered alkaline. But that the liability to these complications is lessened by this treatment must be admitted. Moreover, this treatment seems to exert some influence in abridging the duration of the disease. With reference to these points, the following statistics communicated by Dr. Joln G. Blake may be cited: In 125 cases treated with large closes of the alkalies, the arerage stay in hospital was twentyfive days, and cardiac complications were developed in 18 . In 175 cases treated in other ways, the average stay was thirty-five days, and cardiac complications were developed in 18. "Two deaths oecurred among those treated with the alkalies, although given in the doses recommended by Fuller."' Fuller claims for the alkaline treatment efficiently pursued the power of lessening the febrile movement, relieving the arthritic inflammation, abridging the duration of the disease, and preventing endocarditis and pericarditis. With reference to the latter, which is manifestly the great olject of treatment, he states in a lecture clelivered December, 1862, that his experience embraced, up to that time, one hundred and ninety-four cases, and that " in no single instanee has any heart affection occurred after the patient has been under treatment for twenty-four hours."

The alkaline treatment is consistent with the view which has been presented of the pathology of the disease. Rationally, according to this view, what is most to be desired in the treatmont is the arrest of the produetion of the materies morbi contained in the blood. There are no known means of reaching this end. Next to this, to nentralize and eliminate the morbid principle from the blood, are the rational objects of treatment. It is claimed that the alkaline treatment accomplishes these objects. Assuming the prineiple to be lactic acid, it is nentralized by the introduction of alkalies, and an effect of the treatment is an increase of the secretion of urine. With reference to the latter object, a saline diuretic may be advantageously added.

1 Vide "On a Successful Method of treating Acute Rheumatism by Large and Frequent Doses of Bicarbonate of Potash, by Alfred Baring Garrod, M. D., Trans. Royal Med. and Cliturg. Society, 1855.

¿ Vide Medical and Surgical Reports of the Boston City Hospital, 1871. For an analysis of the cases treated at St. George's Hospital, London, by different remędies, vide article by Dr. Wm. H. Dickinson, in Trans. Med. and Chirurg. Socicty, 1862. The statistics in the paper by Dr. Dickinson afford conclusive evidence of the usefulness of the alkaline treatment as regards diminishing the liability to cardiac complications. Of 48 cases in which a "full alkaline treatment" was employed, in one case only ditl the heart become affected. Per contra, of 113 cases in which other methods of treatment were employed in 35 the heart was affected. Dr. Dickinson's statistics show that a "partial alkaline treatment" is useless. To be successful the alkalies given should be eufficient to render the urine alkaline.

- Vide Medical News and Library, February, 1863. 
To secure as quickly as possible the protective agency against cardiac affections, the alkali selected should be given at once in large doses and repeated at short intervals until the urine gives an alkaline reaction. Fuller advises from one to two drachms of the bicarbonate of either potassa or soda repeated every four hours. To render it more grateful to the palate and stomach, eitric acid or lemon-juice may be added to each dose, which is to be taken during effervescence. This quantity, thus given, is generally well borne, and the urine, as a rule, is rendered alkaline in twenty-four hours. I have given, without inconvenience to the patient, the dose just stated, every two hours, and produced alkalinity of the urine in twelve hours. The quantity of the alkaline remedy should be much diminished as soon as the urine is alkaline, the doses afterward being repeated once or twice only in the twenty-four hours. To secure more diuretic effect than is produced by the alkaline remedy alone, the acetate, nitrate, or bitartrate of potassa, the acetate of ammonia, or small doses of colchicum, may be added. The tartrate of potassa and soda, which is much used as an alkaline remedy in this disease, is inferior to the bicarbonate of potassa or soda, because it must be given for a longer period before alkalinity of the urine is produced. It is well adapted to maintain an alkaline condition of the urine, after this condition has been produced by the biearbonate of potassa or soda. A writer in the London Lancet, Jan. 21, 1865, who had experienced repeated attacks of acute rheumatism, states that he has apparently arrested the progress of the disease by resorting promptly to an alkaline bath, prepared by dissolving in 24 gallons of water at $100^{\circ}$ Fahr. two pounds of the bicarbonate of potassa and one pound of the nitrate of potassa, the period of remaining in the bath being ten minutes.

The alkaline treatment does not preclude other therapentical measures which are apt to be employed with reference to the indications in individual cases. Bleeding, if ever advisable, is indicated only in eases in which the febrile movement is intense, and the patient plethoric. The tincture of aconite is useful as an arterial sedative and an anti-pyretic remedy, the temperature, as well as the frequency of the pulse, being reduced by it. If the thermometer denote an increase of temperature at, or above, $105^{\circ}$ or $104^{\circ} \mathrm{Fahr}$., cold water may be employed by means of sponging the body or the wet sheet. No harm, under these circumstances, need be apprehended from this measure, and, by diminishing the temperature of the body, its efficacy, as a palliative measure, to say the least, is marked. Full doses of quinia, if well tolerated, are useful. Continued purging is never indicated. It confliets with the alkaline treatment, and the necessity for frequent dejections occasions much suffering. Opiates often afford much relief, and should be given according to the amount of suffering from pain, restlessness, and vigilance. They are especially indicated at night. Diluents should be given freely. Carbonated water and lemonade may be allowed ab libitum so long as the stomach is not incommoded. Lemon-juice given in large quantity is one of the remedies for this disease in vogue within late years. It is, in fact, an alkaline remedy, but, taken in sufficient quantity to render the urine alkaline, the stomach often rebels against it, and the alkaline carbonates are to be preferred.

Tonies, and especially the preparations of cinchona, are useful. Nothing is gained by lowering the powers of the system. The diet should be nutritious. The appetite may be encouraged, and wholesome food allowed to the extent of the capacity for digestion. Remedies which compromise appetite and digestion are of doubtful utility. A moderate quantity of wine or spirit is sometimes advisable. In short, having brought the sys- 
tem under the effect of the alkaline treatment, and maintaining this effect, it is an object to preserve and restore the vital forees.

The affected joints claim local measures of treatment. The application of eloths saturated with an alkaline and opiate solution, as recommended by Fuller, is useful. Fuller's solution consists of from four to six drachms of the earbonate of soda or potassa, dissolved in a mixture of an ounce of Battley's solution (for which laudanum may be substituted), two ounces of glycerine and nine ounces of rose-water. 'The soap and opium liniment is a good local application. A liniment containing chloroform has been found useful. I have found the tincture of aconite applied to the parts to afford marked relief. Some practitioners prefer to envelop the joints with dry flannel or cotton wool. In the choice of local applications, the sensations of the patient may serve as a guide. Extension of the limbs by means of an apparatus for that purpose, or by simply a cord, pulley, and weight, in orter to relieve the pressure of the artieulated surfaces upon each other, has been tried to some extent at Bellevue Hospital, and found to afford in some cases marked relief.

Much relief is afforded, in some cases at least, by what may be termed methodic friction. Using some lubrieating emlurocation, the frietion at first over the affected joints, if they be extremely tender, must be as light as possible to aroid giving pain. The force of the friction, however, may be gradually increased, until, at length, the patient is sometimes able to bear without suffering as much pressure as can conveniently be made. Methodic friction, gradually inereased up to as much pressure with the hands as ean be borne without pain, leaves the affected joints more comfortable for several hours, and the operation may be repeated onee or twice daily.

The treatment of rheumatic endocarditis and pericarditis has been already considered. Pleuritis and pneumonitis are to be treated according to the indieations in individual eases, the same as when they occur in other pathological connections. The indieations pertaining to active delirium, convulsions, and coma are derived from the pathological conditions with which these events may be associated, viz., either cerebral meningitis or uræmia.

A plan of treatment in cases of acute rheumatism, advocated in 1850 , by a French physician, Dechilly, has reeently been revived by Dr. Herbert Davies, of London. 'The plin consists in the application of several large blisters near the affected joints. The blisters are to be applied simultaneously, and allowed to remain until they have acted thoroughly. The flow of serum is to be promoted after the removal of the blisters, by linseed-meal poultices. In one of the eases reported by Dr. Davies, it was ealeulated that the amount of surfice blistered was $296 \frac{1}{2}$ square inches. Dr. Davies claims that this local treatment should supersede other measures. He explains its supposed eflieacy by attributing to the blisters the power of eliminating the materies of the disease by means of the serum discharged. IIe states that the urine is rendered alkaline by the operation of the blisters. Of 13 hospital eases, treated after this plan, which are reported by Dr. Davies, the minimum cluration in hospital was 16 days, and the maximum duration 40 days. The average duration was a fraction under 26 days. The report does not contain data for determining the duration of the disease from the attack to convalescence. Of thirteen cases reported by mu, in which the disease was allowed to pursue its course, the minimum duration was 12 days, and the maxinum dura-

Vide Archives de Méd., Nov. 1865. 
tion 50 days, the average duration being a portion over 30 days. Comparing these statisties it is seen that the cases reported by Dr. Davies furnish very little evidence of the disease having been abridged by the treatment pursued. Dr. Davies claims for this plan of treatment that it has a curative effect on endocarditis if this complication have taken place before the treatment is commenced, and that it prevents the occurrence of inflammation of the structures of the heart. He states, that of 50 cases thus treated, in 27 there existed cardiac complication when the treatment was commenced, and in 23 there was no cardiac complication; of the 50 cases 25 were rlischarged frec from evidence of cardiac disease. The average duration of the disease in these 50 cases is not given. ${ }^{1}$

Dr. DaCosta has reported 30 cases treated with the bromide of ammonium in doses of from fifteen to twenty grains every three hours. Of three eases in 23 there was no cardiac complication when this treatment was commenced, and in none of the 23 cases was any cardiac complication subsequently developed. The mean duration of the disease was 22.5 days. $^{2}$ Dr. Charles E. Buckingham, of Boston, Mass., extols, as exerting a curative influence, a remely called by Trousseau the syrup of lime. Dr. Buckingham states that in his experience this remedy has proved more successful than any other." The doses given by him are from ten to forty-five drops, repeated after intervals of from two to six hours, according to the severity of the case and the age of the patient. The remedy should be given in unskimmed milk in quantity from a tablespoonful to four ounces according to the size of the doses. It should not be given in pill or dissolved in water. The urine is not rendered alkaline by this remedy. It is stated that the average duration in a dozen cases treated by Dr. Buckingham with this remedy alone was ten days."

\section{Subacnte and Chronic Articular Rheumatism.}

The term chronic rhemmatism is used in a comprehensive, indefinite sense embracing a variety of affections. It is commonly applied, not alone to cases which present the distinctive features of the disease just considered, save that acuteness of the symptoms is wanting, and there is a tendency to continue indefinitely, but to affections resembling articular rheumatism only in their situations, and to persisting pains in different parts of the body. 'There is confusion as regards the affections grouped under this name, and under the name rheumatic gout, which I shall not undertake to clear up in treating of these affections, and I shall therefore devote but a brief space to their consideration, referring the reader, for a fuller exposition of our present knowledge respecting them, to other works.

Articular rheumatism, in a subacute and chronic form, is frequently presented in medical practice. The local phenomena are, pain of less intensity than in the acute disease, and sometimes only felt during move-

1 The reports by Dr. Davies are contained in Vols. 1 and 2, of clinical lectures and reports by the Medical and Surgical staff of the London Hospital. London, 1864.

2 Vide Penn. Hospital Reports, Vol. 2, 1869.

3 Vide Boston Med. and Surg. Journal, February 28th, and April 4th and 11th, 1867.

- The formula for the preparation of this remedy is as follows: Two ounces of caustic lime mixed with eight ounces of sugar in a mortar. Pour over this mixture a pint (wine measure) of boiling water, and afterwards add enough more boiling water to make up the pint. The preparation is ready for use after it has been filtered. Vide Half-yearly Compendium of Mledical Science, Part I., January, 1868. Philadelphia. 
ments of the affected joints, tenderness on pressure comparatively slight, and more or less swelling of joints not imbelded in muscle. Increase of heat and erythematous redness are frequently wanting. More or less of the articulations may be affected. As in the acute form, different joints are liable to be attacked successively and irregularly. Not infrequently the disease, after a time, becomes concentrated, and remains fixed in a single joint. As in the acnte form, the fibrous tissues entering into ligaments, tendons, bursæ, and aponeuroses are liable to be affecterl. The liability of the joints of the ossicles within the ear to become affected with rheumatism was pointed out by Dr. T. H. Buckler, in 1853.'

As regards the general symptoms, there is little or no febrile movement and the different functions of the body may not appear to be much, if at all, disturbed. The appetite, digestion, and nutrition may be unaffected, and the local manifestations, in these cases, seem to be the only obstacle to the possèssion of health.

Different cases offer a great diversity in the grade of sererity of the discase, in its march, and in its remote consequences. In mild cases, paticnts are able to pursue their usual avocations, complaining only of more or less soreness and stiffness in moving the affected parts. Sometimes inconvenience is experienced only when movements are first made after a period of rest. Some patients experience inconvenience only after irregular intervals, and they are apt to suffer more or less with changes of the weather. In other cases, movements of the affected parts occasion great pain, and in severe cases patients are confined to the bed. Cases of confirmed, long-standing chronic rheumatism are met with occasionally in private practice, and all large hospitals furnish examples in which the disease has led to anchylosis of various joints, with great distortion of the limbs and atrophy of muscles, the patients presenting a truly pitiable spectacle of helplessness ; and yet, in some of these cases, the vital functions may be but little or not at all disturbed. Some writers consider the affection in these cases as a distinct variety of the disease, under the name, "deforming chronic rheumatism."

The constitutional affection is supposed to be the same in subacute and chronic articular rheumatism as in the acute form. A morbid principle in the blood is supposed to be involved in both forms. It is to be presumed that this principle is produced in less quantity when the disease is subacute and chronic, but it continues to be produced for a longer period, or it is reproduced after shorter intervals. In the cases in which anchylosis, distortions of the limbs, and atrophy of muscles have resulted from the long continuance of the disease, these results remain, although the rheumatic disease may not continue.

The chronic form of the disease sometimes follows the acute, but this is not the rule; in the majority of cases the disease is subacnte from the first. The disease in the subacute or chronic, as well as the acute, form involves a diathesis which may be either congenital or acquired. Of the causes which give rise to the diathesis, when it is acquired, and those which, co-operating with the predisposition to the disease, determine its occurrence and render it persistent, we have no positive knowledge.

The diagnosis of subacute and chronic articnlar rheumatism involves discrimination from chronic and rheumatic gout, the diagnostic characters of which will be considered in the next chapter. Subacute or chronic synovitis, not rheumatic, is to be excluded. The points to be considered

1 Vide On Fibro-bronchitis and Rheumatic Pneumonia, by Thomas H. Buckler, M.D., 1853. 
in differentiating the latter from rheumatic arthritis are, for the most part, those which have been stated in connection with the diagnosis of acute articular rheumatism.

A variety of synovitis affecting the knee-joint, and sometimes other joints, occurs in connection with gonorrhœa, and has been called gonorrhoal rheumatism. This is one of the instances in which the term rheumatism is misapplied. Jarwell and others consider this affection as belonging in the eategory of inflammation of joints dependent on purulent infection of the blood. The author just named applies to this pyæmic form of disease the name pyarthrosis. He attributes the pyæmia, in cases of so-called gonorrhœal rheumatism, to inflammation of the prostatic veins. A similar affection of the knee and other joints sometimes occurs after childbed, in consequence of uterine phlebitis.

Another misapplication of the term rheumatism is when syphilitic periostitis is called syphilitic rheumatism. For the consideration of this affection the reader is referred to works treating of syphilis. It may be stated that periostitis presenting all the characters of the affection as one of the tertiary effects of the syphilitic virus is occasionally observed in persons who have not been affected with syphilis.

Subacute articular rheumatism is not necessarily chronic. It may disappear within the limits of the duration of the acute form of the disease. The liability to endocarditis and pericarditis is much less than when the disease is acute, but these and other complications oceur in a certain proportion of cases. The sources of danger are in the liability to cardiac disease and the tendency to become chronic. Chronic articular rheumatism is not dangerous as regards an immediate tendency to a fatal termination. But in view of its continuance for an indefinite period or during life, and the permanent deformities to which it leads in some cases, it is to be considered as a disease of no small importance. The condition of patients in the cases in which it has led to lesions involving anchylosis, with wasting of the muscles, is hopeless as regards recovery from these effects of the disease, although the condition is not incompatible with comfortable general health and a long duration of life.

The treatment of subacute and chronic articular rheumatism embraces the employment of alkalies, but not to the same extent as in the acute form of the disease. They should not be continued steadily for a long period, but intermitted from time to time. The Vichy water is an agreeable alkaline remedy well suited to chronic cases. Alkalies are indicated only during the continuance of the arthritic inflammation; that is, they are not indicated by the effects remaining after the arthritic inflammation has ceased.

The preparations of guaiacum have long been considered useful in chronic rheumatism. 'Their value in certain cases is abundantly' established by clinical experience. Either the simple tincture, the ammoniated tincture, the mixture, or the compound decoction of sarsaparilla, into which guaiacum enters, may be employed. The bichloride or the iodide of mercury is strongly acivocated by Barwell, and in conjunction, inunction with the mercurial ointment over the affected joint or joints until the gums are slightly touched. Mercurial baths and fumigations are recommended by Récamier and Troussean. The iodide of potassium is sometimes highly useful. Colchicum exerts a good effect in some cases, given in small or moderate doses. Fuller regards the hydrochlorate of ammonia as "a remedy of singular efficacy," given in doses of from fifteen' to twenty grains, in combination with bark. The same author has found, in some cases, arsenic serviceable. The salts of quinia are not infrequently 
useful. Sulphur is another useful remedy. All these remedies are supposed to exert, in some way, a modifying, if not a curative, influence upon the disease. The opportunity is often offered of making trial of them in the same cases.

The relief of pain, in certain cases, calls for opiates. They should be employed with as much reserve as is consistent with due regard to the object to be obtained. Aconite will sometimes fulfil the object, and, if so, is to be preferred.

The local treatment is important. Benefit may sometimes be obtained from small flying blisters or the application of the tincture of iodine. Methodic friction or shampooing, as deseribed in connection with the local treatment of the acute form of the disease, conjoined with the use of stimulating embrocations, is often of much utility. Counter-irritation by "firing" has been recommended. Palliation of pain may frequently be procured by the application of the tincture of aconite or the veratria ointment. The local as well as general measures of treatment have reference to two objects, viz., the removal of, first, the inflammatory condition, and, second, the effects of the inflammation. In order to prevent stiffiness and anchylosis, passive motion is advisable as soon as the inflammatory condition subsides.

External measures acting upon the entire surface are, perhaps, in certain cases, not less useful than internal remedies. Reference is had to the simple tepid bath, the alkaline bath, the 'Turkish bath, the sulphur vapor bath, and hydropathic packing. The resourees of treatment should not be considered as exhausted without a fair trial of these measures.

Measures having reference to improvement of the general health and the invigoration of the system form an essential part of the treatment, embracing tonic remedies, with attention to diet, elothing, exereise, ete. It is unnecessary to go into details which are essentially the same in the treatment of most chronic affections. Change from a cold, changeable, and humid climate to one mild, uniform, and dry often proves the most effective measure which can be adopted. The mineral springs are frequently resorted to with advantage by persons suffering from chronic rheumatism. Of the different waters, the alkaline, sulphurous, saline, and chalybeate are each useful in certain cases. Doubtless, however, much of "the benefit derived from watering-places is due to the various incidental hygienic influences.

\section{Muscular Rheumatism.}

The affection to be now noticed is one of the many to which the application of the term rheumatism is, to say the least, of doubtful correctness. There is very little ground for supposing that the essential pathologieal condition, or conditions, existing in the affections which have been eonsidered in this chapter, obtain in so-called muscular rheumatism. This affection, so far as we can julge of its pathological charaeter, is allied to neuralgia ; it is probably a neuralgic affection, and, as such, may be properly ealled myalgia, and might with more intrinsic propriety have been considered under that head than in the present conneetion. I have placed it among the rheumatic affections, because it is, at the present time, and probably will continue to be, commonly known by the name museular rheumatism. Being one of the neuroses, it has, of course, no anatomical characters. It is of frequent occurrence, both as an acute and chronic affection.

The symptomatic features of acute muscular rheumatism are as follows : 
The development is usually gradual. A dull pain is at first felt in certain museles, which increases, and, at length, becomes more or less severe. The pain is comparatively slight when the affected muscles are at rest. In certain positions the patient may be nearly or quite free from suffering, the constant pain which may be felt under these circumstances being of a contnsive character, and spasmodic pains occurring from time to time; but in movements which involve contraction of the affected muscles, the pain is severe, cramp-like, and sometimes so excruciating that the patient can hardly repress loud groans or cries. Voluntary movements which occasion the severe pain are avoided as much as possible, but as it is impossible to maintain rigidly fixed positions of the body as a whole or of its different parts, the affeeted muscles are at times thrown into painful contractions, however much the patient may desire to avoid them. Movements occurring during sleep occasion the attacks of pain, and, hence, the patient is awakened at brief intervals. In some cases the affection changes its seat from eertain muscles to others, but oftener it remains fixed in the muscles first affeeted.

The affected muscles are more or less tender on pressure, the tenderness being diffused over the whole of the space which the muscles occupy, not limited to eertain points as in ordinary neuralgia. The tenderness is usually not great. A side frem the tenderness, there are no local characters, such as swelling, hent, or redness. The local phenomena are not those of inflammation, as in articular rheumatism. If the local symptoms denote inflammation of the muscles, the affeetion is not muscular rheumatism. Uniform firm pressure over the affected muscles is not only here borne, but affords relief in cases of muscular rheumatism.

There is little or no constitutional disturbance beyond that occasioned by the suffering and loss of sleep. The appetite and digestion may not be impaired, and there is no febrile movement.

In the subacute or chronie form of the affection, pain similar to that which belongs to the acute form may be excited only when the affected museles are contraeted with unusual force; the patient experiences suffering when he makes certain violent movements. In other cases, pain is excited by partieular movements when they are first made after a period of rest, and the pain shortly disappears if the movements be continued. The ehronic, oftener than the acute, affection is apt to shift its situation, affeeting now certain muscles, and now those in another part. If the chronic affection remain fixed in partieular muscles, they may become somewhat atrophied in consequence of their being kept as much as possible in a state of quietude.

The duration of acute muscular rheumatism varies from a few hours to a week. Exceptionally the affection continues for several weeks. The duration of the chronic form is inclefinite. It is often a very obstinate affection, persisting for many months. The course of the latter is usually marked by remissions or intermissions. In certain situations, viz., the loins, the walls of the ehest, and muscles of the seapulæ, the chronic affection is apt to be long persisting.

The following are the prineipal situations of muscular rheumatism in the acute and chronie form as enumerated by Valleix :

1. The Muscles of the Head.-Situated in the occipito-frontal muscle, the affection is distinguished from neuralgia affecting the trifacial and the oecipital nerve, by the pain being referred to both sides of the head, by its being excited by movements of the muscle, and comparatively slight 
so long as the muscle is at rest, and by the absence of tendemess in disseminated points. The muscles of the eye are sometimes the seat. Movements of the eye then occasion more or less severe pain. The temporal and the masseter muscle may be affected, causing pain in mastication.

2. The Muscles of the Neck.-In this situation the affection, as it commonly occurs, is known by the significant name rheumatic torticollis. This name is applied to the affection when situated in the muscles of the antero-lateral aspect of the neck. The museles on the posterior part of the neck are sometimes the seat of the affection. Valleix applies to the affection in the latter situation the name cervicodynia. In this situation the affection is to be distinguished from ordinary occipital neuralgia.

3. The Muscles of the Back.-Valleix calls the affection in this situation dorsodynia. Seated in these muscles, the pain is caused especially by movements which approximate the scapulæ.

4. The Muscles of the Loins.-In this situation the affection is commonly known as lumbago. Valleix designates it lumbodynia. This is a frequent seat of the affection in a chronic form. It is to be discriminated from lumbar abscess, lumbo-abdominal neuralgia, and the pains incident to aneurism of the descending aorta.

5. The Thoracic Muscles.-Here the affection goes by the name pleurodynia. In making the diagnosis, pleuritis and intercostal neuralgia are to be excluded. 'This may easily be done by ascertaining the absence of the diagnostic characters of the two latter affections. The pain excited by forced breathing, coughing, and sneezing is not unlike that in pleuritis and intercostal neuralgia. In this situation the pectoral and intercostal muscles are affected.

6. Muscles of the Shoulder.-Valleix entitles the affection here situated scapulodynia. It is seated especially in the deltoid muscles, and it is liable to be confounded with arthritis situated in the shoulder-joint.

7. The Muscles of the Extremities.-Seated in the extremities, the affection is to be discriminated from neuralgia affecting the nervous trunks, and from the affection commonly known as syphilitic rheumatism.

8. The Abdominal Muscles.-The pain and soreness caused by the affection in this situation may suggest as probable or possible the existence of peritonitis. This disease is to be excluded by the absence of the general symptoms, and by firm pressure with the palm of the hand being well borne, as in cases of dermalgia.

9. The Visceral Muscular Siructures.-The tongue, muscles of the pharynx and osophagus may be the seat of muscular rheumatism, giving rise to pain in speaking and in deglutition. Examples, however, are extremely rare. Seated in the stomach, intestines and uterus, as it probably may be, to distinguish the affection from visceral neuralgias is impossible, and in fact to consider it as distinct from the latter is to make a distinetion without any essential differenee. There is reason to believe that the diaphragm may be the seat of muscular rheumatism. It is, perhaps, more reasonable to consider this as the affection, rather than diaphragmatic pleuritis, in certain cases characterized by the instinctive arrest of diaphragmatic movements in consequence of pain, and by the intense suffering caused by coughing and sneezing, febrile movement and pleuritic effusion being wanting. It may be cloubted if the affection be ever seated in the muscular walls of the heart.

In all these situations the most marked diagnostic feature of muscular rheumatism is the connection of cramp-like pain more or less severe, with the movements which bring into play certain museles in the parts to which the pain is referred, pain, irrespective of contractions of the affected mus- 
cles, being comparatively slight and sometimes wanting. This diagnostic feature distinguishes muscular rheumatism, or myalgia, from neuralgic affections seated in the different nervous trunks. Another diagnostic feature is the diffusion of soreness over the affected muscles, whereas, neuralgia, seated in nervous trunks, is characterized by tenderness in circumscribed points. Inflammation affecting muscles is distinguished from muscular rheumatism by the constancy of pain or the absence of the remissions or intermissions which belong to the latter affection, by the existence of swelling and sometimes redness, by the greater degree of , tenderness on pressure, and by the presence of more or less symptomatic fever.

The treatment of the acute form of the affection consists of sinapisms, followed by soothing, emollient applications over the affected muscles, rest, in the position most favorable to avoid contractions of the affected muscles, and the employment of anodynes either internally or by hypodermic injection. In the chronic form, small blisters in succession are useful, but not in the same degree as in cases of ordinary neuralgia. The employment of the induced electrical current I have known to be beneficial in obstinate cases. The constant current has been found, also, highly efficacious. Friction or shampooing, either dry or with stimulating and anodyne liniments, affords relief and promotes a eure. "Firing" is recommended. The bromide of potassium and of ammonium I have found useful. The hydropathic treatment is said to prove successful in some obstinate cases, water being employed locally and generally. The salts of quinia, in full doses, are sometimes efficacious, especially when the affection is intermittent. Measures to invigorate the general health are important in chronic cases. If anæmia exist, preparations of iron should be continued for a long time, in small doses, or until the venous hum disappears from the neck. Anstie recommends, as especially useful, the muriate of ammonia in doses of from twenty to thirty grains.

Persons prone to this affection are apt to have on attack after exposure of a portion of the body to a current of air, especially during sleep, or when the body is perspiring. The susceptibility to this cause of disease is to be overcome by the daily use of the sponge bath, by avoiding an over-abundance of clothing, and by becoming habituated to out-of-door exercise in all kinds of weather.

\section{CHAPTER XI.}

Gout-Anatomical Characters-Clinical History-Pathological Character-Causation-Diagnosis-Prognosis-Treatment. Rheumatic Gout.

THF disease called gout is closely allied to articular rheumatism, and 1 the two affections have been considered by some to be identical. As will be seen, however, they are shown to be essentially distinct forms of disease by points of contrast pertaining to morbid anatomy, clinical history, pathological character, and causation. They are kindred diseases in the same sense as the different eruptive fevers, or typhus and typhoirl ferer, are related to each other; gout and rheumatism may be said to 
belong to the same family, but each has a separate individuality. "The name gout, or its synonym in different languages, is universally applied to the disease to be now considered. In its etymology the name relates to a characteristic feature of the disease, viz., a morbid deposit within and around the joints. Late researches lave tended to enhance the significance of the term by showing that the morbid deposit to which it relates is more constant than has heretofore been supposed.

Gout occurs as an acute, a subacute, and a chronic affection. Writers have made other varieties, such as irregular and misplaced gout. As the subacute form is not always chronic, it is convenient to consider this form when of brief duration and the acute form under one head. The term transient gout will embrace the acute and subacute form, the latter ending too soon to be considered as chronic. Persistent or chronic gout will embrace all cases in which the disease is protracted. It will suffice to consider irregular and misplaced gout incidentally in treating of the clinical history of transient and persistent gout.

Anatontcal Characters.-The anatomical characters distinetive of this disease consist of the presence within and around certain joints, as well as sometimes in other situations, of a morbid deposit ealled tophus or tophaceous matter. Other occasional morbid appearances appear to be incidental to the presence of this deposit, consisting of thickening, ulceration, and sometimes suppuration, being effects of inflammation excited by the presenee of the tophaceous deposit. These effects are by no means constant; they occur in only a certain proportion of cases. A trophy of structures from the pressure of masses of tophus is another effect.

The characteristic deposit is composed of the urate of soda in large proportion, combined with the urate of lime, chloride of sodium, plosphate of lime, carbonate of ammonia, and animal matter. When recent, the deposit is a semi-solid, cream-colored substance, resembling mortar. Under the microscope neelle-shaped crystals are seen in more or less abundance. After a time the deposit becomes hardened, and is then presented in masses which resemble chalk in appearance and are commonly, although erroneously, called chalky coneretions.

The occurrence of chalk-like concretions within and around joints in certain cases of chronic gout has long been known. The articular surfaces of joints opened after death liave been found incrusted with the tophaceous matter; the surrounding ligaments are sometimes conted by it; it may extend to the tendons and their slieaths, and along the tendinous expansions of the muscles. Accumulating in these situations in greater or less abundance, it leads to anchylosis of joints, nodosites, and deformations. Masses of this substance, or chalk-stones, as they have sometimes been called, in some cases cause atrophy and ulceration, thus reaching the surface and becoming exposed. Garrod's researches have shown that the tissues are infiltrated by the morbid matter, and that, when ulceration or erosion of parts occurs, it is secondary to the deposit. The interstitial deposit rarely extends to the osseous structure.' Suppuration is rare. Garrod's researches have also shown that, in cases of transient gout in which there is no persistent swelling or deformity of the affected joints, the characteristic deposit takes place to a greater or less extent. The inference to be drawn from his researches is, that the deposit invariably occurs in gout. This deposit within and around joints is peculiar to gout; that is, it does not occur in any other disease. The deposit in

1 Gout and Rheumatic Gout, second edition, London, 1863. 
masses, varying in size from that of a pin's head to a split pea, not infrequently occurs in the helix of the ear. It is also observed in the straight uriniferous tubes of the kidneys, giving rise to white lines visible to the naked eyc. Garrod is inclined to the opinion, that in the latter situation it occurs only in gouty subjects. It is, however, obscrved in certain cases of renal discase, in which during life the manifestations of gout were wanting. This statement I make from personal observation. I have repeatedly noted white lines in the direction of the straight tubes, and determined by means of the microscope the presence of urate of soda, or uric acid, in cases in which gont had not been manifested.

A form of diseased kidncy was considered by Todd as peculiar to gout, and called by him the gouty kidney. The characters belonging to the so-called gouty kidney are diminution of size, the organ appearing to be shrivelled, the capsules thickened and opaque, and the surface granular. As already stated, these appearances are sufficiently common in this country, disconnected from the phenomena of gout. In the cases in which the characteristic gonty deposit has been supposed to exist in the meninges of the brain, the lungs, and within the vascular system, it is probable that atheromatous and calcareous products have been mistaken for the deposit of gout.

Morbid changes other than those incidental to the tophaceous or gouty deposit are to be regarded as accidental, and are not to be included under the head of the anatomical characters of the disease.

Chinicar History.-Transient gout and persistent gout require separate consideration as regards their clinical history. Having considered these forms of the disease, I shall notice irregular and misplaced gout.

Transient Gout.-Under this head are to be cmbraced all cases in which the disease is of short duration, that is, the duration not sufficiently prolonged for the disease to be considered as chronic. In this form of the cliscase the patient is said to have an attack or fit of gout. The disease is generally acute, but sometimes subacute.

The seizure is generally sudden, occurring, as a rule, during the night, and, in the majority of cases, after midnight. In a certain proportion of cases the seizure occurs without premonitions; the patient may have retired to bed fceling as well as usual. But in some cases there are prodromic phenomena, consisting of cardialgia, gastric flatulency, eructations, and other dyspeptic ailments, together with inability to exert the mental faculties, irritability of mind, and depression of spirits. These phenomena are not sufliciently significant to lead to the expectation of an attack of gout if one or more attacks have not been already experienced; but in persons known to be subjects of the disease they may denote an impending seizurc.

The seizure is marked by pain which, in the great majority of cases, is seated in the metatarso-phalangeal joint of one of the great toes. The pain soon becomes more or less intense; frequently it is excruciating, being compared by patients to pain caused by the gnawing of an animal, a dislocation of the bone, a nail driven into the joint, tearing of the parts with pincers, etc. Such comparisons only express intensity of suffering, for patients have probably never experienced the pain produced by the various causes mentioned. A sense of throbbing in the part accompanies the pain. More or less febrile movement is developed, preceded by shivering. These symptoms continue for several hours, and then the pain and febrile movement subside, the patient is comparatively comfortable, and obtains some sleep. The relief is usually accompanied by 
slight perspiration. In the mean time the affected joint becomes swelled, the skin is reddened and shining, the subcutaneons veins are distended, the heat is raised, and there is exquisite tenderness to the tonch. These appearances are developed or continue after the severe pain has abated.

In some cases a single paroxysm of severe pain only is experienced, but much oftener the seizure is renewed on the following night, and on successive nights for a variable period. The local affection may remain in the joint affected at the first seizure, or the affection may be transferred from this joint to the corresponding joint of the opposite foot. In some cases the inner side of the foot, the instep, or heel become affected, and the affection may extend to the larger joints and to the small joints of the upper extremities. This extension of the local manifestations of the disease rarely occurs until after several attacks have been experienced, and it occurs more especially in cases of chronic gout.

During the continuance of transient gout the symptoms referable elsewhere than to the part or parts affected vary considerably in different cases. Febrile movement is more or less marked in proportion to the acuteness of the local manifestations. The ferer appears to be symptomatic, ancl, as a rule, is less than in cases of acute articular rheumatism. The appetite is frequently impaired or lost, but in some cases is preserved. Dyspeptic ailments are sometimes present and sometimes absent. The urine is generally scanty, high-colored, and deposits a lateritious sediment on cooling. The bowels are generally constipated.

Different cases also differ much as regards the local symptoms. The affection may be called acute when the pain is severe, and swelling, redness, etc., marked, these local symptoms being accompanied by a corresponding amount of febrile movement. On the other hand, the affection is subacute when the pain is notably less severe, the other local symptoms less marked, and febrile movement either slight or wanting. In respect of the seizures of pain, occurring usually in the night-time, they are in some cases paroxysmal, the pain passing off in the day, but in other cases more or less severity of pain continues during the day as well as night; in other words, remissions, rather than intermissions, occur. The local symptoms gradually decline as the fit or attack passes off. Edema of the part or parts is more or less marked at the decline of the local affection, with frequently pruritus, and, in the majority of eases, furfuraceous desquamation of the epidermis. The local symptoms just named are diagnostic of gont as contrasted with rheunatism. Cramp affecting the muscles of the leg, the thigh, and in other parts of the body, is a symptom more or less marked in a considerable number of cases.

The duration of transient gout varies from a few dlays to several weeks. In the majority of eases the paroxysms recur nightly for at least a week. Generally the paroxysms or exacerbations are less and less severe in proportion to the duration. If the disease continue beyond three or four weeks, it is to be considered as persistent or chronic. An attack of acute or subacute gout, more especially the latter, may eventuate in the persistent or chronic form of the disease, or, having continued for a variable period, the disease for the time ceases, recurring at longer or shorter intervals in the vast majority of cases.

Persistent or Chronic Gout.-Gout commencing as either an acute or subacute affection, in a certain proportion of cases continues as a subacute affection for many weeks, months, and even years. Generally this persisting or chronic form of the disease is preceded by the oceurrence of numerous transient attacks, recurring after intervals which become shorter and shorter, until, at length, as it were, they run together. Generally, 
when the clisease is persisting, it has not continuously an uniform severity, but remissions occur from time to time, and, not infrequently, intercurrent acute attacks take place.

In the persistent or chronic form, the local symptoms which characterize the acute affection, viz., intense pain, heat, and redness, are either slight or wanting. There is little or no febrile movement. Disorders of the digestive system are often present, and the general health is more or less impaired. In this form the joints are liable to become stiffened, anchylosed, and deformed, from the abundance of the gouty deposit. Chalk-like concretions are apt to occur within and around the small joints of the hands. In some cases collections of semi-solid matter are felt near the surface, and if they are opened, the matter may be pressed out. Sometimes collections of considerable size open spontaneously. 'The solid concretions, or chalk-stones, sometimes make their appearance through the skin. The gouty matter may be deposited in the bursæmucosa in the neighborhood of joints. In a certain proportion of cases, the number happily not being large, the hands and feet become notably and permanently distorted and crippled, the fingers sometimes presenting an appearance compared by Syclenham to a bunch of parsnips. Nodules of the deposit are frequently seen on the helix of the ear, sometimes also on the eyelids, and occasionally on the face.

In chronic, as in acute, gout, the predilection of the disease as regards the seat of the local manifestation, is for the joints of the great toe. An affection of other joints primarily occurs in a sinall proportion of cases. After the great toes, the order in which parts are liable to become implicated, according to Garrod, is as follows: The heels and ankles, the knees, the smallel articulations of the hands, the elbows, and lastly the shoulders, and hips. There are, however, numerous exceptions to this ortier of sequenee. A blow, sprain, or other injury may determine the seat of the local affection. A point of difference in different cases pertains to the number of joints which become affected. In some cases the local manifestations of the disease, however long it may persist or frequently recur, remain concentrated on a few joints ; in other cases a large number of joints are implicated, and, again, in some cases different joints are affected in successive attacks. If the same and a few joints be always affected, and the disease be long persisting or recur frequently, permanent changes in the affected joints are more likely to occur. Another point of difference in different cases relates to the palpable changes in the affected joints. Some gouty patients escape anchylosis and nodosities, although they suffer from the disease more or less throughout their lives, whereas, in other cases, the hands and feet become deformed and crippled after a comparatively short duration of the disease.

Irregular and Misplaced Goul.-Medical writers have long held the opinion that the local manifestations of gout are liable to be seated in the different visceral organs, viz., the stomach, intestines, lungs, heart, and brain. Disorders referable to these viscera, preceding the ordinary manifestations of gout, are considered as gonty, and it is supposed that gouty disorders of viscera may occur without any affections of the joints either accompanying or ensuing. In such cases gout is said to be irregular and misplaced. Anomalous and latent gout are other terms applied to these cases. Sometimes during an attack of gout, the affection of the joint or joints suddenly disappears, and symptoms denoting an affection of some internal organ supervene. In these cases a transference or metastasis of the gouty manifestations is supposed to take place, and the name retrocedent gout is employed to express such an occurrence. These notions 
are to a certain extent, probably, well-founded, but it is difficult with our present knowledge to say how far they are to be accepted as correct. The difficulty consists in the inability to determine whether antecedent, coexistent, or consecutive affections in certain cases of gout are not associated merely by coincidence. It has doubtless been too much the habit to consider all affections occurring in persons known to have gout, as pathologically of a gonty character, and the gouty diathesis is often considered to exist on insufficient data. Making, however, due allowance for error, it can scarcely be doubted that the constitutional morbid condition which constitutes the disease in cases of gout may determine disorders, sometimes of a grave character, in organs more important to life than those in which the local manifestations are usually seated. Evidence of a pathological connection of these disorders with gout is afforded by complete relief following the development of a gonty affection of the joints, by their oceurrence directly after a gouty affection of the joints has been apparently suppressed, by the absence of any other appreciable causes explaining their oceurrence, and by the indefinite or anomalous character of the disorders.

Disorders of the stomach and intestines, attributed to gout, are, neuralgic pain or spasm and persistent vomiting, accompanied witl more or less prostration, diarrhoa, and, in some cases, symptoms denoting enteritis. It is doubtful if inflammation of the stomach or intestinal canal be ever due exclusively to gout.

Disturbed action of the heart is not uncommon in gouty patients. It sometimes occurs in an alarming degree, but it may be doubted if a purely functional disturbance oceurring in this connection ever proves fatal. Valvular lesions and enlargement of the heart may exist in persons affected with gout, but clinical observation furnishes little or no evidence of the cardiac affections in these cases being dependent on gout. The want of a tendency to endocarditis and pericarditis is one of the points distinguishing gout from rheumatism.

Cough, asthmatic dyspnœa, and bronchitis are attributed to gout. Spasmodic affections of museles, or cramp, in various situations, neuralgia affecting the branches of the fifth pair, the sciatic nerve and other nervous trunks, paroxysmal headache and hysteria are to be added to the list.

Delirium and fatal coma are among the disorders imputed to gout. These symptoms may be due to uramia, or to meningitis, which may ol may not be dependent on gout.

The deposit of the urate of soda in the uriniferous tubes, in eases of gout, has been stated under the head of the morluid anatomy. It is not difficult to understand that this deposit may accumulate and form renal calculi. Clinical observation slows that gouty persons are prone to the formation of renal calculi and to paroxysms of pain caused by the passage of the stones along the ureter, or nephritic colic. The opinion of Todd and others that the contracted kidney may be due to gout has been referred to. However this may be, that renal disease is sometimes developed in persons subject to gout is undoubtedly true. The coexistence of renal disease, in certain cases, may account for the occurrence of some events which have been heretofore considered as being specially connected with gout, viz., vomiting and purging, headuche, neuralgia in different situations, delirium, convulsions, and coma. The existence of renal disease is to be ascertained by means of the urinary changes which have been already considered. A small amount of albumen in the urine is often observed when there are insufficient grounds for concluding that 
the kidneys are diseased; that is, slight albuminuria is incident to gout as well as to a host of other diseases. It is more especially incident to chronic gout.

Cystitis and urethritis have been attributed to gout, but the existence of any special pathological connection may be doubted. This statement is also applicable to certain affections of the skin and to various affections which occur in persons subject to gout. This is not saying that the existence of the gouty diathesis is not to be taken into account in the management of all associated affections. Graves has called attention to the habit of grinding the teeth as peculiar to eases of gout. The habit proceeds from an uneasy sensation in the teeth, which is in this way momentarily relieved. This habit was marked in a gouty patient under my observation.

Pathological Character.-The researches of Garrod have shed much light on the pathology of this disease. His researches appear to show that the pathology involves a particular morbid condition of the blood, viz., an accumulation of uric acid, or the condition which I have designated uricæmia. ${ }^{1}$ The blood in health contains a trace of uric acid, as was first shown by Garrod. In 47 cases of gout, an analysis of the bloodserum by Garrod showed a notable increase of this constituent. Garrod has also shown that, during an attack of acute gout, the uric acid in the urine is not increased but often notably diminished. It is apparently increased, since the deposit of the urates in more or less abundance, when the urine cools, is a common event; but the increase is only apparent, not real, the deposit being due to the scanty quantity of urine. In chronic gout the quantity of uric acid excreted by the kidneys is habitually below the average in health. Garrod has found the uric acid in excess in the blood in cases of lead-poisoning. Further researches are desirable with reference to the question whether it may not be found in excess in other affections.

From the facts just stated, taken in connection with the fact stated under the head of the morbid anatomy, viz., a deposit of the urate of soda taking place within or around the affected joints in all cases, it is rationally concluded that the local manifestations of gout are effeets of the uricæmia. But it is evident that the uricæmin is itself an effect; the prime essential pathological perversions underlie the existence of the morbid excess of uric acid in the blood. Either this excrementitions principle is produced in too great abundance, or, without being unduly produced, it accumulates as a result of insufficient excretion by the kidneys. Probably both explanations are involved. Direct observation, at all events, shows deficient excretion, since it is often less in amount in the urine than in health. Garrod entertains the opinion that the deposit of uric acid in the form of the urates of soda and lime takes place within and around the joints in consequence of diminished alkalinity of the blood. This opinion, if correct, has an important bearing on the treatment.

In treating of rheumatism, it has been stated that an examination of the blood, in that disease, with reference to the amount of uric acid, shows this principle not to be in excess. Here is a capital point of difference between gout and rheumatism, as regards pathological character. The clinical history of gout has afforded several striking points of contrast between the two diseases; these points will be reproduced under the head of diagnosis. Other points of contrast will be presented under the head

1 Vide Part I., page 86. 
of causation. The individuality of each disease is, therefore, sufficiently established.

Causation.-Gout is a diathetic disease; the diathesis in a certain proportion of cases is congenital and inherited. It is a matter of common observation that certain families are predisposed to this disease, and it is sometimes transmitted successively through a series of generations. Scudamore's statisties showed that of 523 patients affected with gout, in 309 the disease had existed in either the parents or grandparents. Of 80 cases reported to a Commission of the French Academy, in 34 the disease had been transmitted, and Garrod states that, in the cases which he has observed, a hereditary predisposition existed in 50 per cent. The diathesis, however, is by no means invariably inherited; in a certain proportion of cases it is acquired; nor, on the other hand, is the disease always transmitted to offspring. There are causes, then, which may produce it in those in whom there is no reason to suppose a congenital predisposition, and in those predisposed by inheritance other causes may be required for its production.

A causative influence relates to age; it occurs very rarely under puberty. Of 515 cases analyzed by Scudamore, with reference to age, in only 5 did the disease occur in persons under eighteen; in 142 cases, the ages at the time of the development or first occurrence of the disease were between 20 and 30 years; in 194 cases, between 30 and 40 ; in 118 cases, between 40 and 50 ; in 38 , betwen 50 and 60 ; and in only 10 eases, between 60 and 66 years. The liability thus diminishes progressively after 50, and the disease is as rare, if not more so, after 70 as before puberty. In the cases in which it commenees between 20 and 30 , it is generally inherited. In the causative influence relating to age is a point of contrast to rheumatism, the latter disease affecting by preference young subjects.

A very marked difference exists between the two sexes in the liability to gout. Of 80 cases submitted to the French Academy, 78 were men and only 2 women. Here is another point of difference from rheumatism, the latter occurling not less frequently in females than in males.

Dietetic and regimenal habits lead to the acquirement of the diathesis, and co-operate with a congenital predisposition in producing the disease. 'To the habitual use of wine or malt liquors the disease is, in many cases, chiefly attributable; on the other hand, the use of distilled spirits seems to exert no influence in the production of the disease. Gout prevails in countries in which wine and malt liquors are largely used, whereas in countries in which the use of spirits predominates the disease is comparatively rare. It is comparatively rare in Scotland, Russia, Poland, Denmark, and in this country, probably in a great measure for the reason just stated. It is proverbially prevalent among those of the opulent class of society who are addicted to luxurious habits. But donbtless the habitual indulgence in the pleasures of the table, exclusive of the use of fermented liquors, contributes largely in this class to the production of the disease. Physical indolence in this class also enters into the causation. The agency of malt liquors alone is strikingly shown by the frequent oceurrence of the disease among a class of laborers in London, employed to raise ballast from the bottom of the Thames, who are accustomed to drink from two to three gallons of porter daily. According to Budd, gout is quite common among this class. The lighter wines are less likely to produce the disease than the stronger varieties, viz., sherry, madeira, port, and the same holds true of malt liquors. There is no reason to suppose that eider tends to produce it. It is evident, from the facts just stated, that the 
causative agent in fermented liquors is not alcohol. According to the use of wine and malt liquors, to high living in other respects, and to indulgence in luxurious ease, a due causative influence, the disease is sometimes developed irrespective of these causes. Examples of the disease are occasionally met with among laborers accustomed to a plain diet, and neither wine nor beer drinkers. My clinical records contain notes of several such cases.

Varions causes may determine the occurrence of an attack of gout, that is, they may act as exciting canses. Attacks are sometimes attributable to prolonged intellectual exertion, anxiety of mind, bodily fatigue, exposure to coll, acts of excessive indulgence in eating or drinking, etc. But in not a few instances the disease occurs without any appreciable exciting canse. The disease is rarer in tropical than in temperate climates. Attacks are more likely to occur in the spring and autumn than at other seasons of the year. Garrod adduces facts which appear to show that impregnation of the system with lead is among the causative agencies. Of 51 male hospital patients, 16 were either painters, plumbers, or workers in lead. The fact that in cases of lead-poisoning the uric acid is found in excess in the blood has already been stated.

Diagnosis.-The difficulties in the way of the diagnosis of gont relates to its discrimination from rheumatism and the affection called rheumatic gout. The distinctive features of the latter will be presented in treating of that affection, and the differential diagnosis of gout and rheumatism only will be here considered. The points involved in this differential diagnosis embrace facts relating to anatomical characters, clinical history, pathological character, and causation, which have been presented under these respective heads. In proceeding to give a summary of the distinctive features of gout, as contrasted with rheumatism, it will be convenient to arrange them under the same heads.

Distinctive Features relating to Anatomical Characters.-Under this head, reference is had to the morbid changes within and around the affecter joints. A distinctive anatomical change is the deposit of a morbid product abounding in the urate of soda, the gouty or tophaceous matter, either in collections of a semi-solid consistence, or in hard concretions commonly called chalk-stones. These are never present in rheumatism, and are pathognomonic of gout. The deposit, however, in a situation and in sufficient quantity to be ascertained during life, occurs in only a certain proportion of cases, and chiefly in chronic gout.

Distinctive Features relating to Clinical History.-In the great majority of cases, the primary local affection is seated in the metatarso-phalangeal joint of one of the great toes. The larger joints are affected subsequently, if at all. The local manifestations tend to the smaller joints of the toes and hands. In acute cases, the pain is more intense than in acute rheumatism, and the tenderness greater. Pain occurs in paroxysms or in marked exacerbations. Wdema, pruritus, desquamation of the cuticlé, and enlargement of the veins, are distinctive of gout. Febrile movement is less marked than in acute rheumatism, and is apparently symptomatic, whereas in rheumatism the fever is apparently essential. Endocarditis and pericarditis are very rarely, if ever, developed in the course of the disease. The deposit of small eollections of the urate of soda on the helix of the external ear is of frequent occurrence in cases of gout, and will serve to establish the diaguosis. They should always be sought for. 
Distinctive Features relating to Pathological Character.-The uric acid in the blood is morbidly increased. This may be ascertained by obtaining serum either from a small quantity of blood, or by means of a small blister, and resorting to what Garrod calls the " uric acid thread experiment." The mode of performing this experiment, as described by Garrod, is as follows: "Take from one to two fluidrachms of the serum of the blood, and put it into a flattened glass dish or capsule ; to this arld ordinary strong acetic acid in the proportion of six minims to each fluidrachm of serum, which eauses the evolution of a few bubbles of gas. When the fluids are well mixed, introduce one or two ultimate fibres, about an inch in length, from a piece of unwashed linen fabric, which should be depressed by means of a small rod, as a probe or point of a pencil. The glass should then be set aside in a cool place, until the serum is quite set and almost dry. Should uric acid be present in the sernm above a small anıount, it will crystallize and during its crystallization will be attracted to the thread, and assume forms not unlike those presented by sugar-candy upon a string. To observe this, the glass containing the dried serium should be placed under a linear magnifying power of about fifty or sixty, procured with an inch object-glass and low eye-piece, or a single lons of one-sixth of an inch focus answers perfectly. To insure perfect suecess, the glasses should be broad and flat; the acetic acid should be neither very strong nor too weak; the glass should not be disturbed during the drying of the serum; the temperature should be that of an ordinary sitting-room, and the glass should be protected from dust."

The foregoing aceount is quoted for the convenience of reference by the practitioner who may find it impracticable to subject the blood-serum to clemical quantitative analysis. For a diagnosis the thread experiment, according to Garrod, suffices. Crystals of uric acid do not form and adhere to the thread in the blood-serum of health. 'The uric acid of the blood is not morbidly increased in rheumatism.

Distinctive Features Relating to Causation.-Gout occurs very rarely under puberty, and chiefly between thirty and fifty years of age. Rheumatism affects especially young subjects. Gout is oftener hereditary than rheumatism. It rarely affects women. The use of fermented liquors, and habits of luxury as regards diet and exercise, exert an agency in producing gout, not exerted to the same extent in the causation of rheumatism. The poorer classes are as liable to rhenmatism, if not more so, than the rich; the reverse is tine of gout.

To determine the existence of the diathesis prior to the arthritic manifestations of the disease, and to recognize the irregular or misplaced manifestations of gout, are objects of diagnosis involving not a little difficulty and doubt. Certain symptoms should excite strong suspicion of the constitutional gonty condition, if occurring within the periocls of life most favorable for the development of the disease, and in a person of a gouty family, and whose habits of life are calculated to co-operate with an innate predisposition. The symptoms referred to are those which belong to dyspepsia, with a scanty secretion of urine which deposits the urates, and the liability to gravel or attacks of nephritic colic. If, with these symptoms, an examination of the blood-serum shows a morbid exeess of uric acid, the development of an attack of gout may be predicted with considerable confidence. The diagnosis of disorders of intermal organs, imputed to gout, or to use a term which has been applied to these cases, of "visceral gout," must rest mainly on their occurrence

1 This account from Garrod's work is somewhat condensed by the author. 
in proximity to attacks of gout, either preceding, accompanying, or following the sudden cessation of well-marked gouty manifestations.

Prognosis.-The immediate danger to life from gout is slight. If it ever per se prove fatal, it is by its irregular or so-called misplaced manifestations; and in many, if not most, of the cases in which death is imputed to gouty affections of vital organs, it is probable, as alrearly intimated, that these affections do not strictly belong to gont, but are to be regarded as coexisting or intercurrent affections. It is, however, an error to suppose that gout is salutary as regards the duration of life. Frequently recurring attacks of transient gout, and the persistence of the disease in a chronic form, impair the constitutional powers, diminish the ability of the system to resist other diseases, and thus shorten life. It is a serious disease in certain chronic cases in which it leads to deformity and rigidity of the affected joints. It has been a favorite notion that the liability to gout lessens the liability to other diseases. There is little foundation for this notion, and, on the contrary, there is ground for the belief that, if gont do not tend to produce other diseases, it favors their inroads upon the system.

A tendency to recurrence is a law of the disease. It is extremely rare that a single attack only occurs. If life be not cut off by some other disease, frequent attacks are almost invariably experienced, and the disease may become chronic. The diathesis is thus rarely if ever extinguished.

Treatuent.-Various therapeutical measures heretofore considered as having a curative influence over gout are now rarely employed. Their disuse has resulted from the clinical study of their effects and improved pathological views. Among the measures referred to are embraced bloodletting, emetics, mercurialization, and continued or often-repeated purgation. It disposes of these measures to say that they are now generally regarded as contra-indicated in both the transient and persistent form of gout. Other measures, long more or less in vogue, are still prized in the treatment of this disease. In the latter list are colchicum and alkaline remedies. The indications for treatment during attacks of transient gout, during the intervals between these attacks, and in cases of persistent or chronic gout, respectively claim separate considerations.

Treatment during attacks of Transient Gout.- T'The great Sydenham, himself for more than thirty years a sufferer from this disease, was led to conclude that an attack should not be interfered with. Regarding it as an effort of nature to get rid of a noxious material, he believed it to be undesirable to arrest or abridge it. The propriety of non-interference is advocated by Trousseau. Few physicians, however, would feel satisfied to fold their hands and await the cessation of the disease, and still fewer patients would be content to forego measures to alleviate their sufferings. Nor is this line of conduct consistent with either reason or experience. Transient gout is a self-limited affection. It is probably rarely, if ever, arrested, and it might not be advisable to arrest it were this practicable. Perhaps its duration is not often abridged by therapeutical interference; but its severity may be lessened, and the suffering connected with it may be materially mitigated by measures which, if judiciously employed, will not do harm. This is a very mild expression of the resources of medicine in cases of transient gout.

Colchicum is an ancient remedy for gout, assuming the hermodactylus of the Grecian and A rabian physicians to have been this drug. It has been not less prized by the majority of physicians in modern times. Its 
special power of controlling the inflammatory phenomena of the disease is undoubted. The objection of some to its use on the score of its renciering the system more prone to a recurrence of the disease rests on insufficient ground, provided the remedy be judiciously employed. Our knowledge of the value of this remedy is derived wholly from experience. Its special mode of operation cannot be explained. A rational olject of treatment, derlueed from the pathological character of gout, so fir as at present known, is the elimination of uric acid; but the researches of Garrod go to show that this remedy does not increase the amount of uric acid excreted by the kidneys, nor does it in all cases increase the quantity of urine. It may be here alded that other rational objects of treatment are, prevention of an undue formation of uric acid or its undue accumulation in the blood, and neutralization of this acid, or keeping it in a state of solution in the blood until it is eliminated through some of the natural emunctories.

In an acute attack of gont, colehicum may be given at first in a tolerably full dose, that is, from half a drachm to a drachm of the wine, or an equivalent dose of the tincture or acetic extract. It should afterward be continued in small doses. It is not judicions ever to carry its use to the extent of producing vomiting or purging. Carried to this extent, it is objectionable on account of its depressing influence, although relief of the gouty inflammation be procured. Its special influence is independent of its operation as an emetic or a cathartic. An emetic of any kind is not indicated, except the attack occur shortly after a full meal and there be reason to suppose that the stomach is overloaded with undigested food. If constipation exist, a free evacuation by means of a saline purgative is desirable. A saline purgative is also appropriate as a depletory measure if the patient be robust or of a full habit, and the febrile movement be high. 'T'he object of purgation should be effected by salines rather than by the colchicum.

Alkaline remedies are rationally indicated with a view of promoting the solubility of uric acicl. For this end the salts of potassa are to be preferred to those of socla, the urate of potassa being more soluble than the urate of soda. Moreover, the salts of potassa have a diuretic action, and thus contribute to another rational object of treatment, viz., the elimination of uric acid by the kidneys. The bicarbonate of potassa may be selected, and the acetate and tartrate are appropriate. The alkaline remedy may be given in eonjunction with the colchicum. The phosphate of ammonia was introduced as a remedy in gout some years since by Dr. Buckler, of Baltimore, its eflicacy being supposed to consist in its forming a soluble salt readily eliminated, by the combination of uric acid and ammonia, the phosphoric acid combining with the soda and forming another soluble salt. Ten grains may be given three or four times daily. This remedy, however, is better suited to the ehronic form of the disease, or to the intervals between attacks of transient gout. This remark will also apply to the benzoic acid, a remedy introduced by Ure, and supposed to be useful by converting the uric into hippuric acid.

The carbonate of lithia, introduced as a remedy in cases of gout and gravel by Garrod, has proved to be of much value.' The urate of lithia is highly soluble, and the remedy, in small doses, acts efficiently as a diuretic. It is a remedy which produces no unpleasant consequences, although continued for a long time. It may be given in five-grain doses

1 This remedy was suggested as a remedy for gout, on theoretical grounds, by Dr. Buckler, in 18i3. Op cit. 
three times daily. An efficient and agreeable mode of administration is to give it largely diluted in carbonated water. 'The citrate of lithia has the alvantage of greater solubility in water than the carbonate. This, as well as other of the remedies given to favor the solubility of uric acid in the blood and promote its elimination, is to be considered as an adjunct to colchicum. My colleague, Prof. W. H. Van Buren, has employed the iodide of lithium as a local application to the affected joints with apparent adrantage.

During the paroxysms of pain, opiates may be given moderately, if well borne. If they occasion unpleasant effects, the succedanea of opium must be relied upon, viz., hyoscyamus, belladonna, and aconite. From its'action upon the skin the Dover's powder is an eligible form of opiate. The inconveniences of opium given by the month may sometimes be avoided by the hypodermic method of administration.

As regards local treatment, the affected joints may be covered with cotton, over which oiled silk should be applied. In addition, relief is sometimes afforded by anodyne applications containing opium, belladonna, or aconite, singly or combined. Chloroform as a local application has been recommended. Trousseau recommends highly tobacco fumigations, not during the paroxysms, but in the intermission, with a view to diminish the suffering when the pain returns.

The diet for one or two days should be considerably restricted, animal food being excluded. Should the attack continue, however, it is important in this, as in other diseases, to provide against the evils of innutrition. A nutritious but unstimulating diet should be directed, embracing milk, farinaceous articles, eggs, fowl, and fish.

The treatment after the attack, is to be considered under the head of the treatment in the intervals between the attacks.

Treatment in the intervals between attacks of Transient Gout.-T'The ends of treatment between the attacks are, the prevention of the recurrence of the disease, the prolongation of the intervals, and diminution of the severity of attacks. The immediate rational objects are, prevention of the undue production of uric acid, its elimination by the natural emunctories, and the promotion of its solubility in the blood. With reference to the two latter objects, it is to be borne in mind that the uric acid is probably accumulating in the blood for some time before gouty manifestations occur, and that probably the deposit of the urates within and around joints precedes, for a greater or less period, the inflammatory phenomena which belong to an attack or fit of the gout.

Removal of the known causes of gout is an essential part of the treatment. Wine and malt liquors, especially the stronger kinds, are to be interdicted. If the use of alcoholics be desirable or allowable, spirits should be substituted. Gin is to be preferred from its diuretic tendency. 'The diet should be plain, wholesome, and restricted to an amount required for nutrition. A certain amount of bodily exercise is an important part of the hygienic management. 'Travelling, a long sea-voyage, and a sojourn in a tropical climate, are in some cases advisable. All exciting causes are, as far as practicable, to be avoided.

If dyspeptic or other symptoms render it probable that an attack is impending, remedies with a view to prophylaxis are indicated. Alkalies should be given in small doses, largely diluted. They should be continued for a short time, then intermitted, and afterward resumed if the indications continue or return. The salts of potassa are to be preferred to those of soda. The carbonate of lithia may also be given. If the near approach of an attack be strongly suspected, colchicum in small doses may be re- 
sorted to. There is reason to believe that this remedy sometimes wards off' an impending attuck.

These remarks lave reference exclusively to the treatment direeted especially to gout. Disorders of any kind, not of a gouty character, of course claim appropriate treatment.

Treatment of Persistent or Chronic Gout.-The removal of causes which produce or promote the gouty diathesis, and of exciting causes, ranks first in importance. This is a sine qua non in the treatment. In addition, in obstinate cases, when circumstances permit, travelling, a seavoyage, and residence in a tropical climate, may be recommended as likely to effect a salutary constitutional change. Hygienic is doubtless rastly more important than medicinal treatment, but remedies are undoubtedly of much utility.

Alkalies, the carbonate of lithia, and other diuretics are inclicated precisely as in the intervals between attacks of transient gout, when the symptoms denote an impencling attack. It is important to bear in nind the injumction to give alkaline remedies in small closes, largely diluted, and to intermit their use after they have been continued for a short time. This injunction is also applicable to the carbonate of lithia and other diuretic remedies. Exacerbations of the discase, which are liable to occur from time to time, may be treated with colchicum in small doses, its effects upon the alimentary caual and circulation being carefully watched. Other remedies, of the value of which, in certain eases, there is abunclance of testimony, are the iodide of potassium, guaiacum, and the phosphate of ammonia. Perhaps the benzoic acid is to be added.

The diet should never be inalequate to nutrition, either as regards quantity, kind, or variety. A plain substantial diet is indicated. The borly should be well nourished, but, of course, highly seasoned dishes and indigestible articles are to be interdicted. Ripe fruits are not only generally allowable but useful.

To relieve stiffness and soreness of the joints, methodic friction or shampooing is highly useful, being careful that undue violence be not used. Small blisters, from time to time, are recommended. Collections of soft gouty matter or "gouty alscesses," as they have been called, if near the surface, may be punctured, and the creany matter gently pressed out. Occasionally ulceration follows, which is troublesome, but leading to no evil results. Deformities, norlosities, and anchylosis due to chalk-stones and struetural changes are effects of the disease which do not admit of removal.

The different mineral waters have been much resorted to in the treatment of gout, particularly the Vichy water. Of the utility of the latter and of other springs, there is much difference of opinion among those who have given more or less attention to an investigation of their effects. The Vichy water is alkaline, its alkalinity being due to the bicarbonate of soda. The imported and the artificial water are used to a considerable extent in this country. But springs which owe whatever therapeutical value they possess to other saline ingredients, such as the sulphate of soda or mannesia, the chloride of sodium or calcium, the chalybeate, and the sulphur springs, are severally advocated as useful in cases of gont. It is diflienlt to eliminate the beneficial influence of hygienic circumstances connected with a residence at watering-places, and thus arrive at a fair julgment of the therapentic value of the water. For the discussion of this matter the reader is referred to other works. I shall content myself with suljoining the rules which according to Garrorl, should guide the practi- 
tioner in advising or in prescribing the exhibition of mineral water in gouty cases:-

"They should be altogether prohibited when there is consideralle structural disease in any important organs, especially in the heart or kidneys ; and even when the organic mischief is slight, the greatest caution is necessary in their use. They should be avoided when an acute attack is either present or threatening.

"The waters should be selected according to the nature of the case. When the patient is robust and of a full habit, the alkaline saline springs; when torpidity of the bowels predominates, the purgative waters; when there is want of vascular action, the saline waters; when the skin is inactive, the sulphur springs; lastly, when debility prevails, then the more simple thermal waters should be chosen. In all cases the use of the waters should be cautiously commenced with, and care taken not to oppress the stomach by giving too much liquid, nor to induce debility or auy other injurious effects by allowing a toolong sojourn in the bath." 1

'The treatment of irregular and misplaced gont involves indications derived from the character and seat of the disorders, in other words, from the symptoms in individual cases. If gout have retroceded, local measures to solicit its return are indicated. For this end, warm and stimulating applications are to be made to the part or parts in which the regular manifestations were seated.

\section{Rheumatoid Arthritis-(Rheumatic Gout).}

The term rheumatic gout is much used by practitioners, but not uniformly in the same sense. It is not infrequently used to denote what the term itself might seem to imply, a combination of the two diseases, rheumatism and gout; this is an incorrect ise of the term. Rheumatism and gout rarely, if ever, occur conjointly. Properly used, the term relates to an affection which is neither rheumatism nor gout, although presenting certain of the characters of either or both of these diseases. The term is also, for 'convenience, applied to cases of either rheumatism or gout, in which the differential diagnosis is not distinctly made; this, of course, is an incorrect use of the term. The affection to which the name rheumatic gout is correctly applied has been otherwise designated by different writers. It was described by Haygarth under the name nodosity of the joints ; Adams, of Dublin, terms it chronic rheunatic arthritis. When seated in the hip, and occurring in the aged, it has been called morbus coxæ senilis. Garrod proposes to call it rheumatoid arthritis, and this seems to me an appropriate name.

This affection is either acute or chronic; generally it is presented as a chronic affection, and is subacute from the first. The anatomical characters which distinguish it from rhenmatism and gout are as follows: $\mathbf{A}$ larger accumulation within the affected joints of synovial liquid, which in the course of the disease is absorbed; absorption of the inter-articular cartilages, eburnation of the ends of the bones denuded of cartilage, and enlargement of the ends of the bones by the deposit of bony matter ; the formation of cartilaginous bodies, which either remain attached to the synovial membrane or are found loose in the cavity of the joint. With these changes is associated more or less thickening of the synovial membrane and ligaments. The ligaments are not infrequently elongated by

1 For a discussinn of mineral waters in general, in gout, and the relative value of different European springs, vide Garrod's work. 
prolonged distension from the synovial liquid, and complete or incomplete dislocations are apt to follow. The so-called chalky deposit of gout is wanting; but rheumatic gout may occur in persons who have this deposit as an effect of attacks of gout which have previously occurred. $\Lambda$ peculiar distortion of the fingers is characteristic of the affection under consideration, viz., an inclination to the ulnar or outer side of the hand. This peculiar clistortion is in some eases marked, existing equally in both hands.

In its acute form, especially when the larger joints are affecter, the affection bears a certain resemblance to acute articular rheumatism. It differs from the latter disease in the febrile movement being less marked, the absence of perspirations, and the greater enlargement of the joints from synovial liquid. The arthritic affection is less migratory and more persisting. There is no liability to pericardial or endocardial inflammation.

In its chronic form the febrile movement is slight. There is little constitutional disturbance connecterl with the affection. The affected joints are painful, especially on motion. There is little or no redness. The enlargement from synovial liquid is at first and for some time great; afterward follow appearanees and local symptoms corresponding to the anatomical changes which have been stated. When these ehanges have taken place, the use of the affected joints is more or less impaired or lost, and if numerous joints are affected the patient is permanently crippled. These cases are frequently ineluded under the name chronic rheumatism.

As already stated the affection is pathologically distinct from both rheumatism and gout. From rheumatism it differs in presenting more of the symptoms and history of a local arthritic affection, and it differs from gout in the absence of uricemia. It is donbtless a constitutional affection, but of its pathological character, aside from the local changes, we have no positive knowledge.

It occurs in persons of all ages, oftener in females than in males, and in all classes of society. Feeble persons are most likely to be attacked by it, but it is sometimes observed in those who had previously enjoyed health and vigor. According to Fuller, "it is remarkably prone to aflect the children of consumptive parents; it attacks the offspring of gouty or rheumatic persons whose health is impaired, and whose nerrous energy is exhausted by the labor and anxieties of business; it fixes upon the girl just arriving at puberty, in whom the uterine functions are ill performed; it invades the stiffening articulations of the woman who has arrived at that time of life which is marked by the cessation of the monthly periods; it shows itself cluring the state of debility which follows a misearriage or a difficult and protracted labor, more especially when accompanied by flooding; it is a frequent attendant upon renal disease, upon the eachexia produced by the syphilis, gonorrhora, or negleeted mereurial action, and it is a common sequel of over-long suckling, of excessive venery, of severe and long-continued mental exercise, and of mental distress and bodily exhaustion." It is difficult to say what amount of causative influence belongs respectively to the several circumstances just named, but the development of the affection when the general health is suffering from some depressing or debilitating cause or causes is a point having an important practical bearing.

The affection is to be discriminated from rhenmatism and gout. From chronic rheumatism the discrimination is not always easy. The diagnostic features are especially those relating to the inter-articular changes which have been stated, viz., the abundant effusion followed by norlosities, crepitus on motion from attrition of the bony surfaces clenuded of cartilage, dislocations, loose or attached cartilaginous growth, and the peculiar 
distortion of the hands. From acute rhematism it is discriminated by the absence of an essential rheumatic fever, of abundant perspirations, of cardiac complications; by the fixedness of the arthritic affection in the joints primarily attacked, and the tendency to become chronic. It is distinguished from gout by the larger joints being primarily affected in a certain proportion of cases; by the smaller joints of the hands being affected without a previons affection of the toes; by the absence of the paroxysms or exacerbations which are characteristic of gonty attacks, by the absence of the chalk-like deposit and of an excess of uric acid in the blood, and by young subjects and women being affected as often, or oftener, than males and middle-aged persons.

Although not involving immediate danger to life, rheumatoid arthritis is a very selious affection from its tendency to continuance and the injury of the affected joints which it occasions. In joints affected for a consiclerable period, permanent, incurable injury is almost sure to follow. Confined to the finger joints, the hands are sometimes rendered nearly useless. Affecting numerous joints, patients are crippled or bedridden as in hopeless cases of chronic rheumatism and gout.

The first and great object of treatment is the improvement of the general health. Tonic remedies, long continued and varied from time to time, in conjunction with a nutritious diet and other hygienic measures calculated to invigorate the system, are to be employed for this object. Remedies which appear to exert a special influence in cases of rheumatism and gont, viz., alkalies and colchicum, are not indicated in this affection. All depressing remedies are contra-indicated. Iodine in the form of either the iodide of iron or the iodide of potassium, guaiacum, and arsenic appear to be highly useful in certain cases. Cod-liver oil may be tried, in conjunction with tonics, if there be a tendency to waste. Chalybeates are especially indicative if the patient be anæmic. The alkaline mineral waters, and those which aet powerfully upon the bowels or kidneys, are hurtful. On the other hand, chalybeate and tonic waters may prove beneficial.

Local measures of treatment are important. Leeching or cupping and severe counter-irritation are certainly not indicated in the great majority of cases. Small blisters and the application of iodine will be likely to be useful if there be much effusion within the joints. Rest is indicated while much effusion exists or when the symptoms denote inflammation; afterward, passive motion, friction, and shampooing constitute, perhaps, the most important part of the treatment.

\section{CH A P T ER XII.}

Scorbutus, or Scurvy-Anatomical Characters-Clinical History-Pathological CharacterCausation - Diagnosis - Prognosis - Prevention - Treatment. Purpura Hemorrliagica. Hæmatophilia.

THIS concluding chapter will be devoted to three affections which, as regards symptomatic events, are closely allied to each other, viz., scorbutus, or scurvy, purpura hemorrhagica, and hamatophilia. 'The first of these affections is of interest and importance especially in view 
of the fact that our knowledge of its pathology and causation, although by no means complete, is sufficient to serve as a basis of eflective treatment, and has rendered it preventable.

\section{Scorbutus, or Scurvy.}

This disease appears to have been known to the ancients, but it began to prevail frequently and extensively when, with improvements in navigation, long voyages were undertaken, and hence it was known as sea scurvy. Iuring the last five or six centuries, it has been pre-eminently the disease destructive to life in armies, navies, and exploring or emigrating expeditions by land and water. Notwithstanding it has been rendered preventable by our knowledge of its pathology and causation, it has prevailed largely and proved greatly destructive to life within the present century. Hanmond states that at Council Bluff's, in 1820, nearly the entire garrison was attacked, and many died; and the efficiency of the United States forees in the Florida and the Mexican war was very materially lessened by its occurrence." It prevailed enormously in the English and French armies in the Crimean campaign. It contributerl not inconsiderably to the mortality of our armies in the late civil war. The inconsistency between these facts and the existing state of knowledge is in part to be explained by the inability always to secure the means of prevention in military operations; but it is in a greater measure to be accounted for by a censurable ignorance or neglect of these means.

Anatomical Characters.-The primary and essential lesions are seated in the blood. The following are the changes which have been ascertained: Diminution of the solid constituents or a relative increase of water, involving, of course, diminished density of the blood ; the proportion of red corpuseles notably diminished; the fibrine augmented, but with more difficulty isolated from the red corpuscles than in health; the albumen diminished, and requiring for its coagulation a greater amount of heat than in health; the inorganic constituents diminished, ehiefly from the reduction in quantity of potassa, lime, and iron. These changes have been ascertained by the researches of Hammond, Becquerel, Rodier, and others. Most, if not all, of them occur in other pathological connections, and are not, therefore, peculiar to scurvy. Doubtless there exist other changes not yet ascertained.

Extravasations of blood are found in the areolar tissue beneath the skin, where they are apparent before death, and also in other situations, viz., between the fibres of the muscles, on the periosteum, into the lungs and other organs, beneath the serous memblanes, and sometimes within serous cavities. The extravasations are evidently secondary to, and dependent upon, the morbid blood-changes. The mode, however, in which the latter give rise to the former is not clearly understood. Fither the blood is so altered that it escapes from the vessels; or, the nutrition of the walls of the vessels is impaired so that they do not offer adequate resistance to the pressure of the blood; or, again, paralysis affecting the vaso-motor nerves may be a pathological element. It is not impossible that each of these conditions enters into the mechanism. The spleen is enlarged and softened.

I Report on Scurry, United States Sanitary Commission, by William A. Hammond, M.D., 1862. 
Clrnical History.-The symptomatic events which are characteristic of the disease are preceded by general weakness and lassitude. Patients are listless, apathetic, and indisposed to either mental or physical exertion. The appetite fails, and pallor of the surface is marked. 'These symptoms progressively increase. The more characteristic events are swelling and sponginess of the gums, which bleed either spontaneously or on slight pressure, hemorrhage from the mucous surface in other situations, and extravasation of blood within and beneath the skin. The fungous apyearance of the gums is chiefly marked around the teeth; it is less marked in situations where the teeth are wanting. The teeth are apt to become loose and sometimes either fall out or may be easily removed. The surface of the lips is notably pale, presenting a striking contrast to the redness of the gums. Hemorihages are frequent from the different outlets, viz., nostrils, mouth, bronchial tubes, vagina, and intestinal canal. The ecchymoses apparent on the cutaneous surface are either in the form of patechiæ, vibices, or patches of variable size; generally these several forms are conjoined. 'They are either limited to, or most abundant on, the trunk and lower extremities. The ecchymoses undergo the same successive alterations in color as when due to contusion. A slight bruise suffices to produce them in any part of the surface of the body. Fetor of the breath is usually a marked symptom.

The skin is dry and rough, compared by Larrey to that of a plucked fowl. The ankles are often œdematous, and some œdema of the face is not infrequent. The patient complains of pain in the loins and in the extremities, more especially the lower limbs. The muscles of the lower limbs are swollen and hard. Palpitation and $d y$ spnœa are excited by slight exertion. Pain referred to the chest is not uncommon. The bowels are in some cases constipated, and in other cases loose, or the latter may succeed the former in the progress of the disease in the same case. The urine is high-colored and speedily becomes fetid. There is absence of febrile movement. The pulse is sometimes slower than in health, but it may become rapid on any exertion. It is always soft and compressible. The temperature of the body is somewhat lowered. The spirits are depressed. The patient frequently longs for fresh vegetables and fruit.

These symptoms are more or less marked. In extreme cases, or in an advanced stage of the disease, the prostration is great. Slight exertion occasions syncope. The patient may fall into a state of collapse. This is apt to follow abundant hemorrhage. Old ulcers and cicatrices sometimes reopen, and bones which had been fractured and united may become separated. Ulcerations are liable to occur within the mouth and over the ecchymoses on the skin. Weakness of vision, day blindness, and night blindness are not uncommon. Inability to sleep is common, but generally the mental faculties, although weakened, remain intact. In cases which are comparatively mild, general debility, mental depression, pain in the back and lower limbs, together with the other symptoms, are more or less marked, but not excessive; and associated with these symptoms are spongy swelling of the gums and ecchymoses beneath the skin.

Scorbutus may exist as the sole affection, but it is frequently combined with other diseases. It is apt to be combined with dysentery, and the latter affection is then called scorbutic dysentery. It may coexist with typhus and typhoid fever. It may be accidentally associated with a variety of diseases which are liable to mask the scorbutic affection. The recognition of its coexistence with other diseases is of vast importance with reference to appropriate treatment. 
Pathological Character.-The essential characteristic blood-change in this affection is not as yet satisfactorily determined. There is donbtless a deficiency as regards certain of the constituents of the blood, but the particular deficiency which belongs specially to the affection is a matter of question. Garrod considers that the special deficiency relates to the salts of potassa. That this deficiency enters into the pathological condition is certain, but that it constitues the pathological condition exclusively or chiefly, in all cases, may be doubted, in view of the fact that supplying the salts of potassa, although highly useful, is not always the most effective method of treatment. With our present knowledge we must be content in saying that, owing to incomplete nutritive supplies, the constitution of the blood is impaired, giving rise to certain ascertained morbid changes common to this and other affections, and to other changes, not yet definitively ascertained, which are peculiar to this affection.

Causation.-Scurvy is a dietetic disease; it is the broadest expression of the causation to say that the discase is due to the want of certain alimentary supplies. A less comprehensive, but probably correct, statement is to say that the discase is caused by an insufficiency of nutritive principles contained in vegetable food. For a long time the disease was attributed to the free use of salted meat ; it is, however, certain, that the discase is not produced by this diet, except in so far as it displaces other articles of food containing principles necessary for the normal constitution of the blood. Scurvy has repeatedly been developed when the diet has been too much restrieted to fresh meat. Garrod's theory of the pathological character of the disease involves the conclusion that it is attributable to a diet wanting the requisite quantity of the salts of potassa, these salts being abundant in vegetable food. Admitting a defieient supply of the salts of potassa to be an important element in the causation, other elements are probably involved. The dietetic principles, the deficiency of which causes this disease, are abundant in various articles of food distinguished as anti-scorbutic, experience having shown that they prevent the development of the disease. The anti-scorbutics will be presently referred to.

Although causes relating to diet are essential to the production of the disease, other causes act powerfully as auxiliaries. A mong the auxiliary causes are, exposure to cold and wet, deficient ventilation, and depressing moral influences. The disease is oftener developed during the winter than during the summer. I have known an instance in which the disease appeared to be produced by great and prolonged mental depression, but probably in this instance, from an utter indifference to food, dietetic canses were involved. The disease is oftener developed in the aged than in young persons; the causes of the disease will act more speedily in persons in whom the constitution has been impaired by previous disease, by injurious medication, and by excesses of any kind. There is reason to believe that the morbid condition of the blood which exists in scurvy is not infrequently produced, to an extent falling short of that requisite to give rise to the striking manifestations of the disease, by an undue restriction of the diet to a few articles of food either from poverty or false notions of dietetics. It is important for the physician to recognize this fact in medical practice.

Diagnosis.-In view of the striking symptomatic features of this disease, and of the fact that generally a greater or less number of persons become affected under circumstances which sufficiently account for its 
development, the diagnosis is easy provided the characters of the disease be marked. In isolated cases the diagnosis involves discrimination from purpura hemorrhagica. This differential diagnosis will be considered in treating of the affection just named.

But the morbid condition of the blood peculiar to scurvy may exist to a greater or less extent when the most striking symptomatic features of the disease are not present, viz., the subcutaneous ecchymoses and hemorrhages from mucous ontlets. Under these circumstances the disease may be overlooked. 'Thus, Hammond, Woodward, and others state that in cases called in army sick-reports cases of general debility, the disease is, not infrequently, incipient or not fully developed scorbutus. The pain in . the back and muscles of the lower limbs is apt to lead the practitioner for a time to consider the disease as muscular rheumatism or myalgia. When associated with other diseases, such as dysentery and the essential fevers, it is liable to be overlooked. 'The appearance of the gums is highly important with reference to the diagnosis, in the cases in which petechixe or vibices are wanting; and when, from the appearance of the gums and other symptoms, the scorbutic condition is suspected, an investigation of the diet may lead to the knowledge of facts which will teud to settle the diagnosis. This investigation is important, not only in army or naval practice, and in public institutions, but in private cases in which the symptoms point to scurvy.

Prognosis.-In cases of scurvy in which ecchymoses are numerous and large, or in which hemorrhage from the mucous ontlets is profuse, the canger to life is great. Life may be destroyed by effusion of blood into the serous eavities or the parenchyma of vital organs. Sudden death sometimes occurs, irrespective of the circumstances just named, from syncope induced by muscular exertion. If the disease be not properly managed, and the causes which have produced it continue in operation, it proves fatal in a large proportion of cases. Before the pathology and causation were as well understood as now, the mortality from this disease among soldiers and seamen was often very large. According to the testimony of Hamilton' and others, this disease contributed, directly and indirectly, to the loss of very many lives during the late civil war. Associated with other diseases, it contributes indirectly to their fatal termination, and it stands in the way of recovery from wounds and surgical operations which otherwise would not prove serious.

Promptly recognized and judiciously treated, the disease may be expected to end in recovery in a pretty large proportion of cases. It has no definite duration. It continues for a longer or shorter period, according to the extent to which it has advanced, the amount and situations of hemorrhagic extravasation, the previous condition of the patient, etc. The recovery is usually gradual, not infrequently tedious, and the system is apt to remain for a long time debilitated.

Prevention.-The prevention of scurvy is to be considered under a distinct head. This disease may be prevented by a diet embracing the

1 For an interesting and instructive account of scurvy as occurring in the armies of the United States in the late rebellion, the reader is referred to the Treatise on Military Surgery and Hygiene, by Frank Hastings Hamilton, M.D., late Lieutenant-Colonel, Medical Inspector U. S. A., Prof. of the Practice of Surgery, with operations, in the Bellevue Hospital Med. College, etc. New York, 1865. Also, to article on "Scurvy in its Medical Aspect," by Sanford B. Hunt, M. D., in Sanitary Memoirs of the War (Medical volume), published by the United States Sanitary Commission. 
requisite variety of alimentary principles, and this is secured by a proper proportion of animal and vegetable food. With the knowledge of this fact and clue attention to its practical application, scurvy should never oceur except under circumstanees which render the means of its prevention unavailable. Its occurrenee is always an occasion for reproacli when artieles of food containing adequate supplies for the healthful eonstitution of the blood can be obtained. It is chiefly in military campaigns and long sea-voyages that there are difficulties in the way of prevention. These difficulties, however, may generally be met by provicling antiscorbutic articles of diet; and, with reference to these difficulties, the knowledge of the numerous artieles which may furnish the needed alimentary princip!es is of vast importance.

Lemon or lime juice has long been known as a most valuable antiscorbutic. Experience has abundantly shown that from one to two ounces per diem of the fiesh juice will prove an effectual preventive against scurvy during very long voyages withont fresh provisions. Orange-juice is also an effectual antiseorbutic. Pure eitric acid, although it appears to be.useful, is not a substitute for the fresh juice of the fruit. One of the most efficient of antiscorbutic vegetables is the potato. It is most efficient when eaten raw. Pickles, onions, raw eabbage or sauerkraut, the water-cress, and other of the cruciferx, are to be reckoned among the valuble antiscorbutic vegetables. Green corn, green apples, and most of the eseulent fruits are preventives. They are more so unripe than ripe. Numerous vegetables which do not enter mueh into ordinary diet may often be used as antiscorbutics in military campaigns when the articles which have been named are not to be obtained. Among the vegetables now referred to, which experience has shown to be effectual in the prevention of scurvy, are the sorrel (rumex acetosella), lamb's quarter (chenopodium album, the bulb of the wild artichoke, a species of cactus known as the agave Americana or maguey, indigenous in Texas, Califormia, and Mexico, the prickly pear (cactus opuntii), and the dandelion (ieontodon taraxacum). ${ }^{1}$

That the antiscorbutie virtue of certain vegetables and fruits depends, to a greater or less extent, on the presence of the salts of potassa is altogether probable. Evidence of this is afforded by the fact that the isolated potash salts are useful in preventing the disease. Hammond recommends the bitartrate of potassa in the following terms: "An ounce taken daily when men are so situated as to render them liable to scurvy, would, we are confident, entirely prevent it." It also is recommended by Hamilton in strong terms.

Finally, in addition to dietetic means, attention to ventilation, cleanliness, avoidance of unnecessary exposure and physical exertions, and to measures calculated to produce cheerfulness, constitutes an important part of the prophylaxis.

Treatment.-The dietetic means of prevention constitute an essential part of the treatment. The juice of lemon, orange, and other fruits should be taken freely, but, of course, not to such an extent as to produce disorder of the stomach. The antiscorbutic vegetables and fruits should enter into the diet in so far as the digestive powers permit, in conjunction with ineat, milk, and farinaceous food. Remedies to improve the appetite and strengthen digestion are indicated, viz., qninia, the bitter infusions or tinctures, and chalybeate preparations. The salts of potassa are use-

' Vide Hamilton and Hammond, op. cit. 
ful. The bitartrate may be selected or the chlorate. The latter has been employed, apparently with advantage, in cases treated at the Blackwell's Island Hospital. Wine or spirit, in small quantities, if they produce an agreeable cordial effect, will be useful. In short, the chief objects of treatment are, the assimilation of alimentary principles needed for the healthy constitution of the blood, and the invigoration of the system.

Particular symptoms or disorders claim appropriate measures in addition to those having reference to the objects just stated. Constipation is to be relieved by mild laxatives or simple enemas, and, on the other hand, diarrhoa, by anodyne and astringent remedies. The dryness of the skin indicates sponging with tepid water or the tepid bath. Pain in the back and limbs may be alleviated by friction with anodyne and mildly stimulating liniments. Stiffness of the joints, which sometimes occurs, is to be prevented or removed by passive movements. The condition of the mouth calls for astringent collutories. Ulcerations in the mouth or on the skin are to be treated locally on general principles. Hemorrhage from the different outlets is to be restriained by hemostatic remedies.

It is hardly necessary to add that the scorbutic condition, when associated with dysentery, typhus or typhoid fever, and any disease, claims the same dietetic and other measures of treatment as scorbutus existing alone.

\section{Purpura Simplex. Purpura Hemorrhagica.}

The term purpura, or the purples, denotes an affection characterized by a truly petechial eruption, or petechiæ. This affection is included among the cutaneous diseases, and is treated of in works devoted to these diseases. A truly petechial eruption consists of small spots, or maculæ, sometimes a mere point, and then called stigmata, the color either being at first or becoming purple or livid, and not disappearing under pressure. The spots are clue to minute extravasations of blood; they are small ecchymoses, and are frequently associated with vibices and still larger extravasations beneath the skin. True petechice are to be distinguished from the characteristic eruption of typhus fever, which is often called petechial.

Petechiæ occur in the affection which has just been considered, viz., scorbutus, associated with vibices and ecchymoses of variable size and form. They occur not infrequently in other diseases, for example, epidemic cerebro-spinal meningitis, typhus, and typhoid fever. They may be accidentally present in the course of any disease. Disconnected from any other affection, and not accompanied by hemorrhage elsewhere than beneath or within the skin, they characterize the variety of purpura called purpura simplex. Accompanied with hemorrhage elsewhere than beneath or within the skin, especially from mucous surfaces, and with graver symptoms than the variety just named, the affection is known as purpura hemorrhagica. As a distinct affection it was first described in 1735, by Werlhof, under the name morbus hæmorrhagicus maculosus, and it has sometimes been called Werlhof's disease. ${ }^{1}$

Purpura hemorrhagica presents hemorrhagic phenomena identical with those which belong to scorbutus. The petechiæ, vibices, and larger ecchymoses apparent on the surface of the body are the same. Extravasation in both affections is liable to occur beneath mucous and serous membranes, into serous cavities, and within the parenchyma of organs. Hemorrhage

I For an analysis of 199 cases of purpura hemorrhagica, vide Pathologie Interne par E. Gintrac, tome troisieme, 1853. 
from the different outlets occurs in both affections. The two affections are by some considered as essentially identical. Our knowledge of their pathological character is not sufficient to base thereon a positive statement concerning their identity or non-identity, but there seems to be suflicient ground for considering them as nosologically distinet affections. With reference to clinical history, causation, and diagnosis, it will suffice to notice the points in which the purpura hemorrhágica differs from seorbutus.

In purpurn there is not that fungus-like swelling of the gums which is a characteristic feature of scorbutus. The gums are either unaffected or but slightly affected. Purpura is not, like scorbutus, distinctly traceable to an insufficient supply of alimentary principles contained in vegetable food. It is met with in isolated cases, whereas, the causes of scorbutus generally affecting masses, the latter affection usually prevails as if it were an endemic. Isolated cases of scorbutus, however, are sometimes observed. It is stated that cases of purpura are most apt to oecur in the summer and autumn, while scorbutus prevails especially in the winter or spring months. There is less depression of the vital powers in purpura than in scorbutus. Swelling of the muscles and stiffiness of the joints are less likely to occur in purpura. Finally, the dietetic treatment which has a special efficacy in seorbutus, is not to the same extent curative in purpura. According full foree to these points of differenee, it must be admitted that they do not establish conclusively the non-identity of purpura and scorbutus, nor do they always suflice to enable the practitioner to make with positiveness the differential diagnosis. The elucidation of the pathologieal relations of purpura to scorbutus requires further study of the changes which the blood undergoes, together with the clinical history and causation. In some cases of purpura simplex, the disease seems to be essentially a febrile disease, the pulse and temperature denoting fever, with anorexia, prostration, ete. Pain in the back, limbs, and joints is a prominent symptom in some of these cases. Cases of this deseription are included under the name purpura simplex, because the eruptive and the continued fevers may be excluded, together with cerebro-spinal meningitis, and other affections in the course of which purpura is liable to oceur as an incidental event.

With or without extravasation from mucous surfaces, the pathological conditions, standing in immediate relation to the hemorrhage, as in cases of scorbutus, relate to an abnormal weakness of the capillary ressels, to morbid blood-changes, or to vaso-motor paralysis, these different conditions being perhaps combined. It has been conjectured that the hemorrhage may depend on multiple capillary embolism.

Simple purpura, when it is the characteristic of a distinct affection, and when it occurs in connection with various diseases, does not in general denote a grave condition. But purpura hemorrhagica involves danger from the same sources as scorbutus, viz., loss of blood from the different outlets of the body and extravasation into serous cavities, the brain, lungs, etc. Exclusive of these sources of danger, recovery, under judieious treatment, may be expected. Occurring in connection with some other important diseases, it adds greatly to the danger from the latter.

The objects of treatment in cases of purpura hemorrhagica are, the restoration of the normal constitution of the bloorl, increase of the vital powers, and arrest or restraint of hemorrhage. With reference to these objects, the diet should be nutritious and varied, the appetite and digestion are to be improved by tonic remedies, together with wine or spirits in small quantity if the immediate effect be good. Of tonic remedies, the dilute sulphuric acid is especially to be recommended. This remedy has the 
advantage of being agreeable to the taste, if added to a proper quantity of water and syrup. It may be given in doses of from five to fifteen drops every two hours. Astringent remedies are to be given with reference to the hemorrhage. The gallic acid and the persulphate or pernitrate of iron have becn employed successfully as hemostatics in this affection. In view of the difficulty of always discriminating purpura from scorbutus, and of there being ground for supposing that the same morbid condition of the blood exists in both affections, the treatment should embrace the dietetic and other measures indicated in cases of scorbutus. The oil of turpentine has been recommended as a valuable remedy in this affection. According to a German author, Henock, ergot, in large doses, is useful in arresting or restraining the hemol'rhage, and he cites this fact in support of the hypothesis that paralysis of the vaso-motor nerves is a pathological element. ${ }^{2}$

\section{Hæmatophilia, or a Hemorrhagic Diathesis.}

A remarkable liability to hemorrhages exists in some persons as a constitutional peculiarity. A congenital hemorrhagic diathesis is sometimes manifested directly after birth by bleeding from the umbilieus, with difficulty restrained, and death often taking place from the loss of blood. The diathesis is generally manifested during, or shortly after, dentition; and is shown by profuse bleeding from slight wounds. Persons having this diathesis in a marked degree continue to lose blood from an insignificant wound, all efforts to arrest the hemorrhage proving ineffectual, until syncope occurs, which may prove fatal; and, if not fatal, the loss of blood induces a notable degree of anæmia. The hemorrhagic diathesis also leads to extensive extravasations within and beneath the integunent, resulting from slight contusions. Hemorrhage, not trammatic, is apt to occur in persons with this diathesis, especially taking place from the nostrils, but sometimes from the bronchial tubes, the stomach, intestinal canal, and the kidneys. Children affected in a marked degree with this diathesis rarcly live to adult age; but in some instances the diathesis diminishes or ceases after childhood; and if it continue in a moderate or slight degree, it may not interfere with good health and longerity. The diathesis is often hereditary, and is sometimes perpetuated in several successive generations, being confined to certain members of a family. Males are affected oftener than females.

The hemorrhagic diathesis probably involves either a deficiency of fibrin in the blood, or a deficient power of coagulation. Two analyses of the blood, in a fatal case, made by M. de Fleury, showed the proportion of fibrin to be 2.640 in 1000 parts $;^{2}$ these results showing that a deficiency of this constituent of the blood does not always exist. Our present knowledge does not enable us to explain why either of the pathological conditions just named exist as a constitutional peculiarity, nor to say what other morbid changes are associated.

The treatment having reference to the diathesis consists of measures to promote assimilation and invigorate the system, namely, a nutritious diet, out-of-door life, and tonic remedies. By these measures we may hope to aid in removing the diathesis. Wounds are, of course, as far as

1 Vide Jaccoud, op. cit.

2 Mémoires et Bulletins de la Snciété Médico-Chirurgicales des Hôpitaux et Hospicés de Bordeaux, 1866. Revicwed in American Journal of Medical Sciences, Jan. 1866, p. 185 . 
HAMATOPHILIA, OR A HEMORRHAGIC DIATHESIS. 1059

possible, to be avoided. The diathesis may constitute an insuperable objection to all surgical operations which are not unavoidable. When hemorrhage occurs, either from wounds or spontaneously, local measures to arrest the flow of blood are to be resorted to if practicable; and hremostatie remedies are to be given internally. In the class of remedies just named, ergot is to be included. 

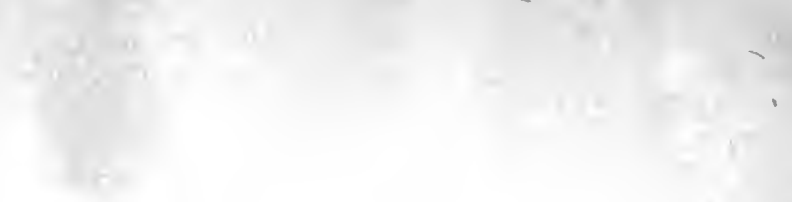

$-\frac{i}{1}$

1

.

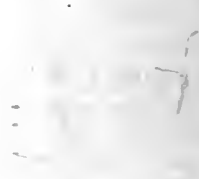

$$
\sqrt{x+2}
$$

14

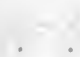




\section{N D E X.}

BORTIVE mensures of treatment, 129

Abscess, retro-pharyngeal, 397 fecal, 381

Abscesses, metastatic, 93, 555

Acholia, 89

Addison's disense, 851

Asthesiometer, 666

Agenesis, cercbral, 661

Ague cake, 588, 920

and ferer, 917

Air, swallowing of, 434

Albumen, increase and dimiuution of, in blood, 79

in urine, modes of testing for, 814

Albuminuria, acute, 802

after scarlutiun, 983

connected with degenerative disease of kidneys, 814

Alcoholism, 772

(Vide-Chronic Bright's Disense.)

Alteratives, 102

Anæmia, 59

Anæsthesia, 666

Analeptic trentment, 129

Analgesia, 666

Anasarcn, 46

Anatomy, morbid, 20

Anatomical characters of disease, 133

Aneurism, thoracic, 861

Angina pectoris, 358

Anthracosis of Lung, 200

Antidotes to corrosive and acrid poisons, 391

Antiscorbutics, 1054

Aphasia, after apoplexy, 614

in cerebritis, 652

in crses of hemiplegin, 682

Aphoni:a, nerrous, 281, 674

Apnoen, 65

mode of dying by, 128

Apoplectic constitution, 610

Apoplexy, 602

anatomical characiers, 602

clinical history, 606

pathological character, 608

crusation, 608

diagnosis, 610

prognosis, 613

treatment, 617

pulmonary, 251

meningeal, 604,612

congestive, 605

serous, 605
Apoplexy-

nervous, 605

from embolism and thrombosis, 605

prevention of, 618

spinal, 624

Arcus senilis, 49

Arthritis, rheumatoid, 1048

Ascaris lumbricoides, 512

vermicularis, 514

Ascites, 540

Asphyxia, 66

Aspirntor in thoracentesis, 163

Asthenia, mode of dying by, 128 nervous, 787

Asthma, 231

clinical history, 231

pathological character, 233

causation, 233

diagnosis, 236

prognosis, 236

hay, 284

treatment, 237

thymic, 279

Ataxia, locomotor, 711

Atelectasis, 201

Atheroma, 49

Atrophy, 27

of lungs, 259

acute, of liver, $569^{\circ}$

progressive muscular, 716

Aura epileptica, 749, 756

Auscultation, intestinal, 475

\section{Bantingism, 82}

B Basedow's disease, 356

Bile passages, disease affecting, 579

Blastema, defiuition of, 34

Blood, morbid conditions of, 56

incrense and diminution of water in, 80

increase and diminution of albumen in, 79

morbid changes of snline constituents of, 89

indeterminate changes of, 100

Blood-globules, changes of, 64 excrementitious principles in, 84

Bloodletting in pleuritis and other acute inflammations, 148

Bothriocephalus, 516

Brain, congestion of, 596

a morbid deficiency of arterial blood in, 601 
Brain-

inflammation and abscess of, 650

induration or sclerosis of, 653

non-inflammatory softening of, 655

hypertrophy and atrophy of, 660

carcinoma of, 657

tubercle of, 656

Breakbone-fever, 1000

Bright's disease of kidneys, acute, 802 chronic, 808

anatomical characters, 809

clinical history, 813

pathological character, 822

causation, 824

diagnosis, 826

prognosis, 827

treatment, 828

Broncliectasis, 198

Bronchitis, 206

acute, affecting the large bronchial tubes, 206

anatomical characters, 206

clinical history. 207

pathological character, 208

causation, 2)

diagnosis, 203

prognosis, 210

treatment, 210

subacute, 211

acute in young children, 211

affecting the small brouchial tubes, 212 epidemic, 216

with exudation of fibrin, 217

chronic, 218

circumscribed, 218

Bronchorrhagia, 246

Bronzed skin-disease, 851

Bulimia, 443

CACHEXIA, definition of, 107

$\mathrm{U}$ malarious, 921

Crecitis, 381

Cæecum, inflammation of, 381

painful tumor of, 382

perforation of, 381

Calcareous degeneration, 49

Calculi, renal, passage along ureter, 799 biliary, 456

Cancer cells, 42

Cancrum oris in typhus fever, 890

Carcinoma, 42

within the chest, 257

of stomach, 411

within the cranium, 657

of kidney, 834

Cardialgia, 433

Carotids, compression of, in epileptic convulsions, 762

Casts of renal tubes, 804,815

Catalepsy, 772

Catarrh, 206

Causes of disease, consideration of, 103

Cell-theory of Virchow, 35

Cephalalgia, 737

Cerebritis, 650
Cervicodynia, 1033

Chicken-pox, 975

Chlorine mixture, 953

Chlorosis, 61

Cholæmia, 88, 581

Cholecystitis, 580

Cholera, sporadic, 483

clinical history, 484

pathological character, 4 S5

causation, 485

diagnesis, 485

prognosis, 486

treatment, 486

infantum, 487

epidemic, 491

anatomical charasters, 492

clinical history, 493

pathological charucter, 497

causation, 498

diagnosis, 501

prognosis, 502

prophylaxis, 503

treatment, 508

Cholerine, 494

Cholesteatoma within cranium, 657

Cholesteræmia, 89, 534

Chorea, 741

saltatorea, 745

scriptorum, 746

Chthonophagia, 444

Circulatory system, diseases of, 315

Cirrhosis of liver, 557,540

of lung, 196

Clergymen's sore-throat, 399

Clinical history of diseases, how obtained, 118

Coagulation of blood in cavities of the heart, 72

in bloodvessels, 73

Colic, hepatic, 456

intestinal, 473

from lead, 477

from copper, 483

nephritic, 799

Collapse of pulmonary lobules, 201, 210, $211,214,226$

Coma, hysterical, 766

nræmic, 818

vigil, 873

Congestion of lungs, 204

of brain, 597

active and passive, 597

Conservative medicine, 127

Constipation, 469

Consumption, pulmonary, 284 galloping, 293

Contagion, definition of, 97

Convulsions, Salnam, 745

uræmic, 818

epileptiform, 754

hysterical, 765

in tetanus, 774

Corpulence, 82

Corpuscles, tuberculous, 37

Cough, nervous, 281

Cowpox, 968 
Cramp, 746

writer's, 746

Oraninm, structural lesions within, 653 tumors within, 656

Croup, 270

iuflaiumatory, 263

caturihal, 266

dipbtheritic, 270

spasmodic, 280

filse, 270

Cruveilbier's disesse, 716 .

Curative measures of treatment, 129

Cysts, renal, 832

Cytoblastema, definition of, 34

Cytoblastions, 37

DEFERVESCENCE, 114

1) Degenerations, 47

Degeneration, fatty, 47

calcareous, 49

lurdaceous or waxy, 50

Delirium tremens, $78 \%$

ebriosum, 598

hysterical, 770

in Bright's disease, 818

Dengue, 1000

Dermalgia, 736

Diabetes, 83

insipidus, 837

mellitus, 839

anatomical characters, 839

clinical history, 810

patbological character, 843

crusation, 843

dingnosis, 844

prognosis, 845

treatment, 846

Diagnosis, definition of, 21,120

consideration of, 120

Diarrhœa, 461

fatty, 461

colliquative, 465

Dinthesis, 107

1)iathetic diseases, 107

Vicrotism of pulse as determined by the sphygmograph, 116

Digestive system, diseases of, 366

Diphtheria, 1002

anatomical cbaracters, 1003

clinical history, 1005

pathological character, 1009

causation, 1010

diugnosis, 1011

prognosis, 1012

treatment, 1018

Dipsomania, 446

Dirt-enting, 444

Diseuse, definition of, 22

elementary,forms of, 132

gross appearances of, 24

microscopical characters of, 24

functional, definition of, 20

dynnmic, 20

individuality of, how determined, 136 clinical and natural history of, 118
Disease-

uniformity of laws of, 119

Diseases, the different aspects under which they are to be considered, 133

Disenses, classificntion of, 135

Disinfection and disinfecting ugencies, 604

Distoma hepaticum, 580

Dorsodynin, 1033

Dropsy, 46

general and local, 46

renal, 47

after scarlatina, 884

cardiac, 46,336

peritoneal, 540

Dropsy, acute inflammatory, 803

in chronic Bright's disease, 813

Duchenne's disease, 711

Ductus communis choledochus, inflammation of, 579,581

Duodenitis, 396

Duodenum, ulceration of, 396

Duramater, henatoma of, 626

Dying, different modes of, 128

Dynamic morbid changes, 20

Dyscrasia, 107

Dysentery, sporadic, 366

anatomical characters, 367

clinical history, 368

pathological character, 369

causation, 370

diagnosis, $3 \overline{7} 0$

prognosis, 370

treatment, 371

epidemic, $3 \overline{7} 4$

chronic, 378

scorbutic, 1052

Dsspepsia, acute, 431

chronic, 432

G CSTASY, 773

E Electricity in paralysis, 703

Electro-muscular sensibility and contractility, 702

Embolism, 71, 326, 330, 685

capillary, 77

of pulmonary artery in rheumatism, 1023

Emphysema of lungs, 222

anatomical characters, 223

clinical history, 2z4

pathological character, 225

causation, 226

diagnosis, 228

prognosis, 230

trestment, 230

Emprosthotonos, 775

Eimpyema, 164

diagnosis, 165

prognosis, 166

treatment, 166

Endemic disenses, 107

Endocarditis, 3:6

anatomical characters, 826

clinical history, 327

pathological character, 327 
Eudocarditis-

causation, 328

diagnosis, 329

prognosis, 380

treatment, 331

ulceration, 331

Enteralgia, 476

Enteritis, acute, 383

anatomical charncters, 383

clinical history, 384

pathological character, 384

causation, 384

diagnosis, 385

prognosis, 385

treatment, 385

subacute and chronic, 386

Enterolithes, 428

Enterorrhagia, 467

Entozoa, 55

intestinal, 512

Epidenic diseases, 108

Epilepsy, 748

Epileptiform convulsions, 754

Epileptoid attacks, 755

Epizoa, 54

Etiology, definition of, 21 consideration of, 103

Excrementitious principles, accumulation of, in blood, 84,87

Expectant treatment of disease, 127

Exudation, definition of, 32 fibrinous, 32

Eyeballs, prominence of, and enlargement of thyroid body associated with functional disorder of the heart, 356

\section{FaCies hippocratica, 123}

Fat, increase and diminution of, in blood, 81

Fatty degeneration, 47

Febricula, 865

Fecal abscess, 381

Fever, ataxic, 862

putrid, high and low, 862

congestive, etc., 862

Chickahominy and camp, 937

breakbone, 1000

gastric, 431

spotted, 640

general pathology of, 862

causes of, 864

simple, 865

typhoid, 867

anatomical characters, 867

clinical history, 870

causation, 881

diagnosis, 884

prognosis, 887

typhus, 888

anatomical characters, 888

clinical history, 889

causation, 892

diagnosis, 894

prognosis, 895

prevention of, 847 .
Fever-
pythogenic, 884

typhus and typhoid, non-identity of, 896

treatment of, 897

relapsing, 906

ana tomical characters, 907

clinical history, 907

causation, 910

diagnosis, 910

prognasis, 911

treatment, 912

erysipelatous, 913

epidemic, characterized by pharyngitis, 915

intermittent, 917

anatomical characters, 917

clinical history, 917

causation, 922

diagnosis, 924

prognosis, $9: 4$

treatment, 925

quotidian, 919 .

tertian, 919

quartan, 919

compound types of, 920

latent, 921

masked, 922

intermittent pernicious, 929

remittent, 933

anatomical characters, 934

clinical history, 935

pathological character and causation, 937

diagnosis, 937

proginosis, 938

treatment, 938

typho-malarial, 934

remittent pernicious, 939

yellow, 940

anatomical characters, 941

clinical history, 942

causation, $946^{\circ}$

dingnosis, 949

prognosis, 950

treatment, 950

Fevers and other general diseases, 861

classification of, 861

eruptive, 954

periodical, 917

Fibrin, coagulation of, within vessels, 73

heart, 72

morbid increase of, in blood, 69

decrease of, 70

qualitative changes in, 71

Fingers, bulbous or clubbed, 292

Fluxes, 45

Fomites, definition of, 108

Formations, new, 51

Functional morbid changes, 20

CALL-STONES, passage of, 456

U Gangrene of lungs, 253

Gastralgia, 452

Gastrio ulcer, 404 
Gastritis, acute, 386

anatomical charncters, 387

clinical history, $38 i$

pathologicul character, 388

calusation, 388

diagnosis, 389

prognosis, 389

treatment, 390

subacute, 391

chronic, 394

Gastro-malaxia, 403

Giastrorrhagia, 446

Genito-urinury system, diseases of, 792

Germ theory of the cllusation of diseases, 108,124

Globules of blood, changes in conformation aud composition of, 154

Globus hystericus, 764

Glosso-pharyngeal nerve, paralysis of, 674

Glottis, œdema of, 277 spasm of, 279

Glycohamin, 82, 839 •

Glycosuria, 839

Goitre, exophthalmic, 356

Gout, 86, 1084

anatomical characters, 1035

clinical history, $1036^{\circ}$

pathological character, 1040

causation, 1041

diagnosis, 1042

prognosis, 1044

treatment, 1044

rheumatic, 1048

irregular and misplaced, 1038

transient, 1036

persistent or chronic, 1037

Granulations of Bayle, 36

Gravel, 801

Graves' disease, 356

Greenhow on Addison's disease, 854

Growths, morbid, 51

Gums, blue discoloration of, in lead-poisoning, 480

\section{IJMATOLOGY, 57}

Homaturia, 835

Ilsmotophilia, 1058

Il eidache, 737

sick, 432,738

Health, definition of, 22

Heart-clot, 71

Heart, valvular lesions of, with enlargement, 333

anatomical characters, 333

clinical history, 335

pathological character, 339

causation, 339

diagnosis, 340

prognosis, 343

treatment, 343

hypertrophy and dilatation of, 334,348 , 349

enlaigement of, without valvular lesions, 347
II eart-

atrophy of, 349

fatty degeneration of, 350

softening of, 352

rupture of, $3 \bar{s} 3$

functional disorder of, 3.53

associated with enlargement of thyroid body and prominence of eye-balls, 256

Heat of body, 113

IJelmintbi, intestiual, 512

IIcmatemesis, $440^{\circ}$

IIemicrania, 737

llemiplegia, 678

Hemoptysis, 247

Hemorhage, pulmonary, 246

Ilemorrhagic diathesis, 10.58

llepatitis, acute, diffuse, 546

anatomical characters, 546

clinical history, 547

puthological character, 548

causation, 548

diagnosis, 648

prognosis, 549

treatment, 549

circumseribed, suppurative, 550

chronic, diffuse, 557

IIermodactylus, 1044

Ilernia within abdomen, 425

Heterologous or heteromorphous products, 40

IIeterotopia, 52

Hippocratic countenance, 123

Histology, morbid, 20

Homologous or homomorphous products, 20

IJum, venous, in anxmia, 63

Hydatids in the kidneys, 833

lungs, 259

liver, 549

Iydramia, 80

Hydrocephalus, 649

Hydronephrosis, 797

Hydro-peritoneum, 540

Ilydrophobia, 778

Hydrops cystidis felleæ, 580

Ilydrorachis, 649

Hydrothorax, 173

IIygienic treatment of disease, 129

Hypergenesis, 52

Ilyperinosis, 69

Ilyperplasia, 26

Hypertrophy, 26

of lungs, 25 ?

Hyperamia, active, 204

passive, 204

of lungs, 204

Hypinosis, 70

Ilypochondriasis, 788

Hypodermic injections of morphin in neuralgia and other affectious, 728 injection of quinia, 926

Hypoglossus verve, paralysis of, 674

Hysteria, 763

Hysterical convulsions, 765

condition, 764 
Hysterical-

coma, 766

delirium, $7 \mathfrak{7 0}$

cough, 281

\section{TCTERUS, 88, 581}

1 Ileus, idiopathic, 429

Impotence, 858

Inanition, 450

Incubation of disease, defiuition of, 119

Induration, 29

Infarctus, 78

Infection, definition of, 97

Inflammation, consideration of, 143

Influenza, 216

Inhalations, 215

Inopexia, 72

Insolation, 618

Intestines, structural affections of, 419

Intestine, invagination of, 419

twisting of, 425

stricture and compression of, 426

obstruction of, from feces, enterolithes, etc., 427

functional obstruction of, 429

affections of, 431

perforation of, in typhoid fever, 875

IDNEY, acute inflammation of, 802, 792

I degenerative lesions of, 809

large white, 809

contructing or cirrhotic, 810

fatty, 811

lardaceous or waxy, 811

cysts in, 832

hydatids in, 833

tuberculosis of, 834

carcinoma of, 835

movable, 835

gouty, $810,822,1036$

IRYNGISMUS STRIDULUS, 279

1 Laryngitis, acute simple, 261. anatomical character's, 261 clinical history, 262

pathological character, 263

causation, 263

diagnosis, 264

prognosis, 264

treatment, 264

subacute, 266

chronic, 266

with exudation, 269

clinical history, 270

pathological charucter, 272

causation, 272

diagnosis, 272

prognosis, 273

treatment, 274

Laryngoscope, 269

Laryngotomy, in cases of thoracic aneurism, 365

Larynx, diseases of, 260

\section{Lorynx-}

topical applications within, 268

morbid growths within, 283

fistulous orifice in, 283

Lead, poisoning by, 477

Lesions of quantity, consistence, ard composition, 25

definition of, 20

classification of, 25

of composition, 31

Leucocytes, 33

Leucocy themia, 66

Lime, syrup of, formula for, 1028

Lithiasis, 801

Liver, acute, diffuse inflammation of, 546 abscess of, 550

pyæmic abscesses of, 555

cirrhosis of, 557

fatty, 562

nutmeg, 565

waxy or lardaceous, 565

cancer of, 567

tuberculosis of, .569

acute atrophy of, 569

torpor of, 563

hydatids in, 572

pigmentary deposit in, 578, 936

hypertrophy of, 578

functional affections of, 586

Liver-fluke, 580

congestion of, 586

Lockjaw, 774

Lotura carnium, 368

Lumbago, 1033

Lumbodynia, 1033

Lumbricoid worms, 512

Lymph, 32

fibriuous and corpuscular,, 34

croupous and plastic, 34

TALACIA, 403, 444

II. Malaria, 922

Malformations, congenital, 25

Malingering, 122

Mania dे potu, 782

Masturbation, 860

Measles (vide Rubeola), 992

Medicine, definition of, 17

different departments of, 18

principles and practice of, 18, 19

conservative, 127

practice of, definition of, 133

Melæna, 467

Melanæmia, 96

Melituria, 839

Meningitis, cerebral, acute, 628

anatomical characters, 628

clinical history, 629

pathological character, 630

causation, 630

diagnosis, 631

prognosis, 631

treatment, 632

rheumatic, 630,1019

chronio, 633 
Meningitis -

tuberculous, 634

spinal, 638

oerebro-spinal, 640

anatomical characters, 640

clinical history, 641

pnthological character, 645

causation, 645

dinguosis, 646

prognosis, 647

treatment, 647

Mental influences in the treatment of disease, 131

Merismopoedia ventriculi, 54,

Mesmeric or magnetic sleep, 774

Miasm, 97

Microsporon audouni, 54

Mind, disorders of, $7: 23$

management of, in disease, 131

Molecular theory of Beunett, 35

Morbilli, 992

Morbus coxø senilis, 1048

Motor oculi communis, paralysis of, 669

Motor externus, paralysis of, 671

Movements, muscular abnormal, 745

Mumps, 400

Murmurs, cardiao mitral, 340

aortic, 341

tricuspid, 341

pulmonic, 342

Mutations in therapeutics, 131

Myalgia, 737, 1031

Myelitis, 661

Myelo-phthisis, 711

Myo-carditis, 832

\section{NECROBIOSIS, 31}

Neoplasms, 51

Nephritis, suppurative, 792

aatomical characters, 793

clinical history, 793

pnthologieal character, 794

causation, 794

dingnosis, 795

prognosis, 796 .

treatment, 797

acute desquamative, 802

anatomical characters, 803

clinical history, 803

pathological character, 805

causation, 805

diagnosis, 806

prognosis, 806

treatment, 807

acute desquamative, after scarlatina, 983

Nerrous system, diseases of, 594

Neuraigia, dorso-intercostal, 202

Neuralgia, 724

diagnosis of, 726

trentment of, 727

intercostal, 202, 784

trifacial, 731

cervico-occipital, 733

cervico-brachial, 733
Neuralgia -

lumbo-abdominal, 734

crural, 735

sciatic, 735

of skin, 736

muscular, 737

Neuritis, 721

Neuroses, 722

Niemeyer's classification of therapeutical iudications, 129

Nomenclature of diseases, 19

Nosology, 135

BESITY, means of diminishing, 82

Obstruction of bowels, 419

Edema, 46

pulmonary, 256

of the glottis, 277

Ophthalmoscope, in disease, 117

Opisthotonos, 775,765

Opium in pleuritis, bronchitis, and other acute infammations, 151,210

Orchitis, variolous, 957

Ovaritis, variolous, 957

Oxaluria, 436, 752

Oxyuris vermicularis, 514

\section{PaChymeningitis, 625}

Palliative measures of trentment, 129

Palsy, wasting or creeping, 716

Pancreas, affections of, 591

Puracentesis in pleuritis, 162 empyema, 166

Paralysis, 665 reflex, 667

of third cranial nerve, 669

of fourth craninl nerve, 670

of fifth cranial uerve, 670

of sixth crauial nerve, 671

of motor portion of seventh cranial nerve, 671

of eighth cranial nerve, 674 .

of ninth cranial nerve, $6 \overline{7} 6$

local, of other than cranial nerves, 675

of the cervical sympathetic nerve, 676

hemiplegic, 678

paraplegic, 689

general spinal, 698

agitans, 747

glosso-labial, 705

general cerebral, 708

myo-sclerotic, 720

treatment of, 701

afte aiphtheria, 1007

from lo $a, 675$

infantle, 711

Paraplegia, 689 reflex, 693

Parnsites, animal and regetable, 53

Parotiditis, 400 in typhoid fever, 874

Pathology, definition of, 18 general and special, 18 medical and surgical, 19 
Pathology -

humoral, 103

relationship to physiology, 23

special, introduction to, 183

Pathophobia, 788

Pericarditis, acute, 316

anatomical characters, 310

clinical history, 317

pathological character, 318

causation, 318

diagnosis, 819

prognosis, 32l

treatment, 322

chronic, 324

pueumo, 325

Peri-hepatitis, 537

Perinephritis, 799

Peripneumonia, 177

Peritonitis, acute general, 526

anatomical characters, 526

clinical history, 527

pathological character, 528

causation, 529

diagnosis, 530

prognosis, 531

treatment, 532

partial or circumscribed, 537

chronic, 537

tuberculous and cancerous, 538

Perspiration, excrementitious principles of, in blood, 87

Pertusssis, 239

clinical history, 239

pathological character, 242

causation, 243

diagnosis, 243

prognosis, 243

treatment, 243

Pharyngitis, $396^{\circ}$

simple acute, 397

chrouic, 398

epidemic, 398

follicular, 399

Pharyngeal-retro, abscess, 397

Phlebitis, 91

portal, 555

Phosphuria, 802

Phthisis, bronchial, 41

pulmonary. (Vide Pulmonary Tuberculosis.)

laryugeal, 267

acute, 36,298

Physician, definition of, 17

Pica, 446

Pigment induration of lnng, 196

Plethora, 58

Pleuritis, acute, 137

anatomical characters, 137

clinical history, 140

pathological character, 143

causation, 144

diagnosis, 145

prognosis, 147

treatment, 148

varieties of, 156

chronic, 156
Pleuritis, cbronic-

anatomical characters of, 156

clinical history, 157 .

pathological character, 158

causation, 158

prognosis, 159

diagnosis, 160

treatment, 161

relations to tuberculosis, 158,159

paracentesis in, 162

suppurative (vide Empyema), 164

Pleuritis with pneumothorax, 167

anatomical characters, 168

clinical history, 168

diagnosis, 168

proguosis, 169

treatment, 170

circumscribed, 172

Pleuritis, diaphragmatic, 964

Pleurodynia, 202, 1033

Pleuro-pneumouitis, 177

Pleurosthotonos, 775

Pneumatoses, 53

Pneumonitis, 174

acute lobar, 175

anatomical characters, 175

clinical history, 178

pathological character, 182

causation, 182

diagnosis, 184

prognosis, 185

treatment, 188

circumscribed, 195

chronic, 196

lobular, 201

interstitial, 197

typhoid, 182

Pneumo-pericarditis, 325

Pneumorrhagia, 251

Pneumothorax, 170

Poisons, 99, 390

Polyæmia, 58

Polydipsia, 445

Polyphagia, 443

Polyuria, 837

Portability of disease, 108

Prodromes of disease, definition of, 119

Prognosis, definition of, 21

consideration of, 122

Prognostics, unfavorable, 123

Proliferation of cells, Virchow's doctrine, 35

Prophylaxis, definition of, 21, 124 consideration of, 124

Prophylactic measures of treatment, 128

Pulmonary gangrene, 253

cdema, 256

congestion, 204

tuberculosis, 284

anatomical characters, 284

clinical history, 287

pathological character, 293

causation, 293

diagnosis, 299

prognosis, 301

treatment, 303 
Pulmonary tuberculosisacute, 312

Purpura hemorrhagica, and simplex, 1056 Pyxmia, 91

Pyarthrosis, 1030

Pyelitis, 792

Pyonephrosia, 793

Pyothorax, 164

Pyrosis, 433, 441

UINIA, hynodermic injection of, 926,932 Quinsy, 396

RABLES, 778

R Regurgitation, gastric, 433

Relapsing tever, 906

Respiratory system, disenses of, 137

Restorative treatment, 129

Rheumatism, acute articular, 1016

clinical history, 1016

patbological character, 1021

causation, 1022

diagnosis, 10:3

prognosis, 1023

treatment, 1024

articular, subncute, and chronic, 1028

gonorrìœal, 1030

syphilitic, 1030

muscular, 1031

Roseoln, 932

Rubeola, 992

clinical history, 992

causation, $99 \bar{J}$

diagnosis, 997

prognosis, 998

treatment, 998

hemorrbagic, 995

nigra, 995

sine catarrho, 994

eruptione, 994

CANITARY measures of treatment, 120

Sarcina ventriculi, $44:$

Scapulodynia, 1033

Scarlatina, 977

anatomical chnracters, 977

clinical history, 977

causntion, 984

diagnosis, 985

prognosis, 986

treatment, 988

maligna, 981

angivosa, 982

heinorrhagic, 982

sine eruptione, 983

Sclerema, 720

Sclerosis of lnng, 169

Scorbutus, 90, 1051

anatomical characters, 1051

clinical history, 1052

pathological character, 1053

causntion, 1053

diagnosis, 1053

\section{Scorbutus-
prognosis, 1054 \\ prevention, 1054 \\ trentment, 1055}

Scrofula, 41

Scybala, 368

Semeiology, definition of, 21

consideration of, 111

Seminnl emissions, juvoluntary, 856

Septicrmia, 94

Sequelæ of disease, 119

Signs, definition of, 112

Siderosis of lung, 200

Smallpox, 954

(Vide Vuriola.)

Softening, 29

Solidism, doctrine of, 103

Somnambulism, 774

Sordes in typhoid fever, 874

Spasm idiopathic, $\mathbf{7 4 6}$

Spcech, loss of, after apoplexy, 614

Spermatorrhœa, 856

Sphygmograph, account of, in health and disease, 114

Spina bifida, 649

Spinal cord, congestion and anæmia of, 624 hemorruage within, 624

epinal meningitis, 638

structural lesions of, 663

Spleen, affections of, 587 removal of, 591

Stomach, structural affections of, 402 induration of, 402

softening of, 403

alcer of, 404

carcinoma of, 411

dilitation of, 414

degeneration of tubules of, 416

inflammation of, 386

(Vide Gastritis.)

functional affections of, 450

Strychnia, effects of, contrasted with tetanus, 776

St. Vitus's dance, 741

Sugar, abnormal accumulation of, in blood, 82

presence of, in urine, 844

Sunstroke, 618

Supra-renal capsules, disease of, 851

Sustaiming measures of treatment, 129

Synuptomatology, definition of, 111

Symptoms, pathognomonic and diagnostic, 111

subjective and objective, 111

TENIE or tapeworms, 516

1 Taches bleuatres, 876

Temperature in disease, 112

Tenesmus, 368

Tetanus, 774

Thallophytes, 54

Therapcutical measures, classification of, 128

indications, Niemeyer's classification of, 129 
Therapeutics, general and special, definition of, 21,125 consideration of, 125

Thermometer in health and disease, 112

Thoracentesis in pleuritis, 155, 162 empyema, 166

Thrombosis, 71

Tic douloureux, 731

Tonsillitis, 396

Tophus, 1035

Tormina, 368

Torticollis, 1033

Toxæmia, 85

Toxicology, 99

Trachea, affections of, 260

Tracheotomy in acute laryngitis, 266 syphilitic laryngitis, 269 diphtheritic laryngitis, 276 œdema of glottis, 278 in diphtheria, 1015

Trance, 773

Transulation, definition of, 32

'Transudations, ' 44

Treatment of diseases, classification of objects of, 128

Trembling, senile, 747 mereurial, 748

Trichinæ spiralis, 521

Trichiniasis, 521

Trichophyt on tonsurans, 54

Tricocephalus dispar, 512

Trifacial nerve, paralysis of, 670 neuralgia of, $i 31$

Trismus, 774

Tubercle, 35

gray, 36

yellow, 37

obsolete, 37

inoculation with, 40

within cranium, 606

in kidneys, 834

Tuberculosis, pulmonary, 284 anatomieal characters, 284 clinical history, 287 pathological character, 293 causation, 293 diagnosis, 209 prognosis, 301 treatment, 803

pulmonary, acute, 312

Tuffnell on treatment of aneurism, 365 Tympanites, 53, 433, 434

Typhlitis, 381

Typhlo-enteritis, 381

Typhoid product, 44

Typho-malarial fever, 934

Typho-mania, 873
TR REMIA, 84

in typhoid fever, 879

in scarlatina, 981

Urea, volumetric analysis of urine for, 815

Uricæmia, 86, 1040

Uric acid, mode of determining presence of, in blood, 1043

Urine, tests for sugar in, 844

VACCINATION, 973

Vaccinia, 968

Varicella, $97 \overline{0}$

Variola, 954

anatomical characters, 955

clinical bistery, 955

ceusation, 959

diagnosis, 962

prognosis, 962

treatment, 963

differential diagnosis of, from scarlatina, rubeola, and roseola, 983

Varioloid, 966

Venom, definition of, 98

Vertigo, 739

Villemin's experiments showing inoculability of tuberele, 40

Virchow, cell-theory of, 35

Virus, 97

Volvulus, 419

niting in dyspepsia, 435

WATERBRASH, 433

Water, increase and diminution of, in blood, 80

Water, employment of, in pleuritis and pneumonitis, 153,189

in typhus and typhoid fever, 898

in scarlatina, 988

Waxy degeneration, 50

Whooping-cough (Vide Pertussis), 239

Wind-sucking, 434

Worms, intestinal, 512

lumbricoid, 512

thread or pin, 514

tape, 516

tricocephalus dispar, 515

trichinal, 521

Wrist-drop, 677

Writer's cramp, 746

$\mathrm{Z}^{\text {YMOSIS, } 98}$ 
I.

\section{PRINCIPLES AND PRAC'TICE OF MEDICINE; DESIGNED FOR THE USE OF STUDENTS AND PRACTITIONERS OF MEDICINE. Fourth Edition, thoroughly Revised.}

\section{In one large and closely printed octavo volume of nenrly 1100 pages: handsome extra} cloth, $\$ 6$; or strongly bound in leather, raised bands, $\$ 7$.

By common consent of the English and Amerionn medical press, this work bas been assigned to the highest position as a oomplete and compendious text-book on the most advanced condition of medical science. At the very moderate price at which it is offered it will be found one of the cheapest volumes now before the profession. A few notices of previous editions are appended.

Admirable and nuequalled.-Western Journal of Flint's. There is no snperfinity of word or thonght,

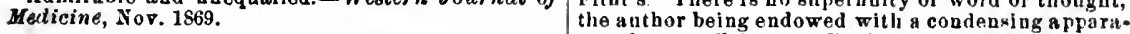

Dr. Flint's work, though clalmleg no higher tille tus of great efficiency. To the student at achool, and thai that of a text-book, is really more. IIe is a man to the "busy practitioner" who lacks time or inclinaof large clinical experience, and hls book is full of such inasterly descriptiona of disease as can only be draw by a mau intimalely acquainted with their various forins. It is a work which we can cordially recommend to onr readers as fully abreast of the Bcience oi the day.-Edinburgh Med. Journal, Oct.'69.

Ove of the best works of the kind for the practitioner, aud the most convenient of sll for the student. -Am. Journ. Med. Sciences, Jan. 1869.

The third edition of this moat excellent book scarce ly needs any commendation from as. The volume, as it stands now, is really a marvel. Not only is it wundertul that any one man can have grasped in his mind the whole scope of medicine with that rigor which Dr. Flint shows, but the condensed yet clear way in which this lo dope is a perfect literury triumph. Dr. Fllnt is pre-eminently one of the strong men, whese right to do this kind of thing is well ad mitted; aud wosuy no more than the truth when we affir that he is rery nearly the only liviog man that cunld du it with such results as the volume before us - The London Practitimer, March, 1869.

This is in some rexpects the best text-book of medicine in our lauguage. - London Med. Times and Gratite, Feb. 6, 1569 .

We do not know of a practical work so solid as tion to question the old and new inasters, and who is willing to be helped towards conclusione, there is no work in onr lauguage so well adapted as the treatise of Profeasor Flint.-Pacific Med. and Surg. Journ. Jan. 1869.

Practical medicine was at sea when thls book ap. peared ahove the horizon as a safe and capacions harbor. It came opportunely, and was greeted with p)easnrable omotions throughont the Jand.-Nashcille Med. and Surg Journ., May, 1569.

Till that day arrives Professor Flint's treatise, of which the third cdltion has jnst appeared, will continue to hold its place in the flrst rank. For perspicuity, atiention to detail, correct pathology, and enlighiened therapentics, it is not excelled by any other sinilar work, and in the last particular excels them all. Large practical experience and thorongh sclentific training are possessed in a high degree by Dr. Fllat. That the nuion of such qualifications should have led to the prodnction of a sacisfactory treatime on the practice of medicine is not a matter of surprise. That such lias been the result in evidenced by the rapid sale of two large editions, and the appearauce of the thild in less than two years from the date of the first publication.-Quart. Journal of Psychological Mecti. cine and Nedical Jurisprudence, Jan. 1869.

II.

Enlarged Edition-Just Issned.

\section{A PRACTICAL TREATISE ON THE}

\section{Diagnosis, Pathology, and Treatment of Diseases of the Heart. Socond Edition, thoroughly Revised and Enlarged. \\ In one handsome octavo volume of 550 pages : extra cloth, $\$ 4$.}

The appearance of a new editlon of this standard wirk calls for uo exlended notlce from the reviener, and yet a compurison of this witl the last issue will blow that in many parts it is snbstautially a new book. Altogether, we are of the opinion that the ad. ditions and changes made in this edition of Prof. Flint's work have materially enhanced its value, and that they will tend atill stronger to conflim the estlmate wlich the professlon in this conntry liave put apon the book, which was al ready accepted as a leading authority in all matters pertainiog to diseases of the beart. $-N$. $\boldsymbol{Y}$. Med. Journal, Oct. 1870 .

On its first issue, ten years ago, this treatlae took Its place iu the front rank of professional literature at home, and has divided the honors with Dr. Stokes 8 work abroad, in the field of which it treats. In this, the secoud edition, the author has embodied the re sults of his experience, larger probably than that of any other American practitioner during the interven. ing vears, and given us what will be recognized, wherever the English language ls read, as a standard autherity on the subject of cardiac diseases. $-\boldsymbol{N}$. $\boldsymbol{Y}$. Med. Gazette, Jan. 2S, 1871.

III.

PHYSICAL EXPLORATION AND DIAGNOSIS OF

\section{DISEASES AFFECTING THE RESPIRATORY ORGANS. SECONO EDITION, REVISED.}

In one handsome octavo volume of 595 pages: extra cloth, $\$ 450$.

Premising this observatlon of the pecessity of each on the ssme subject.-Dublin Medical Press, Feb. 2, student and practltloner making hlmself scquainted 2867.

wlth anscultatiun and percnssion, we may state our honest opinion that Dr. Flint's treatlse is one of the most trastworthy guides whlch he can oonsult. The style ls'clear and distinct, and is also conotse, being free from that tendency to over-refnement and nonecessary minnteness which characterizes many works

Thj 8 is an admirablebook. Excellent in detall and executlon, nothing better conld be desired by the prectitioner. Dr Fliot enriclies hls subject with mnch rolld and not a llttle original observation.Ranking's Abstract, Jan. 1567 . 
A PRACTICAL TREATISE ON THE DISEASES OF WOMEN. By T. Gaillard Thomas, M.D., Professor of Obstetrics and Diseases of Women and Chil * dren in the College of Physicians and Surgeons, N. York; Olstetric Physician to the Strangers' and the Roosevelt Hospitals; Consulting Physician to the N. Y. State Women's IIsspital, \&c. With about Two IInndred and Fifty Illustrations. Third Edition, Enlarged and thoroughly Revised. In one large and handsome octavo volume of 784 pages; leather, $\$ 6 ;$ cloth, $\$ 5$.

The exhaustion of two very large editions in a little more than three years shows that the author has not failed in bis endeavor to supply the admitted want of a work which should. in a moderate compass, furnish a complete view of all the modern aspects of gynacology. Stimalated by the very favorable reception accorded to his labors, he has songht to render the present edition still more worthy than its predecessors. Every portion of the work has been thoroughly revised, several new chapters and a number of new illustrations bave been introduced, and the most painstaking care has been bestowerl to make it a full and trustworthy guide for the student and practitioner. To accommodate the numerous alditions the size of the page has been enlarged, notwithstanding which the number of pages bas been increased by nearly one bundred and fifty; in fact, the present edition contains nearly one-third more matter than the preceding, notwithstanding which it has been kept at the former very moderate price." The work, it is therefore hoped, will continue to maintain its position as the favorite book for consultation by all who have to treat this frequent and important class of diseases.

ON DISEASES PECULIAR TO WOMEN: including Displacements of the Uterns. By IIUGH L. IIongr, M.D., Emeritas Professor of Obstetrics, de., in the Unirersity of Pennsylvania. With origiual illustrations. Second Edition, Revised and Enlarged. In one beantifully printed octavo volume of $52 \mathrm{l}$ pages; extra cloth, $\$ 450$.

As a contribution to the study of women's diseases, on its own merits.-Pacifie Med. and Surg Journ., it is of great value, and is abundantly able to stand oct. 1868.

A CIINICAL EXPOSITION OF TIIE MEDICAL AND SURGICAL DISEases OF WOMEN. By Robert Barnes, M.D., F.R.C.P., Obstetric Physician to St. Thomas's Hospital, \&c. In one large and handsome octaro volume, with numerous illustrations. (Nearly Ready.)

A COMPLETE PRACTICAL TREATISE ON THE DISEASES OF ChildRen. By Lewis J. Smith, M.D., Professor of Morbid Anatomy in the Bellevue IIospital Med College, N. Y. Second Edition. Revised and greatly Enlarged. In one handsome octavo volume of 742 pages; extra cloth, $\$ 5$; leather, $\$ 6$. (Just Issued.)

\section{From the Preface to the Second Edition.}

In presenting to the profession the second edition of his work, the anthor gratefully acknowl. edges the farvorable reception accorded to the first. He has endeavored to merit a continuance of this approbation by rendering the volume much more complete than before. Nearly twenty additional diseases have been treated of, among which may be named Diseases Incidental to Birth, Rachitis, Tuberculosis, Scrofula, Intermittent, Remittent, and Typhoid Fevers, Chorea, and the various forms of Paralysis. Many new formulæ, which experience has shown to be useful, have been introdnced, portions of the text of a less practical nature have been condensed, and other portions, especially those relating to pathological histology, have been rewritten to correspond with recent discoveries. Every effort has been made, however, to avoid an undue enlargement of the volune; but, notwithstanding this, and an increase in the size of the page, the number of pages has been enlarged by more than one hundred.

227 West 49Th StReET, New Yokк, April, 1872.

This excellent work bas already heen fully re- symptoms and pathology of the diseases of early life, This excellent
vewed our pages. We need, therefore, do little and possessing the further advautage, in which it viewed in our pages. the appearance of a new and stands a lone amongst other works on its subject, of more than chronicle No hetter work on child ren's dis- recommending treasmeut in accord with the most reeases conld be placed to the hands of the student, cent therapentical views.-Brit. and For. Med.-Chi. containing, as it does, a very complete account of the rurg. Rev., Jan. 1873.

\section{LECTURES ON THE DISEASES OF INFANCY AND CHILD-}

HOOD. By Charles West, M.D., Physician to the Hospital for Sick Children, \&e. Fourth

American, from the Fifth Revised and Enlarged English Edition. In one large and handsome octaro volume of 656 closely-printed pages. Extra cloth, $\$ 450$; leather, \$5 50 .

Undonbtedly one of the best books on the snbject we have ever seen, and we cheerfilly recommend it to the profession.-N. O. Med. Record, July 1, 1866 .

Dr. West's book is the best that has ever been written in the English langnage on the diseases of in fancy und childhood.-Columbus Review of Medicine and Surgery.

One of the best publications ever issued upon diseases of childrea. - Medical Examiner.

It is one standlng by itsolf on its important snb ject in on $r$ langnage-n napproached, unrivalled.Brit. and Fm. Med.-Chir. Review.

All things considered, this book of Dr. West is by far the best treatise in our language upos $8 \mathrm{ch}$ modifications of morbid action and disease as are witnessed when we have to deal with infancy and childbsod.-London Lancet. 


\section{HE N R Y C. LE A'S}

(LATR LRA \& BAMCHARD's) .

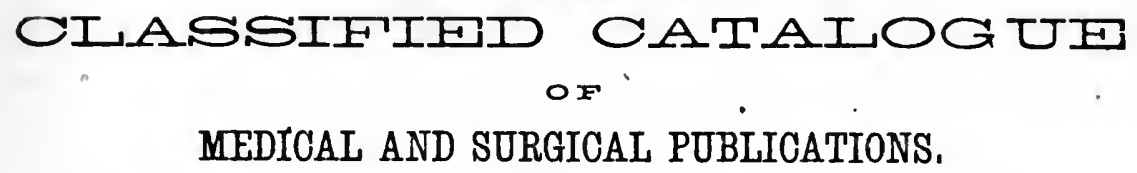

In asking the attention of the profession to the works contained in the following pages, the publisher would state that no pains are spared to secure a continuance of the confidence earned for the publications of the house by their careful selection and accuracy and finish of execution:

The printed prices are those at which books can generally be supplied by booksellers throughout the United States, who can readily procure for their customers any works not kept in stock. Where access to bookstores is not convenient, books will be sent by mail post-puid on receipt of the price, but no risks are assumed either on the money or the books, and no publications but my own are supplied. Gentlemen will therefore in most cases find it more convenient to deal with the nearest bookseller.

An Illustrated Catalogun, of 64 octavo pages, handsomely printed, will be forwarded by mail, postpaid, on receipt of ten cents.

No3. 706 and 708 Sansom St., Philademphia, April, 1873.

HENRY C. LEA.

\section{ADDITIONAL INDUCEMENT FOR SUBSCRIBERS TO}

THE AMERICAN JOURNAL OF THE MEDICAL SCIENCES.

THREE MEDIOAL JOURNALS, containing over 2000 LARGE PAGES, Free of Postage, for SIX DOLLARS Per Annum.

TERMS FOR 1873:

Tan American Journal of the Medicat Sciences, and Five Dollars per annum, Thx Medical News and Library, both free of postage, $\}$ in advance.

$$
\text { OR, }
$$

The American Journal of the Medicai Scinncrs, published quar- six Dollars
terly (1150 pages per annum), with

Thr Medical, News and Lrbrary, monthly (384 pp. per annum), and per annum

The Half-Yeariy Abstract of the Medical Scingers, published Feb. and August ( 600 pages per annum), all free of postage. in advance.

\section{SEPATATE SUBSCRIPTIONS TO}

The American Journal of the Medical Sciences, subject to postage when not paid for in advance, Five Dollars.

The Medical News and Library, free of postage, in advance, One Dollar.

The Half-Yrarly Abstract, Two Dollars and a Half per annum in advance. Single numbers One Dollar. and a Half.

It is manifest that only a very wide circulation can enable so vast an amount of valuable practical matter to be supplied at a price so unprecedentedly low. The publisher, therefore, has much gratification in stating that the rapid and steady increase in the subscription list promises to render the enterprise a permanent one, and it is with especial pleasure that he acknowledges the valuable assistance spontaneously rendered by so many of the old subscribers to the "Journal," who have kindly made 
known among their friends the advantages thus offered and have induced them to subscribe. Relying npon a continuance of these friendly exertions, he hopes to be able to maintain the unexampled rates at which these works are now supplied, and to succeed in his endeavor to place upon the table of every reading practitioner in the United States a monthly, a quarterly, and a half-yearly periodical at the comparatively trifling cost of SIX Dolisars per annum.

These periodicals are universally known for their high professional standing in their several spheres.

\section{THE AYERICAN JOURNAL OF THE MEDICAL SCIENCES, EDITED BY ISAAC HAYS, M. D.,}

is published Quarterly, on the first of January, April, July, and October. Fach number contains nearly three hundred large octavo pages, appropriately illustrated, wherever necessary. It has now been issued regularly for nearly FIFTY years, during almost the whole of which time it has been under the control of the present editor. Throughout this long period, it has maintained its position in the highest rank of medical periodicals both at home and abroad, and has received the cordial support of the entire profession in this country. Among its Collaborators will be found a large number of the most distinguished names of the profession in every section of the United States, rendering the department devoted to

\section{ORIGINAL COM MUNICATIONS}

full of varied and important matter, of great interest to all practitioners. Thus, during 1872 , articles have appeared in its pages from nearly one bundred gentlemen of the highest standing in the profession throughout the United States.*

Following this is the "Review Departuent," containing extended and impartial reviews of all important new works, together with numerous elaborate "AxaLYTICAL ANo Bibliographical Notices" of nearly all the medical publications of the day.

'This is followed by the "Quarterly Summary of Improvements and Discoveries in the Medical Sciences," classified and arranged under different heads, presenting a very complete digest of all that is new and interesting to the physician, abroad as well as at home.

Thus, during the year 1872, the "JourNaL" furnished to its subscribers Fighty-four Original Communications, One Hundred and Twenty-nine Reviews and Bibliograph. ical Notices, and Three Hundred and seven articles in the Quarterly Summaries making a total of about FIVE HUNDred articles emanating from the best professional minds in America and Europe.

That the efforts thus made to maintain the high reputation of the "Journal" are successful, is shown by the position accorded to it in both America and Europe as a national exponent of medical progress :-

Dr. Hays keeps his great Ameriean Quarterly, in matter it contains, and has established for itself a which he is now assisted by Dr. Minis Hays, at the reputation in every country where medicine is culhead of his country's medical periodicals. -Dublin tivated as a science.-Brit. and Fur. Med.-Chirurg. Medical Press and Circular, March 8, 1871. Review, April, 1871.

of English periodicals the Lancet, and of American One of the best of its kind.-London Lancet, Ang. the Am. Journal of the Medical Sciences, are to be 20,1870 .

regarded as necessities to the reading practitioner.$N$ Y. Medical Gazette, Jan. 7, 1871.

The American Journal of the Medioal. Sciences yields to none in the amount of original and borrowed

Almost the only one that circnlates everywhere, all over the Union and in Europe.-London Medical Times, Sept. 5,1868 .

The subscription price of the "American Journal of the Medical Sciences" has never been raised, during its long career. It is still Five Dollaks per annum; and when paid for in advance, the subscriber receives in addition the "MEDICAL NEws aND LIBRARY," making in all about 1500 large octavo pages per annum, free of postage.

\section{II.}

\section{THE MEDICAL NEWS AND LIBRARY}

is a monthly periodical of Thirty-two large octavo pages, making 384 pages par annum. Its "News DeParTment" presents the current information of the day, with Ulinical Lectures and Hospital Gleanings; while the "Library Department" is devoted to publishing standard works on the varions branches of medical science, paged separately, so that they can be removed and bound on completion. In this manner subscribers have received, without expense, such works as "W "'lodd and Bowman's Physiology," "What on Children," "Malgaigne's Surgtery," \&c. \&c. And with January 1873 will be commenced the publication of Dr. McCall

* Cimmunications a re in rited from gentlemon un all parts of the conntry. Elabosate artictes ingeried by tho Editor are paid for by the Pablibuer. 
Anperson's new work "On the Treatuest of Diseages of the Skin, with an Anahysis of Eireven 'Thodsand Consecutive Cases."

As stated above, the subscription price of the "Mrdical News and Library" is ONe Dol.ar per annum in advance; and it is furnished without charge to all advance pajing subscribers to the "American Journal of the Medical Sciences."

\title{
III.
}

\section{THE HALF-YEARLY ABSTRACTT OF THE MEDICAL SCIENCES}

is issued in half-yearly volumes, which will be delivered to subscribers about the first of February and first of August. Each volume contains about 300 closely printed octavo pages, making about six hundred pages per annum.

"RANKING's ABstract" has now been published in England regularly for more than twenty years, and has acquired the highest reputation for the ability and industry with which the essence of medical literature is condensed into its pages. It purports to be " $A$ Digest of British and Continental Medicine, and of the Progress of Medicine and the Collateral Sciences," and it is even more than this, for America is largely represented in its pages. It draws its material not only from all the leading American, British, and Continental journals, but also from the medical works and trentises issued during the preceding six months, thus giving a complete digest of medical progress. Each article is carefully condensed, so as to present its substance in the smallest possible compass, thus affording space for the very large amount of information laid before its readers. The volumes of 1872 , for instance, have contained

\begin{abstract}
SIXTY-FOUR ARTICLES ON GENERAL QUESTIONS IN MEDICINE. NINETY-SIX ARTICLES ON SPECIAL QUESTIONS IN MEUICINE. TWELVE ARTICLES ON FORENSIC MEDICINE. NINETY.THREE ARTICLES ON THERAPEUTICS.

FORTY-TWO ARTICLES ON GENERAL QUESTIONS IN SURGERY.

ONE HUNDRED AND THIRTY.THREE ARTICLES ON SPECIAL QUESTIONS IN SORGERT. EIGHTY ARTIGLES ON MIDWIFERY AND DISEASES OF WOMEN AND CHILDREN. BIGHTEEN ARTICLES IN APPENDIX.
\end{abstract}

Making in all nearly five hundred and fifty articles in a single year. Each volume, moreover, is systematically arranged, with an elaborate Table of Contents and a very full Index, thus facilitating the researches of the reader in pursuit of particular subjects, and enabling him to refer without loss of time to the vast amount of information contained in its pages.

The subscription price of the "Asstract," mailed free of postage, is Two Dolla rs and a Half per annum, pagable in advance. Single volumes, $\$ 150$ each.

As stated above, however, it will be supplied in conjunction with the "Axericas Journal of the Medical Scienceg" and the "Medical News and Library," the whole free of postage, for Six Dollars pkr annux in advance.

For this small sum the subscriber will therefore receive three periodicals costing separately Eight Dollars and a Half, each of them enjoying the highest reputation in its class, containing in all over Two rHousand Pages of the choicest reading, and presenting a complete view of medical progress throughout both hemispheres.

In this effort to bring so large an amount of practical information within the reach of every urember of the profession, the publisher contidently anticipates the friendly aid of all who are interested in the dissemination of sound medical literature. He trusts, especially, that the subscribers to the "American Medrcal Jodrnal" will call the attention of their acquaintances to the advantages thus offered, and that be will be sustained in the endeavor to permanently establish medical periodical literature on a footing of cheapness never heretofore attempted.

\section{PREMIUM FOR NEW SUBSCRIBERS.}

Ang gentleman who will remit the amount for two subscriptions far 1873 , one of which must be for a new subscriber, will receive as a PREMIUx, tree by mail, a cupy of the new edition of 'TanNek's Clinical. MaNUal, for advertisement of which see p. $\overline{5}$, ol of Chambers' Restorative Medicine (see p. 17), or West on Nervous Disorders of Childran (see p. 21).

* * Gentlemen desiring to avail themselves of the advantages thus offered will do* well to forward their subscriptions at an early day, in order to iusure the receipt of complete sets for the year 1873 , as the constant iucrease in the subscription list almost always exhausts the quantity printed shortly after publication.

3 The safest mode of remittance is by bank check or postal money order, drawn to the order of the undersigned. Where these are not accessible, remittances for the "JOURNAL" may be made at the risk of the publisher, by forwarding in REGISTRRED letters. Address,

HENRY O. LEA,

No8. 706 and 708 Sansox Str, Philaderpeis, PA. 
DUNGLISON (ROBLEY), M.D.,

Professor of Institutes of Medicine in, Jefferson Medical College, Philadelphta.

MEDICAL LEXICON; A Dictionary of Medical Science: Containing a concise explanation of the various Subjects and Terms of Anatomy, Physiology, Pathology, Hygiene, Therapeutics, Pharmacology, Pharmacy, Surgery, Obstetrics, Medical Jurisprudence, and Dentistry. Notices of Climate and of Mineral Waters; Formulm for Officinal, Empirical, and Dietetic Preparations; with the Accentuation and Etymology of the Terms, and the Frenob and other Synonymes; so as to constitute a Frenoh as well as English Medical Lexicon. Thoroughly Revised, and very greatly Modified and Augmenterl. In one very large and handsome royal octavo volume of 1048 double-columned pages, in small type; strongly done up in extra cloth, $\$ 600$; leather, raised bands, $\$ 675$.

The object of the author from the outset has not been to make the work a mere lexicon or dictionary of terms, but to afford, under each, a condensed riew of its various medical relations, and thus to render the work an epitome of the existing condition of medical science. Starting with this view, the immense demand which has existed for the work has enabled him, in repeated revisions, to augment its completeness and usefulness, until at length it bas attained the position of $n$ reoognized and standard anthority wherever the language is spoken. The mechanical exsoution of this edition will be found greatly superior to that of previous impressions. By enlarging the size of the volume to a royal octavo, and by the employment of a small hut clear type, on extra fine paper, the additions bnve been incorporated without materially increasing the bulk of the volume, and the matter of two or three ordinary octavos bas been compressed into the space of one not unhandy for consultation and reference.

It would be a work of supererogation to bestow a word of praise upon this Lexicon. We can oniy wonder at the labor expended, for whenever we refer to its pages for information we are keldom disappointed in finding all we desire, whether it be in accentuation, etymology, or definition of terms.-Newo York Medical Journal, November, 1865.

It would be mere waste of words in us to express onr admiration of a work which is вo nniversally and deservediy appreciated. The most admirabie work of its kind in the English langnage. As a buok of reference it is invaluable to the medical practitiuner, and in every instance that we bave turned over its pages for information we have been charmed by the clearness of language and the accuracy of detaif with which each abounds. We can most cordiaily and confidentiy commend it to our readers.Glasgow Medical Journal, January, 1866.

A work to which there is no equal in the English language.-Edinburgh Medical Journal.

It is bomething more than a dictionary, and $80 \mathrm{me}-$ thing less than an encyciopædia. This edition of the well-k nown work is a great improvement on its pre decessors. The book is on $\theta$ of the very few of which it may be said with truth that every medical man should possess it.-London Medical Times, Ang. 26, 1865 .

Few works of the clase ex hibit a grander monument of patient research and of scientlitic lore. The extent of the sale of this lexicon is sutticient to testify to lts nsefulnes8, and to the great service confer red by Dr. Robley Dunglison on the profession, and indeed on ethere, by its isaue.-London Lancet, May 13, 1865.

The old edition, whlch is now superseded by the new, bas beèn nniversally looked upon by the medical profession as a work of immense research and great value. The new has increased usefuiness; for medicine, in all ito branches, has been making such progress that many aew terms a nd aubjects bave reoentiy been introónced: all of which may be fónd futly defined in the present edition. We know of no other dictionary in the English language that can bex $r$ a comparison with it in point of completeness of sobjects and accuracy of atatement. $-N$. Y. Druggists' Circular, 1865.

For many years Dunglibon's Dictlonary has been the standard book of reference with most practitioners in this country, and we can certainly commend this work to the renewed confidence and regard of our readers.-Cincinnati Lancet, A pril, 1865.

It is undoubtedly the most complete and usefal medical dictionary hitherto published in this country. - Chicago Med. Examiner, February, Is65.

What we take to be decidediy the best medical dictionary in the Engiish language. The present edition is brought fuily np to the advanced itate of science. For riany a long year "Dungtion" his been at our elbow, a constant companion and friend, and we greet him in bis replenished and improved form with especiat oatisfaction.-Pacific Med. and Surg. Jour. nal, June 27, 1865.

This is, perhaps, the book of all othere which the physician or burgeon should have on his sheives. It is more needed at the present day than a fow years back.-Cnnada Med. Journạl, July, 1865.

It deservedi $z$ stands at the bead, and cannot be surpassed in excelience.-Buffalo Med. and Surg. Journal, April, 1865.

We can sincerely commend Dr. Dongllson's work 88 most thorough, scientific, and accurate. We have tested it by searching its pages for new terms, which have abounded so much of late in medical nemen. clature, and our search bas been auccessfulin every instance. We have been particularly struck with the fulness of the synonymy and the accuracy of the derivation of words. It is a necessary a work to every enlightened physician as Worcester's Engilsh Dictionary is to every one who would keep np his know ledge of the Engiish tongue to the Btandard of the present day. It ik, to our mind, the most complete work of the kind with which we are acqnainted.Boston Med. and Surg. Journal, J nne 22, 1865.

We are free to confess that we know of no medical dictiouary more complete; no one better, if so well adapted for the use of the atudent; no one that may be consulted with more satisfaction by the medical practitioner.-Am. Jour. Med. Sciences, A pril, 1865.

The value of the present edition has been greatiy enhanced by the introduction of new Bubjects and terme, and a more complete et y nology a ud accentua. tion, which renders the work not outy satiafactory and desirable, but indispensable to the physician.Chicago Med. Journal, Aprii, 1565.

No inteligent member of the profession can or wil be without it. -St. Louis Med. and Surg. Journal. April, 1865 .

It has the rare merit that lt certainly has no rival in the English language tor accuracy and extent of references. - London. Medical Gazette.

GOBLFN (RICHARD D.), M.D.

A DICTIONARY OF THE TERMS USED IN MEDICINE AND

THE COLLATERAL SCIENCES. Revised, with numeron: additions, by JsaAc Hars, M.D., Editor of the "American Journal of the Medical Sciences." In one large royal 12mo. volume of over 500 donble-columned pages; extrs cloth, $\$ 150$; leather, $\$ 200$.

It is the best book of definitlons we have, and ought always to be apontbe stadent's table.-Scuthkrn Med. and Surg. Journal 
NEILL (JOHN), M.D., and SMITH (FRANCIS G.), M.D.,

\section{AN ANALYTICAL COMPENDIUM OF THE VARIOUS}

BRANCHES OF MEDICAL SCIENCE; for the Use and Examination of Students. A new edition, revised and improved. In one very large and handsomely printed royal $12 \mathrm{~m} \ldots$. volume, of about one thousand pages, with 374 wood outs, extra oloth, $\$ 4$; strongly bound in leather, with raised bands, $\$ 475$.

The Compend of Drs. Neill and Snith is incompara- cions factstreasured up in this little volume. A corebly the most valuable work of ita class ever published plete portable library so condensed that the stadent In this country. Attempts liave been made in varions may make it his constant pocket companion. - Westquarters to squeeze Anatomy, Physiology, Surgery, ern Lancet.

the Practlce of Medicine, Obstetrice, Materia Medica, and Chemistry into \& single mannal; but the operation hes signally falled in the hands of all ap to the adrent of "Netll and Smith' 8 " volume, which ts quite 2 miracle of success. The ontlines of the whole are admirably drawn and jllastrated, and the anthors are eminently entitled to the grateful consideration of the student of every class.-N. O. Med. and Surg. Journal.

There are but few students or practitloners of medicine unacquainted with the former editions of this nnassuming though highly instruetive work. The whole science of medicine appenrs to have been sifted, as the gold-bearing sands of El Dorado, and the pre-l

In the rapid course of lectures, where work for tbe atndents is heary, and review necessary for an exa. mination, a compend is not only valuable, but it is almost a gine qua non. The one before us is, in most of the division s, the most n nexceptionable of all books of the kind that we know of. Of course it is neelers for us to recommend it to all last conrse students, but there is a class to whom we very sincerely commend this cheap book as worth its weight in silver-that class is the gradnates in medicine of more then ten years' standing, who have not studied medicine since. They will perhaps find out from it that the ncience is not exactly now what it was when they left it off.-The Stethoscope.

\section{HARTSHORNE (HENRY), M.D., \\ Professor of Hygiene in the University of Pennsylvanta.}

A CONSPECTUS OF THE MEDICAL SCIENCES; containing Handbooks on Anatomy, Physiology, Chemistry, Materia Medioa, Practical Medicinn, Surgery, and Obstetrios. In one large royal $12 \mathrm{mo}$. volume of 1000 elosely printed pages, with over 300 illustrations on wood, extra cloth, $\$ 450$; leather, raised bands, $\$ 525$. (Lately Published.)

The ability of the author, and his practical skill in oondensation, give assurance that this work will prove valuable not only to the atudent preparing for examination, but also to the prac. titioner desirous of obtaining within a moderate compass, a view of the existing condition of the various departments of science connected with medicine.

This work is a remarkably complete one in its way, /less valuable to the beginner. Every medical stadent and comes nearer to onr idea of what a Conspectus who desires a reliable refresher to his raemory when should be than any we have yet seen. Prof. Harts- the pressure of lectures and ot ber collegework crowds horne, with a commendable forethought, Intrusted to prevent him from having an opportunlty to drink the preparation of many of the chapters on special deeper in the larger works, will find this one of the suljects to experts, reserving only angtomy, physio- greatest utility. It is thorough]y trustworthy frore logy, and practice of medicine to himself. As a result beginning to end and as we have before intimated, we have every department worked ap to the latest a remarkably truthful outline sketch of the present date and in a refreshlngly concise and lacid manner. state of medical science. We could hardly expect it There are an immense amount of illnstrations scat- should be otherwlse, however, under the charge of tered throughout the work, and althongh they have such a thorough medical scholar as the author bas often been seen before in the various works npon gen- already proved himself to be. $-\boldsymbol{N}$. York Med. Record, eral and special subjects, yet they will be none the March 15, 1869.

CUDLOW (J.L.), M. D.

A MANUAL OF EXAMiNations upon Anatomy, Physiology, Surgery, Practice of Medicine, Obstetrics, Materia Medion, Chemistry, Pharmacy, and Therapentics. To which is added a Medical Formulary. Third odition, thoroughly revised and greatly extended and enlarged. With 370 illustrations. In one handsome royal 12 mo. volume of 816 large pages, extra cloth, $\$ 325$; leather, $\$ 375$.

The arrangement of this volume in the form of question and answer renders it especially anit. able for the office examination of students, and for those preparing for graduation.

TANNER (THOMAS HA WTES), M.D., \&c.

A MANUAL OF CliNical MEDICINe AND PHYSICAL DIAG. NOSIS. Third American from the Second London Edition. Revised and Enlarged bs Tilbury Fux, M. D., Physician to the Skin Department in University College Hospital,

\&c. In one neat volume small $12 \mathrm{mo}$, , of about 375 pages, extra cloth. $\$ 150$. (Just Issued.)

* * By reference to the "Prospectus of Journal" on page 3, it will be seen that this work is offered as a premium for procuring new subseribers to the "American Jourval of the Medical Sciences."

Taken as a whole, it is the most compact vade mecum for the ase of the advanced etudent and junlor practitioner with which we are acquainted.-Boston Med. and Surg. Journal, Sept. 22, 1870.

It contajus eo much that is valuable, presented in so attractive a form, that it can hardly be spared eren in the presence of more fall and complete works. The additions made to the volume by Mr. Fox very materially enhance 1ts value, and almost make it a new work. Its convenlent size makes it a valuable companion to the conntry practitloner, and if constxutly cerried by h1m, wonld often render him good gervice, and relieve many a doubt and perplexity.Leaven worth Med. Herald, July, $18 \% 0$.

The objections commonly, and justly, urged againat the general rup of "compends," "conspectuses," and other aids to indolence, are not applicable to this little volume, which contalns in concise phrse fust thore practical detalls that sre of most use in daily disg. nosis, but which the yonng practltioner finds it difi. cult to carry always in his memory without gome quickly aocessible means of reference. Altogether, the book is one which we can heartily commend to those who have not opportunity for extensive reading, or who, having read mach, stitl wish an oceasional practical reminder. $-N$. Y. Med. Gazette, Nor. $10,1870$. 
GRAY (HENRY), F.R.S.,

Lecturer on Anatomy at St. George's Hospital, London.

ANATOMY, DESCRIPTIVE AND SURGICAL. The Drawings by H.V. CART ER, M. D., late Demonstrator on Anatomy at St. George's Hospital ; the Disseo. tions jointly by the Autror and Dr. Carter. A new American, from the fifth enlarged and improved London edition. In one magnificent imperial octavo volume, of nearly 900 pages, with 465 large and elaborate engravings on wood. Price in extra clotb, $\$ 600$; leather, raised bands, $\$ 700$. (Just Issued.)

The author has endeavored in this work to cover a more extended range of snbjects than is oustomary in the ordinary text-books, by giving not only the details necessary for the student, but also the application of those details in the practice of medicine and surgery, thus rendering it both a guide for the learner, and an admirable work of reference for the active practitioner. The en. gravings form a special feature in the work, many of them being the size of nature, nearly all original, and having the names of the various parts printed on the body of the cut, in place of figures of reference, with descriptions at the foot. They thus form a complete and splendid series, which will greatly assist the student in obtaining a clear idea of A natomy, and will also serve to refresh the memory of those who may find in the exigencies of practice the necessity of recalling the details of the dissecting room; while combining, as it does, a complete Atlas of Anatomy, with a thorough treatise on systematic, descriptive, and applied Anatomy, the work will be found of essential use to all physicians who receive students in their offices, relieving both preceptor and pupil of much labor in laying the groundwork of a thorough medical education.

Notwithstanding the enlargement of this edition, it has been kept at its former very moderate price, rendering it one of the cheapest works now before the profession.

The illastrations are beautifully executed, and ren-| From time to time, as successive editlons have apder this work an indispensable adjunct to the library peared, we have lad much pleasure in expressing of the surgeon. This remark applies with great force the general judgment of the wondertul excelieuce of to those surgeons practising at a distance from our large cities, as the opportunity of refreshing their memory by actnal dissection is not always attainable.-Canada Med. Journal, Aug. 1870.

The work is too well known and appreciated by the profession to need any comment. No medical man can afford to be without it, if its only merit were to serve as a reminder of that which so soon becomes forgotten, when not called into frequent use, viz., the relations and names of the complex organism of the haman body. The present edition is much improved. -California Med. Gazette, July, 1870.

Gray's Anatomy has been so long the standard of perfection with every student of anatomy, that we need do no more than call attention to the improvement in the present edition.-Detroit Review of Med. and Pharm., Aug. 1870. Gray's Anatomy.-Cincinnati Lancet, July, 1870.

Altogether, it is nnquestionably the most complete and serviceable text-book in anatomy that has ever been presented to the student, and forms a atriking contrast to the dry and perplexing volumes on the same subject through which their predecessors struggled in days gone by. $-N$. $\boldsymbol{Y}$. Med. Record, June 15, 1870 .

To commend Gray's Anatomy to the medical profession is almost as much a work of supererogation as it would be to give a favorable notice of the Bible in the religious press. To say that it is the most complete and conreniently arranged text book of its kind, is to repeat $w$ hat each geceration of students has learned as a tradition of the ellers, and verifled by personal experience.-N. Y. Med. Gazette, Doo. 17,1870 .

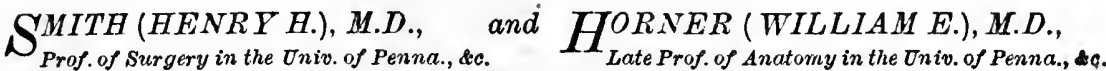
AN ANATOMICAL ATLAS, illustrative of the Structure of the Human Body. In one volume, large imperial octavo, extra cloth, with abont six hundred and fifty beautiful figures. $\$ 4 \mathbf{5 0}$.

The plan of this Atlas, which renders it so pecu- the kind that has yet appeared; and we must add, liarly convenient for the student, and its euperb ar- the very beautifui manner in which it is "got np," tistical execution, have been already pointed ont. We is 80 credituble to the country as to be fiattering to must conglatulate the stadent apon the completion onr national pride.-American Medical Journal. of this Atlas, as it is the most convenient work of

\section{$S^{H A R P E Y}$ (WILLIAM), M.D., and $Q^{\text {UAIN (JONES\& RICHARD). }}$}

HUMAN ANATOMY. Revised, with Notes and Additions, by Joseph Leidr, M.D., Professor of Anatomy in the University of Pennsylvania. Complete in two large ootavo volumes, of about 1300 pages, with 511 illustrations; extra cloth, $\$ 600$.

The very low price of this standard work, and its completeness in all departments of the sabject, should command for it a place in the library of all anatomical students.

HODGES, (RICHARD M.), M.D., Late Demonstrator of Anatomy in the Medical Department of Harvard University.

PRACTICAL DISSECTIONS. Second Edition, thoroughly revised. In one neat royal $12 \mathrm{mo}$. volume, half-bound, $\$ 200$.

The object of this work is to present to the anatomioal student a clear and ooncise description of that which he is expected to observe in an ordinary conrse of dissections. The author has endeavorod to omit unnecessary details, and to present the subje st in the form which many years' experienoe has shown him to be the most convenient and intelligible to the student. In the revision of the present edition, he has sedulously labored to render the volume more worthy of the favor with which it bas beretofure been received. 
WILSON (ERASMUS), F.R.S.

A SYSTEM OF HUMAN ANATOMY, General and Special. Edited by W. H. Gobracht, M. D., Professor of General and Surgical Anatomy in the Medical Col. lege of Ohio. Illustrated with three hundred and ninety-seven engravings on wood. In one large and handsome octavo volume, of over 600 large pages; extra cloth, $\$ 400$; leather, $\$ 000$.

The publisher trusts that the well-earned reputation of this long:established favorite will be more than maintained by the present edition. Besides a very thorough revision by the author, it has been most carefully exanined by the editor, and the efforts of both have been directed to in. troducing everything which increased experience in its use has suggested as desirable to render it a complete text-book for those seeking to obtain or to renew an acquaintance with Human Ana. tomy. The amount of additions which it has thus received may be estimated from the fact that tha present edition contuins over one-fourth more matter than the last, rendering a smaller type sul an enlarged page requisite to keep the volume within a convenient size. The author has not only thus added largely to the work, but he bas ulso made alterations throughout, wherever there sppeared the opportunity of improving the arrangement or style, so as to present every fact in its most appropriate manner, and to render the whole as clear and intelligible as possible. The editor bus exercised the utmost caution to obtain entire accuracy in the text, and has largely increased the number of illustrations, of which there are about one hundred and fitty more in this edition than in the last, thus bringing distinctly before the eye of the student everything of interest or importance.

\section{$H^{E A T H}($ Teacher of Operative Surgery in University College, London.}

PRAC'TICAL ANA'TOMY: A Manual of Dissections. From the Second revised and improved London edition. Edited, with additions, by W. W. KEEN, M. D., Lecturer on Pathological Anatumy in the Jefferson Mediual College, Philadelphia. In one handsome royal 12 wo. volume of 578 pages, with 247 illustrations. Extra cloth, $\$ 350$; leather, $\$ 4$ v0. (Lately Published.)

Dr. Keen, the American editor of this work, in his, Such manuals of anatomy arealways farorite works

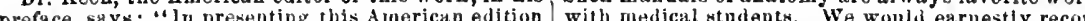
of 'Heath's Practical Auatomy,' I feel that I hare mend this ouo to their attention; it has excellences been instrumental in supplying a want long felt for which make it valuable as a guide in dissectlug, as a real dissector's manual," and this assertion of its. well as in stadying anatomy.-Buffabo Medical and scitor we deem is fully justified, atter an examina- Surgical Journal, Jan. 1871.

tion of its contents, for it is really an excellent work. The first Euglish edition was issued about six years ladeed, we do not hesitate to say, the best of its class ago, and was lavorably rectived not ouly on account with which we are acquainted; resembling Wilson ot the great reputation of its author, but also from in terse aud clear description, excelliug most of the its great value and excelleuce as a guide-book to the co-called practical anatomical dissectors in the scope practical anatomist. The Americau editon has an. of the subject and practical selected matter. - . . dergune some alteratlons and additions which will In readiog this work, oue is furcibly inpressed with no duubt enhance its value materially. The conve. the great pains the author takes to impress the oub- nience of the student has been carefuliy consulted in ject upon the mind of the student. He is full of rare the arrangement of the text, and the directions given and pleasiug little devices to aid memory in main- for the prosecution of certain dissections will beduly taiulng its hold upon the slippory slopes of anatumy. -St. Louis Med. and Surg. Journal, Iar. 10, 1871.

It appears to 28 certain that, as a guide in dissection, aud as a work coutaiulug liacts of anatomy in brief and easily nuderstood lorm, this manual is cumplete. 'This work contains, also, very perfect lisustrations of parts which cau thus be more easily nuderstood and studied; in this respect it compares farorably with works of much gruater preteusion.

This is an excellent Dissector's Manual; one which is not merely a descriptive manual of anatomy, but a guide to the student at the dissecting table, enubling hu, though a begiuuer, to prosecute his work iutelligently, and without assistauce. The American editor has made many valuable alteratious and additions to the origiual work. -Am. Journ. of Ubstetrics, Feb. Is71.

MCLISE (JOSEPH).
SURGICAL ANA'TOMY. By Joseph Machise, Surgeon. In one volume, very large imperial quarto; with 68 large and splendid plates, drawn in the best style and beautifully colored, containing 190 figures, wany of them the size of life; together with copious explanatory letter-press. Strongly and handsomely bound in extra cloth. Price $\$ 1400$.

AB no complete work of the kind has heretofore been published in the English language, the present volume will supply a want long felt in this country of an accurate und comprehensive Atlus of Surgical Anatomy, to which the student and practitioner can at all times refer to ascer. tain the exact relative positions of the various portions of the human frame towards each other and to the surface, as well as their abnormal deviutions. Notwithstanding the large size, beauty and finish of the very numerous illustrations, it will be observed that the price is so low as to place it within the reach of all members of the profession.

We know of no work on eurgical anatowy which can compete with 1.._Lancet.

The work of Maclise on surgical anatomy is of the highest value. In some respects it is the best pablication of its kind we have seen, and is worthy of a place in the libiary of any medicat man, while the ot udent could scarcely make a better Investment than this.-The Western Journal of Mtedicine and Surgery.

No euch lithographic jllustrations of surgical reglone have hitherto, we think, been given. While the operator is shown every vessel and nerve where an operation is contemplated, the exact anatomist is

refreshed by those clear and distinct dissections, whlch every one muat appreciate who has a parificle of enthusiasm. The English medical press has quite exhausted the words of praise, in recolumending this admirable treatise. Thuse who have any curiosity to gratify, in reference to the perfectibuity of the lithographic art in delineating the complex inechanism of the human body, are invited to examine our specimen copy. If auything will induce surgeon and students to patronize a book of such rare value and everyday inportance to them, it wlll be a survey of the artistical skill exhibited in these fac-eimlles of nature.-Bostun Med. and Surg. Journal. 
MARSHALL (JOHN), F.R. S.,

Professor of Surgery in University College, London, \&e.

OUTIINES OF PHYSIOLOGY, HUMAN AND COMPARATIVE. With Additions by Francis Gorner Surte, M. D., Professor of the Institutes of Medi. cine in the University of Pennsylvania, \&c. With numerous illustrations. In one large and handsome octavo volume, of 1026 pages, extra cloth, $\$ 650$; leather, raised bande, $\$ 750$.

In fact, in every respect, Mr. Marshall has present- ern physiological ecience, both haman and compara. od us with a most complete, reliable, and scientific tive, with which we are acquainted. To speak of (1) work, and we feel that it is worthy our warmest commendation.-St. Louis Med. Reporter, Jan. 1869.

This is an elaborate and carefully prepared digest of human and comparative physiology, designed for the use of general readers, but more especially serviceable to the student of medicine. Its style is conoise, clear, and scholarly; Its order perspicuous and exact, and its range of topics extended. The author and his American editor have been careful to bring to the illustration of the subject the important discoveries of modern science in the various cognate departments of investigation. This is especially visible in the variety of interesting information derived from the departments of chemistry and physics. The great amount and variety of matter contained in the work is strikingly illustrated by turning over the copious index, covering tweuty-four closely printed pages in double columns.-Silliman's Journal, Jan. 1869.

We doubt if there is in the English ianguage any oompend of physiolugy more useful to the student than this work. -St. Louis Med. and Surg. Journal, Jan. 1869 .

It quite fulfils, in our opinion, the anthor's design of making it truly educational in its character-which Is, perhaps, the bighest commendation that can be asked.-A m. Journ. Med. Sciences, Jan. 1869.

We may now congratulate him on having com. pleted the latest as well as the best summary of mod- $[25,1868$.

CARPENTER (WILLIAM B.), M. D., F.R.S.,

Examiner in Physiology and Comparative Anatomy in the University of London.

PRINCIPLES OF HUMAN PHYSIOLOGY ; with their chief applications to Psychology, Pathology, Therapeutics, Hygiene and Forensic Medicine. A new American from the last and revised London edition. With nearly three hundred illustrations. Edited, with additions, by Francis Gurney Smith, M. D., Professor of the Institutes of Medicine in the University of Pennsylvania, \&e. In one very large and beantiful octavo volume, of about 900 large pages, handsomely printed; extra cloth, $\$ 550$; leather, raised bands, $\$ 650$.

With Dr. Smith, we confidently believe "that the present will more than sustain the enviable reputaton already attained by former editions, of being one of the fullest and most complete treatizes on the subject in the English language." We know of none from the pages of which a satisfactory knowiedge of the physiology of the human organism can be as well obluined, none better adapted for the use of snch as take up the study of physioiogy in its reference to the institates and practice of medicine. $-\Delta m$. Jour. Mel. Sciences.

We donbt not it is destined to retain a strong hold on public favor, and remain the favorite text-book in our colleges. - Virginia Medical Journal.

The above is the title of what is emphatically the great work on physlology; and we are conscious that it wonld be a useless effort to attempt to add any. thing to the reputation of this invaluable work, and can only say to all with whom our opinion has any influence, that it is onr authority.-Atlanta Med. Journal.

\section{$B^{\text {T THE SAME AUTHOR. }}$}

PRINCIPLES OF COMPARATIVE PHYSIOLOGY. New American, from the Fourth and Revised London Edition. In one large and handsome octave volume, with over three hundred beautiful illustratione. Pp. 752. Extra cloth, $\$ 500$.

As a complete and condensed treatise on its extended and important subject, this work beoowee s necessity to students of natural science, while the very low price at which it is offered places it within the reach of all.

\section{KIRKES (WILLIAM SENHOUSE), M.D.}

A MANUAL OF PHYSIOLOGY. A new American from the third and improved London edition. With two hundred illustrations. In one large and handsome royal 12mo. volume. Pp. 586. Extra oloth, \$2 25; leather, $\$ 275$.

It ie at once convenient in eize, oomprehensive in | lent gnide in the study of physiology in its mest addesign, and concise in statement, and altogether well adapted for the parpose designed. - St. Louis Med. and Surg. Journal. ranced and perfect form. The author has shown in a condensed and conceutrated shape, on a science in which it is necessary at once to be correct and not

The pbysiologieal resder will ind it a post oxsol. liougthened.-Edinburgh Med. ant. Surg. Journal. 
DALTON (J. C.), M.D.

Professor of Physiology in the College of Physictans and Surgeons, Newo York, te.

A TREATISE ON HUMAN PHYSIOLOGY. Designed for the use of Students and Practitioners of Medicine. Fifth edition, revised, with nearly three han. dred illustrations on wood. In one very beantiful ootavo volume, of over 700 pages, extra cloth, \$5 25; leather, \$6 25. (Just Issued.)

\section{Preface to the Fifth Edition.}

In preparing the present edition of this work, the general plan and arrnngement of the previons -ditions have been retained, so far as they have been found useful and alapted to the purposes uf a text-book for students of medicine. The incessant advance of all the natural and physioal sciences, never more active than within the last five years, bas furnished many valuable aids to the special in restigutions of the physiologist; and the progress of physiolngical research, during the same period, has required a careful revision of the entire work, and the modification or rearrangement of many of its parts. At this day, nothing is regarded as of any value in natural gcience which is not based upon direct and intelligible observation or experiment; and, accorl. ingly, the discussion of doubtful or theoretical questions has been avoided, as a general rule, in the present volume, while new fucts, from whatever souroe, if fully established, have been added and incorporated with the results of previous investigation. A number of new illustrations have been introduced, and a few of the older ones, which seemed to be no longer useful, bave been omitted. In all the changes and additions thus made, it has been the aim of the writer to make the book, in its present form, a faithful exponent of the actual conditions of physiological science.

New YoRk, October, Is71.

In this, the standard text-book on Physiology, all that is needed to maintain the favor with which it is regarded by the profession, is the author's assurance that it has been thoroughly revised and brought up to a level with the advanced science of the day. To accomplish this has required some enlargement of the work, but no advance has been made in the price.

The finh edition of this truly valnable work on Human Physiology comes to us with many valuable improvements and additions. As a text-book of physiology the work of l'rof. Dalton has long been well knowa as one of the best which conld be plsced In the hands of student or practitioner. Prof. Dalton hus, in the several editions of his work heretofore published, labored to keep step with the ad vancement fu science and the last edition shows by jtaimprovements on former ones that he is determined to maintain the hlgh staudard of his work. We predict for the present edition increased favor, though this work has long been the favorite standard.-Buffalo Med. and Surg. Journal, April, 1872.

An extended notice of a work so generally and favorably known as this is unnecessary. It is jnstly regarded as one of the most valnable text-books on the aubject la the Eaglish langaage.-St, Louic Med. Arehives, May, 1872.

We know no treatise in physiology so clear, complete, well assimilated, and perfectly digested, as Dalton's. He never writes clondily or dubiously, or tn mere quotation. He assimilates all his material, and from it constructs a hotnogeneons transparent argument, which is always honest and well informed, and hides nelther irath, Ignorace, nor doubt, so far as either belongs to the subject in hand.-Brit. Med. Journal, March 23, 1872.
Dr. Dalton's treatise is well known, and by man highly estcemed in this country. It is, ludeed, a good elementary treative on the subject it professes to teach, and may xafely be put Into the hands of Eoglish students. It has one great merit-it ie clear, and, on the whole, admirabiy illustrated. The part we have always esteemed most highly is that relatiog to Embryology. The diagrams given of the various stages of development give a clearer view of the subject than do those in general use in this conntry; aod the text may be suid to be, upon the whole, equaliy clear.-London Med. Times and Gazette, Murch 23, I872.

Dalton's Physiology is already, and deservedly, the favorite text-book of the majority of American medical students. Tresting a most interesting department of selence ln his own peculis rly lively and fascinating style, Dr. Dalton carries hls reader along withont effort, and at the same time impresses npon bis mind the truths tanght much more successfully than if they were buried beueath a multitude of words.-Kansas City Med. Journal, April, IS72.

Professor Dalton is regarded justly as the anthority In this country on physiological subjects, and the ffth edition of his valuable work fully justifies the exalted opinion the medical world h*s of his labure. This lagt edition is greatly eularged - Virginia Clinical Record, April, IS72.

DUNGLISON (ROBLEY), M.D., Professor of Institutes of Medicine in Jefferson Medical College, Philadelphta.

HUMAN PHYSIOLOGY. Fighth edition. Thoroughly revised and extensicely modifed and enlarged, with five hundred and thirty-two illu trations. In two large and handsomely printed octavo volumes of about 1500 pages, extra cloth. $\$ 700$.

\section{LEHMANN (C. G.).}

PHYSIOLOGICAL CHEMISTRY. Translated from the second edition by Georer E. DAY, M. D., F. R. S., de., edited by R. E. Rogkrs, M. D., Professor of Chemistry in the Medical Department of the University of Pennoylvania, with illustrations selected from Funke's Atlas of Physiological Chemistry, and an Appendix of plates. Complete in two large and handsome octavo volumes, containing 1200 pages, with nearly two hundred illustrations, extra cloth. $\$ 600$.

\section{$B^{\text {T THE SAME AUTHOR. }}$}

MANUAL OF CHEMICAL PHYSIOLOGY. Translated from the German, with Notes and Additions, by J: Crestox Morris, M. D., with an Introductory Essay on -Vital Force, by Professor SAM ER JACksox, M. D., of the University of Penngylvania. With illustrations on wood. In one very handsome octavo volume of 336 pager, extra cloth. $\$ 225$. 
A TTFIELD (JOHN), Ph. D.,

Professor of Practical Chemistry to the Pharmaceutical Society of Great Britain, \&c.

CHEMISTRY, GENERAL, MEDICAL, AND PHARMACEUTICAL; including the Chemistry of the U.S. Pharmacopœia. A Manual of the Aeneral Prinoiples of the Science, and their Application to Medicine and Pharmacy. Fifth Edition, revised by the author. In one handsome royal 12mo. volume. (Neariy Ready.)

We commend the work heartily as one of the best rican work- we allnde to the introduction of the text-books extant for the medical student.-Detroit Rev. of Med. and Pharm., Feb. 1872.

The hest work of the kind in the English language. - N. Y. Psychological Journal, Jan. 1872.

The work is constructed with direct reference to the wants of medical and pharmacentical students and, although an English work, the points of differ ence betwecn the British and United States Pharmacopœiss are indicated, making it as useful here as in England. Altogether, the book is one we can heartily recommend to practitioners as well as studeuts. -N. Y. Med. Journal, Dec. 1871.

It differs from other text-hooks in the following particulars: fret, in the exclnsion of matter relating to compounds which, at present, s re only of interest to the scientific chemist; secondly, in containing the chemistry of every substance recognized officisliy or in general, as a remedial ggent. It will be found a most raluable hook for pnpils, essistants, and others engaged in medicine and phs rmacy, and we heartily commend it to our readers.-Canada Lancet, Oct. 1871 .

When the original English edition of this work was published, we had occasion to express onr high appreciation of its worth, and also to review, in considerable detail, the main features of the book. As the arrangement of subjects, and the main part of the text of the present edition are similar to the former publicatlon. it will be needless for us to go over the ground a second time; we may, however, call attention to a marked ad vantage possessed by the A me-

chemistry of the preparations of the United States Pharmacopeia as well as that relating to the British anthority. - Canadian Pharmaceutical Journal, Nov. 1571 .

Chemistry has borne the name of being a hard $6 \mathrm{nb}$. fect to master by the stndent of medicine, and chiefly because 80 much of it consists of componnds only of interest to the scientific chemist; in this work such portions are modified or altogether left out, and in the arrangement of the subject matter of the work proctical utility is songht after, and we think fnlly attained. We commend it for its clearnens and order to both teacher and pupil.-Oregon Med. and Surg, Reporter, Oct. 1871.

It contains a most admirable digest of what is ape. cially needed by the medical stndent in all that re lates to practical chemistr, and consitutes for him a sond and nsefnl text-book on the subject.

We commend it to the notios of every medical, as well a.s phs.rmaceutical, stadent. We only regret that $w$ had not the book to depend npon in working np the subject of practical and pharmacentical chemistry for the Cniversity of London, for which it geems to as the $t$ it is exactly adapted. This ia paying the book a high compliment. - The Lancet.

Dr. Attfeld's book is written in a clear and able manner; it is a work suigeneris and without a rival ; it wlll be welcomed, we think, by every reader of the 'Pharmacopnia,' and is quite as well snited for the medical student aa for the pharwacist.-The (Ihemical News.

WÖHLER AND FITTIG.

OUTLINES OF ORGANIC CHEMISTRY. Translated with Additions from the Eighth German Edition. By InA Remses, M.D., Ph.D., Professor of Chemistry and Physies in Williams College, Mass. In one bandsome volnme, royal $12 \mathrm{mo}$. of $550 \mathrm{pp}$. extra cloth, \$3. (Just Ready.)

As the numerous editions of the original attest, this work is the leading text-book nnd standard anthority throughout Germany on its important and intrioate subject-a position won for it by the clearness and conciseness wbich are its distinguishing characteristics. The translation has been executed with the approbation of Profs. Wöhler and Fittig, and numerous additions and alterations have been introduced, so as to render it in erery respect on a level with the most advanced condition of the science.

$O^{D L I N G(W I L L I A M)}$

Lecturer on Chemistry at St. Bartholomew's Hospital, \&e.

A CUURSE OF PRACTICAL CHEMISTRY, arranged for the Use of Medical Students. With Illustrations. From the Fourth and Revised London Edition. In one neat royal $12 \mathrm{mo}$. volume, extra cloth. $\$ 2 . \quad$ (Lately Issiced.)

A a work for the practitioner it cannot be excelled. / ganic chemistry, etc. The portiona deroted to a disIt is written plainly and concisely, and givesin a very cnssion of these subjects are very excellent. In no 8 mall compass the information required hy the basy work can the physician find more that is valuable practitioner. It la essentially a work for the physi. and reliable in regard to urine, bile, milk, bone, nrician, and noone who purchases it will ever regret the nary calcnli, tissue composition, etc. The work is outlay. In addition to all that is uaually given in smull, reasonable in price, and well pnblished.connection with inorganic chemiatry, there are most Richmond and Louisville Mrd. Journal, Dec. 1869. valuable contributions to toxicology, animal and or-

YALLOWAY (ROBERT), F.C.S.,

Prof. of Applied Chemistry in the Royal College of Science for Ireland, \&c.

A MANUAL OF QUALITATIVE ANALYSIS. From the Fifth London Edition. In one neat royal 12mo. volume, with illustrations; extra cloth, \$2 50. (Just Issued.)

The success which has carried this work through repented editions in England, and its adoption as a text-book in several of the leading institutions in this country, show that the anthor has sueceeded in the endeavor to produce a sound practical manual and book of reference for the chemical student.

Prof. Galioway's booka are degervedly in high esteem, and this American reprint of the ffth edition (18B9) of his Manual of Qualitative Analysis, will be acceptable to many American students to whom the English edition is not accessible. $-A m$. Jour. of Science and Arts, Sept. 1872.

We regard this volume as a valuable addition to the chemical text-books, and as particularly calcnlated to instrnct the student in analytical researches of the inorganic componnds, the important vegetable acids, and of compounds and various secretions and excretions of animal origin.-Am.Journ. of Pharm., Sept. 1872. 


CHANDLER (CHARLES F.). and
$\begin{gathered}\text { Prof. of Chemistry in the N. Y. Coll. of } \\ \text { Pharmacy. }\end{gathered}$ $\begin{gathered}\text { CHANDLER (WILLIAM H.), } \\ \text { Prof. of Chemistry in the Lehigh } \\ \text { University. }\end{gathered}$

THE AMERICAN CHEMIST: A Monthly Journal of Theoretical, Analytical, and Technical Chemistry. Each number averaging forty large double columned pages of reading matter. Price $\$ 5$ per annum in advance. Single numbers, $50 \mathrm{cts}$.

DF Specimen numbers to parties proposing to subscribe will be sent to any address on receipt of 25 cents.

*** Subscriptions can begin with any number.

The rapid growth of the Science of Chemistry and its infinite applications to other sciences and arts render a journal specially devoted to the subject a necessity to those whose pursuits require familiarity with the details of the science. It has been the aim of the conductors of "Trie AMEricas CuEmist" to supply this want in its broadest sense, and the reputation which the periodical has already attained is a sufficient evidence of the zeal and ability with which they have discharged their task.

Assisted by an able body of collaborators, their ain is to present, within a moderate compass, an abstract of the progress of the science in all its departments, scientific and technical. Important original communications and selected papers are given in full, and the standing of the "Curs1ST" is such as to secure the contributions of lealing men in all portions of the country. Besides this, over one hundred journals and transactions of learned societies in Amerien, Great Britain, France, Belgium, Italy, Russin, and Germany are carefully sorutinized, and whatever they offer of interest is.condensed and presented to the reader. In this work, which forms a special fenture of the "ChE mist," the éditors have the assistance of M. Alsberg, Ph.D., Prof. G. F. Burker, T. M. Blossom, E.M., H. C. Bolton, Ph.D., Prof. T. Egleston, E.M, H. Endemann, Ph.D., Prof. C. A. Goessmann, Ph.D., S. A. Goldschmidt, A.M., E.M., E. J. IInllock, Prof. C. A. Joy, Ph.D., J. P. Kimball, Ph.D., O. G. Masow, H. Newton, E.M., Prof. Frederick Prime, Jr., Prof. Paul Schweitzer, Ph.D., Waldron Shapleigh, Romyn Hitcheock, and Elwyn Waller, E.M. From the thoroughness and completeness with which this department is conducted, it is believed that no periodical in either hemisphere more faithfully reflects the progress of the science, or presents a lnrger or more carefully garnered store of information to its readers.

\section{FTOWNES (GEORGE), Ph.D.}

A MANUAL OF ELEMENTARY CHEMISTRY; Theoretical and Practical. With one bundred and ninety-seven illustrations. A new American, from the tenth and revised London edition. Edited by RoB ERT BRIDGes, M. D. In one large royal $12 \mathrm{mo}$. volume, of about $850 \mathrm{pp}$., extra cloth, $\$ 275$; leather, $\$ 325$. (Lately Issued.)

This work is so well known that it seems almost and nuder the editorship of Mr. Watte, it has resumed superfinous for us to speak abont it. It has been a its old place as the most successful of text-books. favorite text-book with medical students for years, Indian Medical Gazette, Jan. 1, 1869.

and its popularity has in no respect diminished.

Whenever we have beea consulted by medical students, as has frequently occurred, what treatise on chemistry they should procure, we have always re. commended Fownes', for we regarded it as the best. There is no work that combines so many excellences. It is of convenlent size, not prolix, of plain perspicuous diction, contains all the most recent disceveries, and is of moderate price.-Cincinnati Med. Repertory, Aag. 1569.

Large additions have been made, especialiy in the department of organic chemistry, and we know of no ot her werk that has greater claims on the physician. pharmacentist, or student, than this. We cheerfuliy racommend it as the best text-book on elementary chemistry, and bespeak for it the careful attention of students of pharmacy. - Chicago Pharmaclst, Aug. 1869 .

The American reprint of the tenth revised and corrected English edition is now issued, and represents the present condition of the science. No comments are necessary to insure it a favorable reception at the hands of practitioners and etudents. - Boston Med. and Surg. Journal, Aug. 12, 1869.

Here le a new edition which has been long watched for by eager teachers of chemistry. In its new garb,

It will continue, as heretofore, to hold the first rank is a text-book for students of medicine.-Chicago Med. Examiner, Aug. 1869.

This work, long the recognized Ma nual of Chemistry, appears as a tenth edition, under the able editorship of Bence Jones and Henry Watts. The chapter on the General Principles of Chemical Philusuphy, and the greater part of the organic chemistry, have been sewritten, and the whele work revised in accordance with the recent ad vances in chemical knowledge. It remains the standard text-book of chenistry. $-D u b$ lin Quarterly Journal, Feb. 1869.

There is probably net a student of chemistry in this country to whom the admirable manual of the late Professor Fownes is anknown. It has achieved a access which we believe is entirely without a parallel among scientiflc text-books in our language. This success has arisen from the fact that there is no English work on chemistry which combines so many excellences. Of conveniont size, of attractive form, clear and concise in diction, well illusirated, and of moderate price, it would seem that every rejuisite for a student's hand-bonk has been attained. - The Clicmical New8, Feb. 1869.

BOWMAN (JOHN E.), M. D.

PRACTICAL HANDBOOK OF MEDICAL CHEMISTRY. Edited by C. L. Broxa , Professor of Practical Chemistry in King's College, London. Fiftb American, from the fourth and revised English Edition. In one neat volume, royal 12mo., pp. 351, with numerous illustrations, extre oloth. \$2 25.

$B^{Y}$ THE SAME AUTHOR.

INTRODUCTION TO PRACTICAL CHEMISTRY, INCLUDING ANALYSIS. Fifth American, from the ffth and revised London edition. With numerous illastrations. In one neat vol., royal 12mo., extra cloth. $\$ 225$.

RNAPP'S TECH NOLOGY ; or Chemistry Applied to the Arts, and to Manufactures. With American additions, by Prof. WALter R. Johssos. In two very handsome octaro rolnmes, with 600 wocd engravingt, extra cloth, $\$ 60$. 
PARRISH (EDWARD),

Professar of Materia Medica in the Philadelphia College of Pharmacy.

A TREATISE ON PHARMACY. Designed as a Text-Book for the Student, and as a Guide for the Physician and Pharmaceutist. With many Formulæo and Prescriptions. Third Edition, greatly improved. In one handsome octavo volume, of 850 pages, with several hundred illustrations, extra cloth. $\$ 500$; leather, $\$ 600$.

The immense amount of practical information condensed in this volume may be estimated from the fact that the Index contains about 4700 items. Under the head of Acids there are 312 refer. onces; under Emplastrum, 36 ; Extracts, 159; Lozenges, 25; Mixtures, 55; Pills, 56 ; Syrups, 131; 'Tinctures, 138 ; Unguentum, 57 , \&o.

We have examined this large volnme with a good not wish it to be understood as very extravagant deal of care, and find that the author has completely praise. In trnth, it is not so much the best as the exhansted the subject apon which be treats; a more only book.-The London Chemical Nevs.

complete work, we think, it wonld be impossible to find. To the student of pharmacy the work is indispensable; indeed, so far as we know, it is the only one of its kind in existence, and even to the physician or medical student who can spare five dollars to pnrchase it, we feel sure the practical information he will obtain will more than compensate him for the ontlay.-Canada Med. Journal, Nov. 1864.

The medical stndent and the practising physlcian will find the volume of inestimable worth for study and reference.-San Francisco Med. Press, July, $186 \pm$.

When we gay that this book is in some respects

the best which has been published on the subject in

An attempt to furnish anything tike an analysle of Parrish's very valuable and elaborate Treatise on Practical Pharmacy wonld require more space than we have at onr disposal. This, however, is not 80 much a matter of regret, lnasmuch as it would be difficult to think of any point, however minute and apparently trivial, convected with the manipulation of pharmaceutic substances or appliances which has not been clearly and carefally discassed in this rolume. Want of epace prevents our enlarging farther on this valuable work, and we must conclude by a simple expression of our hearty appreciation of $\mathrm{its}$ merits.-Dublin Quarterly Jour. of Medical Science, August, 1S64.

STILLÉ (ALFRED), M. D.,

Professor of Theory and Practice of Medicine in the University of Penna.

THERAPEUTICS AND MATERIA MEDICA; a Systematic Treatise on the Action and Uses of Medicinal Agents, including their Description and History Fourth edition, revised and enlarged. In two large and handsome octaro volumes. (Preparing.)

Dr. Stille's splendid work on therapentics and materia medica.-London Med. Times, April 8, 1865.

Dr. Stille stands to-day one of the best and most honored representatives at home and abroad, of American medicine; and these volumes, a library in themselves, a treasure-house for every studious physician, assure his fame even had he done nothing more.-The Western Journal of Medicine, Dec. 1868.

We regard this work as the best one on Materia sfedica in the English language, and as such it deserves the favor it has received.-Am. Journ. Medical Sciences, July 1868.

We need not dwell on the merits of the third edition of this magniff cently conceived work. It i the work on Materia Medica, in which Therapeutics are primarily considered-the mere natural hlstory of drugs being brietly disposed of. To medical practitioners this is a very valuable conception. It is wonderful how much of the riches of the literature of Materia Medica has been condensed in to this book. The references alone would make it worth possessing. But it is not a mere compilation. The witer exercises a good jadgment of his own on the great doctrines and points of 'Therapeutics. For purposes of practice, Stille's book is almost nnique as a repertory of information, empirical and scientiflo, on the actions and uses of medicines.-London Lancet, Oct. 31, 1868.

Through the former editions, the professional world is well acquainted with this work. At home and

abroad its repntation as a standard treatise on Materia Medica is securely established. It is second to no work on the subject in the Euglish tongue, and, in. deed, is decidedly superior, in some respects, to any other.-Pacific Mfed. and Surg Journal, July, 1868. Stille's Therapeutics is incomparably the best work on the subject.-N. Y. Med. Gazette, Sept. 26, 1868.

Dr. Stille's work is becoming the best known of any of onr treatises on Materia Medica. . . . One of the most valuable works in the language on the snbjects of which it treats.-N. Y. Med. Journal, Oct. 1868.

The rapid exhaustion of two editions of Prof. Stille's scholarly work, and the consequent necessity for a third edition, is sufficient evidence of the high estimate placed apon it by the profession. It is no exaggeration to say that there is no saperior work apon the subject in the English language. The present edition is fully up to the most recent advance in the science and art of therapeutics.-Leavenworth Medical Herald, Ang. 1868.

The work of Prof. Stille bas rapidly taken a bigh place in professional esteem, and to say that a third edition is demanded and now appears before us, suffciently attests the firm position this treatise has mado for itself. As a work of great research, and scholarship, it is safe to say we have nothing superior. It is exceedingly full, and the busy practitioner will find ample suggestions apon almost every inportant point of therapontics. - Cineinnati Lancet, Aug. 1568.

\section{GRIFFITH (ROBERT E.), M.D.}

A UNIVERSAL FORMULARY, Containing the Methods of Preparing and Administering Officinal and other Medicines. The whole adapted to Physicians and Pharmaceutists. Second edition, thoroughly revised, with numerous additions, by Ronert P. Thомas, M.D., Professor of Materia Medica in the Philadelphia College of Pharmacy. In one large and handsome octavo volume of 650 pages, double-columns. Extra cloth, $\$ 400$; leather, $\$ 500$.

Three complete and extended Indexes render the work especially adapted for immediate consul. tation. One, of DisEASES AND THEIR REMEDIES, presents under the head of each disease the remedial agents which have been usefully exhibited in it, with reference to the formulw oontaining them-while another of Pharmaceutical and Botanical Names, and a very thorough GinkRAL INDEX afford the means of obtaining at once any information desired. The Formulary itself is arranged alphabetically, nnder the heads of the leading constituents of the preseriptions.

We know of nove in our language, or any other, so compreheneive in its details. - London Lancet.

one of the most complete works of the kind in any language.-Edinburgh Med. Journal.

We are not cognizant of the exfotence of a parallel work.-London Med. Gazatto. 
PEREIRA (JONATHAN), M.D., F.R.S. and L.S.

MATERIA MEDICA AND THERAPEUTICS; being an Abridgment of the late Dr. Pereira's Elements of Materia Medica, arranged in conformity with the British Pharmacopcia, and adapted to the use of Medical Practitioners, Chemists and Druggists, Medical and Pharmaceutical Students, tc. By F. J. FArRe, M.D., Senior Physician to St. Bartholomew's Hospital, and London Editor of the British Pharmacopoia; assisted by RoBert Bentrey, M.R.C.S., Professor of Materia Mediea ahd Botany to the Pharmaceutical Society of Great Britain; and by RoBert WARINGTON, F.R.S., Chemica) Operator to the Society of Apotheenries. With numerous additions and references to the United States Pharmacopcia, by Honario C. WOOD, M.D., Professor of Botany in the University of Pennsylvania. In one large and handsome octavo volume of $1040 \mathrm{closely}$ printed pages, with 236 illustrations, extra oloth, $\$ 700$; leather, raised bands, $\$ 800$

The task of the American editor hasevidently been, poia, none will be more acceptable to the student no sinecure, for not only hss he given to us all that and practitioner than the preseat. Pereira's Materia ie contained in the abridgment usefut for our pur- Medica had longagoassertod for itself the posttion of poses, but by a carefut and judicious embodiment of being the most complete work on the subject in the over a handred new retusdies has increased the stze of the former work fulty one-tbird, besides adding in the way of its success. Except is the way of refermany new itfastratfons, some of which are origiual. ence, or to those who made a special study of iateria We unhesitatingly say that by so doing he has pro- Medica, Dr. Pereira's work was too fntt, and its peportionatety increased the value, not only of the con- rusal required an amonnt of time which few had at censed edition, bat has extended the appticabitity of theirdisposal. Dr. Farre has very judiciously aratied the great original, and has placed his medical coun- himself of the opportuait of the pubitication of the trymen under lasting obligations to bim. The Ame- new Pharmacopcia, bybringiag out an abridged edirican physician now bas atl that is needed in the tion of the great work. This edition of Pereira is by shape of a complete treatise on materia medica, and no means a mereabridged re-issue, bnt contains many the rnedical studeot has a text-book which, for prac- improvements, both in the descriptive and theratical ntitity and intrinsic worth, stands noparalteled. peutical departments. We can recommend it as a Although of considerabte size, it is none too targe for very excellent and reliable text-book.-Edinburgh the purposes for which it hasbeen in tended, and every nedica man should, in jnstice to himself, spare a place for it npon his book-sielf, resting assured that the more he consults it the better he will be satisfled of its excellence.-N. Y. Med. Record, Nov. 15, 1866.

It will fill a place which no other work can occupy iu the library of the physician, student, and apothecary.-Boston Med. and Surg. Jourhal, Nov. 8, 1866.

Of the many worke on Materia Medica which have appeared since the issuing of the British Pharmaco-

Med. Journal, February, 1866.

The reader cannot fail to be impressed, at a piance with the exceeding vatue of this work as a compeud of nearly all usefui knowiedge on the materia inedica. We are greatly indebted to Professor Wond for bls adaptation of it to our meridian. Without his emendutions and additious it wonld lose much of its ratue to the American student. With them it is an American book. - Pacific Medical and Surgical Journal, December, I\$66.

\section{$E^{L L I S}(B E N J A M I N)$, M.D.}

THE MEDICAI FORMULARY: being a Collection of Prescriptions derived from the writings and practice of many of the most eminent physicians of America and Europe. Together with the usual Dietetic Preparations and Antidotes for Poisons. The whole accompanied with a few brief Pharmaceutic and Medical Observations. Twelfth edition, carefully revised and much improved by Aцвент H. Sмгтн, M. D. In one volume 8v.. of 376 pages, extra cloth, $\$ 300$. (Lately Published.)

This work has remained for some time nut of print, owing to the anxions care with which the Editor has sought to render the present edition worthy a continuance of the very remarkable fuvor which has carried the volume to the unusual honor of a Twectir Edrion. He has sedu. luusly endeavored to introduce in it all new preparations and combinations deserving of confidence, besides adding two new classes, Antemetics and Disinfectants, with brief references to the inhalation of atomized fluids, the nasal douche of Thudichum, suggestions upon the method of hypodermio injection, the administration of anmsthetics, \&o. \&o. To accommodate these numerous additions, he has omitted much which the advince of science has rendered obsolete or of minor importince,

- Dotwithstanding which the volume has been increased by more than thirty pages. A new feature will be found in a copious Index of Diseases and their remedies, which cannot but increase the value of the work as a suggestive book of reference for the working practitioner. Every preonution bas been taken to secure the typographical accuracy so necessary in a work of this nature, and it is hoped that the new edition will fully maintain the position which "ELL1S' ForMULARY" has long occupied.

CYARON (JOSEPH), M.D.,

Professor of Materia Medica and Pharmacy in the University of Pennsylvania, \&c.

SYNOPSIS OF THE COURSE OF LECTURES ON MATERIA MEDICA AND PHARMACY, delivered in the University of Pennsylvania. With three Lectures on the Modus Operandi of Medicines. Fourth and revised edition, extra sloth, $\$ 300$.

CUNGLISON'S NEW REMEDIES, WITH FORMULF F()R THEIR PREPARATION AND ADMINISTRATION Seventh edition, with extensive additions. One vol. Svo., pp. 770; extra cloth. $\$ \$ 00$.

KOYLE'S MATERIA MEDICA AND THERAPEU. Tiss, Edited by Josfra R CARsox, M. D. Witb nivety-eight illustrations. 1 vol. 8vo., pp. 700, extra cloth. 300 .

CHRISTISON'S DISPENSATORY. With copions additions, and 213 large wood-engravings. Bg $\mathbb{R}$
Ealfapelib Griffith, M. D. One vol.8vo., pp. 1000 ; extracloth. $+m$.

CARPENTER'S PRIZE ESSAT ON THE USE OF A rooholic Lrqcors ix Healtr and Diseagr. New edition, with a Preface by D. F. Cospir. M.D., and explanations of scientific words. In one neat $12 \mathrm{~mm}$ volnme, pp. 178, extra cluth. 60 cents.

DE JONGH ON THE THREE KINDS OF COD-LIVER O1L, with their Chemical and Therapentic Properties 1 rol. 12wo., clotb. 75 cents. 
GREEN (T. HENRY), M.D.,

Lecturer on Pathology and Morbid Anatomy at Charing-Cross Hospital Medical School.

.PATHOLOGY AND MORBID ANATOMY. With numerous Illus-

trations on Wood. In one very handsome octavo volume of over 250 pages, extra oloth, \$2 50. (Lately Published.)

The soope and object of this volume can be gathered from the following condensed

We have been very much pleased by our perusal of thology and morbid anatomy. The author shows that this little volume. It is the only one of the kind with he has been not only a student of the teachings of his which we are acquainted, and practitioners as well confreres in thls branch of science, but a practical as students will find it a very useful guide; tor the and conscientious laborer in the post-mortem chutn. information is up to the day, well and compactly ar- ber. The work will prove a useful one to the great ranged, without being at all scanty.-London Lan- mass of students and practitioners whose time for de. cet, 0ct. 7, 1871 .

It embodies in a comparatively small space a clear statement of the present state of our knowledge of pa-

GLUGE'S ATLAS OF PATHOLOGICAL HISTOLOGY. Translated, with Notes and Additions, by Josepr LEIDY, M. D. In one volume, very large imperial quarto, with 320 copper-plate figures, plain and colored, extra cloth. $\$ 400$.

SIMON'S GENERAL PATHOLOGY, as conducive to the Establishment of Ratioual Principles for the Prevention and Cure of Disease. In one octavo volume of 212 pages, extra cloth. \$1 25.

SOLLY ON THE HUMAN BRAIN ; ite Strnctnre, Physiology, and Diseases. From the Second and much enlarged London edition. In one octaro volume of 500 pages, with 120 wood-cnts; extra cloth. $\$ 250$.

LA ROCHE ON YELLOW FEVER, considered in its Historical, Pathological, Etiological, and Therapen-। tical Relations. In two large and handsome octavo volu mes of nearly 1500 pages, extra cloth. $\$ 700$.

HOLLAND'S MEDICAL NOTES ANI REFLEC. Tions. 1 vol. 8vo., pp. 600, extra cloth. $\$ 350$

WHAT TO OBSERVE AT THE BEDSIDE AND AFTER Deatr in Medical Cases. Published under the anthority of the London Society for Medical Obser. vation. From the second London edition. I vol. royal $12 \mathrm{mo}$., extra cloth. \$1 00 .

LAYCOCK'S LECTURES ON THE PRINCIPLES AND Methodg op Medical Observation aNd ReseARCF. For the use of advanced students and junior practitioners. In one very neat roy $212 \mathrm{mo}$. volnme, extra cloth. $\$ 100$.

\section{GROSS (SAMUEL D.), M. D.,}

Professor of Surgery in lhe Jefferson Medical College of Philadelphia.

ELEMENTS OF PATHOLOGICAL ANATOMY. Third edition, thoroughly revised and greatly improved. In one large and very handsome octavo volume of nearly 800 pages, with about three hundrod and fifty beautiful illustrations, of which a large number are from original drawings; extra cloth. $\$ 4^{\circ} 00$.

TONES (C. HANDFIELD), F.R.S., and SIEVEKING (ED. H.), M.D., Assistant Physicians and Lecturers in St. Mary's Hospital.

A MANUAL OF PATHOLOGICAL ANATOMY. First American edition, revised. With three hundred and ninety-seven handsome wood engravings. In one large and beautifully printed octavo volume of nearly 750 pages, extra cloth, $\$ 350$.

$B^{A R C L A Y}(A . W),. M . D$.

A MANUAL OF MEDICAL DIAGNOSIS; being an Analysis of the Signs and Symptoms of Disease. Third American from the second and revised London edition. In one neat octavo volume of 451 pages, extra cloth. $\$ 350$.

WILLIAMS (CHARLES J. B.), M.D.,

Professor of Clinical Medicine in University College, London.

PRINCIPLES OF MEDICINE. An Elementary View of the Canses; Nature, Treatment, Diagnosis, and Prognosis of Disease; with brief remarks on Hygienics, or the preservation of health. A new American, from the third and revised London edition. In one octavo volume of about 500 pages, extra eloth. $\$ 350$.

DUNGLISON, FORBES, TWEEDIE, AND CONOLLY.

THE CYCLOP EDIA OF PRACTICAL MEDICINE: comprising Treatises on the Nature and Treatment of Diseases, Materia Medica and Therapeatics, Diseases of Women and Children, Medical Jurisprudence, dc. \&o. In four large super-royal octavo volumes, of 3254 double-columned pages, strongly and handsomely bound in leather, $\$ 15$; extra oloth. $\$ 11$.

*** This work contains no less than four hundred and eighteen distinct treatises, contributed by sixty-eight distinguished physicians.

FOX (WILSON), M.D., Holme Prof. of Clinical Med., Universily Coll., London.

THE DISEASES OF THE STOMACH: Being the Third Edition of the "Dingnosis and Trentment of the Varieties of Dyspepsia." Revised and Enlarged. With illustrations. In one handsome octavo volume. (In Press.)

The present edition of Dr. Wilson Fox's very admi- Dr. Fox has put forth a volnme of uncommon exrable work difers from the preceding in that it deals with other maladies than dyspepsia ouly. - London Med. Times, Feb. 8, 1573 . cellence, which we feel very sure will take a high rank among works that treat of the stornach. $-A m$. Practitioner, March, 1873. 


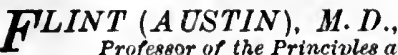

Professor of the Principles and Practice of Medicine in Bellevue Med. College, N. $\boldsymbol{Y}$.

\section{A TREATISE ON THE PRINCIPLES AND PRACTICE OF}

MEDICINE; designed for the use of Students and Practitioners of Medicine. Fourth edition, revised and enlarged. In one large and closely printed octavo volume of ahout 1100 pages; handsome extra cloth, $\$ 600$; or strongly bound in leather, with raised bands, $\$ 700$. (Nearly Ready.)

By common consent of the English and American medical press, this work has been assigned to the highest position as a complete and compendious text-book on the most advanced condition of medical science. At the very moderate price at which it is offered it will be found one of the cheapest volumes now before the profession.

Aduirable and naequalled. - Western Journal of | xcellently printed and bound - and we enconnter Medicine, Nov. 1869.

Dr. Flint's work, though elaiming no bigher title than that of a text-book, is really more. He is a man of large clinical experience, and his book is full of vuch nasterly descriptions of disease as can unly be drawo by a man intimately acquainted with their rarious forms. It is not so long since we had the pleasnre of reviewing his first edition, and we recogbize a great improtement, especially in the general part of the work. It is a work which we can cordially recommend to on roaders as fully abreast of the science of the day.-Edinburgh Med. Journal, Oct.' 69.

One of the best works of the kind for the practitiover, and the most convenient of all for the studeat. -Am. Journ. Med. Sciences, Jun. 1869.

This work, which stands pre-eminently as the adrance standard of medical science up to the present time in the practice of inedicine, has for its unthor one $w$ bo is well and widely known as one of the iesding practitioners of this continent. In fact, it is seldom that any work is ever issued from the pres: more deserving of universal recommendation.-Dominion Med. Journal, May, 1869.

The third edition of this most excellent book scarcely necds any commendation from us. The rolune, as it stands now, is really a marvel: first of all, it is that luxury of America, the ready-cut pages, which the Yankees are 'cute enough to insist npon-nor are these by any means trifies; but the contents of the book are sstonishing. Not only is it wonderful that iny one man can hare grasped in his mind the whole scope of medicine with that vigor which Dr. Fliut 3hows, but the condensed yet clear way in which this 18 done is a perfect literary triumph. Dr. Flint is pre-eminently one of the strong men, whose right to do this kind of thing is well aduitted; and we say ao more than the truth when we affirm that he is very nearly the only living man that could do it with $3 n c h$ results as the volume before as. - The London Practitioner, March, 1868 .

This is in some respects the best text-book of medicine in onr language, and it is highly appreciated on the other side of the Atlantic, inasmuch as the first adition was exhausted in a few montlus. The second edition was little more than a reprint; bit the present has, as the author says, been thoroughly revised. Much valuable matter has been added, and by inaking the type sinaller, the bulk of the volume is uot much increased. The weak point in many American works is pathology, but Dr. Flint has taken peculiar pains on this point. greatly to the value of the book. -London Med. Times and Gaztte, Feb. 6, 1860.

BARLOW'S MANOAL OF THE PRACTICE OF TOND'SCLINICAL LECTURES ON CERTAINACUTE DSEDICINE. With Additions by D. F. Condik, Disensez. In one neat octaro volume, of 320 pages, M. D. I vol. 870., pp. 600 , clotb. $\$ 20$. extra cloth. $\$ 260$.

PAVY $(F$. Wenior Asst. M. D. D., F. R. R. S.

A TREATISE ON THE FUNCTION OF DIGESTION; its Disorders and their Treatment. From the second London edition. In one handsome volume, small octavo, extra.cloth, $\$ 200$. (Lately Published.)

The work befure us is one which deserves a wlde trentise, and sufficiently exhaustive for all practical

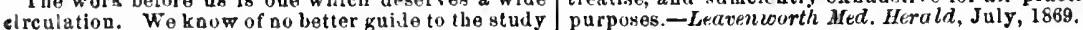
of digestion and lts disorders. - St. Louis Med. and S'urg. Journal, July 10, 1869.

A very valuable wirk on the subject of which it treats. Small, yet it is fnll of valuable information. A thoroughly good book, betag a careful systematic -Cincinnati lifed. Reperiory, Juue, 1869.

BRINTON (WILLIAM), M.D., F.R.S.

LECTURES ON THE DISEASES OF THE STOMACH; with an Introduction on its Anatomy and Pbysiology. From the second and eularged London edition. With illustrations on wood. In one handsome octavo volume of about 300 pages, extra cloth. $\$ 325$.

(YHA.MBERS (T. K.), M.D.,

Consulting Physician to St. Mary's Hospital, London, \&c.

THE INDIGESTIONS; or, Diseases of the Digestive Organs Functionally Treated. Third and revised Edition. In one handsome octaro volume of 393 pages, extra cloth. $\$ 300$. (Lately Published.)

So very large a proportion of the patients applytog | merit, we know of no more desi rable acquisjtion to to every general practitioner siffer from some form a pbysician's library than the bouk before us. He of indigestion, that whatever aids bim in their man. Who should commit its contents to his memury would a cement directly "puts money in his purse," and in. tind its price sn investment of capital that retarned directly does more thro anything else to advance bis him a most usurious rate of interest. $-N$. $\boldsymbol{Y}$. Nredical reputation with the poblle. From this pureiy material point of view, setting aside its higher claims to

\section{$B^{Y}$ THE SAME AUTHOR. (Lately Published)}

RESTORATIVF MEDICINE. An Harveian Annual Oration, delivered at the Royal College of Physicians, London, on June 24, 1871. With Two Sequels. In one very handsome volume, small 12mo., extra cloth, $\$ 100$. 
HARTSHORNE (HENRY), M.D., Professor of Hygtene in the University of Pennsylvania.

ESSENTIALS OF THE PRINCIPLES AND PRACTICE OF MEDI-

CINE. A handy-book for Students and Practitioners. Third edition, revised and im. proved. In one handsome royal $12 \mathrm{mo}$. volnme of 487 pages, clearly printed on small type, oloth, $\$ 238$; half bonnd, $\$ 263$. (Now Ready.)

The very remarkable favor which has been bestowed upon this work, as manifested in the exhaustion of two large editions within four years, shows that it has successfully supplied a want felt by both student and practitioner of a volume which at a moderate price and in a convenient size should afford a clear and compact view of the most modern teachings in medical practice. In preparing the work for a third edition, the author has sought to mointain its character by very nnmerous additions, bringing it fully up to the science of the day, but so concisely framed that the size of the volume is increased only by thirty or forty pages. The extent of the new information thus introduced may be estimated by the fact that there have been two hundred and sixty separate additions made to the text, containing references to one hundred and eighty new anthors.

This little epitome of medical knowledge has al- mulas are appended, intended as examples merely, ready been noticed by $a$. It is a vade mecum of not gs guides for unthinking practitioners. A cum7 ine, inciuding in short space most of what is os value, including in a sort space most of what inessential in the ecience and pructice of medicine. The
third edition is well up to the present dav in the modern methods of treatment, and in the use of newly discovered drugs.-Boston Med. and Surg.Journal, Oct. 19, 1871 .

Certainiy very few volumes contain so much precise information within so stwall a compass. $-N, Y$. Med. Journal, Nov. 1871.

The diseases are conveniently classified; symptoms, causation, diaguosis, prognosis, and treatment are curefuliy considered, the whole being marked by briefuess, but clearnese of expression. Over 250 forplete index facilitates the nse of this little volume in which all important remedies lately lutroduced, such as chloral bydrate and carbolic acid, bave received their fuli share of attention.-Am. Journ. of Pharm., Nov. 1871.

It is an epitome of the whole acience and practioe of medicine, and will be found mest valuuble to the practitioner for easy reference, and especially to the studentin attendance upun lectures, whose time is too much occupied with many studies, to consult the larger works. Such a work must aiways be iu great demand.- Oincinnati Med. Repertory, Nov. 1871.

WATSON (THOMAS), M.D., \&c.

LECTURES ON THE PRINCIPLES AND PRACTICE OF PHYSIC. Delivered at King's College, London. A new American, from the Fifth re. vised and enlarged English edition. Edited, with additions, and several hundred illus. trations, by Henry Hartshorne, M.D., Professor of Hygiene in the University of Pennsylvania. In two large and handsome 8vo. vols. Cloth, $\$ 900$; leather, $\$ 1100$. (Just ready.)

With the assistance of Professor George Johnson, his successor in the chair of Practice of Medicine in King's College, the author has thoroughly revised this work, and has sought to bring it on a level with the most advanced condition of the : ubject. As he himself remarks: "Considering the rapid advance of medical science during the last fourteen years, the present edition would be worthless, if it did not differ mucb from the last"- but in the extensive alterations and additions that have been introduced, the effort of the author has been to retain the lucid and colloquial style of the lecture-room; which has made the work so deservedly popular with all olasses of the profession. Notwithstanding these changes, there are some subjects on which the American reader might reasonably expect more detailed information than has been thought requisite in England, and these deficiencies the editor has endeavored to supply.

The large size to which the work hus grown seems to render it necessary to print it in two volumes, in place of one, as in the last American edition. It is therefore presented in that shape, handsomely printed, at a very reasonable price, and it is hoped that it will fully maintain the position every where hitherto acoorded to it, of the standard and olassioal representative of Eng. lish practical medicine.

At length, after many months of expectation, we huve the satisfaction of finding ourselves this week in possession of a revised and enlarged edition of Sir Thomas Watson's celebrated Lectures. It is a subject for congratulation and for thankfuiness that Sir Thomas Watson, during a period of comparative lelsire, after s long, laborious, and most honorable professional career, while retaining fuli possession of bis bigh mental faculties, should bave employed the oppuriunity to snbmit bis Lectures to a more thorongh revislon than was possibie dnring the esrlier and busier period of his life. Carefully passing in review some of the most intricate and Important patbologica! a nd practleal questions, the results of his ciear insight and his calm judgment are now recorded for the benefil of mankind, in language which, for precision, vigor and classical elegance, has rarely been equalled, and never surpassed. The revision bas evidentiy beev most carefuily done, snd the resuits appear in almosi every page.-Brit. Med. Journ., Oct. 14, 1871.

No words can convey the pleasurahle satisfactlon that we feel in looking over the revised editlon of the admirable lectures of this dlstinguished author. The earnestness which marked lis whole professional career leads him, in s charscteristic manner, to devote his last leisure bours to the correction of his great classic work. The lectures are so well known and so justiy apprecialed, that it is scarcely necessary to do more than call attention to the speciai advantages of the last over previous editions. In the revision, the author has displayed all the charins aud odvantuges of great culture and a ripe experience combined with the soundest judgment and sin-

cerity of purpose. The anthor's rare combination of grest scienlific attuinmeuts combined with wonderful forensic eloquence has exerted extraordiuary influence over the last two generstions of pliysicians. His clinlcal descriptions of most diseases bave never been equalled; and on this score st least bis work wlll live long in the future. The work witl be sought by all who appreciste a great book.-Amer. Journal of Syphilography, July, 1872 .

We are exceedingiy gratified at the reception of this new edition of Watson, pre-eminently the prince of English anthers, on "Practice." We, who read the first edilion as it came to us tardily and in fragments through the "Medical News and Library," chail never forget the gleat pleasure and profit we derived from its grapbic delineations of disease, its vigorous styie and eplendid English. Maturity of yeurs, extenslve observalion, profound research, and yet continnons enthusiasm, have combined tó give us In this latest edition a model of professional excellence in teaching with rare beauty in the mode of conmunicstion. But this classic needs no enjogium of ours. The selection of Prof. Hartshorne as the American editor, is to us peculiarty gratifying, and must insure even larger popularity and more generai sale to Amorican readers. Every guarantee is thus afforded that in every part the book will be found up to the times. Will it do to repert the remark we have keen somewhere: "No library can be considered conplete without it?" Altbough the phrase may not savor of originality, it js, nevertheless, most emphalically true.-Chicago Med. Journ., July, 1872 . 
TLLINT ( A USTIN), M.D.,

Professor of the Prfnciples and Practice of Mediclne in Bellevue Hospital Med. College, N. $\boldsymbol{Y}$.

A PRACTICAL TREATISE ON THE DIAGNOSIS, PATHOLOGY, AND TREATMENT OF DISEASES OF THE HEART. Second revised and enlarged edition. In one ootavo volume of 550 pages, with a plate, extra cloth, \$4. (Just Isszed.)

The author has sedulously improved the opportunity afforded him of revising this work. Portions of it have been rewritten, and the whole brought up to a level with the most advanced condition of soience. It must therefore continue to maintain its position as the standard treatise on tbe subject.

Dr. Flint chose a dificnlt subject for bls researches, and has shown remarkable powers of observation and reflection, as well as great industry, in bis trestment of it. His book must be considered the fullest and clearest practical treatise on those subjects, and shonld be in the hands of ail practitioners and students. It is a credit to American medical literature. -Amer. Journ. of the Med. Sciences, July, 1560.

We question the fact of any recent $A$ merican anthor in our profession being more extensively known, or more deservedly esteemed in this conntry than Dr. Flint. We williugly acknowledge his success, more particnlarly in the volnme on diseases of the lieart, in maklng an extended personal clinical stndy avail- New8.

\section{$B^{Y} T H E$ SAME AUTHOR.}

A PRACTICAL TREATISE O.N THE PHYSICAL EXPLORA. TION OF THE CHEST AND THE DIAGNOSIS OF DISEASES AFFECTING THE RESPIRATORY ORQANS. Second and revised edition. In one handsome octavo volume of 595 pages, extra cloth, $\$ 450$.

Dr. Flint's treatise is one of the most trastworthy (which pervades his whole work lend an additiona] gnides which he can consult. The style is clear and force to its thoroughly practical character, which distinct, and is also concise, being free from that tend. cannot fail to obtain for it a place as a sta udard work ency to over-refinement a ud unnecessary minuteness on disesses of the respiratory system. - London which characterizes many works on the same sub- Lancet, Jan. 19, 1867.

ject.-Dublin Medical Press, Feb. 6, 1867. and his remarks on the diagnosis, especlally in the practitioner. Dr. Fliut enriches his subject with early stsges, are remarkable for their seumen aud much solid and not a littie original observation.great practical value. Dr. Fliut's style is clear and Ranking's Abstract, Jan. 1867.

elegant, and the tone of freshuess and originaity

FULLER (HENRY WILLIAM), M.D., 'Physician to St. George's Hospital, London.

ON DISEASES OF THE LUNGS AND AIR-PASSAGES. Their Pathology, Physical Diagnosis, Symptoms, and Treatment. From the second and revised English edition. In one handsome octavo rolume of about 500 pages, extra cloth, $\$ 350$. Dr. Fuller's work on diseases of the chest was so accordingly we have what might be with perfect jus. Pavorably received, that to many who did not know tice styled an entirely new work from his pen, the the extent of hisengagements, it was a matler of won- portion of the work treating of the heart and great der that it should be allowed to remain three yesrs vessels being excluded. Nevertheless, this voiumeis out of print. Determined, however, to improve it, of almost equal size with the first. -London Medical Dr. Foller wonld not consent to a mere reprint, and Times and Gazette, July $2 \mathrm{C}, 1867$.

WILLIAMS (C. J. B.), M.D., Sentor Consulting Physician to the Hospital for Consumption, Brompton, and

WILLIAMS (CHARLES T.), M.D., Physician to the Hospital for Consumption.

PULMONARY CONSUMPTION; Its Nature, Varicties, and Treatment. With an Analysis of One Thousand cases to exemplify its duration. In one neat octavo volume of about 350 pages, extra cloth. (Just Issued.) $\$ 250$.

Hecan still speak from a more enormous experlence, and a closer study of the morbid processes invoived lu tuberculosis, than most living men. He owed it to himself, and to the importance of the subject, to ombody his views in a separate work, and we are glad that he has accomplished this duty. After ail, the grand teaching which Dr Willisms has for the profession is to be found in his therapeuticai chapters, and in the history of individual cases exteuded, by dint of cure, over ten, twenty, thiriy, and oven forty years. - London Lnncet, Oct. 21, 1871 .

His results are more favorabie than those of any previous autbor; but probahly there is no malady, the treatment of which has been so much improved within the fest twenty years as pulmouary consumption. To ourselves, Dr. Williams's chapters on Treatment are amongst the most vuluable and attractive in the book, and would alone render it a standard work of reference. In conclusion, we would record our opinion that Dr. Widliams's great reputation $3 \mathrm{fully}$ maintalned by this book. It is undonbtedly one of the most valuable works in the language npon any special disease.-Lond. Med. Times and Gaz., Nov. 4,1871 .
LA ROCHE ON PNEUMONIA. 1 vol. 8vo., extra cloth. of 500 pages. Price 830 (I.

BUCKLER ON FIBRO-BRONCHITIS AND RHEU. MATIC PNEUMONIA. 1 vol. 8vo. \$1 25.

FISKE FUND PRIZE ESSAYS ON CONSUMIPTION. 1 vol 8vo,, extra oloth. \$1 00 .
SMITH ON CONSUMPTION ; ITS EARLY AND RE. MEDIABLE STAGES. 1 vol. \$ro., pp. $254 . \quad \$ 225$. SALTER ON ASTHMA. 1 vol. 8vo. \$2 50. WALSHE ON THE DISEASES OF THE HEART AND GREAT VESSELS. Third American edition. In 1 vol. 8v0.. $\$ 20$ pp., cloth. \$3 00 . 
ROBERTS (WILLIAM), M. D.,

A PRACTICAL TREATISE ON URINARY AND RENAL DIS. EASES, including Urinary Deposits. Illustrated by numerous cases and engravings. Serond American, from the Second Revised and Enlarged London Edition. In nne large and handsome octavo volume of 616 pages, with a colored plate; extra cloth, $\$ 450$. (Just Ready.)

The author has subjected this work to a very thorough revision, and has sought to embody in it the results of the latest experience and investigations. Although every effort has been made to keep it within the limits of its former size, it has been enlarged by a hundred pages, many new wood-cnt's have been introduced, and also a colored plate representing the appearance of the different varieties of urine, while the price has been retained at the former very moderate rate. In every respect it is therefore presented as worthy to maintain the position which it has acquired as a leading authority on a large, important, and perplexing class of affections. A few notices of the first edition are appended.

The plen, it will thus be seen, is very complete, an the manuer in which it has been carried out is in the bigbest degree satisfactory. The characters of the different deposits are very well described, and the microscopic appearances they present are illustrated by numerous well executed engravings. It only remains to us to strongly recommend to our readers Dr. Roberts's work, es conlaining an admirable résumé of the present state of knowledge of urinary diseases, and as a safe and reliable guide to the clinical observer.-Edin. Med. Jour.

The most completeand practical treatise n pon renal

diseases we have examined. It is pecnliarly adapted to the wants of the majority of American practitiouers from its clearness and simple announcement of the facts in relation to diagnosis anc treatment of urinary disorders, and contains in condensed form tbe investigations of Bence Jones, Bird, Beale, Hassall, Pront, and a host of other well-known writers upon this subject. The characters of urine, physiological and pathological, as indicated to the naked eye as well as by microscopical and chemical investigations, are concisely represented both by description and by well executed engravings.-Cincinnati Journ. of Med.

$B_{\text {Senior Physician to the W. }}^{A S H}$

RENAL DISEASES: a Clinical Guide to their Diagnosis and Treatment. With illustrations. In one neat royal 12mo. volume of 304 pages. $\$ 200$. (Just Issued.)

The chapters on diagnosis and treatment are very|ment render the book pleasingand convenient.-Am. good, and the student and young practitioner will find them full of valuable practical hints. The third part, on the urine, is excellent, and we cordially recommend its perusal. The author has arranged his matter in a somewhat novel, and, we think, nseful form. Here everything can be easily found, and, what is more important, easily read, for all the dry details of larger books here acquire a new interest from the author's arrangement. This part of the book is full of good work.-Brit. and For. MedicoChirurgical Review, July, 1870.

The easy descriptions and compact modes of stateJourn. Med. Sciences, July, 1870 .

A book that we believe will be fonnd a valnable assistant to the practitioner and guide to the student. -Baltimore Med. Journal, July, 1870.

The treatise of Dr. Basham differs from the rest in its special adaptation to clinical study, aud its condensed and almost aphorismal style, which makes it easily read and easily understood. Besides, the author expresses some new views, which are well worthy of consideration. The volume is a valuable addition to this department of knowledge.-Pacifio Med. and Surg. Journal, July, 1870.

MORLAND ON RETENTION IN THE BLOOD OF THE ELEMENTS OF THE URINARY SPCRETION.

1 vol. 8vo., extra cloth. 75 cents.

TONES (C. HANDFIELD), M. D., Physician to St. Mary's Hospital, \&e.

CLINICAL OBSERVATIONS ON FUNCTIONAL NERVOUS DISORDERS. Second American Edition. In one handsome octavo volume of 348 pagez, extra cloth, \$3 25.

Taken as a whole, the work before ns furnishes a short but reliable acconnt of the pathology and treatment of a class of very common but certainly highly obscure disorders. The ad vanced student will find it a rich mine of valuable facts, while the medical practitioner will derive from it many a suggestive hint to aid him in the diagnosis of "pervous cases," and in determining the true indications for their amelioration or cure.-Amer. Journ. Med. Sci., Jan. 1867.

We must cordially recommend it to the profession of this country as supplying, in a great ineasnre, a defiency which exists in the medical literature of the English language.-New York Med.Journ., April, 1867.

The volume is a most admirable one-full of hints and practical suggestions. - Canada Med. Journal. April, 1867.

$O^{N}$ DISEASES OF THE SPINAL COLUMN AND OF THE NERVES. By C. B. RaDclifF, M. D., and others. 1 vol. 8vo., extra cloth, $\$ 150$.

SLADE (D.D.), M.D.

DIPHTHERIA; its Nature and Treatment, with an account of the History of its Prevalence in various Countries. Second and revised edition. In one neat royal $12 \mathrm{mo}$. volume, extra cloth. $\$ 125$.

HUDSON (A.), M.D., M. R. I. A.,

Physician to the Meath Hospitat.

LECTURES ON THE STUDY OF FEVER. In one vol. 8ro., extra Cloth, $\$ 250$.

LFONS (ROBERT D.), K. C.C.

A TREATISE ON FEVER; or, Selections from a Course of Lectures on Fever. Being part of a Course of Theory and Practice of Medicine. In one nest octaro volume, of 362 pages, extra cloth. $\$ 225$. 
BUMSTEAD (FREEMAN J.), M.D.,

Professor of Venereal Diseases at the Col. of Phys. and Surg., New York, \&c.

THE PATHOLOGY AND. TREATMENT OF VENEREAL DISEASES. Including the results of recent investigations upon the subject. Third edition, revised and enlarged, with illustrations. In one large and handsome octaro volume of over 700 pages, extra cloth, $\$ 500$; leather, $\$ 600$. (Just Issued.)

In preparing this standard work again for the press, the author has subjected it to a very thorongh rerision. Many portions have been rewritten, and much new matter added, in order to bring it completely on a level with the most advanced condition of syphilography, but by careful compression of the text of previous editions, the work has been incrensed by only sixty-four pages. The labor thus bestowed upon it, it is hoped, will insure for it a continuance of its position as a complete and trustworthy guide for the practitioner.

It is the most complete book with which we are ac- much special commendation as if its predecessors had quainted in the language. The latest views of the not been published. As a thoronghly practical book best anthorities are put forward, and the information on a class of diseases which form a large sliare of is well arranged -a great point for the student, and nearly every physician's practice, the volume before still more for the practitioner. The subjects of vis- us is by far the best of which we have knowledge.ceral syphills, syphilitic affections of the eyes, and $N$. Y. Medical Gazette, Jan. 28, 1571 .

the treatment of syphilis by repeated inoculations, are very fully discussed.-London Lancet, Jan. 7, 187 .

Dr. Bumstead's work is already so nniversally know as the best treatise in the English language on venereal diseases, that it may seem almost superfluOux to say iure of it than that a new edition has been issued. But the author's ind nstry has rendered this new edition virtually a new work, and so merits as
YULLERIER (A.), and Surgeon to the Hopital au Midi.
BUMSTEAD (FREEMAN J.)
Profegsor of Venereal Dispecses in the College of Physicians and Surgeons, N. Y.

AN ATLAS OF VENEREAL DISEASES. 'Franslated and Edited by Freeman J. Bumstead. In one large imperial 4to. volume of 328 pages, double-columns, with 26 plates, containing about 150 figures, beautifully colored, many of them the size of life; strongly bound in extra cloth, $\$ 1700$; also, in five parts, stout wrappers for mailing, at $\$ 3$ per part. (Lately Published.)

Antioipating a very large sale for this work, it is offered at the very low price of ThRE DouLARS a Part, thus placing it within the reach of all who are interested in this department of prac. tice. Gentlemen desiring early impressions of the plates would do well to order it without delay.

A specimen of the plates and text sent free by mail, on receipt of 25 cents.

Wewish for once that our province was not restricted to methods of treatment, that we might say something of the exquisite colored plates in this volume. -London Practitioner, Msy, 1869.

As a whole, It teaclies all that can be tanght hy means of plates and print.-London Lancet, March 13, 1869 .

Superior to snything of the kind ever before lssued on this continont.-Canada Med. Journat, March, ' 69.

The practitioner who desires to understand thls branch of medicine thoroughly should obtain this, the most complete and best work ever published.Dominion Med. Journal, May, 1869 .

Thls is a work of master hands on both sides. M. Cullerier is scarcely second to, we think we may traly say is a peer of the illustrious and venerable Rlcord, while in this country we do not hesitate to say that Dr. Bumstead, as an anthority, is without a rival. Assnring our readers that these illnstrations tell the whole history of venereal disease, from its inception to its end, Fe do not know a single medical work,

which for its kind is more necessary for them to have. -Calif rnia Med. Gazette, March, 1869.

The most splendidly illustrated work in the language, and in our opinion far more useful than the French original-Am. Journ. Med. Sciences, Jan.'69. The tifth and concluding number of this magnificeut work has reaclied ns, and we have no hesitation in saying thst jts illustrations surpass those of previons numbers.-Boston Med. and Surg. Journal, Jan. 14, 1669 .

Other writers besides M. Cullerier have given as a good account of the diseases of which he treats, but no oue has furnfshed us with such a complete series of illustrations of the venereal diseases. There is, however, an additional interest and value possessed by the volume before as; for it is an A meriean reprint and translation of 3. Cullorier's work, with incldental remarks by one of the inost emiuent A merican syphilographers, Mr. Bnmstead. - Brit, and For. IItico-Chir. Review, July, 1869.

\section{IILL (BERKELEY),}

Surgeon to the Lock Hospital, London.

\section{ON SYPHILIS AND LOCAL CONTAGIOUS DISORDERS. In} one handsome octavo volume ; extra cloth, \$325, (Lately Published.)

Bringing, as it does, the entire literature of the dis- | to whom we would most earnestly recommend its ease down to the preaent day, and giving with great ghillty the resnlts of modern research, it is in every respect a most desirable work, and one which should find a place in the library of every surgeon.-California Med. Gazette, June, 1869.

Considering the scope of the book and the carefal attention to the manifold aspects and details of it subject, it is wonderfully concise. All these qualities render it an especially valuabie book to the beginzer,

to whom we would most earnestly recommend it
stndy; while It is no less useful to the practlioner. St. Loutis Med. and Surg. Journal, May, 1869.

The most convenient and ready book of reference we have met with. $-N, \boldsymbol{Y}$. Mfed. Record, May 1, 1869.

Most admirably arranged for both stndent and practitioner, no other work on the snbject equals it; it is more simple, more easily stndied.-Buffalo Mtd. and Surg. Journal, March, 1569. octaro volume, with illustrations. (Preparing.) 
WILSON (ERASMUS), F.R.S.

ON DISEASES OF THE SKIN. With Illustrations on wood. Seventh American, from the sixth and enlarged English edition. In onelarge octavo volume of over 800 pages, $\$ 5$.

A SERIES OF PLATES ILLUSTRATING "WILSON ON DISEASES OF THE SKIN;" consisting of twenty beautifully executed plates, of which thir. teen are exquisitely colored, presenting the Normal Anatomy and Pathology of the Skin, and embracing accurate representations of about one hundred varieties of disease, most of thein the size of nature. Price, in extra cloth, $\$ 550$.

Also, the Text and Plates, bound in one handsome volume. Extra cloth, \$10.

such a work as the one before us is a most capital| No one treating skin diseases should be without and acceptuble help. Mr. Wilson has long been held copy of this standard work. - Canada Lanct, as high authority in this department of medicine, and August, 1863.

his book on diseases of the skin has long been re- We can safely recommend it to the profession as garded as one o: the best text-books extant on the the best work on the subject now in exlstence in subject. The present edition is carefully prepared, the English language.-Mredical Times and Gazetie. und brought np in its revision to the present time. In this edition we liave also incinded the beautiful series of plates illnstrative of the text, and in the last edition published separately. There are twenty of these plates, nearly all of them colored to nature, and exhibiting with great fidelity the various groups of
diseases.-Cincinnati Lancet.

\section{$B^{Y}$ THE SAME AUTHOR.}

THE S'TUDENT'S BOOK OF CUTANEOUS MEDICINE and DisEASES OF THE SKIN. In one very handsome royal 12mo. volume. $\$ 350$. (Lately Issued.)

\section{NELIGAN (J.MOORE), M.D.,M.R.I.A.}

\section{A PRACTICAL TREATISE ON DISEASES OF THE SKIN.} Fifth American, from the second and enlarged Dublin edition by T. W. Belcher, M. D. In one neat royal 12mo. volume of 462 pages, extra oloth. $\$ 225$.

Fally equal to all the requirements of students and their value justly estimated; in a word, the work is young practitioners. - Dublin Med. Press.

of the remainder of the work we have nothing beyond anqualified commendation to offer. It ls so far the most complete one of its size that has appeared, and for the student there can be none which can com. pare with it in practical value. All the late disco-
veries in Dermatology have been dnly noticed, and

BY THE SAME AUTHOR.

ATLAS OF CUTANEOUS DISEASES. In one beantiful quarto volume, with exquisitely colored plates, \&c., presenting about one hundred varieties of disease. Extra cloth, $\$ 550$.

The diagnosis of eruptive disease, hewever, nnder / inclined to consider it a very snperior work, comall circumstances, is very difficuit. Nevertheless, $\mid$ bining accurate verbal description with sound vievs Dr. Neligan has certainly, "as far as possible," given of the pathology and treatmeat of eraptive diseases. a faithful and accurate representation of this class of -Glasgow Med. Journal.

diseases, and there can be no donbt that these plates A compend which will very mnch aid the practiwill be of great use to the student and practitionerin tioner in this difficult branch of diagnosis Taken drawing a diagnosis as to the class, order, and species with the beantiful plates of the Atlas, which are reto which the particular case may belong. While markabie for their accuracy and beanty of coloring, looking over the "Atias" we have been induced to it constitutes a very valuable addition to the library examine also the "Practical Treatise," and we are of a practical man.-Buffalo Med. Journal.

\section{HILLIER (THOMAS), M.D.,} Physician to the Skin Department of University College Hospital, \&c.

HAND-BOOK OF SKIN DISEASES, for Students and Practitioners. Second American Edition. In one royal 12mo. volume of $358 \mathrm{pp}$. With Illustrations. Extra cloth, $\$ 225$.

We can conscientiously recommend it to the sindent; the style is ciear and pleasant to resd, the matter 18 good, and the descriptions of disease, whth the modes of treatment recommended, are frequently illastrated with well-recorded cases.-London Med. Times and Gazelte, April 1, 1865.

\section{A NDERSON (McCALL), M.D.}

Physieian to the Dispensary for Skin Diseases, Glasgovo, \&e.

ON THE 'TREA'TMEN'T OF DISEASES OF THE SKIN. With an

Analysis of Eleven Thousand Consecutive Cases. In one vol. 8vo. (Publishing in the Medical News and Library for 1873.)

The very practical character of this work and the extensive experience of the author, cannot fail to render it acceptable to the subscribers of the "A MERICAN Joursal of THE MedictL Sciences." When completed in the "News AND Library," it will be issued separately in a neat octaro volume. 


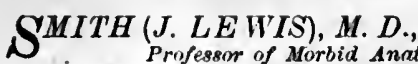

A COMPLETE PRACTICAL TREATISE ON THE DISEASES OF

CHILDREN. Second Edition, revised and greatly enlarged. In one bandsome octavo volume of 742 pages, extra cloth, $\$ 5$; leather, $\$ 6$. (Now Ready.)

From the Preface to tise Second Edition.

In presenting to the profession the second edition of his work, the author gratefully acknow ledges the firorable reception accorded to the first. If has endeavored to merit a continuance of this approbation by rendering the volume much more complete than before. Nearly twenty additional diseases hare been treated of, anong whloh may be named Diseases Incidental to Birth, Rachitis, Tuberculosis, Scrofula, Intermittent, Remittent, and Typhoid Fevers, Chorea, and the various forms of Paralysis. Many new formulw, which experience bas shown to be aseful, have been introduced, portions of the text of a less practical nature have been condensed, and other portions, especinlly those reluting to pathological histolory, have been rewritten to correspond with recent discoveries. Every effort has been made, however, to avoid an undue enlargement of the volume, but, notwithstanding this, and an increase in the size of the page, the number of pages has been enlarged by more than one hundred.

227 West 49th Street, New York, April, 1872.

The work will be found to contain nearly one.third more matter than the previous edition, and it is confidently presented as in every respect worthy to be received as the standard American text-book on the subject.

Eminentiy practicai as well as jndicions in its We regard it as snperior to any other single work teachings. -Cincinnati Lancet and Obs., July, 1872. $\mid$ on the diveases of infancy and childhood.-Detroit

A staodard work that leaves little to be desired.Indiana Journal of Medicine, July, 1872.

We We confess to increased $n$ thusiasm in recommendWe know of no book on this subject that we can lug this second edition.-St. Louis Med. and Surg. more cordialiy recommend to the medical st udent Jurrnal, Ang. 1572.

and the practitioner.-Cincinnati Clinic, June 29,72 .

\section{CONDIE (D. FRANCIS), M.D.}

A PRACTICAL TREATISE ON THE DISEASES OF CHILDREN. Sixth edition, revised and augmented. In one large octavo volume of nearly 800 closelyprinted pages, extra cloth, $\$ 525$; leather, $\$ 625$. (Lately Issued.)

The present edition, which is the sixth, is fully up teachers. As a whole, however, the work is the best to the times in the discussion of ail those pointsin the American one that we have, and in its special adaptapathology and treatment of infantile diseases which thon to American pracilioners it certulniy has no have been brought forward by the Germau and French

WEST (CHARLES), M.D.,

\section{Physician to the Hospital for Sick Children, \&c.}

LECTURES ON THE DISEASES OF INFANCY AND CHILD. HOOD. Fourth American from the fifth revised and enlarged English edition. In one large and hanasome octavo volume of 656 olosely-printed pages. Extra cloth, \$4 50; leatber, $\$ 550$.

Of all the Engilis writers on the disesses of chil- living a uthorities in the diffenlt department of medidren, there is no one so entirely satisfactory to us as cal sclence in which he is most widely known.-

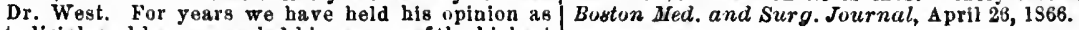
judicial, and have regarded him aa one of the bighest

\section{$B^{Y}$ THE SAME AUTHOR. (Lately Issued.)}

ON SOME DISORDERS OF THE NERVOUS SYSTEM IN CHILD. HOOD; being the Lumleian Lectures delivered at the Royal College of Physicians of London, in March, 1871. In one volume, small 12mo., extra cloth, $\$ 100$.

\section{SMITH (EUSTACE), M.D., Physician to the Northwest London Free Dispensary for Sick Children.}

A PRACTICAL TREATISE ON THE WASTING DISEASES OF INFANCY AND CHILDHOOD. Second American, from the second revised and enlarged English edition. In orie handsome octavo volume, extra cloth, $\$ 250$. (Lately Issued.)

This is in every way an admirable book. The scribed as a practical handbook of the common dis. modest tille which the author has chosen for it scarce- eases of ohildren, so numerous are the affections coniy conveys an adequate idea of the many $\&$ abjects sidered either collaterally or directiy. We are .apon which it treats. Wasting is so coustant an at. acquainted with no sufer gulde to the ireatment of tendant npon the malsdles of childhood, that a trea- children's diseases, and few works give the insight tise upon the wasting dieeasea of children must neces in to the physlological and other peculiarities of chilsarily embrace the consideration of many 8 ffections dron that Dr. Smith's book does.-Brit. Mfed. Journ., of which it is a symptom; and this is excelientiy weil April 8, 1571 . done by Dr. Smith. The book might fairiy be de-

GUERSANT (P.), M. D.,
Honorary Surgeon to the Hospitalfor Sick Children, Paris.

SURGICAL DISEASES OF INFANTS AND CHILDREN. Translated by R. J. Dusanison, M. D. In one neat ootaro volume, extra eloth, $\$ 50$. (Now Ready)

DEWEES ON THE PHYSICAL AND MEDICAL TREATMEAT OP CHILDREN. Elerenth edition. 1 rol. 8vo. of 048 pages. 80 . 
THE OBSTETRICAL JOURNAL of Great Britain and Ireland; Including Midwiferr, and the Diseases of Women and Infaxts. With an American Supplement, edited by WiLliam F. Jenks, M.D. A monthly of about 80 octaro pages, very handsomely printed. Subscription, Five Dollars per annum. Single Numbers, 50 cents each.

Commencing with April, 1873, the Obstetrical Journal will consist of Original Papers by Brit. ish and Foreign Contributors; Transactions of the Obstetrical Societies in England and abroad; Reports of Hospital Practice; Reviews and Bibliographical Notices; Articles and Notes, Editorial, Historical, Forensic, and Miscellnneous; Selections from Journals; Correspondence, de. Collecting together the vast amount of material daily accumulating in this important and rapidly improving department of medical science, the value of the information which it will present to the subscriber may be estimated from the character of the gentlemen who have already promised their support, including such names as tbose of Drs. Atthill, Robert Barnes, Henry Bennet, Thomas Chambers, Fleetwood Chorchile, Matthews Doncan, Graily Hewite, Buaxton licks, Alfred Meadows, W. Leishinan, Azex. Simpson. Tyler Suiti, Edward J. Tilt, SPEncer WELLS, \&c. \&c.; in short, the representative men of British Obstetrics and Gynæ. cology.

In order to render the Obstetrical Journal fully adequate to the wants of the American profession, each number will contain a Supplement devoted to the advances made in Obstetrios and Gynacology on this side of the Atlantic. This portion of the Journal will be under the editorial charge of Dr. WILLIA F. JENKs, to whom editorial communications, exchanges, books for review, \&c., may be addressed, to the care of the publisher.

$*_{*}^{*}$ Gentlemen desiring complete sets will do well to forward their orders without delay.

THOMAS (T. GAILLARD), M.D.,

A PRACTICAL TREATISE ON THE DISEASES OF WOMEN. Third edition, enlarged and thoroughly revised. In one large and bandsome octavo volume of 784 pages, with 246 illustrations. Cloth, $\$ 500$; leather, \$6 00. (Just Issued.)

The author has taken advantage of the opportunity afforded by the call for another edition of this work to render it worthy a continuance of the very remarkable favor with which it bas been received. Every portion has been subjected to a consoientious rerision, several new chapters have been added, and no labor spared to make it a complete treatise on the most advanced condition of its important subject. The present edition therefore contains about one-third more matter than the previous one, notwithstanding which the price has been maintained at the former very moderate rate, rendering this one of the cheapest volumes accessible to the profession.

As compared with the first edition, five new chapters on dysmenorihoea, peri-uterine fuid tumol's, composite tumors of the ovary, solid tumars of the ovaly, and chlorosis, have been added. Twentyseven additional wood-cuts have been introduced, muny subjects have been subdivided, and all have received important interstitial increase. In fact, the book has been practically rewritten, and greatly increased in value. Briefly, we may say that we know of $\mathrm{n}$ o book which 80 completely and concisely represents the present state of gynæoology; none so full of well-digested and reliable teaching; none which bespeaks an author more apt in research and abundant in resources. $-N$. Y. Med. Record, May 1, 1872 .

We should not be doing our dity to the professton did we not tell those who are unaoquainted with the book, how much it is valued by gynæcologists, and how it is in many respects one of the best text-books ou the snbject we possess in our language. We have no hesitation in recommending Dr. Thomas's work as one of the most complete of its kind ever published. .It should be in the possession of every pructitioner for reference and for study.-London Lancet, April $27,1872$.

Our a uthor is not one of those whose views "never change." On the contrary, they have been modifed in many particulars to accord with the progrese made in this department of medical science: hence it has the fresh ness of an entirely new work. No general practitioner can afford to be without it. -St. Louis Med. and Surg. Journal, May, 1872.

Its able anthor need not fear comparison between it and any simllar work in the English language; nay more, as a text-book for students and as a guide f r practitioners, we belleve it is unequalled. In the libraries of reading physictans we meet with it cftener than any other treatise on diseases of women. We conclnde our brief review by repeating the hearty commendation of this volume given when we commenced : If eit her student or practitioner can get but nue book on diseases of women, that hook shonld be "Thomas." - Amer. Jour. Hed. Sciences, April, 1872.
We are free to say that we regard Dr. Thomas the best A merican authority on diseases of women. Several others have written, and written well, but none have so clearly and carefully arranged their text and instruction as Dr. Thomas, - Cincinnait Lancet and Observer, May, 1372.

We deem it scarcely necessery to recommend this work to physicians as it is now wldely known, and most of them already possess it, or will certainly do so. To students we unhesitatingly recomraend it as the best text-book on diseases of females extant.--St. Louis Med. Reporter, June, 1869.

of all the army of books that have appeared of late years, on the diseases of the uterns and its a ppendages, we know of none that is so clear, comprehensive, and practical as this of Dr. Thomas', or one that we should more emphatically recommend to the young practitioner, as his guide.-California Med. Gazette, Jone, 1869.

If not the best work extant on the sabject of which it treats, it is certainly second to none other. So short a time has elapsed since the medical press teemed with commendatory notices of the first edition, that it would be superfuous to give an extended review of what is now firmly established as the American text-book of Gynæcology.-N. Y. Med. Gazette, Jnly $17,1869$.

This is a new and revised edition of a work which we recently noticed at some length, and earnestly commended to the favorable attention of our readers. The fact that, in the short space of one year, this second edition makes Its appearance, shows that the general judgment of the profession has largely conirmed the opinion we gave at that time.-Cincinnati Lancet, Ang. 1869.

It is so' short a time since we gave a full review of the first edition of this book, that we deem it only necessary now to call attention to the second appearance of the work. Its success has been remarkable, and we can only congratulate the author on the brilliant reception his book has received. $-N$. Y. Med. Journal, A pril, 1869. 
ATODGE (HDGH L.), M.D.

Emeritus Professor of Obstetrics, \&c., in the Tninersity of Pennsylvania.

ON DISEASES PECULIAR TO WOMEN; including Displacements of the Uterus. With original illustrations. Second edition, revised and enlarger. In one beautifully printed octavo volume of 531 pages, extra cloth. \$450. (Lately Issued.)

In the preparation of this edition the anthor has spared no palns to improve it with the results of his observation and study during the interval which has elapsed since the first appearance of the work. Considerable additions have thus been made to it, which have been partially accommodated by an enlargement in the size of the page, to avoid increasing unduly the bulk of the volume.

From Pror. W. H. BrFoRd, of the Rush Medical College, Chicrigo.

The book bears the imipress of a master hand, and must, as its predecessor, prove acceptable to the profession. In diseases of women Dr. Hodge has established a school of treatment that has becoine worldwide in fame.

Professor Hodge's work ls truly an original one from beginniag to end, consequently no one can pernse its prges without learning something new. The book, which is by no means a large one, is divided into two grand sectlons, so to speak: Arst, that treating of the nerrons sympathies of the nterus, and, secondly, that which speaks of the mochanical treatment of displacements of that organ. He is disposed, as a nonbeliever in the frequency of inflammations of the nterns, to take strong ground against many of the bighest authorities in this branch of medicine, and the arguments which he offers In support of his positinn are, to sit $y$ the least, well nut. Xumerons wood. cnts adorn this portion of the work, and add incalen. lably to the proper appreciation of the variously shaped instruments referred to by our anthor. As a contribution to the stndy of women's diseases, It la of great value, and is abnndantly able to stand on its own inerits. $-N$. Y. Medical Record, Sept. 15, 1868.

In this point of view, the treatise of Professor Hodge will be indispensable to erery student in its dopartment. The large, fair ty pe and general perfection of workmanshin will render it donbly welcome. -Pacific Med. and Sury. Juzrnal, Oct. 1 s68.

\section{WEST (CHARLES), M.D.}

LECTURES ON THE DISEASES OF TOMEY. Third American, from the Third London edition. In one neat octavo volume of about 550 pages, extra cloth, \$375; leather, $\$ 475$.

The reputation which this volume has ncquired as a standiard book of reference in its department, renders it only necessary to say that the present edition has received a careful revision at the bands of the author, resulting in a considerable increase of size. A few notices of previons editions are subjoined.

The manner of the author is excellent, his descriptlons graphic and persplcuous, and his treatment np to the level of the time-clear, precise, definite, aud marked by strong common sense.-Chicago Afed. Joirnal, Dec. 1861 .

We cannot ton highly recommend this, the seeond edition of Dr. West's excellent lectures on the diseases of females. We know of no other book on this subject from which we have derived as mnch pleasure and instruction. Every page gives evidence of the bonest, earnest, and diligent searcher after truth. He is not the mere compller of other men's ideas, bnt his lectures are the result of ten years' patient investigation in one of the widest field s for women's diseasesSt. Bartholomew's Hospital. As a teacher, Dr. West ls simple and earnest in hls language, clear and comprehensive in his perceptions, and logical in his deductlons.-Cincinnati Lancet, Jan. 1862.

We retarn the author onr grateful thanks for the vast a mount of instruction he has afforded ns. Hls valuable treatise needs no enlogy on our part. His graphie diction and truthful pictures of disease all speak for themselves. - Medico-Chirurg. Reviev.

Most jnstly esteemed a standard work. .

As a writer, Dr. West stands, in onr opinion, $8 \theta$ cond only to Watson, the "Macaulay of Medicine;" he possesses that happy facnlty of clothing instrnctlon in easy garments; combining pleasnre with profit, he leads his pnpils, in spite of the ancient proverb, along a royal road to learning. His work is one whigh will not satisfy the extreme on either side, bnt it one that will pleace the great majority who are seeking truth, and one that will convince the student that he has committed himself to a candid, safe, and valuable gaide.-N.A. Med.-Chirurg Reviev.

We must now conclude this hastlly written sketch with the confident assurance to on readers that the work will well repay perusal. The conscientions, painstaking, practical plyssician is apparent on every

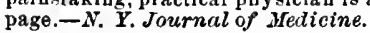

We have to say of it, briefy and decidedly, that it is the best work on the snbject in any language, and that it stamps Dr. West as the facile princeps of British obstetric anthors. - Edinburgh Med. Journal.

We gladly recommend his lectures as in the highest degree instructive to all who are interested in obstetric practice,-London. Lancet.

We know of no treatise of the kind so complete, (avidence of having been carefnlly revised, and la well wortly of the fame it has already obtalned. -Dub. Med. Quar. Jour.

and yet so compact.-Chicago Med. Journal.

BARNES (ROBERT), M. D., F.R.C.P., obstetric Physician to St. Thomas's Hospital, \&e.

A CLINICAL EXPOSITION OF THE MEDICAL AND SURGICAL DISEASES OF WOMEN. In one handsome ootavo volume with ill ustrations. (Pre. paring.)

CHURCHILT ON THE PUERPERAL FEVER AND OTHER DISEASES PECULIAR TO WOMEN. 1 VUl. 8 ro., pp. 450 , extra cloth. 8250.

DEWEES'S TREATISE ON THE DISEASES OF FE-

MALES. With illnstratione. Bleventh Edition, with the Anthor's last improvements and correc. tlons. In one octavo volume of 536 pages, witb platea, extra cloth. $\$ 300$.

WEST'S ENQUIRY INTO THE PATHOLOGICAL IMPORTANCE OF ULCERATION OF THE OS UTERI. 1 vol. 8v0., extra cloth. \&1 25.
MEIGS ON WOYAN: HER DISEASES AND TIEIR REMIEDIES. A Series of Lectures to hls Clask. Fourth and Improred Edition. I rol. Sro, over 700 pages, extra cloth, 8500 ; leather, $\$ 600$. MEIGS ON THE NATURE, SIGNS, AND TREAT. MEN $\Gamma$ OF CHILDBED FEVER. ' 1 vol. svo., pp. $36 \tilde{j}$, extra cloth. 200 .

ASH WELL'S l'RACTICAL TREATISE ON.THE DIS. EASES PECULIAR TO WOMEN. Third American, from the Thitd and rerised London edition I vol. Sro., pp. 525, extra cloth. $\$ 350$. 
RODGE (HUGH L.), M.D., Emeritus Professor of Midwifery, \&c., in the Untversity of Pennsylvania; \&c.

THE PRINCIPLES AND PRACTICE OF OBSTETRICS. Illustrated with large lithographio plates oontaining one hundred and fifty-nine figures from original photographs, and with numerous wood-cuts. In one large and beautifully printed quarto volume of 550 double-columned pages, strongly bound in extra cloth, $\$ 14$.

The work of Dr. Hodge is something more than a We have examined Professor Hodge's work with simple presentation of his particular views in the de- great satisfaction; every topic Is elaborated most partinent of Obstetrics; it is something more than an fully. The views of the anthor are comprehensive, ordinary treatise on midwifery; it is, In fact, a cycio- and concisely stated. The rules of practlce are judl. pædia of midwifery. He has aimed to embody in a cious, and will enable the practitioner to meet every single volume the whole kicience and art of Ohstatrics. emergency of obstetric compllcation with confldence. An elaborate text is combined with accurate and va- -Chicago Med. Journal, Ang. 1864.

ried pictorial illustrations, so that no fact or principle is left unstated or noexplained.-Am. Med. Times, Sept. 3, 1864 .

We should like to anslyze the remainder of this excellent work, but alread y has this review extended beyoud our limited space. We cannot conclude this notice without refering to the excellent finish of the work. In typography it is not to be excelled; the paper is sllperior to what is usally afforded by onr American cousins, quite equal to the best of English books. The engravings and lithographs are most beautifally executed. The work recornmends itself for its originalily, and is in every way a most valuable addition to those on the subject of obstetrics. Canada Med. Journal, Oct. 1864.

It is very large, profusely and elegantiy illustrated, and is fitted to take its place near the works of great obstetricians. Of the American works on the subject

More time than we have had at onr disposal since we received the great work of Dr. Hodge is necessary to do It justice. It is and oubtedly by far the moet original, complete, and carefully composed treatise on the princlples and practlce of Obstetrics which has ever been issued from the American press.-Pacifie Med. and Surg. Journal, July, 1864.

We have read Dr. Hodge's book with great pleqsnre, and have much satisfaction ln expressing onr commendation of it as a whole. 'It is certainly highly instructive, and in the main, we belleve, correct. The great attention which the author has devoted to the mechanism of parturition, taken along with the conclusions at which he has arrived, point, we think, concluslvely to the fact that, In Britain at least, the doctrines of Naegele have been too blindly receired. -Glasgow Med. Juurnal, Oct. 1864.

decidedly the best. $\rightarrow$ Edinb. Med. Jour., Dec. 64 .

*** Specimens of the plates and letter-press will be forwarded to any address, free by mail, on receipt of six cents in postage stamps.

TANNER (THOMAS H.), M.D.

ON THE SIGNS AND DISEASES OF PREGNANCY. First American from the Second and Enlarged English Edition. With four colored plates andillustrations on wood. In one handsome octavo volume of about 500 pages, extra cloth, $\$ 425$.

The very thorough revision the work has undergone women of to-day, so commonly associated with the has added greatly to its practical value, and increased materially its efficiency as a guide to the student and to the young practitiver.-Am. Journ. Med. Sei., April, 1868 .

With the immense variety of subjects treated of and the ground which they are made to cover, the Impossibility of giving an extended review of thistruly remarkable work must be apparent. We have not a single fault to find with it, and most heartily com. mend lt to the careful study of every physician who wonld not only always be sure of his diagnosis of pregnancy, but always ready to treat all the nnmerous ailments that are, unfortunately for the civillzed

iunction. - N. Y. Aled. Record, March 16, 1868

We have mach pleasure in calling the attentlon of our reader's to the volume produced by Dr. Tanner, the.second edition of a work that was, in its original state even, acceptable to the profession. We recornmend obstetrical students, young and old, to have this volume in their collections. It contains not only a fair statement of the signs, symptoms, and diseases of pregnancy, but comprises in addition mach interesting relative matter that is not to be found in any other work that we can name.-Edinburgh Nert. Journal, Jan. 1868.

SWA FNE (JOSEPH GRIFFITHS), M. D.,

0 Physician-Accoucheur to the British General Hospital, \&c.

OBSTETRIC APHORISMS FOR THE USE OF STUDENTS COMMENCING MIDWIFERY PRACTICE. Second Amerioan, from the Fifth and Revised London Edition, with Additions by E. R. Hutceins, M. D. With Illustrations. In one neat $12 \mathrm{mo}$. volume. Extra cloth, $\$ 1$ 25. (Now Ready.)

It is really a capital little compendium of the snb- answers the purpose. It is not only valnable for ject, and we recommend young practitioners to buy It young beglnners, but no one who is not a proficlent and carry it with them when celled to attend cases of in the art of obstetrics should be without it, because labor. They can while away the otherwise tedions it condenses all that is necessary to know for ordihours of waiting, and thoroughly fix in their memo- nary midwifery practice. We commend the book ries the most important practical suggestions it con- most favorabiy.-St. Louis Med. and Surg. Journal, talns. The A merican editor has materially added by Sept. 10, 1870.

his notes and the concluding chapters to the completeness and general value of the book.-Chicago Med. Journal, Feb. 1870.

The manual before ns containsin exceedlngiy small compass-small enough to carry in the pocker-about all there is of nbstetrics, condensed into a nutsheil of A phorisms. 'The illustrations are well selected, and serve as excellent reminders of the cond uct of laborreguiar and diffeult. - Cincinnati Lancet, April, '70.

Thts is a mostad mirable little work, and completely

A studied perusal of this littio book has satisfled us of its eminently practical value. The object of the work, the author says, in his preface, is to give the stndent a fow brief and practical directlons respecting the management of ordinary cases of labor; and also to poiut out to him in extraordinary cases when and how he may act upon his own responsiblilty, and when he ought to send for assistance.-N. Y. Medienl Journal, May, 1870.

WINCKEL (F.).

A COMPLETE TREATISE ON THE PATHOLOGY AND TREATMENT OF CHILDBED, for Students and Practitioners. Translated, with the consent of the author, from the Second German Edition, by James Read CHADWick, M D. In one octavo volume. (Preparing) 
MEIGS (CHARLES D.), M.D., Lutely Professor of Obetetrics, \&c., in the Jefferson Medical College, Philadelphia.

OBSTETRICS: THE SCIENOE AND THE ART. Fifth edition, revised. With one hundred and thirty illustrations. In one beautifully printed octavo volume of 760 large pages. Extra cloth, $\$ 550$; leather, $\$ 650$.

It is to the student that onr anthor has more par- favorably known to the profession that no recomticularly addressed himself; but to the practitiouer we believe it would be equally serviceable as a book of reference. No work that we have met with so thoroughly detalls everything that falle to the lot of the accouchenr to perform. Every detail, no matter how minnte or how trivlal, has found a place.Canadu Medical Journal, July, 1867.

The origlnal edtion is already so extensively and present edition is very.much extended, improved and perfected. Whilst the great practical talents and anlimited experience of the author render it a most valuabie acquisition to the practitioner, it is so con. densed as to constitute a most eligible and exceliout text-book for the student. - Southern Bfed. and Surg. Journat, Juls, 1867 .

\section{RAMSBOTHAM (FRANCIS H.), M.D.}

THE PRINCIPLES AND PRACTICE OF OBSTETRIC MEDICINE AND SURGERY, in reference to the Process of Parturition. A new and enlarged edition, thoroughly revised by the author. With additions by W. V. Keating, M. D., Professor of Obstetrics, \&c., in the Jefferson Medical College, Philadelphia. In one large and handsome imperial octavo volume of 650 puges, strongly bound in leather, with raisêd bands; with sixty-four beautiful plates, and numerous wood-cuts in the text, containing in all nearly 200 large and beautiful figures. $\$ 700$.

We will oply add that the student will learn from To the physician's library it is indispensable, while it ail he need to know, and the practitioner will find It, as a book of reference, surpasced by none other.8tethoscope.

The character and merits of Dr. Ramsbotham's work are so well known and thoroughly established, that comment is unnecessary and praise superfuous. The lilnstrations, which are numerous and accurate, are executed in the highest style of art. We cannot too highly recommend the work to onr readers. - St. Louis Med. and Surg. Journal. to the otudent, as a text-buok from which to oxtract the material for laying the foundation of an ed neation on obstetrical science, it has no superior.-Ohio $\boldsymbol{H}_{t} d$. and Surg. Journal.

When we call to mind the toil we nuderwent in acquiring a knowledge of this subject, we cannot but envy the student of the present day the aid which this work will atford him.-Arn. Jour. of the Med. Seiences.

CHURCHLLL (FLEETWOOD), M.D., M.R.I.A.

ON THE THEORY AND PRACTICE OF MIDWIFERY. A new American from the fourth revised and enlarged London edition. With notes and additions by D. Francis CondiE, M. D., author of a "Practical Treatise on the Diseases of Chil. dren," \&c. With one hundred and ninety-four illustrations. In one very handsome octavo rolume of nearly 700 large pages. Extra cloth, $\$ 400$; leather, $\$ 500$.

In adapting this standard favorite to the wants of the profession in the United States, the editor has endeavored to insert everything that his experience has shown him would be desirable for the A merioan student, including a large number of illustrations. With the sanction of the author, be has added, in the form of an appendix, some chapters from a little "Manual for Midwives and Nurses," recently issued by Dr. Churchill, believing that the details there presented can hardly fail to prove of advantage to the junior practitioner. The result of all these additions is that the work now contains fully one-half more matter than the last American edition, with nearly onehaIf more illustrations; so that, notwithstanding the use of a smaller type, the volume contains almost two hundred pages more than before.

' 'hese additions render the work still more com- has been added which could be well dispensed with. piete and acceptable than ever; and with the excellent style in which the publlshers have presented this edition of Chnrchill, we can cominend it to the profession with great cordlallty and pleasare.-Cin. cinnati Lancet.

Few worke on this branch of medical ecience are equal to it, certainly none excel it, whether in regard to theory or practice, and in one respect It is sn perior to all others, viz., In ite statistlcal informatiou, anj therefore, on these gronnds a most valuabie work for the physician, student, or lectnrer, all of whom will and in it the information which they are seeking.Brit. Am. Journal. An examination of the table of contents shows how thoronghly the author has gone over the ground, and the care he bas taken in the text to present the subjects in all their bearings, will render this new edition even more necessary to the obstetric student than were etther of the former editions at the date of their appearance. No treatise on obstetrics with which we are acquainted can compare favorably with thls, in respect to the amount of material which has been gathered from every source.-Boston Med. and Surg. Journal.

There is no better text-book for students, or work of reference and stndy for the practising physlciun than this. It should adorn and en rich every medical

The present treatise is very much enlarged and amplited beyond the previons editione bnt nothing library.-Chicago Med. Journeal.

DONTGOMERT (W. F.), M. D.,

Professor of Midwifery in the King's and Queen's College of Physicians in Ireland.

AN EXPOSITION OF THE SIGNS AND SYMPTOMS OF PREGNANCY. With some other Papers on Subjects connected with Midwifery. From the second and enlarged English edition. With two exquisite oolored plates, and numerous wood-cuts. In one very handsome ootavo volume of nearly 600 pages, extra cloth. $\$ 375$.

RIGBY'S SYSTEM OF KIDWIFERT. With Notes and Additional Illastrutione. Second American edition. One volnme octavo, extracloth, 422 pages. (2) 5).
DEWEES'S COMPREHENSIVE STSTEM OF MID. WIFERY. Twelfth odition, with the anthor's lest improvements and corrections. In one octaro vol. nub, extrs cloth, of 600 pages. 3350 . 
Y ROSS (SAMUEL D.), M.D., Professor of Surgery in the Jefferson Medical College of Philadelphia.

A STSTEM OF SURGERY: Pathological, Diagnostic, Therapeutic, and Operative. Illustrated by upwards of Fourteen Hundred Engravings. Fifth edition, carefully revised, and improved. In two large and beautifully printed imperial octavo rolumes of about 2300 pages, strongly bound in Jeather, with raised bands, \$15. (Just Ready.)

The continued favor, shown by the exhaustion of successive large editions of this great work, proves that it has successfully supplied a want felt by American practitioners and students. In the present revision no pains have been spared by the author to bring it in every respect fully up to the day. To effect this a large part of the work has been rewritten, and the whole enlarged by nearly one fourth, notwithstanding which the price bas been kept at its former very moderate rate. By the use of a close, though very legible type, an unusually large amount of matter is condensed in its pages, the two volumes containing as much as four or five ordinary octavos. This, combined with the most careful mechanical execution, and its very durable binding, renders it one of the cheapest works accessible to the profession. Every subject properly belonging to the domain of surgery is treated in detail, so that the student who possesses this work may be said to have in it a surgical library.

It must long remain the most comprehensive work on this important part of medicine.-Boston Medical and Surgical Journal, March 23, 1865.

We have compared it with most of our standard works, such as those of Erichsen, Miller, Fergusson, Syme, and others, and we mast, in justice to our author, award it the pre-eminence. As a work, complete in alnost every detail, no matter how minute or trifling, and embracing every subject know $\mathrm{a}$ in the principles and practice of surgery, we believe it stands without a rival. Dr. Gross, in his preface, remarks "my aim has been to embrace the whole domain of surgery, and to allot to every subject its lesitimate claim to notice;" and, we assure our reader, he has kept his word. It is a work which we can most contidently recommend to our brethren, for its utility is becoming the more evident the longer it is upon the shelves of our library.-Canada Med. Journal, September, 1865.

'The first two editions of Professor Gross' System of Surgery are so well known to the profession, and so highly prized, that it would be idle for us to speak in praise of this work. - Chicago Medical Journal, September, 1565 .

We gladly inderse the favorable recommendation of the work, both as regards matter and style, which we made when noticing its frst appearance.-British and Fureign Medico-Chirurgical Review, Oct. 1865.

'T'he most complete work that bas yet issued from the press on the science and practice of surgery.London Lancet.

This system of surgery 1s, we predict, destined to take a commanding position in our surgical literature, and be the crowning glory of the author's well earced tame. As an anthority on general surgical subjects, this work is long to occupy a pre-eminent place, not only at home, but abroad. We have no

hesitation in prononncing it without a rival in onr inguage, and equal to the best systems of surgery in an y language. - N. Y. Med. Journal.

Not only by far the best text-book on the subject, as a whole, within the reach of American stndents, but one which will be much more than ever likely to be resurted to and regarded as a high anthority throad.-Am. Journal Med. Sciences, Jan. I865.

The work contains everytling, minor and major, operative and diagnostic, including mensuration and examination, venereal diseases, and uterine manipnlations and operations. It is a complete Thesaurus of moders surgery, where the student and practilioner shall not seek in vain for whal they desire.San Francisco Med. Press, Jan. 1865.

Open it where we may, we find sound practical in. formation conveyed in plain language. This book is no mere provincial or even national system of sur. gery, but a work which, while very largely indebted to the past, has a strong claim on the gratlicude of the future of surgical science.-Edinburgh Med.Journal, Jan. 1865.

A glance at the work is saffleient to show that the anthor and publisher have spared no labor in making it the most complete "System of Surgery" ever published in any conntry.-St. Louis Med. and Surg. Journal, April, 1865.

A system of surgery which we think unrivalled in onr language, and which wlll indelibly associate bis name with surgical seience. And what, in our opin. ion, enhances the value of the work is that, while the practising surgeon will find all that he requires in it, it is at the same time one of the most valuable trea. tises which can be put into the hands of the sindent seeking to know the principles and practice of this branch of the profession which he designs subsequently to follow.-The Brit. Am. Journ., Montreal.

\section{$B^{Y}$ THE SAME AUTHOR.}

A PRACTICAL TREATISE ON FOREIGN BODIES IN THE AIR-PASSAGES. In 1 vol. 8vo. cloth, with illustrations. pp. $468 . \$ 275$.

SKEY'S OPERATIVE SORGERY. In I rol. Svo. GIBSON'S INSTITUTES AND PRACTICE OF SUR-

cloth, of over 650 pages; with about 100 wood-cuts. $\$ 326$.

COOPER'S LECTURES ON THE PRINCIPLES AND

Practice of Surgery. In I vol. 8 vo. cloth, $750 \mathrm{p}$. \$2. GEKY. Eighth edition, improved and altered. With thirty-four plates. In two haudsome octavo volnmes, about 1000 pp., leather, raised bands. $\$ 600$.

\section{ILLER (JAMES),}

PRINCIPLES OF SURGERY. Fourth American, from the third and revised Edinburgh edition. In one large and very beautiful volume of 700 pages, with two hundred and forty illustrations on wood, extra cloth. \$375.

$B^{Y}$ THE SAME AUTHOR.

THE PRACTICE OF SURGERY. Fourth American, from the last Edinburgh edition. Revised by the American editor. Illustrated by three hundred and gixty-four engravings on wood. In one large octavo volume of nearly 700 pages, extra cloth. $\$ 375$.

$S^{A R G E N T}(F . W),. M . D$.

ON BANDAGING AND OTHER OPERATIONS OF MINOR SURGERY. New edition, witb an additional chapter on Military Surgery. One handsomeroyal 12mo. volume, of nearly 400 pages, with 184 wood-outs. Fxtra cloth, $\$ 175$. 
SHHURST (JOHN, Jr:), M.D.,

Surgenn to the Episcopal Hsopital, Philadelphia.

THE PRINCIPLES AND PRAC'TICE OF SURGERY. In one

very large and bandsome octaro volume of about 1000 pages, with nearly 550 illustrations, extra cloth, \$6 50; leather, raised bands, \$750. (Just Issued.)

The object of the author has been to present, within as condensed a compass as possible, a complete treatise on Surgery in all its branches, suitable both as a text-book for the student and a-work of reference for the practitioner. So wuch has of late years been done for the advance. ment of Surgical Art and Science, that there seemed to be a want of a work which should present the latest aspects of every subject, and which, by its A merican charneter, should render accessible to the profession at large the experience of the praotitioners of both hemispheres. This has been the aim of the author, and it is hoped that the volune will be found to fulfil its purpose satisfactorily. The plan and general outline of the work will be seen by the annexed

\section{CONDENSED SUMMARY OF CONTENTS.}

Chapter I. Inflammation. II. Treatment of Inflammation. III. Operations in general: Anæsthetics. IV. Minor Surgery. V. Auputations. VI. Special Amputations. VII. Effects of Injuries in General: Wounds. VIII. Gunshot Wounds. IX. Injuries of Bloodvessels. X. Injuries of Nerves, Muscles and Tendons, Lymphatics, Bursa, Bones, aud Joints. XI. Fractures. XII. Special Fractures. XIII. Dislocations. XIV. Effects of Heat and Cold. XV. Injuries of the Head. XVI. Injuries of the Back. XVII. Injuries of the Face and Neck. XVIII. Injuries of the Chest. XIX. Injuries of the Abdomen ind Pelvis. XX. Disesses resulting from Inflammation. XXI. Erysipelas. XXII. Pyamia XXIII. Diathetic Diseases: Struma (including Tubercle and Scrofulil); Rickets. XXIV. Venereal Diseases; Gonorrhœa and Chancroitl. XXV. Venereal Diseases continued: Syphilis. XXVI. Tumors. XXVII. Surgical Diseases of Skin, Areolar Tissue, Lymphatics, Muscles, Tendons, and Bursæ. XXVIII. Surgical Disease of Nerrous System (inolnding Tetanus). XXIX. Surgical Diseases of Vascular System (including Aneurism). XXX. Diseases of Bone. XXXI. Diseases of Joints. XXXII. Excisions. XXXIII. Ot thopædic Surgery. XXXIV. Diseases of Head and Spine. XXXV. Diseases of the Eye. XXXVI. Diseases of the Ear. XXXVII. Disenses ot the Face and Neck. XXXVIII. Diseases of the Mouth, Jaws, and Throat. XXXIX. Diseases of the Breast. XL. Hernia. XLI. Special Merniæ. XLII. Diseases of Intestinal Canal. XLIII. Diseases of Abdominal Organs, and various operations on the Abdomen. XLIV. Urinary Calculus. LXV. Diseases of Bladder and Prostate. XLVI. Diseases of Urethra. XLVII. Diseases of Generatire Organs. INdex.

Its auther has evldently tested the writing* and Indeed, the work as a whole must be regarded is expertences of the past and prosent ln the crucible an excellent and concise exponent of modern surof a careful, analytic, and honorable mind, and fajth- gery, and as such it wili be found a valuable textfully endeavored to bring bis work up to the level of book for the student, and a useful book of reforeace the highest standard of practical snrgery He is for the general practitioner.-N. Y. Mfed. Juurnat, frank and definite, and gives us opinjons, and gene- Feb. 1572.

raily sennd ones, instead of a mere résumé of the It gives us great pleasuretocall the attention of the opinions of otbers. He is conservative, but not hide- profession to thisexcellent work. Our knowledge of bound by authority. His style is clear, elegs nt, and its tulented and accomplished author led us to expect scholarly. The wirk is an admirable text book, and from him a very valuable treatise upon subjects to H nseful book of reference It is a credit to American which he bas repeatedly giveu evidence of having proprofessional literature, and one of the first ripe fruits fitably devoted in ucit timeand labor, and rearein no \begin{tabular}{l|l} 
of the sofl fertilized by the blood of our late unhappy & wdy disappointed.-Phila. Hed. Times, Feb. 1, IS72. \\
war. $-\mathrm{N}$. Y. Med. Record, Eeb. 1, 1872.
\end{tabular}

PIRRIE ( WILLIAM), F. R. S. E., Professor of Surgery in the University of Aberdeen.

THE PRINCIPLES AND PRAC'TICE OF SURGERY. Edited by Jorn Neill, M. D., Professor of Surgery in the Penna. Medical College, Surgeon to the Pennsylvania Hospital, \&o. In one very handsome octavo volume of 780 pages, with 31.6 illustrations, extra cloth. $\$ 375$.

$H^{A M I L T O N}(F R A N K \cdot H),. M . D$., Professor of Fractures and Dislocations, \&c., in Bellevue Hosp. Med. College, Neto York.

A PRACTICAL TREATISE ON FRAC'TURES AND DISLOCATIONS. Fourth edition, thoroughly rerised. In one large and handsome octavo volume of nearly 800 pages, with several hundred ill ustrations. Extra cloth, $\$ 575$; leather, $\$ 675$. (Just 1ssued.)

It is not, ef course, onr intention to review in extenso, Hamblton un "Fractures and Dislucations." Eieven years ago such revtew might not have been ont of place; to-day the werk is a an anorlty, go well, so generally, and so farorably known, that it only remalus for the reviewer to say that a new editlon is just out, and it is botter than elther of it 8 predecessors.-Cincinnati Ctinio, Oet. 14, 1571.

Undenbtedly the best work on Fractures and Dislocations in the Engltsh langnage.-Cincinnati Mfed. Repertory, Oct. 1871 .

We have once more before us Dr. Hamilton's admi- $\begin{aligned} & \text { est authority } 16,1871 . \\ & \text { Oot }\end{aligned}$ most complete and rellable work on the subject. As a whole, the work ts without an equal in the literutnre of the profession. - Boston Med, and Surg. Journ., Oct. 12, 1571 .

It is nunecessary at this time tocommeud the book, except to such as are begiuners in the stady of this particular branch of surgery. Every pructical sur. geon tn thle country and abroad knows of lt as a most trustworthy guide, and ene which tbey, in common whih us, would unquallifiedy rocoumead as the blgh. est authority ln any language. $-N$. Y. Med. Reeord, 
FRICHSEN (JOHN E.),

Professor of Surgery in University College, London, etc.

THE SCIENCE AND ART OF SURGERY; being a Treatise on Surgical Injuries, Diseases, and Operations. Revised by the author from the Sixth and enlarged English Edition. Illustrated by over seven hundred engravings on wood. In two large and beautiful octavo volumes of over 1700 pages, extra cloth, $\$ 900$; leatber, $\$ 1100$. (Just Ready.)

\section{Author's Preface to the New American Edition.}

"The farorable reception with which the 'Science and Art of Surgery' has been bonored by the Surgical Profession in the United States of America has been not only a source of deep gratification and of just pride to me, but has laid the foundation of many professional friendzhips that are amongst the agreenble and valued recollections of my life.

"I have endeavored to make the present edition of this work more deserving than its predecessors of the favor that has been accorded to them. In consequence of delays that have unavoidably occurred in the publication of the Sixth British Edition, time has been afforded to me to add to this one several paragraphs which I trust will be found to increase the practical value of the work."'

Los Dos, Oct. 1572 .

On no formeredition of this work has the author bestowed more pains to render it a complete and satisfactory exposition of British Surgery in its modern aspects. Every portion has been sedu. lously revised, and a large number of new illustrations have been introduced. In addition to the material thus added to the English edition, the author has furnished for the American edition such material as has accumulated since the passage of the sheets through the press in London, so that the work as now presented to the American profession, contains his latest views and experience.

The increase in the size of the work has seemed to render necessary its division into two volumes. Great care has been exercised in its typographical execution, and it is confidently presented as in every respect worthy to mantain the high reputation which has rendered it a stand. ard authority on this department of medical science.

These are only a few of the points in which the states in hls preface, they are not confined toany one present edition of Mr. Erichsen's work surpasses its portion, but are distributed geverally through the predecessors. Throughout there is evidence of a subjects of which the work treats. Certainly one of laborious care and solicitude in seizing the passing the most valuable sections of the book seems to no to knowledge of the day, which reflects the greatest be that which treats of the diseases of the arteries credit on the author, and much enhances the valne and the operative proceedings which they necessitate. of his work. Wecan ouly admire the industry which In few text-books is so much carefully arranged inhas enabled Mr. Erichsen thus to succeed, a mid the formation collected,-London Med. Times and Gaz., distractions of active practice, in producing emplatic- Oct. $26,1872$.

ally nus book of reference and study for British pric titioners of surgery.-London Lancet, Oct. 26,1872 .

Considerable changes have been made in this edition, and nearly a hundred new illustrations have been added. It is difficult in a sinall compass to point
out the alterations and additions; for, as the a uthor

The entire work, complete, as the great English treatise on Surgery of our own tlme, ik, we can assn re our readers, equally well adapted for the most jnuior student, and, as a book of refereuce, for the advanced. practitioner-Dublin Quarterly Journal.

\section{$B^{Y}$ THE SAME AUTHOR. (JUSt Issued.)}

\section{ON RAILWAY, AND OTHER INJURIES OF THE NERVOUS} SYSTEM. In a small octavo volume. Extra cloth, $\$ 100$.

DUITT (ROBERT), M.R.C.S., \&c.

THE PRINCIPLES AND PRACTICE OF MODERN SURGERY. A new and revised American, from the eighth enlarged and improved London edition Illus. trated with four hundred and thirty-two wood engravings. In one very handsome octavo volume, of nearly 700 large and closely printed pages. Extra cloth, $\$ 400$; leather, \$5 00.

Ail that the surgical student or practitioner conld perspicnonsly, as to eincidate every important topic. desire-Drublin Quarlerly Journal.

It is a most admirable book. We do not know when we have examined one with more pleasare. Boston Med. and Surg. Journal.

In Mr. Drultt's book, though contalning only some seven hundred pages, both the principles and the practice of surgery are treated, and so clearly and

itself show it to possess marked superiority. We have examined the book most thoroughly, and can say that thls sncces la weil merited. His book, moreover, possesses the inestlinable adrantages of having the subjects perfectly well arranged and classified, and of being written in a style at once clear znd succinct.-Am. Journal of Med. Sciences.

A SHTON (T. J.).

ON THE DISEASES, INJURIES, AND MALFORMATIONS OF THE RECTUM AND ANUS; with remarks on Habitual Constipation. Second American, from the fourth and enlarged London edition. With handsome illustrations. In one very beautifully printed octaro volume of abont 300 pages. $\$ 325$.

BIGELOW (HENRY J.), M. D.

Professor of Surgery in the Mfassachusetts Mfd. College.

ON TIIE MECHANISM OF DISLOCATION AND FRACTURE OF THE HIP. With the Reduction of ibe Dislocation by the Flexion Method. With numerous original illustrations. In one rery handsome octavo volume. Cloth. \$2 50 . (Lately Issued.) 
BRYANT (THOMAS), F.R.C.S., Surgeon to Guy's Iospital.

THE PRACTICE OF SURGERY. With over Five IIundred Engrarings on Wood. In one large and very handsome octavo volume of nearly 1000 pages, extra cloth, \$6 25 ; leather, raised bands, \$725. (Just Rezdy.)

The distinguished reputation of the author and the extended experience which he has enjoyed as surgeon to one of the largest of the London hospitals, are an earnest of the value of lis labors. Though entilled a "Praetiee of Surgery," it will bo seen by the subjoincd summary of tho contents that it is by no means eonfined to operative surgery, but that it presents also a view of the principles which should guide the surgeon in his duily practice. Nearly all of the very full series of illustrations have been prepared expressly for the work.

\section{SUMMARY OF CONTENTS.}

Intronuction.-1. On Repair and Inflaumation. Il. On Traumatic Fever, Septicania, and Pymmia. I11. On Trismus and Tetanus. Iv. Lelirium Tremens. v. Contusions: Wounds of the Senlp, Blood Tumors, Osteitis. vi. Injuries of the Cranium. vil. Conenssion of the Braio. vill. Injuries of the Brain and its Membranes, eomplienting Fracture. 1x. Compression of the Brain. x. Results of Injurics to the Head. XI. On Fructures of the Skull. xil. The Operation of Trephining. xill. Diseases of the Scalp and Cranium. xiv. Spina Bifida. xv. Injuries of the Spine. xvi. Intra-Spinal Inflammation, Spinal Paralysis, Railwny Concussion. xvil. Fractures, Disloeations, and Wounds of the Spine. xvill. Curvature of the Spine. xix. Injuries and Diseases of the Nerves. xx. Surgieal Affections of the Nose. xxI. Surgical Affections of Larynx and Trachea. Xxir. Surgery of the Chest. Xxil. Wounds of the IIeart. Xxiv. Diseases of the Arteries. XXv. Aneurism. xxvr. Ligature of Arteries. Xxvir. Injuries and Diseases of the Veins. xxvill. Affections of the Lips,'etc. xxix. Disenses of the Juws, ete. xxx. Affeetions of the Pharynx. xxxy. Injuries of the Abdomen. xxxil. Hernia. xxxin. Varieties of Hernia. xxxiv. Trusses. xxxv. Surgery of the Anus. xxxvi. Diseases of the Integuments: Wounds. xxxvil. Poisoned Wounde. xxxvir. Isurns. xxxıx. Skin Grafting. xL. Boils, etc. xlı. Gangrene, ete. Xlıl. Ulcers. XuII. Mortification. XLIV. Erysipelas. xLV. Diseases of the Lymphatics. xLVI. Diseases of the Kidney. xlvir. Diseases of the Bladder. xuvin. Diseases of the Prostate. x xix. Urinary Deposits. L. Stone in the Bladder. Li. Lithotrity. LII. Lithotomy. Lin. Stone in the Female Bladder. Lir. Stricture of the Urethra. LV. Retention of Urine. Lvi. Affections of the Penis. Lvir. Hæmatocele, etc. Lvil. Diseases of the Testicle. Lix. Sterility. Lx. Affections of the Female Genitals. Lxi. Ovariotomy. LxII. Venereal Disease. Ixur. Syphilis. Lxiv. Tumors. Lxv. Anatomy of Tumors. Lxvi. Tumors of the Breast. Lxvir. Diseases of the Thyroid Gland. Lxvin. Wounds of the Joints. Lxix. Dislocations. Lxx. Dislocations of the Upper Extremity. Lxxi. Disloca. tions of the Lower Extremity. Lxxir. Pathology of Joint Diseases. Lxxir. Diseases of Special Joints. LxxIv. Treatinent of Joint Disease. IXXv. Excision and Amputation in Joint Disease. IXXVI. Osteo-arthritis. LXXYil. Diseases of the Bones. LXxviI. Tumors of Bone. LXXIX. Fraetures. Lxxx. Fractures of the Upper Extremity. Lxxxi. Fraetures of the Lower Extremity. I.XXXı. Complieated Fractures. Lxxxin. Gunshot Injuries. Lxxxıv. Feigned and IIysterical Dis. ease. Lxxxv. Affections of the Museles and Tendons. Lxxxvi. Ganglions. Ixxxvir. Orthopredic Surgery. Lxxxvil. Anæsthetics. Lxxxix. Shock. xc. Amputation. xcr. Special Amputations. xcr. Elephantiasis. Xcill. Affections of the External Ear. Xciv. Parasites.

\section{WELLS (J. SOELBERG),} Professor of Ophthalmology in King's College Hospital, \&c.

\section{A TREATISE ON DISEASES OF THE EYE. First American} Edition, with additions; illustrated with 216 engravings on wood, and six colored plates. Together with selections from the Test-types of Jaeger and Snellen. In one large and very handsome octavo volume of about 750 pages: extra cloth, $\$ 500$; leather, $\$ 600$. (Lately Jssued.)

In this respect the work before $\mathrm{ns}$ is of mnch more (mend it to all who desire to consult a reatly good service to the general practitioner than those heavy compllattons which, in giving every person's views, too often neglect to specify those which are most in accordance with the author's opintons, or in geveral acceptunce. We have no hesitation tn recommending this treatise, r6, on the whole, of all Euglinh works on the subject, the one best adapted to the wants of the genersl practitioner.-Edinburgh Med. Journal, March, 1870 .

A trentise of rare merit. It is practical, compreheusive, and yet concise. Upon those subjects usually fonnd difficult to the student, he has dwelt at lengih and entered into full explanation. After a careful perusal of its contents, weican unhesitutingly com-

mend it to all who desire to consult a reatiy good
work on ophthalmic science. The American edition of Mr. Wells' treatise was superintended in t ts passage through the press by Dr. I. Minis Hays, who has added some notes of his own where it seemed desirable. He has also introduced more than one hundred new addltional wood-cuts, and added selections from the test-types of Jaeger and of Snellen. - Leavenzoorth Med. Heruld, Jan. 1870.

Withont donbt, one of the best works apon the sab. ject whlch has ever been published; it is complete on the subject of which it treats, and is a necessary work for every physician who atlempty to treat dikeases of the eye-Dominion Med. Journal, Sept. IS69.

\section{LAWSON (GEORGE), F. R. C. S., Engl.,}

Assistant Surgeon to the Royal London Ophthalmic Hospital; Moorfields, de.

INJURIES OF THE EYE, ORBIT, AND EYELIDS: their Immediate and Remote Effects. With about one hundred illustrations. In one very hand. some octavo volume, extra oloth, $\$ 350$.

It is an admirable practical book in the highest and best sense of the phra6e.-London Medictl Times anit Gasette, Ming 18, 1867. 
CAUR FNCE (JOHN Z.), F.R.C.S., Editor of the Ophthalmic Review, \&c.

A HANDY-BOOK OF OPHTHALMIC SURGERY, for the use of Practitioners. Second Edition, revised and enlarged. With numerons illustrations. In one very handsome octavo volume, extra cloth, $\$ 300$. (Lately Issued.)

For those, however, who must assume the care of tion of the optical defects of the eye, the publisher dior much pressed for time to study the classic works on psges of Suellen's test-types, so generally used to test the acuteness of vision, and which are difficnlt to uh. the subject, or those recently published by Stellwag, tain in this country. The volnme has been conside. Wells, Buder, and others, Mr. Lanrence will prove a tain in this country. The volnme has been conside. safe and trustworthy guide. dence of the profession since the appearance of his edition.-Am. Journ. Med. Sciences, Jan. 1870.

last. To the portion of the book devoted to a descrip-

\section{WALES (PHILIP S.), M.D., Surgeon O.S.N.}

MECHANICAL THERAPEUTICS: a Practical Treatise on Surgical Apparatus, Appliances, and Elementary Operations: embracing Minor Surgery, Band. aging, Orthopraxy, and the Treatment of Fractures and Dislocations. With six hundred and forty-two illustrations on wood. In one large and handsome octavo volume of-about 700 pages: extra cloth, $\$ 575$; leather, $\$ 675$.

A Naval Medical Board directed to examine and report upon the merits of this volume, officially states that "it should in our opinion become a standard work in the hands of every naval surgeon;" and its adoption for use in both the Army and Navy of the United States is sufficient guarantee of its adaptation to the needs of every-day practice.

\section{THOMPSON (SIR HENRY),}

Surgeon and Professor of Clinical Surgery to Dniversity College Hospital.

LECTURES ON DISEASES OF THE URINARY ORGANS. With

illustrations on wood. In one neat octavo volume, extra cloth. $\$ 225$.

These lectures stand the severe test. They are in. on which Sir Henry Thompson speaks with more an. structive without being tedious, and simple without thority than that in which he has specially gathered being diffuse; and they include many of those prac- his laurels; in addition to this, the conversational valuable to the yonng practitioner.-Elinburgh Med. Journal, April, 1869.

style of instruction, which is retained in these printed
lectures, gives them an attractiveness which a syslectures, gives them an attractiveness which a sys.
tematic treatise can never possess.-London Medical

Very few words of ours are necessary to recommend Times and Garette, April 24, 1869. these lectures to the profession. There is no subject

$B^{Y}$ THE SAME AUTHOR.

ON THE PATHOLOGY AND TREATMENT OF STRICTURE OF THE URETHHA AND URINARY FISTULA. With plates and wood-cuts. From the third and revised English edition. In one very handsome octavo volume, extra cloth, $\$ 350$. (Lately Published.)

This classical work has so long been recognized as a standard authority on its perplexing snbjects that it should be rendered accessible to tine American profession. Having enjoyed the advantage of a revision at the hands of the author within a few months, it will be found to present his latest views and to be on a level with the most recent advances of surgical science.

With a work accepted as the authority upon the ably known by the profession as this before ns, mnet rubjects of which it treats, an extended notice wonld create a demand for it from those whe wonld keep be a work of supererogation. The simple announce- themselves well np in this department of surgery.ment of another edition of a work 80 well and favor- $\mid$ St. Louis Med. Archives, Feb. 1870.

TAYLOR (ALFRED S.), M.D., Lecturer on Med. Jurisp. and Chemistry in Guy's Hospital.

MEDICAL JURISPRUDENCE. Seventh American Edition. Edited by John J. Reese, M.D., Prcf. of Med. Jurisp. in the Univ. of Penn. In one large octavo volume. (Preparing.)

The present edition of this valuable manual lo a partment of medicine for stndents and the general great improvement on those which have preceded it. practitlonerin ourlanguage._Boston Sred. and Surg. It makee thas by far the best guido-book in this de- Journal, Dec. 27, 1866.

$B^{Y}$ THE SAME AUTHOR. (Nearly Ready.)

THE PRINCIPLES AND PRACTICE OF MEDICAL JURISPRUDENCE. Second Edition, Revised, with numerous Illustrations. In two very large octavo volnmes.

This great work is now recognized in England as the fullest and most.anthoritative treatise on every department of its important sabject. In laying it, in its improved form, before the American profession, the publisher trusts that it will assume the same position in this country. 
TUKE (DANIEL HACK), M.D.

Joint author of " The Manual of Psycholngical Medicine," \&c.

IILUSTRATIONS OF THE INFLUENCE OF THE MIND UPON THE BODY IN HEALTH AND DISEASE. Designed to illustrate the Action of the Imagination. In one handsome octavo volume of 416 pages, extra c'oth, $\$ 325$. (Now Ready.)

The object of the author in this work has been to show not only the effect of the mind in causing and intensifying disease, but also its curative influence, and the use which may be made of the imagination and the emotions as therapeutic agents. Scattered facts bearing upon this subject have loug been familiar to the profession, but no altempt bas hitherto been made to collect and systematize them so as to render thein available to the practitioner, by establishing the seve. ral phenomena upon a scientific basis. In the endenvor thus to convert to the use of legitinate medicine the means which huve been employed so successfully in many systems of quackery, the author has produced a work of the highest freghness and interest as well as of permanent value.

BLANDFORD (G. FIELDING), M. D., F. R.C P., Lecturer on Psychological Medicine at the Sehool of St. George's Hospital, \&c.

INSANITY AND ITS TREATMENT: Lectures on the Treatment, Medical and Legal, of Insane Patients. With a Summary of the Laws in force in the United States on the Confinement of the Insane. By Isalc RAY, M. D. In one very handsome octavo volume of 471 pages: extra cloth, \$3 25. (Just Issued.)

This volume is presented to meet the want, so frequently expressed, of a coinprehensire trea. tise, in moderate compass, on the pathology, diagnosis, and treatment of insanity. To render it of more value to the prictitioner in this country, Dr. Kay has added an appendix which aftords information, not elsewhere to be found in so accessible a form, to physicians who may at any moment be called upon to take action in relation to patients.

It ealisfies a want which must have been sorely actually seen in practice and the appropriate treat. felt by the busy general practitioners of this country. ment for them, we find in Dr. Blaudford's work a It takes the form of a manal of clinical description considerabje advance over previous writings on the of the various forms of insanity, with a description subject. His pictures of tbe varions forms of mental of the mode of examining pemsons suspected of in- disease are so clear and good that no reader can fail 8anity. We call particular attention to this feature to be struck with their superiority to those given in of the book, as giving it a unique value to the gene. irdinary manuals iu tbe Euglish fanguage or (so far ral practitioner. If we pass from theoretical conside- as our own readiugexiends) in any other.-Londun raiions to descriptlons of the varieties of insanity as Practitioner, feb. 1871.

\section{WINSLOW (FORBES), M.D., D.C.L., \&c.}

ON OBSCURE DISEASES OF THE BRAIN AND DISORDERS OF THE MIND; their incipient Symptoms, Pathology, Diagnosis, Treatment, and Pro. phylaxis. Second American, from the third and revised English edition. In one handsome octavo volume of nearly 600 pages, extra cloth. $\$ 425$.

CEA (HENR $Y$ C.).

SUPERSTITION AND FORCE: ESSAYS ON THE WAGER OF LAW, THE WAGER OF BATTLE, THE ORDEAL, AND TORTURE. Second Edition, Enlarged. In one handsome volume royal 12mo. of nearly 500 pages; extra cloth, $\$ 275$. (Lately Published.)

We know of no singie work which contains, in 80 interesting phases of hnman society and progress. . . sinull a compass, so much illustrative of thestraugest operations of the human mind. Foot-notes give ihe authority for each atatement, showing vast reseurch and wonderful indnstry. We advise our confrêres to read this book and ponderits teachings. - Chicago Med. Journat, Ang. 1870.

As a work of curions inquiry on certain ontlying points of obsolete law, "Superstition and Force" is oue of the most remarkable books we have met wlth. -Irondon Athenæum, Nov. 3, 1866.

He has thrown a great deal of light upon what must be regarded as oue of the most instructive as well as The fulness and bread th with which he bas carried out his comparative survey of this repulsive field of history ['Torture], are $8 \mathrm{nch}$ as to preciude our dotag justice to the work within our present limits. But here, as throughout the volume, there will be found a wealth of lliustration and a critical grasp of the philosophical import of facts which will render Mr. Lea's labors of sterling value to the historical atu. dent.-London Saturday Review, Oct. \$, 1870.

As a book of ready reference on the subject, it is of the highest value.-Westminster Revitw, Oct. 1867.

$B^{Y}$ THE SAME AUTHOR. (Late'y Published.)

STUDIES IN CHURCH HISTORY-THE RISE OF THE TEMPORAL POWER-BENEFIT OF CLERGY - EXCOMMUNICATION. In one large rogal 12 mo. volume of $516 \mathrm{pp}$. extra cloth. $\$ 275$.

The story was never told more calmly or with literary phenomenon that the head of one of the frst greuter tearuing or wiser thought. We doubt, indeed, if auy other study of this tield can be compared with thts tor clearness, accuracy, and power. - Chicago Examiner, Dec. 1870.

Mr. Leu's latest work, "Studies in Church Hlstory," fuity sustains the promise of the tirst. It deals with three subjects - the Temporal Power, Benefit of Clergy, and Excommunication, the record of which has a peculiar importance tur $t$ he Englisb student, and is a chapter on $\Delta$ ucied Law likely to be regarded as f nal. We can hardity pass from our mention of such works as thexe-wib which that on "Sacerdotal Colibacy" should ve included-withont noting the

Ilterary phenomenon that the head of one of the first
A merlcan honses is also the writer of some of its unist orlginal books. - London Athenceum, Jan. 7, 1871

Mr. Lea has done great honor to himself and thle conntry by the admirable works he has written on ecclesiological and coguate subjects. We hare atready bud occaslon to commend his "Superstition and Force" and his "Histury of Sacerdotal Cellbacy." The present volume is fully as adinirubie in its inethod of dealiug with topics aud in tha thoruughneso¿ quality so frequently iacking in American authorswith which they are investigated.-N. I. Journal of P'sychat Medicine, July, 1870. 
INDEX TO CATALOG OE.

American Jonrnal of the Medical Sciences Anerlcan Chemiat (The)

Abstract, Half-Yearly, of the Med Sciences Anatomical Atlas, by Smith and Horner

Anderson on Diseases of the Skin

Ashton on the Rectum and Anus.

Attfeld's Chemistry

Ashwell on Diseases of Females .

Ashburst's Surgery

Barnes on Diseases of Women

Bryant's Practical Surgery

Blandford on Insanity

Basham on Renal Disease

Brinton on the Stomach

Bigelow on the Hip

Barclay a Medical Diagnosis.

Barlow's Practice of Medicine

Bowman's (John E.) Prsctical Chemistry Bowman's (John E.) Medical Chemistry

Buckler on Bronchitis

liuinstead on Venereal

Bumstead and Cullerier's Atlas of Venereal Cerpenter's Human Physiology

Carpeuter's Comparative Physiology

Carpenter on the Use and Abuse of Alcohol Carson's Sy nopsis of Mfateria Medica.

Chambers on the Indigestions

Chambers's Restorative Medicine

Christison and Griffith's Dispensatory

Chnrchill's System of Midwifery .

Churchill on Puerperal Fever

Condie on Diseases of Children

Cooper's (B. B ) Lectures on Surgery

Cullerier's Atlas of Venereal Diseases

Cyclopedia of Practical Medicine .

Dalton's Human Physiology .

De Jongh on Cod-Liver Oil

Dewees's System of Midwifery

Dewees on Diseares of Femiles

Dewees on Diseases of Children.

Druitt's Modern Surgery

Dunglison's Medical Dictionary .

Dunglizon's Human Physiology

Dunglison on New Remedies

Bllis's Medical Formulary, by Smith

Erichsen's System of Surgery

Erichseu on Nervous Inj uries

Flint on Respiratory Organs.

Flint on tlie Heart

Flint's Practice of Medicine

Fownes's Elementary Chemistry

Fox on Diseases of the Stomach

Fulleron the Lungs, \&c.

Green's Patbology and Morbid Anatomy Gibson's Surgery

GInge's Pathological Histology, by Leidy “ ”

Galloway's Qualitative Analy sis .

Gray's Anatomy

Griffth's (R. E.) Universal Formulary

Gross on Foreign Bodies in Air-Passages

Gross's Principles and Practice of Surgery.

Grose's Pathological A uatomy

Guersant on Surgical Diseases of Chlldren

Hartshorne's Essentials of Medicine

Hartshorne's Conspectus of the Atedical Sciences

Hamilton on Dislocations and Fractures

Heath's Practical Anacomy.

Hoblyn'B Medical Dictionary

Bodge on Women

Hodge's Obstetrics

Hodges' Practical Diseection

Holland's Medical Notes and Refections

Horner's Anatomy and Histology

Hudson on Fevers

Hill on Venereal Diserses

Hilller's Handbook of Skin Diseases

Jones and Sieveking's Pathological Anatomy Joneo (C. Handfeld) on Nervone Disorders Rirkes Physlology

Knayp's Chemical Technology

Lea's Superstition and Force
PAGB

1 Lea"s Studies in Charch Hlstory.

11 La Roche ou Yellow Fever.

Laurence and Moon's Ophthalmic Surgery

Lawson on the Eye aycock on Medical Observation Lebmann's Physiological Chemistry, 2 vole. uebmann's Chemical Physiology.

uld low's Manual of Examinations yons on Fever . . . . . . . 18

Maclise's Surgical Anatomy . . . . 7 Marsball's Physiology - • • • •

Meigs's Obstetrics, the Science and the Art . : 25

Meigs's Lectures on Diseases of Women . . 23

Moigs on Puerperal Fever - - • $\quad$ - 23

Miller's Principles of Surgery : $\quad \cdot \quad 26$

Montgomery on Pregnancy . . . . . 25

Molland on Urinary Urgans . . . . : 27

Morland on Urmis - 18

Noill and Smith's Compendlam of

Neligan's Atlas of Di seases of the Skin $: \quad 2^{20}$
Neligun on Disesses of the Skin $:-20$

Ubstetrical Journal . • • • • : 22

Odling's Practical Chemistry . . . . 10

Prize Essays on Consumption - • • $\quad$ - 17

Pirrie's Syatem of Surgery $:{ }^{-}:{ }^{27}$

Pereira's Mat. Medica and Therapentics, abridged 13

Quain and Sharpey' Anatomy, by Leidy . . 6

Ranking's Abstract

Radcliff and others on the Nerves, \&c. $\quad \cdot 18$

Roberts on Urinary Disesses.

Rigby's Midwifery.

Rokitansky's Pathological Anatomy : $: \quad$ : 14

Royle's Materia Medica and Therapeutics . . 13

Salter on Asthma.

wayue's Obstetric Aphorlsms : . 24

Sargent'B Minor Surgery . • • • 28

Sharpey and Quain's Anstomy, by Leidy - - 6

imou's General Pathology .

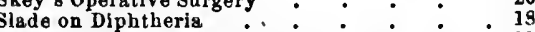

Smith (J. L.) on Children * . * 21

inith (H. H.) and Horver's Anatomical Atlas

(Edward) on Consumption .

olly on Anatomy and Diseases of the Brain :

Stille's Therapeutics.

unuer's Manual of Clinical Medicine.

Taylor's Medical Jurisprudence : $\quad \cdot \quad \cdot \quad \cdot 30$

Trylor's Principles and Practice of Med Jurisp. 30

Tuke on the Influevce of the Mind . . . 31.

Thomas on Diseases of Females . • • .2

Thompson on Urinary Organs - : : $\quad$ : 30

Todd on Acute Diseases : $\quad: \quad \cdot \quad \cdot \quad \cdot \quad: 15$

Wales on Surgical Operations .

Walsbe on the Heart . . . . . . 17

Watson's Practice of Physic. . . . . 16

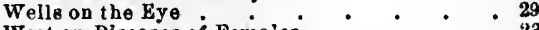

West on Diseases of Femaies - • - • 23

West on Diseases of Children

West on Nervons Disorders of Children $\quad: \quad: 21$

What to Observe in Medical Cares : $: \quad$ : 14

Williams's Principles of Medicine • . $\quad 14$

Williams on Consumption $\cdot \bullet^{-} \cdot \bullet^{-} 17$

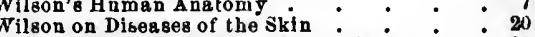

Wilson's Plates on Diserses of the skin : : 20

Wilson'e Handbook of Cutaneons Medicine : 20

Wilson on Spermatorrhas . . . . . 19

Winglow on Brain and Mind : : : : 81

Wïhler's Organic Chemistry . . . . 10

Zelssi on Venereul . $\quad \cdot \quad \cdot \quad \cdot \quad \cdot \quad \cdot \quad \cdot 19$

$\cdot \quad \cdot \quad 15$ 



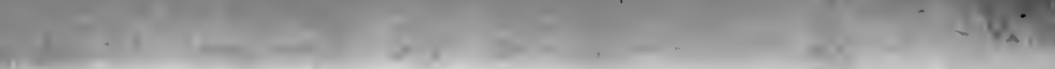

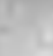

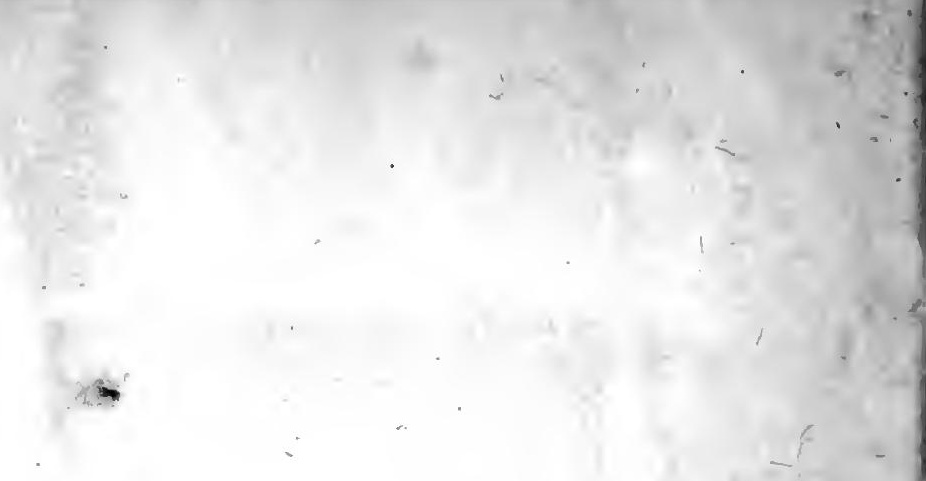

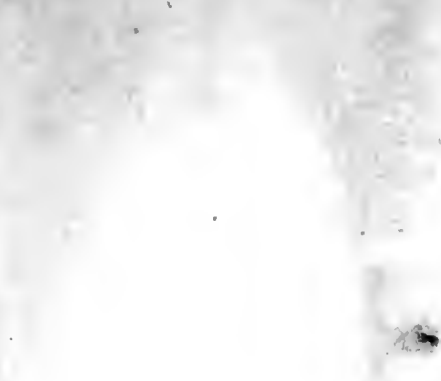

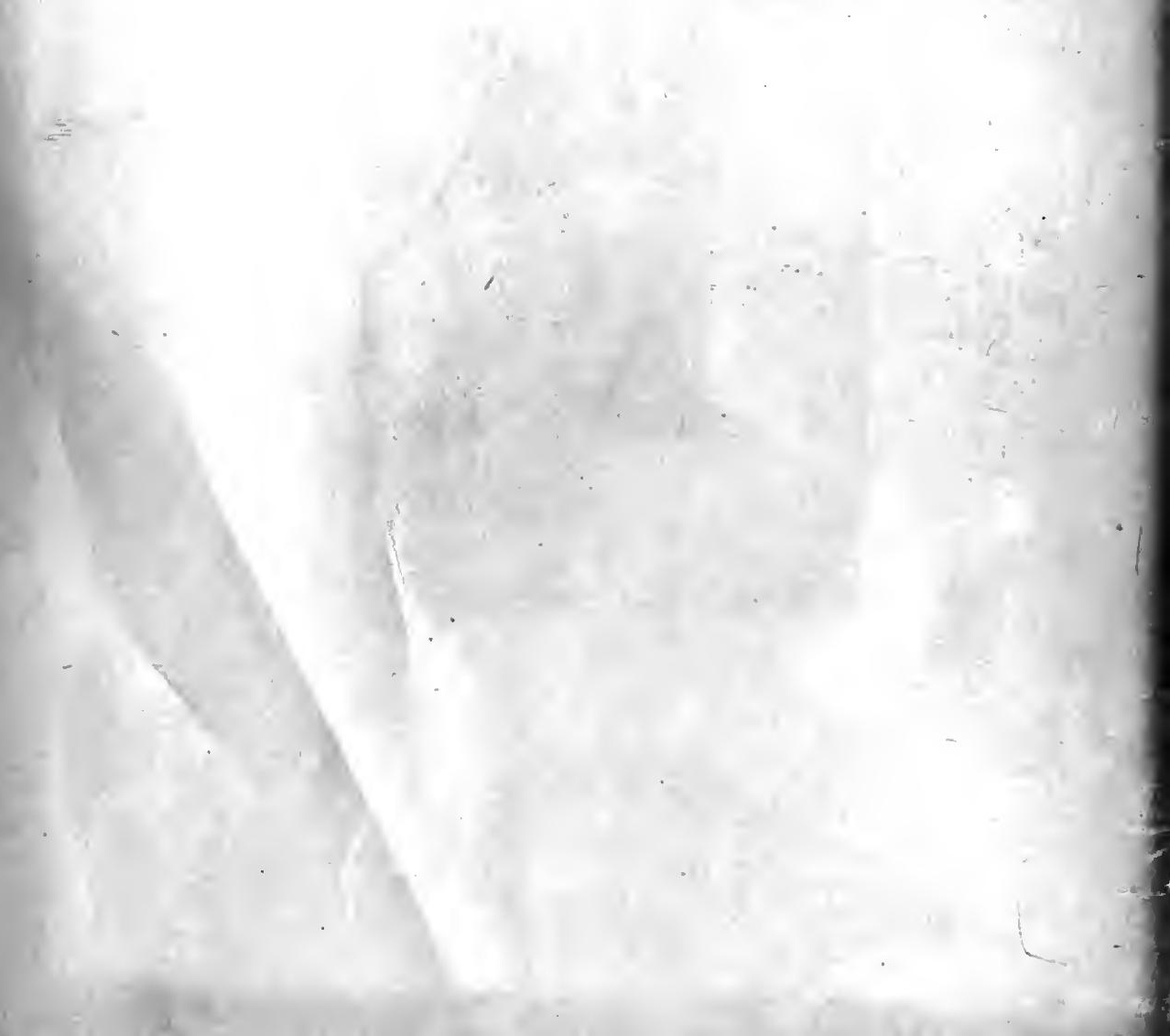




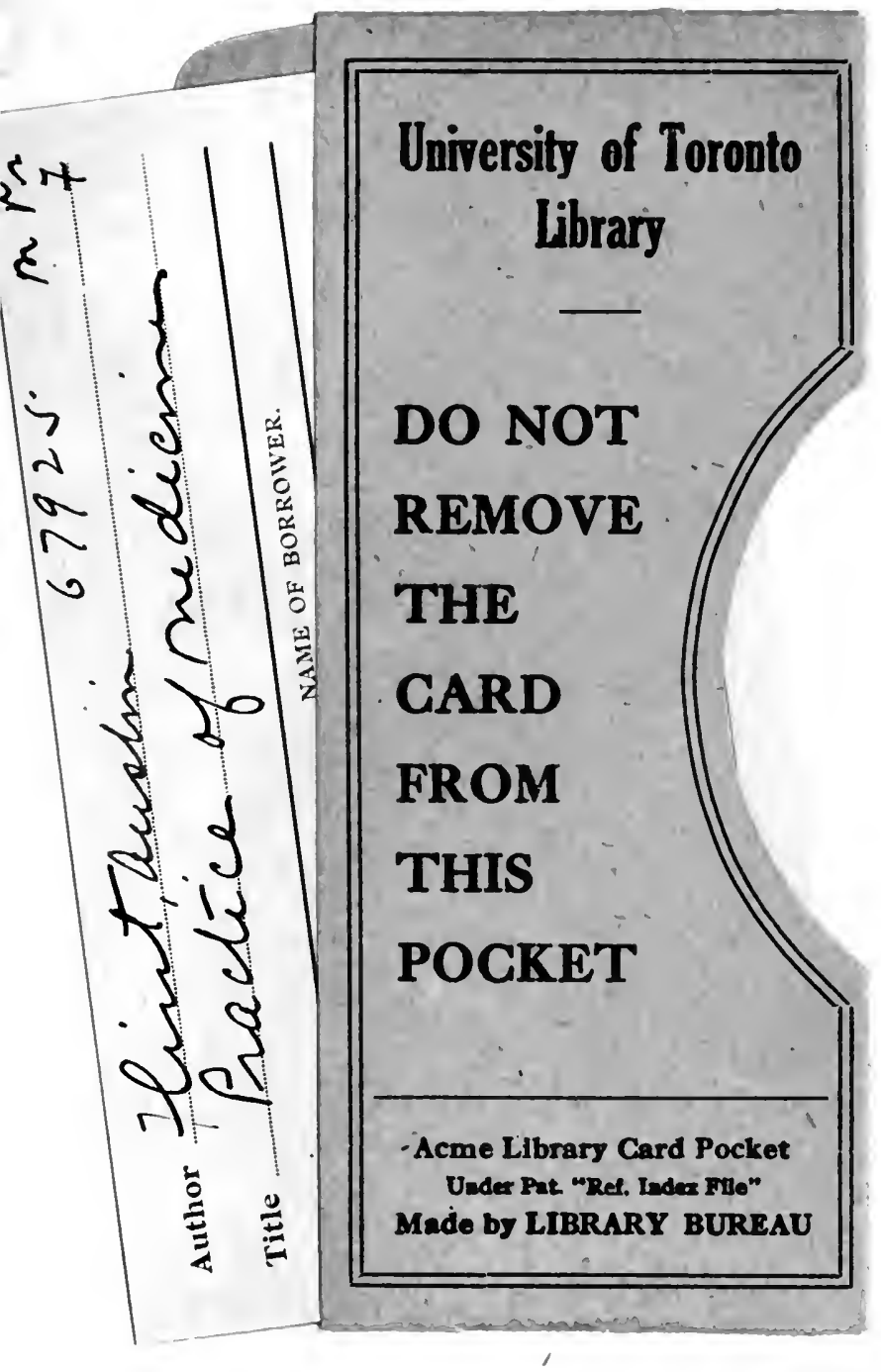




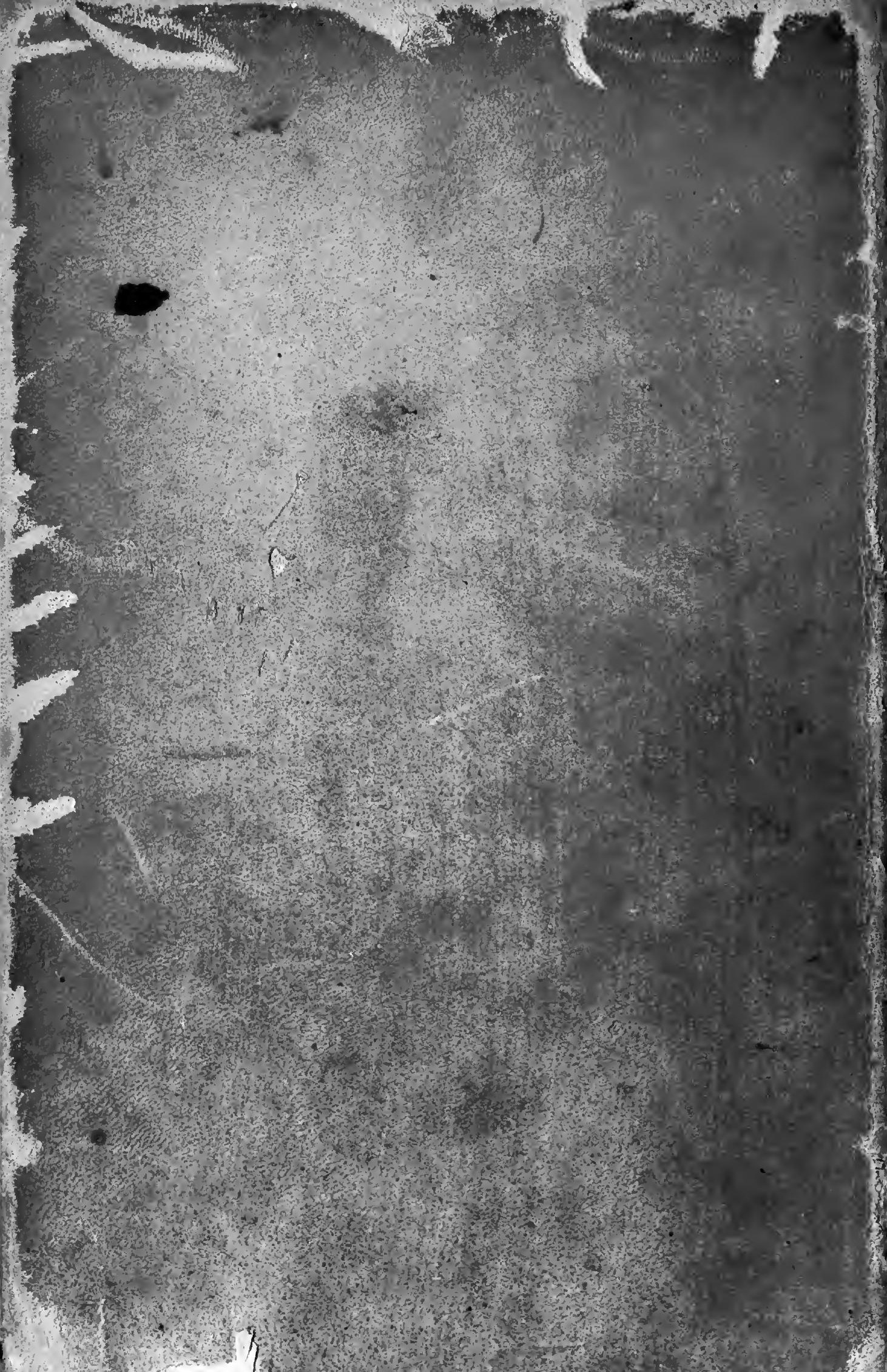

